
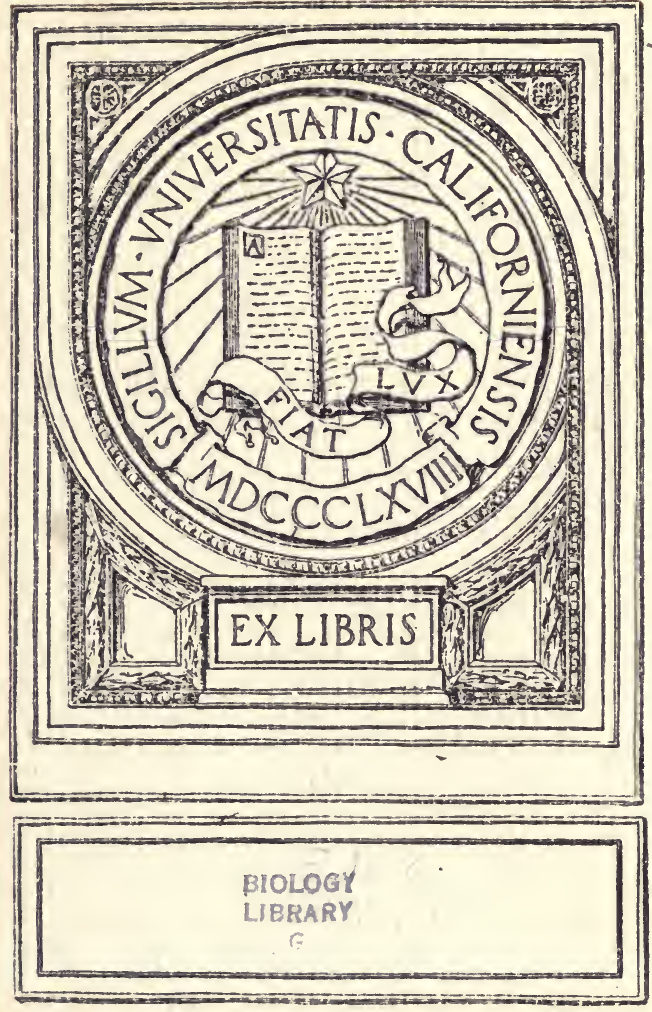

BIOLOGY

LIBRARY 

Digitized by the Internet Archive in 2007 with funding from Microsoft Corporation 


\title{
VETERINARY MEDICINES
}

\section{THEIR ACTIONS AND USES}

\author{
BY \\ FINLAY DUN
}

FORMERLY LECTURER ON MATERIA MEDICA AND DIETETICS AT THE FDINBURGH VETERINARY COLLEGE

EXAMINER IN CHEMISTRY TO THE ROYAL COLLEGE OF VETERINARY SURGEONS

REVISED AND EDITED BY

JAMES MACQUEEN, F.R.C.V.S.

AND

HAROLD A. WOODRUFF, M.R.C.V.S.

ROYAL VETERINARY CULLEGE, LONDON

Twelfth Édition

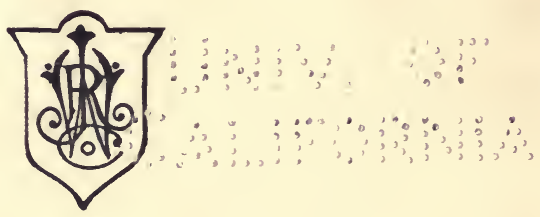

New York

WILLIAM R. JENKINS CO.

PUBLISHERS

851-853 Sixth Avenue

1911

[All Rights Reserved] 
SFq1

$D^{8}-$

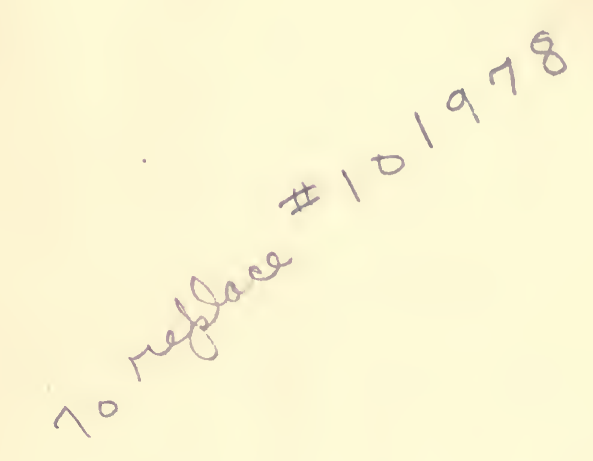




\section{PREFACE TO THE TWELFTH EDITION}

THIs Edition has been thoroughly revised and brought up to date. The pharmacology has been largely rewritten by my colleague and joint editor, Professor Woodruff ; many additions and alterations have been made in other portions of the text ; and a few of the older and least useful drugs have been deleted.

To Professor Lander, D.Sc., for revising the chemical formulæ, and to Professor German Sims Woodhead, for again undertaking the revision of the pages on bacteriology, I desire to tender my grateful thanks. Of the many valuable works consulted in the preparation of this edition, mention must be made of Cushny's Pharmacology, Dixon's Manual of Pharmacology, Kaufmann's Traité de Thérapeutique et de Matière Médicale Vétérinaires, Gobert's ThérapeutiqueVétérinaire Appliquée; Mollereau and Porcher's Vade-mecum du Vétérinaire; Winslow's Veterinary Materia Medica and Therapeutics, Bocquillon-Limousin's Formulaire des Médicaments Nouveaux; Squire's Companion to the British Pharmacopoia, and Merck's Annual Reports of recent advances in Pharmaceutical Chemistry and Therapeutics.

\section{J. MACQUEEN.}

Royal Vetperinary College,

London, 25th April 1910. 



\section{PREFACE TO THE TENTH EDITION}

A NEW Edition of this standard work on Veterinary Materia Medica has been wanted for some time. Prior to his lamented death, Mr. Finlay Dun, recognising the urgency of the demand, had commenced the preparation of the Tenth Edition, which, on the plans indicated by his MS. and marginal notes, has now been completed. Numerous important alterations, rendered necessary by the publication of the new British Pharmacopoeia (1898), have been made and many new remedies have been inserted. The body of the work has been rearranged, and while every article has been revised, no change has been made in the principles enunciated by the author. To provide space for the various additions and alterations without enlarging the volume, the text in places dealing with the Chemistry of Drugs has been curtailed. Revision has been carried out with a constant regard to practical utility, and in the hope that Dun's Veterinary Medicines will continue to serve effectually the requirements of students and practitioners.

The Editor desires gratefully to acknowledge his indebtedness to Professor German Sims Woodhead, Cambridge University, for revising and extending the pages on bacteria and antitoxins; and to Professor John F. M'Fadyean, Principal, Royal Veterinary College, for permission to insert the directions for using mallein, tuberculin, and black-quarter vaccine. Much valuable information 
has been obtained from Guinard's Thérapeutique et Pharmacodynamie (1899), Delaud and Stourbe's Pharmacie et Toxicologie Vétérinaires (1900), Cagny's Formulaire des Vétérinaires, Hale White's Materia Medica (fourth edition), Squire's Companion to the British Pharmacopoeia (1899), Martindale and Westcott's Extra Pharmacopœia (ninth edition), Coblentz's The Newer Remedies (1899), and Hare's Practical Therapeutics.

\section{J. MACQUEEN.}

Royal Veterinary College,

London, 10th April 1901. 


\section{PREFACE TO THE EIGHTH EDITION}

\section{(ABRIDGED)}

THe First Edition of Veterinary Medicines was published in 1854, while I was Lecturer on Materia Medica and Dietetics at the Edinburgh Veterinary College. The work continues a text-book at the British Veterinary Colleges, is used by Veterinarians and Agriculturists, and meets with increasing demand in the United States of America and in the Colonies.

The increased bulk of the present volume results from the introduction of the recently discovered antiseptics and antipyretics of the benzol series, and other new remedies ; from details of various British and foreign experiments made with alkaloids and other medicines; and from fuller and more systematic treatment of the actions of various important drugs.

As in previous editions, the general actions and uses of Veterinary Medicines, and the more important principles and practice of Pharmacy, are dealt with in the Introduction. The several drugs, discussed in alphabetical order according to their English names, occupy the bulk of the volume. Under each individual medicine the space allotted to preparation and properties has been curtailed, while careful revision has been made of the matter relating to the actions on the several domesticated animals, the curative uses, doses, and medicinal forms. 
In preparing the present edition the following works have been consulted:-Dr. Lauder Brunton's Pharmacology, Therapeutics, and Materia Medica; Robertson's Equine Medicine; Williams' volumes on the Principles and Practice of Veterinary Medicine and Surgery; and the Journal of Comparative Pathology and Therapeutics. Hertwig's Praktische Arzneimittellehre für Thierärzte, and Moiroud's Traité Élémentaire de Matière Médicale et de Pharmacologie Vétérinaire-for many years the standard works on Veterinary Pharmacology in Germany and France respectively. Further valuable information has been derived from the Lehrbuch der Arzneimittellehre für Thierärate, von Dr. Eugen Fröhner; Traité Thérapeutique et de Matière Médicale Vétérinaires, par M. Kaufmann; Précis de Thérapeutique de Matière Médicale et de Pharmacie Vétérinaires, par Paul Cagny ; as well as from Pathologie et Thérapeutique Spéciales des Animaux Domestiques, par Friedberger et Fröhner.

FINLAY DUN. 


\section{CONTENTS}

INTRODUCTION,

I. THE ACTIONS AND USES OF MEDICINAL AGENTS,

II. VETERINARY MEDICINES, . • • • 152

The Alkaline Metals, Аmmonium, Рotassium,

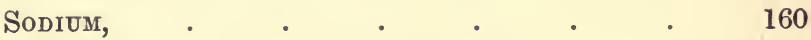

The Alkaline Earths, Calcidm, Magnesium, Barium, Aluminium, $\quad$ - $\quad$. 203

The Metals, Bismuth, Lead, Zinc, Copper, Silver, Iron, Antimony, Arsenic, Mercury, . $\quad$. 224

The Non-Metals, Phosphorus, Bromine, Chlorine,

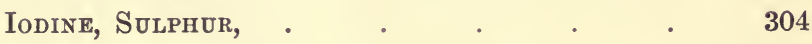

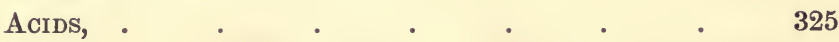

Carbon Compounds, Alcohols, Ethers, . $\quad 349$

III. MEDICINES DERIVED FROM THE VEGETABLE

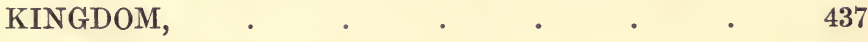

MEDICINES DERIVED FROM THE ANIMAL KINGDOM, . $\quad$. $\quad$. $\quad$. $\quad$. $\quad 669$

IV. VETERINARY PHARMACY, . . . . 700

INDEX OF DISEASES AND REMEDIES, . . $\quad 739$

INDEX OF MEDICINES, . $\quad$. $\quad$. 804 



\section{VETERINARY MEDICINES}

\section{THEIR ACTIONS AND USES}

\section{N T R O DU C T I O N}

Veterinary Materia Medica, in the extended sense of the term, treats of every agent which is used for the relief or cure of disease or injury, or for the preservation of health, among the domesticated animals. The full consideration of a subject so large and diversified would, however, fill several volumes, and the present work has been restricted to the description of drugs, their characters and properties, their pharmaceutical preparations, and their actions and uses among veterinary patients. Medicines or drugs, although derived from the mineral, vegetable, and animal kingdoms, possess many actions in common, and are prepared for use by similar chemical and pharmaceutical processes. In this work the first section is devoted to general observations on Pharmacology, which treats of the actions of drugs, and Therapeutics, or the application of remedies to the cure of disease. A description of the medicines, arranged according to their source, occupies the body of the volume, which is completed by a section on Pharmacy, the administration of medicines, and an index of diseases and remedies. 


\section{SECTION I}

\section{THE ACTIONS AND USES OF MEDICINES}

The general and medical management of the domestic animals has greatly improved during the past forty years. The laws of health, the causes and nature of disease, as well as the actions and uses of remedies have been more fully studied. The beneficent curative effects of fresh air, diet, suitable surroundings and good nursing are more thoroughly realised. Preventive treatment also receives more attention alike from stock-owners and practitioners. Disease accordingly is not only less prevalent, but it is generally less serious, and the attacks of shorter duration. In Great Britain such equine maladies as specific ophthalmia, canker of the feet, and mange, are now seldom seen, while colic and inflammation of the bowels are not nearly so common as they were forty years ago. Cattle plague, contagious pleuropneumonia, rabies, and sheep-pox have been exterminated. The prevalence of glanders or farcy, swine-fever, anthrax, and bovine tuberculosis should be greatly limited by the measures now being adopted in dealing with these disorders.

Fuller and more definite knowledge of the actions of remedial agents has been obtained by systematic experiments and clinical observations not only on the lower animals but on man himself. Numerous illustrations might be adduced of the practical benefits of such investigations. Magendie's experiments with the Java upas antiar and nux vomica demonstrated that these strychnine-containing plants violently stimulate the spinal cord, producing tetanic convulsions. In virtue of this stimulation of the cord, and its reflex functions, carefully regulated doses of this drug have been utilised to restore disturbed co-ordination of the gastro-intestinal functions, and to relieve some form 3 of paralysis. Experiments on animals also have demonstrated the action of digitalis and strophanthus as cardiac stimu- 
lants, and hence have led to their use in strengthening and steadying the weak and overtaxed heart. It has been proved in the same way that ergot of rye, and its active constituent, ergotin, have the power of causing contraction of the arterioles, hence their employment for the arrest of internal hæmorrhage. Belladonna, and its active principle atropine, have been shown to diminish sensibility of the ends of the vagi and sensory nerves, and from this results their value in quickening slow, irregular heart rhythm, diminishing excessive bronchial secretion, and relieving certain forms of pain. When the precise action of medicines is recognised their practical use is obviously rendered not only safer, but more effective.

The Study of Bacteriology within the last twenty years has done much to throw light on the diagnosis, prevention and treatment of disease both in men and animals, and is apparently destined to do much more. A number of diseases classified as zymotic, and comprising anthrax, glanders, tuberculosis, black-quarter, strangles, swinefever, tetanus, with typhoid, eruptive and other fevers, have been shown to depend upon the introduction into the body of micro-organisms belonging to the lower class of vegetable fungi which, in susceptible subjects, multiply rapidly, and produce chemical ferments, alkaloidal poisons, and deadly albumoses. Pasteur, Koch, and others have investigated the life-history of many of these diseaseproducing microbes, the pathogenic conditions to which they give rise, and the methods by which their invasion may be averted or counteracted.

Rabies differs somewhat from the other types of disease here mentioned. Although the most elaborate searches have been made for organisms of bacterial character, these have been unsuccessful. It has been suggested from time to time that organisms more resembling protozoa might be present, but it was not until Negri described what are now called 'Negri-bodies,' that any advance was made in this quest. Lying in the protoplasm of the nerve cells or in their branches, or in some cases outside them, they are seen as oval, triangular, or slightly spindle- or sausage-shaped bodies, which, taking on an eosin ground stain, are studded 
with basophile granules, rods, and 'circles.' These bodies are from $0.5 \mu$ to $20 \mu$ in diameter, the size increasing with the time that the disease has run, the larger forms being met with in less susceptible animals, the smaller in specially susceptible animals, and in those cases in which the disease runs a very rapid course. In some cases these bodies may be constricted in the middle, or, if the bodies are somewhat elongated, there may be two or three constrictions. These bodies may be found in any of the nerve cells of the central nervous system in cases of rabies, but they are most numerous and are found most readily in the cells of Ammon's horn and in the Purkinji cells of the cerebellum.

It has been suggested that a small central corpuscle, surrounded by no clear space, may correspond to a protozoan nucleus; this, however, can be little more than a suggestion. The presence of these Negri bodies certainly appears to be specific to rabies and hydrophobia. Such bodies are present in large numbers even at an early stage of the disease, but are then so small that they may easily escape detection; they may be so small, indeed, that they pass through the pores of a Berkefeld filter. Later, and this of course is best seen in more chronic cases, they attain a considerable size.

These bodies may be simply the result and not the cause of disease, but many who have examined them acknowledge that they may be protozoan in character.

Microbes, when cultivated in media containing chemicals, or when repeatedly passed by inoculation through the bodies of certain animals, become weakened, and lose their virulence. Attenuated cultures or vaccines, properly employed, confer on certain animals more or less protection against poisonous doses of the natural virus subsequently introduced by infection, or by experimental inoculation. In districts of France, Russia, Austria, and Switzerland, where anthrax abounds, cattle and sheep for many years have been vaccinated with attenuated anthrax virus, the mortality among the vaccinated is stated to be less than one-tenth of that which occurs amongst the unvaccinated stock. Pasteur, by repeated injection of attenuated rabies 
vaccine, rendered dogs and other animals insusceptible to the action of lethal doses of rabies virus, and Pasteur's method applied to persons bitten by rabid animals continues to afford protection against hydrophobia in 99 per cent. of the cases timeously submitted to this treatment. Pigeons repeatedly inoculated with small doses of snake poison for periods of three months withstand seven times the ordinary fatal dose of snake poison. The modified black-quarter virus, obtained by drying and heating the muscle of an animal that has suffered from this disease, also exerts a distinct effect in protecting even susceptible animals against attacks of this disease; whilst cultivations of the swine erysipelas bacillus, when similarly modified by heat, have been used with great success as a protective inoculation agent against the ravages of swine erysipelas (Rouget du porc).

The manner in which these vaccines effect their protective powers has been variously explained, but the most satisfactory view is, that small repeated doses of the cultivated organism or its products modify the functions of the cells on which they specially act, and thus confer upon them a tolerance against deadly doses of the same or allied poisons (Bacteria and their Products, by G. Sims Woodhead, M.D.). Going further than this, however, it must now be recognised as a result of the observations of Behring, Roux, Ehrlich, and numerous later workers, that this tolerance is due in great part to the production, during the reaction between the cell and the toxin, of an antitoxin which at first stored in the cell, soon overflows its boundaries and passes into the blood, where it appears to be stored up in the fluid elements, always ready to combine with any toxin that may be produced in or introduced into the tissues. It would appear that an antitoxin can only be produced in response to the action of what is spoken of as an exotoxin, a toxin that is thrown out into the culture medium, i.e. is set free from the organism by which it is formed, in somewhat the same manner as alcohol is set free by yeast. This toxin, separated from the bodies of the micro-organisms that formed it, and injected into an animal, induces the formation of a specific antitoxin. The antibody produced by the bacilli which produce this toxin gives rise to an 
anti-bacterial substance, a specific substance that has far less power to neutralise the toxin, but far greater power to destroy the bacilli. The only two true antitoxins as yet brought into play in practical medicine are the diphtheria antitoxin and the tetanus antitoxin, though similar substances are now used in the treatment of snake-bite and of pneumonia, and one or two similar diseases.

Antibacterial substances, however, now constitute a very important class of therapeutic agents, the whole of the vaccines, whether dead or living, being of this type. In the case of the new tuberculin, the bodies of the bacilli are broken down in order to allow the whole of the elements contained in the body of the tubercle bacillus to come into play. Typhoid vaccine, cholera vaccine, and others are all of the same class. The poisons thus locked up in the bacteria are sometimes spoken of as endotoxins.

From the fact that these antitoxic substances are stored up in the fluid constituents of the blood, it has been found possible to produce antitoxins in one animal, and then by drawing off its blood, and allowing the clot to separate from the serum to obtain a fluid containing a large quantity of antitoxin which, injected into a second patient, acts upon the toxin, neutralising it just as surely and completely as if it were acting upon the original patient. This has gradually developed into a definite system of treatment-the antitoxin treatment. It has been worked out most thoroughly in diphtheria in the human subject, and in tetanus in veterinary medicine; whilst fair results have also been obtained using a similar method in the treatment of snake poisoning. In Diphtheria, the best results have been obtained (1) because the local lesion, i.e. the false membrane, etc., is usually well marked before the constitutional disturbances, i.e. the toxic effects, make their appearance; and (2) because the poison is not only formed slowly, but takes some time to produce its effects upon the nervous tissues to which it usually attaches itself.

To produce diphtheria antitoxin all that is necessary is to inject subcutaneously the toxic products of the diphtheria bacillus grown in slightly alkaline broth, or still better, in broth containing a certain proportion of blood plasma, 
into some animal, preferably a horse. This should be followed by a rise of temperature, and by swelling at the seat of injection. Unless this reaction is obtained no antitoxin will be formed: Gradually increasing doses of the toxin must be injected from time to time, care being taken to obtain a distinct reaction after each injection, and to make the fresh injection before the effects of the last one have completely passed away. After a time it will be found that it is very difficult to obtain a local reaction, or a rise of temperature, even when large quantities of toxin are injected. During the whole of this period it will be found that the antitoxic value of the blood is rising more or less rapidly, and at last there is sufficient antitoxin present to make it valuable for the treatment of diphtheria patients. (The antitoxin in the serum injected into a patient suffering from the effects of diphtheria poisoning, combines with the toxin formed by the diphtheria bacillus, and so prevents it from exerting its deleterious action upon the tissues of the patient.) The diphtheria bacilli, with their poison neutralised, now behave like ordinary non-pathogenetio organisms, and are rapidly destroyed by the tissues, and the patient recovers. The power of this antitoxic substance may be realised when it is borne in mind that doses of antitoxic serum containing 12,000 to 30,000 units may be injected with safety into a diphtheria patient. A single cubic centimetre of the serum of the treated horse may contain a thousand units, each one of these units being sufficient to neutralise toxin sufficient to kill, within four days, one hundred half-grown guinea-pigs ; the amount of neutralising and protective power thus obtained is therefore enormous, and we can readily realise why the antitoxin treatment of diphtheria has proved so successful.

In tetanus the principle of production of antitoxin is the same as in diphtheria, but the treatment has not been so successful, because the disease in this case does not manifest itself locally in the first instance, the poison has already attacked the nerve centres before the necessity for treatment makes its appearance. The damage has already been done, and antitoxin, though it can neutralise the poison, and so prevent further damage being done, cannot make good the 
ravages already carried on. Tetanus antitoxin, however, like diphtheria antitoxin, acts as'a most powerful prophylactic, and it is in this character that its greatest value as a therapeutic agent must be sought.

Calmette's antivenin has also been found to be of great use in the treatment of snake bite. It is prepared in the same way as are the other antitoxins. By treating a horse with gradually increasing doses of the mixed venom taken from various snakes-cobra, black snake, and others-it is found that, if sufficient care be taken to give small enough preliminary doses, and to allow the animal to regain weight and condition after each injection, large doses may be given later; the horse then becomes not only immune against the action of these large doses, but his blood contains an antivenin which, held in solution in the serum, may be injected into other animals, where it acts not only as a prophylactic, but also as a curative agent against snake bite, even of the most virulent types.

The products of micro-organisms, however, have been utilised in other most important fashions. Tuberculin (which consists essentially of the products of the tubercle bacillus grown in beef peptone broth containing a five per cent. solution of glycerin, the bacilli destroyed and then filtered out by means of a Pasteur-Chamberland filter, the whole concentrated by evaporation at a low temperature) is now used for the diagnosis of tuberculosis, as it is found that these products, when injected subcutaneously, appear to co-operate with the poisons already in the body, and to cause a reaction of the tissues, and a rise in temperature which, together, give evidence of the presence of a tubercular process. Exactly the same thing happens in the case of mallein, which is prepared in the same way from the glanders bacillus as the tuberculin is prepared from the tubercle bacillus. A small quantity of this substance injected subcutaneously in a healthy animal with a normal temperature gives rise to little or no local swelling, and a very slight rise of temperature, whilst a similar quantity injected into a horse suffering from glanders gives rise to a local swelling of considerable size, which goes on increasing up to the thirty-sixth or forty-eighth hour, or even longer, 
and is accompanied by a rise of temperature to $104^{\circ} \mathrm{F}$. In certain cases where the temperature is high to begin with, even where the disease-tuberculosis or glandersis well advanced, no characteristic reaction is obtained; but in almost all these cases the diagnosis can be made without the aid of the specific products.

It is evident, then, that bacteria are coming to play a greater and greater part in both diagnosis and treatment of disease, just as they have come to.play such an important rôle in our conception of its production.

Recent studies of the life of the simplest animal structures, such as amœba, and the manner in which they are acted upon by medicinal agents, have thrown much light on therapeutics as applied to the higher animals. The complex albuminoid material termed protoplasm, which is the ever-present constituent of living cells, is coagulated and precipitated by heat, and dissolved by alkalies. It is precipitated by small quantity, and dissolved by excess, of most mineral acids. Organic alkaloids resemble acids in lowering the temperature at which heat coagulates albumin. Protoplasmic movements, as illustrated in the amœba, are retarded or arrested by cold. Heat, slight electric shocks, and common salt, even in diluted solution, first quicken them ; but a higher temperature, a stronger electric current, or prolonged exposure to a saline solution, tetanises them. Protoplasm has the power of absorbing and storing oxygen; and the chemical energy developed from this oxidation is capable of conversion into mechanical energy and movements. Protoplasm has also the power of carrying and transferring oxygen to other substances, and appears to contribute largely to the diffusion of oxygen, and interchange of gases, constantly occurring between the blood, the intercellular fluid, and the cells, and constituting what is termed internal respiration.

Infusoria have both their rhythmical and ciliary movements increased by heat and diminished by cold. Weak saline solutions increase their movements; while strong saline solutions alter the amount of water they contain, and cause them to shrivel, by withdrawing water from their substance. (See 'Salt Action.') 
Leucocytes are affected in much the same way as amœbæ. Their movements are notably arrested by the cinchona alkaloids. Quinine injected into the circulation has been found to diminish the migration of leucocytes from the blood-vessels.

Red Corpuscles are markedly affected by any change in the salt content of the blood serum in which they are bathed. These cells may be considered to be masses of colloid material surrounded by a membrane, this membrane being permeable by water, but having a selective power with respect to the salts it will allow to pass through. Blood serum normally contains 0.9 per cent. of sodium chloride, and the corpuscles may be assumed to contain the same amount. A salt solution of this strength is called an isotonic solution, one of greater concentration is hypertonic, a more dilute one being hypotonic. Red corpuscles in an isotonic salt solution (' normal saline ') are unaffected, for although osmosis takes place, the rates of diffusion, in and out, are the same. Cells placed in a hypotonic solution swell up, for salt diffuses out of them and water enters until the envelope, no longer able to contain the extra fluid, bursts, setting free the hæmoglobin. If the cells are placed in a hypertonic salt solution, water diffuses out to dilute the more concentrated fluid, while salt diffuses in, but the net result of the loss of fluid from the cell is that it shrinks and becomes crenated. Thus any excess of water in the blood serum at once results in an increase in the size of the red cells, which imbibe fluid, swell up, and burst. On the other hand, if an excess of salt (sodium chloride) is put into the bloodstream so that the serum becomes hypertonic, water diffuses out of the red cells to dilute the serum, the red cells becoming smaller and shrivelled, or crenated.

The important blood constituent hæmoglobin, like protoplasm, has great capacity for taking up oxygen, thus becoming converted into oxyhæmoglobin, which, however, holds its added oxygen loosely, and parts with it readily, as it slowly circulates through capillary vessels. The hæmoglobin also combines with other substances as well as with oxygen-as with hydrocyanic acid and carbon monoxide, forming tolerably stable compounds; and these can neither 
take up oxygen from the air in the lungs, nor give it off in the tissues, which hence become asphyxiated. Addition to the blood of such drugs as alcohol, chloroform, quinine, morphine, nicotine, and strychnine, likewise, in various degrees, diminishes the amount of oxygen absorbed, and of carbonic acid given off by the blood.

It is exceedingly interesting, in this connection, to note how easily the non-nucleated blood corpuscles of the mammalian animal take up, but also give up, oxygen. It would appear that these red blood corpuscles, like the plasma itself, are capable of carrying in loose combination an enormously greater quantity of oxygen than they require for their own special purposes. Leucocytes, on the other hand, nucleated cells, although circulating in the blood have no oxygen carrying power beyond what they require for their own use, the presence of nuclear substance of the cell, in mammals at any rate, appears to be associated with the using up of oxygen rather than with its transportation, and we find that in blood diseases, even where the number of red cells in the blood remains high, the oxygen transporting power of the blood to other tissues may be interfered with by the presence of an increased number of nucleated cells, the leucocytes, this interference varying with the increase.

\section{FERMENTS-BACTERIA-BACTERIAL PRODUCTS-VACCINES}

Ferments determine the healthy nutrition of plants and animals, as well as their decay and many of their diseases. They are exemplified by the yeast which raises bread and converts the starch and sugar of barley into beer or spirit, the rennet which coagulates milk, the filamentous fungus which causes ringworm, and the bacillus which induces the deadly anthrax. Ferments are divisible into two classes:-

1. Enzymes or unorganised ferments are exemplified by diastase, which causes germination in barley and other seeds, ptyalin from saliva, pepsin from the stomach, trypsin from the pancreas, with histozyme, a recently discovered ferment present in blood, and believed to be the chief agent in the reduction of albuminoids. These, like the 
toxins, may be divided into two groups-the endoenzymes and the exoenzymes. The former appear to be the result of the functional activity of the cell, and to be so closely associated with the tissues that they seldom appear in the fluids of the body in anything but small quantities. Vernon points out, however, that certain of these endoenzymes, such as maltase, are elaborated in larger quantities. The exoenzymes, like the exotoxins, are set free much more freely, and may be thrown out into the fluids in considerable amounts whenever they are required: 'Probably the endoenzymes and the exoenzymes are formed and are bound up in the tissues in a similar manner, only the linkage binding the exoenzymes is more readily snapped under an appropriate chemical or nervous stimulus than that binding the endoenzymes.' Both forms appear to be elaborated in larger quantities during increased functional activity of the tissues by which they are formed, the endoenzymes, on the one hand, being stored up in the cells, the exoenzymes, on the other, readily making their way into the fluids surrounding the tissues.

In the blood there are diastatic enzymes, e.g. amylase which converts starch into dextrin and maltose, and a glucase or maltase which converts these products into glucose :-indeed, it is maintained that there are several of the amylase group. Corresponding to these, numerous antibodies of various types are also probably present in the blood.

2. Organised or formed ferments, such as yeast, mycoderma vini, moulds, and bacteria, are living vegetable organisms of parasitic habit.

The reduction of complex carbon compounds into simpler forms is the work of both classes of ferments. Their effects are produced, it is believed, in one of two ways :-(1) By abstraction of water, as in the conversion of starch into sugar, or the splitting up of glucosides-changes chiefly effected by enzymes, and analogous to the effects of heat in conjunction with diluted mineral acids or alkalies.

(2) The breaking up of the fermentible body is sometimes effected by transfer of oxygen from its hydrogen to its carbon, as in alcoholic and lactic fermentations, and in putrefactive 
processes-a mode of reduction usually effected by the organised ferments.

The organised ferments, which are the causes of putrefaction and of various diseases, have been classified as-

1. Yeasts, or sprouting fungi, which consist of ovoid cells, multiplying by budding, are represented by the torula cerevisiæ, mycoderma vini, and, according to most authorities, also include the aphthous patches of thrush found in the mouth of young animals.

2. Moulds, or filamentous fungi, occur in threads, which are agglomerated into masses or tufts, multiplying by budding and formation of spores, and exemplified by the common moulds which appear on moist objects, and by those which cause such skin diseases as favus and ringworm.

3. Bacteria, Microbes, or Schizomycetes, are the lowest forms of vegetable life, but the most widely distributed, in air, earth, and water. They consist of round, oval, or cylindrical cells, so minute that they can only be examined with high powers of the microscope. They multiply chiefly by division, occasionally by spore formation. Their multiplication is effected with enormous rapidity, a single individual cell sometimes producing a million in twenty-four hours. Some are fixed, others are motile. For their formation and growth they require organic matter, moisture, salts, and a moderate temperature; some, further, need oxygen; some thrive without it. They speedily exhaust the nutriment obtainable from the substance on which they grow, or form in it matters inimical to their life; but where one species languishes and perishes, others frequently spring up and flourish.

Bacteria are divided into two groups-(1) Non-pathogenic or saphrophytic. Many of these feed and live on dead animal or vegetable matter, and their great function is the conversion of complex into simpler forms. They are exemplified by the bacillus of hay infusion, the bacterium termo found in all putrid fluids, and the bacilli developing the saccharine and lactic fermentations.

(2) Pathogenic or parasitic bacteria live on or within the bodies of living plants or animals, and when in large numbers interfere with nutrition and cause disease. Their pathogenic power is proved 
beyond all question by taking cultures from any of the specific disease products, growing them in suitable media for several generations, and inoculating these on living subjects, when the original disease is reproduced. But pathogenic and non-pathogenic are only relative terms. The organisms, usually harmless, under certain conditions become harmful; while those that are pathogenic by cultivation and otherwise sometimes lose their toxic power, and live and reproduce themselves amongst dead vegetable and animal matter. The bacilli of anthrax, hog cholera, and tetanus illustrate these varying states of activity and change of habit. Microbes which have had their activity reduced or destroyed may, however, under certain conditions regain it.

Both pathogenic and non-pathogenic bacteria are divided into three classes, each distinguished by the form assumed : (a) Micrococci or round cells, such as the sarcina found in the stomach, or the cocci which arrange themselves in clusters or in chains, and cause strangles in horses, and pus formation in all animals ; (b) Bacilli or rod-shaped bacteria, as those of anthrax and glanders, as well as the short ovoid bacillus of pneumonia; (c) Spirilla or thread-like bacteria, as of relapsing fever in man and the comma-like organisms of cholera.

In recent years, as the result, in great measure, of the search for pathogenic bacteria, and aided by methods of precision devised for the detection of bacteria in the tissues and for their cultivation outside the body, a large number of pathogenic protozoal and other animal organisms have been described, their history and the methods and channels by which these organisms pass through various phases of development in various hosts. Following Manson and Ross's work on the intermediate phases of development of the malaria organism of man in certain anopheline hosts (mosquitoes) came Bruce's observations on the Nagana or tsetse-fly disease of Southern Central Africa, by which he was able to prove that the trypanosome associated with this condition found an intermediate host in the tsetse-fly (Glossina morsitans). He demonstrated that the tsetse-fly might carry the trypanosome from an animal, 
the buffalo, that appeared to be immune to the disease, to other species intensely susceptible to the action of this organism, the horse and the dog. The importance of these lower mammals as reservoirs or harbourers of the parasite, and of insects as vectors, or carriers, was thus made clear. Long before this, William Williams of Edinburgh had maintained most stoutly that ticks were in some way or other associated with the transmission of the infective material of Redwater fever from one bovine to another, an opinion he later translated into partial demonstration. This opinion has now been confirmed beyond doubt by numerous observers. Bruce, applying his reservoir theory to Malta fever, found that goats, apparently healthy, provided a continuous supply of the Micrococcus melitensis, which, distributed through milk, infects susceptible human beings. Similarly, the rat forms a 'reservoir' for the plague bacillus, this organism, moreover, being carried from rat to rat by the rat flea, and thence even to the human subject by the same vector. Following these observations, there have been described during the last ten years a regular swarm of protozoal, blood and other parasites, which are carried principally by arthropodsinsects, ticks, crustaceans-from one animal to another. In certain instances the carrier seems to act as an intermediate host, some phase of alternation of generation, usually the sexual phase, being here passed through. In connection with human diseases we have mosquitoes (Stegomyia fasciata) harbouring and carrying the infective material of yellow fever. Malaria we have already mentioned. Sleeping sickness appears to be the result of infection by an organism very like that of Nagana, but carried to the human subject by another tsetse-fly, the Glossina palpalis. Nuttall points out that many of the hæmatozooal parasites belonging to the genus Piroplasma are transmitted by ticks; as are also certain relapsing fevers of the fowl and the human subject. Amongst these he mentions the Redwater in cattle, transmitted by certain species of Boophilus, of Ixodes, of Rhipicephalus; Rhodesian fever in cattle, Redwater in sheep, malignant jaundice in dogs, biliary fever in horses, spirochaetoses in cattle, 
in man, and in fowls, and Rocky Mountain spotted fever in man, all of which are carried by similar organisms, and species of Argas and Ornithodoros.

It is evident, then, that these arthropod-borne diseases are coming more and more into prominence, especially in connection with tropical diseases, and Manson goes so far as to say that in parasite-ridden Africa bacterial disease is a comparatively negligible quantity. Again it has been pointed out that in the United States alone the annual loss to cattle breeders through these tick diseases is already known to amount to more than eight million pounds sterling. Other blood-inhabiting parasites, such as spirochætes and organisms of the piroplasmal form, are all carried by these arthropod hosts. The spirochrtes found in rats, fowls, geese, cattle, sheep, horse, the bat, and man, appear to be transmitted in this way, as do also the piroplasmata associated with Redwater fever in cattle and sheep, Texas fever in cattle, Heart water fever, malignant jaundice in the dog, biliary fever in horses, mules, and donkeys. Monkeys are also affected by piroplasmosis, transmitted apparently by similar carriers. It is evident, then, that a careful study of the life-history, not only of the parasites themselves, but of the arthropod carriers of these organisms, will, in the near future, add considerably to our stock of knowledge of the diseases both of animals and man. No account of this aspect of disease in animals and man would be complete which did not include some notice, however short, of the parasitic amœbæ. At one time it was supposed that these amœboid organisms were of little importance, certainly from the pathogenic point of view. It is now being realised, however, that these simple amœboid parasites have a much wider distribution and a much more important rôle in the production of disease in man and animals than was at one time supposed. These amœbæ, first brought into prominence in connection with dysentery, appear to multiply not only by fission, but also by a process of internal division corresponding closely with spore-formation. Under certain conditions, they assume what Geddes calls the resting or cystic stage. Walker (Jour. Med. Res., Boston, vol. xvii., 1907-8, p. 379) describes no fewer than eleven 
animals, in addition to man, from which he has been able to isolate these parasitic amœbæ, cultivating them from the contents of the intestine in from twenty-five to one hundred per cent. of the animals he examined. From this he gathers that these parasites must have a wide, if not universal distribution amongst vertebrates. He states: 'Amœbæ were found in the intestinal tract of every species from which cultures were made, from amphibians to man.' In certain cases, these parasitic amœbæ may undoubtedly invade the tissues of their hosts. This has been proved both in the human subject and in the pig. In the former in cases of tropical abscess, in the latter in hog cholera (Theobald Smith).

Both organic and organised ferments, in breaking up complex and vegetable animal bodies, frequently produce substances of great activity. The emulsin-the ferment of bitter almonds-gives rise to a hydrocyanated oil. The myrosin of mustard seed develops the acrid mustard oil. The protoplasm of particular plants produces their respective alkaloids, some of which are active poisons. Certain mushrooms produce the poisonous alkaloid muscarine; putrefying yeast yields sepsin; from putrefied maize is obtained an extract which contains one substance which tetanises, and another which narcotises. Animal bodies invaded by bacteria undergo decomposition, and the bacterial cells, according to their kind, elaborate their respective alkaloids, some of which are poisonous. Under healthy conditions they are excreted, but if retained they are injurious. In the healthy muscles of living animals, after active exertion, there are found alkaloids allied to xanthin and creatin. During digestion of fibrin by pepsin an alkaloid is formed. Bouchard has stated that the alkaloids formed in the intestines of a healthy man in twenty-four hours would suffice to kill him if they were all absorbed and excretion stopped.

The pathogenic, like the putrefactive bacteria, when they invade the bodies of higher organisms, act as ferments, cause disintegration of living tissues, and formation of alkaloidal toxins, and, besides, produce globulins or albumoses, often as deadly as the alkaloids. Special local effects are 
produced by the action of these poisons, notably congestion, inflammation, and necrosis ; but the soluble poisons carried in the blood-stream further develop general effects. Thus, the anthrax bacilli cause local odema and pustule, and further lead to nervous collapse. The diphtheria bacillus causes special throat lesions, while the toxin it develops, circulating widely, impairs the functions of the great nervecentres.

In all these reactions between toxins and the tissues or enzymes and the tissues or the complex proteid bodies of the tissues we appear to have a similar uniformity of type of action. Whenever an animal is immunised against a bacterium, against blood corpuscles, against a serum or against enzymes and the like, an 'antibody' is formed which possesses the peculiar property of linking itself on to the substance that has been injected, and against which the organism is now 'immunised.' This ' antibody' substance, which has a variety of names but which we will speak of as the immune body, may be produced in very large quantities. It may, however, be present in minute quantities in the blood of animals which have apparently not been subjected to any special treatment. Such an immune body is spoken of as being 'stable,' as it has the power of resisting a comparatively high temperature over a prolonged period. This stability, however, is not the same in all cases. In the blood of the treated or infected animal it is usually associated with a second substance, also variously named, to which we will apply the term 'complement,' the name by which it is now most generally known. This complement is present in the blood of all animals, though it varies in character in different species, and, so far as we know, is not dependent upon the action of any specific substance. Without the presence of this complement the immune body appears incapable of combining with the material by which it is produced. With the complement, however, the combination readily takes place. This can be proved very easily, for it has been found that the complement is what is called a 'labile' substance, i.e. it is easily and rapidly destroyed at a temperature of $60^{\circ} \mathrm{C}$. Under ordinary conditions the amount of this complement is not 
increased during the process of immunisation. Advantage has been taken of the fact of this difference of stability between these two substances to analyse the action of both bodies. For example, if the blood serum of an animal immunised against the red blood corpuscles of another animal be mixed with a suspension of these same red blood corpuscles in normal saline solution, it will be found that, owing to the combination of this serum with the red blood corpuscles, they are broken down, and the hæmoglobin is set free into the serum.

If, now, the serum of the immune animal be heated to $55^{\circ} \mathrm{C}$. for half an hour, and then be added to the red blood corpuscles, they are not dissolved, and no hæmoglobin is liberated. If, however, a few drops of fresh serum be added, the breaking down takes place immediately. The heated serum contains the immune body, but the complement has been destroyed. Along with the fresh serum, however, complement is added, and the immune body, linking the complement on to the substance of the red corpuscle, brings about the breaking down of the corpuscle. Without the complement, which is the real attacking body, the corpuscle remains intact, just as it does when there is no immune body by which the attacking complement may be linked on or brought into direct relation with the corpuscle.

In relation to the prevention and cure of the diseases depending upon bacteria, it must be noted that these pathogenic organisms have their activity retarded or destroyed by exposure to high temperatures, by the action of chemical solutions, by being reproduced for several generations in the bodies of certain animals, and in other ways. Anthrax virus is thus attenuated by heat; rabies virus by being grown in the bodies of rabbits. These attenuated viruses are used as protective vaccines. The vaccines of anthrax and rabies, as well as those of black-quarter, swine erysipelas, and fowl cholera, when injected into the bodies of animals liable to these disorders, render the vaccinated subjects immune. This is demonstrated by the animals operated upon suffering no harm when subsequently inoculated with doses of the unattenuated bacterium or its products, which would destroy unprotected animals. 
Vaccines exerting a curative as well as preventive effect may also be obtainable. This is a justifiable expectation, for human patients who have been exposed to the contagium of smallpox, if promptly vaccinated with cow-pox lymph, have the smallpox attack favourably modified. Perhaps still more to the point are Pasteur's experiments with rabies. He inoculated dogs and rabbits with lethal doses of rabies virus, and thereafter with repeated doses of vaccine. No serious results followed, although control animals inoculated with rabies virus, but not with the vaccine, died.

The general method of fighting these pathogenic bacteria at present at our command consists in strict isolation of animals affected by such specific diseases, and fully recognising and acting on the fact that infected subjects and their discharges are apt to distribute the virus. Remedial measures must be adopted early-if possible, before the bacteria have multiplied, and before the toxins are produced. In such cases as rabies, which have a long incubation stage, there will be time for the protective operation of vaccines, which appear to confer on the tissues a tolerance of the bacteria, and enable them successfully to cope with the intruders and their products. Every endeavour must be made to maintain in its fullest vigour both the part primarily attacked and the system generally, with the view that the healthy tissues may, if practicable, destroy the parasites and their products. The excreting channels, moreover, will be maintained in healthy action, in order that waste and diseased materials, as well as the organisms themselves, may be removed. Disinfectants will continue to be used so that the bacteria and their spores shall be destroyed, and the spread of the disease prevented.

Anaphylaxis. When the serum of a horse is injected into a rabbit, a specific antiserum body similar to a specific antitoxin is produced by the tissues of the rabbit, and we may say that an antibody has been formed. During the period that this process is going on, however, the rabbit appears to become, during one phase (the negative phase of Wright and others), very much more susceptible to the action of the horse serum. Indeed, the horse serum appears to become intensely toxic. There has been no change in 
this horse serum itself, but there has been a change in the infected animal; it has become hypersensitive to some substance or substances (probably enzymes) contained in that serum.

Attention was first drawn to this by Behring, who found that during the process of the production of antitoxin certain animals, instead of becoming more resistant to its action, became much less resistant. This anaphylaxis or hypersusceptibility should be borne in mind in connection with the treatment of diphtheria or tetanus, and as large a dose as possible of the antitoxin should be given at once, so that a second dose may not be necessary, as it is quite possible that this phase of anaphylaxis may come to play an important part in the condition of the patient where antitoxic serum is given by repeated injections.

\section{CLASSIFICATIONS OF MEDICINES}

Medicines are drawn from the three great natural kingdoms, and are characterised by various physical and chemical properties: but these characters do not afford sufficiently accurate or definite indications of the actions' of drugs on living bodies, and hence are not of much service in practical classifications. The atomic weights of inorganic elements are of little or no value in determining on what organs or in what way inorganic elements and their compounds act as medicines. The soluble salts of the heavy metals are certainly generally active poisons, though neither similar chemical composition nor similar chemical reaction necessarily confers similar physiological effects. Substances which crystallise in the same form have, however, somewhat similar actions, and on this isomorphous basis the elements have been arranged into nine groups, in each of which it is noted that the intensity of action increases with the atomic weight (Sir Lauder Brunton). The same base, united with different acids, produces salts which exhibit very different actions, as illustrated in the several compounds of sodium and potassium. Equally diverse physiological effects are produced by compounds resulting from conjoining the same acid with different bases. Such irritant 
corrosive substances as caustic soda and sulphuric acid, entering into chemical combination, produce a neutral, comparatively mild saline. Organic, like inorganic bases, are notably modified by the acid radicles with which they unite. Thus, amyl-hydride is an anæsthetic ; when oxygen is introduced, as in amyl-alcohol, or amyl-acetate, spasm is added to the anæsthesia; amyl-iodide notably increases secretion, while amyl-nitrite lessens arterial pressure. It is hence evident that the action of a compound medicine cannot be inferred from a knowledge of the action of the substances that combine to form it. On the contrary, a compound substance exerts special actions of its own, these depending on the proportion of its components, and upon its own physical qualities.

Very important investigations have been made by Crum Brown, Fraser, Schroff and Jolyet, in artificially modifying the chemical constitution, and thus changing the physiological actions of drugs. When strychnine, brucine and thebaine, which act upon the spinal cord as powerful convulsants, are converted by addition of methyl into methyl-strychnine, methyl-brucine and methyl-thebaine, they act upon the ends of motor nerves as paralysants. Indeed, methyl, when combined with other alkaloids, as quinine, morphine, atropine and codeine, renders these also powerful paralysers of motor nerves.

'The study of the natural orders of plants affords some general information as to their physiological actions. Thus, the Ranunculaceæ furnish many acrid irritants, such as aconite, podophyllum and stavesacre. The Solanacer yield narcotics, such as tobacco and dulcamara; while the suborder, Atropaceæ, are paralysers of nerve terminals. The seeds of many Umbelliferæ yield carminative volatile oils. 'These general botanical characters do not, however, afford sufficient data for the accurate classification of drugs. Edible as well as poisonous plants occur in many natural orders. Plants of different orders and genera sometimes closely resemble each other, while plants of the same genus may have very different properties. Thus, one species of Strychnos yields strychnine, which stimulates the motor centres of the spinal cord, inducing tetanic convulsions, 
while another yields curare, which paralyses the peripheral endings of motor nerves. But even the same drúg sometimes yields antagonistic active principles. From opium are obtained the soothing anodyne morphine, the convulsant thebaine, and the emetic apomorphine. Calabar bean yields eserine which depresses, and calabarine which stimulates the spinal cord. Jaborandi yields pilocarpine and its antagonist jaborine, the former stimulating, and the latter paralysing the ends of secretory nerves.

The grouping of medicines according to their actions has not hitherto been of much more practical value than their chemical or botanical classification. The precise actions of many medicines are only now becoming definitely known. Many, moreover, have a variety of actions, and hence have to be included in several groups. Alcohol, for example, is stimulant, irritant, narcotic, and sedative, as well as nutrient, antiseptic, and antipyretic. Opium is narcotic, anodyne, and hypnotic; but it also stimulates certain patients, and tetanises others.

Disregarding the classifications hitherto adopted, students and practitioners will find it.advantageous to study the actions of medicines upon the chief organs and functions of the body :-

\section{Actions of Medicinal Agents.}

I. Local and general actions : absorption and distribution.

II. Elective affinity between drugs and particular tissues or cells : elimination.

III. Effects on different classes of patients.

IV. Modifying influences of Climate and Temperature, Habit, Idiosyncrasy, Disease, and Surroundings, etc.

\section{Curative Systems : Allopathy, Homœopathy.}

On Protoplasm, Blood, and Bacteria.

Antiseptics: Disinfectants: Germicides: Deodorisers : Antiperiodics. 


\section{On the Surface of the Body.}

Counter-irritants : Rubefacients : Vesicants : Pustulants : Caustics.

Setons : The Actual Cautery :

Astringents : Styptics :

Demulcents : Emollients : Diluents.

On Muscles.

Muscular Stimulants : Muscular Poisons.

On the Nervous System.

The Brain. Cerebral Stimulants : Exhilarants.

Cerebral Depressants : Soporifics : Narcotics : Anodynes : Antispasmodics : Anæsthetics.

The Spinal Cord. Spinal Stimulants and Depressants.

Motor Nerves. Stimulants: Paralysers.

Sensory Nerves. Stimulants: Local Sedatives: Local Anæsthetics.

On the Eye and other Special Senses.

Mydriatics dilate the Pupil.

Myotics contract the Pupil.

On the Respiratory Organs.

Errhines or Sternutatories: Respiratory Sedatives: Expectorants : Disinfectants.

On the Circulatory Organs.

Cardiac Stimulants :

„Tonics :

, Sedatives :
Vaso Dilators :

, Constrictors.

On the Digestive System.

The Salivary Glands and Fauces. Sialagogues: Antisialics : Refrigerants.

The Stomach. Gastric Tonics : Stomachics: Antacids : Emetics : Anti-emetics : Gastric Sedatives.

The Intestines. Purgatives: Carminatives: Intestinal Astringents : Antiseptics.

The Liver. Direct Cholagogues : Indirect Cholagogues. Worms. Athelmintics: Vermicides: Vermifuges. 
On the Skin.

Diaphoretics : Sudorifics : Anhydrotics: Parasiticides.

On the Urinary Organs.

The Kidneys : Diuretics.

The Bladder : Lithontriptics : Astringents : Disinfectants :

Sedatives : Tonics.

On the Organs of Generation.

Aphrodisiacs : Anaphrodisiacs .

Ecbolics :

Agents acting on the Mammary Glands.

On Tissue Change and Temperature.

Restoratives : Tonics : Hæmatinics : Alteratives :

Febrifuges : Antipyretics.

Blood-letting.

Poisons and Antidotes.

\section{THE ACTIONS OF MEDICINES}

LOCAL AND GENERAL ACTIONS-ABSORPTION-ELECTIVE AFFINITY BETWEEN DRUGS AND PARTICULAR TISSUES OR CELLS-EFFECTS ON DIFFERENT CLASSES OF PATIENTS -MODIFYING INFLUENCES OF CLIMATE AND TEMPERATURE, HABIT, IDIOSYNCRASY, DISEASE, SURROUNDINGS, ETC.

I. Every medicine is possessed of certain effects or actions on living animals, as distinctive as its colour, taste, or chemical properties. Such actions, when exerted in health, are termed physiological actions; when exerted in the treatment of disease they are termed therapeutic or curative actions. These actions cannot, however, be regarded as twofold or distinct, for the physiological action determines and is merged in the curative results. A horse eats some indigestible food, and in consequence suffers from spasm of the bowels, for which a dose of purgative medicine may be prescribed. The purgative exerts its physiological action by 
increasing intestinal secretion and peristalsis ; the irritant is thus swept away, and spasm and pain are removed. A dose of physic prescribed for a horse with itching and swollen legs produces the physiological effects of emptying the bowels, and clearing the body of irritant waste matters with the curative result of relieving or removing the itching and swelling of the limbs. Hunting horses frequently, after a hard day, have stiff limbs, with puffy joints and tendons ; diligent hand-rubbing and subsequent bandaging mechanically and physiologically stimulate the activity of the local circulation, with the therapeutic effect of restoring the parts to their normal state. All the physiological actions produced by medicines may not be favourable to the curative result desired, but subsidiary, useless, or harmful effects may be diminished or neutralised by judicious selection and combination of remedies. Some medicines are chiefly local and direct in their action. A strong acid applied to the skin irritates and, it may be, destroys it. A hot fomentation or poultice in contact with a painful surface soothes it, and relieves local congestion and pain. The primary action of local irritants is frequently followed by secondary and remote effects. In sore throat the application of a blister directly irritates and inflames the skin, and reflexly, or through the nervous system, it relieves congestion and pain of the respiratory membrane. In horses a large cantharides blister, used for its local effect, may, owing to absorption of the active principle of the fly, occasionally produce febrile symptoms, and congestion of the urinary passages by which the irritant is excreted.

The general effects of most medicines are only produced when they enter the blood, and the more rapidly a medicine enters the circulation the more immediate and powerful are its effects. The short time required for absorption, distribution, action and elimination is well illustrated in the rapidly fatal effects of such poisons as prussic acid and strychnine. Yellow prussiate of potash injected into the trachea was detected two minutes later in the jugular vein (Colin); injected into one of the jugular veins of a horse it appeared in the other in twenty-five seconds, and in a few minutes was exhaled from the mucous and serous membranes 
(Hering). Barium chloride traversed the circulation of a horse in twenty seconds, and injected into the jugular vein of a dog it reached the carotid artery in seven seconds. When the foot of an Albino rat was immersed for a few seconds in chloroform containing one per cent. of atropine, absorption occurred, and dilatation of the pupils followed in from two to five minutes (Waller).

Medicines may be administered by injection into the subcutaneous areolar tissue, the trachea, veins, glands, muscles, rectum, uterus, udder, and large serous cavities; but the most frequent and generally the most convenient mode of administration is by the mouth, whence, speedily reaching the stomach and small intestine, medicines are absorbed and enter the circulation. Administered in a tolerably concentrated but soluble form medicines do not generally require to be acted upon by the ferment-containing secretions of the digestive canal; but the digestion of mashes and many restorative foods is promoted by the alkaline saliva, which also favours the hydration and solution of some drugs. The acid, pepsin-containing, gastric juice dissolves proteids, as well as iron, mercurial and other salts. The alkaline bile emulsifies fats and resins, and activates some agents, such as aloes; the pancreatic fluid furthers digestion of starch, albumin and fats ; and specially refractory substances are more thoroughly reduced by the alkaline intestinal juices. Medicines taken up by the vessels of the gastro-intestinal mucous membrane are conveyed to the liver where they may be stored up, detained for a time, neutralised or modified, eliminated in the bile, or passed unchanged into the general circulation.

Absorption by the healthy buccal membrane is possible, but, excepting the most volatile agents, such as prussic acid, medicines introduced into the mouth are either quickly swallowed or rejected, and consequently contact with its thick epithelium is too brief to permit of penetration. Gastric absorption varies with the species, the age of the animal, and the state of the stomach as regards contents, digestive phenomena, and freedom from disease. Very active in dogs and pigs, gastric absorption in cattle and sheep occurs only in the abomasum. In horses, absorption by 
the stomach has been doubted or denied since Bouley and Colin published the results of their experiments with strychnine. Bouley, after dividing the pneumogastric nerves, administered lethal doses of strychnine without injurious effect. Colin found that after tying the pylorus, large doses of strychnine introduced into the stomach did not poison; but later experiments seem to prove that poisoning does not occur when after an interval the ligature is removed, and the contents of the stomach are allowed to pass into the bowel. Schiff considers that absorption of the strychnine is sufficiently gradual to allow of its being proportionately eliminated in the urine, and that the drug does not accumulate in the blood in sufficient quantity to cause poisoning.

In certain animals (dog, pig, ox, sheep) the empty stomach is supposed to absorb more rapidly than the stomach filled with food: but the empty stomach is less vascular, and its corrugated lining is protected by a thick mucus, which probably retards absorption. Medicines given on a full stomach or mixed with the food are exposed to attenuation and the action of the secretions formed in the digesting stomach, and, if unstable, they may undergo rapid alteration, and fail to produce general effects. Medicines intended to act directly on the stomach should be given to the animal fasting, and nutrients should be given with the food or soon after feeding. In gastric impaction medicine given by the mouth seldom acts with the desired rapidity. It mixes with the ingesta, but owing to the movements of the stomach being either diminished or suspended, the food does not pass into the intestine, or the drug does not reach the absorbent surface in sufficient quantity, and in consequence its action is delayed.

Absorption by the small intestine in all the domestic animals is very active. A strychnine salt injected into the duodenum produces its effects in three or four minutes, and ferrocyanide of potassium similarly used may be found in the blood in five to six minutes (Kaufmann). The cæcum and large colon absorb rapidly, and in cases of tympany treated by puncture advantage may be taken of the canula to introduce medicines directly into the intestine. The 
rectum and floating colon rapidly absorb soluble medicines and nutrients introduced by the anus ; and in gastric disease rectal injection is often preferable to administration by the mouth. Anæsthesia may be induced by chloral or ether thrown into the rectum, but this method is unsatisfactory. To ensure speedy absorption, and to prevent ejection of medicines introduced through the anus, the solution should be warm and concentrated to a few ounces. Larger quantities will be retained and absorbed, but the injection should not be bulky if immediate effects are desired.

The respiratory tract furnishes probably the most actively absorbent mucous surface in the body. Long used for anæsthetic inhalations, it is occasionally employed for the administration of remedies in cases of purpura hæmorrhagica and parasitic bronchitis. Soluble drugs, introduced by insufflation, are absorbed by the nasal lining, and medicines in aqueous or alcoholic solution, not too concentrated, are well borne and very rapidly absorbed by the tracheal, bronchial, and pulmonary mucous membranes. Drugs dissolved or suspended in oil are less tolerated, although Levi, the chief authority on intratracheal administration, maintains that small injections of oil are absorbed. Emulsions, however, are safer, so long as the quantity is small and injection is made slowly. Intratracheal injections are especially risky in bronchitis and pneumonia. Beyond a slight gain in time, intratracheal injection has no advantage over hypodermic administration, except in cases where direct or local action is required (Guinard).

Although seldom employed in practice for the administration of medicines, the female generative tract-the uterus, especially after parturition, absorbs very rapidly. Potassium iodide has been found in the urine in from two to four minutes after injection into the womb. The healthy vagina absorbs very slowly, but when congested its absorbent power is much increased. Absorption from the lining of the galactophorous sinus of the cow's udder is fairly active. The absorbent power of the conjunctiva is well known. Cocaine appears to promote conjunctival absorption of solutions of atropine, eserine, and strychnine, and of other drugs.

The peritoneum, pleura, and the synovial membranes of 
joints and tendons absorb very rapidly. Potassium prussiate injected into the peritoneal cavity was found six minutes later in the urine; and strychnine similarly used caused death in less than four minutes. Anæsthesia can be readily induced in dogs by intra-peritoneal injection of chloral and morphine.

Drugs, even when volatile, pass very slowly and imperfectly through the unbroken skin, except when applied with the assistance of an electric current (cataphoresis and dielectrolysis), but absorption readily occurs from open wounds and abraded skin surfaces. Clean-cut fresh wounds, free from bleeding, absorb more rapidly than wounds which are irregular, bruised, or bleeding. Absorption by granulation tissue is less active, though occasionally undesirable effects follow the careless application of toxic antiseptics to wounds in process of healing. Drugs incorporated with oil, lard, or lanoline, penetrate the skin more readily than do ointments made with vaseline. Absorption of aqueous solutions is hindered or prevented by the sebaceous matter, but salts which are dissolved by the secretion of the skin may produce general effects. Experiments prove that agents which are soluble in sebaceous matter, or which emit during their application vapour or gas, as guaiacol, alcohol, benzine, potassium iodide, mercurials, carbolic acid, etc., pass more or less rapidly through the skin. But the intact epidermis of the horse, ox, and dog, offers considerable resistance to penetration ; and in practice, lotions, liniments, or ointments carefully used seldom endanger life. Slow absorption may occur, but owing to more rapid elimination the drug does not accumulate in sufficient quantity to excite toxic symptoms. The subcutaneous areolar tissue absorbs nearly as rapidly as the respiratory mucous membrane. Hypodermic injections, properly prepared, are promptly taken up unchanged by the blood-vessels and lymphatics.

II. Between certain organs, tissues, or groups of cells, and certain medicines, there appears to be a special elective affinity. A medicine absorbed and in circulation, owing to its chemical affinity, becomes arrested in contact with certain cell groups, where it fixes itself and tends to replace some of the normal nutritive elements. From the common 
stream of blood each tissue takes up its appropriate nutrient materials, and, in like manner, it appears to select its own medicines. The characteristic effects are not developed until medicines come into actual contact with the special organs, or, it may be, the particular cells, on which alone they operate. Curare does not exert its paralysing power until it reaches the intramuscular endings of the motor nerves. Magendie found that strychnine does not excite its notable tetanic convulsions until it is in contact with the spinal cord. Indeed, when a frog or other small animal, immediately after receiving a full dose of strychnine, has the spinal cord removed or destroyed from above downwards, tetanic symptoms are prevented in the same order.

On the particular part on which they act-as, for example, on the nerve-centres or nerve-endings that control bloodvessels or glandular secretions-some medicines exert stimulant, others depressant or paralysing effects. These effects, as already indicated in the case of drugs acting locally, frequently produce reflexly indirect or remote effects. The same medicine sometimes acts differently when given in different doses. Thus, alcohol and ether in small doses are stimulants, but in large doses are depressants.

Within the living body most medicines not only effect changes, but themselves coincidently undergo changes, notably of oxidation or deoxidation. Thus, many salts of tartaric, acetic, and other organic acids are converted into carbonates. Morphine has its chemical constitution altered, and in an animal habituated to its use, it is all broken down by the tissues so that none is excreted, and none can be found by analysis on post-mortem. It has, in fact, been destroyed. The activity of medicines depends materially on their solubility, the rapidity of their absorption, and the period during which they remain within the body-in other words, on the speed of their excretion. Some drugs, as lead, mercury, silver, and digitalis, are apt to be retained for a considerable period, and hence have more or less continuous or cumulative effect. Unusual activity of such excreting channels as the bowels or kidneys hurries most medicines out of the body, and hence diminishes their action. 
In a variable but usually short period, medicines, generally in a modified form, are eliminated by the bowels, kidneys, skin, salivary and mammary glands, or pulmonary mucous membrane. Digitalis, for example, after exerting its action mainly on the heart and arterioles, is removed by the kidneys. Alcohol and its analogues are got rid of by the skin and kidneys, and also pass away through the respiratory mucous membrane. During their excretion medicines exert their in-contact effects on the excretory organ and passages. Thus, aloes and neutral salts, after stimulating the secretion and movements of the bowels, are in part absorbed into the blood, and thence are returned into the bowels, causing further purgation. Nitre, and small doses of salines and ethers, chiefly removed through the kidneys, produce diuresis. Terebene and various balsams during their excretion by the pulmonary membrane or urinary passages exert their antiseptic properties.

\section{The several species of veterinary patients are differ-} ently affected by many medicines. These differences, however, are in degree rather than in kind, and depend upon differences in organisation and function. On the circulatory, respiratory, and urinary systems, which nearly resemble each other in man and the domestic animals, medicines act tolerably uniformly. Thus, aconite, digitalis, and nitre, produce very similar effects in men, horses, dogs, and cattle. Greater diversity, however, occurs in regard to medicines acting on the nervous, digestive, and cutaneous systems, which differ considerably in the several species of animals. Rabbits and monkeys seem to possess a special resistance to the action of atropine. Apomorphine, which promptly causes vomiting in dogs, has no emetic action on the pig (Feser). Morphine is an excellent hypnotic for the dog; but in the cat and pig it causes excitement and convulsions.

The more highly any organ or system of organs is developed the more susceptible does it become to the action of medicines, and, it may be added, to diseases also. This general law explains why the highly-developed human brain is specially susceptible to the effects of such cerebral medicines as opium and chloral, and why frogs, whose spinal 
system is better developed than their brain, are so susceptible to strychnine, which acts specially on the cord. The human cerebrum, the seat of intelligence, is more than seven times the weight of the mesencephalon and cerebellum. In the domestic animals the cerebrum is only five times the weight of the posterior parts of the brain, whilst the cord is relatively larger than in man. These differences of development explain how such medicines as opium, chloroform, and chloral cause in man blunted intellectual function and deep stupor, while in the lower animals, with less marked depression of brain function, they conjoin more marked deranged motor function and convulsions.

The Horse has a small stomach, and capacious, highlyvascular intestines, adapted for absorption of nutriment from bulky vegetable food. Nearly two-thirds of the water in the ingesta pass off by the bowels, while in man only five per cent. is removed by this channel, and the amount is still less in dogs and cats. Vegetable purgatives, notably aloes, appear more suitable than mineral purgatives. Except in very rare diseased states, attempts at vomition are not easily excited in horses. Tartar emetic, of which a few grains cause immediate emesis in dogs, has no such physiological effect on either horses or cattle. According to some authorities, this insusceptibility of the horse to the action of emetics is ascribable to an inaptitude of the vagus nerve to receive and convey the special irritation, but more probably it is due to imperfect development of the vomiting centre. Actual vomition in horses is hindered by the small stomach not being readily compressed between the diaphragm and abdominal muscles, and by the stout band of muscular fibres which surrounds its œesophageal opening. The many sedatives available in human and canine practice operate uncertainly and imperfectly on horses. The kidneys of horses are easily stimulated; in ordinary circumstances they remove about one-seventh of the fluid ingesta, while the same organs in man drain away 54 per cent., and in dogs nearly 50 per cent. of the fluid discharges. Sudorifics are less prompt than in man, and are apt to act on the kidneys, unless the animal be well clothed.

In Cattle the peculiarities of the action of medicines are 
chiefly referable to the construction of their alimentary canal, and to their phlegmatic temperament. The stomach of these ruminants is quadrisected, is extensively lined with cuticular mucous membrane, and, as regards its first three divisions, is less vascular, and in function is less chemical and more mechanical than the corresponding portion of the alimentary canal of men, dogs, or horses. The first and third compartments always contain food, often in large quantity. These facts explain why cattle require large doses of all medicines, why considerable quantities of irritant and corrosive poisons can be given them with comparative impunity, and why purgatives, even in large doses and in solution, are so tardy and uncertain in their effects. Several times a day, for about an hour at a time, in animals of this class, the solid food is returned from the first and second stomachs for more thorough mastication and insalivation. Imperfect or suspended rumination is a cause, as well as a common effect of stomach disorders in cattle. Their kidneys and skin are less easily affected than the corresponding organs in horses ; and their dull, phlegmatic disposition resists the action of both stimulants and tonics. It is a prevalent notion that medicines, when poured slowly down a cow's throat, pass, like the ruminated food, direct to the fourth stomach. But experiments made at slaughterhouses on both cattle and sheep, prove, however, that neither animal can be induced to exert this voluntary effort in behalf of medicines, which in all cases, no matter how slowly they are administered, fall partly into the first and second stomachs, whence they shortly pass onwards through the third and fourth stomachs, especially if given, as they always ought to be, with a considerable quantity of fluid.

Sheep closely resemble cattle in the way in which they are affected by most medicines ; they usually require about one-fourth of the dose suitable for cattle. This animal is best drenched by being backed into a corner, its head being steadied between the operator's knees, while the medicine is cautiously poured into its mouth.

On Dogs medicines generally operate much in the same way as on man; but to this rule there are some remarkable 
exceptions. Dogs, for instance, take six or eight times the dose of aloes usually given to human patients, but are seriously injured by half as much calomel or oil of turpentine as is prescribed for a man. The opinion generally held, that medicines may be given to dogs in the same doses as to man, cannot therefore be safely entertained without many reservations. In dogs, while the stomach is relatively large, the alimentary canal is short and straight, and purgatives consequently act with greater rapidity than in other veterinary patients. Another peculiarity is the facility with which they can be made to vomit. Indeed, vomition in dogs is often produced by their eating various grasses, by their swallowing nauseous or unpalatable matters, or by their overloading the stomach. The kidneys are excited with more difficulty than in horses or cattle. On Pigs the actions of medicines are similar to their actions on men and dogs.

Sir Lauder Brunton points out several curious differences in the actions of drugs on several of the lower animals. Morphine convulses frogs, but, even in large doses, has no effect on pigeons, except in reducing their temperature. Belladonna quickens cardiac action in man, dogs, and horses, by paralysing the vagus, which controls or restrains heart action. But in rabbits the vagus has hardly any appreciable effect in regulating the heart-beats, and these animals accordingly take large doses without having the rapidity of the circulation increased. The rabbit's heart not being controlled by the vagus, a marked difference also occurs in the action of amyl-nitrite on rabbits as compared with dogs. Such observations are not only most interesting in themselves, but greatly further the understanding of the actions of drugs.

The action of medicines is influenced by the age of the patient, and as a rule very young animals are more susceptible than adult animals. Young dogs are much 'more easily affected by morphine than adult dogs. Kittens, however, seem to be able to withstand larger doses of morphine than fully grown cats (Guinard). The young tolerate chloroform anæsthesia better than the old, and poisons such as strychnine and digitalis may be given in larger doses to young animals than to adults. 
IV. Climate and Temperature modify considerably the actions of medicines. Heat increases the power of antiseptic solutions. Narcotics are generally believed to act more powerfully in warm than in cold climates. This fact, as well as other differences in medicines observed in hot as compared with cold climates, may depend upon slight differences in animal temperature, and in the varying amount of excretion effected by the skin and kidneys.

Moderate warmth favours chemical reactions and protoplasmic movements - two conditions intimately connected with the actions of medicines. 'Alexander von Humboldt first observed that warmth not only acted as a stimulant to the heart, increasing the power and rapidity of its contractions, but noticed that warmth increased the rapidity with which alcohol destroyed the irritability of a nerve, and potassium sulphide that of a muscle. . . Many, if not all, muscular poisons act more quickly with increased temperature. . . Rabbits poisoned with copper or potassium salts also die more quickly when placed in a warm chamber than when left at the ordinary temperature' (Brunton). On the other hand, however, narcotic poisoning by alcohol or chloral is retarded when the animals are in a warm atmosphere.

Habit.-The continued use of a medicine sometimes alters the degree of its action. Caustics and irritants, which exercise only topical action, exhibit, on their repeated application, gradually increasing activity. But many medicines, when continuously administered, have their ordinary power considerably diminished. Thus, arsenic-eaters sometimes use with perfect impunity twelve or fifteen grains of arsenic daily-a quantity sufficient to poison three or four unhabituated persons. A like tolerance is observable among horses which have been accustomed to receive arsenic. Opium, and most general stimulants, when administered for some time, gradually lose their effects. Virginian deer, from habit, are said to thrive on tobacco; some monkeys, feeding on strychnine-containing nuts, are stated to become insusceptible to strychnine (Wood). The tolerance thus induced by the habitual use of a medicine occasionally depends on retarded absorption or quickened excretion; sometimes, as in the case of many alkaloids, on the liver 
acquiring greater power for the detention, modification, destruction or excretion of the drug, while frequently the tissues, by repeated contact, become progressively accustomed to its influence, and more or less resistant.

Individual Idiosyncrasies, which in the human subject render some poisons almost innocuous, and some simple medicines deadly poisons, are less frequent and notable among the lower animals. Those of most frequent occurrence among veterinary patients are either an increased or a diminished susceptibility to the action of purgatives and diuretics. Most medicines act with greater certainty and effect upon-well-bred animals, whether horses or dogs, than upon coarsely-bred mongrels. The prescription even of a blister or a colic draught demands consideration of the temperament, breeding, and condition of the patient.

Diseases modify the action of many medicines. - Altered structure and functions, and increased temperature occurring in most serious disorders are important modifying factors. A congested or inflammatory condition of the alimentary canal, or even an overloaded stomach, retards absorption, and the consequent activity of medicines given by the mouth. Acute fever, on account of increased arterial pressure, is also unfavourable to absorption. When excretion is hindered, medicines, however, are usually retained longer in the system, and some accordingly act more powerfully. Conversely, when excretion is active, as in diuresis, diabetes, or diarrhœa, such medicines as opium, belladonna, and alcohol, being rapidly got rid of, do not manifest their full activity. Influenza, low fevers, and any considerable inflammation of mucous or cutaneous surfaces, withstand reducing remedies badly, and require for their successful treatment the early exhibition of restoratives, tonics, and stimulants. Even the comparatively slight and temporary requirements for the changing of the coat render horses in spring and autumn notably less enduring and less able to stand lowering treatment. Full doses of sedative medicines induce less depression in acute inflammation than in health ; large quantities of opium and chloral hydrate have comparatively slight effect in tetanus, enteritis, or other painful disease ; while excessive doses of purgatives and stimulants 
are well borne in parturient toxæmia of cattle, and in other cases in which there is depression of nervous force.

The surroundings of the patient materially alter the action of remedies. Diseases, whether in horses, cattle, or dogs, occurring in large towns, and in filthy, overcrowded, and badly-ventilated premises, are notoriously liable to assume chronic and untoward forms, and are less responsive to curative measures. Medioines can only act effectually when seconded by proper sanitary arrangements. Overheated, imperfectly ventilated stables lower the vitality of their inmates, by retarding excretion, and favouring absorption of noxious exhalations. Such facts demand consideration alike in the treatment and prevention of disease. Frequently a horse with influenza, bronchitis, or pneumonia, is thrown back for days by being stripped and taken out of his box in cold weather. One meal of coarse, indigestible food, even of moderate amount, sometimes retards recovery from gastric derangements, and, indeed, from most debilitating diseases. Constipation or torpidity of the bowels interferes.with the absorption, and hence with the satisfactory operation of all medicines. Exposure to cold seriously injures patients which have received full aperient doses of salts or of turpentine, or which have been freely dressed with mercurial ointment. Foul air and disordered digestion retard the healing even of simple wounds. On the other hand, gentle exercise encourages the action of most eliminatives; quiet favours the effects of soothing remedies; generous diet considerably aids in the benefits of restoratives, tonics, and stimulants. Actively depleting treatment, as by purgatives, is more easily borne in winter than in summer, in the country than in the town, in well-bred animals in good condition than in coarser subjects which have been indifferently nourished.

CURATIVE SYSTEMS : ALLOPATHY, HOMEOPATHY

The difficult question is frequently asked-How do medicines cure disease? Endeavour is made, guided by biological laws, to adjust or restore to harmony any irregularity or deviation from health which may have affected 
the organism or any of its parts. The complex composition and diverse functions of the bodies of the higher animals are liable, however, to be altered and disturbed in many different ways, and such alterations or aberrations cannot be restored by any one curative system or formula. As already indicated, medicines have special actions on different organs or groups of cells, and affect them in very different ways, and hence would seem to produce their curative effects, not in one, but in many ways. There have been from the very earliest times, before the causes of disease were known or the actions of drugs understood, innumerable systems of treatment. A very ancient one was 'the doctrine of signatures,' which taught among other things that the external shape or contour of a plant was suggestive of its use in medicine, and so we still have such plant names as liverwort and lungwort. Galen came later and enunciated his celebrated hypothesis, but it was as fantastic and unreasonable as the last. Then the doctrine 'Contraria contraribus curantur' came into vogue, and of course still obtains to a considerable extent, e.g. constipation is cured by a purgative, diarrhœa by an astringent. Two such systems of counteraction have been propounded-(1) the antipathic, whereby medicines were believed to overcome morbid conditions or symptoms by a superior and antagonistic force ; (2) the allopathic, whereby effects are produced which, although they may sometimes be unnatural, overcome the disease. But diseases, it has been affirmed, may not only be cured by counteractions, but by similars. Upon the old saying that ' like cures like,' Homœopathy ${ }^{1}$ is based,

${ }^{1}$ Homøopathy ( $8 \mu_{0} \circ o s$, homoios, like or similar; and $\pi \dot{\theta} \theta 0 s$, pathos) was propounded by the German physician Hahnemann in his Organon der rationellen Heilkunde, published in 1810. This system teaches that the cure of a disease is effected by infinitesimal doses of such medicines, as would induce, if given to a healthy subject in large quantity, symptoms similar to the disease. Cinchona is declared to cure such fevers as ague and intermittents, because it produces some such febrile symptoms when given to healthy individuals in considerable doses; aconite is regarded as the appropriate remedy for reducing inflammatory fevers, because in large doses it produces symptoms which are thought, by homœopathists, to resemble those of inflammation; while strychnine is selected as a remedy for paralysis, because in large doses it appears to produce paralytic symptoms. This doctrine, if sound, would stamp most disorders as hopelessly incurable; for 
and its votaries declare that diseases are to be treated by small doses of such medicines as in large doses produce symptoms similar to the disease to be cured. The pathological conditions which underlie and produce the symptoms, and which a rational cure generally aims if possible at removing, are ignored. The homœopathic dictum of similia similibus curantur does not bear investigation; at best it is only capable of narrow and occasional acceptance. The symptoms of ague and intermittent fever are certainly similar to those produced by cinchona bark, which is an accepted cure for ague, and the illustration on which

it is only in a few exceptional cases that any similarity can be detected between the symptoms produced by large doses of the remedy and those of the disease for which it is given. No known medicines, for example, are capable of developing symptoms such as those of thick-wind, roaring, pleurisy, strangles, distemper, or rabies, yet fifteen or twenty remedies are prescribed homœopathically for each of these diseases.

Mr. Dudgeon's translation of the Organon of Medicine states that, 'the symptoms of each individual case of disease must be the sole indication, the sole guide to direct us in the choice of a curative remedy.' Now, symptoms, although sometimes requiring special treatment, are but the visible signs and results of derangement and disease; whilst their removal, which is all that is aimed at in homœopathic treatment, does not always ensure the removal of the conditions on which they depend. Thus, rheumatism, pleurisy, enteritis, worms, and many other disorders, frequently remain unchecked after the symptoms have been relieved. Instead of thus vainly attempting the removal of symptoms, it were therefore more rational to remove at once the morbid condition-the source of the evil. No curative system directing its efforts, as homœopathy does, merely against the symptoms of disease, can ever rest upon a safe or scientific basis; for it is notorious that, under varying modifying influences, the same diseases sometimes induce very dissimilar symptoms, and would consequently, according to this system, require dissimilar treatment. On the other hand, diseases essentially different sometimes manifest similar symptoms. Thus, stupor and vertigo result sometimes from an excessive and sometimes from a deficient quantity of blood sent to the brain; difficulty of breathing from too much as well as from too little blood circulating through the lungs; vomiting from irritation of the stomach, or from irritation of the vomiting centre; diarrhœa from crudities in the alimentary canal, or irritant matters in the blood.

Not only are the principles on which homœopathy is said to be based untenable, but the details of the system are inconsistent and ridiculous. The homœopathic doses are so small that they are of ten incapable of detection either by the microscope or by chemical analysis, and are sometimes so inconceivably minute that the mind can form no idea of them. It is admitted even by homœopathists, that millions of such doses may be swallowed by a healthy individual without inconvenience: but in disease the body is stated to become so susceptible to their action that much risk 
Hahnemann founded his system. But many diseases exhibit no symptoms accurately similar, as the homœopathists insist they should be, to those produced by the medicine prescribed for their cure. Numerous drugs, moreover, cause symptoms wholly unlike those of the diseases in the treatment of which homœopathists use them. The homœopathic selection of so-called appropriate remedies, on the presumption that 'like cures like,' is based upon a fallacy, while the minute, finely triturated, and subdivided doses are too attenuated to affect veterinary patients. The practice of homœopathy has, however, developed wholesome discussion, has suggested some useful

is incurred by their insufficient dilution! Medicines such as charcoal, sand, and calcium carbonate, which, in doses of several drachms, have only slight mechanical effects, when given in fractional parts of a grain are thought to produce very powerful effects, and cause many hundred symptoms. The extraordinary powers supposed to be conferred on these and other medicines, even when given in doses of inconceivable minuteness, are chiefly ascribed to the magic influence of careful and continued triturations and often-repeated shakings, performed according to most precise directions. Some homœopathic authorities declare that there is little difference of activity between different dilutions of the same medicine; and it is said that, if the medicine be well selected, it matters little whether the tenth, hundredth or thousandth of a grain be used (Gunther and Haycock). There is probably some truth in this observation, for, with most medicines, especially when administered to the lower animals, all the dilutions mentioned would be equally harmless. But homoopathists assert that, in spite of the errors which their opponents discover in the system, it is nevertheless very successful in the cure of disease. In judging, however, of homoopathy as a system of practical medicine, it must be regarded as made up of two distinct parts:-1st, The original and peculiar part of the system, consisting in the use of medicines selected in accordance with a law embodied in the axiom similia similibus curantur, and administered in infinitesimal doses, usually varying from one grain to one-millionth of a grain, and carefully prepared according to certain precise directions ; and 2nd, Attention to diet and regimen - the only effectual and rational part of homœopathy, the true source of all its boasted cures, and that department of medical treatment which has always been insisted upon by rational and successful practitioners, of human and veterinary medicine. The value of medicines given homœopathically has never been satisfactorily shown, and never can be until two series of cases, as nearly as possible alike, be treated-the one in the usual homoopathic fashion, the other with the same attention to diet and regimen, but without the globules. In comparative experiments, made at the Edinburgh Veterinary College, in the treatment of pleuro-pneumonia and other diseases, it appeared that those cases treated by diet and regimen alone were as speedily and effectually cured as those treated with the globules in addition, so long as the globules were given only in homøopathic doses. 
experiments on the actions of medicines, has helped to show the evils of heroic and indiscriminate drugging, has taught the advantage of simple prescriptions, exemplified the power of nature to cure, when not too much interfered with, and demonstrated the powerful influence of diet and regimen in the successful treatment of disease.

ACTION OF MEDICINAL AGENTS ON FERMENTS, BACTERIA, AND THEIR PRODUCTS

ANTIZYMOTICS-ANTISEPTICS-DISINFECTANTS-GERMICIDES

-DEODORANTS-PARASITICIDES

Many agents inhibit or retard the action of ferments, and some effectually destroy them. These agents have been designated :-

Antizymotics.-Substances which arrest fermentation. They act chiefly in two ways:-(1) Chemically, by direct or indirect oxidation. Chlorine, iodine, bromine, and potassium permanganate act in this way. (2) Arresting proliferation or catalysis of ferments; such is the mode of action of corrosive sublimate and many other metallic solutions, of carbolic, boric, salicylic, and benzoic acids, and of temperatures above $200^{\circ}$ Fahr. Antizymotics include antiseptics and disinfectants, and nearly allied to these are deodorants.

ANTISEPTICS are remedies which arrest putrefaction. They kill or prevent the development of those bacteria which produce septic decomposition. The action of antiseptics seems to prove that living tissues differ in degree only from dead tissues and their disintegration products. Antiseptics such as ether, chloroform, carbolic acid, and toluol, differ from mercury, silver, and similar metallic antiseptic substances in that whilst they kill bacteria readily, the enzymes formed by these bacteria can carry on their work almost to the full in the presence of saturated aqueous solutions of these germicidal substances. As a whole, however, Vernon maintains ' that there is no sharply defined demarcation between the reaction of living organisms and that of enzymes.' 


\section{Disinfectants or germicides destroy the specific poisons} of communicable diseases. Their special function is to kill, or arrest the development of, those germs or bacteria which produce disease.

DEODORISERS or deodorants destroy smells. Some of the most disagreeable smells, and those most injurious to the higher animals, result from putrefaction, hence their cause is removed by effectual antiseptics. Smells consisting mainly of sulphuretted hydrogen are neutralised by chlorine; those from ammoniacal gases by hydrochloric and nitric acids. Offensive odours may also be attracted and absorbed by freshly-burnt charcoal or dried earth.

Enzymes or separable ferments generally have their action arrested or are destroyed more readily than the organised ferments, amongst which there is much difference in viability. Microzymes may be as sensitive, but usually are not so sensitive, to the action of antiseptics as bacilli. The bacillus anthracis is more easily killed than some others. The spores of all species are specially resistant, and for their effectual destruction require prolonged exposure to tolerably strong solutions of potent antiseptics.

The action of watery solutions of various drugs on the several enzymes has been carefully examined by Wernitz, and his experiments are corroborated and quoted by Sir Lauder Brunton. Corrosive sublimate stands pre-eminent in the certainty of its effects, even in very diluted solution. The action of emulsin was arrested by 1-65,000th part, of diastase by 1-50,000th part, of ptyalin by 1-52,000th part, of pancreatin by 1-21,600th part; but it took 1-1766th part to arrest the action of pepsin, and 1-720th part to arrest that of rennet. Minute quantities of copper sulphate; chlorine, iodine, and bromine, and also bleaching powder and sulphurous acid, readily destroy these organic ferments. Salicylic and benzoic acids and chlorinated lime are also effectual, usually in proportions of about 1-1000th part. Borax is effective generally with 1 to 100 , although 1-3580th part arrests the action of the intestinal ferment invertin. Aluminium acetate, carbolic acid, and glycerin in the order mentioned are weaker still. Chloroform, thymol, eucalyptol, and mustard oil have little, if any, action, even in 
saturated solution. A temperature over $125^{\circ} \mathrm{Fahr}$. weakens or destroys the enzymes.

Drugs act differently on different ferments. While 1-52,000th part of corrosive sublimate, as already indicated, arrests the action of ptyalin, 1 part in 720 is needful to arrest the action of rennet, which is, however, destroyed by 1-1000th part of borax, 1 part of which in 100 is required to destroy ptyalin. For destruction of rennet, bromine and chlorinated lime are specially effective. Creosote, although it has small effect on the enzymes, in solution of 1 part to 500 destroys yeast cells, and, in solution of half that strength, kills bacteria. The mould fungi are destroyed by the same agents which kill yeast and bacteria.

Bacteria of different sorts exhibit some differences in their susceptibility to different antiseptics; indeed, the susceptibility somewhat varies in the same bacteria when raised in different media. It is more easy to prevent than to arrest development of bacteria. As already pointed out, the spores have much greater resisting powers than the fully-developed bacteria. The fully-developed bacteria are destroyed by exposure for an hour to a temperature of $150^{\circ}$ to $168^{\circ} \mathrm{Fahr}$, but the spores require more prolonged exposure at $212^{\circ} \mathrm{Fahr}$. Moist heat, having a greater power of softening and penetrating the spore envelope, is more effectual than dry heat. Milk containing the bacillus of tubercle or other specific disorder may be rendered innocuous by five minutes' boiling.

Experiments bearing on the power of different substances to prevent the development of bacteria in various stages and solutions, to kill them, or to arrest development and reproduction of their spores, have been carried out by many good observers. The plan of procedure has generally been to add to carefully prepared sterilised fluids in test tubes, known quantities of the disinfectant to be tested, and then introduce a drop of liquid containing bacteria or their spores. Such experiments show that the agents which most promptly and effectually arrest the action of enzymes also prove the most destructive to bacteria. Of corrosive sublimate, 1-5805th part kills the developed parasite; 1-25,258th part prevents the development of bacteria taken from 
meat infusions; but it requires a strength of 1 in 2525 to prevent reproduction of spores in unboiled meat infusion, and still stronger solutions to penetrate and kill the spores. Chlorine, chlorinated lime, bromine, iodine, and quinine stand next in activity. Sodium sulphite is about 1-10th the strength of quinine. Compared with corrosive sublimate, it requires twenty times the amount of thymol, salicylic acid, or potassium permanganate to prevent development of bacteria, and sixty times the amount to prevent reproduction of spores. Sodium hyposulphite has very little action. Carbolic acid did not stand so high as expected. The fresh blood of an animal just dead from anthrax must be mixed with its own bulk of a 1 per cent. solution in order to destroy the bacilli, and enable it, without harm, to be injected into another animal. A half per cent. solution fails to destroy the bacilli.

Koch's experiments with anthrax spores constitute reliable tests of the value of disinfectants. Solutions of the several substances, of specified strength, were placed in tubes, their mouths stopped, as is generally done, by cotton wool ; and threads steeped in fluids containing bacilli and their spores were carefully introduced. Some of these threads were removed from day to day, and subjected to microscopic examination. Even after one hundred days' exposure to the antiseptic, some threads still exhibited bacilli. Chlorine water, freshly made, bromine 2 per cent. solution, iodine 1 part in 7000 , corrosive sublimate 1 per cent. in water, were found effectually to destroy these anthrax spores with which they had been in contact one day. Formic acid, specific gravity 1120, destroyed all spores after four days' exposure. In five days all spores were killed by 5 per cent. watery solutions of chlorinated lime and ferric chloride. One per cent. of arsenic in water, and the same proportion of quinine in acidulated watery solution, were effective in ten days. Oil of turpentine took five days, ether thirty days. The results with carbolic acid were disappointing; a 1 per cent. solution had not much effect on the spores, even when exposure was prolonged for fifteen days ; and a five per cent. solution was required to secure their destruction in one day. Like salicylic, boric, 
and benzoic acids, sodium chloride, and many metallic solutions, carbolic acid was thoroughly effectual in destroying microzymes which had not formed spores, and from its volatility it is frequently more serviceable than fixed antiseptics. The infected threads, subjected to the influence of even the least active of those antiseptics, produced only scanty and retarded crops of bacilli. Such reliable antiseptics as corrosive sublimate, bromine, and iodine, when dissolved in alcohol, ether, or oil, were not nearly so effectual as when dissolved in water.

The following table by Miguel indicates the minimum quantity of each antiseptic required to prevent putrefaction of a litre of neutralised beef bouillon :-

Hydrogen peroxide gm. 0:05 Mercuric chloride gm. 0.07 Silver nitrate . . gm. 0.08 Iodine . . . . gm. 0.25 Copper sulphate - gm. $0 \cdot 90$ Salicylic acid . . gm. 1.00 Potassium bichromate . . . gm. $1 \cdot 20$

Zinc chloride . . gm. $1 \cdot 90$ Carbolic acid . . gm. $3 \cdot 20$ Potassium permanganate . gm. $3 \cdot 50$

Tannin . . . gm. $4 \cdot 80$ Arsenious acid . gm. $6 \cdot 00$ Boric acid . . . gm. $7 \cdot 50$ Sodium salicylate $\mathrm{gm} .10^{\circ} 00$ Sodium borate . gm. $70 \cdot 00$ Barium chloride . gm. $95 \cdot 80$ Alcohol . . . . gm. 95.00 Potassium iodide gm. $150 \cdot 00$ Sodium chloride . gm. $165 \cdot 00$ Glycerin . . . gm. 225.00 Sodium hyposulphite. . . gm. $275 \cdot 00$

According to Christmas and Respaut, the best mixture, as regards solubility and antiseptic power, consists of carbolic acid, grammes 9 ; salicylic acid, gramme 1 ; lactic acid, grammes 2 ; and menthol, gramme 0.10 ; employed in solution of one to four per cent.

The uses of antiseptics.-In surgery, zinc and iron chlorides, pitch and tar, tinctures of myrrh, benzoin, and other balsams have long been used empirically both in human and veterinary practice. But Lord Lister has explained and systematised the antiseptic treatment, and rendered it both certain and successful. He studied fermentative processes, and the analogous actions of microorganisms on living tissues; and in 1867 inaugurated the aseptic treatment of wounds. Two conditions, he insists, 
are essential in dealing with wounds, whether accidental or made by the surgeon: (1) Nothing septic must be left in them ; and (2) Nothing septic must be allowed to get into them. Acting on these principles, wounds which for an hour or two have remained exposed, and into which the ubiquitous dust-particles and organisms have been introduced, should first be thoroughly cleansed of mechanical irritants, and then washed, and, if need be, syringed with hydrogen peroxide or a watery solution of corrosive sublimate, zinc chloride, or carbolic acid. The wound thus rendered aseptic, and the removal of discharges, if need be, provided for, it is to be protected from access of organisms by antiseptic dressings. Wounds become septic not so much from the germs found in the atmosphere as from those found on the hands of the operator.

Any wound from a clean knife, or howsoever produced, if it is perfectly aseptic, is as quickly as possible covered with the dressing. This consists of lint, tow, oakum, or other such absorbent substance, saturated with an effectual germicide solution of carbolic acid, which has the advantage of being volatile, of perchloride or biniodide of mercury, or of boric or salicylic acid. These dressings are removed daily, or as required, and are continued till healthy granulations cover and protect the wound. Further, to prevent wounds from being inoculated by septic germs, the knives, probes, ligatures, sponges, drainage tubes, and other appliances used in connection with them, should lie in an antiseptic solution until required, while the operator, whose hands should be most scrupulously clean, must repeatedly moisten his hands with a similar solution.

Wounds kept strictly aseptic heal quickly; if otherwise properly treated inflammation rarely appears, inasmuch as the conditions determining irritation are avoided ; pain consequently is slight and of brief duration; suppuration does not occur, its organismal causes being excluded ; blood-clots are preserved from harmful decomposition, and are gradually replaced by new tissue; portions of damaged structure without hurt are removed; septicæmia cannot occur when micro-organisms and their products have been destroyed or prevented from entering the protected wound. Serious 
operations, such as opening the chest or abdomen, exposing joints and tendon sheaths, can be undertaken with greatly diminished risk when proper antiseptic precautions are adopted.

Carbolic acid is the antiseptic most frequently used in veterinary surgery. Its volatility carries it into the recesses of wounds, and thus in many cases increases its efficacy. It is applied, according to circumstances, in the proportion of one part dissolved in twenty to forty of water; but besides watery solutions, liniments and antiseptic lints are used. It is employed alone or in conjunction with corrosive sublimate. As an effectual non-poisonous antiseptic and parasiticide, Fröhner and other German authorities strongly recommend creolin, which is a derivative of coal-tar. Salicylic acid destroys some ferments, but is not volatile; it is used especially for the dressing of ulcers and eczema. Boric acid is non-volatile, non-irritant, and is often serviceable for superficial wounds, where frequent dressings are unnecessary, where the more active carbolic acid has for some time been persevered with, and where granulations and growth of skin require encouragement. The watery solution of sulphurous acid is cheap and effectual. Chlorinated soda solution is sometimes used. Sanitas, which consists of camphoraceous bodies and peroxide of hydrogen, is a non-poisonous and valuable antiseptic. Iodoform readily parts with its 90 per cent. of iodine, conjoins antiseptic and local anæsthetic actions, and stimulates granulation. It is applied to ulcers and chronic foul wounds. Such poisonous dressings require, however, to be used with some care for dogs, as they are liable to lick them. Iodine tincture undiluted or diluted according to requirements, proves a useful stimulant and antiseptic ; zinc chloride, chinosol, lysol, izal, formalin, naphthol, and thymol are also employed.

Antiseptics used internally are neither so certain nor so effective as when used externally. Bacteria within the living body are not easily reached or destroyed ; and, moreover, medicines such as corrosive sublimate and carbolic acid, which readily destroy the micro-organisms, are also liable to poison the patient. Cash, however, has found that the 
continued administration of minute doses of corrosive sublimate render animals capable of resisting the deadly effects of the subsequent inoculation of anthrax. Polli of Milan found that dogs, which for five days previously had received daily doses of sodium sulphite, suffered comparatively little inconvenience from the inoculation of fotid pus, which destroyed, with gangrene and typhoid symptoms, dogs not previously protected by the antiseptic.

To prevent decomposition of the contents of the digestive canal, allay irritation and vomiting, and relieve diarrhœa and dysentery, such antiseptics as salol, dermatol, naphthol, tannoform, tannalbin, lysol, creolin, creosote, formalin, carbolic, salicylic, and sulphurous acids are administered. Alcohol, ether-oils, and other members of the fatty series of carbon compounds, are prescribed. Most of the bodies of the aromatic series are also antiseptics; but carbolic and salicylic acids are more active than salol, exalgin, thallin, and resorcin. Creolin and lysol are the antiseptics most frequently prescribed in Germany. The notable efficacy of mercurials in many gastro-intestinal disorders depends upon their antiseptic properties. In the treatment of phthisis in human patients, inhalation of spray, containing very minute quantities of corrosive sublimate, has been used with some success.

The uses of disinfectants.-Perfect cleanliness of the animals and their surroundings, with sunlight and abundance of pure air and water, are the chief purifying agents requisite, so long as animals are in perfect health. When, however, contagious disease occurs, it is necessary to destroy the specific micro-organisms produced, and prevent their diffusing and attacking healthy subjects. Pure air dilutes, but it also diffuses, and does not destroy these contagious organisms.

Water, like air, mechanically dilutes noxious matters, and hastens their oxidation. Sewage freely mixed with running water is hence rapidly decomposed and robbed of injurious properties. Decomposing organic and contagious matters, insufficiently diluted with water, instead, however, of being deprived of their activity, are apt to get distributed, and are liable to assume more dangerous forms. Hence, in purifying 
foul or infected places, solid accumulations should be mixed with some fitting antiseptic, and removed without the addition of water. Infected stables, sheds, market-stances, trucks, and ships should be swept out, and, if need be, scraped; and dry or semi-solid filth, which proves so ready an absorbent of contagious virus, should be mixed with disinfectants, and cleared away. The partially cleansed surfaces should then be well washed with carbolic soap and water, or corrosive sublimate solution; brickwork subsequently lime-washed, and woodwork sprinkled with crude carbolic acid in the proportion of six ounces to the gallon of water.

It is of paramount importance to attack the infecting micro-organisms as soon as they are produced, and before they have opportunity for distribution. Animals affected with contagious diseases should accordingly be immediately isolated, provided with attendants who shall have nothing to do with the healthy stock, their droppings at once disinfected, their skin and feet washed with some disinfectant, whilst antiseptic medicine should be given internally.

Sheds or stables occupied by infected animals should be fumigated with chlorine, sulphurous acid, or formaldehyde. The former is the more effectual, and is evolved gradually by treating bleaching powder with diluted sulphuric acid, or more freely by mixing common salt and black oxide of manganese with sulphuric acid. A pound of sulphur, mixed with about one-fourth part of charcoal, and placed in a chauffer or on a shovel of hot cinders, fumigates a shed about 100 feet long and 20 feet in breadth and height. Neither chlorine nor sulphurous gas, properly managed, should cause pulmonary irritation, either to the animals or their attendants. Carbolic acid in its impure liquid form is conveniently applied with a brush over the doors, walls, and mangers; and carbolic powder should be scattered daily over the floors and manure heaps.

Carbolic acid sprinkled about the boxes, sheds, and enclosures of the Jardin d'Acclimatation, in Paris, proved successful in preventing the spread of cattle plague in 1865 . Similar treatment has secured the like immunity from attacks of contagious pleuro-pneumonia and foot-and- 
mouth disease. Repeated instances have been reported where foot-and-mouth disease has been arrested, after a portion of the herd has been attacked, by washing twice a week the walls, floors, doors, and other woodwork of the infected premises with carbolic acid, confining the animals for several weeks to their sheds or boxes, and keeping them surrounded by an atmosphere abounding in the tar acids. By similar disinfection, the progress of influenza and of strangles in large studs has frequently been arrested. Nocard has shown that when a cow aborts, whether from accidental cause, or from virus introduced from subjects which have previously aborted, further cases of the mishap may be prevented by corrosive sublimate injections into the vagina, washing the external organs with a similar solution, and disinfecting and burying or burning the aborted calf and membranes. Incalvers standing with those aborting should have the external organs and tail washed daily with an antiseptic solution.

Burning is the only absolutely safe method of destroying the bodies of anthrax subjects, from which removal of the hides is dangerous to persons employed or, it may be, to other animals. Cattle plague subjects should be either burned or deeply buried; while for the diseased organs of tuberculous patients the furnace is the only safe tomb.

A high temperature, as already indicated, destroys infective particles. Koch, as above stated, found that the bacilli of anthrax and swine fever, even when bearing spores, were deprived of pathogenic power when exposed for four hours to a temperature of $216^{\circ}$ to $220^{\circ} \mathrm{Fahr}$.; while exposure for five minutes to boiling water, or, better still, to steam heat, is equally effective. The power of steam depends-(1) on its latent heat ; (2) on its moistening ; (3) on its condensing ; (4) on its penetrating. It is most effective when employed under pressure, and when its entrance into the chamber is occasionally interrupted, so that cold air in the interstices of bulky and non-conducting bodies may be displaced. Russell, Glasgow, exposed all infected washable articles, for three-quarters of an hour in a chamber, to steam heat, along with soap and soda, and found that this treatment destroyed bacilli of anthrax and swine fever, tuberculous pus, and also 
the ova of lice. This method should, where practicable, be adopted in the case of rugs and other articles used by infected animals.

Conveyance of contagium by attendants is prevented by sprinkling their clothes and boots with weak carbolic solutions. After handling animals affected by contagious disease, or making post-mortem examinations of such subjects, the hands should be cleansed first with soap and water, and then washed with a 4 per cent. solution of carbolic acid, or with a solution of 12-15 grains corrosive sublimate to a quart of water, which very effectually destroys any adhering bacilli.

So soon as the premises in which animals affected with contagious disease have lived can be emptied, more thorough disinfection should be carried out. To this end, doors and windows having been closed, formic aldehyde, chlorine, or sulphurous acid should be freely evolved, and the place kept shut for several hours. Walls, floors, and woodwork should subsequently be scraped, and washed with corrosive sublimate solution or other disinfectant.

\section{Different disinfectants are suitable for different purposes.} For putrefying or contagious matters mixed with water, the best are mineral salts, of which the most effective and cheapest are corrosive sublimate; zinc chloride, in the familiar form of Burnet's fluid; and iron chloride, or formalin. For sewage disinfection, or where there is much water, aluminium sulphate, followed by lime, can be recommended. Sulphites promptly remove smells, and are most effectual when conjoined with the tar acids. The mixture of sodium sulphite and carbolic or cresylic acid, although effectual for deodorising, has a feeble power in preventing the putrefaction of night soil, for which potassium permanganate is most useful. Common salt, although ineffectual in checking decay when once established, or in neutralising bad smells, is a cheap preserver of many animal substances. It preserves and disinfects skins. Iodine is volatile and penetrating. It is used in many sick-rooms and hospitals, conveniently dissolved in the light diffusible pentane. The solution contains 20 grains to the ounce; an ounce suffices for four cubic feet of space; distributed by a spray pro- 
ducer, it volatilises rapidly ; it leaves, when freely used, a film of iodine, and effectually destroys smells and noxious organic matter. Its expense, however, precludes its general use in veterinary practice.

The uses of deodorisers.-Objectionable smells are largely made up of sulphuretted hydrogen, phosphuretted hydrogen, and nitrogen gases, with sulphurous and ammoniacal compounds. Some popular deodorisers only cloak and overpower, instead of neutralising or destroying offensive smells. Of this description are fumigations with aromatic and balsamic substances, such as camphor, cascarilla, and lavender, the burning of brown paper, the sprinkling of scents and essences. Odours depending upon gases are readily removed by effectual chemical neutralisers: sulphuretted hydrogen, by chlorine ; ammoniacal emanations, by hydrochloric and nitric acids. Smells from decomposing organic matters are usually most effectually got rid of by arresting decomposition by suitable antiseptics. For destroying the intolerable smell from cochineal dye-works, no deodoriser has been found so satisfactory as sulphurous acid. For deodorising the contents of privies, without detracting from the manurial value, a mixture of common salt and carbolic acid, or eight parts of calcined dolomite mixed with two of peat or of wood charcoal, can be recommended.

Powerful mineral antiseptics, such as the zinc and iron chlorides, especially when used in concentrated solution, are not good deodorisers. Not being volatile, they can only destroy the odorous particles brought into immediate contact with them. Iodine, dissolved in spirit, although an expensive, is an elegant and effective deodoriser. Cresylic and carbolic acids are good deodorisers, and are volatile, but have the disadvantage, that when used in concentrated form, they may cause the evolution of sulphuretted hydrogen. A mixture of dry sodium sulphite with carbolic acid is effectual, and moderate in cost, and should be placed in vessels about the premises. Creolin-one part in 50 to 100 of water-is largely used as a deodorant for cattlesheds, stables, piggeries, and kennels. Chlorinated lime, in the familiar form of bleaching powder, although possessed of small antiseptic power, is a prompt and effectual deodor- . 
iser, can be employed either for solid or liquid impurities, gives off chlorine, and never causes any disagreeable combinations; but breaking up instead of preserving organic matters, it diminishes the value of manure with which it is mixed. It is applied as powder, or in solution containing from 2 to 5 per cent., to the walls, wood-work, and floors of the places requiring purification, or sheets soaked in the solution are suspended about the premises.

PARASITICIDEs are killers of parasites, whether animal or vegetable. The group includes germicides, or killers of micro-organisms, and vermicides, which will be subsequently noticed. They are referred to here as they mainly consist of antiseptics. The many varieties of ringworm produced by fungi are destroyed by antiseptic solutions, by phenol oils, and tincture of iodine. Scab and mange caused by various acari are treated by sulphur ointments, solutions of carbolic acid, creolin, or arsenic, or by tobacco infusion. The strongyli invading the bronchial tubes of young cattle and sheep, and causing hoose or husk, are destroyed by inhalation of diluted sulphurous acid, or chlorine, or by turpentine, chloroform, or terebene, given intratracheally.

ANTIPERIODICS are medicines which mitigate or prevent intermittent intensity of the symptoms of certain diseases. Such periodical recrudescence is less marked in the lower animals than in man, but is sometimes observable in the pyrexia of influenza in horses and distemper in dogs. These exacerbations usually occur in specific disorders, and are believed to result from the recurring development of fresh crops of micro-organisms or their products. Cinchona, quinine, iodine, arsenic, alkalies, and salicin, are the most effective antiperiodics.

\section{REMEDIES ACTING ON THE SURFACE OF THE BODY}

RUBEFACIENTS - VESICANTS - PUSTULANTS - CAUSTICS SETONS-THE ACTUAL CAUTERY-ASTRINGENTS-DEMULCENTS-EMOLLIENTS-DILUENTS

Irritants, or Counter-irritants, applied to the skin, produce nervous and vascular reaction, and reflexly induce certain 
remote effects. They relieve or remove congestion and pain, and, by stimulating functional activity, promote repair. It is not always easy, however, to explain how these curative results are produced.

Heat and cold both relieve tension, and hence pain; but they produce their effects in different ways. Cold reflexly contracts arteries, constringes the tissues, and hence lessens the quantity of blood flowing to an inflamed part. Warmth dilates capillaries adjacent to the seat of inflammation, and hence relieves stasis of blood and venous congestion. Cold lessens the amœboid movements of the white corpuscles, but to be effective it must be used in the earlier stages of inflammation, and continuously for a considerable time.

A mild blister acts in the same way as a warm poultice, viz., it dilates the congested or inflamed capillary network, and thus relieves congestion, inflammation, and pain. It certainly increases circulation through the part, promoting cell growth and hastening absorption. It thus restores healthy action in most inflamed organs, in swollen glands and joints.

Irritation or inflammation of the skin surface, as indicated, frequently relieves or removes congestion or inflammation, and pain, but it also has an effect on adjacent or deeperseated parts. For such purposes blisters are applied in most animals, in sore throat, bronchitis, pleurisy, inflamed joints, etc. In fact, the action of irritants applied to the skin may be described as general or systemic, local on the part to which they are applied, and reflex in subjacent or distal parts.

The general effects produced by moderate stimulation of sensory nerves are (1) vaso-constriction, with a rise of bloodpressure ; (2) quickening of the heart; and (3) quickening of respiration with a slight rise of temperature. Excessive stimulation causes great vaso-dilatation, fall of blood pressure, and inhibition of the heart, in other words, a condition similar to 'surgical shock.' The local effects have been described.

The special reflex action (counter-irritation) in subjacent organs is probably due to a relation between the sensory nerves supplying the stimulated skin area, and the trophic 
nerves supplying the subjacent viscera. This theory is supported by the fact that optimum effects are obtained when the skin area stimulated is supplied from the same segment of the spinal cord as that which supplies the viscus to be acted upon reflexly. Thus, when the chest walls are blistered in a case of pleurisy, so soon as the skin becomes hot and tender, a stimulus is conveyed by the afferent nerves to the vaso-motor medullary centres, and thence is reflected by the vaso-motor nerves, causing the pleural capillaries to dilate, thus diminishing tension and pain.

The several classes of irritants used externally differ materially in the intensity and duration of their effects.

RUBEFACIENTS produce slight redness and vascular dilatation or congestion, and are represented by ammonia solution, iodine, and mild preparations of cantharides; by alcohol, ether, and chloroform, if evaporation be prevented by oiled silk or other means; by turpentine and other volatile oils, as well as by smart friction and moderate heat. Owing to the colour of the skin and abundance of hair, reddening in veterinary patients is, however, less obvious than in man.

MASSAGE or friction with graduated pressure, as in kneading or shampooing, exerts many of the effects of counterirritants. It promotes the circulation of the blood and lymph, hastens absorption of exudates, stimulates the cutaneous nerves and reflexly the central nervous system, and eases pain. Massage is indicated at the beginning of the treatment of recent strain of ligament or tendon, wrenched joints, œdema, contusion, paralysis, myositis, and muscular atrophy. After removing the hair, the surface of the part is anointed with lard, lanoline, or oil, then rubbed and kneaded for ten or fifteen minutes and always in the direction of venous return. Massage is contra-indicated on septic swellings, abscesses, etc.

VESICANTS are more active; cause exudation of plasma, which, collecting under the epidermis, raises vesicles or blisters. Vesicles contain a fluid consisting of about 78 parts of water, 18 of albumin, with a little fibrin, and 4 of salts. Steam and boiling water rapidly produce a large amount of effusion. Blisters, by whatsoever agent raised, 
after some days generally dry up, protecting the parts until the new epidermis forms. Biniodide of mercury, cantharides, mustard, acetic acid, turpentine, and ammonia, are the vesicants commonly used in veterinary practice.

Pustulants inflame the deep-seated cutaneous tissues, especially the orifices of the sweat glands, cause exudation of leucocytes and raise pustules. These are the effects of croton oil, tartar emetic, mercury biniodide; and also of cantharides, mustard, and other active vesicants, when applied with sufficient friction.

CAustics combine with the water and albumin of the tissues, with which they are brought into contact, kill the cells, and cause the separation of a slough. Those producing extensive sloughing receive the title of escharotics. Caustics are exemplified by the concentrated mineral acids, glacial acetic, carbolic, and chromic acids, concentrated alkalies, antimony chloride, arsenic, bromine, and the soluble salts of the heavy metals.

Caustics are used to destroy parasites or virus in infected wounds, and for this purpose penetrating fluid caustics are sometimes preferable to solid. They are employed for opening abscesses, and for removing warts and other growths, especially when so deep-seated and vascular that they cannot be safely extirpated by the knife. (See also Astringents and Styptics.)

But besides these more direct actions, they develop more complex and vital reparative effects. Applied, for example, to indolent or callous ulcers, they stimulate the trophic nerves and blood-vessels in the zone adjacent to the dead or dying cells, promote healthy nutrition, and thus hasten healing. Lightly used, they condense soft, spongy, exuberant granulations, and hasten the cicatrisation of sinuses. These beneficial effects on morbid processes thus result, not only from direct action on the diseased tissues, but also from indirect reflex action on surrounding parts; in other words, from counter-irritation.

SETONS are sometimes substituted for blisters or firing, and are frequently preferred to firing on account of their being less apt to blemish. The seton consists of a piece of tape or cord, and is usually inserted by means of a seton needle. 
To prevent slipping out, its ends are tied together, or knotted. It is usually moved daily ; and if severe effects are desired, it is smeared with blistering ointment. Setons act chiefly on the comparatively insensitive subcutaneous cellular tissues.

A rowel acts in much the same manner as a seton. A wound is made in the skin with a bistoury or rowel scissors, and is kept open by the insertion of a disc of leather, or a pledget of tow, which, to increase counter-irritation, is smeared with blistering ointment.

Acupuncture is effected by needles three to six inches in length, introduced into fleshy parts, with a rotary movement. Occasionally anodynes are deeply injected into muscles for the relief of rheumatic pain.

The Actual Cautery, or thermo-cautery is still much used in veterinary practice as a counter-irritant. It is generally applied at a red heat, and the higher the temperature, the less the pain attending its application. It is employed for some of the purposes of active vesicants, and also of caustics. In the treatment of chronic inflammation of bones, joints, ligaments, and tendons, for which it is chiefly used, it modifies the nutrition of the diseased part (counterirritation). Deep cauterisation with penetration, as in pyropuncturing bony outgrowths, increases the activity of the inflammatory process and hastens consolidation. Moderate cauterisation, as in linear or transcurrent firing, promotes absorption of exudate and resolution. The fired skin does not, as was once believed, form a permanent bandage around the parts; for a short time indeed after the operation the skin is corrugated and tightened, but it soon resumes its natural elasticity, and does not embrace the subjacent parts more firmly than in health. The firing of healthy limbs, with the popular idea of strengthening and bracing them up, is now deservedly discountenanced, and any benefits apparently accruing really result from the rest which the operation necessitates. In nervous, excitable horses, firing occasionally produces irritative fever, especially if several parts are fired at the same time.

The uses of counter-irritants.-In influenza, bronchitis, and other depressing disorders of horses, in order to rouse 
the action of the heart and avert lung congestion, rubefacients, such as soap liniment or mustard paste, are sometimes rubbed into the chest, abdomen, or legs, and when the surface is warmed, as it generally will be in ten to fifteen minutes, the dressing is washed off. Counter-irritants are in common use in certain stages of inflammation of the joints, air passages, intestines, and their investing membranes. They are more beneficial in laryngitis and bronchitis affecting the larger tubes, and in pleurisy, than in pneumonia. In the outset of inflammatory attacks, by reflex action, they lessen hyperemia, chiefly by stimulating the dilated paralysed capillaries, thus favouring resolution. In more acute stages, when blood-plasma and red and white corpuscles are escaping through the walls of the distended vessels, fomentations and poultices are generally more suitable than irritants. When the urgency of the febrile symptoms has somewhat abated, counter-irritants are, however, again useful in promoting absorption of inflammatory products, and they frequently invigorate enfeebled, over-distended capillaries, and substitute higher formative for lower debased action.

Blisters act more powerfully on horses than on cattle, act uncertainly, and yet require to be used with special caution on dogs, which are apt to bite and rub the blistered parts, and thus induce sloughing. For general purposes in canine practice, iodine is a most useful counter-irritant. The action of turpentine on the skin of horses is peculiar. Applied over a considerable surface, it produces such intense irritation that some animals for a short time become unmanageable, a result the more remarkable as turpentine acts but slightly on the more delicate human skin.

The choice of a counter-irritant and the mode of using it are determined by various conditions. For prompt but temporary effects, as in combating chill, rousing nervous depression, or overcoming such functional disturbance as occasions colic, mustard and other rubefacients are specially indicated. To act more permanently on parts in which nutrition has been more seriously impaired, cantharides is the appropriate counter-irritant. Where bone, cartilage, or ligament has been chronically affected, still more profound 
and permanent effects result from the use of mercuric biniodide ointment, the hot needle-iron, or setons.

In inflammatory diseases of the chest in horses mustard is preferable to cantharides. In well-bred sensitive animals a pound of mustard flour made into a paste with cold water is rubbed into the sides and washed off in half an hour. In the heavier less sensitive breeds paper is laid over the mustard dressing, and the horse-rug loosely applied. Some horses show considerable restiveness, and even pain. So soon, however, as tenderness and swelling are notable externally, as they usually are in a few hours, the chest symptoms abate. No other remedy affords such prompt and effectual relief in these cases.

Before a blister is applied, the skin should be well washed with soap and water, and the hair, when long or thick, removed. The effect of the blister may be hastened and increased by subjecting the part to smart friction, or the action of hot water, and by rubbing the agent well in, taking care to spread it over the surface of the part diseased. Violent, deep-seated action is seldom desirable. Better curative results are usually attained by moderate and continuous effects kept up by repeated applications.

Counter-irritants may generally be applied directly over the inflamed area, when removal of fluid or inflammatory products is desired; but should seldom be applied to extensive acutely inflamed parts, or to tissues immediately continuous with them.

When vitality is low, or the skin irritable, blisters are apt to cause sloughing. When inordinate local irritation has been produced, it may be abated by fomentations, while undue constitutional excitement is removed by opiates, diluents, a mash diet, and salines. On the next or second day after a blister has been applied, the part should be dressed with zinc ointment, lard, vaseline, oil, glycerin, or acetate of lead lotion.

Astringents condense the living tissues. Many produce their effects by coagulating or precipitating albumin. These comprise alum, chalk, salts of the heavier metals, acids, and alcohol, with tannic acid, and such tannin-containing substances as oak-bark and catechu. All caustics used in 
small quantity, or diluted solution, are astringent. Agents, such as ergot, digitalis, and adrenalin, have no coagulant power on albumin, but constringe the tissues, in virtue of their action on the walls of the nutrient arterioles. Experiments made by Stockman with gallic acid show that it has no claim to any special coagulant action nor any effect in lessening, like ergot, the calibre of blood-vessels, either by peripheral or central action. The remote effects of vegetable and other astringents have been overestimated. So soon as their chemical affinities have been satisfied by union with a base or with albumin, they must evidently lose their power of coagulating or precipitating albumin; and it is therefore difficult to comprehend how they can exert astringent effects upon either the respiratory or the urinary mucous membrane.

Astringents are used to diminish excessive, and modify faulty, secretion, to combat congestion of cutaneous and mucous surfaces, and to arrest limited recent superficial inflammation. These results appear to be obtained in several ways. Usually some change is effected in the vascular walls, or in the perivascular connective tissues, not always, however, by narrowing the vessels, for such notable astringents as alum and tannic acid dilate vessels; while silver nitrate acts on the cement substance of the endothelium. Their efficacy is often well seen in circumscribed inflammation of the conjunctiva or fauces. Solutions of tannin, eucalyptus gum, or alum, in spray or gargle, or inhalations of turpentine vapour, mixed with air, arrest the inordinate secretion, and relieve the congestion of sore throat and bronchitis. Their application in disorders of the digestive organs will receive special notice under that heading. As injections and suppositories, they are used in irritable and inflamed conditions of the vagina and uterus. The uterus and rectum, when prolapsed, are washed with astringent antiseptics, in order to diminish their irritability and swelling, and to facilitate their safe return. They condense exuberant granulations, lessen discharges from wounds and ulcers, which they usually coat with a protective film of albumin.

STYPTICS are astringents specially used to arrest bleeding. 
Some, like matico, tow, lint, or pressure, mechanically check blood-flow from superficial vessels ; others, like most astringents and caustics, coagulate albumin, and thus plug the leaking vessels. When thus used, the blood is removed by a piece of lint or a sponge, and the part lightly pressed, so that the blood-vessels may be more readily seen, and the caustic applied to them with precision, and with as little destruction as possible of surrounding textures. The effect of the styptic may be seconded by equable pressure and application of cold. Others again, like adrenalin, ergot, digitalis, ether-spray, and antipyrine in tannic acid solution, contract capillary vessels, while lead acetate and calcium chloride act by increasing the coagulability of the blood, the former also contracting arterioles. Astringent and caustic agents are also used as coagulants to prevent the flow of synovia from open joint capsules and bursæ.

DEMULCENTS soothe and protect parts with which they come into contact, act chiefly mechanically, and closely resemble emollients. They include gums, mucilage, linseed, cotton-wool and collodion, fuller's earth, starch, treacle, gelatin, albumin, fats, oils, glycerin, and milk. They take the place of mucus and other natural demulcents, where these are deficient or wanting. They lubricate or defend abraded or irritable parts from external injury, acrid discharges, and poisonous matters. They are employed in solution, spray, draught, or enema, to relieve dry, irritable conditions of the skin, respiratory, digestive, and urinogenital membranes by local application.

Emollients soften, soothe, and relax the parts to which they are applied. They resemble demulcents, and include many of the articles specified in that class, as well as those substances which absorb and retain heat and moisture. They are represented by fomentations, poultices, and spongio-piline, and by folds of lint, flannel, or woollen cloth, wrung out of hot water, and covered with waterproofing. (See Poultices and Fomentations.) Fats, oils, lanoline, vaseline, paraffin, with soap and other liniments, are also emollients. Fatty emollients rubbed into the skin soften and supple it; and when applied with smart and continued friction, they also increase tissue changes, 
and hasten removal of deposits. In the form of watery vapour, simple or medicated emollients relieve irritability and congestion of the respiratory mucous membrane. Not only do they reduce tension and relax tissues to which they are immediately applied, but, acting on adjacent vasomotor centres, they dilate collateral blood-vessels; and mainly in this way fomentations and poultices relieve irritation and inflammation of the throat, lungs, and other deep-seated organs. In the earlier stages of inflammation they promote resolution; and in all stages they relieve heat, tension, pain, and spasm. Although serviceable for softening and cleansing septic wounds, they should not be used for wounds in process of healing by first intention or adhesion.

Diluents are allied to demulcents and emollients, are liquid or solid substances used along with more active agents in order to diminish their activity. They include most demulcent drugs.

\section{MEDICINES ACTING ON MUSCLE}

\section{ACTION OF MEDICINES ON MUSCLE}

The three varieties of muscle, striped or voluntary, unstriped or involuntary, and cardiac muscle, are affected quite differently by drugs, and must be considered separately. The chief characteristic of muscle is the power of contraction when stimulated. The striped muscles are all connected with the central nervous system by means of nerve fibres of two kinds, the one sensory or afferent, conveying impressions from the muscle to the nerve centre, the other motor or efferent, transmitting impulses from the centre to the muscle. These motor impulses result in contraction of the muscle, and these contractions and the succeeding relaxations (passive) are accompanied by chemical changes in the muscle which set free the necessary energy. Oxygen is used up whilst sarcolactic acid and carbon dioxide are formed. These waste products, and the accompanying fatigue consequent on repeated violent contractions, can be removed 
experimentally by washing out the muscle with a current of blood or serum, either of which supplies it with food at the same time, or with normal saline solution containing a trace of alkali. In practice, removal of these waste products is hastened by shampooing the muscles, or massage, the effects of which, in overcoming fatigue, are fully recognised. In like manner thorough grooming and diligent hand-rubbing of the limbs of horses after hard work lessen fatigue, and prevent subsequent stiffness and swelling of joints.

But muscle fibres are susceptible to other stimuli than impulses along the motor nerves, for thermal or electrical stimulation will cause contraction even when all the nerve fibres in the muscle have been destroyed. Similarly, drugs can affect the muscle fibres themselves, some stimulating to stronger and quicker contraction, others depressing, lessening the power of contraction, and making the reaction slower.

Muscular spasms consist of irregular purposeless contractions of either voluntary or involuntary muscles, usually depending on faulty action of the higher co-ordinating centres. They may, however, be due to some change in the muscle itself, or to the direct action of some poison. Spasms may be either tonic, as in tetanus of muscle, or clonic, as in tremor or rigors. Spasm of involuntary muscles, as illustrated by that of the blood-vessels, bronchi, or intestines, is antagonised by nitrites, such as amyl-nitrite and nitrous ether (see Antispasmodics). When a muscle, or its motor nerve, receives an abnormal number of stimuli, so that it has no time to relax between them, instead of contraction being followed by relaxation, prolonged tonic contraction or tetanus ensues. This occurs with poisons like strychnine and with tetano-toxin.

'Rapid alternation of contraction and relaxation, or tremor, may affect either- $(a)$ a few bundles of muscular fibres; (b) a single muscle; or (c) groups of muscles' (Brunton). Such tremor may occur when the muscle is at rest, or when it is in motion. This form of insubordination may probably result from the number of stimuli from the nerve-centre being either too few or too many. 
The agents which act on striped muscle tissue may be arranged in the following groups :-

1. Diminishes the excitability of the muscle, as well as its capacity for work. This group contains salts of potassium, lithium, and ammonium, the cinchona alkaloids, chloroform, chloral, and alcohol, in large doses. This action is of little practical importance, for it is either insignificant in the living animal or is overshadowed by some other more marked action of the drug.

2. Diminishes the total amount of work, whilst leaving the irritability unaffected, until, with large doses of the drug, the depression ends in death of the muscle. This effect is produced when the drug is brought in contact with the muscle, but not when medicinal doses are given by the mouth. The group contains apomorphine, saponins, salts of copper, zinc and lead, emetine and cocaine.

3. Alters the form of the muscle curve, as exhibited by veratrine and barium, and to a similar, although less extent, by strontium and calcium salts, digitalis, oxycolchicine, and glycerin. This effect is produced only by large or even poisonous doses of most of these agents.

4. Increases the irritability, as is notably done by physostigmine.

5. Increases the capacity for work. The agents belonging to this group cause rapid restoration of the muscle after fatigue, and are represented by creatin, hypoxanthin, caffeine, and glycogen. These substances must hence be regarded, not only as nerve stimulants, but as direct muscle restoratives.

Voluntary muscles differ from involuntary, not only in structure, but in other particulars. Their contraction and relaxation are more rapid. The nerves in voluntary muscles terminate in end-plates, while the terminal twigs in involuntary muscles form a plexus round the fibres.

Involuntary muscle fibres are directly stimulated to contract and their tonus increased by barium, and to a less extent by veratrine and lead. That this action is direct 
is shown by the fact that the arterioles in the lungs, which are practically without vaso-motor nerves, are constricted when a solution of barium chloride is perfused through them. The opposite effect, namely, direct relaxation of unstriped muscle fibres, is brought about by nitrites, such as amylnitrite, and with these the lung vessels become widely dilated.

\section{MEDICINES ACTING ON THE NERVOUS SYSTEM}

ON THE BRAIN.-CEREBRAL STIMULANTS-EXHILARANTSCEREBRAL DEPRESSANTS - SOPORIFICS - NARCOTICS ANODYNES-ANTISPASMODICS-AN ZESTHETICS.

ON THE SPINAL CORD.-SPINAL STIMUlantS AND DEPREsSaNTS. ON MOTOR NERVES.-STIMULANTS-PARALYSERS.

ON SENSORY NERVES.-STIMULANTS-LOCAL SEDATIVESLOCAL AN ESTHETICS.

\section{ELECTRICITY.}

The nervous system of the higher animals comprises :-

I. The brain, which takes cognisance of external impressions, co-ordinates movements, and originates mental or psychical ideas. Relatively to other parts of the nervous system, the brain of man is more highly developed than that of animals, and most drugs, accordingly, act upon it more powerfully than upon the less developed brain of the horse, ox, or dog. The brain consists of (1) the medulla, pons, iter, corpora quadrigemina and third ventricle, which may be looked upon as continuing the spinal cord forward and giving off cranial in place of spinal nerves; (2) the cerebellum, which is chiefly concerned in the maintenance of equilibrium; and (3) the cerebral hemispheres. The cerebral hemispheres are concerned with the highest functions of judgment and will power, and are the seat of the intelligence. This is especially the part of the brain which is most highly developed in man. The cerebrum controls and co-ordinates movement, and in the grey matter of the cerebral cortex are the motor cells from which these impulses spring. 
II. The Spinal Cord and Medulla have for their functions (1) the control of reflex actions ; and (2) the conducting of impulses to and fro between the brain and the various organs and tissues of the body. The cells of the grey matter are chiefly concerned with the former, whilst the white nerve fibres outside them are the conducting media.

In the medulla, in the floor of the fourth ventricle, are situated the vital reflex centresthose concerned with respiration, cardiac inhibition and vaso-motor control-also the vomiting * centre. In the cord itself are centres for micturition, defæcation, parturition, muscular tone, and vaso-motor control.

These reflex centres of the cord, whilst able of themselves to carry out reflex movements, are more or less under the control of the higher centres of the brain.

III. Sensory nerves, or afferent nerves, distributed to all parts of the body, convey impressions to the brain or cord.

IV. Motor and secretory or efferent nerves. From the cerebro-spinal axis arise nerves which give motion to muscles, and convey other efferent impulses to glands.

V. Lastly, there are the sympathetic ganglia and nerve fibres, supervising functions such as vaso-motor control, peristalsis, and many others performed by involuntary muscle, and the secretion of some glands.

Concerning the functions and diseases of the nervous system much has still to be discovered, while the effects of medicines acting upon the brain and cord still require much investigation.

\section{ACTION OF MEDICINES ON THE BRAIN}

Medicines affecting the brain directly act in one of two ways, either to stimulate or to depress. They pick out, as a rule, some part of the brain as the special seat of action, 
but gradually the effect tends to spread to all parts of the central nervous system. How the effects are produced is not easy to decide, but there are various modes of action which are of importance. Some drugs are absorbed easily, and without any chemical change are readily soluble in the fatty material of the brain, whilst being relatively insoluble in water. All these act as narcotics. Others, again, chemically combine with the nerve cells, for example the toxin of tetanus, which unites with the cells of the spinal cord to form a non-toxic inert substance. Still others act on nerve - cells by their osmotic effects, such as salines circulating in the blood.

Thus, by various slight physical and chemical changes the cells of the nervous system are affected, and, on the one hand, may become quicker to respond to excitation (stimulated), or less quick to respond (depression), and so less able to perform their functions.

The motor areas of the cerebral cortex have their excitability lowered by alcohol, chloral, and cold. The depression caused by cold, unless extreme, or applied for a long period, is followed, however, by reaction. Bromides of potassium and ammonium, without disturbing the relations of one centre to another, appear to have a marked effect in lowering general brain activity. Still more prompt and powerful are anæsthetics which abolish all motor action. Atropine in moderate doses at first increases, but in large doses diminishes, motor excitability.

The motor centres have their excitability increased by mechanical irritation, as by the point of a needle, which produces epileptic convulsions. Similar convulsions also ensue when the vessels of the brain are surcharged with venous blood, as in asphyxia, but these are medullary in origin, from the convulsive centre related to the respiratory centre. Camphor and other volatile oils cause excitement and constant movements, succeeded, after large doses, by clonic convulsions and death. The active principles of cocculus indicus, cicuta virosa, and cenantha crocata, as well as cinchonidine and quinine, have similar convulsant effects. The action of these agents is not confined to the brain motor centres, but also extends to those in the medulla. 
Cerebral Stimulants. - The functions of the brain generally are stimulated by a large group of agents, sometimes termed brain stimulants or exhilarants, and exemplified by caffeine, theobromine, cocaine, atropine, and oil of turpentine. Of these, the first two especially affect the psychical side, whilst the others are motor stimulants primarily. Ammonia vapour, liquor, or carbonate, applied to the nostrils, reflexly stimulates the heart, and then acting upon the vaso-motor centres, also increases general blood pressure, and the circulatory improvement stimulates the brain. Other circulatory stimulants act similarly. Brisk exercise has much the same stimulating effect on the cerebral as on other arteries and capillaries. Mastication and sucking in young animals have been shown by experiment to increase circulation in the carotids and cerebral arteries. Chewing tobacco, betel-nut, or, indeed, anything else, smoking, sipping alcoholic stimulants, or even tea, coffee, or cold water, have similar effects in dilating the human cerebral arteries. Placing the head on a lower level than the rest of the body favours brain circulation, and hence wards off syncope.

The functional activity of the brain is lowered by large or repeated doses of alcohol, which, after apparent exhilaration, and, it may be, delirium, produce narcosis, sleep, and sometimes death. Other agents causing depression, after more or less preliminary excitement, are chloroform and chloral, opium and cannabis indica. Bromides of potassium and ammonium, without preliminary excitement or disturbed function, diminish brain activity. Accumulation of lactic acid, and probably other elements of tissue waste, appears to have an effect similar to that of the bromides in lowering the activity of the nerve-cells.

SOPORIFICS or HYPNOTICS are agents which induce sleep. Many hypnotics lessen functional activity of the nerve-cells of the brain and spinal cord, while others impede the impressions transmitted through the nerves and special sense organs to the cerebrum. Full doses further depress the functions of the respiratory and vaso-motor centres in the medulla, as evidenced by slower respiration, dilatation of surface-vessels, and lowering of arterial tension. During 
sleep, certain parts remaining in a state of partial unregulated activity, induce the phenomena of dreams, which occur in the domesticated animals as well as in man.

A perfect hypnotic has not yet been found even for human patients. Those prescribed are liable to be uncertain, and injuriously disturb motor, organic and trophic functions. Hypnotics act still less satisfactorily on the lower animals, on account of the want of development of the cerebrum. Dogs and pigs are, however, brought under their influence more readily than horses or ruminants. The agents most trusted are opium and morphine, chloral hydrate, croton chloral, hyoscine, cannabis indica, and bromides. Opium and morphine prove of special value, not only in depressing cerebral functional activity, but also in antagonising pain and irritation which interfere with sleep; but not to an equal extent in all animals, for in the cat, and to a less extent in the horse, excitement and restlessness are caused. Bromides diminish conduction of impressions, and hence notably quiet cerebral excitement. Chloral is a powerful hypnotic, but in large doses it is a local irritant, and sometimes also produces dangerous delirium and convulsions. It induces sleep mainly by its depressant action on the brain, and by dilating vessels generally. Chloralamide is prescribed for human patients as a safe and effectual hypnotic. Paraldehyde, even in full doses, has none of the disadvantageous secondary effects of opium or chloral, causes quiet sleep in dogs, but is rather nauseous, and not one-third of the strength of chloral. Sulphonal is more active, but being insoluble, hypnosis is slowly established. It has been used especially in cases of motor unrest. Large doses diminish excitability of the reflex functions of the spinal cord, and also of peripheral sensations. Trional is allied to sulphonal, but is more soluble and active; and in experiments on dogs it produced sleep more rapidly. Hypnone, urethane, and amylene hydrate, though ineffective in the horse, are useful hypnotics for the dog. Warmth to the body and legs, and comforting warm drinks withdraw blood from the brain, and hence favour the anæmia which occurs in sleep.

NARCotics are drugs which disturb the relation of the 
mental faculties with the external world by bringing about cerebral depression. This disturbing effect is produced by full doses of such agents as alcohol, ether, and chloroform, as well as by most of the agents described as hypnotics. After a variable amount of excitement, locomotor incoordination ensues, and the animal staggers in its gait. Then there is drowsiness, stupor, and, with the full action of the drug, coma. Where the effect is still further developed, fatal paralysis of the respiratory centre occurs. The action of these agents is best explained, although not completely, by the theory suggested by Meyer and Overton. These observers have pointed out that the narcotic agents such as chloral, chloroform, ether, and those of the fatty carbon series are alike in being readily soluble in fats and oils, and not so easily soluble, or even insoluble in water. Nervous tissue is largely made up of the fatty compounds cholesterin and lecithin, and as a consequence these narcotic substances circulating in the blood tend to leave this watery fluid and become concentrated in the fatty substances of the nervous tissues. This alters, and in fact lessens, the activity of the nerve-cells, and so accounts for the narcotic or even anæsthetic effect. This theory does not fully explain the action of these drugs, nor is it true of every agent, but, generally speaking, the more soluble in fat and insoluble in water a drug of this series is, the more powerful is its narcotic or anæsthetic action.

ANODynes or analgesies are agents which relieve pain by diminishing excitability of nerves or nerve-centres. Pain may originate in the hippocampal region, which Ferrier regards as the central seat of sensation, and some abnormal excitement of these nerve ganglia is believed to occur in hysteria. It may depend upon stimulation of the grey matter of the cord, through which painful impressions are conveyed. It may begin in the trunk of a nerve, but frequently its origin is in the peripheral endings of the sensory nerves.

Pain, thus produced in various ways, requires diverse treatment. Its cause should, if possible, be discovered and removed. When merely local, it is combated by local anodynes, such as belladonna, with its alkaloid; by cocaine, 
veratrine, carbolic acid and other phenols ; by menthol and thymol, local blood-letting, heat and moisture as by poultices and fomentations, and by cold, in the form of ice or cold water. Counter-irritants also act reflexly as analgesics.

When pain is not localised, general anodynes are administered, either by the mouth or hypodermically. Many act mainly as sedatives or paralysants of the brain centres. Chloral hydrate, Indian hemp, hemlock, bromides, and anæsthetics are types of this class. Others with little or no central action paralyse sensory nerves or nerve-endings, and so prevent painful impulses being received. These are exemplified by atropine, cocaine, and aconite. Opium and morphine produce their anodyne effects by depressing sensory nerve-cells of the brain and cord, although they have little or no effect on sensory nerve-endings and so are of little use applied locally. Several of the newly-discovered bodies of the benzol or aromatic series conjoin antiseptic and anodyne properties. Such are salol, a salicylate of phenol ; antifebrin, which controls many varieties of pain; while exalgin is still more generally effectual. Salicylic acid and salicylates have a special power of controlling the pain of acute rheumatism. Electricity applied along the course of the stimulated nerves, and, in acute rheumatism, nervestretching, are sometimes tried. Dividing the nerves supplying the seat of injury, as is done in navicular and some other diseases of the feet, prevents the feeling of pain, but of course does not arrest local inflammation or other mischief. In horses, as in other animals, the giving of a dose of physic and the administration of tonics are often effectual, probably owing to their relieving irritability of the cerebrospinal centres, by removing waste material, and by a general improvement in tone.

ANTISPASMODICS are agents which prevent or lessen muscular spasm, which is an irregular painful contraction of voluntary or involuntary muscles. In the medulla oblongata, where it joins the pons, and related to the respiratory centre, is a centre which, when stimulated, causes general convulsions and spasms. These are excited by direct irritation of the centre with chemical agents, by contact action of such poisons as strychnine, nicotine, picro- 
toxin, and ammonia, by rabies and other animal toxins, as well as by the altered condition of the blood in asphyxia, and by sudden anæmia of the medulla from copious blood-letting. This spasm centre appears to be in an abnormal state in epilepsy, when its inordinate action is directly controlled by bromides. The tonic spasms of tetanus, and strychninepoisoning, are relieved by bromides, chloral hydrate, and nicotine.

But spasms are more frequently local than general. They have been defined as a kind of insubordination, in which the individual muscles or nerve-centres act for themselves, without reference to those higher centres which ought to co-ordinate their action for the general good of the organism. Spasm may be due, therefore, to an excess of action in the muscles or local centres, or diminished power of the higher co-ordinating centres. As a rule, it is due to diminished action of the co-ordinating or inhibitory centres, rather than to excess of action in the motor centres. It is, therefore, a disease rather of debility and deficient co-ordination than of excessive strength. Local irritation is frequently the cause of spasm.

Excessive exertion develops in the muscles of locomotion, especially when employed in unwonted work, waste products, which produce spasm or cramp. Both cause and effect are frequently removed by smart friction. In the intestine, cramp may be due to the presence of a local irritant, which ought, in the normal condition, to produce increased peristalsis, and thus ensure the speedy removal of the offending substance. 'From some abnormal conditions, the muscular fibres around the irritant contract excessively, and do not pass on the stimulus to those adjoining. From this want of co-ordination, painful and useless spasm occurs. In order to remove it we apply warmth to the abdomen, so as to increase the functional activity both of the muscular fibres and of the ganglia of the intestine. Peristalsis then occurring instead of cramp, the pain disappears, and the offending body is passed onwards and removed. Or we give, internally, aromatic oils, which have a tendency to increase the ordinary peristalsis ; or, yet again, we may give opium or other anodyne for the purpose of lessening the sensibility of 
the irritated part' (Brunton). In the treatment of colic in horses, these several modes of attack are usually conjoined. A diffusible stimulant such as ether or alcohol is given to improve the local circulation and to increase the powers of the higher nerve-centres, and thus bring the disturbed lower centres and the muscles into subordination ; an anodyne is associated to lessen local excitability ; while a purgative is, besides, administered in order to remove the indigestible food, which is usually the cause of the mischief.

The convulsions of epilepsy, as already mentioned, are warded off by bromides, while endeavour is also made to remove the conditions which produce them by the administration of arsenic or iodides. Chorea, depending probably upon some lesion of the sensori-motor ganglia at the base of the brain, is treated by arsenic and iodides, and when the patient is anæmic by iron and a generous oleaginous diet. Spasm affecting the heart is usually controlled by the judicious use of alcohol, ether, digitalis, or nitrites. These nitrites, exemplified by amyl-nitrite, nitro-glycerin, and nitrous ether, are pre-eminently relaxers of spasm of involuntary muscles. They relieve the heart and bloodvessels in angina pectoris of human patients, and the dyspnœa of bronchitis, as well as intestinal cramp in all animals. In overcoming spasm of particular parts, it is, as already indicated, important to exalt the power of the controlling centres of the brain and spinal cord by such stimulants as alcohol, ether, camphor, and bromo-camphor, and thus regulate or co-ordinate the lower disturbed centres. This twofold stimulation of the higher central and lower topical centres is also exerted by valerian, asafœtida, musk, and volatile oils. Other antispasmodics as borneol and menthol, instead of exalting nervous excitability, lessen irritability, and paralyse motor, sensory, and reflex centres of the brain and cord, and thus often relieve spasm. In the successful treatment of spasmodic diseases which generally depend, as already indicated, on deficient and imperfect nervous power, restoratives, tonics, and good hygiene are essential factors.

ANAESTHETICS are substances that produce insensibility to pain, diminish muscular action and other phenomena. They 
are allied to anodynes, but act more promptly and powerfully. Certain volatile drugs, brought into contact with the nerve-cells, reduce or arrest for considerable periods their functional activity, probably in virtue of chemical action.

Local anæsthetics produce paralysis of the peripheral endings of sensory nerves. They include cocaine, stovaine, eucaine, holocaine, ethyl-chloride, methyl-chloride, carbolic acid, antipyrine, iodoform, orthoform, and extreme cold. Cocaine is generally preferred. The part is painted or injected with a solution of the hydrochloride, until the requisite insensibility is secured. Cocaine proves particularly serviceable in diminishing irritability, and facilitating examinations of the eye and larynx, as well as for the performance of minor operations.

General anæstheties, when inhaled, are carried by the blood-stream to the centres of the brain and cord, which they paralyse, producing unconsciousness. They comprise nitrous oxide gas, chloroform, ether, and other substitution compounds of the methane $\left(\mathrm{CH}_{4}\right)$ and ethane $\left(\mathrm{C}_{2} \mathrm{H}_{6}\right)$ series. Nitrous oxide produces its effects rapidly, induces a general anæsthesia, which can, however, only be kept up for a very short time-one to two minutes-because of the asphyxia which is produced. This is shown by the venous condition of the blood, cyanosis of mucous membranes, with contraction of arterioles and rise of blood-pressure, and there is hence no risk of its causing syncope. Admixture with oxygen prevents asphyxia, but lessens and delays the anæsthesia. In human practice, anæsthesia is often induced by nitrous oxide, and subsequently maintained by chloroform or ether. Chloroform is generally used both in human and veterinary practice. It is the most effectual and, rightly used, the safest known anæsthetic. It acts in smaller quantity, more rapidly, and with less excitement than ether. Ether, first employed in America, is preferred by many English practitioners, on the plea that it is less apt than chloroform to impair cardiac action. But it has the disadvantage of greatly increasing bronchial secretion and salivation, of causing more irritation and excitement than chloroform, while its administration requires the use of an inhaler. 
The A. C. E. Mixture consists of one part of absolute alcohol, two of chloroform, and three of pure ether. It is much used on the continent of Europe, and is stated to be more stimulant than chloroform, and less likely to depress heart action. A mixture of equal parts of ether and chloroform is used in various parts of France and Germany. The Austrian Government has advised one part of chloroform with six of ether in winter, and with eight of ether in summer. The theoretical arguments in favour of these mixtures have not been justified in practice. Alcohol, chloroform, and ether volatilise at different rates and at different temperatures, so that the composition of the vapour inhaled does not remain constant; at first the animal receives chiefly ether, and this necessitates a high concentration of vapour to air. Later, the chloroform becomes the chief constituent of the vapour, and the former concentration becomes highly dangerous. Since there is nothing to indicate this variation in composition, the mixture increases, instead of lessening, the risk. Chloral hydrate is given by the mouth or rectum, or by intravenous, or intraperitoneal injection. It depresses the heart and vaso-motor centres, and consequently the vessels dilate and blood pressure falls. METHYLEnE $\left(\mathrm{CH}_{2} \mathrm{Cl}_{2}\right)$, containing an atom more of nitrogen and an atom less of chlorine than chloroform, causes more rapid anæsthesia, but requires to be used in larger amount. The pure drug has also the disadvantage of being costly, and that usually sold is stated to be a mixture of chloroform and alcohol. METHYLAL, which has also been used, acts quickly and effectually on dogs without apparent injurious after-effects, and is also serviceable for local anæsthesia.

Anæsthesia is generally divided into four stages-I. Stimulant ; II. Narcotic ; III. Anæsthetic ; IV. Paralytic.

I. The stimulant stage is characterised by symptoms of inebriation, more marked in the case of ether than of chloroform ; excitation of cerebral and cardiac functions ; vigorous animals struggle; the pulse is accelerated usually from nervousness or fear, and respiration is irregular and quickened; the pupil is dilated; the special senses and general sensibility are blunted. This stage usually continues from 
one to three minutes, but is shortened when the drug is given quickly in full doses.

II. The narcotic stage is marked by paralysis of the motor centres, the voluntary muscular system gradually becomes relaxed, the force and volume of the pulse are lowered, the functions of the higher brain centres are impaired, but reflex functions are slightly if at all affected. This stage, usually reached in less than five minutes, is that suitable for anodyne and antispasmodic effects, and for moderating violent and irregular labour pains.

III. The anæsthetic stage exhibits complete muscular relaxation, unconsciousness, and insensibility; the frequency and force of the pulse are decreased, although it remains quite regular; respiration is regular but somewhat shallow, the functions of the cerebrum and spinal cord are paralysed, the oculo-palpebral reflex is impaired or abolished, the pupil is slightly contracted, but the centres of the medulla presiding over respiration and heart action are only slightly affected. This complete insensibility may be safely maintained for an hour or two by small doses of the anæsthetic, and is the condition requisite for the performance of serious surgical operations.

IV. The paralytic stage occurs when the functions of the medullary centres are abolished. It includes two distinct phases-suspension of respiration, and subsequently suspension of cardiac action. Implication of the respiratory centre is indicated in animals by irregular sighing or shallow breathing, with long pauses between the respiratory movements. This is partly due to direct depression of the medullary centre and also to anæmia of the centre owing to failing circulation. Up to this point the animal is in no serious danger. But if anæsthesia is further pushed, the heart centre is paralysed, the pulse beats very quickly then stops, blood pressure falls to zero, and usually within two minutes heart action also ceases.

The action of anæstheties was very fully elucidated by two series of investigations undertaken at the instance of the Nizam of Hyderabad. The first, made in the spring of 1888, under the supervision of Surgeon-Major Lawrie, comprised 141 experiments, chiefly on dogs ; while the second, made 
in the later months of 1889 , under the direction of Sir Lauder Brunton, included 571 experiments on dogs, monkeys, horses, goats, cats, and rabbits. Chloroform, ether, and the A.C.E. Mixture were used. The investigations demonstrate that the action of these anæsthetics is much the same on man and on the animals mentioned; that lethal doses, of chloroform or ether, whether poisoning be slow or rapid, usually arrest the respiratory before the cardiac action ; and that the heart in uncomplicated cases stops two to six minutes after respiration. Consequently, as was taught by Simpson and Syme, the careful observation of the respiration is the safeguard in the administration of anæsthetics. Although the patient is safe so long as the anæsthetic vapour continues to be eliminated by respiration, whenever the heart stops, unless artificial respiration is had recourse to, the chances of resuscitating the animal are small. This important practical point was established by numerous experiments. It should be noted that in the early stages of chloroform administration, the heart may be inhibited to a serious degree, or even cease to beat, by irritation and stimulation of the vagus centre in the medulla. Thus, if concentrated chloroform vapour is given at first, sudden inhibition of the heart may cause death. When anæsthesia is established this danger ceases.

In the use of chloroform, and indeed of all anæsthetics, in animals, these investigations emphasise the necessity of constantly watching the breathing, and, moreover, ensuring that nothing shall in any way interfere with it. The animal should be in the recumbent position-the head placed so that air passes directly into the lungs ; no girths, straps, or pressure must interfere with respiratory movements. Monkeys, encased in plaster of Paris, or bound with abdominal bandages, died quickly. The paralysed tongue, dropping back upon the larynx of the unconscious patient, may cause suffocation. Respiratory failure is also hastened by having the limbs of the subject firmly bound; while struggling, or shallow, gasping breathing, by filling the lungs with the volatile vapour, intensifies its effects.

Deaths occurring during anæsthesia, both in men and animals, have been ascribed to syncope, or surgical shock, 
and in order to elucidate this matter numerous experiments were made on dogs and monkeys. When full anæsthesia was produced, teeth were extracted, nails evoluted, incisions made into the abdomen, portions of intestine ligatured, and the testicles sharply struck; but in no case was any marked effect produced on the heart action. To test the effect of chloroform on animals with enfeebled heart, dogs and monkeys were fasted, others were freely bled, while others were given grain doses of phosphorus during several days, in order to produce fatty degeneration of the heart muscle. But neither syncope nor heart-shock was observable when these subjects were deeply chloroformed ; respiratory failure invariably preceded cardiac failure; and when breathing was stopped by full doses, the animals, like others in perfect health, were restored by artificial respiration. Occasionally, however, as mentioned above, death occurs suddenly through inhibition of the heart by vagal stimulation during the earlier stages; and experiments prove that healthy horses can be killed in a few minutes by rapid administration of concentrated chloroform vapour.

The post-mortem appearances of animals dying under anæsthesia consist in general congestion of the lungs, liver, kidneys, and spleen, which is also puckered, and two or three times larger than usual. The left heart may be empty and the right heart distended with blood.

Anæsthetics are used in painful, delicate, or protracted operations, as in castration, neurectomy, excisions of portions of the hoof, and other operations on the foot ; reduction of herniæ, and removal of tumours ; extraction of firmly-fixed teeth, especially in dogs and cats ; in tetanus, and strychnine poisoning; in difficult parturition, especially in the mare ; and in destroying injured, useless, or old animals.

Administration to horses may be made while the animal is standing, but more safely and effectually when he is cast and secured. A sponge or piece of lint, saturated with the anæsthetic, is placed in a tolerably close-fitting nose-bag, which is adjusted to the head. Inhalers, bags or muzzles for the purpose have been designed by Cox, and other veterinary surgeons. Many practitioners, when the horse is cast, place the lint, moistened with chloroform, over one 
nostril, that on the upper side being preferable, while, to prevent undue evaporation, a napkin is laid over both nostrils. Endeavour is sometimes made to dilute the chloroform vapour with about ten volumes of air, as in Wallis Hoare's method, but as far as possible air should be excluded, as diluted chloroform vapour acts slowly, and the stage of excitement is prolonged. Undue excitement and struggling can be overcome by giving the anæsthetic freely or by previous subcutaneous injection of morphine. But chloroform anæsthesia can be most satisfactorily induced in horses by slow administration, beginning with half an ounce poured on a warm sponge and adding a drachm or two at short intervals until the requisite degree of insensibility is reached, which is ascertained by testing the conjunctival reflex. Small quantities of the drug suffice to maintain insensibility safely for an hour or more, provided a careful watch, as already stated, is kept on the respiration. An ounce and a half to three ounces of chloroform properly given, without waste, will fully anæsthetise an average horse or ox in from five to ten minutes. Four times the quantity of ether is required. Young animals are more readily anæsthetised than old ones. In giving chloroform to the horse standing, a leather muzzle which fits lightly over the nose and mouth of the animal is used. In the muzzle is placed a sponge on which the drug is poured, the commencing dose being one to one and a half ounces. The horse is securely held by two ropes attached to the head-collar, but struggling and violence are exceptional.

Dogs are very susceptible to the action of anæsthetics, but, with rational precautions, may be kept under their influence for an hour or longer. It has frequently been stated that chloroform is not so safe as ether; but the Hyderabad experiments seem to disprove this. The dog should be fasted. for two or three hours. The drug may be placed on a sponge, or on lint, in a wire muzzle covered with a towel ; or it may be given through an inhaler. For small animals Hobday's apparatus may be used, by means of which a fixed safe percentage of chloroform is given. During anæsthesia free respiration by the mouth is advisable, and the tongue must be prevented from falling back so as to 
obstruct entrance of air into the larynx. Savage dogs are coaxed into a kennel, or covered with a packing case, and pieces of cotton waste or blotting paper, saturated with the drug, are then introduced. Small dogs, cats, rabbits, etc., are speedily and safely anæsthetised when placed under a bell jar or tin pail enclosing cotton wool saturated with chloroform. In dogs the last reflex is furnished by the upper incisor gum, which when irritated induces quick elevation of the lower lip. This reflex is abolished in complete anæsthesia.

The aspect of an animal perfectly anæsthetised is that of an inert body, in which movements of the thorax and heart alone show that life is not yet extinct (Guinard). When anæsthesia has been pushed too far, inhalation of the drug must immediately be stopped, and any impediment to breathing of fresh air removed. The mouth should be widely opened and the tongue pulledforward; if breathing has ceased, artificial respiration must at once be adopted. In such a case unless the lungs are surcharged with the anæsthetic, as when it has been given in large quantity or too concentrated, and for some considerable time, artificial respiration, properly employed within thirty seconds after natural breathing has ceased, will revive most animals in two or three minutes. In the Hyderabad experiments some animals were recovered fifty, but none sixty, seconds after natural breathing had stopped. In narcosis occurring in ordinary practice, recovery, however, need not be despaired of so long as cardiac movements continue. Artificial respiration should be persisted with for at least half an hour after natural breathing has ceased. Insufflation of air through a tube inserted in the trachea may be tried. Inhalation of amyl-nitrite, or ammonia; hypodermic injections of ether, strychnine, adrenalin solution, or hot brandy ; and the continuous galvanic current, the positive pole being placed in the rectum, and the negative moved rapidly over the chest wall, are recommended in the hope of stimulating the arrested respiratory movements; and bleeding from the jugular is enjoined to relieve the right heart. Hobday recommends the administration of a dose of prussic acid to exert an energetic, but transitory, stimulation of the respiratory centre. 
Spinal Anæsthesia.-Intraspinal injections of sterilised solution of cocaine, stovaine, tropacocaine, novocaine, or other suitable anæsthetic, may be used to produce anæsthesia of the posterior portions of the body, prior to operations on the hind limbs and pelvic organs. This method, introduced in human surgery by Dr. Boer, was first tried on veterinary patients by Guille and Sendrail. Under the influence of lumbar anæsthesia, neurectomy, tenotomy, and cauterisation of the hind limbs, oophrectomy, the reduction of inverted uterus, castration, urethrotomy, and some hernial operations can be painlessly performed, and without the risks attending general anæsthesia. Injection is made through the lumbosacral space at the point of intersection of two lines, one median uniting the last lumbar and first sacral vertebræ, the other transverse, connecting the summits of the internal angles of the haunch. The skin of the region is shaved and carefully disinfected, and a sterilised hollow needle or fine trocar and cannula is implanted perpendicularly, to a depth of one half to three inches (dog or cat), or four to six inches (horse), at the point of intersection of the two lines in the dog and cat, and about three-fourths of an inch in front of this point in the horse. The entrance of the needle into the subdural space is indicated by the escape of cerebro-spinal fluid. Recently attempts have been made in the dog to enlarge the area of anæsthesia by injecting the drug through the occipito-atloid ligament, but at this situation the operation is more dangerous to the patient than lumbar injection. In practising injection at the poll, the dog's head should be held well elevated, and care must be taken to prevent puncture of the cord. Doses.-Solution of cocaine ( 2 per cent.), dog and cat, $m_{v}$ to $m_{15}$; horses, $m_{x x}$ to $m_{x l v}$; solution of stovaine ( 5 per cent.), dog, $m 20$ to $m 30$; cat, $m 10$ to $m 20$; horse, $3 \mathrm{j}$ to $3 \mathrm{ijss}$.

\section{ACTION OF DRUGS ON THE SPINAL CORD AND MEDULLA}

SPINAL STIMULANTS-SPINAL DEPRESSANTS

As on the brain, so on the spinal cord and medulla drugs act in one of two ways, namely, to stimulate or to depress. 
It is impossible to find a drug whose actions are confined to the cord alone, for in some degree, and with different doses, the other parts of the central nervous system are affected. At the same time there are drugs whose chief action is on the spinal cord. By stimulation is meant an improvement in the carrying out of the normal function; the centre becomes more sensitive and quicker to act. By depression is meant a delay in action, and a lessening of the power to carry out the normal function.

SpINAL Stimulants increase the functional activity of the cord. This they do by either direct excitation of the part, or by the depression of those cerebral centres which control the spinal centres. (See Alcohol.) The direct excitants apparently act much in the same manner as mechanical irritation or electricity. They seem to increase conductivity and lessen all resistance between the nerve-cells. Small doses heighten reflex excitability ; large doses cause tetanic convulsions ; whilst very large doses eventually bring about depression and paralysis. Spinal stimulants include strychnine, brucine, and thebaine, as well as calabarine, caffeine, absinthe, and ammonia. They are used in cases of collapse, of general debility, in certain forms of paralysis, and to rouse sluggish action, as of the bowels. A pure medullary stimulation would be shown by quickening and deepening of respiration, rise of blood pressure from vaso-motor stimulation and slowing of the heart from stimulation of the vagus (cardio-inhibitory) centre.

SPINAL DEPRESSANTS comprise most of the agents which depress the brain, such as the hypnotics, narcotics, and anæsthetics, but in addition there are some drugs which especially act on the medulla and cord. Thus hydrocyanic acid, turpentine, and camphor, after more or less preliminary stimulation, depress the medulla and cord. Others, such as physostigmine, emetine, carbolic acid, and the antipyretics, depress from the first. For practical purposes, to lessen reflex action and counteract the effects of spinal stimulants such as strychnine, the general narcotics and hypnotics like chloral and bromides should be preferred.

Spinal depressants are prescribed to lessen increased excitability of the cord, as in tetanus, chorea, and some forms of paralysis. By diminishing the conducting power 
of the grey matter of the cord, they impede the transmission of painful impressions. Sir Lauder Brunton propounds an explanation of the nature of inhibition. $\mathrm{He}$ believes that nervous stimuli consist in vibrations in nervefibres or nerve-cells, analogous to the vibrations of light or sound. When two waves of light or sound fall upon each other so that their crests coincide, the intensity of the light or sound is increased; but when they fall so that the crest of one wave occupies the trough between the two preceding or succeeding waves, such two waves of light cause darkness, or two such waves of sound cause silence. Moving the one wave forward or backward upon the other intensifies or diminishes the vibrations of light or sound. 'Supposing nervous stimuli to consist of vibrations like those of light and sound, the action which any nerve-cell would have upon the others connected with it would be stimulant or inhibitory according to its position in relation to them.' If nerveforce, as believed, consists of vibrations similar to those of light or sound, the relative position of nerve-cells in action will often determine a stimulant or inhibitory result. If one nerve-current meets another in such a way that the waves of which they consist coincide, the nervous action will be doubled, but if they interfere the nervous action will be abolished. If they meet so as neither completely to coincide nor to interfere, the nervous action will be somewhat increased, or somewhat diminished, according to the degree of coincidence or interference between the crests of the waves. The relation of these waves to one another may be affected by the distance each travels and the rate of transmission.

This hypothesis seems to explain why different doses of poisons sometimes produce very different results. The phenomena of strychnine poisoning thus appear to depend upon the nervous vibrations being thrust crest upon crest, when intense convulsions occur ; while, from one or another wave dropping half a length behind, the interval of rest or relaxation follows. In like manner may be explained the similar effects of cold and heat. Cold retards, while heat accelerates, transmission of vibrations, and either agent may thus alter one of the waves, causing coincidence and 
consequent stimulation, or separation by a half or a quarter of a wave and consequent inhibition or restraint.

\section{ACTION OF DRUGS ON THE NERVES}

\section{PARALYSERS-STIMULANTS-ELECTROTHERAPY}

Nerves may be acted upon in various parts of their course ; in the nerve centres in which they originate ; in their cords or trunks ; or in their minute endings distributed in muscles or glands, or on the surface of the skin (sensory endings). Motor nerves have their excitability more readily disturbed or destroyed than sensory nerves. Injuries of compound nerves frequently arrest motor function, but leave the sensory function slightly, or only temporarily, impaired. The nerve trunks are much less susceptible than the end plates, and are only acted upon by strong solutions directly applied to them.

Nerve-endings-motor, secretory, or sensory-are acted upon by many drugs, and these effects can be shown by experiments on isolated organs. When an impulse is conveyed by a nerve to a nerve-ending, it is thought that some chemical change takes place in the ending which produces a substance acting on the cells of the muscle or gland to cause either increased or decreased activity. Instead of an impulse by way of its nerve fibres, the nerve-ending may be acted upon in a similar fashion by various drugs applied locally, or brought to it in the blood stream.

Increased excitability of motor nerve-endings is produced by aconitine, physostigmine, pilocarpine, and pyridine. Alcohol, ether, and chloroform, applied directly to nerves, first increase and then diminish their irritability.

PARALYSERS of motor nerve-endings have their most powerful representative in curare, which when injected prevents stimulation of the nerve from producing a contraction. Numerous other agents act similarly, of which the best known is conine. The nerve-endings in secretory glands and unstriped muscle are also stimulated by drugs like pilocarpine and physostigmine, whilst atropine and hyoscyamine have the reverse effect. 
Sensory nerves are readily affected by many drugs; their local effects are comparatively easy to determine; but when the drug enters the circulation many structures are liable to be affected, and definite results are difficult to obtain. Much trustworthy information has, however, been got by experiments on frogs, chiefly by ligaturing the sciatic artery of one leg, injecting into another part of the body the drug to be tested, and by pinching, pricking, heat, or electricity, noting the difference in sensation between the poisoned limb and the ligatured unpoisoned limb. By these and other experiments it has been demonstrated that nervous sensibility is diminished by aconite, veratrine, belladonna and atropine, carbolic acid, and chloroform. Hydrocyanic acid exerts topical paralysing effects on sensory nerves. Notable reduction of the sensibility of sensory nerves is likewise effected by several members of the aromatic series of carbon compounds, such as exalgin (methyl-acetanilide), antifebrin (acetanilide), and antipyrine (phenazone). Diminishing excitability of sensory nerves, such agents relieve pain, and are accordingly anodynes. Others exert marked paralysing effects on the terminals of cutaneous nerves, temporarily destroy sensibility, and hence are useful local anæsthetics. Among these are cocaine and its allies, ether spray, cold, in the form of ice or freezing mixtures, and carbolic acid.

The irritability of sensory nerves is increased by topical or cants. Aconite and veratrine, whether applied locally, irritarried through the circulation, produce at first peculiar numbness and tingling of the torigue and lips, and indeed of all parts supplied by the fifth nerve, to be followed by depression and lessened sensibility.

Electrotherapy constitutes an important method of treatment in human medicine, but in veterinary practice its application is limited to the occasional use of Faradism or Galvanism. The faradic, alternating, or induced current is serviceable in peripheral paralysis where muscular stimulation is required, as in the treatment of equine facial, radial, or crural paralysis, and of similar conditions in the dog. The Galvanic, Voltaic, or constant current is most useful in cases where definite chemical change in the tissues is required. Insulated needles placed in the tissues and tra- 
versed by a constant current induce decomposition and this process of electrolysis has been employed for the removal of tumours. Cauterisation, hæmostasis, and the amputation of diseased organs, tongue, eyeball, etc., are sometimes effected by means of a platinum wire or other suitable instrument heated by a constant current, which may be obtained from one of Faure's portable Accumulators. Galvanism is also used to improve nutrition and prevent further wasting in muscular atrophy, and in the treatment of paralysis of central-cerebral or medullary-origin.

Slight electric currents stimulate both motor and sensory nerves and muscles; more powerful or long-continued currents exhaust, paralyse, or tetanise.

Like nux vomica and other excito-motors, electricity stimulates depressed nervous action, controls disordered action, and hence improves impaired nutrition. For strains of muscles, after the primary inflammation and effusion are relieved by fomentations and rest, faradism over the seat of injury lessens pain and stiffness. A current of suitable strength applied for six or eight minutes, and repeated if needful twice daily, frequently benefits and sometimes removes muscular rheumatism, and is also serviceable in chronic articular rheumatism, which has resisted other treatment. In patellar luxation, when, owing to muscular contraction, replacement is very difficult, a prolonged application of the induced current is helpful. The current increases the contraction, but when it is discontinued the muscles quickly relax and reduction can be readily effected.

Paralysis depends upon various conditions, functional and molecular, and hence demands very different methods of treatment. Electricity, however, is often useful in diagnosing its seat and extent, and also in abating or removing the depressed or disordered conditions on which it depends. Torpidity of the bowels, resulting from imperfect intestinal peristalsis, is sometimes overcome by faradisation. To stimulate contractions in muscular atrophy one electrode is placed over the principal local nerve-centre, or nerve of the wasted part; while the other is moved over the altered muscles, for ten or fifteen minutes twice daily. Cases of roaring have thus been treated. One electrode is applied 
to the jugular furrow above the larynx, while the other is moved over the surface of the larynx and down the trachea. Only feeble currents should be used. The application is continued for five to fifteen minutes, and repeated two or three times a day.

Clonic spasms, represented according to their cause or site by trifling tremors or violent convulsions, are sometimes treated by electricity. The current may be directed to the faulty centre in the brain or spinal cord, to the nerve trunk the conductivity of which is morbidly affected, or to the local centres which are acting abnormally. Chorea in dogs, especially when of the chronic paralytic type, has been benefited by electric treatment.

To facilitate the passage of the current the skin of the affected region, as well as the rheophores, should be moistened with salt solution. Under the influence of the induced current the horse is restless, sometimes struggling violently, and the muscular contraction, which is always considerable, may provoke defæcation and emission of urine. In all cases before applying the current the horse should be carefully secured.

Electro-therapeutics as applied to the domestic animals deserves more extended practical study. The primary conditions for its rational and safe employment consist in a thorough understanding of the instruments used, and a knowledge of the strength of currents and their proper distribution to the parts to be influenced. Batteries, coils, and apparatus for veterinary purposes are now manufactured by Messrs. Arnold, West Smithfield, London; and the advantages of the various forms of electricity are fully discussed in Electrical Treatment, by Wilfred Harris, M.D., London, 1908.

\section{ACTION OF MEDICINES ON THE EYE}

In the local treatment of the cornea and conjunctiva appropriate astringents are zinc sulphate and silver nitrate. When the surface of the cornea is abraded, lead salts are unsuitable, as they form an insoluble albuminate, which may cause opacity ; while alum and potassium permanganate are 
undesirable, on account of their tendency to dissolve the corneal cement. The antiseptics generally used are mercuric chloride and boric acid, the latter frequently conjoined with an equal quantity of sodium sulpho-carbolate. The anodynes preferred are atropine, cocaine and eucaine. The sensitiveness of the eye is increased by strychnine. It is diminished, and local anæsthesia produced, by cocaine, which, accordingly, is serviceable in some examinations, as well as in operations on the eyes. The lachrymal secretion is increased by pungent odours from such volatile oils as mustard and onion, and by physostigmine. It is diminished by atropine.

The pupil is dilated by belladonna, atropine, homatropine, cocaine, daturine, and hyoscyamine. Such dilators are termed mydriatics. The pupil is contracted by calabar bean, eserine, opium, morphine (see opium), pilocarpine, and nicotine. They are termed myotics. Anæsthetics, and some narcotics in full doses, first contract, and afterwards from circulation of venous blood dilate, the pupil. The action of most mydriatics and myotics is purely local. Most mydriatics are anodynes, and hence are useful to allay irritation, inflammation, and pain. They are used in iritis to prevent adhesions. Dilating the pupil, they facilitate examination of the lens for cataract and of the retina. Myotics are used alternately with mydriatics to discover adhesions of the iris, and to break them down, to restrict the passage of light in painful diseases of the eye ; and to lessen intra-ocular tension, as in the earlier stages of glaucoma.

\section{ACTION OF MEDICINES ON RESPIRATION}

\section{ERRHINES AND EXPECTORANTS}

Respiration consists in the alternate enlargement and diminution of the cavity of the chest, whereby air is alternately inspired and expired. These movements, so essential to the life of all the higher animals, are chiefly presided over by a nerve-centre or group of ganglionic cells, situated in the medulla, posterior to the vaso-motor centre, and extending into the anterior part of the spinal cord. This centre is 
normally stimulated by venous blood as well as by afferent impulses from the lungs by way of the vagi, and inspiratory and expiratory movements are thence co-ordinated. The diaphragm is drawn back, the intercostal, scaleni, and other muscles raise the ribs, and air enters the lungs, distending the elastic walls of the air-cells. In ordinary circumstances, the chest then contracts, largely passively, with little muscular exertion and air is expired. Expiratory effort, although scarcely realised in ordinary breathing is, however, evoked in coughing and sneezing, as well as in producing vocal sounds. Inspiration and expiration thus alternate, in healthy adult horses at perfect rest, from twelve to sixteen times, in cattle about fifteen to twenty times, in sheep from thirteen to eighteen times, and in dogs from fifteen to twenty times per minute.

The respiratory centre is stimulated in various ways. Changes in the air breathed, such as an increase of $\mathrm{CO}_{2}$, or an excess of this gas in the blood, will stimulate the centre and cause quicker and deeper breathing. Similarly, deficiency of hæmoglobin, or loss of blood by hæmorrhage, increase respiratory activity. Then there are various drugs which directly stimulate the centre, notably strychnine, ammonia, atropine, thebaine, substances of the digitalis group, and caffeine. It is first excited and then depressed by chloroform, ether, and alcohol. Its activity is diminished, with consequent slow and shallow respiration, by opium, eserine, and aconite, and by the narcotics and depressants of the central nervous system generally. The centre can also be affected reflexly, for as we have seen afferent impulses from the lungs and bronchioles by way of the vagus determine normal respiratory rhythm. Thus any irritant gas inhaled, or irritant drug excreted by the bronchial mucous membrane, will affect the centre, deepen respiration and may cause coughing or sneezing. Other sensory (afferent) impulses, such as cold to the skin, a blow, or pain anywhere, usually stimulate the respiratory centre, often after a momentary inhibition with holding of the breath.

When respiration is paralysed, as in narcotic poisoning, subcutaneous injection of strychnine is sometimes useful. The changes in calibre of the bronchial tubes are determined 
by contractions and relaxations of the bronchial muscle (unstriped). Afferent impressions conveyed in the vagus fibres affect the respiratory centre, and at the same time efferent (motor) impulses in the vagus efferent fibres may determine a contraction of the muscle with lessening of the calibre. Such a spasmodic contraction occurs in asthma. It can be produced experimentally by drugs like pilocarpine and physostigmine which stimulate vagal nerve-endings, or by barium chloride acting directly on the muscle. The vagal nerve-endings are depressed, and consequent relaxation and dilatation of the tubes produced by atropine, hyoscyamine, and lobelia. The muscle is directly depressed and relaxed by the nitrites.

ERRHINES or sternutatories, when applied to the nostrils, cause irritation, sneezing, and increased secretion. They include tobacco in a finely divided state, hellebore, ipecacuanha euphorbium, and saponin. Errhines, although now seldom used, were formerly prescribed to cause counter-irritation in diseases of the eye and head, and to expel, by inducing sneezing, foreign substances lodged in the nostrils, facial sinuses, and respiratory passages.

\section{EXPECTORANTS increase and facilitate ths removal of} secretions from the air-passages. The healthy respiratory mucous membrane is moistened and protected by a thin, slightly adhesive solution of mucin, which is gradually moved outwards by the cilia. Cold applied to the surface of the body, dust and foreign particles, and micro-organisms, as in cases of influenza, readily excite irritation of the respiratory tract, and alter the amount and character of the mucus.

While the irritated membrane is dry and vascular, as in the earlier stages of catarrh and bronchitis, the breathing of warm, moist air, diffused from a steam kettle, or nose-bag containing steamed hay, beneficially dilates the congested vessels, and promotes secretion. In such cases, and notably in laryngitis, heat and moisture should also be applied externally by means of poultices, or fomentation with flannel or woollen waste wrung out of boiling water, covered with thin waterproofing, and kept in place by a properly adjusted hood. Further counter-irritation may subsequently be needful. In the dry stage of inflammation of the respiratory 
membrane, or when the secretion becomes thick and adhesive, so that irritating coughing is set up, expectorants increasing secretion and assisting its expulsion are indicated. They may produce their action in various ways. Some agents, such as jaborandi and pilocarpine, stimulate the secretory nerve-endings in the mucous glands and so increase secretion. Others are absorbed into the system and excreted to a greater or less extent by the bronchial mucous membrane, and their local effect is to increase secretion. Such are the alkaline carbonates and the iodides. Still another class are drugs which are irritants in the stomach and produce vomiting in larger doses, such as ipecacuanha, saponins, tartar emetic, and squill. These in small doses all increase bronchial secretion. Then in addition to the foregoing are the balsams and volatile oils, which, besides being partly excreted in, and increasing, bronchial secretion, also exert antiseptic effects locally. Terpine, oil of turpentine in a vaporised state, creosote, or the old popular remedy of the fumes of burning tar, prove effectual in moderating irritable congestion and continued secretion in many cases of bronchitis by their disinfectant action, and by causing expulsion of the irritant. A relaxed throat generally indicates the existence of a similar condition throughout the respiratory tract. An appropriate remedy is a combination of terebene and an acid given as an electuary, which exerts beneficial effects both topically and generally.

Expulsion of the respiratory secretions is produced by increased activity of the cilia, which are believed to be stimulated by ammonia solutions; by coughing; and by increased activity of the respiratory centre, which, as already stated, is stimulated by ammonia salts, as well as by ipecacuanha, belladonna, and strychnine.

Influenza colds, so common amongst horses, and notoriously infectious, no doubt depend upon specific microorganisms, which attack the upper, sometimes the lower air-passages, and not infrequently also induce gastro-intestinal catarrh, and other complications. Occasionally such seizures may be checked or mitigated in their early stages by moistening the parts first affected with solution of carbolic or sulphurous acid or eucalyptus oil, applied in the form of 
spray, gargle, or inhalation. Similar treatment proves beneficial in the later stages, by lessening congestion or by exerting antiseptic effects. The washing out of the nostrils is conveniently done by an ordinary syringe, by Higginson's enema apparatus or by Rey's nasal funnel.

Cough is a modified, usually involuntary, respiratory act, whereby gaseous, liquid and solid substances are forcibly removed from the air-passages. This sudden expulsive expiratory effort is brought about by faulty mucus or other irritant, lodged in the pharynx or larynx, by irritation of the lower air-passages, and reflexly, by impressions produced on surfaces other than the respiratory mucous tract, as by cold applied to the skin, or by nasal, buccal, pleural, gastric, or hepatic irritation.

Soothing gargles and electuaries, even if they do not actually reach the seat of irritation, frequently abate cough. Mechanically acting mucilaginous or saccharine substances may be rendered more effectual by combination with morphine, which diminishes irritability of the respiratory centre, and decreases secretion of mucus. The latter result is still more notably effected by atropine. A combination of these alkaloids is hence specially valuable where there is troublesome cough and profuse secretion of mucus. A somewhat different effect is obtained by conjoining morphine and apomorphine, which, with diminished excitability of the respiratory centre, produce increased bronchial secretion, and are hence serviceable where there is cough, and the membrane is dry, or coated with thick, sticky mucus.

A comfortable loose box, with abundance of pure fresh air, at a temperature of about $60^{\circ} \mathrm{Fahr}$., in several ways benefits the patient suffering with respiratory disease. More perfect aëration of blood is secured, while the cool, pure air, moreover, contracts dilated vessels, combats congestion, and hence will often remove cough, especially when depending upon irritation of the larynx, trachea, or larger bronchi. But while in many stages of respiratory disease the breathing of cool air is grateful and beneficial, draughts and cold must be scrupulously guarded against, and the body and legs of the patient kept comfortably warm, with extra covering, in 
order to promote free circulation in the superficial vessels, and thus antagonise congestion of the internal organs.

Cough depending upon gastric derangement, not uncommon in young animals, is often relieved by antacids. Cough resulting from bronchial filariæ is abated by the usual soothing remedies, and sometimes removed by turpentine administered in drench or intratracheally, or by inhalation of diluted chlorine, vapour of iodine, or sulphurous acid, which is rendered still more effectual for destruction of the parasites when conjoined with carbolic vapour.

In dogs with bronchitis or pneumonia, when the breathing is difficult, relief is often obtained by giving an emetic of ipecacuanha and squill. Venous congestion is overcome, and the state of the bronchial secretions improved. These good effects may often be maintained by the subsequent use of frequently repeated doses of ammonium carbonate, which is also serviceable earlier, or when the patient is too weak to justify the use of an emetic. In dogs recovering from acute attacks, or suffering from chronic bronchitis, cod liver oil is often useful, possibly on account of its furnishing readily assimilable nourishment for the delicate epithelial cells.

\section{ACTION OF MEDICINES ON THE CIRCULATION}

Medicines can affect the circulation by acting on either the heart or the vessels. There are many ways in which the heart can be affected, and it must be remembered that normally the rhythm of the heart is under the control of two sets of nerves, namely the vagus and the sympathetic. The vagus has its centre in the medulla and its fibres end in the heart wall, where they connect with the intra-cardiac ganglia from which non-medullated fibres ramify in the heart wall. Whether stimulated centrally or peripherally the vagus causes inhibition, slowing the beat and lessening the heart force. The sympathetic has the reverse effect, and stimulation quickens and strengthens the heart beat. But in addition to these direct nervous effects, any changes in blood pressure affect the cardiac rhythm, a fall in pressure producing a quicker pulse and vice versa. Then again cardiac muscle is directly acted on by several drugs, some strengthening and 
others decreasing its power. One part of the muscle-a band between auricles and ventricles known as the excitomotor area-is concerned in cardiac rhythm, and if stimulated causes quickening of the heart beat.

The blood-vessels are regulated in calibre and blood pressure is maintained by the muscle (unstriped) in the arteriole walls under the control of the vaso-motor centres and nerves. The vessels can be dilated and blood pressure lowered by drugs which either relax involuntary muscle directly or which depress the medullary and spinal centres. On the other hand, stimulation of the vaso-motor centres or of the muscle in the vessel walls causes a constriction and consequent rise of pressure.

The heart beat may be slowed by stimulating the vagus, either centrally in the medulla, or peripherally in the heart. Thus such agents as strychnine or ammonia, or concentrated chloroform vapour, when first administered, slow the pulse rate of the normal heart by stimulating the medulla, whilst digitalis acts on the peripheral endings to produce the same effect.

The heart beat may be quickened by agents which either (1) depress the vagus either centrally or peripherally, or (2) stimulate the sympathetic accelerator mechanism. Thus agents like chloroform, chloral, or any of the narcotics by central depression, quicken the heart during the safe stage of anæsthesia or narcosis. Atropine, on the other hand, paralyses the vagal nerve-endings in the heart, and so causes quickening. Again, adrenalin and cocaine afford examples of agents which stimulate the sympathetic and so increase both force and frequency of the beat.

The heart beat may be strengthened (1) by the last-named group of agents, namely those which stimulate the sympathetic accelerator mechanism ; (2) by agents which stimulate cardiac muscle, such as barium, digitalis, and strychnine, and these in small doses slow the beat somewhat. The excito-motor area is stimulated by larger doses of most of the drugs which stimulate cardiac muscle generally, and so the heart beats become very frequent. There are, in addition, some agents which specially attack this area from the first, notably caffeine and its allies, so that with small doses the 
pulse rate is greatly quickened. With excessive doses the rate gets so quick as to produce the condition of delirium cordis, where, with very frequent incomplete beats the output of blood falls down to zero. This occurs with toxic doses of digitalis and aconitine. There are also many indirect ways by which the heart and circulation may be affected. For example, alcoholic, etherous, and ammoniacal solutions, especially when given in tolerably concentrated form, immediately irritate the mouth, throat, stomach, and other parts with which they come in contact, produce reflex acceleration of the heart, and thus their effects often anticipate their subsequent actions resulting from their actual conveyance in the blood stream to the heart and other organs.

Cardiac stimulants are used to counteract failure of the heart's action from shock, physical injury, overwork, or depression dependent on disease. Stimulants, when acting favourably, under the circumstances produce a more vigorous heart-beat-the pulse, previously slow, is accelerated; or if quick, unequal, or weak, it becomes slower, stronger, and more regular. The heart pulsating more forcibly, and propelling at each contraction a larger volume of blood, arterial pressure is increased. A combination of two stimulants, acting as indicated in more ways than one, is often more effectual than any single drug. Hence alcohol is frequently conjoined with ether, ammonia, or aromatic volatile oils. Strychnine is prescribed with caffeine; and in serious cases hypodermic injections may be needful. Heat used in the form of warm drinks, and also externally, as warm rugs, fomentations, or poultices, is a heart stimulant, especially when the applications are made to the chest.

VASO DILATORS dilate the peripheral vessels (arterioles), and thus accelerate the blood-flow through them. This they do either by depressing the vaso-motor centres or by relaxing involuntary muscle. Prominent amongst remedies acting in this way are alcoholic solutions, ether, nitrous ether, amylnitrite, nitro-glycerin, and nitrites, which, by dilating peripheral vessels, lower blood pressure. Alcoholic solutions combining the twofold action of stimulating the heart and dilating vessels, usefully combat chill, equalise circulation, 
and prevent or relieve congestion. Horses brought in chilled and exhausted are frequently saved from congestion and inflammation of internal parts by the timely use of a stimulating drink, the good effects of which are further ensured by an extra rug, and flannel bandages to the legs. More permanent dilatation of external vessels is effected by frequently repeated doses of nitrous ether and ammonium acetate, with which camphor may also be conjoined. In combating chronic inflammation, vascular dilators are also serviceable, and their operation is further promoted by hot applications, friction, and counter-irritation.

HeART Tonics produce their effects more gradually and slowly than heart stimulants. Although large doses may induce violent, excitable, irregular heart action, repeated moderate doses prolong the diastole, and render the contractions slower, stronger and more regular. Most, besides, contract the muscular coat of the arteries, and hence are vascular tonics. On the muscular coat of the digestive canal they are also liable to act, producing nausea, spasms, and sometimes diarrhœa. Heart tonics comprise digitalis and its constituents, sparteine, cactus, strophanthus, veratrine, convallaria majalis, squill, nux vomica, and strychnine.

Digitalis has hitherto been the heart tonic generally used, notwithstanding the disadvantage of its preparations being of irregular strength, and its active principles, digitalin and digitoxin, usually consisting of several bodies, differing considerably in their actions. The difficulty is overcome only by physiological standardisation of the drug and its preparations. Digitalis is prescribed where one or other ventricle, from weakness and dilatation caused by reducing disease, or from incompetence of the auriculo-ventricular valve, is unable to drive the blood into the arteries. In hardworked horses compensating hypertrophy gives increased propelling power, and hence sometimes mitigates the results of valvular disease. When dilatation occurs, and the mitral valve is insufficient to close the orifice, blood is liable to regurgitate into the left auricle, retarding the blood flow from the lungs, and leading to general venous congestion. Heart tonics, notably digitalis, relieve this condition by 
imparting to the contractions the needful regularity and strength ; while, moreover, by slowing the beats, the ventricle is more completely filled. In dilatation of the right side of the heart usually depending upon serious attacks of influenza, bronchitis, or emphysema, heart tonics are seldom so beneficial as in mitral disease. In the various diseases for which digitalis has been used, strophanthus may be given. It has little effect on peripheral vessels, is a better diuretic than digitalis, but is much more toxic to the heart, so that minimal doses should be used. In heart failure, whether depending on nervous asthenia or on muscular weakness, strychnine in continued small doses is specially useful. Where there is marked irritability, it may be conjoined with cocaine, and, in anæmia, with preparations of iron. Where there is cardiac pain, nitrites are prescribed. Where blood pressure is abnormally high, it is usually desirable in vigorous subjects to relieve venous congestion by purgatives or diuretics before even the most cautious use of heart tonics is attempted. Lymphangitis, or inflammatory œdema, attacking usually the lymph glands and vessels of the hind limbs of the heavier breeds of horses, and occurring chiefly in well-fed, hard-worked animals after a day's rest, is probably caused by local infection and connected with imperfect oxidation, the consequent formation of sarcolactic acid, obstruction and congestion of veins, capillaries, and lymph vessels. The resulting acute inflammation is combated by hot fomentations, a smart purgative, antiseptics, and saline diuretics, while the tediously chronic œdema, which is apt to follow, is removed by friction, vasodilators, and circulatory stimulants, and regular exercise.

Cardiac Sedatives lessen the force and frequency of the heart's action. For such purposes aconite, gelsemium, and antimonials, or the anæsthetics and narcotic agents, are chiefly prescribed. In veterinary patients aconite is effectual, especially when given in small doses, at intervals of two or three hours. It is chiefly used in antagonising violent palpitating action of the heart, or lowering the quick, full, bounding pulse, and other febrile symptoms of laryngitis, laminitis, acute lymphargitis, and other local inflammations. In cases of hæmorrhage the narcotic agents are 
often useful by lowering blood pressure and allaying excitement and movement.

VASo-Constrictors contract blood-vessels, lessen the flow of blood through the vessels, and hence limit local inflammation, prevent escape of lymph and consequent œdema, and arrest hæmorrhage. They may act either by stimulation of the vaso-motor nerves or centres, or by directly stimulating the involuntary muscle in the arteriole walls to contract. They are represented by ergot, lead acetate, and notably adrenalin, full doses of digitalis or strychnine, and other heart tonics, and topical application of cold. Ice or refrigerant lotions applied to circumscribed spots contract the arterioles, and even considerable arteries, and thus relieve congestion, inflammation, and pain. In like manner, ice, when swallowed, arrests bleeding from the stomach, reflexly checks bleeding from the lungs, and, moreover, acts as a cardiac sedative. When the bleeding vessels cannot be reached, either directly or reflexly, ergotin is injected hypodermically.

\section{MEDICINES ACTING ON THE DIGESTIVE SYSTEM}

ON the Salivary Glands.-Sialagogues-antisialicsREFRIGERANTS.

ON the Stomach. - antacids - Gastric tonics STOMACHICS-BITTERS-EMETICS-ANTI-EMETICS-GASTRIC SEDATIVES.

ON THE INTESTINES.-PURGATIVES-CARMINATIVES-INTESTINAL ASTRINGENTS-ANTISEPTICS.

ON THE LiVER.-HEPATIC STIMULANTS-CHOLAGOGUESHEPATIC DEPRESSANTS.

On the Pancreas and Spleen.

ON WORMS.-ANTHELMINTICS-VERMICIDES-VERMIFUGES.

SIALAGOGUES are drugs which increase the secretion of saliva. This alkaline fluid comes from the secreting glandular cells, which are replenished with fresh materials from the blood-vessels of the glands. The process of salivation is regulated by a nerve-centre in the medulla, and subsidiary nerve-centres (ganglia) in the several glands. By food or 
other substances moved in the mouth, by irritation of the stomach, or even of the nostrils, stimulation is conveyed by their respective nerves to these ganglia, and reflexly salivation ensues. In this way the presence of food in the mouth and the movements of the jaws naturally provoke salivation. In like manner, through nerves of taste, distributed within the mouth, acids, alkalies, bitters, ethers, mustard, ginger, and other pungent substances reflexly increase secretion of saliva. Tartar emetic, and in fact all emetics, exert similar effects reflexly by acting on the stomach. Jaborandi, calabar bean, and their alkaloids, with muscarine and nicotine, produce salivation when injected into the blood by stimulating the peripheral ends of the secreting nerves within the glands or nerve-cells in the ganglia, and are termed specific sialagogues. Another group, including mercury, and potassium iodide, induce their effects by stimulating the gland cells, to a slight extent, during excretion.

The salivary and buccal secretions moisten the mouth and fauces, and hence facilitate mastication and swallowing, and lessen or prevent thirst. The ptyalin of the saliva, moreover, helps the digestion of starch in the stomach, whilst still the secretion of gastric juice is small. Graminivora secrete proportionally large quantities of saliva for the moistening of the dry food, on which they chiefly live. The horse in twenty-four hours secretes $84 \mathrm{lbs}$. In all animals the fluid is more alkaline the larger the amount of the starch food.

Antisialies are medicines which lessen the salivary secretion. Borax and potassium chlorate frequently remove the faulty irritable conditions of the mucous membrane, which lead to over-secretion. Opium and morphine diminish irritability of the nerve-centres, while atropine is the most effective paralyser of the peripheral endings of secreting nerves. The fermentative action of ptyalin is diminished by alcohol, alkalies, and acids, and checked by 1 per cent. solution of carbolic acid.

Refrigerants, in contact with the buccal and pharyngeal membrane, induce a sensation of coolness, and allay thirst, which is locally manifested by dryness of the mouth and 
fauces. Thirst is quenched by washing out the mouth with water, or lubricating the dry throat with bland mucilaginous fluids, sucking portions of ice, which horses with sore throats soon learn to do, or swallowing slowly slightly acidulated drinks, which, by stimulating secretion of saliva, moisten the parched membrane. But thirst also depends upon a deficiency of fluid in the body, and excess of soluble or saline substances in the blood-conditions which are remedied by ingestion of water or other diluents. The extreme thirst which occurs in horses affected with polyuria, or diabetes insipidus, is best controlled by a combination of iodine and opium.

\section{ACTION OF DRUGS ON THE STOMACH}

The stomach of the horse is small relatively to his size ; the cardiac portion is lined with stratified epithelium, and secretes no digestive fluid. The pyloric portion, which occupies about one-half of the viscus, is the active digestive part, and is lined with a vascular villous membrane, in which lie the gastric glands. Gastric absorption in horses has been questioned or denied since Bouley and Colin published their experiments with strychnine; but probably slow absorption does take place from the right sac in a normal condition. In ruminants, the first three compartments of the subdivided stomach are lined with cuticular mucous membrane, are chiefly occupied in the reception, maceration, and subdivision of the bulky fibrous herbage, which constitutes their principal diet. This thick epithelial covering, and the amount of food always lodged in these three stomachs, explain the tardy action of many medicines administered to ruminants, and their taking with impunity large doses of irritants. The fourth stomach is lined with vascular velvetlike mucous membrane, and secretes the gasíric juice, while from its walls slow absorption takes place. In the dog and pig the stomach and digestive organs resemble those of man, and in both animals absorption commences in the stomach.

Secretion of gastric juice is stimulated by gentle mechanical and chemical irritation, by introduction of suitable food into the stomach, and by stimulation of the nerves of taste 
in the mouth, for example by means of bitters, dilute acids, alcohol and ether. When indigestion occurs from presumed insufficiency of the gastric juice, two modes of treatment are available-(1) dilute acids, bitters, and a little spirit are given to stimulate secretion; but (2) where, from reducing disease or other causes, the stomach is enfeebled, a substitute for the gastric juice should be given in the form of diluted mineral acid, preferably hydrochloric, conjoined with pepsin. Moreover in all such cases, the food must be readily digestible. For horses, well-prepared mashes are substituted for dry corn and hard fibrous hay; while calves, foals, or puppies, when their undiluted milk disagrees, should have it mixed with water, or, better still, with lime water, sodium citrate, or with linseed tea, in order that the tough curd may be more easily broken down.

ANTACIDS.-Certain forms of dyspepsia depend upon, or are aggravated by, undue gastro-intestinal acidity, which is counteracted by antacids. These comprise the alkaliespotash, soda, and ammonia ; the alkaline earths-lime and magnesia ; and carbonates and bicarbonates of these bases. The neutral salts which these alkalies form with vegetable acids, notably the tartrates and citrates, after acting primarily as salines, are converted in the blood into carbonates, and secondarily exert alkaline effects on the tissues and secretions with which they are brought into contact. It is thus that they are serviceable in rheumatism, eczema, some cases of pyrexia, and in counteracting acidity of the urine. As an antacid, the volatile ammonia is less permanent than the fixed alkalies. Potash and its salts are more active than soda and its corresponding salts. Lime salts, being protective and astringent, and not readily absorbed, are indicated in diarrhœa; magnesia salts, being laxative, are appropriate where acidity concurs with torpidity of the bowels. Lithium carbonate, present in Baden-Baden and Bath mineral waters, is prized in human medicine as a solvent of urinary calculi and deposits.

Horses fed irregularly, or too closely restricted to dry food, frequently suffer from gastric acidity, instinctively lick the lime-washed walls of their stables, or eat earth, and are usually promptly relieved by antacids and suitable feeding. 
Calves carelessly managed manifest the same disposition to allay their discomfort by eating earthy matters. Antacids given with or just after meals neutralise this gastric and intestinal acidity. After producing local and direct effects on the digestive organs, they undergo absorption, and produce remote antacid effects on the blood and urine.

GASTRIC TonICs or stimulants, sometimes called stomachics, aid gastric digestion and improve the appetite. Such results occur when, in experiments, the stomach walls are gently irritated, and are also produced by small doses of stimulants and bitters. In certain conditions of gastric irritation, where an excess of a weak, inefficient juice is secreted, stimulants or bitters are, however, unsuitable, and small doses of mineral acids, or of gastric sedatives, such as salts of bismuth, or prussic acid, are prescribed.

EMETICs are agents which cause vomiting. This is effected by firm compression of the stomach between the diaphragm and the abdominal muscles, and by the simultaneous contraction of the longitudinal fibres which pass from the œsophagus round the gastric walls. When the stomach is thus compressed from behind, and drawn forward, the familiar spasmodic movements of retching result. When concurrently, however, with these movements, the cardiac orifice is dilated, the contents of the stomach are thrown up, and vomiting occurs. Sir Lauder Brunton thus describes the phenomena of vomiting :- ' Uneasiness is felt ; the inspirations become deeper; several swallowing movements are made, which sometimes carry down sufficient air to distend the stomach moderately. After several deep inspirations, there suddenly comes one which is deeper still. Then, instead of this being followed by expiration, the glottis shuts to prevent the escape of air ; the diaphragm again contracts still more deeply into the abdomen, and pulling the ribs together, the abdominal muscles forcibly contract; the left half of the stomach is drawn upwards, and the cul-de-sac flattened out ; the cardiac orifice dilates, and the contents of the stomach are forcibly expelled. The pylorus remains firmly contracted, and allows but little escape into the intestines.'

The movements of vomiting are modified respiratory and 
ruminating actions, and are presided over by a. nerve-centre in the medulla. The ganglia regulating respiration and vomition lie close together. Certain cells probably take part in both actions, and are acted upon by the same agents. 'Emetics usually quicken the respiration considerably before they produce vomiting, and, if injected into the veins, they not only quicken the respiration, but prevent the condition of apnœa being produced by vigorous artificial respiration. On the other hand, the desire to vomit may be lessened, to some extent, by taking frequent and deep inspirations, and narcotics which diminish the excitability of the respiratory centre also lessen the tendency to vomit.'

That the vomiting centre, rather than the stomach itself, is the prime factor in the production of emesis, is evident from Magendie's famous experiment of removing the stomach of a dog, attaching to the severed œsophagus a pig's bladder filled with fluid, which, when tartar emetic was injected into the veins, was compressed between the abdominal muscles and the diaphragm, and emptied of its contents by vomiting.

When the fauces of men, dogs, or other animals which vomit readily, are tickled with a feather, or when the interior of the stomach is irritated mechanically, or by a solution of mustard, the stimulus is conveyed by afferent nerves to the vomiting centre with which the special motor impulses are correlated. Many other parts of the body, through their afferent nerves, have communication with the vomiting centre, and hence vomiting is produced, not only by irritation of the fauces and stomach, but by irritation of the brain, lungs, liver, and gall ducts, the intestines, kidneys, and bladder, sometimes even by pain or injury of the extremities.

Dogs, cats, and pigs vomit as readily as men. Indeed, in dogs, vomiting is induced by most disagreeably-tasted, nauseous, or acrid substances, and sometimes is brought on purposely by the eating of grass. But horses, ruminants, rabbits, and guinea-pigs rarely if ever vomit, and are insensible to the action of powerful emetics. In horses emesis only occurs from extreme distension and spasm of the stomach, from dilatation of the lower part of the gullet, from complete obstruction of the intestines, and from the action of large doses of aconite, which, however, induce 
retching and discharge of excessive secretion of saliva rather than true vomiting. The insusceptibility of horses to the action of emetics is due apparently to some undiscovered peculiarity of the nervous mechanism concerned in vomition in most other animals. The horse's inability to regurgitate matters from the stomach, even when attempts to vomit are excited, depends upon several conditions-on the smallness of the stomach, which prevents it, even when tolerably full, from being grasped and squeezed between the abdominal muscles and the diaphragm; on the strong horse-shoe-like band of fibres which guards the cardiac orifice; and on the greater length of that portion of the œsophagus between the diaphragm and stomach, which bends on itself, and thus more securely obstructs the cardiac orifice when the tube, under the influence of emetics, is shortened by the contraction of its longitudinal fibres. The contents of the horse's stomach, even if discharged upwards, owing to the position and length of the soft palate, would pass out by the nostrils, and not by the mouth. As cattle naturally ruminate, it might be supposed that they might also readily perform the analogous act of vomiting; but the substances which cause emesis in other animals have no such effect on cattle or sheep. This, in part, depends upon the large size of the subdivided stomach, which cannot be grasped and compressed between the' abdominal walls and diaphragm. In horses and ruminants, the arrangement of the digestive organs thus virtually preventing vomiting, the vomiting centre would not be required; if it ever existed amongst earlier races, it has become dwarfed or ineffective, as seems evident from the notable tolerance which horses have of tartar emetic. Sir John $\mathbf{M}^{6}$ Fadyean suggests that in ruminants the power to vomit has perhaps been merged into the habit of rumination.

Emetics are divisible into two classes :-

(1) Those which mainly act locally on the stomach, such as copious draughts of tepid water, ipecacuanha, solutions of salt, mustard, alum, and ammonium carbonate, with copper and zinc sulphates.

(2) Those which act, through the circulation, on the vomiting centre, such as apomorphine. Muscarine, digitalis, pilocarpine, and the saponins, such as 
senega, are emetics by peripheral stimulation of the alimentary tract, although not used medicinally.

Emetics, acting locally, stimulate the vomiting centre reflexly from the stomach. Those of the second class may be carried direct to the vomiting centre ; but some are also attracted to the stomach, and thus, in part, at any rate, act reflexly. Tartar emetic injected into the blood is believed thus to act in both ways. The effects of local emetics are not usually of long duration, ceasing as a rule when the cause of irritation is expelled, and leaving little depression. Some, however, are more persistent, and are followed by nausea, depression, and increased secretion of saliva and sweat, as well as of mucus, alike from the digestive and respiratory tracts.

Emetics are used on dogs and pigs for removing from the stomach foreign bodies, acrid, irritating, undigested food, and poisons. Where prompt and effectual results are desired, as in cases of poisoning, apomorphine or copper and zinc sulphates are most suitable. By relaxing the longitudinal fibres of the gullet, and exciting anti-peristaltic movements, they are also serviceable in expelling obstructions from the fauces and upper part of the œsophagus. They expel bile from the gall ducts, and gall bladder, and force inspissated mucus and small gall stones into the intestine, thus relieving jaundice resulting from obstruction. By clearing out both the stomach and biliary system, they remove biliousness, and sometimes arrest epileptic seizures. In animals which vomit easily it is better that irritants lodged in the anterior parts of the digestive tube should be promptly got rid of by the mouth, rather than make the longer and more tedious route through the intestines, running risk of absorption, and thus probably doing further mischief. By stimulating the respiratory as well as the vomiting centre, emetics beneficially promote secretion and expectoration in the dry stage of catarrh and bronchitis, and sometimes in congestive as well as spasmodic asthma. In respiratory disorders, ipecacuanha and squill are often conjoined, and, where there is cardiac depression, ammonium carbonate is prescribed, alone or in combination. Relaxing muscular fibre, they were wont to be given to assist in the reduction of dislocations, but for such 
purposes anæsthetics are much more effectual. Their paralysing effect on muscle explains why emetics in excessive doses often fail to cause vomiting.

Emetics are contra-indicated in gastric inflammation, cerebral congestion, and hæmorrhagic conditions, and they should be cautiously used in pregnancy and hernia.

A safe and convenient emetic for a medium-sized dog consists of a teaspoonful each of common salt and mustard dissolved in three ounces of tepid water. More prompt and certain effects are produced by two or three grains of copper or zinc sulphate dissolved in a couple of ounces of warm water, rolled in a piece of meat, or mixed with other food. Greater depression follows the administration of three grains tartar emetic and ten grains ipecacuanha, given dissolved in three or four ounces of tepid water. Apomorphine, the most prompt and certain of emetics, acts by whatever channel it enters the body, and produces full effects on dogs in doses of one-tenth to one-fifth of a grain.

To check vomiting, which occasionally proves troublesome in dogs, three methods of relief are indicated-(1) the removal, by appropriate means, of the irritation of the fauces, bronchi, stomach, or other part which excites the reflex act ; (2) lessening irritability of the gastric nerves by giving small pieces of ice, or atropine, cocaine, carbolic acid, creosote, silver nitrate, bismuth subnitrate, or hydrocyanic acid; (3) quieting over-activity of the irritable vomiting centre by morphine, chloral, potassium or ammonium bromide, or amyl-nitrite.

\section{ACTION OF DRUGS ON THE INTESTINES}

PURGATIVES-CARMINATIVES-INTESTINAL ASTRINGENTS AND ANTISEPTICS

\section{Purgatives or Cathartics cause intestinal evacuations} by stimulating the muscular coat, and accelerating the peristaltic movements of the bowels; by increasing secretion from the intestinal mucous membrane; and also by limiting absorption of the intestinal fluids, or by setting up exosmosis of fluid from the blood and tissues into the lumen of the bowel. 
Intestinal movements are dependent on the ganglia of Auerbach's plexus, situated between the outer longitudinal and inner circular layers of muscle. Secretion is believed to be influenced by Meissner's plexus, lying in the submucous coat; but these ganglia, immediately regulating intestinal movements and secretions, are controlled by cerebro-spinal centres and nerves, notably by the vagi, which, when irritated, cause increased peristalsis, and by the splanchnics, which diminish intestinal movements. The blood-supply of the intestine is mainly regulated by the splanchnics and vagi, but also in part by the lumbar portion of the cord.

Purgatives vary in the degree and method of their action. The purgative oils, such as linseed and castor oils, act in virtue of their fatty acids, linoleic and ricinoleic acids, set free on saponification in the duodenum. These acids mildly irritate the bowel and so increase peristalsis gently and without griping. Their mechanical lubricant effects assist this action throughout the alimentary tract.

Another group of purgatives are the anthracene group, all derivatives of anthraquinone. They include aloes, rhubarb, and senna, cause moderate purgation by increasing peristalsis, but do not inflame the bowel wall to any extent.

The drastics are much more irritant, and cause inflammation of the intestine with the outpouring of considerable inflammatory exudate. In consequence of this condition, absorption is prevented, peristalsis is very violent, and severe purgation, often with griping, follows.

The last group of agents are those which act by stimulating the unstriped muscle in the bowel wall to contraction. These are exemplified by physostigmine and barium, and their action is produced whether given by the mouth or by hypodermic or intravenous injection.

Purgatives are frequently classified clinically as follows :-

Laxatives or aperients, such as small doses of oil, magnesia, sulphur and treacle, with fruits, roots, and green vegetable food.

Simple purgatives, such as full doses of oils, aloes, various species of rhamnus, which produce more copious, softened, or fluid evacuations. 
Drastic purgatives, such as croton oil, colocynth, elaterium, gamboge, and podophyllum, which greatly increase peristalsis, violently stimulate intestinal contractions, causing more or less pain, promptly produce copious, fluid discharges, and in large doses may cause serious intestinal irritation and inflammation. Eserine and barium chloride exert similar effects.

The term hydragogue is often applied to those agents which greatly increase the fluid contents of the fæces, examples being the more active salines in large doses and the drastics.

Cholagogue purgatives, such as mercurial preparations, podophyllum, and euonymin, remove bile, and will be further noticed.

Saline purgatives consist of neutral salts of the alkalies and alkaline earths, such as magnesium sulphate and citrate, sodium sulphate, potassium tartrate, and bitartrate.

The salines produce purgation by greatly increasing the amount of fluid in the bowel, and this they do by drawing water from the blood and tissues through the bowel wall. These salts in solution undergo dissociation to a greater or less extent, and their purgative action largely depends on their rate of absorption, which varies with each salt. Potassium and sodium ions are freely absorbed, but magnesium very slowly and with difficulty. Thus a magnesium salt, other things being equal, will remain longer in the bowel, and will exert its osmotic effects to draw out more water than a sodium or potassium salt. Again, the $\mathrm{Cl}$ ions are very rapidly and easily absorbed, the $\mathrm{NO}_{3}$ ions less rapidly, and the $\mathrm{SO}_{4}$ ions hardly at all. Thus chlorides are not nearly so effective as sulphates in producing purgation. Since in magnesium sulphate neither of the ions is absorbed to any extent, this salt is an excellent saline purgative, for it remains in the bowel and exerts its osmotic power until sufficient water has been drawn out to make it isotonic. Salines act especially on the small intestines, but only very slightly increase the secretion of bile or pancreatic fluid. When the accumulated fluid mechanically distends and stimulates the intestine, extra peristalsis is excited. These salines excite very little intestinal secretion when injected 


\section{PRECAUTIONS IN THE USE OF CATHARTICS}

into the blood, or subcutaneously. The amount of fluid secreted has been measured by Sir Lauder Brunton, who experimented on cats with concentrated solutions of Epsom salt tied into a loop of intestine. In four hours he found that from 42 to 56 minims of serous fluid were outpoured for every inch of surface acted on. In cattle or horses upwards of 12 square feet of intestine must often be directly stimulated by even a moderate dose of physic. A secretion of 50 minims to the inch would give a discharge of nine pints of fluid. Such considerations illustrate the depurative and febrifuge effects of an active cathartic.

The intestines of the horse are voluminous, presenting about 550 square feet of vascular mucous membrane. Purgatives and other irritants hence require to be used with much caution. For a day previous to the exhibition of a purgative, the animal, if possible, should be restricted to mash diet or green food. The dose should be moderate, and its effect may be accelerated and increased by administering it while the animal is fasting, by occasional gentle exercise, until it begins to operate, and by the repeated use of clysters. This last auxiliary, when properly employed with sufficient perseverance, is indeed so effectual in promoting the action of the bowels that one of the most successful of army veterinarians was wont to trust almost entirely to its use, seldom giving, except in extraordinary cases, any purgative medicine whatever. In serious, obstinate impaction of the large intestines, a flexible tube, six feet long, should be screwed on to a Read's pump, and copious enemata introduced into the colon.

For horses, aloes is the best cathartic. Linseed and castor oils are tolerably good, but less certain; while croton is much too drastic, unless in small amount, and largely mixed with some bland oil. Salines in cathartic doses are irregular, and sometimes act with unexpected violence. Senna, colocynth, buckthorn, and other drugs used as purgatives for men and dogs have little effect on horses.

With a warm mash the previous night, and subsequent abstinence from solid food, a moderate dose of aloes given in the morning, assisted by further mashes and occasional draughts of tepid water, purges most horses in ten or twelve 
hours. Without this desirable preliminary preparation, purgation seldom occurs within eighteen or twenty hours. In acute febrile cases absorption is usually tardy, and the action of the purgative is hastened by combination with a small dose of calomel, nux vomica, or tartar emetic. A horse should never have purgative medicine when his strength is reduced as in the advanced stages of inflammatory disease of the air-passages, in influenza and other debilitating epizootics, and seldom when the bowels are congested or inflamed. Horses affected by bronchitis may die from superpurgation, induced by three or four drachms of aloes ; and similar susceptibility to the action of moderate doses may also be observed in influenza, purpura hæmorrhagica, and laminitis.

In cattle and sheep the magnitude of the quadrisected stomach, the large amount of food which it always contains, the relatively small size of the true digestive compartment, and, compared with the horse, the greater length but smaller capacity of the intestines, explain the tardy, uncertain action of purgatives and some other drugs. For these ruminants saline cathartics are preferable, and their action is materially hastened by encouraging the drinking of water, rendered palatable by sweetening it with treacle. In obstinate constipation, or torpidity of the bowels, gamboge, croton, and calomel are often useful. Purgation may usually be produced in cattle in twelve to sixteen hours; but cases frequently occur where, in spite of treatment, the bowels remain unmoved for several days. The best purgatives for sheep are common and Epsom salts and castor oil, in doses of about one-fourth of those given to cattle. Calomel and croton are apt to act too violently. As sheep drink sparingly their medicine should be given with a liberal quantity of fluid.

The dog, on account of his small stomach and short alimentary tube, and the concentrated nature of his food, is peculiarly susceptible to the action of purgatives. Jalap, with a little calomel or a mixture of equal parts of syrup of buckthorn and castor oil, is most generally approved of, and usually operates in from five to eight hours. Aloes acts more slowly and uncertainly, while saline medicines are 
apt to cause vomiting, or, if retained, to purge with undue violence.

Pigs are affected by cathartics much in the same way as men and dogs, and are best physicked by administering three or four ounces of Epsom salt dissolved in water, or a like amount of linseed or castor oil.

The uses of purgatives are numerous. Few medicines are applied to so many important purposes.

(1) They empty the alimentary canal of undigested food, fæces, bile, unabsorbed poisons, and worms. Sweeping away partially digested food, they diminish the amount of bloodmaking materials, and thus diminish plethora and obesity. In horses fully two-thirds of the fluid ingesta, under ordinary circumstances, is removed by the bowels, and this large amount is greatly increased when physic is given. They remove noxious gases and fluids, micro-organisms, ptomaines, and other intestinal toxic matters which are the causes of dyspepsia, colic, and diarrhœa ; and which, moreover, secondarily or reflexly produce nervous depression, and other serious effects on the system.

Constipation is usually dependent in great part on deficient peristalsis, and hence, when of frequent occurrence, is often advantageously combated by conjoining a little nux vomica with the cathartic. When the general vigour of the patient is defective, the aperient may be conjoined with iron or arsenic; and where there is venous congestion, with digitalis or belladonna. Horses restricted to dry food are frequently affected with constipation, and in such cases the diet should be varied with an occasional mash, a little linseed cake or green food, while water ad libitum should be allowed at least four times daily. The bulky and comparatively indigestible nature of the horse's food induces copious alvine evacuations, which are passed usually at intervals of four or five hours. Impaired intestinal action, or obstruction, hindering or arresting these frequent evacuations, causes more serious and rapidly fatal results in horses than in dogs, or ruminants, in which the bowels naturally act less frequently. Torpidity or obstruction which has resisted ordinary treatment is now usually relieved-even in horses-by the hypodermic injection of a grain of eserine 
and one or two grains of pilocarpine; or by intravenous injection of a solution of barium chloride, or arecoline hydrobromide. Constipation, troublesome in dogs kept in the house, or on the chain, is best treated with a dose of oil, and prevented by attention to diet.

Diarrhœa, at its outset, is usually most effectually treated by a dose of oil, containing a little laudanum or hyoscyamus - a combination which removes the cause of irritation, and prevents irregular peristalsis and griping.

(2) Purgatives, notably salines or hydragogues, increase the amount of intestinal fluid, hinder its absorption, and thus purge the blood of waste products, relieving febrile attacks, and lowering blood-pressure.

The blood, thus left in a state of concentration, speedily recuperates itself, absorbs water and lymph from the tissues, thus relieving œdema, dropsy, and lymphangitis. To secure this special action, such salines as Epsom salt and alkaline tartrates are specially useful, and their efficacy is increased when they are prescribed in tolerably concentrated form, and given when there is comparatively little fluid in the alimentary canal.

(3) Purgatives lower fever temperature, but how this effect is produced is not definitely known. They diminish the force of the circulation, and may in this way lessen the production of heat, and, moreover, hasten removal from the body of waste or other deleterious matters, which are a frequent cause of fever. In animals in health purgatives do not, however, produce any appreciable lowering of temperature.

Carminatives are agents which regulate peristalsis and assist the expulsion of gases from the stomach and intestines. When digestion is in any way interfered with, the contents of the stomach are liable to undergo excessive or irregular fermentation, giving rise to large quantities of gas composed chiefly of carbon dioxide, marsh gas, and hydrogen, with some sulphuretted hydrogen. Formation of these gases is favoured by accumulation of mucus on the walls of the stomach, which prevents secretion of gastric juice, and by want of the normal stomach movements. These gases cause uncomfortable distension, and often provoke spasm and pain. (See Antispasmodics.) 
Carminatives are closely allied to Antispasmodics, and include the aromatic volatile oils of the umbelliferæ, labiatæ, and other orders, with ginger, mustard, and peppers, alcohol, ethers, and chloroform. Carbonic acid gas is neutralised by solution of ammonia and by solution of chlorine or chlorinated lime.

These agents, therefore, are used to expel flatus, relieve spasm, and prevent or lessen pain. Their effects mainly depend upon their controlling irregular peristalsis. They stimulate contraction of the distended stomach, and thus promote escape of gas by either the cardiac or pyloric opening. Regulating, in like manner, intestinal peristalsis, they displace and expel gases from other parts of the canal. They are usefully conjoined with purgatives. In cattle, owing to the large amount of food in the first stomach, it is sometimes difficult to remove accumulations of gas, by either carminatives or antispasmodics, the use of a gag fixed in the mouth, or even by the probang. Where these means fail, and distension is so great as to interfere with breathing or circulation, it is necessary to remove the gas by opening the rumen, with either a trochar and canula, or a tolerably large knife. In serious distension, threatening rupture of the large intestines, in horses, the gas is liberated by puncture of the cæcum or colon with a special trochar and canula.

InTESTINAL ASTRINGENTS diminish excessive or unduly fluid intestinal evacuations. They are specially used to antagonise various forms of diarrhœa. Some, like opium and chloral, lessen the excessive peristalsis on which diarrhœa generally in great part depends. Some, like antacids, neutralise acids which provoke both peristalsis and increased secretion. Some, like creosote, check fermentation and putrefaction, and thus arrest formation of irritants. Others, like catechu and tannin-containing substances, coagulate albumin, and consequently dry up both discharge of mucus and of blood. Others, like copper and iron sulphates, usually conjoin antiseptic and astringent actions. Many cases of diarrhœa, whether continuous or alternated with constipation, are best checked by castor oil, administered with a few drops of opium tincture. Where the diarrhœe still persists, opium in moderate doses is given. Where active peristalsis 
occurs after eating, drinking, or the excitement of quick work, as in some nervous horses and dogs, liquor arsenicalis is useful, or strychnine may be prescribed. Undue relaxation of the bowels, occurring in irritable horses during active work, is mitigated by careful attention to diet, by using the best food in digestible form, allowing water in small quantity at a time but frequently, and withholding water for several hours previous to putting the animal to quick work.

Intestinal antiseptics or disinfectants are sometimes prescribed in the treatment of disorders of the bowels, and of diseases which are believed to depend on the presence of pathogenic bacteria or their toxins in the intestine. Naphthol, salol, iodol, dermatol, lysol, creolin, creosote, carbolic acid, salicylates, iodine, iodides, tannoform, thymol, tannalbin, tannic acid, and terebene are the disinfectants generally employed. Experiments show that repeated small doses of beta-naphthol, salol, or creolin, rapidly diminish the number of micro-organisms expelled with the fæces ; and the administration of these or other intestinal antiseptics in hæmoglobinuria, diarrhœa, dysentery, and ulcerative enteritis, some cases of tetanus, and in distemper and other infectious diseases, deserves further trial. To ensure full effects within the bowel, the agent selected should be given encased in keratin, which is unaffected by the gastric secretion.

\section{ACTION OF MEDICINES ON THE LIVER}

\section{CHOLAGOGUES}

The liver is the largest gland in the body. It not only secretes and excretes bile, but part of the bile, mingled with the food materials, is again taken up from the intestine and again excreted. The liver, moreover, forms glycogen, and is concerned with the general metabolism of the body, the breaking up of the red corpuscles, and the formation of urea. Medicines taken up by the vessels of the small intestine enter the liver, where they may be retained, destroyed, or neutralised. Some are excreted in the bile. Arsenic, copper, and mercury are retained, and morphine, atropine, 
strychnine, veratrine, antipyrine, cocaine, and other alkaloids, are detained and their action is delayed. This important power of the liver to delay the action of or actually to destroy poisons, whether elaborated in the vital processes or introduced from without, is illustrated in Lautenbach's experiments. One-twentieth of a drop of nicotine does not kill a frog, but half that dose suffices when the liver has been removed.

The bile has various functions. It promotes absorption and assimilation of fats. It moistens the intestinal walls, and excites contraction of their muscular coat, thus acting as a natural laxative.

Direct Cholagogues increase the formation of bile, and are represented first and foremost by bile salts themselves, put into the blood stream. Others are dilute nitro-hydrochloric acid, sodium salicylate and benzoate, turpentine, podophyllum, euonymin. Some of these drugs augment the quantity of bile without altering its quality; others, like sodium salicylate, increase the quantity and fluidity; others, such as toluylendiamine, increase the solid parts, rendering it so viscid that it cannot readily pass through the bile ducts, and hence becomes reabsorbed, and may produce jaundice. Healthy dogs with biliary fistulæ, liberally fed with fats and oils, were found to secrete more bile than when freely fed on albuminoids or carbo-hydrates. It is of little practical importance to increase biliary secretion, although often necessary to remove excess of bile from the intestines. This can be done by the administration of purgatives.

INDIRECT ChOlagogues remove bile from the body mainly by increasing intestinal action. Excess of bile is apt to lodge in the small intestine, and become reabsorbed. Effectually to get rid of it, the bowels must be freely moved, preferably by a purge, which will produce sufficient fluid to wash out the small intestine. The drugs which effect this are calomel and other purgative mercurial salts, given with a cathartic, such as aloes, jalap, podophyllum, or sulphates of magnesium and sodium. Their effects are increased by active exertion. In dogs and other animals that vomit, emetics effectually remove bile by compressing the liver between the diaphragm and the abdominal muscles, diluting the bile with abundant 
mucus, and promptly discharging it by the mouth as well as by the rectum.

Owing to the low blood-pressure in the portal vein, and also the low pressure at which bile is secreted, there is little vis a tergo to overcome obstruction in the gall ducts, and hence the bile flow is rather liable to stagnation, with consequent increased reabsorption. This is apt to occur in human patients living largely on albuminoid food, and not taking sufficient brisk exercise. It also occurs in cattle forced for exhibition, and in all animals as a concomitant of intestinal catarrh. It is frequent among horses suffering from influenza, and the circulation of bile accounts not only for the yellow membranes, but also, in great part, for the dulness and languor characterising such complaints. The removal of this superfluous bile in these cases is suitably effected by half a dose of physic, or by some calomel or grey powder, followed by or conjoined with salines. Nitro-muriatic acid and iron salts, which experience shows to be subsequently serviceable, owe their good effects, at least in part, to their action on the liver. In jaundice, Robertson prescribed a purgative, followed by salines, and subsequently administered twice daily a bolus of inspissated ox bile, alternately with aromatic spirit of ammonia.

The pancreas has been termed an abdominal salivary gland, but its secretion not only converts starch into sugar, but also digests proteids, and breaks up and emulsifies fat. Not much is accurately known regarding the action of drugs upon the pancreas. Its secretion is increased when ether is introduced into the stomach, and diminished in dogs by atropine and vomiting. Calomel and salicylic acid check decomposition of pancreatic juice.

Few investigations have yet been made regarding the action of drugs on the spleen.

\section{MEDICINES WHICH KILL OR EXPEL WORMS}

ANTHELMINTICS—VERMTCIDES-VERMTFUGES

ANTHELMINTICs are agents which kill or expel intestinal worms. They include vermicides, which kill the parasites, 
and vermifuges, such as purgatives, which, without necessarily killing, detach them from the walls of the canal, and wash them away with the mucus in which they are usually imbedded.

The parasites most frequently infesting the alimentary canal are-bots, the larvæ of the Gastrophilus equi, found in the stomach of the horse; various tape-worms and round worms, occurring in the intestines and stomach of most animals ; and flukes, which invade the liver, bile-ducts, and intestines of sheep, and occasionally of cattle and deer.

The appropriate vermicides are-

1. For bots, green food, a combination of aloes, asafœtida, turpentine, and ether ; iodine tincture, or carbon bisulphide.

2. For tape-worms, areca nut, male fern, kamala, kousso, pomegranate root bark, turpentine, and chloroform.

3. For ascarides, popularly known as round worms, the remedies used are tænicides, with santonin, oil of chenopodium, bitters, arsenic, lysol, and creolin.

4. For strongyli or thread-worms, turpentine and essential oils, thymol, tannin, and tannin-containing substances, lysol, carbolic acid, naphthol, turpentine oil, with enemata of common salt, ferric-chloride solution, or lime water.

5. For flukes infesting the liver and bile-ducts of sheep, and sometimes of cattle and other animals, the treatment consists in maintaining the patient's strength by good feeding ; furnishing common salt and soluble iron salts, which exert general tonic effects and limited vermicidal action, and giving a dose of physic, which hastens the removal of flukes which have migrated into the intestines. Prevention is ensured by keeping the flock on sound pastures, free from the developmental forms of the parasite.

Bots in horses complete their larval stage in spring, and their discharge is then readily promoted by the laxative fresh grass. During autumn or winter they are dislodged with difficulty, and unless numerous, and causing much irritation, their removal is seldom attempted ; but animals seriously infested with them require liberal feeding. A considerable number of the larvæ may be dislodged by giving, after twelve hours' fast, two drachms each of aloes and asafœtida, dissolved in hot water, to which is added, when 
cold, half an ounce each of oil of turpentine and ether. The mixture is administered in gruel or linseed tea, and repeated on several consecutive days. Carbon bisulphide, in halfounce doses, given for several days before feeding, and followed by a purgative, causes discharge of the dead bots ; and one ounce of tincture of iodine diluted with two ounces each of glycerin and water kills bots lodged in the stomach.

Tape-worms of the three species infesting horses are usually expelled by aloes, turpentine, and oil. Friedberger and Fröhner place first on their list of tænicides three to five drachms of male fern extract. Gamgee recommended two drachms of asafœida, a drachm each of powdered savin and calomel, with thirty drops of male fern extract, made up with treacle and linseed meal, given at night, and followed by a purge next morning. Whatever remedies are used, it is essential that the bowels be emptied as thoroughly as possible by twelve to fifteen hours' fasting, or by a gentle aperient, in order that the vermicide shall be brought into contact with the head of the worm.

Dogs in some localities, in the proportion of fifty to every hundred, are infested with tape-worms. The most effectual remedy is powdered areca nut, the dose being two grains per pound of body weight. Amongst other remedies are male fern extract, now reputed the most certain remedy for tapeworm in man; aloes, pomegranate root bark, or kousso, followed by a purge; the American remedy, emulsion of pumpkin seed; tenaline, a registered preparation of areca nut ; and kamala, obtained from a Euphorbiaceous plant, is effectually used in India. A drachm of turpentine in two ounces of castor or linseed oil is frequently used.

Sheep, and especially lambs, are frequently infested with tapeworms, which grow very rapidly, and sometimes do widespread mischief. Areca nut, or extract of male fern, in the dose suitable for large dogs, is most effectual. Poultry harbour various species, for which areca nut followed by a laxative is the best remedy.

Ascarides are more readily removed than tape-worms. British practitioners usually treat the Ascaris equorum with drenches containing one to two drachms of aloes, and half a drachm each of chloroform and turpentine, given fasting on 
two consecutive mornings, and repeating the treatment a week later. German authorities recommend three or four doses of one drachm of tartar emetic, conjoined with bitters, at intervals of three hours, or a drench of arsenic, aloes, or absinthe, thrice daily, either prescription being followed by an aloetic purge.

The Oxyuris curvula, met with in the colon and rectum of the horse, is removed by similar prescriptions, and when confined to the rectum is still more readily dislodged by enemata of quassia decoction or other bitters, creolin solution, lime water, or solution of common salt.

The Ascaris marginata, the most common lumbricoid of dogs, is killed by three to five grains of santonin, the active crystalline principle of artemisia or wormwood. This agent is, however, dangerous in young animals, and it should be prescribed with great care. Turpentine and oil, gentian and other bitters, oil of chenopodium, and various other medicines also remove round worms. The effect of vermicides, as already indicated, is greatly increased by first emptying the intestine by fasting, or by a purgative, in order that the drug may act more directly on the parasites. Occasional doses of salines and mineral tonics remove superfluous mucus, which shelters the worms. The spread of parasitism is prevented by isolating infested animals, destroying their excreta, and by keeping healthy animals in uncontaminated quarters, and supplied with pure water and sound food.

The Strongylidæ include many species, two of which infest the horse, and, imbedding themselves in the mucous membrane usually of the large intestine, are difficult to expel. Various strongyles invade the fourth stomach and intestine of cattle, sheep, and goats, and not infrequently concur with other strongyles infesting the bronchi. Other species attack dogs, cats, pigs, and poultry; while the $S$. pergracilis is the cause of disease in grouse. Empyreumatic coal-tar oils, lysol, creolin, thymol, and chloroform are the remedies used.

Some cases of parasitism, unfortunately, are beyond the reach of anthelmintics. Trichinæ get immured in the muscles; the palisade worms (Sclerostomum armatum) develop aneurisms; Scl. tetracanthum, which causes fatal 
enteritis in many horses, becomes encysted in the mucous coat of the cæcum, colon and rectum, and is thus protected from the action of medicinal agents. Several species of Uncinaria burrowing in the mucous coat of the bowels of dogs and cats produce a pernicious anæmia (Friedberger). The treatment of such cases is limited to a dose of aloes, with antiseptics, nutritive food, and tonics, to sustain failing strength.

\section{MEDICINES ACTING ON THE SKIN}

\section{DIAPHORETICS—SUDORIFICS-ANHYDROTICS}

The skin, in the domesticated animals, besides being protective and tactile, secretes sweat and sebaceous matter, exerts to a very slight extent a modified respiratory function, and, on account of its constant and large secretion of fluid, is an important factor in regulating animal temperature. The sweat is a clear colourless fluid containing only about two per cent. of solids, of which sodium chloride is the chief. There are also traces of fatty acids, but there is little or no excretion of nitrogenous waste products except under pathological conditions. In cases of kidney disease, urea is excreted to some extent by the skin. In the dog and rabbit it has been found that removal of the hair and varnishing of the skin causes rapid lowering of temperature and death, due to the great loss of heat from the skin, the vessels of which are dilated. This result can, however, be prevented by wrapping the animals in cotton wool, and since neither of these animals sweat naturally, death cannot in any case be due to non-excretion of poisonous waste products through the skin. Thus the only important effect of the secretion of sweat is to cause a loss of heat from the skin by evaporation of the fluid.

The sweat glands, placed in the subcutaneous adipose tissue, number 2000 to 3000 on every square inch of the surface of men and horses. Their activity is regulated by the special centres which are situated in the spinal cord. The amount of natural perspiration depends mainly upon the dryness and temperature of the air. Sweating in men 
and horses begins, even while they are at rest, at a little over $80^{\circ}$ Fahr. It is chiefly determined-(1) by anything which increases heat production in the system, such as muscular exercise; (2) by increased activity of the sweat glands; and (3) by increased circulation of blood. The taking of food, the drinking of warm water or other bland fluids, and the administration of strong tea and coffee, by raising arterial pressure increase blood circulation through the cutaneous vessels, and promote perspiration. The sweat glands are stimulated by various aromatic and volatile substances which are excreted by them. The sweat centres are stimulated by ammonia salts, ipecacuanha, opium, camphor, nicotine, and antimony salts, by mental emotions and nausea, by a venous condition and high temperature of the blood, and reflexly by warmth to the surface, warm drinks, and alcohol.

Diaphoretics or sudorifics are agents which increase the skin secretions. They include (1) agents which stimulate the sudoriparous glands, or nerves connected with them, such as pilocarpine, jaborandi, physostigmine, and warmth to the surface; (2) agents which increase superficial blood supply, including such vascular stimulants as alcohol, ethers, and ammonia acetate solution, vaso-dilators such as amylnitrite, sweet spirit of nitre, and such nauseants as ipecacuanha and tartar emetic. Diaphoretics are less prompt and certain in veterinary than in human patients. Horses are made to sweat more readily than cattle, while the skin of horses and cattle is more easily acted upon than that of sheep or pigs. In the dog and cat there are no sweat glands in the skin except on the hairless pads of the feet, so that diaphoretics are without action on these animals. In all animals the readiest way of promoting copious cutaneous secretion is by heavy clothing, warm diluents, and keeping the animal in a dry atmosphere of about $70^{\circ}$, and administering small and repeated doses of ammonia acetate solution, or sweet spirit of nitre. Friction or grooming with suitable brushes beneficially excites the action of the skin in all animals. Warm and vapour baths, at temperatures varying from $100^{\circ}$ to $120^{\circ}$ Fahr., are useful diaphoretics.

Hydrotherapy affords a ready means of producing dia- 
phoresis in the lower animals, as well as in man. The patient may be enveloped in a sheet saturated with either cold or tepid water. Over this are placed three or four large horse-cloths. The legs should be subjected to similar treatment, or rolled in warm bandages. After the patient has been thus clothed for half an hour or an hour, he will steam and perspire very freely. The sheet and rugs should then be removed, and the animal dried by hand-rubbing, and comfortably clothed. This practice has been successfully adopted both with horses and cattle. The evil effects of chills are thus counteracted, colds are cut short, and rheumatism, especially in gross subjects, removed. Hydrotherapy should not, however, be adopted unless with due consideration, and under competent supervision. Protracted or violent diaphoresis, howsoever produced, proves debilitating.

Diaphoretics are used for the following purposes :-

(1) They restore checked cutaneous secretion, and hence equalise irregularities of circulation, counteract congestion of internal organs, and lower abnormal temperature. They are hence often serviceable in cutting short chills, colds, and simple febrile attacks, especially amongst horses.

(2) They help to remove injurious waste products of metabolism, which are apt to accumulate, particularly in febrile, inflammatory, and rheumatic disorders. These depurative services are especially valuable when the eliminating functions of the kidneys, bowels, or pulmonary membrane are impaired. In such cases the skin may be made to undertake a vicarious duty, and excrete waste matters usually removed by other channels.

ANHYDROTICS are drugs which lessen cutaneous secretion. Their effects appear to be induced (1) by diminishing the activity of the sweat glands ; (2) by lessening excitability of the sweat centres ; or (3) by acting on the circulation, usually by stimulating the respiratory centre, and thus overcoming that venous condition of the blood which in weakness and disease is a frequent cause of sweating ; (4) by cooling the skin either with refrigerant lotions or cool air, or ventilated cellular clothing in place of thick rugs which prevent radiation of heat. 


\section{MEDICINES ACTING ON THE URINARY ORGANS}

\section{ON THE KIDNEYS : DIURETICS}

Diuretics are agents which cause increased secretion of urine.

The amount of urine is liable to much variation, depending mainly on the nature of the food, the quantity of water drunk, and the proportion of fluid removed by the bowels and skin. Horses during the twenty-four hours pass from two quarts to two gallons, or on an average about ten pints. Secretion is augmented during digestion, especially when the diet is rich in proteids, by such food as heated oats or musty hay, and by vetches, particularly when animals are unused to them. More urine is passed during rest than when the horse at active work is losing fluid freely by the skin and lungs. Major-General Smith, from a series of examinations of the urine of horses, finds the specific gravity averages 1036, and that $3 \frac{3}{4}$ ounces of urea are excreted in the twenty-four hours. Cattle pass 10 to 40 pints of urine per diem, the specific gravity ranging from 1007 to 1030 . Sheep pass 10 to 30 ounces of alkaline urine having a specific gravity of 1006 to 1015 . Pigs excrete 3 to 14 pints of urine, which may be acid or alkaline. The quantity of urine, 15 to 35 ounces, excreted by the dog depends upon the diet and the size of the animal. The specific gravity ranges from 1016 to 1060 .

The urinary secretion is increased by a variety of conditions, notably by raising the pressure of blood in the Malpighian tufts; by cardiac stimulation, especially in cases where there is valvular disease and consequent venous congestion of the kidney; also by contraction of the bloodvessels of other vascular areas, as when cold diminishes cutaneous activity. Then too, any condition which increases the percentage of water in the blood (hydræmia), such as saline infusions, causes diuresis. Irritation of the medulla in the floor of the fourth ventricle experimentally produced by mechanical injury, or naturally produced by circulation of venous blood, greatly increases secretion, 
owing, it is believed, to stimulation of the vaso-motor centre which controls the renal arteries.

The proportion of the several urinary constituents is altered by different conditions. Urea, uric acid, and hippuric acid are increased by nitrogenous food, by common salt, phosphoric acid, leucin, and glycocoll, and are also augmented during the early stages of most acute diseases. The percentage is diminished by alcohol, turpentine, arsenic, and large draughts of water. Horses at rest pass a maximum of uric acid and a minimum of the less perfectly oxidised hippuric acid, but these proportions are reversed during and immediately after exertion, when disintegration of proteid tissues freely uses up oxygen and increases production of carbonic acid.

Albumin is always a pathological constituent of urine, but occurs in convalescence from febrile disorders, temporarily in horses receiving excess of albuminoids, and also in hæmoglobinuria in horses, and red water in cattle. It appears especially in inflammatory conditions of the kidney ; and is likewise produced by full doses of cantharides, which drug also causes hæmaturia. Such exudation of albumin, more apt to appear suddenly and temporarily in horses than in man, is lessened by careful dieting, the animal only receiving easily digested low diet or even milk alone, and by arbutin, the active principle of uva ursi, and also by keeping the bowels and skin in proper action, clothing the patient comfortably, but avoiding active diuretics. Bile constituents are occasionally found in the urine of the lower animals, but sugar is rarely present.

Diuretics may be classified as follows :-

1. Saline diuretics, such as potassium acetate and nitrate, liquor ammonii acetatis, the alkalies, saline infusions. All these induce a condition of hydræmia, i.e. of excess of fluid in the blood, and this excess the kidneys promptly endeavour to remove. Thus the tissues are drained of fluid and this action is particularly useful in cases of dropsy and the like.

2. Drugs which act by increasing the blood flow through the kidneys. Of these there are two groups, $(a)$ consisting of drugs which, whilst they do not lower general blood pressure, yet dilate the renal vessels, examples being caffeine and the 
purin bodies, the volatile oils, nitrites during excretion ; and $(\beta)$ drugs which are only diuretics in case of failure of the normal circulation, such being digitalis and strophanthus. These agents so improve the general circulation in cases of heart disease that the kidneys receive supplies of fresh arterial blood, and so are able to turn out larger quantities of urine.

3. Cold applications to the skin, cold air, and anything which lessens skin secretion.

The selection of a diuretic must in great part depend upon the purpose for which it is given. A diuretic ball, commonly used for horses standing for several days in the stable, or affected with swollen or itching legs, is made with half an ounce of nitre, resin, and soft soap, and may be repeated as required. The same ingredients dissolved in a pint of water make a diuretic drink for the cow. For a medium-sized dog, Stonehenge advises six grains of nitre, a grain of digitalis, and three grains of ginger, made into a pill with linseed meal and water. Another useful combination for dogs consists of thirty minims of spirit of nitrous ether, and five grains of potassium nitrate in a little water. Diuretic effects are best ensured by conjoining several drugs, by giving small and repeated doses, by encouraging the animal to drink water, thin gruel, or other bland fluids, and otherwise promoting excretion of the medicine by the kidneys rather than by the skin or bowels.

Diuretics are used-

(1) To increase the proportion of water in the urine, thus preventing deposition of its solids in the kidneys or bladder, and mechanically washing out such solids and other debris when they have been formed. Along with medicinal diuretics, diluents in such cases are freely supplied.

(2) To hasten expulsion of waste products and poisonous matters from the body, as in febrile or rheumatic disorders, or when poisonous drugs are required to be excreted, or when the kidneys are acting tardily. In these, as in other cases, a combination of diuretics is desirable, and digitalis, turpentine, or oil of juniper is often usefully conjoined with nitre. In human practice caffeine is prescribed.

(3) To remove excess of fluid from the tissues or serous 
cavities as in hydrothorax or ascites. When dropsy is connected with cardiac disorder, digitalis, and other drugs which act on the vascular system, are indicated, their efficacy being rendered more certain by combination with some saline diuretic, such as nitre. Copaiba is added to the prescription when the liver is affected. In dropsy connected with chronic kidney disease, nitrous ether and oil of juniper are preferred ; but they should be used with extreme caution.

Calomel augments secretion of urea, and hence promotes secretion of urine. In excessive or too frequently repeated doses diuretics are apt unduly to stimulate the kidneys and urinary organs, and provoke strangury, inflammation and hæmaturia. In cystitis, urethral disease, or obstruction, to prevent alkaline decomposition of the urine, antiseptics, such as benzoic and salicylic acids, or urotropine are prescribed.

\section{MEDICINES ACTING ON THE BLADDER}

LITHONTRIPTICS-URINARY SEDATIVES, TONICS, AND ASTRINGENTS

The movements of the urinary bladder are mainly reflex and regulated by a centre in the lumbar portion of the spinal cord, but in all the higher animals there is also a certain amount of voluntary control. Most drugs influencing the bladder appear to come into actual contact with it, and produce their effects reflexly. Thus irritant agents, such as cantharides, which are excreted in the urine, irritate the bladder and cause frequent micturition. Some horses will not urinate while in harness; others will not while the rider is in the saddle. As with other animals, the desire to urinate is suggested, and the act facilitated, by seeing or hearing other animals staling, or even by the sound of flowing water. If, as is often the case, the horse is in the habit of being whistled to when urinating, the act will be encouraged by whistling to him. Control of the sphincter of the bladder is frequently lost after injuries to the posterior part of the spinal cord or in paralysis of the hind parts of the body. Partial loss of control may be caused by nervous debility, and will disappear with improved general condition. 
Horses and dogs are liable to suffer from urinary deposits, which are sometimes found in the kidney, but more commonly in the bladder, and in male animals in the tract of the long urethra. In horses, as in other herbivora, urinary deposits consist mainly of calcium and magnesium salts, sometimes derived directly from drinking water, from earthy matters mixed with fodder or grain, or from lime salts, abundant in clovers and other fodder, which unite with the carbonates produced by oxidation of the vegetable acids also present in the food. These calcareous deposits are sometimes in a finely-divided sabulous state; sometimes they are aggregated into masses or calculi. Whether occurring as sediment, gravel, or stone, they cause more or less difficulty, straining, and pain in urination; the stream is interrupted, and from irritation of the lining membrane of the passage the urine usually contains excess of mucus ; while the portions last discharged are often turbid. When such symptoms are caused by a calculus in the bladder, medical treatment is unavailing. No medicine can be safely given in sufficient amount or sufficiently concentrated to dissolve calcareous urinary deposits within the body. Hence a stone which cannot be naturally discharged can only be removed by a surgical operation. When small it may be extracted by lithotomy; when large or of awkward shape, it should be crushed and removed in pieces. Calcareous sediment can usually be got rid of in great part, or entirely, by giving liberal supplies of barley water, linseed tea, or other diluents ; or with a syringe and flexible catheter the bladder may be filled with tepid water, and deposits thus washed out. Successive quantities of water may be introduced until they come away tolerably clear.

LITHONTRIPTICS are defined as remedies which prevent deposit of solids from the urine, or cause their resolution. In veterinary patients, as already indicated, they cannot resolve calculi, although they may promote their removal, and may check their formation. Such preventive treatment in the case of horses mainly consists in furnishing abundant, regular, and pure supplies of drinking water. Waters rich in calcareous matters are theoretically more liable to deposit such earthy constituents, especially under 
conditions where their carbonic anhydride is diminished. A weekly mash, containing any simple saline, somewhat lessens the tendency to these urinary deposits; and it is further important to remove conditions which interfere with regular urination or any obstruction to the outflow. It is accordingly advisable, from time to time, to wash out the horse's sheath with soap and tepid water, and thus get rid of accumulating sebaceous matter.

Bulls and oxen, and still more frequently rams and wethers, when liberally supplied with albuminoid food, and having little or no exercise, are liable to deposits, chiefly of ammonio-magnesian phosphates, in the bladder and curved or tortuous urethra. Amongst feeding sheep, fatal uræmic poisoning may thus be produced. The patients must be turned up, and endeavour made by manipulation to displace the deposits which block the urethra. Where these means fail to effect a passage, the vermiform appendage may be excised, or the canal may be opened, when a full stream of urine will be discharged, and with it a considerable amount of deposit. Prevention is effected by withholding or reducing the allowance of cake and corn, supplying soft laxative food, raising the sheep and moving them about at least thrice daily, so as to encourage urination, and by prescribing potassium bicarbonate.

Dogs, when freely eating animal food, suffer occasionally from deposits of uric acid and acid urates, the tendency to which is combated by suitable diet, diluents, and salts of potassium and lithium, both of which form soluble salts with uric acid, but the lithium having a lower atomic weight, unites with a larger proportion of uric acid.

Vesical and URINARY Sedatives are agents which lessen irritability of the bladder and urinary passages, and thus remove straining and pain. Diluents, such as linseed tea or other mucilaginous drinks, are often serviceable. Irritability when caused by the presence of calculi is diminished by the administration of alkalies. In cystitis, rugs wrung out of hot water and laid over the loins, and hot fomentations to the perineum, afford much relief. Irritability of the nerve-centres is soothed by opium, belladonna, and hyoscyamus. Chronic inflammatory conditions are relieved by such 
astringents as uva ursi, buchu, and Pareira root. Copaiba, sandalwood oil, urotropine, and terpenes are excreted in considerable amount by the kidneys, and exert their antiseptic and astringent effects throughout the urino-genital tract. Relaxed and hæmorrhagic conditions may be treated by salicylic, or benzoic acid, or by injections of astringents such as a dilute solution of silver nitrate (half a grain per ounce), and iron sulphate, alternated by salicylic acid.

VeSICAL AND URINARY Tonics are agents which increase the muscular tone of the bladder. Strychnine and cantharides strengthen the sphincter muscle and thus prevent involuntary escape of urine. Belladonna acts upon the sensory nerve-endings, and so is believed to lessen the sensibility.

MEDICINES ACTING ON THE ORGANS OF GENERATION AND THE MAMMARY GLANDS

APHRODISIACS-ANAPHRODISIACS-ECBOLICS, OR OXYTOCICS

The sexual function is regulated by two nerve-centres which influence and react on each other.

(1) The cerebral is believed to lie in the crus cerebri, and is stimulated reflexly by the special nerves of smell, sight, or hearing. (2) The spinal centre, situated in the lumbar region, regulates the vascular supply of the erectile genital tissues. Irritation of this centre causes turgid rigidity. Erection is also produced reflexly by local stimulation of the genital organs, as well as by irritation of the bladder, prostate, and lower intestine.

APHRODISIACS are agents which increase sexual desire. Deficient sexual activity usually depends upon want of general vigour, and the rational treatment consists in the administration of tonics-notably iron, strychnine, and yohimbine, which have a special effect in stimulating the spinal sexual centres. Cantharides exerts aphrodisiac influences mainly by irritating the urinary mucous membrane, and hence is a very uncertain and unsafe remedy. Alcohol, although stimulating the cerebral sexual centre, appears to paralyse the lumbar vaso-motor centres, and hence interferes with the proper performance of the generative act. 
ANAPHRODISIACS are agents which diminish the sexual passion. Some, as applications of ice, or cold water, act locally on the organs themselves; others, as potassium iodide, and bromide, purgatives, and hemlock, act generally on the genital nerve centres. A spare diet and steady work exert anaphrodisiac effects. Irritation of the genital lumbar plexus is produced reflexly by distension of the bladder with acid urine, by accumulation of filth around the prepuce, by ascarides, and even by fæces in the rectum. Removal of such causes of irritation accordingly diminishes undue sexual excitement.

ECBoLICS.-The involuntary muscular fibres of the uterus have the power of rhythmical contraction, but are besides controlled by higher nerve-centres in the lumbar portion of the cord, and in the brain. Experiments have demonstrated that stimulation of the cerebellum, crura cerebri, corpora striata, and optic thalami produces uterine contractions.

Ecbolics cause expulsion of the contents of the uterus. They include ergot, hydrastis, savin, and thuja ; but ergot is the only one in general use. It induces uterine contractions even when all nervous connections have been divided, but it also acts on the special centre. It is occasionally used in veterinary patients-particularly in the bitch-to hasten parturition when no obstruction is present, but when expulsive power is deficient. As it induces persistent contraction of the uterus, with consequent arrest of placental circulation, it must be used sparingly and cautiously during parturition. It is serviceable, however, subsequently in promoting contraction and checking hæmorrhage. Prompt contraction of the flaccid uterus, with arrest of dangerous bleeding, is best secured by subcutaneous injection of ergotin, and also by injection of warm water.

The local irritation of metritis and leucorrhœa can be relieved by injection of warm water, which is rendered still more effectual by addition of potassium permanganate, creolin, carbolic acid, or chinosol. Suppositories of opium and belladonna may be subsequently introduced.

Agents acting on the Mammary Glands.-A full stream of healthy blood passing through the mammary glands is 
essential to the abundant secretion of good milk. Animals which are to milk well must accordingly be well fed. Their diet must contain a sufficient proportion especially of albuminoids and fatty matters, which furnish the casein and cream of the milk. There are no drugs of much practical value as galactagogues, or increasers of milk. Jarborandi exerts only a temporary effect. Many drugs, however, pass into the milk, communicating to it their flavour and medicinal properties. Volatile oils promptly taste the milk of any animal to which they are given. Fixed oils and salines administered to milking mothers, purge the sucking offspring. Acids, diuretics, opiates, potassium iodide, arsenic, and other active drugs given to suckling mothers frequently exhibit notable effects on their susceptible progeny.

By careful selection of good milking breeds, and by suitable management, the quantity of milk yielded by first-class dairy cows is many times that obtained from cattle in their natural or semi-feral state, which furnish only sufficient for the rearing of one calf. But the highly developed mammary organs of these improved dairy animals become increasingly susceptible to disease, and less amenable to treatment. Acute inflammation, usually of bacterial origin, frequently attacks the udder, causing much constitutional disturbance, and necessitating the administration of purgatives, and febrifuges, and the application of antiseptics. In order to relieve its weight, the inflamed udder should be suspended by a broad web passed over the loins, and provided with holes for the teats. The web will conveniently support a light poultice of spent hops, or an antiseptic compress, which is often advantageously applied. A teat-syphon is generally useful to withdraw the milk or exudate, and to introduce disinfectants. The inflamed parts are dressed with belladonna, which paralyses the terminations of the nerves, and slightly diminishes lacteal secretion; and also relieves tension, lessens pain, and relaxes the sphincters of the teats.

With the view of hastening the drying of cows, belladonna is sometimes applied topically to the udder, and is also administered ; but as milk secretion is not entirely dependent on nerve influence, atropine and belladonna, which act only on nerve-endings, are of little real value for this purpose. 
The desired object is more practically attained by restricting the cow to dry food, milking her at gradually lengthening intervals, and, where the result has to be quickly secured, giving a dose of purgative medicine.

\section{REMEDIES ACTING ON TISSUE CHANGE}

\section{RESTORATIVES-TONICS-H EMATINICS-ALTERATIVES-}

FEBRIFUGES-BLOOD-LETTING

The various structures of healthy animal bodies are continually undergoing reconstruction, change, and devolution. Fresh materials or restoratives, in sufficient abundance, and containing in suitable proportion the constituents of the several tissues, are required. By digestion and assimilation, the food materials are prepared for their special uses. But these complex nutritive processes sometimes become deranged. Some fault occurs in the digestive enzymes; some want of activity or correlation overtakes the presiding nervous centres ; some delay takes place in the prompt and effectual removal of waste products by the bowels, kidneys, or skin. Hence arise muscular and nervous depression, expressed by dulness, debility, and diminished capacity for exertion. For such weakened, relaxed, unfit conditions, the appropriate remedies are tonics. ${ }^{1}$ Within the living organs and tissues themselves, further subtle reparative processes constantly occur, and certain drugs, termed alteratives, modify these remoter tissue changes in a manner not fully understood. The maintenance of a tolerably uniform temperature is essential to the performance of normal tissue changes in warm-blooded animals. In fever, however, the temperature is increased, and the agents employed for its reduction are antipyretics, or febrifuges.

Restoratives.-The bodies of all animals, especially when at work, undergo disintegration and waste, and their growth and repair hence require continual recuperation. Food must be provided in sufficient amount, of suitable quality,

1 Strictly stated, the word tonic refers to an agent which increases muscle tonus, but in therapeutics the term has a wider significance, implying increased functional activity and improved general tone. This latter meaning is indicated where the term occurs in this book. 
and with its several constituents in fitting proportion, to furnish appropriate nutriment for every tissue. Water, which constitutes four-fifths of the total weight of most animals, is being constantly removed by the lungs, skin, kidneys, and intestines, and, unless restored at short intervals, thirst and impaired health ensue. Not only are water and watery fluids requisite for the normal nutrition of the tissues, and for dissolving and carrying away their waste products, but in sick animals they also assist the removal of the products of disease. Even more imperative is the need of pure air to oxygenate the blood, maintain internal respiration and normal tissue change, and remove waste products. Animals require, either in the form of food, or when the subjects of disease, as restorative medicines, varying supplies of many constituents-phosphorus, specially for blood, bone, brain, and nerves ; sulphur, for the skin and bile acids ; fats, for cell-growth generally; iron, for the blood globules; salines, for the healthy restoration of the blood and most other parts.

These requirements, essential in health, are even more important in animals affected by disease. Food then requires to be given with especial care, and in an easily digested form, for in all serious diseases the digestive functions are impaired. In many febrile complaints, of horses and cattle, the ordinary grains and dry fodder, being imperfectly digested and assimilated, are apt to produce or aggravate gastric derangement. Animals suffering from febrile and inflammatory disorders should therefore be restricted to mashes, gruels, or cooked food, to which extra nutritive value can be given as required by addition of milk, eggs, or meat extracts. Food should never be allowed to lie long before a sick animal. If not promptly eaten, it should be removed, and in a couple of hours, or less time, replaced by a fresh supply. During and after attacks of debilitating diseases patients fed, as they should be, on small quantities of rapidly digested fare, obviously require food more frequently than in health. With returning appetite a convalescent occasionally greedily eats more than can be easily digested, and against this contingency well-intentioned attendants require to be warned. Relapses of colic and lymphangitis sometimes occur by 
allowing horses, immediately after recovery, their full allowance of dry corn and hay.

Unless when affected with diarrhœea, dysentery, or diabetes, animals do not injure themselves by taking too much water or watery fluids, but are often rendered uncomfortable by undue restriction. A supply of water should always be within the patient's reach. Cold water seldom does harm, and is more palatable and refreshing than when tepid. Salines, chalk, and other simple medicines, sometimes given with the water offered to sick horses, should be sparingly added, or administered in some other way.

Much mismanagement occurs with regard to the ventilation and temperature of the habitations of sick animals. Even for horses or cattle accustomed to comfortable boxes, a temperature of $60^{\circ}$ to $65^{\circ} \mathrm{Fahr}$. is sufficiently warm. Avoiding draughts, cool air should be freely admitted. No restorative or tonic is so effectual as cool, pure air, and it is especially needful in diseases of the respiratory organs and in zymotic cases. Sunlight is also an essential factor of health, especially in young animals. It increases the capacity of the blood and tissues for oxygen, favours healthy excretion, and is an excellent disinfectant.

A comfortable bed greatly conduces to the restoration of most sick animals. A sick, exhausted horse, who to his disadvantage would continue to stand if kept in a stall, will often at once lie down and rest if placed in a comfortable box. In febrile and inflammatory attacks, and during recovery from exhausting disease, alike in horses and cattle, a warm rug or two, and flannel bandages to the legs, help to maintain equable temperature and combat congestion of internal organs; but at least twice daily these rugs and bandages should be removed, the skin wisped over, and the clothing at once replaced. In fever, when the skin is hot and dry, great comfort results, a natural moist state of skin is secured, and more active blood purification and restoration ensue, from sponging the body several times a day with tepid water acidulated with vinegar, quickly drying, and at once putting on the clothing.

Attention to the position of the patient is frequently important in the treatment of disease. The debilitated horse 
allowed to lie long on his side is apt to suffer from congested lungs or even hypostatic pneumonia. The horse with severely injured knees is advantageously placed in slings. The cow, in the unconscious stage of milk fever, if permitted to lie on her side, speedily becomes tympanitic, and her chances of recovery are seriously impaired. In this and other cases, in which she has to lie even for a few hours, she must be propped up in a natural position on her breast-bone.

Mechanical and physiological rest are great restoratives. The pain accompanying most injuries and diseases, and greatly aggravated by performance of the natural functions of the part, instinctively enjoins as much rest as possible. Mechanical rest is frequently secured by splints and bandages, applied in all animals in cases of fractures of long bones of the limbs, and serious muscular and tendinous strains. Slings are also of similar service in horses suffering from fractures, open joint, and occasionally in laminitis. An inflamed part, when practicable, should be raised above the level of surrounding parts. Any pressure likely to interfere with circulation should be removed. To relieve irritation, pressure, and tension, the inflamed udder of the cow is suspended. In irritable and inflammatory states of the digestive organs, the simplest and most digestible food is given, and as little action as possible exacted from the stomach and bowels. When the kidneys are diseased, their work should be lightened, by promoting the action of the skin and bowels. Emollients and demulcents, as well as opium, belladonna, and other agents which paralyse the endings of sensory nerves, exert their curative effects mainly by ensuring physiological rest. But when acute disease has passed away, the gradual use of an affected part generally does good. Exercise in such circumstances proves a health restorer, improving appetite, and stimulating most of the bodily functions.

The medicinal restoratives in common use are as follows : Linseed, in the form of gruel, tea, or cake, proves a soothing, palatable, digestible, laxative combination of food and medicine. Cod-liver oil, especially in dogs and cats, conveniently supplies assimilable fatty matters. Iron salts, possessing tonic and hæmatinic, as well as restorative properties, are 
specially serviceable in anæmia; phosphates are prescribed for ill-thriving, weakly young animals ; salines are indicated in skin eruptions and itching, often met with amongst hardworked, liberally-fed horses ; pepsin, peptonised milk, liquor pancreatis, and malt extract, are sometimes administered to foals, dogs, and calves, when the natural digestive ferments are deficient or faulty.

Tonics.-When digestion is enfeebled, nutrition impaired, circulation languid, or waste products not promptly removed, there is apt to ensue weakness, want of energy, and unfitness for work-conditions treated for the most part by tonics. They are defined as remedies which impart tone or strength to the parts on which they specially act. They are allied to nutrients and restoratives. They resemble stimulants; but their effects are more slowly and gradually produced, are more permanent, and not succeeded by subsequent depression. While stimulants usually call forth strength previously latent, tonics frequently give strength. The same drugs, in different doses, often appear, however, in two or more of these classes. Alcohol, for example, is nutrient, tonic, and stimulant. Iron salts, according to their dose and the state of the patient, are nutrient, tonic, and astringent, but, used improperly, are sometimes irritant.

Tonics exert their curative effects in one or more of the following ways :-

1. By influencing digestion directly, usually increasing gastric secretion. Gentian, calumba, chiretta, and other bitters are chiefly serviceable as gastric stimulants or stomachics.

2. By indirectly assisting digestion, by improving the chemical and physiological condition of the blood, secretions, and tissues. The most important members of this class are hæmatinics, noticed below.

3. By acting on other special organs and structures, notably on the heart, nervous centres, or liver.

In using tonics it is accordingly essential to discover what part or organ is primarily and chiefly at fault. When digestion is enfeebled, gastric or intestinal tonics are prescribed. When the pulse is soft and weak, with a tendency to local congestion and œdema, cardiac and vascular tonics are used. 
When nervous funcíions are imperfectly performed, nervine tonics are appropriate. Cold, in the form of baths, douches, and sponging, proves a valuable tonic, applicable for general as well as local purposes, relieving irritability, bracing up soft, flabby textures, and equalising circulation.

HeEMATINICS, or blood tonics, constitute an important group of tonics, which increase the number of red corpuscles and the quantity of hæmoglobin in the blood. The red blood corpuscles are formed chiefly in the red marrow of bones, especially in the vertebræ and ribs. Red corpuscles are in great part destroyed by the liver and spleen, and it hence appears possible that disorder of these organs is a cause of the anæmia, which is very common amongst badly reared young animals. Anæmia is of two kinds:-(1) where there is a normal number of red cells all more or less deficient in hæmoglobin (chlorosis) ; (2) where there is a definite shortage of red cells. The former variety is frequently due to the insufficient absorption of iron to supply the hæmoglobin, whilst the latter may result from hæmorrhage or from hæmolysis due to disease toxins, or poisons such as lead. Then again, anæmia may often be due to malnutrition, unsuitable or insufficient food, digestive diseases, or the effects of parasites. In order to restore iron and fatty matters which are deficient in anæmic blood, daily doses of soluble iron salts are prescribed, while easily assimilated fatty matters, such as boiled linseed, or linseed cake, are given to horses and cattle, and cod-liver oil to dogs. An improvement of general health is further effected by judicious feeding and comfortable quarters. The anæmia resulting from debilitating disease requires similar treatment. To ensure their good effects, tonics are generally given in moderate doses, two or three times daily, for several days, or for weeks, and throughout their administration the bowels should be kept in a normal state.

Alteratives are defined as drugs which influence the amount and kind of tissue change going on in different organs and cells. Their results are usually readily recognised, but the way in which they are produced is difficult to explain. With the better understanding of pathological processes and the pharmacological actions of drugs, this term is falling 
into disuse as being too indefinite. At the same time, many drugs with definite and well-understood action have widespread and remote effects on metabolism in other organs, and this might be called an alterative effect. Thus purgatives, by removing waste materials and fluid from the intestine, drain off further waste products from the blood, and cause a flow of lymph from the tissues into the bloodvessels, and, so to speak, wake up metabolism in these tissues. Again, an agent like iodine, which in the form of iodide is diffused throughout the system, witness its excretion in so many channels, combines with the protoplasm of the cells, and then is gradually set free from it and excreted. This combination with and elimination from, the cell, stirs up its activities and promotes metabolism. The complex chemical changes taking place in living protoplasm are being gradually analysed by physiological experiment, and are seen to consist in large measure of a great number of simple chemical combinations and alterations, each brought about by a ferment-the new product at each step being handed on to another ferment for further alteration. These minute processes taken in the mass constitute the vital processes, and in fact constitute life, and drugs put into the blood or lymph may hasten or inhibit any of these minute but multitudinous changes, and so alter the rate or quality of the process. This, it will be seen, covers a great part of the field of drug action, and therefore the term alterative is too indefinite to have any real significance.

The agents formerly credited with chief value as alteratives are Iodine, Mercury, and Arsenic.

FEBRIFUGES are agents which lower the temperature of the body in fever. Their effects are more notable when the temperature is abnormally high. Animal heat is chiefly produced by oxidation, in the muscles, both voluntary and involuntary, and in glands, especially when they are in a state of activity. It is given off by the skin and lungs, in small amount by radiation; in still larger amount by contact with cold water or cold air, the latter abstracting heat with especial rapidity when it is damp or in motion, and also by the excretion of fæces and urine. Owing to diminished activity of the cerebro-vaso-motor centre, and conse- 
quent dilatation of the surface vessels, loss of heat is greater when animals are asleep than when awake. Conversely, more heat is produced when the animal is in active motion, and blood circulates freely through the heat-producing apparatus of the muscles and glands. Small animals, having a cooling surface relatively larger than their internal heating appliances, are more rapidly cooled than large animals. A centre in the corpus striatum appears to regulate the production of heat (thermotaxis), and certain agents (antipyretics) appear to develop their effects by acting on this centre.

Febrifuges may be divided into two classes-those which lessen production of heat, and those which increase loss of heat; and these again are subdivided, as shown in the following table :-

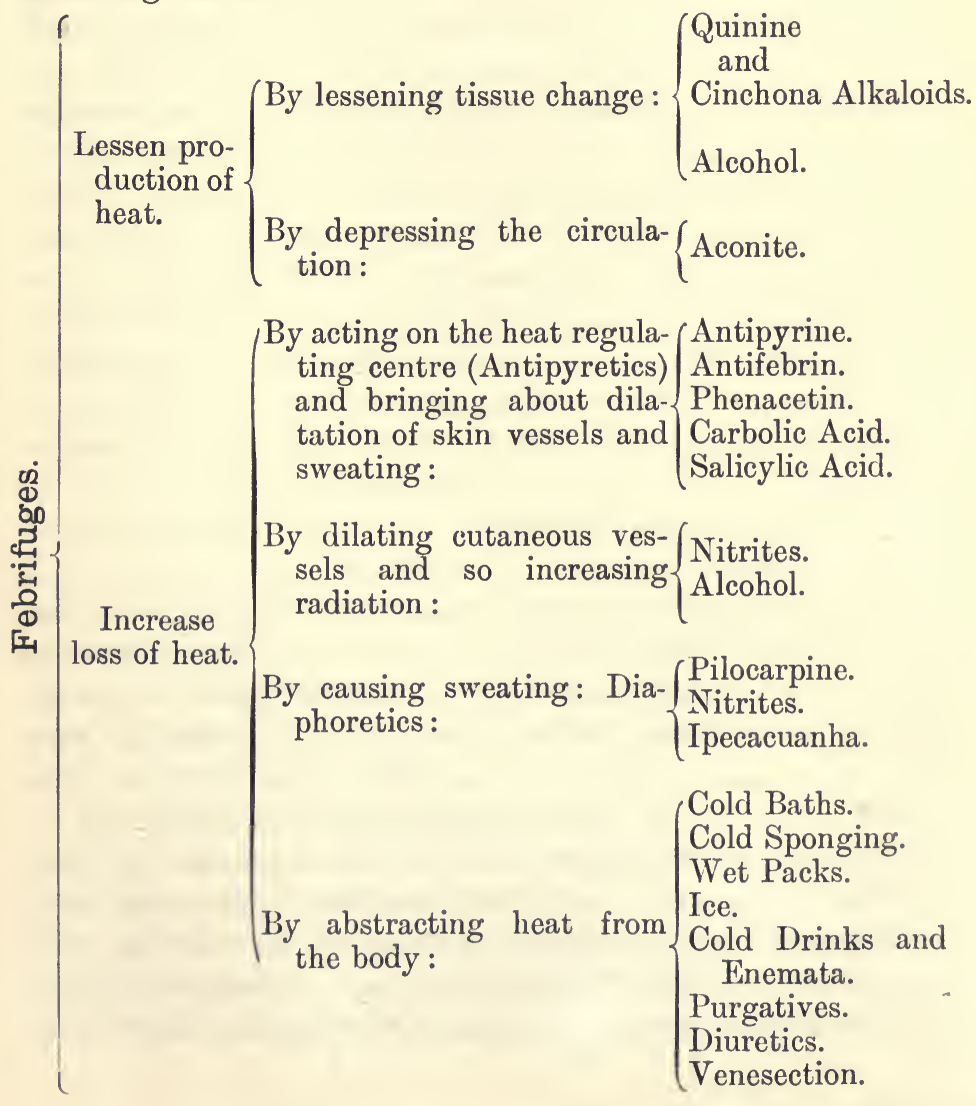


The production of heat (thermogenesis) is lessened in various ways:-The use of cold water, chiefly in the form of cold bath or cold packing, diminishes abnormal thermogenesis. Water absorbs and parts with heat quickly; it is capable of application locally or generally, continuously or intermittently. Cold water taken into the system, by whatever channel, increases the activity of healthy vital processes, notably metabolism, elimination, and leucocytosis, and thus both lessens abnormal heat formation and increases heat loss.

Quinine and its allies lessen metabolism in the tissues, prevent the oxidation of protoplasm, and the movement of leucocytes, and so prevent the formation of heat. Thus experiments show that these agents will still lower temperature if loss of heat by radiation from the skin is prevented by clothing and rugs. Alcohol probably acts to some extent in the same way.

Aconite also, and other agents which depress the circulation, lower abnormal temperature by preventing the formation of heat.

The loss of heat (thermolysis) is increased, as set forth in the above table, chiefly in the following ways :-

(1) By the antipyretics of the coal tar series of hydrocarbons. These agents are experimentally found to act on the thermotaxic or heat-regulating centre in the brain. In health heat formation and heat loss balance one another in such a way that the normal temperature of the animal is preserved, and these drugs have little or no reducing effect on this normal temperature. In fever the regulating centre is still acting, but now its mean point has been raised to an abnormal level. Thus exertion causes a fevered animal to sweat and lose heat, but this does not result in lowering of the temperature, for metabolism and heat formation go on to an extent sufficient to keep up the temperature to its former abnormal level. In other words, the centre now aims at keeping the temperature at the suitable temperature for the particular disease, and appears to have been geared up to work at that level. The antipyretics such as phenazone (antipyrine), and acetanilide (antifebrin), act on the centre and bring it down again to its normal level. As a conse- 
quence, heat must be got rid of, and this is brought about by dilatation of the skin vessels, and by sweating.

(2) By dilating the cutaneous vessels, and augmenting radiation of heat from the body, as is effected by alcohol, volatile oils, and nitrites.

(3) By promoting secretion of sweat, and thus increasing cooling evaporation, as is effected by diaphoretics.

(4) By directly removing heat, as is effected by hydrotherapy, cold baths, cold affusion, and by purgatives and diuretics.

Febrifuges are used to lower abnormal temperature, whether caused by prolonged exposure to heat or by febrile disease. Cinchona and its alkaloids are the remedies most trusted in the treatment of the fevers of animals. They mitigate the pyrexia, cut short the attack, and prevent the exacerbations which characterise such cases. Antipyrine, although it has little action on the temperature of healthy animals, reduces abnormal temperature quickly, and usually permanently. For veterinary patients it is one of the best of the new fever medicines. It has been successfully used in influenza, and is given both by the mouth and hypodermically. Salicylic acid and salicylates are specially serviceable in combating the fever and pain of acute rheumatism. Alcohol, in its several forms, exerts a twofold effect, diminishing oxidation and also dilating cutaneous vessels, and thus accelerating cooling. Bleeding, both general and local, judiciously used, lowers abnormal temperature, by relieving local inflammation. Purgatives doubtless act in like manner, and in some cases, moreover, relieve gastric derangement, and remove disease products. Horses and cattle are sometimes quickly sponged with cold or tepid water, rapidly dried, and comfortably clothed. Heat is thus directly removed; the cooling functions of the skin, which are impaired in most febrile attacks, are re-established, and this action may be further stimulated by the administration of ammonia salts, ethers, and alcohol. Very essential adjuncts for ensuring the effects of antipyretics are perfect rest, comfortable quarters, and a temperature of about $60^{\circ} \mathrm{Fahr}$.

BLOOD-LETTING promptly and directly affects tissue 
changes. A full bleeding diminishes the activity of all vital functions, excepting the production of blood corpuscles. The heart-beat is quickened, but its force is lessened; arterial tension is lowered; absorption is increased; sensibility to pain is diminished, owing to reduced activity of the peripheral centres. When blood is lost rapidly or freely, sighing, yawning, fainting, and convulsions ensue, and artificial anæmia is produced. In healthy subjects, however, these effects quickly disappear, and the blood is rapidly restored to its normal state.

Until within the last forty years, blood-letting was freely practised, and very generally abused. Venesection is now seldom employed even in cases of acute congestion and inflammation, which it is especially fitted to control. It may be practised either generally or locally. In robust animals suffering from acute congestion of the lungs, especially when accompanied by venous stasis, blood-letting affords prompt and frequently permanent relief. Alike in horses and cattle, it is serviceable where fever is acute, with a firm, incompressible, or full, slow pulse, as in laminitis, as well as in acute rheumatism. In some cases of lymphangitis in horses it is also useful. Dogs are so readily brought under the influence of emetics and nauseants that bleeding is less needed in them than in horses and ruminants.

Blood may generally be taken from full-grown horses or cattle to the extent of three or four quarts. The amount drawn should be accurately measured. The circumstances of the case materially affect the amount of blood to be drawn. It should flow freely until its abstraction has made a decided impression on the volume and strength of the pulse, or until the earliest symptoms of nausea and fainting are apparent. The jugular vein on either side is generally selected as the vessel on which it is most convenient and safe to operate. Excepting in expert professional hands, the fleam is safer than the lancet, which in restive horses may make an ugly gash. When practicable, the horse should be bled with his head erect, for in this position the nauseating effects, which indicate that no more blood can be spared, are most noticeable. When sufficient blood has been taken, the edges of the wound of the skin are brought accurately 
together, and secured by a pin, round which is wound some aseptic thread, or tow.

Blood-letting, although sometimes valuable in the earlier stages of acute inflammation in vigorous animals, is injurious in young or weakly subjects, in the later stages of disease, in epizootic and eruptive fevers, and, indeed, wherever the pulse is small, quick, or weak. A pulse of this character indicates debility, and bleeding in such cases increases exudation and effusion instead of preventing them, while it unnecessarily weakens the patient and retards recovery. While blood is being drawn, the finger should, in all animals, be placed upon a prominent artery, and if the pulse is observed to become rapidly weaker, it is evident that the treatment is unsuitable. Such mischance should, however, rarely happen, for when there is any question as to the propriety of blood-letting, such a reducing remedy should be avoided.

Local blood-letting is not much practised among the lower animals. Lancing the tumid gums of teething horses is seldom necessary, especially if soft food is supplied, as it should be in such cases. In laminitis some practitioners pare the horny sole, and open the vessels of the sensitive sole, encouraging the flow of blood by immersing the foot in hot water or in a warm poultice; but in such cases the heat and moisture are generally of more benefit than the bleeding. Cupping and leeches are not used in veterinary practice.

\section{POISONS AND ANTIDOTES}

Antidotes are agents which counteract the effects of poisons. A poison is an agent which is capable of determining definite modifications of protoplasm. In the popular acceptation of the term, a poison is a drug, whether animal, vegetable, or mineral, which, in small quantity, destroys health and life ; but it differs from a medicine only in the degree or intensity of its effects. Indeed, many valuable medicines, when given in large doses, become active poisons, whilst many poisons, properly administered, prove valuable medicines.

Antidotes may prevent the action of the poison, or may 
mitigate or arrest its effects. When a lethal dose has been swallowed, endeavour should be made, before it has time to enter the circulation, promptly to remove it by the stomachpump, stomach syphon, or by an emetic. It is advisable, however, in all cases to empty the stomach, and thus remove unabsorbed portions of the poison, before giving any fluid which favours solution and absorption, or even before administering the antidote. Some antidotes, such as charcoal, and demulcents, mechanically envelop the particles of the poison, or ensheath and protect the mucous surfaces, and thus retard absorption. Many enter into chemical combination with the poison, forming comparatively insoluble inert compounds (chemical antidotes). Thus, albumin forms, with corrosive sublimate and other metallic salts, insoluble albuminates. Freshly precipitated ferric oxide converts arsenious acid into an insoluble iron arsenite. When poison has been introduced into a wound, as by the bite of a rabid dog, or by the fang of a serpent, a ligature, if possible, is placed so as to prevent or retard absorption, and the wound is forthwith thoroughly washed with antiseptics and cauterised or excised.

The action of poisons, even after absorption, may, moreover, be controlled and counteracted by remedies which antagonise their lethal tendencies (physiological antidotes). Hypodermic injection of antivenomous serum neutralises the poison of cobra. Opium lessens the irritation and pain caused by irritants. Artificial respiration frequently sustains life throughout the stage of deadly narcosis induced by curare, prussic acid, or anæsthetics. But still more definite antagonism occurs between certain drugs. The stimulant and convulsant effects of strychnine on the spinal cord are opposed by chloral hydrate and tobacco, which lessen the excitability of the cord. The fatal depression of the cardiac and respiratory centres, produced by large doses of aconite, is antagonised by alcohol, atropine, digitalin, and by strychnine. Between pilocarpine and atropine the antagonism is very marked in their actions on the vagus, heart, muscular tissues, and iris, as well as on secretion.

Two explanations are given of this antagonism-(1) By 
chemical action, the drug first given is supposed to combine with the tissues immediately acted on, and to this combination the second drug may be added, developing another and less active compound; or otherwise, from such compound the second drug may displace the first. (2) The two antagonistic drugs may act independently of each other on the tissues, producing opposite effects - the one exciting, the other, it may be, paralysing. This latter physiological view seems to meet with most general approval (Brunton). In the case of poisons not rapidly fatal-such as lead, mercury, savin, or yew-an important curative measure consists in hastening their removal from the body by the organs through which they are chiefly excreted.

\section{Poisons}

\section{Antidotes}

Sulphuretted Hydro-) gen

Chlorine cautiously inhaled.

Chlorine, Bromine
Iodine Vapour $\cdot\left\{\begin{array}{l}\text { Ether : alcohol. } \\ \text { Steam inhalation. } \\ \text { Opium : starch. }\end{array}\right.$

Ammonia Vapour . Vinegar vapour.

Carbon Monoxide $\cdot\left\{\begin{array}{c}\text { Fresh air and artificial respiration : } \\ \text { transfusion. }\end{array}\right.$

Nitrous Oxide

Artificial respiration; tongue drawn

forward; intermittent pressure over cardiac region if heart action failing.

Coal Gas

Charcoal Fumes

Carbonic Acid

Marsh Gas
Artificial respiration.

Alternate warm and cold douches to the head and neck.

- Encourage circulation by friction.

Mustard plasters over surface.

Alkalies: sodium or potassium bicarbonate.

Sulphuric Acid Hydrochloric Acid Nitric Acid Phosphoric Acid $\left.\begin{array}{l}\text { Magnesia : chalk : plaster } \\ \text { Soap : milk : eggs whisked }\end{array}\right\}$ in oil. Olive or Almond oil. The alkalinity of the blood impaired by acids is restored by intravenous injection of sodium bicarbonate. 
Oxalic Acid and Oxalates

Tartaric Acid Acetic Acid

Chalk, whiting, or wall plaster, with water. Carbonates of lime and magnesia.

Alternate cold and warm affusions to the spine.

Artificial respiration: ether and

Hydrocyanic Acid . camphor subcutaneously.

Potassium Cyanide.

Atropine injection, repeated every half-hour.

Mixed salts of iron: ferrous sulphate, ferric perchloride: magnesia: tracheotomy.

Potassium hydrate and Carbonate. Sodium hydrate, Carbonate and Chloride Ammonia Solution Calcium Oxide

Vinegar : lemon juice.

Other dilute acids.

Milk : oil : acidulated drinks.

Stimulants.

Aconite

Alcohol : ammonia : ether hypodermically.

Digitalis : atropine : warmth.

Acorns ; Oak Shoots ;) Fern

Oil : salines : laxative diet.

Strong coffee, and cold douches to Alcohol . . $\left\{\begin{array}{l}\text { the head: camphor: ether: am- } \\ \text { monia. }\end{array}\right.$

Anæsthetics- $\quad$ Artificial respiration: amyl-nitrite : Chloroform, Ether, ammonia : adrenalin chloride sol. : etc. hydrocyanic acid.

Cold douche to head and neck.

In patients that do not vomit; wash out the stomach with tanAntimony . . n nic or gallic acid, followed by milk, white of egg, or other demulcents. 
Arsenic

Wash out stomach with large amount of warm water, introduced by stomach syphon or pump.

Give dogs zinc sulphate or other emetic.

Iron oxide, moist, made by precipitation of ferric chloride solution by sodium carbonate or ammonia : milk : oil.

Stimulants and coffee : tannic acid.

Caffeine, subcutaneously injected.

Atropine-

Belladonna .

Hyoscyamus

Stramonium

Sustain action of motor centres by interrupted electric current, and

. occasionally moving the animal.

- Artificial respiration, if needful : animal charcoal.

Physostigmine given cautiously.

Barium Chloride $\quad\{$ Epsom salt: Glauber's salt.

\{ Sulphuric acid diluted.

Calabar Bean- $\quad\left\{\begin{array}{l}\text { Stimulants : chloral. } \\ \text { Atropine : strychnine. }\end{array}\right.$

Physostigmine $\cdot$ Artificial respiration, if necessary.

Cantharides. Barley water, gruel, and other demulcents.

Avoid oils and fats.

Oil : sodium sulphate.

Carbolic Acid

Creosote

Saccharated lime : stimulants.

Sulphate of lime.

Warmth.

Keep patient moving.

Strychnine and caffeine, subcutaneously.

Cocaine

Chloral : amyl-nitrite.

Inhalation of chloroform.

Tannic and gallic acids : demulcents.

Colchicum

Stimulants.

$\begin{gathered}\text { Conium : Conine- } \\ \text { Cicuta Virosa }\end{gathered} .\left\{\begin{array}{l}\text { Tannic acid. } \\ \text { Potassium iodide. }\end{array}\right.$

Enanthe .

Strong coffee.

- Stimulants. 
Croton Oil . Demulcents : stimulants.

Artificial respiration.

If there be a wound, ligature, if possible, above it, and incise and suck strongly.

Loosen ligature from time to time, but avoid letting too much poison into the blood at a time.

Digitalis-

Digitalin

Tannin : stimulants.

Aconite, subcutaneously.

Perfect quiet.

Ergot .

Tannin : stimulants.

Fungus-infested or mouldy fodder or grain Substitute sound food: laxatives: eucalyptol: menthol: other antiseptic volatile oils.

Ether : stimulants : saline antiseptics.

Gelsemium

Atropine : stimulants.

Artificial respiration.

Insects' Venomous

Stings

Apply ammonia and oil : solution of - carbolic acid : sal volatile.

Iodoform

Stimulants : starch : diaphoretics : hot baths.

Stimulants : coffee.

Laburnum . $\quad$ Alternate hot and cold douches to chest.

Lobelia

STannin : stimulants.

Strychnine, hypodermically.

Epsom salt : dilute sulphuric acid.

Lead Salts ..$\{$ Potassium iodide : occasional dose of castor oil.

White of egg, in large amount.

Subsequently wash out stomach.

Give demulcents: milk and soap :

Copper treacle : magnesia : moist iron peroxide.

Foment: poultice.

Morphine, if needful.

Lead . $\quad$ Magnesia sulphate : sodium sulphate.

Mercury . . Potassium iodide : olive oil. 
Morphine-

Opium

Other Narcotics

Empty stomach by syphon or pump, or wash out stomach with sol. potassium permanganate.

Warm coffee : ammonia: emetics : alcohol.

Maintain activity of motor centres by keeping patient moving, and by electric shocks : tannin : zinc sulphate.

Strychnine hypodermically sustains action of heart.

Atropine in small doses subcutaneously.

Artificial respiration, if needful.

Nitro-Benzol .

Amyl-Nitrite .

Stimulants.

Alternate hot and cold douche.

Artificial respiration.

Nitro-Glycerine . $\left\{\begin{array}{l}\text { Ergotin : atropine subcutaneously. } \\ \text { Cold to }\end{array}\right.$

Cold to head.

Copper sulphate : emetics : charcoal.

Phosphorus . . Oil of turpentine, old and oxidised.

Avoid fats, fatty oils, and alkalies.

Picrotoxine : Cocculus Indicus

Pilocarpine : Jaborandi. Atropine.

Quinine. $\quad . \quad \begin{aligned} & \text { Tannic or gallic acid : coffee. } \\ & \text { Stimulants : artificial respiration. }\end{aligned}$

Savin ..$\left\{\begin{array}{c}\text { Epsom salt: demulcents : ethereal } \\ \text { stimulants: }\end{array}\right.$

Ligature limb: excise wound, and sear with hot iron: antivenin : potassium permanganate applied Snake-bite $\cdot\left\{\begin{array}{l}\text { petar lancing wound. } \\ \text { after lation }\end{array}\right.$

Alcoholic stimulants : ammonia.

Artificial respiration.

(Chloroform : chloral: tobacco infuStrychnine : Brucine sion: apomorphine for dogs.

Nux vomica. . Potassium bromide : tannin : paraldehyde. 


Tobacco . $\quad\left\{\begin{array}{l}\text { Warm stimulants. } \\ \text { Tannin: strychnine: solution of } \\ \text { iodine. }\end{array}\right.$

Turpentine Oil . Demulcents : Epsom salt.

Veratrine- Stimulants : warm coffee : emetics.

While Hellebore . \{Perfect quiet: opium : tannin.

Stimulants: laxatives: ammonia

Yew acetate.

Demulcents.

Zine Salts

White of egg: acetate of ammonia emetics. 


\section{SECTION II \\ Inorganic Materia Medica}

\section{WATER}

Aqua. Hydrogen Oxide or Monoxide. $\mathrm{H}_{2} \mathrm{O}$.

WATER exists in the solid, liquid, and gaseous forms. It is transparent, neutral, colourless, odourless, and tasteless. A minim weighs $\cdot 91$ grain; a fluid ounce, $437 \cdot 5$ grains. It is the standard of comparison for specific gravities of liquids, its specific gravity being represented as 1 or 1000 . It solidifies, freezes, or crystallises at $32^{\circ} \mathrm{Fahr}$., expanding and giving out latent heat ; it reaches its greatest density at $39^{\circ} \cdot 2 \mathrm{Fahr}$.; it slowly volatises at all temperatures; at $212^{\circ} \mathrm{Fahr}$. it boils, rising in steam, and increasing in bulk 1700 times. A cubic inch of water becomes a cubic foot of steam. When the solid ice melts, heat is absorbed or becomes latent; when the liquid water boils, or gives off gas, still more heat is absorbed. A cubic foot of water on conversion into steam renders latent 900 units of heat. The melting ice and evaporating water, thus abstracting heat from bodies in contact with them, are valuable refrigerants.

Water is almost a universal solvent; it readily dissolves many mineral matters, gases, and organic substances. From soils and rocks through which it passes it takes up salts, especially of calcium, magnesium, and sodium, and occasionally of lead. It absorbs atmospheric air, carbonic acid, and other gases, some adding to its sparkling, refreshing, and palatable qualities, others rendering it disagreeable and unwholesome. Gases are more soluble in cold than in hot water; solids, conversely, are generally more quickly and freely dissolved by hot than by cold water. Organic matters are present, especially in river and marsh waters, causing them to spoil rapidly when kept, and sometimes to 
produce diarrhœa and dysentery in animals drinking them. Surface drainage and sewage are apt to contain vegetable and animal parasites, bacteria, spores and ova, which give rise to dangerous diseases in animals as well as in man.

Even in potable waters the nature and proportion of the solid constituents differ materially. Glasgow derives from Loch Katrine the purest water supply of any large city in the world, containing only three-fourths of a grain of organic and one and a half grains of inorganic matters to the gallon. The water of the Thames, supplied to part of London, contains about three grains of organic and sixteen grains of inorganic matters to the gallon. When the mineral constituents, consisting of salts of calcium and magnesium, exceed $\frac{1}{5000}$ th part, the water is said to be hard, and is unsuitable for many pharmaceutic and domestic purposes ; it curdles or precipitates soap, instead of forming with it a froth or lather ; it forms a brown encrustation on the kettles or furnaces in which it is boiled; it is not so well liked by animals, and is apt to cause diarrhœa and other digestive derangements, especially in subjects unaccustomed to it. When the salts do not amount to $\frac{1}{5000}$ th part the water is considered soft.

The presence of the more dangerous organic and organised impurities is discovered by several simple tests-(1) Half a pint of the water is well shaken in a clean, wide-mouthed bottle; when sewage is present an offensive smell will be discovered on removal of the stopper or cork. (2) In a tumbler of water two or three drops of sulphuric acid are placed, and sufficient potassium permanganate to render the water pink. When allowed to stand for fifteen minutes, the water, if containing organic impurity, will have become colourless. (3) Sewage contaminated water usually contains common salt, which may be discovered by silver nitrate producing milkiness $(\mathrm{AgCl})$, which is not removed by a few drops of nitric acid.

For purifying water various methods are adopted. Subsidence and decantation get rid of grosser mechanical particles. Filtration through sand, charcoal, gravel, or spongy iron removes coarse and organic impurities, but filters may become infected, and are not usually proof against bacteria. 
Alum, even in minute amount, clears turbid water. Oxidation gradually destroys disagreeable or dangerous foulness ; hence a running stream, contaminated even by sewage several hundred yards higher up, may again become clear and wholesome. Alkaline permanganates, by similar oxidation, promptly destroy organic contamination. Boiling destroys most noxious living vegetable and animal matters, drives off carbonic acid gas, and thus throws down calcium carbonate, the cause of temporary hardness. Sodium carbonate, or lime, as in Clarke's process, diffused through hard water, which is then allowed to settle, abstracts carbonic acid gas, and causes subsidence of calcium and magnesium carbonates, and also reduces the permanent hardness produced by calcium sulphate. For chemical and pharmaceutical purposes, aqua destillata is requisite, and distillation leaves behind all impurities except a trace of organic matters, and one to two per cent. per volume of air. Such distilled or other pure water is understood to be used when 'water' is ordered in prescriptions.

Mineral waters are unfit for general use on account of their undue proportion of mineral matters or gases, or from their being at a higher temperature than that of the locality in which they are found. The most common mineral waters are those containing iron and salines. Sea water has a specific gravity of 1027 ; imperial pint contains about 312 grains of solid matters, of which about 240 grains are common salt.

ACTIONS AND UsES.-Wader is nutrient, diluent, antipyretic, diuretic, and detergent. Introduced into the body in excess of its requirements, it is removed usually within six hours, chiefly by the kidneys, and in less amount by the skin and bowels. When given cold, the kidneys perform the main excretory office; but when used hot, water is an adjuvant diaphoretic, cathartic, and (warm) in dogs and other carnivora, an emetic. Water applied topically, as in the form of hot fomentation, or the familiar warm-compress, is emollient and anodyne, abates congestion of circumscribed inflammation and wounds, and its beneficial effects are also reflexly propagated to adjacent parts. At high temperatures water is an irritant, but dry steam mixed with 
air is emollient and soothing. Cold water is refrigerant and tonic. Ice is a prompt and effective refrigerant; it controls congestion and inflammation, and arrests hæmorrhage from the stomach, lungs, and other parts. Baths are used not only for comfort and cleanliness, but for the cure of disease.

Water constitutes from fifty-five to eighty per cent. of the weight of the higher animals, and is essential for digestion, absorption, secretion, excretion, and indeed for every vital process. It is largely present in every kind of food, facilitating its digestion and assimilation, and replacing the loss of fluid constantly taking place by the skin, lungs, and kidneys. Insufficient and excessive supplies are alike injurious ; but animals in health, and with constant free access to water, rarely take more than is good for them. Excepting for a few hours previous to any great exertion, and when hungry, overheated, and prostrated, the horse in health should not be restricted in his water supply. Indeed, in many well-managed modern stables a limited amount of water is constantly at the horse's head, and the daily quantity thus drunk is actually less than when the animal is allowed to slake his thirst three or four times daily. Although a moderate amount of water is essential for digestion, an excessive quantity injuriously dilutes the intestinal contents, and favours acid fermentation.

Horses, especially if tired and hungry, before having a little hay-which, being eaten slowly, is in such circumstances preferable to grain-should receive some water, or, better still, a gallon of gruel. In some cab and carrying establishments, each hard-worked horse, on his return to the stable, is provided with a supply of oatmeal gruel, which is found not only to help condition, but to diminish attacks of colic and other gastro-intestinal derangements. Acopious draught of water, taken immediately after a rapidly-eaten meal, hurries the imperfectly digested food too rapidly into the intestines, where it is apt to set up colic and inflammation. Very cold water, freely drunk, especially by hungry, exhausted horses, is a frequent cause of gastro-intestinal disorder; and in many establishments throughout winter steam or hot water is introduced into the horse-troughs, or 
the buckets are filled and brought into the stable several hours before they are required for use.

Water, judiciously used, is a valuable diluent, febrifuge, and evacuant, serviceable in febrile and inflammatory diseases. When given moderately cold, it is more palatable and satisfying than in the tepid state in which it is sometimes presented to sick horses. Rendered feebly bitter with a little cascarilla or quassia infusion, secretion is encouraged and thirst is more effectually quenched. Small portions of ice placed in the mouth are sucked by most animals, and promote secretion, abate thirst, and also relieve congestion and irritation. Horses greedy of water, and especially those with defective wind or liability to acidity or diarrhœa, should be supplied frequently with small quantities, while, further to relieve thirst, the food should be damped. After a cathartic dose, and until the physic has ceased to operate, even moderate draughts of cold water in many horses may be followed by griping. Calves and lambs, feverish and purging, sometimes die if they have free access to water.

As a diluent, water mechanically relieves choking and coughing ; dilutes corrosive and irritant poisons ; assists the action of diaphoretics, diuretics, and purgatives. Tepid water is a convenient auxiliary emetic for dogs and pigs. Injected into the rectum, warm water allays irritability of the bowels, and urino-genital organs, and promotes the action of the bowels. Water, whether cold or hot, checks bleeding; but is most effectual at a temperature of about $120^{\circ}$ Fahr. A good scrubbing with tepid water and soap is a very essential preliminary to the successful treatment of mange or scab. It removes scales and dirt, abounding especially in inveterate cases, and hence facilitates access of the special dressings to the burrows of the parasites.

Hot fomentations moisten, soften, and relax dry and irritable textures, and relieve tension, tenderness, and pain. Applied early, and continued for several hours, they control or relieve congestion and inflammation of strains and contused wounds. Their external application, by reflex action, often soothes irritated or inflamed internal parts. In this way fomentations allay the pain of colic and inflammation of the bowels. Steaming the head and throat in like 
manner often relieves catarrh, sore-throat, and strangles. Williams insisted on the value of steaming, and hot fomentations in laryngitis and bronchitis, and preferred fomentations to counter-irritants in pneumonia and pleurisy.

Soothing watery vapour, medicated, if need be, is readily evolved from a steam-kettle, from a well-made bran-mash placed in a roomy nose-bag, or from a bucket containing freshly-steamed hay.

Water-dressings, or compresses, consisting of several folds of lint or tow, saturated with hot water, and covered with oiled skin or mackintosh cloth to retard evaporation, or a sheet of well-soaked spongio-piline, are frequently substituted for poultices, and are usually preferable, on account of their lightness and cleanliness.

Cold water is a useful refrigerant. When the acute congestion, heat, and tenderness of bruises, strains, and wounds have been so far abated by hot applications, cold exerts wholesome, refrigerant, tonic, and constringing effects. Linen bandages, constantly wetted, relieve chronic strains, jars, and windgalls of the legs of horses. Cold water is also serviceable, after disinfection, in broken knees, synovitis and arthritis, open or closed, and other injuries of the limbs. Continuous irrigation is readily effected through a small indiarubber pipe, connected with a water tap, or brought from a supply tank on a higher level. (See Irrigation.) Cold water similarly supplied keeps at low temperature the swabs around the coronets and feet of horses suffering from laminitis. Rugs dipped in cold water and applied to the chest walls, are successfully employed in acute congestion of the lungs, brought on by over-exertion, and especially in hot weather.

Cold affusion to the head and neck is a powerful stimulant, serviceable in megrims, sunstroke, phrenitis, convulsions, syncope, and the comatose stage of parturient toxæmia in cattle, as well as in poisoning with alcohol, chloroform, opium, and prussic acid. The shock is increased when very cold water is used, and when it falls on the patient from a height of two or three feet. Such cold affusion must not, however, be long continued, as it quickly abstracts animal heat. Equally effectual results are more safely 
attained by alternately douching with cold and warm water.

Ice in small pieces, placed in the mouth, is readily sucked by most animals, and often relieves congestion of the throat, and irritability of the stomach, especially in dogs. Applied usually in a bag or bladder, it is serviceable in inflamed and prolapsed uterus and rectum, herniæ, in the early treatment of flexor strains and wrẻnched joints, in hæmorrhage, which sometimes occurs shortly after parturition, as well as in parturient coma in cows. Two parts of ice mixed with one of salt form a powerful freezing mixture of the temperature of $4^{\circ} \mathrm{Fahr}$. Snow or ice is applied to retard the sudden rise of temperature and consequent gangrene in frost-bite, to arrest circumscribed congestion and inflammation, to check bleeding and convulsions. Ice maintained in contact with the skin for six or eight minutes diminishes sensation, and facilitates the performance of a few minor operations; but for inducing local anæsthesia, cocaine is preferable.

\section{OX Y G EN}

Oxygen is a colourless, odourless gas, slightly heavier than atmospheric air, and forming about one-fifth of its volume. Twenty-five volumes of water dissolve one volume of oxygen. It has wide chemical activity.

Actions AND USES.-Oxygen has slight effect on the unbroken skin, but stimulates denuded skin and mucous surfaces. Oxygenated solutions have been applied to atonic wounds and ulcers. Such solutions, when swallowed, aid oxidation of waste products in the alimentary canal. The inhalation of oxygen has been recommended in dyspnœa arising from asthma, bronchitis, pneumonia, and other respiratory diseases, as well as in cardiac failure. In impeded respiration with stagnation of air in the alveoli the inhalation of oxygen by promoting diffusion, may be beneficial. Only limited quantities can be retained by the blood; the serum, when saturated, retains one-fifteenth of the amount the red globules can take up, and Durig has shown that in rest oxygen exerts no influence on metabolism. Oxygen is largely used as an insufflation for the udder in 
parturient toxæmia (milk fever) of cows, for which it is regarded as a specific.

OzONE.-When electric sparks are passed through air, the molecules of oxygen, represented by two atoms $\left(\mathrm{O}_{2}\right)$, are split up, and rearranged in triatomic molecules, constituting ozone $\left(\mathrm{O}_{3}\right)$. It is also produced by the slow oxidation of phosphorus in the presence of water, and by the action of protoplasm. It is unstable, being readily converted into oxygen. It is distinguished by its peculiar smell, and by its decomposing potassium iodide solution, and when mixed with damp starch, producing the blue starch iodide.

ACTIONS AND USES.-Ozone oxidises more actively than oxygen, destroys the coagulability of albumin, decomposes many organic substances, and kills micro-organisms. In virtue of its chemical actions it is a powerful irritant. When inhaled it induces excitement, succeeded by exhaustion and sometimes by convulsions. It has been used for most of the cases in which oxygen has been given, notably for the destruction of bacteria in diphtheria and other similar diseases (Brunton).

\section{HYDROGEN PEROXIDE}

Liquor Hydrogenil Peroxidi-Hydrogen Peroxide Solution. An aqueous solution of hydrogen peroxide, $\mathrm{H}_{2} \mathrm{O}_{2}$, prepared by the interaction of water, barium peroxide, and a dilute mineral acid at a temperature below $50^{\circ} \mathrm{Fahr}$. ; containing nine to eleven volumes of oxygen (B.P.).

Peroxide of hydrogen is a clear, odourless liquid, with a bitter taste. It renders the saliva frothy, mixes with water in any proportion, and when heated it decomposes into water and oxygen. Commercial peroxide of hydrogen tends to become acid on keeping, but Merck's Perhydrol, containing thirty per cent. of oxygen, keeps well in the original bottles. Samples of peroxide that have become acid may be neutralised by the addition of wodium bicarbonate, or borax. Hydrogen peroxide is a pormful nonpoisonous germicide, deodorant, and hæmostatic. Applied locally it bleaches the 
skin and mucous surfaces; injected under the skin it decomposes, with liberation of oxygen, producing subcutaneous emphysema. Intravenous injections are said to be anæsthetic. It has no action on soluble ferments, but it destroys organised ferments. It is of great value in the treatment of suppurating wounds, abscesses, fistulæ or sinuses. In contact with blood or pus it decomposes with effervescence, a reaction that is sometimes useful in diagnosis. Injections of three per cent. solution of perhydrol may be employed in retention of fœtal membranes, in vaginitis and metritis, and impaction of the facial sinuses. Peroxide solution is also used as a disinfectant for abscess cavities and to facilitate the removal of clinging wound dressings. As a hæmostatic, undiluted commercial peroxide or a solution of one part perhydrol in ten parts of physiological salt solution may be used. Perhydrol diluted with nine times its weight of boiled water has been employed as a substitute for a 1:1000 mercuric chloride solution in the treatment of suppurating joints, tendon sheaths, and compound fractures. A solution (one to three per cent.) may be used as an udder injection, an ounce for each quarter, in parturient toxæmia and contagious mammitis, and as a spray for keratitis and corneal ulcer.

Internally, solutions of peroxide (one or two per cent.) have been administered in dyspepsia, diarrhœa, and gastrointestinal catarrh. Peroxide is incompatible with permanganates, charcoal, chromic acid, and mercuric chloride. Caution is required in injecting peroxide in quantity into large wounds, serous and other cavities, as it is quickly decomposed; and 'if more oxygen is formed than the blood can dispose of, gas emboli are produced, and these lodging in the lungs or brain may cause death.' (Hale White.)

Doses (hydrogen peroxide 10 vols. per cent.).-Horses and cattle, $\zeta^{j}$. to $\zeta_{\mathrm{ij}}$; d dogs, $3 \mathrm{j}$. to $3 \mathrm{ij}$.

\section{SALT ACTION-THE IONIC THEORY}

Osmosis. - If two solutions of a salt of different strengths, say sodium chloride, are separated by an animal membrane, water will pass through the membrane from the less con- 
centrated solution to dilute the stronger one, whilst, on the other hand, salt will pass from the more concentrated to the weaker solution, until, as a result of both these processes, the two solutions on opposite sides of the membrane become of one uniform strength, i.e. each contains the same number of molecules in the same volume.

Not only does this occur when the solutions contain the same salt, but two solutions of different salts will also tend to diffuse so that there are the same number of molecules of each on either side of the membrane. The rate at which osmosis takes place is proportional to the difference in molecular concentration of the two solutions.

Consider the effect of a membrane allowing the passage of water but holding back the molecules of the dissolved substance. Any membrane will do this for substances known as colloids, such as albumin, gum, or starch, and when tested with these it is said to be semi-permeable. There are, moreover, membranes which prevent the passage of, or are semi-permeable for, salts, and in fact the cell walls and cell membranes in plants and animals often possess this property. A cell thus surrounded and containing some small amount of salt in solution in its contents, will, if immersed in a very weak salt solution or in pure water, take up water to dilute the salt solution inside it and become tense and turgid. A plant, for example, when immersed in water or a very dilute salt solution takes up water, its stems stiffen and become firm because of the osmotic pressure in its cells drawing the water in. If, on the other hand, the cell be immersed in a concentrated solution of a salt, water now leaves the cell to dilute the salt solution round about it, and the cell shrinks. If the solution outside has the same molecular concentration as the cell contents then no apparent diffusion of water either in or out will take place. Such solutions in equilibrium (because equimolecular) are called isotonic, the more concentrated of two is called hypertonic, and the less concentrated hypotonic. As used in pharmacology the word isotonic signifies a solution of equal osmotic pressure to that of the blood. Thus, ' normal saline solution' is a solution of sodium chloride isotonic with the blood. When red blood corpuscles are placed in such a 
solution no change is apparent, but if placed in a concentrated salt solution (hypertonic) water diffuses out of the red cells and they become crenated or shrunken. If the salt solution is very dilute or if water is used (hypotonic) the cells take up water, swell up and burst, the hæmoglobin being set free into the plasma. In the case of red corpuscles then, it is understood that the outer layer or envelope of the corpuscle is semi-permeable, and that it will allow water to pass in or out, but not salts. This is not quite true, because the cell envelope has a vital selective power, and is permeable for certain substances. Thus urea in solution can pass through the membrane, and so also can free acids, alkalies, and ammonium salts. With these exceptions, however, the corpuscles are semi-permeable. The osmotic pressure of the blood varies in different animals to a slight extent and so ' normal saline solution' should be varied for the particular species. In mammals, however, sufficient accuracy is obtained by using a solution containing $0.9 \%$ of common salt as being isotonic with the blood.

The osmotic pressure of the blood varies a little at different times, and reaches a maximum shortly after a full meal. The variation is, however, very slight, for the kidneys at once proceed to correct it, by excreting urine more concentrated or less concentrated, to suit the case. Thus the salt content, and therefore the osmotic pressure, of the urine, varies considerably at different times during the day.

Normal saline solution is the ideal fluid for saline injections, for irrigation of the body cavities, the abdomen, the stomach, etc., for the reason that, being isotonic, it does not damage cells. The toxic action of distilled water when drunk is simply due to the fact that, being hypotonic, it is taken up by the cells of the stomach mucous membrane which swell up, die, and may be cast off, so causing catarrh. Water got from melted snow or ice containing no dissolved salts has the same harmful effect.

When a hypertonic solution of a salt is given by the mouth and absorbed into the blood, or if such a solution is injected directly into the blood stream, the effects on the corpuscles would be to produce crenation and destruction, but for the fact that the tissues are at once drawn upon for 
water to dilute this salt in the blood plasma, and thus a condition of hydræmia, i.e. excess of water in the blood, is brought about. This stimulates the kidneys and there is accelerated excretion of dilute urine. Such salts are therefore used as saline diuretics.

Other salts taken by the mouth in concentrated solutions do not cause diuresis for the reason that little of the salt is absorbed into the blood. In other words, the intestinal wall has a vital selective power and whilst it allows the passage of many salts (e.g. the saline diuretics) it resists the passage of others. These latter remain in the intestine, the wall of which acts as a semi-permeable membrane, water is drawn out from the blood and in turn from the tissues, and so the intestinal contents become very liquid. These salts, therefore, are used as saline purgatives.

The Ionic Theory.-In the succeeding pages frequent references will be found to the action of ions, and the actions of many drugs are only explainable by an understanding of the theory of ionisation.

When a salt, such as sodium chloride, is in watery solution of a known strength the osmotic pressure of that solution can be theoretically calculated from its molecular concentration. It is found experimentally, however, that the osmotic pressure is higher than this theoretical result, and from analogies between the behaviour of gaseous compounds and solutions of salts it is believed that the increase of pressure is due to dissociation of the salt, into chlorine and sodium ions. The whole of the molecules of the salt are not dissociated, but a large number are, and thus the solution contains $\mathrm{NaCl}$ molecules and $\mathrm{Cl}$ and $\mathrm{Na}$ ions, and since for the purposes of osmosis these ions are just as effective as molecules, the osmotic pressure of the solution rises. Acids, bases, and salts all undergo dissociation in solution to a greater or less extent, and depending on the degree of dissociation the osmotic pressure of the solution is higher than if no ionisation occurred. The ions are not present in the solution just in the form of sodium and chlorine, for they have none of the specific characters of these elements. They are believed to be held bound by being charged with electricity, the metallic ion having a positive or + charge, the acid ion a negative or 
- charge. Distilled water will not convey an electric current, but if an acid or a salt is added to it the current will flow, from the positive to the negative pole. This current is conveyed by the ions, the positively charged metallic or basic ones being attracted to the negative pole, and vice versa. In the case of sodium chloride, the sodium ions are attracted to, and liberated at the negative electrode and at once react with the water forming $\mathrm{NaOH}$ sodium hydroxide, making the water alkaline in reaction, and setting free bubbles of hydrogen gas. If copper sulphate is used copper is deposited on the negative electrode, whilst the $\mathrm{SO}_{4}$ ion reacts with water to form sulphuric acid at the other. These substances of which sodium chloride and copper sulphate are examples, are called electrolytes because they promote the electrolysis of water when a current is passed through their solution. On the other hand, cane sugar added to water has no such property, for it does not undergo dissociation. Alcohol and chloroform are similarly devoid of action as electrolytes because they are not ionised.

The importance of this theory is that it is the ions of a salt and not the molecules as a whole which act on the cells and tissues of the body. Thus $\mathrm{KOH}$ and $\mathrm{NaOH}$ both dissociate in solution and both possess strong caustic action. This action is not due to the potassium, $K$, or the sodium, $\mathrm{Na}$, but to the $\mathrm{OH}$ group in each. Alcohol, on the other hand, $\mathrm{C}_{2} \mathrm{H}_{5} \mathrm{OH}$, does not dissociate and therefore has no free $\mathrm{OH}$ group and no caustic action. Again, whilst HCN or KCN are both extremely toxic by dissociation with liberation of the $\mathrm{CN}$ ion, potassium ferrocyanide is but slightly toxic since no free $\mathrm{CN}$ ion is found in its solution.

The rate of absorption of different acid or basic ions is greatly different and this has a profound effect on the action of the drug containing them. Thus the intestinal wall resists the passage of magnesium, $\mathrm{Mg}$, ions, whilst sodium, $\mathrm{Na}$, and potassium, $\mathrm{K}$, are readily absorbed. Again, whilst $\mathrm{Cl}$ and $\mathrm{NO}_{3}$ ions are readily allowed to pass through, the $\mathrm{SO}_{4}$ group is not absorbed to any extent. Thus we can premise at once that sulphates will be more effectual saline purgatives than chlorides, and that magnesium sulphate will be more efficient 
than sodium sulphate, and this is found to be the case in practice.

From what has been said above it will be obvious that it is not possible to describe the actions of all the compounds of, say, a metal under one head, for the acid ion may be the predominant partner, as for example, in the cases of potassium cyanide and potassium bromide. Again the physical behaviour of a compound is often of as great importance as its chemical constitution. Thus a strict chemical classification of drugs is not of any value as a guide to their actions. The chemical and the physical properties of the drug and the vital reaction of the body towards it constitute the specific action of that agent and this must be determined for each individual agent and to some extent for each kind of animal.

\section{AMMONIUM AND ITS MEDICINAL COMPOUNDS}

Ammonit Chloridum. Sal-ammoniac. Chloride of Ammonium. $\mathrm{NH}_{4} \mathrm{Cl}$.

This salt, from which most ammonium compounds are derived, may be formed by neutralising crude solution of ammonia or ammonium carbonate with hydrochloric acid, and purifying the product (B.P.). The salt thus prepared occurs in inodorous colourless crystals, or in translucent, tough, fibrous masses. It has a saline, acid taste, a slightly acid reaction ; is soluble in one part boiling, or three of cold water, and in 60 parts alcohol (90 per cent.). During solution it abstracts much heat, and is consequently an ingredient of many freezing mixtures. Heated it sublimes unchanged. Mixed with lime or potash it evolves ammonia.

ACTIONS AND USES.-Expectorant, diuretic and refrigerant. Large doses, injected intravenously or subcutaneously, exhibit the stimulant and subsequent paralysing effects of ammonium salts. Given by the mouth, two ounces administered to a horse caused muco-enteritis (Moiroud); two drachms destroyed a small dog in an hour. The alimentary mucous membrane was found congested and swollen (Orfila). The symptoms described as occurring in dogs are muscular 
weakness, slow breathing, violent action of the heart, and tetanic spasms' (Christison).

MEDICINAL Doses stimulate the alimentary and respiratory mucous membranes, promote theirsecretions, and relieve gastric as well as bronchial catarrh, especially in patients where pyrexia has not been serious, or has abated. They were recommended for torpidity of the liver and for rheumatism, but have proved of little benefit.

Doses.-Horses, 3ij. to $3 \mathrm{jv}$; cattle, $3 \mathrm{jv}$. to $\mathrm{j}_{\text {. }}$; sheep and pigs, grs. $\mathrm{xxx}$. to $3 \mathrm{j}$. ; dogs, grs. iii. to grs. x. ; cats, gr. i. to grs. v. In bolus, pill or drench.

One part of chloride dissolved in ten to twenty parts of water is used as a stimulant gargle, and five parts salammoniac, eight parts sodium sulphate, and five parts potassium nitrate in sixteen parts of water, form a refrigerant lotion for inflammatory swellings, bruises, and sprains. A cooling mixture, stated to lower the thermometer from $50^{\circ}$ to $10^{\circ} \mathrm{Fahr}$., is made with four ounces each of sal-ammoniac and nitre, dissolved in eight ounces of water; but for ordinary refrigerant purposes, six or eight times this amount of water may be used.

Lrquor Ammoniж Fortis. Strong Solution of Ammonia. Caustic Ammonia. Hartshorn.

An aqueous solution containing 32.5 per cent. by weight of ammonia, $\mathrm{NH}_{3}$. It may be obtained by heating a mixture of ammonium chloride and slaked lime, and passing the resulting ammonia into distilled water (B.P.).

Traces of ammonia exist in the air, and in rain. It occurs in the excretions of living animals, and is evolved from the putrefaction and destructive distillation alike of vegetable and animal matters. But the coal beds are the great commercial source of ammonia and its compounds. Coal, when distilled in the making of gas, leaves a waste liquor, which if treated with hydrochloric acid, yields ammonium chloride or sal-ammoniac. .

Properties.-The liquor ammoniæ fortis is colourless, pungent, and caustic. Specific gravity 0.891 . One fluid drachm contains $15 \cdot 83$ grains of gaseous ammonia. Purity 
is ensured when the sample, diluted with four times its volume of distilled water, gives no precipitate with solution of lime, or ammonium sulphide, and, when treated with an excess of nitric acid, it is not rendered turbid by silver nitrate or barium chloride. It is very strongly alkaline, and unites with fats and oils, forming soaps and liniments.

For most medicinal and pharmaceutical purposes the liquor ammoniæ fortis is too concentrated, and a diluted solution is made by adding to one volume of liq. ammon. fortis, two volumes of distilled water. This medicinal solution is entitled liquor ammoniæ, contains 10 per cent. by weight of ammonia $\mathrm{NH}_{3}$, and has the specific gravity 0.959 .

A spirit of ammonia of corresponding strength, containing 10 per cent. of gas in rectified spirit, is recognised by the U.S.P.

Aromatic spirit of ammonia, popularly known as salvolatile, is a solution of liquor ammoniæ fortis and ammonium carbonate in rectified spirit and water, flavoured with oil of nutmeg and lemon.

Actions and Uses of Ammonia.-Unlike caustic potash and soda, liquor ammoniæ does not dissolve the epidermis, and consequently does not cauterise, but if evaporation be prevented, it passes through the epidermis, irritates the dermis and vesicates. Ammonia causes topical irritation. Tolerably concentrated solutions abstract water from the tissues, liquefy their albumin, and saponify their fats. They hence act as corrosives. Ammonia gas entering the airpassages is irrespirable, and causes suffocation by paralysis of the glottis. Strong solutions swallowed produce gastro-enteritis, while, from absorption, paralysis of the brain centres and coma may ensue. Reflexly, when applied to the nostrils or stomach, it stimulates the vasomotor centre, raises blood-pressure, stimulates the circulatory and respiratory nerve-centres, and promotes secretion alike from the mucous surface and skin. It is administered as an antacid, diffusible stimulant, and antispasmodic, and used externally as a stimulant and counter-irritant.

Toxic EFFECTS.-Hertwig found that half an ounce of the strong solution, given diluted, had no bad effects on horses, 
but that one ounce proved fatal in sixteen hours, and three ounces in fifty minutes, the latter quantity causing violent cramps and difficult breathing. Half a drachm introduced into the stomach, and retained by tying the œsophagus, destroyed a dog in twenty-four hours, causing much uneasiness, agitation, and stupor, and leaving after death slight redness of the mucous membrane of the stomach (Orfila). These effects are caused partly by the corrosive irritant action in the stomach, and also partly by the passage of some of the vapour into the air passages with resulting asphyxia. The most effectual antidotes are vinegar and diluted acids, with diluents and demulcents.

Medicinal Uses.-Its antacid and stimulant properties recommend ammonia in indigestion, tympanites, and spasmodic colic in ruminants. Stimulating the vaso-motor and respiratory centres, it is valuable in antagonising syncope in influenza and similar complaints. As in human practice, ammonia fumes are occasionally used to rouse animals from shock, collapse, or chloroform intoxication, but must be used cautiously lest excessive irritation of the respiratory mucous membrane be produced. It is a promptly-acting antidote in poisoning by opium, aconite, digitalis, and other narcotic and sedative drugs. It may be administered much diluted in the usual way, injected subcutaneously and intravenously, and also applied externally in the treatment of snake-bites ; but its success is uncertain, especially in the case of the cobra and other venomous snakes. On account of its promoting bronchial secretion, and assisting in its expulsion, ammonia is serviceable as a stimulating expectorant. To develop its more general effects, it is frequently prescribed with alcoholic stimulants, as in the convenient form of aromatic spirit of ammonia.

EXTERNAL USES. - In the form of liniment of ammonia, or of compound liniment of camphor, ammonia proves a useful counter-irritant for muscular strains and rheumatism, for stiff joints, for sore throat and bronchitis, and for preventing the rapid chilling of fomented surfaces. A pledget of lint saturated with ammonia, applied to the skin and covered with oiled silk quickly vesicates. It relieves the irritation caused by nettles, and by bites and stings of insects. 
Doses, ete.-Of liquor ammoniæ as a diffusible stimulant and antispasmodic, horses take $\mathrm{f} 3 \mathrm{ij}$. to $\mathrm{f} 3 \mathrm{iv}$.; cattle, $\mathrm{f} 3 \mathrm{iv}$. to $\mathrm{f} z \mathrm{z}_{\text {. ; }}$ sheep and pigs, f $3 \mathrm{ji}$. ; and dogs, $\mathrm{m}_{\mathrm{v}}$. to $\mathrm{m}_{\mathrm{x}}$. The aromatic spirit is given in proportionally larger doses. In order to sustain their transient effects they require to be repeated at intervals of two or three hours. On account of their pungency, they must be largely diluted with water, or, better still, with cold gruel or mucilage. A useful stimulant draught, either for horses or cattle, is made with half an ounce each of liquor ammoniæ, sweet spirit of nitre, and tincture of gentian, given in a quart of ale or of cold gruel. For external application the liquor ammoniæ is generally used, mixed with five to ten parts of oil. A convenient stimulating liniment is made with one part each of medicinal solution of ammonia, oil of turpentine, and water, mixed with four to six parts of linseed oil. A drachm of liquor ammoniæ fortis, with half a pint of soap liniment, makes a useful stimulant embrocation for sore throat. The B.P. liniment of ammonia consists of one part solution of ammonia (10 per cent.), one part of almond oil, and two parts of olive oil. The popular 'White Oil ' is made with one ounce of camphor, four ounces of rectified spirit, a pint of olive oil, and two ounces solution of ammonia.

Ammonit Carbonas. Carbonate of Ammonia. Ammonium Carbonate.

A variable mixture of Ammonium hydrogen carbonate, $\mathrm{NH}_{4} \mathrm{HCO}_{3}$, with ammonium carbamate, $\mathrm{NH}_{4} \mathrm{NH}_{2} \mathrm{CO}_{2}$, produced on heating ammonium sulphate with calcium carbonate (B.P.).

It occurs in colourless, translucent, fibrous, crystalline masses, with a pungent alkaline taste, and a strong ammoniacal odour. Soluble in four parts of cold water ; rather less of tepid water ; in two hundred of alcohol ; and in five of glycerin. Decomposes in boiling water, with evolution of ammonia and carbonic acid; sublimes when heated, and when exposed to the air becomes opaque, friable, and covered with a white efflorescence.

Actions AND USES.-The carbonate closely resembles 
liquor ammoniæ, but is less volatile, less powerful, and rather more permanent in its effects. Orfila records that two and a half drachms given to a dog caused gastric inflammation, tetanic convulsions, and death.

Medicinal Uses.-It is given to all animals in atonic dyspepsia ; conjoins the actions of an antacid and diffusible stimulant; in small doses promotes secretion of gastric juice, and in larger relieves flatulence and spasm. It is prescribed with nux vomica to stimulate the neuro-muscular apparatus in obstruction of the bowel. A few doses materially help the extra rug, warm bran mash, and other hygienic remedies in combating chill, blowing, and other premonitory symptoms of disease of the air-passages in hardworked horses. It stimulates both cardiac and respiratory functions, and hence is prescribed in influenza, and in the later stages of various acute debilitating inflammatory complaints, in many of which it may be substituted for or conjoined with alcoholic stimulants. In respiratory disease, while sustaining the action of the heart, it promotes secretion and expectoration, and hence relieves bronchial congestion, being especially serviceable when the lower bronchi are choked with tough mucus, and cardiac action is weak. It is sometimes given to dogs as a stimulant emetic ; acts without nausea, and usually promptly ; but as it is somewhat uncertain is best used in conjunction with ipecacuanha, or other emetic. It sometimes averts epileptic fits in weakly dogs. It neutralises the poisons of wasp stings and insect bites. A useful dressing for removing the scales of psoriasis is made by adding one part to ten of unguentum simplex.

Doses, etc.--Horses take $3 \mathrm{ij}$. to $3 \mathrm{iv}$.; cattle, $3 \mathrm{iij}$. to $3 \mathrm{vj}$.; sheep and pigs, grs. xv. to grs. lx. ; dogs, grs. iij. to grs. viij. It is given either in bolus with linseed meal, or dissolved in gruel, which, to prevent coughing from liberation of ammonia, must be used cold. Where prompt stimulant effects are required, ammonium carbonate is conjoined with alcohol or ether; where febrifuge and expectorant effects are sought, it is prescribed with spirit of nitrous ether, potassium chlorate, and camphor ; while in chronic gastric derangements it is given with gentian, ginger, or cinchona bark. 
Smelling salts are prepared by adding to the carbonate half its weight of solution of ammonia, and mixing some bergamot, lavender, or other aromatic oil.

Liquor Ammonit Acetatis. Solution of Ammonium Acetate. Mindererus Spirit.

Is prepared by dissolving one ounce of ammonium carbonate in ten times its weight of distilled water, neutralising with acetic acid, and adding sufficient distilled water to produce one pint of the solution (B.P.). It is clear, colourless, and nearly odourless, but has a mawkish, unpleasant taste. Incompatibles, potash, soda, and their carbonates, acids, lead and silver salts, and lime water.

ACTIONS AND USES.--Ammonium acetate, although not so powerful a stimulant as liquor ammoniæ or the carbonate, is a valuable diaphoretic and antipyretic, and a mild diuretic and expectorant. It is much used in febrile and inflammatory attacks, especially affecting the respiratory organscatarrh, bronchitis, and pneumonia, and in influenza, strangles, and purpura. In these and other cases it abates fever, promotes cutaneous and bronchial secretion, and helps to clean the tongue and improve the appetite. In the onset of local inflammation in horses, when pyrexia is considerable, two to four ounces of liquor ammonii acetatis are given, with a drachm of potassium nitrate or chlorate three to four times daily. When the bowels are confined and the urine high-coloured, two to three ounces of Epsom salt are added to the febrifuge mixture. When bronchial secretion is scanty the acetate is conjoined with ipecacuanha or potassium iodide, and its beneficial effects may be increased by inhalations of moist warm air and by hot applications to the chest. When the smaller bronchial tubes are choked with mucus, balsams, turpentine, and squill are useful additions, along with moderate external stimulation. When there is sore throat and cough, belladonna extract and camphor are serviceable adjuncts. In convalescence, when the appetite is indifferent, powdered gentian or cinchona bark may be combined or alternated with acetate and salines. When the patient is weak and exhausted, alcohol and ether are fitting additions. 
For cattle similar prescriptions are suitable, given usually in somewhat larger doses. In dogs, the diuretic action of ammonium acetate is more notable than the diaphoretic. A convenient antipyretic and expectorant is made with liquor ammonii acetatis fziv.; spiritus ætheris nitrosi f $\mathrm{f}_{\mathrm{ij} . \text {; }}$ spiritus camphoræ $\mathrm{f} z \mathrm{i}$. For large dogs the dose is half a fluid ounce; for small animals a fluid drachm, in either case given diluted with five or six parts of water. This mixture is adapted for special canine cases by additions similar to those indicated for horses.

Doses, etc.-For horses and cattle the dose of liquor

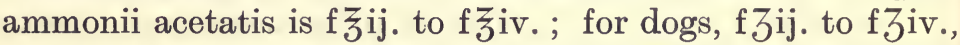
given in five or six parts of water, diluted spirit, or linseed tea. Many horses and cattle readily take it in their drinking water. Like the chloride, the solution of the acetate is sometimes used externally as a refrigerant discutient.

General Action of Ammonium Salts.-They resemble potassium and sodium salts, but being more volatile are more prompt and powerful. 'Ammonium is considerably modified by the acid radicle with which it is combined. All the ammonium salts have an action on the spinal cord, motor nerves, and muscles, and, in advanced poisoning, paralyse these structures. They do not, however, affect all these structures with equal readiness. The organ first affected, and consequently the symptoms of poisoning, vary with the salt employed. Some salts affect the spinal cord first, others the motor nerves. They appear to form a series, at one end of which the members stimulate the spinal cord, and have no marked paralysing action on the motor nerves; while those at the other end have no marked stimulating action on the cord, but, on the contrary, have a marked paralysing action both on the cord and on motor nerves. At the stimulating end of this series are ammonia and ammonium chloride, and at the paralysing end ammonium iodide; whilst the bromide, phosphate, and sulphate lie between ' (Brunton).

To determine the true action of the ammonium radicle $\left(\mathrm{NH}_{4}\right)$ a salt must be used in which, as in ammonium chloride, the acid radicle effects can be neglected. If such a salt be injected subcutaneously, the chief effect of ammonium is 
seen to be on the central nervous system. The spinal cord is markedly stimulated, reflex irritability is increased, and tetanic convulsions may be caused. Blood pressure rises and respiration becomes accelerated, whilst the heart may be slowed from stimulation of the vagus (inhibitory centre). With excessive doses paralysis of the central nervous system results, and the animal dies of asphyxia. Given by the mouth ammonium salts are more readily absorbed than the same salts of potassium or sodium, but they do not become sufficiently concentrated in the blood to cause convulsions, or even marked effects on the central nervous system. Moreover, the mucous membrane of the lungs is impermeable to ammonium, so that ammonia vapour inhaled only affects the nervous system reflexly through its local action.

In their primary stimulation and secondary paresis, ammonium salts resemble the mono-hydric alcohols and ethers ; but they act more markedly on the cord and motor centres, and less on the higher cerebral centres. Ammonium salts increase the secretion of the bronchial and intestinal glands, and also of the sweat glands and kidneys, iby which they are mainly excreted. In the blood of mammals ammonia is converted almost entirely into urea, in the blood of birds into uric acid.

\section{POTASSIUM AND ITS MEDICINAL COMPOUNDS}

Potassium salts are obtained from (1) carnallite, a chloride of potassium and magnesium $\left(\mathrm{KCl} \cdot \mathrm{MgCl}_{2} \cdot 6 \mathrm{H}_{2} \mathrm{O}\right)$ overlying the rock-salt in the mines of Stassfurt in Saxony ; (2) from the crude potashes got from wood ashes; and (3) from the argol deposited during the fermentation of wine (p. 188). Most are soluble in water. They are identified in solution by their negative reaction with the several group tests for the metals, while moderately strong neutral solutions rather slowly form, with sodium hydrogen tartrate, a white crystalline precipitate $\left(\mathrm{KHC}_{4} \mathrm{H}_{4} \mathrm{O}_{6}\right)$, soluble in hydrochloric acid and in caustic potash. Evaporated to dryness, and ignited with alcohol, they produce a distinctive violet-coloured flame, which gives on the spectrum two lines-one intense on the red, the other transient on the violet. 
ACTIONS AND USES.-The alkalies comprise salts of potassium, sodium, lithium, and ammonium. They are the chemical opposites of the acids. They neutralise acids, dissolve albumin, and saponify fats, and in virtue of these properties are irritant and caustic. They are destitute of astringency, and in dilute solution relax and soothe the tissues with which they come into contact. They are also used to neutralise excessive acidity developed from undue fermentation, in which case being administered an hour after eating. They promote elimination of lactic acid in rheumatism, and to some extent prevent the precipitation of uric acid in the bladder. The alkalies and their salts in solution exert osmotic effects in contact with animal membranes, and hence promote both catharsis and diuresis. When absorbed, they increase the alkalinity of the blood and urine, encourage oxidation processes, and promote other alterative effects.

Potassium salts are protoplasmic poisons when applied directly to the tissues, and in sufficiently strong solution for a sufficient time they destroy muscles, nerves, and nervecentres. They are more soluble, more readily absorbed and diffused, and more quickly excreted than sodium salts, but less rapidly than those of ammonium. When a salt, such as potassium chloride, in which the acid ion has little action, is injected intravenously or subcutaneously the characteristic action of potassium is produced. It attacks especially the heart, causing paralysis of that organ, depresses the whole central nervous system and paralyses the reflex centres of the cord. Large doses cause a rapid fall of blood pressure and pulse rate ; whilst general symptoms are great depression, muscular weakness, and quick breathing.

Given by the mouth these actions are not produced, for the drug is excreted as fast or faster than it is absorbed. Thus potassium salts given per os exert only 'salt action' (q.v.), except where the acid ion-as in the case of the bromide or iodide-is itself physiologically active.

They occur in plants and animals, and are essential constituents of the food of both. Their removal from the food of dogs impairs nutrition and growth more decidedly than deprivation of the corresponding sodium salts. In animal bodies potash salts occur chiefly in the solid textures, notably 
in the muscles; sodium salts in the nutrient fluids. Ringer teaches that potash salts have a high diffusive power, rapidly enter the blood, increase its alkalinity, promote oxidation and tissue metamorphosis, are solvents of albuminoids, and in one or another of these ways help to abate febrile and inflammatory attacks. They are alteratives, and antidotes to poisoning by barium salts. They are quickly excreted, mainly by the kidneys; increase chiefly the watery parts of the urine, neutralise its acidity, and often exert soothing effects on the urino-genital mucous surfaces. In febrile complaints they are eliminated in amounts three or four times larger than in health, and in larger proportion than the soda salts, which are excreted more largely during convalescence.

Recollection of the uses of the several potassium salts is facilitated by dividing them into three groups. First: Salts which are corrosive, antacid, antilithic, and alterative-such as the hydrate and carbonates. The salts of the weaker vegetable acids-tartrates and citrates-in their passage through the body are decomposed into carbonates, rendering the urine alkaline. Second: Salts which are cathartic, diuretic, alterative, febrifuge, and refrigerant on account of their salt action-such as the sulphate, acetate, tartrate, nitrate, chlorate, and permanganate. Third: Salts which exhibit prominently the actions of their acid ion or salt radical constituent-such as potassium sulphide, iodide, bromide, bichromate, and cyanide.

Potassium Hydroxide. Potassa Caustica. Hydrate of Potash. Caustic Potash. KOH.

Potassium Hydrate Solution. Liquor Potassæ. Caustic Potash Solution.

When impure potassium carbonate is boiled with calcium hydrate, calcium carbonate $\left(\mathrm{CaCO}_{3}\right)$ is precipitated, and potassium hydrate $(\mathrm{KOH})$ remains in solution, twenty-seven grains being present in the fluid ounce of the liquor potassæ. This is a dense, oily-like fluid, of specific gravity $1 \cdot 058$, colourless and odourless, with an intensely acrid, alkaline, soapy taste, and an alkaline reaction. Boiled with oils and 
fats, it forms soaps; mixed with acids, it forms neutral, soluble, crystallisable salts. It softens and dissolves soft animal and vegetable tissues. Although little used in medicine, it is of much importance in chemistry and pharmacy. When boiled until a drop removed on a stirrer becomes hard on cooling, and poured into pencil-like moulds, there are formed the grey or white deliquescent, hard, crystalline sticks of caustic potash. This agent may be taken as the representative of the caustic alkalies-other members of the group being sodium and lithium hydratesand in a less degree the carbonates and bicarbonates of these metals. All these act by virtue of their $\mathrm{OH}$ (hydroxyl) ion produced on dissociation in solution, and not by reason of the metal ion. Thus $\mathrm{KOH}=\mathrm{K}-\mathrm{OH}$ and the degree of dissociation determines the severity of the action. With the bicarbonate $\mathrm{KHCO}_{3}=\mathrm{KOH}+\mathrm{CO}_{2}$ and the $\mathrm{KOH}$ again becomes $\mathrm{K}-\mathrm{OH}$. The carbonates and bicarbonates, however, dissociate less readily than the hydroxides and hence are milder and less caustic in action. (See Salt Action.)

Actions AND UsES.-Full doses of potassium hydrate are actively dehydrating, irritant, and corrosive. Medicinal doses are antacid and diuretic. Externally, potassium hydrate, whether in substance or in concentrated solution, is a penetrating caustic. The corrosive and caustic action is due to the power caustic potash has of dissolving albumin, and to its great affinity for water.

Toxic EFFEcTs.-Large doses, when swallowed, soften, corrode, and inflame the œsophagus and stomach, sometimes so severely as to cause perforation; while great depression, and often collapse, accompanies the local lesions. Hertwig records that two drachms, dissolved in six ounces of water, killed a horse, with symptoms of colic, in thirtytwo hours. Orfila gave a dog thirty-two grains, which caused violent vomiting, restlessness, and death in three days. Post-mortem discovered the mucous coat of the œsophagus and stomach red and black from extravasation of blood, with a perforation measuring three-quarters of an inch near the pylorus, surrounded by a hard thickened margin (Christison). The blood is dark-coloured and generally fluid, owing to the solvent action of the alkali. Smaller 
or more diluted doses gradually impair digestion and assimilation, and destroy life by inanition. The antidotes are diluted acids which form mild salts, and oils which produce soaps-the latter serving as demulcents, and in men and dogs as auxiliary emetics. Irritation is also relieved by milk and gruel.

Medicinal Uses.-Diluted solution of caustic potash has been used both internally and externally as an antidote for the poison of snakes. Half a drachm, repeated twice daily, has been prescribed for sheep affected with vesical and urethral calculi ; but the carbonate is milder and equally effectual. It is occasionally added to cough mixtures when bronchial secretion is scanty and tenacious.

Caustic potash is used for eradicating warts and fungous growths, and cauterising poisoned wounds. On account of its deliquescence and liability to spread, it must, however, be applied cautiously, and any excess of alkali neutralised by subsequent washing with a weak acid. Mixed with onethird lime, and moistened with alcohol, constituting Vienna paste, it is less deliquescent and more manageable.

Potassium Carbonate. Potassii Carbonas. Carbonate of Potash. $\mathrm{K}_{2} \mathrm{CO}_{3}$.

Potassium Bicarbonate. Potassii Bicarbonas. Potassium Hydrogen Carbonate. $\mathrm{KHCO}_{3}$.

Potassium carbonates are got by several processes-(1) the American pot or wood ashes, in their partially purified condition of pearl ashes, contain about eighty per cent. of potassium carbonate, with twenty per cent. of potassium sulphate and chloride, which, being less soluble, are got rid of by dissolving the pearl ashes, with brisk agitation, in an equal weight of water, pouring off the solution, and evaporating it to dryness. (2) From the sulphate they are obtained by a process similar to that followed in making sodium carbonate. (3) A pure carbonate is got by burning potassium tartrate with charcoal.

The carbonate occurs in crystals, as a crystalline powder, but more generally in grains. It is white, opaque, and inodorous, with a strong alkaline taste, and an alkaline 
reaction. It is soluble in its own weight of water at $60^{\circ}$ Fahr., deliquesces rapidly in the air; but as it gradually absorbs carbonic acid, it again slowly dries up. Exposed to a red heat, it loses water of crystallisation to the amount of sixteen per cent.

Potassium bicarbonate, or acid carbonate of potash, is prepared by passing carbonic anhydride into a strong aqueous solution of the neutral carbonate. It occurs in transparent, colourless, right rhombic prisms; has a mild, saline, and slightly alkaline taste; dissolves in about four times its weight of water at $60^{\circ} \mathrm{Fahr}$.; when heated to redness, it gives off carbonic acid and water, and is converted into the neutral carbonate. It is distinguished from the neutral carbonate by its milder, non-acrid taste, its less solubility in water, its more abundant effervescence with hydrochloric acid, its not deliquescing when exposed to the air, and by giving, in diluted solution, no precipitate with Epsom salt or corrosive sublimate.

ACTIONS AND USES.- The two carbonates have the soluble alkali group actions and differ only in degree. Both resemble the hydrate, but have their activity tempered and diminished by combination with carbonic acid, which partly neutralises the caustic $\mathrm{OH}$ group set free by dissociation. The neutral carbonate, in concentrated solution, has much of the corrosiveness of the hydrate. Two drachms given to a dog caused vomiting, great agony, and death in twenty-five minutes (Orfila). Three ounces are said to be fatal to horses or cattle (Kaufmann). Its antidotes are the same as those of caustic potash. The bicarbonate has virtually no irritant or corrosive action, and so is often preferable as an antacid, and, in virtue of its liberating carbonic acid, exerts soothing effects on the irritable gastric membrane. It is less of an alkali and more of a pure saline. Both carbonates are antacid antidotes for overdoses of acids, and are alterative and diuretic.

Medicinal Uses-Potassium bicarbonate is occasionally substituted for sodium bicarbonate to aid the emulsifying of fats, and, on account of the evolution of carbonic acid, to soothe the irritable stomach. Given before meals, it was thought to increase gastric secretion. The experiments of 
Pawlow on dogs have, however, shown conclusively that alkalies lessen the secretion of gastric juices. In many cases of indigestion there is an irritated mucous membrane which continues to secrete a weak slimy juice. By inhibiting this useless secretion, and allowing the gastric glands a resting interval, alkalies do greatly benefit these cases. They should be given one or two hours before a meal. Given after meals, they neutralise excess of acid, resulting from undue secretion of gastric fluid, or from such acid fermentation of starch, sugar, or fats as occurs among carelessly fed calves. In rheumatism, small repeated doses of alkaline bicarbonates sometimes prove beneficial, apparently by neutralising excess of sarco-lactic acid, and encouraging the action of the kidneys. In such cases they are conjoined, according to circumstances, with oil of turpentine, salicylic acid, quinine, or potassium iodide. Similar antacid treatment is also successful in nettle-rash, and occasionally in eczema, a diluted solution being also applied externally to raw, weeping, painful, or itching surfaces. Potassium carbonates are specially suitable for preventing uric acid deposits, which occasionally occur in dogs ; they render the urine less acid, and since hyperacidity is the chief factor in the deposition of uric acid crystals, they prevent the formation of calculi. The potassium salt is much more soluble than the sodium urate. Calculi and deposits occur in the bladder and urethra of highly-fed rams and wethers. In the treatment of these cases, Litt recommends castor oil, f $z^{\mathrm{ijj}}$. to f亏viij., with belladonna extract, grs. viij. to grs. xvj., followed by potassium bicarbonate, 3ss. to $3 \mathrm{j}$., freely dissolved in water or other diluent. As diureties, the carbonates are less certain than the nitrate or acetate.

Externally, the carbonates are applied as stimulants and detergents. Used with soap and hot water, they soften and remove skin incrustation, whether consisting of sebaceous matters, thickened scales, abnormal discharges, or dirt. Diluted with 100 to 200 parts of water, along with a little glycerin, the carbonate forms a soothing dressing for the earlier weeping stages of eczema, especially in dogs. The itching parts should be kept continually wetted, while, to prevent evaporation, the wet lint should be covered with 
gutta-percha tissue. Cases of itching which are not relieved by alkaline lotions should be dressed with a dilute acid, and such alternation is sometimes successful, when neither the alkaline nor acid treatment alone succeeds. The bicarbonate proves a serviceable injection in leucorrhœa in all patients. In Cape Colony a ley made from wood ashes has been used successfully as a remedy for sheep scab, either alone or mixed with sulphur.

Doses, etc.-Of either carbonate, horses and cattle take

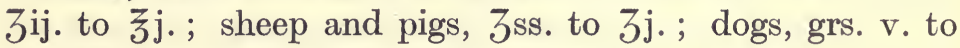
grs. xxx., repeated several times a day, liberally diluted with water. For improving gastric secretion they are given about an hour before eating; but in most dyspeptic cases acids are more permanently effectual.

Potassa Sulphurata.-Sulphurated Potash. Potassium Sulphide. A mixture of salts of potassium, of which the chief are sulphides. (B.P.)

One part of sulphur and two of potassium carbonate are mixed and heated until fusion occurs, poured on a stone slab and cooled. There is produced a liver-brown, bitter, acrid, alkaline substance which is odourless when dry, but when moistened smells of hydrogen sulphide. It readily dissolves in water, forming a yellow solution.

ACTIONS AND USES.-It conjoins the action of a sulphide and a potassium salt. Large doses are irritant and narcotic. Medicinal doses are laxative, and, like other sulphides, stimulate the secretions of the skin and respiratory mucous membrane, and are alterative. Externally, it is occasionally applied as a substitute for sulphur in the treatment of chronic skin diseases, and as a rubefacient, resolvent, and antiparasitic.

Toxic EFFECTS.-Two ounces are stated to have destroyed a horse (Bouchardat); six drachms and a half, introduced into the stomach of a dog, and retained by ligature on the sosophagus, occasioned death with tetanic symptoms in seven minutes; a drachm and a half in small fragments, introduced into the subcutaneous areolar tissue of dogs, caused extensive inflammation, coma, and death in thirteen hours 
(Christison). It appears to act much in the same manner as sulphuretted hydrogen, decomposing the hæmoglobin of the blood, and causing nervous and muscular paralysis.

Medicinal Uses.-It has been used in chronic cough, rheumatism, and skin diseases, in doses of 3i. to 3iij., for horses and cattle, and grs. ij. to grs. x. for dogs. Once a panacea for all kinds of poisoning, it is now used only in poisoning by lead, which it converts into a black, insoluble, and almost inert sulphide.

Potassium Sulphate. Potassii Sulphas. Sulphate of Potash. $\mathrm{K}_{2} \mathrm{SO}_{4}$.

Potassium Bisulphate. Bisulphate of Potash. $\mathrm{KHSO}_{4}$.

Potassium sulphate is got from certain salt mines, and from the mineral kainite, which is a double sulphate of potassium and magnesium. It occurs in transparent, colourless, rhombic prisms, which have a sharp, saline, bitter taste, are hard and difficult to powder, and dissolve in ten parts of cold water, and in four parts of boiling water.

The bisulphate is the residue in the preparation of nitric acid from nitre and sulphuric acid. It is colourless, crystalline, and soluble, with an acid taste, and an acid reaction. It is distinguished from the neutral sulphate by its small flat prisms, its greater fusibility and solubility in water, its acid taste and reaction, and its decomposing carbonates with effervescence-a property which has led to its being occasionally substituted for tartaric acid in making effervescent powders.

ACTIONS AND UsES.-They are cathartic, cholagogue, and diuretic. As cathartics they cause both hydragogue and peristaltic actions, while as diuretics they are less certain than the nitrate or acetate. On account of its hardness and inaptness to absorb moisture, potassium sulphate is used for facilitating trituration of such tough vegetable substances as opium, ipecacuanha, and jalap.

Potassium Iodide. Potassii Iodidum KI. (See Iodine and Iodides.)

Potassium Bromide. Potassii Bromidum. KBr. (See Bromine and Bromides.) 
Potassium Nitrate. Potassii Nitras. Nitrate of Potash. Nitre. Saltpetre. $\mathrm{KNO}_{3}$.

In the East Indies, Persia, Egypt, Spain, and other dry climates, a brown incrustation, consisting largely of nitre, covers considerable tracts of country! It is dissolved in water, mixed with impure potassium carbonate, and purified by repeated solution and crystallisation. By decomposing sodium nitrate with potassium chloride, nitre is also prepared.

Properties.-White, opaque, crystalline masses, or transparent, colourless, anhydrous, slender, six-sided prisms, with a sharp, cooling, saline taste, undergoing no alteration in the air, deflagrating when thrown on flame. It is soluble in $3 \frac{1}{2}$ parts of cold water, and one-third of its weight of boiling water; during solution much heat is abstracted; it is insoluble in alcohol. Warmed in a test-tube, with sulphuric acid and copper filings, it evolves ruddy fumes of nitric peroxide; heated to fusion, the melted mass forms, on cooling, the hard, white, fibrous sal-prunelle. None of its common impurities interfere with its medicinal actions.

ACTIONS AND USES.-Nitrates show very similar 'salt action ' to that of the chlorides, but are more irritant. Large doses of potassium nitrate irritate both the bowels and kidneys. Medicinal doses are diuretic, diaphoretic, and feebly cathartic, and therefore alterative and febrifuge. It is excreted by the bronchial membrane, the skin and kidneys, increasing the secretions of these organs. Used externally, it acts like other simple salts in solution, and is slightly stimulant and refrigerant.

Toxic EFFECTs. - Large doses cause, in man and carnivora, fatal gastro-enteritis, with vomiting, weakness, and arrest of circulation, partly depending on reflex action, partly on direct action on the heart (Brunton.) Guttmann, experimenting, chiefly upon dogs, states that, in common with other potash salts, poisonous doses, besides in-contact irritation, paralyse the spinal cord, cause dyspnca, and occasionally convulsions and muscular weakness, first overtaking the hind extremities, and lessen the frequency and force of the heart-beat, which in fatal cases ceases in diastole. 
This toxic effect of potassium is not likely to occur except when the drug is given intravenously or hypodermically. Given by the mouth, only the irritant effects of the salt are produced. Although an ounce has proved fatal in human patients, two ounces have no permanent injurious effect on horses or cattle. Morton, indeed, gave a healthy horse 2 pounds, dissolved in 6 pounds water, and found that it acted both on the kidneys and bowels, but that its effects ceased in twenty-four hours. Moiroud, however, found that half a pound given to horses, and two or three drachms to dogs, inflame the alimentary canal and urinary organs, causing depression and death, usually within twenty-four hours. Kaufmann states six ounces as the toxic dose for horses and cattle, six drachms for sheep, and seventy-five grains for dogs.

Medicinal Uses.-It is soluble, diffusible, and quickly absorbed. It promotes bronchial, cutaneous, and urinary secretion, and is especially used as a diuretic. Clinical experience accords it notable alterative and febrifuge properties. In conjunction with ammonium acetate solution, it is prescribed in catarrhal disorders, in which it has the twofold advantage of promoting discharge from the dry respiratory membrane and abating fever. American practitioners use nitre freely in laminitis, which, owing to careless feeding and long fasts, is still common in America; they give two ounces, dissolved in a pint of water, repeated frequently, and state that fever and pain are abated and exudation controlled. One fourth of this dose would be safer, and perhaps equally effectual. Repeated doses, conjoined with quinine, have been given in purpura. It is serviceable in rheumatism, being frequently prescribed with the carbonate or iodide, or with salicylic acid.

Nitre, when dissolving in water, abstracts heat, and is hence sometimes used externally as a refrigerant ; its cooling effects are increased by admixture with sal-ammoniac. Five ounces each of nitre and sal-ammoniac, dissolved in sixteen of water, reduce the temperature from $50^{\circ}$ to $10^{\circ}$ Fahr. For such purposes ice, however, is cheaper, and more convenient.

Doses, etc.-As a diuretic, horses take そss. to $\jmath^{j}$. ; cattle, 
そj. to $\zeta_{\mathrm{ij}}$.; sheep, $3 \mathrm{j}$. to $3 \mathrm{ij}$; pigs, $3 \mathrm{ss}$. to $3 \mathrm{j}$; dogs, grs. v. to grs. xx. Soap, resin, with other diuretics, and free solution in water, hasten and increase the action of nitre on the kidneys.

A diuretic mass is thus made :- Take soap and nitre, of each lbs. ij. ; resin, lbs. iij. ; Venice turpentine, lbs. ij. ; oil of turpentine, $f \zeta$ viij. Melt the soap and resin over a slow fire; remove the mixture from the heat, and when it has somewhat cooled stir in the other constituents. The dose of this mass is $z \mathrm{ij}$. The balls are made up with a little linseed meal or flour.

As an alterative and febrifuge nitre is given in about half the doses used to cause diuresis, is repeated several times a day, and is generally conjoined with other medicines. A febrifuge and laxative ball for the horse is prepared with an ounce of nitre, a drachm of aloes, and twenty grains of calomel. For a horse with catarrh and impaired appetite, a useful draught is made with Epsom salt two ounces, and nitre and ammonia acetate solution, of each an ounce, dissolved in gruel or ale. Catarrhal symptoms and sorethroat are relieved by four drachms nitre and one drachm each of ipecacuanha, camphor, and belladonna extract, made into bolus, and repeated every four or six hours. An ounce each of potassium nitrate and carbonate, with two drachms iodide, are useful in rheumatism. For cattle similar combinations are serviceable. For them a convenient alterative is made with two ounces each of nitre, sulphur, and ginger, given in treacle and water, or in ale.

For the dog a good febrifuge consists of five grains each of nitre and Dover's powder, and one grain calomel, placed upon the tongue, or bolted in a piece of meat, or made into a pill. Cats take about half the dose requisite for dogs.

\section{Potassium Chlorate. Potassii Chloras. Chlorate of Potash. $\mathrm{KClO}_{3}$.}

Chlorine gas is passed rapidly into a strong solution of potassium carbonate and calcium hydrate. The hypochlorite first formed is decomposed by the heat evolved. The mass, when charged with chlorine, as indicated by its 
acquiring a pink colour, is boiled, and the crystals formed in cooling are purified by re-solution in boiling water. They are colourless rhomboidal plates, have a cool saline taste, are soluble in sixteen parts of cold water, and in two parts at $212^{\circ}$ Fahr. The salt readily parts with its oxygen; thrown on red-hot coal it deflagrates; triturated with sulphur or phosphorus it explodes. Explosive gases are also evolved when it is heated with sulphuric or hydrochloric acid.

ACTIONS AND USES.-Potassium chlorate is antiseptic, sialagogue, and diuretic ; used externally, it is antiseptic, mildly stimulant, and refrigerant. It is less soluble than sodium chlorate, which it closely resembles.

Medicinal Uses. - The chlorate was formerly thought to act by liberating oxygen in the tissues and blood. That this is erroneous is proved by the fact that ninety per cent. of the drug can be recovered in the urine. At the same time chlorates are slowly reduced with liberation of oxygen in the presence of putrid decomposing organic matter. The salt after absorption is excreted in the saliva, from mucous surfaces, and by the kidneys, and if putrefaction is going on in these situations the oxidising and antiseptic effects of the drug are produced. Poisonous doses highly oxidise the hæmoglobin of the blood, converting it into methæmoglobin, which holds oxygen firmly, and thus interferes with aëration of blood in the remote capillaries. Respiration accordingly is impaired, blood-pressure falls, hæmaturia and asphyxial convulsions precede death. A small quantity mixed with recently-drawn blood causes it to assume a chocolate colour and to give the spectrum of methæmoglobin, and many red cells are destroyed. Used as a wash or gargle, it stimulates the salivary and buccal glands, moistening the dry, parched mouth. It soothes and heals aphthous eruptions and ulcerations of the mouth and throat; while in catarrh, sorethroat, and bronchitis it thins the secretions and promotes expectoration.

It is readily absorbed, and in febrile and blood-poisoning cases has been believed to exert antiseptic effects, depending upon its saline properties, and on its readily parting with oxygen and chloric acid. But this explanation is not altogether satisfactory, for, as already stated, it is excreted 
in great part unchanged, small doses being removed by the kidneys, and larger by the bowels. Like other salines, in febrile and inflammatory cases, whether in horses or cattle, it is believed to lower pulse and temperature, clean the tongue, improve appetite, gently stimulate the bowels, and render the evacuations more natural and less coated with mucus. It is frequently prescribed with Epsom salt, gentian, or ether. Hard-worked horses, overdone or suffering from catarrh, are usually benefited by half an ounce, given night and morning, with gentian and ether. In influenza of horses, it may be given with sweet spirit of nitre and camphor. In the treatment of purpura, Williams prescribed it with iron salts, in the belief that it increasedas it does outside the body-the coagulability of blood. $\mathrm{He}$ gave an ounce daily, divided into two or three doses, but after the second day found that smaller doses sufficed. It is rapidly eliminated in the urine-rendering it acid even in herbivora-and also in the sweat, bile, milk, and saliva.

Solutions of six to twenty grains to the ounce of water and glycerin are used as antiseptic washes for unhealthy wounds of the mouth.

Doses, etc.-Horses take $3 \mathrm{j}$. to $3 \mathrm{iv}$. ; cattle, $3 \mathrm{ij}$. to $3 \mathrm{vj}$. ; sheep and pigs, grs. xx. to grs. lx. ; dogs, grs. v. to grs. xv., repeated two or three times daily, given in either bolus or solution, alone or conjoined with other salines, bitters, tonics, or stimulants. Most horses of their own accord will take an ounce daily, dissolved in water or gruel. As a soothing electuary for sore-throat it is conjoined with camphor, belladonna, and treacle.

\section{Potassium Permanganate. Potassii Permanganas.} $\left(\mathrm{KMnO}_{4}\right)$.

Potassium permanganate is obtained by the interaction of manganese dioxide, potassium hydrate, and potassium chlorate, occurs in dark purple crystals, with a sweet, astringent, disagreeable taste. It is readily soluble in cold water, producing a deep-red solution. So readily does it part with oxygen that when mixed with such easily oxidised substances as sugar and glycerin it takes fire or explodes 
spontaneously. The solution also readily evolves oxygen and hence is an effectual bleacher and deodoriser.

ACTIONS AND UsEs.- The permanganates, in virtue of their power of oxidation, are deodorisers, and also topical stimulants. Strong solutions are irritant and caustic. Their power of breaking up various unstable organic substances is further illustrated when they are mixed with the cobra poison, which, thus treated, loses its deadly power, and may with impunity be injected subcutaneously. When an animal has been bitten by a cobra, fifteen minims of a one per cent. solution, injected round the bitten part, is a reliable antidote. When swallowed it does not seem to exert the alterative or febrifuge effects of the nitrate or chlorate. Concentrated solutions produce gastro-enteritis. It is an antidote to poisoning by opium.

Potassium permanganate, although it has not the antiseptic power of corrosive sublimate, effectually destroys bacteria, and Koch found that a five per cent. solution arrested development of the spores of anthrax soaked in it for one day. It is used to deodorise and disinfect fœtid wounds, the nostrils in ozæna, the mouth in aphtha, the throat when ulcerated, the uterus in retention of the fœetal membranes, and also to cleanse hands and instruments that have been in contact with offensive or contagious matters. As a disinfectant permanganate fails in many cases, because, being non-volatile, it cannot penetrate below the surface.

Permanganate solutions are frequently placed in shallow vessels about buildings to be deodorised : or cloths, saturated with one part of the fluid to fifty or sixty of water, are suspended. But for thorough disinfection such a non-volatile body is not so trustworthy as chlorine, sulphurous acid, or the volatile tar acids. Effectual results are, however, obtained when the permanganates are brought into immediate contact with the injurious organic particles. Thus, four ounces of permanganate added to 100 gallons of stalesmelling, unsightly rain-water left in a foul cistern, usually precipitates all impurities, and after some hours the clarified water becomes sweet and fit for use. The rapidity with which a known quantity of the permanganate solution parts 
with oxygen and loses its purple or pink colour, is a test of the amount of organic contamination in water, other fluids, or even in air. Its expense, however, precludes its general use in veterinary practice.

DoSES, etc.-Potassium permanganate has been given to horses and cattle as an alterative and febrifuge in drachm doses ; but observation does not justify its preference to the nitrate or chlorate. For antiseptic and deodorant purposes one part permanganate is dissolved in 50 to 1000 parts of water. A 1 to 1200 solution is used for intravaginal and intrauterine injection after difficult parturition, and in cases of retention of the fœtal membranes.

Potassium Acetate. Potassii Acetas. Acetate of Potash.

Potassium Citrate. Potassii Citras. Citrate of Potash.

Potassium acetate is prepared by fusing the product of the interaction of acetic acid and potassium carbonate. It occurs in white, foliaceous, satiny masses, or in granular particles, very deliquescent, and freely soluble in water and alcohol. In their actions and uses the acetate and citrate closely resemble the nitrate. Without action on the gastric juice, they render the urine and other secretions strongly alkaline. Like other alkaline salts containing a vegetable acid, when they enter the body they are mainly converted into the carbonate, and are eliminated in the urine, producing diuresis. The acetate and citrate of potassium are the inorganic diuretics most frequently prescribed in human medicine. Doses for horses and cattle, $3 \mathrm{jv}$. to $\bar{\jmath}$. ; sheep and pigs, grs. $x x x$. to $3 j$. ; dogs, grs. v. to grs. $x x x$.

Acid Potassium Tartrate. Potassii Tartras Acidus. Cream of Tartar. $\mathrm{KHC}_{4} \mathrm{H}_{4} \mathrm{O}_{6}$.

Potassium Tartrate. Potassii Tartras. $\mathrm{K}_{2} \mathrm{C}_{4} \mathrm{H}_{4} \mathrm{O}_{6} \cdot \mathrm{H}_{2} \mathrm{O}$.

The crude tartar or argol, obtained from the interior of wine-casks, when purified by solution and crystallisation, occurs in white, hard, crystalline masses, with a sharp acid taste. When administered it takes up water with avidity, 
and is slowly absorbed; although it does not cause intestinal irritation or peristalsis, doses of several ounces given to horses or cattle render the fæces fluid, and are mildly laxative. Smaller doses, like those of the alkaline salts of most organic acids, are converted in the body into the carbonate, and excreted mostly in the urine, causing diuresis.

The normal potassium tartrate is obtained by neutralising acid potassium tartrate with potassium carbonate. It occurs in small, colourless four- or six-sided prisms. It resembles the acetate and nitrate; in small doses is diuretic, in larger purgative. Robertson used to recommend it with magnesium or sodium sulphate for anæmic young horses affected with congested liver. Doses, horses and cattle $3 \mathrm{jv}$. to $\bar{\jmath}$ j.; sheep and pigs, $3 \mathrm{jv}$.; Dogs, grs. $\mathrm{xxx}$. to $3 \mathrm{j}$. ; cats, grs. v. to grs. xv.

\section{SODIUM AND ITS MEDICINAL COMPOUNDS}

Sodium salts abound in the ashes of marine and maritime plants. They occur native in the Chili nitre beds and in borax, but their chief commercial source is the chloride obtained from rock-salt deposits, or from the evaporation of sea-water. They are soluble, with the single exception of the antimoniate, which separates very slowly from solution. They are distinguished by their negative reaction with the several group tests, and by their communicating to the flame of burning alcohol a bright yellow colour.

Actions AND Uses.--Sodium salts, chiefly as albuminates, chlorides, carbonates, and phosphates, are constituents of the blood, bile, serous fluids, and indeed of all animal secretions and textures. The more readily soluble salts, in small doses and diluted solution, are chiefly excreted by the kidneys; while the less soluble, in larger doses and more concentrated solution, are removed by the bowels.

Like potassium salts, they may be grouped as follows :1. The hydrate, carbonates, and salts of organic acids, which in the body are converted into carbonates, are antacid, alterative, and slightly diuretic. The hydrate and carbonate are caustics. Sodium ethylate solution contains 18 per cent. 
of the solid salt $\left(\mathrm{C}_{2} \mathrm{H}_{5} \mathrm{ONa}\right)$, is a colourless, syrupy liquid, becoming brown by keeping, and is used as a caustic.

2 . The chloride, sulphate, and nitrate act as soluble crystalloids, are mildly antiseptic, febrifuge, and refrigerant; small doses are slightly diuretic, while large doses are saline purgatives.

3. The borate, benzoate, hyposulphite, sulpho-carbolate, chlorate, salicylate, and valerianate resemble their acid radicle rather than their base.

Sodium Hydroxide. Sodium Hydrate. Caustic Soda. $\mathrm{NaHO}$.

Sodrum Carbonate. Sodii Carbonas. Carbonate of Soda. $\mathrm{Na}_{2} \mathrm{CO}_{3} \cdot 10 \mathrm{Aq}$.

Sodium Bicarbonate. Sodii Bicarbonas. Bicarbonate of Soda. $\mathrm{NaHCO}_{3}$.

Sodium hydrate and solution of caustic soda resemble in their preparation and general properties the corresponding potassium compounds, but are little used in veterinary practice.

The carbonate was formerly obtained by lixiviating the ashes of marine plants, and from the native sesqui-carbonate or natron found as an efflorescence on the margins of lakes in warm climates. It is now obtained from common salt, by heating it in furnaces with sulphuric acid; the sulphate thus prepared is converted into sulphide, and thence into carbonate, by roasting with coal or slack and limestone; lixiviating, calcining, and crystallising. From a saturated solution of this soda ash there separate large transparent, colourless, laminar, rhombic crystals of hydrated carbonate $\left(\mathrm{Na}_{2} \mathrm{CO}_{3} .10 \mathrm{Aq}\right.$.). The water may be driven off by heating to $120^{\circ}$ Fahr., when the dried granular sodium carbonate remains. The carbonate in its several forms is alkaline to taste and reagents, efflorescent, and soluble in one or two parts of water.

The bicarbonate produced by saturating the carbonate with carbonic anhydride occurs as a white crystalline powder, or aggregation of irregular opaque scales, has a saline, slightly 
alkaline, not unpleasant taste, is soluble in about ten parts of cold water, and is distinguished from the carbonate by its feeble alkalinity, and its giving a colourless instead of a coloured precipitate with corrosive sublimate. Soda water, as ordinarily sold, is simply aerated water, but it should contain in every pint thirty grains of bicarbonate, and be saturated with carbonic acid gas, dissolved under pressure of four atmospheres.

ACTIONS AND USES. - Sodium carbonate and bicarbonate are antacids, expectorants, and diuretics. They differ only in the degree of their action, and resemble the corresponding potassium salts. They increase the alkalinity of the blood, promote secretion of bile, assist in the elimination of mucus, and exert a slight laxative effect.

Medicinal Uses.- Small doses given a quarter to half an hour before meals improve the secretion of gastric juice in many cases of dyspepsia. (See Potassium Carbonate.) Given with or after food, they aid the emulsification and digestion of fats, and neutralise the acid of the gastric juice, as well as any acid produced by excessive fermentation of food. They are hence sometimes serviceable in relieving indigestion and flatulence, their efficacy being increased by administration with aromatics or stimulants. Calves fed on stale skim milk, and suffering from atonic dyspepsia, are often relieved by one to two drachms of sodium bicarbonate and 30 grains of bismuth carbonate, dissolved in each meal of milk. Hæmoglobinuria has been successfully treated with half a pound to a pound sodium bicarbonate daily given in doses of two ounces every hour. Normal alkalinity of blood and muscle is believed to be secured, and further solution of hæmoglobin arrested (Dieckerhoff). They are antidotes in poisoning by mineral and other acids; being less irritant than the corresponding potassium salts, they are preferred for improving the secretion of gastric juice and neutralising acids in the alimentary canal, while potassium carbonates are more effectual antacids in rheumatism or acidity of the urine, and are more active diuretics.

Sodium carbonate solutions applied locally lessen irritation of urticaria, lichen, and other skin eruptions, but are not so effectual as potassium carbonates. In more chronic 
cases the alkaline dressings are alternated with tar, or oil of cade. Leucorrhcea is usually arrested by two or three injections of diluted sodium bicarbonate. A stronger solution abates the pain of burns.

Doses, etc.-Of the carbonate, horses and cattle take $3 \mathrm{jj}$.

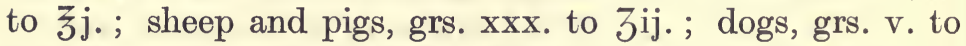
grs. $x x x$. The bicarbonate, although less active than the carbonate, is more convenient for general use, and is given in double these doses, either in bolus or solution. It is frequently given dissolved in the drinking water to dyspeptic, diabetic, or febrile horses.

Borax. Sodium Biborate. Sodium Pyroborate. $\mathrm{Na}_{2} \mathrm{~B}_{4} \mathrm{O}_{7}$. $10 \mathrm{Aq}$.

Borax occurs native in certain Austrian mineral waters, as an incrustation on the edges of various lakes in Thibet and Persia, and in streams in Southern California. As crude borax or tincal, it is imported from Calcutta in greenish pieces, moistened with oil to prevent efflorescence. It is purified by calcining and recrystallising. Borax is now got by calcining together, in proper proportions, boric acid and sodium carbonate.

In colourless crystals, with a saline, cooling taste, soluble in 17 parts of cold and two of hot water, and in one of glycerin ; insoluble in alcohol. Heated, it melts in its water of crystallisation, and swells into the porous borax usta ; at a red-heat it becomes the transparent glass or anhydrous borax used as a flux.

ACtions AND Uses.-Borax is antiseptic, parasiticide, diuretic, and alkaline, and is used to relieve irritation of the skin and mucous membranes. By the mouth it exerts its antiseptic and antacid effects in the stomach, but larger doses are irritant and cause vomiting and diarrhœa. Injected into the blood stream toxic doses bring about muscular weakness and collapse.

It has notable antiseptic powers ; one part in 100 of water arrests the action of emulsin, diastase, and ptyalin ; while one part in 1000 of water prevents the action of rennet; it requires, however, according to Koch, one part in forty-eight 
of water to kill developed bacteria, and it has no effect on spores.

It has been prescribed in the same doses as the bicarbonate in gastric irritation. It is a useful antiseptic in aphthous and ulcerated conditions of the mouth and fauces, is sometimes conjoined with potassium chlorate, and applied either in powder or with 'Sanitas,' or glycerin and water. It allays irritation and itching in many cases of erythema, eczema, and psoriasis. It is preferable to more active and poisonous remedies for dogs, which are apt to lick their dressings. In acute eczema one part each of borax and alum is used, dissolved in fifty parts of water. It is an effectual injection for leucorrhœa. It is a good solvent for salicylic and benzoic acids. Glycerin of borax is made with one part of borax and six of glycerin. Mel Boracis is composed of 2 parts borax, 1 of glycerin, and 16 of honey.

Doses.-Horses and cattle, 3iij. to 3vj.; sheep and pigs, 3 j. to 3 iij. ; dogs, grs. v. to grs. xxx.

Sodium Perborate, prepared by the action of boric acid on sodium peroxide, is a white powder, soluble in water, and employed as a substitute for hydrogen peroxide in the dressing of wounds.

Sodium Sulphate. Sodii Sulphas. Sulphate of Soda. Glauber's Salt. $\mathrm{Na}_{2} \mathrm{SO}_{4} \cdot 10 \mathrm{Aq}$.

The sulphate effloresces on the surface of the soil in various parts of India, occurs in masses in Spain, and is a constituent of sea-water, of many aperient mineral waters, of various plants, and of several animal secretions. When two parts sodium chloride are heated with one part sulphuric acid, hydrochloric acid is evolved, and sodium sulphate crystallises from the solution. It occurs in colourless, transparent, oblique prisms, which effloresce on exposure to air, have a saline, bitter taste, and at $60^{\circ} \mathrm{Fahr}$. are soluble in less than their own weight of water.

Actions AND USES.-According to the dose it is laxative or cathartic, slightly diuretic, febrifuge, and indirect cholagogue. Externally it is refrigerant.

Unlike the magnesium sulphate, it has no toxic effect when 
injected into the circulation. Like other saline purgatives, it has a low diffusive power, for the $\mathrm{SO}_{4}$ ion is absorbed to a very slight extent; it impedes absorption of fluids present in the intestines, draws out fluid from the blood, increases peristalsis, and thus augments the quantity and fluidity of the dejections. Bile poured into the intestine is hurried along and excreted, its reabsorption being prevented, and the same occurs with sodium phosphate. While large doses of Glauber salts are removed by the bowels, small doses, freely diluted, pass off, in great part unchanged, by the kidneys. Although little used for horses, it is still prescribed for cattle and sheep, for the same purposes as Epsom salt, with which it is sometimes conjoined. Catharsis is seldom, however, produced within twenty-four hours. It is an antidote in poisoning by carbolic acid and barium chloride. In dogs it acts both as an emetic and purgative.

DOSES, etc.-As a purgative cattle take lb. j. to lb. jss. ; sheep, ऊij. to そiv., given with ginger and treacle, and succeeded by liberal supplies of chilled water. As a febrifuge for horses $\jmath_{j}$. to $ろ \mathrm{jv}$. may be given.

Sodrum Sulphite. Normal sulphite. Sodii Sulphis. $\mathrm{Na}_{2}$ $\mathrm{SO}_{3} .7 \mathrm{Aq}$.

Sodium Hyposulphite. Hyposulphite of Soda. Sodium Thiosulphate. Sodii Hyposulphis. $\mathrm{Na}_{2} \mathrm{~S}_{2} \mathrm{O}_{3} \cdot 5 \mathrm{Aq}$.

Sodium sulphite may be obtained by the interaction of sulphurous acid and sodium carbonate. In colourless crystals, efflorescent, inodorous, with a saline taste.

Sodium hyposulphite may be prepared by dissolving sulphur in a boiling solution of sodium sulphite and crystallising. The hyposulphite is very soluble in water, and fusible. It is more permanent than the sulphite.

ACTIONS AND USES.-The sulphite and hyposulphite are antiseptics, deodorisers, and insecticides. In the presence of acids, without and also within the body, they give off sulphurous acid, which they therefore resemble. They destroy ferments and bacteria, and remove offensive smells by abstracting oxygen from organic matter to become themselves converted into sulphates. These bactericidal 
and deodorant properties are greatly increased when they are used along with the tar acids.

General Actions.-Sodium sulphite and hyposulphite, when given by the mouth are antiseptic, in the mouth and stomach they limit fermentation and, in the dog and cat, may cause emesis from the sulphurous acid liberated by the gastric juice. They are slowly absorbed and, if in excess, cause depression of the medulla and weakening of the heart with symptoms of dyspepsia, muscular debility, and restlessness. They are excreted mostly as sulphates in the urine, although a considerable amount (30 per cent.) of the hyposulphite is excreted in the urine unchanged. Polli, of Milan, made upwards of three hundred experiments with acid sulphite, mostly upon dogs, and found that it materially diminished the effects of animal poisons. He gave dogs 225 grains daily for a fortnight; very moderate doses were detectable in twenty-four hours in the blood, liver, and urine; two ounces of blood drawn from dogs, which for five days had received daily with their food thirty grains of sulphite, kept fresh for three weeks ; while blood taken from dogs similarly fed, but receiving no sulphite, became putrid within a few days. Full doses, given previous to death, retarded or prevented putrefaction of the body.

Polli injected fifteen grains of fœit pus from an abscess into the thighs of two dogs, and next day repeated the injection. Both dogs were stupefied, reeled, and tottered when made to walk, while their pulse and breathing were much quickened. For five days previously both dogs had been treated exactly alike, with this difference only, that one had received daily thirty grains of sodium sulphite, which was continued throughout the experiment. In four days after the injection this dog was again eating, and the wound in his thigh healing. The other, receiving no sulphite, daily became worse, gangrene set in, and in ten days he died, exhausted.

Medicinal Uses.-These experiments held out great hope that septicæmia might be prevented or cured by sulphites. But repeated careful clinical observation has not justified the high expectations formed of them, and their administration does not appear to arrest or materially alter the 
course of febrile, contagious, or infectious diseases. Amongst horses they have been given in febrile attacks, and in such cases many American practitioners have given favourable reports of them. They relieve acidity and flatulence occurring in badly-fed young calves. The sulphite, mixed with treacle and placed within the lips, diminishes irritation, smell, and acrid discharge in foot-and-mouth disease. Used alone, and occasionally with potassium chlorate, it has been given to young cattle to prevent attacks of black-leg, halfounce or ounce doses for this object being administered with the ordinary food for three or four days consecutively during every fortnight. It is difficult to estimate the precise value of such preventives, adopted, as they often are, in conjunction with more careful feeding and management. In distemper in dogs, ten to twenty grains of sulphite, or hyposulphite, are given twice daily to regulate the bowels and abate the fever.

A solution of ten grains hyposulphite to the ounce of water is an excellent application for corneal ulcers and recent opacities.

DOSES, etc.-Of the sulphite, horses and cattle take $\zeta$ ss. to ろij.; sheep and pigs, 3ss. to 3iv.; dogs, grs. xx. to $3 \mathrm{ij}$. Of the hyposulphite, somewhat smaller doses suffice. Either drug is prescribed in powder or solution, and may be repeated several times daily. Having little taste, they may usually be taken mixed with the food or drinking water. They may be conjoined with ginger, gentian, camphor, or ammonium carbonate.

Sodrum Chloride. Sodii chloridum. Chloride of Sodium. Common salt. $\mathrm{NaCl}$.

Salt is found in extensive rock deposits in Poland, Spain, and other parts of Europe, and in this country in Cheshire and Worcestershire. It exists in variable amount in every soil, and hence in every water, is the largest saline constituent of the ocean, and is present in the tissues and fluids of plants and animals. It is obtained for medicinal and domestic purposes by quarrying the solid beds of rock-salt, or by evaporating brine springs or sea-water. 
It forms crystals, which vary in size according to the rapidity of their formation. When pure, it occurs in small, white, crystalline grains, or transparent cubical crystals free from moisture, and has a purely saline taste. From the presence of magnesium and calcium chlorides, many samples are deliquescent. It is soluble in about two and threequarter parts of water, and insoluble in alcohol.

Actions AND Uses.-Salt is an essential article of food; small doses are restorative, stomachic, and antiseptic ; larger doses are irritant, cathartic, and emetic ; it is used externally as a stimulant, antiseptic, and refrigerant.

General Actions.-So essential is the regular or frequent use of salt for the maintenance of health, that animals, especially vegetable feeders, in a state of nature instinctively travel many miles to saline springs, the sea-shore, or incrustations or beds of salt. Boussingault, experimenting on its dietetic value, selected six cattle, as equal as possible in weight and appearance, and fed them in exactly the same manner, except that three received each $1 \cdot 2$ ounces of salt daily, whilst the other three got none. In about six months the skin and hair of those without salt became rough, dry, and staring, presenting a striking contrast to the smooth, shining coats of the others, which, although little heavier than their neighbours, were more lively, and of so much better appearance that they brought a somewhat higher price. The cattle receiving salt exhibited throughout greater appetite and relish for their food, consumed it in a shorter time, and also drank larger quantities of water.

A piece of rock salt should constantly lie in the horse's manger, the ox's crib, and the sheep's trough. It is specially necessary when the diet consists largely of cooked grains or roots, in the preparation of which part of the salt is apt to be dissolved out. The condiment not only gratifies the palate, but also, as indicated, serves important nutritive purposes. It increases secretion of saliva and gastric juice, furnishes hydrochloric acid for the gastric juice, and soda salts for the bile ; alters the rate of diffusion of fluids through membranes, while four to six parts per 1000 are present in the blood serum, contributing to the solution of the globulins. During convalescence from acute disease the chloride and 
other sodium salts are removed from the body in unusual amount, and most animals then instinctively take salt freely. Besides furnishing an essential constituent of the animal fluids and solids, it appears to assist in the assimilation of nutritive matters. On the absorption of calcium salts it has a marked effect, for when withheld from dogs with fractured limbs repair and union are tardy. It is excreted by the kidneys, skin, and mucous membranes.

In common with cathartic salines, it acts chiefly physically by osmosis, though it is absorbed much more readily and is therefore a less effective purgative than magnesium sulphate. It impedes absorption of fluid from the bowels, which thus become mechanically distended with fluid. Their contents are softened and peristalsis is encouraged. The blood not only does not obtain from the bowels its usual supply of fluid, but pours serous secretion into the bowels. Within an hour or two after administration of a saline cathartic the blood becomes concentrated, and thereafter gradually recoups itself from the tissues, thus promoting excretion of fluid and waste materials.

On horses the cathartic action of common salt is uncertain, often violent, and usually accompanied by considerable irritation of the kidneys. On dogs it usually operates both as an emetic and cathartic. Small and freely diluted doses increase the secretion of urine and the proportion of urinary solids. On pigs it acts as a purgative, but is not so safe or certain as oil, jalap and calomel, or aloes.

TOXIC EFFECTS. - Cases are recorded of pigs eating about four and a half ounces daily for several days. They suffered from flatulence, diarrhœa, vertigo, convulsions, and paralysis, and died in eight to twenty-four hours after showing those symptoms. The mucous membrane of the stomach and bowels was found after death highly injected and inflamed. Sir Charles Cameron reported the poisoning of thirty-one pigs conveyed by rail in a salt-truck, from the sides of which they had licked the salt. For many hours they had been deprived of water. They appeared in a state of asphyxia ; emetics and subsequently stimulants were ordered, and eleven recovered. The carcases of those that died exhibited 'signs of gastrointestinal inflammation; the brain was greatly congested, 
and there was considerable extravasation of blood in the cerebellum and medulla' (Veterinarian, 1871). Even cattle and sheep, for which it is generally a suitable cathartic, occasionally suffer from overdoses. Dangerous effects have been produced by several ounces given to young and delicate calves, for which oil is a more suitable purge.

Medicinal Uses.-For vigorous eattle and sheep common salt is a useful purgative, resembling in its effects Epsom and Glauber salts. It is, however, more soluble, moderate doses are more quickly absorbed, and hence it is frequently desirable to conjoin common and Epsom salts. Full doses of such salines cause thirst, induce the animal to drink water or other bland fluids freely, thus softening and carrying onwards the hard, dry, impacted food, which is apt to accumulate in the first and third stomachs of ruminants. For such patients salt is administered to unload the bowels in impaction of the rumen and omasum, as well as in the earlier stages of diarrhœa depending on over-feeding, or kept up by the presence of irritating matters in the canal. It is also given for its indirect effects on general metabolism and nutrition, for it not only beneficially empties the stomachs and bowels, but frees the blood of peccant matters. It is indicated in the later stages of pleurisy, peritonitis, and pneumonia.

Small and repeated doses are stomachic, are prescribed for all animals suffering from indigestion and irregular appetite, and may be conjoined with gentian, ginger, or spirits and water. It obviates to some extent the effects of damp and badly kept fodder, and, given with nutritive dry food, prevents or retards the progress of ascites in sheep so often caused by flukes and other parasites. Systematically given, salt lessens the liability to intestinal worms, and an enema of two or three ounces to a pint of water often brings away ascarides from the rectum. It is frequently added to laxative clysters. A solution of 80 grains pure sodium chloride in 20 ounces of boiled water cooled to blood heat, is injected subcutaneously into the peritoneal cavity, or intravenously, in collapse from hæmorrhage, serious operation, or from exhausting disease. Injections of a quart or more may be made daily with marked benefit in many cases. 
Dissolved in ten to twenty parts of water, it proves a serviceable antiseptic and stimulant gargle in relaxed and ulcerated sore-throat of horses and other patients. Saltwater baths exert curative effects on animals as well as on man. For stuping or cleansing wounds, a one per cent. warm watery solution, in virtue of its stimulant and antiseptic properties, is preferable to plain water, and less apt to sodden the parts or weaken their vitality. Salt solutions are applied cold as stimulants and refrigerants for strains and chronic inflammation of the joints and feet, particularly amongst cattle and sheep. For a cooling mixture, one part each of salt, nitre, and sal ammoniac is dissolved in thirty to forty parts of water ; or one part of salt is mixed with two of pounded ice. Such freezing mixtures require, however, to be used warily, for their prolonged application dangerously lowers vitality.

For preventing and arresting putrefaction, salt is cheap and effectual. For antiseptic purposes it is advantageously conjoined with carbolic acid. To disinfect skins, a pound of salt and two ounces of carbolic acid are used, dissolved in a gallon of water. Waste chlorides are used to preserve for manure the meat seized at markets as unfit for human food. Salt alone or mixed with soot, or gaslime, and loam forms an excellent top dressing for pastures infested with parasites.

Doses, etc.-As a purgative the ox or cow takes $1 \mathrm{~b} . \frac{3}{4}$ to

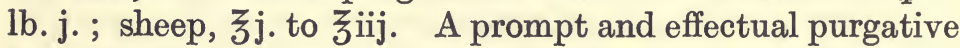
consists of half doses of common and Epsom salts, dissolved in about two quarts of warm water, with two ounces of powdered ginger, anise, or other aromatic, and a pound of treacle. Some cattle readily drink the mixture thus sweetened, and the trouble of drenching may be saved. To hasten and increase the effects of salines other purgatives are sometimes added. Along with half a pound each of common and Epsom salts, dissolved in water with aromatics and treacle, may be given ten or twenty croton beans in

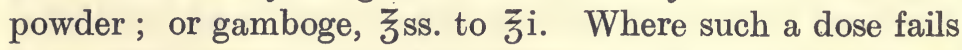
to act in twenty or twenty-four hours, it may be repeated, or a pint or two of linseed oil may be substituted for the salts. But large doses of drastic physic are, however, to be avoided, for they induce nausea and depression, which prevent purga- 
tion. When a patient has had two full doses without effect, clysters should be tried, with a free allowance of water containing salt and treacle.

As a stomachic and alterative for horses or cattle, two or three ounces of salt are given, usually with aromatics, bitters, or vegetable tonics. As an emetic for the dog, the dose varies from one to four drachms, dissolved in tepid water. A still more effectual, readily obtained emetic for a medium-sized dog consists of a tablespoonful of salt and half a teaspoonful of mustard flour, dissolved in three or four ounces of water. More prompt results are secured by adding a few grains of zinc or copper sulphate, or of tartar emetic.

Nitrate of soda, much used as a manure, has irritant and cathartic properties, somewhat resembling those of common salt, but also showing typical irritant nitrate action. It has sometimes killed both horses and cattle that have licked it, or eaten grass strongly saturated by a large, recently applied dressing.

A number of cattle died rather suddenly on a farm in South Lincolnshire, and an inquiry into the circumstances of the case left no doubt that death arose from nitrate of soda which the animals obtained from bags which had been used in conveying this fertiliser into the field, and had afterwards been left lying about. On a farm in Massachusetts, eleven cows out of a herd of fifteen died as a result of having had given to them nitrate of soda in mistake for common salt. The fifteen cows had been out at grass all day, and were driven to the stalls in the evening, when an attendant gave to all except two what he supposed to be common salt, thoroughly mixed with a small quantity of wheat bran. Apparently, all the animals ate heartily. Two hours later the cows attracted notice by the noise they were making, and when examined they were found to be uneasy and evidently in pain, and several died within an hour or two. The only skilled advice obtainable was that of a medical practitioner who, thinking the case was one of arsenic poisoning, prescribed accordingly, but before morning eleven of the cows were dead. An examination of samples of material collected from the mangers revealed the presence of large quantities of 
nitrate of soda, but no common salt. An analysis of the contents of the stomach failed to discover any trace of arsenic, lead, or other mineral poison. Similar cases have been recorded in the Archives Vétérinaires. A horse had drunk freely of a solution of nitrate of soda and exhibited many of the symptoms that were subsequently noticed in the Massachusetts cattle; the administration of strong infusions of coffee and alcohol and irritant clysters relieved the animal, which in a few days completely recovered. In another case four bags of nitrate of soda had been placed on a grass field and allowed to remain some five hours before being applied. Meanwhile, some of the nitrate had become washed by rain from the bags upon the ground. Three days later cows were turned into the pasture and obtained the salt by licking it from the herbage. They showed symptoms of poisoning, but ultimately recovered. One other case worth noticing is that in which two hundred bags which had contained nitrate of soda were washed in large tubs of water close to which the cattle passed on their way to the pasture. A few hours afterwards many of the cows were seen to be ill, and within eight hours two of them had died. These proved to be the first two that had left the stalls, and consequently had time to drink more of the solution than the others.

Liquor Sod ex Chlorinates. Solution of Chlorinated Soda. Solution of Sodium Hypochlorite. $\mathrm{NaCl}, \mathrm{NaClO}$.

Admixture and subsequent filtration of solutions of sodium carbonate and chlorinated lime produce the B.P. liquor sodæ chlorinatæ, known also as Labarraque's disinfecting fluid. It is a colourless alkaline liquid, with an astringent taste, and a feeble odour of chlorine. Like the analogous solution of bleaching powder, it contains chlorides, and hypochlorites, and, acted on by air or acids, gives off chlorine and chlorine compounds.

ACTIONS AND Uses.-It is antiseptic, stimulant, and antacid, and is also used as a disinfectant and deodorant. It is applicable to the same purposes as solution of chlorinated lime and liquor chlori.

It arrests the action of yeast and other ferments, and kills 
bacteria. When administered internally, it has been credited with the power of oxidising urea and other products of tissue metamorphosis, and of hastening their excretion. It has been prescribed as an antiseptic in fever and purpura in horses, and as an antidote in poisoning by hydrogen sulphide, the hydro-sulphides, and prussic acid. It is to be remembered, however, that chlorine is much more poisonous to the higher animals than to bacteria so that its internal use should be restricted or avoided.

It is chiefly serviceable as an external antiseptic for stimulating and deodorising foul wounds and ulcers, checking excessive noisome discharges from the skin or mucous surfaces, controlling the earlier stages of eczema, and for douching from an atomiser relaxed and irritable sore-throat. As an antiseptic, although more expensive, it is for some purposes preferable to chlorinated lime, inasmuch as, upon exposure, it becomes converted into common salt-itself a valuable antiseptic, and more permanent and convenient than the deliquescent, moist calcium chloride, which remains when bleaching powder is used.

Doses, ete.-Of the B.P. solution, which contains about $2 \frac{1}{2}$ per cent. of available chlorine, horses and cattle take $f \exists j$. to $f z^{i j}$. ; sheep and pigs, $f 3 j$. to $f 3 i j$. ; dogs, $m_{x v}$. to $m_{x x x}$., dissolved in water.

Fluoride of sodium, obtained by saturating hydrofluoric acid in water with sodium carbonate, has been used to liquefy the exudate and other deposits in cystitis and mammitis. It is antiseptic. For contagious mammitis, an ounce of a solution of one part sodium fluoride in a thousand parts of boiled water, is injected, three or four times daily, into the affected quarter, after drawing the teat. Later, or in a week from the first injection, a solution of 1 to 500 may be used until recovery is well established. Injections of sodium fluoride may be alternated with injections of solution of sodium iodate ( 5 per cent.), or boric acid (4 per cent.).

\section{CALCIUM AND ITS MEDICINAL SALTS}

Calcium belongs to the group of alkaline earths, which includes also barium and strontium. Its salts resemble 
chemically and physiologically those of magnesium. Carbonate of lime is the great source of the calcium salts. They are detectable in solution by their yielding no precipitate with hydrochloric acid, hydrogen sulphide, or ammonium hydro-sulphide; a white precipitate with an alkaline carbonate; an immediate and abundant white precipitate with oxalic acid, insoluble in acetic but soluble in hydrochloric and nitric acids ; but no precipitate with ammonia, which precipitates the compounds of aluminium and magnesium. Calcium salts give a reddish-yellow tinge to flame.

Calcium compounds have little affinity for animal textures, and are slowly absorbed and diffused. As with other metals, the oxide and carbonate nearly resemble each other, and are antacid and desiccant. The phosphate, or glycerophosphate is prescribed as a restorative and tonic in many forms of malnutrition. The hypophosphite is recommended in anæmia and debility. The chloride has been given, though seldom with success, to reduce enlarged lymphatic glands, and to promote the healing of ulcers. The presence of calcium is necessary to the coagulation of milk by rennet, and also to the clotting of blood. For this reason the chloride is often given for a few days to increase the plasticity of the blood and thus lessen risk from hæmorrhage in operations. Calx chlorinata is irritant, disinfectant, and deodorant, owing to its chlorine content. Calx sulphurata, consisting of calcium sulphide with calcium sulphate and carbon, has been credited with the power of checking formation of carbuncles, boils, and acne pustules. Calcium sulphate rendered nearly anhydrous by heat, is employed in making plaster bandages for fractures.

\section{Calcium Oxide. Lime. Quick-lime. Calx. $\mathrm{CaO}$.}

When limestone, chalk, marble, or other form of calcium carbonate is mixed with coal and thoroughly burned, its carbonic acid is driven off, and the metallic oxide or quicklime is left. It occurs in greyish-white irregular masses, has an alkaline, caustic taste, and great affinity for water. It combines with about 24 per cent. of water, giving off much heat, and forming calcium hydroxide, or slaked lime, 
$\mathrm{Ca}(\mathrm{OH})_{2}$. A pint of water at $32^{\circ} \mathrm{Fahr}$. dissolves 13.25 grains of lime; a pint at $69^{\circ} \mathrm{Fahr} ., 11.6$ grains ; a pint at $212^{\circ}$ Fahr., 6.7 grains. The presence of sugar increases fully twelve times the solubility of lime in water. Limewater (aqua Calcis) is prepared by adding two ounces of washed Calcium hydroxide to one gallon of distilled water, agitating briskly, allowing undissolved matters to subside, and, after a few hours, syphoning off the clear solution. As it readily absorbs carbonic acid, it should be kept in closely-stoppered bottles.

ACTIONS AND USES.-Lime is irritant, astringent by contact, antacid, and sedative. It is a natural constituent of the animal textures, in which it occurs mainly in combination with phosphoric and carbonic acids; but, being present in most articles of food, extra supplies are seldom required. When swallowed, there is probably deposited on the gastric mucous membrane a film of carbonate, which is dissolved by hydrochloric or lactic acid, slowly absorbed as chloride or lactate, reconverted in the blood into carbonate, held in solution by the free carbonic acid, and ultimately excreted by the kidneys, increasing the alkalinity of the urine, diminishing its irritant qualities, and perhaps exerting astringent effects on the urinary mucous membrane. Lime, especially when unslaked, and in contact with mucous and abraded skin surfaces, attracts water, forms a coating of carbonate, and possibly combines with albumin, and, in considerable amount, it irritates and superficially corrodes. Orfila mentions that $1 \frac{1}{2}$ drachms administered to a small dog caused vomiting and considerable irritation, which lasted for about a day; and that three drachms caused vomiting, pain, languor, and death in five days.

MEDicinal Uses.-Lime-water is used as an antacid and sedative in indigestion and diarrhœa in all classes of patients. Young animals, with which undiluted milk happens to disagree, are usually benefited by mixing the milk with onefourth to one-half of lime-water, which prevents acidity and coagulation of the casein in large tough masses. Limewater is appropriate for gastric derangement, while the less soluble chalk, retaining longer its antacid and desiccant properties, is better adapted for intestinal acidity. When 
acidity concurs with constipation, sodium bicarbonate is preferable in the proportion of a drachm to the pint of milk. Lime-water is occasionally given as an antidote in poisoning by the mineral acids and by carbolic and oxalic acids. In the form of enema, it brings away ascarides lodged in the lower bowel. Mixed with oil, glycerin, or vaseline, with a little boric or carbolic acid, it is. applied in aphtha and is occasionally substituted for zinc oxide in cases of eczema. Scalds and burns are often treated with Carron oil which consists of lime-water mixed with an equal quantity of linseed oil. The more recent treatment consists in the immediate application of layers of cotton wadding with gentle and equable pressure. Lime in solution (limewash) is used for cleansing and deodorising stables, cowhouses, and piggeries.

DOSES, etc.-Of quicklime, horses and cattle take 3i. to 3ij.; sheep, grs. xx. to grs. xxx. ; dogs, grs. v. to grs. xx. Of lime-water the larger patients take $f \mathrm{z}_{\mathrm{iv}}$. to $\mathrm{f} \zeta \mathrm{v}$.; and the smaller, f $3 \mathrm{ij}$. to $\mathrm{f} z \mathrm{i}$., given alone, or with glycerin, oil, or milk. Two ounces each of lime-water and gentian infusion, repeated twice or thrice daily, often checks diarrhœa in feeble calves; half the dose answers for sheep. For calves and dogs, saccharated lime-water is used as an antacid and stomachic. It is made by rubbing an ounce of slaked lime with two ounces of sugar, transferring the mixture to a bottle containing a pint of water, shaking, and separating the clear solution with a syphon. It conveniently renders the milk alkaline, without diluting it as does lime-water.

Calcium Carbonate. Calcii Carbonas. Carbonate of Lime. Chalk. $\mathrm{CaCO}_{3}$.

Calcium carbonate occurs in the several forms of limestone, marble, calcareous spar, and chalk. When chalk is triturated with a little water, agitated with a larger quantity of water, and the mixture allowed to stand, the coarser fragments and foreign matters subside. The clear liquid is poured or syphoned off, and slowly deposits an impalpable powder, which, when dried in blocks, constitutes whiting, and when in smaller conical rolls is creta præparata. Precipitated 
chalk is obtained by the interaction of calcium chloride and sodium carbonate. It is a dull-white, micro-crystalline, tasteless powder, adheres to the tongue, owing to its porosity and its affinity for water, and effervesces with acids. Calcium carbonate is a constituent of the bones of animals, of shells, and of corals. It is the common source of hardness in drinking water, which, when pure, holds about two grains dissolved in the gallon; but carbonic acid increases the solvent power of the water. Sixteen grains are sometimes taken up, and in chalk districts much more; but when such hard waters are boiled, the carbonic anhydride is driven off, and calcium carbonate is deposited in the kettles and boilers.

ACTions AND USES.-Chalk is a cheap and convenient antacid, much used for the domesticated animals in the treatment of indigestion and chronic diarrhœa. It resembles lime, but is less irritant, and its effects extend beyond the stomach, throughout the intestines, neutralising acidity, and leaving a protecting film of carbonate, thus acting much in the same way as the bismuth salts. It is an antidote for oxalic, carbolic, and the mineral acids. In a dry and finelydivided state it is used as a desiccant and astringent for abraded skin surfaces, burns and ulcers.

Doses, etc.-For horses, $\zeta^{i}$. to $\zeta_{\mathrm{ij}}$.; for cattle, $z_{\mathrm{ij}}$. to Ziv. ; for sheep, Zij. to $3 \mathrm{iv}$. ; for pigs, $3 \mathrm{i}$. to $3 \mathrm{ij}$. ; for dogs, grs. viij. to grs. $\mathrm{xxx}$. It is conveniently given in bolus, or suspended in milk, gruel, or mucilage. When administered in large or frequently-repeated doses the bowels should be kept open, in order to prevent its accumulation in the intestines. It is frequently conjoined with catechu, kino, and other vegetable astringents to arrest excessive discharges ; with ginger and other carminatives, to control indigestion and diarrhœa; with opium or belladonna, to diminish irritability or pain ; and with salicylic acid, or bismuth, in diarrhœa and hæmorrhagic gastritis in dogs. The following formulæ prove serviceable as antacids, carminatives, and mild astringents:-For horses, half an ounce each of chalk, gentian, and ginger is made up in the usual way with linseed meal and treacle, or with glycerin ; or chalk zi., opium $3 i$., and creosote $m_{x}$., are made into bolus ; or again, half an ounce each of chalk, catechu, and ginger are conjoined with 
one drachm of opium. For cattle, similar prescriptions are given, frequently in draught. For sheep, the like combinations are used, in about one-fourth the dose. For dogs, a convenient pill is made with chalk and ginger, of each grs. $x$. with opium, grs. ij.; and aromatic confection, q.s. A draught of similar action is made with chalk, grs. x., laudanum and ether, of each $m_{x v}$., given in a little milk or soup. Any of these prescriptions may be repeated several times daily. Where it is sought to act chiefly upon the stomach, such medicines are fittingly given dissolved in starch solution as prepared by the laundress, or in spirits and water, administered, according to circumstances, either hot or cold. Where the effects are to be extended throughout the intestines, the drugs are generally given in the solid form. For dyspeptic and diabetic horses suffering from acidity, a piece - of chalk should be placed in the rack or manger, or in the drinking water.

\section{Calcium Phosphate.}

Calcii Phosphas.

Phosphate of Lime. $\mathrm{Ca}_{3}\left(\mathrm{PO}_{4}\right)_{2}$.

Calcium phosphate is prepared by roasting bone ash until animal and carbonaceous matters are removed, dissolving the residue in diluted hydrochloric acid, precipitating the phosphates by ammonia solution, and washing. Thus purified, it is a light, tasteless, white, amorphous powder, insoluble in water, but soluble without effervescence in hydrochloric and nitric acids.

ACTIONS AND USES.-Calcium phosphate is present in bones, nerves, and other animal textures ; occurs abundantly in the intercellular fluid, and wherever cell-growth is most active ; and is hence an essential constituent of food. Its absence in the dietary is shown by Chossat to induce softening of the bones and general wasting; it is deficient in the bones of pregnant animals. Milne-Edwards found that, when supplied to dogs whose bones had been intentionally fractured, more rapid union occurred.

From its local salt-action, and the soothing effect of the deposition of an insoluble calcium salt, it is specially useful in chronic diarrhœa, anæmia, and other forms of mal- 
nutrition, especially in young animals. It is frequently given to rapidly-growing, rickety subjects, and conjoined with iron, for anæmic, badly-nourished, scouring animals, beneficially restoring the phosphates, which in such cases are sometimes too freely removed. For pregnant animals in a weakly state it is also prescribed. This treatment is suggested by the known effects of phosphorus on metabolism and nutrition, but for this purpose the hypophosphites, or glycerophosphates, which are easily absorbed are better than the phosphates, whilst preparations of free phosphorus are the most reliable. Bran and bruised oats owe in part their notable dietetic value for young stock to the large amount of calcium phosphate which they contain.

Doses, etc.-Horses take $3 \mathrm{ij}$. to 3 iv.; cattle, $3 \mathrm{iv}$. to 亏ij.; sheep and pigs, 3 j. to 3 ij. ; dogs, grs. v. to grs. $\mathbf{x}$. Small doses are preferable to large, which sometimes derange the bowels. They are conveniently given mixed with food, and in combination with an iron salt.

Calx Chlorinata. Chlorinated Lime. Calcium Hypochlorite. Bleaching Powder. $\mathrm{CaCl}_{2} \mathrm{O}_{2}, \mathrm{CaCl}_{2}$.

It is obtained by exposing slaked lime to the action of chlorine gas until absorption ceases.

Properties. - It is a dull-white powder, with a characteristic smell, and an acrid taste. When exposed to the air it becomes moist and gradually decomposes. When heated or mixed with an acid, chlorine is rapidly given off. It is partially soluble in water, a portion of the lime remaining undissolved. The solution evolves chlorine copiously upon the addition of an acid. The intensity of its odour and the degree of its solubility are simple approximative tests of its strength and purity, and a good specimen yields at least 33 per cent. of chlorine.

ACTIONS AND USES.-Bleaching powder is irritant, stimulant, astringent, antiseptic, and alterative; it is seldom given internally, but externally it is stimulant, antiseptic, deodorant, disinfectant, and parasiticide, and owes its several actions mainly to the hypochlorous acid and chlorine it so readily evolves. 
Toxic EFFECTS.- - Hertwig gave horses and cattle one ounce to two pounds ; sheep and goats one to eight drachms ; and dogs half a drachm to four drachms. The smaller quantities produced scarcely any effect; the larger, besides local irritation, caused acceleration of the pulse, difficult breathing, increased warmth in the mouth, weeping eyes, abundant secretion of urine, having a curious odour of chlorine or prussic acid, and a white sediment, frequent copious alvine discharges, and in dogs, vomiting. In horses the effects usually began in twenty or thirty minutes, and lasted two to five hours. Considerable doses, given repeatedly, did not impair appetite, but caused thirst and gradual emaciation.

Medicinal Uses. - Youatt recommended bleaching powder for hoven in cattle, and tympanites in horses, in doses varying from two to four drachms, and ascribed its supposed good effects to its decomposing the gases evolved in the alimentary canal. But repeated careful trials made at the Edinburgh Veterinary College indicate that it is of little, if any, service in the majority of cases of hoven or tympanites, whether in cattle or horses. It is an antidote in poisoning by hydrogen sulphide and ammonium hydrosulphide ; and, with a respirator filled with bleaching powder, Roberts explored in safety the sewers of the Bastille, which had not been opened for thirty-seven years, and were full of hydrogen sulphide and other noxious gases.

Externally, it is irritant and antiseptic, and is used as a stimulant and deodorant for unhealthy wounds, for removing the fœtor of foul discharges, and for destroying the fungus of ringworm, the parasites of mange, and for grease. Diluted solutions have been recommended for checking conjunctival ophthalmia, and other circumscribed and superficial inflammations ; but are not so effectual as lead or zinc lotion. Although it does not arrest the action of enzymes, or prevent the reproduction of developed bacteria or their spores as promptly and effectually as corrosive sublimate, it is superior as an antiseptic to sulphurous acid, bromine, iodine, salicylic acid, and the permanganates (Wernitz and Koch). Its active gaseous products, moreover, readily attack and break up the products of putrefaction, and it hence proves an effectual deodoriser. 
Chlorinated lime, in powder or in 10 per cent. solution, is spread about cow-houses and premises where contagious or epizootic diseases prevail. When free and rapid evolution of the hypochlorous and chlorine gases is desired, vessels containing the powder are set about, and sulphuric acid poured over them ; a more gradual evolution is effected when cloths saturated with a strong solution are suspended inside the infected dwellings. Solutions varying from one to ten per cent. are employed for disinfecting hides, flesh, or excreta of diseased animals. It has the disadvantage of a sickly smell, which is disliked both by horses and cattle; it is gradually converted into calcium chloride, which, having great affinity for water, leaves the floors, walls, and other surfaces to which the deodoriser has been applied in a very moist state; while its property of decomposing ammonia, urea, and such other unstable nitrogen compounds, greatly diminishes the agricultural value of any manure to which it is added. Scattered about stables or cow-houses, it drives away flies; while neither rats nor mice frequent places where it is sprinkled, especially when mixed with sulphur.

Doses, etc.-Horses take $3 \mathrm{iv}$. to $\zeta_{\mathrm{j}}$.; cattle, $3 \mathrm{vj}$. to 3ij. ; sheep, 3ss. to 3i. ; dogs, grs. ii. to grs. xv., given either in bolus, or with cold gruel, mucilage, or milk.

\section{Calcium Chloride. Calci Chloridum. $\mathrm{CaCl}_{2} 2 \mathrm{H}_{2} \mathrm{O}$.}

Chloride of calcium, the salt formed by neutralising hydrochloric acid with calcium carbonate, carefully desiccated at a temperature not exceeding $392^{\circ}$ Fahr. (B.P.). It occurs in white odourless scales or masses, with a saline taste ; deliquescent ; soluble in an equal weight of water, and in three parts alcohol. Calcium chloride is believed to increase the coagulability of the blood and is sometimes administered as a hæmostatic in hæmorrhage from the lungs, or stomach, and, with or without addition of sterilised solution of gelatin, a few days before operations in which bleeding may be profuse. It is also employed externally as a styptic.

Doses.-Horses and cattle, $3 \mathrm{jv}$. to $ろ \mathrm{j}$.; dogs, grs. v. to grs. $\mathrm{xxx}$. 


\section{MAGNESIUM AND ITS MEDICINAL SALTS}

Magnesium salts are obtained from dolomite or magnesian limestone ; magnesite, a native carbonate ; talc, meerschaum and other silicates, and from sea-water.

Magnesium salts when swallowed are not rapidly absorbed, and do not cause any toxic effects. The oxides and carbonates are antacid and laxative; the sulphate is purgative and febrifuge.

Magnesium Oxides. Magnesia: Calcined Magnesia. MgO. Light Calcined Magnesia. Magnesia levis. Heavy Calcined Magnesta. Magnesia ponderosa. (B.P.)

Light Magnesium Oxide, $\mathrm{MgO}$, is prepared by exposing light magnesium carbonate to a dull-red heat. Heavy magnesium oxide, $\mathrm{MgO}$, is prepared by exposing heavy magnesium carbonate to a dull-red heat. Magnesium oxide is a bulky white powder differing from heavy magnesia only in its greater lightness, the volumes corresponding to the same weight being to each other in the ratio of three and a half to one (B.P.). Magnesia is odourless, with a slightly earthy taste; insoluble in water, but readily dissolved by acids.

Magnestum Carbonates. $3\left(\mathrm{MgCO}_{3}\right), \mathrm{Mg}(\mathrm{HO})_{2}, 4 \mathrm{H}_{2} \mathrm{O}$.

Light Magnestum Carbonate. Magnesii Carbonas levis. Heavy Magnestum Carbonate. Magnesii Carbonas ponderosus.

When sodium carbonate is mixed with. a solution of magnesium sulphate, magnesium carbonate and magnesium hydrate are precipitated. The manner of preparation affects the weight, but not the other properties. Diluted solutions, boiled, yield the light carbonate. Tolerably concentrated solutions, mixed without heat, yield the heavy carbonate, which is three and a half times heavier than the other, more dense, loose, and granular; and, under the microscope, is found to be partly amorphous, with intermixture of numerous slender prisms. Both varieties are white, odourless, and tasteless, sparingly soluble in water, but readily dissolve in 
dilute mineral acids. Fluid magnesia usually contains in every ounce ten grains of carbonate, dissolved in water charged with carbonic acid gas.

Actions AND Uses.-Magnesia is antacid and laxative. The oxide and carbonate are sparingly absorbed, chiefly as chlorides. Thus they combine the action of the saline laxatives with that of the very mild alkalies. Their laxative effect and absence of causticity distinguish them from corresponding lime salts.

Magnesia does not purge either horses or cattle, but is a gentle laxative for dogs and eats, to which it is occasionally prescribed with calomel, jalap, or buckthorn. Its laxative effects are increased when the bowels contain acid secretions. For foals and calves suffering from acidity and flatulence, acids and bitters are usually the appropriate treatment; magnesia, however, is sometimes prescribed, but must not be too freely or continuously used, as it is apt to concrete and accumulate in the bowels. It is an antidote in poisoning by oxalic and the mineral acids. It removes arsenic from solution, and for this purpose is most effective in the form of the gelatinous hydrate made by adding caustic potash to a solution of the sulphate. It is occasionally applied as a desiccant.

Doses, etc.-Foals and calves three or four months old take, as an antacid, 3 ss. to $3 \mathrm{i}$. ; dogs and cats, grs. v. to $3 \mathrm{j}$. It is given suspended in milk or gruel, and conjoined with carminatives.

Magnesium peroxide, a white powder, insoluble in water, alcohol and ether, and containing about thirty per cent. of magnesium peroxide $\left(\mathrm{Mg} \mathrm{O}_{2}\right)$, or about ten per cent. of active oxygen, has been found serviceable in affections of the stomach and intestine associated with much fermentation.

Doses.-Small dogs, grs. jss. to grs. v. ; large dogs, grs. vj. to grs. xv., in milk.

Magnesium Sulphate. Magnesii Sulphas. Epsom Salt. $\mathrm{MgSO}_{4} \cdot 7 \mathrm{H}_{2} \mathrm{O}$.

Magnesium sulphate is present in various rocks and soils, in the proportion of fifteen to twenty grains in the pint of 
sea-water, and in some mineral springs. It derives its vernacular name from the mineral springs of Epsom. It may be prepared by the interaction of the native magnesium carbonate and diluted sulphuric acid; or by purifying the native sulphate (B.P.).

Properties.-Epsom salt occurs in transparent, colourless, minute, rhombic prisms. It has a cooling, saline, nauseous, bitter taste ; is insoluble in alcohol, but soluble in its own weight of water. It resembles zinc sulphate, from which, however, it is distinguished by its saline, bitter taste, by absence of metallic astringency, and by its neutral solution giving no precipitate with hydrogen sulphide. Epsom salt is distinguished from Glauber salt by its neither efflorescing when exposed to air, nor communicating any yellow colour to the flame of alcohol. From oxalic acid (for which it has been sometimes mistaken) it is distinguished by its finer and more needle-like crystals, its bitter taste, devoid of acidity, and its precipitating alkaline carbonates without effervescence.

ACTIONS AND USES.-Epsom salt is purgative, alterative, and febrifuge, and is also feebly diuretic and diaphoretic. As a purgative it resembles common and Glauber salts, and is more active than potassium bitartrate or sodium phosphate.

General Actions.-When swallowed, it causes outpouring of fluid from the walls of the small intestines, most quickly produced, and abundant when the bowels have been partially emptied by several hours' fasting. Neither pancreatic fluid nor bile is materially increased. But Epsom salt, like other salts in solution, becomes dissociated into its basic and acid ions, namely $\mathrm{Mg}$ and $\mathrm{SO}_{4}$, and experiment shows that both these penetrate the intestinal mucous membrane very slowly and with difficulty. As a consequence, when a strong solution (hypertonic) of magnesium sulphate is introduced into the intestine the salt remains there, both ions exerting their osmotic pressure and drawing fluid from the blood to dilute the solution in the bowel. Thus by the osmotic action of magnesium sulphate the fluid contents of the bowels are increased, producing more or less mechanical distension, and provoking, like other salines, 
slight peristalsis. There is a tendency to cause griping, which is relieved by conjoining carminatives ; while effectual removal of the intestinal fluids is attained by using with the saline some aloes, oil, or calomel. Meanwhile, a certain amount of the salt undergoes slow and gradual absorption; but this is checked when purgation occurs. - After several hours the absorbed salt is excreted by the kidneys, but not before it has produced some amount of hydræmia by draining water from the tissues ; thus more or less diuresis ensues.

Free outpouring of fluid from the intestinal walls, and the subsequent more limited excretion from the kidneys, necessarily remove both fluid and saline matters from the blood, and within a few hours this loss is in great part made good by absorption of fluid from the tissues. Mainly in this manner result the febrifuge and alterative effects of salines, in reducing abnormal temperature, and removing dropsical effusions.

Sir Lauder Brunton demonstrated, experimentally, the effect of Epsom salt in causing outpouring of mucous fluid from the intestinal walls. He placed four ligatures round the intestines of a cat, so as to make three separate closed sacs from five to seven inches long. Into the two outside sacs water alone was introduced; into the central one were injected seven grains Epsom salt, dissolved in 105 minims of water. The cat was killed four hours later, and although the two outside sacs were quite empty, the middle one, into which the purgative had been injected, contained 320 minims of pale amber fluid, of the nature of a secretion rather than an albuminous exudation. In two similar experiments, 425 and 250 minims of fluid were found, four and five hours after injection of 85 and 90 minims of saturated solution of Epsom salt. No congestion or inflammation was noticed. The loops on either side, which had been filled with the same quantity of water, were empty. Croton oil, elaterium, and gamboge, tested by like experiment, all caused an effusion of liquid, but none so abundant as Epsom salt, which yielded 42 to 56 minims persquare inch of intestine acted on by the purgative. Again, in the case of the vegetable purgatives the fluid was more of an inflammatory nature, and the mucous membrane was congested and inflamed.

W. Rutherford's experiments on dogs demonstrate that 
magnesium sulphate has no stimulant action on the liver. It nevertheless notably counteracts ' biliousness,' alike in men and animals, by sweeping away bile, which is generally present in the duodenum, and which, unless removed, becomes re-absorbed.

Epsom salt injected into the circulation does not produce intestinal secretion, rather it drains fluid from the intestine into the blood, to be excreted later by the kidneys. Five grains to the pound of body-weight were proved by Matthew Hay to produce powerful toxic effects in cats and other animals, paralysing first the respiration and afterwards the heart, abolishing sensation, or paralysing the sensori-motor reflex centres. This effect is a specific action of magnesium and is not produced by other salines.

The several domesticated animals are differently affected by Epsom salt. On horses, unless given in combination, it acts uncertainly. Full doses sometimes cause violent catharsis, and occasionally produce considerable diuresis ; but two to three ounces, repeated daily, are laxative and febrifuge. On dogs the purgative effect is irregular, and often accompanied by vomiting. For cattle and sheep it is a convenient and effectual cathartic, inducing copious fluid evacuations, usually in twelve or fifteen hours.

Medicinal Uses.-For ruminants Epsom salt is the purgative in general use. In indigestion, constipation, and in the earlier stages of many cases of diarrhœe, it clears the bowels of undigested, fermenting food, of irritant matters, and occasionally of worms. Horses liberally fed on cut, dry food, or tough, over-ripened green fodder, are subject to overloaded bowels, and in such cases, with restriction to fluid food, and in conjunction with one or two doses of linseed oil and copious clysters, two ounces Epsom salt, repeated twice daily, help to moisten, soften, and expel the dry, impacted ingesta. Emptying the bowels, and removing waste products from the blood, it lowers abnormal temperature and blood-pressure, and relieves febrile and inflammatory conditions.

Although not a desirable purgative for horses, it is a useful febrifuge. One to three ounces given in influenza, pneumonia, and indeed in most febrile and inflammatory dis- 
orders, improve the appetite, clean the buccal membrane, lessen fever, and help to establish and maintain a healthy and regular action of the bowels. For such febrifuge purposes, whether in horses or cattle, it is given once or twice daily, but should be withheld or diminished in amount, whenever the bowels become unduly relaxed, or where flatulence or spasm follows its use. It acts more certainly and regularly when given in solution than in bolus. It is often conjoined with nitre and other salines, and during convalescence from acute disorders with powdered gentian and other carminatives. Epsom salt is one of the best antidotes in poisoning by barium chloride, and salts of lead ; it converts them into insoluble sulphates, and further evokes action of the bowels, which in lead-poisoning is apt to be impaired and tardy. In smaller and repeated doses it acts as a diuretic, but is seldom specially used for that purpose. It is frequently added to laxative clysters. A saturated solution is a valuable application for recent strains, wrenched joints, articular rheumatism, and orchitis.

DOSES.-As a cathartic, cattle take lb. j. to lb. jss.; calves

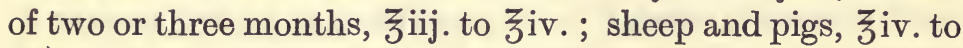
亏vi.; dogs, 3ii. to 3iv. One-fifth to one-eighth of these doses is often effectual in removing indigestion, keeping up the action of other cathartics, and as a febrifuge or alterative. Epsom salt is given dissolved in ten or fifteen parts of water. To conceal its nauseous, bitter taste it is administered with treacle, or with sulphuric acid, in the proportion of about five drops to every ounce of salt. To expedite its purgative action and prevent nausea and griping, there is usually added some carminative, such as a drachm of ginger to the ounce of salt. To ensure prompt and full purgation in cattle or sheep, equal weights of Epsom and common salt are preferable to either given alone ; treacle and a full dose of ginger are added, and solution is effected in a liberal amount of tepid water. In impaction of the third stomach, and obstinate constipation of cattle, it is sometimes advisable to add to the saline mixture twelve or fifteen croton beans, a drachm of calomel, or half an ounce of gamboge, and to follow this with repeated doses of treacle and ginger. In torpidity of the bowels it is conjoined with half a dose of aloes and 
thirty grains of nux vomica. For febrifuge and alterative purposes, Epsom salt is conjoined with nitre, mineral acids, gentian and other bitters.

\section{BARIUM CHLORIDE-BARII CHLORIDUM}

Barium occurs in nature in combinations analagous to those of strontium; as carbonate in the mineral witherite and as sulphate in barite or heavy spar.

Barium Chloride. $-\mathrm{BaCl}_{2} 2 \mathrm{H}_{2} \mathrm{O}$, Terra ponderosa salita, discovered by Scheele, may be prepared by dissolving the native carbonate in hydrochloric acid. It occurs in flat or tabular crystals, colourless and transparent, with bevelled edges. Taste, acrid and bitter ; reaction, neutral. Soluble in two and a half parts of cold water, in 400 parts absolute alcohol, and slightly soluble in rectified spirit. The salt keeps well either solid or in aqueous solution.

ACTIONS AND USES.-Barium chloride has a specific action on muscle, whether striped or unstriped, causing strong contraction. Given by the mouth it causes vomiting in small animals, colicky pains, contraction of the muscle of the stomach and intestines, and so purgation. Small doses, whether given intravenously or by the mouth, stimulate the muscles of the intestine, uterus, and bladder, causing tonic contraction. Excessive doses induce general muscular spasms, dyspnœa, sweating, incoördination, and paralysis. Death may occur suddenly from spasm of the heart. Barium quickens the heart, steadies its rhythm, and increases the volume of blood forced from the ventricle. Blood pressure is enormously raised, an effect which is due to the action of the drug on the muscular coat of the vessels. In animals, large doses over-stimulate and arrest the heart in systole. It thus resembles digitalis in its local action, but having no effect on the vagus, does not slow the heart. Barium chloride has little therapeutic effect on sheep, but on horses it increases the power and causes strong contraction of the muscular coat of the bowels. It causes no irritation of the gastric mucous membrane, exerts its action on the whole length of the gut, and acts very quickly, especially when injected intravenously. As soon as the bowels have been 
evacuated the effects of the drug cease, and even during its operation there is little or no constitutional disturbance. Large doses may cause pain by exciting intestinal spasm. An underdose has no apparent effect; but an overdose may be followed by fatal spinal paralysis. The activity of the chloride depends on its mode of administration. Given intravenously in suitable dose, the bowels respond in a minute or two, and fæces may continue to be passed at intervals of fifteen minutes for five or six hours. Administered in drench or in bolus it is less active. In some horses it has a powerful action on the heart. It paralyses the central nervous system, and a second dose administered within twelve hours of the first is not without danger (Veterinarian, 1895).

Zschokke experimented with the drug on apparently healthy horses. He gave three horses, $\mathrm{m}_{150}$; two, $\mathrm{m}_{105}$; one horse, $M 75$; and another, $m 225$, of a ten per cent. aqueous solution injected into the jugular vein. Within thirty seconds all the subjects exhibited masticatory movements of the jaws, and in less than three minutes and a half there was expulsion of gas followed by fæces. The horse that received the largest dose groaned and strained.

Röder injected into the jugular of a horse three grammes of the chloride dissolved in thirty grammes of water. In a minute loud borborygmus was heard, and in two minutes profuse sweating occurred, followed by defæcation, with violent straining. In half an hour the horse was all right.

Barium chloride, as a remedy for colic in horses, was introduced in 1895 by Dieckerhoff. In seven months he treated 400 cases, with only one death. Cadiot recommends the drug after an experience of its effects in 32 cases, with three deaths, two from gastric rupture, and one from volvulus. Mollereau used it successfully in 38 consecutive cases, and then lost three suddenly.

In cattle practice, and particularly in the treatment of tympanites, Kreutzer obtained excellent results from the administration of barium chloride in doses of grains lxxv. for calves ; $3 \mathrm{jv}$. for cows ; and $3 \mathrm{v}$. for bulls, in drench.

The toxic effects are salivation, champing of the jaws, dyspnœa, loss of pulse, distension of the superficial veins, sweating, tremors, muscular cramps, incoördination, con- 
vulsions, and paralysis of the central nervous system. In poisoning, the best antidote is magnesium sulphate, or sodium sulphate, which is given with the object of converting the poison into the insoluble and harmless barium sulphate.

Although not often followed by a fatal result, it should be noted that intravenous injection of barium chloride sometimes produces symptoms of poisoning, and occasionally sudden death. Dieckerhoff attributed the single mishap in his 400 cases to barium poisoning. Röder mentions the death of a horse in two minutes after an intravenous injection of twelve grains; and Mollereau has no doubt that the drug caused the immediate death of three of his patients. It gives unquestionably good results in many cases, but in others its effects are uncertain and unsafe. In most cases of abdominal pain the drug should be used cautiously, or as a last resort, after having exhausted other treatment, perhaps less active, but infinitely less dangerous.

Doses, Intravenous.-Horses, according to size, grs. v. to grs. xx., in two and a half to six drachms of sterilised water. When the patient is weak, or his pulse is over 70 , only half a dose should be given; or half a dose at the first injection, followed by two quarter doses at intervals of from ten to twenty minutes. Administered in this way, the drug is quite as effective as when given in one dose. Mollereau prescribes $3 \mathrm{j}$. of a one in fifteen aqueous solution; and Zschokke gives $m 75$ of a ten per cent. solution.

Drench.-Horses, 3iss. to 3iij. ; ponies, grs. xl. to 3j., in a quart of water.

\section{ALUM. ALUMEN}

Potassium Alum (Aluminium and Potassium Sulphate). $\mathrm{Al}_{2}\left(\mathrm{SO}_{4}\right)_{3}, \mathrm{~K}_{2} \mathrm{SO}_{4}, 24 \mathrm{H}_{2} \mathrm{O}$. Or-Ammonium Alum. $\mathrm{Al}_{2}\left(\mathrm{SO}_{4}\right)_{3},\left(\mathrm{NH}_{4}\right)_{2} \mathrm{SO}_{4}, 24 \mathrm{H}_{2} \mathrm{O}$.

Produced by the combination of aluminium sulphate with potassium sulphate, or with ammonium sulphate (B.P.).

The alums are a series of double salts in which aluminium sulphate is conjoined with potassium, sodium or ammonium 
sulphate. They are found in limited quantity on the surface of soils and rocks, especially in volcanic districts, and are largely prepared from aluminium clay, shale, or schist, which mainly consists of aluminous silicate and iron sulphide. Near Paisley, where alum is extensively manufactured, the schist lies between the coal and limestone strata. When slowly roasted it absorbs oxygen, and the sulphur is converted into sulphuric acid, which unites with the iron and aluminium. Water is added, and a large portion of the less soluble iron sulphate crystallises out.

To make potash alum this residual solution is treated with potassium chloride, usually obtained as a by-product from the soap-boilers, saltpetre refineries, and glasshouses ; double decomposition results. The remaining iron sulphate is converted into iron chloride, which continues in solution; while potassium sulphate unites with aluminium sulphate to form potash alum, which crystallises, and is further purified by repeated solution and crystallisation.

PROPERTIES. - The alums occur in transparent, colourless cubes, or octahedral crystals, have a sweet, acidulous, astringent taste, act like acids on colouring matter, and, when heated, fuse and part with their water of crystallisation. They are soluble in one-third of their own weight of water at $212^{\circ}$ Fahr., and in seven parts of cold water. Freely soluble in glycerin : insoluble in alcohol.

ACTIONS AND USES.-Alum is slightly irritant, astringent, and antiseptic, and is chiefly used externally as an astringent styptic, desiccant, and caustic.

Toxic EFFECTs.-One or two ounces given to dogs cause vomiting. But when the œsophagus was tied, and vomiting prevented, Orfila found that two ounces occasioned death in five hours, with great exhaustion; the intestines were found extensively inflamed. Devergie found that four drachms of dried alum killed a dog when the gullet was tied. An ounce introduced into the areolar tissue of a dog's thigh caused excessive suppuration, and death in fifteen hours. Moiroud states that large doses given for some time exhaust the digestive organs, diminish cutaneous transpiration, and produce grave disorders. Several ounces given daily to cows, and continued for a week or two, do not produce any 
obvious bad effects. Thus the action of alum in excessive doses is simply to produce gastro-enteritis, and this because very little of the drug is absorbed. Alum is decomposed in its passage through the intestines, rendering the fæces odourless and somewhat firmer. Over-doses are decomposed and neutralised by small and repeated doses of sodium carbonate, followed by demulcents.

Medicinal Uses.-Alum closely resembles copper and zinc sulphates, but is less active. It is occasionally given to the dog as an emetic. Applied in a dry state, it absorbs water from the tissues, and hence acts as a mild caustic. It has little or no effect upon the unbroken skin. It coagulates albumin and gelatin, and hence invests the abraded skin and mucous membranes with a protective film, diminishes their blood supply, and thus relieves congestion, and lessens secretion. In virtue of its astringent properties, it is used as a lotion for aphthous or ulcerated conditions of the mouth; as a gargle and spray for sore-throat; as a dressing for blistered surfaces, as a stimulant injection for leúcorrhœa ; and in many of these cases it may be conjoined with glycerin and borax. Alum is occasionally prescribed with opium in diarrhœa and dysentery, but vegetable astringents are preferable. In America, large doses have been given to horses infested with bots. With the object of precipitating lead salts in the intestine, it has been prescribed as an early antidote in lead-poisoning. Alum was formerly given for polyuria in horses, but iodine and iron sulphate are more effectual remedies. To arrest the flow of synovia from open bursæ, or joints, finely powdered alum, mixed with two or three parts of wheat flour, or a powder composed of equal parts of dried alum, iron sulphate, and myrrh, is a valuable application. A saturated, aqueous solution is a useful styptic for hæmorrhage. For simple conjunctivitis and keratitis, alum ( 5 grains) and morphine ( 2 grains), in an ounce of water, form a useful collyrium, but it is said to dissolve the intercellular cement substance of the cormea, and so increase the size of ulcers and lead to perforation. An ointment, made with one part of alum to three or four of lard, or vaseline, will be found useful as a dressing for ulcerated heels and slow-healing wounds. Glycerin of alum, 
composed of alum, $\zeta_{j}$., water 3 iij., and glycerin, $\zeta v j .$, is used for chapped teats and eczema. Alumen exsiccatum, the dried alum of the B.P. is potash alum deprived of its water of crystallisation by heat. It is a white powder, forming spongy opaque masses on exposure to moisture. Slowly soluble in water, it should contain 45 to 46 per cent. less water than alum. It is a useful caustic and astringent for exuberant granulations, warts, and superficial sores. An albuminate, made by thoroughly mixing an ounce of dried alum with the whites of six eggs, is employed as an adhesive in setting fractures, and as a styptic for open joints.

DOSES, etc.-Astringent for horses and cattle, $3 \mathrm{ij}$. to $3 \mathrm{jv}$. ; sheep and pigs, grs. xx. to $3 \mathrm{ij}$. ; dogs, grs. x. to xxx., given in bolus or in solution. Alum is incompatible with alkalies, and their carbonates, lime salts, phosphates, salts of lead and mercury, and tannin-containing bodies.

Kaolin, a native aluminium silicate, is an excellent desiccant, mild astringent and protective, much used for chafed shoulders, harness-galls, and simple abrasions. Aluminium sulphate is occasionally used as a caustic, antiseptic, and astringent.

\section{Chloralum. Solution of Aluminidm Chloride.}

When aluminium sulphate and calcium chloride are dissolved together, double decomposition ensues, and there remains in solution an impure hydrated aluminium chloride -a colourless, oily fluid, with a sweet astringent taste, sold as chloralum.

ACTIONS AND UsEs.-Like many metallic chlorides, chloralum is corrosive, astringent, and antiseptic. It has been recommended in influenza in horses, in dysentery in cattle, and in distemper in dogs. Drachm doses suffice for horses or cattle, $m_{\mathrm{v}}$. to $m_{\mathrm{x}}$. for dogs. It may be administered either in bolus made with meal, or dissolved in water or gruel. Externally it may be applied to the purposes for which alum or zinc sulphate is serviceable. As a disinfectant it has been more used on the Continent than in this country. As a deodoriser it is more effectual than alum, but less effectual than tar oils, sodium sulphite, or bleaching powder. 
Diluted solutions destroy the parasites of mange and scab, and kill fleas and ticks.

\section{BISMUTH AND ITS SALTS}

Bismuth and its salts belong to the group comprising phosphorus, and the salts of antimony, and arsenic. Formerly, owing probably to defective preparation, and the presence of arsenic or antimony, their administration in full and continued doses produced gastro-intestinal irritation, and fatty degeneration of the liver and other organs. But pure salts of bismuth, being sparingly soluble, and absorbed to a very slight extent if at all, have little, if any, toxic effect.

Bismuth oxycarbonate, prepared by the interaction of bismuth nitrate and ammonium carbonate; bismuth oxide, prepared by boiling the oxynitrate with solution of sodium hydroxide ; and bismuth subnitrate or oxynitrate, obtained by the interaction of bismuth nitrate and water, have similar characters and reactions. The carbonate is whitish, the oxide is brownish-yellow, and the subnitrate is white. They form heavy powders, are insoluble in water, but soluble in diluted nitric acid.

Bismuth salicylate, prepared by the interaction of bismuth nitrate and sodium salicylate, occurs as a white amorphous powder, odourless and tasteless, insoluble in water, alcohol and glycerin, and containing about 65 per cent. of bismuth oxide $\left(\mathrm{Bi}_{2} \mathrm{O}_{3}\right)$.

Bismuth benzoate, is obtained by heating the nitrate in glycerin and water, and adding a solution of sodium benzoate, and washing and drying the precipitate. It is a white, insoluble powder, employed externally as a desiccant, and internally as an intestinal disinfectant.

Actions AND UsEs.-Externally, applied to wounds, bismuth salts are desiccant, protective, slightly astringent, and antiseptic. The salicylate has been used as a substitute for iodoform. With starch, boric acid, or iodoform, the oxynitrate is employed to relieve irritation and itching of the skin. The oxide, in ointment made with eight parts of vaseline, is sometimes applied after firing or blistering, but ointment of zinc oxide is quite as useful. In human surgery 
bismuth salts applied to wounds have sometimes given rise to toxic symptoms. These are salivation and swelling of the gums and tongue, then vomiting and diarrhœa, and albumin has generally been found in the urine (Cushney).

Internally, the oxynitrate and carbonate are astringent, antacid, protective, and absorbent. The subnitrate mixed with equal parts of charcoal and salol forms an excellent dressing for otorrhœa of the dog, and cracked heels in horses. It allays irritation in dyspepsia, vomiting, and in gastrointestinal catarrh. It acts by its oxide and by its acid, which is antiseptic. The salicylate is a more powerful antiseptic than the subnitrate. In the bowel it is rapidly split up into salicylic acid and bismuth oxide. But as free salicylic acid irritates the mucous membrane, bismuth benzoate, which is not irritant, is usually preferred as an intestinal disinfectant.

Doses, etc.-Subnitrate, Horses, 3ij. to 3jv. or more ; dogs, grs. v. to grs. xxx., in mucilage, milk, or bolus.

Salicylate or benzoate, foals and calves, grs. xv. to grs. $x l$ : dogs, grs. v. to grs. xv. ; The B.P. liquor bismuthi et ammonii citratis, containing three grains of bismuth oxide in each drachm, is a convenient preparation for special cases.

Dermatol, or bismuth subgallate, prepared from bismuth trinitrate, glacial acetic acid, and gallic acid, is a bright yellow powder, odourless, tasteless, and insoluble. Dermatol is absorbent, astringent, and feebly antiseptic. It is employed externally as a dry dressing for wounds. Two parts each of dermatol and zinc oxide, and twenty parts of vaseline form an ointment, which promotes cicatrisation of cracked heels. Internally, dermatol has been prescribed for diarrhœa and gastro-intestinal catarrh. Doses, dogs, grs. v. to grs. x. ; calves and foals, grs. x. to grs. xx. Xeroform (bismuth tribromophenol) is a neutral, odourless, tasteless, and insoluble yellow powder, containing about fifty per cent. of bismuth oxide. It is employed as a substitute for iodoform, and occasionally as an intestinal antiseptic. Airol (bismuth oxy-iodo-gallate), a light-greyish, or yellow powder, tasteless, odourless, and insoluble in water, alcohol, or ether; has been used as a substitute for iodoform, and by Fröhner as a pigment, made with airol, glycerin, gum and talc, for dressing small surgical wounds. Orphol (bismuth beta-naphthol), a 
light-brown powder of agreeable taste, and containing about three per cent. of beta-naphthol. It is a useful astringent and antiseptic in intestinal catarrh of foals, calves, and dogs. Doses.-Grs. x. to grs. xxx., in boiled milk, or in electuary. Thioform (bismuth dithio-salicylate) occurs as a bulky, yellow powder, odourless, tasteless, and insoluble. It is antiseptic and desiccant, and in the form of paste made with glycerin or vaseline is used in the treatment of wounds, eczema, and conjunctivitis. Pugh (Sevenoaks) prescribes thioform in gastro-intestinal inflammation and ulcerative diarrhœa. Combined with opium it alleviates the tenesmus of white scour in calves, and also that which follows the enteric form of distemper in the dog. It is a valuable intestinal antiseptic, sedative, and astringent.

\section{LEAD AND ITS MEDICINAL SALTS}

Lead. Plumbum. Pb.

Lead is chiefly obtained by roasting galena, the sulphide $(\mathrm{PbS})$. It has a blue-grey colour, and a peculiar odour when rubbed ; is soft and fusible, melting at $617^{\circ} \mathrm{Fahr}$. ; is readily cut and rolled into sheets or pipes. It has the specific gravity of 11·4. Exposed to air it oxidises, loses its metallic lustre, and becomes dull and opaque. In contact with air and soft water a soluble basic carbonate is gradually formed, and water thus contaminated is dangerous.

ACTions AND Uses.-The salts of lead resemble those of tin. Metallic lead is devoid of medicinal or poisonous action. Shot-an alloy of lead, with two per cent. of arsenic-is occasionally used by the lower order of dealers, temporarily and mechanically to relieve the distressed breathing of broken-winded horses, by weighting the stomach and so pulling the abdominal viscera away from the diaphragm. Four ounces of metallic lead were given to a dog at the Veterinary School of Lyons without effect. Introduced into the animal body, in frequently repeated although small quantity, the metal is apt, however, to become oxidised, and cause poisoning. Painters, plumbers, and other persons working with lead frequently suffer from lead-poisoning, and 
such poisoning also occasionally occurs in the domestic animals.

The soluble salts-such as the nitrate and acetate, as well as the peptonates formed in the stomach-unite with albumin to form a precipitate which is very slightly soluble in excess of the salt. Thus lead salts do not penetrate deeply but are among the most astringent of salts. Small to moderate diluted doses do not irritate either the skin or mucous membranes, but are astringent, desiccant, and analgesic. They have a sweet rather than a corrosive taste. When swallowed they act as gastro-intestinal astringents, are absorbed very slowly, chiefly as albuminates, permeate the tissues, contract arterioles to some extent, raise bloodpressure, and slow the heart movements. Larger or longcontinued small doses irritate and then paralyse voluntary and involuntary muscles through the motor-nerves, and also affect the central nervous system. This action is illustrated by the vomiting induced in dogs and cats, the cramp of the intestinal and other muscles in man and animals, and the paresis developed subsequently by toxic doses. These effects appear to result from the local action of the lead in the several textures affected. Hence arise, in chronic cases, degeneration of the implicated muscles, chronic interstitial nephritis, and cirrhosis of other internal organs. Certain animals exhibit more prominently some of the actions of lead. Muscular weakness and paralysis are well marked in frogs and rabbits, are less pronounced in cats, and are absent in dogs. Elimination is slowly effected in the urine, bile, and intestinal mucus (Brunton).

Medicinal Uses.-Lead is used medicinally only for its astringent effects, and that when locally applied. It is specially valuable as an intestinal astringent and styptic with opium. It is of no use in cases of bleeding from the lungs or kidneys, for it does not come in contact with the part in sufficient concentration.

Lead-poisoning, or plumbism, in the lower animals exhibits symptoms analogous to those in man, and frequently continues, in more or less aggravated form, for several weeks, or even months. Digestion is impaired, appetite becomes capricious and irregular; there are spasms and 
subsequently torpidity of the bowels. These symptoms simulate those of gastric distension in horses and impaction of the third stomach of cattle-disorders for which leadpoisoning has been mistaken. Distinctive features shortly, however, present themselves. Anæmia is a marked feature, probably because of the destruction of red cells, but partly from malnutrition. Along the margin of the gums appears a grey or blue line of lead deposited in the connective tissue, blackened by hydrogen sulphide present in the mouth, or by sulphur in the food. Colic and constipation are not so invariably present in the lower animals as in human patients. The extensor muscles of the limbs are cramped and paralysed earlier and more seriously than the flexors. The affected muscles gradually waste. The cause of the paralysis of muscles is generally thought to be peripheral neuritis, and experimentally lead has often been found to cause nerve degeneration in animals. In some cases, however, degeneration of motor areas in the spinal cord has been found. In cattle lead-poisoning occasionally proves a cause of abortion. Amaurosis and amblyopia are not infrequently caused by lead, probably inducing optic neuritis. In animals, too, chorea, convulsions, and delirium are easily set up by lead-poisoning, and these symptoms often arise with great suddenness. They appear to be due to a direct action on the cells of the cerebrum.

Shenton, a veterinary surgeon practising in Derbyshire, in 1861, had eleven horses poisoned, and several cattle, and thus described the conditions which came under his observation :- 'There was a rough, staring coat, a tucked-up appearance of the abdomen, and a slightly accelerated pulse ; in fact, symptoms of febrile excitement, which usually, however, passed away in about a week. About this time large quantities of grey-coloured mucus were discharged from the nostrils, and saliva from the mouth; but at no time was there any enlargement of the submaxillary, lymphatic, or salivary glands. Neither was there constipation of the bowels, which appears to be nearly always present in lead-poisoning in man. (Fits and partial paralysis came on at intervals, and when the animals got down they often struggled, for a long time ineffectually, to get up again. 
The breathing up to this period was tranquil, but now became so difficult and laboured that the patients appeared in danger of suffocation. The pulse was in no case above sixty or seventy, and I ascribed the difficulty of respiration to a paralysed state of the respiratory apparatus.) The animals did not live more than two or three days after these symptoms appeared. The post-mortem appearances varied little. The lungs and trachea were inflamed, the lungs engorged with large quantities of black blood, the trachea and bronchi filled with frothy spume. In all but two cases the villous portion of the stomach presented isolated patches of increased vascularity, and in all cases the intestines, and especially the large ones, were inflamed. The blind pouch of the cæcum was nearly gangrenous. There was nothing remarkable about the liver, spleen, or kidneys, except that they were of a singularly blue appearance. The brain and spinal cord were not examined.' (According to Mosselman and Hebrant, the effects of the poison in horses are especially manifested upon the vagus nerve, inducing roaring and dyspnœa.)

In 1863 , three milch cows were poisoned by eating sheet lead, which had been used for lining tea-chests, and which was afterwards carelessly thrown on the manure heap, and thence spread on the pastures. Besides failure of milk and appetite, grinding of the teeth, and dulness, several curious symptoms are mentioned. The head was rested against any convenient object as if the animal were asleep, while the eyes were nearly closed, and were little sensitive to light or to movements of the finger. The gait was weak and tottering, while for an hour or two at a time the cows, although persistently standing on their hind limbs, went down on their knees, propping themselves against the wall. They survived four or five days. From the fourth stomach of one cow a pound of sheet lead was removed; the lining membrane was thickened, and of a brown colour. The mucous membrane of the stomachs and bowels was abnormally vascular, and exhibited in places patches of ecchymosis. The liver was pale, clay-coloured, compact, and contained little blood.

Watson records the poisoning of three cows, which languished for several months, and died from eating grass on 
which bullet spray from the Rugby rifle butts had fallen. Fragments of the lead were found adhering to the coats of the stomach, and the poison was also detected in the intestines, liver, and kidneys (Veterinarian, 1864). Broad also records cases of cattle poisoned by picking up bullet spray. The animals were described as dull and tucked-up, the eyes staring, the gait unsteady, the appetite good, but the bowels constipated; emaciation, with œedema under the jaw, made rapid progress. Portions of bullet spray were found in the second and third stomachs; both large and small intestines were pale-blue and bloodless (Veterinarian, 1865). Tuson reported similar symptoms from licking red paint, which he found retained for twenty-eight weeks in a cow's stomach. Cox, of Hendon, had several sheep which became emaciated and paralysed from eating ${ }^{\circ}$ the splashes of lead bullets, which were found in the stomachs in thin flakes, readily soluble in the gastric fluids (Taylor). Birds are occasionally poisoned by eating berries contaminated by the smoke of lead smelters.

Herapath reported interesting cases of lead-poisoning which followed the erection of smelting furnaces on the Mendip hills in 1853. Lead oxides, carbonate, and sulphate were found on the herbage, hedges, and hay. On the live stock 'the effects of the metal were, stunted growth, leanness, shortness of breathing, paralysis of the extremities, particularly the hinder ones ; the flexor muscles of the forelegs affected, so that they stood upon their toes; swelling of the knees ; but no constipation or colic, as in the human species; in a few months death followed. In the young the symptoms were more conspicuous and the mortality greater. Lambs were yeaned paralytic; when three weeks old they could not stand, although they made great efforts to do so ; in attempting to feed them from a bottle they were nearly suffocated from paralysis of the glottis ; twenty-one died early out of twenty-three. (Colts also died, and those that lived could not be trotted 150 yards without distressed breathing.) Pigs confined to the stye were not injured, but if allowed to roam were soon affected. The milk of cows and sheep was reduced in quality and quantity, and cheese made from the former had less fat in it. In the milk of both 
minute traces of lead were found. The dead subjects showed the mucous surfaces to be paler than natural; a blue line appeared in the gum of the lower jaw, which Dr. Taylor said in court was not caused by lead poison, as it did not occur as in the human subject, on the upper edge of the gum, but where the gums first come into contact with the teeth, about three-sixteenths of an inch below the top edge. Herapath dissected out this line, which was about three-quarters of an inch in length, and the thickness of sewing cotton, and, by aid of carbonate of soda and the blow-pipe, reduced a spangle of lead from it, quite visible to the jury without the aid of a microscope.'

Lead is readily found in the bodies of animals thus poisoned. It has been detected in the blood, the contents of the stomach and intestines, the brain and spinal cord, the muscles, lungs, spleen, and liver. Lead enters the bodies of animals in their food or water, portions of metal are picked up, or paint is licked. The poison is sometimes brought to the farm in street manure. Water, especially soft water, is liable to contamination by conveyance through leaden pipes or storage in leaden cisterns. The hounds at the royal kennels at Ascot some years since suffered from paralysis from drinking water contaminated by passing through new lead pipes. At Claremont the late Louis Philippe and his suite had symptoms of lead-poisoning, although the amount of lead did not reach half a grain to the gallon. But in some of the Yorkshire towns where lead-poisoning occurred from new pipes, the contamination did not exceed one twenty-fifth of a grain per gallon. On lead pipes or vessels the conjoined action of air and soft water is liable to produce a crust of carbonate $\left(\mathrm{PbCO}_{3}\right)$, with variable proportions of hydrate $\mathrm{Pb}(\mathrm{OH})_{2}$. This crust crumbles away as a crystalline powder, partly dissolved and partly suspended in the fluid. Leaden vessels, or vessels soldered with lead, must therefore be used with caution for storage, especially for any length of time, of water, saccharine or acetic solutions, or other fluids likely to dissolve the metal. This caution is especially applicable to soft waters and to those rich in chlorides, nitrites, nitrates, and nitrogenous matters yielding ammonia. Hard waters, abounding in carbonates, sul- 
phates, or phosphates, are less liable to contamination, as their acid, uniting with the lead, forms an insoluble crust, which protects the metal from further action of air or water. But even such hard waters are not absolutely safe from lead contamination. A piece of iron, a patch of soft solder, or a few carbonaceous or other impurities in the lead, are liable to set up galvanic action, and thus dissolve the metal. Great care should therefore be taken to prevent lime, mortar, nails, or in fact any foreign body, getting into leaden cisterns, which should further be emptied and cleaned out frequently, especially when new.

In acute poisoning an emetic or the stomach pump is promptly used, followed by a soluble sulphate, such as magnesium sulphate, to form the insoluble lead sulphate, then by demulcents, such 'as albumin, oils, gruel, etc., and appropriate agents to combat the collapse. In chronic poisoning, the lead, whether deposited in the tissues or lodged in the digestive canal, should be excreted as rapidly as possible. If in the intestine, by the action of magnesium sulphate, and if in the tissues, by means of potassium iodide. This agent is believed to combine with the lead albuminate in the tissues, forming the more readily excreted lead iodide, which is removed by the kidneys. Diuretics should be given at the same time, and a nutritious strengthening diet.

Lead Oxide. Plumbi Oxidum. Litharge.

Lead Carbonate. Plumbi Carbonas. White Lead.

There are five oxides of lead. Litharge $(\mathrm{PbO})$ is a yellowish-red, scaly powder, prepared by the action of air on melted lead.

Lead carbonate, or white lead, may be prepared by the interaction of lead, water, and carbonic anhydride, in the presence of vapours of acetic acid (B.P.). It is insoluble in water; entirely soluble in diluted acetic acid.

Actions AND Uses.-Litharge and white lead are used topically as desiccants and astringents. Mixed with linseed oil, glycerin, or vaseline, they form antiseptic, astringent, protective coverings, useful in burns, herpes, and moist eczema. 
Animals, however, are prone to eat or lick such dressings, and to obviate risk of poisoning, zinc oxide and carbonate are hence usually substituted.

\section{Lead Oleate. Lead Plaster. Emplastrum Plumbi.}

The common sticking or diachylon plaster is prepared by boiling together gently, by the heat of a steam bath, one pound litharge, two pounds olive oil, and sixteen ounces of water, keeping them simmering for four or five hours, stirring constantly until the product acquires a proper consistence for a plaster, and adding more water if necessary (B.P.). In this process the oil is decomposed in the same manner as fats are acted upon by steam heat, or by alkalies in the preparation of soap; lead oleate rises to the surface, and glycerin remains in solution. Lead plaster is sold in rolls, about a foot in length, of a yellow-white colour, and a faint, sweet, soapy odour. Although brittle when cold it becomes soft and adhesive when heated.

Actions AND UsES.-Lead plaster is adhesive, free from irritant properties, and the lead is not liable to absorption. For bringing together the edges of small incised wounds it is generally used spread on linen or calico, and thus applied it besides affords protection and support. Lead plasters are rendered more adhesive, and consequently better adapted for most veterinary purposes, by melting with every pound four ounces of pitch or resin and two ounces of hard soap.

\section{Lead Iodide. Plumbi Iodidum. $\mathrm{PbI}_{2}$.}

When equal parts of lead nitrate and potassium iodide are dissolved, and the solutions mixed, double decomposition occurs, potassium nitrate remains in solution, and lead iodide is precipitated in brilliant, golden-yellow, crystalline scales, or in a fine, bright yellow, heavy powder. It is tasteless, colourless, sparingly soluble in cold water, but readily dissolved by 200 parts boiling water, entirely soluble in solution of ammonium chloride.

Actions AND Uses.- It resembles other lead salts ; is occa- 
sionally prescribed for enlarged glands to promote reduction, and applied as a dressing for ringworm and for indolent tumours, being used in the form of ointment or plaster. Emplastrum Plumbi Iodidi is made with two ounces lead iodide, one pound lead plaster, and two ounces of resin.

Lead Acetate. Plumbi Acetas. Sugar of Lead. Neutral Acetate. $\mathrm{Pb}\left(\mathrm{C}_{2} \mathrm{H}_{3} \mathrm{O}_{2}\right)_{2} 3 \mathrm{Aq}$.

Strong Solution of Lead Subacetate. Liquor Plumbi Subacetatis Fortis. Goulard's Extract. (B.P.)

Two lead acetates are used in medicine-the neutral acetate or sugar of lead, and the tribasic, which occurs in Goulard's Extract.

Sugar of lead is obtained by dissolving lead oxide or lead carbonate in acetic acid. Lead acetate occurs in minute needle-like crystals, which are slightly efflorescent, have an odour of vinegar, and a sweet, astringent taste. It is soluble in less than three parts of cold water, and in thirty parts of alcohol (90 per cent.).

The liquor plumbi subacetatis, or Goulard's Extract, is prepared by boiling 5 ounces of lead acetate and $3 \frac{1}{2}$ ounces lead oxide, in powder, in a pint of water, for half an hour, constantly stirring ; then filter, and, when the liquid is cold, add to it more distilled water, until the product measures 20 fluid ounces (B.P.). It is a colourless, transparent, alkaline liquid with a sweet, astringent taste. It becomes turbid on exposure to air. From a solution of the neutral acetate it is distinguished by its alkalinity, and by its producing an opaque white jelly when mixed with mucilage of gum acacia. The diluted solution of lead subacetate, or Goulard's water, is made with 2 drachms of strong solution of lead subacetate, 2 drachms alcohol (90 per cent.), and $19 \frac{1}{2}$ ounces of distilled water.

ACTIONS AND USES.-The acetates exhibit the physiological actions of other soluble lead salts. They are prescribed as internal astringents and styptics, and externally as astringents and analgesics. They are less corrosive and astringent, and more soothing, than zinc or copper acetates. The 
greater solubility of Goulard's Extract renders it more active than the sugar of lead, and it is preferable for external application on account of its not drying or crystallising.

Toxic EFFEcTs.-Hertwig gave a pound of sugar of lead to horses, and observed nausea, colic, a quick, small, hard pulse, stiffness of the limbs, paralysis of the optic nerve, and sometimes of other parts, insensibility, and often death. Even more energetic effects occur in cattle. Prinz observed that half an ounce given daily for three days produced in cows fever, with a quick, throbbing pulse, colic, and other symptoms of abdominal pain ; in one case mania, but in none death. Mecke found that eight ounces, dissolved in water, and given in divided doses during two days, destroyed nine cattle-the first on the second, the last on the fourteenth day after the poison had been given. Early in 1857 a farmer near Glasgow lost eight cows from their boiled food having been stored in a large tub obtained from a chemical manufactory, and impregnated with sugar of lead. The symptoms were similar to those above recorded. Dogs receiving half an ounce, retained by tying the œesophagus, suffered intense intestinal irritation, and died, occasionally in nine hours, but sometimes only after two or three days (Orfila). Owing to chemical action the membrane of the stomach is grey, of a macerated appearance, and sometimes very vascular, especially in lingering cases. These effects are due to the local irritation of the mucous membrane of the stomach and intestine, set up by concentrated solutions or large doses. They are not due in any measure to systemic lead poisoning, for little lead is absorbed from the alimentary tract unless given for long periods and in small doses.

Medicinal Uses.-Lead acetate is administered to check hæmorrhages, especially from the stomach and intestine. Formerly it was prescribed for purpura in horses and redwater in cattle ; but for these purposes it is useless, primarily because of its non-absorption. Half-drachm doses of sugar of lead and opium, given daily, sometimes check the dangerous diarrhœa and dysentery which attack badly-managed anæmic cattle in autumn and early winter. Scouring lambs 
are equally benefited by eight or ten grains each of lead. acetate and opium. In many of these cases, besides being given by the mouth, it is also added to starch injections.

Externally it is applied to check superficial, circumscribed inflammation; to soothe and heal burns, bruises, and irritable moist ulcers; to cool and relieve strained, inflamed tendons and joints ; in conjunction with a dose of physic, to abate the itching of nettle-rash and erythema; to remove the irritation and heal the excoriations of herpes ; to limit the surrounding inflammation of acne ; to arrest irritation and discharge in eczema, in such cases being advantageously alternated with dilute alkalies, sulphur or citrine ointment, or yellow wash. It is applied in mucous or catarrhal inflammation of the eye, but is unsuitable when there is abrasion or rupture of the cornea, as insoluble lead albuminates and sulphates are formed, causing opacity, which is difficult to remove.

Doses, etc.-Of the lead acetates, horses and cattle take 3ss. to 3 i. ; calves and sheep, grs. x. to grs. xx. ; pigs, grs. ij. to grs. vj. ; dogs. grs. j. to grs. iv., given in bolus or solution, repeated once or twice daily. For external application, sugar of lead is used in powder, ointment, or dissolved in twenty to forty parts of water, with a little vinegar to increase its solubility. Goulard's Extract, diluted with four to six parts of linseed or olive oil, is a cooling application for blistered or contused surfaces. An equally serviceable astringent and anodyne is made with one part of extract to six or eight of vaseline or glycerin. Equal parts of extract and spirit, diluted with eight or ten parts of water, make a useful refrigerant astringent. One part of lead. acetate and three-quarter part zinc sulphate, dissolved in thirty or forty of water, constitute the familiar white lotion, a serviceable astringent, analgesic, and antiseptic.

\section{ZINC AND ITS MEDICINAL COMPOUNDS}

Metallic zinc is obtained by roasting zinc blende, which is a native sulphide, or calamine, which is a native carbonate. Zinc is a bluish-white metal, brittle at low and 
high temperatures, but between $212^{\circ}$ and $300^{\circ} \mathrm{Fahr}$. it is ductile and malleable. Its salts are colourless.

ACTIONS AND USES.-Zinc salts unite with albumin to form an insoluble precipitate, and hence are astringent. As solids and concentrated solutions, several are irritant and caustic, and being deliquescent they tend to spread. Although not affecting the unbroken skin, when applied to mucous membranes small doses are astringent, large doses are irritant. The chloride, nitrate, and iodide are readily soluble and diffusible, and hence are active and corrosive. The sulphate and acetate, although less energetic, have more activity than the less soluble oxide or carbonate. The sulphate and acetate are prompt emetics for dogs and other animals that vomit, and unlike tartarised antimony, they cause little depression of the circulation. They produce emesis chiefly by local action on the stomach. Continued full doses produce symptoms allied to those of acute poisoning by copper or lead, due to the local irritant effect in the stomach and intestine. They are absorbed very slowly as albuminates, and produce no characteristic effects, but injected intravenously, depression of the central nervous system and of muscle tissue is produced as with copper. They are eliminated more rapidly than mercury, lead, or copper, in small quantity by the kidneys, but chiefly by the liver and intestinal glands (Bartholow).

\section{Zinc Oxide. Zinci Oxidum. Oxide of Zinc. ZnO.}

Oxide of zinc may be prepared by exposing zinc carbonate to a dull red heat, or from metallic zinc by combustion. It is a soft, nearly colourless, tasteless, inodorous powder, insoluble in water, but soluble without effervescence in acids and in alkalies. When heated it becomes yellow, but if free from iron nearly loses its colour on cooling.

ACTIONS AND USES.- It is a desiccant, protective, and hæmostatic, but, being insoluble in water, it acts mechanically unless dissolved by an acid, when it is mildly astringent. It is occasionally prescribed in catarrh and bronchitis for the arrest of profuse secretion, is said to be a nerve sedative, and is given to dogs in epilepsy and chorea. 
It relieves cutaneous tenderness and itching. In erythema, in which it is often desirable to avoid moist dressings, it is dusted over the tender surface mixed with four to six parts of kaolin, 'Sanitas ' powder, or starch flour. In many cases of erythema it is usefully conjoined with glycerin, vaseline, or soft soap. Acute vesicular eczema is often successfully treated by a thorough soaking with mercurous oxide wash and the subsequent in-rubbing of zinc oxide ointment or oleate. When there is much tenderness or itching such dressings are mixed with or followed by application of orthoform. After cleansing the meatus the ointment is useful in canker of the ear of dogs, and in diseases that simulate it.

Doses, etc.-Horses and cattle take 3ij. to 3iv.; dogs, grs. ij. to grs. vj., given in bolus or solution. For external use aqueous solutions are made with equal parts of zinc oxide and borax or other alkaline salt or glycerin added to ensure solution, with ten to thirty parts of water. It is also used with subnitrate or oxide of bismuth. Liniments are prepared with one part of oxide to five or six of olive oil, or other fatty matter. Unguentum zinci is made with three parts of zinc oxide, and seventeen parts of benzoated lard. Some cutaneous complaints, in which ointments freely used are apt to impair secretion and excretion, and others which should not be wetted, are satisfactorily treated by pastes or powders. Zinc oxide suits well for such purposes, and may be applied mixed with kaolin, magnesium carbonate, starch, iodoform, or boracic acid.

Peroxide of zine, or zine perhydrol (Merck), is a white powder, insoluble in water, and composed of equal parts of zinc peroxide $\left(\mathrm{ZnO}_{2}\right)$, and zinc oxide $(\mathrm{ZnO})$. It is said to combine the disinfectant properties of oxygen with the astringent effects of zinc oxide. Applied to open wounds it is neither caustic nor irritant ; and in powder or ointment it is valuable as a dressing for contusions, ulcers, dermatitis, and eczema. For operation wounds it may be used in place of iodoform. A pomade, made with five to twenty parts of zinc peroxide and a hundred parts of vaseline, or lanoline, is a convenient application for recent superficial wounds and bruises. 


\section{Zinc Carbonate. Zinci Carbonas. Carbonate of Zine.}

Calamine, the native carbonate, is an important ore of zinc. The B.P. carbonate-white, tasteless, and insoluble in water-is prepared by the interaction of zinc sulphate and sodium carbonate, and is a mixture of carbonate and oxide with water of crystallisation, $\mathrm{Zn} \mathrm{CO}\left(\mathrm{Zn} \mathrm{H}_{2} \mathrm{O}_{2}\right)_{2} \mathrm{H}_{2} \mathrm{O}$. Its uses are identical with those of the oxide.

Zinc Sulphate. Zinci Sulphas. Sulphate of Zinc. White Vitriol. $\mathrm{ZnSO}_{4} 7 \mathrm{H}_{2} \mathrm{O}$.

Zinc blende, the native sulphide when roasted, yields a crude sulphate. The B.P. salt is formed by the interaction of diluted sulphuric acid and zinc. It occurs in colourless, transparent, tabular crystals, isomorphous with those of Epsom salt, with a styptic metallic taste, and efflorescent in dry air. It is soluble in 2.5 parts of alcohol (90 per cent.), and in two parts of water.

ACTIONS AND UsES.- It is irritant, emetic, astringent, and antiseptic internally. It is used externally as a stimulant, astringent, and antiseptic.

Toxic Actions.-Powerful astringent effects are produced on horses by sixty to seventy-five grains; three to five drachms dry the buccal and gastro-intestinal secretions, and cause nausea, colic, and efforts to vomit (Tabourin). Two horses had each half an ounce daily for a fortnight without marked effect, but larger doses impaired appetite, and caused nausea and diuresis. Somewhat larger doses produce similar effects on cattle. Dogs receiving seven to thirty grains promptly vomit; but the act is seldom accompanied by the nausea and depression produced by tartar emetic. Orfila found that seven and a half drachms were vomited by dogs in a few seconds, but produced no lasting bad effects. When vomiting, however, was prevented by ligature of the œsophagus, much smaller quantities sufficed to destroy dogs, in about three days, from gastro-enteritis. Thirty grains in solution, injected into the veins, depressed the action of the heart and destroyed life in a few seconds (Christison). Repeated doses are detected in the spleen, liver, fæces, and 
urine. Unlike lead or mercury, it exhibits no cumulative action.

Medicinal Uses.-As a gastric tonic it resembles, but is inferior to, iron and copper sulphates. As an astringent compared with lead or silver salts, it contracts capillary vessels more powerfully. It is sometimes prescribed with opium in diarrhœa and dysentery, but is less serviceable than copper sulphate or lead acetate. It may be given for acute chorea in dogs in good condition: but iron is better in chronic cases associated with debility. For diminishing excessive discharge, or arresting hæmorrhage from the alimentary canal, frequent small doses are given with sulphuric acid and opium. As a safe and prompt emetic it is prescribed for dogs and pigs to empty the stomach of undigested food, foreign bodies, and poisons.

Externally, it is much used as a stimulant and astringent for wounds, ulcers, conjunctivitis, relaxed sore throat, irritable conditions of the mucous membrane of the uterus or vagina, vesicular and pustular skin eruptions, and interdigital inflammation in sheep. Where powerful astringent and caustic effects are sought, the zinc sulphate is conjoined with that of copper or iron, or with both. Malcolm, in his successful treatment of canker in the foot of the horse, uses a dressing of equal parts of zinc, copper, and iron sulphates, with carbolic acid, and sufficient vaseline to form a paste.

DOSEs, etc.-As an emetic, grs. ii. to grs. v. for cats; grs. jv. to grs. viij. for dogs ; and grs. $x$. to grs. xv. for pigs, are given in two or three ounces of water. As an astringent for horses and cattle, 3ss. to 3 ij. ; for sheep, grs. x. to grs. xx. ; for dogs, grs. j. to grs. iij. are given, either in the solid or fluid state. Externally, it is used in powder or solution, usually made with thirty to sixty parts of water. Three-quarters of an ounce of zinc sulphate and an ounce of lead acetate, with 30 to 40 ounces of water, constitute the white lotion so familiar in veterinary practice, but for some purposes this strong solution requires further dilution.

Zinc sulpho-carbolate is sometimes used as a mild antiseptic and astringent, two to five grains being dissolved in an ounce of water. 
Zinc Chloride. Zinci Chloridum. Butter of Zinc. $\mathrm{ZnCl}_{2}$.

Chloride of zinc is produced by the interaction of hydrochloric acid and zinc. It occurs in colourless, opaque rods or tablets, very deliquescent and caustic ; soluble in water, alcohol, and ether.

ACTIONS AND UsES.-It is an irritant and corrosive poison. Medicinal doses are antiseptic and astringent; but it is seldom given internally. Externally, it is applied as a stimulant, astringent, caustic, and parasiticide. It is also used as an antiseptic, disinfectant, and deodoriser.

SURGical UsEs.-From its strong attraction for water, and its coagulating albumin, it is an energetic caustic. It resembles mercuric and antimony chlorides. It is used to control luxuriant granulations, unhealthy ulceration, and foot-rot in sheep, and for such purposes is applied in substance. To remove malignant growths and to destroy the walls of sinuses, it is applied, usually mixed with two parts of flour made into a paste with glycerin and water. Unlike arsenic or mercury salts, it is not liable to undergo absorption and produce constitutional mischief. Strong solutions, containing 10 per cent., secure the aseptic state of foul or envenomed wounds, and are serviceable where repeated dressings are inadmissible, and the volatile carbolic acid cannot be conveniently used. Socin's paste, consisting of five parts zinc chloride, thoroughly mixed with fifty parts each of zinc oxide and water, is used as an antiseptic protective for surgical wounds which cannot be bandaged. Solutions of two to three per cent. are used for ordinary astringent purposes and for the destruction of vermin. Almond recommends a $2 \frac{1}{2}$ per cent. solution as an application for follicular mange of the dog.

Like mercuric chloride, it is serviceable for keeping animal tissues for dissection. Its antiseptic effects are exerted even in the presence of considerable quantities of water, which interferes with the efficacy of the tar acids. Besides preventing and arresting putrefaction, it also decomposes hydrogen sulphide, ammonia, and other offensive products of decay. Burnett's disinfecting and antiseptic fluid contains twentyfive grains zinc chloride in every fluid drachm, and is 
ordered to be used in the proportion of one pint to five gallons of water.

Zinc Acetate. Zinci Acetas. Acetate of Zinc. $\mathrm{Zn}\left(\mathrm{C}_{2} \mathrm{H}_{3} \mathrm{O}_{2}\right)_{2}$ $3 \mathrm{H}_{2} \mathrm{O}$.

Zinc acetate is prepared by neutralising acetic acid with zinc carbonate. It crystallises in colourless, odourless, pearly plates, which have a sharp, disagreeable, metallic taste, and are soluble in 2.5 parts of water.

Actions AND UsES.-The acetate closely resembles the sulphate. It is an emetic and nerve tonic, but is seldom used internally. Externally, as a stimulant and astringent it dries excessive serous discharges, relieves erythema, eczema, and impetigo, as well as conjunctivitis and other superficial inflammations. Tuson recommended a solution for saturating at short intervals the wash-leather bandages applied to the jarred, swollen legs of hunters. According to the purpose for which it is required, forty grains to an ounce are dissolved in a pint of water.

Doses.-Horses and cattle, $3 \mathrm{j}$. to $3 \mathrm{ij}$. ; dogs, grs. ii. to grs. v.

\section{COPPER AND ITS MEDICINAL SALTS}

Copper $(\mathrm{Cu})$ is a brilliant red metal, found native near Lake Superior in North America, crystallised in octahedrons or cubes. It has a specific gravity of 8.95 , a nauseous styptic taste, and unpleasant odour, especially when rubbed. The principal copper ores are pyrites, which is a double sulphide of copper and iron, and the carbonate or malachite. Its chief officinal salts are the sulphate, nitrate, iodide, and acetate. Copper forms two series of salts, the cuprous and cupric, the latter the more stable, and, when hydrated, having a green or blue colour.

ACTIONS AND USES.-Salts of copper, like those of other heavy metals, form sparingly soluble albuminates. In virtue of their combining with the albumin of the tissues, they are antiseptic, astringent, irritant, and caustic. When they are absorbed, these topical effects are more widely 
extended, and more general astringent, antiseptic, tonic, or irritant effects are produced.

Copper salts are allied to those of zine and silver, and some of their actions also resemble those of iron, lead, and mercury. The sulphate, nitrate, and other soluble salts have slight action on the unbroken skin, but combine with albumin, and hence constringe and irritate the abraded skin and mucous surfaces. When swallowed they irritate the stomach, producing emesis in many animals. After exerting, according to dose or state of concentration, astringent or irritant effects on the alimentary mucous membrane, they are slowly absorbed as albuminates, and as the still more soluble peptonates. They are chiefly stored in the liver, to a less extent in the kidneys and spleen. They probably unite with various tissues, and modify their nutritive and functional activity. Like many other metallic salts, they are excreted slowly in the bile, in the mucus of the stomach and bowels, to a slight extent in the sweat, but mainly in the urine.

Copper in the metallic state is devoid of poisonous action. Drouard gave ounce doses finely divided to dogs of different sizes and ages, but none experienced any inconvenience. If a double salt of copper (copper albuminate) is injected into the blood-stream the specific effect of the metal is seen. There is awkwardness and staggering gait, then paralysis of locomotion, and finally of the heart and lungs. Thus copper, like zinc and lead, paralyses the central nervous system, and also has a direct toxic action on muscle. Given by the mouth this typical action is not produced, for the drug is absorbed too slowly. Two drachms of oxide caused in dogs vomiting and diarrhœa. The more soluble salts are active irritants. Dogs tolerate for a week or two daily doses of 10 to 15 grains of the sulphate, or acetate, but 40 to 60 grains induce loathing of food, diarrhœa, and in some instances death by collapse. Cupreous poisoning also results in cows, pigs, and dogs, from the use of food or drink boiled in copper vessels, and allowed to remain in them while cooling. Acid and fatty matters are most apt thus to be contaminated, especially if long in contact with copper, and exposed at the same time to air and moisture. The prominent symptoms are, impaired appetite, constipation, alter- 
nated with diarrhœa, colic, imperfect nutrition, muscular weakness and trembling, and occasional hæmoglobinuria or hæmaturia. All these symptoms arise from the gastroenteritis set up, and are similar to the effects produced by any corrosive. The antidotes consist of white of egg, washing out the stomach, administering demulcents, and allaying irritation and pain, if need be, by morphine.

Chronic poisoning occasionally occurs among animals depastured in the neighbourhood of copper-smelting works, but such effect may in part depend upon the arsenic present in copper ores. This is shown by gradual unthriftiness, emaciation, and increasing weakness, and is relieved by removal of the cause, and good feeding.

Copper Sulphate. Cupri Sulphas. Cupric Sulphate. Blue Vitriol. Blue Stone. $\mathrm{CuSO}_{4} 5 \mathrm{H}_{2} \mathrm{O}$.

Copper sulphate is got by dissolving the black oxide in sulphuric acid, by boiling metallic copper with sulphuric acid, and on the large scale by roasting copper pyrites $\left(\mathrm{CuFeS}_{2}\right)$, when both the copper and iron are oxidised into sulphates; at the red-heat used the iron sulphate is decomposed, and the copper sulphate crystallised from a hot watery solution. Blue vitriol made from pyrites always contains iron, which does not, however, interfere with its medicinal uses. It occurs in large blue triclinic prisms, with a styptic metallic taste. Exposed to the air, it effloresces and becomes covered with a greenish-white powder of carbonate. It is almost insoluble in alcohol, but soluble in $3 \frac{1}{2}$ parts of cold water, and in $2 \frac{1}{2}$ of glycerin.

ACTIONS AND USES.- - In moderate doses or weak solutions it is antiseptic, astringent, and tonic in the alimentary tract. Even small doses induce emesis in dogs and other carnivora. Large doses and concentrated solutions are irritant and caustic. Externally, applied to a mucous membrane or a wound, it combines with the albumin of the tissues with which it comes into contact, and is used as a stimulant, astringent, and caustic. Like many other metallic salts, it arrests the action of enzymes and of organised ferments, and has an inhibitory action on parasites such as worms. 
Toxic EFFEcTs.-Hertwig records that large doses (above twelve drachms for horses and cattle, one drachm for sheep or swine, and half a drachm for dogs) cause indigestion and impaired appetite ; in carnivora, vomiting and diarrhœa ; the evacuations are tinged green or blue, and mixed with blood; and fatal inflammation of the stomach and intestines usually follows. Drouard found that 60 grains retained in the stomach of a dog killed it in half an hour, but left little appearance of inflammation. Mitscherlich found that two drachms speedily killed dogs, leaving 'blueness of the villous coat of the stomach, mingled with brownness, the apparent effect of chemical action.' A drachm applied to a wound caused in dogs rapid prostration, and death in four hours. Injected into the jugular vein, it speedily reduces and arrests the action of the heart, 15 grains proving fatal in twelve seconds (Christison). In poisoning by copper salts, the appropriate antidotes are white of egg and milk, which form insoluble innocuous albuminates; iron filings, which attract and fix the copper; or potassium ferrocyanide, which produces a comparatively insoluble and harmless salt.

Medicinal Uses.-Copper sulphate is given to dogs and cats as a promptly-acting effectual emetic, useful in narcotic poisoning. It acts chiefly on the stomach. With phosphorus it forms a stable, inert compound, the copper from a salt being deposited in the metallic form on the particles of phosphorus, so that copper sulphate is a valuable antidote in phosphorus poisoning. It is prescribed for all animals in atony of the stomach and excessive catarrhal discharges from the alimentary canal. In chronic diarrhœa and dysentery it is prescribed with opium. Given in bolus, administered fasting, and repeated daily for a week, it is a useful general vermifuge for the horse. As a nerve tonic it has been prescribed for weakly dogs affected with epilepsy and chorea, but the results have not been reassuring.

Externally, it is applied as a stimulant, astringent, and disinfectant. In ophthalmia, as an antiseptic stimulant; as a spray and gargle for ulcerated sore-throat ; as an antiseptic stimulant and caustic for sluggish wounds, discharging and parasitic skin diseases, exuberant granulations, grease, 
canker, cracked heels, sinuses, fistulæ, and in foot-rot in sheep, and as a styptic for arresting hæmorrhage from superficial vessels.

Doses, etc.-As a tonic and astringent, horses take $3 \mathrm{i}$. to 3ij. ; cattle, 3i. to 3 iv. ; sheep, grs. xx. to grs. xxx. ; pigs, grs. v. to grs. x. ; and dogs, gr. $\frac{1}{4}$ to grs. ij. These doses, repeated twice daily, are administered either in bolus or dissolved in some mucilaginous solution; and as tonics are best given along with food, or immediately after feeding. Unless in very small doses, copper sulphate should not be given for more than ten days, as it is apt to interfere with appetite, and even cause nausea. As a prompt emetic for the dog, grains ii. to grains x. are given dissolved in water, gr. ss. to grs. iii. for cats, and grs. viii. to grs. xxiv. for pigs. Externally, the powder or a watery solution is applied, and the crystals are used as an escharotic. A useful caustic injection for sinuous wounds is made with two ounces each of copper and zinc sulphates, three ounces of lead acetate and a pint of vinegar. Shepherds make an ointment for foot-rot with equal weights of powdered blue vitriol, gunpowder and lard. A more convenient and adhesive application is prepared by carefully mixing over a slow fire one part of powdered blue vitriol with one of lard and two of tar. A resinate of copper may be made by boiling 12 parts of copper sulphate in 250 parts of water, and adding 25 parts of powdered resin. The solid resinate, dissolved as required in methylated alcohol and glycerin, is used as an injection for quittor and similar wounds.

Copper Iodide. Cupri Iodidum. Cuprous Iodide. $\mathrm{Cu}_{2} \mathrm{I}_{2}$.

It is the by-product in one of the processes for making iodine, and is also obtained by mixing solutions of cuprous sulphate and potassium iodide. It is a fawn-coloured salt, has a disagreeable, styptic, coppery taste, and evolves an odour of iodine. It was introduced in the belief that it conjoined the actions of its two constituents; but large doses, in which its characteristic actions should be most obvious, produce the effects of other soluble copper salts. It has been recommended as a stimulating tonic in chronic 
œdema of the legs, and as an astringent in ill-conditioned ulcers and inveterate grease.

Copper Acetate. Cupri Acetas. Cupri subacetas. Verdigris. AErugo.

Copper subacetate may be prepared by subjecting plates of copper to the vapour of acetic acid. It occurs either in amorphous masses or powder, is blue or green, according to the mode of preparation, and has the taste and odour of a copper salt. It remains unchanged in air ; when heated, it gives off water, acetic acid, and acetone, leaving a residue of oxide and metal.

ACTIONS AND UsES.-The acetate, like other copper salts, is an irritant poison, emetic, antiseptic, astringent, and tonic, but is rarely prescribed internally. It is used externally as a caustic, stimulant, astringent, and antiseptic.

Drouard exhibited 12 grains of the subacetate to a strong dog fasting, and observed aversion to food, efforts to vomit, diarrhœa, listlessness, and death in twenty-two hours. Paralysis of the hind extremities was also observable in some cases, but in none was the stomach much inflamed. Cupric acetate is still more active. Orfila found that 12 to 15 grains given to dogs, produced besides gastric irritation, convulsions, tetanus, sometimes insensibility, and death within an hour. The toxic dose for the horse is two ounces. Hertwig records that one ounce administered to a horse caused colic, with acceleration of the pulse ; and that two ounces, given some hours after, aggravated these symptoms, causing first acceleration and then depression of the pulse, debility, and, after six hours, convulsions and death. Prescribed internally, the doses of the acetate are the same as those of the sulphate. The external uses are also the same. It is applied in the form of solution or ointment, the latter made with one part of the salt to eight or ten of lard or of resin ointment. A useful dressing for footrot in sheep is made with one part of acetate to three or four parts of lard, oil, or tar. Liniment of Verdigris, or Mel Egyptiacum, is made with 9 ounces of verdigris, 6 ounces of alum, and $1 \frac{1}{2} \mathrm{lbs}$. of honey, or treacle, heated together over 
a water-bath until the mixture assumes a brown colour. It is employed as a dressing for open joint, for thrush, canker, and other foot cases.

\section{SILVER AND ITS MEDICINAL COMPOUNDS}

Silver Nitrate. Argenti Nitras. Lunar Caustic. Lapis Infernalis. $\mathrm{AgNO}_{3}$.

When metallic silver is gently heated with diluted nitric acid, and the solution evaporated, silver nitrate crystallises in colourless right rhombic prisms. To form the familiar sticks or pencils, the salt is fused and run into moulds. Toughened caustic is prepared by adding, before fusion, one part potassium nitrate to nineteen parts silver nitrate. Mitigated caustic is made by fusing together one part of silver nitrate, and two parts of potassium nitrate, and pouring into moulds.

Argenti nitras is devoid of odour, has a disagreeable metallic taste, is permanent in air, but blackens on exposure to light or in contact with organic matters. It is soluble in its own weight of cold water, ether, and glycerin, and slightly soluble in rectified spirit. It blackens the cuticle, parts readily with oxygen, and thus corrodes soft animal tissues.

Like other silver salts, it is distinguished by giving, with hydrochloric acid, a curdy-white precipitate of silver chloride (AgCl), insoluble in nitric acid, but soluble in ammonia, and darkened by exposure to light.

ACTIONS AND USES.- It is antiseptic, astringent, irritant, and corrosive, is used as a caustic, and administered as an astringent and nerve tonic. It induces emesis in animals capable of vomiting. Large doses cause gastro-enteritis, corrosion of the mucous membrane of the stomach and intestine, and then prostration and collapse.

Toxic EFFECTs.-Like iron, zinc, and copper salts, which it resembles, silver nitrate readily unites with albumin. It is absorbed slowly as an albuminate; and the astringent, irritant, and caustic actions of the solid nitrate or a strong solution, are confined to the parts with which it comes into contact. When vomiting is prevented, 30 to 60 grains 
given to dogs cause prostration, weakness of heart action, intestinal irritation, and sometimes convulsions, paralysis, and death from shock. When administered for some time it is deposited in the skin and blackens it ('argyria '), and has also been detected in the liver, spleen, pancreas, and bones. As in the case of arsenic, antimony, or phosphorus, chronic poisoning is accompanied by fatty degeneration. Rosenstern, experimenting on the vessels of the mesentery of frogs, with weak solutions of various astringents, found silver nitrate most powerful; lead acetate followed next in order, requiring for production of a given effect a solution five times as strong; ferric chloride acted only feebly; alum caused dilatation. Silver nitrate is slowly excreted in the albuminous secretions and in the bile, mainly by the bowels, very little, if any, by the kidneys. Undue irritation, produced whether internally or externally, is diminished by solution of common salt, which forms the insoluble and inert chloride; milk and eggs should also be given. For pigmentation there is no certain remedy, although potassium iodide may be tried.

Medicinal Uses.-As a tonic it is prescribed in chronic nervous diseases, especially amongst dogs. Like arsenic, it is sometimes used to check chronic gastric irritation. Alone, or in combination with opium, it is given as an astringent in chronic diarrhœa and dysentery in dogs; while enemata of 2 to 5 grains to the ounce of distilled water or of starch gruel are occasionally also used.

Applied to irritable, relaxed, discharging skin or mucous surfaces, it coagulates mucus and albumin, constringes dilated vessels, produces a white film of chloride, which quickly deepens in colour, from the reduction of the salt to the sulphide and metal. The solid nitrate or strong solution rubbed into the skin raises blisters. The eschar remaining, after a free dressing, gradually cracks and peels off, leaving usually a healthy surface beneath. The solid nitrate acting superficially, and readily localised, is for many purposes preferable to fluid caustics, or to the deliquescent caustic potash. It is serviceable for destroying ringworm, warts, and other neoplasms, and checking the progress of indolent boils. Silver nitrate rolled in a piece of tissue 
paper is sometimes substituted for corrosive sublimate in treating sinuses not easily reached with the knife, and a few days after its introduction causes sloughing of the hard walls of the canal, and leaves a healthy granulating surface. It forms one of the most effectual remedies for the interdigital inflammation and discharge of contagious foot-rot in sheep.

A light dressing of the solid caustic, or of a weak solution, promotes a healthier condition of indolent wounds and ulcers, represses over-luxuriant granulations, often arrests the irritability of circumscribed attacks of erythema, eczema, or pruritus, and is an excellent dressing for chronic sore teats in cows. Solutions of 10 to 20 grains to the ounce of water destroy the parasites of mange and scab.

A solution containing half a grain to two grains to an ounce of distilled water abates the pain and congestion of conjunctivitis, and stimulates and heals the inflamed, suppurating eyelids of weakly dogs. It removes opacity of the cornea, if recent and produced by accident, but is of little avail in dense opacity of the cornea, resulting in horses from repeated attacks of ophthalmia. Solutions of 10 to 30 grains to the ounce of water are sometimes used, with a spray producer, to control laryngeal ulceration, follicular tonsillitis, and pharyngitis in dogs.

Doses, etc.-Of the nitrate horses and cattle take grs. viii. to grs. xv.; sheep, gr. ij. to grs. iv.; pigs, gr. j. to grs. ij. ; dogs, gr. $\frac{1}{8}$ to gr. j. These doses may be repeated two or three times daily, and are given in bolus or pill. When astringent effects are to be directed upon an ulcerated or discharging portion of intestine, the bolus should be made with kaolin, and given coated with keratin. The oxide, having no topical irritant effect, is sometimes substituted for the nitrate as a nerve tonic. For external purposes the sticks of nitrate are sometimes coated with wax to preserve them from the decomposing action of air and light; and are held in quills or forceps to prevent their blackening the fingers. An ointment is occasionally made with grs. v. to grs. viij. to the ounce of 
vaseline. Solutions require to be protected from light, and kept in bottles with glass stoppers. Silver nitrate is incompatible with highly oxidised bodies, and forms explosive compounds with creosote and morphine.

Argentum Colloidale. Collargolum. Soluble metallic silver prepared from the directions of Dr. Credé, by Heydens' Chemical Company. Colloid silver occurs in small hard masses of a greenish-grey lustre, containing 97 per cent. of silver. Reduced to powder, it is soluble in 20 parts of water and in albuminous fluids. It is antiseptic and, except in excessive doses, non-poisonous. This soluble silver is stated to be of great value in the treatment of septic diseases in man. In veterinary practice it has been used as a remedy for equine purpura hæmorrhagica, strangles, and omphalo-phlebitis of foals and calves, with the object of destroying microorganisms or their toxins in the blood and tissues. Dieckerhoff obtained very satisfactory results in four cases of purpura treated by intravenous injection of one per cent. aqueous solution in doses of 50 grammes. Further investigation is necessary to determine the proper dose and frequency of administration. To one patient, in ten hours, Dieckerhoff gave five injections of $7 \frac{1}{2}$ grains silver colloid dissolved in water. The solution does not irritate the vein. It may be administered subcutaneously, or in pill or bolus mixed with sugar of milk and glycerin. A solution (one or two per cent.) may be used to spray or inject wounds. An ointment is employed for skin diseases. For intravenous injection a one per cent. solution in sterilised water is employed in doses of $3 \mathrm{vj}$. to 3 xii. for horses; $3 \mathrm{j}$. to $3 \mathrm{ij}$. for foals and calves; and $m_{x x x}$ to $3 \mathrm{j}$. for dogs. These doses may be repeated twice a day. Actol, or silver lactate, is a white, tasteless, and odourless powder, soluble in twenty parts of water. An aqueous solution of 1 in 800 forms an active germicide. Itrol, or silver citrate, occurs as a light powder, without odour or taste, slightly soluble in water (3800 parts). Less irritating than the lactate, it is employed as an antiseptic lotion ( 1 in 5000 of water), or ointment ( 1 to 50 of lanoline) for wounds and skin eruptions. Argentol, a compound of silver and quinaseptol, is used as a substitute for iodoform. 
Protargol, a silver albumose, containing eight per cent. of silver, is a yellow powder, readily soluble in water and in glycerin ; insoluble in alcohol. The solution is not affected by heat, albumin, sodium chloride, caustic soda, or hydrochloric acid. It is said to be absolutely non-irritating. An aqueous solution ( 1 to 10 per cent.) forms a penetrating antiseptic of great value in the treatment of vaginal catarrh, keratitis, conjunctivitis, and otorrhœa. Argonin, a combination of silver casein and alkali, containing 4 per cent. of silver, is a white powder, insoluble in cold water, but freely soluble in hot water. Solutions of one to five parts in 100 of water, are powerful germicides (Coblentz).

\section{IRON AND ITS MEDICINAL SALTS}

InoN. Ferrum. Ferrum redactum. Fe.

Iron is a lustrous grey metal, tenacious, malleable, ductile, the least fusible of the useful metals, but readily welded at a white heat. It is attracted by the magnet, and becomes itself magnetic. It is widely diffused in rocks and soils, and is present in the structures of plants and animals. Small quantities occur uncombined, probably of meteoric origin. Its chief ores are the oxides, comprising magnetic ore and hæmatites ; the carbonates or clay ironstone, and blackband; and the bisulphide or pyrites.

Iron forms three compounds with oxygen $-\mathrm{FeO}, \mathrm{Fe}_{2} \mathrm{O}_{3}$, and $\mathrm{Fe}_{3} \mathrm{O}_{4}$-the last being a compound of the other two. Iron forms two series of salts--the lower proto or ferrous salts, in which it is divalent and magnetic; and the higher per or ferric salts, in which it is trivalent and nonmagnetic.

The ferrous salts are reducing agents, are chiefly grey or green, and in solution give, with hydrochloric acid and sulphuretted hydrogen, negative results; with ammonium hydrosulphide, a black precipitate of hydrated sulphide $\left(\mathrm{FeS} . \mathrm{H}_{2} \mathrm{O}\right)$; with caustic alkalies, white or grey precipitates of hydrated protoxide, $\mathrm{Fe}(\mathrm{HO})_{2}$, rapidly becoming green and then brown ; with potassium ferrocyanide, a white precipitate, gradually becoming blue by oxidation; with potassium 
ferricyanide, a precipitate dark-blue from the first, $\mathrm{Fe}_{4}[\mathrm{Fe}$ $\left.(\mathrm{CN})_{3}\right]_{3}$.

The ferric salts are oxidising agents, are mostly brown or red, and in solution exhibit, with hydrochloric acid, a negative reaction; with sulphuretted hydrogen, give a white precipitate of sulphur; with ammonium hydrosulphide, the black ferrous sulphide, together with sulphur; with caustic alkalies, a brown-red precipitate of ferric hydrate; with potassium ferrocyanide, a deep-blue precipitate of Prussian blue, at once goes down ; with potassium ferricyanide, no precipitate, but an olive or brown discoloration; with solution of galls, neutral solutions yield a blue-black precipitate-the basis of writing ink; with potassium sulphocyanide, an intense blood-red colour.

ACTIONS AND Uses.- Iron and its salts were the first mineral substances employed in medicine; they have been used for three thousand years ; but although anciently and extensively prescribed, a good deal has still to be learned regarding them.

The presence of iron seems to be essential to life of practically all forms of protoplasm. In the bodies of the higher animals iron occurs chiefly in the blood. About 9 grammes is obtained from the blood of a horse or ox of about $1000 \mathrm{lbs}$. live-weight. In the hæmoglobin and oxyhæmoglobin the iron performs much the same functions as it does in the ferrous and ferric oxides in the soils. In the lungs, hæmoglobin takes up oxygen, and becomes oxyhæmoglobin, which readily parts with oxygen as it circulates through the capillary vessels. Thus maintaining the healthy activity of these blood constituents, iron is said to act as a hæmatinic or blood tonic. Its curative effects are specially manifested in anæmia, in which the number of red corpuscles and amount of hæmoglobin are seriously reduced, sometimes to the amount of one-fifth of their normal proportion, impairing tissue oxidation and functional activity. In chlorosis, where there is a deficiency of hæmoglobin but a normal number of red cells, it is especially valuable. Clinical observation testifies that full doses of iron restore the pallid soft textures to their normal colour and firmness, and improve general health. These curative results depend upon 
the medicine being absorbed, and directly furnishing iron to the hæmoglobin, restoring its deficiency, and aiding the formation of red blood corpuscles from leucocytes.

A prolonged controversy has waged on the question of the absorption of iron salts, and although clinical experience of the remarkable value of iron in cases of anæmia, especially chlorosis, warranted the assumption that the iron given was absorbed and supplied iron to the blood, yet many authorities have denied that the drug was absorbed at all. Bunge notably was of this opinion, and he affirmed that the beneficial action of inorganic iron in any of its salts was simply to remove from the alimentary tract, by combination and precipitation, the excess of alkaline sulphides there as a result of dyspepsia. He believed that these sulphides united with and precipitated the organic iron contained in food which would normally have been absorbed and would have supplied the necessary iron to the blood. Thus on this theory inorganic iron was only useful by making it possible for organic food iron to be absorbed. This theory has now been given up, by its author among others, and it can be definitely stated that a small part of the inorganic iron given by the mouth is absorbed. Iron salts are converted into the chloride in the stomach and then into the albuminate. As the latter it is absorbed in the duodenum and carried to the spleen and liver in which organs it is stored. It appears probable that this iron stimulates the blood-forming tissues, such as the red marrow, and it supplies the needs of the blood and other tissues whilst the excess is excreted chiefly by the mucous membrane of the large intestine.

Neither ferrous nor ferric salts dissolve or pass through the epidermis. Both, except the albuminate of iron, coagulate albumin, and exert astringent effects on mucous and denuded skin surfaces, and also coagulate blood. Soluble iron salts, which do not coagulate albumin, such as the albuminate or double tartrate of iron and sodium, when injected into the circulation produce metallic poisoning, characterised by muscular and nervous depression, dyspnœic breathing, vomiting, cardiac weakness, and gastrointestinal, and to a less extent, renal inflammation. But 
when iron salts are swallowed no such effects are produced, for absorption is too slow and the quantity too small.

It would thus appear that iron salts are absorbed, but only partially and slowly from the alimentary tract; only small proportions of the doses prescribed can be used by the red blood corpuscles ; the absorbed portions, as occur with so many other metallic salts, accumulate in the liver, spleen, bone marrow, and lymphatic glands, and are again returned to the intestine to be excreted.

The numerous salts of iron possess much the same kind of action, but differ considerably in the degree of their activity. Comparing the ferrous with the ferric salts, the latter are darker coloured, more soluble and stable, as well as more irritant and astringent. Small dogs are injured by 4 or 5 grains of ferric chloride, but swallow without harm 40 grains of ferrous sulphate. The more soluble ferric salts are notably irritant, astringent, and corrosive. In the earlier stages of convalescence, where the stomach is irritable, in young patients, and especially in dogs, ferrous iodide or the carbonate, in the conveniently keeping saccharated form, is usually better borne than the ferric chloride, or even the ferrous sulphate. But in order to secure the full tonic effects of iron it is essential that the bowels be maintained in a natural state, and an occasional laxative should be given to counteract the constipating effect of the drug. Where prompt astringent effects are to be produced, full doses of the chloride or other soluble ferric salt are given.

The salts of iron chiefly used in veterinary practice, and hence demanding special notice, are the saccharated carbonate, ferrous sulphate, and iodide, with the ferric oxide and chloride.

Metallic iron, as filings or pulvis ferri, is occasionally given in cases of poisoning with salts of mercury and copper. Ferrum redactum, or reduced iron, is a greyish-black powder, containing 75 per cent. of metallic iron with iron oxide. Tasteless, and without astringency, it is frequently prescribed for young, unthrifty animals as a hæmatinic, which is less apt to derange digestion than the sulphate. Iron arsenate has been prescribed in squamous and herpetic skin diseases, in about the same doses as arsenic, and is also 
applied externally. Citrate of iron and quinine, conjoining the tonic properties of its components, and the less astringent citrate of iron and ammonium are occasionally used for dogs, in doses of 4 to 10 grains. Dialysed iron and amorphous quinine have been conjoined. The phosphate $\left(\mathrm{Fe}_{3} \mathrm{P}_{2} \mathrm{O}_{8}\right)$ is sometimes prescribed in diseases of the bones, in diabetes, and in nervous exhaustion. It is occasionally given to delicate dogs and foals, along with other phosphates, in the form of Squire's Chemical Food, which contains the phosphates of iron, calcium, sodium, and potassium, and in nervous depression with quinine and strychnine in the preparation known as Easton's Syrup.

The acetate, lactate, citrate, and tartrate, are very soluble, readily absorbed, and only slightly astringent, are sometimes preferred to the less expensive sulphate and carbonate.

Ferratin, a brownish-red powder, prepared from alkaline solution of tartarated iron and albumin, and containing 7 per cent. of iron, is prescribed as a tonic for dogs, in doses of grs. 5 to grs. 30 .

Iron Carbonate. Ferri Carbonas. Ferrous Carbonate. $\mathrm{FeCO}_{3}$.

Saccharated Iron Carbonate. Ferri Carbonas Saccharatus.

The ferrous carbonate occurs in clay iron ore and in many mineral waters. It is prepared by mixing solutions of iron sulphate and ammonium carbonate. It is greyish-green, has a chalybeate, inky-taste, and dissolves with brisk effervescence in hydrochloric acid. Exposed to the air, it rapidly absorbs oxygen, gives off carbonic anhydride, and becomes converted into ferric oxyhydrate-a change constantly taking place along the banks of chalybeate streams.

The saccharated carbonate is greatly more stable. It is made by rubbing the freshly-prepared carbonate with sugar in a porcelain mortar. It occurs in small, coherent, grey lumps, has a sweet, feebly chalybeate taste, and should contain about 20 grains of ferrous carbonate in a drachm. It is readily soluble, is a mild chalybeate, especially convenient in canine practice, and administered for the same purposes as the sulphate. Doses, horses and cattle, $3 \mathrm{j}$. to 
3jv. ; sheep and pigs, grs. xx. to 3j. ; dogs, grs. ii. to grs. $\mathbf{x v}$., repeated two or three times a day.

Iron Sulphate. Ferri Sulphas. Ferrous Sulphate. Green Vitriol. Copperas. $\mathrm{FeSO}_{4} \cdot 7 \mathrm{H}_{2} \mathrm{O}$.

Iron sulphate may be prepared by the interaction of dilute sulphuric acid and iron.

Ferrous sulphate occurs in bluish-green, oblique, rhombic prisms, which, exposed to the air, gradually oxidise, becoming opaque, and covered with a brown coating of the normal and basic ferric sulphate ; an excess of sulphuric acid retards this oxidation. It has an inky, styptic taste ; is insoluble in rectified spirit, but soluble in less than two parts of cold water ; exposed to a temperature of $212^{\circ} \mathrm{F}$., until aqueous vapour ceases to be given off, it parts with six-sevenths of its water of crystallisation, and forms the exsiccated sulphate. This dried sulphate is a white powder, slowly but entirely soluble in water.

ACTIONS AND UsES.-Topically applied, it is astringent and antiseptic. When swallowed it is a hæmatinic, astringent, and, in large doses, irritant. It is twice as active as the oxides and carbonates, but it is not so astringent, corrosive, or irritant as the ferric chloride or nitrate. As an antiseptic it is not so powerful as the ferric chloride, but has about the same power as the sulphates of alumina and zinc. Gohier administered $10 \frac{1}{2}$ ounces to a horse, 6 ounces to a donkey, and 3 ounces to a six-months foal. All were nauseated; no appreciable increase of iron appeared in the excretions from either bowels or kidneys. The three subjects died the following day, and their intestines were found to be gangrenous (Kaufmann).

Medicinal Uses.-Iron sulphate is administered to all veterinary patients in anæmia, and especially when it is connected with dyspepsia. It improves the appetite, diminishes exhausting discharges, and abates glandular enlargements. It is specially beneficial in anæmia occurring in young horses, cattle, or sheep, kept throughout the late autumn or winter on grass that has lost its nutritive value, or in young stock that have been reduced by restriction to indigestible, 
poor straw fodder. Along with concentrated, good food, the iron salt in many such cases is advantageously conjoined or alternated with quinine and other bitter tonics, nux vomica, acids, and occasionally with arsenic.

In conjunction with nutritive and oleaginous diet, iron is given to horses and cattle to improve condition. It is said to abate nasal gleet and leucorrhœa. It is prescribed with aloes for atonic torpidity of the bowels, and for destroying intestinal worms. Combined with iodine, it arrests diabetes insipidus in horses. It is one of the remedies given to check the earlier progress of liver-rot in sheep. Chorea and epilepsy, when connected, as they often are, with anæmia, are benefited by iron. In hæmorrhagic cases, as in purpura, it is prescribed with a mineral acid, and alternated with quinine. Given after a laxative, it aids recovery of cattle and sheep from red-water.

In convalescence from debilitating disorders it is regarded as a valuable hæmatinic. In the several forms of influenza and bronchitis, Robertson prescribed ferrous sulphate and nux vomica, of each half a drachm, with four drachms of powdered gentian, in bolus. In irritability, chronic catarrh, or hæmorrhagic conditions of the urinary bladder, such as accompany or follow epizootic disorders in horses, it has been prescribed with sulphuric acid and alternated with salicylic acid. A course of iron and quinine is advised in convalescence from nephritis. Although itself devoid of purgative effect, iron sulphate is stated to increase the activity of most cathartics with which it is combined. The sulphate in solution, 2 to 10 per cent., is much used as an astringent antiseptic. Mixed with sulphates of lime and alumina, Tuson's disinfectant powder is formed, which, when moistened, gives off sulphurous anhydride.

Doses, etc.-Horses take 3ss. to $3 \mathrm{ij}$. ; cattle, $3 \mathrm{i}$. to $3 \mathrm{iv}$. ; sheep, grs. $\mathrm{x}$. to grs. $\mathrm{xxx}$. ; pigs, grs. v. to grs. $\mathrm{xx}$. ; dogs, grs. ij. to grs. $x$; cats, gr. $\frac{1}{6}$ th to gr. j. The smaller doses are given as hæmatinics and tonics, the larger as astringents. Two and a half parts of the dried sulphate are equal to four of the sulphate. The drug is administered in bolus, in solution in water-gruel, infusion of calumba, or ale, or mixed with soft food, and repeated twice or thrice daily. 
As a tonic for horses and cattle, one to two drachms iron sulphate, and half an ounce each of gentian and ginger, are made into bolus, or dissolved in a pint of ale or gruel. Such proportions make three or four doses for sheep and eight or ten for dogs. Preparations of iron intended to act as tonics should be given during or shortly after meals. Full doses introduced into the empty stomach, especially of dogs, are apt to cause dyspepsia. To obviate gastric irritation or constipation, and maintain the continued good effects of iron tonics, after being used for a week or ten days they should for several days be withheld, or replaced by other tonics. Constipation and the dark colour and foetor communicated to the dejections are abated by appropriate diet, combination with Epsom or Glauber salt, or by an occasional laxative.

\section{Iron Iodide. Ferri Iodidum. $\mathrm{FeI}_{2} 4 \mathrm{Aq}$. (Not official.)}

When iodine, iron wire, and distilled water are gradually heated together, combination occurs, and the solution, filtered and evaporated, yields tabular green crystals, which are inodorous, have a styptic, metallic taste, and are soluble in about their own weight of water and alcohol. When heated, iron iodide gives off violet-coloured fumes of iodine, and, exposed to the air, it deliquesces and acquires a redbrown colour. This oxidation is retarded by keeping the solution in contact with fresh iron wire, in well-stoppered bottles, secluded from light, or by boiling the freshlyprepared solution in syrup.

ACTIONS, USES, AND DOSES.-It is a hæmatinic, alterative, and astringent. Poisonous doses are irritant, and produce the effects of iron rather than of iodine. Thus Cogswell found that three drachms caused in dogs vomiting and purging, while one drachm in concentrated solution killed a rabbit in three hours and a half, with the symptoms and post-mortem appearances of poisoning with other soluble salts of iron.

Besides being used for the same hæmatinic purposes as the sulphate, it is given to promote absorption of glandular enlargements, especially in young and weakly animals ; it is 
serviceable in swellings of joints, and has been commended for its efficacy in polyuria and nasal gleet in horses. The doses are the same as those of the sulphate. It is prescribed in bolus, saccharated powder, and in the form of the official syrupus ferri iodidi. This syrup, containing about $5 \frac{1}{2}$ grains of ferrous iodide in each drachm, is frequently given in the later stage of canine distemper and bronchitis, in doses of $m_{x}$. to $m_{x x x}$.

Iron Peroxide Moist. Ferri Peroxidum Humidum. Moist Ferric Oxide, $\mathrm{Fe}_{2} \mathrm{O}_{3} \cdot\left(\mathrm{H}_{2} \mathrm{O}\right)$, with about 86 per cent. of uncombined water.

Red or ferric oxide is found native in the several varieties of hæmatite, ochre, red chalk, and specular ore. The hydrate may be prepared by mixing together three ounces of liquor ferri perchloridi and one ounce of sodium carbonate diluted with water. This moist ferric oxide, freshly prepared, is the best antidote for arsenic. Twelve parts are stated to neutralise one part of arsenious acid. It mechanically absorbs the dissolved poison, and further converts it into an insoluble arsenite. The antidote should be followed by a saline purgative. In human patients doses of a dessertspoonful of the peroxide are given every five or ten minutes. Another arsenic antidote is the ferri oxidum hydratum cum magnesia, made by mixing a solution of magnesia with a solution of iron persulphate (U.S.P.).

Iron Perchloride. Ferri Perchloridum. Ferric Chloride. $\mathrm{Fe}_{2} \mathrm{Cl}_{6}$. Liquor Ferri Perchloridi Fortis.

Iron perchloride is prepared by boiling the metal in hydrochloric acid and water, and adding a little nitric acid to ensure production of the higher chloride. The green crystals, having a great affinity for water, quickly melt. The strong solution, liquor ferri perchloridi fortis, is orange-brown, odourless, with a styptic taste, and miscible with water and alcohol in all proportions. Specific gravity about $1.42 ; 110$ minims contain $22 \frac{1}{2}$ grains of iron. Diluted with three measures of distilled water, it constitutes the medicinal 
solution, liquor ferri perchloridi, which for ordinary purposes is as effectual as the tincture. Tinctura ferri perchloridi, known as tincture of steel, is made by mixing five ounces each of strong solution of ferric chloride and alcohol ( 90 per cent.) and adding ten ounces of distilled water. This tincture has a red-brown colour, an ethereal odour, and an acid, chalybeate taste.

ACTIONS AND USES.--Iron perchloride, whether in watery or alcoholic solution, is one of the most soluble, irritant, and corrosive preparations of iron. It is prescribed as a hæmatinic, antiseptic, astringent, and styptic, and is used topically as an antiseptic, astringent, and caustic.

MEdicinal Uses. - The solutions of the chloride are serviceable in most of the cases for which the sulphate has been recommended. They conjoin, with general tonic effects, marked astringent action. They are prescribed in atonic dyspepsia, and for the removal of intestinal worms. In relaxed throat, which accompanies and follows catarrhal fever in horses, half-drachm doses, with an equal quantity of glycerin and six or eight ounces of water, are given every two hours, administered slowly, as a gargle, or applied as a spray. Solutions are used in anæmia and the several conditions connected with it. When debility and anæmia are associated symptoms, the salts of iron, more especially the tincture of the perchloride, have a marked effect in promoting absorption of inflammatory products. In influenza and purpura in horses the chloride improves the appetite, and is credited with tonic effects both on the blood and bloodvessels. In hæmorrhagic eases it is sometimes prescribed with turpentine, in purpura with quinine, and sulphuric acid, alternated with occasional doses of potassium chlorate. Liquor ferri pernitratis, containing $3 \frac{1}{3}$ grains of iron in 110 minims, is sometimes preferred to the chloride, in obstinate diarrhœa, dysentery and hæmaturia. In rheumatism, especially in weakly subjects, it is useful, and may be alternated with salicylic acid. After the bowels have been freely opened it is serviceable in red-water in cattle.

The chloride is particularly suitable in cases of distemper and rheumatic lameness in weakly dogs, and, with or without arsenic, benefits most attacks of chorea and many of epilepsy. 
In dogs, as in other animals, it promotes recovery from exhausting diseases.

Externally, it is used as an antiseptic and astringent. It coagulates albumin, and carefully employed is a valuable styptic. Tow or wool saturated with a solution of the liquor and applied directly to the orifice of the bleeding vessel, is usually effectual. Diluted with six or eight parts of water the medicinal solution is injected into the uterus in cases of post-partum hæmorrhage, and into the nose in epistaxis. Two drachms to a pint of water, injected into the rectum, destroy and bring away ascarides. The growths of actinomycosis, after scraping, are sometimes dressed with the strong liquor, and subsequently with weaker solutions. The strong solution has been applied, with beneficial results, in grease, and canker of the horse's foot.

Although not so effectual an antiseptic as corrosive sublimate, it readily yields part of its chlorine, and arrests the actions of ferments. A solution of five per cent. in water in two days retarded the growth of anthrax bacilli, and in six days effectually destroyed both bacilli and their spores, which was effected, however, in two days by corrosive sublimate (Koch). As caustics, the liquor ferri perchloridi fortis, and the solid chloride are sometimes used.

Doses, etc.-Of the medicinal liquor and tincture, horses and cattle take $\mathrm{f} \zeta \mathrm{ij}$. to $\mathrm{f} \zeta \mathrm{j}$. ; sheep, $\mathrm{m}_{\mathrm{xx}}$. to $\mathrm{m}_{\mathrm{xxx}}$. ; pigs, $m_{x}$. to $m_{x x}$; dogs, $m_{j}$. to $m_{x}$. The smaller doses suffice for hæmatinic or tonic purposes, are repeated two or three times daily, are diluted with at least ten parts of water, ale, or gruel; are sometimes conjoined with mineral acids, quassia, calumba, and other bitters, or with alcohol or ether. They are incompatible with ammonia, alkalies, or their carbonates, and with tannin-containing substances. The larger doses mentioned are given when powerful astringent effects are required.

\section{ANTIMONY AND ITS MEDICINAL SALTS}

The salts and preparations of antimony in their physiological as well as their chemical relations resemble those of arsenic and bismuth. They combine with albumin, are 
precipitated by acid solutions, and consequently exert their irritant effects on parts where they meet with acid secretions, as in the stomach and around the orifices of the sweat glands. With the exception of the chloride, which is a powerful escharotic, antimony salts, locally applied, produce inflammation of isolated spots, causing first papules, and subsequently pustules. The solubility and volatility of the several preparations mainiy determines their activity. In animals that vomit they cause emesis, cardiac and vascular depression, and increased secretion. Large doses produce gastro-enteritis, and depression of the spinal cord and brain. Given for a considerable period, they induce fatty degeneration. The geese in the duchy of Brunswick, fed for their fatty livers, receive daily doses of antimonious oxide. Tartar emetic is almost the only antimonial prescribed internally.

\section{Antimonious Oxide. Antimonii Oxidum.}

The medicinal oxide, also known as native white or flowers of antimony $\left(\mathrm{Sb}_{2} \mathrm{O}_{3}\right)$, is often used as a paint instead of white lead. It is prepared, by direction of the B.P., by pouring solution of the chloride into water, and decomposing the precipitated antimony oxy-chloride with sodium carbonate. It is a greyish-white, tasteless, heavy, crystalline powder, insoluble in water, but soluble in hydrochloric, tartaric, and acetic acids, and forms, by oxidation with nitric acid, antimony tetroxide, and antimony pentoxide. An imitation of the patentJames's powder is made by thoroughly mixing one part of antimony oxide and two parts of calcium phosphate.

ACTIONS AND USES.-Antimony oxide is chiefly important on account of its employment in the preparation of tartar emetic, which it closely resembles in its actions and uses. It has, however, no advantages over the latter, and is less easily absorbed.

Antrmonious Sulphide. Antimonium Nigrum Purificatum.

The native sulphide or stibnite $\left(\mathrm{Sb}_{2} \mathrm{~S}_{3}\right)$, the most abundant ore of antimony, when purified by fusion, washing and drying, occurs in dark-grey, metallic, heavy, brittle cakes, or as a heavy, greyish-black, crystalline powder, devoid of 
odour and taste, insoluble in water, and known as black antimony. The orange-red sulphurated antimony of the B.P. is obtained by boiling black antimony with sublimed sulphur and caustic soda, neutralising the solution with sulphuric acid, and washing the precipitated mixture. The following sulphides are used in the arts, and have occasionally been employed in medicine :-Glass of antimony, a red, transparent body, consisting of about eight parts of oxide and one of sulphide; liver of antimony, a double sulphide of antimony and potassium ; and Kermes mineral, a red-brown powder containing a variable proportion of oxide and sulphide.

ACTIONS AND USES.-Being uncertain, irregular, and often violent remedies, the antimony sulphides are now seldom used in either human or veterinary medicine. Their irregular action mainly depends on their variable composition and their insolubility in water. They once had the reputation of being expectorant, and anthelmintic, and were given to horses and cattle in doses of one to three drachms, usually along with sulphur or nitre. They cause emesis in dogs. The orange sulphide has been recommended for mammitis. It is transformed in the stomach into the double chlorides of potassium and antimony. In contagious mammitis four to six drachms may be given three times a day.

Soldtion of Antrmonious Chloride. Liquor Antimonii Chloridi. Butter of Antimony. $\mathrm{SbCl}_{8}$.

When native sulphide is boiled with about five times its weight of hydrochloric acid, hydrogen sulphide is evolved, and the chloride remains in solution-a transparent, yellowred liquid, with a specific gravity of $1 \cdot 47$. The colour darkens by exposure, depending upon oxidation of the iron chloride, which is sometimes added intentionally. Containing excess of hydrochloric acid, it has an acid reaction, and fumes on exposure to air. Addition of water separates a white precipitate of basic oxy-chloride ( $\mathrm{SbOCl}$ ), which, if persistently washed, yields the oxide. The true butter of antimony - a hard, white, crystalline, fusible solid-is got by evaporating and then distilling the commercial solution. 
ACTIONS AND Uses.-Although less used than formerly, the chloride in solution is still employed as a desiccating caustic for thrush, canker, and luxuriant granulations; and for wounds of the feet in cattle, and foot-rot in sheep. Its application causes little pain; but, except in cautious hands, it is, however, too energetic for general use. As it cannot be diluted with water without undergoing decomposition, it should be mixed with three or four parts of tincture of myrrh.

Antimony Tartrate. Potassio-tartrate of Antimony. Potassium Antimonyl Tartrate. Tartar Emetic. $\left[\mathrm{K}(\mathrm{SbO}) \mathrm{C}_{4} \mathrm{H}_{4} \mathrm{O}_{6}\right]_{2} \mathrm{H}_{2} \mathrm{O}$.

Prepared by setting aside a mixture of antimonious oxide, and acid potassium tartrate, made into a paste with a little water, until combination has taken place, and then purifying by crystallisation from water (B.P.).

Properties.-Tartar emetic is sold as a white powder, and in colourless, transparent crystals, exhibiting triangular facets, becoming opaque when opposed to the air, and crepitating and blackening when heated. It is devoid of odour, has a sweet, metallic taste, is insoluble in strong alcohol, sparingly soluble in weak spirit ; dissolves in about seventeen parts of water, and in three parts boiling water. The watery solution is slightly acid; is decomposed by strong acids, alkalies, alkaline earths and their carbonates, and by solution of tannic acid, but not by gallic acid. Iron oxide, the most common impurity, communicates to the salt a yellow or brown colour; cream of tartar diminishes its solubility. If purchased in crystals instead of powder, impurities are more readily discoverable.

ACTIONS AND UsES.- Tartar emetic applied to the skin causes eruption of isolated papules, which shortly become pustules. Poisonous doses produce gastro-enteritis, nervous depression, or in small repeated doses, fatty degeneration. Medicinal doses given to dogs, cats, and pigs are emetic, expectorant, and depressant. Their physiological effects on horses, cattle, and sheep are not so well marked. It is occasionally used externally as a counter-irritant. 
General Actions.- Tartar emetic in substance or in concentrated solution acts as an irritant upon the alimentary mucous membrane. In the stomach it is partly decomposed by the acid of the gastric juice, soluble chlorides of antimony and potassium being formed (Kaufmann). It probably combines with albumin. Horses and cattle when receiving even poisonous doses, do not vomit, and doses of one to four drachms sometimes given medicinally do not produce the nausea and depression which are the notable results of medicinal doses in man, dogs, cats, or pigs. Dogs receiving two to four grains are nauseated, and vomiting occurs usually within fifteen minutes. Emesis depends upon irritation both of the stomach and vomiting centre, but more especially of the former. Reflexly, from the stomach, from the effects of the emesis, as well as by acting directly on the heart and vessels, it produces in men and carnivora cardiac and vascular depression, with lowered blood pressure; it slightly increases but subsequently diminishes the number of the respirations; reduces temperature, relaxes voluntary and involuntary muscles; and increases secretion from the skin, bronchial and gastro-intestinal membranes. Poisonous doses cause purging and gastro-enteritis, and when their action is very violent, after slightly exciting, they paralyse both the motor and sensory tracts of the spinal cord. In many of its actions it resembles phosphorus and arsenic notably in arresting the formation of glycogen, and in causing fatty degeneration of the liver and other organs. It is eliminated in the mucus of the stomach and bowels, and also in the bile and urine. It increases the excretion of urea. As with arsenic, animals receiving small doses acquire a condition of tolerance, and with impunity take doses which would otherwise prove dangerous.

THE Toxic EFfects, like the general actions, are less marked in herbivora than in dogs, cats, and pigs, which are affected much in the same way as men. According to Kaufmann, dogs are poisoned by three to six grains. But these and even larger doses are apt to be speedily ejected by vomiting if the dogs are left to themselves. If the œsophagus, however, be tied so as to prevent vomiting, such doses, and sometimes even one grain, cause nausea, accelerated and 
difficult respiration, fluid dejections, gastro-intestinal inflammation, and death in a few hours. Taylor, in his volume On Poisons, records that three to six grains injected into the jugular vein of dogs caused death in eight or ten hours. Fröhner states that half a grain to a grain injected intravenously proves fatal in half an hour, while small quantities applied to the broken skin also kill.

Pigs are not so susceptible. Hertwig mentions that ten to twenty grains cause nausea and vomiting, but act neither very rapidly nor very certainly; that one drachm in solution, given to a boar nine months old, caused vomiting, dulness, and uneasiness, which continued for three days ; but that two drachms given to a similar animal dissolved in half a litre of water, within an hour and a half caused vomiting five times, loss of appetite, thirst, spasms, prostration, and death the following day. Poultry swallowing one to three grains in bolus vomit freely.

Horses take, per os, without injury, thirty to forty times the quantity of tartar emetic which would prove fatal to a man or dog. Without notable effect they may be given one to four drachms in bolus, and such doses may be repeated night and morning for several days without causing impaired appetite, nausea, or gastric derangement. This insusceptibility of horses to the action of tartar emetic was demonstrated by experiments made by Dun and Barlow :

Case I.-On 9th September, about 10 A.M., a brown mare, unfit for work on account of lameness, with the pulse 38 and respirations 7 , got three drachms of tartar emetic in a ball made up with treacle and linseed meal. In the evening the pulse was unaffected, and the dose was repeated.

10th. - The pulse was 40, the respirations 7, appetite good, bowels and kidneys regular. A dose of four drachms was given morning and evening.

11 th. - At 10 A.M. the pulse was 42, respirations 7, appetite and bowels quite normal. Got an ounce in a ball as before. In the evening the pulse was 40 , no perceptible nausea, appetite good, bowels and kidneys regular. Dose of an ounce repeated.

12th.- In the morning the pulse was 37 , somewhat weaker than yesterday, but still firm. The appetite was very good, and there was no change in the state of the kidneys or bowels. Got a dose of an ounce. In the evening the pulse was 40 , and the patient in other respects as in the morning. Gave an ounce, being five ounces 6 drachms in four days.

13 th. - At 10 A.M. the pulse was 35, the appetite good, and the bowels and kidneys normal. About 1 A.м. the animal had dropped or lain down, and while lying the pulse was somewhat irregular, varying between 60 and 70. The respirations were quiet. At 12 the animal was lifted, when the pulse fell in a few minutes to 55, and the respirations to 6 . The appetite 
still remained very good. Gave ten drachms in the usual way. In the evening the pulse was 40 , the respirations 6 , the appetite and evacuations natural. Gave fourteen drachms.

14th.-10 A.M. No change from last night. Got an ounce; but when having it put over, the animal ran back and went down. At 1 she was raised, still continued to eat, and at 1.30 got another dose of an ounce. She remained down all day, and appeared nauseated. The pulse was not quite regular, probably owing to occasional struggling, but reached about 60 when at its maximum. Respirations about 12 . At 6.30 the animal was still eating and drinking, but only sparingly; was much nauseated and lying pretty quiet, with the lips much retracted, and the pulse 75 and weak.

15th.-10 A.M. Found dead, having taken ten ounces and six drachms of tartar emetic in six days. Barlow made the following notes of the post-mortem examination :-The muscular tissue in every part of the body was unusually flaccid, although rigor mortis was well established. The right lung, which was lowermost as the animal lay, was much congested in its deeper and central parts ; the several margins were comparatively pale ; at the anterior part of the anterior lobe there was much emphysema. The left lung was perfectly healthy, and not at all emphysematous. The bronchial tube and smaller bronchi in both lungs contained frothy mucus. The pleura and pericardium were in every way healthy. The heart was very large, its cavities being filled with firmly coagulated blood. At the junction of the villous and cuticular coats, the stomach was much contracted, and exhibited a slight blush, not amounting to redness. The cuticular coat was marked with several indentations, such as are produced by bots; but in all other respects the stomach was perfectly healthy. The intestines were also perfectly healthy both within and without. The liver was in a state of cirrhosis, such as is often seen in old and worn-out horses. The organs of urination and generation were quite normal. The brain was healthy, but the subarachnoid spaces contained a considerable quantity of fluid.

CASE II.-A mare, about 16 hands high, and in good health and condition, got three drachms of tartar emetic daily, in bolus, for five days, and then four drachms daily for thirteen days-making in all ten ounces and three drachms in eighteen days - but without exhibiting any physiological effect. The animal was destroyed by cutting the carotid artery, but the post-mortem examination revealed nothing at all abnormal. It may be mentioned that, on the twelfth day of experiment, twenty ounces of urine were removed, and found to contain a perceptible but not very large quantity of antimony.

CAsE III.-A black mare, of sound, healthy constitution, took ten ounces and a half of tartir emetic (in doses of four drachms, repeated twice and thrice a day) during ten consecutive days; yet she was in no way affected by it; her pulse and respiration were scarcely at all altered; her appetite throughout was voracious; her evacuations natural in appearance and quantity; and her condition considerably improved. She was poisoned by a fluid drachm of Fleming's tincture of aconite. On dissection not a single morbid appearance referable to the tartar emetic was observed. The stomach and intestines were carefully examined, and found 'beautifully healthy.'

CAsE IV.-A healthy, well-bred horse was given ten ounces of tartar emetic in solution, and after showing a good deal of nausea, uneasiness, and pain, died in about six hours. The only notable appearances on postmortem examination were softness and increased vascularity of the intes- 
tines, analogous to what is seen in patients that have died while affected by diarrhoea. Neither in this nor in any of the other cases were the lungs congested or inflamed, as is said to have occurred in Magendie's experiments.

Fröhner's experiments show similar results. Healthy horses, he states, take one or two drachms without causing any notable effect except increased discharge from the bowels; but these doses if continued are said to slow the pulse and cause palpitation, lassitude, diarrhœa, polyuria, and, when further continued, great weakness. An ounce in pill or electuary was not fatal, but in solution caused spasms, and death in eight days; while sixty grammes (nearly two ounces) in solution proved fatal in two and a half hours. Hertwig records that four drachms in solution in water induced colic, trembling, and acceleration of the pulse; the symptoms after a few hours abated, but death resulted after six or eight days. Much greater activity is, however, observed when tartar emetic is administered to horses intravenously. One drachm thus given raised the pulse to 120 beats per minute, caused difficult breathing, purging, sweating, flow of tears and saliva, eructation, retching, and muscular spasms. Two drachms further produced severe fits, vertigo, paralysis, and death in one and a half to three hours, but without action of the bowels. Kaufmann states that horses receiving fifteen grains intravenously in a few minutes exhibited efforts to vomit, great inquietude, dilatation of the nostrils, and lowering of the neck, as if suffering abdominal pain.

Cattle, like horses, take large doses with impunity. Hertwig and Viborg gave quantities varying from two to ten drachms, and Gilbert gave ten drachms in solution-all without effect. Dun repeatedly administered an ounce twice a day to cattle, and, except in a few cases where purgation occurred, did not observe any evidence of its action. Balfour, Kirkcaldy, has given half a pound in solution without any very obvious effects. Sheep exhibit similar insusceptibility. Viborg gave one drachm, and Gilbert three drachms in solution, and four in the solid state, without effect. But Gilbert found that four to six drachms in solution destroyed one-year-old sheep. Intravenous 
injection of five to six grains produces, however, lassitude, small, frequent pulse, difficult breathing, and purgation (Fröhner).

No very satisfactory explanation has been given of this insusceptibility of horses, ruminants, and also of rabbits. It is evidently due in part to imperfect development of the vomiting centre; but the gastric functions have also, doubtless, some connection with it, and also difficulty of absorption, as is shown by the drug being about ten times more active when administered intravenously than when given by the mouth.

Antidotal treatment consists in the removal of any unabsorbed poison by promoting vomiting or using the stomach pump, and subsequently giving large quantities of tannic acid, or of tannin-containing solutions, which form an insoluble compound, and thus delay absorption. Demulcents abate gastric irritation, which, with irritation of the vomiting centre, may also be relieved by morphine and chloral, while tendency to collapse is treated by stimulants.

Medicinal Uses.-As tartar emetic in safe doses produces no very marked physiological actions in horses and cattle, it can scarcely exert any marked curative effects on these animals. The febrifuge and sedative virtues formerly ascribed to it were doubtless the result of other medicinal or hygienic remedies with which it was used. As a vermifuge it is still occasionally given with aloes to horses, and with Epsom salt to cattle; but although increasing the activity of purgatives, it has no special anthelmintic action.

When the stomach of the dog, cat, or pig is to be emptied of undigested food, irritants, or poisons, ipecacuanha, mustard, or zinc sulphate, being more prompt and less nauseating, is preferred. But tartarised antimony is occasionally used as a nauseating emetic for robust subjects at the outset of febrile and inflammatory complaints. It promotes bronchial and gastric secretion, and relieves engorgement of the stomach, liver, throat, and chest. It hence mitigates the early acute catarrhal symptoms of distemper, and relieves bilious attacks in pampered, overfed dogs.

As a counter-irritant, unless employed with much caution, it is apt to induce painful, deep-seated inflammation, slough- 
ing, and blemishing, and is consequently unsuitable either for horses or dogs. For cattle, however, it is sometimes applied in chest diseases and chronic rheumatism. Unlike cantharides, it does not irritate the kidneys; but if it be absorbed, it produces in dogs, cats, and pigs nauseating effects similar to those which follow its administration by the mouth.

DOSES, etc.-When given to horses or cattle with the view of producing sedative, alterative, or expectorant effects, $3 i$. to 3iv. are administered three or four times daily, in either bolus or solution. It may be conjoined with nitre, camphor, Epsom salt, calomel, or aloes. As an emetic for dogs or cats, gr. i. to grs. iv., and for pigs, grs. iv. to grs. x. are given in bolus, rolled in a piece of meat, or dissolved in hot water. Doses considerably less than one grain, conjoined with ipecacuanha, have been.prescribed for carnivora as antipyretics and expectorants in acute attacks of bronchitis and pneumomia.

Externally it is occasionally used in the form of saturated watery solution or of ointment, which is made with one part of tartar emetic and four of lard. The ointment is sometimes added to ordinary blisters to increase their activity. Antimonial wine, prepared by dissolving forty grains tartar emetic in an ounce of distilled water, and adding sufficient sherry to form twenty ounces, is seldom used in veterinary practice. Doses.-Dogs, $m_{\mathrm{v}}$. to $\mathrm{m}_{\mathrm{lx}}$.

\section{ARSENIC}

Acidum Arseniosum. Arsenicum Album. Arsenious Anhydride. Arsenium. $\mathrm{As}_{4} \mathrm{O}_{6}$.

Arsenic is obtained by roasting certain arsenical ores. The crude arsenious anhydride is conducted into condensing chambers, and purified by sublimation.

Properties.-The anhydride or white arsenic, used in medicine, as well as in the manufacture of glass, colours, and shot, occurs as a heavy white powder, consisting of minute glassy fragments and octahedral crystals. It is odourless and tasteless, rough and gritty between the teeth, 
and if held in the mouth shortly causes irritation. When long kept it loses its transparency, and becomes opaque. At a temperature of $400^{\circ} \mathrm{Fahr}$. it is volatilised. Sprinkled on ignited charcoal it emits a characteristic garlic odour. Arsenic is soluble in one hundred parts cold, and in ten parts boiling water, and in five parts of glycerin. Moderately soluble in solutions of alkaline carbonates, and in diluted hydrochloric acid (B.P.). Boiled in water for two or three hours, the ordinary crystalline arsenic is converted into the vitreous form, and in each pint 219 grains are dissolved. Solubility is diminished by organic matters, but increased by acids, alkalies, and alkaline arsenites.

ACTIONS AND USES.-Arsenious acid is a corrosive irritant poison, killing either by gastro-enteritis or by nervous paresis. Continued doses cause fatty degeneration. It is prescribed as a gastro-intestinal stimulant and alterative, acting especially on the digestive and respiratory mucous membranes and skin, as an aid to growth and nutrition, as an antiperiodic, and as a tonic in nervous diseases. It is used externally as a parasiticide, antiseptic, and caustic.

General Actions.-Arsenic belongs to the trivalent group, which also comprises phosphorus, antimony, and bismuth. These agents diminish oxidation, decompose albuminoid tissues, produce fatty degeneration, and impair the glycogenic functions of the liver. With the exception of bismuth, the members of this group are irritant, corrosive poisons. The specific 'arsenic ' action is due to the negative ion of arsenious acid.

Locally applied, arsenic causes congestion, heat, and pain, with destruction of tissue, but the slough is preserved by the antiseptic effect of the poison. It is absorbed from any mucous, serous, or skin-abraded surface, and exerts its effects by whatever channel it enters the body. Small medicinal doses directly stimulate the stomach and promote gastric secretion. Beginning with small doses, animals shortly acquire a tolerance of arsenic, and take with impunity doses which would otherwise prove fatal. Full doses irritate the surfaces with which they come into contact ; cause gastrointestinal inflammation; in dogs and other carnivora vomiting, diarrhœa, weakened cardiac action, reduced 
temperature, cramps, delirium, coma, and death. Its power to produce fatty degeneration is not so great as that of phosphorus, but fatty degeneration is often observed in the epithelial lining of the stomach and of the alveoli of the lungs of animals poisoned by arsenic. The glycogenic function of the liver is impaired by continued doses, and in pigs, fowls, and rabbits fatty degeneration in three or four weeks invades most of the soft textures and attacks the bones. It is eliminated by the kidneys, and to a less extent by the liver, skin, and glands generally. Five to six hours after administration it may be found in the urine and milk. It is longest retained by the bones.

All arsenical compounds are poisonous, and the most soluble are the most active. Deadliest of all is arseniuretted hydrogen, which has occasioned the death of three chemists, who unfortunately inhaled it. Orfila found that the sulphides, in doses of forty to seventy grains, destroyed dogs in two to six days, and had much the same effect whether they were swallowed or applied to a wound. Metallic arsenic, although itself innocuous, unites so readily with hydrogen and oxygen that it speedily acquires poisonous activity.

The toxic dose for the horse is liable to considerable variation. Kaufmann's estimate is 240 to 720 grains. Berthe gave a mare affected with inveterate mange two, and afterwards three, drachms without injury. Beissenhirz gave one, four, three, two, and eight drachms, on successive days ; and death occurred twenty-four hours after the last dose. Hertwig gave eight horses doses, beginning with 20 grains and gradually increasing to a drachm; continued the administration in some cases for thirty days, in others for forty days, but observed no bad effects, either during the use of the poison or afterwards ; the pulse became a little stronger and harder, and some of the animals improved in condition. Percivall gave a horse affected with glanders one drachm in bolus with linseed and treacle, and increased the dose by 20 grains daily for seventeen days, when the animal received 380 grains in one dose. By the eighteenth day the animal had taken upwards of seven ounces of arsenic. Yet no physiological effect was obvious, no loss 
of appetite, no uneasiness or pain, and no alteration of the pulse or respiration.

Such comparatively negative effects are doubtless explained by the drug being given in insoluble form, and hence being only partially and slowly absorbed. Much smaller doses occasionally act with greater violence. Thus, according to Gerlach, twenty grains cause acute diarrhœa; and Percivall mentions that two glandered horses, receiving five grains daily in bolus, were attacked, one on the eighth, the other on the ninth day, with shivering, loss of appetite, nausea, purging, and other symptoms of abdominal irritation and prostration of strength. One died, the other recovered. These different effects depend partly on varying susceptibility ; on the amount of food in the alimentary canal ; and mainly on gradually acquired tolerance which enables animals receiving arsenic regularly to take with impunity, at one dose, as much as would kill a patient unused to the drug. Large doses, moreover, produce inflammatory changes in the lining of the alimentary canal which greatly retard absorption. Arsenious anhydride is much more active in solution than in substance. Thirty grains given daily, in solution of potassium carbonate, destroyed a horse in four days. Applied to wounds, Kaufmann states, it is much more active than when swallowed.

Baldwin reports the case of six horses poisoned by drinking from a pail in which some arsenical sheep-dipping mixture had been dissolved. Two died, and post-mortem examination revealed inflammation of the mucous coat of the stomach, and patches of inflammation extending throughout the intestines. The others suffered from dulness, colicky pains, and purging ; the pulse was upwards of 70 , and wiry; the extremities cold; the visible mucous membranes highly injected. One mare was ill for three or four days. The treatment consisted of opiates and lime-water (Veterinarian, 1858).

Eleven cart horses were poisoned at Edgeware from drinking water containing arsenic. They had been drawing heavy loads of building materials fully eight miles from London, were tired, and their stomachs empty, which doubtless accounts for the rapid and serious results. Although 
arsenic is not known to have proved fatal in the human subject under seven hours, one of the horses dropped and died ten minutes after drinking, and several were dead within an hour. The symptoms were colic, staggering gait, pallid membranes, cold ears, pulse 40 to 60 , quickened breathing, and latterly coma. Brandy and ammonia were the remedies prescribed (Veterinarian, 1874).

Cattle take with less danger even larger doses than horses, for the comparatively insoluble poison mixes with the food in the rumen, and hence slowly reaches the abomasum and intestine, while the small amounts thus gradually introduced into the circulation are continuously excreted, and toxic effects are delayed or prevented. Blake records the death of fourteen in-calf cows, which had an arsenical dip powder mixed with their hay. Symptoms of poisoning appeared in twenty hours, and consisted of 'acute abdominal pains, profuse diarrhœa, heavy breathing, staggering gait, cold extremities,' etc. 'Thirteen of the cows died within three days, while one survived a week (Veterinarian, 1892). Kaufmann fixes the toxic dose at four to eight drachms.

Sheep are poisoned by 75 to 90 grains. A strong sheep received an ounce of arsenic in water containing a handful of common salt, and after exhibiting most of the symptoms mentioned as occurring in horses, died on the fifth day. Hertwig states that 5 to 10 grains given in solution to healthy sheep produced gastro-intestinal irritation; that a second dose of 10 to 20 grains, given twenty-four hours after, caused death; and that, on examination, the poison was found in the blood, urine, lungs, liver, and muscles. But dogs have eaten with impunity the carcases of sheep poisoned by arsenic.

Dogs, eats, pigs, and poultry are more quickly and powerfully affected than horses and cattle, and, relatively to their weight, exhibit about the same susceptibility as human patients, in whom 2.5 grains is the smallest dose known to have proved fatal. Dogs to which Dun administered 3 to 10 grains in solution within a few minutes exhibited nausea, vomiting, moaning, difficult breathing, a wiry, rapid pulse of 120 or upwards, and shortly passed black fæces with considerable pain; while death with convulsions followed in 
six to thirty hours. Dogs receiving a quarter of a grain to a grain, repeated twice daily, and continued during eight to fourteen days, exhibit gradually diminishing appetite and increased vomiting. From the sixth to the tenth day, diarrhœa, lowered temperature, rapid emaciation, and painful cough ensue, and death occurs in twenty to thirty days. Half an ounce of Fowler's solution injected into the jugular vein of a dog, although it caused immediate vomiting, proved fatal in eighteen hours, and left the stomach and intestines reddened and injected. Kossel gave sodium arsenite to dogs in doses of $1 \frac{1}{2}$ to 3 grains for ten days, and found that the amount of nitrogen excreted rose, even in inanition, to 48 and 60 grains, and in healthy dogs receiving arsenic reached 110 to 120 grains.

Pigs have been poisoned by 1 to 15 grains, and poultry by half a grain to a grain.

Chronic arsenical poisoning, with symptoms of indigestion, thirst, wasting, and chronic disease of the bones and joints, is sometimes met with amongst both cattle and horses near the tin and copper smelting works of Cornwall and Wales. Arsenical green paper left in the way of animals has sometimes been eaten in quantities sufficient to cause death. Rabbits at shows have been destroyed by nibbling the bright green prize cards. An aged donkey is reported to have died in three hours, poisoned by eating green paper.

The post-mortem appearances of poisoning by arsenic, although very similar in all animals, differ with the concentration of the poison and the severity and duration of the case. In the horse the cuticular portion of the stomach is not usually much altered ; but the villous portion is intensely inflamed, especially if the drug has been given in powder. The mucous membrane is softened, easily separated, and sometimes eroded or perforated. Except in rapidly fatal cases, patches of inflammation with extravasation will be found in the small intestine, colon, and rectum. The serous membranes are ecchymosed. In chronic cases fatty degeneration affects the epithelial tissues, liver, heart muscle, and brain, and owing to the antiseptic power of the drug cadaveric changes are retarded. In most cases the lesions are fairly characteristic ; but the discovery of arsenic in the 
organs of the body affords the only positive proof of arsenical poisoning.

In treating acute cases of poisoning, the stomach must be washed out with copious draughts of tepid water, and emptied, in carnivora by emetics, such as mustard or zinc sulphate, in horses or cattle by the stomach pump. The best chemical antidote is prepared by precipitating ferric chloride solution with ammonia, washing the precipitate with warm water, and administering it moist and freshly made. Some authorities recommend precipitation of two to three ounces of liquor ferri perchloridi, with one ounce of sodium carbonate diluted with water; these quantities, freshly prepared, suffice to neutralise ten grains of arsenic, converting it into insoluble iron arsenite. Dialysed iron is nearly as effectual as the ferric oxide or carbonate. These iron antidotes should be given as soon as possible, in repeated doses, at intervals of ten minutes, until a quantity at least twelve times greater than that of the poison has been swallowed. Magnesia in its hydrated or gelatinous form, prepared by precipitating a solution of Epsom salt with caustic potash, also greatly diminishes the solubility of arsenic. Perhaps the best agent is made by adding magnesia to iron sulphate, for this contains the iron hydrate and magnesium sulphate which helps excretion. Insoluble powders, charcoal and clay, envelop the particles of poison, and retard absorption ; but such agents to be of service must be given before, along with, or immediately after the poison. Oils, lard, glycerin, mucilage, lime-water, white of egg, and milk, exercise similar mechanical effect, and some of these bodies also slightly diminish the solubility of arsenic. Demulcents and opium are given to combat gastro-intestinal irritation, whilst collapse is prevented by warmth and stimulants, alcohol, digitalis and strychnine. In chronic cases oleaginous laxatives and enemata relieve griping and constipation; easily-digested, nutritive food helps to sustain the powers of life, and occasional diuretics hasten excretion of the poison by the kidneys.

Medicinal Uses.-Arsenic is administered to animals as a gastric stimulant in dyspepsia and gastralgia, and in chronic catarrhal diarrhœa, where imperfectly-digested food 
is hurried through the intestines. In such cases it is frequently conjoined with antacids and opium. It is often given as an anthelmintic.

Chronic diseases of the air-passages, notably catarrh and ozæna in horses, are often benefited by a course of arsenic. It relieves irritable cough, as well as thick and broken wind. Robertson was confident of its value in roaring; in abating the dyspnœa and cough of thick wind, he enjoined its use daily for ten days or a fortnight, when some other remedy was directed to be substituted; and in broken wind, frequently with advantage, prescribed two to three grains twice a week for months.

As an alterative, modifying tissue changes and improving general nutrition, it has been given in the earlier stages of tuberculosis, and in chronic rheumatism, chorea, and epilepsy. In dourine, trypanosomiasis, and piroplasmosis arsenic is prescribed with iron, or with oil of turpentine. In febrile attacks manifesting periodicity arsenic is sometimes as effectual as quinine. Sir Lauder Brunton believes that its efficacy in phthisis depends upon its hastening the removal of the effused products of pneumonia which form a suitable nidus for the bacillus tuberculosis. In anæmia medicinal doses probably increase both the white and red corpuscles, especially when conjoined, as it usually is in such cases, with iron. When mixed with freshly-drawn blood it retards coagulation and putrefaction, and preserves the globules, and possibly exerts similar effects internally. Full doses freely diluted are prescribed thrice daily, immediately after meals, for dogs suffering from chorea, and are sometimes also serviceable in epilepsy. Its value in these disorders appears to depend on some action on the central nervous system, as yet unexplained. It stimulates the dermis, hastens removal of morbid epidermal cells, and is hence useful in chronic eczema, psoriasis, impetigo, scab, and mange, and in such cases is used both internally and externally. In chronic scaly skin complaints it has been prescribed with mercury and iodine.

In Styria it is eaten by the peasantry, with the view of improving the complexion, producing plumpness, increasing general vigour, and preventing breathlessness in running or 
climbing. In various parts of England, as well as in Southern Europe, small doses are sometimes regularly given to horses, and as it diminishes oxidation, muscle waste, and production of carbonic acid, active exertion is performed with a minimum of fatigue. So long as it is used cautiously and regularly, the animals appear to be in excellent health, and have fine sleek coats ; but when the arsenic is discontinued they fall off in appearance, and for many months are greatly more difficult to keep in condition. This practice should not be tolerated, for it is attended with much risk of poisoning, and is, moreover, liable to injure the horse's constitution.

Externally, arsenic is occasionally used to eradicate warts and destroy fistulæ and malignant tumours. For foot-rot, Williams advised that the affected sheep be slowly driven through troughs containing a tolerably strong solution of arsenic and alkaline carbonate. In solution it is sometimes applied to remove the scurfiness of psoriasis. Used incautiously, it causes sloughing and blemishing, and if absorbed may produce constitutional disturbance. In virtue of its antiseptic properties it is employed in powder or in solution with soap for the preservation of skins and natural history specimens.

For sheep dips arsenic is much used. Such dips destroy ticks and keds more effectually than solutions of tobacco, spirit of tar, alkaline and non-poisonous dips, and are safer and more convenient than mercurial baths or ointments. Two to two and a half pounds of arsenic, with about the same quantities of soda ash or impure sodium carbonate, soft soap, and sulphur, are dissolved in a hundred gallons of water. Three, four, and even five pounds of arsenic are sometimes used without untoward results. In many parts of England, pearl ash or impure potassium carbonate is substituted for the soda ash, and makes a more cleansing and softening ley. Some flock-masters double or quadruple the quantity of soap, which, with the alkaline carbonate, aids in dissolving the arsenic, while the sulphur whitens and softens the fleece, and also for a considerable time prevents attacks of flies, which are further deterred by addition of a pint or two of naphtha, or of impure carbolic acid. The ingredients 
are best dissolved in five to ten gallons of boiling water; cold water is added to make up a hundred gallons, which, with careful dripping, will dip about a hundred sheep. The sheep, held in the dip for forty to sixty seconds, is lifted on to a sparred drainer placed over a second tub, or over a trough communicating with the dipping tub, and the wool well squeezed with the hands, or with a scraper. The head must of course be kept out of the dip.

Serious and fatal consequences sometimes, however, result from the use of arsenical dipping mixtures. A Lincolnshire breeder, twenty hours after dipping 150 half-bred Leicester sheep, lost eleven, and several some days later. A greatly more serious case occurred at Burton, in Northumberland, during the summer of 1858. Mr. Black of Burton, purchased from Mr. J. Elliot, chemist, Berwick-on-Tweed, fifteen packets of dipping mixture. Every packet contained twenty ounces each of arsenic and soda ash, and two ounces of sulphur, and was directed to be dissolved, with four pounds of soft soap, in three or four gallons of boiling water. With forty-five gallons of cold water subsequently added, this made sufficient for fifty sheep. Mr. Black had 869 sheep dipped in the usual manner; the apparatus and arrangements were good, and the dripping was satisfactory. In two days, however, the sheep began to die; they were seized much in the same order as they had been dipped, and within a month 850 had perished. In many cases the symptoms appeared suddenly, and Mr. Bird, veterinary surgeon in attendance, records that several died in twenty minutes after he had observed them eating or ruminating, and apparently well. The symptoms were dulness and nausea, frothing at mouth, bloodshot eyes, pain in the bowels, discharge of black and bloody urine, laboured breathing, blackening of the skin, with the wool falling off in patches, especially about the back and loins. Post-mortem examination discovered the bowels inflamed, and covered with patches of extravasated blood, the lungs blackened and inflamed, the liver, black, soft, and friable, the spleen congested, the bladder empty. On analysis arsenic was found in the stomachs and bowels.

The case came to trial at Newcastle in February 1859, and 
the jury found a verdict for Mr. Black, with damages amounting to $£ 1400$. Mr. Black's case rested mainly on the fact that his sheep had been carefully dipped in the usual manner, and according to the printed instructions sent with each packet of the dipping mixture. It was sought to be proved that the mixture might in some way have been improperly made up, and was of such strength that the poison had become absorbed through the skin. The poisoning of a donkey, which had carried the skins of the dead sheep, some sores and gangrenous patches on the hands and arms of several of the men employed in the dipping, were also adduced as evidence of the undue strength of the mixture.

In defence of Mr. Elliot, it was shown on the other hand, that thousands of sheep had without accident been dipped in mixtures of the same strength as that sold to Mr. Black ; that, indeed, on the same day as the Burton sheep were dipped another gentleman in the neighbourhood, without any bad effect whatever, used eight packages of the same mixture made in the same way and at the same time. John Gamgee and Stevenson Macadam made various experiments, using, in two instances, arsenic in the proportion of 28 and 68 ounces for fifty sheep, instead of the 20 ounces present in Mr. Elliot's dip. An Oxfordshire sheep-dipper, who annually passed through his hands several thousand sheep without losing one, for years employed $2 \frac{1}{2} \mathrm{lbs}$. of arsenic for fifty sheep, or exactly double the strength of Elliot's mixture. In 1859, Dun made experiments with dips three and four times the strength of Elliot's ; some of the sheep were immersed for several minutes, and had these concentrated solutions well rubbed into the skin. Dun abstained in several instances from pressing or drying the wool, dipped the same sheep twice within two hours, and several times within a week, and yet failed in destroying or injuring in the smallest degree any one of the sheep subjected to these several trials. These experiments were subsequently repeated and verified.

Arsenical sheep-dipping mixtures obviously are not absorbed through the sound skin. Their danger depends on the poisonous fluid being retained by the fleece, from which it drips on the grass or other food over which the animals 
stray. In this manner undoubtedly the serious mortality at Burton can be explained. The sheep were rapidly dipped at the rate of eighty per hour ; and, according to the usual calculation, each sheep carries away in its fleece, even after it has been reasonably drained, about a gallon of the fluid, which, of Elliot's strength, would contain nearly 200 grains or arsenic-a quantity sufficient, if swallowed, to poison several sheep.

Sheep after dipping are turned out hungry, and at once begin to eat; while the drippings fall on the grass, which in the Burton case, appears to have been still further contaminated by rain, during the night following the dipping, freely washing the solution out of the fleeces on to the pastures. Here it was found in three sods, removed ten days after and examined by Douglas Maclagan, who failed, however, to find any arsenic in sods brought from an adjoining pasture, where no dipped sheep had grazed. It is obvious how the donkey, two oxen, and two horses shared the fate of the sheep ; whilst the drippings, left in the yards before the flock was turned out, would account for the alleged mortality amongst the poultry.

It is an error to suppose that sheep, pigs, or other animals refuse to eat food over which arsenical dipping mixtures with their nauseous soft soap and alkali have fallen. Horses, pigs, and poultry sometimes die from gaining access to yards where recently-dipped sheep have been confined. Two colts were poisoned by eating vetches carelessly left in a yard where some sheep had been placed to drip.

The practical precautions suggested by such cases are :Yards into which freshly-dipped sheep are to be turned should previously be cleared of all green food, hay, and even fresh litter; if perfectly empty they are still safer. When the dipping is finished, they should be cleaned, washed, and swept, and any of the unused dipping solution at once poured down the drains. Obviously, however, no such poison should be run into drains emptying into pools or streams accessible to live stock. Dipped sheep should remain, if possible, in an airy, exposed place, as on a dry road, or in a large open yard. Overcrowding should be avoided, and every facility given for rapid drying, which is greatly expedited by fine, clear, 
sunny weather. On no account should sheep be returned to their grazings until they are dry, and there is no risk of their poisoning the pastures.

Doses, etc. Of Arsenic.-Horses and cattle take grs. ij. to grs. $x$. ; sheep, gr. j. to grs. ij. ; and dogs, gr. $\frac{1}{15}$ to gr. $\frac{1}{10}$. When it is desired to produce local action on the stomach and intestines, small doses are given before food; when its absorption is required, it is administered immediately after meals. It is usually given once daily, and continued for a week or ten days, when a change of prescription is often desirable. When it causes acceleration or hardness of the pulse, injection of the conjunctiva, indigestion, diarrhœa, or other physiological action, the drug should be discontinued, or the dose materially reduced.

It is most active and uniform in its effects when prescribed in solution with diluted acid or alkali. The Liquor Arsenici hydrochloricus contains one grain of arsenic in 110 minims. Liquor Arsenicalis or Fowler's solution-made with arsenious anhydride, potassium carbonate, compound tincture of lavender and distilled water-is the preparation most frequently used in veterinary practice. It contains one grain of arsenic in 110 minims or about $4 \frac{1}{2}$ grains to the ounce. The dose for horses and cattle is from half an ounce to an ounce and a half. Dogs may be given 2 to 10 minims.

The Liquor Arsenii et Hydrargyri Iodidi or Donovan's solution, of which 110 minims correspond to one grain each of arsenious iodide and mercuric iodide, is useful in chronic skin and rheumatic complaints. The dose for horses and cattle is $3 \mathrm{iv}$. to $3 \mathrm{j}$. Arsenical preparations are frequently administered in the drinking water, or in the mash. For hypodermic or intra-tracheal injection minimum doses of Fowler's solution diluted with two parts of distilled water may be prescribed.

Cacodylic acid (Dimethyl arsenic acid, $\mathrm{AsO}\left(\mathrm{CH}_{3}\right)_{2} \mathrm{HO}$ ) is an arsenical compound in which the arsenic is in organic combination. It contains 54 per cent. of arsenium. It is a white crystalline substance, soluble in four parts of alcohol, and readily soluble in water. Its salts, cacodylate of soda or sodium dimethylarsenate, which is very soluble in water and cacodylate of iron, have been employed in the treatment 
of tuberculosis, trypanosomiasis, piroplasmosis, and certain skin diseases. The cacodylates are believed to promote assimilation and to improve nutrition, and in anæmia the sodium salt is said to increase the number of red corpuscles in the blood. In dourine hypodermic injections of sodium cacodylate, grs. $x v$. to grs. $x x x$., increasing to grs. xlv. per day, have given very good results.

DoSEs (sodium cacodylate).-Horses, grs. viii. to grs. xxx. ; dogs, gr. $\frac{2}{3}$ to gr. $1 \frac{1}{2}$ per day, hypodermically. These doses freely diluted may be administered daily for five to ten days, then discontinued for a week, and repeated if necessary.

Atoxyl, the sodium salt of para-aminophenylarsenic acid $\mathrm{NH}_{2} \mathrm{C}_{6} \mathrm{H}_{4} \mathrm{AsO}(\mathrm{OH})_{2}$, a white crystalline powder, soluble in six parts of cold water, and in 125 parts of alcohol; and arrhenal or disodium methylarsenate, a colourless crystalline salt, very soluble in water, have been prescribed in the treatment of sleeping sickness in man, and of the various trypanosomiases (dourine, nagana) and piroplasmoses in animals. Koch states that atoxyl in trypanosomiasis is equal in value to quinine in malaria. Arrhenal, given as a substitute for cacodylate of soda, has proved very beneficial in the treatment of bovine and canine piroplasmosis. Atoxyl is much less toxic than arsenious anhydride ; and as it is decomposed in the stomach it should be administered subcutaneously. Foals infested with sclerostomum tetracanthum have been successfully treated by atoxyl administered subcutaneously in doses gradually increasing from three grains to fifteen grains per day.

The administration of atoxyl to the dog requires great care. Woodridge gave a bulldog one cubic centimetre of a 10 per cent. solution on alternate days, and on the day following the fourth dose the dog refused food and had a rolling gait ; next day the dog's temperature was subnormal ( $97^{\circ} \mathrm{Fahr}$.), his skin was livid in patches, and he died during the following night.

Doses, ete. (atoxyl).--Horses, grs. ii. to grs. iv., gradually increasing to grs. $x$. or grs. xii. per day, in at least twenty parts of sterilised solution of sodium chloride. Before injection the solution should be warmed to blood heat; and the minimum dose may be injected daily for a week, then 
the dose may be cautiously increased until toxic symptoms appear, when the medicine should be suspended for a few days, or if desirable, the injections may be continued in diminishing doses until the degree of tolerance peculiar to the patient has been ascertained. Then the optimum dose may be given once a week for the remainder of the treatment.

Doses of arrhenal, horses, grs. viii. to grs. xxx.; dogs, gr. $\frac{1}{2}$ to grs. ii. per day, given hypodermically in twenty parts of boiled water.

\section{MERCURY AND ITS MEDICINAL COMPOUNDS}

Mercury. Hydrargyrum. Quicksilver. $\mathrm{Hg}$.

From its liquidity and volatility this metal is named mercury; to its silvery appearance it owes its synonym hydrargyrum; to its liquidity and metallic lustre such appellations as aqua argentum, aqua metallica, and quicksilver. Although occasionally found in metallic globules, its most important source is the sulphide or cinnabar ( $\mathrm{HgS})$. When the ore is roasted or heated with iron or lime, sulphur is got rid of and mercury distils over.

Mercury is easily distinguished by its liquidity and silverwhite lustre. It is tasteless and odourless; freezes at $-40^{\circ} \mathrm{Fahr}$., forming octahedral crystals ; slowly volatilises at all temperatures, and boils at $662^{\circ} \mathrm{Fahr}$., forming a dense, colourless gas. Its specific gravity at $60^{\circ}$ is $13 \cdot 6$, its atomic weight 200 . Triturated with fatty or saccharine substances, as in the preparation of mercurial ointments, liniments, and pills, the metal loses its fluidity and globular structure, is reduced to the condition of a dark-grey powder, while a small portion is oxidised.

Mercury forms two series of salts-the mercurous and the mercuric.

Mercurous salts in solution treated with hydrochloric acid, produce a white precipitate of calomel $\left(\mathrm{Hg}_{2} \mathrm{Cl}_{2}\right)$. Silver and lead have similar white, insoluble chlorides ; and these three are distinguished by their reaction with ammonia solution, which blackens the mercurous chloride, dissolves the silver chloride, but leaves the lead chloride unaltered.

Mercuric salts treated with hydrochloric acid give negative 
results. To this acidulated solution hydrogen sulphide, cautiously added, throws down a white precipitate $\left(\mathrm{HgCl}_{2}+\right.$ $2 \mathrm{HgS})$, which, on further addition of the precipitant, becomes brown and then black (HgS), and is insoluble in dilute acids and ammonium sulphide. Caustic potash yields a yellow precipitate of oxide $(\mathrm{HgO})$, insoluble in excess. Potassium iodide gives a precipitate yellow at first, but rapidly becoming red $\left(\mathrm{HgI}_{2}\right)$, soluble in excess of the potassium salt.

ACTIONS AND USES.-Mercury, mercurous salts, and mercuric salts differ in their local effects, largely depending on their solubility. Metallic mercury has no topical irritant action, but is slowly absorbed, and produces its general effects if finely divided, as in the case of mercurial ointment, or in the state of vapour. The chief mercurous salts are insoluble in water, and are but slowly dissolved by the tissues. Hence they have a very slight topical action, and are only slowly absorbed. But the more soluble mercuric salts readily unite with albumin, the albuminate is dissolved by excess of proteid, and so these salts are corrosive, act as irritant poisons, and are quickly absorbed. When absorbed, all mercurials produce, however, the distinctive actions of the drug, and if continued, the condition of mercurialism. Calomel, grey powder, and blue pill, are prescribed to develop mild mercurialism, to lessen putrefaction in the intestine, and to act as purges. They are cathartics, increasing both secretion and peristalsis by the irritation they set up, and especially when conjoined with a small amount of any cathartic. They are also diuretic. Corrosive sublimate, the biniodide, and the double cyanide of mercury and zinc are effectual germicides. Ointments of mercury and of mercuric iodide are applied as antiparasitics, absorbents, and counter-irritants. Mercurials are excreted chiefly by the kidneys and intestine, but also in the saliva, bile, and sweat. They pass into the milk of nursing females ; and Gasparin has seen lambs die from mercurialism when the ewes had been freely dressed with ointment. Elimination begins tolerably rapidly, but is slow and irregular, and is often not completed for months after administration has ceased.

Mercury, so long as it remains uncombined, like other 
metals is devoid of physiological action. Several pounds which were formerly given to human and veterinary patients for the removal of obstruction of the bowels, produce only mechanical effects. In a state of fine division it is, however, oxidised and dissolved, and hence acquires activity. Mercurial vapours thus speedily become poisonous, as was strikingly illustrated in the case of the Triumph man-of-war and the Phipps schooner, which received on board several tons of quicksilver, saved from the wreck of a vessel near Cadiz in 1809. From the rotten bags the mercury escaped, and within three weeks two hundred men were salivated, two died, and all the animals-cats, dogs, sheep, fowls, a canary bird, and even the rats, mice, and cockroaches were destroyed (Pereira). Men working with the metal frequently suffer from mercurial poisoning. Out of 516 workmen employed at the quicksilver works at Idria, 122 were, in 1856, affected with dyspepsia, scrofula, anæmia, neuralgia, mercurial gout, tremor, and caries. The finely-divided mercury so pervaded the atmosphere that cows feeding in the neighbourhood of the furnaces suffered from excessive secretion of saliva, became unthrifty, and aborted; the calves were also often ailing; while trout in adjacent reservoirs, contaminated by the waste products of the furnaces, lost their red spots and became sickly.

Mercurialism or chronic poisoning in all animals consists in a persistent condition of anæmia, prostration, and wasting ; secretion and excretion are increased. The abundant flow of saliva so notable in man is not, however, observed to the same extent amongst the lower animals. The mouth becomes tender; the gums red, soft, and swollen; the breath fœtid. There is impaired appetite, nausea, gradual loss of condition, œdema, and general weakness. The heart action is enfeebled and respiration impaired. The fæces, increased in quantity, are largely mixed with mucus, and are very offensive. There may be diarrhœa and attacks of colic. The joints are often stiff and painful. The urine is often increased, and it may contain albumin and casts. There is marked pallor of the mucous membranes, anæmia, and a fast, weak pulse. In man there occur peculiar tremors, passing into paralysis, and affecting certain muscles and groups of muscles. Percivall 
and Williams describe a red skin rash resembling red-mange, and occurring especially in cattle and dogs.

Different species of animals show a diminishing susceptibility to mercurialism in the following order: birds, cats, sheep, cattle, dogs, pigs, and solipeds (Kaufmann). Although generally less susceptible, individual horses exhibit different degrees of susceptibility. Percivall mentions that ten grains of calomel given daily to a four-year-old horse made the mouth so sore by the fifth day that he 'cudded' his hay ; while a mare received six drachms of calomel, two ounces of blue pill, and had mercurial ointment well rubbed into her thighs, without suffering either from sore mouth or salivation. Mercurialism occasionally results from one large dose, if this is slowly absorbed, when it is apt to be violent and difficult to control, but is induced more certainly and with less danger by small and repeated doses of calomel, or any mild mercurial, and its production is hastened by using the medicine both externally and internally.

Acute mercurial poisoning is caused by the soluble salts such as the perchloride. There are all the signs of acute gastro-enteritis, and then collapse and death from exhaustion in from a few hours to a few days. If the latter, characteristic signs of mercurialism are seen before death.

The patient under the effects of mercury must be protected from cold and wet. To arrest excessive action the drug must be withheld; its excretion hastened by administration of potassium iodide, followed by a saline purge ; the mouth, if sore, washed repeatedly with solution of chlorinated lime or alum; anæmia and wasting combated by good food, iron salts, and quinine. In acute poisoning by irritant mercurial salts, the preliminary treatment consists in repeated full doses of albumin and other demulcents. Common salt helps the solution of mercury, and should not be used as an emetic in poisoning with mercury salts.

\section{Mercury Ointment. Unguentum Hydrargyri.}

Mercury or blue ointment of good quality cannot be made on the small scale without immense labour and loss of time. It is composed of one pound of mercury, one pound of lard, 
and one ounce of suet. Trituration is continued until metallic globules cease to be visible. A good ointment contains about one per cent. of its mercury in a state of oxide, and infriction and exposure during application increase this more active oxidised portion, which is dissolved by the salts and fatty acids of the skin. This strong ointment is usually diluted with two or three parts of hog's lard or soft soap ; or used in the form of unguentum hydrargyri compositum, which contains ten parts mercury ointment, six parts each of yellow wax and olive oil, and three parts of camphor.

ACTIONS AND USES.-Mercurial ointment, when merely laid on the surface of the skin, exerts slight topical effect, and is only very gradually absorbed. When applied with smart friction it is more quickly absorbed, and, in considerable amount, irritates, vesicates, and induces constitutional effects. Two ounces of the strong ointment, rubbed daily into the skin of a horse, salivates in four or five days: but according to Bouley, three ounces may, without fatal effects, be thus applied daily for a week. Lafosse produced, however, grave constitutional effects in cattle by rubbing three ounces into the throat (Kaufmann).

Mild dressings encourage the healing of indolent ulcers, and promote a healthier action in psoriasis and other persistent scaly skin disorders. For the successful treatment of psoriasis and allied complaints, Williams recommended the internal as well as the external use of liquor arsenii et hydrargyri iodidi. Although not superior to many safer remedies, it is sometimes used for destroying ringworm fungi, and killing lice, the acari of mange and scab, and other skin parasites, and for such purposes is frequently conjoined with tar oils, sulphur, or iodine. As a counter-irritant and absorbent the strong ointment is employed in reducing swelling of joints and tendons.

Mercurial ointment is apt, however, to be used too freely and indiscriminately. When incautiously used it not only causes undue irritation, but is apt to produce poisoning after being absorbed. Many cattle and sheep, dressed for skin complaints and skin parasites, suffer from mercurial poisoning, and some of these cases terminate fatally. In Lincolnshire a lot of scabby sheep were treated with blue 
ointment, and forty died, with symptoms of short breathing, a peculiar grunt indicative of pain, and drooping head (Taylor). The maximum amount of the strong ointment to be used for horses is two ounces, for cattle an ounce, for sheep half an ounce, for dogs thirty grains to a drachm. For topical purposes a second dressing, if required, should not be applied for two or three days.

\section{LinIment of Mercury. Linimentum Hydrargyri.}

Mercuric Oleate. Hydrargyri Oleas.

One part of mercury ointment, one-third part of strong ammonia solution, and one and a half of camphor liniment, thoroughly mixed, constitute the B.P. liniment. Its effects are similar to those of the ointment. Two liniments suitable for persistent scaly skin diseases, enlarged glands, and chronic indurations are subjoined :-

Mercurial ointment, 2 ounces.

Camphor, . . 1 drachm.

Oil of tar, . . 4 ounces.

Linseed oil, . 4 ounces.
Mercurial ointment, 2 ounces. Creosote, . . 1 drachm. Liquor ammoniæ, 2 ounces. Linseed oil, . . 6 ounces.

The oleate is prepared with one ounce mercuric chloride, two ounces hard soap, one drachm oleic acid, and a sufficient quantity of distilled water. One part mixed with three parts of benzoated lard is diffusible and penetrating. It promotes absorption, and, used repeatedly, acts as a counterirritant. It is applied in articular inflammation, exostoses, indurations of the udder, and other glandular and indolent swellings. Diluted solutions destroy ringworm and favus, and are also fatal to animal parasites.

\section{Mercurial Plaster. Emplastrum Hydrargyri.}

Is made with olive oil, 56 grains ; sulphur, 8 grains ; mercury, 3 ounces ; and lead plaster, 6 ounces. The olive oil is heated, and the sulphur gradually added until they unite. With this mixture triturate the mercury until globules are no longer visible; then add the lead plaster, previously liquefied, and mix thoroughly (B.P.). This plaster is occasionally used as a stimulant for glandular and chronic enlargements, wind-galls, and other bursal swellings. 
Mercury with Chalk. Hydrargyrum cum Creta.

Powder.

Grey

This mixture is made by triturating together one part mercury with two of prepared chalk until globules disappear, and a uniform grey colour is acquired. Grey powder should be free from grittiness, and insoluble in water ; but its chalk dissolves in hydrochloric acid, leaving the mercury in a finely-divided state.

Grey powder is antacid, laxative, and alterative. It is given with good effect to young calves and foals suffering from indigestion and diarrhœa, in doses of five to fifteen grains, repeated once or twice a day, conveniently placed on the patient's tongue, or administered with a drachm of ginger, in spirit and water, milk, or gruel. To allay gastric irritation, caused by constipation and putrefactive changes in the intestine, and as an alterative for dogs, one to three grains are prescribed two or three times daily; but such treatment is only advisable for robust patients.

\section{Mercury or Blue Pill. Pilula Hydrargyri.}

This pill, so much used in human medicine, is seldom employed in veterinary practice. It consists of two parts mercury, three of confection of roses, and one of liquorice root. Mayhew recommended, as a cathartic for a mediumsized dog, five grains blue pill, six grains powdered colchicum, and ten grains colocynth extract. Five grains blue pill and eight grains compound extract of colocynth, flavoured with a few drops of oil of peppermint or of cloves, make a convenient laxative for a large dog, or two or three doses for a small one. The activity of mercury pill in part results from its containing a small proportion of oxide, which is readily dissolved by the acid gastric juice.

\section{Mercurous Oxide. Hydrargyri Suboxidum. $\mathrm{Hg}_{2} \mathrm{O}$.}

Mercurous, black, grey, or lower oxide is prepared by decomposing calomel $\left(\mathrm{Hg}_{2} \mathrm{Cl}_{2}\right)$ with caustic potash solution, and washing with water. It is a heavy black powder, devoid of taste or odour, insoluble in water and alkalies, but soluble 
in nitric and acetic acids. It is unstable, and on exposure to light or gentle heat decomposes into mercuric oxide and metallic mercury. It is less active than the mercuric oxide, and is frequently used as a stimulant for unhealthy wounds and ulcers, in the form of the black wash (lotio hydrargyri nigra), made by mixing 30 grains calomel, half an ounce of glycerin, $1 \frac{1}{4}$ ounces of tragacanth mucilage, and sufficient lime-water to make 10 ounces of lotion.

Red Mercuric Oxide. Hydrargyri Oxidum Rubrum. Red Precipitate. $\mathrm{HgO}$.

Yellow Mercuric Oxide. Hydrargyri Oxidum Flavum.

Red mercuric oxide is obtained by heating mercurous nitrate until acid vapours cease to be evolved. Yellow mercuric oxide is obtained by the interaction of mercuric chloride and sodium hydroxide (B.P.). The red oxide occurs in orange-red crystals, which become yellow when powdered and brownish-black when heated, recovering, however, their original colour on cooling. It dissolves sparingly in water, but readily in hydrochloric acid. The yellow oxide is contained in lotio hydrargyri flava or yellow wash.

Mercuric oxide is nearly as active and irritant as corrosive sublimate. Two or three grains given to dogs cause fatal gastro-enteritis. Eight to fifteen grains caused colic in horses, and one or two drachms enteritis and death (Hertwig). It is applied externally as a stimulant and caustic for indolent ulcers, over-luxuriant granulations, chronic scaly eruptions, and glandular enlargements, being used in the form of powder, lotion, or ointment. Red precipitate ointment is made with $\frac{1}{4}$ ounce mercuric oxide and $2 \frac{1}{4}$ ounces of yellow paraffin ointment.

Mercurous Chloride. Hydrargyri Subchloridum. Calomel. $\mathrm{Hg}_{2} \mathrm{Cl}_{2}$.

Calomel is found native in Carniola and Spain, but in too small amount to be of commercial value. It is obtained by heating a mixture of mercurous sulphate and sodium 
chloride. The calomel, rising in vapour, may be condensed in fibrous cakes on the cool part of the vessel, or conducted into a large chamber, where it falls in fine powder. Traces of corrosive sublimate are removed by washing with boiling distilled water. The powder is dried at a temperature not exceeding $212^{\circ}$ Fahr.

Calomel is a dull-white, heavy, and nearly tasteless powder, sometimes rendered yellowish by prolonged trituration. It is inodorous, insoluble in water, alcohol, or ether. It is slowly decomposed by light; volatilises unchanged when heated; and yields mercurous oxide when acted on by alkalies or lime water.

ACTIONS AND UsES.-Calomel, being insoluble in water, has no in-contact effect on the skin, but it is in part dissolved by the gastric secretions. It irritates the gastric membrane and glands, full doses producing emesis in man and carnivora. Similar irritation is exerted on the mucous membrane of the small intestines, and it is consequently a cathartic. It does not increase secretion of bile, but, acting as an indirect cholagogue, it removes bile lodged in the duodenum. Like other mercurials, repeated doses cause mercurialism, and increase secretion of the salivary, pancreatic, and intestinal glands, and also of the kidneys. The earlier stages of mercurialism are produced, with the view of altering metabolism and nutrition, and promoting especially absorption of fibrinous exudation. Externally, it is used as a desiccant, stimulant, and anti-parasitic.

General Actions.-Tuson showed that calomel and distilled water, digested in a glass vessel at $100 \cdot 2^{\circ} \mathrm{Fahr}$. (the temperature of the stomach), when mixed with either pepsin or a 2 per cent. solution of hydrochloric acid remained unaltered, even after twenty-four hours ; but when both pepsin and hydrochloric acid were used, solution speedily occurred, and a black precipitate of mercury sulphide was formed. The mucus and fats of the canal, and also the bile, further aid solution. It is chiefly absorbed as an albuminate, which, although insoluble in water, dissolves in excess of albumin and in sodium chloride solution. Small proportions even of small doses of calomel are absorbed, and most of that absorbed, as well as that unabsorbed, is excreted by the bowels 
in the state of metallic sulphide and sulphate exerting an antiseptic effect, and usually imparting a green colour to the fæces by preventing decomposition changes in the bile. The opinion that calomel undergoes partial conversion into the higher chloride before absorption is not supported by evidence, and such a change is unnecessary, for the proteid present is sufficient to dissolve it and form the albuminate. It is likely that leucocytes may take up particles of the salt and transport them through the bowel wall, just as they do when calomel is injected subcutaneously.

Toxic EFFEcTs.-Calomel is less irritant than mercuric chloride or nitrate, but irritant effects, usually followed by constitutional disturbance, are produced in horses by three or four drachms; in cattle by two or three drachms; in sheep by fifteen to thirty grains ; in dogs by six to thirty grains. Hertwig found that these doses, within twenty-four or thirty-six hours, and in dogs in less time, caused occasional colic and copious discharge of fæces, which contained bile, and were greyish-green in cattle, but darker in dogs. Such doses, especially if repeated daily for three or four days, further induce fluid and stinking evacuations, fœtid breath, soreness of the mouth, rapid impairment of appetite and condition, and fatal low fever and dysentery.

At the Edinburgh Veterinary College a healthy donkey was given a drachm of calomel daily in three separate doses. About the sixth day the animal became excitable, and the pulse rose to 85 . By the eighth day secretion of saliva was augmented, the breath was fotid, the gums red and tender, and appetite impaired; but the fæces and urine were normal. By the twelfth day these symptoms became more aggravated, the pulse softer and less frequent, the strength much reduced. On the fourteenth day administration of the calomel was suspended, but death occurred two days later. The animal had received fourteen drachms in fourteen days. Postmortem examination discovered the teeth loose, the mucous membrane of the mouth and air-passages blanched, while that of the stomach and intestines was softened, easily torn, and in many places thickly covered with mucus. The liver was rather friable, but the kidneys, spleen, and lungs were healthy. 
Dogs weighing $30 \mathrm{lbs}$. to $40 \mathrm{lbs}$. receiving three or four grains night and morning were salivated in a week, and died in nine days. The most notable appearance was inflammation of the large intestine. Hertwig considers that dogs and swine, on account of their often getting rid of the drug by vomiting, are less easily affected than the other domesticated animals, and that horses are less susceptible than cattle.

The action of calomel on the liver was investigated by a Committee of the British Medical Association. They experimented chiefly on dogs with fistulous openings into the duodenum, and arrived at the conclusion that neither calomel nor blue pill affects secretion of bile, unless it purges or impairs health, when the quantity of bile is diminished. Röhrig curarised dogs, maintained life by artificial respiration, and placed a glass tube in the gall duct. Calomel, even in twenty-grain doses, did not re-establish the secretion when it had ceased, but had a marked power in increasing and maintaining it beyond the natural time for its cessation.

W. Rutherford and Vignal, also experimenting on curarised dogs, found that doses of ten, five, or even two grains of calomel, placed in the duodenum of fasting subjects, produced purging; they did not, however, increase secretion of bile, but actually diminished it. These experiments justify the conclusion that calomel and mercurials have no special cholagogue action; they do not stimulate secretion of bile, as they notably do of saliva and pancreatic fluid. The increased intestinal action sweeps out bile lodged in the duodenum, as well as in the hepatic ducts, and thus prevents its reabsorption, and, moreover, abates congestion of the portal system. Calomel, therefore, although not increasing secretion of bile, notably hastens its expulsion.

Medicinal Uses.-Few remedies have been applied to so many and diversified uses, but neither in veterinary nor in human medicine is it as much used as formerly. Gastric irritation, intestinal catarrh, as well as bilious diarrhœa, are frequently treated with small doses, either used alone or conjoined with chalk or opium. For foals and calves calomel may be substituted for grey powder, when the bowels are 
irregular and the discharges malodorous. In such cases, like other mercurials it owes its good effects to its combination of antiseptic and eliminative properties. Although useful in biliousness and congestion of the liver, and, along with laxatives, removing waste bile from the duodenum, it has no special remedial effect in jaundice, arrested secretion of bile, or chronic liver complaints. Such cases are fittingly treated by nitro-hydrochloric acid and other liver stimulants. That type of influenza in horses complicated with gastric derangement and yellow mucous membranes is often successfully treated in the early stages with calomel grs. xx., and opium 3ss., with or without oil, repeated twice or thrice, at intervals of twelve hours, and alternated with or followed by salines.

It is a useful adjuvant eathartic. Four drachms of aloes, with half a drachm of calomel, purge most horses as effectually as eight drachms of aloes. Such a combination is advantageously used in lymphangitis and other cases where prompt and full catharsis is desired. But as an adjuvant purgative it is more serviceable in cattle than in horses. As an anthelmintic, combined sometimes with santonin, and followed by a laxative, it removes entozoa. In acute rheumatism calomel is given with a purgative; in chronic cases, and also in malarial disorders, small doses are sometimes used with quinine sulphate. Dogs and pigs frequently have calomel conjoined with jalap or with oils as a cathartic and febrifuge, and occasionally with ipecacuanha as an emetic.

As an alterative and febrifuge it is not as much used as formerly, but some practitioners still prescribe it in acute localised inflammation, particularly in pneumonia, pleurisy, peritonitis, laminitis, iritis. It is most effectual when such cases are complicated with gastric derangement, and its curative action probably depends upon its combination of antiseptic, cathartic, and diuretic effects, these latter being increased by the laxatives and salines with which it is usually conjoined. In chronic inflammation it reduces enlarged glands, removes recently formed deposits, and sometimes prevents adhesions, in iritis and pleurisy. For such objects, small, frequently repeated doses are used, conjoined with 
opium to delay excretion, while constitutional effects may be hastened by in-rubbing of the ointment. In muco-enteritis, whether in horses or cattle, Barlow sometimes used half a drachm of calomel with an ounce of laudanum, repeated at intervals of one or two hours, until three or four doses were taken. Calomel, chalk, and opium are frequently prescribed in dysentery.

Calomel has diuretic effects; it notably increases the diuresis caused by resinous or saline drugs.

Externally, calomel destroys the acari of scab and mange, kills lice, abates the itching of those eczematous eruptions of the limbs of underbred cart horses, and of eczema in dogs. Although of small benefit in soothing the itching of urticaria, it relieves the irritation of prurigo, removes the scales and heals the cracks of psoriasis, hastens removal of warts, and is one of the best remedies for thrush in the horses' frog. It must be used discreetly, for if freely applied it may be absorbed, and cause untoward constitutional effects.

DoSEs, etc.-As an alterative and febrifuge, horses and cattle take grs. x. to 3 i. ; sheep and pigs, grs. v. to grs. xxx. ; dogs, gr. i. to grs. iij., usually given two or three times a day, frequently with an equal weight of opium, to prevent too rapid removal by the bowels. As a cathartic, calomel is not used alone, and the dose is consequently regulated by that of the drug with which it is combined. For the horse a full purgative consists of calomel $3 \mathrm{i}$., with aloes $3 \mathrm{iv}$.; for cattle, 3i. to $3 \mathrm{ij}$., with Epsom or common salt, lb. 1, or oil, Oj. ; for dogs, grs. ij. to grs. iv., with jalap, grs. xx. to grs. xl. As a vermifuge for the horse, the following combination is given before feeding for three or four consecutive mornings :-One drachm each of calomel, extract of male fern, and aloes, with four drachms of ginger, made into bolus with linseed meal and treacle. As an emetic for dogs or pigs, two or three grains are given, with an equal quantity of tartar emetic, or with grs. xv. to grs. xx. of ipecacuanha. To allay irritation, and promote healthy cutaneous action, or to destroy skin parasites, calomel is used in powder, or in ointment, 1 in 10 of vaseline, conjoined sometimes with iodine, boric acid, or wood-tar oils. 
Mercuric Chloride. Hydrargyri Perchloridum. Bichloride of Mercury. Corrosive Sublimate. $\mathrm{HgCl}_{2}$.

Corrosive sublimate and calomel are both chlorides of mercury ; corrosive sublimate contains twice as much chlorine as calomel, is the higher, per-, or mercuric chloride $\left(\mathrm{HgCl}_{2}\right)$, and is a soluble and actively corrosive poison; while calomel, the lower or mercurous chloride $\left(\mathrm{Hg}_{2} \mathrm{Cl}_{2}\right)$, is an insoluble, and therefore comparatively mild agent. By using, in speaking or writing, the vernacular names, risk of mistaking these chlorides is diminished.

Corrosive sublimate may be prepared by heating a mixture of mercuric sulphate, sodium chloride, and a little black oxide of manganese.

It occurs in heavy, colourless masses of prismatic crystals, devoid of odour, but with an acrid, metallic taste. When heated it sublimes without decomposition, leaving only a trace of fixed residue. It is soluble in about three parts of alcohol (90 per cent.), four parts of ether, two parts of glycerin, two of boiling water, and sixteen of cold water, or nearly four grains to the ounce. Its solubility is increased by the presence of certain salts, such as the chlorides of sodium and ammonium. Its antiseptic properties recommend it for preserving anatomical preparations. It is not subject to intentional adulteration.

ACTIONS AND USES.- It is a corrosive and irritant poison ; is occasionally prescribed as an alterative and antiseptic; repeated doses cause mercurialism. Externally, it is used as an antiseptic, astringent, caustic, and parasiticide.

Corrosive sublimate precipitates albumin, the precipitate being dissolved by excess of proteid, and hence in powder or concentrated solution is irritant and corrosive, for the protective scum of albumin is removed, and the drug penetrates farther. Dissolved in water, it is the most energetic antiseptic ; one part in 25250 prevents the development of bacteria taken from meat infusion; one part in 10250 prevents the development of spores in boiled meat infusion ; one part in 6500 prevents reproduction of spores (Koch). Spores placed in one per thousand solution in water are effectually destroyed in one to two days. Solution of one 
to two grains to the ounce of water effectually destroys vegetable and animal parasites infesting the skin. It must, however, be used with caution, for it not only irritates and corrodes locally, but is readily absorbed, and hence liable to produce constitutional effects.

TOXIC EFFECTS.--Swallowed in strong solution it is an irritant corrosive poison, producing gastro-enteritis and collapse. Smaller or more diluted doses produce mercurialism. Seven or eight grains destroyed dogs in seven to thirty hours; four drachms dissolved in three pounds of water killed a horse in twelve hours ; two drachms caused in cattle great emaciation, and death in fourteen days; one drachm proved fatal to a sheep within twelve hours (Hertwig). Larger quantities, however, appear to be tolerated when the poison is first given in small doses. Thus, Percivall, experimenting upon a horse, commenced with ten grains, and gradually increased the dose to five drachms before the appetite or pulse became affected. Injurious effects occur whatever the channel by which poisonous doses enter the body. Shepherds using strong solutions for foot-rot or for scab have suffered from its irritant and also from its constitutional effects. Dogs dressed with it for mange have occasionally died from gastro-enteritis and collapse.

Compared with arsenical poisoning, the acute symptoms with large doses come on more rapidly; there is more chemical and corrosive action, whilst in chronic cases salivation usually ensues.

Post-mortem examination discovers the mucous lining of the alimentary canal softened and bluish-grey ; where large doses have been given it is disorganised by chemical action ; where death is postponed for a day, patches of inflammation -and sloughing are found; the kidneys are inflamed, the tubules plugged with casts, and other urinary organs are congested ; the lungs are usually spotted with effused blood.

The treatment consists in the free use of albumin, which forms an insoluble mercuric albuminate. One egg suffices to neutralise four grains of sublimate. When eggs cannot be had, wheat or barley flour, milk, or other albuminoids should be given, followed by astringent solutions. Unless the drug has caused vomiting, the stomach must be emptied either 
by emetics (avoiding common salt), the stomach pump, or syphon.

Medicinal Uses.-For internal use, milder mercurials are preferred, and it is dangerous to use it for the production of the alterative effects of mercury. For horses it has been prescribed in chronic skin eruptions, and swollen, œdematous legs resulting from repeated attacks of lymphangitis. Half or even a quarter of a grain, repeated every three hours, sometimes arrests the slimy, bloody, reducing discharges of persistent diarrhœa and dysentery in cattle. Conjoined with opium, hemlock, and salines, it has been advised in rheumatism.

As an effectual antiseptic it is used for many surgical purposes. Its antiseptic power is diminished or destroyed in presence of albumin, with which it forms an insoluble albuminate. To prevent this change Laplace suggested the addition of tartaric acid to sublimate lotions. Fifteen grains of corrosive sublimate, seventy-five grains of tartaric acid and thirty-five ounces of distilled water, form a useful antiseptic solution. Foul wounds washed with one part mercuric chloride dissolved in 500 to 1000 of water, are rendered aseptic. Instruments, sponges, towels, as well as the hands, are disinfected by washing in a one to a thousand solution. But sublimate lotions damage most metal instruments, and irritate and roughen the operator's hands. Solutions of average strength destroy the fungi of ringworm, kill lice, and acari, and allay the itching of urticaria. For the lastnamed disease Robertson prescribed mercuric chloride grs. xii., diluted hydrocyanic acid $3 \mathrm{iv}$., glycerin $3 \mathrm{ij}$., and water 3x. A solution of one part in 1000 or 2000 of water is injected into the uterus in metritis, and in cases of abortion. The spread of contagious abortion may be prevented by washing with the solution, twice daily, the external genital organs and the tail of aborted cows. Warm solutions are much more active than cold. An aqueous solution of one part in 500 is used to arrest necrosis in bone and cartilage, and 10 to 15 grains rolled in tissue paper (or a sublimate crayon), and introduced deeply into sinuses or fistulæ in four to eight days, slough out the fibrous walls. As a synovial styptic, sublimate in fine powder is applied to the 
wound in cases of open joint. In conjunctivitis and keratitis a solution containing one-tenth to half a grain per ounce of distilled water is used as an astringent antiseptic.

For most external purposes the B.P. liquor hydrargyri perchloridi containing 10 grains in 20 ounces of distilled water, will be found convenient. Two parts of sublimate and four of glycerin form a 'stock solution,' which may be diluted as required. Van Swieten's solution is composed of one part mercuric chloride, 900 of water and 100 of alcohol. Lotio hydrargyri flava, or yellow wash, is made with 40 grains corrosive sublimate and a pint of lime water. To prevent accidents which sometimes attend the use of uncoloured lotions, pastils, lotiforms, and compressed dises, containing mercuric chloride and sodium chloride and coloured with magenta or methyl-violet, may be used. Wool, lint, gauze, and other wound dressings impregnated with corrosive sublimate dissolved in glycerin or water are frequently employed.

With suitable precautions, watery solutions are used for disinfecting infected premises, and the carcases of animals which have died of anthrax or other contagious disease. Sublimate solution has, however, one great disadvantage in that it is not volatile, but, on the contrary, coagulates albumin, and so forms a protective surface layer, beyond which the drug does not penetrate. Thus it is inferior to phenol for disinfecting anthrax blood, fleeces, or other material containing albumin.

Doses, etc.-Horses and cattle take grs. v. to grs. viij.; sheep and large pigs, gr. j. ; dogs, gr. $\frac{1}{16}$ to gr. $\frac{1}{8}$. It is best given freely dissolved in water or other simple fluid. To relieve itching, especially amongst dogs, one grain corrosive sublimate and one minim prussic acid are dissolved in an ounce of water. An ointment containing one part of sublimate to twenty or thirty parts of fatty matters is sometimes used cautiously for skin complaints, and for destroying skin parasites.

Ammoniated mercury, or ' white precipitate,' is an opaque, white, insoluble powder, made by mixing corrosive sublimate solution with excess of ammonia solution, and washing and drying the precipitate $\left(\mathrm{NH}_{2} \mathrm{HgCl}\right)$. It is devoid of irritant 
action. An ointment, made of one part precipitate to nine of white paraffin ointment, is used as a parasiticide, and as a mild mercurial in skin diseases.

Mercuro-zine cyanide, a combination of mercuric cyanide and zinc cyanide, has been much used in human surgery. It is a white powder, recommended by Lord Lister as a nonirritating and very powerful antiseptic.

Mercurous Iodide. Green Iodide. Hydrargyri Iodidum Viride $\mathrm{Hg}_{2} \mathrm{I}_{2}$. (Not official.)

Mercuric IodIDE. Red Iodide. Hydrargyri Iodidum Rubrum. Biniodide of mercury. $\mathrm{HgI}_{2}$. (B.P.)

Mercurous iodide is an unstable salt, prepared by triturating together iodine and an excess of mercury with a little alcohol, or by mixing solutions of mercurous nitrate and potassium iodide. Although not so active as the higher iodide, twenty grains destroyed a rabbit within twenty-four hours, and a drachm a pointer dog in five days (Cogswell).

Mercuric or red iodide, formed by the interaction of mercuric chloride and potassium iodide, is a vermilioncoloured, heavy, crystalline powder, with a disagreeable metallic taste. It is almost insoluble in water, sparingly soluble in cold alcohol, but soluble in ether, acids, solution of potassium iodide, and most saline fluids.

ACTIONS AND Uses.-Mercuric iodide is as irritant as mercuric chloride or nitrate. But the presence of iodine increases the solubility of albuminates, and, compared with the chloride, the iodide is more quickly absorbed and excreted. Twenty grains given to a rabbit induced gastroenteritis, collapse, and death in twenty-four hours. It is used internally, combined with potassium iodide, to arrest the growths of actinomycosis and scirrhous cord, and solutions are applied externally as antiseptics, and ointments as stimulants, counter-irritants, and caustics.

The biniodide, dissolved in water, by the aid of potassium iodide, in ether, and alcohol is frequently used for the same antiseptic and stimulant purposes and in the same strength as the perchloride. The B.P. ointment, of 20 grains iodide to 480 grains of benzoated lard, is not sufficiently strong for 
veterinary purposes, and a more effective preparation is made by mixing one part iodide with six or eight of lard. This 'red ointment' is very effectual for condensing and reducing splints, spavins, ring-bones, and other bony enlargements. It penetrates deeply, arrests chronic inflammation, and aids removal of chronic swellings and indurations of strained tendons, bursæ, and joints. It is occasionally used as a counter-irritant in sore-throat, chronic cough, and roaring, relieving cases that have resisted milder blisters. When effusion has occurred in pleuro-pneumonia in horses, and other irritants are ineffectual, an ounce of biniodide ointment ( 1 to 16 ) rubbed (if need be) into each side is stated sometimes to give relief. It is frequently applied with advantage to the rheumatic joints of cattle. Repeated dressings are serviceable in arresting induration of the udder, lymphatic, and other glands. When absorbent and counterirritant effects are to be produced, it is sometimes used diluted with iodine ointment. The strong red iodide ointment requires to be used with care, otherwise it destroys the hair-roots, causes sloughing and blemishing, and produces constitutional effects.

\section{Mercuric Nitrate. Mercury Nitrate. $\mathrm{Hg}\left(\mathrm{NO}_{3}\right)_{2}$.}

Unguentum Hydrargyri Nitratis. Citrine Ointment.

When mercury is dissolved in diluted nitric acid, and the solution boiled gently for fifteen minutes, there is produced the B.P. hydrargyri nitratis liquor acidus, a colourless, strongly acid, caustic solution.

The unguentum hydrargyri nitratis, or citrine ointment, is prepared by mixing a cold solution of mercury in nitric acid with lard and olive oil. It has a lemon-yellow colour, a nitrous acid odour, and is apt to spoil unless well made and kept in earthenware vessels secluded from light. One part of this ointment and four parts of soft yellow paraffin, constitute the B.P. diluted mercuric nitrate ointment.

ACTIONS AND UsES.- The stronger mercuric nitrate solutions are caustics. They and the ointments are applicable for many of the purposes of corrosive sublimate, and used for reducing and destroying malignant growths, and stimulating 
such specific sores as canker in the feet of horses and foot-rot in sheep. Milder solutions and citrine ointment are good remedies for eczema, especially after heat and pain have been subdued and desquamation has set in, and are usefully alternated with tar preparations. With good feeding and alkaline washes, they are applied in those cases of pityriasis not uncommon amongst cattle in poor condition. They destroy lice and other skin parasites, and the cryptogamic growths of ringworm. Being easily absorbed, if too freely applied they induce the usual specific effects of mercury. Diluted with olive or almond oil, or lard, citrine ointment relieves irritable, swollen, discharging conditions of the eyelid.s.

\section{PHOSPHORUS}

A solid non-metallic element obtained from Calcium Phosphate. (B.P.)

Phosphorus is prepared by the digestion of bones in sulphuric acid; the acid calcium phosphate remaining in solution is evaporated, mixed with charcoal, and distilled, when phosphorus comes over, and is condensed under water. Two allotropic forms occur. The first is wax-like, easily cut with a knife, of a yellow-white colour, volatile, readily oxidisable and inflammable, luminous, and soluble in carbon disulphide, eighty parts of olive oil, twenty-five parts of chloroform, and in sixty of oil of turpentine. It is an irritant corrosive poison. The second-prepared by keeping the yellow phosphorus for a considerable period at a temperature of $450^{\circ} \mathrm{Fahr}$. in an atmosphere of carbonic anhydride or nitrogen-is red, amorphous ; at ordinary temperatures has little affinity for oxygen ; is not volatile, luminous, or soluble in carbon disulphide; heated to the boiling point it reverts to the vitreous form. It is innocuous when given by the mouth, but both forms are poisonous if injected under the skin.

ACTIONS AND USES.-Phosphorus is an active member of the group of pentad elements, comprising nitrogen, antimony, arsenic, and bismuth. Phosphorus is slowly dissolved and absorbed, unchanged, but best if in finely divided form or 
dissolved in oil, stimulates growth of bones, and is occasionally prescribed as a nerve tonic, alterative, and aphrodisiac. These effects on the nervous system are by no means reliable. Its effect on bone formation is more important. With minute but continued doses cancellous bone becomes denser and the outer compact layer of bone is thickened. If an excess is given the bones are deformed, the medullary cavity enlarged, and an appearance like rickets is produced. Full doses when swallowed cause gastro-enteritis. Repeated doses break up the albuminoid textures by accelerating normal autolysis and induce fatty degeneration.

TOXIC EFFECTS. - A piece of yellow phosphorus, or a strong solution applied to the skin, abstracts oxygen and produces limited inflammation, sometimes terminating in gangrene. When swallowed most of it is slowly dissolved by the bile and any fatty matters with which it comes into contact, whilst some of the drug is converted into phosphoretted hydrogen gas. It exerts on the alimentary tract its local irritant effects; minute doses are gastro-intestinal tonics; larger doses cause diarrhœa and emesis in animals that vomit; while fifteen grains cause gastro-enteritis in horses and cattle. Often these acute symptoms may pass off to reappear in two or three days' time, when the drug is being excreted. There is a peculiar characteristic smell of garlic in vomited material which may be phosphorescent in the dark. Larger doses, such as thirty grains in horses or cattle, and half a grain to a grain in dogs or men, produce paresis, convulsions, coma, and death usually within two or three days. The paresis occasionally affects the heart, causing sudden death. Moderate to full doses, repeated several times daily, within a few days produce fatty degeneration of the albuminoid tissues, hypertrophy of connective structures, and acute cirrhosis. The toxic doses are : horses, grs. xv. to grs. xxiv. ; dogs, grs. $1 \frac{1}{2}$ to grs. jv. It is excreted by the kidneys and lungs, chiefly as phosphorus and phosphoric acids. But these acids and the salts they form have not, however, the specific action of phosphorus.

The antidotes consist in emptying the stomach by emetics (copper sulphate, ipecacuanha), or the pump, or by washing it out:with potassium permanganate solution, which forms 
the less active phosphoric acid, administering mucilaginous fluids, but avoiding milk, oils, and eggs, which dissolve the poison, and prescribing frequently repeated doses of old turpentine and of copper sulphate. Turpentine forms with it the non-poisonous terebinthino-phosphoric acid, a nonpoisonous solid body. Copper sulphate unites with it to form copper phosphide which is insoluble, whilst some of the metal is precipitated on the particles of phosphorus, so hindering absorption.

Medicinal Uses.-Small doses promote tissue growth, and replace spongy texture of bones with denser tissue. Phosphorus is prescribed in rickets, bone softening, after fractures, and in chronic malnutrition. It has been substituted for arsenic in persistent skin disorders. It is empirically administered in epilepsy, chorea, and general paralysis, and in such cases it is sometimes applied as a topical irritant.

Doses, ete.-Horses and cattle take gr. ss. to grs. iii.; sheep and pigs, gr. $\frac{1}{100}$ to gr. $\frac{1}{20}$; dogs, gr. $\frac{1}{200}$ to gr. $\frac{1}{60}$, given in pill or electuary, or dissolved in almond oil or vaseline. Phosphorus may be prescribed in the form of elixir phosphori, made with compound tincture of phosphorus and glycerin, and containing gr. $\frac{1}{50}$ in each drachm. The minimum dose should be administered daily for a week, then suspended for a week, and repeated if required. For external purposes a liniment is made with one part phosphorus to one hundred parts olive or almond oil. The paste for the destruction of rats usually consists of one part phosphorus to sixty each of water and flour.

Lecithin, a natural phosphoglyceride, containing phosphorus in organic combination, is found in yolk of egg, the brain, liver, suprarenal gland, milk, beans, peas, etc. Lecithin obtained from yolk of egg is a brownish-yellow resin-like mass, with a characteristic odour; insoluble in water, soluble in alcohol, ether, and chloroform. It is readily and completely assimilated by the tissues, stimulating cell-multiplication, improving appetite, increasing weight and promoting growth in young animals. Its administration is indicated in all derangements due to lack of organic phosphorus. It is recommended in the treatment of 
anæmia, rickets, diabetes, tuberculosis, and of nervous affections associated with lowered vitality. In convalescence following canine distemper it is especially valuable. Doses for the dog, gr. j. to grs. v. ; in pill or mixed with the food.

Glycerophosphoric ACID. Glycerophosphates.

Glycerophosphoric acid may be prepared by heating glycerin with phosphoric acid, and subsequently neutralising the product with calcium carbonate. It is a pale, yellowish, syrupy fluid, with a sour taste, seldom employed medicinally. Its salts, the glycerophosphates of calcium, iron, potassium, sodium, magnesium, and quinine, are frequently prescribed and are of great value in the treatment of debilitating and nervous diseases. Glycerophosphate of calcium, prepared by heating together bicalcic phosphate, phosphoric acid, and glycerin, is a light white powder, soluble in 20 parts cold water, forming a neutral solution. Recent investigations suggest that this salt merits extended trial in tuberculosis, rickets, and other diseases depending on malnutrition ; and in all cases requiring the administration of a tonic restorative the glycerophosphate is preferable to the phosphate as it is much more easily assimilated. The glycerophosphates of sodium and potassium are alkaline syrupy liquids which are usually prescribed in combination with other salts. Glycerophosphate of quinine is especially indicated in cases associated with indigestion and low fever; and glycerophosphate of iron, which occurs in greenish-yellow scales, soluble in five parts of cold water-promotes nutrition by furnishing phosphoric acid and iron in a very assimilable form. It is an excellent tonic (Kaufmann). Doses of calcium and iron glycerophosphates, horses, 3iij.; dogs, grs. v. to grs. xv. per day. Quinine glycerophosphate is prescribed in the same doses as quinine sulphate. Hypodermically, a five per cent. solution of the calcium salt, or a twenty per cent. solution of the sodium salt may be injected in doses of $m \mathrm{v}$. to 3ij. per day in the dog. Glyphocal emulsion with almond oil (Squire and Sons), containing the glycerophosphates of calcium, iron, potassium, and sodium, with pepsin 
and diastase, is a valuable tonic and restorative for weakly, unthrifty foals and puppies, in doses of $3 \mathrm{jv}$. to $z \mathrm{ij}$. per day.

\section{BROMINE AND BROMIDES}

Bromine. Bromum. A liquid, non-metallic element obtained from sea-water, and from some saline springs. (Not official.)

Bromide of Potassium. Potassin Bromidum. KBr. (B.P.)

Bromine, like its analogues chlorine and iodine, has a great affinity for hydrogen, removes it from its several combinations, and hence is antiseptic and indirectly an oxidising agent. It is very diffusible. These three halogens are antiseptic, disinfectant, and topically irritant and stimulant.

BRomine is a dark, red-brown, volatile liquid, has a strong disagreeable odour and taste, and produces a yellow colour when added to cold solution of starch : soluble in thirty parts of water. It is occasionally used as a caustic in malignant and fungoid diseases, one part being dissolved in ten to fifteen parts of rectified spirit. Diluted Hydrobromic Acid containing ten per cent. by weight of hydrogen bromide, is devoid of irritant action, and is occasionally prescribed as a nerve sedative, but is not so convenient or effectual as potassium bromide.

THE BRomides, including those of potassium, sodium, and ammonium, do not differ materially in their action. They have very slight topical effect on the skin or mucous surfaces, but are rapidly absorbed, and readily decomposed, the potassium bromide forming in the stomach sodium bromide and potassium chloride. They are quickly eliminated by all the excreting channels, chiefly by the kidneys, but also to some extent in the milk, sweat, and from the lungs. Large doses act as depressants of the spinal cord and reflex portions of the brain, impairing afferent conductivity of nerves. They are devoid of true hypnotic action, such as that of chloral hydrate, but diminish cerebral excitability by reducing the activity of the reflex parts of the brain.

Potassium Bromide is the salt in general use. It is prepared by heating bromine and caustic potash with charcoal, and contains 67 per cent. of bromine. It occurs in colourless, 
cubical, odourless crystals, which have a pungent saline taste; soluble in two parts cold water, and in two hundred parts of rectified spirit. Horses receiving about an ounce, or dogs 45 grains, become listless, exhibit muscular feebleness, unsteadiness of gait, impaired reflex movements; the pulse is feeble, respiration slowed, rectal and cutaneous temperatures are diminished, and secretion of urine increased. Some of these effects, notably cardiac and muscular depression, however, are possibly due in part to the potassium rather than to the bromine. In addition these salts develop the usual 'salt-action,' and so vomiting, diuresis, and sometimes diarrhœa may occur. In animals receiving repeated full doses of bromides, mechanical irritation of the cortical substance of the brain fails to produce epileptic convulsions (Brunton). From the lessened movement induced there may be a fall of temperature. Full doses continued for some time induce bromism, a form of chronic poisoning, characterised by depression of the cerebral faculties, increased secretion from the mucous glands, feebleness, anæmia, and wasting, dilatation of the pupils, and eczematous eruptions produced as portions of the drug are excreted through the skin. Toxic doses kill by asphyxia.

Potassium bromide is used in nervous disorders to allay excitement and relieve spasm, and thus indirectly may produce sleep. It alleviates and wards off epileptic convulsions in dogs, whether connected with distemper or other cause, and is serviceable in violent cases of chorea. It has no constant or decided influence in controlling the spasms of tetanus in horses. Kaufmann records that Vogel gave nine horses suffering from tetanus three to six ounces daily without moderating the spasms. It is of little use in asthma, sometimes checks persistent vomiting, and has considerable anaphrodisiac effect. It is occasionally used with digitalis to quiet cardiac excitement. Sodium bromide only differs in not producing the depressant effects on the heart and muscles which potassium bromide may induce. Strontium bromide has the advantage of disturbing digestion less than potassium bromide. Hydrobromic acid has the same bromide action as the salts, but is irritant locally.

DOSES, etc.-Of potassium bromide horses and cattle take 
3iv. to zi.; dogs, grs. v. to grs. lx., in bolus, electuary, or watery solution, repeated three or four times daily. Where cerebral excitement is great, and sleep is sought, chloral or other hypnotic is conjoined with the bromide. Bromide of zine has been introduced for epileptic cases in the belief that it unites the actions of bromine and zinc. Bromol, or Tribromophenol, a crystalline product of the action of bromine on phenol, is used as a caustic, disinfectant, and intestinal antiseptic. It is soluble in alcohol, ether, and glycerin, and not affected by the gastric secretion.

\section{CH L O R I N E}

Chlorine may be obtained by the interaction of hydrochloric acid and manganese peroxide. It is a yellow-green gas, with a peculiar suffocating odour and an astringent taste. Water charged with two volumes of chlorine gas constitutes the liquor chlori-a yellow-green, chlorinesmelling liquid, readily decomposed by air and sunshine.

ACTIONS AND USES.-Chlorine, whether as gas or in solution, is irritant, antiseptic, deodorant, disinfectant, and parasiticide.

One part in 8540 of a watery solution arrests the action of ptyalin on starch paste ; one in 7411 part arrests the action of diastase ; one in 27167 part arrests the action of pepsin. Although not so active as corrosive sublimate, one 22768 part kills developed bacteria ; but one 1431 part is required to prevent their reproduction, and 1008 to prevent reproduction of spores. One part to 1500 prevents development of anthrax bacilli. The bleaching, antiseptic, and other actions of chlorine result from the breaking up of complex organic substances by the chlorine seizing their hydrogen, while the nascent oxygen thus liberated exerts active oxidation.

Applied to the skin or mucous surfaces, it causes irritation, which is relieved by lime-water, white of egg, soap, or diluents. One part in 100,000 of air inhaled for some time causes great irritation of the bronchial mucous membrane, and hæmorrhage from the lungs: Irritation of the air-passages, induced by the insufficiently diluted gas, is counteracted by inhala- 
tion of ether, weak ammonia, or the vapour of warm water or of alcohol.

Medicinal Uses.-Diluted chlorine gas has been used as an inhalation, or the freshly-prepared solution applied in spray, to increase bronchial secretion, to stimulate and disinfect ulcerated sore-throat in horses, and abate the discharge and fœtor from disease of the facial sinuses. Both gas and solution are used for the destruction of strongyli infesting the air-passages of calves and lambs, and the liquor chlori, and the equally effectual but less irritant sulphurous acid solution, are also employed intratracheally. As a mouth wash for dogs with foul condition of the gums and teeth, the mixture of chlorine and its oxides obtained by the action of strong hydrochloric acid on potassium chlorate with subsequent dilution in water, is very useful.

For disinfecting or deodorising, the materials for evolving chlorine should be placed in earthenware vessels in the upper parts of the boxes or sheds in order to facilitate diffusion of the heavy vapour. One part each of common salt and manganese black oxide, intimately mixed, are treated with two measures of oil of vitriol, diluted with two measures of water. For more gradual production of the gas, bleaching powder is mixed with potash alum. Where chlorine is used for thorough disinfection, the buildings must be cleared of animals; large volumes of gas liberated ; sunlight admitted to intensify the action; and the walls and woodwork washed with a strong watery solution. It may be fittingly used in conjunction with the tar acids, but is incompatible with sulphurous acid.

\section{O I N E}

IoDUM. A solid non-metallic element, obtained from the ashes of seaweeds, and from native iodides and iodates. (B.P.)

Iodine is present in sea-water, and is thence taken up by sea plants and animals. It is prepared from kelp, and 
from the mother-liquors of the Chili nitre mines iodine is also obtained.

Properties.-Iodine occurs in soft, friable, black or blueblack, rhombic prisms or octahedrons of a metallic lustre. Its specific gravity is 4.95 . It has an acrid, disagreeable taste, and a pungent, unpleasant odour, resembling that of chlorine or sea-water. Applied to the skin it produces a brown stain, readily removed by alkalies. At the temperature of the atmosphere it slowly evaporates ; at $237^{\circ} \mathrm{Fahr}$. it melts ; at $392^{\circ} \mathrm{Fahr}$. it boils, volatilising entirely in violetcoloured, irritating, antiseptic vapours, nine times as heavy as air. With water it forms a brownish-yellow solution, containing, however, only $\cdot 05$ per cent. It is dissolved by twelve parts of rectified spirit, and still more readily by ether, volatile oils, chloroform, carbon disulphide, and also by solution of potassium iodide, and other salts. It readily unites with metals; the iodides of the alkalies closely resemble iodine in their actions ; the iodides of the heavy metals exhibit chiefly the properties of the base.

Iodine is easily distinguished by its characteristic odour, by the brown stain it leaves on the fingers, by the violetcoloured vapour it evolves when heated, and by the blue colour it forms with cold solution of starch. This starch test is inapplicable when iodine is in combination, from which, however, it is readily set free by a drop of weak chlorine solution, or of diluted nitric acid. Iodine is liable to intentional adulteration as well as accidental impurities.

ACTIONS 'AND USES.- Iodine resembles the other halogens, alike in chemical and physiological actions. Its notable affinity for hydrogen, and its combining with albumin, determine its stimulant, irritant, and caustic effects, as well as its antiseptic, alterative, and resolvent actions. It acts notably on mucous membranes, skin, and lymphatic glands. It is almost a specific for diabetes insipidus in horses. Full doses persisted with produce a state of debility and emaciation termed iodism. Externally, it is applied as an antiseptic, stimulant, counter-irritant, and parasiticide. It is employed for the several purposes of an antiseptic, deodorant and disinfectant.

General Actions.- - It is an active antiseptic, whether used 
in the gaseous, fluid, or solid state. One part in 4125 parts of water arrests the action of diastase and ptyalin ; one part in 7817 arrests the action of pepsin; one part in 7000 destroys both bacilli and their spores (Wernitz and Koch). Iodine stains the skin yellow-brown, and is almost the only substance that penetrates the unbroken skin; after local application traces can always be found in the urine; strong solutions cause hyperæmia, irritation, and desquamation of the cuticle ; but the irritant action can readily be regulated. The skin and nutritive processes may be stimulated; absorption of inflammatory products is assisted, and the congestion and pain in some distal part may be relieved. (See Counter-irritants.) A gradual and fairly prolonged effect is produced when iodine is brought into contact with either skin or mucous surfaces; for it is volatile, slowly penetrating, and so active for considerable periods on parts with which it is placed in contact. Large doses if swallowed cause gastro-enteritis, and if inhaled produce rhinitis, laryngitis, and bronchitis. In the stomach it is converted into iodides and iodates; and it differs from the action of iodides only in the fact that it is more irritant in the stomach. Medicinal doses are absorbed, stimulate glandular activity, and promote metabolism. In the tissues iodine may again be set free, and combine with serum albumin, but iodine albuminates are unstable, and hence readily removed. This appears to explain the action of iodine and its compounds in the liquefaction and absorption of pathological products. It combines with lead, mercury, or other metals present in the body, and hastens their removal. It is excreted by the mucous surfaces and glands, notably in the saliva, perspiration, bronchial secretion, milk, and urine, while full doses during excretion irritate the excreting channels.

Although the element itself is less active than chlorine or bromine, its compounds are more active, probably because they are more readily decomposed. The iodides of potassium, sodium, and ammonium chiefly produce the effects of the iodine, but are less irritant, less active as thyroid gland stimulants, although more prone to affect the kidneys than the element itself. The iodides of iron, copper, lead, and mercury exhibit in addition the actions of their power- 
ful bases. In stimulating the skin and mucous surfaces iodine shows some resemblance to arsenic and sulphur.

TOXIC EFFECTS.--Hertwig gave horses forty to sixty grains of solid iodine twice daily for fourteen days, with the effect of causing slight diarrhœa, with black evacuations and increasing emaciation. Dick repeatedly gave larger quantities for several weeks, without observing any other symptom than the total refusal of water. To one horse he administered for three weeks doses of two drachms per day, and towards the end of the experiment doses amounting to two ounces daily. Several ounces have also been given to cattle with the like negative results. In many of these cases the iodine, having been given in the solid form, must have been slowly, perhaps only partially, dissolved and absorbed, and, during tardy solution, may in great part have been neutralised by contact with starch food.

Dogs receiving two or three drachms of solid iodine speedily get rid of it by vomiting ; but when the œesophagus is tied such doses cause fatal gastro-enteritis in two to seven days, leaving numerous yellow spots and little ulcers in the stomach, and a peculiar rose tint of the liver (Cogswell). Hertwig found that such doses killed every dog to which they were given, inducing sero-sanguineous exudation and hæmaturia. Fröhner states that intravenous injection causes solution of the red globules, inducing hæmoglobinuria, anæmia, lung œdema, hæmorrhagic inflammation of the pleura and peritoneum, and bloody discharges from the bowels and kidneys.

Iodism, produced by prolonged administration of full doses, is characterised by loss of appetite, an irritable, catarrhal condition of the mucous membranes of the nostrils, eyes, throat, and digestive organs, a vesicular skin eruption, abstinence from water, diminution of the urinary secretion, languor, inaptitude for exertion, elevation of temperature and emaciation. Such effects are produced in some animals by full doses given for eight or ten days. But in ordinary practice iodism is exceedingly rare in the lower animals, although not uncommon in man. Where it occurs, it is arrested by withholding the medicine, exhibiting starch, in order to convert any unabsorbed iodine into the innocuous 
starch iodide, and giving mineral tonics, bitters, and nutritive diet.

Medicinal Uses.-Iodine is prescribed as an alterative and resolvent in enlargement of glands, and of the udder, in chronic rheumatism, especially involving the joints, in hydrothorax and ascites, and in persistent cases of psoriasis. Some American practitioners conjoin iodine with carbolic acid in febrile attacks, especially when depending upon malaria. Dry, congested conditions of the respiratory mucous membrane are sometimes relieved, and secretion of mucus encouraged, by inhalation of steam or warm air, medicated with a little iodine tincture, and by potassium iodide internally. Similar inhalations are also beneficial in checking muco-purulent discharges from the nostrils or sinuses of the head, and in infective sore-throat. In purpura hæmorrhagica, Dieckerhoff recommends the intra-tracheal injection of eight to twelve drachms, twice daily, of a solution of one part iodine, five of potassium iodine, and one hundred of water. Injections carefully and slowly made are not followed by coughing. Many British veterinary surgeons testify to the value of this treatment in reducing the swellings of the disease.

Iodine is pre-eminently useful in diabetes insipidus, or polyuria, affecting horses in which twenty or thirty pints of urine are sometimes passed daily, thirst is insatiable, and strength and flesh are rapidly lost. Iodine given night and morning seldom fails to arrest this disease in two or three days. How it does so is not satisfactorily explained. Its antiseptic effects may control excessive production of injurious enzymes. But neither quinine nor iron, although possessed of notable antiseptic properties, is as effectual as iodine in this form of diabetes. Neither potassium nor iron iodide is as trustworthy as the crude drug. Dollar has experimented with various more correct chemical combinations, but finds none so reliable as iodine 3 ss., iron sulphate 3ij., and powdered gentian 3iv., made into bolus with treacle, syrup, or meal and water. This is repeated once, in bad cases twice, daily. Rarely are more than six doses required to effect a cure.

Externally, iodine is used as an antiseptic, stimulant, and 
resolvent for chronic synovitis, bursal swellings, muscular pains, slight strains of tendons, thickening of periosteum, and indurations of the udder and other glands. It is also used as a counter-irritant in sore throat in horses, in limited lung consolidation, especially in dogs, and in subacute attacks of pleurisy. It is a serviceable antiseptic and deodoriser for unhealthy and malignant wounds. Indolent ulcers are sometimes healed by application of a piece of lint, spread with simple cerate, sprinkled with one to five grains of iodine, and covered with a piece of oiled silk or tinfoil. But excess of iodine must be avoided, otherwise corrosive effects are produced. Dilute iodine solutions are injected, as stimulating antiseptics and promoters of adhesion, into cysts and abscesses from which the contents have been withdrawn. Conjoined with potassium iodide, and oil of turpentine, it is injected into the trachea in hoose in calves. A solution of one drachm in sixteen drachms of rectified spirit is employed after withdrawing the dropsical fluid, as an injection for hydrocele, thoropins, and bursal distensions. On account of its penetrating the hair follicles and ducts, it is serviceable in persistent cases of mange, eczema, and psoriasis, in all forms of ringworm, and in such cases is sometimes mixed or alternated with wood-tar oils, sulphur, or mercurials. Like most effectual antiseptics, it destroys skin parasites, œstrus larvæ, and entozoa. It is an effective but expensive deodoriser and disinfectant.

Doses, etc.-For horses, grs. xx. to $3 \mathrm{i}$.; cattle, $3 \mathrm{ss}$. to 3iss. ; sheep, grs. xv. to grs. xl. ; pigs, grs. x. to grs. xx. ; dogs, grs. iij. to grs. viij. Such doses are repeated once or twice daily, given a couple of hours after feeding, in order to diminish the proportion otherwise converted into the mild, insoluble starch iodide, continued for a week or ten days, withheld for a day or two, and, if necessary, again resumed. Larger doses, although they may be given with impunity, do not ensure better curative results.

Iodine is administered in bolus ; but, handy though this form is for horses and dogs, it is less certain than a good aqueous solution, such as is obtained by mixing two parts of iodine and one of potassium iodide with six or eight of water. The potassium iodide ensures perfect solution and full action 
of the iodine. This concentrated solution is diluted with water as required; the dose is easily ascertained, for the iodide is about half as powerful as the iodine. For intratracheal injections, iodine grs. xxxii.; potassium iodide, grs. clx., are dissolved in three ounces of distilled water, and of this solution $m_{x x x}$. diluted with $m_{x l} l_{\text {. }}$ of distilled water are injected daily for two or three days, when the quantity of diluent may be gradually reduced and the dose of the pure solution gradually increased to four or five drachms.

For external purposes the tincture and compound aqueous solution are generally suitable. When gentle stimulation and absorption are desired weak solutions are used. As a counter-irritant about two parts iodine and one of potassium iodide are dissolved in sixteen of water or fatty matters. For reducing bony enlargements iodine ointment is sometimes mixed in equal proportion with mercury biniodide ointment. For wounds ten grains each of iodine and potassium iodide to an ounce of water usually suffice. Two parts iodine, one part potassium iodide, four wood-tar oil, and thirty-two of lard or oil, make a serviceable mange dressing.

Tincture of iodine is made with half an ounce each of iodine, potassium iodide, and water, and sufficient alcohol (90 per cent.) to produce twenty ounces of the tincture. Liquor iodi, or Lugol's solution, contains iodine two, potassium iodide three, and water forty parts. Unguentum iodi contains 20 grains each of iodine and potassium iodide, 60 grains of glycerin, and 400 grains of lard. Glycerin of iodine contains 1 of iodine to 50 of glycerin. An injection for bursæ is made with 16 grains each of iodine and potassium iodide, dissolved in an ounce of sterilised glycerin, or distilled water. Tinctura iodi decolorata is made with 250 grains of iodine dissolved, with a gentle heat, in $5 \frac{1}{2}$ ounces of rectified spirit; when cold, add 10 drachms of strong solution of ammonia; and, when decolorised, dilute with sufficient spirit to form 1 pint. Tinctura iodi oleosa is composed of iodine one, rectified spirit nine, and castor oil two parts. This tincture applied as a pigment does not crack the skin (Extra Pharmacopocia).

Iodipin (Merck) is a compound of iodine with oil of sesame. It is supplied in two strengths, one containing ten per cent., 
the other twenty-five per cent. of iodine. Iodipin (10 per cent.) is a light yellow liquid with a sp. gr. of 1025 ; while the 25 per cent. iodipin is a thick, oily fluid with a sp. gr. of 1227. Both preparations are soluble in ether and chloroform, and insoluble in alcohol. Given by the mouth iodipin is stated to pass unchanged through the stomach, and in the intestine a small proportion is decomposed, the greater part being absorbed as an intact fat. On the oxidation of iodipin the iodine is taken up by the alkalies of the blood and tissues. Administered subcutaneously iodipin is slowly but regularly absorbed and oxidised. Iodine may be detected in the urine within fifteen minutes after the first dose, and it continues to be excreted for several weeks following the last injection. Iodipin is employed as a substitute for the alkaline iodides. It is a valuable medicine in the treatment of actinomycosis, shoulder abscess, scirrhous cord, bronchitis, pulmonary emphysema, purpura, and acute infectious diseases. It is also used as a routine antiseptic.

Doses.-Iodipin 25 per cent., subcutaneously, horses and cattle, $z_{j}$. to $z_{\mathrm{ij}}$. per day; dogs, iodipin 10 per cent., per $o s$, one to three teaspoonfuls per day, administered in milk, or mixed with gum acacia and treacle and given as an electuary.

Potassium Iodide. Potassii Iodidum. Potassic Iodide. KI.

The iodide is prepared by slowly adding iodine to a solution of potash, until it acquires a brown colour, evaporating to dryness, mixing the residue with one-tenth of its weight of powdered charcoal, and fusing in a red-hot crucible. The fused mass is dissolved in hot water, filtered and evaporated until a film appears on the surface, when it is set aside to crystallise.

Properties.-Cubical crystals, colourless, generally opaque, with a faint odour of iodide, a saline taste, decrepitating when heated, fusing at a red-heat, at a higher temperature volatilising unchanged, soluble in two-thirds of its weight of cold water, in twelve parts of rectified spirit, and in three parts of glycerin. These agents dissolve iodine freely, and are hence useful vehicles for its exhibition. 
Actions AND Uses.-Potassium iodide closely resembles iodine, but is less powerful and devoid of local irritant action. Medicinal doses are antiseptic, alterative, deobstruent, expectorant, and diuretic. Sodium iodide has the same actions.

It is readily soluble, and is quickly absorbed ; in the tissues it undergoes decomposition; the iodine, when liberated, apparently combines with albuminoids, and acts specially on the thyroid gland and, in fact, on glands generally, modifying nutrition, hastening metabolism, and promoting absorption. It is doubtless in this way that it also unites with lead and mercury albuminoid compounds deposited in the tissues, renders them soluble, carries them into the circulation, and causes their elimination. It is quickly excreted by the mucous and skin surfaces, in all the secretions, but chiefly by the kidneys. Full doses increase both the solids and fluids of the urine.

Toxic EFFects.-Large doses, such as three ounces in horses or cattle, or a drachm in dogs, enfeeble the heart, and also the spinal and cerebral functions. It causes iodism, especially when it is mixed with iodates.; but this chronic poisoning is much rarer in animals than in man. Dogs receiving two or three drachms dissolved in water vomited, showed great depression, and died in a few days from the effects of the salt action and irritation; rabbits were similarly affected by one drachm; three drachms, injected beneath the skin of the back of a dog, caused extensive subcutaneous inflammation, and death in three days. Iodine is detected after death in the blood and urine, in the brain and spinal cord, in most of the internal organs, and even in the muscles and bones (Cogswell).

Medicinal Uses.- - It is given, either alone or with iodine, to promote absorption of morbid products, as in lymphangitis in horses, pleuritic and other serous effusions, enlarged glands and lung consolidations in all animals. For such purposes full doses are generally prescribed twice or thrice daily for a fortnight, and, where the lesions are superficial, iodine and soap liniments are also used externally. It is useful in cases of enlarged thyroid - simple goitre-where the secretion is scanty and the gland grows to supply the 
deficiency. The iodide stimulates the gland to normal activity and so removes the hypertrophy. Trasbot, from fourteen years' study of the action of potassium iodide, was satisfied that in bronchitis, pneumonia, and pulmonary congestion, especially in horses, a few doses diminish the frequency and force of the pulse, the difficulty and quickness of breathing, and the abnormal temperature. These benefits are ensured, and congestion and dryness of the bronchial membrane relieved, by conjoining the iodide with ammonium acetate solution. Like digitalis, potassium iodide is serviceable in troublesome cough and in chronic cardiac cases. These latter effects probably depend mainly upon its action as a potassium salt. In roaring, Robertson prescribed potassium iodide and arsenic. Atheroma and aneurism, in man, are often benefited by potassium iodide, but this is due to the action of iodides in cases of syphilis, the common cause of these two conditions. Thomassen and Nocard, after extensive experience, relied implicitly on the iodide in actinomycosis, especially in those hitherto unsatisfactory cases affecting the tongue of cattle. Ninety grains in about a pint of water are given daily for eight or nine days ; within that period swelling and pain abate, the animal is able to eat, and the cure is 'always successful,' usually within a month. Similar treatment, though less certain, has proved beneficial in discomycosis of the testicular cord, withers, and poll, and in cases of chronic abscess of the shoulder. Dieckerhoff recommended the intratracheal injection of dilute iodine solutions in equine purpura hæmorrhagica, and other German practitioners have approved of the treatment. The solution used consists of five parts potassium iodide, one part iodine, and 100 parts water. Five drachms of the solution are injected into the trachea with a hypodermic syringe. It constitutes the chief agent in Schmidt's treatment of parturient toxæmia in cows. Grains 110 to 160 of the iodide are dissolved in 35 ounces of boiling water ; and a fourth part of the solution, cooled to blood heat, is injected into each teat. Asthma, rheumatism, and eczema are frequently benefited by a course of the iodide. In chronic poisoning with lead and mercury it removes the metal from the tissues and from the 
body. It should not be prescribed with bismuth subnitrate, liquorice, nitrous ether or any drug containing starch.

Externally, usually with soap liniment and laudanum, it is applied to painful, swollen rheumatic joints, and to the inflamed udder in cows and ewes. A solution of five grains in one ounce of water is recommended as an application for recent corneal opacities. It is much used for increasing the solubility of iodine, both in water and alcohol.

Doses, etc.-Horses and cattle take $3 \mathrm{j}$. to $3 \mathrm{vj}$. ; sheep and pigs, grs. xx. to grs. lx.; dogs, grs. jv. to grs. xx., repeated two or three times a day, and given either in bolus or solution, in water or spirit. Its effects are increased when it is given with common salt, more iodine being thus liberated. A convenient solution for intratracheal use is made with 30 grains of iodine, $2 \frac{1}{2}$ drachms potassium iodide, dissolved in 4 ounces of distilled water. The dose is $m 30$ to $\mathrm{m} 60$, mixed with an equal measure of water.

Iodide of sulphur is a stimulant and parasiticide. It is prepared by mixing, in a Wedgwood or glass mortar, four parts iodine with one of sublimed sulphur, and gently heating until the mixture liquefies. The red-brown liquid, as it cools, becomes a grey-black crystalline mass, insoluble in water and alcohol, but soluble in glycerin and fats, with eight or ten parts of which it is mixed for liniments or ointments, which are suitable for chronic scaly skin complaints, ringworm, and mange.

\section{S U L P H U R}

Sulphur, or brimstone, is one of the most ancient articles of the Materia Medica. It occurs in many animal substances as sulphates, and notably also in bile and the albuminoids ; in the strong-smelling volatile oils of the Cruciferæ and Umbelliferæ; in various mineral waters as hydrogen sulphide, and in the pyrites or metallic sulphides, from which it is extracted by roasting. The extensive supplies of sulphur required in the manufacture of sulphuric acid, sulphurous acid, gunpowder, lucifer matches, and vulcanised indiarubber, are, however, chiefly obtained from the native 
sulphur, occurring as a product of volcanic action in beds of blue clay in Sicily and Italy.

The crude sulphur is purified by distillation, and when run into wooden moulds forms the stick or roll sulphur, which is yellow, crystalline, and so bad a conductor of heat that it often cracks when held in the warm hand.

Sublimed sulphur, also called flowers of sulphur, is prepared by distilling the crude sulphur, and conducting it in the state of vapour into large chambers, where it condenses in a fine yellow powder consisting of spherical granules.

Precipitated sulphur, or milk of sulphur, is prepared by boiling sublimed sulphur with slaked lime, when calcium sulphide and thiosulphate are formed, and when treated with diluted hydrochloric acid are decomposed, sulphur being precipitated in a finely divided yellowish-white powder.

Sulphur vivum, caballum, or horse sulphur, the residue left in the subliming pots, must be used with caution, for, besides other impurities, it contains arsenic.

Properties.-Sulphur occurs in three allotropic forms, manifesting differences in physical condition, specific gravity, fusing point, solubility in carbon disulphide, and electric affinities. Most varieties have a yellow colour, a specific gravity of about 2 , have little or no taste, are insoluble in water and cold alcohol, and are freely soluble in benzol, carbon disulphide, fixed and volatile oils. All varieties. melt at $240^{\circ} \mathrm{Fahr}$., are entirely volatilised by heat, inflame at $500^{\circ}$ Fahr., burning with a pale blue flame, and giving off suffocating fumes of sulphurous anhydride $\left(\mathrm{SO}_{2}\right)$. As it is raised from the melting to the boiling point it exhibits various curious changes.

Actions AND UsES.-Sulphur applied to skin or mucous surfaces is a feeble, mechanical stimulant, and an irritant in proportion to the amount of sulphide liberated. The addition of an alkali increases the proportion of sulphide present, and therefore the action of the drug. It destroys parasites infesting the skin. Administered internally it is laxative and alterative.

General Actions.- It destroys fungi on vines, and kills similar parasites affecting plants and animals. This toxic effect depends not so much on the sulphur acting as sulphur, 
as on the alkaline sulphides, sulphuretted hydrogen, and sulphurous acid, into which it is gradually converted. Sulphur when swallowed is slowly acted upon by the alkaline secretions of the intestines, and small quantities are converted into sulphides, which irritate and stimulate the intestinal mucous membrane. A further change liberates sulphuretted hydrogen. Thus a part of the sulphur taken by the mouth is absorbed in the form of sulphides. During excretion this imparts its disagreeable smell to the breath, secretions of the skin, and bowels. The mutton of sheep receiving daily several ounces of sulphur is stated to acquire a distinct sulphurous flavour. The greater part, however, is removed unchanged by the bowels, and of that absorbed the bulk is excreted in the urine as sulphates. The alkaline sulphides are sometimes substituted for sulphur, and, like it, are laxative and parasiticide.

Toxic Actions.--One pound given to horses causes colic, purging, prostration, and sometimes fatal gastro-enteritis (Moiroud). A horse affected with glanders received doses beginning with an ounce, and gradually increased by addition of an ounce daily until the sixteenth day, when he had got 136 ounces. Diarrhœea supervened on the seventh day ; but appetite remained throughout unimpaired, the urinary secretion unaffected, the pulse and breathing normal. By the third day the perspiration had a sulphurous smell, and a piece of paper, moistened with lead acetate and laid on the skin, became grey. The muco-purulent discharge from the nostrils increased daily; the patient, though well fed, became gradually emaciated, and so debilitated that by the seventh day he was unable to rise. After the tenth day, the blood, even in the arteries, became dark-coloured, thin, and slow to coagulate. On the seventeenth day the animal was destroyed. The mucous lining of the stomach, colon, and cæcum was reddish-blue, soft, and easily torn. The lungs, muscles, and intestinal contents smelt strongly of hydrogen sulphide, but the blood had no such odour (Hertwig).

Medicinal Uses.-Sulphur is given to the several domestic animals as a laxative where more powerful purgatives might irritate-as in pregnancy, convalescence from acute diseases 
in young animals, and in piles. Its alterative and stimulant effects on the skin have led to its use in rheumatism, eczema, and cutaneous diseases. Some practitioners affirm that it benefits dry, congested conditions of the respiratory membrane by stimulating its epithelial cells and increasing movements of the cilia. It has no special vermicide action. The piece of roll sulphur frequently placed in the dog's trough, being insoluble in water, has no effect in preventing, as is popularly believed, distemper and other canine disorders.

Sulphur dusted on the skin slightly stimulates, but when dissolved by admixture with an alkali or oil, and smartly rubbed in, it more actively stimulates the cells of the rete Malpighii, and thus hastens desquamation; while it also increases the skin circulation. It thus promotes a healthier action in chronic eczema and psoriasis, and in such cases sulphur dressings are with benefit conjoined or alternated with iodine or tar acids, and are aided by the internal use of sulphur and arsenic. Infriction of sulphur ointment is stated to relieve the pain of rheumatic muscles and joints.

For the prompt and effectual cure of mange and scab it is essential to reach the female parasites in their burrows. Hair or wool must be closely clipped or shaved ; the affected parts freely rubbed with soft soap, allowed to remain on for twenty minutes or half an hour; crusts and scales are thus softened, and removed by subsequent thorough scrubbing with warm water. These preliminary measures are requisite to enable any parasiticide to reach the sarcoptes. Küchenmeister demonstrated that the parasites, although they lived for several days in sulphur ointment, perished in fifteen minutes in mixtures of sulphur and potash solution. This combination quickly produces hydrogen and other toxic sulphides. Numerous formulæ are in use. Two parts sulphur and one part potassium carbonate are dissolved, with the aid of gentle heat, in ten or twelve of lard or oil. A still more effectual sarcopticide is made by addition of two parts of benzine. Sulphur iodide is very useful for such cases. In chronic mange and scab a second or third soaping, scrubbing, and dressing, at intervals of a week, may be requisite; and in inveterate cases some of the penetrating tar oils, or a mercurial, may be used. Sulphur 
is a useful antidote in poisoning by lead and mercury oxides.

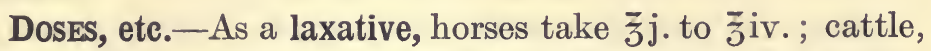

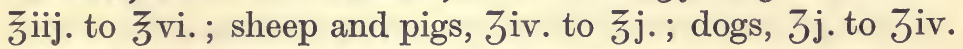
As an alterative, one-fourth of these doses may be given. The precipitated being more finely divided than the sublimed sulphur, is somewhat more certain and active as a laxative, and is in addition free from admixture with arsenic and other impurities. Sulphur is conveniently administered suspended in gruel, or treacle and water, or dissolved in milk or oil, and is often conjoined with aromatics, salines, or mercurials. For horses or cattle a laxative mixture is made with one to two ounces each of sulphur and cream of tartar, dissolved in water, with half a pound of treacle ; one-third of this dose suffices for sheep and pigs ; one-sixth part for dogs. A convenient alterative for horses or cattle consists of an ounce each of sulphur and ginger, and half an ounce of nitre, repeated once or twice daily. For torpid liver in house dogs, Garrod's sulphur lozenges may be used.

The ointment usually consists of one part of sulphur and four of vaseline or lard; one-fourth part mercury ointment is sometimes added. A liniment is made with one part of sulphur and six or eight of linseed or other fixed oil ; one part of tar oil or of Barbados tar is often added. Inveterate cases of grease have been treated with eight parts of sulphur, four of potassium carbonate, one of carbolic acid, with thirtytwo each of lard and olive oil. The dressing is freely rubbed in, allowed to remain on for two or three days, and then washed off with soap and warm water. For itch papules and vesicles in human patients, Fox recommends a drachm of sulphur, eight grains each of ammoniated mercury and creosote, twenty minims chamomile oil, thoroughly mixed with two ounces lard. This prescription answers well for similar cases in dogs.

\section{ACIDS-ACIDA}

The mineral acids, with acetic and tartaric acids, resemble each other in their actions and uses, and may be conveniently grouped together. Boric, sulphurous, carbolic, salicylic, 
tannic, and hydrocyanic acids differ chemically and physiologically, and will be dealt with separately.

Acids are hydrogen salts which in presence of an alkali exchange for it their hydrogen, or a portion of it. They redden various blue and violet colouring matters, and most have a sour taste. In virtue of their affinity for basic substances and for water, acids, especially when concentrated, form new compounds with the animal tissues. Their primary effects are (1) to unite with and neutralise the free alkali which is present in most normal tissues, thus acting as protoplasmic poisons; (2) the stronger often displace weaker acids ; (3) they precipitate albumin, while all the mineral acids, except nitric, again dissolve albumin. When introduced directly into the blood, they, moreover, destroy the red cells, decompose hæmoglobin, forming methæmoglobin, a substance which parts with oxygen much less readily. They coagulate myosin, and hence cause muscular rigidity, whilst even one in twenty thousand lessens the height of a muscle contraction. The stronger acids, especially when concentrated, are caustics and escharotics. Weaker and more diluted acids act as rubefacients, cause temporary congestion, and, if freely or continuously applied, inflame the dermis, producing vesication. They destroy enzymes and check proliferation of organised ferments, in proportion to their acidity.

Acids, when swallowed, are corrosive, irritant poisons. As with other irritants, they are more active and fatal in horses and dogs than in cattle or sheep, in which their toxic effects are diminished by admixture with the bulky food usually present in the stomachs of these ruminants. 'In cases of acute poisoning, where death has not occurred too quickly, much albumin, hæmatin, and indican have appeared in the urine, and fatty degeneration of the liver, muscles, and kidneys has been found ' (Sir Lauder Brunton). Their appropriate antidotes are alkaline bicarbonates, or calcium and magnesium carbonates, given with such diluents and demulcents as milk, oil, and linseed gruel, and followed by opium and fluid nutrients.

Acids in the mouth reflexly increase the saliva from the parotid and submaxillary glands, and effectually moisten the 
fauces and allay thirst. They further exert some astringent and antiseptic effects by precipitating a scum of proteid on the mucous membrane. If not neutralised before they reach the intestines, they increase especially the pancreatic secretion, and to a less extent the bile. The pancreas is stimulated to secrete by the absorption into the blood stream of secretin. This substance is developed by the action of acids on the duodenal mucous membrane, the cells of which manufacture prosecretin, the antecedent of secretin. Thus the pancreatic secretion is proportional to the amount of acid leaving the stomach in the chyme, and if this is naturally deficient, a mineral acid must be given by the mouth.

Acids assist digestion in several ways.

(1) They furnish the gastric juice with its acid constituent, which, unlike the pepsin, is not capable of reproduction, and without which the digestive power of the gastric solvent is impaired. Hydrochloric acid, being the chief natural gastric acid, is generally prescribed when the acidity of the gastric fluid is believed to be deficient, as it sometimes is in young animals living chiefly on milk, in febrile, enfeebled, or old subjects, or in those suffering from gastric catarrh. To aid digestion, acids are given shortly after food. Where there is want of appetite and irregular action of the bowels, acids are given conjoined with bitters.

(2) Acids, by their astringent action, check gastric secretion when this is excessive, as it appears to be in cases of indigestion, where the fluids in the mouth are sour, not only after, but before feeding, and where animals instinctively lick the walls, or eat alkaline earthy matters. In such cases the alkaline treatment frequently adopted affords temporary relief ; but a laxative, followed by acids, generally removes the conditions on which the dyspepsia depends. To such patients acids are given before feeding, and a protective film of albumin is deposited on the mucous membrane so that useless secretion is arrested.

(3) Acids acting antiseptically check fermentation, and thus prevent formation of gases and irritating organic acids. This is an important use of the hydrochloric acid normally 
present. In this way they are serviceable not only in indigestion, but in certain cases of diarrhœa.

Before reaching the general circulation, acids must pass through the liver, where they alter processes of tissue change, and check formation of urea (Brunton). As hepatic tonics and stimulants, nitric and nitro-hydrochloric acids are preferred.

When they enter the blood, their acidity has been neutralised, and they are present as salts. The blood is made less alkaline, but cannot become acid during life or it would cease to be able to carry the carbon dioxide from the tissues. To prevent this dangerous acid intoxication in carnivora, the ammonia liberated from the tissues as a result of the breaking down of proteid food combines with the acid in the portal vein and the resulting ammonia salts are excreted in the urine. In herbivora there is little available ammonia but more potassium and sodium in the blood, and so these fixed alkalies have to combine with the dangerous acid and are excreted in the form of salts in the urine, causing some amount of diuresis. Thus acids reduce the alkalinity of the blood more seriously in herbivora than in carnivora and man. They are excreted from the body in part through the intestinal mucous membrane, but chiefly by the kidneys in combination with ammonia and other bases. Full or repeated doses diminish, however, the alkalinity, or increase the normal acidity of the urine, by the presence in it of acid salts, and these cause some irritation in the urinary passages. Acetic, citric, and tartaric acids, being readily oxidised into carbonates, exert a primary acid, but a secondary alkaline effect, notably on the urine.

On account of their astringent effect on the gastric mucous membrane, acids should not be prescribed for more than a week or ten days at a time. They must be given freely diluted, and are often conjoined with bitters, iron salts, and alcoholic stimulants.

The several acids, although possessing properties in common, have distinguishing characteristics. Hydrochloric being volatile, and possessing, whether in the gaseous or fluid state, a strong affinity for water, is most destructive to vegetation, browning and shrivelling plant tissues. Nitro- 
hydrochloric, sulphuric, phosphoric, and hydrochloric acids are the most powerfully corrosive. Nitric acid does not so readily re-dissolve the precipitated albumin, and hence is scarcely so penetrating as other mineral acids. Sulphuric acid, when applied to the skin or swallowed, causes blackening or browning of the parts with which it comes in contact ; nitric acid leaves a yellow stain; hydrochloric, a white film of precipitated albumin.

Sulphurous, boric, benzoic, lactic, and salicylic acids, are used for their antiseptic rather than for their special acid properties. Carbolic acid is also an antiseptic, and an alcohol rather than an acid. Arsenious acid is not a true acid, but an anhydride. Tannic and gallic acids, although they have acid reactions, are glucosides. The striking actions of hydrocyanic acid distinguish it from all other acids. These acids will accordingly receive separate notice under their English names.

\section{SULPHURIC ACID}

Acidum Sulphuricum. Oil of vitriol. An acid produced by the combustion of sulphur or pyrites, and the oxidation and hydration of the resulting sulphurous anhydride by means of nitrous and aqueous vapours. It should contain about 98 per cent., by weight, of hydrogen sulphate. $\mathrm{H}_{2} \mathrm{SO}_{4}$. (B.P.)

PROPERTIES.-The strong acid of commerce is oily-looking, colourless, odourless, with an intensely acid and acrid taste. It freezes about $30^{\circ} \mathrm{Fahr}$., boils at $640^{\circ} \mathrm{Fahr}$., absorbs moisture from the air, and hence, if kept in unstoppered bottles, speedily becomes diluted. Specific gravity $1 \cdot 843$. It has great affinity for water, mixes with it in all proportions with evolution of much heat. Thus, combining with water and albumin, it decomposes and chars organic substances and soft animal tissues. When heated with charcoal, sulphur, or metals, it rapidly parts with oxygen, and is converted into sulphurous acid.

The acidum sulphuricum dilutum, or medicinal acid, should contain 13.65 per cent. of hydrogen sulphate. The 
acidum sulphuricum aromaticum contains tincture of ginger $10 \mathrm{Fl}$. ounces; spirit of cinnamon half an ounce;

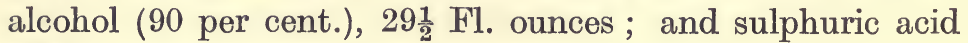
3 Fl. ounces.

Its impurities-nitric acid, lead, arsenic-seldom interfere with its medicinal uses.

Incompatibles, alkalies, their carbonates, lead and calcium salts.

ACtions AND USES.-Sulphuric acid is a corrosive, irritant poison when given in concentrated form ; is used medicinally as a refrigerant, antiseptic, and astringent ; and externally as a caustic, stimulant, and astringent. In aqueous solution-5 to 10 per 1000 -it diminishes the secretion of mucous membranes, neutralises the alkalinity of the saliva, bile, and pancreatic juice, and after absorption it diminishes the alkalinity of the blood. It is excreted in the urine as a sulphate.

Toxic EFFEcts.-Its local actions depend upon its affinity for water and bases, and its coagulating albumin. Swallowed in concentrated form, it almost immediately produces retching, with emesis in animals that vomit. The vomited matters stain and corrode, are acid, often dark, viscid, and bloody, and contain shreds of mucous membrane. The lips, mouth, and fauces are red, inflamed, and swollen. From irritation and swelling of the throat breathing is frequently difficult, and when a strong acid has been swallowed by human patients, or given to rabbits experimentally, death from suffocation has resulted in an hour, or even less time (Taylor). "In other cases there is great abdominal pain, rapidly increasing prostration, and death usually in twelve to twenty hours. The mouth, fauces, gullet, and stomach exhibit brown and black stains, and patches of corrosion, and there is sometimes perforation of the stomach. When the acid has been diluted, and death does not occur for several hours, the digestive mucous membrane is softened, swollen, and inflamed, but not so blackened or charred as when the acid has been concentrated, and death more rapid. Injection into the veins proves fatal by coagulation of blood and thrombosis (Orfila). 
Sulphuric and other acids are sometimes given by grooms and carters, with the idea of improving the appearance of horses. Acute poisoning occasionally occurs from overdoses, chronic irritation of the bowels not infrequently results; and horses which for a season have thus been senselessly doctored, usually continue for months and even for years thriftless, and difficult to keep in health.

The antidotes are alkaline bicarbonates, soap, chalk, or magnesia, given diluted in milk or water, in small quantity at short intervals. Demulcents as milk, oil, linseed tea, are subsequently administered. Where breathing is difficult, tracheotomy should be performed.

Medicinal Uses.-Sulphuric acid is prescribed as a tonic, astringent, and hæmostatic. It is given in chronic diarrhœa and dysentery, usually with laudanum in starch gruel or mucilage. In influenza in horses, with a tendency to œdema, or purpura, thirty drops of the medicinal acid are sometimes given in gruel or ale several times a day, with an ounce each of ether and powdered cinchona bark. In purpura Robertson prescribed $m_{x x}$. to $m_{x x x}$., with grs. $x x x$. iron sulphate, in cold water thrice daily. In relaxed and ulcerated sorethroat, a diluted solution, slowly given, exerts a beneficial influence as a local astringent. Sulphuric acid checks bleeding from the stomach, abates the itching of nettle-rash and lichen, and is an antidote in poisoning by lead, carbolic acid, and alkalies.

Externally it may be used for cauterising irregular, sinuous, and poisoned wounds, and as a styptic and astringent. Three parts strong acid, thoroughly mixed with one of asbestos, and rubbed to fine powder, have been used for removing cancerous and other growths. It is used to destroy warts, which, from their shape or situation, cannot readily be removed by knife or ligature. It hastens disintegration of necrosed bone. Mixed with linseed oil, it is sometimes applied to shrink the skin and reduce umbilical hernia; but ligatures, clamps, or needles are safer and more effectual. A mixture of one part in 12 to 20 of alcohol is employed as an application for seborrhœic dermatitis. It is occasionally added to blistering ointments, but, unless in small amount, is apt to cause blemishing. A 
weak solution is useful in the treatment of chronic pruritus of the tail. A few drops, given with Epsom salt and other saline purgatives, diminish their disagreeable taste and rather increase their activity.

DosEs, etc.-Of the medicinal acid horses take $\mathrm{f} 3 \mathrm{j}$. to

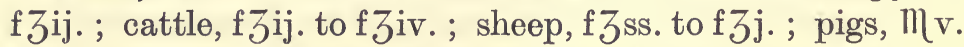
to $m_{x x}$. ; dogs, $m_{i j}$. to $m_{x}$., repeated several times a day, given freely diluted, and often conjoined with aromatics and bitters. As an external astringent, ten to twenty drops of medicinal acid are mixed with an ounce of water.

\section{SULPHUROUS ACID}

Acidum Sulphurosum. An aqueous solution containing 6.4 per cent. hydrogen sulphite, $\mathrm{H}_{2} \mathrm{SO}_{3}$, corresponding to 5 per cent. by weight of sulphurous anhydride, $\mathrm{SO}_{2}$. (B.P.) Sulphurous anhydride may be prepared by burning sulphur in air or oxygen, or by boiling sulphuric acid with carbon, mercury, or copper (B.P.).

The aqueous solution is colourless, has a pungent sulphurous odour, reddens litmus, bleaches colouring matter, leaves no residue when heated, and has the specific gravity 1.025. It is distinguished by its odour; from its salts it is liberated by hydrochloric acid.

Acrions.-Sulphurous acid is antiseptic, disinfectant, and deodorant, and is used as a parasiticide. Concentrated doses, whether in the gaseous or liquid state, are irritant.

General Actions.-Its value in medicine depends on its affinity for oxygen or powerful reducing action, and its arresting the growth of micro-organisms, and not mainly on its acid properties. A solution of one part in 8000 of water destroys diastase and pytalin; but although smaller quantities arrest the action of emulsin and myrosin, one part in 1317 is required to destroy pepsin. Developed bacteria are killed by one part in 2000 of water ; but to prevent reproduction of the spores one part in 325 is needful. Christison found that one-fifth of a cubic inch, diluted with 10,000 volumes of air, destroyed the leaves of plants in forty-eight 
hours. It prevents putrefaction of the gelatin used in paper-making, and destroys the effluvia of the cochineal dye manufacture. Its antiseptic properties are shared by the sulphites and thiosulphates.

Medicinal Uses.-Dewar, Kirkcaldy, greatly extended its application in human medicine and surgery. With solution, fumigation, and spray, he successfully treated nasal catarrh, sore-throat, bronchitis, typhoid fever, as well as wounds. In rheumatism he directed the bed-clothes to be exposed to the vapours of burning sulphur, and laid over the patient, when refreshing perspiration was evoked. In analogous cases amongst the lower animals, sulphurous acid has also proved useful. Robertson employed it to check the muco-purulent discharge of equine influenza. Williams recommended its inhalation in nasal gleet. It is serviceable in catarrh, pharyngitis and laryngitis in horses when the membrane is irritable and relaxed, and the discharges are profuse. It has been prescribed in hoven in cattle and tympanites in horses ; but two-ounce doses of the solution do not give the prompt relief which can be obtained by giving ammonia or ether. In calves, flatulent from hasty or careless feeding, ounce doses, however, arrest undue fermentation. For dogs, $\eta_{x x x}$. to $m_{l x}$., in water, check gastric irritation and vomiting. In husk or hoose of calves and lambs the parasites are destroyed by two fumigations made at an interval of a few days. The affected subjects are placed in a loose box; sulphur is burned six feet distant from them, to dilute the gas before it is breathed; unless bronchial irritation is excessive, they may remain in the medicated atmosphere ten to fifteen minutes. The solution, used alone or with 'Sanitas' fluid, or glycerin, is a good antiseptic dressing for wounds. It is useful in the early irritable stage of eczema, especially in dogs. In limited mange, ringworm, and scab the solution is used as a parasiticide.

Officers of health, both in Britain and America, bear testimony to the efficacy of sulphurous acid as a disinfectant. Outbreaks of foot-and-mouth disease are believed to have been arrested by it. The gas is readily evolved in the stable or premises to be disinfected by scattering flowers of sulphur 
over a few embers in a shovel, iron basin or brazier. It burns best when previously mixed with about one-fourth part of finely-divided charcoal, or when each charge of one and a half pounds of sulphur is treated with an ounce of alcohol, which in burning furnishes a large amount of steam, and thus increases the penetrating and disinfectant power of the gas. Sulphurous acid, even when diluted, causes irritation and coughing when breathed either by men or animals, and consequently for thorough disinfection of infected premises animals must be removed, doors and windows closed, and one and a half pounds sulphur burned for each thousand feet of cubic space. Articles of saddlery and clothing should be cleansed by steam-heat, or washed with corrosive sublimate solution. Such articles, freely exposed to sulphurous gas, are bleached, and eventually damaged, from condensation of sulphurous acid.

Doses, etc.-Of the B.P. solution horses and cattle take

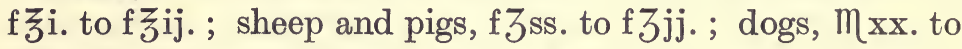
mlx., given every three or four hours, diluted with water or other cold bland fluid. It may be continued until the system is saturated and the skin gives off its odour. It is conjoined as required with aromatics, alcohol, ether, or opium. For surgical purposes it is diluted usually with three or four parts of water; with this the lint or other dressing is kept saturated; admixture with glycerin renders it more soothing. For disinfectant purposes it may be used with carbolic acid, but not with chlorine or bleaching powder, which neutralises it.

\section{HYDROCHLORIC ACID}

Acidum Hydrochloricum. Muriatic Acid. Spirit of Salt.

A liquid containing 31.79 per cent. of hydrogen chloride, $\mathrm{HCl}$, and 68.21 per cent. of water (B.P.).

Hydrochloric acid is obtained by dissolving in water the gas produced by the interaction of sulphuric acid and sodium chloride. It is colourless, intensely sour and acrid, emits white, pungent fumes of the gas, and has the specific gravity $1 \cdot 160$. The B.P. acidum hydrochloricum dilutum is 
made by mixing six fluid ounces of the stronger acid with water until the mixture at $60^{\circ} \mathrm{Fahr}$. measures twenty fluid ounces. It has the specific gravity 1.052 , and contains 10.58 per cent. of hydrogen chloride. The test for hydrochloric acid is silver nitrate, with which it produces a curdy white precipitate, insoluble in nitric acid, but soluble in excess of ammonia. Its chief impurities are sulphuric and sulphurous acids, nitrous compounds, chlorine, iron, and occasionally traces of arsenic.

Actions AND USES.-Concentrated doses are corrosive and irritant; medicinal doses are astringent, antiseptic, and tonic. Topically it is used as a caustic, stimulant, astringent and antiseptic.

TOXIC EFFECTs.-Concentrated solutions have a strong affinity for the water, bases and albuminoids of the tissues. They leave upon them a white film. When swallowed they cause gastro-enteritis. Independently of irritant or corrosive effects, they appear to destroy life by neutralising the alkali of the blood. Rabbits and herbivora are stated to suffer in this way more readily than dogs or other carnivora. Seven or eight grammes per kilogramme of body-weight may be given to rabbits in twenty-four hours without serious results, but nine grammes prove fatal in a few hours, causing frequent laboured breathing, quick pulse, imperfect power of moving, and death, depending upon fatal diminution of the alkali in the blood, determining first stimulation, and soon paresis of the respiratory centre. That these toxic effects directly depend upon neutralisation of the alkali in the blood appears to be demonstrated by experiments, in which animals nearly dying from acid poisoning promptly revived when sodium carbonate was injected into the veins. The alkaline antidote proves effectual even when three times the ascertained fatal dose of acid has been administered (Phillips).

Medicinal Uses.-Hydrochloric acid, made into an electuary with glycerin, treacle, or honey, or diluted with water (2 to 5 per 1000), exerts astringent and antiseptic effects on irritable, relaxed, or ulcerated condition of the mouth and throat. Stimulating the mucous membrane of the mouth, it reflexly evokes secretion of saliva, moistening 
the parched mouth and abating thirst. Like other acids, it specially stimulates the pancreatic, and other alkaline secretions. Hydrochloric acid is the special acid of the gastric juice; in herbivora it amounts to 1 to 2 per 1000 . When the natural acid of this fluid is deficient, digestion is performed tardily and imperfectly, the food ferments and acrid acids are evolved. For obviating or removing such conditions, hydrochloric acid is specially suitable; it aids digestion, especially of albuminoids, controls acid fermentation common in young animals, and due to lactic acid and other organisms in the milk, and hence often checks diarrhœa. In young calves or foals, digesting their food indifferently, and scouring, a few drops of hydrochloric acid may be given with the milk, exerting both antiseptic and digestive effects ; and acids are usually preferable to alkalies, being given either immediately before or about an hour after feeding. Acids conjoined with bitters are also useful for convalescents from exhausting disease, for show beasts that have been systematically over-fed, and for young and weakly, as well as for old, enfeebled subjects. The acid treatment is equally appropriate in the totally different gastric condition of undue acidity depending upon excessive but weak secretion, for here the astringent effects tend to rest the mucous membrane; but in such case the acid should be administered half an hour before feeding. Given alone or with ferric chloride, it promotes a healthier state of the bowels in animals infested with intestinal worms, and sometimes expels ascarides. Like other mineral acids, it increases the acidity of the urine during excretion.

Externally it is used to destroy warts, and, as a caustic and antiseptic for wounds, for foot-rot in sheep, and occasionally as a styptic. A tepid solution, diluted until only faintly acid to the tongue, is sometimes used, instead of vinegar and water, for rapidly sponging the skin of febrile patients.

DosEs, etc.-Of diluted or medicinal acid, horses take f3ss. to f3ij.; cattle, f3ij. to f3iv. ; sheep and pigs, $m_{x v}$. to $m_{x x}$. ; dogs, $m_{\text {iij. }}$ to $m_{x x}$., usually prescribed with forty or fifty parts of water; often given along with bitters and iron salts. 


\section{NITRIC ACID}

Acidum Nitricum. Aquafortis. A liquid containing 70 per cent. by weight of hydrogen nitrate, $\mathrm{HNO}_{3}$, and 30 per cent. of water. (B.P.)

Nitric acid is prepared by the interaction of sulphuric acid and potassium or sodium nitrate. Specific gravity 1.42. The B.P. Acidum Nitricum Dilutum contains $17 \cdot 44$ per cent. of hydrogen nitrate. $\mathrm{HNO}_{3}$. Specific gravity $1 \cdot 101$.

Properties.-Nitric acid, in tolerably concentrated solution, is colourless; emits pungent, corrosive, suffocating fumes ; has an intensely sour taste ; oxidises, corrodes, and dissolves many organic substances; has great affinity for water ; in imperfectly stoppered bottles it quickly increases in quantity and diminishes in strength ; diluted with water it evolves much heat.

Its tests are the production of an orange-red colour with a solution or crystal of morphine or brucine; copper, mercury, and some other metals deoxidise strong solutions, with evolution of ruddy nitric peroxide fumes $\left(\mathrm{NO}_{2}\right)$; it gives a yellow stain of picric acid to wool and to the skina discoloration deepened by alkalies, and removed from the skin only by its desquamation.

Actions AND Uses.-Nitric acid is irritant and corrosive, and especially destructive when in concentrated solution and containing the volatile nitrous acid. It leaves yellow or brown stains on the skin and throat, but in the stomach this discoloration is usually obscured by inflammation or extravasation of blood. Besides acting like the other mineral acids, it exerts oxidising effects, notably when used locally, and probably also when given internally. It has some reputation as a hepatic stimulant and tonic, frequently indicated in horses recovering from influenza, jaundice, and other debilitating disorders, and is alternated with arsenic, in eczema and chronic skin diseases, but is not so good for these affections as dilute nitro-hydrochloric acid.

Externally the medicinal acid is applied for extirpating warts, fungous, and malignant growths which cannot be 
removed by the knife ; for dissolving the hardened scurf, and promoting a healthier condition of skin in mallenders and chronic eczema ; and as a caustic in poisoned wounds, necrosis, canker, and foot-rot. As an escharotic it is generally applied on a glass rod ; surrounding tissues are protected by lard or oil, and undue action arrested by subsequent washing with an alkaline solution. Freely diluted in hot water, it abates the itching of nettlerash. Dissolved in two or three hundred parts of water, it is used for sponging the skin, and for relieving the tenderness and tension of piles in dogs. Nitric acid preserves putrescible substances, and prevents evolution of hydrogen sulphide more effectually than either hydrochloric or sulphuric acid; but as a disinfectant, it cannot be recommended, owing to its action on organic and metallic substances, and the irritant effects of its fumes.

DOSES, etc.-Of the diluted medicinal acid, horses or cattle take $\mathrm{f} 3 \mathrm{i}$. to $\mathrm{f} 3 \mathrm{ij}$. ; sheep and pigs, $\mathrm{m}_{\mathrm{x}}$. to $3 \mathrm{j}$. ; dogs, $m_{\text {ij. to }} m_{x x}$. It must be largely diluted with water or other bland fluid, and is often conjoined with bitters. For external application, a drachm of strong acid in a pint of water suffices for all except escharotic purposes. An ointment is occasionally used, made by melting together in a glass vessel a pound of olive oil, four ounces of lard, and when the mixture is nearly concrete, adding six drachms of nitric acid, and stirring briskly with a glass rod till the whole solidifies. A paste made with sulphur and lard is used for extirpating warts, and stimulating patches of scurfy skin.

\section{DILUTED NITRO-HYDROCHLORIC ACID}

Acidum Nitro-Hydrochloricum Dilutum. NitroMuriatic Acid. Aqua regia. An aqueous solution of free chlorine, hydrochloric, nitric and nitrous acids.

The diluted nitro-hydrochloric acid of the B.P. is prepared by adding to twenty-five fluid ounces of distilled water in a glass-stoppered bottle three fluid ounces of nitric acid and four of hydrochloric, and allowing the mixture to stand for fourteen days before it is used. It contains free chlorine, and has the specific gravity 1.07. 
Action, Uses, AND Doses.-The strong acid is very corrosive and irritant, but it is not used as a caustic. Medicinal doses are said to exert special tonic and stimulant actions on the liver and intestinal glands, and are used in hepatic torpidity, catarrhal jaundice, rickets, and occasionally in equine influenza. The presence of free chlorine may help to explain this action on the liver. It is prescribed with the same precautions as to dilution as nitric acid. Doses, horses and cattle, $3 \mathrm{j}$. to $3 \mathrm{ij}$.; sheep and pigs, $m_{\mathrm{x}}$. to $3 \mathrm{j}$. ; dogs, $\mathrm{m}_{\mathrm{ij}}$. to $\mathrm{m}_{\mathrm{xx}}$., in drench.

\section{PICRIC ACID}

Acidum picricum or trinitrophenic acid, prepared by the action of fuming nitric acid on phenol, occurs in yellow, shining, odourless crystals, having a very bitter taste; slightly soluble in water; very soluble in alcohol, ether, and chloroform. Heated slowly with free access of air picric acid burns; heated rapidly it explodes. It precipitates albumin in acid solution, stains the skin, and hair, or wool yellow, and with bases forms salts, of which the most important in veterinary practice is potassium picrate. Picric acid is a powerful antiseptic and vermicide, and a very active desiccant. Applied externally in suitable cases it promotes the formation of epidermis and hastens the cicatrisation of cutaneous wounds, and in solution or ointment it has been used in the treatment of eczema, pruritus, and burns. It is seldom prescribed as an anthelmintic as it is liable to produce fatal gastro-enteritis. The toxic dose for the dog is ten grains (Kaufmann).

Picrate of potash, which is much less irritant and toxic than the acid, has been employed as a vermicide in sheep and pigs.

Doses (picric acid).-Lambs, grs. jss to grs. iijss ; (picrate of potash), lambs, grs. vii. to grs. xv. per day ; young pigs, grs. iijss to grs. viii., per day, in gruel, mucilage, or in bolus.

\section{CONCENTRATED PHOSPHORIC ACID}

Acidum phosphoricum concentratum, a liquid containing 66.3 per cent. of hydrogen orthophosphate $\mathrm{H}_{3} \mathrm{PO}_{4}$, with 
33.7 per cent. of water. Prepared by treating, with water and nitric acid, the residue left after burning phosphorus in air (B.P.). Specific gravity $1 \cdot 5$. It is a colourless, sour, syrupy liquid, with an acid reaction. In diluted solution it gives, with ammonia-silver nitrate, a canary-coloured precipitate, soluble in ammonia and dilute nitric acid. The acidum phosphoricum dilutum contains 13.8 parts of hydrogen orthophosphate and 86.2 parts of water. It is a colourless liquid of specific gravity $1 \cdot 08$.

Compared with the other mineral acids it is less corrosive, but it may be used for many of the purposes for which nitric and hydrochloric acids are employed. Internally, phosphoric acid is believed to be less apt to derange digestion when prescribed for a considerable time. In human medicine it has been given in diabetes and various cachectic conditions, to supply a supposed deficiency of phosphates, but with little success. In canine practice it is sometimes useful in the convalescent stage of distemper. Doses (dilute acid), horses and cattle, $3 \mathrm{j}$. to $3 \mathrm{jv}$.; sheep and pigs, $m_{x x x}$. to $3 \mathrm{j}$. ; dogs, $\prod_{\mathrm{v}}$. to $\mathrm{m}_{\mathrm{xxx}}$.

\section{CHROMIC ACID}

\section{Chromic Anhydride $\left(\mathrm{CrO}_{3}\right)$.}

Acidum chromicum produced by the interaction of sulphuric acid and potassium bichromate, occurs in crimson, deliquescent, needle-shaped crystals, and is very soluble in water and in ether. The liquor acidi chromici is made with one part of acid and three of water. It readily parts with oxygen, oxidises organic matter, coagulates albumin, destroys low organisms, and decomposes ammonia and sulphuretted hydrogen, and though not prescribed internally, it is used as a caustic, antiseptic, deodoriser, and disinfectant, and to harden the replacing horn after operations on the horse's foot. In the treatment of 'canker,' Sheather has been very successful with pure chromic acid applied on carbolised cotton wool. For snake-bites, Kaufmann recommends the injection round the wounds of four or five drops of a one per cent. solution of chromic acid. 


\section{HYDROBROMIC ACID}

\section{Acidum Hydrobromicum Dilutum.}

Diluted hydrobromic acid is an aqueous solution containing 10 per cent. by weight of hydrogen bromide $(\mathrm{HBr})$. Prepared by the distillation of potassium bromide with concentrated phosphoric acid. A colourless, odourless acid liquid. Specific gravity 1·077. It is occasionally employed in human medicine as a nerve sedative, and it has been recommended as an anodyne for nervous diseases of dogs in doses of $m_{\text {xx }}$. to $\mathrm{m}_{\mathrm{lx}}$. (See Bromides.)

\section{CARBONIC ACID}

Acidum Carbonicum. Carbon Dioxide. Carbonic Anhydride. Choke Damp. After Damp. $\left(\mathrm{CO}_{2}\right)$.

When air, containing more than $\frac{1}{200}$ parts by volume of carbonic acid, is inhaled, by either man or the domestic animals, discomfort and languor are produced. An atmosphere containing $\frac{1}{12}$ promptly prevents removal of the carbonic acid from the blood, interferes with oxidation of the tissues, and hence impairs their functions ; $\frac{1}{9}$ shortly causes unconsciousness and anæsthesia; $\frac{1}{5}$ does so more rapidly, renders the blood markedly venous, but if respiration of the mixture has been continued only for a few minutes, recovery gradually occurs on removal to the fresh air. If the amount of oxygen is increased in the mixture whilst the percentage of carbon dioxide remains the same, asphyxia does not occur, but only narcosis from the direct action of carbon dioxide in depressing the central nervous system. Asphyxia, when it occurs, is referable to the absence of, or decrease in, the amount of oxygen. Equal parts of carbonic acid and air quickly produce death by asphyxia, exhibiting the three stages characteristic of such poisoning, namely-(1) dyspnœa, (2) convulsions, and (3) paralysis. Examination after death discovers general venous congestion, the blood dark-coloured, the right side of the heart much distended with blood, the brain congested, and sometimes exhibiting exudation and extravasation. In treating such cases, endeavour is made to oxygen- 
ate the stagnating venous blood by bringing the animal into a pure atmosphere, stimulating respiratory effort by dashing cold water over the head and neck, employing artificial respiration, and, if the action of the heart is failing, relieving its engorgement by drawing blood from the jugular vein. Carbonic acid in solution applied to the skin or stomach produces mild irritation and stimulation. Effervescent drinks increase gastric and intestinal secretion, hasten metabolism, and are excreted from the kidneys more rapidly than corresponding quantities of non-aërated water.

\section{ACETIC ACID}

Glacial acetic acid is prepared by heating sodium acetate with sulphuric acid. One hundred parts by weight should contain ninety-nine parts of hydrogen acetate, $\mathrm{CH}_{3}$.COOH. Specific gravity $1 \cdot 058$. The glacial acid is mobile, oily, and colourless, with a pungent acetous odour and taste, and a corrosive action upon organised tissues. It boils at $243^{\circ}$ Fahr., distils unchanged, is combustible, miscible in all proportions with water and alcohol, crystallises into radiating pearly plates when cooled, and remains crystalline until the temperature rises above $60^{\circ} \mathrm{Fahr}$.

Acetic acid (acidum aceticum) is a product of the destructive distillation of wood, or of the oxidation of ethylic alcohol. One hundred parts by weight should contain thirty-three parts of hydrogen acetate, and sixty-seven of water. Specific gravity 1.044. It is colourless, strongly acid, with a pungent odour. Acetic acid is about onethird the strength of the glacial acid (B.P.). An imperfectly purified acid containing some residual tarry matters is sold as pyroligneous acid. The acidum aceticum dilutum is acetic acid diluted with distilled water until it has a specific gravity of $1 \cdot 006$. It should contain $4 \cdot 27$ per cent. hydrogen acetate.

Acetic acid, even when considerably diluted, reddens litmus, dissolves volatile oils, resins, camphor, and most alkaloids, and unites with bases to form the crystallisable and soluble acetates, which are distinguished by the acetous odour they emit when heated with sulphuric acid; the pleasant odour of acetic ether they evolve when heated with 
alcohol and sulphuric acid; and the red-brown colour they produce in neutral solution when treated with ferric chloride - a colour which changes on boiling to a brown precipitate of basic acetate of iron.

Vinegar (acetum) is diluted acetic acid, sometimes containing traces of colouring matter, mucilage, alcohol, ethers, sulphuric acid, and calcium sulphate. Besides being got from the destructive distillation of wood, it is also obtained from the oxidation of impure alcohols, by exposing them to the air at a temperature of about $80^{\circ} \mathrm{Fahr}$., and in contact with a ferment. In this way vinegars are manufactured in this country from malt, grain, cider, or solutions of sugar or spirit ; in France, by exposing the poorer wines in half-filled casks ; and in Germany, by what is termed the quick vinegar process, from weak spirit mixed with about 1-1000th part of yeast, or beetroot juice, and allowed slowly to trickle at a temperature of from $75^{\circ}$ to $80^{\circ} \mathrm{Fahr}$. over a large surface of wood shavings previously soaked in vinegar. There is shortly formed on the surface of the shavings a gelatinous mould-the mycoderma aceti-which favours attraction of oxygen from the air, supplies it to the alcohol, and hastens its conversion into acetic acid.

Actions AND Uses.-Acetic acid is corrosive, irritant, and vesicant. It is seldom used internally. Diluted, usually in the form of vinegar, it is employed externally as a stimulant and refrigerant, and in pharmacy as a solvent.

TOXIC EFFECTS.-An ounce of acetic acid destroyed a medium-sized dog in an hour, with symptoms of uneasiness, abdominal pain, vomiting, and collapse ; a quarter of an ounce was fatal in five to nine hours; four or five ounces of vinegar in ten to fifteen hours (Christison). Horses take six to twelve ounces of vinegar, and cattle three or four pounds, without apparent injury (Hertwig). A goat weighing $44 \mathrm{lbs}$. got five ounces, and died in twenty-six hours, the lesions consisting of hyperæmia and œdema of the lungs, and inflammation of the mucous lining of the digestive tract, the kidneys, and liver (Fröhner).

Antidotes.-Magnesia, soap and water, lime water, chalk and water.

EXTERNAL Uses.-Rubbed into the skin, acetic acid 
speedily causes redness, and eruption of large blisters resembling those produced by boiling water; but as a vesicant, mustard or cantharides is preferable. As an astringent or caustic it is rarely used. Dissolving albumin, fibrin, and gelatin, it removes warts as well as corns in the human subject, softens scurf, destroys cryptogamic parasites and acari, and hence is sometimes found in prescriptions for mallenders, ringworm, scab, and mange. Along with either hot or cold water, vinegar is used for contusions and strains, and for sponging the skin and checking excessive perspiration in febrile disorders. Once in high repute as an antidote for many poisons, vinegar is now employed only in poisoning by the alkalies and alkaline carbonates. It dissolves the active principles of various drugs, and enters into the composition of aceta, or vinegars of cantharides, ipecacuanha, and squill. Oxymel is made by heating together forty ounces of sugar or honey, and five each of acetic acid and distilled water.

\section{TARTARIC ACID, $\mathrm{H}_{2} \mathrm{C}_{4} \mathrm{H}_{4} \mathrm{O}_{6}$.}

Acidum tartaricum is prepared from acid potassium tartrate. The crystals are colourless, monoclinic prisms, longer than those of citric acid, with an acid taste, soluble in less than their own weight of water, and less than three times their weight of alcohol (90 per cent.). Either watery or spirituous solution not too diluted, when stirred with potassium acetate, yields a white crystalline precipitate.

Tartaric acid is devoid of irritant and poisonous properties. Christison gave drachm doses to cats without causing them apparent inconvenience. Like other organic acids, it is burnt within the body, and the resulting carbonates exert their usual alkaline effects. It closely resembles citric acid, and, like it, is used as a cooling antipyretic. Doses, horses and cattle, 3 ij. to 3 jv.; dogs, grs. x. to grs. $\mathbf{x x x}$.

\section{LACTIC ACID, $\mathrm{CH}_{3}$.CHOH.COOH.}

Acidum lacticum produced by the fermentation of lactose, from the action of the lactic acid bacillus, is a colourless, 
syrupy liquid, which the B.P. states contains 75 per cent. of hydrogen lactate, with 25 per cent. of water. Specific gravity $1 \cdot 21$. It is miscible in all proportions with water, alcohol and ether.

It is present in the gastric juice, while sarcolactic acid, which is isomeric with it, is found in the juice of flesh and in the bile. It has a special power of dissolving false membranes, and in human patients has accordingly been used as spray or vapour in the treatment of croup and diphtheria. Diluted and sweetened, it is occasionally substituted for hydrochloric acid in dyspepsia. It is a good intestinal antiseptic, and is prescribed for diarrhœa following distemper of the dog, and, with bismuth subnitrate, for muco-enteritis in calves. Doses, horses and cattle, $3 \mathrm{ij}$. to $3 \mathrm{jv}$.; dogs, mxx. to $3 j$.

Lactic acid, as a remedy for disease arising within the intestine, has received considerable attention since Metchnikoff attributed the remarkable longevity of the inhabitants of Bulgaria (where deaths at ages of 110 to 120 are fairly common) to their habit of drinking sour milk. Soured milk, containing active lactic acid bacilli, and lactic acid derived from the lactose of the milk, by inhibiting the growth of intestinal bacteria and arresting putrefaction, is believed to prevent those diseases which are due to absorption of toxins from the bowel. Various proprietary preparations, Lactillin, Lactone, Trilactine, Lactobacilline, etc., containing lactic acid bacilli are now supplied for souring sterilised milk prior to its administration.

\section{CITRIC ACID, $\mathrm{C}_{3} \mathrm{H}_{4} \cdot \mathrm{OH} \cdot(\mathrm{COOH})_{3} \cdot \mathrm{H}_{2} \mathrm{O}$.}

Acidum citricum, or hydrogen citrate, obtained from the juice of the fruit of various species of citrus, occurs in large, colourless, trimetric prisms. Soluble in water, glycerin, alcohol, and ether. A solution of citric acid, thirty-five grains in an ounce of water, forms a convenient substitute for lemon-juice, which has been used as a refrigerant in canine distemper. Citric acid is employed in the preparation of the granular effervescing citrates. Doses, horses and cattle, 3ij. to 3jv.; dogs, grs. x. to grs. xx. 


\section{OLEIC ACID, $\mathrm{CH}_{3}\left(\mathrm{CH}_{2}\right)_{7} \mathrm{CH}: \mathrm{CH}\left(\mathrm{CH}_{2}\right)_{7} \mathrm{COOH}$.}

Acidum oleicum, or hydrogen oleate, is obtained by the saponifying action of alkalies and subsequent action of acids, or by the action of superheated steam upon the olein of fats. Usually not quite pure (B.P.). It is a straw-coloured liquid, occasionally with a faintly rancid smell, and a slight acid reaction. Becomes brown and more acid on exposure to the air. Insoluble in water; readily soluble in alcohol (90 per cent.), chloroform, or ether. Specific gravity, 0.890 to 0.910 . Oleic acid is used in pharmacy for dissolving various metallic oxides and the alkaloids morphine, aconitine, atropine, veratrine, and cocaine. It is used in the preparation of mercuric oleate and of various ointments. (Squire.)

\section{BORIC ACID}

ACIDUm Boricum. Hydrogen Borate. Boracic Acid. $\mathrm{H}_{3} \mathrm{BO}_{3}$.

Boric acid is obtained from borax by the action of sulphuric acid. In volcanic regions in Italy and in the Lipari Islands, through natural fissures or holes bored in the earth, there issue vapours and jets of steam, which are passed through water, and the solution, subsequently evaporated, yields colourless, pearly, lamellar crystals of boric acid. They are feebly acid, and bitter, with a sweetish after-taste, and dissolve in thirty parts of cold water, in three of boiling water, in sixteen of rectified spirit, and five of glycerin, and communicate a green colour to an alcoholic flame. The addition of borax increases the solubility of boracic acid. Aiding the fusion of other bodies, it is much used as a blowpipe test: mixed with seven parts of acid potassium tartrate, it constitutes the soluble cream of tartar of the shops. When heated, three molecules of water are driven off, and anhydrous vitreous boric acid remains $\left(\mathrm{B}_{2} \mathrm{O}_{3}\right)$.

ACTIONS AND USES.-Boric acid is a non-volatile, non-irritating but rather feeble antiseptic, which has more effect on organised than on chemical ferments. Ingested, it is readily absorbed by the digestive mucous membrane, and 
in the blood it is transformed into borate of soda. Small doses produce no appreciable effect on circulation or respiration; but large doses ( 1 gramme per kilogramme of live weight) cause gastro-enteritis, with nausea, vomiting, muscular feebleness, albuminuria, and collapse. These symptoms may follow the injection of strong solutions into wounds, or into the uterus. It is occasionally prescribed in fermentative diarrhœa in foals, calves, and dogs, but estimation of the aromatic sulphates in the urine, the measure of intestinal putrefaction, shows that it has little effect on this. In weak solution it is useful for washing out the stomach and for douching wound cavities after operations. It is excreted in the saliva, sweat, and urine, and exerts slight antiseptic effects in cystic catarrh.

As an antiseptic it is much less powerful than carbolic acid. Koch found that one part in 1250 of water hindered, and one part in 800 prevented, development of anthrax bacillus. The carcase of a horse, which had lain four months in a Californian soil rich in borax, was completely preserved and free from odour (Robottom). It is used for the preservation of milk, fish, and other articles of food. Being non-volatile, its effects are confined to the parts with which it comes into actual contact. Even in concentrated form, it is not so irritant and caustic as carbolic acid, and hence is adapted for wounds which for some time have been treated with carbolic acid, and in which granulation has become tardy. A foul wound or ulcer of moderate size, after thorough washing with corrosive sublimate or zinc chloride, or repeated dressing with carbolic acid, may usually be kept aseptic by boric acid. Extensive wounds as those of the withers and poll after operation may be stuffed with crystallised boric acid, which acts as a powerful antiseptic (Busy). A warm 3 per cent. solution has been used to irrigate the chest in septic pleurisy. In the form of lotion or ointment, spread on gauze or lint, it proves a soothing dressing for burns and blistered surfaces ; as a spray, it relieves aphthous, irritable, ulcerated throat, and, like borax, checks excessive salivary or pharyngeal secretion. Alternated with weak alkaline lotions or zinc oxide dressings, boric acid, conveniently mixed with six or eight parts of starch and one 
part of iodoform abates the erythema and itching of eczema in dogs and horses. Catarrhal conjunctivitis and keratitis are benefited by spraying the surface three or four times a day with a solution containing three to six grains boric acid, one drachm of aqueous solution of opium, and an ounce of water.

Doses, etc.-Horses and cattle take 3ii. to $3 \mathrm{v}$.; foals and calves, grs. $\mathrm{xx}$. to grs. xxx. ; dogs, to which it is usefully given in distemper, grs. $\mathrm{v}$. to grs. $\mathrm{xx}$.

A saturated solution is made with one part of acid to sixteen of water, and for surgical purposes is used diluted as required. A few grains of salicylic acid are sometimes added. An ointment is prepared by melting nine parts white paraffin ointment, and adding one part boric acid in fine powder. Boric dressings are made by soaking lint, gauze, cotton wool, or oakum in a saturated boiling watery solution, from which the acid crystallises, adhering to the fibrous material. Four to ten layers of lint are applied, either wet or dry, over the wound; and in order to prevent evaporation and access of micro-organisms, a piece of mackintosh is laid on the lint and secured by a bandage. Unless the wound discharges are excessive, a properly applied dressing does not require to be disturbed for several days. A few layers of boric lint applied wet to a wound or ulcer, and covered with oiled silk or mackintosh, acts' as an antiseptic compress, and its effects may be kept up by pouring the lotion from time to time between the folds of lint. Boric gauze is charged with 20 per cent. of boric acid.

Boro-glyceride is made by heating ninety-two parts glycerin with sixty-two boric acid. Solutions of one to twenty of hot water are used as antiseptic gargles, lotions for purulent ophthalmia, urethritis, and dressings for wounds.

\section{FORMIC ACID-FORMATES}

Formic or aminic acid $\left(\mathrm{H}_{2} \mathrm{CO}_{2}\right)$ is obtained by oxidation from various organic substances (albuminoids, fatty bodies, acid fats, sugar, etc.). It is a volatile, colourless fluid, with an irritating odour and a caustic taste; very soluble in 
water. By saturation with carbonates, or oxides, it forms salts which are known as formates. Formic acid is antiseptic, caustic, and musculo-tonic. It improves the nutrition of the tissues, promotes muscular energy, and is specially valuable in convalescence following influenza, pneumonia, strangles, purpura, or other systemic disease. As a general tonic it is prescribed as a formate or as a combination of glycerophosphates and formates of soda, calcium, strychnia, and quinine.

Glyphocal with formates (Squire and Sons), a compound elixir of calcium, iron, magnesium, sodium, and potassium glycerophosphates, pleasantly flavoured, and combined with the formates, is a valuable tonic for young horses and dogs. It may be given in doses of 3 iij. to $3 \mathrm{vj}$. to horses, and $m_{x}$. to $m_{l x}$. to dogs, with the food, or drinking-water. Formic acid is seldom employed as an antiseptic, but occasionally a solution of one part acid in three of alcohol is used as a caustic.

\section{CARBON DISULPHIDE}

Carbonis disulphidum, $\mathrm{CS}_{2}$, may be prepared by the combination of carbon and sulphur at a high temperature, the product being subsequently condensed and purified (B.P.). It is a clear, colourless, inflammable liquid, with a characteristic odour, and soluble in alcohol, ether, chloroform, the fixed and volatile oils, and in five hundred parts of water.

Carbon bisulphide is little used in veterinary practice, but it was suggested by Perroncito as an excellent agent for the destruction of bots in the horse's stomach and strongyles in the intestine. In foals and aged horses, Taar employed this remedy with excellent results, and found that while one dose was sufficient for foals infested with gastrophilus larvæ, a second dose followed by a purgative was necessary for those with worms. He states that autumn is the best time for this treatment, as then the parasites are immature. Doses, horses, $3 \mathrm{ij}$. to $3 \mathrm{jv}$. ; foals, $3 \mathrm{j}$. to $3 \mathrm{ij}$., enclosed in gelatin capsules. If necessary the dose may be repeated in two hours, or after an interval of two or three 
days. The dead parasites may be found in the fæces in from sixteen to twenty hours after the administration of the first dose. Occasionally a patient, after receiving carbon bisulphide, shows symptoms of colic. This may be prevented by walking the animal for an hour after giving the medicine.

\section{CHARCOAL-CARBON}

Two varieties of charcoal, or carbon, are used in medicine and pharmacy-wood charcoal, or carbo ligni, and animal charcoal, or carbo animalis. The former is prepared by piling billets of hard wood into heaps, covering them with turf and sand, and leaving a few apertures for admission of air. The pile is ignited : after the flame has risen through the whole mass, the openings are closed, and combustion proceeds slowly, without access of air. The high temperature dissipates moisture, breaks up the complex vegetable matters into simpler forms, producing empyreumatic gases and tarry fluids, and leaving a charred residue of about onefifth the weight of the original wood, and consisting of chemically pure carbon and ash, with oxygen, hydrogen, and traces of nitrogen retained in the porous mass. Oils or resins, when burned in a deficiency of air, produce lamp black-a finely-divided, amorphous carbon.

Animal charcoal, also known as bone or ivory black, is chiefly prepared from bones, which are boiled to separate fatty matters, and heated in close vessels until the ammoniacal gases cease to be disengaged. The fixed residue contains about 10 per cent. of carbon, 88 per cent. of calcium phosphate and carbonate, and 2 per cent. of iron carbide and silicide-mineral matters which separate the carbon particles and enhance their absorbent properties.

Both vegetable and animal charcoal are remarkable for their porosity, are brown-black, insoluble and inodorous, readily absorb moisture, gases, and most vegetable colouring matters. Animal charcoal is distinguished by its greater density, its incombustibility, its bitter taste, its large proportion of phosphates, and also by its greater absorbing power.

ACTIONS AND USES.-Charcoal is a desiccant, antiseptic, disinfectant, and deodorant, and is used as a decoloriser in 
pharmacy, sugar-refining, and other arts. One volume of good boxwood charcoal absorbs into its pores 100 volumes of ammonia gas, $\mathbf{5 0}$ of hydrogen sulphide, and $\mathbf{1 0}$ of oxygen. The oxygen thus retained decomposes and deodorises noxious gases which come into contact with the charcoal. Air laden with sewer gases is purified by passing it over charcoal. Brown sugar in solution filtered through animal charcoal is deprived of colouring matter; crude spirit is robbed of its fusel oil; foul water is cleansed of organic impurities. Urine heated with it parts with colouring matters, urea, and uric acid, but not with any sugar it may contain. Vegetable acids, alkaloids, and their salts are sometimes purified by charcoal; but for these pharmaceutic purposes it has the disadvantage of retaining, not only colouring particles, but portions of the drugs - a property, however, which renders it serviceable as an antidote in poisoning with opium, aconite, strychnine, and even prussic acid. The charcoal mechanically envelops and holds the poisonous particles; half an ounce, swallowed immediately after a grain of morphine or strychnine, is stated to prevent absorption.

Sprinkled over meat or game, or placed in barrels of water intended for long keeping, it retards putrefaction. Unlike chlorides of mercury and zinc, or other powerful antiseptics, it does not, however, attack or destroy organised germs. Whilst passing through the alimentary canal, it checks fermentative changes, lessens acridity and fœtor of the fæces ; probably also removes mucus, and exerts some degree of healthy stimulation. As an absorbent and deodoriser, it is occasionally used as a poultice, and is often sprinkled over suppurating or foul wounds. Equal parts of charcoal, gum arabic, and resin constitute a useful hæmostatic.

Doses, ete.-For the horse, Ziv. to zi.; for cattle, zi. ; for sheep and pigs, 3i. to 3iij. ; and for dogs, gr. x. to grs. lx. It is usually given suspended in gruel or other mucilaginous fluid. To preserve its activity charcoal must be kept dry and clean. Raised to a low red-heat shortly before it is used, gases and organic matters are burned out of its pores, and its efficacy is much increased. The charcoal fouled in sugar-refining and other processes is thus cleansed for repeated use. 


\section{A L COHOL}

The more important mono-hydric alcohols used in medicine and pharmacy are :-

Methyl-alcohol, $\mathrm{CH}_{3} . \mathrm{OH}$, from distillation of wood. Ethyl- " $\quad \mathrm{C}_{2} \mathrm{H}_{5} \cdot \mathrm{OH}$, ", fermentation of grape sugar. Propyl- " $\quad \mathrm{C}_{3} \mathrm{H}_{7} \cdot \mathrm{OH}, \quad, \quad$ grapes. Butyl- " $\mathrm{C}_{4} \mathrm{H}_{9} . \mathrm{OH}, ", \quad$ beet. Amyl- " $\quad \mathrm{C}_{5} \mathrm{H}_{11} \cdot \mathrm{OH}, ", \quad$ potatoes.

These alcohols are hydroxides of the radicles of the methane or marsh gas series $\left(\mathrm{CH}_{4}\right)$. In their production the hydrocarbon has one of its atoms of hydrogen $(\mathrm{H})$ displaced by the radicle hydroxyl $(\mathrm{OH})$. Thus, methane $\left(\mathrm{CH}_{4}\right)$, losing one atom of $\mathrm{H}$, and assuming one $\mathrm{OH}$, becomes $\mathrm{CH}_{3} . \mathrm{OH}$, or methyl-alcohol, popularly known as wood spirit. Ethane $\left(\mathrm{C}_{2} \mathrm{H}_{6}\right)$, losing one atom of $\mathrm{H}$, and taking up one $\mathrm{OH}$, becomes $\mathrm{C}_{2} \mathrm{H}_{5} . \mathrm{OH}$, ethyl-alcohol, or spirit of wine. Each of these alcohols, when oxidised by removal of $\mathrm{H}_{2}$, in the form of a molecule of water $\left(\mathrm{H}_{2} \mathrm{O}\right)$, yields an aldehyde ; while by substitution of $\mathrm{OH}$ for $\mathrm{H}$, the aldehydes are converted into acids. Thus, ethyl-alcohol $\left(\mathrm{CH}_{3} \cdot \mathrm{CH}_{2} \cdot \mathrm{OH}\right)$ is converted into aldehyde $\left(\mathrm{CH}_{3} \mathrm{COH}\right)$, and thence into acetic acid $\left(\mathrm{CH}_{3} \mathrm{CO} . \mathrm{OH}\right)$. In each of the alcohols, moreover, the $\mathrm{H}$ in the $\mathrm{OH}$ can be replaced by an alcohol radicle, when an ether is formed; thus, ethyl-alcohol $\left(\mathrm{C}_{2} \mathrm{H}_{5} . \mathrm{OH}\right)$ yields common ether $\left(\mathrm{C}_{2} \mathrm{H}_{5} \cdot \mathrm{O} \cdot \mathrm{C}_{2} \mathrm{H}_{5}\right)$.

Absolute alcohol. - Ethyl hydroxide $\left(\mathrm{C}_{2} \mathrm{H}_{5} . \mathrm{OH}\right)$, with not more than one per cent., by weight, of water ; obtained by the removal of water from less concentrated ethylic alcohol and subsequent distillation. Specific gravity from 0.794 to $0 \cdot 7969$. Very volatile and hygroscopic at ordinary temperatures (B.P.). Absolute alcohol is a mobile, colourless fluid, with a spirituous odour, and an intensely fiery taste. It has great affinity for water, takes it from any substance with which it is in contact, and thus exerts its notable power of preserving both vegetable and animal matters,

Rectified spirit (spiritus rectificatus), or alcohol (90 per. cent.). A liquid containing ninety parts by volume of ethyl hydroxide, and ten parts by volume of water ; obtained by 
the distillation of fermented saccharine liquids. Specific gravity 0.8340 . By weight it contains 14.35 per cent. of water, and 85.65 per cent. of ethylic alcohol. It burns with a blue, smokeless flame. Leaves no residue when evaporated, and remains clear when mixed with water (B.P.). It dissolves iodine, camphor, balsams, castor oil, most alkaloids, and deliquescent salts, but not the carbonates. It is used in the preparation of all the spirits, and a large number of the tinctures of the Pharmacopœia, 1898.

Diluted alcohol.-The four official liquids obtained by diluting alcohol (90 per cent.), or rectified spirit, with distilled water, contain respectively $70,60,45$, and 20 per cent. by volume of ethyl hydroxide (B.P.). They may be prepared as follows :-

(1) Alcohol (70 per cent.). -With 100 fluid ounces of alcohol (90 per cent.) mix 31.05 fluid ounces of distilled water. Specific gravity 0.8900 .

(2) Alcohol (60 per cent.).-With 100 fluid ounces of alcohol (90 per cent.) mix $53 \frac{2}{3}(53 \cdot 65)$ fluid ounces of distilled water. Specific gravity 0.9135 .

(3) Alcohol (45 per cent.). - With 100 fluid ounces alcohol (90 per cent.) mix $105 \frac{1}{3}(105 \cdot 34)$ fluid ounces distilled water. Specific gravity 0.9436 .

(4) Alcohol (20 per cent.).-With 100 fluid ounces of alcohol (90 per cent.) mix $355 \frac{3}{4}(355 \cdot 8)$ fluid ounces of distilled water. Specific gravity $0 \cdot 9760$.

The non-official proof spirit (spiritus tenuior) contains 57.09 per cent. by volume of ethylic alcohol. Specific gravity 0.920 . In the B.P. 1898, proof spirit has been replaced by alcohol ( 60 per cent.).

Ethyl bromide and ethyl chloride are volatile liquids, and occasionally used to produce both local and general anæsthesia.

Methylic alcohol $\left(\mathrm{CH}_{3} \mathrm{OH}\right)$. - This spirit is obtained by the destructive distillation of wood. It is also prepared from beet sugar refuse. Methylic alcohol when pure has many of the properties, and is applied to many of the purposes of ethylic alcohol. It burns with a pale flame, is not rendered turbid by water, boils at $140^{\circ} \mathrm{Fahr}$., and has an empyreumatic odour depending upon the presence of acetone (di-methyl- 
ketone) and oily matters. Specific gravity 799 to 803 . From ethylic alcohol it is distinguished by heating with sulphuric acid and potassium bichromate, when it yields formic acid; ethylic alcohol, similarly treated, yields acetic acid.

Methyl chloride, a gaseous compound, prepared from methylic alcohol and hydrochloric acid, is employed as a local anæsthetic.

Methylated spirit (spiritus methylatus) is a mixture of nine parts alcohol (90 per cent.) with one part wood naphtha or methylic alcohol. The methylated spirit on sale in oil and other shops contains petroleum oil, which is added to render the mixture distasteful to drinkers.

Whisky (sp. frumenti) is obtained from fermented grain by distillation. It contains from 50 to 58 per cent. by volume of ethylic alcohol.

Brandy (sp. vini gallici), a liquid distilled from wine, should contain $43 \frac{1}{2}$ per cent., by volume, of ethylic alcohol.

Champagne, the fermented juice of the grape, contains from 10 to 13 per cent., by volume, of ethylic alcohol, and owes its peculiar bouquet to traces of fragrant ethers. Port contains 20 to 30 per cent.; sherry, 16 to 22 per cent.; and claret, hock, and burgundy, 8 to 12 per cent. of ethylic alcohol.

Rum, a spirit of about the same strength as brandy, is obtained by distillation of a fermented solution of molasses. Hollands, Geneva, and gin are prepared from fermented malt, with a small quantity of juniper berries. They contain from 51 to 59 per cent. of alcohol.

Ales and porter are made by infusing malt in water, allowing it to stand until the starch is in great part converted into dextrin and sugar, by means of the ferment diastase; boiling the solution with hops, and adding yeast to cause fermentation, which, however, must be carefully controlled. The dark colour of porter depends on a part of the malt being roasted. Stout, or porter and ales contain from 2 to 9 per cent. of ethylic alcohol.

Amylic alcohol.-A liquid consisting principally of isoprimary amylic alcohol, and prepared by shaking commercial fusel oil with a saturated solution of common salt, separating 
the oily layer, distilling, and collecting the portion which distils between $257^{\circ}$ and $289^{\circ} \mathrm{Fahr}$. Fusel oil is present in all crude ethyl alcohols, is obtained from the distillation of potatoes, and is distinguished by its offensive flavour. It is sparingly soluble in water, but readily soluble in other alcohols, ether, and essential oils. Amylic alcohol, when oxidised, yields valerianic acid. It is used for the manufacture of amyl-nitrite.

Actions AND Uses.-Ethyl-alcohol, commonly known simply as alcohol, according to dose and concentration, is a local irritant, refrigerant, mild astringent, and antiseptic. It is diffusible, and readily absorbed, and its effects are produced on a wide range of organs. Large doses paralyse the nerve-centres, and kill by respiratory arrest. Medicinal doses are diffusible stimulants, and are prescribed as carminatives, antispasmodics, and cardiac excitants. Diluted ethylic-alcohol is a readily assimilable food, and hence a restorative and tonic ; it is antiseptic, diaphoretic, and antipyretic. Causing a fuller stream of blood to circulate through the glands, it increases their secretions, notably that of the kidneys, by which the unassimilated portions of the drug are chiefly excreted. Both ethylic and methylic alcohols are used as preservatives of vegetable substances, and pharmaceutically as solvents.

GENERAL Actions.-Alcohol in tolerably concentrated solution withdraws water from albuminoid solutions, and precipitates them. Applied to a mucous surface, it precipitates a thin white pellicle of albumin, thus exerting irritant and slight astringent effects. Owing to its rapid evaporation, alcohol, when applied to the skin, causes a sensation of cold ; but if applied with friction, or if evaporation be prevented by a covering of oiled silk, it passes into the skin, inducing a sensation of warmth. It is also used as an antiseptic, both for the surgeon's hands, instruments, and the operation area. When swallowed, a similar warming effect is produced in the stomach. Moderate doses dilate the vessels, redden the mucous membrane, and increase the secretion of gastric juice. If not too concentrated, alcohol is readily absorbed through any of the ordinary channels of administration. Orfila poisoned dogs by 
causing them to breathe air charged with it. Being highly diffusible, it quickly enters the blood. It appears to form with the hæmoglobin a compound of diminished capacity to take up and give off oxygen. Full or large doses, accordingly, interfere with oxidation of blood and tissue. Its effects vary considerably with the dose and its concentration ; but even small doses quickly affect the central nervous system. There is an apparent stimulation, as shown by alertness and motor activity, but with larger doses this primary stimulation is succeeded by dulness, depression, passing into stupor and coma. Schmiedebergs' theory with regard to the mode of action of alcohol is that the drug $a b$ initio exercises a depressant effect. This depression first affects the cerebrum, in other words, the judgment and controlling centres. This allows free play to the more reflex centres, for all inhibition is lessened, in fact one may say the brake has been taken off. As the depression spreads it affects the centres in the inverse order of development, and leaves the vital medullary centres till the last.

On the circulation alcohol, in small doses, exerts a definite stimulant effect, quickening the heart and increasing its force, probably by direct action on the cardiac muscle. Blood-pressure is also raised to some extent. With larger doses both these effects are overcome, the heart being made slower, and pressure falling. Respiration is indirectly stimulated, for by causing loss of heat, alcohol promotes quicker breathing to supply oxygen for compensatory heat production.

Observations on various classes of animals demonstrate that alcohol in full doses lowers animal temperature. This mainly results from (1) lessened oxidation ; (2) vaso-motor paresis ; and (3) increased skin-circulation, and consequent increased evolution of heat. As with most of the other effects of alcohol, the lowered temperature is not, however, of long duration. Magnan has shown that puppies lose $3^{\circ}$ to $7^{\circ} \mathrm{Fahr}$. when alcohol is added to their food. "The experiments of Binz and Bouvier on septicæmic fever in animals gave singular results. In rabbits, for instance, after injecting septic fluid, and inducing a febrile reaction ranging from $102^{\circ}$ to $105^{\circ} \mathrm{Fahr}$., about three drachms of 
alcohol diluted with water were introduced into the stomach by means of a tube ; fifteen minutes afterwards the temperature was $104 \cdot 1^{\circ}$, in forty-five minutes $102 \cdot 4^{\circ}$, in ninety minutes $100.9^{\circ}$, in two hours and a half $100.6^{\circ}$ Fahr. The following day the pyrexia returned, and increased till the death of the animal. Very large doses lower the temperature as much as $8.5^{\circ}$ Cent., and if narcosis was induced before fever began, quite prevented its development' (Phillips). Other experiments also show that doses sufficient to cause unconsciousness dangerously reduce temperature of birds to the amount of $5^{\circ}$, of $\operatorname{dogs} 3^{\circ}$, of rabbits $10^{\circ}$, and of man himself $2^{\circ}$ to $3^{\circ} \mathrm{Fahr}$.

Digestion and nutrition, although injured by large, are improved by small doses. Small quantities increase both the oxygen absorption and the discharge of carbonic acid from the lungs, supply the necessary amount of energy, and so check metabolism of fat, but increase that of proteid, thus increasing secretion of urine. If alcohol in small doses is continued for a long period a tolerance is acquired, proteid is spared, and the nitrogenous excess in the urine ceases. Mere traces are removed by the lungs, skin, and kidneys, but by far the larger proportion undergoes combustion in the body, and, like other hydrocarbons, and especially when used with suitable food, alcohol proves a source of heat and vital energy. Schulinus' experiments on these points are conclusive, and have been repeatedly verified. Animals receiving measured quantities of alcohol, so soon as full effects were produced, were killed by bleeding and insufflating air into the vessels. Fractional distillations of the blood and viscera were made separately, and repeated investigations have demonstrated-(1) that alcohol in small or moderate quantity does not localise itself in any particular organ, such as the brain or liver, but diffuses itself equally in the system ; (2) that only the blood contains proportionately more than other tissues ; (3) of the alcohol taken, the greater part is decomposed within the organism, and the amount eliminated by the lungs, skin, and kidneys, is insignificant (Phillips). Binz' experiments show that not quite three per cent. of moderate doses is excreted unchanged. Alcohol is a true food, rapidly assimilated, and 
yielding abundance of energy at little cost to the tissues, and it is in general use in fevers, although it is by no means an indispensable remedy (Mitchell Bruce).

The dietetic value of alcohol has been the subject of much controversy. Its consumption in great part within the body explains how men and animals kept on somewhat deficient diet, on which weight would be lost, nevertheless maintain their weight, when receiving, in addition, daily small doses of alcohol. But for ordinary nutrition it is a food which, although quickly assimilated, is rapidly used up, and, unless carefully employed, has the disadvantage of impairing oxidation and excretion. For permanent repair of waste, and maintenance of strength during severe continued exertion, it cannot compare with suitable food. This was strikingly illustrated in the Ashantee War, when soldiers, on exhausting marches, who received rations of rum, although temporarily improved in vigour soon flagged again ; while those receiving beef-tea were equally refreshed in the first instance, and did not experience the secondary depression which overtook those who preferred the alcohol.

Different classes of animals are somewhat differently affected by alcohol. Man, whose intellectual centres are about eight times larger than his motor centres, has his intelligence quickly acted on by doses which are insufficient to impair his motor functions. Amounts corresponding to 0.4 to the 1000 of the total weight of the body disturb human intelligence ; while 2.40 per 1000 of weight are needed to impair motor functions. In dogs the cerebral are about five times the weight of the motor centres. Alcohol has no notable effect until 1.5 to the 1000 of body weight is taken, and the prominent results then occurring are disturbance not of intelligence, but of motor function. Horses and cattle receiving full doses exhibit excitement and perversion of motor function ; they prance, strike with the feet, are unsteady in their gait, and drag the hind limbs. Muscular twitching and convulsions occasionally occur.

Toxic Actions.-Large doses paralyse the nerve-centres in the inverse order of their development, the cerebral (judgment and volition) being first affected, the respiratory and cardiac (vital centres) last. Thus alcohol affords an 
illustration of the so-called 'law of dissolution.' Its effects are generally divided into four stages-(1) stimulant, (2) narcotic, (3) anæsthetic, and (4) paralytic. With ethylalcohol these stages are usually distinctly marked. With methyl-alcohol the excitement is more intense, the subsequent stages follow more rapidly; but if the doses are not lethal the effects pass off more quickly. With œnanthic and caprylic, which are non-fermented alcohols, the stages are less regular, and are marked by convulsions. Ethyl and methyl alcohols are not so deadly as most of the others. Their toxic dose is eight grammes to the kilogramme of the live weight of the animal. The toxic dose of any crude spirit is less than that of the same spirit after rectification, and the impure spirit produces greater excitement and heavier stupor.

Large concentrated doses produce rapid narcosis with a minimum of preliminary excitement. Hertwig gave an old but serviceably sound horse eight ounces of ethyl-alcohol of specific gravity $\cdot 825$. He became much excited and uneasy, pranced, staggered, and after two minutes fell, struck out vehemently with his feet, rolled his eyes - the pupils at first were contracted, but afterwards were dilated. He rapidly became insensible, and died in about ten minutes. The heart continued to beat for several minutes after death. Four to six ounces produced similar motor excitability, but did not prove fatal.

Dogs about twenty pounds weight, retaining four or five ounces of whisky, given quickly, soon become comatose without any appreciable excitement, and die within a few minutes. Percy injected strong alcohol into the empty stomach in dogs, and they almost immediately fell over insensible; respiratory and cardiac movements ceased within two minutes; the blood was found charged with alcohol. Similar sudden fatal paralysis occurs in men swallowing quickly full draughts of whisky. Dogs receiving one to two ounces of rectified spirit, the œsophagus being afterwards ligatured, become uneasy, endeavour to vomit, are unable to control their movements, become comatose, and die in a quarter to half an hour. One to two drachms induced in dogs reeling and stupor, which continued for 
about half an hour. Harley found that half an ounce of alcohol, with an equal quantity of water, injected into the portal vein of dogs, so seriously interfered with the glycogenic function of the liver that the urine within three hours became diabetic.

Cattle and sheep, and indeed all ruminants, are less susceptible to the actions of alcohol than dogs or horses. Hertwig mentions that when brandy is given to sheep and goats, they soon become fond of it, taking six or eight ounces at a time without exhibiting serious symptoms.

Chronic poisoning by alcohol, with impaired nervous power and fatty degeneration, common in human patients, is unknown in the lower animals.

The antidotes are tea and coffee, or the alkaloid caffeine, cold douches, enforced movement, and other means of warding off the deadly stupor. Strychnine and alcohol are mutually antagonistic, and hypodermic injections of strychnine may be given if collapse occur.

Medicinal Uses.-Few remedies are more frequently and extensively used than the various alcoholic liquids. They stimulate gastric secretion, and improve appetite in atonic indigestion; they act as carminatives in flatulence ; control the spasms of intestinal colic ; and check persistent diarrhoa especially when they are conjoined with sodium benzoate or vegetable astringents. They equalise irregular circulation in chills, and antagonise cardiac feebleness, especially of a temporary character, such as results from debilitating disease, poisoning by sedatives, snake-bite, or shock. Many cases of milk fever in cows, when stupor is approaching, or even during the earlier excitable stages, are benefited by six to ten ounces of whisky, given at intervals of one or two hours.

In influenza amongst hard-worked horses, when the pulse is quick and weak, the breathing hurried and embarrassed, and the temperature above $102^{\circ} \mathrm{Fahr}$., no treatment is more successful than a few ounces of whisky, diluted with gruel or water, repeated every two or three hours; a saline draught administered thrice daily; the body well clothed, and mustard, if need be, applied to the throat. Many cases of bronchitis and pneumonia, after a few days' illness, and 
especially during the period when inflammatory products are being removed, are benefited by moderate, frequently repeated doses of alcohol. The restorative sustaining power of alcohol in the second stage of such disorders is well attested and generally conceded.

The antiseptic and antipyretic actions of alcohol are well exhibited in septicæmia. This is illustrated by an experiment of Binz, who produced septic fever by injection of pus in two young healthy dogs of equal weight, one of which, treated with three doses of two drachms of alcohol introduced into the stomach, shortly recovered, while the other which remained without treatment, died on the second day. His experiments with rabbits, already quoted, also testify to the notable antipyretic effects of alcohol. Not only does it lower high temperature, but in such cases it also helps to maintain the failing cardiac action, and to surmount the critical nervous depression. It is serviceable in horses fevered and enfeebled with protracted strangles, or debilitated by purpura ; in all animals suffering from parturition metritis ; and in dogs exhausted from distemper; as well as in most other forms of systemic poisoning. During convalescence from enervating disorders, as well as in chronic cases connected with mal-assimilation, alcohol is beneficial in all classes of patients as a gastric tonic, general stimulant, and a readily assimilable food. Anæmic subjects are frequently treated with sound ale or spirit and water, conjoined with iron salts and oleaginous food.

The more important medicinal effects follow within ten or fifteen minutes after administration. As already indicated, the weak pulse should become stronger and firmer, the quick pulse slower, breathing should be more natural, the skin more moist, and temperature lower-in a word, there should be remission of the more prominent morbid symptoms. If such favourable effects are not produced, alcohol is not a suitable remedy, or the dose has been wrongly estimated. When unsuitable, or given too frequently, or in quantity too large, whether in health or disease, alcohol hinders oxidation, retards excretion, impairs digestion, and causes dryness of skin and mucous surfaces.

Alcohol somewhat resembles in its actions and uses the 
volatile oils, ethers, and ammonia, and is frequently prescribed with one or more of these allied remedies.

EXTERNAL USES.-In a state of concentration, alcohol, rubbed into the skin, acts as a rubefacient. Coagulating albumin, it is occasionally applied to arrest bleeding. Beaten up with white of egg, it is used in veterinary as well as in human practice to harden the epidermis and prevent excoriation of parts exposed to pressure. Weak solutions, conjoined with a half to one per cent. of carbolic or salicylic acid, exert anodyne effects, relieving itching in erythematous and other skin diseases. Nettlerash is often treated with dilute spirituous solutions, to which one per cent. of petroleum benzene may be added. For surgical purposes it is employed as a refrigerant and antiseptic. As a stimulant and refrigerant for bruises, wounds, and strains, it is popularly used throughout Scotland in the familiar form of whisky and water. A cooling lotion is made with an ounce each of rectified spirit, vinegar, and ammonium chloride, dissolved in a quart of water. As a refrigerant, ice, however, is generally more convenient and effectual. A convenient solvent for the active principles of many drugs, alcohol is largely used for making tinctures and extracts.

Doses, etc.-Of rectified spirit, horses and cattle, $\mathrm{f} z \mathrm{i}$. to Ziv.; ; sheep, fzj.; pigs, fZiv.; and dogs, fZjj. or more, diluted with six or eight parts of water or other bland fluid. Whisky, gin, or brandy, in larger doses may be substituted for rectified spirit ; sherry, port, ale, or stout may be given during convalescence. The suitable doses and the frequency of their repetition are mainly determined by the condition of the patient, and the purpose intended to be served. Alcohol acts promptly, but its effects are transient. It is less apt to excite or disagree when given with the food or drinking water. Mixed with oatmeal or linseed gruel, there is little risk of misappropriation.

To intensify one or another of the effects of alcohol, it is often conjoined with other medicines-with ether or ammonia, when more prompt and powerful stimulation is desired; with opium or chloral hydrate, when anodyne effects are required, or spasms are to be combated; with digitalis, when the full effects of a cardiac tonic and stimu- 
lant are sought; with ammonium acetate, when diaphoresis is to be encouraged; with red cinchona, creosote, or sodium benzoate, when antiseptic and astringent effects are to be produced in the intestine.

\section{ETHER}

EтнеR. A volatile liquid, prepared from ethylic alcohol, by interaction with sulphuric acid, and containing not less than 92 per cent. by volume of ethyl oxide. $\left(\mathrm{C}_{2} \mathrm{H}_{5}\right)_{2} \mathrm{O}$. Formerly termed sulphuric ether. (B.P.)

Ether is prepared by heating in a glass flask, connected with a Liebig's condenser, one part of sulphuric acid, and five of rectified spirit, which is added gradually. Every alcohol, by substitution of an alcohol radicle for the $H$. in the hydroxyl $(\mathrm{OH})$, forms a corresponding ether. Thus, ethyl-alcohol $\left(\mathrm{C}_{2} \mathrm{H}_{5} \cdot \mathrm{OH}\right)$ yields $\mathrm{C}_{2} \mathrm{H}_{5} \cdot \mathrm{O} \cdot \mathrm{C}_{2} \mathrm{H}_{5}$.

The B.P. ether (ethyl-ether) contains about eight per cent. of alcohol and water ; is a colourless, very volatile, inflammable liquid, with a strong and characteristic odour. Specific gravity, 0.735. It should boil below $105^{\circ}$ Fahr.; yields a vapour two and a half times heavier than air, but half that of chloroform ; is miscible in all proportions with chloroform and alcohol (90 per cent.); and is soluble in ten volumes of water. It readily dissolves fats, volatile oils, balsams, resins, and, next after chloroform, is the best solvent for alkaloids.

Purified ether is ether from which most of the ethylic alcohol and water have been removed by washing with distilled water, and subsequent distillation in the presence of calcium chloride and recently calcined lime. Specific gravity not below $\cdot 720$, and not exceeding $\cdot 722$.

ACTIONS AND USES.-Ether is an active member of the alcohol series. It is exceedingly volatile. Applied externally it is irritant, refrigerant, and a local anæsthetic. Given internally, it is quickly absorbed; acts specially on the central nervous system; primarily, and in small doses, stimulating; secondarily, and in larger doses, paralysing, and when inhaled producing anæsthesia. Poisonous doses 
almost immediately cause inebriant narcosis; and kill by paralysis of respiration. It is prescribed as a general stimulant, carminative, and antispasmodic, and is also expectorant, diaphoretic, diuretic, antiseptic, and parasiticide. Next to chloroform it is the most generally used anæsthetic.

General Actions.-Applied to the skin, ether evaporates and abstracts heat. If evaporation be prevented, redness, irritation, and even desquamation occur. Continued or repeated application produces local anæsthesia, which, if maintained too long, may kill the frozen part, and form a slough. . Swallowed in medicinal doses, it irritates and so reflexly stimulates the mucous surfaces of the mouth and alimentary canal, increasing secretions and movements, and hence developing sialogogue, carminative, and antispasmodic actions. But full doses, especially if undiluted, are so rapidly vaporised and so irritant that they cause gastrointestinal distension and nausea, occasionally with vomiting in carnivora, and in dogs and rabbits have produced rupture of the stomach. Ether is quickly absorbed from the intestines, and still more rapidly when introduced by inhalation into the lungs, and promptly acts upon the central nervous system. Small doses stimulate; larger doses, after brief stimulation, paralyse and anæsthetise the centres, first of the brain, next those of the spinal cord, and eventually those of the medulla, killing by respiratory arrest. The glycogenic functions of the liver are said to be stimulated, rendering the animal temporarily diabetic. It is removed almost solely by the lungs.

General anæsthesia can be induced in the horse by administration of ether by the rectum, but is produced more readily by inhalation. Anæsthesia by ether is produced more quickly in cats than in dogs or horses, which are generally made insensible in eight or ten minutes. A donkey is stated to have been fully affected in four minutes, another in five minutes, and a third in three minutes and a half, the last remaining insensible to pain for about half an hour. The effects may be safely maintained for as long as may be necessary.

Compared with chloroform, ether has the disadvantage 
of having a more disagreeable taste. As it has to be given in more concentrated state, it is also more irritant. Larger doses have to be used. Its vapour is dangerously inflammable. On account of its extreme volatility it cannot be used in hot climates. It is, however, less liable to paralyse the heart or vaso-motor centre. Ether and chloroform may be used together, or the anæsthesia produced by chloroform may be maintained by ether. But experiments show that respiration and heart action are more quickly arrested when ether is first given, and anæsthesia subsequently carried on by chloroform, than when chloroform alone is used. Ether is preferable when heart action is feeble, when anæsthesia has to be kept up for a considerable period, when operations likely to be attended with collapse are undertaken in pregnant animals, and in those dogs which are thought for any reason to be specially liable to cardiac failure during chloroform anæsthesia.

Ether resembles most of the bodies of the alcohol series. Like alcohol, it has a twofold stimulant and paralysant action; but it acts more promptly, its effects pass away more quickly, and, in virtue of its volatility, it is markedly anæsthetic. It stimulates more powerfully than ethylacetate or spirit of nitrous ether, neither of which is used as an anæsthetic. Its stimulant properties are somewhat similar to those possessed by turpentine and the other volatile oils.

Medicinal Uses.-Ether, diluted with a little spirit and water, is a prompt and effectual carminative in indigestion in all animals. It checks undue gastric fermentation, expels flatus, and overcomes irregular, violent, gastrointestinal movements. In colic in horses this antispasmodic action is frequently aided by conjoining the stimulant with such anodynes as opium, Indian hemp, and belladonna. Horses with gastric distension are frequently roused and the action of the bowels is promoted by ether, which, in urgent cases, may be hypodermically injected. It is sometimes given for the expulsion of intestinal worms, and especially of ascarides, which, when in the rectum, are readily dislodged by enemata of diluted ether. Such enemata also relieve spasmodic affections of the intestines. 
As a prompt and powerful diffusible stimulant ether is useful in collapse, whether caused by shock, hæmorrhage, or exhausting disease. With alcohol, it is used in parturient apoplexy in cows, and in such cases, when the patient is unable to swallow, it is advantageously given hypodermically. Chills and rigors which usher in many attacks of disease, are sometimes checked by a dose or two of ether. It equalises irregular circulation, restores imperfect action of the skin and kidneys, and gives tone to the enfeebled heart. Hard-worked horses, especially in towns, prostrated by catarrhal fever, are thus benefited, even from the outset of the attack, by ether, given with alcohol and salines. In convalescence from inflammatory and exhausting diseases in all classes of patients, conjoined frequently with aromatics and bitters, it improves the appetite, strengthens the feeble pulse, and sometimes allays cough.

As a stimulant it may be safely prescribed repeatedly wherever it reduces the number and increases the strength of the pulse, lowers excessive temperature, and promotes secretion. It should be avoided where there is much vascular excitement and inflammatory fever. As ether taints the carcase and spots the flesh its employment as a medicine for cattle should be guarded.

As an anæsthetic it is used for the same purposes as chloroform, and is administered in the same manner and with similar precautions.

Local anæsthesia is usually induced by applying the ether from a spray producer. For this purpose the ether must be tolerably pure, for water, if present, gets frozen and blocks the instrument. About an ounce usually suffices for the painless opening of abscesses and fistulæ, or for tenotomy. The effects, however, are more superficial and transient than those of cocaine, and are more apt to be followed by irritation and reaction, which retard healing of surgical or other wounds. A minor degree of local anæsthesia sometimes allays neuralgic pains and those of toothache and earache.

Doses, etc.-The B.P. ethyl-ether, containing eight per cent. of alcohol and water, is generally used for medicinal 
purposes ; but good methyl-ether, on account of its cheapness, is sometimes substituted. As a stimulant horses take

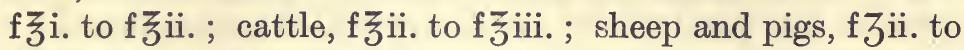
$\mathrm{f}$ Ziv.; dogs, $\mathrm{f}$ Zss. to $\mathrm{f} 3 \mathrm{i}$. It is administered in ten parts cold water or diluted spirit, is sometimes sweetened with sugar or treacle, or flavoured with aromatics. Combination with opium, Indian hemp, or belladonna, increases its antispasmodic and anodyne effects. Where immediate results are required, as in violent attacks of colic, or in collapse, two to four drachms are injected hypodermically, or deeply into muscles.

As an anæsthetic, ether made from ethyl-alcohol is generally preferred. The larger animals take $f \zeta$ iii. to $f \zeta$ viii. : the smaller, $f$ ziv. to $f z i$.

SPIRIT OF ETHER, or spiritus ætheris, is a mixture of ten ounces ether and twenty ounces of rectified spirit, and is used as a stimulant and antispasmodic.

ETHEREAL OIL, oleum æthereum, or oil of wine, produced towards the close of the distillation of ether, is an oily, yellow, neutral liquid, containing ether mixed with various hydrocarbons, has a bitter taste and aromatic odour, is soluble in ether and alcohol, and possessed of anodyne and hypnotic properties.

SPIRITUS ÆTHERIS CoMpositus, or Hoffmann's anodyne, consists of oil of wine, ether, and alcohol (90 per cent.).

ACETiC ETHER, or ethyl-acetate $\left(\mathrm{C}_{2} \mathrm{H}_{5} \cdot \mathrm{C}_{2} \mathrm{H}_{3} \mathrm{O}_{2}\right)$, is prepared by distilling rectified spirit with sodium acetate and sulphuric acid. It is a colourless, neutral liquid, with a fragrant odour. Freely soluble in alcohol, ether, or chloroform. Specific gravity $\cdot 900$ to $\cdot 905$. Its actions nearly resemble those of ether. It is used as a stimulant, carminative, and antispasmodic, but is unsuitable as an anæsthetic.

Contractile Collodion is prepared by mixing one part of gun-cotton with thirty-six of ether and twelve of rectified spirit. The solution, preserved in well-corked bottles, is clear, colourless, inflammable, evolves a strong ethereal odour, and on exposure to the air, dries rapidly, leaving a thin transparent film, insoluble in water or spirit. It is hence a useful substitute for sticking-plaster; repeated applications are laid on with a fine brush at intervals of a 
few seconds, and produce a protective covering, holding together the edges of slight wounds. Flexible collodion adheres still more firmly, gives support without splitting or cracking ; is made by mixing forty-eight parts of collodion, two parts Canada balsam, and one part castor oil, and is kept in well-corked bottles. A styptic, antiseptic protective is made by thoroughly mixing with one hundred parts of collodion, five parts each of tannic and benzoic acids, and ten of carbolic acid. As a stimulating, antiseptic protective for abraded skin or mucous surfaces, and notably for sore teats in cows and ewes, one hundred parts of collodion are mixed with two of glycerin and one of carbolic acid. One part of iodoform or chinosol mixed with ten parts of collodion, has been strongly recommended as a protective adhesive for small wounds. Collodion is occasionally used for coating boluses.

\section{SPIRIT OF NITROUS ETHER}

Spiritus Atheris Nitrosi. Sweet Spirit of Nitre. An alcoholic solution of ethereal compounds containing ethyl nitrite, aldehyde, and other substances. (B.P.) An alcoholic solution of ethyl nitrite $\left(\mathrm{C}_{2} \mathrm{H}_{5} \cdot \mathrm{NO}_{2}\right)$, containing five per cent. of the crude ether. (U.S.P.)

Liquor ethyl nitritis. Solution of ethyl nitrite. A mixture of ninety-five parts, by volume, of absolute alcohol, with five parts, by volume, of glycerin, containing, when freshly made, 3 per cent., by weight, of ethyl nitrite (B.P.).

When rectified spirit, sulphuric and nitric acids, with various practical safeguards, are heated with copper wire, the nitric acid radicle $\left(\mathrm{NO}_{3}\right)$ is deoxidised by the copper; the resulting nitric peroxide or nitrous acid radicle $\left(\mathrm{NO}_{2}\right)$ displaces the hydroxyl in the alcohol $\left(\mathrm{C}_{2} \mathrm{H}_{5} \cdot \mathrm{OH}\right)$, and there is formed ethyl nitrite $\left(\mathrm{C}_{2} \mathrm{H}_{5} \cdot \mathrm{NO}_{2}\right)$, which distils over with a portion of the alcohol. This saline ether, when diluted with about three times its bulk of rectified spirit, constitutes sweet spirit of nitre. As usually prepared, it is unstable, and readily loses strength, but the B.P. solution of ethyl nitrite keeps well, and is easily prepared. Spirit 
of nitrous ether should be kept in a cool, dark place, and in small bottles.

Properties And Tests. - Sweet spirit of nitre is a limpid liquid with a very slight yellow tinge; it is inflammable, has a peculiar, penetrating, apple-like odour, and a sweetish, cooling, sharp taste. Specific gravity 838 to 842 . It should not effervesce, or only feebly, when shaken with sodium bicarbonate. A good, freshly-prepared specimen should yield seven volumes of nitric oxide gas, and, even when kept, should yield not less than five times its volume. It should contain between $2 \frac{1}{2}$ and $1 \frac{3}{4}$ per cent. of ethyl nitrite. Many veterinarians obtain from the wholesale chemist nitrous ether of guaranteed strength, and dilute it, as required, with spirit of such sort and strength as they desire.

ACTIONS AND USES.-Sweet spirit of nitre conjoins the actions of the alcohol and ethyl nitrite of which it consists. It is hence a general stimulant, and a relaxer and paralyser of non-striped muscle. It relieves acute fever, and the difficult breathing of bronchitis and asthma. It is antispasmodic, diaphoretic, and diuretic. Large doses are narcotic.

Medicinal Uses.-Consisting of an irritant-alcohol and a vaso-dilator, ethyl nitrite, when swallowed it stimulates the circulation of the stomach and intestines; is hence stomachic, carminative, and antispasmodic, and is prescribed to animals in indigestion, tympanites, and colic. It is quickly absorbed, and, on account of its contained alcohol, proves a valuable cardiac and general stimulant, serviceable in cases of prostration and convalescence from debilitating disorders. But its properties as a diffusible nitrite, relaxing spasm of involuntary muscles, also come into operation. It dilates arterioles, thus decreases arterial tension, and hence is of special value in the acute stages of fever, whether depending upon specific conditions or local inflammation where the heart is overburdened. It relaxes the muscular fibres of the bronchial tubes, and thus relieves the spasmodic contraction and difficulty of breathing which characterise catarrh, bronchitis, and some forms of asthma. It is specially indicated when there is difficulty of breathing, as in influenza of horses. During excretion, notably by the skin and kidneys, its 
twofold constitution is further useful-the alcohol stimulates secretion, while the ethyl nitrite dilates the lumen of contracted vessels, and thus diaphoresis and diuresis are promoted in fevers, local inflammations, rheumatism, and other disorders.

DOSES, etc.-As a stimulant and antispasmodic horses

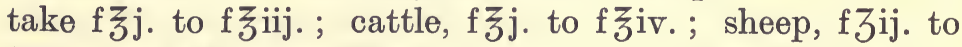

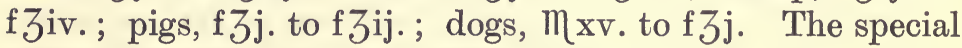
action depending on the costly nitrous ether, its percentage should be guaranteed in all purchases, and the drug should be preserved in properly stoppered bottles. As it is readily decomposed, even by water, it should be diluted or mixed with other medicines only immediately before it is administered. It is usually given in cold water, beer, or linseed tea.

For antispasmodic purposes, it is conjoined with opium, belladonna, hyoscyamus, or chloral hydrate. For colic in horses, two to four ounces are given with two or three drachms of aloes, one to two ounces of laudanum in certain cases being added, and the whole dissolved in a pint of cold gruel, ale, or water. Two to four ounces of the spirit, with about half the dose of laudanum, repeated every hour, counteract the spasmodic pains which occasionally follow parturition. As a general stimulant, and for relieving dyspnœa in inflammation of the respiratory passages, and in catarrhal fever in horses, two ounces each of sweet spirit of nitre and ammonium acetate solution are conjoined with a drachm of belladonna extract. The good effects are sometimes maintained by repeating the draught at intervals of two or three hours. To combat serous exudation in horses or cattle, two or three ounces of spirit of nitrous ether are usefully conjoined with half a drachm each of iodine and potassium iodide. Diuresis is determined by combination with nitre or oil of turpentine. Diaphoresis is developed when the patient is kept well clothed in tolerably warm quarters, and the medicine given in small and frequently repeated doses.

For dogs with catarrh, sore-throat, or distemper, a soothing mixture is made with two ounces spirit of nitrous ether, an ounce spirit of camphor, and one ounce of liquid 
extract of glycyrrhiza and two ounces of water, the dose ranging, according to the size and condition of the patient, from two to four fluid drachms.

\section{CH L O R O F O R M}

Chloroformum, or Trichloromethane, $\mathrm{CHCl}_{3}$, to which has been added sufficient absolute alcohol to produce a liquid having a specific gravity not less than $1 \cdot 490$, and not more than $1 \cdot 495$. Trichloromethane may be prepared by distilling together ethylic alcohol, chlorinated lime, slaked lime and distilled water (B.P.).

Chloroform was discovered in 1832, about the same time, by Soubeiran and Liebig; its effects on the lower animals were described by Dr. Glover in 1842; while its valuable anæsthetic properties were first discovered and applied by the late Sir James Y. Simpson in 1847. Since then it has been largely and successfully used for the alleviation of human and animal suffering during surgical operations, parturition, and various diseases.

Properties.-Chloroform is a limpid, colourless, neutral, pungent fluid, with a sweet taste, and a characteristic odour. At ordinary temperatures it entirely volatilises. It is slowly decomposed by sunlight, and hence must be kept in the dark. Its vapour is four times heavier than air. It should boil between $140^{\circ}$ and $143^{\circ}$ Fahr. Though not spontaneously inflammable, it can be burned round a wick saturated with alcohol, forms a green, sooty flame, and evolves hydrochloric acid. It is soluble in two hundred parts of water, and in alcohol (90 per cent.), ether, oil of turpentine, olive oil, and carbon bisulphide; insoluble in glycerin. It readily dissolves iodine, bromine, volatile oils, wax, resin, and many organic active principles.

IMPURITIES.-Chloroform carelessly prepared or imperfectly purified contains volatile organic oils, which, if inhaled, induce nausea and headache. Such specimens have an unpleasant, pungent odour when evaporated from the back of the hand, and are blackened by agitation with sulphuric acid. Samples containing more than the one per 
cent. of alcohol authorised to check decomposition have their specific gravity proportionally lowered, lose bulk, notably when shaken with water, and moreover become cloudy at temperatures approaching $32^{\circ} \mathrm{Fahr}$. Traces of sulphuric acid are discovered by the usual barium chloride test ; chlorine and hydrochloric acid by silver nitrate. The purity of chloroform is also judged by its odour when evaporated, its behaviour when agitated with sulphuric acid, its reaction with litmus, and its specific gravity, which is lowered by the ordinary adulterations.

ACTIONS AND USES.-Chloroform precipitates albumin, and is a topical irritant. It is antiseptic, and destroys the lower forms of plant and animal life, and hence is parasiticide. Small to moderate doses, swallowed or otherwise absorbed, produce slight and temporary irritation and stimulation, and hence are carminative and antispasmodic. Full doses quickly and powerfully paralyse the cerebro-spinal nervous system, causing muscular relaxation, insensibility to pain, and unconsciousness. It kills by respiratory and cardiac arrest. The paralysant and anæsthetic effects are most rapidly induced when the drug is inhaled. Chloroform is the anæsthetic most used in this country, alike for human and veterinary patients. Applied topically, it is rubefacient, refrigerant, anodyne, and a local anæsthetic.

General Actions.-Chloroform is allied chemically and physiologically to alcohol, ether, and other bodies of the alcohol series. It dissolves the essential constituent of nerve-centres, nerves, and red blood corpuscles, and retards oxidation of blood. Applied to the skin, it evaporates, causing a sensation of cold; but if evaporation be prevented, it irritates. Hence, when swallowed, it stimulates the flow of saliva, excites gastric secretion, in men and dogs occasionally causing emesis, and develops carminative and antispasmodic actions. By whatever channel it is absorbed, it acts on the nerve-centres somewhat in the same manner as alcohol, but its stimulant action is slight and brief. When the vapour is inhaled anæsthesia is quickly produced. Its effects are divisible into four stages-1. Stimulant ; 2. Narcotic; 3. Anæsthetic ; 4. Paralytic.

The vapour inhaled first stimulates and subsequently 
paralyses the parts with which it comes into contact. Acting as an irritant on the nasal and laryngeal mucous membrane, it first slows the breathing, and also reflexly the pulse, often causing coughing at the same time. The fear and excitement caused by the restraint and administration induce irregularity and quickening of respiration, and usually also of the circulation. If given slowly narcosis gradually supervenes, the muscles relax, reflexes are lost, and there is a fall of blood-pressure, but the respiratory movements are sustained steadily, as in the third stage of anæsthesia. Still fuller effects paralyse the medullary centres, respiration becomes slower and feebler, and stops, while pulsation and blood-pressure are lowered. "The nervous system,' Sir Lauder Brunton states, 'is paralysed in the following order-first, the cerebral hemispheres; next, the grey matter of the cord ; next, the white matter ; next, the reflex power of the medulla oblongata; next, the automatic power of the respiratory centre ; and lastly, the cardiac ganglia.'

Medical men and veterinarians generally concur in the belief that chloroform is the most convenient and effectual anæsthetic, and these conclusions have been fully justified by two series of investigations carried out at Hyderabad in 1888 and 1889, and comprising upwards of seven hundred experiments, chiefly on dogs and monkeys, but also on horses, goats, and rabbits. Chloroform was the anæsthetic chiefly used, but ether and mixtures of chloroform and ether were also given. Careful records of every experiment were made, and tracings of the pulse and blood-pressure, registered by a manometer, have been reproduced by photography. The experiments were undertaken to make clear the manner in which chloroform acts, and especially to determine how overdoses kill. With these objects, the chloroform was administered in many different ways, and under very various conditions. Some of the animals were fasted for twenty-four hours; others were fed with flesh or farinacea; some had coffee, wine, or other stimulants shortly before inhalation; most were healthy, a few had cardiac disease, and some had fatty degeneration of the heart, purposely produced by administration of 
phosphorus. Morphine, strychnine, and atropine, singly and in combination, were administered subcutaneously to various subjects before the chloroform was inhaled. The drug was given with and without an inhaler, and in almost every conceivable way.

Notwithstanding these different conditions, the train of effects followed in regular order. Preliminary excitement, with more or less struggling, occupied from one to two minutes, but gradually gave place to increasing insensibility, unconsciousness, and muscular relaxation. Fuller anæsthesia, suitable for the performance of operations, was reached in two or three minutes from the beginning of inhalation, breathing became quiet and regular, blood-pressure was slightly lowered, and reflex actions were impaired and abolished. When the effects were further pushed, respiration ceased in six or seven minutes from the commencement of the experiment. About one and a third minutes later, the pulse, after being greatly quickened, ceased; while two or three minutes later the heart itself stopped. In no case did the heart stop before the breathing. Even when poisonous doses were used, two to six minutes elapsed between the time that respiration ceased and the heart stopped. A somewhat shorter interval occurred, however, -in two cases, in which the inhalation was very slow and prolonged ; in four cases, complicated with asphyxia ; and in ten cases, in which morphine, atropine, or strychnine had been previously injected subcutaneously; but even in these sixteen cases the heart continued to beat for one minute after respiration ceased. Even in those animals debilitated by twenty-four hours' fasting, by blood-letting, or by fatty degeneration of the heart caused by phosphorus, the pulse and heart continued to beat after respiration ceased. Neither serious surgical operations nor bruising of delicate parts during full anæsthesia, or even while the animal was partially conscious, produced syncope or notable direct action on the heart.

These experiments testify that chloroform may be administered to animals with perfect safety, provided there is no interference with breathing. They further indicate that the large proportion, if not all, the fatalities from chloroform 
result from asphyxia, depending upon some fault in its administration. As Sir Lauder Brunton has aptly observed, suffocation and anæsthesia are a deadly combination. There is one effect of chloroform, not recognised by the Commission, which may be dangerous and even cause death. If concentrated vapour is given early during the administration the vagus centre in the medulla is so stimulated and excited that inhibition of the heart may result. That such is the cause is proved by the fact that it cannot be produced if the vagi are cut, and further, a dose of atropine given beforehand paralyses the vagus endings in the heart, and so prevents any inhibition. Schäfer has suggested the use of atropine as a routine measure in chloroform administration, but the better method is to give the drug slowly. Dangerous restriction of air may result from the inhaling apparatus unduly closing the nostrils. Ingress of air may be arrested by pressure on the neck or chest, by the animal being placed in an awkward position, by the tongue dropping on to the larynx, or by vomited food entering the windpipe. In forcibly restraining the early struggling, breathing is sometimes interfered with, and the deep inspirations which usually subsequently occur will convey into the lungs and distribute to the nerve centres unduly large quantities of the anæsthetic vapour. The experienced practitioner is always specially watchful of cases in which there is much struggling; he pushes the administration as rapidly as is safe, in order quickly to produce perfect anæsthesia; but so soon as this is effected he allows several full draughts of air. At this stage the napkin, nose-bag, or inhaler may be removed, for so soon as full anæsthesia is produced, the insensibility is readily maintained by small quantities of the drug, given occasionally.

The further precautions mainly consist in the chloroformist bestowing undivided attention on two points :-(1) He must watch the breathing, in order that the patient's safety shall be maintained by fresh air entering the lungs, thus preventing any dangerous accumulation of the drug, and the arrest of natural respiration ; (2) he must maintain throughout the operation the requisite degree of anæs- 
thesia, and accordingly, from time to time, he should test the cornea, for returning reflex activity, which indicates that more chloroform should be given. With these precautions, anæsthesia may be safely continued for an hour or more. Dogs, without harm, have been kept under chloroform for two hours, and chloroformed deeply on consecutive days. Fröhner mentions that sheep will stand two hours' anæsthesia, repeated daily for eight days, about an ounce of the drug being used each day.

Whenever respiration is impaired, unduly slow, shallow, or gasping, the administration of the drug should be stopped, fresh air freely allowed, the tongue drawn forward, and the head placed on a lower level than the rest of the body. But if natural respiration ceases, not a moment must be lost in adopting artificial respiration. The Hyderabad experiments show that every animal was revived, in which artificial respiration was used within thirty seconds after natural respiration ceased, and in some cases in which it was used after fifty seconds, but in none in which the treatment was delayed for sixty seconds after respiration stopped. When the pulse and heart had ceased to beat, neither artificial respiration nor other remedies, however promptly used, were effectual in restoring life. In such emergencies, faradic stimulation of the phrenic nerve and inhalation of amylnitrite may aid in restoring respiratory movements.

Chloroform depresses all muscular tissue and notably cardiac muscle. Thus with prolonged administration the contractile power of the heart gradually diminishes, and the pulse becomes weaker, until the heart stops in a condition of extreme dilatation, unable to propel the blood any longer. Compared with ether, chloroform has several advantages as an anæsthetic: it is more agreeable to the taste, is less irritant, about one-fourth of the quantity suffices, it produces less preliminary excitement, the effects continue longer after inhalation ceases, and it is less inflammable. Many practitioners, however, prefer ether, as it does not so readily paralyse either the heart or the vasomotor centre. In protracted and serious operations, especially in dogs or cats, it is accordingly desirable in many cases to use ether. In some parts of the Continent the 
A.C.E. mixture of absolute alcohol one part, chloroform two, and pure ether three parts, is preferred. (See Anæsthesia.)

MEDicinal Uses.-Chloroform is used to anæsthetise horses and other animals during castration, firing, and other painful operations. Parturition in the lower animals is usually performed so easily, and with so little apparent pain, that chloroform, in the majority of cases, is unnecessary. Where false presentations have to be rectified in the mare, it is sometimes, however, impossible, without anæsthesia, to keep the animal quiet, or to abate violent uterine throes; while in bitches it is also sometimes requisite when the pups have to be reduced before they can be extracted. Amongst cows and ewes, labour pains sometimes continue for hours, and other preparations for parturition appear to be complete; but the neck of the uterus remains firmly closed, often in spite of medicines and manipulation. Chloroform inhaled in amount insufficient to produce complete anæsthesia usually relaxes the rigid muscle. Partial anæsthesia generally controls labour pains occurring prematurely, moderates irregular, tumultuous contractions, such as are sometimes met with in first parturitions, and abates afterpains, the chloroform inhalation in some of these cases being advantageously aided by hypodermic injection of morphine.

Inhalation of chloroform has been advised for relaxing intestinal spasm, and hence aiding reduction of herniæ. It has been recommended in tetanus in horses, and relief is usually obtained so long as anæsthesia continues; but the temporary benefit is more than counterbalanced by the disturbed and excited state caused by the administration of the drug and by the excitement which succeeds the anæsthesia. The shoeing of irritable and vicious horses is greatly facilitated if chloroform be given so as to produce partial anæsthesia. Inhaled or swallowed, it is occasionally prescribed to check the symptoms of chorea and epilepsy in dogs.

When swallowed, it relieves gastric irritation, spasm, and pain, and also exerts antiseptic effects on the contents of the canal. Chronic irritability of the bowels in weakly foals and calves, after removal of the irritant by castor oil, is usually benefited by a dose of chloroform or of spirit of 
chloroform and laudanum, repeated two or three times daily. Similar treatment is sometimes useful in removing worms. Horses affected with epizootic catarrh and spasmodic cough are relieved by chloroform, conjoined with belladonna extract, or laudanum, dissolved in cold linseed gruel, and swallowed slowly, so as to ensure more prolonged anodyne effect on the irritable nerve-endings.

As a local anæsthetic it has not such direct paralysing effects on sensory nerves as ether, cocaine, iodoform, or even as carbolic acid, or cold. It is sometimes, however, applied to allay the pain of neuralgia, rheumatism, and local inflammation. Its anodyne and antiseptic properties have suggested its use, with carbolic acid and vaseline in stomatitis and actinomycosis. Mixed with a little spirit, it is a cleanly means of destroying lice or fleas infesting dogs, cats, or fowls. As a liniment it is sometimes useful in mammitis in cows. It is occasionally added to anodyne enemata for the relief of irritation of the lower bowel and urino-genital organs. Its high diffusive power, which it retains when mixed with spirit, renders it a useful vehicle for the subcutaneous injection of morphine, atropine, and other alkaloids. It is a solvent for guttapercha, and the solution is occasionally employed as a substitute for collodion.

DosEs, etc.-To produce anæsthesia in horses and cattle, $\mathrm{f} \xi \mathrm{jj}$. to $\mathrm{f} \xi \mathrm{jv}$. are required; $\mathrm{f} \zeta \mathrm{iv}$. to $\mathrm{f} \xi \mathrm{j}$. for sheep and pigs ; $\mathrm{f} Z \mathrm{j}$. to $\mathrm{f} \zeta \mathrm{j}$. for dogs. Anæsthesia is readily maintained by repeated small doses. The chloroform vapour may be given alone, without intentional admixture of air or diluted with from ten to thirty volumes of air.

Horses can be chloroformed standing or after castingthe recumbent position being generally preferred. Administration may be effected by pouring chloroform-a drachm or two at a time-on a warm sponge, which is placed close to a nostril, and carefully covered with a large towel folded several times; or more conveniently by using one of the many special muzzles now on sale. Roalfe Cox's waterproof canvas bag is very simple, inexpensive, and efficient. The leather muzzle patented by Messrs. Carlisle and Bell is furnished with a perforated tin box to contain the saturated sponge, and provided with straps which allow 
of the adjustment of the muzzle to fit most horses. Gresswell's nose cap, Dowell's inhaler, and Krohne and Sesemann's modification of Junker's apparatus can be recommended. Inhalers properly used prevent waste of chloroform. The vapour being inhaled in tolerably concentrated form, the initial stage of excitement is shortened, the reduced quantity required to produce anæsthesia diminishes risk from respiratory and cardiac paralysis; while the irritation and nausea apt to ensue from the use of larger doses are avoided. The subcutaneous injection of morphine or of a mixture of atropine and morphine, fifteen minutes before giving chloroform, diminishes preliminary excitement and intensifies the anæsthesia. Partial or limited anæsthesia induced by inhalation of minimum doses of chloroform is useful in difficult parturition, and as a means of restraint when firing, castrating, or performing simple operations on horses standing. Dogs may be chloroformed by dropping the drug on cotton wool or a sponge placed over the nostrils, or by using an inhaler, which ought to cover only the upper jaw. During administration it is of utmost importance to keep the dog's mouth open, which can be effected without difficulty by a small gag. Cats, lap-dogs, and other small animals, may be chloroformed under a bell-jar or an inverted box containing bits of blotting paper or cotton wool charged with chloroform. Administered by the mouth as a stimulant, antispasmodic, and analgesic, the dose for horses or cattle is $f 3 i$. to $f 3 i j$. ; for sheep and swine, $m_{x x}$. to $m_{x l}$., and for dogs, $m_{v}$. to $m_{x}$. These doses are given with syrup, mucilage, whisked egg, or weak spirit, and repeated at intervals of two or three hours:

AquA-ChLoroformi, consisting of one part chloroform to four hundred of water, is used as a carminative, antiseptic, and stimulant, and as a convenient medium for giving unpalatable drugs.

SpIRIT of Chloroform, also called chloric ether, is made by dissolving one fluid part of chloroform in nineteen fluid parts of alcohol ( 90 per cent.). It has the specific gravity $\cdot 871$, and a warm ethereal odour and taste. It is an effectual stimulant, antispasmodic, and anodyne, resembling ether and sweet spirit of nitre. Diluted with water, or any bland 
cold fluid, it is prescribed for horses in doses of $f \zeta i$; for

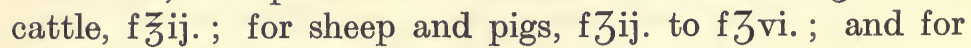
dogs, $m_{x x x}$. to $3 \mathrm{ij}$.

The B.P. Tinetura Chloroformi et Morphinæ Composita, is composed of chloroform, tinctures of capsicum and Indian hemp, oil of peppermint, hydrocyanic acid, morphine hydrochloride, glycerin and alcohol. Ten minims contain $\frac{1}{11}$ grain of morphine, $\frac{1}{2}$ minim dilute hydrocyanic acid, and $\frac{3}{4}$ minim of chloroform.

CHLORODYNE, a popular anodyne in human medicine, is made from different formulæ; one is stated to contain ten parts each of chloroform, ether, Indian hemp, and morphine, two parts capsicum tincture and prussic acid, three parts aconite and hyoscyamus tinctures, one part of oil of peppermint, five parts hydrochloric acid, and fifty of simple syrup. It is an effectual anodyne and antispasmodic, frequently prescribed to relieve especially gastro-intestinal and bronchial irritation.

\section{CHLORAL HYDRATE-CHLORAL HYDRAS}

Chloral hydrate, or trichlorethylidene glycol, $\mathrm{CCl}_{3} . \mathrm{CH}$ $(\mathrm{OH})_{2}$, is obtained by the addition of water to the liquid chloral produced by the action of dry chlorine gas on ethylic alcohol (B.P.).

Chloral hydrate occurs in colourless crystals ; is neutral, aromatic-with a melon-like odour-bitter, pungent, and permanent in air. It melts at about $133^{\circ}$ Fahr., and boils at $205^{\circ} \mathrm{Fahr}$. It is soluble in less than its own weight of water, alcohol, and ether, and in four parts of chloroform. Triturated with camphor it forms a liquid. The caustic alkalies, and, in less degree, the alkaline carbonates, decompose it into chloroform and an alkaline formate. A hundred grains dissolved in an ounce of distilled water, and mixed with thirty grains of slaked lime submitted to careful distillation, should yield not less than seventy grains of pure chloroform. Inferior specimens, besides being of imperfect strength, are apt to contain chlorinated organic impurities, which render them yellow and cloudy, acrid and irritating, imperfectly soluble 
in water, on which they float as oily drops, while, instead of hypnosis, they produce nervous excitement.

ACTIONS AND USES.-Like other members of the alcohol group, chloral hydrate is antiseptic and a topical irritant. When absorbed it has a sedative hypnotic action on the central nervous system. Toxic doses, after slight and temporary stimulation, notably depress and paralyse the cerebro-spinal centres. Medicinal doses are hypnotic, analgesic, and feebly anæsthetic. It is used topically as a stimulant, anodyne, and antiseptic.

General Actions.-It destroys micro-organisms; one part in a thousand hinders development of anthrax bacilli; it has about the same antiseptic strength as carbolic acid. Personne exhibited at the Academy of Sciences, Paris, the body of a dog perfectly preserved in chloral hydrate for fifty-five days. Solutions exceeding 20 per cent. are topical irritants, and hence when swallowed cause a burning sensation in the throat, and sometimes vomiting and purging. Diluted solutions are readily absorbed. The drug acts on the central nervous system apparently without undergoing decomposition into chloroform, which is not discovered in the blood tissues, or expired air of animals receiving chloral, and only appears in the urine when that fluid contains sufficient free alkali to decompose the chloral. Small doses produce drowsiness and lower blood pressure. Fuller or repeated doses slow circulation and respiration, and produce sleep, usually natural and deep, from which the animal awakes without discomfort. Anodyne and antispasmodic actions are likewise produced. Anæsthesia cannot safely be induced by giving the drug by the mouth, but is produced by intravenous, intraperitoneal, or rectal injection. Larger doses lessen reflex irritability and sensibility, cause shallow respiration, a marked fall in blood pressure, weakening of the heart muscle, and lower temperature, sometimes to the extent of $6^{\circ}$ or $8^{\circ} \mathrm{Fahr}$. This fall of temperature is due to the great loss of heat from the dilated cutaneous vessels. By moderate doses, and during safe anæsthesia, the pupil is contracted; but it is dilated when the doses are dangerously large, or the anæsthesia deep or long continued. Death results from cardiac and 
respiratory paralysis. The heart is arrested in diastole, with the right cavities distended. There is no paralysis of muscles or motor nerves. The drug is eliminated by the lungs, skin, and kidneys.

Toxic EFFECTS.-Richardson, in an extended series of experiments, found that fish and pigeons were narcotised by one and a half to two grains ; mice by one-third of a grain ; rabbits weighing eighty-five ounces, by thirty grains. One hundred and eighty grains produce fatal effects in man, but dangerous symptoms have occasionally been developed by one-fourth of that amount. Dollar gave a horse suffering from spasmodic colic two ounces in water; the spasms were speedily removed, but for twelve hours the patient remained very dull and sleepy. Mavor gave a horse four ounces in water ; in five minutes he fell insensible, sweated freely, his muscles relaxed, his pupils dilated; his pulse, at first accelerated, gradually became normal, respirations were quickened, until in an hour they numbered thirty-six. The temperature, from $100^{\circ}$ Fahr., fell in two hours to $95 \frac{2}{5}$, but two hours later rose to $97 \frac{1}{5}$. In half an hour he was in a quiet sleep, lasting one and a half hours, when he attempted but failed to rise, and shortly again slept, the breathing being slow and heavy, the skin cold, the sphincters relaxed. Four hours after receiving the draught he was restless, shivering, but disposed to feed, continued in this state for several hours, and suffered next day from bronchitis, from which he gradually recovered. A healthy horse was given four ounces in ten ounces of water; in half an hour he was restless but drowsy, passing fæces frequently; his pupils dilated. He continued in this state for fully three hours, when he was slightly delirious, but gradually became quiet. Eight hours later the effects had passed away.

Fröhner records. that horses receiving, by the mouth or rectum, three to four ounces, or intravenously six to ten drachms, became intoxicated, staggered, fell, and lay unconscious for several hours with the muscles relaxed. Sleep may be maintained by repeated doses, he states, for several days, or even weeks. Nocard kept a horse affected by tetanus under its influence for thirty days, administering daily one and a half to two ounces. The lethal dose is 
four to six ounces when given by the mouth or rectum, but one-third these quantities is fatal when introduced intravenously. Cattle are affected in much the same manner, and by similar doses. The lethal dose for dogs is two to six drachms. In them preliminary excitement is more marked than in horses or cattle.

The treatment of poisoning consists in maintaining the temperature by warm clothing, hot applications, stimulants, and hot coffee. Although chloral is an antidote to strychnine, the antagonism of strychnine to chloral is not so marked.

Medicinal Actions.-Chloral hydrate quiets irritability and causes sleep. Conjoined with morphine, it is prescribed to relieve gastro-intestinal irritation and spasm, but as it is a topical irritant it must not be used where there is congestion or inflammation. Small doses are serviceable in canine asthma, and in violent paroxysmal coughing, both in dogs and horses. It quiets the excitability and spasms of chorea, epilepsy, and hysteria, and temporarily relieves those of tetanus and rabies. It antagonises the tetanic convulsions of strychnine. Administered to rabbits along with lethal doses of strychnine, sleep is produced, and the creature recovers. Chloral is also antagonistic to physostigmine, but to act as an effectual antidote the slower-acting chloral must be given before, at the same time, or within two minutes after these quickly-acting convulsants. It has been given with benefit in the outset of those cases of parturient toxæmia in cows in which there is intense nervous excitement, and violent cramp of the muscles of the hind extremities. Pugh (Sevenoaks) prescribes chloral in all cases of undue fermentation in the digestive tract, and finds that large doses are tolerated in colic and milk fever. Conjoined with bromides, it is indicated in cases of meningitis. A like combination abates the sleeplessness frequently occurring in canine distemper, and allays irritability and straining in disorders of the intestines and urino-genital organs.

French veterinarians use it as an anæsthetic, injecting a solution into the peritoneal cavity. Kaufmann declares that it yields to no other anæsthetic when injected in- 
travenously. But intravenous injection is troublesome, and attended with considerable danger. Cagny anæsthetises horses by injecting hypodermically two to three grains of morphine with a half to one grain atropine, and shortly giving an enema containing eight drachms of chloral hydrate. Anæsthesia occurs in about an hour, and is maintained during operations by inhalation of ether or chloroform. But the anæsthesia produced by chloral, however administered, is neither so complete nor lasting as that obtained by inhalation of chloroform. Equal parts of chloral and camphor, mixed with six or eight parts of vaseline or simple ointment, form an analgesic dressing which relieves the pain of neuralgia and the itching of various skin complaints. A diluted solution is sometimes applied as an antiseptic stimulant to foul wounds. It should not be prescribed where there is weak, irregular action of the heart or congested lungs.

Chloral hydrate resembles various other drugs. As a hypnotic, it is allied to sulphonal, paraldehyde, and morphine. Like bromides, it quiets excited cerebral centres. For the relief of pain and spasm it is usefully conjoined both with morphine and atropine. Although allied in composition to chloroform, it is not effective as a local anæsthetic, and as it cannot be inhaled, general anæsthesia is produced only when full doses are swallowed or injected into the rectum, veins, or peritoneal cavity. In relieving spasm and lowering arterial pressure it bears some resemblance to amyl-nitrite.

Doses, etc.-For horses and cattle, そi. to そij. ; for sheep and pigs, 3ss. to 3ij. ; for dogs, grs. v. to grs. xxx. ; cats, grs. ij. to grs. xv., repeated every two or three hours, administered in mucilage or syrup. Intratracheally, eight to thirty grains in water may be injected as an antispasmodic ; twenty to eighty grains in difficult parturition, and one hundred and sixty grains in tetanus (Levi). For enemata an ounce or two ounces mixed with mucilage may be used. On account of its irritating in-contact effects, it should not be used hypodermically. Continued use of the drug does not establish tolerance, as in the case of alcohol or opium. For relief of general irritability it is prescribed with bro- 
mides, opium, or belladonna ; for relief of pain, with opium, belladonna, or camphor. The B.P. syrupus chloral contains ten grains chloral hydrate in each drachm.

ButYl-Chloral Hydrate is obtained by the addition of water to the liquid butyl-chloral produced by the action of chlorine gas on aldehyde $\left(\mathrm{C}_{4} \mathrm{H}_{5} \mathrm{Cl}_{3} \mathrm{O} \cdot \mathrm{H}_{2} \mathrm{O}\right)$. It forms pearly crystalline scales, which are pungent, acid, and disagreeable to the taste. Soluble in fifty parts of water, in one of glycerin, or alcohol, and in twenty of chloroform. It resembles chloral, but is less powerful, has less depressant action on the heart, has been said to paralyse especially the fifth nerve and parts supplied by it, and has been prescribed in human medicine in facial neuralgia, migraine, and as a hypnotic instead of chloral in weak heart (Brunton).

Chloralamide has been obtained by the action of formamide on chloral. It occurs in colourless crystals, slightly bitter, soluble in nine parts of water, and in two of alcohol. When administered it appears to be converted into chloral, which it resembles, but is less depressant to the circulation so that blood pressure is not lowered to the same extent. It is a topical irritant, causing diarrhœa when full doses are swallowed. Dogs receiving seven to ten grains per kilogramme of body-weight in five minutes become restless, moan, and lose the power of movement. These symptoms continue about an hour, and are followed by drowsiness, and sometimes by sleep (Kaufmann). Neither as a hypnotic nor as an analgesic is it as effectual as chloral. Fröhner states that it is frequently substituted for chloral, but that it is milder, and does not act so notably on digestion or circulation. He prescribes it in cramps and excitement in dogs, and especially in distemper. Doses.Horses and cattle, そjss. to そijss ; sheep, grs. xlv. to 3 iii. ; dogs, grs. x. to grs. xlv.

ChLORETONE (trichlor-tertiary-butyl-alcohol) may be prepared by the interaction of chloroform, acetone, and an alkali. It occurs in white crystals, with a camphor-like taste; sparingly soluble in water, soluble in ten parts glycerin, and dissolving readily in alcohol. It is hypnotic, local anæsthetic, and antiseptic. Its hypnotic action is believed to be exerted through the central nervous system 
and has no influence on the heart. Administered by the mouth it produces sedative and antiseptic effects upon the gastric mucous membrane, and is a very useful remedy for obstinate vomiting in the dog. It may also be prescribed for epileptic fits, chorea, and colic, and to quieten patients during operation under local anæsthesia. If a dose of chloretone be given to the dog an hour before inducing general anæsthesia, much less of the anæsthetic will be required, vomiting does not so readily occur and there will be less struggling. Combined with boric acid, as in borochloretone (Parke, Davis and Co.), it forms a valuable antiseptic and soothing application for wounds. Doses for the dog, grs. v. to grs. $x$.

\section{S U L P H O N A L}

Dimethyl-methane-Diethylsulphone $\left(\mathrm{CH}_{3}\right)_{2} \mathrm{C}\left(\mathrm{SO}_{2} \mathrm{C}_{2} \mathrm{H}_{5}\right)_{2}$. A product of the oxidation of Mercaptol, obtained from Acetone and Mercaptan (B.P.).

Sulphonal is a complex compound of the methane series, crystalline, colourless, inodorous, nearly tasteless, neutral, melting at $258^{\circ}$ Fahr., sparingly soluble in cold water, soluble in ninety parts of cold rectified spirit, in ether, and chloroform.

ACTIONS AND USES.-In man it is ' a useful hypnotic, producing sleep, and in most cases having no disagreeable after effects, even when used continuously for a length of time' (Brunton). In men and dogs it is more effectual as a sleep producer than paraldehyde, but less so than morphine. Dogs, whether healthy or sick, receiving it either by the mouth or subcutaneously, sleep quietly for six to ten hours (Fröhner). Kaufmann hypodermically injected dogs weighing ten kilogrammes with two grammes, and reports ataxia, shortly followed by calm and profound sleep, and without any untoward effects. He recommends it in canine subjects in neuroses characterised by agitation and hyper-excitability. Horses and cattle resist its hypnotic action as they do that of opium. Instead of calming and paralysing their higher cerebral centres, it primarily and prominently 
stimulates their motor centres. Fröhner has given it to horses, cattle, and sheep without observing any definite hypnotic effect. Large doses (150 to 200 grammes), he states, produce in horses excitement, muscular trembling, spasmodic movements, paresis of the spinal cord and muscles, and are excreted uncombined in the urine. Death is caused by hæmorrhagic ulcerative gastro-enteritis. $\mathrm{He}$ records that, while 0.5 gramme per kilogramme of bodyweight killed horses and cattle, dogs stood one gramme per kilogramme of body-weight. Horses and cattle receiving 25 to 75 grammes suffered from weakness and trembling, but without narcosis ; sleepiness was apparent for a day, but was alternated with greatly increased reflex activity, and even with fits, while numbness sometimes lasted a week.

Doses, etc.-Horses take $3 \mathrm{jv}$. to $\bar{j}$. in electuary or in mash. A dose of sulphonal is sometimes given thirty minutes before casting a nervous horse for operation. Dogs may be given grs. x. to grs. xl., in pill, hot milk, or soup. To ensure hypnosis a couple of doses should be given, at intervals of two or three hours.

URETHANE or ethyl carbamate $\left(\mathrm{C}_{3} \mathrm{H}_{7} \mathrm{NO}_{2}\right)$, introduced as a hypnotic for man, occurs in brilliant prismatic crystals, having a faint odour of paraffin and a salt taste; very soluble in water, alcohol, and ether. Applied to the tissues urethane exerts no appreciable action, but after absorption, moderate doses produce in the dog drowsiness, followed by sleep more or less profound. Old dogs, however, are not easily brought under the influence of this drug, and according to Fröhner, horses, even after receiving large doses ( 2 to 15 ounces) appear to be quite insusceptible to its hypnotic action. In dogs toxic doses (800 grains) cause transient general excitement, followed by deep sleep, during which the reflexes disappear, the heart's impulse weakens, temperature falls, respiration becomes gradually slower, then stops, and death takes place by asphyxia. Urethane may be prescribed as a hypnotic for dogs suffering from painful wounds, fractures, and nervous diseases accompanied by agitation, vertigo, or convulsions. It is a functional antagonist of strychnine (Coze). Sleep induced by urethane 
lasts from one to six hours, according to the dose administered and the susceptibility of the patient. Doses, grs. xx." to grs. cl. per day, in simple syrup, pill, or powder.

- THypnone or Acetophenone $\left(\mathrm{C}_{8} \mathrm{H}_{8} \mathrm{O}\right)$ is a volatile, colourless liquid, with a fragrant odour ; insoluble in water, but soluble in glycerin, almond oil, alcohol, ether, chloroform, and benzine. It is a local irritant. In the horse it does not induce sleep, but it is a serviceable hypnotic for the dog, administered in doses of $m_{\mathrm{v}}$. to $m_{\mathrm{xxx}}$., in gelatin capsules, emulsion, or in glycerin and spirit.

Amylene Hydrate $\left(\mathrm{C}_{5} \mathrm{H}_{2} \mathrm{O}\right)$, a colourless, oily liquid, with an odour of camphor and an acrid taste, and soluble in eight parts of water, is a powerful hypnotic for the dog, inducing profound sleep, lasting several hours, without sensibly disturbing the heart or modifying blood pressure, but lowering the temperature of the body. Thirty minims of amylene hydrate produce the same effects as fifteen grains of chloral (Gobert). It may be given to dogs in doses of $m \times x$. to $m l$., in capsule, or dissolved in alcohol and water.

\section{PARALDEHYDE}

A polymeric modification of Aldehyde. $\left(\mathrm{C}_{2} \mathrm{H}_{4} \mathrm{O}\right)_{3}$.

Paraldehyde is a colourless fluid, soluble in ten parts of water, still more soluble in glycerin, and of a disagreeable, persistent, nitrous odour and taste.

Actions AND Doses.-It is antiseptic, hypnotic, and slightly diuretic. It hinders fermentation, and flesh placed in a two per cent. solution has been kept fresh for two months. It is a more effectual hypnotic than hypnone or urethane, but does not produce sleep in man or dogs as readily as opium or chloral, while horses are brought under its soporific effects with still greater difficulty. Fröhner, experimenting on horses, found that 200 grammes (about 7 fluid ounces) produced only slight trembling, vertigo, and staggering; 450 grammes induced powerful trembling, yellow-red colouring of the visible mucous membranes, the presence of hæmoglobin in the urine, but no sleep; the effects passed off in an hour; 500 grammes caused staggering, 
excitement, pawing, plunging, difficult breathing, dulness, diminished sensation, yellow membranes, solution of the red globules, with discharge of hæmoglobin in the urine, great weakness continuing for twelve hours, while weariness, anæmia, and emaciation persisted for a week.

Dogs receiving three to four grammes per kilogramme of body-weight had difficulty in balancing themselves, and cerebral narcosis followed later. Medicinal doses of fifteen to thirty minims, after brief excitement, cause sleep, lasting six or eight hours. But its effects are by no means certain when the patient is excited or pained. It is an antidote to strychnine poisoning. In human practice it is prescribed in nervous insomnia, and as a hypnotic in cardiac cases. On account of its local irritant effects it is unsuitable for hypodermic injection. Doses, for the dog, $m_{\text {vi. to }} m_{x x x}$., in simple syrup or in capsule.

\section{A M Y L-NITRITE}

\section{Amyl-Nitris. Nitrite of Amyl. $\mathrm{C}_{5} \mathrm{H}_{11} \mathrm{NO}_{2}$.}

A liquid produced by the interaction of nitrous acid, and amylic alcohol which has been distilled between $262^{\circ}$ and $270^{\circ} \mathrm{Fahr}$. It consists chiefly of iso-amyl nitrite, $\mathrm{C}_{5} \mathrm{H}_{11} \mathrm{NO}_{2}$, but contains other nitrites of the homologous series (B.P.). Amyl-nitrite is a yellow, ethereal, limpid, volatile liquid, with a fragrant odour. Specific gravity, 0.870 to 0.880 . Nearly insoluble in water ; soluble in rectified spirit, ether, and chloroform, and is itself a solvent for oils. It speedily deteriorates unless kept in well-stoppered bottles in a cool dark place.

ACTIONS AND USES.- - It has in marked degree the actions of a nitrite, relaxing and paralysing non-striped muscle. It is hence an antispasmodic of involuntary muscle, dilates arterioles, and is prescribed chiefly in angina pectoris and asthma.

General Actions.-Whether in the test tube or in the body, it converts the hæmoglobin of the blood into methæmoglobin, which does not readily part with oxygen; internal respiration is accordingly interfered with; con- 
vulsions and asphyxia ensue; and the blood acquires a chocolate hue. This has occasionally occurred in animals, but is not easy to produce. The chief action of this, as of the other nitrites, is to paralyse involuntary muscle (unstriped) all over the body. From paresis of their muscular wall the arterioles are rapidly and greatly relaxed and dilated, and blood pressure is diminished. From the fall of blood pressure there is less work for the heart to do, and so a nitrite indirectly stimulates an overworked heart. The unstriped muscle of the bronchioles is also similarly affected and the tubes dilated. Secretion of sweat and urine is increased, and the urine contains sugar. Human patients receiving one to two minims within a few minutes are flushed; perspiration overspreads the head and neck, extending sometimes over the body; there is general vascular dilatation; arterial pressure is reduced; the temperature falls, the pulse becomes soft, quickened, and dicrotic; breathing, at first accelerated, becomes slower and shallower. Similar symptoms are produced in dogs, in which the temperature may be lowered $3^{\circ}$ or $4^{\circ}$ Fahr. The antidotes are stimulants, alternate hot and cold douches, artificial respiration, and inhalation of oxygen.

Medicinal Uses.- It has been successfully used in spasmodic breathing occasionally occurring as a sequel of sore-throat and bronchitis. In such asthmatic cases in horses and also in dogs it was used by Robertson. Experiments on rabbits made artificially epileptic have shown that it not only prevents the impending fit, but arrests it when it has begun. It hence deserves more extended trial in epilepsy in dogs and young cattle. Richardson found that amyl-nitrite, promptly administered to rabbits and frogs, which had received lethal doses of strychnine, relieved the tetanic spasms and generally ensured recovery. For tetanus in horses it has been frequently given, but seldom with lasting benefit. In horses and dogs it is useful as an inhalation in bringing about recovery from deep chloroform anæsthesia.

DOSES, etc.-Horses and cattle take $m_{x}$. to $m_{l x}$; d dogs, $m_{i}$. to $m_{\nabla}$. A minimum dose should first be tried. When given hypodermically half doses generally suffice. Repeated 
use does not interfere with its efficacy. It is inhaled, administered on a piece of sugar, or in draught with rectified spirit or ether. Ether, chloral, or full doses of alcohol intensify its effects.

Sodium nitrite, obtained by fusing sodium nitrate with metallic lead, has the same actions as amyl-nitrite, is given in the same doses, and is more stable and convenient. Its effects are not so rapidly produced, but persist longer, often continuing for half an hour.

Trinitroglycerin, Nitro-glyeerin, or glonoin, $\mathrm{C}_{3} \mathrm{H}_{5}\left(\mathrm{NO}_{3}\right)_{3}$ is prepared by dropping pure glycerin into a mixture of sulphuric and nitric acids kept cool by ice, and washing it in water. Specific gravity, $1 \cdot 6$. It is a colourless, transparent, explosive liquid, soluble in alcohol, ether, and oils. Its actions resemble those of amyl and sodium nitrites, but are more powerful and persistent, while full doses affect the voluntary as well as the involuntary muscles. It is used for the same purposes as amyl-nitrite. The preparation generally preferred is the B.P. alcoholic solution, liquor trinitrini, of which 110 minims contain one grain of nitroglycerin. The dose for human patients is a half to two minims. For dogs suffering from spasmodic asthma or epilepsy, a teaspoonful of a solution of $m 30$ of liquor trinitrini in ten ounces of water may be given as required. Chocolate tablets containing one-hundredth of a grain of nitroglycerin are now obtainable.

\section{FORMALDEHYDE-FORMALIN}

Formaldehyde (H.COH) is a gaseous body obtained by subjecting methyl alcohol to oxidation. It is a very powerful disinfectant and germicide. A concentrated (35 to 40 per cent.), aqueous solution is known as formalin, or Formol, a colourless liquid, with a strong, pungent odour and neutral reaction, which is largely used as a disinfectant, deodorant, and preservative in solutions of from one to five per cent. Formalin is stated.to retard the healing of surgical wounds. Undiluted it is irritant and caustic to tissues, and corrosive to surgical instruments. Strong solutions applied to the skin may induce poisoning. A solution of one in five 
hundred may be used as an antiseptic wash for the hands, operation area, and accidental wounds. A 2 per cent. solution is recommended as an application for ringworm. Stronger solutions ( 2 to 5 per cent.) are employed in the treatment of canker of the horse's feet ; to preserve histological, botanical, and pathological specimens, and to disinfect stables and cowsheds. Walter and Schlossmann having made critical tests of the efficiency of various methods of disinfection, consider that formaldehyde in presence of sufficient water, deserves preference over other disinfectants. Rational and practical disinfection requires rapid action with thorough penetration, but without injury of the objects under treatment. To meet these conditions, glycoformal, a mixture of an aqueous solution of formaldehyde and glycerin, has been introduced. The glycerin, attracting moisture, ensures the effectiveness of every particle of formaldehyde. Glycoformal, applied by means of an atomiser, may be used to disinfect stables (Coblentz).

Lysoform is a combination of formaldehyde and soap, containing 18 to 20 per cent. of formalin. It is a clear liquid miscible with water, to which it imparts a slight milky appearance. Solutions of 3 per cent. do not irritate the hands or damage instruments.

Urotropine (Hexamethylenetetramine, $\mathrm{C}_{6} \mathrm{H}_{12} \mathrm{~N}_{4}$ ), obtained by combining ammonia and formaldehyde, is a colourless, crystalline powder, soluble in water and alcohol ; insoluble in ether ; and employed as a solvent of uric acid and urates. It is said to undergo decomposition in the kidneys, and to act as a powerful urinary disinfectant. Doses, for the dog, grs. iii. to grs. $x v$. per day, given well diluted in water.

Formaldehyde forms an important constituent of the following powders:- -glutol, a combination of gelatin and formaldehyde, dried and reduced to powder, and employed as an antiseptic dry dressing for wounds: amyloform, a condensation product of formaldehyde with starch, forming a non-poisonous, white, odourless, insoluble powder, introduced as a substitute for iodoform ; and amyloiodoform, a compound of starch, iodine, and formaldehyde, employed as an antiseptic dry dressing. 


\section{TAN NOFOR M}

Tannoform (Merck) or Methyl-ditannin $\left(\mathrm{C}_{29} \mathrm{H}_{20} \mathrm{O}_{18}\right)$ is a condensation product of tannic acid and formaldehyde, obtained by adding formol to an aqueous solution of tannin, and precipitating with hydrochloric acid. It occurs as a pale, rose-coloured, almost tasteless powder, odourless, insoluble in water, but soluble in alcohol, and in dilute caustic potash or soda, and in ammonia. It readily mixes with cold milk or cold water, but with hot liquids it forms tough masses.

Actions AND Uses.-Tannoform combines the astringent properties of tannic acid with the antiseptic effects of formaldehyde, and therefore it is astringent, desiccant, deodorant, and antiseptic.

Administered by the mouth, it is unaffected by the gastric juice and passes through the stomach unaltered. In the alkaline medium of the intestine it is split up into its components, and acts as an astringent antiseptic. In the treatment of diarrhœa, gastro-intestinal catarrh, and mycotic enteritis of foals, calves and dogs, Schaefer, Foth, Schünoff, and other veterinarians, report most favourably of its value. Foals, less than a month old, suffering from intestinal catarrh with profuse discharge, have been successfully treated with doses of 45 to 60 grains of tannoform, administered in cold linseed gruel, or milk. For diarrhœa or scour in calves it has been recommended as a specific, in doses of 15 to 45 grains, with $\frac{1}{6}$ th grain of calomel, made into a paste or linctus with honey or treacle. It does not cause gastric disturbance or loss of appetite, and is harmless and well borne even when given in large doses. Satisfactory results have followed the administration of tannoform in the treatment of dogs affected with necrosing gastritis or so-called 'Stuttgart disease.'

Externally tannoform is largely used alone, or mixed with boric acid, starch, or alum, as a dry dressing for wounds, burns, harness galls, cracked heels, thrush, canker of horses' feet, and wounds of the claws of cattle. For superficial wounds healing under a scab, Fröhner strongly recommends tannoform as a substitute for the more expensive iodoform. 
After thorough disinfection, recent wounds carefully powdered with tannoform are quickly covered with a protecting crust, suppuration seldom occurs, and cicatrisation speedily follows. When granulations are too prominent they are touched with copper sulphate, and covered with a mixture of equal parts of tannoform and alum. For cracked heels and mallenders the powder should be rubbed into the fissures with a pledget of cotton-wool, or the finger, until moisture disappears.

Tannoform, being odourless and non-poisonous, is especially valuable in canine practice. It is extensively employed in the treatment of moist eczema, otorrhœa, dogbites, and operation wounds. In moist eczema the skin is first cleansed with an antiseptic solution, and then dusted with pure tannoform. Abrasions and small wounds may be dressed with a pomade composed of one part tannoform and six parts of lanoline, or vaseline.

Doses, ete.-Horses and cattle, ऊiv. to $\jmath_{j}$. ; foals, grs. xxx. to grs. xc. ; calves and pigs, grs. xlv. to 3ij.; dogs, grs. x. to grs. 1. These doses may be repeated three or four times a day, and are conveniently given in cold linseed gruel, or milk, or in electuary. For otorrhœa, after cleansing the ear, tannoform may be insufflated, or employed in alcoholic solution (5 to 10 per cent.). Tannoform mixed with collodion forms a useful protective for small sutured wounds.

\section{BENZOL, BENZENE, OR AROMATIC SERIES OF CARBON COMPOUNDS}

The benzol, benzene, or aromatic series of carbon compounds includes a number of antiseptics and antipyretics. The lowest members of this series contain six carbon atoms, five of which have their affinities satisfied by hydrogen, constituting the organic radicle phenyl $\left(\mathrm{C}_{6} \mathrm{H}_{5}\right)$. The hydride is benzene $\left(\mathrm{C}_{6} \mathrm{H}_{5} \mathrm{H}\right)$. Substitution of hydroxyl $(\mathrm{OH})$ for one hydrogen atom produces the phenol-carbolic acid $\left(\mathrm{C}_{6} \mathrm{H}_{5} \mathrm{OH}\right)$. A like substitution of one or more of the hydrogen atoms for other organic radicles forms other aromatic bodies. Still further variety results from the union 
of molecules of the same or of different members of the group; two benzene molecules form naphthalene $\left(\mathrm{C}_{10} \mathrm{H}_{8}\right)$; a benzene and pyridine molecule quinoline $\left(\mathrm{C}_{9} \mathrm{H}_{7} \mathrm{~N}\right)$, which is allied to quinine; indeed, it is generally believed that many of the organic alkaloids are closely related to this aromatic series. The higher members appear to be the most active. Rearrangement of the atoms of these bodies, and substitution of various radicles, will doubtless produce other valuable substances.

The lower members of the series are the most useful medicinally. They are antiseptic, usually antipyretic, frequently analgesic. They affect the central nervous system in a characteristic manner, at first, and in moderate doses, causing great irritability, with tremors, convulsions, and paralysis. Later, there is great depression of the medullary centres causing marked muscular weakness, paralysis, and collapse. They exhibit a marked contrast to the lower members of the fatty or marsh-gas series $\left(\mathrm{CH}_{4}\right)$, which chiefly affect the higher cerebral nerve centres, are at first indirect stimulants by removal of inhibition, and later anæsthetics.

Benzol or Benzene, a mixture of homologous hydrocarbons, obtained from light coal tar oil. It contains about 70 per cent. of benzene, $\mathrm{C}_{6} \mathrm{H}_{6}$, and 20 to 30 per cent. of toluene, $\mathrm{C}_{6} \mathrm{H}_{5} \mathrm{CH}_{3}$. It is an ethereal, inflammable liquid, with an acrid and bitter taste, the odour of coal-gas, and the specific gravity 888 . It is insoluble in water, soluble in alcohol and ether, and a useful solvent for sulphur, phosphorus, chlorine, iodine, many alkaloids, fats, resins, and caoutchouc. Benzene is a perfectly distinet body from benzin, petroleum benzin, or petroleum ether, which is a purified distillate obtained from American petroleum-a paraffin of the marsh-gas series, consisting chiefly of $\mathrm{C}_{5} \mathrm{H}_{12}$, and sometimes used as an anæsthetic, anthelmintic, and parasiticide.

Benzol or coal-tar benzene is irritant, antiseptic, and parasiticide. Its irritant effects are especially notable on mucous and skin-abraded surfaces, or when applied with friction. Moderate doses, when swallowed, produce salivation, slight temporary pyrexia, and obstinate constipation. 
It is excreted in the urine, to which it imparts the odour of violets. Larger doses accelerate cardiac and respiratory movements, while toxic doses cause nervous depression, muscular trembling, convulsions, lowered temperature, and loss of sensibility. Benzene is an effectual poison for various skin parasites in all classes of patients, and is usually applied after a thorough wash with soap and water, either undiluted, or in young and delicate subjects, and in cats, dogs, and fowls, which are sensitive to its irritant effects, mixed with one to three parts of olive oil, or vaseline. Benzol alone or mixed with oil is sometimes used in the treatment of follicular mange, prurigo, and urticaria.

Doses.-(Vermifuge), horses and cattle, $3 \mathrm{jv}$. to $\mathrm{z}_{\mathrm{ij} . \text {; }}$ sheep and calves, $3 \mathrm{j}$. to $3 \mathrm{jv}$. ; dogs, $m_{\mathrm{x}}$. to $3 \mathrm{j}$.

\section{A NTIPYRINE}

Phenazone. Phenazonum. Commonly known as 'antipyrine.' Phenyl-dimethyl-pyrazolone. $\quad \mathrm{C}_{6} \mathrm{H}_{5}\left(\mathrm{CH}_{3}\right)_{2}$ $\mathrm{C}_{3} \mathrm{HN}_{2} \mathrm{O}$.

Phenazone is obtainable from phenylhydrazine by interaction with acetoacetic ether, and the subsequent interaction of the resulting phenyl-methyl-pyrazolone with methyl iodide (B.P.). It occurs in colourless, odourless, scaly crystals, with a bitter taste. Soluble in about one part and a half of water, alcohol, or chloroform, and in forty parts of ether. A one per cent. aqueous solution yields a white precipitate with tannin, and is coloured yellow by concentrated nitric acid. Incompatibles-spirit of nitrous ether, tannic acid, vegetable astringents, corrosive sublimate, chloral, and salicylates.

ACTIONS AND USES.-Antipyrine is an active member of the benzol or aromatic carbon group, and, like others of the series, it is antiseptic, antipyretic, and analgesic. It is a local anæsthetic and hæmostatic. Strong doses irritate the mucous surfaces, and hence when swallowed sometimes cause vomiting, and other evidences of gastro-intestinal disturbance in men and dogs. Fröhner reports that dogs 
receiving two drachms exhibited excitement, and subsequently paralysis of the central nervous centres, tetanic or epileptic convulsions, cyanosis, muscular weakness, paralysis beginning in the hind-quarters, with serious lowering of temperature. Although elimination by the kidneys begins within half an hour after administration, it is slowly excreted. The urine of dogs receiving full doses, for several days contained the drug, as evidenced by its being coloured red-brown by ferric chloride solution. Fröhner further states that, although medicinal doses have little effect in lowering the temperature of healthy animals, they reduce abnormal temperature usually within half an hour, and their effects continue two or more hours. Kaufmann records that one gramme (15.4 grains) given hypodermically reduced the temperature of dogs from $38.9^{\circ}$ to $38.2^{\circ}$ Cent., of horses from $38 \cdot 7^{\circ}$ to $38 \cdot 2^{\circ}$ Cent., and of rabbits from $40^{\circ}$ to $37^{\circ}$ Cent. These antipyretic effects are ascribed to the action of the drug on the heat-regulating centre in the brain. In addition to the antipyretic effect there is depression of the central nervous system, so that pain is lessened and there is less reflex irritability. It produces its actions whether it is swallowed or introduced subcutaneously or intratracheally, and seems to develop no untoward effects. The antipyretics affect the red corpuscles, causing shrinking, loss of hæmoglobin, and the conversion of the latter into methæmoglobin in the plasma.

It has been prescribed for the several domestic animals to reduce fever and relieve pain. German veterinarians commend it for influenza in horses, as well as muscular and articular rheumatism ; but as an anti-rheumatic it is not as effectual as the salicylates. In doses of four to six drachms, it has been employed with marked benefit in acute laminitis. Dogs are brought under its antipyretic action more effectually than cattle or horses, and it deserves to be more generally used in canine practice. French veterinarians prescribe it as a carminative in irritable conditions of the central nervous system, and as a general analgesic as well as a local anæsthetic in neuralgic cases. A five per cent. solution is frequently used to check capillary hæmorrhage, and 
hypodermically for local pains. Its hæmostatic power is greatly increased by admixture with tannin solution.

Doses, ete.-Horses take 3ij. to 3iv.; cattle, 3iij. to 3 vi. ; sheep, grs. xxx. to $3 i$. ; dogs, grs. iv. to xx., given in bolus, drench, electuary, or in the drinking water, and repeated as may be required. For hypodermic or intratracheal injection in horses the dose is 40 grains dissolved in four drachms of water. As some animals are very susceptible to its depressant effects, it is well to begin with small doses, and repeat twice or thrice daily.

Acetopyrine, or acetosalicylate of antipyrine, is sometimes employed as a substitute for antipyrine. It is antipyretic and analgesic. It occasions neither gastric nor cardiac disturbance, and does not affect the kidneys. It constitutes a good antipyretic without accessory depression of the heart. It promotes the secretion of the pancreas, and, after a time, that of the skin. It has proved beneficial in rheumatism, influenza, and pneumonia, in which it eases pain and lowers temperature. It may be prescribed in doses about one-third less than those of antipyrine.

\section{ACETANILIDE OR ANTIFEBRIN}

Acetanilidum. $\mathrm{CH}_{3}$.CO.NH. $\mathrm{C}_{6} \mathrm{H}_{5}$. Phenyl-Acetamide. (Antifebrin is a registered name.)

Acetanilide, one of the most serviceable of the benzol or aromatic carbon series, is a crystalline substance, prepared by the interaction of glacial acetic acid and aniline. It is colourless, odourless, with a slightly burning taste, oily to the touch, soluble in 190 parts of water, four parts rectified spirit, forty parts glycerin, freely soluble in ether, benzol, and chloroform.

ACTIONS AND USES.-It is antipyretic, sedative, diuretic, and feebly antiseptic. Even in excessive doses it rarely causes toxic symptoms in animals, although in man it is more toxic than antipyrine, and more liable to produce dangerous collapse. It is neither irritant nor nauseous, and has, moreover, the merit of cheapness. Compared 
with antipyrine, antifebrin is more energetic and lasting in its effects. Given in large doses it depresses the heart, alters the colour of the blood to a brownish red, and to some extent reduces the hæmoglobin to methæmoglobin. According to Lepine antifebrin exerts a destructive action on the red corpuscles. Elimination of the drug by the kidneys occurs within twenty-four hours of its administration, and frequent large doses darken the urine and increase the excretion of urea and uric acid. The diuretic action of antifebrin suggests its employment in hæmoglobinuria. Fröhner and other German authorities testify to its febrifuge effects both in horses and dogs, and prescribe it in febrile cases, cramps, neuralgia, and rheumatism. Ordinary doses have no appreciable action on temperature in health, but rapidly lower the temperature in fevers, pneumonia, and influenza, probably by diminishing the production of heat and by increasing heat dispersion. For relieving pain acetanilide is less useful than opium, or cocaine, and as an antiseptic it cannot be recommended.

The doses for horses and cattle are 3ij. to 3jv.; sheep, grs. xxx. to 3 j. ; dogs, grs. ii. to $x$. ; cats, gr. $1 \frac{1}{2}$ to grs. iv., given in powder, pill, syrup, or electuary, or in water containing alcohol, three times daily", in critical cases every third hour.

RESORCIN or metadihydroxybenzene, resorcinol (U.S.A.), a product of the distillation of galbanum, ammoniacum, asafotida, or extract of Brazil wood, with caustic alkalies, is usually obtained from benzene or phenol. It occurs in colourless, crystalline plates, which become pink on exposure to the air. It has a harsh, sweet taste, and is freely soluble in water, alcohol, and oils. It coagulates albumin, and a one per cent. solution is an effectual antiseptic. Concentrated solutions irritate the skin and mucous surfaces, but it is not so irritant as its analogues, carbolic acid and creosote, while, like them, it has a slight topical, anæsthetic effect. Lethal doses, given to dogs and rabbits, cause clonic convulsions, dyspnœa, and paralysis. Much less toxic than phenol, it is eliminated in the urine. Its antipyretic action is of short duration, and it has a dangerous 
tendency to cause collapse. As an intestinal antiseptic, calves and foals suffering from diarrhœa or gastric catarrh receive thirty to sixty grains, dogs, grs. ii. to grs. v., while horses may be given from $3 \mathrm{jv}$. to $3 \mathrm{vj}$. These doses may be repeated two or three times in twenty-four hours. It is used as an antiseptic in surgical cases-in inflammation of the eyes, mammæ, urino-genital organs, and other sensitive parts, and as a hæmostatic in capillary bleeding. Kaufmann commends it in erythema, herpes, and eczema, especially of the seborrhœal type, in dogs. The solutions and ointments applied contain one to ten parts per hundred.

HYDROQUINONE, chemically para-dihydroxybenzene, resembles resorcin, but is about four times stronger, and is soluble in twenty parts of water, and in alcohol and ether. As it is excreted in the urine, it exerts a stimulant and antiseptic action on the urino-genital mucous membrane.

Pyrocatechin, or ortho-dihydroxybenzene, resembles resorcin in its actions and uses, but is about three times stronger.

Pyrogallic ACID, or trihydroxybenzene, is obtained by heating gallic acid, and is readily soluble in water and alcohol. It is more irritant than most of the series, but is a doubtful antiseptic. A few grains produce in dogs, as well as in man, vomiting, purging, and collapse. It decomposes the red corpuscles, causing shrinking of the cells and escape of the hæmoglobin into the plasma, where it is converted into methæmoglobin. Mixed with fatty matters or starch, it is sometimes used as a caustic. A 15 per cent. ointment has been applied with good effects in psoriasis, but the drug should not be given internally.

SACCHARIN, glusidum, or benzoyl-sulphonimide, is derivable from toluene of coal-tar. It is a colourless, crystalline powder, two hundred and twenty times sweeter than sugar, soluble in four hundred parts of water, twenty-five of alcohol, forty-eight of glycerin, and slightly in ether or chloroform. It is antiseptic, very stable, passes through the body unchanged, and is eliminated in the urine. As it is inconvertible into sugar, it is used in tablets for sweetening food, and flavouring the medicines of human patients 
suffering from diabetes, being given in quantities of one-fifth to half a grain. An elixir is made with saccharin, twentyfour grains, sodium bicarbonate, twelve grains, rectified spirit, one drachm, distilled water, seven drachms. Twenty minims contain one grain of saccharin.

SALOL, or phenyl salicylate, is prepared by the interaction of salicylic acid and phenol. It occurs as a crystalline white powder, with an aromatic smell and insipid taste. Insoluble in glycerin or water, but soluble in ten parts alcohol, less than one of ether, chloroform, or benzene; and in the fixed oils. The actions and uses are those of its two components. It is antiseptic, analgesic, antipyretic, and an intestinal disinfectant. It is not so irritant as salicylic acid, and only poisons in large doses. It is eliminated in the urine as salicylic acid and sulphophenol. By the saliva, the pancreatic and intestinal juices, it is resolved into salicylic and carbolic acids, which, with their products, can be detected in the urine. It checks undue fermentation in the digestive canal, and is serviceable in diarrhoa. Fröhner recommends it as an anti-rheumatic, especially in dogs ; but neither in muscular nor arthritic rheumatism does it appear to be so effectual as sodium salicylate. Kaufmann advises its use as a substitute for iodoform in surgical cases, notably in diseases of the urino-genital organs, and in otorrhœa, stomatitis, and ozæna. Horses take 3iii. to 3 vi. ; dogs, grs. iii. to grs. xv., in pill or electuary. In acute rheumatism these doses may be repeated two or three times a day. For the several purposes of a non-irritant antiseptic it is used as powder, liniment, or ointment, and with collodion as an antiseptic adhesive. A useful dressing for catheters consists of one part salol, and fifteen parts each of castor oil and almond oil (G. B. Browne).

SALACETOL, a salicylic ester of acetone, introduced as a substitute for salol, which contains phenol. It contains about seventy-five per cent. of salicylic acid, occurs in shining crystals, insoluble in water, freely soluble in alcohol, ether, and chloroform. It is employed as a disinfectant for the digestive and urinary tracts. Doses, horses, 3 ii. to 3 vi. ; dogs, grs. iv. to grs. $\mathrm{xx}$.

Pyoktanin (methyl violet) is a powerful antiseptic, pro- 
ducing little irritation. Soluble in seventy-five parts of water, and twelve of alcohol. A solution of one in one thousand destroys the vitality of anthrax bacilli; and a solution of one in two thousand arrests the development of pyogenic organisms. Methyl violet has been used in the treatment of keratitis and suppurative conjunctivitis.

Methylene Blue, a complex derivative of aniline, occurs in dark blue crystals or crystalline powder of a bronzelike tinge. Slightly soluble in water, alcohol, and glycerin, it has been prescribed as an anodyne in painful nervous affections, to paint on and eradicate warts, and as an antiseptic dressing for ulcers of the lips in dogs and cattle -for which purpose Cadiot employs a solution of ten parts dissolved in fifty parts each of alcohol and glycerin.

TrypanbluE or Trypanblau $\left(\mathrm{C}_{34} \mathrm{H}_{24} \mathrm{~N}_{6} \mathrm{O}_{14} \mathrm{~S}_{4} \mathrm{Na}_{4}\right)$, derived from toluidine and sodium amido-naphtholsulphonate, has been recently used by Nuttall, Hadwen, Jowett, and others in the treatment of canine and bovine piroplasmosis and of East Coast fever. For some time another similar dye, trypanred or trypanroth has been tried as a remedy for trypanosomiasis with varying results. Trypanblue given by the mouth has no appreciable action in piroplasmosis, but when given intravenously or subcutaneously it destroys the piroplasmata in the blood stream. Nuttall employed a one to five per cent. solution of trypanblue in cold sterilised water, in doses of 2 cubic centimetres for small dogs ; 15 cub. cent. for large dogs ; and 150 cub. cent. to 200 cub. cents. for cattle. Hypodermic injections of trypanblue may be followed by local irritation, abscess formation, and sloughing of portions of the skin, and to prevent these effects Jowett advises administration by intravenous injection. Experiments with this agent on dogs tend to show that it may prove useful as a prophylactic for piroplasmosis.

NAPHTHALENE is prepared from tar and tar oils, and occurs in colourless, soft, peculiar-smelling, but tasteless crystals. Soluble in ether, alcohol, fats and oils ; insoluble in water. It is antiseptic, feebly antipyretic, and parasiticide. Moderate doses are non-poisonous, but when given for several months they cause wasting and diarrhoa, with ulceration of the 
cornea, opacity of the lens, and spots on the retina (Kaufmann). It is used as an intestinal antiseptic, and vermicide, is serviceable in diarrhœa and dysentery, in protracted cases of influenza and purpura, and is prescribed either with mucilage or castor-oil. In the bowel it is decomposed into alpha- and beta-naphthols. Being excreted in part in the urine, it exerts antisepsis in diseases of the bladder. It is used for antiseptic dressings, and in all animals for the destruction of skin parasites. The dose for horses and cattle is $3 \mathrm{jss}$ to $3 \mathrm{jv}$. ; calves, grs. $\mathrm{xxx}$. to $3 \mathrm{j}$. ; for sheep and dogs, grs. iss. to grs. xv., administered in electuary or bolus. Externally it is applied with vaseline, or glycerin.

The NAPHTHoLs are prepared from naphthalene. Alphanaphthol is stated to be more irritant and less toxic than beta-naphthol. The $\beta$ variety, which is chiefly used, is a colourless, crystalline, phenol-smelling powder, almost insoluble in water, but very soluble in alcohol, ether, chloroform, olive oil, and vaseline. Its actions and uses are the same as those of naphthalin, and also closely resemble those of resorcin. It is antiseptic and antiparasitic. One part in 3000 prevents the development of the microbes of anthrax and glanders. For such antiseptic purpose it is five times more effectual than carbolic acid. It is irritant in contact with mucous membranes whether in vapour or in solution; hence small doses stimulate the mucous and glandular secretions. Somewhat larger doses induce nausea, diarrhœa, and dysuria. Powerful doses, exceeding one gramme per ten kilogrammes of body weight, cause epileptiform convulsions in cats and horses, but in dogs there occur instead coma and reduced temperature. Rabbits relatively to their weight will stand three times the dose borne by dogs and cats. Naphthol is administered internally to destroy tape- and round-worms (Willenz), as well as putrefactive and infective germs lodged in the intestines. As it is not readily soluble, full doses exert antisepsis throughout the greater portion of the intestinal tract, lessening the smell and irritant action of the fæces, and hence checking diarrhœa. Externally it is used as an antiseptic and parasiticide, one part being mixed with six of oil or twenty of vaseline. One part $\beta$ naphthol with two of camphor 
forms Naphthol Camphor, a viscid, non-toxic antiseptic, which, mixed with an equal part of glycerin or olive oil, is used as a dressing for auricular catarrh and small wounds. Doses of Naphthol, horses, 3jss. to 3jv.; dogs, grs. v. to grs. xv. Sometimes conjoined with bismuth salicylate and given suspended in mucilage.

HYDRONAPHTHOL is a useful antiseptic and germicide, soluble in one hundred parts of water and twenty of oil, and often conveniently applied as a dry dressing, mixed with twenty to thirty parts of fuller's-earth.

NAPHTHALOL (Betol), prepared from $\beta$-naphthol-sodium, phosphorus oxychloride, and sodium salicylate ; and alphol, the salicylic ester of $a$-naphthol, are sometimes employed as intestinal antiseptics. In the bowel betol and alphol are decomposed into salicylic acid and naphthol. Benzo-naphthol, the benzoate of $\beta$ naphthol, has been recommended as a gastro-intestinal disinfectant. It is split up into naphthol and benzoic acid (Coblentz). Benzo-naphthol is very slightly toxic. Horses take grs. xxx. to grs. lx.; dogs, grs. iv. to grs. viii., in bolus, pill, or electuary.

- Phenacetin (para-acetphenetidine is produced by the action of glacial acetic acid on para-phenetidine, a body obtained from para-nitro-phenol (B.P.). It is white, tasteless, odourless, occurs in glistening, scaly crystals, sparingly soluble in cold water, soluble in twenty parts of alcohol, and in chloroform and glycerin; insoluble in acid or alkaline solutions. Like antifebrin and antipyrine, it lowers temperature, diminishes pain, and depresses the heart. Although its action is less rapid, it is more prolonged, and less liable than either of these analogues to produce collapse, while in human patients it has also slight soporific effects (Brunton). Fröhner and other German observers state that grs. iii. to grs. vii. given to dogs in a febrile condition lower the temperature $2^{\circ}$ Fahr., also slow the pulse, and relieve respiratory difficulty. These effects continue for four hours. Horses and cattle take 3ij. to 3iv.; dogs, grs. iii. to grs. $\mathrm{xv}$., in bolus or electuary; repeated at intervals of two or three hours.

Piperazin $\left(\mathrm{C}_{4} \mathrm{H}_{10} \mathrm{~N}_{2}\right)$, an organic base, produced by the interaction of sodium glycol, and ethylene-diamine hydro- 
chloride. It occurs in white deliquescent scales, very soluble in water. Outside the body it dissolves uric acid and insoluble urates; but when administered its action is exceedingly doubtful. A small part only is excreted combined with the stronger acids rather than with uric acid in the urine. The granular citrate is generally used. Doses of five to fifteen grains in carbonated water, taken twice daily, were stated to break up cystic concretions in human patients, but this reputation has not been maintained.

ChINASEPTOL, diaphthol, or ortho-oxy-quinolin-metasulphonic acid, occurs in yellowish crystals, which are soluble in water. Solutions of one or two per cent. are powerfully antiseptic, but their use in surgery is restricted owing to their effect on instruments. It is rapidly absorbed, as an alkaline diaphtholate, from the intestine and excreted by the kidneys-disinfecting the urine and urinary passages. Medicinal doses are free from toxic effects. Horses take 3j. to 3ij. ; dogs, grs. x. to grs. xxx., in bolus or electuary.

EXALGIN (Methylacetanilide) occurs in nearly colourless crystals, is odourless, almost tasteless, slightly soluble in water and freely in alcohol. It is slightly antiseptic, distinctly antipyretic, and markedly analgesic. As an antipyretic it resembles antifebrin; it impairs perception of painful impressions; but large doses are dangerous, and produce epileptiform convulsions, dyspnœa, stupor, and death. Small, perfectly safe doses, such as one or two grains in human patients, or half a grain in dogs, repeated hourly, relieve neuralgic pain ; but for the pain of muscular rheumatism it is not so effectual as salicylates. It does not appear to produce any disagreeable secondary symptoms. It has been used with benefit in epilepsy and chorea in man, and may be serviceable in such eases in dogs. It is generally administered dissolved in weak spirit.

QUINOLINE is an oily liquid produced from glycerol, sulphuric acid, aniline and nitro-benzene. It is related to cinchonine and quinine, from which it may also be obtained. It is colourless or yellow, bitter and acrid, almost insoluble in water, but soluble in alcohol, ether, chloroform, and benzene. Quinoline and its salts, tartrate and salicylate, are antiseptic and antipyretic, but other drugs of this aromatic 
series are safer and more effectual, for quinoline is very apt to cause collapse. Dogs and cats, receiving three to six grains per kilogramme of body weight, exhibit increased secretion of saliva and bile, and suffer from vomiting and general enfeeblement.

THALuINE is a synthetically prepared base chemically known as tetrahydroparamethyl-oxyquinoline. It is crystalline and colourless, has a piquant, bitter, anise-like taste, is soluble in seven parts of cold water and a hundred of alcohol. Both the watery and alcoholic solutions are inflammable. It combines with acids, and is used as a sulphate or acetate. It is antiseptic and antipyretic. It resembles antifebrin, but Brunton considers it is less effectual. T. Fraser regards it as probably the most trustworthy antipyretic of the series. Kaufmann and others testify to its febrifuge effects on veterinary patients, and state that it causes neither digestive nor nervous disturbance. Moderate doses given to horses and dogs, in one hour reduce abnormal temperature $3^{\circ}$ Cent., and such reduction is stated to be maintained for several hours. It slows respiration, diminishes the number of the pulsations, and lessens arterial pressure. These effects appear to depend upon the depression of the heat regulating nerve centres with consequent increased loss of heat by way of the dilated skin vessels. Thalline is stated to communicate a dark-red colour to the blood, from the formation of methæmoglobin, and sometimes causes a red eruption on the tongue (Kaufmann). It is slowly excreted in the urine, which acquires a greenish-brown hue, and is coloured purple by ferric chloride solution. Friedberger prescribes it in contagious pneumonia in horses, both by the mouth and hypodermically. Horses take 3i. to 3iii.; cattle, 3ii. to 3iv.; sheep and pigs, grs. xv. to grs. $x x x$. ; dogs, grs. ii. to grs. vi., cats, gr. i. to grs. ii., given, as the sulphate, in bolus or solution. Hypodermically, one-third of those doses may be given.

Pyridine $\left(\mathrm{C}_{5} \mathrm{H}_{5} \mathrm{~N}\right)$ is obtained from the destructive distillation of bones and other organic substances, and is one of the empyreumatic constituents of tobacco smoke. It is a typical member of the alkali-like bases found in coal- 
tar, and from it various bodies of the aromatic series are prepared. It is a colourless, strong-smelling, volatile, antiseptic liquid. Soluble in water, alcohol, ether, and oils. It diminishes the reflex activity of the medulla and spinal cord, notably of the respiratory centre, acting like the alkaloid conine, to which it is chemically allied, and causes death by asphyxia; but is so rapidly eliminated by all the excretory channels that large doses are required to kill. As an inhalation it has been used in asthma and other cases of difficult breathing.

Chinosol, or oxyquinoline sulphonate of potassium, is a derivative of coal-tar. It occurs as a yellow, crystalline powder, with a faint odour, soluble in water, insoluble in alcohol or ether. Its solution is unaffected by albumin. Chinosol is antiseptic, antipyretic, styptic and disinfectant. A solution of one part in forty thousand prevents bacterial development, and a grain dissolved in six drachms of water is stated to be equal in antiseptic power to a one in forty solution of carbolic acid. Applied to wounds, the alkaline discharges decompose it and liberate oxyquinoline, which oxidises and disinfects. In the treatment of surgical cases solutions varying from one in twelve hundred to one in sixty have given most satisfactory results. Mixed with boric acid, zinc oxide, or starch, it forms an excellent dry dressing, and with collodion it is used for sealing small wounds. Hobday considers that its action is better marked when used in lotion than in powder. Concentrated solutions should not be used to disinfect instruments as the drug attacks steel. Chinosol has been used as an udder injection in parturient apoplexy. For this purpose fifteen grains are dissolved in a pint of water, and a fourth part injected into each quarter. Chinosol can be obtained in tablets containing five, eight, and fifteen grains.

ACETOzone, or Benzoyl-Acetyl-Peroxide, occurs as a white crystalline powder, sparingly soluble in water, and decomposed by alkalies. As it acts on organic matters, alcohol or glycerin should not bo used in the preparation of solutions. Acetozone is a powerful antiseptic, deodorant, and germicide, and is said to be non-toxic and harmless to animal tissues. It has been prescribed in diarrhœa, 
dysentery, contagious abortion, and in distemper of the dog. For the destruction of parasites in the intestine a filtered solution of twenty grains in a quart of water may be used. Externally, a solution of five grains to a pint of water is employed as a lotion for wounds and ulcers, and as a dressing for eczema and mange.

\section{CARBOLIC ACID}

Acidum Carbolicum. Phenol. $\mathrm{C}_{6} \mathrm{H}_{5} \mathrm{OH}$.

Carbolic acid is an occasional constituent of the urine of most animals, may be extracted from some plants, and is one of the many products of coal-tar. Cannel coal is its most prolific source; but it also occurs in other coals, as well as in bitumen and petroleum. It is obtained from coaltar oil by fractional distillation (B.P.).

Carbolic acid occurs in small, colourless, deliquescent crystals, having a peculiar odour and sweetish pungent taste. Exposed to moist air it may acquire a pinkish tinge. Specific gravity $1 \cdot 060$ to $1 \cdot 066$. It is devoid of acid reaction, is liquefied at $60^{\circ} \mathrm{Fahr}$. by ten parts of water, and completely dissolved by twelve hundred parts of cold water. It is freely soluble in glycerin, most volatile oils, alcohol, ether, alkaline solutions, and acetic acid. It has a caustic action on the skin and mucous membranes, coagulates albumin, and liquefies camphor. With an equivalent of sulphuric acid, it forms sulpho-carbolic acid, which produces a series of definite, stable, soluble, crystallisable salts-the sulphocarbolates, which have no action of carbolic acid. With nitric acid, it forms explosive picric acid $\left(\mathrm{C}_{6} \mathrm{H}_{2}\left(\mathrm{NO}_{2}\right)_{3} \mathrm{OH}\right)$, an antiseptic, and much used as a yellow dye. When carbonic anhydride is passed through heated dry powdered sodium phenate, salicylic acid is produced.

Carbolic acid is distinguished by its odour. Bromine water forms, even in very dilute aqueous solutions, paleyellow crystalline needles of tribromo-phenol. An aqueous solution, even if containing $\frac{1}{1000}$ part, when treated with a drop or two of ferric chloride solution, produces a purple colour. 
According to Martindale, the following commercial varieties are in general use :-(a) Absolute phenol; (b) Carbolic acid, in crystals and in liquid, containing 10 per cent. of added water : the crystals are soluble in fourteen parts of water; (c) No. 2 carbolic acid, also in crystals and in liquid, soluble in eighteen parts of water; $(d)$ No. 4 carbolic acid, a pale straw-coloured liquid, containing about 10 per cent. of phenol, and nearly 90 per cent. of cresols; (e) No. 5 carbolic acid, a dark-coloured liquid of uncertain strength. Carbolic disinfectant powder contains 15 per cent. of phenols mixed with a dry powdered earth.

The B.P. acidum earbolicum liquefactum, or phenol to which distilled water has been added in the proportion of ten parts of water to one hundred of phenol, is a colourless or slightly pink liquid, with the taste, odour, and properties of the pure acid. The empyreumatic red-brown liquid commercial acid contains the uncrystallisable, acrid methylphenol or cresol $\left(\mathrm{C}_{6} \mathrm{H}_{4} \cdot(\mathrm{OH}) \cdot \mathrm{CH}_{3}\right)$. Calvert's carbolic powders consist of 20 to 30 per cent. of carbolic acid, incorporated with refuse from the alum works. M'Dougall's disinfecting powders contain about 33 per cent. of calcium carbolate and 59 per cent. of magnesium sulphite. A mixture of carbolic acid and bleaching powder has been patented. Blast furnace residual oils consisting of 20 to 35 per cent. of phenoloids, soluble in caustic soda, resemble wood-tar products, and are used for preserving timber.

ACTIONS AND USES.-Carbolic acid belongs to the benzol or aromatic series of carbon compounds, which are notable for their antiseptic and antipyretic properties (p. 394). It is a general protoplasmic poison, and closely resembles creosote and creolin. Large doses are irritant and narcotic poisons. It is used as an antiseptic, antiparasitic, occasionally as a local anæsthetic, and also as a caustic. It is administered in various contagious diseases, with a view to prevent or arrest the development of micro-organisms. It is employed as a disinfectant.

\section{General Actions.-It coagulates albumin and destroys} micro-organisms. It is not nearly so active as corrosive sublimate, chlorine, iodine, or sulphurous acid in arresting the action of ptyalin, pepsin, diastase, and other organic 
ferments, or in killing or preventing the development of bacteria; but Koch's experiments show that about one part to 500 prevents the growth of anthrax and other bacilli. Oats, barley, beans, and lentils, soaked in a one per cent. solution, do not germinate. Milk is maintained unchanged by $\frac{1}{54}$ th part of acid. The bacilli of tuberculosis are killed in twenty-four hours by a 5 per cent. solution. The development of putrefactive organisms is arrested by a 4 per cent. solution which is also fatal to the organisms of pus. Warm aqueous solutions are more powerfully antiseptic than solutions in alcohol, oil, or glycerin ; in fact, carbolised oil is only feebly antiseptic and not reliable as a disinfectant. The probable reason for this is that phenol is so readily soluble in oil that it will not leave the oily solvent to attack the protoplasm of the bacteria. As carbolic acid gradually volatilises, not only may fresh infection occur, but spores and organisms, the development of which has been arrested, may regain activity.

A strong solution applied to the skin, or to a mucous surface, coagulates albumin, acts as a topical irritant and slight caustic; anæsthetises not only the skin, but the underlying structures; causes a stain at first white, but shortly becoming brown; and leaves a dry, roughened surface, from which the shrivelled epidermal scales subsequently peel off.

Full doses when swallowed, besides producing local effects, cause salivation, and in carnivora usually vomiting, with gastro-enteritis and collapse from shock, which may end fatally. It is absorbed, and like other members of the benzol series, in small doses, it first very briefly stimulates and subsequently paralyses the medulla and spinal cord, and involves also the cerebral centres. The respiratory and vaso-motor centres are first stimulated, quickening respiration, raising blood-pressure, and accelerating the pulse ; but as paralysis is developed, respiration is slowed, and blood pressure falls. Stimulation of the sweat-centre increases perspiration. Implication of the cerebral centres gives rise to restlessness, irregular movements, convulsions, and anæsthesia. These symptoms are followed by depression, weakness, staggering gait, then collapse and coma. Moderate 
doses kill by paralysis of respiration, but larger doses besides cause cardiac paralysis. It is excreted in part by the lungs, salivary glands, and skin, mainly by the kidneys, and chiefly in the form of alkaline carbolates, detectable, two or three hours after administration, by bromine water. The urine has a dusky green or olive-brown hue, and for a considerable time resists putrefaction ; but, if it stands long, it becomes amber-brown, depending upon hydroquinone and other phenol products undergoing further oxidation. Excretion is tolerably rapid; carbolic acid can seldom be found in any notable amount in the urine either of men or animals twenty-four hours after the exhibition of the last dose.

Toxic EFFECTS.-Two drachms prove immediately fatal to dogs, and kill full-grown cats in two minutes (Sansom). Cullen found that one drachm given to small dogs caused excitement, dilated pupils, shallow, stertorous breathing, convulsions, and death in ten minutes. Friedberger found that fifteen grains killed dogs in a few hours. Three or four drops placed under the wings of sparrows caused excitement, restlessness, and death in half an hour; toads, earthworms, beetles, ants, and fleas were promptly poisoned (Lamaire). Two drachms repeatedly given to a donkey had no very notable effect. Half-ounce doses are dangerous for horses ; ounce doses are fatal (Kaufmann). Poisonous doses immediately cause dogs, rabbits, and other animals to reel, move in jumps, and fall ; they tremble and show muscular weakness, cough, and froth at mouth; the pulse is small, quick, irregular, and intermittent; temperature is lowered; albuminuria and hæmaturia are occasionally present; shallow, gasping, difficult breathing, collapse, paralysis, more or less anæsthesia, and occasionally convulsions, precede death.

By whatever channel it is introduced into the body, its characteristic effects are produced. Dressings used in human surgery sometimes cause nausea, vomiting, giddiness, high-coloured urine, and occasionally collapse, and even death. Scabby sheep too freely dressed may suffer from congested and inflamed lungs, linger for weeks, and even then die. Sheep, dogs, and cats are particularly susceptible ; even a single dressing, incautiously applied 
over a large surface, or the free use of carbolic lotions for wounds or during operations, may produce dulness, trembling, and disinclination for food, continuing for several days. Stronger dressings within a few minutes cause excitement, blowing, unsteady gait, and occasionally fatal collapse. A considerable skin surface, freely wetted, is said to have produced 'gradual failure of the heart's action'; whilst in other cases the dog has fallen into a state of marasmus, with sunken eyes, fœetor of the breath, formation of sordes on the teeth, 'tarry' fæces, and total loss of appetite, followed by death in six to twelve days.

The Post-Mortem Appearances are-brown discoloration and corrugation of the membrane of the mouth and fauces, and sometimes of the stomach; strong solutions leave patches of redness and inflammation in the stomach and small intestine. The kidneys are sometimes congested, occasionally inflamed. The blood is dark-coloured and feebly. coagulated, but the corpuscles are unchanged. Chronic poisoning sometimes produces granular and fatty degeneration of the liver, heart, and kidneys (Mosselman). When death occurs within a day after the poison has been taken, a smoky phenol odour pervades the body, and the poison may be discovered in most of the internal organs. It may also be detected in the urine by means of bromine water, but where life has been prolonged beyond twentyfour hours, the volatile drug may not be discoverable. Cullen records that the vessels of the brain are full of fluid blood; while serous effusion is generally observable on the surface of the brain and within the ventricles. The lungs, in cases that have survived several days, are sometimes ecchymosed.

ANTIDotes.-Where the poison has been swallowed, any unabsorbed portion should be removed by the stomachpump, or by an emetic. Pharyngeal and gastric irritation is allayed by albumen, milk, or alcohol, and by demulcent drinks, saccharated lime, olive or castor oil, camphor, or vinegar. The patient should be kept warm. Neutralisation of the poison, by conversion into the sulpho-carbolates, and excretion by the kidneys, are hastened by administration of sulphates, either by the mouth or intravenously, 
conveniently given in the form of solution of sodium sulphate. The escharotic action of pure carbolic acid is quickly neutralised by alcohol or oil.

For SuRgical PuRposes carbolic acid is the antiseptic most frequently and generally used in this country. According to Lister, it has a powerful affinity for the epidermis. Carbolic acid penetrates deeply into its substance, and mingles with fatty materials in any proportion. Corrosive sublimate solution, on the other hand, cannot penetrate in the slightest degree into anything greasy. Preparatory to an operation the parts, and the hands of the operator and his assistants, are washed with a 5 per cent. warm, aqueous solution, and the instruments, sutures, and other appliances are placed in a solution of the same strength. During operation a one to forty solution is used for washing sponges, and rinsing instruments, and for irrigating the wound. With similar antiseptic precautions, human surgeons have reduced the mortality of capital operations by more than one-half, and equally favourable results have been obtained by veterinarians.

To prevent and arrest attacks of micro-organisms, carbolic acid is applied, in all classes of patients, to most accidental or surgical wounds. Incised or lacerated wounds are washed or swabbed according to their condition, with a one to forty or one to twenty solution, before and after being secured with stitches or sutures. Broken knees and open joints are cleansed, and at intervals irrigated with carbolic lotions. When wounds for several days have been treated with the stronger carbolic solutions, an aseptic condition may be maintained, and healing usually hastened by milder dressings of boric, salicylic, or sulphurous acid. Added to Carron oil, it allays pain, and prevents or limits suppuration of burns and scalds. Over-reaches and other bruises, after being thoroughly cleansed, are dressed with a watery solution, and covered with a few folds of carbolised lint or wool, secured by a bandage. Similar treatment is serviceable in necrosis of the coronary band, occurring in hardworked horses in wet, cold weather. A saturated watery solution is used in foot-rot in sheep. Ulcers are stimulated, and their healing promoted, by thorough soaking with 
strong carbolic lotion, and afterwards coating the sores with collodion. Sinuses of the poll, withers, or coronet, cleansed and disinfected with a five per cent. carbolic solution, and provided with a dependent opening, frequently heal if protected by carbolic gauze from fresh infection.

Injuries of the uterus or vagina, resulting from parturition, and metritis in all animals, are treated with carbolic solutions, 1 to 3 per cent., with the effect of abating irritation, discharge, and straining. No treatment is so effectual in metritis in ewes, the prevalence of which might be materially lessened if shepherds would observe greater cleanliness, and wash their hands with an antiseptic fluid before rendering assistance to lambing ewes. Such precautions are specially needful where post-mortem examinations have been engaged in, where dead lambs, which have lain about for some days, have been skinned, or where cases of metritis have been handled. In foals and calves at birth, after ligaturing the cord and washing the parts with corrosive sublimate solution, a five per cent. solution of phenol painted over the navel, and repeated daily for a week, effectually prevents septic infection, necrosis, and the extension of infection to joints and other parts.

Carbolic acid is seldom used as a caustic. Concentrated solutions are applied to boils to cause their abortion, and are sometimes injected into tuberculous, cancerous, and melanotic tumours to arrest extension. Injected into the warbles on cattle or horses, it kills the parasitic larvæ. Painted on the skin, strong solutions cause superficial local anæsthesia, sufficient for the opening of abscesses, and solutions of 8 or 10 per cent. are occasionally applied as topical stimulants and rubefacients for sore-throat and rheumatic joints.

Medicinal UsES.-Carbolic acid has been prescribed in most diseases produced by micro-organisms. Its use for this purpose, however, is very limited, for whether absorbed from the alimentary tract, or injected hypodermically, it produces toxic symptoms in doses much too small to render the blood an antiseptic medium. The same is true of every antiseptic without exception, and it is hopeless to look for a drug which can be given in such quantity as to be anti- 
septic in the blood, without poisoning the cells of the animal. There are specific diseases in which certain drugs have a special affinity for, and a deterrent or destructive effect on the causal organism, for example, quinine on the malarial parasite, and iodine on actinomyces, but there is no one drug useful as a blood and tissue antiseptic, and the employment of carbolic acid internally has been attended with disappointing results. It has been administered in foot-and-mouth disease, in which the glycerin of phenol and other solutions are also applied locally with zinc and lead lotions. For tetanus, Bacelli recommends subcutaneous injection of a 3 per cent. solution in distilled water, and this treatment in the United States has proved satisfactory. In France carbolic acid is frequently given as an antithermic and internal antiseptic; and for contagious equine pneumonia a favourite remedy consists of sixty grains each of phenol and camphor with a sufficiency of honey or treacle and powdered liquorice to form an electuary. The severity of catarrhal influenza of horses is materially abated, while its spread is checked by administration of antiseptics and by spraying the nostrils and throat with a one per cent. phenol solution. In such cases, and also in chronic bronchitis and pharyngitis, air or steam, medicated with carbolic acid, is used as an inhalation. In tedious cases of strangles and in purpura it is prescribed with iodine or iron, or both, and is also applied externally.

Stomatitis and ulcerations of the mouth and throat are treated with a two to four per cent. solution of the acid conjoined, sometimes with iodine, or with tannin and glycerin. Actinomycosis, after the diseased surface has been scraped, is dressed with four parts of carbolic acid and one of iodine, dissolved in six or eight parts of glycerin (Walley). Added to the ordinary prescriptions used in dyspepsia, diarrhœa, and dysentery, carbolic acid or creolin checks fermentative changes and lessens acridity and fotor of the excreta. With oil of turpentine and opium tincture, it is used for intratracheal injections in calves suffering from parasitic bronchitis. Foals and other animals infested with Scl. tetracanthum and other strongyles, have been successfully treated with carbolic acid conjoined with turpentine 
and dialysed iron. Carbolic solutions relieve the itching and swelling occasioned by bees, wasps, mosquitoes, and ants, and, promptly used, prevent mischief from dissection wounds.

Carbolic acid checks the parasitic growth of ringworm, but is not so effectual as iodine or ferric-chloride dressings. Alternated with other remedies, it is often serviceable in that form of eczema popularly known as grease. In these and other cases where there are foul discharges, it proves a useful addition to lead, zinc, or other appropriate dressings. In patchy eczema of dogs, attended with discharge, the acid is sometimes applied mixed with starch powder or fuller'searth. For most eczema cases the wood-tar oils are, however, more suitable than those derived from coal-tar. Diluted solutions are used for destroying ticks, keds, lice, and the acari of scab and mange. Follicular mange in dogs may be successfully treated by shaving the hair, scrubbing thoroughly with soap and water, and painting the skin with a solution of one part of acid to twenty of water or glycerin, but to avoid dangerous absorption, too large a surface must not be dressed at a time.

Crude carbolic acid is used for the disinfection of stables, kennels, cow-houses, piggeries, and poultry pens ; of railway horse-boxes, cattle-trucks, and loading-places, and of cattle vessels and landing-stages. Carbolic disinfecting powders are conveniently sprinkled daily throughout the stables of many omnibus, cab, and carrying establishments of London, Liverpool, and other large towns. Thus employed, carbolic acid is not injurious or distasteful either to the animals or their attendants. It drives away flies and vermin ; arresting decomposition, it prevents unpleasant smells; fixing ammonia, it increases the value of manure with which it has been mixed. To ensure purification of infected premises, the antiseptic must be freely and frequently used in powder, fluid, spray, or vapour, or in several of these forms. The vapour is readily evolved by sprinkling the acid on live coals or on a hot metal plate. During the prevalence of infectious disease, the walls and woodwork of stables, cowsheds, etc., may be smeared with the crude acid. To destroy germs or limit their distribution, animals infected with contagious 
disease should be sponged daily with a two per cent. carbolic solution, and all discharges should be treated with a five per cent. or stronger solution. Infection may thus be prevented spreading to healthy subjects, which, by daily administration of antiseptics, may, moreover, be rendered less liable to suffer from disease.

Doses, etc.-Horses and cattle take $m_{x x x}$. to $\mathrm{m}$ clx.; sheep and large pigs, $m_{x}$. to $m_{x x}$; dogs, $m_{i}$. to $m_{\text {iii. }}$ Carbolic acid should not be given to cats, and as it communicates a disagreeable odour to the flesh, it should not be administered to cattle or sheep intended for immediate slaughter for human food. The B.P. liquefied acid is best for internal use. It may be made into bolus with meal, but is more readily absorbed, more regular in its effects, and less likely to develop local irritation, when given well diluted, either in water or in glycerin and water. One part by weight of acid rubbed in a mortar with ten of glycerin forms a convenient compound, readily miscible with water or other solvent. Brown discoloration of the urine need not prevent the continued use of the drug. Such discoloration results more frequently from external applications which favour rapid oxidation. An ointment is made by rubbing in a mortar about one part of acid with twenty of vaseline or lard. The liniment usually contains one part of acid shaken up with twenty to forty of olive or rapeseed oil. For dusting irritable surfaces it is mixed with starch, lycopodium, and occasionally with charcoal and plaster of Paris. Watery solutions ( 3 to 5 per cent.) are, however, most convenient and penetrating, and are best fitted for antiseptic purposes. Lister's strong solution consists of 50 parts each of carbolic acid and glycerin (or alcohol), and 900 parts of water. For intratracheal injection a one per cent. solution in alcohol and water may be used in doses of $2 \frac{1}{2}$ to 5 drachms. Subcutaneously the horse may be given $\bar{\jmath}$. to $\bar{j}$ ij. of a one or two per cent. solution of phenol in 20 parts of glycerin and 80 parts of water. Camphorated carbolic acid, used as a germicide dressing for sluggish sores, consists of twelve parts phenol, four of camphor, and one of water ; liquefied by trituration. Iodised phenol is made with one ounce of liquid carbolic acid and forty grains of iodine. Carbolic oil, 
made with one of phenol, four of castor oil, and twelve of almond oil, is used for oiling catheters and instruments after they have been disinfected. Aseptic Chinese twist for sutures, is prepared for use by stretching the twist, boiling for ten minutes, rewinding on a glass slide, and preserving in 5 per cent. carbolic solution. Carbolised tow contains tar, and 5 per cent. of phenol. Absorbent carbolised wool contains 5 per cent., and carbolised lint and gauze 5 to 10 per cent., of phenol. Carbo-sapol consists of fifty parts carbolic acid, twenty-five parts yellow hard soap, and twenty-five parts soft soap. The ingredients are heated together on a water bath until a clear solution is obtained. It readily mixes with water, and a one per cent. solution does not attack the hands. Its antiseptic power is nearly equal to that of corrosive sublimate (Coblentz). Phenyform, a condensation product of phenol and formaldehyde, is a white, tasteless, and odourless powder, insoluble in water, ether, chloroform, and oils, soluble in alcohol, acetone, and alkalies. It is a good antiseptic for wounds, and a cheap substitute for iodoform.

Sulpho-carbolic or sulpho-phenic acid $\left(\mathrm{H}^{-\mathrm{C}_{6}} \mathrm{H}_{4} \mathrm{O} . \mathrm{HSO}_{3}\right)$ is prepared by the action of sulphuric acid on warm phenol. It crystallises slowly, and forms colourless, deliquescent needles, having less odour than carbolic acid ; at $400^{\circ} \mathrm{Fahr}$. it becomes red; at $540^{\circ} \mathrm{Fahr}$. it boils. It is antiseptic and disinfectant, and soluble in water, alcohol, ether, and glycerin. With the oxides or carbonates of the alkalies, earths, or metals, it forms crystalline, soluble, almost odourless, usually colourless, sulpho-carbolates, which do not give any evidence of the actions of carbolic acid. The sodium salt may be used as either a surgical or medical antiseptic. The iron, zinc, and copper salts exhibit the actions of their bases. These sulpho-carbolates are excreted by the kidneys in great part unaltered; they probably do not readily give up their carbolic acid in the body, and certainly have not fulfilled the expectations formed of them when they were introduced as antiseptics. Sulpho-carbolic acid is stated to form $33 \frac{1}{3}$ per cent. of aseptol, an antiseptic which is soluble but inactive in glycerin, alcohol, or oil. An aqueous solution of 3,5 , or 10 per cent. is used as a 
dressing for wounds. It is less caustic than phenol, and it may be administered as an intestinal antiseptic.

\section{CREOSOTE}

Creosotum. Creosote. A mixture of guaiacol, creosol, and other phenols, obtained in the distillation of wood-tar (B.P.).

Tar obtained from hard woods yields 20 to 25 per cent. of creosote. The process of extraction is tedious and complex, requiring repeated distillations and the removal of the lighter oils. It is a mixture of phenol, cresol, phlorol, guaiacol, and 20 to 50 per cent. of creosol. It yields creosotic acid, which in its properties and uses closely resembles salicylic acid.

Creosote is a mobile, oily, neutral, or only faintly acid fluid, colourless and transparent when first prepared, but, unless very pure, soon becoming brown. Specific gravity 1.079. It has a strong, persistent, smoky odour, and a pungent, acrid taste, with a sweet after-taste. It requires for solution one hundred and fifty parts of water, but readily dissolves in alcohol, ether, acetic acid, and volatile oils. Dropped on white filtering paper, and exposed to a heat of $212^{\circ} \mathrm{Fahr}$., it leaves no translucent stain (B.P.).

Impure carbolic acid and other coal-tar oils, frequently mixed with or substituted for the more expensive wood creosote, are distinguished from it by their greater solubility in water; by their solidifying in acicular crystals at low temperatures ; by their not affecting a ray of polarised light, which creosote turns to the left ; by their producing a clear jelly when shaken with collodion, which does not affect wood creosote; while their watery solution gives a blue colour, with a neutral iron perchloride solution, which gradually browns the watery solution of wood creosote.

ACTIONS AND USES.-Creosote belongs to the aromatic series of carbon compounds. Containing so many phenols and guaiacol, it has a complex action, but it resembles carbolic acid, and is a more active germicide. It is occasionally administered to arrest gastro-intestinal fermentation, 
and diminish fotor of the discharges. It may be used as an antiseptic, hemostatic, and analgesic, and for the destruction of skin parasites and bacteria. In large doses it is an irritant poison and cardiac paralysant, just like carbolic acid.

1. General Actions.-When undiluted, it coagulates albumin and destroys the epithelium of mucous membranes, and even of the skin, producing corrugation and a white stain or scar. Diluted it is astringent and antiseptic, irritates slightly, and then causes analgesia and feeble anæsthesia. Although in saturated solution it has little effect on enzymes, one part in five hundred of water arrests the action of yeast, while one in one thousand kills bacteria (Bucholtz). It is absorbed, and communicates its odour to the various tissues. Large doses, given internally, cause nausea ; in carnivora, vomiting, colicky pains, and diarrhœea, with muscular paralysis, especially involving the heart, and depressing the central nervous system. It is excreted partly by the lungs, but chiefly by the kidneys.

Toxic EFFECTs.-Three drachms given to horses caused slight and temporary feverishness, and imparted to the breath a creosote odour (Hertwig.) Thirty drops given to dogs caused uneasiness, copious salivation, vertigo, muscular twitching, enfeebled and fluttering action of the heart, laboured breathing, diminished sensibility, dulness, and stupor. The symptoms came on within a few minutes, and continued for two or three hours. For a day or two, however, irritability of the stomach, occasional vomiting, and dulness were still observable. Two dogs got two drachms each, and died within three hours, evincing, besides the symptoms above mentioned, violent convulsions and complete coma. From the prominence of the convulsions in these cases, it is probable that the creosote was largely mixed with carbolic acid. The antidotes have been enumerated (p. 412).

Medicinal Uses. - It is prescribed in some forms of indigestion, to arrest undue fermentation, and in chronic vomiting in dogs. With chalk and catechu mixture, or a little laudanum and an aromatic, it helps to check diarrhœa and dysentery in cattle. A few drops inhaled with steam 
benefit chronic bronchitis and lung complaints, when accompanied by excessive or fœtid discharges. Parasites lodged in the air-passages are destroyed by creosote, administered by the mouth, or, more effectually, by inhalation, or intratracheal injection. In gangrenous equine pneumonia creosote has been given intratracheally with some advantage in relieving distressed breathing and irritable bowels. It has been tried in purpura in horses, but without any very striking results. Nasal gleet, with enlarged glands and fœtid discharge, is sometimes, however, benefited by a daily drachm of creosote, made into bolus with linseed meal.

For external purposes, carbolic acid has superseded creosote as an antiseptic for wounds, but as a stimulant and escharotic in caries, fistula, canker, thrush, and footrot, creosote with an equal part of tincture of iodine, is still used. Diluted with alcohol and water, or with vinegar, it is occasionally used to relieve itching and remove scurf in chronic eczema, prurigo, and psoriasis. It destroys parasites infesting the skin. For mange and scab, Gerlach advised an ounce of creosote, dissolved in fifteen ounces of spirit and forty of water. For intractable follicular mange, after washing the dog with soap and water, or, better still, shaving him, Hunting advises inunction of a mixture of one part of creosote and fourteen of olive-oil, the penetrating power being increased by the addition of two parts solution of caustic potash. Human patients suffering from toothache depending on caries are often relieved by a drop of creosote cautiously placed in the hollow of the tooth.

Doses, etc.-For horses, $m_{x x x}$. to $m_{l x x x}$; for cattle, f3i. to $3 j v$. ; sheep, $m_{x x}$. to $m_{x x x}$; pigs, $m_{v}$ to $m_{x v}$. ; dogs, $m_{i}$. to $m_{v}$. It is given in bolus or pill with syrup; in emulsion, with mucilage, or oil ; in six parts of alcohol, or in aqueous solution one per cent.; or conveniently shaken up with milk. As a stimulant or escharotic, it is applied with a camel's-hair brush; is used in solution in spirit or acetic acid, or as an ointment, made with one part to eight of lard or simple ointment. For skin diseases, equal parts of creosote and sulphur may be made into an ointment with lard, or a liniment with oil. A few drops are sometimes added to turpentine, ammonin, or other 
embrocations. For intratracheal injection in parasitic bronchitis $m 5$ to $m 10$ creosote are dissolved in $m 60$ to m100 of a mixture of equal parts of alcohol and water; and in equine pneumonia, five drachms of a solution of one part creosote and forty to fifty parts each of alcohol and water, may be used.

Guaiacol (methyl-pyrocatechin), $\mathrm{CH}_{7} \mathrm{O}_{2}$, a colourless, refractive liquid, with a characteristic odour, is obtained by distillation from beech creosote, or from guaiacum resin. It is closely allied in antiseptic properties and general actions to creosote, for which it may be substituted. Commercial guaiacol is an oily liquid containing about 90 per cent. of pure guaiacol, which is crystalline. It is soluble in eighty-five parts of water, and freely in alcohol, acetone, ether, oils, and glycerin. It is more germicide than phenol, and much less toxic. Applied to the skin in fever it markedly lowers temperature, but as an antipyretic it has been little used. As a local anæsthetic its action is slow, but more durable than that of cocaine. It anæsthetises both inflamed and healthy tissues. A one per cent. solution in castor oil is used as an anæsthetic instillation for the eye ; and fifteen grains pure guaiacol dissolved in four or five drachms of sterilised neutral oil or glycerin form an anæsthetic injection which is useful in minor surgery, half a drachm to two drachms being injected into the area of operation. Guaiacol has been prescribed in phthisis and pneumonia of man, and in most cases the temperature was considerably reduced. It may be administered in the same doses as creosote. The carbonate (Duotal), a neutral crystalline powder, is sometimes prescribed in bronchitis and rheumatism, and as an intestinal antiseptic. It passes unchanged through the stomach into the bowel, where it is split up (Coblentz).

\section{CRESOL-CREOLIN}

Cresol is a coal-tar product of complex composition, containing naphthalene, xylenol, phloral, leucoline, anthracene, paracresol, pyridine, with sodium carbonate, sulphate and chloride, and traces of carbolic acid. 
Creolin, or cresols and hydrocarbons saponified with resin and alkalies, is one of the most useful of the benzol or aromatic carbon series. It is a dark-brown, oily liquid, of a tar-like odour and an aromatic taste ; neutral or slightly alkaline; soluble in ether, chloroform, and in ninety-five parts of strong alcohol; mixed with forty or more parts of water it forms an opaque milky emulsion.

ACTIONS AND UsEs.-Creolin is antiseptic, disinfectant, deodorant, parasiticide, astringent, and styptic. Its antiseptic power has been investigated by Fröhner, Albrecht, Eisenberg, and others. Bacteriological tests prove it to be more prompt and effective than carbolic acid in the destruction of the microbes of glanders, anthrax, fowlcholera, etc. A 1 per 1000 solution is stated to kill cholera bacteria in ten minutes, and arrest development of typhus bacilli in one hour; while a 1 per 1000 solution of carbolic acid requires four days to kill the microbes of cholera, and had no effect on those of typhus in twenty-six days (Fröhner). In solutions of 3 to 5 per cent. creolin destroys nearly instantaneously the bacteria of glanders, anthrax, and mammitis, as well as pyogenic organisms. The virulence of tubercle bacilli is destroyed in a few minutes by a 3 per cent. solution, while a 5 per cent. solution is fatal to anthrax spores in twenty-four hours. Kaufmann states that as a bactericide it is ten times as powerful as carbolic acid. It coagulates albumin, but, unlike carbolic acid and creosote, moderately strong solutions do not irritate the skin, or the mucous membranes of the mouth and nose. It is quickly absorbed, and large doses have been given to animals without causing intoxication or serious injury. It rapidly impregnates with its empyreumatic phenol odour the sweat, milk, and urine, by which it is excreted, partly as naphthalene, partly as sulphuric acid. It usually colours the urine brown.

Employed with ordinary care, creolin is not poisonous ; but as the proportion of phenols and cresols in different samples probably varies, caution should be exercised in its administration. Horses have received in emulsion from one and a half to three fluid ounces without ill effect, and cattle still larger doses. A medium-sized dog had a drachm 
and a half, and another thirty minims daily for four weeks without injury. Sheep and goats have taken with impunity two to six fluid drachms. Very large doses have been given by the mouth without provoking serious symptoms. The in-rubbing even of concentrated solutions is well borne. Fröhner records that at the Berlin Veterinary College upwards of 1000 mangy dogs have been treated with creolin lotions without a mishap. A thousand horses suffering from mange have been dressed with a 10 per cent. solution ; sheep suffering from scab have been dipped in a $2 \frac{1}{2}$ per cent. solution ; while 20,000 sheep are stated to have been washed. with creolin dips in Prussia in 1888. Further testimony of its innocuous and non-irritant character is adduced by Späth and two of his colleagues, who took daily from thirty minims to two fluid drachms without loss of appetite, nausea, or disturbance of circulation or secretion. The larger doses diminished the amount of intestinal gas, the fæces lost their distinctive smell, and the urine contained less indican, and, although kept for several days in a warm room, did not decompose.

Medicinal Uses.-At the several Continental veterinary schools Creolin-Pearson or Jeyes' Fluid, is much used, and is characterised as the cheapest and best antiseptic and disinfectant. It is not often prescribed internally, but, as already indicated, in gastro-intestinal derangements it is effectual in checking undue fermentation, and lessening the fœtor and acridity of the excretions. As a surgical antiseptic, it has the merit of being non-irritant and nonpoisonous. It does not dry or harden the operator's hands, or spoil the steel instruments, as carbolic acid does. With glycerin and water it is a serviceable gargle in aphthous ulcerated conditions of the mouth and throat. It is inhaled in ozæna, strangles, bronchitis, pneumonia, and purpura. In the form of injection it is useful in retention of the fœtal membranes, metritis, cystitis, and otorrhœea. It destroys skin parasites, whether animal or vegetable, in all animals, and is recommended in chronic eczema. It is used for the several purposes of a disinfectant. Creolin has been thoroughly tested by Albrecht and Fröhner, who report most favourably of its antiseptic value in the treat- 
ment of accidental and surgical wounds, skin affections, and diarrhœa.

Doses, etc.-Creolin, horses and cattle, f3ij. to f3vi.; dogs, $m_{v}$. to $m_{x x}$; calves, sheep, and pigs, $m_{x x x}$. to $m_{l x}$., in five to ten ounces of water. For external purposes, one to five parts are mixed with a hundred of water, solution being facilitated by addition of a little glycerin or soft soap. As a dry dressing it is used with boric acid, zinc oxide, or kaolin. Creolin solutions lose their efficacy when long kept, and hence should be made up fresh every week. For mange or scab Fröhner advises the dressing of the worst parts with a lotion of one part each of creolin and methylated spirit and eight parts of soap, and subsequently placing the patient for two or three minutes in a bath of two or two and a half per cent. of creolin, and, if necessary, repeating the treatmont in a week.

Cyllin is a non-toxic antiseptic, containing about sixty per cent. of oxidised hydrocarbons, free of phenol, emulsified with neutral tar oil. Solutions of one to two hundred of water are employed for operation wounds, and a solution of one to four hundred of water for mucous membranes. It is often prescribed as an intestinal antiseptic.

Lysol, stated to be the product of a solution of tar oils in a neutral soap, is a clear, brown, syrupy liquid, containing about 50 per cent. of cresols. Mixed with water it forms a clear, saponaceous liquid. Cadeac, Guinard, and others state that as an antiseptic it is more powerful than carbolic acid. A one or two per cent. aqueous solution is used as a wound dressing, as a bath for instruments and ligatures and for disinfection of the hands. A one per cent. solution has been prescribed as a vermicide, especially for strongyles, and as an udder injection in parturient toxæmia; and a three per cent. solution is serviceable as a dressing for parasitic affections of the skin. Izal is said to consist of an emulsion containing 40 per cent. of a tar oil produced by a special process in the manufacture of coke. A solution of one part in two hundred of water is used as a disinfectant and antiseptic. Solutol, an alkaline solution of sodium cresol, and Solveol, a solution of cresol in sodium cresotate, are used as disinfectants. Solutol is unsuitable 
for surgical uses because of its caustic alkalinity. Solveol is nearly odourless, and miscible with water in all proportions, forming a solution which is free from the greasiness of lysol and creolin. As a germicide, an aqueous solution of one part in two hundred, is stated to be equal to a 2 per cent. solution of carbolic acid (Coblentz).

\section{O D F O R M}

Iodoformum. Tri-iodomethane. A product of the action of iodine on ethylic alcohol in the presence of solution of potassium carbonate. $\mathrm{CHI}_{3}$ (B.P.).

It occurs in shining, lemon yellow, hexagonal crystals ; is volatile, has a persistent, disagreeable odour and taste ; is very slightly soluble in cold water, but is soluble in 10 of ether, 12 chloroform, 95 rectified spirit, 10 collodion, 60 vaseline, 14 eucalyptus oil, and in glycerin, fixed and volatile oils.

ACTIONS AND UsES.-It contains 96 per cent. of iodine with which it readily parts, exerting antiseptic and parasiticide actions. It has only a very limited power of destroying bacteria which grow and multiply on media charged with the drug. It, however, diminishes the virulence of micro-organisms by acting on their products which, according to Lister, it chemically alters or destroys. Local suppuration invariably produced by subcutaneous injection of cadaverin does not occur when iodoform is added to this toxin. Behring found that cultures of pyogenic organisms injected into the peritoneum caused death in twenty-four hours, but had no effect when the culture was mixed with a little iodoform. Although proved to be a feeble germicide, iodoform has rendered excellent service in clinical practice. It is not so irritant as iodine. It resembles chloroform and bromoform, and, like them, is a local anæsthetic. Applied to a wound it remains long unconsumed; and under the combined influence of heat, moisture, and fat it is slowly dissolved and decomposed with liberation of free iodine upon which its antiseptic power probably depends.

It has little effect on the unbroken skin, but slightly 
irritates abraded skin and mucous surfaces. It is a more effectual local anæsthetic than chloroform or bromoform, inasmuch as it is neither so irritant nor so volatile. It is slowly absorbed, forms iodides and iodates, and produces many of the effects of iodine ; but when swallowed in large doses it is more lethal, and it appears probable that some iodoform is absorbed unchanged for the toxic effects are not those of iodides. In dogs and cats it induces gastric derangement, vomiting, and muscular spasms, and then lowered temperature, impaired heart action, albuminuria, and narcosis, preceded by lessened excitability of the cord and motor areas of the brain ; but in rabbits narcosis is not produced. In man the most characteristic effects of iodoform poisoning are violent delirium and mania, much more rarely deep sleep and stupor without excitement. Fröhner records that for each kilogramme of body-weight dogs are poisoned by fifteen grains given by the mouth, twenty to thirty grains given subcutaneously, or seven grains injected into a serous cavity. An old cow which received an ounce and a half died in thirty-six hours, with spasms and narcosis. Chronic poisoning induces emaciation and fatty degeneration of muscles and glands. Iodoform is slowly excreted in the urine chiefly as sodium iodide.

It is rarely prescribed internally. Its chief use is as an antiseptic dressing for unhealthy wounds, being especially useful when dressings cannot be conveniently changed, or where they cannot be properly applied, as in wounds of the rectum, bladder, and generative organs. It is used in burns, eczema, canker in horses' feet, and fistulæ. Small doses maintain wounds in an aseptic state, promote granulation, and allay irritation. Wounds, after cleansing with carbolic or zinc chloride solution, are dusted with the powder, used alone or mixed with boric acid, and covered with iodoform or carbolic gauze, or moistened with an ethereal or oily solution. Oil of eucalyptus, and balsam of Peru or Tolu, are convenient solvents, which also cover the disagreeable smell and taste. As a parasiticide it is used for the same cases as iodine. Ethereal solutions are injected in ozæna and ulcerated sore-throat. Pencils made with 50 to 70 per cent. of iodoform, mixed with starch and gum, are used in 
wooden holders for dressing wounds; while injections and suppositories made with eucalyptus oil and cacao butter, are serviceable as deodorisers and local antiseptics in painful conditions of the rectum and urino-genital organs. Although not liable to cause injury by absorption, dogs, unless muzzled, are prone to lick the dressed surfaces, and in this way are sometimes poisoned. Iodoform emulsion-containing one part iodoform moistened with alcohol, two parts boiling water, and seven of glycerin-is a valuable injection for sinuous wounds.

Iodol (tetra-iodopyrrol) $\left(\mathrm{C}_{4} \mathrm{I}_{4} \mathrm{NH}\right)$, containing 89 per cent. of iodine, is a light-brown, tasteless, amorphous powder, with a faint thymol-like smell, insoluble in water, but soluble in three parts of alcohol, and also in ether, chloroform, glycerin, and collodion. It resembles iodoform, and being less irritant, is preferable for internal use. Horses and cattle take grs. $x v$. to grs. xxv.; dogs, gr. i. to grs. iii. It may be given suspended in glycerin or mucilage.

Aristol (di-thymol-iodide) $\mathrm{C}_{20} \mathrm{H}_{24} \mathrm{I}_{2} \mathrm{O}_{2}$, is a compound of iodine and thymol, conjoins the properties of its two constituents, and resembles iodoform, but is devoid of its disagreeable taste and toxic effects. It contains 46 per cent. of iodine. It is soluble in fixed oils, ether and collodion, but not in water, alcohol or glycerin. It is used for the same purposes as iodoform, but is a feeble antiseptic. It dries excessive skin secretions, and is useful for burns, and cracked heels.

Europhen, containing 28 per cent. of iodine, is a light yellow powder, insoluble in water and glycerin, but soluble in alcohol, ether, and oil. It is non-poisonous, has very little odour, and is used as a dry dressing in eczema and otorrhœa in dogs. Iodo-salicylic acid, containing 50 per cent. of iodine, and di-iodo-salicylic acid, containing 66 per cent. of iodine, are anodyne, antipyretic, and antiseptic, and are used internally as substitutes for salicylates in the treatment of rheumatism. Horses take $3 \mathrm{j}$. to $3 \mathrm{ij}$., dissolved in alcohol, ether, or oil.

Loretin, losophan, sanoform, sozoiodol, and periodate are odourless iodoform substitutes. All contain iodine, and are used as antiseptics in powder or solution, or mixed with 
vaseline or collodion. Iodoformin, a white, odourless powder, insoluble in water, alcohol, ether, and chloroform, containing 75 per cent. of iodoform; and iodoformal, the product of the action of ethyl iodide on iodoformin, having a strong odour of coumarin, are sometimes employed in place of iodoform.

\section{PRUSSIC OR HYDROCYANIC ACID}

Acidum Hydrocyanicum Dilutum. An aqueous solution containing 2 per cent. by weight of hydrogen cyanide, HCN. (B.P.)

Prussic acid was so called from being first obtained from Prussian blue. Its title of hydrocyanic acid is derived from its being composed of hydrogen and the compound radicle cyanogen. It is one of the products of the distillation of coal, and traces are found in imperfectly purified coal-gas. The leaves and kernels of various stone fruits of the apple and almond natural orders, when crushed and moistened, undergo a species of fermentation, their albuminoid emulsin decomposing the glucoside amygdalin, and producing hydrocyanic acid, benzaldehyde, and glucose.

$$
\begin{aligned}
& \text { Amygdalin. Water. } \begin{array}{c}
\text { Prussic } \\
\text { Acid. }
\end{array} \quad \begin{array}{c}
\text { Benzalde- } \\
\text { hyde. Glucose. }
\end{array} \\
& \mathrm{C}_{20} \mathrm{H}_{27} \mathrm{NO}_{11}+2 \mathrm{H}_{2} \mathrm{O}=\mathrm{HCN}+\mathrm{C}_{7} \mathrm{H}_{6} \mathrm{O}+2 \mathrm{C}_{6} \mathrm{H}_{12} \mathrm{O}_{6} \text {. }
\end{aligned}
$$

Medicinal hydrocyanic acid may be prepared by the interaction of diluted sulphuric acid and potassium ferrocyanide.

The anhydrous acid is obtained by cautiously distilling the medicinal acid, and collecting the vapour in a receiver kept cold by ice. It is a colourless, very volatile, inflammable liquid, and, alike in its gaseous and liquid forms, is a most active, deadly poison.

The medicinal acid, when freshly prepared, contains two per cent. of anhydrous acid, but on account of its volatility it is apt to lose strength. This may be obviated by keeping it in stoppered bottles, tied over with some impervious covering, laid down inverted and in a dark place. It is colourless, with a diffusible, peculiar odour. Specific gravity, 997 . It only slightly reddens litmus. Evapor- 
ated on a platinum capsule it leaves no residue. Scheele's acid, which is frequently used in veterinary practice, contains four per cent. of hydrogen cyanide.

ACTIONS AND USES.-Prussic acid first briefly stimulates and then paralyses all nerve structures with which it comes into contact. A few drops of the diluted acid applied to mucous or skin surfaces diminish or arrest sensation. It is hence used as an analgesic for relief of irritation and pain, especially of the skin, stomach, and throat. It is quickly absorbed. Full doses paralyse the cerebro-spinal axis, kill almost instantaneously by cardiac arrest, or somewhat less suddenly by respiratory arrest.

General ACTIONS.-It stops protoplasmic movements both in plants and animals, kills infusoria, checks oxidation, and arrests fermentation (Brunton). The anhydrous acid applied to the skin, after momentary irritation, paralyses and anæsthetises. The diluted acid applied to the skin or mucous surfaces quickly penetrates and paralyses the nerveendings, impairing and destroying tactile sensation and sensibility to pain. If the surfaces are freely moistened, these effects usually continue for one or two hours. Its volatility and rapidity of diffusion ensure quick absorption, and poisonous doses promptly paralyse the central nervous system. In moderate doses after absorption the primary stimulant action is seen. Respiration becomes quicker and deeper; the heart beats slower from vagal stimulation, but blood-pressure rises from the general vaso-constriction. In a short time this stimulation passes into depression, respiration becomes slow and weak, blood-pressure falls, and the heart becomes progressively weaker. The further effects depend on the dose. If small, the volatile drug becomes diffused and excreted and recovery takes place. When a large dose has been given, the respiratory, vasomotor, and spasm centres of the medulla, and the peripheral afferent nerves, are early and notably affected; and in all mammalia death usually occurs within a few minutes. When poisoning is not immediate, the central nervous system is affected ; there is giddiness, staggering movements, and a few slow inspirations, followed by rapid expirations and irregular heart action ; convulsions, coma, anæsthesia, 
and paralysis of voluntary muscles supervene, and death from paralysis of the cardiac or respiratory centre. Unlike other acids, prussic acid does not coagulate albumin or break down the formed elements of the blood. The potassium and other cyanides are soluble, readily yield their cyanogen, and are active poisons, though less so than the free acid; but the ferro-cyanides and other double cyanides are greatly less active and non-toxic.

Toxic Actions.-Horses have been poisoned in one or two minutes by ten to twenty minims of anhydrous acid, injected hypodermically. Trousseaux and Pidoux placed a piece of cotton wool, on which six minims of anhydrous acid had been dropped, in the nostrils of two horses. In ten seconds they dropped as if dead, and continued for an hour to exhibit grave nervous symptoms-convulsions, spasms, vertigo, paralysis, and stupor. Horses swallowing four to five drachms of the two per cent. medicinal acid may die within an hour. Coleman gave an aged horse repeatedly, at intervals of several days, one to three drachms of acid, containing about four per cent. of anhydrous acid, and noted much excitement, the pulse raised to 100 , and in one experiment to 160 , laboured breathing and tetanic contraction of the muscles; but the effects gradually passed away. Six ounces of medicinal acid given to an old elephant caused only slightly laboured breathing. The horse can be quickly killed by intrathoracic injection of one or two drachms of Scheele's acid.

Dogs, eats, and rabbits, which had one to four drops of anhydrous acid placed on the tongue or within the eyelids, in ten to thirty seconds made three or four hurried inspirations, a convulsive expiration, often a cry, had tetanic convulsions, and died in one to three minutes. Air saturated with the gas killed one dog in ten seconds, another in five, and a cat in two seconds. Guinea-pigs inhaling it for one second die in fifteen seconds. Strong rabbits inhaling it for three seconds die in thirty seconds; but birds are not susceptible, and frogs are still less so. Dogs and cats receiving forty to sixty minims of the two per cent. acid were sometimes poisoned almost as rapidly as with the anhydrous. More frequently, however, life is prolonged for several 
minutes, and death is preceded by giddiness, impaired voluntary movement, dilatation of the pupil, a slight rise and subsequent fall of blood-pressure, slowing of the pulse, rapid failure of respiration, and tetanic convulsions. The heart continues to beat for several minutes after respiration has ceased. Experimentally, two ounces were found to cause rapid death of Greenland whales, when discharged by an ingenious device into the wound made by the harpoon.

Post-mortem discloses variable appearances. Animals dying almost instantaneously from cardiac arrest have the blood of an arterial hue, as if, from dilatation of the remote capillaries, it had passed through them without change. When the respiratory centre of the medulla has been paralysed, causing death somewhat more slowly by respiratory arrest, the appearances are those of suffocation. For some hours after death the blood remains fluid, of a blue colour, and occasionally evolves the peculiar odour of the acid.

ANTIDOTES.-Prussic acid is usually so rapidly fatal that the animal is often dead before any remedial measures can be adopted ; but so volatile is the poison, and so rapidly is it removed from the body, chiefly by the lungs, that if the animal lives for half an hour it will generally recover. Artificial respiration, and subcutaneous injection of ether, sometimes save animals that have had lethal doses. Cautious hypodermic injection of small doses of atropine sulphate stimulates the cardiac and respiratory centres, and may thus avert mortal paralysis. Inhalation of ammonia and douching alternately with cold and warm water applied to the head and neck, have also been advised. The chemical antidote is a mixture of a ferrous and ferric salt, administered with magnesia or potassium carbonate, and forming the insoluble Prussian blue. But to be effectual the antidote must be swallowed before the rapidly-acting poison is absorbed.

Medicinal Uses.-By paralysing the ends of the sensory nerves, the acid allays the irritation of urticaria, prurigo, and other itching skin complaints. In like manner it sometimes relieves gastrodynia and chronic vomiting, being conjoined in such cases with ice, bismuth, and morphine; while in irritable conditions of the throat it is prescribed with 
cocaine, chlorodyne, morphine, or atropine. In the symptomatic treatment of tetanus and nervous diseases it is given to allay excitement. For destroying the strongyles of hoose in calves, and soothing consequent irritation, Williams prescribed $m_{x}$. to $m_{x x}$. of acid, conjoined with sodium carbonate and gentian. Hobday suggested the use of hydrocyanic acid as an antidote in cases of respiratory failure under chloroform. A full dose frequently starts respiration with a few deep gasps.

Doses, ete.-Of the B.P. two per cent. acid, horses and cattle take $m_{x x}$. to $m_{l x}$. ; sheep, $m_{x}$. to $m_{x v}$. ; pigs, $m_{i j}$. to $m_{\text {vij. ; dogs, }} m_{\mathrm{ij} .}$. to $m_{\mathrm{iv}}$; ; given in water sweetened with syrup. As the soothing effects are transient, suitable doses may be repeated three or four times daily, but their effects should be carefully watched; while, to prevent mistakes which are apt to occur with such a poisonous, colourless liquid, it is often made up with compound tincture of cardamoms. For external use it is diluted with two hundred parts of water, a few drops of glycerin being added to retard evaporation. Lotions must be cautiously applied, especially where the skin is broken. But the potassium or other soluble cyanide being stable and not volatile, is more convenient for most external uses.

\section{PETROLEUMS OR PARAFFINS}

Hard paraffin. Paraffinum durum. A mixture of several of the harder members of the paraffin series of hydrocarbons ; obtained by distillation from shale (B.P.).

Liquid paraffin. Paraffinum liquidum. A clear, oily liquid, obtained from petroleum, after the more volatile portions have been removed by distillation (B.P.).

Soft paraffin. Paraffinum molle. A semi-solid mixture containing soft members of the paraffin series of hydrocarbons; obtained by purifying the volatile portions of petroleum (B.P.).

The petroleums or paraffins are hydrocarbons, produced by the decomposition of vegetable matter. They are obtained from the destructive distillation of coal, from bituminous shales, and from the oil-wells found in various parts of 
the world. They occur as gases, fluids, and solids, and many are used in the arts and in medicine. The simplest of the series is marsh gas, methane, fire-damp, or light carburetted hydrogen $\left(\mathrm{CH}_{4}\right)$ - the inflammable gas which causes coalpit explosions.

When rock-oil is distilled, ethane and other gaseous paraffins are first evolved, are collected, and in great part liquefied by a condensing pump, and yield the liquid cymogene, which, on account of the cold produced by its rapid evaporation, is used in freezing machines. Proceeding with the fractional distillation, the products which come off below $170^{\circ} \mathrm{Fahr}$., and consist chiefly of pentane and hexane, are sold as petroleum spirit, or petroleum ether, and used for making varnishes, for dissolving indiarubber, and for singeing lamps. The next portion of the distillate, coming off about $212^{\circ} \mathrm{Fahr}$., is heptane, and is used for illuminating purposes, under the names benzoline, paraffin oil, and mineral sperm oil. For safe use this oil, when placed in an open saucer at $100^{\circ} \mathrm{Fahr}$., should not kindle when a light is brought near its surface. At higher temperatures there come off hexadecane and other paraffins, richer in carbon, constituting such soft solids as vaseline and the soft petroleums, while still higher temperatures produce the hard paraffins, cereses, or paraffin waxes. These soft and hard paraffins are also obtained by distillation from shale, the liquid portions being separated by refrigeration, and the solid products purified by melting and filtration. They are frequently substituted for oils, lard, and wax in the making of ointments and protectives. Liquid paraffin is useful as a base for hypodermic and intratracheal injections of iodine. Mixed with sulphur and olive oil it is employed for psoriasis and eczema, and with oxide of zinc or iodoform as a protective for wounds.

Rangoon petroleum, obtained from wells on the Caspian shores, and the analogous Barbados or mineral tar, found in the island of Barbados floating on the surface of springs or pools, and in Trinidad forming extensive beds or lakes, are of the consistence of treacle, of a dull green-brown colour, with a petroleum odour and a bitter taste.

ACTIONS AND UsES.-The petroleums belong physiologi- 
eally to the fatty or alcohol series of hydrocarbons. Methane, ethane, and the gases low in the series, and the more volatile liquids are more easily absorbed and excreted than the heavier liquids and solids, and are stimulants, anæsthetics, and inebriant narcotics.

Petroleum benzin, or petroleum spirit, is a topical irritant, germicide and vermicide, a stimulant and antispasmodic. On the skin its irritant action is very similar to that of oil of turpentine. It must be distinguished from benzine $\left(\mathrm{C}_{6} \mathrm{H}_{6}\right)$ obtained from the distillation of coal-tar, which has a higher specific gravity and a higher boiling-point. Petroleum benzin is seldom prescribed internally because of its irritant effects on the digestive tract. The dose for horses and cattle is $f \zeta$ ss. to $f \zeta j$.; for dogs, $f \zeta \frac{1}{2}$ to $f \zeta j$., given in milk, olive oil, or gruel.

Animals are sometimes poisoned by the refuse oils from petroleum works contaminating the drinking water. Cases have been recorded of cattle suffering from diarrhœa, wasting, and anæmia, and their intestinal glands being found saturated and darkened with the oil.

Petroleum spirit and other paraffin oils, in virtue of their diffusive, solvent, stimulant, and antiseptic actions, are applied in limited scaly skin complaints, as in oldstanding cases of grease in horses, to destroy vermin, remove scurf and dissolve accumulated sebaceous matters, to stimulate the dermis, and promote growth of hair. For such purposes they are frequently used in conjunction with alkalies, three parts of petroleum and alkali to one hundred of bland oil, or vaseline. The application of petroleum benzin to the skin demands care, as it may cause poisoning.

Vaseline, the petrolatum of the U.S.P., is prepared by heating rock-oil in iron retorts, and filtering the residual heavier oils through animal charcoal. It is red, yellow, or white, according to the proportion of colouring matter retained. It has the consistence of summer butter, is tasteless, odourless, and neutral. It melts about $95^{\circ} \mathrm{Fahr}$., and boils about $300^{\circ} \mathrm{Fahr}$. It is insoluble in water, glycerin, cold alcohol, and ether, but is soluble in chloroform, carbon disulphide, and in fixed and volatile oils. A handy solvent is made with one part of castor oil and eight of water. Vaseline 
dissolves bromine, iodine, sulphur iodide, and carbolic acid, as well as fixed and volatile oils and alkaloids, and is a serviceable base for ointments, liniments, and pomades. Vaseline is not absorbed by the skin, and it is preferable to lanoline or lard in ointments which are intended to act superficially. It has the advantage of being nearly free from greasiness ; it is not oxidisable, and hence does not become rancid. Ointments prepared with it accordingly keep better than those made with animal or vegetable fats. Neutral or white vaseline is used as a lubricant and emollient for irritable, inflamed, or blistered mucous and skin surfaces. It is used for making up boluses, and as a protective for leather and cutlery.

Bangoon and Barbados tars were at one time prescribed in chest diseases and as anti-emetics, but are not now used internally. Externally, they are applied for the same purposes as wood-tar, and particularly in the treatment of skin complaints, thrush, canker, and other diseases of the feet. Coal-tar differs from Barbados tar in having a stronger and more offensive sulphurous smell, but it deserves its popular credit as an antiseptic and stimulant adhesive for diseases of the feet. The alcoholic solution of coal-tar, sold as liquor carbonis detergens, is useful in eczema, one or two drachms in a pint of warm water being applied either alone or with liquor plumbi diacetatis. The B.P. liquor picis earbonis, prepared with two ounces of soapbark, twenty ounces of rectified spirit, and four ounces of coal-tar, is also used for diseases of the skin. 


\section{SECTION I I}

Medicines derived from the Vegetable Kingdom

\section{ALOES}

AloE.-Inspissated juice from the transversely-cut bases of the leaves of various species of Aloe, evaporated to dryness. Nat. Ord.-Liliaceæ.

Alors, $\mathrm{C}_{17} \mathrm{H}_{18} \mathrm{O}_{7}$. - A crystalline substance extracted from aloes by solvents and purified by recrystallisation. (B.P.)

The several species of Aloe, which yield the various commercial aloes, are succulent, liliaceous perennials, having short woody stems; strong, thick, fleshy, amplexicaul light-green leaves, with sharp, serrated edges, and a stout spine projecting at the apex; while on a slightly branched scape is carried a raceme of yellow, scarlet, or white tubular pendulous flowers. Underneath the leathery cuticle, and exterior to the loose, mucilaginous pulp, lie elongated thin walled cells, which contain the yellow, bitter, purgative juice.

Barbados Aloes.-Aloe Barbadensis, or Curaçoa aloes, the product of the aloe vera, aloe chinensis, and probably other species, is the variety chiefly used in veterinary practice. It is imported from Barbados and most of the West Indian islands. A dwarf variety is cultivated; the leaves, measuring a foot to two feet in length, are chopped off close to the stem; are placed for twelve to twenty hours in tubs with their cut ends down; from the longitudinal vessels the juice trickles, is collected in casks, and heated for four or five hours, sediment and impurities being carefully kept back. When sufficiently concentrated, the juice is poured into gourd shells, and the opening closed by a portion of shell let in, and secured in its place by a piece 
of coarse cloth nailed over it. The gourds, when filled, usually weigh from 10 to $40 \mathrm{lbs}$. Barbados aloes has a liver brown colour; a brown, opaque, earthy fracture; a disagreeable, bitter, persistent taste, and a strong and disagreeable odour, especially when breathed upon-an odour generally likened to that of the human axilla. It is hard, tough, and difficult to pulverise ; small fragments are translucent, and of an orange-brown hue; its powder is dull olive-yellow, and darker than that of other varieties. It is almost entirely soluble in alcohol (40 per cent.).

Socotrine (also known as East Indian, Bombay, or Zanzibar aloes) is chiefly imported from Bombay and other Indian ports. It is stated to be the product of the leaves of Aloe Perryi, and probably also of other species. It occurs in red-brown pieces of variable size; darkens on exposure; breaks usually with a smooth resinous fracture ; thin fragments are translucent and orange-red or orangebrown; the odour, though strong, is somewhat agreeable ; the taste is bitter.

CAPE Aloes (brought from Cape Town and Natal) is chiefly got from the Aloe ferox, Africana, or Spicata, or from hybrids obtained by crossing these with other varieties. The better qualities have a dark-brown or olive-green resinous appearance, a compact structure, a vitreous, conchoidal, dark-green fracture, and a strong and rather disagreeable sour odour. It is very brittle, and easily reduced to a gamboge-yellow powder. The better qualities of Cape are little, if at all, inferior to Barbados or to Socotrine aloes. Gamgee's experiments show that, compared with Barbados, Cape aloes causes equally copious but less watery discharges, while its action is sooner expended.

Properties. - The several varieties have a specific gravity of $1 \cdot 364$, are resinoid, rather brittle, their external surface is duller and darker than a freshly-made fracture. The temperature at which the juice is concentrated accounts for such marked differences in opacity, as the dull opaque Barbados and the translucent East Indian. All have an intensely bitter and persistent taste, and a strong and more or less disagreeable odour, much increased when the specimen is breathed upon or heated. When held in the hand 
for a few minutes, aloes softens and becomes adhesive. At a low red-heat it is partially fused, froths up, chars, and burns. Temperatures exceeding $150^{\circ} \mathrm{Fahr}$. alter its composition and impair its purgative property. Moistened with rectified spirit, a thin stratum, examined under the microscope, exhibits numerous crystals. It is almost entirely soluble in boiling water, which deposits, however, as it cools, 60 to 80 per cent. of a brown resin. Good specimens are almost entirely soluble in alcohol (40 to 60 per cent.). The watery solution, when cold, reddens litmus, is deepened in colour by alkalies, blackened by ferric chloride, and yields a yellow-grey precipitate with lead acetate.

Composition.-Aloes contains from 25 to 30 per cent. of an active yellow, crystalline, neutral bitter principlealoin, which is noticed more in detail at the end of this article; and about the same proportion of an equally soluble, uncrystallisable aloin, into which the crystallisable form is convertible by heat; and a pale-yellow, mobile, mint-flavoured volatile oil, of which only an ounce is obtained from $400 \mathrm{lbs}$. of aloes. Besides mineral matters and albumin, aloes contains about 30 per cent. of a transparent brown resin, almost entirely soluble in rectified spirit, occurring in large amount in inferior samples, in which it is formed at the expense of the aloin, usually by exposure of the juice to high temperatures during inspissation.

ACTIONS AND USES.-Aloes is purgative, and belongs to the anthracene group; a bitter tonic in small doses, insufficient to increase the action of the bowels.

General Actions.-Given by the mouth, it is dissolved in the gastric fluid, and emulsified and saponified by the bile and pancreatic juice. The presence of bile greatly assists its action, and aloes given in enema is inactive unless bile is injected with it, or the aloes is dissolved in glycerin, which takes the place of the bile. Joseph Gamgee made seven drachms of Cape aloes into a ball with sixty minims of glycerin, rolled it in tissue paper and gave it to a horse, which, in thirty-three minutes, was killed by dividing the carotid artery. An hour later the ball was found entirely dissolved; the distinct odour of aloes in the stomach and 
duodenum had not, however, extended to the large intestine. Aloes enters the circulation, communicates its bitterness and purgative properties to the milk and other secretions, and is excreted chiefly by the intestinal glandular apparatus, and also in less amount by the kidneys, when it causes diuresis. It is prone to cause hyperæmia of the kidneys, uterus, and pelvic organs. While in contact with the intestines it produces both peristalsis and increased secretion. It acts chiefly on the large intestine, which in part explains its rather slow effects. It causes copious evacuations, but not such fluid discharges as usually follow full doses of salines, gamboge, or croton. It is not so irritant as croton, colocynth, elaterium, or podophyllum. It increases the secretion of bile. W. Rutherford introduced aloes into the duodenum of a fasting dog, and found that, although only slight purgation ensued, all the bile constituents were increased. It is said to produce evacuations which possess a peculiar disagreeable odour (Hertwig).

Six drachms of Barbados aloes, dissolved in twenty-four ounces of water, and injected into the jugular vein of a horse, caused nausea, frequent straining, and colic-which, however, was only of short duration-and, after twelve hours, purgation. Administered intravenously, it sometimes acts more powerfully on the kidneys than the bowels. Moiroud injected four drachms, dissolved in diluted alcohol, into a vein of a horse, and next day eight drachms, dissolved in a similar manner ; but instead of catharsis, observed only diuresis.

The several varieties differ somewhat in the degree of their action. Barbados aloes, although not the most expensive, is generally preferred by veterinarians. It is the most active and uniform in its effects, but not more drastic than Socotrine, while it is certainly less liable than Cape aloes to produce diuresis. Every sort is most effective when freshly powdered, and hence, except for immediate use, should be kept in pieces, preserved from moisture in oiled silk or in tin. A temperature approaching $150^{\circ}$ Fahr. applied, whether in the extraction of the juice, or in making it up for use, impairs activity by converting the active aloin into inert resin. 
In the horse, a cathartic dose generally causes in a few hours dryness and increased warmth of the mouth; a rise of one or even two degrees in temperature occasionally occurs; the pulse is somewhat quickened; nausea, colic, and copious secretion of urine may result. Such diuresis occurs sometimes with good Barbados aloes, especially when the bowels are constipated, and more commonly with inferior specimens of Cape and other kinds, in which the aloin has been converted into resin. Combination with jalap, calomel, and other purgatives, usually counteracts this diuretic tendency. Combination with ginger or other aromatic, or with hyoscyamus or belladonna, wards off nausea and straining. The purgative effect is usually accelerated and increased by giving the drug in solution, or conjoined with oil or calomel, and combination with an alkali or a small dose of an iron salt increases its action.

The time required for the operation of aloes differs considerably in different horses, being modified by various circumstances, especially by the previous feeding. Four to six drachms generally operate in sixteen to twenty-four hours. The degree and continuance of the action are also liable to variation; in some horses purging is over in two or three hours ; in others it extends over twenty-four hours. When aloes fails to move the bowels it is seldom wise to prescribe another dose until forty-eight hours have elapsed. A second dose too closely following the first is liable to cause nausea or superpurgation. If more physic should be deemed absolutely necessary, oil and a little calomel may be given, and enemata should be diligently used. In order to prevent superpurgation, which even ordinary doses of aloes and other cathartics occasionally produce in horses, it is important, until purging has ceased, that the quantity of cold water allowed be carefully regulated, and that the animal, although at walking exercise, be not put to work. Neglect of such precautions may lead not only to superpurgation but to enteritis and congestion of the feet.

Ruminants are neither promptly nor powerfully purged by aloes alone. When given to cattle, even in the fluid state, and in doses of several ounces, it fails to produce copious evacuations. Hertwig mentions that, in an experi- 
ment, a cow got six ounces of aloes, partly in solution, partly in electuary; but although uneasiness and loss of appetite were observed, the bowels remained unmoved. Gilbert also gave six ounces, with an infusion of four ounces of senna leaves, without effect. Sheep and goats take doses varying from two drachms to an ounce without being speedily or effectively purged. This tardy and uncertain action has been attributed to the drug being delayed by the gastric contents and to peristalsis of the bowel being more difficult to excite in ruminants.

For the dog, aloes, when given alone, is neither so speedy nor so safe a cathartic as calomel, jalap, buckthorn, or castor oil. It has also the disadvantage of occasionally producing irritation of the rectum; but this may, in great part, be overcome by combining it with other purgatives. The dose required to purge a dog is large when compared with that administered either to man or to the horse. The doses of most medicines for men and dogs are very similar ; but man is purged by an eighth or tenth of the aloes requisite to physic the dog. Aloes is a good though slowly acting cathartic for swine. It operates usually in about twelve or fifteen hours.

Medicinal Uses.-Aloes is the purgative in general use for horses. In dyspepsia, where the appetite is capricious, the bowels irregular, the coat staring, or where there is itching with swelling of the limbs, a dose of aloes is prescribed, and generally followed by salines, acids, or bitters. In torpidity of the bowels it is conjoined with nux vomica, which stimulates peristalsis. In flatulent or spasmodic colic it is prescribed either in bolus or rubbed down with warm water, and followed with volatile oils, ethers, ammoniacal or other stimulants and antiseptics. Dick recommended four or five drachms of aloes dissolved in a quart of hot water, with an ounce each of oil of turpentine and laudanum. Some colic cases are relieved by conjoining with the aloes twenty minims of tincture of aconite. In obstinate torpidity, eserine sulphate, conveniently given hypodermically, promptly produces in most cases copious evacuations. When the bowels are overloaded with indigestible food, aloes is frequently given, but linseed oil and calomel are 
often preferable. Whatever physic is used, enemata of water should be thrown into the colon with an extra long tube, in quantities of six to eight gallons. In obstruction, obstinate torpidity, and severe colic, copious injections introduced into the large intestine are very important adjuncts. Aloes, in virtue of its bitterness and power of increasing peristalsis, is a useful anthelmintic. For entozoa it is administered with oil of turpentine, ether, santonin, sometimes with iron or copper sulphate. Although aloes is effectual in sweeping out excess of bile lodged in the intestines, it is unsuitable in jaundice or torpidity of the liver, in which the bile requisite for its solution is deficient. In such cases, salines, oils, and calomel are preferable, or the aloes may be prescribed with ox bile, which greatly assists its action.

Aloes promotes excretion of waste products, and hence usefully relieves febrile symptoms, rheumatic attacks, skin irritation, swollen limbs, and inflamed joints. It is effectual alike in preventing and removing lymphangitis; while it also hastens the removal of cdematous swellings, when not depending upon debility or serious disease of internal organs. Removing excreta, and withdrawing, by derivation or counter-irritation, blood from congested or inflamed parts, it relieves congestion and inflammation of the brain and spinal cord, full doses being usually requisite ; while it is also serviceable in the onset of paralysis, especially in subjects in gross or high condition, or suffering from gastrointestinal derangement. Repeated doses lessen the formation of superfluous blood and fat, are given both professionally and empirically to promote condition-an object usually, however, more safely and effectually secured by judicious feeding and well-regulated exercise.

To cattle and sheep, in constipation and indigestion, as well as in febrile and inflammatory complaints, aloes is occasionally given; but, as already stated, it is less reliable in ruminants than in horses. If used for cattle and sheep, it should be combined with salines, gamboge, or croton, and given in drench. For dogs it is sometimes prescribed in the same class of cases for which it is given to horses; but calomel and jalap, or some of the oils, act more promptly. 
Aloes should be avoided in irritation or inflammation of the alimentary canal, and in piles or hæmorrhage from the rectum ; in bronchitis, inflammatory affections of mucous membranes or the skin, in inflammation of the kidneys, and in influenza. In such cases if used at all, it must be with great caution; for in these diseases the intestinal mucous membrane is unusually irritable, and superpurgation is readily induced. During pregnancy, both in the mare and bitch, the violent operation of aloes must be carefully avoided. Some practitioners give it to both foals and calves, but for these young animals linseed or castor oil, or a mixture of the two, is more suitable.

As a bitter aloes is occasionally prescribed in enfeebled and relaxed conditions of the alimentary canal, and where there is suspicion of intestinal worms. It is sometimes applied externally, as a gentle stimulant and desiccant, and is an ingredient of the once famous friar's balsam. (See Benzoin.)

Doses, etc.- - Horses receive $3 \mathrm{ij}$. to 3 viij., the dose depending upon the rapidity and degree of catharsis required. For foals several months old, the dose may be readily ascertained by allowing grs. $\mathrm{v}$. for every week of the patient's age.

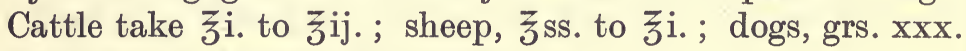
to 3 iss. ; and swine, $3 \mathrm{j}$. to $3 \mathrm{iv}$.

As a tonic, the dose of aloes for any of the domesticated animals is about an eighth or tenth of that given as a purgative. Tonic doses may be administered daily, or every second day, in combination with other bitters and aromatics. A convenient laxative for the horse is made with two drachms each of aloes, gentian, and ginger, made into a ball with treacle. Another is prepared with a drachm each of aloes and iron sulphate, and half an ounce of ginger, made up with treacle and linseed meal. Either of these may be repeated daily, or every second day.

Aloes is generally administered in ball or in watery solution. A ball for immediate use is made with freshlypowdered aloes, mixed with about one-eighth of ginger, and made up with soft soap, lard, glycerin, or vaseline. The physic mass of the Edinburgh Veterinary College was composed of equal weights of Barbados aloes and treacle, 
with two ounces of ginger to every pound of aloes. The addition of ginger, or some such aromatic, hastens catharsis, and diminishes nausea and griping. The ingredients were mixed over a slow fire, and constantly stirred until properly melted, care being taken to prevent the temperature rising above $120^{\circ} \mathrm{Fahr}$. The mass should be kept in air-tight jars, the balls being made up as required. Another good and less bulky mass is prepared by adding to melted aloes about one-fourth of its weight of rectified spirit, or oil of turpentine, which keeps the mass soft and moist. Aloetic balls made with lard, oils, or soap are only suitable for immediate use, and, if kept for several weeks, become dry and hard. Drying may, however, be retarded by adding a little glycerin and an ounce of potassium carbonate or acetate to every pound of the compound. Twenty grains each of powdered aloes, jalap, ginger, and soap, made into a pill, with glycerin or vaseline, is a good purge for a large dog, and will make two doses for a small one.

Watery infusions for immediate use are prepared by rubbing down the aloes in hot water, avoiding a temperature exceeding $120^{\circ} \mathrm{Fahr}$. Tinctures made by macerating the drug in alcohol (60 per cent.) for seven days, and afterwards filtering, may be of such strength as suits the practitioner's convenience. The B.P. tincture is prepared with extract of Barbados aloes, liquid extract of liquorice, and alcohol (45 per cent.). Extracts made with the view of removing a portion of the resin have nothing to recommend them. Aloes is slowly dissolved when introduced into the rectum, and hence exerts little laxative effect; but one or two drachms are occasionally ordered as an enema for the horse, dissolved, with soap and a drachm of potassium carbonate, in two quarts of warm water.

AlorN.-The active crystalline principle aloin was first discovered in Barbados aloes, and subsequently in the other varieties. An analogous crystalline substance has been separated from Natal aloes, and named nataloin, while from Socotrine aloes socaloin has been obtained. These three aloins are generally believed to be isomeric, although some authorities regard them as a homologous series. They are derivatives of anthraquinone, and so are related 
to the active principles of rhubarb and senna and other members of the anthracene group of purgatives.

Pure barbaloin is odourless; its taste, at first slightly sweet, soon becomes intensely and permanently bitter, and distinctly aloetic. It is entirely combustible, burning with a yellow flame and much smoke. It yields, by destructive distillation, an aromatic volatile oil, and a resinous residue. It is neutral to test paper, is soluble in rectified spirit, but less so in cold water, an ounce of which dissolves about a grain of aloin. The solvent power both of water and alcohol is greatly increased by heat. Barbaloin is also dissolved by acetic acid and alkalies, forming with the latter orange-yellow solutions, which deepen in colour by oxidation. It is insoluble in ether, oil of turpentine, and chloroform. Watery solutions rapidly darken by exposure to air and light; and when heated above $150^{\circ}$ Fahr., the aloin is oxidised, decomposed, and converted into a resinous substance of little activity.

ACTIONS AND UsES.-Aloin has been used with growing favour in medical practice, and with some practitioners it has entirely superseded aloes. The dose for an adult is from one to two grains, or about one-third of the quantity of Barbados aloes usually prescribed. Drachm doses made up with flour and glycerin were administered to six threeparts-bred carriage horses, four and five years old, 15 to 16 hands high, in good health and condition, and prepared with one bran mash given four hours previously. No effect was observable on the pulse, temperature, appetite, or secretion of urine; the bowels were relaxed to a slight extent in two of the animals, when they were exercised twenty-four hours after receiving their ball; whilst in two of the subjects of experiment fulness and itching about the joints disappeared, although no sensible effect was observed on the bowels. Drachm doses of aloin, conjoined with half an ounce each of gentian and ginger, proved serviceable in abating febrile symptoms, and removing heat and fulness of the limbs in hard-worked or grossly-fed horses. Two drachms of aloin given to strong five and six years old hunters, well prepared by mashes for upwards of twenty-four hours, caused, in thirteen or fourteen hours, abundant fluid evacuations. Nothing notable was observed as to the pulse or temperature; there was less dulness or loss of appetite than usually accompanies the full action of the ordinary aloetic ball; there was no nausea or griping; the purging usually continued six or eight hours. In these 
horses, which were in the country, it will be noted that two drachms of aloin operated several hours earlier, without impairment of appetite or spirits, and with the certainty and effect usually following six drachms of Barbados aloes.

F. Smith and C. Rutherford made several experiments with aloin on healthy horses. Five grains were given hypodermically to one subject, and eight grains were injected into the right jugular of another. Neither case exhibited any evidence of gastro-intestinal disturbance. In a third case twelve grains were injected hypodermically; in three hours the animal lay down, apparently pained, and the bowels were moved. During the next four hours were noted dulness, uneasiness, scraping, circling round the box, bowels repeatedly moved, but the droppings were hard and dark, and the effects gradually wore off. A fourth case received twenty-four grains injected hypodermically. In an hour and a half rumbling in the intestines was heard, and hard pellets were passed. To these symptoms were shortly added dulness, scraping, circling round the box, pain, exhaustion, and further passage of hard fæces. These effects continued for nine hours from the exhibition of the drug, but gradually passed away without the bowels being notably relaxed.

A strong shorthorn cow received by the mouth two drachms dissolved in hot water, with an ounce of ginger ; the bowels were only slightly relaxed; but three drachms operated tolerably freely in twenty hours. Two drachms, with half a pound Epsom salt, acted as rapidly and effectually as one and a quarter pounds Epsom salt. English terriers, twenty pounds weight, are little affected by doses of twenty grains given in bolus ; even drachm doses have scarcely any effect on pointers and setters ; but when two or three grains of calomel, or twenty to thirty grains of jalap are added, full effects occur in six or eight hours.

Old horses, familiar with the smell of aloes, and induced to swallow it with difficulty, show much less antipathy to the inodorous aloin. Definite and uniform in composition, concentrated in form, aloin should come into more general use as a cathartic for horses. 


\section{B U CKTHORN S}

The recently-expressed juice of the ripe berries of Rhamnus catharticus, or purging buckthorn.

The dried bark of Rhamnus frangula or black alder. (Not official.)

The dried bark of Rhamnus purshianus or sacred bark (B.P.). Nat. Ord.-Rhamneæ.

The buckthorns are shrubby, spinous trees, eight or ten feet high. The berried fruit and barks contain a glucosidal body, three resins, one concentrating the activity of the drug, with mallic and tannic acids.

The berries of $R$. catharticus are about the size of black currants, contain an acrid, nauseous, bitter juice, which is evaporated, strained, and gently heated with sugar, ginger, and pimento, forming a mild cathartic syrup, of which dogs take $f z i$. to $f z_{i j}$; and cats, fziv. to $f z i$. A little senna confection, jalap, or castor oil renders this syrup more prompt and certain.

The bark of Black Alder, when fresh, acts as a gastrointestinal irritant; but when dried and kept for twelve months, oxidation of the resinoid active matters appears to occur, and the fluid extract prepared from the bark may be used as a cholagogue laxative.

Cascara Sagrada, or sacred bark, brought from the North Pacific coast, has a persistent, nauseous, bitter taste; contains cascarin, a volatile oil, an acid, a glucoside, and several resins. It is useful in habitual constipation; it produces peristalsis, especially in the large intestine, but, unless in very large doses, does not materially increase the glandular secretions. It is generally used as the liquid extract, which American practitioners prescribe for dogs and cats as a stomachic bitter and tonic, in doses of $m_{v}$. to $m_{x}$., and as a laxative in doses of $m_{v}$. to $3 j$., mixed with mucilage or syrup. Cascara evacuant (Parke, Davis and Co.) is prescribed in doses of $3 \mathrm{ij}$. to $3 \mathrm{iv}$. for well-bred horses ; $m_{x}$. to $3 j$. for dogs ; and $m_{i}$. to $m_{x}$. for cats. 


\section{CASTOR OIL}

Oleư Ricini. The oil expressed from the seeds of Ricinus communis. (B.P.) Nat.Ord.-Euphorbiaceæ.

The castor oil plant, or Palma Christi, is generally considered to be Jonah's gourd. Cultivated in the colder parts of Europe, it is an annual shrub, four or five feet high ; in Spain and Sicily it reaches a height of twenty feet; in the southern latitudes of India, in Central Africa, and various parts of North and South America, it becomes a large tree. The natural order Euphorbiaceæ, besides the castor oil and croton, includes a tall Brazilian tree, the coco-purgatif, which yields the oil of Danda, or assu juice, resembling castor oil, but greatly more active.

Of the seeds, which are contained in capsules, two varieties are met with, one the size of beans ; the other, and commoner, somewhat smaller. Both have the shining yellowwhite epidermis, mottled with red-brown streaks and spots. The seeds comprise upwards of 25 per cent. of ligneous husk, 8 per cent. of moisture, and nearly 70 per cent. of kernel, containing about 50 per cent. of oil. They contain small quantities of the vegetable proteid poison (tox-albumin) ricin. Castor oil contains glyceryl ricinoleate, palmitate, stearate, cholesterin; an alkaloid, ricinine, which is not purgative; and a resin, but is free of ricin.

Castor oil is manufactured in London, largely imported from the East Indies and America, and in smaller quantities from Italy, the West Indies, and Australia. Various modes of extraction and purification are employed. The carefully shelled seeds are crushed in a hydraulic press, the oil purified by rest, filtration, and bleaching. In the East Indies mucilage and albumin are got rid of by heating the expressed oil with boiling water, and straining it through flannel. In America, the seeds, deprived of husk, are exposed to gentle heat, in order that the oil may be more readily expressed; the crude oil is freed from mucilage and albumin by boiling with water until perfectly transparent when cool ; 25 per cent. of best oil is thus got. In Jamaica the bruised seeds are boiled with water, and the oil skimmed 
off as it rises to the surface- a process which yields, however, an inferio and dark-coloured specimen. The Continental plan of extracting the oil by alcohol or carbon bisulphide is expensive and inconvenient.

ProperTIES.-Oil obtained by these various methods differs slightly in activity, but considerably in colour, flavour, solubility, and keeping properties. Cold-drawn castor oils, prepared by expression alone, or with only a very slight degree of heat, are generally preferred.

Castor oil, when fresh and well prepared, is viscid, almost colourless, and of a faint oily odour and taste. Although lighter than water, it is one of the heaviest of the fixed oils, its specific gravity being $0 \cdot 950$ to $0 \cdot 970$. Exposed in a thin layer it thickens, gets rancid, and after a time entirely dries into a varnish-like film. Castor oil and alcohol are mutual solvents ; the oil is soluble in one volume of absolute alcohol and five of alcohol (90 per cent.), and in ether ; is easily miscible with other oils; saponifies with alkalies, yielding glycerin, palmitic, and other fatty acids, and the special ricinoleic acid. Thus saponification, caused by the alkaline secretions of the bowels develops, as in the case of croton oil, the irritant, active principle from the bland oil.

IMPURITIES.-Castor oil is adulterated with croton oil to increase its activity, with lard and bland oils to reduce its cost. Inferior sorts are dark-coloured, but become translucent by exposure to sunlight and filtration through animal charcoal ; while the disagreeable acrid taste and odour may in great part be removed by repeated agitation with water containing calcined magnesia and coarse animal charcoal.

ACTIONS AND USES. - Castor oil seeds are irritant and purgative, and have caused fatal gastro-enteritis both in human patients and in animals. This irritant action is due to the presence of the tox-albumin ricin. This substance is extremely toxic if injected intravenously, less so given subcutaneously, and is largely neutralised by the gastric juice, and rendered harmless when given by the mouth. It may, however, escape this neutralisation, and will then exert all its toxic effects. It causes vomiting and diarrhœa, and post mortem the mucous membrane of the intestine is found inflamed, and there are hæmorrhages into the serous 
membranes. In vitro ricin has a characteristic action on blood, causing an agglutination or clumping of the red corpuscles, so that they will not pass through a filter. Ricin is interesting because it was by means of experiments with this agent that Ehrlich discovered many facts relating to immunity and serum-therapy. A decoction of the leaves is applied by the women of South Africa to their breasts to increase the lacteal secretion. The oil is a mild purgative, closely resembling linseed and the other fixed oils.

Medicinal Uses.-The oil, emulsified mainly by the alkaline bile, is in part absorbed; but the greater amount, little changed, passes through the bowels, increases both sccretion and peristalsis, rarely causes griping, and imparts to the dejections a glazed appearance.

For horses it is a mild cathartic, prescribed in diarrhœa, dysentery, enteritis, and pcritonitis ; in hernia, advanced pregnancy, affections of the kidneys and bladder; in purpura and bilious influenza, when more drastic purgatives might unduly irritate, or where repeated doses require to be given. It has no cholagogue action, nor is it a vermicide ; its occasional effect as a vermifuge depends upon its purgative action. Foals and calves, for several days after birth, sometimes have no movement of the bowels, and the removal of obstructing masses of meconium, and a natural action of the intestine, are best secured by administration of castor oil and enemata.

In cattle practice it is useful in diarrhoea, and inflammation of the digestive organs, and, united with Epsom salt, in doses of eight to twelve ounces of each, produces prompt and certain effects. For young calves it is the best of purgatives.

In the dog it is more active than in man, and for delicate subjects a mixture of equal quantities of castor and olive oils is often used. It occasionally causes emesis, an effect which results from its nauseous oleaginous taste, and not from any specific emetic action. This may be obviated by giving oil of good quality and mixed with an egg, mucilage, and a little ether. It proves a safe and easy purge for pigs, and also for poultry.

The bruised seeds are much used by native Indian farriers 
for the cure of mange. For enemata it is generally superseded by rape or linseed oil. As an external demulcent it is unsuitable on account of its tendency to become rancid. A few drops applied to the conjunctiva lessen irritation after removal of a foreign body from the eye. Castor oil is often employed with cocaine.

DoSES, etc.-Castor oil seeds are occasionally given to the dog or pig to the number of six or eight, triturated with linseed meal, made into bolus, or rolled in a piece of meat. The dose of oil for the larger quadrupeds is about

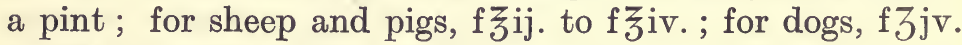

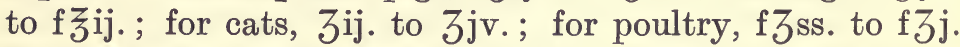
It may be given alone or mixed with linseed oil, with gruel, milk, or aromatics ; to increase its activity it is combined with a small quantity of oil of turpentine or of croton; to remove the cause of undue irritation, as in diarrhœa and dysentery, it is prescribed with laudanum, or chloroform, and warm starch gruel. For delicate or pampered dogs, a palatable laxative emulsion is made by shaking together equal parts of castor oil and syrup of buckthorn with ten to twenty minims of spirit of nitrous ether.

\section{CROTON SEEDS AND OIL}

Croton Seeds. Semina Crotonis. The seeds of Croton tiglium. (Not official.)

СвотоN OIL. Oleum Crotonis. Oil expressed from the seeds of Croton tiglium. (B.P.) Nat. Ord.-Euphorbiaceæ.

The Croton tiglium is a tree growing on the Indian continent, in Ceylon, and in many islands of the Indian Archipelago. Its oval-shaped fruit or nut is somewhat larger than a hazel, and contains three seeds about the size of French beans, resembling castor oil seeds, brown, but unmottled, and when shelled weighing on an average three grains each. They are odourless, with a taste at first mild and mucilaginous, but soon becoming hot and acrid. When heated they yield irritating fumes. The thin, brittle, external shell constitutes fully one-third of the weight of 
the seed. The seed kernel contains 50 to 60 per cent. of fixed oil.

The oil is viscid, of a brownish-yellow colour, with a peculiar nauseous odour and a persistent acrid taste. This acridity is due to the presence of a variable small amount of free crotonoleic acid. It is freely soluble in ether, chloroform, light petroleum spirit, and oils, fixed and volatile. Specific gravity, 0.940 to 0.960 . When the oil comes into contact with the alkaline intestinal secretions and the bile, it is saponified, producing glycerin and free crotonoleic acid. The residual cake left after expression of the oil, owing to the variable amount of oil it contains, is dangerous, and should not be used. Croton tiglium seeds contain a toxalbumin, Crotin, which is similar to but less toxic than ricin (p. 449). Crotin is not found in the oil.

ACTIONS AND USES. - Croton oil containing free crotonoleic acid irritates the living textures with which it comes into contact. The oil, undiluted, and in various degrees of dilution, is occasionally used as a counter-irritant, and when rubbed into the skin produces an eruption which becomes pustular. It is a drastic, hydragogue cathartic. Full doses cause gastro-enteritis and much prostration. If the free acid be removed the irritant effects on the skin, mouth, and stcmach, are prevented, although the purgative action remains.

General Actions.-Croton oil, undiluted and freely used, produces pustules, seriously and deeply inflames the skin, leaving permanent blemishing, and causing besides pyrexia, sometimes purgation. It resembles tartar emetic in its action on the skin. Used with an alkali, the irritant action is more readily developed, and its effects intensified.

Purgation is produced when the oil is freely applied to the skin, or enters the body by any other channel. When full doses are given, the drug is excreted not only by the bowels, but by the kidneys, inducing diuresis, with much irritation. As a drastic and hydragogue cathartic, croton resembles gamboge and elaterium. It operates more speedily than aloes, and produces more frequent, full, and fluid dejections. For horses, croton is, however, too violent for general use. For cattle it is sometimes valuable, 
operating with certainty when most other purgatives fail, and, if carefully used, is rarely attended with evil consequences. For sheep it is too irritating and depressing to be generally prescribed. For dogs and pigs it is an effectual drastic purge, requiring, however, as in other patients, to be used with much caution. W. Rutherford and Vignal have shown that, although causing great dilatation of the vessels of the intestinal mucous membrane it has no special cholagogue action.

Toxic EFFECTs. - Forty seeds destroyed a horse in seven hours, with acute gastro-enteritis; half that quantity usually produced fatal inflammation (Hertwig). Morton administered twenty bruised seeds to two horses and observed superpurgation, accelerated pulse and respiration, injected mucous membranes, collapse, and death in eighteen and twenty-four hours. Medicinal doses sometimes cause, alike in horses and dogs, unexpected and serious irritation. In India the seeds are occasionally used to poison horses. Orfila gave a dog three drachms, which killed him in three hours; one drachm was also fatal; while Hertwig found that ten or twelve grains induced violent purgation, gastro-enteritis, and death in four to seven hours, if vomiting was prevented by tying the œsophagus. About the same quantity of the bruised seed or oil, which proves fatal when given internally, has the like effect when placed in the areolar tissues, or applied to a wound. Hertwig states that eight drops injected into the jugular vein killed a horse, while two drops killed a dog. Moiroud records that twelve drops injected into the veins of a horse produced in a few minutes alvine evacuations, while thirty drops were quickly fatal. Fifty drops in alcoholic solution, applied to the belly of a small horse, induced, for two days, alvine evacuations of normal consistence, but three or four times more abundant than usual. Thirty drops had similar effects on sheep, fifteen to twenty on dogs (Hertwig).

Post-mortem examination discovers inflammation of the small and large intestines. In horses poisoned, the cæcum and colon are especially affected, usually exhibiting much extravasation of blood, and occasionally patches of erosion ; 
sometimes the lungs are congested, and occasionally they are inflamed.

Medicinal UsEs.-Croton is used as an active hydragogue cathartic for cattle suffering from gastric impaction and other forms of constipation, and from indigestion associated with phrenitis or other disordered state of the nervous system. It is serviceable where bulky medicines are inadmissible, where animals are unmanageable, or have difficulty in swallowing, where it is requisite promptly to produce copious fluid evacuations and extensive counter-irritation. It is contra-indicated in young and delicate subjects, in all debilitating complaints, and wherever any portion of the alimentary canal is in an irritable or congested state. The effects of overdoses are abated by demulcents and opium, given by the mouth and rectum, by hot cloths to the abdomen, and, if need be, by stimulants to counteract depression.

As a counter-irritant its effects may be regulated by diluting it. When undiluted it is too irritant either for horses or dogs; but is less apt either to purge or blemish cattle, to which it is occasionally applied in laryngitis, chronic glandular enlargements, and articular rheumatism.

Doses, etc.-Ten or twelve seeds, which, allowing three grains for each, weigh from thirty to thirty-six grains, form the dose for the horse, fifteen to twenty seeds for cattle, three or four for sheep, two or three for pigs, and one or two for dogs. The dose of croton oil for the horse is $m_{x}$. to $\mathrm{m}_{\mathrm{xx}}$; ; for cattle, $\mathrm{f} \zeta \mathrm{ss}$. to $\mathrm{f} 3 \mathrm{j}$.; for sheep and swine, $m_{v}$. to $m_{x}$. ; and for the dog, $m_{i}$. to $m_{i i j}$. The bruised seeds and the oil are administered made into bolus with linseed meal, or dissolved in castor or linseed oil or mucilage. They are less irritating and more certain and regular when conjoined with other purgatives. In obstinate constipation or torpidity of the bowels of cattle, half doses are given, with twenty or thirty grains of calomel, a pound of salts, or a pint of linseed oil ; and few purgative mixtures are more effectual. The oil is sometimes dropped on the tongue, but, unless freely diluted, is apt to irritate both tongue and fauces. For external purposes it is dissolved in six or eight parts of bland oil or soap liniment. Small quantities 
added to blistering ointments promote their activity, but in horses also increase their tendency to blemish.

Linimentum crotonis is made with one ounce croton oil, $3 \frac{1}{2}$ ounces of oil of cajuput, and $3 \frac{1}{2}$ ounces of alcohol (90 per cent.). A tincture-prepared with $1 \frac{1}{2}$ ounce of bruised croton-seeds and 20 ounces of rectified spirit is occasionally used as a counter-irritant.

\section{ELATERIU M}

Elaterium. A sediment from the juice of the fruit of Ecballium elaterium, the squirting cucumber. Nat. Ord. -Cucurbitaceæ.

Elaterin. $\mathrm{C}_{20} \mathrm{H}_{28} \mathrm{O}_{5}$, the active principle of Elaterium (B.P.).

Elaterium occurs in light, friable, flat, or slightly curved opaque cakes, about one-tenth of an inch thick, pale green or greyish-green in colour, odour faint, taste bitter and acrid. It should contain 20 to 25 per cent. of the active principle elaterin, which is neutral, insoluble in water, soluble in 115 parts of alcohol (90 per cent.), in 220 of ether, and very soluble in chloroform.

Elaterium is a very active irritant on mucous membranes, large doses causing gastro-enteritis and hæmorrhage. Medicinally it is a powerful drastic, hydragogue cathartic, much too energetic for general use. Its action is followed by depression. It has been prescribed in the treatment of abdominal dropsy in dogs. Its action on horses or cattle has not been ascertained.

DOSES, etc.-(Elaterium for dogs, gr. $\frac{1}{20}$ to gr. $\frac{1}{2}$; (Elaterin) gr. $\frac{1}{60}$ to gr. $\frac{1}{10}$ in mucilage, milk, or pill (Kaufmann). To facilitate dispensing, the compound powder of elaterin, containing 5 grains of the active principle and 195 grains of milk sugar may be used.

Euonymin, an extract obtained from the root-bark of Euonymus atro-purpureus (Celastraceæ), is employed as a liver stimulant and laxative for the dog, in doses of grs. ii. to grs. $v$. 


\section{G A M B O G E}

Gambogia. A gum-resin obtained from Garcinia Hanburii (B.P.). Nat. Ord.-Guttiferæ.

Gamboge, imported from Singapore, Siam, and Ceylon, is the produce of a moderate-sized diceious tropical tree, and is obtained from incisions into the middle layer of the bark, or by breaking the leaves and branchlets, when the yellow milky juice exuding is collected in leaves, in cocoanut shells, or in joints of bamboo, is transferred into flat earthen vessels, and dried in the sun. It occurs in cylindrical, solid or hollow rolls, four to eight inches long, two to three inches in diameter, and in cakes; breaks easily with a conchoidal reddish-yellow fracture; powder bright yellow; odourless, taste very acrid. It is feebly soluble in water, makes with it a yellow emulsion, and is soluble in alcohol and ether. It consists of 15 to 20 per cent. of soluble gum, and about 70 of an active orange-yellow resin, gambogic acid. It is largely used as a pigment.

ACTIONS AND USES.- It is a powerful irritant and drastic hydragogue cathartic, inferior in activity only to croton and elaterium. Slightly diuretic, it colours the urine yellow.

It undergoes solution in the alkaline intestinal juices, and in large doses causes gastro-enteritis. Moiroud gave horses six to twelve drachms, and found the dejections frequent and fluid, the pulse irregular, the animal shivering and anxious. Two drachms killed a sheep, two ounces and a half had little effect upon a cow, but five ounces caused dysentery, which continued for seventeen days.

Gamboge is too drastic and uncertain to be safely given to either horses or dogs. It causes profuse watery discharges and increased peristalsis, and although W. Rutherford's experiments demonstrate that it has no special stimulant action on the liver, like all purgatives acting on the small intestine it is a cholagogue, in the sense that it promptly moves onwards the bile in the duodenum, and thus prevents its reabsorption. It has no direct vermicide effect, but produces diuresis, especially when given in small 
doses dissolved in alkalies. For ruminants it is safe, speedy, and manageable when in combination with other purgatives. Half a pound each of Epsom and common salts and an ounce of gamboge form a prompt and effectual purgative in indigestion, gastric impaction, and brain diseases of cattle. Although neither gamboge nor aloes is particularly certain when used alone, an ounce of each, rubbed down and given in solution, constitute an effectual purge for ordinary cattle cases. Applied to wounds it is said to promote healing (Rey).

Doses, ete.-For cattle, $\jmath_{\text {ss. }}$ to $\jmath_{\mathrm{j}}$; ; for sheep, grs. xx. to grs. xxx., given in combination with other purgatives, and in solution; dogs, grs. ii. to grs. viii.

\section{J A L A P}

JALAPA. The dried tubercules of Ipomœa purga (Hayne). (B.P.) Nat. Ord.-Convolvulaceæ.

JALAP AE RESINA. Jalap resin, obtained from Jalap digested and percolated with alcohol (90 per cent.).

Jalap derives its name from Xalapa or Jalapa, a town in Mexico, whence it was first obtained. The hardy climbers yielding it grow on the Andes 6000 feet above sea-level, are cultivated in Southern India, and in sheltered spots in this country produce their salver-shaped crimson or light-red flowers. The perennial root-stock throws off underground shoots, which at intervals send down roots, gradually thickening, becoming irregularly oblong or ovoid, ranging in size from a walnut to an orange, invested with a thin, brown, furrowed, wrinkled cuticle, and presenting within a dirty yellow colour, with dark-brown concentric lines. The larger roots, or tubercules, are divided into halves or quarters or gashed to facilitate drying. They are tough, and difficult to reduce to powder, which has a pale-brown colour, a faint, disagreeable odour, and a taste at first sweet and mawkish, but afterwards acrid and nauseous. Water dissolves the sugar and mucilage without the cathartic, resinous principle, which is, however, readily soluble in rectified spirit. 
With starch, cellulose, uncrystallisable sugar, and gum, jalap contains 9 to 11 per cent. of the active resin, consisting chiefly of the glucoside convolvulin, which differs from the jalapin of scammony in being insoluble in ether. Jalap resin occurs in dark-brown fragments, readily reduced to powder, with a sweetish odour, and acrid taste. Soluble in rectified spirit, but not in water or in oil of turpentine.

ACTIONS AND UsES.-Jalap is a hydragogue cathartic, and a vermifuge. The ordinary jalap closely resembles the larger-rooted male jalap, or orizaba root, the smaller paler tubercules of the tampico root, the dried root of Convolvulus scammonia, and the roots of Bryonia alba and B. dioica, as well as the Kaladana seeds used roasted as a purgative by the nations of Hindustan. It is more active than senna, the leaves of Cassia acutifolia, and the other anthracene purgatives, but less powerful and irritant than the drastics, gamboge, podophyllum, elaterium, and colocynth.

Jalap has very gentle cathartic action on either horses or cattle. Two or three ounces given to the horse have slight effect on the bowels, but increase the activity of the kidneys (Moiroud). White reports administering half a pound to horses without causing purgation. Dun repeatedly gave cows four ounces without perceptible effect. Probably the B.P. jalap resin would be more energetic. For dogs, cats, and pigs it is, however, a good purgative, although full doses occasionally produce nausea and sometimes vomiting. It is prescribed for most purgative purposes, acts tolerably speedily and certainly, produces full watery discharges, and is specially effective when given with a grain or two of calomel. As with several other purgatives the presence of bile in the intestine is necessary for its action.

Doses, etc., of Jalap powder.-Dogs take $3 \mathrm{i}$. to $3 \mathrm{ij}$. ; cats, grs. iv. to grs. xx. ; pigs, 3i. to 3iv. It is best given in combination with calomel. Dogs, if fasted for six hours, are effectively purged in three or four hours by 3 ss. to 3 i. of jalap, with two or three grains of calomel, made into bolus with any convenient excipient. 


\section{PODOPH Y L L UM}

The dried rhizome and roots of Podophyllum peltatum. Nat. Ord.-Berberidaceæ.

The Podophyllum, May apple, or mandrake, is a perennial herbaceous plant, plentiful in the Northern States of America, where its subacid fruit is eaten under the name of wild lemons. The root is imported in flattened cylindrical pieces of variable length, one-fifth to one-third of an inch thick; marked with irregular tuberosities giving off brittle brown rootlets. It is reddish-brown externally, white within, and breaks with a short fracture. The powder has a yellow-grey colour, a disagreeable odour, a bitter, sub-acrid, nauseous taste.

Podophyllum resin, the active principle, is prepared from a strong tincture, made by exhausting the root with rectified spirit. It is a pale-brown amorphous powder, soluble in alcohol and ammonia, and consisting of an inert, fatty, resinous acid, and two amorphous, bitter, active resinspodophyllotoxin and picropodophyllin, the former being the more powerful.

ACTIONS AND USES.-Both root and resin are topical irritants and drastic purgatives. The resin is dissolved by the alkaline secretions of the duodenum; stimulates glandular secretion and peristalsis, in full doses causing spasm ; carried into the liver, it increases secretion of bile. In dogs and cats, as in human patients, it is an emetic. In horses and dogs repeated doses reduce the force and frequency of the pulse, even when the bowels are only slightly affected. It is eliminated by the bowels, in smaller amount by the kidneys.

General Actions.-The root has long been used by the American Indians as an emetic and anthelmintic. Its actions being supposed to resemble those of calomel, it has been styled vegetable mercury. The cholagogue action of podophyllum was investigated by W. Rutherford. Moderate doses introduced into the duodenum, whether of fasting or recently-fed dogs, increased secretion both of the fluid and solid constituents of the bile. Podophyllum directly 
stimulates the hepatic cells, but does not increase the blood-supply of the liver. Excessive doses are imperfectly absorbed, and do not increase biliary secretion. In common with other purgatives acting upon the small intestine, it sweeps out food, which, when absorbed, stimulates the liver; while, moreover, it carries away bile poured into the canal, and thus prevents its reabsorption.

'In the domestic animals the cathartic effects of podophyllum are produced tardily, and moderate doses are apt to cause nausea and griping. The depressant action on the heart and central nervous system is probably due to shock from its irritant effects on the small intestine. Anstie, experimenting with alcoholic solutions containing one to two grains podophyllum, injected into the peritoneum of dogs, cats, and rats, produced in ten to fifteen hours vomiting, bloody fæces, hurried, shallow breathing, and death from exhaustion.

Howell found podophyllum to be a prompt and effectual purgative for dogs, acting usually in four hours. One drachm to one drachm and a half, with two drachms ginger, moved the bowels of horses in six to eight hours. The action was prompt and effective, and unattended with noticeable griping, even when the resin was given without preparation, and water was allowed ad libitum.

Dun was unable to obtain such marked results. Repeatedly he gave healthy horses, prepared by mashes, two drachms podophyllum without perceiving any increased action of the bowels. Two drachms resin, with one or two drachms of aloes, added to determine, if possible, its action on the bowels, produced little more softening of the discharges than might be expected from the aloes alone. Three healthy shorthorn cows were each given three drachms, and another cow received half an ounce, without producing any laxative effect. Doses of one grain, given in pill or in meat, to English terriers weighing about twenty pounds, produced no notable effect upon the bowels; while two grains acted as a gentle laxative, but only after six or eight hours.

To a well-bred hunter under treatment for injury of the psoas muscles, and fed for twenty-four hours on bran, 
Dollar administered two drachms podophyllum in a ball, and two ounces Epsom salt in solution. Scarcely any perceptible action was observed on the bowels; and two days later two drachms podophyllum and one drachm calomel were given, also without purgative effect, but with a reduction in the pulse of nearly ten beats per minute.

A powerful cart-horse, under treatment for sand-crack, and previously restricted for twenty-four hours to a mash diet, received four drachms podophyllum resin in a ball. Although no purgation followed, there was nausea, and in two hours the pulse, soft and somewhat weakened, fell from 36 to 24 , and did not recover its natural force or number until next day. The appetite continued impaired for a week.

A Scotch terrier, eight months old, received half a grain of the resin in a pill, without apparent effect ; and on the following day a grain, which in the course of an hour caused nausea and vomiting. Considerable dulness remained for twenty-four hours.

A bull terrier bitch, thirty-six pounds weight, received four grains in a pill, without showing any notable symptoms, and on the following day had a further dose of six grains, which in twelve hours produced great uneasiness, griping, and gentle catharsis. During the two following days the bitch refused food, and for a week continued dull and listless.

A French poodle, suffering from mange and constipation, had a pill containing two grains podophyllum resin, half a grain calomel, and twenty grains jalap. No effect was observable at the end of twelve hours, when the dose was repeated, and after eight hours the dog was briskly purged. Half the above dose was repeated every second day for a fortnight, with the result of gently moving the bowels. In all these cases the pulse was reduced in number and in strength, the urinary secretion was unchanged, and the fæces were little altered in colour (Dollar).

Medicinal Uses.-These experiments demonstrate that for veterinary patients podophyllum resin is a tardy and uncertain purgative, especially when used alone, and there 
are many more reliable agents. In combination, however, with aloes, jalap, or calomel, it relieves torpidity or congestion of the liver; while its nauseant and sedative effects may occasionally be used for lowering cardiac action in acute lymphangitis, rheumatism, and other inflammatory disorders in robust patients. Although possessed of vermifuge powers, depending upon its purgative effect, it does not appear to have any special vermicide action. For human patients it is prescribed, both in this country and America, in habitual constipation, hepatic congestion, in some forms of sick headache, and, in smaller doses, as an alterative in skin diseases and rheumatism. Half a grain to a grain of the resin slowly empties the bowels.

Doses, etc.-For cholagogue or sedative purposes, horses and cattle take $3 \mathrm{j}$. to $3 \mathrm{ij}$. of podophyllum resin, with aloes, calomel, nitre, or Epsom salt. For dogs, gr. j. to grs. ij. ; with calomel, gr. j. to grs. ij., may be conjoined with half a dose of jalap or olive oil. Nausea and griping are obviated by admixture of ginger or other carminative, and of henbane or cannabis indica. The B.P. tincture contains two grains of podophyllum resin in each fluid drachm.

\section{R H U B A R B}

Rhei Radix. The erect rhizome, or so-called root, deprived of more or less of its cortex, and dried, of Rheum palmatum, Rheum officinale, and probably other species. Collected in China and Thibet (B.P.). Nat. Ord.-Polygonaceæ.

The perennial, herbaceous rheums grow on the hill ranges of the interior of China and Thibet; the roots, after five years' growth, are collected in summer, are cleaned, peeled, cut into round or flat reddish-yellow pieces, and each piece is usually bored with a hole, through which a cord is run in order to dry it hanging in the sun. The powder is bright yellow-brown, has a strong, peculiar, aromatic odour, with a bitter astringent taste, and when chewed is gritty, from the presence of calcium oxalate crystals. It is dissolved by ether and rectified spirit, and less readily by cold and hot 
water, forming with the latter an orange-coloured solution. The East Indian rhubarb is coarser and less aromatic. English rhubarb, the produce of R. raponticum, generally cultivated for its familiar leaf-stalks, the pleasant acid taste of which is due to the presence of malic and oxalic acids, is grown extensively for its roots near Banbury, and is sometimes mixed with or substituted for the Chinese; but it is softer and more mucilaginous, has less aroma and grittiness, contains fewer crystals of calcium oxalate, and is deficient in purgative power.

Rhubarb is one of the anthracene group of purgatives to which belong also senna and aloes. The active principles in these drugs are derivatives of anthraquinone. Among other constituents rhubarb contains (1) chrysophanic acid, in the proportion of about 2 per cent., extracted by ether or alcohol, insoluble in water, and occurring, as its name indicates, in brilliant yellow crystals. It appears to have tonic properties, and is one of the most effectual remedies for ringworm of the human scalp. (2) Chrysophan, a bitter soluble glucoside, which, when boiled with sulphuric or hydrochloric acid, splits into chrysophanic acid and sugar. (3) Phæorretin and other resinous bodies appear to confer the cathartic properties. (4) Rheotannic acid imparts astringency. (5) Mineral substances are largely present, consisting chiefly of calcium oxalate, 35 per cent.

Actions AND UsES. Rhubarb is stomachic, tonic, mildly cathartic, and afterwards astringent. Small and repeated doses improve the appetite, correct slight gastric derangement ; in virtue of their tannin may even diminish secretion and peristalsis; by their chrysophanic acid impart to the fæces a yellow-brown hue, and may be detected in the blood, urine, and occasionally in the milk. Larger doses, in dogs and cats, as in human patients, are mild eathartics, stimulate the peristaltic movements, especially of the stomach and small intestine, and are said to increase secretion of bile. Even small doses, insufficient to purge fasting dogs, slightly increase all the constituents of bile (W. Rutherford). In horses and cattle rhubarb has scarcely any purgative effect; a pound has been given to cattle without moving the bowels, while half a pound to a pound 
caused in horses only slight laxative effects after thirtysix hours (Moiroud). On skin or mucous surfaces it acts as a mild astringent.

DOSES, etc.-As a stomachic, repeated several times a day, horses take そj.; cattle, そij.; sheep, 3 j. ; dogs and cats, grs. $x$. to grs. xx. As a laxative, dogs take 3j. to 3iij., usually combined with one or two grains of calomel, or with twenty grains of jalap. Rhubarb is used in powder, infusion, syrup, and tincture. The compound powder, or Gregory's mixture, prepared by mixing thoroughly six parts magnesia, one part ginger, and two parts rhubarb-all in fine powder-is an excellent stomachic and antacid, and is given in doses twice as large as those of the simple rhubarb. In diarrhoa in calves and foals it exerts carminative, laxative, and subsequently astringent effects. When the bowels are persistently relaxed, two drachms each of rhubarb and magnesia, with half a drachm of opium, may be given night and morning in well-boiled wheat-flour gruel, with one or two tablespoonfuls of brandy or sweet spirit of nitre. One-third to one-half this quantity is prescribed for diarrhœa in lambs.

Syrupus Rhei is made with two parts each of rhubarb and coriander, twenty-four of sugar, eight of alcohol (90 per cent.), and twenty-four of water; and the compound tincture with two parts of rhubarb, one-fourth part each of coriander and cardamoms, two of glycerin, and eighteen of alcohol (60 per cent.).

\section{OPI U M}

The juice obtained by incision from the unripe capsules of Papaver somniferum, inspissated by spontaneous evaporation (B.P.). Nat. Ord.-Papaveraceæ.

MorphIne. Morphia. Morphina. An alkaloid prepared from opium.

Morphine Hydrochloride. Morphinæ Hydrochloridum (B.P.).

Morphine Tartrate. Morphinæ Tartras (B.P.).

Morphine Acetate. Morphinæ Acetas (B.P.). 
Codeine. Codeina. An alkaloid obtained from opium or morphine (B.P.).

APOMORPHINe HydrochloRIDE. Apomorphinæ Hydrochloridum (B.P.).

Herorn. Diacetylmorphine. A morphine derivative.

Opium, one of the most ancient articles of the Materia Medica, derives its name from the Greek word omós (opos), signifying juice. The stem, unripe capsules, and other succulent parts of any species of poppy, contain a milkwhite narcotic juice, which, as it dries, becomes darker in colour, and constitutes opium. The fresh purple petals of the Papaver rhœas, or corn rose, which contains no morphine, and only minute traces of rhœadine, are used as colouring agents, but not medicinally. The roots of some species contain a cathartic principle. The nearly ripened poppy heads, or capsules, gathered about twelve days after the petals fall, when digested in hot water produce a mucilage used for anodyne purposes ; when dried they yield less morphine and codeine than when green, and besides contain the feebly alkaline crystalline rhœadine. Within the capsules are numerous white or brown reniform seeds, devoid of narcotism, but yielding a bland drying oil, similar to that of flax or rape. The cake or residue left after expression of this oil is used for cattle food.

The Papaver somniferum-the common white or garden poppy - is a native of the warmer parts of Asia, but it also thrives in this country. It flowers from May to July, and the capsules ripen about two months later. It is two to four feet high ; has a round, smooth, erect stem, with a few hairs on the extremities and peduncles ; large, sessile, glaucous green leaves, with cut and wavy margins; large terminal white, red, or purple flowers, drooping before they open ; and globose capsules about the size of a duck's egg, and containing numerous kidney-shaped white or brown seeds. Of the several varieties the white-flowered have hitherto been preferred, but the darker-flowered, especially the purple, are now stated to yield a larger quantity and better quality of opium. 
The several varieties-of which the most notable are Turkey, East Indian, and Egyptian, owe their characteristics to differences in soil and climate, and also to the time and manner of collecting and making up the juice. Any suitable variety of opium may be employed as a source of tincture of opium and extract of opium of the respective alkaloidal strengths, provided that when dry it contains not less than $7 \frac{1}{2}$ per cent. of anhydrous morphine ; but when otherwise used for officially recognised purposes, opium must be of such a strength that, when dried and powdered, it shall yield not less than 9.5 to 10.5 per cent. of morphine (B.P.).

Turkey or Smyrna opium, mostly of fine quality, and highly prized in the English market, is chiefly collected in the north-western districts of Asia Minor. It occurs in round, flattened pieces, usually weighing from half a pound to two pounds, covered with poppy leaves and the chaffy seeds of the rumex. It is soft, moist, and ductile, and when minutely examined is seen to be made up of small tears. Its odour is peculiar, but not disagreeable; its taste bitter; its recent fracture pale liver-brown. It readily yields its active principles to water, forming a red-brown solution, and to alcohol of all strengths, forming darker-coloured tinctures. Good samples in a fresh state contain an average of 10 per cent. of morphine.

East Indian opium, a large proportion of which is imported to China, is of uniform and good quality.

Egyptian opium, being generally grown on moist soils and collected before the capsules are ripe, is inferior to the best Turkey opium, and contains on an average about 6 per cent. of morphine.

Characters.-Opium occurs in irregular, red-brown or red-black masses, which weigh from four ounces to two pounds, when fresh, plastic, and internally moist, coarsely granular or nearly smooth, and reddish- or chestnut-brown, but becoming harder on keeping, and darkening to blackish brown (B.P.). Odour strong and characteristic; taste bitter, persistent, and disagreeable. Turkey opium, recently imported, contains 10 to 15 per cent. of water, and is moist 
and plastic ; when long kept, or artificially dried, it is hard, and easily reduced to a brown powder, which is apt, unless carefully preserved, to absorb moisture. When heated it softens, and at high temperatures burns with a strong, peculiar odour. Cold water dissolves about 60 per cent. of a good dried specimen, and forms a red-brown solution, including most of the active constituents. Rectified spirit dissolves about 80 per cent., and forms a dark-brown tincture, which includes all the active principles. Acids, when strong, decompose opium, but when diluted are excellent solvents for it. The watery solution reddens litmus, owing to the presence of meconic and other acids, and is precipitated by vegetable astringents, salts of calcium, lead, copper, and other metals.

IMPURITIES.-Inferior specimens of opium are distinguished by narrowly examining their consistence, texture, colour, odour, and taste. They are sometimes dry, hard, and resinous, or oleaginous and waxy ; their fresh fracture devoid of the characteristic red tint and agreeable aromatic odour; while water and alcohol dissolve them imperfectly. Of the several substances used for adulterating, the most common are starch and molasses, the bruised leaves and chips of the poppy, the juice, pulp, or extract of the prickly pear, and opium from which the morphine has been extracted. Inorganic matters, such as sand, clay, and mud, may be detected by inspection, especially if the specimens be dried. But the official and most certain test of quality or purity is the proportion of morphine. One hundred grains of good opium should yield 9.5 to 10.5 grains of anhydrous morphine; but picked specimens have produced 22 per cent.

Composition.-Opium is a complex substance. Besides 15 to 25 per cent. of water, it contains 50 of gum, pectine, wax, and albumin; 2 to 6 of ash; traces of an aromatic volatile oil; while combined with meconic, thebolactic, phosphoric, and sulphuric acids are a number of alkaloids in variable proportions, together amounting to about 20 per cent. The most important are morphine, codeine, narcotine, and thebaine. There are also two neutral bodies, meconin and meconiasin. 
Subjoined is a list of these opium alkaloids, arranged according to their chemical composition :-

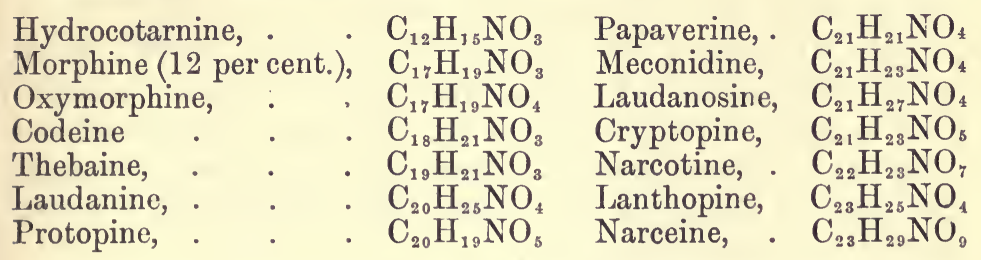

Some of these alkaloids, such as codeine and oxymorphine, are derivatives of morphine. (1) The codeine series is prepared from morphine by the addition of alcohol radicles ; others are got by (2) oxidation; (3) others by dehydration.

Opium alkaloids differ greatly in their action. Morphine is analgesic, and more or less hypnotic. Thebaine is stimulant and convulsant, and allied to strychnine. The best known of the others-oxymorphine, papaverine, codeine, and narcotine-form a series, in which the first resembles the narcotic morphine, and the last the tetanising thebaine.

Morphine and its salts are got by macerating opium in successive portions of water, which dissolve the morphine meconate; calcium chloride is added to the solution; calcium meconate precipitates, and morphine hydrochloride remains in solution, from which, when concentrated, the morphine salt crystallises, is subjected to pressure in flannel or stout calico, thus removing narcotine and colouring matter, and is redissolved in hot water, and repeatedly crystallised. By the use of animal charcoal colouring matter is removed; while, to get rid of codeine, ammonia is added to the watery solution, when pure morphine is precipitated.

Morphine crystallises in minute, transparent, right rhombic prisms, usually arranged in tufts. It has an intensely bitter taste and an alkaline reaction. It is soluble in ether, benzol, and chloroform; dissolves in 1000 times its weight of cold water, in 400 of boiling water, and still more readily in oils, caustic alkalies, and weak acids, with which it forms crystallisable and usually soluble salts.

Morphine hydrochloride $\left(\mathrm{C}_{17} \mathrm{H}_{19} \mathrm{NO}_{3} \cdot \mathrm{HCl} \cdot 3 \mathrm{H}_{2} \mathrm{O}\right)$ is preferable to the alkaloid on account of its solubility, is the salt 
in common use, and is prepared by diffusing morphine in hot distilled water, gradually adding hydrochloric acid, and setting aside the solution to crystallise. It is a snow-white powder, consisting of broken-down crystals, which, when entire, are needle-like prisms clustering in radiated groups. It is without odour, but has the intensely bitter taste of morphine. It is soluble in twenty-four parts of cold water, one part of boiling water, eight parts of glycerin, and in fifty parts of rectified spirit.

Morphine acetate and tartrate are sometimes used, and are prepared in a similar manner to the hydrochloride. The acetate is a white powder, almost entirely soluble in two and a half parts of water, in five of glycerin, and in one hundred of rectified spirit. The tartrate is soluble in eleven parts of cold water, but not in alcohol.

Codeine is methyl-morphine. Morphine $=\mathrm{C}_{17} \mathrm{H}_{18} \mathrm{NO}_{2}$ $(\mathrm{OH})$; codeine $=\left(\mathrm{C}_{17} \mathrm{H}_{18}\left(\mathrm{CH}_{3}\right) \mathrm{NO}_{3} \mathrm{H}_{2} \mathrm{O}\right)$. It is present in opium in the proportion of $\frac{1}{4}$ to 1 per cent. It is a colourless, bitter alkaloid, crystallising in trimetric crystals, soluble in eighty parts cold water, in less than two parts of alcohol and chloroform, in ammonia and dilute acids. Unlike morphine, it is insoluble in cold, weak, caustic potash, and is unaffected by ferric chloride. Like the other opium alkaloids, it exhibits the twofold stimulant and hypnotic action, but its hypnotic power is slight, and, like methyl compounds of the alkaloids, it notably stimulates the motor centres, and full doses cause tetanic convulsions similar to those produced by strychnine or picrotoxin. Codeine depresses sympathetic nerve cells more effectively than morphine, and so prevents inhibition of the intestinal movements. Thus it often causes vomiting and purgation when given to dogs and cats, although its continued use lessens irritability of the digestive tract. When given for several days to dogs, cats, or rabbits, arsenic or other irritants administered cause neither vomiting nor purging. It also diminishes the production of hepatic sugar, and is hence prescribed in canine diabetes mellitus. The dose for the dog is gr. $\frac{1}{12}$ to gr. $\frac{1}{2}$.

Apomorphine hydrochloride, $\mathrm{C}_{17} \mathrm{H}_{17} \mathrm{NO}_{2}, \mathrm{HCl}$, is the hydrochloride of an alkaloid obtained by heating morphine hydro- 
chloride or codeine hydrochloride in sealed tubes with hydrochloric acid (B.P.). It occurs in small, greyishwhite, shining, acicular crystals, soluble in sixty parts cold water, and more soluble in rectified spirit. It is neutral to litmus solution. It is a prompt and effectual emetic in animals that vomit, acting on the vomiting centre. When gr. $\frac{1}{4}$, dissolved in water, is swallowed by either man or dog, repeated vomiting occurs, but is not followed by so much nausea as usually follows the use of tartar emetic. In dogs and cats, freshly prepared solutions have the advantage of producing emesis usually in three or four minutes, when used hypodermically in doses of gr. $\frac{1}{8}$ to $\frac{1}{20}$. It increases bronchial salivary, and intestinal secretions. It appears to act specially on the medulla, stimulating the vomiting centre to produce emesis and also the respiratory centre causing quickened respiration. Large doses in cats, dogs, as well as in rabbits, cause excitation of the higher brain-centrescerebrum and cerebellum-producing inco-ordinate manège movements and tetanic spasms. Subsequently the excitement gives way to depression, loss of reflexes, difficult breathing, and muscular paralysis. Friedberger states that sucking lambs, suffering from gastric obstruction produced by wool, are reported to have been promptly relieved by $1 \frac{1}{2}$ to 3 grains, given hypodermically. F. Smith states that 2-grain doses given to horses produce intense delirium and nervousness, constant movement of the limbs, sweating, and every appearance of approaching dissolution. The B.P. Injectio Apomorphinæ Hypodermica contains 1 grain of apomorphine hydrochloride in 110 minims. Dose, as an emetic for the dog, gr. $\frac{1}{10}$ to gr. $\frac{1}{5}$; small dogs, gr. $\frac{1}{12}$ to gr. $\frac{1}{8}$; cats, gr. $\frac{1}{30}$ to gr. $\frac{1}{12}$, given subcutaneously.

Thebaine or Paramorphine is present to the extent of 0.3 per cent., is obtained in minute, colourless, rectangular prisms, melting at $380^{\circ} \mathrm{Fahr}$., has an alkaline taste and reaction, is almost insoluble in water, but soluble in fortyfive parts of rectified spirit, and in ether and chloroform. It has very slight hypnotic action, prominently exhibits the excitant effects of opium, stimulates the motor tract of the spinal cord, and causes, like strychnine, muscular rigidity and convulsions. One to two grains, 
injected hypodermically, produced fatal tetanus in dogs (Harley).

Narcotine exists in opium in quantities varying from 4 to 6 per cent., and is got by treating the insoluble residue left in the preparation of morphine with diluted acetic acid, precipitating the solution with ammonia, and purifying with hot alcohol and animal charcoal. It is a feeble base, and is distinguished from morphine by having no bitter taste, no reaction on vegetable colouring matter, and no effect on ferric chloride. Inappropriately named, it is devoid of narcotism; is tonic and antiperiodic ; and has been used in India as a substitute for quinine in the treatment of malarial fevers. Large doses are convulsant.

Narceine occurs as a light, colourless, bitter, asbestos-like body, made up of soft, needle-like crystals, soluble in 100 parts of boiling water, 400 of cold, and rather more soluble in glycerin and diluted hydrochloric acid. Somewhat contradictory opinions are expressed regarding its actions. In dogs, grs. v., subcutaneously injected, produced calmative and hypnotic effects, similar, it is said, to those induced by a grain of morphine. Poisonous doses arrest respiratory movements, but do not cause convulsions.

ACTIONS AND USES.-Opium has a complex and somewhat variable composition, and its alkaloids have different, and some of them opposite, effects ; hence its actions are liable to variation; although in the main opium acts in the same manner as morphine, its chief alkaloid. The effects differ in the same individual according to the dose, and in man and the lower animals according to the relative development of the several parts of the central nervous system. In man, the higher brain centres, on which the drug acts primarily and prominently, are paralysed, and the patient is usually calmed, sleeps, and, where large doses are given, becomes comatose. In the lower animals opiates stimulate in addition the reflex centres of the cord, and instead of being quieted and hypnotised, the subject at first is excited, exhibits irregular, involuntary movements, tetanic convulsions, and, as death approaches, coma, from which, however, it can be readily roused. This action is explained by the fact that the higher (cerebral) controlling centres being 
depressed the lower reflex centres are free and unchecked. Opiates kill by respiratory arrest. Medicinal doses are analgesic and antispasmodic, diminish the several secretions, excepting those of the skin, and, in combination with other drugs, are sometimes used as calmatives for horses and dogs. They are frequently applied locally to relieve irritability and pain, but experimentally it has been found that they have no effect on either sensory or motor nerve-endings, so that local application is of little value.

General Actions.-When administered by the mouth, opiates slightly augment the secretion of the salivary and gastro-intestinal glands with which they are brought into contact. Full doses cause some amount of gastric irritation, occasionally producing vomiting in man and dogs (partly by central action), but shortly, and more notably and permanently, they diminish gastro-intestinal secretion and movement.

After absorption, full doses induce primary, usually brief, stimulation, followed by depression and paresis of the central nervous system. In veterinary patients the prominent phenomena are agitation, unrest, inco-ordinate, generally manège, movements, diminished sensibility to pain, indisposition for voluntary movement, and, in toxic doses, convulsions, coma, and death by respiratory arrest. Moderate doses dilate the blood-vessels of the skin and quicken heart action, and this is more notable in horses than in man. But repeated full doses slow heart action in all animals. Moderate doses affect respiration and render it shallow, slow, and then irregular. The rectal temperature is temporarily raised; skin temperature from increased transpiration is reduced; but lethal doses, depressing the heat-producing centres, eventually lower the general temperature. Opium, morphine, and narceine diminish all secretions excepting those of the skin and kidneys, their effect on the latter organs being variable. By lessening reflex activity, and depressing all sensory nerve cells, they are notable antispasmodics. The effect on the iris varies in the different animals. In man the pupil is typically contracted to pinhole size. In the horse and cat it is dilated throughout, whilst in the 
dog little change is seen, but the action is a central one and the pupil is not affected by topical applications.

Different animals are somewhat differently affected by opiates, depending upon the relative development of different parts of their central nervous system. The higher the development, the greater is the susceptibility of an organ to the operation of the drugs which act upon it. Compared with the lower animals, the cerebrum of man is relatively heavier and more highly organised than the mesencephalon, the cerebellum, and the spinal cord ; and this explains why opiates given to man in relatively small doses quickly and without marked stimulation paralyse the human cerebral centres, inducing sleep, and, in large doses, coma. Horses, with less development of these higher brain centres, have relatively more development of the locomotor centres and of the reflex centres of the spinal cord, and upon these lower centres opiates in equine subjects exert their primary stimulant effects. Full doses produce, at first, restless, involuntary movements of the head and limbs, pawing, or walking continuously round the box ; followed by sleepiness, disinclination to move, and when moved staggering. Excessive doses cause tetanic convulsions.

Ruminants, like horses, are usually excited and restless. Cattle bellow, digestion is deranged, and tympanites frequently supervenes. Sleep is not quickly or readily induced, excepting by full and repeated doses.

Dogs exhibit effects intermediate between those observed in man and in horses. Relatively to their body-weight, they take eight or ten times the doses prescribed for man. They show more preliminary excitement than man, but less involuntary muscular movement than the horse, and during drowsiness and sleep muscular twitchings occur. Sleep, however, is never very profound ; the dogs are easily awakened ; they dream, and appear to have hallucinations, and after full doses remain stupid for a day. There is some salivation, in the majority of cases vomiting occurs, and often defæcation. After twenty minutes or so the narcosis is sufficiently pronounced to permit of even major operations, such as amputations and laparotomy. Although the skin is congested and hot, there is no notable increase of secretion 
such as occurs in man and occasionally in horses. Cats are as much excited as horses or cattle, more so than dogs, and hypnosis is produced with difficulty.

Rabbits also have their motor and spinal centres prominently affected, and convulsions are more common than hypnotism. Mice receiving a fraction of a grain of morphine are restless, have spasms, breathe irregularly, but sleep only when dangerous doses are given. Birds are curiously insusceptible; relatively to their weight they take three hundred times the dose given to man, do not sleep, or show any alteration of the pupil. Pigeons swallowing ten grains, or receiving hypodermically three grains, are seized with inco-ordinate movements, laboured breathing, and convulsions ; the temperature falls sometimes five or six degrees, and usually they die. Mixed with the food of poultry, opium retards digestion, the crop remaining full for three times the normal period. Cold-blooded animals are even less susceptible than birds. In proportion to their weight, frogs take with impunity a thousand times the dose that would poison a man, and one or two grains cause convulsions, paralysis, and respiratory arrest.

TOXIC EFFECTS.-Opium and its preparations annually destroy in Great Britain upwards of one hundred human lives, three-fourths being children under five years. In the domesticated animals accidental poisoning with opiates occurs occasionally, intentional poisoning very rarely.

Horses without danger may be given by the mouth about one hundred times as much opium as would poison a man. Hertwig mentions that two to four drachms produce slight stimulation, and that an ounce in solution caused first increased liveliness, and, after two hours, dulness, diminished sensibility, slower circulation, less frequent evacuations, and stupor-symptoms which continued for twelve hours, but entirely disappeared the following day. Two ounces and a half induced similar effects, with convulsions and death in about twenty hours. Four drachms powdered opium caused little effect for seven hours, and then only acceleration of the pulse. Even four ounces of laudanum are stated to have had little effect.

Dun gave a strong, healthy cart-horse one ounce powdered 
opium dissolved in water; the pulse in eight minutes fell from 44 to 34 beats per minute; the superficial muscles were relaxed, the nasal mucous membrane blanched, and the animal was dull and dejected. After half an hour four drachms were given, increasing the dulness and lowering the pulse to 32. Half an hour later the animal, continuing in the same state, was destroyed by cutting the carotid artery. A mare, aged and rather feeble, had drachm doses in solution thrice a day. She exhibited dulness, loss of appetite, torpidity of the bowels, diminished force of the pulse, and died on the fourth day, having received nine doses. One drachm, given thrice a day to a healthy donkey, induced after the sixth dose acceleration of the pulse to 88, restlessness, vertigo, nausea, champing of the jaws, and death on the third day.

In horses, Harley and Mavor hypodermically injected four grains of morphine acetate, and recorded marked acceleration of the pulse, increase alike of its force and volume, restlessness, pawing, augmented moisture of mouth and skin, elevation of temperature, and slight dilatation of the pupils. Twelve grains, dissolved in three drachms of water, injected by three punctures, produced light drowsiness, followed after three hours by excitement, restlessness, and slight delirium, continuing about six hours. Thirty-six grains, in seven drachms of water, introduced by three punctures into a seven-year-old hunter in good condition, caused drowsiness and stupor, coming on in fifteen minutes, and continuing for three hours, slight muscular tremors, awkward, staggering gait, leaning against the sides of his box, dilated and fixed pupils, blindness and insensibility to light; the respiration, at first slow and sighing, gradually became accelerated. The dilatation of the pupil is opposed to the contraction so constantly seen in man. After the third hour restlessness and delirium set in, continuing for seven hours; he walked rapidly, and even ran round his box; his pulse was 96, full and thrilling; the skin damp with perspiration; the membranes of the eyes, nose, and mouth intensely injected. For twenty-four hours the effects continued ; the secretions were, however, unaffected, but the horse was left exhausted. 
F. Smith and C. Rutherford made a series of experiments with alkaloids at Aldershot, and furnished the following notes :-A bay gelding had three grains morphine injected hypodermically, and in fifteen minutes had shaking of the head, which continued more or less for a couple of hours ; but there were no other appreciable symptoms. A bay gelding, fed on hay and grass, with a pulse of 38 , respirations 12 , and pupil half an inch vertical diameter, had five grains morphine injected. In forty minutes the pulse was 42 , respiration and pupils unchanged. He walked occasionally round his box, threw back his ears ; a patch of perspiration appeared on the breast, around the seat of injection; the head was frequently shaken; the animal was easily startled. The effects wore off in about an hour and a half.

In experiments with morphine and atropine injected together, C. Rutherford records that the horses were more readily excited than when morphine was given alone. They moved almost constantly round the box, had rhythmical movements of the head, and dilated pupils. Morphine five grains, injected with fifteen minims atropine solution, caused, in fifteen minutes, uneasiness, walking round the box, throwing up and shaking of the head, slight pawing, dilated pupils, nervousness, and excitability. These symptoms continued for one and a half hours when they gradually abated; but the animal for six hours still remained easily excited, and a 'little on the move.'

Twelve grains morphine acetate, dissolved in a pint of water and swallowed by a horse, had no effect beyond increasing the pulsations eight beats. One hundred grains of the acetate, swallowed in solution, caused convulsions and killed the horse in three hours (Wood). Kaufmann states that forty-five to seventy-five grains of the hydrochloride, given hypodermically, poison horses.

Ruminants are not so susceptible, especially to opiates given by the mouth. Cows and sheep, to which Dun gave respectively an ounce and four drachms of opium, exhibited dryness of the mouth, occasional nausea and restlessness, acceleration, and subsequently slight slowing of the pulse. Fröhner hypodermically injected a cow with fifteen grains morphine hydrochloride; she lowed hoarsely, became ex- 
cited, and tympanitic ; but the effects passed off in an hour. Kaufmann gives one to two drachms as the toxic dose for cattle, and fifteen to thirty grains for sheep and goats. Swine, receiving one or two drachms of opium, become first lively and then dull and sleepy, with hot skin and constipated bowels.

Dogs, swallowing moderate doses, usually become stupid and drowsy, but occasionally are rendered delirious, especially by large doses. The pupil is not dilated, as is frequent in the horse and cat, nor continuously contracted, as in man, but is contracted while the dog is asleep or narcotised. Two to three drachms of opium cause, within a few minutes, increased force and frequency of the pulse; followed by nausea, a staggering, unsteady gait, twitching of the limbs, clonic spasms, stertorous breathing, drowsiness, stupor, and sometimes death. The symptoms continue from three to fifteen hours, and most animals surviving the latter period recover. Harley injected twenty minims laudanum under the skin of a bitch about $25 \mathrm{lbs}$. weight; she was nauseated ; in fifteen minutes she vomited ; had spasms of the diaphragm, the bowels acted, mucus ran from the mouth ; within an hour the pulse had fallen from 120 to 78, and was irregular ; the animal lay quiet, but did not sleep or show narcotism. Twenty minims more were injected; the pulse fell to 72 , and was regular; respirations 16, and regular; half an hour later she closed her eyes and was drowsy, continued so for an hour, but did not actually sleep.

Morphine acetate, half a grain, subcutaneously injected into a bitch weighing $25 \mathrm{lbs}$, in a few minutes caused vomiting and urination. She lay motionless, her nose on the rug, her fore and hind limbs fully extended. For upwards of three hours she was so completely narcotised that the eyes were insensible to light, the pupils much contracted; the pulse fell from 120 to 50, and became irregular; the respirations went down from 20 to 14, and were shallow; the muscles were flaccid. Two to three grains, subcutaneously injected, killed dogs of $12 \mathrm{lbs}$. to $16 \mathrm{lbs}$. in ten or twelve hours; doses insufficient to kill develop in most dogs excitant instead of soporific effects. 
The spinal cord is more notably affected than the brain; vomiting, nausea, restlessness, and delirium are the usual symptoms.

Post-mortem examination discovers the results of asphyxia. The blood is fluid and dark-coloured, but it does not yield on analysis any indications of the poison. There is general venous engorgement; the lungs and brain are markedly congested. The ventricles of the brain and subarachnoid spaces contain more fluid than usual.

ANTIDOTES.-Opium is not very quickly absorbed; when a poisonous dose has been swallowed, the stomach should be emptied as soon as possible, preferably by the stomach pump, and washed with solution of potassium permanganate. Administration of nitrites, and of small doses of atropine, hypodermically, maintain cardiac action; but the atropine must be used cautiously, as full doses are apt to intensify paralysis both of the heart and cord. Even better than atropine is caffeine, and the lethal tendency can be further combated by strychnine, used hypodermically, and by artificial respiration. Coma is prevented by giving strong coffee and stimulant enemata, and keeping the patient moving. Tincture of galls and other chemical antidotes are of little avail.

Medicinal USES.-Opium and morphine are used to relieve symptoms rather than to remove the conditions on which they depend. Small doses are nerve stimulants, and retard digestion and metabolism. The Cutch horsemen share their opium with their jaded steeds, and increased capability of endurance is observed alike in man and beast. In the lower animals, as already indicated, they do not produce the general calmative and hypnotic effects which characterise their use in man. In human patients opiates cause notable vascular dilatation in the skin, and hence relieve congestion and inflammation (Brunton). But in the lower animals this vascular dilatation occurs only when toxic doses are used (Fröhner), and accordingly the antipyretic and anti-inflammatory power of opiates must be considerably less than in man. Moreover, owing to the greater excitant action in veterinary patients, the administration of opium does not blunt the perception of pain as 
effectually as it does in human patients. When, however, a morphine salt is injected hypodermically, fifteen to thirty minutes previous to the inhalation of chloroform or ether, anæsthesia is intensified and prolonged, and the anæsthetic is economised.

In gastritis and gastro-enteritis, whether produced by disease or by swallowing acrid poisons, opiates are anodyne, astringent, and hæmostatic. Obstinate chronic vomiting, in either dogs or pigs, whether depending upon irritation of the stomach or of the vomiting centre, is generally relieved by a few grains of opium, given with chloroform or chloral hydrate. In gastric irritability it is sometimes prescribed with bismuth subnitrate. When, in weakly, young, growing animals, food is hurried too rapidly through the digestive canal, opium checks excessive secretion and peristalsis, and, conjoined with mineral acids or arsenic, should be given shortly before feeding.

Diarrhœa, whether occurring from congestion of the alimentary mucous membrane, or as a symptom of other ailments, is often checked by a laxative, which carries away offending matters. Occasionally, however, the bowels continue irritable and relaxed; opium in such cases abates irritability, diminishes excessive secretion, and is adminisstered with well-boiled starch gruel, sometimes conjoined with an antacid, sometimes with acids, bitters, or vegetable astringents. For such purposes the following recipes are used, alike in horses and cattle :-A drachm each of powdered opium, kino, gentian, and sodium carbonate; or a drachm of opium, a drachm of catechu, or half a drachm of tannin, with half an ounce of chalk. These drugs may be made into bolus with treacle or meal and water, or dissolved in ale or gruel, and given twice daily, or as required. An ounce of laudanum, thirty drops sulphuric acid, two drachms powdered catechu, with an ounce of ginger, aniseed, or fenugreek, make an astringent anodyne drench for diarrhœa in cattle, which may be given in gruel, ale, or spirit and water. Another useful prescription for relaxed bowels consists of an ounce each of laudanum, decoction of oak bark, ginger, and sodium bicarbonate, given several times daily in starch gruel. One-third of this dose 
suffices for calves of six months. For dogs, three to eight drachms laudanum, two to three drachms chalk, one drachm aromatic confection, and two drachms gum acacia, are added to seven ounces of water, and of this mixture one or two tablespoonfuls may be given every time the bowels are relaxed.

For dysentery, whether in horses, cattle, or dogs, opium is of service in allaying pain and straining, and may usually be freely given, along with antiseptics, both by the mouth and rectum. A drachm each of opium and tannin, with half a drachm powdered copper sulphate, may be repeated twice a day, for either horses or cattle. Whilst febrile symptoms continue, any such opium mixture must be used cautiously. In gastro-intestinal cases, opium is generally contra-indicated when secretion is impaired, or the liver and kidneys act imperfectly.

In enteritis in horses, Robertson used half a drachm each of powdered opium and camphor, with five minims Fleming's tincture of aconite, in a pint of gruel, with or without a dose of oil. In the rapidly fatal muco-enteritis amongst the heavier breeds of hard-worked horses, opium and calomel were prescribed in the earlier stages, and opium, belladonna, chloral hydrate, and ether in the second stages; but more prompt measures are needful to avert the deadly passive congestion and hæmorrhage, and morphine and atropine, sometimes used with ergotin, are hypodermically injected, and repeated every two hours.

In peritonitis, and hernia, to arrest intestinal movements, full doses of opium are administered. Aritagonising muscular spasm, opium or morphine is valuable in spasmodic colic in horses, being usually conjoined with ether, sweet spirit of nitre, chloral hydrate, chloroform, spirit of ammonia, or oil of turpentine, and with laxatives as aloes, and linseed or castor oil. For general service four or five drachms of aloes are rubbed down in a quart of tepid water, and, when the solution is nearly cold, an ounce each of laudanum and ether is added; while for large horses the dose of the anodyne and stimulant may be doubled. If more convenient, the aloes may be given in bolus, the laudanum and ether in draught. 
As an antispasmodic for the dog, Stonehenge advised half a drachm to a drachm each of laudanum and ether, given in an ounce of camphor mixture. The following case illustrates the antispasmodic effect of morphine on the dog :-A collie poisoned with strychnine, and so convulsed that recovery seemed impossible, had five grains morphine injected hypodermically; the muscular spasms ceased, he slept for twenty-four hours, and recovered (F. Smith).

Diseases of the respiratory organs, with shallow, embarrassed breathing, are unsuitable cases for full doses of either opium or morphine, which is apt still further to depress the respiratory centre, and lead to death by apnœa. Pleurisy, however, may be treated by large and more frequently repeated doses than bronchitis or pneumonia. Opiates, when absorbed, diminish excitability of the respiratory centre, and thus relieve cough, and pain of the throat and chest. Belladonna and opium, although in large doses opposed in their effects on the respiratory centre-the former acting as an excitant, the latter as a depressantin medicinal doses are sometimes advantageously conjoined in allaying bronchial irritability. In the catarrhal epizootics of horses, after a few doses of salines, half a drachm each of opium and belladonna extract, conjoined with an ounce of spirit of chloroform, ether, or sweet spirit of nitre, and repeated two or three times daily, frequently abates vascular congestion and cough. A similar prescription is useful in asthma-a common complaint in dogs; but in this, as in other diseases, more prompt and certain effects are obtained by the hypodermic injection of morphine and atropine.

Rheumatism is sometimes advantageously treated with opium, prescribed in the earlier and more acute stages with calomel and salines, and in chronic cases with turpentine and other stimulants, smart friction and warm clothing. Neuralgic pains occurring in horses, and causing puzzlingsometimes frequently shifting-lameness, are relieved, and occasionally removed, by morphine injected deeply into the affected muscles.

American practitioners prescribe opium and also morphine 
by the mouth and hypodermically, in combating the rigidity and pain of spinal meningitis. Tetanus, occurring in young animals, is sometimes treated by opium, conjoined with antiseptics and chloral or conium; while in the more serious cases amongst adults, spasms and morbidly acute sensibility have been checked for several hours by the hypodermic injection of morphine. In hysteria it is prescribed with potassium bromide, and camphor.

Opiates are of service alike in mares, cows, and bitches, in allaying post-partum irritability, straining, and bleeding, for which they are sometimes conjoined with chloral or chloroform. Morphine, given either by the mouth or hypodermically, alone, or, better still, conjoined with atropine, is often effectual in arresting premature labour pains. Some practitioners recommend opiates in epilepsy and chorea, but they are of little use in either. They were formerly used in polyuria of horses, but are not so effectual as iodine. Although powerless to arrest tuberculosis, they are often serviceable in relieving the accompanying cough and diarrhœa.

Opiates are contra-indicated in acute fever, in congestive and inflammatory diseases of the brain or kidneys, and in obstinate constipation. Full doses, depresssing respiratory functions, prove injurious when there is a tendency to death by suffocation.

Externally, opium has been used to relieve the pain of wounds, bruises, boils, blistered and cauterised surfaces, and superficial inflammation of the eye, skin, and joints. For such purposes five to ten drops each of the tincture (or aqueous solution), and Goulard's extract, may be mixed with an ounce of water. With borax or alkaline carbonate, it is useful in abating the irritation of prurigo and acute eczema. For hæmorrhoids, opium is conjoined with gall ointment. It is the anodyne chiefly relied on for injections and suppositories in enteritis and dysentery, as well as in irritation and inflammation of the uterus, kidneys, bladder, and rectum. As a clyster, opium is used in about the same quantities as are given by the mouth. Not only does opium relieve pain, but peristalsis is lessened and the animal quietened. 
Doses, etc.-Of opium, horses take 3 ss. to $3 \mathrm{ij}$. ; cattle, 3 ij. to 3iv.; sheep, grs. x. to grs. lx.; pigs, grs. v. to grs. xx. ; dogs, gr. i. to grs. vj. ; cats, gr. ss. to grs. ij. Of morphine salts, horses and cattle take grs. iii. to grs. xv.; sheep, gr. ss. to grs. ii. ; pigs, gr. $\frac{1}{10}$ to gr. ss. ; dogs, gr. $\frac{1}{10}$ to gr, $\frac{1}{4}$, given in pill or dissolved in diluted spirit, slightly acidulated either with hydrochloric or acetic acid. In canine practice lozenges containing gr. $\frac{1}{10}$ opium, or gr. $\frac{1}{36}$ morphine hydrochloride, will be found convenient. Morphine is unsuitable for cats. For hypodermic and intratracheal injections, the B.P. Injectio Morphinæ Hypodermica, containing 5 grains morphine tartrate in 110 minims, should be used. Stronger solutions of the acetate and hydrochloride are also prepared. Tablets, lamellæ, pellets, and dises, containing from gr. $\frac{1}{4}$ to several grains of a morphine salt are very convenient, especially for hypodermic use.

Tolerance alike of opium and morphine is as quickly acquired in the lower animals as in man. It is increased not only by frequent use but by acute pain. Special susceptibility is observable in young animals, in which, accordingly, reduced doses must be used. Although opium and morphine resemble each other, the crude drug is more apt to cause gastric disturbance and constipation, while the alkaloid, when used hypodermically or intratracheally, is more effectual in arresting pain.

The several actions are altered, intensified, or repressed by combination with other drugs. Hypnosis and quieting of nervous excitability are determined by combining the opiate with cannabis indica, chloral hydrate, and bromides ; antispasmodic effects, by conjunction with ethers or volatile oils; the checking of intestinal secretion, by prescription with lead acetate, tannic or sulphuric acid ; sudorific action is promoted by ipecacuanha, pilocarpine, ammonium acetate solution, diluents, and warm clothing; anodyne properties are increased by combination with atropine, and occasionally with aconite or prussic acid; while in malarial fevers, Indian and American practitioners prescribe opiates with quinine.

Veterinarians do not use so many preparations as are 
employed in human medicine. Crude opium is given to horses and dogs made into bolus or pill, and no other solid form is necessary. To reduce it to powder, it is first dried in a vapour bath, and its trituration is facilitated by mixture with potassium sulphate, or other hard salt. The extract, containing 20 per cent. of morphine, though somewhat less bulky than crude opium, is seldom prescribed. Dover's powder, the pulvis ipecacuanhæ compositus, consists of one part each of powdered opium and ipecacuanha, and eight parts potassium sulphate, added to facilitate trituration and intermixture of the vegetable matters. It contains 10 per cent. of opium, and is given to dogs in doses of grs. v. to grs. xv. A watery solution, made by rubbing down opium in hot water, and giving both dissolved matters and residue, has the merit of being cheaper than the tincture, and is more prompt and effectual than the solid drug.

The B.P. tincture of opium, popularly known as laudanum, is made with three ounces of opium and equal parts of distilled water and alcohol (90 per cent.). It is now standardised to contain 0.75 per cent. of anhydrous morphine. An ounce contains the soluble matter of nearly 33 grains of opium, or about one grain in 15 minims. For immediate effects laudanum is usually preferable to solid opium. The dose for horses and cattle is $f z j$. to $f$ ziij.; for sheep and pigs, $f 3 \mathrm{ij}$. to $f 3 \mathrm{vj}$. ; for dogs, $m_{v}$. to $m x \mathrm{xl}$. The vinegar and wine of opium are seldom used in veterinary practice ; but the Ammoniated tineture, known as Scotch paregoric, containing one grain of opium in 96 minims is sometimes given. Tinetura eamphoræ composita or English paregoric, made with tincture of opium, benzoic acid, camphor, oil of anise, and alcohol (60 per cent.), contains $\frac{1}{4}$ grain opium in each fluid drachm. The liquid extract of opium contains gr. $\frac{3}{4}$ of morphine in 110 minims; or gr. i. of opium in 15 minims. Equal parts of laudanum and soap liniment make an excellent anodyne, much used externally.

Heroin (diacetyl-morphine), derived from morphine, is a white crystalline powder, alkaline, odourless, with a bitter taste; soluble in acidulated water. The hydrochloride, soluble in two parts of water, is sometimes employed as a substitute for morphine in the treatment of respiratory 
diseases (cough, bronchitis, pneumonia, etc.). In many cases it is preferable to morphine, as it does not constipate, and very small doses suffice. For the dog it is five times more toxic than morphine. Dose.-Dogs, gr. $\frac{1}{24}$ th to gr. $\frac{1}{6}$ th, in pill in solution. For horses heroin is generally prescribed as a compound glycerol, containing $\frac{7}{16}$ th grain of heroin in each drachm, in doses of about an ounce two or three times a day. Dogs take $m_{x}$. to $m_{x x x}$.

\section{B ELLA D O N A}

Belladonn molia. Belladonna Leaves. The fresh leaves and branches of Atropa belladonna, collected when the plant is in flower. Deadly Nightshade. Nat. Ord.Atropaceæ or Solanaceæ.

Belladonne Radix. The root of Atropa belladonna, collected in the autumn and dried.

Atropina. Atropine. An alkaloid obtained from belladonna leaves or root. $\mathrm{C}_{17} \mathrm{H}_{23} \mathrm{NO}_{3}$.

Homatropin ж Hydrobromidum. Homatropine Hydrobromide. $\mathrm{C}_{16} \mathrm{H}_{21} \mathrm{NO}_{3} \cdot \mathrm{HBr}$. The hydrobromide of an alkaloid prepared from tropine (B.P.).

Belladonna grows wild in most parts of Great Britain, especially about old walls, edges of plantations, and shady places; but the great demand for its preparations has led to its extensive cultivation. It has a fleshy, wrinkled, branching, perennial root, a round, branched, reddish, downy, annual stem, three to five feet high; broadly ovate, acute, entire, smooth leaves, supported on short leaf-stalks of a sombre-green colour, and having a faint bitter taste ; pendulous, dark-purple, bell-shaped flowers, appearing in June or July ; a round, violet, berried, mawkish-tasted fruit, the size of a small cherry, and containing numerous kidney-shaped seeds. The plant has greatest activity towards the end of June and throughout July, when flowering is over, and before the fruit and seeds are ripe.

The active alkaloid atropine occurs in the plant as a bimalate. The leaves contain $0 \cdot 46$ per cent., the younger roots as much as $0 \cdot 60$ per cent. It is prepared from a strong 
tincture. It occurs in colourless acicular crystals, is volatile, has a bitter taste, is soluble in 300 parts water, readily soluble in alcohol and ether, and in chloroform. The more soluble sulphate, obtained by neutralising atropine with diluted sulphuric acid, is used in preference to the alkaloid. It gives a citron-yellow precipitate with gold perchloride. An alcohol solution of mercuric chloride added to a crystal or strong solution of atropine causes a precipitate, which becomes red on standing or when boiled. It powerfully dilates the pupil.

Atropine can be split up into tropic acid and tropine, and this base, reunited with other acids, forms tropeines. When combined with oxytoluylic acid, the crystalline homatropine is produced, and is used as the soluble hydrobromide. It is similar to but weaker than atropine, and the solution is preferred by oculists, as its effects, although as promptly and effectually produced, are not so inconveniently persistent as those of atropine sulphate. Isomeric with atropine is hyoscyamine, whilst hyoscine differs only slightly in its formula. They occur along with atropine in belladonna and other plants of the order Solanaceæ.

Actions ANd Uses.-Belladonna and atropine, combined with glycerin, alcohol or chloroform, which promote penetration, and, applied topically, paralyse sensory nerveendings, and hence relieve irritability and pain. Mainly by paralysing the terminals of the third nerve, they dilate the pupil, and are serviceable in relieving congestion and inflammation of the eye, and in facilitating its examination. Atropine is readily absorbed by mucous surfaces, and exerts its physiological effects on the organs with which it is brought into contact. Toxic doses, after stimulating, paralyse the spinal cord, brain, and medullary centres, causing irregular movements and delirium, with coma from impaired respiration, and kill by asphyxia. Medicinal doses stimulate respiratory and cardiac action, and hence combat collapse, respiratory depression, and poisoning by ptomaïnes. They antagonise spasm, are used both topically and internally in cases of intestinal and urinary colic and in constipation. They diminish the secretion of the salivary, sweat, gastric, mucous, and mammary glands, but not 
that of the kidneys, by which atropine is quickly excreted unchanged.

General Actions.-Atropine depresses the functions of sensory nerve-endings, and hence allays irritability and pain when applied to the itching or erythematous skin, when injected subcutaneously into a tender muscle, or into the neighbourhood of an irritated nerve, and also when carried in large doses to internal organs. Moderate or large doses increase heart action, by paralysis of the vagus-endings in the heart, while they raise blood-pressure by stimulating the medullary centre. They powerfully stimulate the respiratory centre. These cardiac and respiratory actions explain the value of atropine in the treatment of collapse, difficult or impaired breathing, and weakness from toxins and other sedative poisons.

Paralysing the special secretory nerve-endings of glands, atropine is a powerful antisecretory, diminishing or arresting secretion, and exerting this effect in whatever way it is used. Although devoid of action on voluntary muscles, moderate and large doses paralyse involuntary muscles, probably indirectly through the local nervous mechanism, and thus prevent griping and relax spasm of the hollow organs. A similar action on the bronchial muscles prevents spasmodic contraction of the bronchioles in asthma. In almost all animals atropine dilates the pupil, renders the eye bright, dry, and injected, paralyses the power of accommodation, and slightly increases intraocular tension. These effects result alike from the local and internal use of atropine, and depend upon its paralysing the third nerve terminals, and perhaps stimulating the sympathetic. Dilatation of the pupil reaches its maximum in dogs in twenty to twenty-five minutes, in herbivora in thirty-five to forty-five minutes. The effects of full doses of atropine last several days, but those of homatropine, although as quickly produced, pass off more rapidly.

Full doses cause dryness of the mouth, thirst, difficulty in swallowing, quickened pulse, delirium, with a tendency to irregular movements. The brain centres are stimulated, but the ends of motor nerves are paralysed, and hence result the concurrence of delirium, irregular movements, and 
lassitude. Like alcohol, atropine exerts primary stimulant and secondary paralysant action. It stimulates, then depresses the respiratory centre, and death results chiefly from paralysis of respiration. Small doses stimulate, but large paralyse the motor areas of the brain and the centres of the medulla. The vaso-motor medullary centres, as well as the peripheral vaso-motor ganglia, are stimulated by small, but paralysed by large doses. Small doses consequently raise blood-pressure and temperature. Continued use does not confer any tolerance as occurs with opium or arsenic.

Belladonna resembles hyoscyamus and stramonium, but is more active. It is somewhat similar to opium in its antispasmodic and anodyne effects; but the distinctions between the two are marked and various. Fairly large doses of belladonna or atropine stimulate and subsequently paralyse the central nervous system, and produce delirium, restlessness, and continued movements, followed by depression and coma; while similar doses of opium or morphine paralyse more particularly the brain centres causing narcosis and coma. Belladonna paralyses the vagus-endings and inhibitory mechanism of the heart, and hence accelerates the pulse, while opium slows it by central action. Atropine stimulates, while morphine depresses the respiratory centre. Atropine dilates, morphine contracts the pupil. The secondary effects of belladonna ally it to hemlock, which it also resembles in dilating the pupil, and paralysing the ends of sensory nerves. Atropine has no direct physiological antagonist, but morphine, caffeine, Calabar bean, prussic acid, and especially pilocarpine oppose some of its actions. Methyl and ethyl atropine, although paralysing the ends of motor nerves and retaining the specific effects of atropine on the eye, heart, and respiratory centre, have no tendency to tetanise.

Toxic EFFEcTs.-Horses were subjected to experiment by Hertwig. Twenty received four to six ounces of the dry pulverised herb, with meal and water, in four separate doses, at intervals varying from four to eight hours. In four or five hours, and on the succeeding day, he observed dulness, languor, uneasiness, dilated pupils, and a feverish 
mouth ; inappetence, impaired digestion, gas abundantly evolved from the stomach and intestines. The pulse about 90, was small, hard, and scarcely perceptible; breathing was short, quick, and accompanied by flapping of the nostrils; sensibility slightly diminished, but no drowsiness. Some of the cases exhibited abdominal pain; others imperfect power in the hind extremities; others terminated fatally in thirty to fifty hours after receiving the first dose ; but in most the symptoms gradually subsided, and in thirty-six to forty-eight hours the animals were perfectly well. Two to three ounces of the dried root acted on horses in a similar manner, and six ounces usually proved fatal.

With atropine sulphate, subcutaneously injected, Harley and Mavor made an extended series of experiments on a healthy six-year-old horse and a weakly two-year-old thoroughbred. One-twelfth of a grain dissolved in water caused in about half an hour acceleration of the pulse from 32 to 42 beats; half an hour later a further rise of ten beats was noted. The tongue and mouth were dry, and the temperature increased. The pupils began dilating after thirty-five minutes, and reached their maximum in an hour, when the iris was scarcely visible. The symptoms gradually receded, and in two to three hours had disappeared.

One-sixth of a grain caused restlessness and dryness of the mouth, and in thirty-five minutes an increase of 34 beats in the pulse, which was full, soft, and compressible, and only fell to its original number after six hours; the dilated pupils returned to their normal state after three hours; the secretions were unaffected.

One-fourth of a grain in twelve minutes increased the pulsations from 38 to 56, producing also slight irregularity ; the pupils gradually dilated, and in an hour reached their fullest expansion. These effects on the pulse and pupil, with dryness of the mouth and lips, continued unabated during three hours. For eighteen hours the animal remained dull and quiet.

Half a grain in twelve minutes fully dilated the pupils ; the pulse rose to 68 ; the mouth, tongue, and lips became 
dry; the horse gaped occasionally, and stood perfectly quiet; after three hours he showed considerable nervousness, and was restless when disturbed; for six hours the pulse continued weak and compressible, but the effects gradually declined.

Two grains, given subcutaneously, after fifteen minutes raised the pulse 35 beats, and weakened it, and caused dryness of the mouth, yawning, restlessness, and nervousness. The animal was confused, misjudged distances, and appeared under the influence of illusions; the membrane of the eye was injected. Occasional hiccough, tremulousness, and twitching of the superficial muscles continued for fourteen hours, when the symptoms gradually declined ; but the pupils remained dilated for twenty-four hours. Urine was frequently voided, and in increased amount; the mucus of the bowels and the bile were slightly augmented, the skin and the respiratory functions were not disturbed.

These and other experiments demonstrate that the maximum stimulation of the heart results from doses insufficient to produce nervous excitement. Medicinal doses seem to dull the cerebro-spinal nervous system, but over-doses cause increased sensibility to external impressions, wakefulness, and, in extreme cases, delirium.

F. Smith and C. Rutherford, experimenting on healthy horses with liquor atropinæ sulphatis, containing one grain of the alkaloid to 100 of water, injected into the chest quantities ranging from 15 to 30 minims without producing any notable effects. Injections of 30 to 60 minims reduced the pulse two or three beats, but had scarcely any effect on the pupil. Injections of 120 to 180 minims slightly slowed the pulse and rendered it softer, and increased the size of the pupil. Most of the subjects were quiet, some slightly sleepy, but all easily roused. Two experiments were made by introducing intratracheally $\frac{3}{10}$ grain of atropine sulphate, which caused immediately a gulp and cough; but the only further effects were, dryness of the mouth and slightly increased fulness and quickening of the pulse, which continued for about three quarters of an hour. 
Cattle, according to Hertwig, are as susceptible to the action of belladonna as horses. He states that two to four ounces of the root caused in cows violent symptoms, lasting forty-eight hours, and that large doses are dangerous.

Dogs receiving full doses of belladonna exhibit less marked cerebral, but more pronounced and prolonged cardiac effects than horses. This apparently depends upon the heart in dogs being more under the regulating influence of the vagi and inhibitory ganglia, which the drug specially paralyses. Harley found that while $\frac{1}{2}$ grain of atropine sulphate doubled the pulse-rate in horses, $\frac{1}{4}$ grain trebled it in dogs. Doses of $\frac{1}{96}$ to $\frac{1}{4}$ grain raised the dog's pulse in a few minutes from 120 to 400 , the beats continuing strong and regular; the pupils were so fully dilated that vision was confused; the mouth and nose were dry and hot. The larger doses further caused slowness and unsteadiness of movement, but no loss of sense or intelligence.

A Scotch terrier weighing $16 \mathrm{lbs}$. received $\frac{1}{80}$ grain atropine sulphate, injected under the skin; in four minutes the pulse rose from 118 to 280 ; the respirations advanced from 19 to 30 ; the pupils dilated to their full extent, the mucous membranes were dry, the animal excited and whining; these effects continued four hours. Dogs weighing $15 \mathrm{lbs}$. and $16 \mathrm{lbs}$. were killed in three hours by $\frac{3}{4}$ grain, with symptoms of prostration, rapid and feeble pulse, irregular and shallow respiration, muscular twitchings, and paralysed sphincters, death occurring in convulsions.

Hertwig found that 30 to 50 grains of the dried herb or root, given to dogs, in thirty minutes dilated the pupil, so that the iris was out of view, and rendered the eye insensible to bright light. Vomiting sometimes occurred, the nose became dry and hot, and the gait tottering from inability to move the hind extremities. In one to three hours the symptoms began to abate, but diminished irritability of the iris remained even after twenty-four hours. Orfila poisoned dogs with 15 grains of extract. Christison found that half an ounce of the watery extract given by the mouth killed dogs in about thirty hours, half that quantity introduced into a wound killed in twenty-four 
hours, while even smaller doses were more speedily fatal when injected into the jugular vein.

Rodents, such as rabbits, guinea-pigs, and rats, as well as pigeons, do not exhibit the marked acceleration of the pulse so remarkable in dogs and cats. Rabbits require 15 grains of green extract to poison them, pigeons 2 grains. The mydriatic action is much less marked in rabbits than in dogs or cats, and is scarcely noticeable in birds.

The post-mortem appearances are those of asphyxia. The blood is dark-coloured, and coagulates slowly ; the ventricles are generally empty and firmly contracted.

The antidotal treatment consists largely in treating the symptoms presented. In the early stages with excitement and hyperæsthesia it may be advisable to use morphine or other sedatives. It must be remembered, however, that the stimulating effect of large doses of atropine is soon followed by its depressant sedative action, and the combination of atropine and morphine may produce dangerous results on the respiratory centre. Stupor, if impending, is combated by moving the patient about, by the administration of alcohol, ammonia, and strong coffee, or by the galvanic battery. Subcutaneous injection of caffeine is recommended for human patients, with the cautious use of eserine, and artificial respiration (Brunton). Pilocarpine is the direct antagonist of atropine with regard to secretion and involuntary muscle, but is of little service as an antidote to toxic doses of atropine. As atropine is rapidly eliminated in the urine, recovery usually results if dangerous symptoms can be relieved, and life preserved for a few hours. Where urination is difficult, the catheter should be used.

MEDICINAL UsEs.-Stimulating the respiratory centre, abating excessive mucous secretion, combating spasm of involuntary muscles, and soothing irritability, belladonna and atropine are serviceable in catarrh, pharyngitis, laryngitis, and bronchitis in all patients, and are used in the several forms of inhalation, spray, electuary, and hypodermic injection. In influenza in horses, they besides beneficially stimulate the weakened heart. Robertson prescribed belladonna, with small doses of aconite, in the 
acute stages of respiratory diseases in horses; but it is chiefly indicated in the second stages, when secretion is over-abundant, swallowing difficult, and the throat irritable.

The noisy respiration accompanying some cases of catarrh, and the loud, prolonged, spasmodic cough of laryngitis, are usually relieved by its use. Along with ether or ammonium carbonate, belladonna abates the distressed breathing and cough occurring in bronchitis, as well as in distemper of dogs. In asthma it is useful, by preventing spasm of the bronchial muscle and the consequent constriction of the tubes. In cardiac syncope belladonna and atropine are also useful. In colic cases, as well as in enteritis, belladonna is conjoined with opium, and is stated to be specially indicated when the glandular structures of the bowels are implicated. Belladonna is given in obstinate constipation and obstruction of the bowels, with the view of relieving griping, and aiding the action of laxatives; and in irritable, spasmodic conditions of the rectum it is used in the form of suppository. For controlling the symptoms of tetanus in horses, 3i. to 3iij. of extract may be placed within the teeth several times a day. Neither the crude drug nor the alkaloid confers permanent benefit in epilepsy or chorea.

Whether prescribed internally, or as an injection, belladonna allays irritation of the bladder, rectum, and uterus. The extract is sometimes applied to the neck of the uterus to promote dilatation in cases of delayed parturition. In acute mammitis belladonna given internally, and applied locally, is a serviceable anodyne. By diminishing the activity of the secretory nerves, it checks the flow of milk, and allays congestion and inflammation. Belladonna and atropine are antidotes in poisoning by opium, Calabar bean, pilocarpine, and anæsthetics.

In examinations and diseases of the eye atropine sulphate or homatropine hydrobromide is used for dilating the pupil, assisting in the detection of cataract, and in testing the condition of the refracting media. For ophthalmic purposes solutions containing grs. ij. to grs. iv. to the ounce of distilled water are employed, 
and are sometimes conjoined with cocaine. They prevent prolapse of the iris in lesions of the cornea, suoh as perforating ulcer, which is frequent in dogs. In iritis mydriatics relieve congestion, and prevent or break down adhesions between the iris and the capsule of the lens, in such cases being generally alternated with myotics.

External topical applications often increase the effects produced by internal administration, belladonna plasters being applied to the spine in tetanus, to the loins in irritation of the kidneys or bladder, and to the throat in spasmodic cough. Belladonna and atropine diminish congestion, irritability, and pain in wounds, glandular swellings, and injuries of the œsophagus, in rheumatism, and in those hyper-sensitive conditions of the skin and muscles which sometimes occur in horses and hounds from severe exertion. To secure these results active preparations should be applied or deeply injected, as near as possible to the painful spot. In cases of emergency atropine sulphate, administered hypodermically, is preferable to any belladonna preparation.

Doses, etc.-Of the dried leaves horses and cattle take

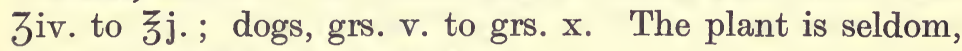
however, used in the crude form.

An active green extract is made by the B.P. process, by which $100 \mathrm{lbs}$. fresh leaves and young branches yield 6 to $7 \mathrm{lbs}$. of extract. The dose for horses is $3 \mathrm{j}$. to $3 \mathrm{ij}$.; for

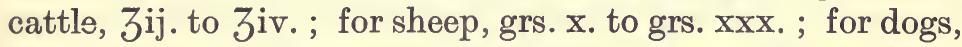
gr. i. to grs. v. In catarrhal and influenza cases the extract is frequently prescribed with alcohol, ether or camphor. A convenient electuary is made with liquor ammonii acetatis, camphor, and treacle.

The liquid extract, made with belladonna root and rectified spirit, contains $\frac{3}{4}$ grain of alkaloids in 110 minims. The tincture is made with 2 ounces of liquid extract and sufficient alcohol (60 per cent.) to form 30 ounces. The B.P. tincture is now standardised to contain 0.05 per cent. of alkaloids. The succus is obtained by bruising the fresh leaves and young branches of belladonna, pressing out the juice, and adding to every three volumes of juice one of 
alcohol (90 per cent.). An ounce is the dose for the larger animals, $m_{\mathrm{v}}$. to $m_{\mathrm{x}}$. for the smaller. The tincture is given in similar doses.

The linimentum belladonnæ, prepared with liquid extract 10 , rectified spirit 7 , camphor 1 , and water 2 , contains 0.37 per cent. of alkaloids. Veterinarians frequently extemporise an ointment by melting over a water bath one part of extract and four of vaseline, and adding, as the mixture cools, half a part of camphor.

For external application, equal parts of belladonna extract and glycerin may be used. It mixes readily with water. The plaster is made by evaporating four ounces of the liquid extract until it is reduced in weight to one ounce, and adding five ounces of resin plaster previously melted (B.P.).

Atropine, being sparingly soluble in water, the sulphate is generally used, and is about fifty times the strength of the green extract. Subcutaneously the dose of atropine sulphate (one in twenty) for horses and cattle is gr. $\frac{1}{2}$ to gr. $1 \frac{1}{2}$; dogs, gr. $\frac{1}{60}$ to gr. $\frac{1}{6}$ (Trasbot). The B.P. liquor atropinæ sulphatis in general use, consists of grs. $17 \frac{1}{2}$ of atropine sulphate, grs. 2 of salicylic acid, and ऊiv. of distilled water, and contains one grain of the salt in 110 minims. For horses and cattle the dose is $\mathrm{f} 3 \mathrm{i}$. to $\mathrm{f} 3 \mathrm{ij}$. ; for dogs, $\mathrm{m}_{\mathrm{i}}$. to $m_{v}$. When used hypodermically, the minimum or still smaller doses should be prescribed. Injectio atropinæ hypodermica contains grs. jv. of atropine sulphate in $\xi^{j}$. of water. Specially prompt and marked antispasmodic and anodyne effects are secured, as stated, by conjoining about equal proportions of atropine and morphine solutions. For ophthalmic and other purposes, tissue papers containing $\frac{1}{250}$, and lamellæ containing $\frac{1}{100}$ and $\frac{1}{120}$ grain atropine sulphate are convenient. The ointment is composed of atropine, grs. 10, oleic acid, grs. 40, and lard, grs. 450 .

Homatropine hydrobromide, as already indicated, is sometimes substituted for atropine sulphate for internal administration, and still more frequently for eye cases. To dilate the pupil, a solution, containing grs. iv. homatropine hydrobromide, to the ounce of water or castor oil, may be employed. 


\section{A F F EIN E}

Cafreina. An alkaloid usually obtained from the dried leaves of tea, Camellia thea, or the dried seeds of Coffea arabica. $\mathrm{C}_{8} \mathrm{H}_{10} \mathrm{~N}_{4} \mathrm{O}_{2}, \mathrm{H}_{2}$ O. (B.P.) Nat. Ord.-Ternströmiaceæ or Rubiaceæ.

Caffeine and theine are now considered identical, and the same alkaloid is also got from the seeds of the Guarana or Paullinia Sorbilis, the leaves of Ilex paraguayensis, as well as from the Kola nut. Caffeine (Trimethyl-xanthine) is homologous with theobromine (Dimethyl-xanthine), which is obtained from the nibs of the Theobroma cacao. Caffeine occurs in colourless, inodorous, acicular crystals, soluble in seventy parts cold water, in one of boiling water, seven of chloroform, and in forty of alcohol. Treated with a crystal of potassium chlorate, and a few drops of hydrochloric acid, and the mixture evaporated to dryness in a porcelain dish, a reddish residue results, which becomes purple when moistened with ammonia.

Caffeine citrate, obtained by adding caffeine to a hot solution of citric acid, is a white, inodorous powder, with an acid taste and reaction. It is soluble in thirty-two parts of water, and in twenty-two of alcohol.

ACTIONS AND USES.-Cadeine stimulates and subsequently paralyses the nerve-centres of the cerebrum, cord, and medulla. In dogs, cats, rabbits, and rats, full doses, hypodermically injected, do not, as in man, act prominently on the brain, but chiefly affect the spinal cord, exalt reflex excitability, and cause muscular rigidity, convulsions, and tetanus (Phillips). Large doses swallowed by dogs, moreover, sometimes cause vomiting, and gastro-intestinal irritation.

Like theobromine, it exerts a restorative effect on both voluntary and involuntary muscles, by direct action on the muscle fibres, enabling them to perform increased work. On account of its stimulating the medulla and cardiac centres, moderate doses increase respiration and pulse rate, and raise blood-pressure; and, resembling strychnine and veratrine, caffeine antagonises heart and lung inability and 
paresis. It hence steadies and strengthens the quick action of the weak heart in exhausting diseases, thus acting like digitalis, but more promptly and with more notable diuresis. As a nerve stimulant, caffeine has been given in indigestion in horses, milk-fever in cows, and to dogs prostrated with distemper. It is excreted in the urine and is a diuretic, increasing both the amount of the urinary solids and fluids, but especially the latter. It is prescribed in cardiac, hepatic, and renal dropsies, and is used as an antidote for the cadaver alkaloids and ptomaines, for the paralysis of curare, and for the narcoses of morphine, chloroform, and alcohol. Coffee is not identical in action with caffeine, for besides 0.50 of the alkaloid, it contains aromatic oils and tannic acid. Tea, well diluted with milk, is sometimes serviceable for horses, and still more so for foals, calves, and dogs reduced by acute disease.

Doses, etc.-For horses and cattle, grs. xxx. to grs. cxx. ; for dogs, according to size, gr. ss. to grs. ij. When swallowed, caffeine is liable to produce gastro-intestinal irritation, and hence should be given hypodermically in the minimum doses mentioned. Hypodermic injection: caffeine, grs. xxx.; sodium benzoate, grs. xxx. ; distilled water, 3 jss. Caffeine citrate, doses for horses and cattle, $3 \mathrm{ij}$. to $3 \mathrm{jv}$.; for dogs, gr. i. to grs. vj., according to the patient's weight.

Theobromine (Dimethyl-xanthine) is the active principle of cacao seeds (Theobroma cacao), which also contain about 45 per cent. of the concrete oil, cacao butter. Theobromine is a white crystalline powder, with a bitter taste, nearly insoluble in water, alcohol, and ether. It is very slightly irritant to the digestive mucous membrane, has little if any influence on blood-pressure, and is a prompt and powerful diuretic, directly stimulating the renal epithelium. In cardiac odema and ascites of the dog, grs. iv. to grs. xxx., divided into four or six doses, in pill, syrup, or powder, may be given daily for five or six days ; and after an interval the medicine may be repeated if required.

Diuretin, a compound of sodium theobromine and salicylate of soda, is frequently preferred to theobromine as a diuretic. It is freely soluble in water, and may be prescribed in the same doses as theobromine. 


\section{CANNABIS INDICA}

Indian Hemp. The dried, flowering, or fruiting tops of the female plant of Cannabis sativa, grown in India, from which the resin has not been removed (B.P.). Nat. Ord.-Cannabineæ.

The Cannabis sativa cultivated in India, and also in the southern states of America, attains a height of four to ten feet. The stalks, leaves, female tops, fruit, and exuding resin are used in making the extract, the preparation generally prescribed. The most active extract is stated to be obtained from the resinous juice, cannabin or churrus, which, although exuding from various parts of the plant, appears to be more potent when got from the female flowering tops. Churrus, mixed with tobacco and treacle, is smoked in the East as an intoxicant, producing dreamy narcosis. The larger leaves and fruit, with adhering resin, constitute bhang, which is sometimes given to horses on long journeys. Gunjah consists of the leaf-stalks, with adhering brown leaves, dried, flowering, and fruiting tops and resinous exudate. Haschisch is an Arabian preparation obtained from the tops of the female plants after flowering. In India, bhang and gunjah are given to vicious horses when being shod, or when undergoing surgical operations.

Indian hemp, for pharmaceutical use, occurs in compressed, rough, dusky green masses, consisting of the branched upper part of the stem bearing the leaves and pistillate flowers or fruits, matted together by a resinous secretion. The upper leaves are simple, alternate, 1-3-partite, the lower are opposite, and digitate. The fruit is one-seeded, and supported by an ovate-lanceolate bract (B.P.). The composition of cannabis indica is still uncertain, but the following constituents have been obtained :-

Cannabin, a glucoside ; cannabinol, an oil which is said to be the active principle ; cannabene, a volatile oil ; tetanocannabine, and a resinous body cannabinon, insoluble in water, soluble in alcohol, ether, chloroform, fats, and volatile oils.

The extract is directed to be prepared by exhausting Indian hemp in coarse powder, with alcohol (90 per cent.), 
by percolation, and evaporating the percolate to the consistence of a soft extract (B.P.).

Actions AND Uses.-Indian hemp is a deliriant-narcotic, hypnotic, anodyne, and antispasmodic. Bhang is used in India by all classes as a pick-me-up, and in larger amount to induce pleasing, dreamy narcosis. Similar effects are stated to be produced in horses ; the flagging appetite is improved, capacity for exertion increased, exhaustion and restlessness are overcome. Gunjah is a more active anodyne and narcotic. It has long been used in India to dull sensibility during surgical operations. Christison stated that for energy, certainty, and convenience, Indian hemp is the next anodyne to opium, and often equals it. R. Rutherford for several years in India used gunjah largely in the treatment of equine colic, and he states that it relieves spasm and pain as quickly as opium, and without arresting the action of the bowels or producing delirium. American practitioners sometimes prescribe it in diarrhœa, occasionally conjoining it with chloroform and aromatic ammonia. F. Smith prescribed the extract, in the treatment of colic, enteritis, laminitis, and other painful affections, with excellent results. In India C. Rutherford administered the extract in graduated doses of one drachm to an ounce, in bolus, to twenty-six geldings and mares of various stamps and temperaments. Eight animals received one drachm; in from one to two hours, usually after a little preliminary excitement, they became sleepy, but were readily roused; there was no uniform effect on the pulse or breathing. The appetite was not affected. In geldings the penis was drawn. A prominent feature was excitement when trotted, most moving as if drunk. Usually within six or eight hours the effects passed off.

Two drachms administered caused no preliminary excitement. The temperature usually receded about one degree, the pulse fell in one subject 8 beats per minute, respirations were reduced about two movements. Food was neglected, trotting was very unsteady. Two hours later drowsiness appeared, the eyes were heavy, the lower lip pendulous, the penis drawn sometimes as much as four inches. The effects continued ten or twelve hours. 
Several animals receiving three drachms of cannabis extract, and others six drachms, manifested very similar symptoms. The dulness and sleepiness, however, came on more quickly, and were more marked, than when smaller doses had been given. When moved, the subjects showed unsteadiness on the fore-limbs, staggering, inability to trot straight, the penis protruding. The fæces were discharged at unduly long intervals, and in reduced amount. The effects continued from twenty to twenty-six hours. Eight drachms in bolus with linseed meal were given to a nervous, kicking five-year-old. For two hours he tossed his head incessantly, subsequently stood very quiet, but when touched became excited, ate grass slowly, but left portions between the lips. By the seventh hour he became very sleepy, took no notice of any one, he was unsteady on his forelegs, the body swayed backwards and forwards, the muscles about the elbow and flank quivered, the penis was drawn about nine inches. There was no movement of the bowels for sixteen hours. By the twenty-fifth hour he brightened up; and in thirty hours was again normal. A second horse received eight drachms cannabis extract ; in three and a half hours he became sleepy, with penis drawn three to four inches. An hour later the head was down, the eyelids almost closed, the lower lip pendulous. He was unsteady on his legs, swayed backwards and forwards, took no notice of flies on face or body, when trotted, did so sideways and very unsteadily, the pulse and respiration were lowered. For six hours he remained in the same quiescent sleepy state, noticing nothing, and did not lie down during the night. By the twenty-fourth hour he got brighter, but was still unsteady on his limbs. No dung was passed for thirty hours, when some dry, small pellets were discharged. Although continuing quiet, he gradually became lively, and in thirty-five hours was perfectly recovered. Hobday states that doses of ten grains to two drachms of extract, administered to dogs, although quickly causing stupor, with paralysis of the hind-limbs - which might continue one or two days-had never proved fatal, these effects gradually passed off, and their removal was expedited by cocaine.

Indian hemp has proved serviceable in abating the 
delirium occurring in some cases of parturient apoplexy, and in tranquillising the involuntary spasms of chorea in dogs. In the form of suppository it is sometimes substituted for opium in the treatment of irritable or painful conditions of the rectum and urino-genital organs. F. Smith and other veterinarians bear evidence to the narcotic and analgesic effects of the thick, treacle-like, semi-solid extract obtainable in India. An ounce, it is stated, very shortly renders the horse so sleepy that he can be roused with difficulty, and may be severely pricked with a needle without wincing. The Indian extract is devoid of the excitant effects which opium exerts on many horses.

Doses, etc.-Of the extract, horses and cattle take $3 \mathrm{j}$. to 3iv. ; dogs, gr. j. to grs. iij. in bolus or pill. Larger doses may be given without danger. F. Smith prescribed one ounce, sometimes, two, three, or more ounces of the extract, for horses affected with colic, enteritis, or laminitis. It is often advantageously conjoined with chloral hydrate. The B.P. tincture is made with one ounce of the extract and a pint of alcohol (90 per cent.). It contains one grain of extract in twenty-two minims. The tincture is inconvenient to use, as water precipitates the resin. It may be given to horses in doses of $\xi_{j}$. to $\xi_{i v}$., or more, mixed with mucilage, a drachm to the ounce of tincture, before adding water or other vehicle. As the quantity of Cannabinol contained in different samples of Indian hemp varies considerably, only standardised preparations should be used.

\section{N UX - V O M I C A}

Nux-Vomica. The dried ripe seeds of Strychnos nuxvomica. Nat. Ord.-Loganiaceæ.

StrychNine. Strychnina. An alkaloid obtained from the dried ripe seeds of Strychnos nux-vomica, and other species of strychnos (B.P.).

The Strychnos nux-vomica grows on the southern coasts of India, in many islands of the Indian Archipelago, and in the northern parts of Australia. It is a moderate-sized tree, with crooked stem, irregular branches, tough white 
wood, known in commerce as snake-wood; grey or yellow bark-the poisonous, false Angustura bark; a globular berry, about the size of a small apple, containing, amid soft gelatinous pulp (which birds are said to eat with impunity), five round, disc-shaped, ash-grey seeds, about an inch in diameter. The seeds have a central scar or hilum on one surface, are covered with short satiny hairs, have an intensely bitter taste, and are tough and horny. Nux-vomica contains two poisonous alkaloids-strychnine and brucineeach present to the amount of about one per cent.; the soluble, amorphous strychnic or igasuric acid, which is allied to mallic acid; sugar, fat, loganin, and igasurine.

Strychnine $\left(\mathrm{C}_{21} \mathrm{H}_{22} \mathrm{O}_{2} \mathrm{~N}_{2}\right)$ is prepared by splitting the nuxvomica seeds, steaming and reducing them to powder, which is digested with spirit and water. The spirit is recovered by distillation. To the watery extract lead acetate is added, which precipitates acid and colouring matters. The filtered solution is treated with ammonia, which precipitates the alkaloids.

Strychnine occurs in trimetric prisms, is colourless and inodorous ; it requires for solution 5760 parts of cold water and 2500 parts of hot water, but its intensely bitter taste is appreciable even when diluted with 30,000 parts of water. It is soluble in 150 parts of spirit, and in 6 parts of chloroform, and nearly insoluble in ether. It is not coloured by nitric acid, and leaves no ash when burned with free access of air. It forms crystalline, colourless, intensely bitter salts, of which the hydrochloride is official.

It is readily recognised. On a white plate a crystal is dissolved in pure sulphuric acid without change of colour, but when the dissolved alkaloid is made to mingle with a drop or two of an oxidising solution, such as that of potassium permanganate, there is produced a characteristic violet coloration. Its extreme bitterness, and the tetanic spasms produced in frogs and other small animals by solutions containing the $\frac{1}{5000}$ th part of a grain are valuable corroborative tests.

BRUCINE or BRUCIA $\left(\mathrm{C}_{23} \mathrm{H}_{26} \mathrm{~N}_{2} \mathrm{O}_{4} \cdot 4 \mathrm{H}_{2} \mathrm{O}\right)$ is associated with strychnine in the seeds. It occurs in colourless prismatic crystals; is almost as bitter as strychnine, but is more 
soluble in water. It is reddened by nitric acid and chlorine. Strong sulphuric acid containing nitric acid colours brucine a rich rose, but on adding potassium permanganate, the play of colours observed with strychnine is not produced.

ACTIONS AND USES.-Nux-vomica, strychnine, and brucine are stimulants of the motor centres of the spinal cord, and of other motor centres. Full doses produce tetanic convulsions, which cause death from asphyxia, or from subsequent paralysis and exhaustion. Medicinal doses are gastric, vascular, and nerve-tonics, and anti-paralysants. They destroy enzymes and other vegetable and animal organisms.

General ACTIONS.-The alkaloids differ from each other and from nux-vomica mainly in the degree of their action. Strychnine is fully fifty times as active as the powdered nux, and nearly twenty to thirty times as active as brucine. The St. Ignatius' bean yielded by the Strychnos Ignatiaa tree grown in the Philippine Islands-contains strychnine and brucine. Akazga, the ordeal plant of the West Coast of Africa, belongs to this order, and yields akazgine, which is analogous to strychnine. Other trees of the same order also yield tetanising poisons. Allied to this group are thebaine and some other opium alkaloids; gelsemine, the alkaloid obtained from the rhizome and rootlets of yellow jasmine ; calabarine, an alkaloid found in Calabar bean ; and picrotoxin, a neutral principle prepared from the seeds of Anamirta paniculata (Cocculus indicus). The methyl compounds of strychnine and brucine, instead of stimulating spinal and motor centres, and producing convulsions, resemble curare, and paralyse the ends of motor nerves.

Strychnine lessens oxidation of protoplasm and of blood, and checks fermentation, but not nearly so effectually as quinine. Low organisms, wetted with weak watery solutions, have their activity increased; wetted with strong solutions, their activity is diminished and they are destroyed. It stimulates the whole central nervous system, but especially the spinal cord, exalting its reflex excitability, and it also stimulates other reflex nerve centres. Small to moderate doses promote secretion of saliva, improve appetite, stimulate the intestinal muscular coat and increase peristalsis, and hence assist in overcoming constipation. 
They stimulate the medulla with the respiratory, cardiac, and vaso-motor centres, and hence increase the rapidity and depth of the respirations, the number and force of the pulsations and raise blood-pressure, although the heart itself may be somewhat slowed by vagal inhibition. Larger doses cause muscular twitching, hyperæsthesia, greater acuteness of the special senses, and clonic convulsions. The extensor muscles usually overcome the flexor muscles, producing opisthotonos. Death results from asphyxia, occurring during a spasm, or from paralysis and collapse, occurring during a period of relaxation. Strychnine is absorbed rapidly from the small intestine and rectum, and still more rapidly from the bronchi and cellular tissues. It has been detected in the spinal cord, brain, liver, spleen, and blood. It is tardily excreted unchanged, or as strychnic acid, in the urine, in which it has been found an hour after administration, and has also been detected forty-eight hours later. Owing to its slow elimination strychnine should be regarded as a cumulative medicine.

Toxic Actions.-Poisonous doses within a few minutes produce in all animals trembling and twitching of voluntary and also of involuntary muscles, and violent spasms, usually lasting one to two minutes, gradually becoming more frequent and severe; the glottis, diaphragm and other muscles of respiration being involved, death is caused by asphyxia. The symptoms (and mode of death) resemble those of tetanus, but are suddenly developed, intermittent, and more rapidly fatal. The temperature is raised sometimes two degrees.

The several classes of animals differ in their susceptibility to the actions of strychnine. Horses and cattle are not so readily affected as men and dogs. Poultry are said to be less easily affected than other birds; while guinea-pigs and some monkeys seem quite insusceptible to its action, at least so long as it is given by the mouth (Wood).

Horses after swallowing six grains of strychnine had twitching of the muscles, and were poisoned by twelve grains in about twelve minutes (Tabourin). Five grains in bolus produced, after six hours, abdominal pain, laboured breathing, acceleration of the pulse from 42 to 60 , excite- 
ment when touched, and tetanic spasms. Twelve hours later the pulse was 96 , and subsequently rose to 120 . Bloodletting and fomentations gave no relief, and in a convulsive paroxysm the horse died. The membranes of the brain and cord were injected, the lungs engorged. The toxic dose is stated by Fröhner and Kaufmann to be three to five grains. Half a grain, given hypodermically, induced in half an hour general muscular rigidity. Ten drachms of nux-vomica in powder caused muscular tremors but in solution proved fatal in ten hours (Hertwig). Coleman gave a mare two ounces in a drench; within an hour, and after the animal had drunk some water, she had violent tetanic symptoms, and died half an hour later. Ounce doses, given a glandered horse, caused tetanic spasms, but were not fatal. Moiroud states that the fatal dose of nuxvomica for a horse is one to two ounces.

Cattle withstand larger doses than do horses when the poison is given by the mouth, whether in solution or bolus. Macgillivray gave an old cow thirty grains strychnine, and, shortly after, sixty grains, both doses in solution, with the result of a few spasmodic tremors, which continued for about twenty minutes. . Dun gave a small red cow, affected with pleuro-pneumonia, grs. xv. strychnine, suspended in two ounces of oil, at 12 o'clock. At 12.30 the pulse had risen from 70 to 78 , regurgitation was observable in the jugular veins, quivering and twitching affected the facial muscles, particularly during inspiration. At 12.45 the pulse numbered 84, and the symptoms were aggravated. Grs. ij. were given, dissolved in diluted acetic acid ; and in a quarter of an hour the animal was very uneasy, and attempted to vomit ; the pulse was 94, full and strong; the pupils much dilated. At 1.30 the nausea and efforts to vomit were much increased, the breathing more laboured; the animal lay down, and the pulse shortly fell to 58. At 2.15 the nausea was diminished, and the pulse 92 . Grs. xxx. were then given in acetic acid and water. At 2.20 the pulse was 100, sharp and distinct. The muscles were affected by frequent spasms. At 2.25 the pulse was 140 , and the animal very sensitive to light, sound, and external impressions. It reeled and fell. At 2.30 the pulse had risen to 160 , the limbs were very 
rigid, the eyes protruding, involuntary spasms more general, frequent, and severe. Two minutes later she died quietly. Much smaller doses are fatal when strychnine is quickly absorbed. Kaufmann states that the toxic dose for cattle is $3 \frac{1}{3}$ to $6 \frac{1}{2}$ grains, but even a grain, given hypodermically, may prove disquieting. Tabourin records the death of a cow in twenty minutes from four grains placed in the areolar tissues.

Sheep are destroyed by half an ounce nux-vomica in about thirty minutes, but goats appear to be less susceptible. Pigs were violently convulsed by fifty grains of nux-vomica (Tabourin), and poisoned by $\frac{1}{6}$ to $\frac{3}{4}$ grain of strychnine (Kaufmann).

Dogs are destroyed in two minutes by gr. $\frac{1}{6}$ strychnine, and in twelve minutes by gr. $\frac{1}{8}$ (Christison). An English terrier was poisoned in twenty-four minutes by gr. $\frac{1}{2}$; a greyhound in one hour and a half by grs. iij.; another greyhound in thirty-three minutes by gr. ss. Kaufmann fixes the toxic dose at $\frac{1}{12}$ to $\frac{1}{3}$ gr., but $\frac{1}{20}$ gr. may prove fatal in small animals. Dogs have been poisoned with grs. viij. of nux-vomica, and eats with grs. v. Dogs moan and whine, are uneasy, nauseated, sometimes vomit, tremble, have muscular twitchings and general spasms, during which the head is drawn upwards and backwards, and the rectal temperature is raised $2^{\circ}$ to $4^{\circ} \mathrm{Fahr}$. The tetanic convulsions continue one to two minutes, cease for several minutes, but recur with increased force until death results.

Post-mortem appearances vary with the severity and duration of the case. Asphyxia renders the blood dark-coloured and unusually fluid; there is venous engorgement; congestion of the lungs and of the cerebral and spinal meninges ; dilatation of the vessels of the medulla, and sanguineous extravasation into the grey matter. When the patient has survived for several hours, the intestines occasionally present patches of redness and congestion. Where spasms have been severe and rapidly fatal, the left side of the heart is firmly contracted, and contains little, if any, blood. The tetanised muscles quickly undergo rigor mortis, which sometimes continues longer than usual. In dogs destroyed with $\frac{1}{5}$ grain of strychnine, Dun found the buccal mucous 
membrane blanched; the left auricle, and also the intestines, continued to contract for nearly an hour after death, while the cerebral and intestinal vessels were congested with dark venous blood.

ANTIDOTES.-The stomach should be emptied with as little delay as possible; if convulsions have begun, the patient should be anæsthetised, the stomach well washed out, and chloral hydrate given. Hughes Bennett first showed the antidotal power of chloral hydrate. He found that the minimum fatal dose of strychnine for rabbits was $\frac{1}{28}{ }^{\text {th }} \mathrm{gr}$. per pound of body-weight. Twenty rabbits received more than this poisonous dose; fifteen of these, to whom chloral was given, recovered. But a few days later, on receiving the dose previously given, without the chloral, all died. French authorities advise the chloral to be given intravenously. Strychnine tetanus is also antagonised by tobacco, and, less effectually, by such motor paralysers as curare, conium, opium, Calabar bean, and paraldehyde.

Medicinal Uses.-As bitter tonies, nux-vomica and strychnine are prescribed in loss of appetite and atonic dyspepsia. Their good effects probably depend upon their checking irregular fermentation, diminishing excessive secretion, as in catarrhal conditions, and perfecting coordination between the several functions of digestion and assimilation (Brunton). It is probably mainly in this way that they relieve many cases of broken-wind.

Small doses, especially when combined with acids, are often effectual in checking chronic relaxed and hypersecreting conditions of the bowels, where these are not complicated with irritation. Larger doses, increasing peristalsis, overcome chronic constipation, whether connected with acute indigestion, inflammation, or febrile attacks, and are usually prescribed with aloes or salines. They are beneficial in weak, dilated conditions of the heart; during their excretion they stimulate the urinary organs, while aphrodisias is occasionally produced. In convalescence from acute disease they improve appetite and general tone. Strychnine, subcutaneously injected, is sometimes serviceable in maintaining activity of the respiratory and heart centres in collapse and narcotic poisoning. 
Nux-vomica and strychnine are prescribed in many cases of paralysis. Where paralysis depends on losś or diminution of nerve force, due to debility after some systemic disease, or to prolonged inaction following an injury, strychnine will give tone to both muscles and nerves. Where, however, there is degeneration or destruction of nervous tissue causing paralysis no treatment is of any avail. Subcutaneously injected over the larynx strychnine has been recommended for roaring in early cases. In epizootic cerebro-spinal meningitis it has been used in one-grain doses repeated two or three times a day, but the mortality in this disease is extremely high and the benefits to be derived from strychnine are very doubtful.

In cattle practice, nux-vomica and its alkaloids are used, in indigestion and in chronic paralysis. Two bullocks suffered from chronic paralysis, one had to be carted home from the grass field. He was dull; his pulse 55, and rather weak; his hind extremities and tail had lost their power of movement, their sensation was impaired; the sphincter ani was relaxed, and both fæces and urine were passed involuntarily. Purgative medicine was given, and operated next day, without, however, any abatement of the paralysis. Two drachms nux-vomica were prescribed night and morning for ten days; but little improvement being notable, the dose was increased to three drachms thrice a day. This treatment being continued for ten days, the patient was able to walk round the house in which he was confined, and rapidly recovered. The other bullock exhibited similar symptoms, was treated in the same manner, and with like satisfactory results.

A week or two before parturition, cows, especially if in low condition, occasionally lose the power of their hind limbs, and are unable to stand. Little can then be done beyond propping the patient in a suitable position, turning her several times daily, and allowing laxative, nutritive diet. Within two or three days after parturition, most of these cases gradually regain the use of their limbs; but when defective nervous power continues, nux-vomica or strychnine is used with success. 
In canine practice, strychnine is a valuable tonic in atonic indigestion, in some cases of asthma and chronic bronchitis, in convalescence from exhausting disease, in chorea, and in paralysis resulting from distemper or other causes, but it should be very carefully prescribed. It is frequently conjoined or alternated with iron salts, or prescribed in the form of Easton's Syrup, or syrup of phosphate of iron, with quinine and strychnine: one drachm of which contains 1 gr. ferrous phosphate, $\frac{4}{5} \mathrm{gr}$. of quinine sulphate, and $\frac{1}{32} \mathrm{gr}$. of strychnine.

Strychnine is used for the destruction of rats, mice, and other vermin, and for the poisoning of wolves and other wild animals. It constitutes the active ingredient of various ' infallible' insect and vermin destroyers, which are usually made up with starch, sugar, and about ten per cent. of strychnine.

DOSES, etc.-Of the powdered nux-vomica, horses take grs. xxx. to grs. cxx.; cattle, grs. lx. to grs. ccc.; sheep, grs. x. to grs. xl. ; pigs, grs. x. to grs. xxx. ; dogs, gr. ss. to grs. ij. The powder has the disadvantage of not being very soluble. The extract, containing 5 per cent. of strychnine, is six to eight times as active as the powder. The B.P. standardised tincture is sometimes used. It contains $\frac{1}{4}$ gr. strychnine in 110 minims.

Strychnine is greatly more uniform and more readily absorbed than the crude drug, and is fifty times more powerful. The hydrochloride, on account of its solubility, in 35 of water or 60 of alcohol, is preferable to the alkaloid. The dose for the horse, except in special cases-when much more may be given-is gr. $\frac{1}{12}$ to grs. $i j \frac{1}{2}$; for cattle, gr. $\frac{1}{12}$ to grs. v. ; for sheep, gr. $\frac{1}{30}$ to gr. $\frac{1}{12}$; for dogs, gr. $\frac{1}{100}$ to gr. $\frac{1}{50}$. The B.P. Liquor Strychninæ hydrochloridi contains one grain of strychnine hydrochloride in 110 minims.

Strychnine, although conveniently given by the mouth, is more prompt and active when administered hypodermically, and when thus used the minimum doses, dissolved in about 100 parts of a mixture of distilled water and alcohol, should first be tried. Nux-vomica and strychnine are generally given twice a day, and as anti-paralysants the doses 
may be cautiously and gradually increased, until slight muscular twitchings are produced. Tablets and lamellæ containing $\frac{1}{60}$ th and $\frac{1}{100}$ th gr. of strychnine sulphate are convenient for hypodermic injections. Strychnine arsenite has been recommended in some of the Continental veterinary schools for the treatment of persistent nasal discharges.

\section{CALABAR BEAN}

Physostigmatis Semina.-The ripe seeds of Physostigma venenosum. Nat. Ord.-Leguminosæ.

Physostigmine Sulphas. $-\left(\mathrm{C}_{15} \mathrm{H}_{21} \mathrm{~N}_{3} \mathrm{O}_{2}\right)_{2}, \mathrm{H}_{2} \mathrm{SO}_{4}, x \mathrm{H}_{2} \mathrm{O}$. Eserine Sulphate. The sulphate of an alkaloid obtained from Calabar bean. Western Africa (B.P.).

Calabar bean is a large reddish-brown, oblong, reniform seed, usually about an inch long, three-quarter inch broad, and half an inch thick. The testa, hard, thick, and rough, encloses two cotyledons, between which there is a large cavity. The bean has no characteristic taste, and no odour (B.P.). Its activity depends upon the presence of two alkaloids-(1) Physostigmine or Eserine, occurring in colourless crystals, soluble in alcohol, benzol, chloroform, and diluted acids, and partially in water. It paralyses nerve centres, and stimulates muscular fibre. (2) Calabarine, soluble in water and alcohol, but not in ether. It causes strychnine-like convulsions.

Eserine Sulphate occurs in yellowish-white minute crystals, becoming red by exposure to air and light, having a bitter taste, highly deliquescent, very soluble in water and in alcohol (90 per cent.). The aqueous solution is neutral to litmus, and, applied to the eye, causes contraction of the pupil. Eserine sulphate contains about 70 per cent. of the alkaloid. Solutions keep better when mixed with a grain or two of salicylic acid.

General Actions.-Calabar bean and its chief alkaloid stimulate motor nerve-endings supplying voluntary and involuntary muscles, but depress the central nervous system. Eserine exerts no topical action on the skin or mucous 
membranes. It relieves obstruction by stimulating the neuro-muscular apparatus of the stomach and intestines. Given by the mouth, or applied locally, it contracts the pupil and slightly diminishes intraocular pressure-effects due to stimulation of the third nerve, or of the circular muscular fibres of the iris, or of both. This myosis results in horses in twenty-five to thirty minutes ; and in less than half that time in carnivora; but is not produced, even by conjunctival injection, in birds, frogs, and fishes.

Excessive doses after this stimulation paralyse muscular fibre, especially the unstriped variety, and also motor nerves. Moderate doses have little effect on voluntary muscles, but full doses induce local twitching, best marked at the elbow and stifle, followed by general trembling and spasmodic contractions ; at the same time the animal sweats, salivates, blows, strains, and discharges fæces and urine, and all his organs provided with unstriated muscle participate more or less in the clonic convulsions. Its stimulant effects on motor nerve-endings and on unstriped muscle in part explain its action on the circulation. Small to moderate doses contract minute blood-vessels, and reduce the force and frequency of the heart movements. Kaufmann mentions that a single full dose reduces the pulse of the dog from 100 to 40 beats per minute. Fraser believes that its action on the heart is threefold-(1) it stimulates peripherally the cardio-inhibitory branches of the vagus; (2) it depresses the cardiac motor ganglia; and (3), in large doses, it paralyses the cardiac muscular fibres. Respiration is temporarily quickened, apparently from stimulation of the vagi in the lungs, but in fuller doses is slowed from paresis of the medullary respiratory centre. Moderate doses stimulate the liver, the involuntary muscles of the bronchial tubes, so lessening their calibre, also the muscular fibres of the uterus, and bladder, and increase gastric and intestinal peristalsis, quickly causing free and fluid evacuations from the bowels, and besides, inducing in man and carnivora retching and vomiting. The secretion of saliva, sweat, and mucus is increased.

Poisonous doses disturb voluntary motility and paralyse the spinal cord. Hence results the characteristic curare- 
like paralysis affecting motor and reflex functions, which, involving the medulla, kills by respiratory arrest (Brunton). According to Fraser, death sometimes results from cardiac paralysis, the heart stopping in diastole. Convulsions occasionally occurring from the use of the bean and commercial physostigmine are due to the presence of calabarine. The brain in most animals appears to be irritated, cats and guinea-pigs poisoned exhibiting cerebral excitement, becoming timid, and running wildly about. It is rapidly absorbed and quickly excreted mainly in the urine, bile, and saliva.

Between physostigmine and its analogues interesting points of contrast are noted. It resembles pilocarpine in its action on the heart, eye, and glandular secretions, but it does not cause such profuse flow from either the salivary, bronchial, skin, or intestinal glands. Again physostigmine has a more marked effect than pilocarpine on striated muscle. Physostigmine, like pilocarpine, probably induces secretion by stimulating the peripheral endings of secretory nerves. Atropine is its physiological antagonist, paralysing muscles, stimulating the respiratory and cardiac medullary centres, and dilating the pupil.

Eserine, in virtue of its promptly and effectually stimulating the muscular fibres of the intestines, is of great value in the treatment of obstruction and obstinate constipation. This was first pointed out by Dieckerhoff, and has been demonstrated by F. Smith and C. Rutherford, who made an important series of observations on horses, using physostigmine freed from the convulsant calabarine :- 'The earliest indications of the action of the drug are loud intestinal murmurs, passage of flatus, with slight colicky pain ; shortly this is followed by evacuation of the contents of the rectum, and the motions then pass at intervals of a few minutes, each becoming gradually softer, more watery, less formed in balls, until the stage when the evacuations are moist and fluid, exactly representing cows' fæces. All this time the abdominal disturbance has become greater, the animal lies down, but seldom rolls, the intestinal murmurs are louder, the passage of flatus almost continuous, straining marked, facces are voided with great rapidity, often ejected with force, 
and several ounces of a brown-coloured fluid will at this time accompany each motion. About two to two and a half hours from the time of injection the effects are commencing to pass off, and during this short time an almost incredible amount of fæces will have been excreted.

'A horse received $1 \frac{1}{2}$ grains of eserine, subcutaneously; it acted in twenty-five minutes, and produced in the first hour seven evacuations, in the second hour seven, the effect passing off in two hours and ten minutes.

'A horse received $1 \frac{1}{2}$ grains of eserine, hypodermically, which took twelve minutes to act, producing seven evacuations in the first hour, and then terminating.

' Another horse received $1 \frac{1}{2}$ grains of salicylate of eserine, hypodermically, producing a free action of the bowels in one hour. This case terminated fatally from ruptured stomach; and thus it was demonstrated that eserine could act upon the large intestine, in spite of the shock to the abdominal nervous system which a ruptured stomach causes.

'A pony received 1 grain of eserine, hypodermically; three evacuations were produced in fifty minutes, and in eighty minutes from the time of injection eight evacuations had occurred. The case was a fatal one, the cause of obstruction being due to a small diaphragmatic hernia. Had the gut not been nipped so tightly, there is reason to believe the increased peristalsis might have withdrawn it.

'A horse received $1 \frac{1}{2}$ grains of eserine by injection into the trachea; it took seventeen minutes to act, and produced in the first hour twelve evacuations, weighing $11 \mathrm{lbs} .13 \mathrm{oz}$, and a considerable quantity of flatus. The action then passed off.

' A horse received 1 grain of eserine, hypodermically; it took forty-two minutes to act, and produced only one evacuation in one hour, accompanied by a considerable quantity of flatus.

' The same horse received 1 grain of eserine and 3 grains of pilocarpine by injection into the trachea ; it took twentyone minutes to act. In the first hour, counting from time of injection, it produced fourteen evacuations, weighing $30 \mathrm{lbs} .6 \mathrm{oz}$. ; in the second hour four evacuations, weighing 
7 lbs. $6 \frac{1}{2}$ oz.; and in the third hour two evacuations, weighing 2 lbs. $13 \frac{1}{2} \mathrm{oz}$; ; in three hours a total of $40 \mathrm{lbs} .10 \mathrm{oz}$. of ingesta.

'In comparing these two cases, the value of pilocarpine as an addition to eserine is clearly demonstrated.

'A horse received $1 \frac{1}{2}$ grains of eserine by the trachea ; it acted in forty-one minutes, and produced in the hour five evacuations; during the second hour four evacuations. The weight was unfortunately not obtained, but the quantity of ingesta completely filled a stable bucket. The case was one of most obstinate constipation, and had previously received 6 drachms of aloes, which acted at the expiration of the usual time.

'Another horse received by the trachea 1 grain of eserine with 3 grains of pilocarpine, and this combination acted in one and a half hours, producing, in two and a half hours from time of injection, eight evacuations, weighing $26 \mathrm{lbs}$, exclusive of loss. The pilocarpine produced its salivating effects in four minutes from the time of injection.' (Veterinary Journal, 1888.)

R. Rutherford gave a horse, weighing about 950 lbs., 5 grains commercial eserine, which within half an hour caused profuse perspiration, convulsive breathing, with violent action of the diaphragm. About two hours later, when the symptoms were abating, he gave 3 grains more, and death followed in half an hour.

Kaufmann records that on opening the abdomen of a horse which had received a full dose of physostigmine, energetic contractions of the large intestine were seen. Animals poisoned exhibit pallor, contraction, and hardness of the large intestine; the urinary bladder is empty and contracted, and the uterus also contracted.

Antidotes.-As the bean is not very soluble, the stomach should be evacuated either by an emetic or the stomachpump. Ipecacuanha has been recommended as an antidote. Physostigmine is antagonised by moderate doses of atropine. Fraser found that rabbits, receiving one and a half the lethal dose, recovered, if atropine was given simultaneously, in doses of gr. $\frac{1}{20}$ to gr. i. While small doses act as antidotes, larger hasten a fatal result. The atropine specially counter- 
acts the cardiac paralysis. To a less extent physostigmine antagonises the poisonous action of atropine; chloral also somewhat opposes physostigmine.

Medicinal Uses.-Physostigmine or eserine is myotic, expectorant, and a gastro-intestinal stimulant. The observations of $\mathrm{F}$. Smith and C. Rutherford testify to its value in combating intestinal torpidity and impaction. They administered physostigmine hypodermically and intratracheally, and preferred the latter method on account of its enabling more fluid to be introduced, occasioning less loss of the drug, and causing less inconvenience to the patient. Cases of intestinal obstruction in all animals may be safely treated by eserine, conjoined with pilocarpine and anodynes, by enemata, and abstention from solid food. French and German practitioners have arrived at similar conclusions. Kaufmann and others recommend physostigmine as an 'intestinal anæmiant' in congestion, atony, colic, and torpidity of the digestive tract, especially of the large intestine, and also for the expulsion of concretions and foreign bodies. They further note its use in producing contraction of the uterus. In doses of half a grain to a grain per day it has been used with success in the treatment of intestinal catarrh with inappetence in horses. For retention of meconium in foals doses of gr. $\frac{1}{12}$ to gr. $\frac{1}{6}$ hypodermically have proved beneficial. It is also valuable in obstinate constipation in dogs, for which the sulphate may be given in doses of gr. $\frac{1}{60}$ to gr. $\frac{1}{12}$ per day. Eserine has been given, subcutaneously and intravenously to cattle affected with indigestion and gastric impaction, but although causing considerable, sometimes painful, disturbance of the bowels, it does not produce copious evacuations. It has been tried in tetanus, but the relief afforded is merely transient. Recoveries attributed to the use of eserine should only be accepted with reserve.

The extract and eserine sulphate or salicylate have been prescribed in epilepsy, chorea, and other spasmodic nervous affections, and, as an antidote, in poisoning by strychnine and atropine. They should not, however, be given to pregnant animals, in which untimely stimulation of the uterus may lead to premature expulsion of its contents. 
In solution eserine is applied as a myotic to relieve congestion and inflammation of the conjunctiva and cornea, and, alternated with atropine, to prevent or break down adhesion (synechia) caused by iritis. In chronic dropsical conditions of the joints and bursæ of tendons in the horse, after evacuation of the fluid by an aspirator, or a trocar and canula, Stottmeister, instead of the iodine solutions frequently used, recommends injection of a grain to a grain and a half eserine dissolved in $\mathrm{m} 80$ to $\mathrm{m} 160$ of distilled water, applying, subsequently, for two or three days, ice or refrigerant lotions to abate inflammation.

Doses, etc.-The powdered bean is given to horses and cattle in doses of grs. Xv. to grs. $\mathrm{xxx}$. ; to dogs, gr. $\frac{1}{4}$ to gr. $\frac{1}{2}$. As already indicated, the diverse character of the two alkaloids present in the extract, as well as in the bean, renders it desirable to use eserine, which is conveniently employed in the form of sulphate, of which the dose per os for horses is grs. ii. to grs. iii., and for dogs, gr. $\frac{1}{20}$ to gr. $\frac{1}{10}$. Intratracheally, (horses) gr. ss., in $\mathrm{Ml}$. to Mlx. of water ; and subcutaneously, gr. j. to gr. jss., in Mlx. to mlxxx. of water. In intestinal obstruction more prompt and certain effects are obtained by addition of 2 to 3 grains of pilocarpine. The hydrobromide and salicylate of eserine are sometimes used. An anæsthetic collyrium may be made with cocaine hydrochloride grs. iv., eserine sulphate grs. ii., and distilled water $ろ \mathrm{j}$.

Eseridine $\left(\mathrm{C}_{15} \mathrm{H}_{23} \mathrm{~N}_{3} \mathrm{O}_{3}\right)$, an alkaloid obtained from Calabar bean, has similar properties to physostigmine, but only onesixth its activity.

\section{HEMLOCK}

Hemlock Leaves. Conii Folia. The fresh leaves and young branches of Conium maculatum, collected when the fruit begins to form (B.P.). Nat. Ord.-Umbelliferæ.

Hemlock Fruit. Conii Fructus. The dried, full-grown unripe fruits of Conium maculatum (B.P.).

Hemlock grows wild in hedges and waste places in most parts of Europe. The flowering stem is two to five feet high, 
round, hollow, jointed, smooth, branching towards the top, and covered with purple spots. The large bi- or tri-pinnate leaves are glabrous and dark-green, have clasping petioles of varying length, a nauseous, bitter taste, and a strong, peculiar odour, which is characteristic of all parts of the plant, and aptly compared to that of mice or of cats' urine. The fruit resembles that of anise, is of a brown colour, about one-eighth of an inch in length, broadly ovate, the two mericarps, generally separated, each marked with five ribs. Nine pounds of fruit produce an ounce of the alkaloid conine, which, with a bitter oleo-resin and a non-poisonous volatile oil, is found stored chiefly in cubical cells in the endocarp.

The leaves gathered in June, when the fruit begins to form, are rapidly dried in stoves at about $120^{\circ} \mathrm{Fahr}$., and preserved in tins, bottles, or jars, excluded from light. By drying, they lose three-fourths of their weight, and one-half of their volatile principle, of which scarcely a trace remains after they are kept twelve months (Royle). Long keeping of the fruit and leaves, and their exposure to temperatures exceeding $120^{\circ} \mathrm{Fahr}$., account for the inertness of many hemlock preparations.

In addition to the active principle, conine, hemlock contains varying proportions of methyl-conine, which acts on the spinal cord, paralysing reflex action; conhydrine, which is said to be much less active than conine; and coniic acid.

Pure conine $\left(\mathrm{C}_{8} \mathrm{H}_{16} \mathrm{HN}\right)$ may be obtained from the fruit or leaves by distillation with caustic potash. It is a yellowish, oily liquid, with an intense odour of mice, and a peculiar acrid taste. Specific gravity 885 . Soluble in 100 parts of water; and readily dissolved by alcohol or ether. Nitric acid dropped on conine produces a blood-red colour, sulphuric acid a purple-red, passing to olive-green. Its chief salt is the hydrobromide, which contains about 60 per cent. of the alkaloid. It is soluble in two parts of water and in three of rectified spirit (Squire).

The leaves and fruit of hemlock are distinguished by their appearance, and, if triturated with diluted caustic potash solution, evolve the characteristic odour of mice. Fool's 
parsley (Æthusa cynapium), water hemlock or cowbane (Cicuta virosa), the fine-leaved water hemlock (Phellandrium aquaticum), the water parsnip (Enanthe crocata), are Umbelliferæ with physiological actions similar to those of conium maculatum, and when freely eaten have poisoned many of the domestic animals. Of wholesome dietetic Umbelliferæ, parsley, parsnip, and celery are illustrations. The natural family is rich in aromatic carminative seeds.

ACTIONS AND UsES.-Hemlock and its alkaloids have many points of resemblance to curare and nicotine. The chief action of conine is to paralyse motor nerve-endings, so giving rise to more or less complete paralysis, with weak, staggering gait and then a gradual failure of respiration.

General Actions.-Hemlock was the state poison of the Athenians, the death-potion of Socrates. The alkaloid conine especially paralyses motor nerve-endings and so produces weak, staggering ataxic gait, and then complete paralysis. At the same time certain nerve cells are paralysed as with nicotine, so that vomiting and diarrhœa may occur from paralysis of the inhibitory sympathetic ganglion cells in the abdomen. Similarly the heart beats quicker from depression of vagal ganglion cells and blood-pressure falls from vaso-dilation. On the central nervous system it exerts a primary, brief, stimulant action, followed by depression. Thus there is twitching, tremor, quickened respiration, then slower, shallow breathing (diaphragm paralysed or centre?), asphyxial convulsions, and death. Death results from paralysis of respiration. It is excreted by the kidneys. It acts more powerfully on man and carnivora than on herbivora. Goats with impunity eat considerable quantities of the fresh leaves (Kaufmann). Its physiological antagonists are nux-vomica, strychnine, and other tetanisers.

Toxic Actions.- Harley and Mavor gave a two-year-old thoroughbred colt six, eight, and twelve ounces of succus conii without appreciable effect. Sixteen ounces produced in twenty-five minutes dulness and stupidity, drooping and swollen eyelids, but no change in the pulse or pupils. 
A few minutes later the colt went down upon his knees, appeared to require special efforts to keep himself on his legs, stumbled, and walked slowly when led; but in two hours the symptoms had entirely disappeared. Moiroud poisoned a horse with half a pound of the dried leaves given as a decoction, and observed nausea, spasmodic twitching of the muscles of the extremities, cold sweats, dilatation of the pupils, and dulness. In Italy asses eating hemlock have sometimes been so thoroughly paralysed that, supposing them to be dead, the peasants have begun to remove the skin (Matthiolus).

Cattle poisoned lie as if lifeless, with slow, feeble pulse, cold extremities, and dilated pupils. Sheep become giddy, listless, and sometimes die. When other food is scarce lambs will crop hemlock with fatal results. Fifteen grains of the succus injected into the blood-vessels of a full-grown mouse produced, in half an hour, paralysis, continuing for five hours. Christison found that an ounce of the extract swallowed by dogs proved fatal in forty-five minutes; ninety grains applied to a wound had the same effect in an hour and a half; while twenty-eight grains caused death in two minutes, when injected into the veins.

Gerrard recorded the poisoning of pigs which strayed into an orchard and ate growing hemlock. They lay prostrate and unable to rise, pulse imperceptible, the body cold, the eyes amaurotic, and when left alone they lapsed into a comatose state. There were no convulsions, and no pain was apparent when they were pricked with a pin. In fifteen hours two died, and two a few hours later. Examination discovered the blood throughout the body, and especially in the large organs, dark-coloured and fluid, the result of the fatal asphyxia; the intestines distended with gas ; the mucous coat of the stomach, particularly its cardiac portion, much congested, while similar spots of congestion were observed throughout the intestines.

Conine is generally used in the form of hydrobromide. One drop applied to the eye of a rabbit arrested respiration in nine minutes; three drops in the eye of a cat killed it in a minute and a half; five drops swallowed by small dogs began to operate in thirty seconds, and proved fatal in one 
minute. Still smaller quantities injected into the veins poisoned with even greater rapidity (Christison).

The antidotes are tannic acid, the cautious administration of coffee and other stimulants, ammonia to the nostrils, stimulating enemata, enforced exercise, and artificial respiration.

Medicinal Uses.-Hemlock is occasionally given to relieve the muscular spasm of chorea. It is of no avail in tetanus in horses, nor, as demonstrated by experiment, in strychnine poisoning. Spasmodic cough connected with muscular irritability, such as occasionally occurs in epizootic sore-throat and bronchitis in horses, is sometimes relieved by inhalation of steam medicated with hemlock, or by swallowing slowly an electuary of succus conii, glycerin, and ammonium acetate. Injections and suppositories are applied in irritable, painful conditions of the urino-genital organs. Conium ointment, made with two ounces of succus conii and three-quarter ounce of lanoline, is employed as an anodyne in acute mammitis of the cow.

Doses, etc.-Neither the dried leaves nor the fullyripened dried fruit are to be depended upon. The fresh leaves and young branches, and preparations promptly obtained from them without heat, are, however, reliable, of which the best is the succus. Three parts of juice are mixed with one of rectified spirit, allowed to stand for seven days, and then filtered and bottled. This succus has a dark sherry colour, an agreeable odour, and acid reaction; one fluid ounce yields thirty grains of soft extract. Horses and cattle take $\mathrm{f} z \mathrm{ij}$. to $\mathrm{f} z \mathrm{iv}$.; sheep and pigs, $f z_{\text {ss. to }} \mathrm{f} \boldsymbol{z}_{\mathrm{j}}$.; dogs, $\mathrm{f} Z$ ss. to $\mathrm{f} Z \mathrm{j}$. Its analgesic and anti-spasmodic effects are increased by using it with opium or chloral-hydrate. Conine employed hypodermically by Harley and Mavor, frequently produced irritation, which hindered its absorption. For subcutaneous or intratracheal injection the hydrobromide, which contains 60 per cent. of conine, should be used. Doseshorses, gr. i. to grs. ii.; dogs, gr. $\frac{1}{30}$ to gr. $\frac{1}{12}$, dissolved in 20 to 60 minims of water containing a few drops of alcohol. Much larger doses have been given to dogs without producing ill effects (Wooldridge). 


\section{H Y OSCYAMUS}

Hyoscyamus or Henbane Leaves. The fresh leaves and flowers, with the branches to which they are attached, of Hyoscyamus niger; also the leaves and flowering tops, separated from the branches and carefully dried. Collected from the flowering biennial plants (B.P.). Nat. Ord.-Solanaceæ.

Henbane grows wild in most parts of this country, and is cultivated at Mitcham and Hitchin. The large, sinuate, usually decurrent yellow-brown leaves are rough, hairy, and clammy, with a fotid, narcotic odour, and a nauseous, bitter taste. There are two varieties, an annual and a biennial ; the latter alone recognised by the B.P., is larger, stronger, more branched, clammy, and active. One hundred pounds of the fresh plant when dried weigh $14 \mathrm{lbs}$., and yield about $4 \mathrm{lbs}$. of extract.

The active principle, hyoscyamine $\left(\mathrm{C}_{17} \mathrm{H}_{23} \mathrm{NO}_{3}\right)$, in its impure form is an oily liquid, becoming brown on exposure, but it can be slowly crystallised into colourless, translucent needles. It is soluble in 120 of water, and readily dissolves in alcohol, chloroform, and dilute acids. It resembles daturine, the active principle of Datura stramonium, is identical with duboisine, the active alkaloid of Duboisia myoporoides, and is isomeric with atropine. Its relationship to atropine is very close, and, in fact, it is quite easily converted into atropine. It is decomposed, and its physiological action neutralised by caustic alkalies. Henbane also contains hyoscine, or scopolamine, which is a cerebral and spinal sedative.

AcTiONS AND USES.-Hyoscyamus closely resembles belladonna and stramonium. Locally applied, it paralyses the endings of sensory nerves. It dilates the pupil, although not so certainly and fully as atropine. Full doses of the drug or its alkaloid stimulate the cerebral centres to a somewhat less extent than atropine, and paralyse the ends of motor nerves. There are produced dryness of the mouth, restlessness, general convulsions, paralysis, and stupor, alternated with a peculiar form of delirium, in which a constant desire for movement is accompanied by lassitude, 
failure of the action of the heart, and of breathing, and death from asphyxia (Brunton).

Toxic EFFECTs.-Horses receiving an infusion made with three to four ounces of the leaves have dilatation of the pupils, spasmodic movements of the lips, acceleration and subsequently depression of the heart-beats, but no symptoms of acute poisoning. Dogs are acted on exactly as by belladonna. Cats become dull and drowsy, the mouth and nose dry, the pulse accelerated, the pupils dilated, and the power of walking or springing impaired.

Medicinal Uses.-Hyoscyamus is prescribed with cathartics to prevent their griping. It is mainly excreted by the kidneys, and occasionally is used as an anodyne in irritable conditions of the kidneys and bladder. It is prescribed in human practice in cases of mania and nervous or muscular excitement, and has been used with some success in epilepsy and chorea in dogs. It is occasionally substituted for opium as a topical anodyne. For the human subject, hyoscine or scopolamine is a useful hypnotic, producing, after a brief stage of excitement, a condition like natural sleep. In horses and dogs, according to Fröhner, this effect is not easily produced, though moderate doses cause marked mydriasis.

Doses, etc.-Of the tincture, horses and cattle take $3 \mathrm{ij}$. to $f z_{j}$. ; dogs, $m_{x}$. to $m_{x l}$. The extract is six times the strength of the tincture. Hyoscyamine, usually prescribed as the sulphate, which is freely soluble in water, is about one hundred times more active than the extract, and is sometimes used hypodermically. Doses, horses, gr. i. to grs. ii. ; dogs, gr. $\frac{1}{50}$ to gr. $\frac{1}{20}$. Of Hyoscine hydrobromide the dose for the horse is gr. $\frac{1}{6}$; for the dog, gr. $\frac{1}{60}$ to gr. $\frac{1}{30}$.

\section{COCAIN E}

Cocaina. An alkaloid obtained from the leaves of Erythroxylum coca and its varieties.

Cocaine Hydrochloridum. The hydrochloride of an alkaloid obtained from Coca leaves. $\mathrm{C}_{17} \mathrm{H}_{21} \mathrm{NO}_{4} \cdot \mathrm{HCl}$. Nat. Ord.-Linaceæ.

The alkaloid, of which the leaves yield 26 per cent., is 
prepared by agitating an acidulated alcoholic extract with ether. It occurs in colourless prisms, almost insoluble in water, insoluble in glycerin, soluble in ten parts rectified spirit, and in twelve of olive oil. The hydrochloride, in colourless acicular crystals, or crystalline powder, is readily soluble in water, alcohol, and glycerin. It is insoluble in olive oil, and nearly insoluble in ether. Its watery solution has a bitter taste, producing on the tongue a sensation of tingling, followed by numbness, and when applied to the eye dilates the pupil. It gives a yellow precipitate with gold chloride, and a white precipitate with ammonium carbonate, soluble in excess of the re-agent. Cocaine is associated in the plant with coca-tannic acid, and with several other alkaloids, amongst which are cocamine and cinnamyl-cocaine - and a volatile constituent which gives aromatic fragrance to the fresh leaves. All these alkaloids contain a pyridine derivative known as ecgonine, and in cocaine this is combined with benzoic acid and methyl alcohol. Many artificial cocaine substitutes have been produced, all containing the ecgonine molecule.

ACTIONS AND USES. - Cocaine paralyses the sensory nerves with which it comes in contact, and is thus a local anæsthetic. It is also antiseptic. Small to moderate doses stimulate the central nervous system, are tonic, and diminish metabolism. The South American Indians, on long marches, not only chew coca leaves, but give them to their horses, with the effect of diminishing thirst, hunger, and sense of fatigue. Although topically anæsthetic and anodyne, large doses, swallowed or injected subcutaneously, exert this central stimulant effect, causing excitement, restlessness, increased reflex irritability, and then tonic or clonic convulsions. Subsequently they paralyse the nerve-centres, impair co-ordination, causing aimless gyrating movements, muscular spasms, and death from respiratory failure.

General Actions.-Solutions of 4 to 10 per cent., applied to a mucous surface, within one minute cause pallor and vascular contraction. After two or three minutes the endings of the sensory nerves are paralysed, so producing local anæsthesia lasting ten minutes. A few drops of a 
5 per cent. solution, placed within the eyelids, produce anæsthesia, constrict the vessels, lessen the power of accommodation, and dilate the pupil. This dilatation is more notable in men and dogs than in horses and cattle (Fröhner). It is caused by stimulation of the sympathetic fibres supplying the dilator muscle of the iris. When swallowed, cocaine diminishes the sensations of hunger and thirst. Large or repeated doses quicken circulation, increase blood-pressure, breathing, and temperature, and heighten reflex irritability by their effect on the central nervous system, and in the case of the heart, by depression of the vagal endings. Still larger doses cause trembling and timidity, impair coordination and equilibrium; animals cannot walk straight, have muscular trembling and rotatory convulsions, and die from paralysis of respiration. It is excreted by the kidneys ; but does not appear, however, to alter the proportion of the urinary constituents. Applied to the mammary gland it diminishes secretion of milk.

Horses receiving 60 to 80 grains injected subcutaneously, or about 005 gramme per kilogramme of body-weight, according to Fröhner, are restless, paw with the fore feet, neigh, and exhibit timidity and excitement, the pulse rises to $90-96$, temperature is increased, salivation occurs, the bowels are frequently moved, and the pupil dilated. After fifty minutes the animal is in a state of frenzied excitement, with greatly augmented reflex activity. Two hours elapse before these effects disappear. In cows like effects were produced by hypodermic injection of similar doses. One drachm is stated to have produced excitement bordering on madness, and continuing for four hours, but gradually passing off, and leaving no injurious effects.

In dogs, doses consisting of 015 to 02 gramme per kilogramme of live-weight produce psychical excitement, muscular spasms, rhythmical contractions of the skeletal muscles, circus-movements, wild galloping, tetanic and clonic spasms, epileptiform fits, dyspnœa, rolling, and loss of co-ordination. The spasms and more prominent symptoms do not, however, occur when potassium bromide, ether, or amyl-nitrite has previously been given. Large doses subsequently paralyse the central nervous system in the 
same order as they have previously stimulated the parts, implicating first the brain, then the corpora quadrigemina, the spinal cord, and lastly the medulla. Injected hypodermically, twelve to fifteen grains kill small dogs in ten minutes (Hobday).

MEdicinal Uses.-Cocaine hydrochloride is a convenient and effectual local anæsthetic. Its effects are confined to the skin or mucous surface moistened with it, are more easily regulated than those of ether spray, are unaccompanied by pain, and may be kept up for considerable periods without injuriously affecting the nutrition of the parts. Anæsthesia may be produced within five minutes, and, when insensibility is secured, it usually continues for twenty to thirty minutes. For application to the sound skin cocaine should be dissolved in oil of cloves which ensures deeper penetration. Twenty minims of a 4 or 5 per cent. solution dropped into the eye within ten minutes diminish sensibility, so that a thorough examination can be made of the organ ; the irritability and pain of conjunctivitis, iritis, and ulceration of the cornea are abated; chaff or other foreign bodies imbedded in the cornea can be removed without provoking pain or reflex movements ; warts can be excised, torn lids stitched, and injuries of the eye painlessly treated. Indeed, after several applications of the cocaine solution, the eyeball of the horse has been removed, without symptoms of pain, and without the necessity of casting the patient. In examinations and operations in connection with the larynx, cocaine is equally serviceable, and for such cases a stronger solution is generally used. Applied to the skin, along the course of the plantar nerves, and still more effectually when injected subcutaneously, it abolishes sensibility sufficiently for the painless performance of neurectomy. R. Rutherford, after closely clipping or shaving the hair, finds that half an ounce of a 20 per cent. solution, in fifteen or twenty minutes anæsthetises the limbs even of irritable horses sufficiently for the performance of firing without casting, and for the painless insertion of setons. In combination with a small proportion (five to ten per cent.) of solution of adrenalin, it is very serviceable in operations on mucous membranes, the uterus, vagina, 
and rectum, for the removal of tumours, and the opening of abscesses. Doses of grs. ii. to grs. v. cocaine hydrochloride, in solution (two to five per cent.), subcutaneously injected, are used to allay rheumatic and other local pains, and to assist in the diagnosis of lameness in horses. Cocaine is also employed in lumbar anæsthesia. For this purpose a two per cent. solution may be injected in doses of $m_{x v}$. to $m l x$. for the horse and ox, and $m_{v}$. to $m_{x x x}$. for the $d o g$.

In order to preserve cocaine hydrochloride solutions, which, when long kept, are liable to spoil, 1-200th part of boric acid should be added to them when freshly made. The B.P. injectio cocainæ hypodermica, is made with 33 grains cocaine hydrochloride, $\frac{1}{2}$ grain salicylic acid, and 6 drachms distilled water. One hundred and ten minims contain about ten grains of cocaine. The ointment consists of 20 grains cocaine, 80 grains oleic acid, and 400 grains of lard. Tablets containing $\frac{1}{10}$ and $\frac{1}{6}$ grain are now obtainable.

Holocaine, a cocaine substitute, obtained by combination of phenacetin and paraphenetidin, is employed as the hydrochloride, which is soluble in one hundred parts of water. In ophthalmic practice a few drops of a 1 per cent. solution produce anæsthesia in fifty seconds, the effects lasting for five to fifteen minutes. The solution is antiseptic but does not dilate the pupil.

Acoine, derived from guanin, is less poisonous and acts longer than cocaine. It is antiseptic as well as anæsthetic. A solution for hypodermic injection is composed of one part acoine, eight parts of sodium chloride, and a thousand parts of distilled water.

Tropacocaine (benzoyl-pseudo-tropine), employed as the hydrochloride, occurs with cocaine and other bases in Java coca leaves. Prepared synthetically, it is a white crystalline powder, readily soluble in water. Used in solution (2 to 3 per cent.) it is a powerful local anæsthetic, more rapid in action and less toxic than cocaine. It has been employed in lumbar anæsthesia, by Becher, in doses of one grain for the dog, and four to eight grains for the horse.

Eucaine (beta-eucaine) hydrochloride is a good substitute for cocaine. It is more active and much less toxic than cocaine. Readily soluble in water, it is free of irritant action. 
Solutions can be sterilised by boiling without undergoing decomposition. Solutions of 2, 5, and 10 per cent., with or without addition of solution of adrenalin, are employed as local anæsthetics for minor operations. A mixture of equal parts of eucaine and cocaine has been recommended as the best and safest local anæsthetic.

Stovaine (ethyl-dimethyl-aminopropinol-hydrochloride), occurring in white lustrous scales, soluble in water, alcohol, and acetic ether, is said to be two or three times less toxic and more easily eliminated than cocaine. Solutions of 1 to 2 per cent. are used hypodermically to produce local anæsthesia. Half a drachm to a drachm of a 6 per cent. solution, injected at four or five points of the conjunctiva, ensures painless excision of the eyeball in horses and dogs. Instillation of a few drops of a 4 per cent. solution greatly facilitates the removal of foreign bodies from the eye. For intraspinal anæsthesia a 5 per cent. solution is injected in doses of 3 ijss. in horses, $m_{x x x}$. in dogs, and $m_{x}$. to $m_{x x}$. in cats. Solutions of stovaine can be sterilised by boiling.

Novocaine (para-aminobenzoyl-diethylamino-ethanol hydrochloride) is a non-irritant local anæsthetic, in colourless spicular crystals, soluble in an equal quantity of water and in thirty parts of alcohol. Solutions of 1 and 2 per cent. produce satisfactory local anæsthesia for minor operations on horses and dogs. Instillations of a 5 to 10 per cent. solution are employed for operations on the eye. Anæsthesia is complete in from seven to ten minutes. Novocaine ( 3 per cent.) is serviceable in the diagnosis of lameness, and combined with solution of adrenalin, it forms an excellent hæmostatic anæsthetic for injecting round the base of tumours prior to extirpation. Solutions keep well even when exposed to light, and they may be boiled several times without losing anæsthetic value.

Orthoform (methylic ether of paramido-meta-oxybenzoic acid), another cocaine substitute, occurs as a white, odourless, and tasteless powder, slightly soluble in water. According to Guinard and Souliere, Orthoform is more analgesic than anæsthetic. Applied to wounds and ulcers only a small part is dissolved by the discharge. Absorption is exceedingly slow, so that local applications may be regarded as 
non-toxic, and, moreover, the local anæsthesia is maintained for several hours. This renders orthoform of great service in canine practice for preventing irritation and pain, and so allowing the patient to rest. When swallowed, or injected subcutaneously, it is quickly absorbed and acts as a powerful nerve depressant, blood pressure being lowered and heart action and respiration much increased. Large doses given to dogs, hypodermically or by the mouth, cause nausea and vomiting. Orthoform is employed as a local anæsthetic ; and as an antiseptic dressing, for recent cracked heels, broken knees, and superficial wounds, in powder, or in ointment (10 to 20 per cent.) made with lanoline. Mixed with collodion it is used as an anodyne, antiseptic, adhesive protective for small operation wounds.

\section{J A B O R A N D I}

Jaborandi Folia. The dried leaflets of Pilocarpus jaborandi. (B.P.) Nat. Ord.-Rutaceæ.

Pilocarpine Nitrate. Pilocarpinæ Nitras $\left(\mathrm{C}_{11} \mathrm{H}_{16} \mathrm{~N}_{2} \mathrm{O}_{2}\right.$ $\mathrm{HNO}_{3}$ ). The nitrate of an alkaloid obtained from Jaborandi leaves (B.P.).

The shrubs yielding jaborandi are natives of Brazil. The leaflets have a slightly aromatic odour and a bitter, pungent taste, and when chewed they increase secretion of saliva. The leaflets are about four inches long, and contain an acrid resin, an essential oil consisting in part of a dextrogyrate terpene $\left(\mathrm{C}_{10} \mathrm{H}_{16}\right)$, and an amorphous, liquid, colourless alkaloid, pilocarpine $\left(\mathrm{C}_{11} \mathrm{H}_{16} \mathrm{~N}_{2} \mathrm{O}_{2}\right)$, which is soluble in alcohol, ether, chloroform, ammonia, and dilute acids, and forms crystallisable salts, the nitrate being chiefly used. Another alkaloid, jaborine, has been described as occurring in much smaller proportion, and was stated to be a basic decomposition product of pilocarpine, and antagonistic to it in its actions, although more recent work has cast doubt upon this. Pilocarpine nitrate is a white, crystalline powder, soluble in nine parts of water, and in fifty parts of cold alcohol (90 per cent.).

ACTIONS AND USES.-Pilocarpine and jaborandi leaflets 
have no notable in-contact effect on the skin or mucous membranes, but when absorbed they stimulate glandular secretion more promptly, energetically, and generally than any other known drugs. The salivary, lachrymal, bronchial, gastric, pancreatic, and intestinal secretions are increased, the milk, bile, and urine, to only a slight extent. The cutaneous perspiratory glands are not so actively stimulated in the lower animals as in man. They stimulate the efferent nerve-endings of involuntary muscles, while large toxic doses impair the irritability of voluntary muscles and motor nerves by their central action. They are prescribed as eliminatives in catarrhal, pneumonic, and rheumatic cases, and in torpidity and obstruction of the bowels-in the latter being conjoined with physostigmine.

General Actions.-Pilocarpine stimulates the peripheral terminations of efferent nerves going to glands and to involuntary muscles. In the lower animals secretion of saliva is early and prominently increased. Horses subcutaneously injected with three to four grains in two or three minutes are freely salivated; within one hour three and a half pints of saliva have been collected; during the next hour about half that quantity, but an hour later the secretion was nearly normal (Kaufmann). The nasal and lachrymal secretions are augmented. So much bronchial mucus is outpoured that a distinct râle is audible, and in poisonous doses the accumulation of fluid and œdema of the membrane, together with the lessened calibre of the tubes due to contraction of the bronchial muscle, cause dyspnœa, which is sometimes fatal. The intestinal glands are stimulated, rendering the dejections more abundant, soft, and shortly semi-fluid. Small and moderate doses slightly increase the secretion of urine, and also of milk. In man pilocarpine produces profuse sweating, but in the lower animals even full doses only render the skin moist. By its stimulation of the skin growth of hair is said to be encouraged (Fröhner).

Pilocarpine stimulates the peripheral terminations of the motor nerves distributed to involuntary muscles, and secondarily, and especially in large doses, paralyses them. Given by the mouth, or injected locally, the circular fibres 
of the iris are contracted by stimulation of the third nerve, but frequently the pupil is subsequently dilated. The muscles of the stomach and intestines are in a state of active peristalsis, occasionally accompanied by vomiting; colic, and diarrhœa. The bladder contracts, and urine is passed at short intervals. Contractions of the uterus and movements of the spleen are also produced. After slight and temporary quickening from stimulation of the sympathetic fibres, heart action is slowed and blood-pressure lowered, by reason of vagus stimulation becoming dominant. The temperature, which at first rises, subsequently falls several tenths of a degree. Fröhner states that a single dose in from two to four hours will reduce the weight of a horse by forty to sixty pounds.

Horses receiving two to four drachms of the leaves infused in hot water, in fifteen to twenty minutes exhibited profuse salivation, continuing for three hours, but without notable diaphoresis, altered circulation, or increased temperature. Carriage horses to which Dun gave two to four drachms, in fifteen minutes salivated abundantly, and the discharge continued for two or three hours ; very slight diaphoresis occurred for twenty minutes; no change was noticeable in the pulse, temperature, or quantity of urine excreted. Dollar injected hypodermically $1 \frac{1}{2}$ grains pilocarpine in ten parts water into the shoulder of an aged horse $15 \frac{1}{4}$ hands ; in six minutes marked salivation set in, the saliva pouring out of the mouth; the secretion from the buccal glands also appeared to be augmented. These effects continued for fully an hour and a half; the pulse was lowered in force, and was slowed two to three beats; the skin, previously dry, became moist, but there was no distinct sweating. F. Smith reports that in horses, in about ten minutes after a subcutaneous injection of three grains, there is constant ' champing of the jaws, whilst saliva flows from the mouth, sometimes in quite a stream. There is no attempt at sweating; the sweat glands of the horse are perfectly insensible to the action of pilocarpine. The involuntary muscles of the intestinal canal are stimulated, and the rectum is repeatedly emptied.'

Horses are poisoned by the subcutaneous injection of five 
grains (Kaufmann). Cattle, however, tolerate much larger quantities. Feser subcutaneously injected a cow and a bull with doses ranging from three to eighteen grains. The larger doses produced abundant secretion of viscid saliva, frequent, short, laboured respiration, tympanites, intestinal irritation, colic, and profuse diarrhœea, but only slight and temporary diaphoresis. Still larger doses increased the œdema of the lung and paralytic tympany of the rumen, and also weakened heart action. But much larger doses, reaching to forty-five grains, were tolerated when given by the mouth. Compared with physostigmine, pilocarpine, although stimulating more powerfully intestinal glandular secretion, had much less effect on intestinal muscular fibre, and two to four times the dose is stated to be required to produce purgation in cattle.

Dogs and cats are more sensitive to the drug than horses or cattle. A dog of $25 \mathrm{lbs}$. weight was prostrated for two days by three-quarters of a grain, and Fröhner records that this dose killed by pulmonary œedema a dog weighing $132 \mathrm{lbs}$. Half a grain caused profuse salivation, continuing for six hours, and increased action of the bowels and kidneys. Half a drachm to a drachm of the leaves, infused in water, produced in English terriers, of 20 to $25 \mathrm{lbs}$. weight, abundant salivation, but no notable diaphoresis. The physiological antagonist of pilocarpine is atropine, which arrests glandular secretion and paralyses the nerve-endings of involuntary muscles. It is hence the appropriate antidote in poisoning by pilocarpine.

\section{MEDICINAL UsEs.-The prompt and general eliminative} action of pilocarpine has suggested its use for the absorption of pleuritic and other effusions, and the removal of products of tissue waste. It has been prescribed for rheumatism, especially when affecting muscles, and in chronic eczema. Kaufmann testifies to its value as an expectorant in catarrh, pneumonia, and complaints resulting from exposure to cold. In such cases it may be usefully combined with other expectorants. Friedberger and Fröhner advise its subcutaneous injection in acute brain inflammation, hydrocephalus, and laminitis. In nephritis it beneficially removes by other channels the albuminoid waste usually got rid of 
by the kidneys. In virtue of its increasing both intestinal secretion and peristalsis, it is serviceable in torpidity and obstruction of the bowels, and may even relieve volvulus and invagination. In these gastro-intestinal cases it is conjoined with physostigmine, which stimulates muscular contractions more powerfully than pilocarpine.

Doses, etc. - Of the fresh leaves, horses or cattle take $3 \mathrm{ij}$. to 3iv.; sheep, pigs, or large dogs, 3 ss. to 3 i., given as an infusion. But pilocarpine nitrate or hydrochloride is more certain and effective, and is prescribed, hypodermically or intratracheally, to horses and cattle in doses of grs. ij. to grs. vi.; to dogs, gr. $\frac{1}{12}$ to gr. $\frac{1}{3}$; to cats, gr. $\frac{1}{60}$ to gr. $\frac{1}{20}$, dissolved in water, 1 grain of the salt to 20 minims of water containing a drop or two of alcohol. As a myotic a few drops of a two per cent. solution may be used.

\section{URARE}

Curara. Wourara. Wourali. Urari. The South American arrow poison. An extract from one or more species of Strychnos, mixed with some mucilaginous juice, and owing its activity to an alkaloid, curarine $\left(\mathrm{C}_{10} \mathrm{H}_{15} \mathrm{~N}_{3}\right)$. (Not official.)

Curare is a black-brown substance, with a very bitter taste, and imperfectly soluble in water. It appears to vary somewhat in composition, and two varieties have been described.

The drug, and its twenty-times more active alkaloid curarine, by whatever channel they enter the body, paralyse the peripheral endings of motor nerves. The nerves of the voluntary muscles of the limbs are first affected, then those of the trunk and head, until the only movement is that of respiration. Later and with larger doses this becomes enfeebled and finally fails. Intelligence and consciousness remain unimpaired. Horses are poisoned by 32 to 48 grains, dogs by gr. $\frac{5}{6}$ to gr. $3 \frac{1}{3}$ of curare given subcutaneously. Experimental investigations demonstrate that the poison affects simply the nerve terminals in the muscles, the effect being similar to fatigue; that paralysis is removed when the drug is washed out of the muscle ; that it acts less powerfully 
on the vaso-motor system of rabbits and cats than of dogs ; applied to the conjunctiva it dilates the pupil of birds (the iris having striated muscle), but not of mammals (iris unstriated); while the reverse obtains in the case of atropine. Although the blood becomes charged with carbonic acid, the motor nerves are so paralysed that asphyxial convulsions do not occur. The heart continues to beat after the breathing ceases, but the poison is quickly eliminated by the kidneys, and artificial respiration persisted with accordingly prevents death, even when lethal doses have been given. The rapid excretion of the poison, unchanged by the kidneys, is strikingly illustrated by the fact that the urine of a frog, poisoned by curare, injected subcutaneouşly into a second frog, paralyses it, and its urine will even paralyse a third (Brunton).

It is allied to hemlock and conine, and to methylstrychnine, methyl-brucine, and methyl-thebaine. Some of its effects are antagonised by strychnine. It has been given in chorea and epilepsy ; but in neither of these has its efficacy been established. In tetanus, Wiedmann, employing acid solution of curare, which had previously been tested on rabbits, injected a quantity equal to 3 ijss. of the drug into the facial subcutaneous tissue, and in twelve hours the horse was able to eat. Injections into the neck were equally satisfactory.

The doses for horses and cattle are from gr. ss. to gr. j.; for dogs, gr. $\frac{1}{20}$ to gr. $\frac{1}{5}$. It acts much more powerfully when injected intravenously, hypodermically, or intratracheally, than when swallowed. Any considerable amount of food in the stomach retards and minimises its effect when given by the mouth.

\section{I G I TA L IS}

Foxalove. The dried leaves of Digitalis purpurea. Collected from plants commencing to flower (B.P.). Nat. Ord.-Scrophulariaceæ.

Digitalis grows wild in this country, and in many parts of the Continent, on gravelly, sandy soils, in young plantations, on hedge sides, and hill pastures. Other species have 
probably the same properties as the $\mathrm{D}$. purpurea, recognised by the B.P. It is herbaceous, biennial or perennial, with numerous drooping, purple-spotted, occasionally white flowers, an erect stem one to five feet high, and large alternate ovate-lanceolate, crenate, rugose leaves, downy, especially on their paler lower surfaces, and tapering into winged foot-stalks. Leaves of the second year's growth are preferred as they are richer in the active principles. The leaves are dried in baskets, in darkness, over stoves, and are then of a dull-green colour, with little smell, but a nauseous, bitter, slightly astringent taste. They should be used when fresh; twelve months' keeping greatly diminishes their activity. Both the roots and seeds are bitter, and probably active, but less so than the leaves.

The chemistry of digitalis is still somewhat uncertain, but the following active principles can be extracted :-

(1) Digitalin, or digitalinum, a bitter glucoside, almost insoluble in water, but readily soluble in alcohol. Pure digitalin and the commercial varieties are topical irritants and muscle poisons, and hence notable cardiac poisons. The three undermentioned non-nitrogenous substances have also been isolated.

(2) Digitoxin is a crystalline body, insoluble in water, but soluble in ether, chloroform, and alcohol. It is the most active of the several glucosides which constitute digitalin.

(3) Digitalein is bitter and amorphous, and insoluble in water. Soluble in alcohol, chloroform, and in acetic acid. Digitoxin and digitalein act in the same manner as digitalin.

(4) Digitonin is soluble in 600 parts of water, and insoluble in alcohol, benzine, ether, and chloroform (Kaufmann) ; resembles saponin, the active principle of quillaia, the Chili soap bark ; is a powerful irritant, local anæsthetic, and muscular paralysant; and hence is in some degree antagonistic to digitalin, digitoxin, and digitalein.

These non-nitrogenous bodies, in very variable proportion, are obtainable from the plant grown in different climates and circumstances, and also from different parts of the same plant. The tincture contains the first three in solution, while the infusion holds them in suspension and thus is an active preparation. They readily yield products of 
decomposition, especially when exposed to high temperatures, and several of these products are convulsants like picrotoxin.

Actions AND USES.-Digitalis is a topical irritant and contractor of muscle, especially of the unstriped variety. Medicinal doses are vascular and cardiac stimulants and tonics, and are prescribed to increase the force and coordinating power of the heart, and relieve congestion of veins and capillaries. They are diuretic. In healthy animals small doses of digitalin lower rectal temperature from $\frac{4}{10}$ ths to $\frac{5}{10}$ ths of a degree. Large doses are muscle poisons : they contract spasmodically and even tetanically the heart and other muscles, and kill usually by cardiac paralysis.

General Actions.-Digitalis, in contact with living tissues, is an irritant. Injected under the skin or into the trachea it irritates and inflames. Placed in the mouth, besides a sensation of bitterness, it causes salivation and redness. Introduced into the stomach and bowels, it induces irritation and nausea; in carnivora, vomiting, colic, and diarrhœa. It is absorbed slowly, often taking several hours to produce its effects, and contracts muscular fibre, notably of the heart and arterioles. It also stimulates the medulla and so increases the inhibitory action of the vagus. Properly regulated doses thus strengthen and prolong the cardiac diastole, both auricles and ventricles are more fully dilated, systole is more vigorous, and consequently the heart is more perfectly emptied. The muscular fibres of the arterioles have their tonicity increased. Blood-pressure accordingly is raised. Such doses, while increasing the volume of the pulse, diminish the pulse-rate of healthy horses three to five beats, and of dogs ten to fifteen beats per minute, and these effects last from six to twelve hours. The action on the heart is more notable on the dog and sheep than on the horse and ass.

The action on the circulation may be divided into the following stages :-

1. Medicinal doses cause a fuller stream of blood to be thrown into the circulation, blood-pressure rises, the pulse is slowed, but increased in volume. These effects depend on 
the two factors, (1) contraction of muscular fibre, and (2) stimulation of the inhibitory vagus roots in the medulla, and of the nerve-endings in the heart itself. This represents the therapeutic stage.

2. Following these effects, there is often a stage of excessive inhibition with very marked slowing of the pulse, lessened outflow from the heart, and a temporary fall of blood-pressure.

3. Larger doses now bring about quickening of the heart beat and a second rise of pressure, these effects being produced by paralysis of the vagus inhibition, and by a marked increase in the irritability of the cardiac muscle. The rhythm becomes quicker, but the auricles and ventricles contract independently of each other, and the heart action becomes very irregular.

4. Still larger doses produce rapid fall of blood-pressure, extreme irregularity of the heart (delirium cordis), sudden stoppage of the heart, and death. The heart usually stops before the respiration.

Neither digitalis nor digitalin has any very direct action on the brain or spinal cord, the stimulation being confined to the medulla, nor any marked effect on sensory or motor nerves. They temporarily quicken, and more notably and permanently slow, respiration. By increasing general blood-pressure, a somewhat fuller stream of blood passes through the kidneys, and thus diuresis is tardily produced. This is especially well marked in cases of valvular disease of the heart. The venous congestion and stagnation of blood in the kidney is relieved and fresh arterial blood is pumped through it. No direct irritation of the kidneys occurs; but large doses, constricting arterioles, while at the same time lessening the heart's force, diminish renal excretion, and the drug consequently being longer retained, its general effects are intensified, and its so-called cumulative action developed. This can be avoided if the effects on the circulation are not allowed to get beyond the therapeutic stage.

Toxic Actions. - The toxic doses of the powdered leaves are thus stated by Kaufmann:-For horses, 400 to 480 grains; for dogs, 80 to 130 grains; for cats, 30 grains. 
The toxic dose of amorphous digitalin for horses is $2 \frac{1}{2}$ grains ; for dogs, $\frac{1}{3}$ grain; and for cats, $\frac{1}{6}$ grain.

A horse was poisoned in twelve hours by two ounces of dried powdered leaves (Moiroud). One ounce, and in some cases six drachms, given to horses in bolus, caused, in three to ten hours, loss of appetite, frequent urination, fluid fæces, sometimes tinged with blood, a pulse at first full and increased, but afterwards small, slow, and irregular, contraction of the pupil, difficulty of breathing, languor, and, after twelve or sixteen hours, death (Hertwig). Bouley and Reynal, administering large doses to horses, observed quickened circulation, diuresis, abrupt and energetic heartbeats with a vibratory thrill, and subsequently a bellows murmur, with intermittence, the pulse, as death approached numbering 120 to 140 . Smaller doses, after slight acceleration, lowered pulsations 20 or 25 beats per minute, and rendered the several cardiac sounds particularly distinct.

The following cases, in which Dun gave full medicinal doses of digitalis to healthy horses, illustrate its effects on the heart, its nauseating action, and its irritation of the digestive organs.

In February, powdered digitalis was given to three horses in good health, and receiving daily $12 \mathrm{lbs}$. hay, $5 \mathrm{lbs}$. oats, and $5 \frac{1}{2} \mathrm{lbs}$. bran. On the $20 \mathrm{th}$ they each received a drachm of the powder at 12 noon, and another drachm at 6 P.M. ; on the 21 st and 22 nd one drachm at 6 A.M., at 12 noon, and 6 P.M. ; and on the $23 \mathrm{rd}$ a drachm at 6 A.M.-in all, nine doses of a drachm each in three days.

No. 1. Brown Mare, 3 years old :-

Feb. 20, 12 noon, pulse 38, respirations 8

$\begin{array}{llllll}\text { " } 21, & \text { " } & \text { " } & 34, & \text { " } & 6 \\ \text { " } 22, & 23, & \text { " } & 28, & \text { ", } & 7 \\ & \text { " } & 28, & , & 7\end{array}$

On the evening of the 22 nd she became dull and refused her food. 23rd, 10 A.M., still dull, without appetite, pupil contracted, passing flatus, with small quantities of fluid fæces ; 4.30 P.M., pulse 32 , more distinct than at noon, pupil considerably contracted, rather less dulness. On the 25th, two days after the medicine was withdrawn, the mare was eating and perfectly well again.

No. 2. Bay Gelding, 3 years old :-

Feb. 20, 12 noon, pulse 36, respirations 7

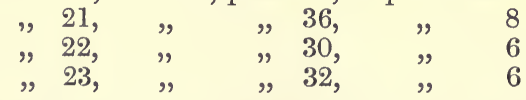

23rd, 12 noon.-Pulse, both yesterday and to-day, slightly irregular; no appetite, very dull and stupid, with the pupil somewhat contracted. 
4.30 P.M., pulse 34 , tolerably firm, but unequal ; eating a little, and scarcely so dull. No more digitalis being given, the animal recovered its appetite, and by the 26th was well again.

No. 3. Brown Mare, 3 years old :-

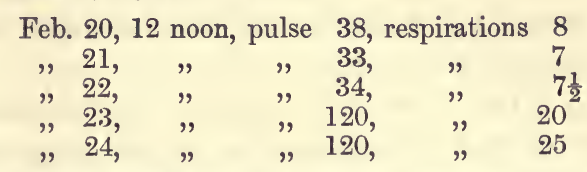

Towards the evening of the 22nd the mare became dull and would not feed. 23rd, 10 A.M., very much nauseated; nose, mouth, and ears cold ; abdomen tympanitic, with colicky pains, and occasional pawing; pupil somewhat.contracted; pulse firm at axilla and heart, but not very perceptible at jaw. Had four drachms of carbonate of ammonia and clysters occasionally, the stimulant being repeated at two o'clock and four. At 4.30 P.M. she was down, much pained, attempting to roll ; pulse 82 , but unequal. 24th, 12 noon, pulse, imperceptible at jaw, about 120 ; respirations 25 , and very much laboured; lips retracted and saliva dripping from the mouth; enormous abdominal tympanites and much pain; rapid sinking; died on 25th at 11 A.M.

Post-mortem examination made next morning at 9.30. Voluntary muscles unusually pale; spots of ecchymosis in the areolar textures, between the muscular fibres and in places underneath the skin. Lungs and pleuræ healthy ; anterior extremity of lungs contained more blood than posterior; venæ cavæ contained the usual amount of dark non-coagulated blood; bronchial tubes inflamed for about six inches along their anterior ends; windpipe inflamed halfway up the neck, and containing flakes of greenish pus mixed with mucus; no froth here or in bronchi. Heart pale, friable, containing a small clot of blood in its left ventricle. A rent of eight inches long was found in the inferior curvature of the stomach, through which food had passed into the omentum; the mucous membrane of the stomach was quite healthy; the organ itself very large, but collapsed, in consequence of the rupture; the intestines were pale and flaccid, and contained enormous quantities of food and gas, but their mucous membrane was quite healthy. The kidneys and generative organs, with the brain and spinal cord, were perfectly healthy.

According to Salvisberg, ruminants can support large doses by the mouth of digitalis leaves without showing any reaction. If, however, the drug be administered intravenously the typical actions of digitalis are produced. In treating pericarditis, Dottl found that doses of 150 grains of powdered digitalis given by the mouth induced uterine pains in pregnant animals and premature labour.

Dogs receiving one or two drachms were nauseated, and, when vomiting was prevented, moaned, and exhibited abdominal pain, green-coloured fluid dejections were passed, the pulse was feeble and indistinct, breathing irregular and distressed, spasmodic efforts were made to empty the bladder, muscular debility preceded death (Tabourin). 
Pigs poisoned by decoction of the leaves are reported to be languid, attempt to vomit, strain, and pass small quantities of fæces ; whilst after death the mucous coat of the stomach and small intestine is inflamed, the kidneys slightly congested, the bladder empty.

MEDicinal Uses.-Digitalis exerts its curative effects in one or more of the following ways-first, by strengthening the action of the heart ; second, by lessening the frequency of the heart-beats ; third, by correcting irregular action of the heart.

When the heart is enfeebled or acting irregularly, as in horses suffering from influenza or other exhausting disease, in cattle convalescing from pleuro-pneumonia or pericarditis, in dogs debilitated by distemper, digitalis improves cardiac co-ordination and expulsive power, and gives tone to relaxed capillaries, rendering the quick, weak irregular pulse-beat slower, stronger, and steadier. Difficulty of breathing and dropsical effusion resulting from imperfect action of the heart, especially if due to valvular disease, are usually relieved, and general as well as cardiac nutrition is improved. In such cases digitalis is usefully conjoined with potassium chlorate or nitrate, or with alcohol, or ether. Palpitation in horses resulting from over-exertion, or from fast work performed shortly after a full meal, occasionally persists for several days; the violent, irritable impulse of the heart, accompanied by lifting of the flanks, comes in paroxysms; repeated doses usually control such inordinate, tumultuous, functional disturbance. In the more violent of these cases digitalis may be conjoined with small doses of aconite, and in other cases with belladonna. In dilatation of the heart, with mitral insufficiency, carefully regulated doses of digitalis abate the dyspnœa, cold extremities, venous pulse, and œdema. In dilatation or hypertrophy of the left ventricle-common in hard-worked, aged horses-even when accompanied by slight valvular disease, the full, intermittent pulse is usually moderated, and the breathing relieved by digitalis.

In pericarditis, after the more acute symptoms have been subdued by salines, digitalis frequently lessens the embarrassed breathing and reduces the excessive pulse rate. In 
endocarditis, occurring occasionally in cattle, it renders the heart-beat more regular, and gives fulness to the small thready pulse. Digitalis, though often given, should not be advised in cases of internal hæmorrhage, for the increased flow of blood from the heart and the rise of blood-pressure tend to increase the bleeding.

In equine pneumonia, especially in the second stages, digitalis frequently relieves engorgement, probably by propelling blood in fuller stream into the abdominal and other vessels. Promoting circulation, it moreover aids oxygenation of blood, and hence is also useful in congestion and purpura. It is a frequent constituent of cough mixtures.

Digitalis relieves many cases of dropsy by regulating faulty heart-action, stimulating dilated arterioles, as well as by inducing diuresis. In pleuritic effusion, Robertson gave horses digitalis, grs. xx. to grs. xxx.; potassium nitrate, $3 \mathrm{ij}$.; powdered cantharides, grs. iv. to grs. $\mathrm{x}$., made into bolus, and repeated twice daily for a week. Diuresis is determined by prescribing digitalis with salines - a combination often useful in cardiac dropsy.

The chief indications for the use of digitalis are an enfeebled, irritable, jerking, or irregular heart, deficient arterial pressure, venous engorgement, and scanty secretion of urine. Under its influence the pulse gains force and amplitude, respiration becomes less difficult, and urination is increased. It is more suitable for chronic than acute cases. As with other tonics, it is best tolerated in those weak and irritable states of the heart in which it is most serviceable. It is of little use in difficulty of breathing or dropsical conditions chiefly dependent on lung disease. It does harm in aortic disease or in hypertrophy, where the pulse continues strong, firm, and regular; or in enfeebled circulation dependent on advanced fatty degeneration. Nausea or irritability of the digestive organs, coldness of the extremities, unwonted force of the pulse-beats, indicate that the medicine should be stopped, or given in reduced amount. The effects of overdoses are combated by alcohol or other stimulants, injections of ether or strychnine; emetics and purgatives; and by keeping the patient perfectly quiet. 
Doses, etc.-Of the powdered leaves, horses take grs. $x$. to grs. $\mathrm{xxx}$. ; cattle, 3ss. to $3 \mathrm{j}$.; sheep and pigs, grs. v. to grs. x. ; dogs, gr. i. to grs. iv., in bolus or pill. These doses may be administered daily for a week, and are advantageously conjoined with potassium iodide, caffeine, or arsenic. Digitalis and digitalin are cumulative, and care should be exercised in their administration. It is preferable to begin with full doses and gradually diminish the quantity. The infusion is made by digesting for fifteen minutes 60 grains of dried leaves with 20 ounces of distilled water. The tincture is made by maceration and subsequent percolation of $2 \frac{1}{2}$ ounces dried leaves with one pint alcohol ( 60 per cent.), B.P. It contains $54 \frac{1}{2}$ grains to the fluid ounce, is about sixteen times the strength of the infusion, and when standardised is the most suitable preparation for cardiac cases. Horses and cattle take fZii to fZiv.; sheep, f Zss. to $\mathrm{f}_{3 \mathrm{j} .}$; dogs, $\mathrm{m}_{\mathrm{ij}}$. to $\mathrm{m}_{\mathrm{xxx}}$.

In commerce four varieties of digitalin are met with(1) Homolle's, or French ; (2) the German; (3) Nativelle's ; and (4) digitalin (Kiliani). One-twelfth grain digitalin is equal to $15 \frac{1}{2}$ grains of the powdered leaf, and the dose for the horse is gr. $\frac{1}{12}$ to gr. $\frac{1}{6}$; for the dog, gr. $\frac{1}{60}$ to gr. $\frac{1}{30}$. The several preparations are administered by the mouth. Even when diluted they are apt to irritate if given hypodermically or intratracheally. They are not always of uniform strength. For hypodermic or intratracheal injection the physiologically standardised Digitalone should be used.

\section{STROPHANTHUS}

The dried. ripe seeds of Strophanthus kombé, freed from the awns (B.P.). Nat. Ord.-Apocynaceæ.

The ripened follicles contain upwards of a hundred oval acuminate seeds, about three-fifths of an inch long and one-sixth of an inch broad, covered with silky hairs ; odour characteristic, taste very bitter. They contain 8 to 10 per cent. of an active, bitter, crystalline glucoside, strophanthin, which is soluble in water and rectified spirit, insoluble 
in chloroform or ether. Similar seeds are got from the S. hispidus. A paste prepared from strophanthus seeds is used in Africa as an arrow poison.

ACTIONS AND UsEs.-The seeds and their active principle are muscle poisons. They augment the contractile power, especially of striated muscles. They resemble digitalis and the bodies of that group. They are prescribed as cardiac tonics and diuretics.

Fraser has carefully investigated the actions of strophanthus and digitalis. The former, he reports, is more soluble, and hence more rapid in its actions ; but it is also more quickly eliminated, and its effects are hence less durable, and the cumulative results credited to digitalis are not observed. Its efficacy does not, however, seem to be impaired by repetition. Full doses produce less gastrointestinal disorder and less marked vascular contraction. Strophanthus acts more notably on striated muscle, digitalis on unstriated; strophanthus has less diuretic action, and may with safety be given more frequently and in larger doses than digitalis. Both increase the length and power of the heart systole, and hence strengthen and co-ordinate enfeebled or irregular action. Fröhner has experimented on various animals, and concludes that the lethal dose of strophanthus tincture is about half a gramme ( $7 \frac{1}{2}$ minims) per kilogramme of body-weight. Horses tolerate 100 grammes, dogs 10 to 20 minims of the tincture. Full doses, he states, are irritant, narcotic, producing hæmorrhagic gastro-enteritis, colic, diarrhœa, cramp, with some stupor. The cardiac action manifests two stages(1) diminution of pulse-rate, with rise of temperature ; (2) increase of pulse-rate, with diminution of temperature.

Medicinal Uses.-Strophanthus is prescribed to slow, strengthen, and steady feeble or faulty heart action. Combining cardiac tonic and diuretic effects, it is commended by Fröhner in valvular disease, hydrothorax, hydropericarditis, ascites, and chronic nephritis.

DoSES.-Of the tincture, made with one part of seed to forty of alcohol (70 per cent.), horses and cattle take f3iv. to $\mathrm{f} 3$ vi., and dogs $\mathrm{m}_{\text {ii. }}$ to $\mathrm{m}_{\mathrm{xxv}}$. 


\section{SQ U I L L}

ScIlla. The bulb of Urginea scilla, divested of its dry, membranous, outer scales, cut into slices, and dried (B.P.). Nat. Ord.-Liliaceæ.

The large bulbs of this Mediterranean plant, when sliced and dried, have a faint odour and disagreeable, mucilaginous, bitter, acrid taste. The slices are easily reduced to powder. The active principle is a glucoside - scillain or scillitoxinwhich is soluble in water, acetic acid, and alcohol.

ACTIONS, USES, AND DOSES.--Squill and its active principle, in full doses, are irritants, causing vomiting and purging; absorbed into the blood, they lower the pulserate and raise blood-pressure; they are expectorant and diuretic. They resemble digitalis, acting as heart tonics, and producing diuresis. Large doses, or small doses too long continued, induce urinary irritation and hæmaturia. Squill is prescribed chiefly in those catarrhal and bronchial cases in which secretion is defective. Robertson gave horses the syrup in $f$ Ziv. doses; dogs take $m_{x}$. to $m_{x v}$., conjoined, as the exigencies of the case require, with ammonium acetate solution, or camphor electuary. The vinegar and tincture are used in about half the dose of the syrup. Powdered squill is sometimes added to electuaries. Horses may be given 3ij. to 3iv.; cattle, 3iii. to 3vi.; dogs, gr. i. to grs. viii.; cats, gr. $\frac{1}{2}$ to gr. i., in bolus, pill, or drench.

\section{B R O O M}

Scopari Cacumina. The fresh and dried tops of Cytisus scoparius (B.P.). Nat. Ord.-Leguminosæ.

The tops and other parts of the shrub contain a natural glucoside, scoparin $\left(\mathrm{C}_{21} \mathrm{H}_{22} \mathrm{O}_{10}\right)$, which has diuretic properties and a volatile, oily, poisonous alkaloid, sparteine $\left(\mathrm{C}_{15} \mathrm{H}_{26} \mathrm{~N}_{2}\right)$, which much resembles conine in some of its actions. It slows the rhythm and lessens the force of the heart, but by contracting arterioles, it causes a slight rise of blood-pressure, and acts as a diuretic. The sulphate of 
sparteine is occasionally prescribed in dropsy associated with heart disease, in doses of grs. viii. to grs. xlv. for horses, and gr. $\frac{2}{3}$ to grs. $1 \frac{1}{2}$ for dogs. It is given in solution, in pill, or electuary. For hypodermic injection a solution of the sulphate (one part in fifty of distilled water) may be used in appropriate doses.

\section{B U CH.U}

Buchu Folia. The dried leaves of Barosma betulina (B.P.). Nat. Ord.-Rutaceæ.

Buchu is a shrub two to four feet high, and a native of the Cape of Good Hope. The leaves are smooth, dull yellow-green, with a strong, penetrating odour, a bitter aromatic taste, and varying in different species from half an inch to an inch and a half in length. Oil glands are distinctly visible in the leaves, especially near the margin. They contain a volatile oil, a bitter substance, and mucilage.

ACTIONS AND UsEs.-Buchu is a mild, stimulating bitter, expectorant, and diuretic, and a disinfectant of the urinogenital mucous membrane. The oil or active principle is excreted by the kidneys and bronchial mucous membrane. Robertson gave it to allay irritability in cystitis, using it either alone or with borax or benzoic acid. The tincture of buchu-made with one of buchu to five of alcohol (60 per cent.) is seldom prescribed.

The dose of the infusion for horses or cattle is zi. to ziv. ; for dogs, 3j. to 3ij. The infusion is made with one part leaves and twenty parts of boiling water. Animals readily take this infusion when it is mixed with linseed tea or barley water. It is sometimes advantageously conjoined with belladonna, opium, hyoscyamus, potassium bromide, or saline diuretics.

Bearberry leaves - the leaves of Arctostaphylos Uva-ursi - contain the bitter neutral extractive arbutin, which within the body is in part converted into hydroquinone, and is employed as a diuretic astringent, and antiseptic, in chronic vesical irritation. 
Pareira - the root of chondrodendron tomentosum, containing the active principle buxine, although not very reliable, is also used for the same purposes as buchu and uva-ursi.

\section{ACONITE}

Aconite.-Monkshood. Wolfsbane. Blue Rocket. Aconitum. The root of Aconitum napellus. Collected in the autumn from plants cultivated in Britain, and dried. Nat. Ord.-Ranunculaceæ.

Aconitine.-An alkaloid obtained from Aconite root, and having the formula $\mathrm{C}_{33} \mathrm{H}_{45} \mathrm{NO}_{12}$. (B.P.)

Botanists have numbered twenty-two species, and upwards of a hundred varieties of aconite, which are common throughout the cooler mountainous countries of both hemispheres. Some species are eaten as vegetables, some are bitter tonics; but others, as the Aconitum ferox, sinense, and napellus, are sedative poisons. The last of these, the common officinal species, is a doubtful native of Britain, but often grown for its flowers in gardens and shrubberies. Its several varieties are herbaceous, with perennial, tapering, carrot-shaped, brown roots, with lateral rootlets, from which, after the first year's growth, are formed one or more oval tubers, at first nourished by the decaying parent root; several annual, erect, glabrous stems two to five feet high ; numerous alternate dark-green leaves ; long-stalked, helmetshaped blue or purple flowers, which form loose terminal racemes, and appear in June or July; and dry, black, angular seeds, which ripen about the end of August.

Aconite root, from which the tincture, liniment, and alkaloid are prepared, varies from two to four inches long, and from half an inch to nearly an inch thick at the crown, which is knotty; is brown externally, but pinky white within; conical, rapidly tapering, prominently marked with the bases of the rootlets, and of an earthy odourcharacters which distinguish it from the larger, longer, more uniformly cylindrical, white, pungent, bitter root of horse-radish, for which aconite root has sometimes been fatally mistaken. According to Schroff, Vienna, the root 
is six times as active as the other parts, and should be taken up after the plant has flowered in autumn, when it is in perfection, or before the new stem rises in spring, cut into small pieces, and dried at a low temperature. The leaves are less active than the root, but more so than the flowers, fruit, or stem. Any part of an active aconite, when slowly chewed, produces a peculiar sensation of tingling, and numbness of the lips and tongue.

The chief active principle-aconitine $\left(\mathrm{C}_{33} \mathrm{H}_{45} \mathrm{NO}_{12}\right)$-is obtained by a tedious process from the powdered root. It occurs in colourless, hexagonal rhombic prisms, nearly insoluble in water, but readily soluble in alcohol, chloroform, and ether. Its salts are crystalline. Aqueous solutions of aconitine when heated are hydrolised with the formation of acetic acid and benzaconine. This latter substance may be further split into benzoic acid and aconine. Both the plant and the ordinary pharmaceutical preparations of aconite may contain these decomposition products, and even samples of 'aconitine' as sold may vary considerably in pharmacological action because of a varying content of benzaconine and aconine: Benzaconine is very much less toxic than aconitine; it slows the heart, but has no paralysing action on sensory nerve-endings. Aconine is still less active and is practically non-toxic. In the plants the alkaloids are united with aconitic acid $\left(\mathrm{C}_{6} \mathrm{H}_{6} \mathrm{O}_{6}\right)$.

Actions AND Uses.-A local anodyne, acting specially on the peripheral endings of sensory nerves, on the heart, and on respiration; aconite kills by respiratory arrest. Its physiological actions as a cardiac and respiratory depressant, together with a certain central action, render it a febrifuge; it is also diaphoretic. It is prescribed in acute febrile conditions and in the earlier stages of acute local inflammation. It is used topically to relieve pain.

General Actions.-Locally applied, in virtue of its action on sensory nerve-endings, aconite produces first irritation, tingling and twitching, and subsequently numbness and anæsthesia. It is not absorbed through unbroken skin, unless made up with some substance, like alcohol or oil, which is volatile and penetrating. Aconite tincture is rapidly absorbed and quickly passes into the tissues, as is 
shown by the blood of a poisoned dog, five minutes after the drug has been administered, being transferable into the veins of another dog without producing the phyșiological action of the poison.

Full medicinal doses administered by the mouth induce salivation, champing of the jaws, movements of swallowing, and nausea, and cause in dogs and cats vomiting, and in horses, ruminants, and rabbits retching and eructation of frothy mucus. The topical irritant action is exerted not only on the stomach, but sometimes on the bowels, which are affected by spasms and diarrhœa, while the secretions of the skin and kidneys are also increased. Within fifteen to twenty minutes the strength and frequency of the heartbeats are reduced, and blood-pressure is lowered. These effects on the circulation appear to depend upon the inhibitory action of the vagus due to stimulation of the vagus centre in the medulla. This can be proved experimentally by cutting the vagi when the heart resumes its normal rate and strength. The vaso-motor centre is also probably stimulated at the same time, but the cardiac depression is such that the blood-pressure falls. From the impaired circulation temperature is lowered, and general muscular weakness ensues. There is some evidence to show that aconitine acts to some extent on the heat-regulating centres in the brain. Kaufmann records that subcutaneous injection of aconitine, in moderate doses in dogs, lowered the temperature from $38.5^{\circ}$ Cent. to $36.7^{\circ}$ Cent.; while intravenous injection in horses reduced the temperature from $37 \cdot 4^{\circ}$ Cent. to $37 \cdot 1^{\circ}$ Cent. Partly from the reduced circulatory force, and partly from the drug directly depressing the respiratory centre, breathing is slow and deepened, and exhibits a distinct expiratory effort. When large or repeated doses have been given, aconite exerts a direct action on the heart muscle, lessening its power of conducting impulses from auricle to ventricle. Thus the contractions of the auricles and ventricles are independent and arhythmic, cardiac action becomes irregular, and often much quickened, but tension remains low (delirium cordis); the breathing becomes still slower, shallower, and more laboured; after every two or three respirations there is a distinct pause in 
expiration. Convulsions, mainly due to asphyxia, sometimes precede death, which generally results from failure of respiration. The brain and special senses are unaffected. The pupil, which in the earlier stages of poisoning is sometimes dilated and sometimes contracted, during the later stages remains dilated. Aconite is removed from the body chiefly in the urine, augmenting both its solid and fluid parts.

Toxic EFFEcTs.-Aconite exerts tolerably uniform effects upon all animals, especially when injected hypodermically. Horses have been poisoned within two or three hours by 120 to 150 minims of Fleming's tincture, given by the mouth. Cattle, however, sometimes receive large doses without fatal effects. Dogs weighing $40 \mathrm{lbs}$. are killed usually within half an hour by 50 to 60 minims of Fleming's tincture ; cats by 10 minims. But half these doses are liable to produce alarming symptoms. Full medicinal doses sometimes leave untoward effects ; pulse, blood-pressure, and breathing may continue reduced for ten or twelve hours, while nausea and impaired appetite may remain for several days.

The following experiments on animals were made at the Edinburgh Veterinary College by Barlow and Dun :-

A black mare, $15 \frac{1}{2}$ hands high, previously used for slow work, and in good health, got, at 12.40 P.M., one fluid drachm of Fleming's tincture of aconite. At 1 she was nauseated, had eructations of frothy mucus, with attempts to vomit, which increased till 1.30, when she went down. The pulse, which was 35 before administration of the poison, was now 60 , and very weak; she continued down till 7 P.M., when she was destroyed, in consequence of being unable to stand.

An aged chestnut cab horse, 16 hands high, and useless from quittor, was tied up for ten minutes, to ensure perfect quietude. The pulse was then found to be 56, and the respirations 12. The animal had a good appetite and regular evacuations. At 10 o'clock he got ninety minims of Fleming's tincture of aconite in a linseed meal ball, the head being still kept tied up for fifteen minutes. In half an hour he fed greedily on potatoes and beans, but no change was observable. At 1 P.M. he got tifty minims of the same tincture in four ounces of water. At 1.15 he appeared to be making continual efforts to swallow something; his mouth was closed; and, after such attempts at swallowing, air and fluid were regurgitated up the gullet, causing a rattling noise, as of air-bubbles mixed with water. At 1.20 the pulse was 50 ; symptoms of actual nausea appeared; the muscles on the side of the neck and throat were contracted, the muzzle brought near to the breast, the lips retracted, and the mouth slightly opened. Fits of retching came on every two minutes, and increased in violence during the next ten or fifteen minutes. 1.30.-During each paroxysm of retching the mouth 
was opened, the lips retracted, and four or five ounces of frothy mucus discharged on the ground. The pulse had fallen to 40 , and become weak. On account of the retching, the respirations could not be counted. Sweating broke out over the body; the mucous membranes of the mouth, nose, and eyes were pallid, and there were fibrillary twitchings of the muscles, especially about the head and neck. 2 P.M.-Pulse 38, and weak ; the respirations not easily counted, but probably about 9 ; in other respects no change. The animal passed fæces and urine freely; and, shortly after taking a pint of cold water, lay down somewhat relieved, with the retching scarcely so frequent. At 2.30 the pulse was somewhat weaker; the breathing irregular, interrupted, and sighing; and the animal unable to rise. The labial and nasal muscles were contracted, causing retraction of the lips, and disclosing the gums blanched, and the teeth covered with frothy mucus. Two bottles of strong ale were given, with half an ounce of spirit of ammonia. At 3 P.M. the pulse was 35, and still weaker than before; respiration was somewhat accelerated, probably owing to the animal being down; profuse sweating continued and the retching, though somewhat subsided, still came on about every ten minutes. The animal remained down without much change until about 6 , when the nausea was somewhat diminished, but the pulse so weak as to be scarcely perceptible. He was raised with difficulty, and stood, blowing much, for fifteen minutes. At 7 there was little change ; the pulse remained imperceptible, the respirations about 20, and there was no appetite for food or drink. He was left with the expectation of finding him dead next morning, but at 7 A.M. he was up and eating. His pulse was 65 , his respirations 10 , and his appearance very haggard and reduced. He continued in much the same state for a week, never regained his former look or appetite, for two days was unable to rise or stand, and became much wasted. He was destroyed by six drachms of prussic acid; but, on post-mortem examination, every part except the lungs seemed healthy. These organs, more especially the right lung, were extensively studded with patches of extravasated blood about the size of walnuts, which, in those parts connected with the pulmonary tissue, were more or less softened, and emitted an odour characteristic of heated, decomposed blood. The rusty fluid produced from the softening had in various places passed into the bronchi, imparting to their frothy mucus a brown colour.

A cat of average size got seven minims of Fleming's tincture of aconite. In two minutes severe retching came on, with a copious supply of saliva, probably arising from paralysis of the fauces; and in five minutes painful vomiting and involuntary muscular contractions of a most active kind, with perverted action of the voluntary muscles, causing the animal to leap up the wall and turn somersaults backwards. In this, as in most other cases, the pupil, at first somewhat contracted, ultimately became dilated. The pulse was reduced in volume and strength, shortly becoming very weak; the breathing was gasping. The vomiting and inordinate muscular action continued until within two or three minutes of death, which took place twenty minutes after the administration of the poison. No morbid or peculiar post-inortem appearances were observable.

A medium-sized Scotch terrier got thirty minims of Fleming's tincture. In five minutes painful and active vomiting came on, which must have effectually emptied the stomach. The retching and vomiting continued, however, for half an hour, when the animal was so exhausted and paralysed in its hind extremities as to be unable to walk, except by supporting itself on its fore-limbs and dragging the hind-quarters. It gradually recovered, however, in about two hours. In some cases a drachm of Fleming's tincture has destroyed dogs with as much rapidity as an equal quantity of prussic acid. 
After death the lungs are collapsed, and contain little blood; the trachea and bronchi contain excess of frothy mucus, accumulating owing to paralysis of the respiratory muscles and glottis; the cavities of the right heart are greatly distended with blood ; the left heart is nearly empty ; there are ecchymoses of the lungs, pleura, and endocardium ; the digestive organs are normal.

ANTIDOTES.-If the patient is seen immediately after swallowing the poison, endeavour should be made to empty the stomach by an emetic or the stomach-pump. Tannin and potassium iodide to precipitate the unabsorbed aconitine and alcoholic and ammoniacal stimulants are given. Ether, digitalis, or atropine should be used hypodermically to antagonise the sedative effects of aconite on the heart and breathing. Warmth, and infriction of the chest-walls with stimulating liniment also assist in maintaining cardiac and respiratory action.

Medicinal Uses.-Aconite is more used by British than by German practitioners. Fröhner states that there are other safer febrifuges. Cagny indicates its more general use in France, and characterises it as the grand vaso-motor sedative, slowing the circulation in acute fever. Kaufmann designates it a very precious febrifuge in the early stages of all internal inflammatory maladies, especially of the airpassages. Medicinal doses, as already stated, within ten or fifteen minutes reduce the number and lessen the force and tension of the pulsations, lower abnormal temperature, and relieve pain. In virtue of these actions, carefully regulated doses are beneficial in fever and acute inflammation in robust patients, as in the earlier stages of pleurisy, enteritis, peritonitis, mammitis, lymphangitis, laminitis, and acute rheumatism. Pharyngitis in horses, accompanied by high fever, is sometimes controlled by a moderate dose, followed at intervals of an hour by half-doses, repeated until five or six have been given. In the more common epizootic sore-throat of influenza, aconite is useless, and indeed injurious on account of its depressant effect on the heart. Although serviceable in pharyngitis, laryngitis, and pleurisy, it is too reducing a remedy to be used in most cases of bronchitis or pneumonia. 
Conjoined with a purgative, aconite is sometimes prescribed in spasmodic colic. In enteritis in horses, within five minutes after aconite tincture is swallowed the pulse falls from 100 to 70 beats per minute, and this effect is usually succeeded by gradual abatement of fever and pain. Robertson prescribed in enteritis $m_{v}$. Fleming's tincture, and 3 ss. each of camphor and powdered opium in a pint of gruel. R.Rutherford finds aconite specially useful in laminitis. The patient, he urges, should be hobbled and thrown, especially when all four feet are affected. A full dose, followed by four or five half-doses, given at intervals of one to two hours, abates violent cardiac action, fever, and pain. In acute rheumatism it usually relieves both febrile symptoms and local pain. In small, frequently repeated doses, either alone or with hemlock, it usually controls and steadies tumultuous, excessive, or irregular action of the hypertrophied heart, especially in plethoric patients. Although administered for other purposes, it frequently leads to the expulsion of intestinal worms.

Paralysing sensory nerves, aconite is used externally as a local anodyne in neuralgic and rheumatic affections, and for swollen and painful joints. As with other anodynes, it is more effective in combating irritative than inflammatory pain. It frequently relieves the itching of dermatitis and eczematous eruptions in horses and dogs. More rapid absorption and greater anodyne effect are secured by adding a little chloroform to the aconite tincture or liniment. The external application of aconite, it must be remembered, demands, however, almost as much care as its internal use.

Doses, etc.-The plant is not used in the crude state. The extract, unless very carefully made from an alcoholic solution, is apt to be of defective or irregular strength. The B.P. tincture ( 1 in 20 ), now made with two-fifths of the proportion of root ordered in the B.P. of 1885 , is convenient alike for internal and external use. For horses, the dose varies from $m_{x x v}$. to $3 j$.; for cattle, $\mathrm{f} \zeta$ ss. to $\mathrm{f}$ Ziss. ; for sheep and pigs, $m_{x}$. to $m_{x x}$; for dogs, $m_{i j}$. to $m_{x}$. Fleming's tincture ( 1 in $1 \frac{1}{2}$ ), still used in veterinary practice, is twelve times stronger than the B.P. tincture, and, on account of its concentration, requires to be used carefully. The dose 
for horses is from $m_{v}$. to $m_{x x}$; f for cattle, from $m_{x}$. to $m_{x x x}$; for sheep, $m_{i j .}$ or $m_{i i j . ;}$ and for dogs, from $m_{\text {ss. }}$. to $m_{\mathrm{j}}$. Either tincture should be given in several ounces of cold water. The effects of full doses sometimes continue for twelve or fifteen hours. Small and repeated doses are preferable to larger doses at longer intervals. The first may be a full dose, and may be followed by five or six half-doses, repeated, as the case appears to require, at intervals of from half an hour to two hours. The antipyretic effects which should thus be produced are usually kept up by salines and other treatment. Used hypodermically, less than half the above quantities suffice. Walley taught that the activity of aconite is increased by giving it in combination with alkaline carbonates. The liniment of aconite ( 1 in $1 \frac{1}{2}$ ), made with powdered root, camphor, and rectified spirit, is occasionally used. It should not be applied to a wound.

Aconitine is one of the most potent of sedative poisons. Headland records that $\frac{1}{300}$ th of a grain in solution in water suffices to destroy a mouse; $\frac{1}{10} \overline{0}$ th of a grain kills a small bird after a few minutes, and $\frac{1}{50}$ th almost instantaneously; $\frac{1}{20}$ th to $\frac{1}{10}$ th kills cats, the latter quantity in twenty minutes or half an hour. Half a grain, given to a shepherd's dog weighing $30 \mathrm{lbs}$., began to operate in three or four minutes, and proved fatal in sixty-five minutes. The toxic dose for an adult man is $\frac{1}{50}$ th grain ; for the horse gr. $\frac{1}{6}$ th, and for the dog, gr. $\frac{1}{30}$ th. Mavor and Burness subcutaneously injected over the scapula of a horse $\frac{1}{20}$ th grain, and noted in a few minutes champing of the teeth, salivation, fits of retching, and reduced number and force of the pulsations.

F. Smith and C. Rutherford made four experiments on healthy horses with aconitine. One grain of the alkaloid was dissolved in one ounce of water, and 10 minims, containing $\frac{1}{48}$ th grain, were injected hypodermically into the anterior region of the chest of two geldings. Within ten minutes there were produced biting and licking at the site of puncture, persistent shaking of the head, yawning, pawing ; increase of pulse in one subject to the number of ten beats, in the other of two beats; no change of temperature occurred. The effects disappeared in one and a half hours. 
Two horses had injections of 15 minims of the above solution with 15 minims of water, the dose containing $\frac{1}{32}$ nd grain aconitine. The same effects resulted; but pawing and movements of the head were more marked; both subjects coughed and sneezed; while one occasionally belched, ground its teeth, and showed indications of pain ; the pulse, previously 38 and soft, rose to 52 beats, and was firmer; there was no change of temperature, and no increased secretion from skin, bowels, or kidneys, and in about two hours the symptoms passed off.

These and other experiments indicate that for hypodermic use $\frac{1}{60}$ th to $\frac{1}{20}$ th grain of aconitine nitrate is a sufficient dose for the horse. As with other preparations, administered either hypodermically or otherwise, the effects may be increased and maintained by repeating half the dose three or four times, at intervals of half an hour or an hour.

\section{PECACUANHA}

IPECACUANHA. Ipecacuanhæ radix. The dried root of Psychotria ipecacuanha (B.P.). Nat.Ord.-Rubiaceæ.

The Psychotria ipecacuanha is a Brazilian shrub two or three feet high. The root, the only officinal part, occurs in twisted, knotted pieces, two to four inches in length, of the thickness of a quill. The tough, white, internal woody matter is inert; the brittle brown bark, marked with unequal rings, contains the active principle. The powder is grey-brown, has an acrid bitter taste, a faint, nauseous odour, and communicates its properties to hot water, alcohol, and diluted acids. Besides other plant constituents, it contains an odorous volatile oil, the amorphous red-brown tannin called ipecacuanhic or cephaëlic acid, and the alkaloids emetine $\left(\mathrm{C}_{30} \mathrm{H}_{42} \mathrm{O}_{2} \mathrm{~N}_{5}\right)$ and cephaeline. Emetine is a colourless powder, slightly bitter, soluble in ether, alcohol, and chloroform, but not in caustic potash. It neutralises acids, forming crystalline salts. A volatile alkaloid has also been isolated.

ACTIONS AND USES.-Ipecacuanha and emetine are topical irritants and emetics. The drug is thus got rid of, and, as 
a rule, no further effects are seen. When, however, small doses are absorbed they dilate blood-vessels, reduce bloodpressure, increase secretion, notably from the bronchi, intestines, skin, and liver. They are used as expectorants and diaphoretics, and occasionally, in small doses, as stomachics.

Ipecacuanha powder, and emetine, like tartar emetic, when applied locally, irritate the skin and mucous membranes: When swallowed by dogs or other carnivora, they produce similar in-contact irritation, stimulate the ends of the vagus, causing vomiting, and when absorbed into the blood likewise produce emesis, again probably by direct action on the gastric mucous membrane. Full doses induce gastro-enteritis, with congestion, and œdema of the respiratory mucous membrane and lungs. W. Rutherford found that 60 grains of ipecacuanha powerfully stimulated the liver of dogs; 3 grains given to a dog weighing $17 \mathrm{lbs}$. produced no purgation, but increased the mucus secreted from the small intestine. Gobert states that 100 grammes are toxic for the horse. It is more active when given in solution than in bolus. It is eliminated by the kidneys, intestinal mucous surface, and the skin (Binz).

Medicinal Uses.-As an emetic for dogs, cats, or pigs, it acts more slowly and gently than zinc or copper sulphate, its effect is more prolonged than that of apomorphine, and it is less nauseating than tartar emetic. As an anti-emetic, drop doses of the vinum, conjoined with morphine or chlorodyne, are sometimes serviceable in dogs. Given in doses insufficient to cause emesis, or used in horses or other animals which do not vomit, it promotes secretion of bronchial mucus, and hence is serviceable in the dry stages of catarrh and bronchitis. Dollar frequently gave a drachm of powdered ipecacuanha with an ounce of ammonia acetate solution, in ten ounces of water, repeating the dose several times daily. Following the practice of human medicine, American practitioners prescribe it as a remedy for dysentery, in half-drachm doses, for horses and cattle, and Robertson also recommended it in these cases, in conjunction with opium.

DoSES, etc.-Of the powder, as an emetic, dogs take grs. $x$. 
to grs. $\mathbf{x x v}$; ; cats, grs. iv. to grs. xii. ; pigs, grs. $\mathbf{x v}$. to grs. xlv., given in tepid water, either alone or with half a grain to a grain of tartar emetic. Some practitioners use Dover's powder, or its pharmaceutical imitation, made by triturating together one part each ipecacuanha and opium, and eight parts potassium sulphate. Of this expectorant and diaphoretic, horses and cattle take 3 i. to 3 iij.; sheep, grs. $x x x$. to 3 i. ; dogs, grs. x. to grs. xv. ; cats, grs. ii. to grs. v., repeated several times daily, the patient supplied with plenty of diluents, and kept comfortably clothed, and in an atmosphere of about $60^{\circ}$ Fahr. The wine is prepared with an ounce of the liquid extract of ipecacuanha and ten fluid ounces of sherry. Doses for the dog, $m_{x v}$. to $3 \mathrm{i}$.

Emetine, when inhaled even in minute amount, irritates the mucous membrane of the air-passages, and induces symptoms analogous to hay-fever. The toxic doses of emetine are gr. iss. to grs. viii. for the dog, and gr. $\frac{1}{3}$ for the cat. Two grains swallowed by a dog caused violent vomiting, increased secretion of mucus from the respiratory and alimentary membranes, inflammation of the stomach and intestines, stupor, and death in twenty-four hours (Magendie). It is eliminated by the mucous membranes and liver, increasing secretion of bile. Large doses lower temperature, relax voluntary muscles, and kill by cardiac paralysis. Emetine hydrochloride (or hydro-bromide) has been prescribed as a gastric stimulant for cattle and sheep, and as an emetic for dogs. Doses-Cattle, grs. iii. to grs. vi.; sheep, gr. i. to grs. ij. ; dogs, gr. $\frac{1}{6}$ to gr. i. Administered subcutaneously, the emetine salt is dissolved in equal parts of water and alcohol.

\section{ERATRINE}

Veratrina. An alkaloid, or mixture of alkaloids, obtained from Cevadilla, the dried ripe seeds of Schœnocaulon officinale (B.P.). Nat. Ord.-Liliaceæ.

It is prepared from Cevadilla by precipitation with ammonia. It is pale grey, amorphous, odourless, bitter, and acrid, insoluble in water, but soluble in spirit, in ether, 
and in diluted acids. In nitric acid it dissolves, yielding a yellow solution, and, warmed with hydrochloric acid, produces a blood-red colour. Commercial specimens are said to consist of veratrine and two other alkaloids, cevadine and cevadilline, resembling jervine, an alkaloid of green hellebore.

ACTIONS AND USES.-Veratrine is a topical irritant and subsequent paralysant, especially of the heart and other muscles, and is sometimes used to relieve rheumatic and neuralgic pains, and as a parasiticide and vermin-killer.

General Actions.-Rubbed into the skin or placed upon a mucous surface, it causes irritation, with a peculiar, prickling, warm sensation, and then numbness, similar to that produced by aconite, and depending upon irritation, followed by paralysis of sensory nerve-endings. When inhaled it induces violent sneezing; when swallowed in considerable doses it causes gastro-enteritis, shown by nausea, vomiting, and purging, followed by collapse. It is a nervo-muscular poison, first exciting afterwards depressing, and paralysing the peripheral terminations of motor and sensory nerves. It acts directly on voluntary muscle fibres and produces prolonged muscular contraction, followed by paralysis. Minimum doses increase muscular power. Its effect on the heart muscle is similar to that of aconitine. Under the influence of large doses the heart's action becomes slower and weaker, then irregular and intermittent, and finally arrested.

Toxic EFFECTs.-Magendie found that one grain of veratrine acetate killed a dog in a few seconds when injected into the jugular vein, and in nine minutes when injected into the peritoneum. One or two grains swallowed by dogs caused great uneasiness, nausea, vomiting, violent purging, slowness of respiration, slowness and irregularity of circulation, extreme prostration of strength, spasmodic twitching, and subsequently paralysis of the voluntary muscles, especially those of the extremities, and death from respiratory arrest, usually amid convulsions. Horses swallowing 15 to 45 grains, or when given grs. vj. hypodermically, are salivated, sweat profusely, have trembling of external muscles, and violent contractions of the gastro- 
intestinal muscles, with efforts to vomit. Similar doses in cattle produce emesis (Kaufmann). The appropriate antidotes are stimulants, such as ammonia, alcohol, or atropine, also warm coffee, potassium carbonate solution, and perfect quiet ; tannin, acetate of ammonia, and demulcents.

MEDicinal USES.-For its febrifuge and analgesic actions it has been prescribed in such febrile diseases as acute pneumonia, pleurisy, peritonitis, rheumatism, and laminitis ; but it must be used with extreme caution, and in most cases safer and more effectual remedies may be used. In persistent cases of shoulder rheumatism in horses, Friedberger recommends $\frac{1}{2}$ to $1 \frac{1}{2}$ grains, dissolved in alcohol, to be deeply injected into the affected muscles daily, beginning with the smaller amount, and gradually increasing it, intermitting the treatment every fourth or fifth day, and walking the patient after each injection until the general excitement produced abates. Kaufmann prescribes it in muscular atony and in chronic intestinal catarrh. Muller recommends it hypodermically in locomotor paralysis; and Berre considers it is the best gastric stimulant for cattle affected with plenalvia, or indigestion. It is given by the mouth in doses of three to five grains; or hypodermically, in doses of gr. $\frac{5}{6}$, every two hours.

Externally, the ointment, made with 1 of veratrine, 4 of oleic acid, and 45 of lard, is used to relieve rheumatic and neuralgic pains, and as an insecticide.

Doses, etc.-Horses and cattle per os take gr. i. to grs. v., but hypodermically not more than gr. $\frac{5}{6}$ for a first dose, in 60 to 100 minims of diluted alcohol. Veratrine should not be given to the dog.

\section{I N C H O N A}

Red Cinchona Bark. Cinchonæ Rubræ Cortex. The dried bark of the stem and branches of cultivated plants of Cinchona suceirubra. Nat. Ord.-Cinchonaceæ (Rubiaceæ). 
Quinine Sulphate. Quininæ Sulphas. The sulphate of an alkaloid obtained from the bark of various species of Cinchona and Remijia (B.P.).

QUININe Hydrochloride. Quininæ Hydrochloridum. The hydrochloride of an alkaloid obtained from the bark of various species of Cinchona and Remijia (B.P.).

The evergreen trees or tall shrubs which yield the medicinal barks were originally grown on the slopes and in the valleys of the Andes, but are now cultivated in British India, Ceylon, Java, and Jamaica. The bark, in 1639, was brought from Peru to Madrid, distributed by the Jesuits, and hence received the names of Peruvian and Jesuits' bark. Of thirty-six known species, there are many varieties, yielding barks distinguished as pale, yellow, and red.

The pale cinchonas, some of which are got from the stem and branches of the Cinchona officinalis and C. condaminea, are usually in single and double rolls, and yield more cinchonine than quinine.

The yellow barks obtained from the C. calisaya and other species are commonly met with in flat pieces, eight to fifteen inches long, two to three wide, and two to four lines thick. They consist mostly of liber, are furrowed and brownishyellow externally, fibrous and yellow-orange within. The transverse fracture shows numerous short fibres; the powder is cinnamon-brown; the odour aromatic; the taste bitter without astringency. Good specimens yield 5 to 6 per cent. of quinine.

The official or red bark is the produce of the C. succirubra ; imported in quilled or more or less in-curved pieces, two to twelve inches long, one-tenth to one-fourth inch thick. The pieces are red, rough, wrinkled, and coated with epiderm externally; finely fibrous, with a granular fracture, and brick-red or deep red-brown internally; no marked odour; taste. bitter and somewhat astringent. It yields 5 to 6 per cent. of alkaloids.

The cuprea barks from the Remijia-a genus nearly allied to cinchona and cascarilla-are now largely imported; are dense, with a thin, longitudinally striated 
epidermis, and a smooth pale red inner surface; and besides quinine and quinidine, contain a special alkaloid, cinchonamine, but no cinchonidine (Phillips).

Properties.-The cinchona barks occur in quills, stripped from the smaller branches, and curled into single or double rolls, and in flat pieces from the larger branches or trunk. They are dried in the sun, or on hurdles over fires. Their colour varies from deep yellow to red-brown, and is deepened by moisture. They have a faint odour, and a bitter, usually astringent taste. They are soluble in cold and hot water, and in alcohol ; their best solvents are alcohol (70 per cent.), and diluted acids. The tests of quality and value are the general appearance, fracture, colour, odour, taste, and percentage of the alkaloids, which are the active principles.

Composition.-Besides ordinary plant constituentslignin, starch, gum, resin, mineral matters, with traces of a volatile oil-cinchona bark contains (1) a series of active alkaloids ranging from 3 to 5 per cent.;

(2) chinic and chinovic acids, with which the alkaloids are naturally united, but which have no very marked physiological actions; (3) tannins, recognised as cincho-tannic acid, constituting 1 to 3 per cent. of the bark, and conferring astringency; (4) a glucoside, chinovin; (5) a colouring matter, cinchona red.

Quinine $\left(\mathrm{C}_{20} \mathrm{H}_{24} \mathrm{~N}_{2} \mathrm{O}_{2}\right)$ is present in all the cinchona and Remijia barks. It is in the form of sulphate that quinine is generally prescribed in this country. From a watery solution of the sulphate the alkaloid may be precipitated by ammonia. It occurs in delicate acicular crystals, inodorous and intensely bitter. It requires for solution 900 parts of cold water, but is readily soluble in alcohol, ether, chloroform, ammonia, and diluted acids. It forms colourless, bitter, crystallisable salts, remarkable, like the alkaloid, for tonic and febrifuge properties. Quinine and its salts turn a ray of polarised light to the left. Aqueous solutions, acidulated, even when extremely diluted, exhibit blue fluorescence. Treated with chlorine or bromine solution, and then with a drop of liquor ammoniæ, a green coloration is produced.

QuINIDINE, is isomeric with quinine, but crystallises in 
larger prisms, is dextro-rotatory, and its salts are more soluble and of nearly the same activity.

Cinchonine $\left(\mathrm{C}_{20} \mathrm{H}_{24} \mathrm{~N}_{2} \mathrm{O}\right)$ is present in the bark of various species of Cinchona and Remijia. It is obtained from the mother-liquors, after crystallisation of sulphate of quinine. Like quinine, it is used in the form of sulphate, which occurs in hard, colourless prisms, having a feebly bitter taste. It is anhydrous, dextro-rotatory; soluble in alcohol, and in thirty parts of water; its acidulated watery solution exhibits no fluorescence. It is the least active of the cinchona alkaloids, requiring to be given in double the dose of quinine.

Cinchonidine, an alkaloid isomeric with cinchonine, is obtained from the mother-liquors of the crystallisation of sulphate of quinine. Like the other alkaloids, it is used as a sulphate, and is considerably more active than cinchonine.

Quinine and cinchonine, when heated with excess of a mineral acid, are converted into amorphous isomeric alkaloids, termed respectively quinicine and cinchonicine.

Quinine Sulphate $\left[\left(\mathrm{C}_{20} \mathrm{H}_{24} \mathrm{~N}_{2} \mathrm{O}_{2}\right)_{2}, \mathrm{H}_{2} \mathrm{SO}_{4}\right]_{2}, 15 \mathrm{H}_{2} \mathrm{O}$, contains $74 \frac{1}{2}$ per cent. of the alkaloid. In filiform silky white crystals of an intensely bitter taste. Soluble in about 800 parts water, entirely soluble in water acidulated with a mineral acid, and in alcohol.

QUININE HydRochloRIDE $\left(\mathrm{C}_{20} \mathrm{H}_{24} \mathrm{~N}_{2} \mathrm{O}_{2}, \mathrm{HCl}, 2 \mathrm{H}_{2} \mathrm{O}\right)$ contains 83 per cent. of the alkaloid. In crystals resembling those of the sulphate but somewhat larger. Soluble in thirty-five parts cold water, and in three parts cold alcohol (90 per cent.), and very soluble in boiling water.

AcID QUININE HYDROCHLORIDE, is a white crystalline powder, soluble in less than its own weight of water, yielding a somewhat acid liquid (B.P.).

ACTIONS AND USES.-Cinchona bark is astringent from the presence of tannin, and antiseptic, tonic, and antipyretic owing to its alkaloids. These alkaloids have in concentrated form the several actions of the crude drug, but not its astringency. They differ only in the degree of their action. Quinine is the most powerful and most generally used. Small doses very briefly stimulate, large doses at once depress the functions of the organs with which they are brought into contact. 
General Actions.-Quinine and its salts combine with albumin, and have antiseptic properties nearly as marked as those of carbolic, benzoic, and salicylic acids, camphor, eucalyptol, or chloral-hydrate. One part to 830 hinders, one part to 625 prevents, development of anthrax bacilli (Koch). It diminishes fermentation, especially when depending upon such organised ferments as the alcoholic, lactic, or butyric. It checks oxidation, and lessens protoplasmic and amœboid movements. Similar effects doubtless occur when quinine is administered, and afford explanation of most of its curative effects.

Quinine in the mouth, from its bitterness, reflexly increases the flow of saliva. Small or moderate doses thus increase appetite and stimulate the stomach; but large doses impair appetite, and may induce näusea and vomiting. Its effects on intestinal secretion and movement are unknown; but it does not increase secretion of bile. It is absorbed from mucous, serous, and areolar surfaces, especially when in perfect solution, and its systemic effects are notable fifteen to twenty minutes later. Small doses stimulate, large doses depress. Small to moderate doses at first reduce the calibre of the blood-vessels, and briefly increase the strength of the circulation, but large doses weaken cardiac action and diminish blood-pressure. Moderate doses may, at first, quicken respiration, but it is afterwards weakened; large doses slow and eventually paralyse it. Death results from respiratory failure. The brain functions are stimulated at first by small, but depressed by large doses. Sensory and motor nerves are affected only when the drug is locally applied.

Tissue change is diminished. Experiments on dogs have shown that less oxygen is taken up, while less carbonic acid and albuminoid waste materials, especially urea and uric acid, are excreted. Temperature, notably in febrile cases, is lowered. These effects seem to depend upon the characteristic action of quinine in lessening the activity, after transitory stimulation, of all forms of protoplasm, and may be connected with its property of diminishing the capacity of the red cells, to give up oxygen, and on its diminishing the number, contractility, and movements of 
the white blood-corpuscles. It diminishes all secretions except the urinary, which is increased. Repeated full doses contract the spleen and also the uterus, sometimes exciting abortion, but whether this is due to idiosyncrasy in a certain number of animals is not definitely known. That the drug has no specific ecbolic action appears to be proved by Wood's experiments on healthy pregnant cats. The headache, impaired sight and hearing, and other symptoms of cinchonism produced in man by large or repeated doses, have not been distinctly recognised in the lower animals.

Cinchona bark as a bitter tonic, resembles gentian root, cascarilla bark, calumba root, and hydrastis. The antiseptic and febrifuge properties of quinine ally it to various substances of the aromatic carbon series, while the antimalarial actions resemble those of arsenic.

MEDICINAL UsES.-The bark and its alkaloids are prescribed for all classes of patients as bitter stomachies and tonics. They improve appetite, check abnormal gastro-intestinal fermentation, and counteract relaxed conditions of the intestine and accumulations of mucus, which prove favourable to the development of worms. In troublesome cases of atonic indigestion in horses, where alkaline treatment had failed, Robertson frequently gave 20 to 30 grains of quinine sulphate, with half a drachm to a drachm of nitric or hydrochloric acid. Weakly foals and calves affected by relaxed bowels, after a dose of oil, are often much benefited by a few doses of cinchona bark, hydrochloric acid, and spirit. Few tonics are so effectual as bark or quinine in improving appetite and muscular strength, and hastening convalescence from debilitating disease. In anæmia they are advantageously joined with iron salts. They are often used in the earlier stages of tuberculosis, in septicæmia, and pyæmia in all animals; in influenza, protracted cases of strangles, purpura, and other similar diseases in horses, in septic metritis in cows and ewes, and in lingering cases of distemper in dogs-their beneficial effects in these and other diseases probably depending on the action of quinine on micro-organisms or their products. Drachm doses, conjoined with iron salts, repeated night and morning, are certainly very useful in the treatment of purpura. The 
sulphate, in doses of 240 to 350 grains, repeated if requisite, is strongly recommended as a remedy for red water in cattle.

Quinine is often employed in malarial diseases, and in rheumatism, being given either by the mouth or hypodermically, frequently conjoined with salicylic acid or potassium iodide. Dollar has successfully treated cases of rheumatism and sciatica in horses, which have resisted other remedies, by hypodermic injection into the affected muscles of half a drachm of quinine sulphate in solution, and has not found undue irritation or abscess follow the operation. Like other bitters, when administered with cathartics, it generally increases their activity. Alternated with cod-liver oil and iron, quinine is the best tonic for weakly dogs and those suffering from chorea.

The alkaloids are seldom used as antiseptics for wounds, but occasionally in the form of spray or gargle are applied to relaxed or suppurating throat.

Doses, etc.-Cinchona bark is prescribed in the following doses :-For horses, 3iij. to ろi.; cattle, $3 \mathrm{vj}$. to ろij.; sheep and pigs, 3 i. to 3 iv.; dogs, grs. xx. to 3 i. ; cats, grs. v. to grs. xx., repeated twice or thrice daily for several days. If nausea or vomiting supervene, as occasionally happens in dogs, the dose should be considerably reduced or intermitted for a day or two. It is administered in bolus, pill, or solution, and is often conjoined with camphor, gentian, ginger, spirit, or ether. The infusion is made by digesting one part red bark with one-fourth part aromatic sulphuric acid and twenty parts water, and straining. The tincture, now standardised, is made by maceration and percolation of 4 ounces red bark in one pint of alcohol (70 per cent). It contains 1 per cent. of alkaloids. The compound tincture, made with tincture of cinchona, orange peel, serpentary, cochineal, saffron, and alcohol (70 per cent.), is standardised to contain 0.5 per cent. of alkaloids. The liquid extract of cinchona contains 5 per cent. of alkaloids. The salts of quinine are prescribed in the following doses:- Horses and cattle (tonic), grs. xxx. to grs. xc.; (febrifuge) grs. clx. to grs. ccxl. ; sheep and pigs, grs. viii. to grs. xxx.; dogs and cats, gr. i. to grs. $x$. Cinchonine sulphate is given in double these quantities. These doses, in bolus, pill, or solution, are 
administered two or three times daily. Given in the fluid form, their solubility is increased and their bitterness diminished by prescribing them in an acidulated solution. They are also conveniently exhibited in milk. Any tendency to nausea or vomiting is abated by combination with hydrobromic acid. Quinine is contra-indicated in pregnant females and in patients with heart disease.

Intratracheal injection, horses, grs. ii. to grs. viij. quinine hydrochloride, in two drachms distilled water. For intratracheal injection, a convenient solution of the sulphate ( 80 grains) is made with tartaric acid ( 40 grains) in 4 drachms of distilled water. Another equally useful solution is made with 15 grains quinine hydrochloride and $2 \frac{1}{2}$ drachms distilled water, containing a minim of diluted hydrochloric acid. Quinine lactate, which is soluble in ten parts of water, is sometimes preferred for hypodermic administration. The cinchona alkaloids form comparatively insoluble compounds with bile, and hence before their administration any excess of bile should be cleared away by a laxative. They are often conjoined with other bitter tonics, and with capsicum, camphor, valerian, or salts of iron. The citrate of iron and quinine is sometimes used in canine practice, but it is better to prescribe a reliable quinine salt with a salt of iron. The tincture of quinine, made with the hydrochloride and the tincture of orange, contains one grain of the salt in 55 minims. The ammoniated tincture of quinine, made with the sulphate, solution of ammonia, and alcohol (60 per cent.), contains about one grain in 55 minims. Quini-chloral, a thick oily mixture of quinine and chloral, soluble in water and in alcohol, is stated to be superior, as a germicide, to corrosive sublimate.

\section{SALICYLIC ACID-SODIUM SALICYLATE}

Acidum Salicylicum. A Crystalline Acid, $\mathrm{C}_{6} \mathrm{H}_{4} \cdot \mathrm{OH} . \mathrm{COOH}$, obtained by the interaction of sodium carbolate, and carbonic anhydride; or from natural salicylates, such as the oil of winter-green (Gaultheria procumbens) and oil of sweet birch (Betula lenta). (B.P.)

Salicylic acid was originally prepared from salicin, a crystalline glucoside obtained from willow and poplar 
barks. It can also be extracted from the stems, leaves, and rhizomes of violets. In these plants, and in the volatile oils of the winter-green and various Spireas, it occurs as a methyl salicylate. But the commercial source is sodium phenol, through which carbonic acid gas is passed for several hours. The mixture is raised to $482^{\circ}$ Fahr., the residue is dissolved in a limited quantity of water, and treated with hydrochloric acid, when salicylic acid is precipitated, and is subsequently crystallised. Commercial specimens frequently contain cresotic acid.

Properties.-Salicylic acid occurs as a soft, light, colourless or faintly pink powder, consisting of minute acicular crystals; but it may be crystallised in bold four-sided prisms. It is odourless, but when inhaled irritates the nostrils. It has a taste at first sweet, but subsequently bitter. It is soluble in 538 parts of water, 120 of olive oil, 200 of glycerin, $3 \frac{1}{2}$ of rectified spirit, 2 of ether, and 8 of lard ; and its solubility is increased by admixture with sodium borate or phosphate. It fuses at $311^{\circ}$ Fahr., volatilises without decomposition below $392^{\circ}$ Fahr., but above that is decomposed into phenol and carbonic acid gas. Sodium salicylate, $\mathrm{C}_{7} \mathrm{H}_{5} \mathrm{O}_{3} \mathrm{Na}$, may be obtained by the interaction of salicylic acid and sodium carbonate. It occurs in small, colourless scales, or in tabular crystals; odourless, taste sweetish and saline; soluble in 10 parts water, and in 6 parts of rectified spirit.

ACTIONS AND USES.- Salicylic acid belongs to the benzene or aromatic series of carbon compounds, and in chemical constitution and physiological action is allied to benzoic and carbolic acids. It is antiseptic, antiperiodic, antipyretic, irritant, and astringent, and is specially useful in the treatment of acute rheumatism. The acid, its alkaline salts, and salicin have similar actions, but salicin is now little used.

General Actions.-Salicylic acid, as an antiseptic, is less penetrating, but rather more powerful than carbolic acid or creolin. It prevents fermentation and putrefaction. Watery solutions are more active germicides than the alcoholic or oily ; they have no appreciable action on the intact skin, unless applied for some time; but on a mucous membrane, 
or a wound, they irritate, and coagulate albumin. Left in contact with the skin the horny epidermis is softened and easily removed, but without irritation. Full doses of the powder or concentrated solution are in-contact irritants, provoking, when inhaled, sneezing and coughing, and when swallowed vomiting in carnivora and diarrhœe in all animals. Sodium salicylate is devoid of irritant action, but has very similar antiseptic power to that of the acid. Although not very soluble, the acid and its salts are tolerably quickly absorbed, and the acid occurs in the blood as an alkaline salt. The general effects are similar to those produced by carbolic acid, but in some individuals (men) and in dogs, full continued doses cause nausea, occasional vomiting, and giddiness-symptoms which resemble those of cinchonism. Further administration causes slowness and weakness of the heart, marked dyspnœa and depression of the central nervous system, with convulsions and collapse. In healthy animals the temperature is not affected, but in rheumatic and malarial fevers abnormal temperature is reduced, sometimes to the extent of several degrees. This effect is probably due to the dilatation of the vessels of the skin and the consequent loss of heat. W. Rutherford found that the acid and its soda salt, like benzoic acid and benzoates, are hepatic but not intestinal stimulants, and slightly increase the flow of bile. They are eliminated more quickly in vegetable than in flesh feeders (Fröhner). They are excreted in the perspiration, saliva, and urine, in which they appear as salicylates, and in combination with glycocoll as salicyluric acid. They communicate to the urine a brown or green coloration, and retard its decomposition.

Toxic doses are borne better by graminivora than granivora. Fröhner records that a healthy horse, weighing 1000 lbs., received during three days 300 grammes (about $9 \frac{1}{2}$ ounces). Slight dyspepsia resulted from irritation of the alimentary mucous membrane, but no toxic symptoms. A healthy sheep of $70 \mathrm{lbs}$, during three days had 50 grammes ( $1 \frac{1}{2}$ ounces), but remained perfectly healthy. The like negative results also occurred in the case of a sheep of $65 \mathrm{lbs}$., which received in three days 65 grammes (2 ounces) of sodium salicylate. Dogs are not so tolerant; toxic effects 
were produced by 1 gramme $(15.04$ grains) for each 5 kilogrammes of body-weight. A dog $10 \mathrm{lbs}$. weight received 0.8 gramme in repeated doses during six hours; he vomited, had muscular trembling, and weakness of the hind limbs. A dog of $60 \mathrm{lbs}$. had 4 grammes, in divided doses, which caused weakness and cramp of the hindquarters. A dog of $10 \mathrm{lbs}$. had 5 grammes sodium salicylate subcutaneously, and exhibited dyspnœa, irregular pulse, dilated pupils, dulness, vomiting, lameness of the hindquarters, convulsions, and fatal paresis. A dog of 18 lbs. was fatally asphyxiated by eight grammes injected into the rectum.

MEDICINAL USES.-The acid and its alkaline salts are serviceable in arresting dyspeptic fermentation and diarrhœa in young animals. When administered in cases of gastrointestinal irritation, the sodium salicylate, not being irritant, is preferable to the acid.

The salicylate treatment is almost a specific for acute rheumatism in human patients. It frequently relieves the pain before the temperature or fever is abated. The beneficial results have been ascribed to the breaking up of lactic acid products. But British veterinarians have not found the treatment so effectual either in horses or cattle. In animals such attacks are chiefly of a chronic type, on which salicylic acid has not such marked effect as in the acute cases, and it may be that the doses prescribed have not been large enough, or given with sufficient frequency, or for a sufficient period. To foals suffering from lameness Hinterholzer gave two teaspoonfuls sodium salicylate twice a day, and as a prophylactic measure in pregnant mares he prescribed sixty to seventy grains morning and evening for a few days before the date of foaling. Fröhner uses both the acid and the sodium salt in muscular and arthritic rheumatism in all animals, and states that chronic cases are benefited by continued doses, that good results need not be despaired of until the drug has been persisted with for ten or fifteen days, while to prevent relapse the administration is continued for some days after the symptoms have been relieved. Dogs are benefited especially where the joints are prominently affected. 
In horses, as in human patients, a few, frequently repeated doses arrest attacks of acute nasal catarrh when given in the earlier stages, and appear to have a similar power in gastro-intestinal and urinary catarrh. Fröhner recommends it in cystitis. Robertson used it in equine influenza accompanied with gastro-intestinal symptoms. Other practitioners testify to its value in purpura and also in strangles. In zymotic and malarial fevers it is not so effectual as quinine. Feser has shown that it exerts no antipyretic effect in septic or pyæmic fever. Conjoined with tannic acid it is prescribed for obstinate diarrhœa in calves.

In antiseptic surgery, salicylic acid is sometimes substituted for or alternated with carbolic acid or creolin. It is serviceable in the treatment of canker and open joint, for abating the itching and discharge of eczema, for dressing sores on the teats of cows, washing out the uterus in metritis, and with alcohol as an injection in otorrhœa. Its antiseptic effects are increased by admixture of boracic acid.

Doses, etc.-Horses and cattle take 3 iv. to $\zeta i$. ; sheep and goats, 3 j. to 3 iij. ; swine, grs. xlv. to grs. lxx.; dogs, grs. v. to grs. xxx. of sodium salicylate, administered with mucilage or glycerin, in bolus, electuary, or drench. Salicylic acid and salicin are used in similar amounts. The larger doses are given in fevers; the smaller, repeated frequently, in rheumatism, in the muscular form of which a solution may be injected deeply into the affected part.

For surgical purposes convenient solutions are made by dissolving one part each of salicylic acid and borax in thirty to fifty parts of water. Ointments and liniments are prepared with one part acid, mixed in a heated mortar, with twenty to twenty-five of vaseline or bland oil. Salieylic collodion, for protecting small wounds, consists of salicylic acid one part and flexible collodion nine parts. Salicylic cream, employed as a pigment for surgical wounds, is composed of two parts salicylic acid, and ten parts of glycerin. The B.P. ointment consists of one part acid, and forty-nine parts of white paraffin ointment. Lint, cotton-wool, or jute, soaked in 4 to 10 per cent. hot, watery solution, made with borax to ensure solubility, absorbs the acid, and is used as an antiseptic covering for wounds and 
burns in the same manner as carbolic or boric lint. Being unirritating, salicylic lint is applied directly to abraded surfaces without the intervention of any protective. Iron salicylate is antiseptic and astringent.

Dithion, or di-thio-salicylate of sodium, a greyish-white powder, freely soluble in water, is antiseptic and antipyretic. In foot-and-mouth disease it has been given internally, and applied externally as a dusting-powder, and as a lotion ( $2 \frac{1}{2}$ to 5 per cent.) to the sores of the mouth, feet, and udder.

Methyl salieylate $\left(\mathrm{CH}_{3} \mathrm{C}_{7} \mathrm{H}_{5} \mathrm{O}_{3}\right)$, a product of the distillation of salicylic acid with methyl alcohol and sulphuric acid, is a colourless liquid with an odour of honey and a nauseous taste. It forms nine-tenths of oil of wintergreen or Gaultheriæ Oleum, a volatile oil obtained from the leaves of Gaultheria procumbens, an American evergreen. Methyl salicylate is employed externally as a local anodyne for muscular and articular rheumatism; for which it may also be administered in doses of $3 \mathrm{ij}$. to $3 \mathrm{jv}$. to horses and cattle ; and $m_{\text {iij. to }} m_{x v}$. to dogs. Oil of wintergreen is also applied to rheumatic joints, and, diluted with alcohol, it has been used as a dressing for wounds.

Aspirin (acetyl-salicylic acid), formed by the action of acetic anhydride on salicylic acid, is a crystalline powder, soluble in 100 parts of water, in dilute alkalies, and in alcohol and ether. It passes through the stomach unchanged, and in the bowel is split up, salicylic acid being liberated. It is used as a substitute for sodium salicylate, and is largely prescribed in the treatment of muscular and articular rheumatism, neuralgia, and pleurisy. Doses, dogs grs. ii. to grs. x. ; horses, 3ij. to 3jv. In special cases larger doses may be given.

\section{GENTIAN}

Gentian es Radix. The dried rhizome and roots of Gentiana lutea (B.P.) Nat. Ord.-Gentianaceæ.

The Gentiana lutea, or yellow gentian, has a perennial, often forked root, and an annual herbaceous stem, which rises three or four feet, and bears axillary whorls of yellow 
flowers. It abounds in most parts of temperate Europe, thrives best between 3000 and 5000 feet above the sealevel, and is extensively cultivated in the mountainous districts of the Alps, Vosges, and Pyrenees. All parts of the plant are bitter and tonic, but only the rhizome is officinal. It occurs in cylindrical, usually more or less branched, often twisted, pieces, or in longitudinal slices, marked by transverse annular wrinkles and longitudinal furrows, and varying in length and thickness. It has a peculiar aromatic and rather disagreeable odour, and a taste at first sweet, but afterwards bitter. When moist, it is tough and flexible ; when dry, brittle, and easily pulverised. The powder is yellow, with a shade of brown, and readily yields its bitterness to water, alcohol, and ether.

Gentian root contains gentianose, a sugar, which, in Southern Bavaria and Switzerland, is fermented into a drinking spirit; a large amount of pectin, a little volatile oil and fat, the yellow crystalline gentianin, or gentianic acid $\left(\mathrm{C}_{14} \mathrm{H}_{10} \mathrm{O}_{5}\right)$, which is inert; and about $0^{\circ} 1$ per cent. of an intensely bitter glucoside, gentiopicrin $\left(\mathrm{C}_{20} \mathrm{H}_{30} \mathrm{O}_{12}\right)$, obtainable in colourless crystals, which are soluble in water and alcohol. In its actions gentiopicrin is nearly allied to quinine.

Roots of other Gentianaceæ are frequently mixed with those of G. lutea ; but this is not of much importance, since all are possessed of similar properties. Admixture, however, sometimes occurs of poisonous roots, such as monkshood, belladonna, and white hellebore, which may be distinguished by the absence of the pure bitter taste and the bright yellow colour so characteristic of true gentian. Gentian powder, especially that met with abroad, is stated to be occasionally adulterated with yellow ochre, easily detected by heating the suspected specimen with a little sulphuric acid, filtering, and testing for iron.

ACTIONS AND UsES.-Gentian is a pure bitter, and is prescribed as a stomachic and tonic for all veterinary patients. It resembles calumba, chiretta, quassia, and lupulus or hops. It is virtually devoid of astringency. It may be taken as a type of these simple bitters all of which produce similar effects clinically, although their exact mode of 
action is disputed. Given by the mouth, the bitter taste causes a reflex flow of saliva, and by stimulating the nerves of taste there is, first of all, improved appetite, and then in about thirty to forty minutes an increased reflex secretion of gastric juice. But increased gastric secretion of acid and pepsin means more efficient gastric digestion, and more than that, pari passu with the improvement in the stomach, there is increased outpouring of pancreatic juice and of bile, for this depends on the amount of hydrochloric acid escaping from the pylorus into the duodenum. Thus a bitter in the mouth, by stimulating the nerves of taste, starts a series of reflex secretions which make the whole process of digestion more efficient. Another effect of this group of drugs is an increase in the number of leucocytes in the blood, and in this way absorption of the products of digestion may be helped to some extent.

Medicinal Uses.-Gentian improves the appetite and general tone. In atonic indigestion it is particularly useful amongst young animals, and in such cases is often conjoined with ginger and sodium bicarbonate. In relaxed and irritable states of the bowels, and where intestinal worms are suspected, after administration of a laxative, gentian and hydrochloric acid are often serviceable. For horses suffering from simple nasal catarrh few combinations are more effectual than an ounce of powdered gentian, two drachms nitre, with two ounces Epsom salt, dissolved in a pint of water, linseed tea, or ale, and repeated night and morning. In inflammatory complaints, after the acute stage is passed, such a prescription also proves serviceable. Where the bowels are constipated or irregular, or febrile symptoms are insufficiently subdued, a drachm of aloes is sometimes conjoined with the gentian. Where more general tonic effects are sought, iron sulphate is alternated with gentian and salines. Two to four drachms of gentian, with an ounce of ether or sweet spirit of nitre, given three or four times daily in a bottle of ale, proves an excellent stomachic and stimulating tonic in influenza and other epizootics, helps convalescence from exhausting disorders, and is a useful restorative for horses, jaded, overworked, or suffering from loss of appetite or slight cold. 
For cattle the above prescriptions are as serviceable as for horses, but require to be given in somewhat larger doses. For sheep gentian is a very useful stomachic, and when prescribed with salt arrests for a time the progress of liverrot. It is a valuable bitter tonic for dogs prostrated by reducing disorders.

Doses, etc.-For the horse, $3 \mathrm{ij}$. to $\overline{3}_{\mathrm{i}}$; for cattle, $\bar{\zeta}^{\mathrm{i}}$. to $3 \mathrm{ij}$. ; for sheep, $3 \mathrm{i}$. to $3 \mathrm{iij}$.; for pigs, 3 ss. to $3 \mathrm{i}$.; for dogs, grs. v. to grs. xx., repeated twice or thrice daily. The carefully-prepared Pharmacopœia extract, infusion, and tincture, flavoured with orange-peel and aromatics, are frequently used in veterinary practice. The powder is prescribed in bolus, prepared with treacle, glycerin, and meal, or in infusion, made by digesting the powder during several hours in hot water, and decanting the clear fluid. A small addition of diluted alcohol ensures more thorough solution and better keeping.

\section{A L U M B A}

Calumbe Radix.-The dried sliced root of Jateorhiza columba; growing in Eastern Africa (B.P.). Nat. Ord.-Menispermaceæ.

Calumba root occurs in irregular, flattish, circular or oval slices, with a yellow centre and a brownish-yellow cortex. The fracture is short, odour feeble, taste bitter (B.P.). It contains a crystalline, neutral, bitter principle, calumbin ; an alkaloid, berberine; calumbic acid; and 33 per cent. of starch.

Actions AND USES.-Calumba is a bitter and gastric stimulant. It promotes secretion of gastric juice and improves the appetite in a manner similar to gentian. As it contains no tannin it is devoid of astringency and may be prescribed with preparations of iron. Like quassia, calumba infusion may be used to destroy worms in the horse's rectum.

DOSES, ete.-Of the infusion (one of calumba to 20 of cold

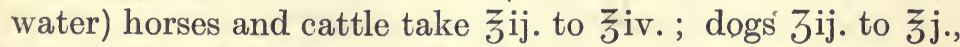
twice or thrice daily. The tincture, made with one of calumba root to 10 of alcohol (60 per cent.) is also pre- 
scribed. The doses are about one-third of those of the infusion.

Chiretta, the dried Indian plant, Swertia Chirata, collected when in flower, is closely allied in actions and uses to calumba. It contains an active bitter principle, chiratin, but no tannin. The infusion and tincture of chiretta are prescribed in the same doses as calumba or gentian.

\section{A S A R I L L A}

Cascarillm Cortex. The dried bark of Croton eluteria (B.P.). Nat. Ord.-Euphorbiaceæ.

Cascarilla bark is principally imported from the Bahama Islands in quills about the size of a drawing pencil, and varying from two to four inches in length. Its outer surface is fissured, and usually covered with a light-coloured lichen ; its inner surface is smooth and light-brown. It has a strong, pungent, rather nauseous taste ; its aromatic odour is increased by heat, and recommends it as a constituent of fumigatory pastilles. It contains the neutral crystalline bitter cascarillin $\left(\mathrm{C}_{12} \mathrm{H}_{18} \mathrm{O}_{4}\right), 15$ per cent. of two resins, tannin, and 1.5 of a pungent volatile oil, one portion of which is isomeric with oil of turpentine.

ACTIONS AND USES.-Cascarilla is an aromatic, bitter stomachic, and carminative, allied to cusparia (Angustura bark) and resembling cinchona, but less active, and occasionally used in indigestion, diarrhœa, and convalescence from exhausting diseases.

Doses, etc.-For horses, $3 \mathrm{ij}$. to $3 \mathrm{iv}$. ; for cattle, $\xi_{\mathrm{i}}$; f for sheep and swine, $3 \mathrm{i}$. to $3 \mathrm{ij}$. ; and for dogs, grs. $\mathrm{x}$. to grs. $\mathrm{xl}$., given in bolus, infusion, or tincture, which is made with one of cascarilla to five of alcohol (70 per cent.).

\section{OAK BARK}

Quercus CorTex. The dried bark of the smaller branches and young stems of Quercus robur (Q. peduculata). Collected in early spring from trees growing in Britain. (Not official.) Nat. Ord.-Cupuliferæ.

Bark from smaller branches of young trees is more astringent than thicker pieces of older growth; the interior 
finer fibrous portions than the external rougher cortical. Oak bark contains a bitter crystalline substance, querein, and owes its astringency to 10 or 15 per cent. of quercitannic acid, which differs somewhat from gallo-tannic acid, and does not, by oxidation, yield gallic acid. The infusion has a powerful astringent taste, reddens litmus, gives a blue-black precipitate with ferric salts; and with gelatin solution a white flocculent precipitate, which resists putrefaction better than that of gallo-tannic acid. Acorns-the fruit of the oak-are collected in many parts of England for feeding sheep and pigs, are credited with a nutritive value approaching that of beans, but on account of their astringency require to be used sparingly.

ACTIONS AND USES.-Oak bark is astringent, resembling galls and catechu. It is prescribed to check chronic otorrhœa, diarrhœa, dysentery, and other excessive mucous discharges. For weakly, scouring calves the infusion is given once or twice daily as required, with warm starch gruel, to which may be added aromatics, gentian, spirit, ether, or chloroform, or where there is griping, laudanum. It lacks the tonic properties of cinchona and gentian, and constipates when given too frequently or freely. Infusions are applied to dry and constringe hyper-secreting and relaxed surfaces, and to relieve piles in dogs.

Doses, etc.-Horses take 3ij. to Ziv. ; cattle, žss. to zij. ; sheep and pigs, 3 ss. to $3 \mathrm{ij}$.; dogs, grs. x. to grs. xxx., administered in infusion made with one or two ounces of bark to the pint of water.

\section{QUASSIA WOOD}

Quassia Lignum. The wood of the trunk and branches of Picræna excelsa (B.P.). Nat. Ord.-Simarubaceæ.

The dense, tough, white quassia wood, the produce of a handsome tree, is imported from Jamaica and other West Indian islands in billets of varying length, and is met with in yellow-white chips or raspings. Quassia has no odour, but an intensely bitter taste, dependent on a neutral crystalline principle, quassin $\left(\mathrm{C}_{10} \mathrm{H}_{12} \mathrm{O}_{3}\right)$. It contains no tannin. 
ACTIONS AND USES.-Quassia is a bitter stomachic and tonic. It resembles gentian and calumba. It. is prescribed for the several domestic animals in dyspepsia, loss of appetite, and convalescence from debilitating disorders. Although it has no appreciable vermicide effect when given by the mouth, when used as an enema it destroys both ascarides and lumbrici. Large doses are irritant. The infusion is a narcotic poison for flies and other insects, and is used as a parasiticide in skin diseases.

DosEs, etc.-The B.P. infusion, prepared by macerating one part of chips for fifteen minutes with one hundred parts cold water, is administered alone, or with salines, acids, or iron salts, with which, unlike most vegetable bitters, it mixes without decomposition or discoloration. Of the infusion, horses and cattle take $\mathrm{f} z \mathrm{ij}$. to $\mathrm{f} \xi \mathrm{iv}$.; sheep and pigs, $\mathrm{f} 3 \mathrm{iv.}$; dogs, $\mathrm{f} 3 \mathrm{j}$. The tincture is not used by veterinarians.

\section{MUSTARD}

Sinfpis. The dried ripe seeds of Brassica nigra and Brassica alba, powdered and mixed (B.P.). Nat. Ord.Cruciferæ.

The mustard plants are annuals, one to two feet high, with yellow cruciform flowers, and pods containing several brown seeds. They are indigenous in most parts of Europe and extensively cultivated in Durham, Yorkshire, and Lincolnshire. An abundant wild variety, familiarly known as charlock and kellocks, is sometimes used for adulterating the better sorts. The black mustard seeds are red or greyish-brown, about the size of millet; the greenish-yellow powder has a pungent oily taste, and when triturated with water yields a pungent odour. The white mustard seeds are double the size of the black, and lighter in colour. Inodorous when entire or powdered, and almost inodorous when triturated.

Black and white mustard seeds contain about 25 per cent. of a yellow, tasteless, non-drying fixed oil, similar to that of rape, and consisting of olein, stearin, and glyceride of erucic or brassic acid; 20 per cent. of mucilage, chiefly found in 
the epidermis ; 4 of inorganic matters, and 10 to 15 of myrosin, a ferment similar to diastase or the emulsin of bitter almonds, usually more abundant in white than in black mustard seeds, and coagulated and rendered inactive when heated above $140^{\circ}$ Fahr. Black mustard, besides, contains about $2 \frac{1}{3}$ per cent. of the crystalline potassium myronate or sinigrin; white mustard contains an allied principle, sinalbin. When dissolved in water, as in making mustard flour into paste, the ferment myrosin decomposes the crystalline bodies, and there are produced two acrid, irritant oils - the pungent volatile oil of mustard, allylisothiocyanate $\left(\mathrm{C}_{3} \mathrm{H}_{5} \mathrm{NCS}\right)$ from the black mustard, and the fixed oil-acrinyl-isothiocyanate- $\left(\mathrm{C}_{8} \mathrm{H}_{7} \mathrm{NSO}\right)$ from the white mustard.

Actions AND UsEs.-Unbruised mustard-seeds, being only partially and gradually digested, have little effect when swallowed. When the ground seeds are mixed with water the pungent, acrid oils are evolved; large doses of the flour are accordingly irritant; medicinal doses are stomachic, carminative, and stimulant. It is, however, rarely used internally, excepting as a locally acting emetic for the dog, cat, or pig. For this purpose a dessert-spoonful of mustard flour is given, dissolved in several ounces of water. Small doses are slightly laxative and diuretic; whilst large doses may cause purging and collapse.

As an external irritant, mustard is much used as a rubefacient and vesicant. The paste made with cold water, and rubbed into the skin of the horse, within twenty minutes causes congestion, heat, and tenderness, with subsequent swelling. Reflexly, the activity of conterminous and subjacent parts is roused. In two to six hours vesication occurs ; twenty-four hours later some of the vesicles will have run together, others being ruptured. From repeated, prolonged, or injudicious use in irritable states of the skin, there occasionally ensue active inflammation, sloughing, and destruction of the hair-roots.

Compared with cantharides, mustard acts more promptly, but unless used freely or repeatedly it is less permanent. It is used to control functional disturbance rather than to repair structural damage; it causes more swelling of sur- 
rounding parts, but less exudation of serum; applied repeatedly, especially to the extremities of the horse, it is more apt to affect the skin deeply, and hence produce sloughing; unlike cantharides, it has no tendency to act upon the kidneys. It is almost as prompt, and is more manageable than very hot water. For horses it is less irritating and burning than oil of turpentine. It is not so severe or so apt to cause suppuration as euphorbium or croton oil. For cattle it is an excellent blister, often acting promptly when other agents have slight or tardy effect, and seldom causing injury or blemishing. On dogs and sheep it acts powerfully, and must be used with caution.

MEdicinal UsES.-In all veterinary patients suffering from catarrh, sore-throat, laryngitis, bronchitis, pneumonia, or pleurisy, mustard, applied in the early congestive stage, lessens pain and relieves difficult breathing. It is more serviceable in chronic than acute bronchitis. In pleurisy, mustard liniments alternated with fomentations are often applied at intervals throughout the attack, but are specially indicated after the tenth day, when such counter-irritation seems to promote absorption of exudate. During the later stages of pneumonia mustard is of little use ; but occasional dressings are sometimes serviceable in sustaining the action of the heart and promoting absorption. It is frequently rubbed over a considerable area immediately external to the congested, painful, or inflamed parts ; in about fifteen minutes it is washed off, and in an hour or two, if required, another application may be made.

Mustard dressings are serviceable in acute indigestion, colic, and enteritis, especially of horses. Mustard is of service in chronic rheumatism, especially amongst cattle, in the second stages of inflammation of joints and tendons, in enlargement of glands, and occasionally as a stimulant in chronic scurfy skin diseases. Flying blisters, applied over the chest or abdomen, or below the knees and hocks, especially when the limbs are cold, arouse vitality, and overcome congestion in the later stages of pneumonia, in parturient apoplexy of cattle, and in poisoning by narcotics. With stimulants administered internally, mustard is rubbed over the region of the heart to counteract syncope. Applied over 
the kidneys, it promotes diuresis. It is occasionally used for maintaining or increasing the effects of cantharides, but in horses considerable caution is necessary in applying the one irritant soon after the other.

Mustard is specially indicated where extensive counterirritation has to be speedily produced and stimulation of the kidneys avoided. Cantharides or mercuric iodide ointment is preferable in chronic disease of joints, and where structural changes have occurred in bone, cartilage, or tendon. Neither mustard, nor indeed any blister, can be directly applied to parts extensively or deeply inflamed without causing much irritation, and probably sloughing.

Doses, etc. - If used as a stomachic, carminative, or mild stimulant, horses take $3 \mathrm{iv}$. to $3 \mathrm{vi}$.; cattle, 3 ss. to $\boldsymbol{\zeta}_{\text {j. ; }}$ sheep and pigs, 3 j. to $3 \mathrm{ij}$.; dogs, grs. x. to grs. xx. To prevent irritation of the fauces, it is given in the form of pill, bolus, or electuary. Larger doses, especially in solution, act as emetics in dogs, cats, and pigs.

Externally, it is used generally as a paste made as for the table, with cold water. Hot water or admixture of spirit, acid, or alkali coagulates the ferment, and impairs its action. A mustard paste made with water produced, in six minutes, effects similar to those produced in fifty minutes with mustard mixed with vinegar. Extra activity is secured by using black and white mustard seeds, in about equal amount, ground unmixed with bland ingredients, or by adding to the paste made from the mustard of the shops a little oil of turpentine. The freshly-made paste is usually applied directly to the skin, with friction; after fifteen or twenty minutes it may be washed off with tepid water, and, if required, again applied two or three times. Such repeated moderate external warming is usually more serviceable than one violent dressing, whether for diminution of congestion, relief of pain, or even for removal of exudate.

For veterinary patients little use is made of plasters prepared by spreading mustard upon calico or paper; of leaves consisting of powdered mustard seeds and guttapercha solution spread upon cartridge paper and dried; or of poultices usually made with equal parts of mustard and linseed meal, well stirred with four parts of hot water. 
The B.P. volatile oil of mustard, distilled from black mustard seeds after maceration with water, is antiseptic and antipyretic, and one of the most poisonous of the volatile oils. It is soluble in alcohol, and in fifty parts of water. Rabbits are killed in two hours by a drachm, in fifteen minutes by half an ounce, with symptoms of gastro-enteritis, loss of sensation and muscular power, difficult breathing, and collapse. Diluted and applied externally, it is a prompt and powerful vesicant.

\section{FIBROLYSIN}

Fibrolysin (Merck) is described as composed of Thiosinamine (obtained from mustard oil) and salicylate of soda. It occurs as a clear, colourless liquid, with a faint odour and a slightly acrid taste. Fibrolysin has the reputation of possessing the power to soften pathological fibrous tissue and to prevent its contraction. Its use is indicated in thickened and contracted tendons, indurations, fibrous ankylosis, adhesions, corneal opacities, etc. No ill effects have been noted from its administration; and it is stated to improve the appetite and general health. Rahne, Kratzer, Train, and other veterinary surgeons, report favourably of its use in 'induration of the thigh ' in a mare ; 'tendinitis fibrosa' of both forelimbs with thickening and shortening of the flexor tendons; fibroid swelling of a horse's hock, and enlarged fetlock joints. Fibrolysin may be administered hypodermically, intravenously, or by intramuscular injection into the substance of the gluteal muscles. The injections should be repeated daily, or at intervals of two or three days, according to the case. Fibrolysin is supplied in sterile glass ampullæ, each containing 11.5 c.c., in aqueous solution, or sufficient for one injection for the horse.

\section{Y R R H}

MYrRha. A gum resin obtained from the stem of Balsamodendron myrrha and probably other species (B.P.). Nat. Ord.-Burseraceæ.

Myrrh is imported from the coasts of the Red Sea. With olibanum or frankincense it has long been used in making 
incense, perfumes, holy oils, and unguents for embalming. It exudes spontaneously, as a yellow-white oily substance, from perforations or cracks in the cortical layer of the trunk or branches of several species of the shrubby thornlike balsamodendrons. The best sorts are in irregularshaped, semi-translucent, red-brown tears, or masses of tears, which deepen in colour when breathed on. They are brittle, and easily powdered ; their fracture is irregular, shining, oily, and occasionally dotted with opaque white markings. Myrrh has a slightly bitter, acrid taste, and an agreeable aromatic odour. When heated, it softens, froths up, and burns, leaving a dark spongy ash. Powdered, with water it forms an emulsion, but it readily dissolves in rectified spirit. It consists of 50 to 65 per cent. of soluble gum, chiefly arabin ; 23 per cent. of a resin, myrrhin, soluble in alcohol, ether, and acetic acid, and 2 per cent. of a pale yellow volatile oil, myrrhol $\left(\mathrm{C}_{10} \mathrm{H}_{14} \mathrm{O}\right)$, isomeric with thymol and carrol, and some bitter substances.

ACTIONS AND UsES. - Its antiseptic volatile oil and slightly irritant resin render myrrh a topical stimulant. When swallowed it increases the secretions of the gastro-intestinal glands, producing stomachic, carminative, and mild laxative effects. During elimination it stimulates the mucous lining of the respiratory and urinary tracts, and is hence prescribed as a disinfectant, expectorant, and diuretic. It is sometimes used as an antiseptic, mildly astringent vulnerary, and a flavouring agent. It resembles the fragrant gum resin olibanum, produced by several species of Boswellia, and the concrete resin of the Elemi tree imported from Manilla. It is less stimulant than the balsams and fœtid gum resins.

Doses, ete.-Horses and cattle take $3 \mathrm{ij}$. ; sheep and pigs, 3ss. to 3i. ; dogs, grs. x. to grs. xx., repeated several times daily, in bolus, decoction, or tincture, with vegetable tonics, or with aloes. The tincture of myrrh is thus, prepared :-Macerate 4 ounces of myrrh in 16 ounces of alcohol (90 per cent.), for seven days in a closed vessel ; shake frequently, filter, and add sufficient alcohol to make one pint. 


\section{PEPPERS}

The black and white peppers in daily domestic use are obtained from the brown wrinkled berries of an East Indian perennial climbing plant-the Piper nigrum, of the natural order Piperaceæ. They are imported from the Malabar coast, the islands of the Indian Archipelago, and the West Indies. The pendulous spike, bearing twenty to thirty berries, is gathered as it begins to redden, shortly before ripening, and is dried in the sun. The berries rubbed off, and ground without separating their outer covering, yield black pepper. To prepare the milder white pepper, the best and soundest ripe berries are steeped in water, and stripped of their pungent outer covering before they are ground. Long pepper, the produce of Chavica Roxburghi, is brought from Singapore and Batavia, and consists of small, closely-attached berries, arranged on cylindrical grey spadices one or two inches long.

The peppers when ground have a hot, pungent, spicy taste, and owe their properties to $1 \cdot 6$ to $2 \cdot 2$ per cent. of a volatile oil-isomeric with oil of turpentine $\left(\mathrm{C}_{10} \mathrm{H}_{16}\right)$, a soft, pungent resin, and 2 to 3 per cent. of the colourless, crystallisable, neutral piperine $\left(\mathrm{C}_{17} \mathrm{H}_{19} \mathrm{NO}_{3}\right)$, which is isomeric with morphine, and when boiled with caustic potash yield an active oily alkaloid, piperidine $\left(\mathrm{C}_{5} \mathrm{H}_{11} \mathrm{~N}\right)$.

Cubebs, or Cubeba, is the dried, partially ripened fruit of the Piper Cubeba, cultivated in Java and other islands of the Indian Archipelago. The berries are stalked, and lighter coloured than those of common pepper, are globular, rough, and wrinkled, with a strong odour, and pungent, aromatic, bitter taste. They contain a volatile oil, a resin, and the neutral crystalline cubebin, which is devoid of any marked action.

Piper angustifolium, a shrub found in moist regions throughout Brazil and Peru, yields matico leaves, much used in America as a styptic dressing, and also occasionally administered for the arrest of internal hæmorrhage.

Pimenta, pimento, Jamaica pepper, or allspice, closely resembles the true peppers; is the dried, unripe berry of Pimenta officinalis, an evergreen West Indian tree of the 
natural family Myrtaceæ. The berries are about the size of those of the Piper nigrum, have the same penetrating aromatic odour, and hot, pungent taste, but are more truly aromatic and less acrid. They contain an acrid fixed oil, and about 6 per cent. of volatile oil, with traces of an alkaloid, having the odour of conine (Flückiger). Oil of pimento contains about 70 per cent. of aphenol, eugenol, and is sometimes substituted for oil of cloves.

Capsicum - the dried ripe fruit of Capsicum minimumis also known as Chili pepper, chillies, Guinea or pod pepper. The red pods are filled with numerous small round or ovoid red-brown seeds. Both pericarp and seeds are pungent, and when ground constitute the familiar Cayenne pepper, which owes its pungent acridity and irritant properties to an acrid non-volatile substance, capsaïcin $\left(\mathrm{C}_{9} \mathrm{H}_{14} \mathrm{O}_{2}\right)$, and an alkaloid, capsicine, resembling conine in odour.

ACTIONS AND USES.- The peppers are irritants, stimulating stomachics, carminatives, and rubefacients. Large doses, especially in carnivora and omnivora, are irritant poisons, inflaming the alimentary tract and sometimes also the urinogenital mucous membrane. That they are especially poisonous to pigs is a popular error. Properly regulated doses promote salivary and gastric secretions, are stomachic and carminative, and during their excretion stimulate the urino-genital mucous membrane. Rubbed into the skin they cause redness, irritation, and swelling. The several peppers differ in the intensity of their action. The black is more active than the white and long peppers, which are of nearly equal strength. Pimento is less active, while capsicum is more irritant than black pepper. In virtue of its stimulant in-contact effect, and its rendering the urine antiseptic, cubebs checks irritation and discharges from the urino-genital mucous membrane.

Black pepper (the variety chiefly used in veterinary practice) is administered in simple indigestion, and for obviating the disagreeable taste and nauseating effects of various drugs. It is not now given as a sialogogue, nor for the object of increasing sexual appetite, which, when defective, may usually be restored, not by irritating drugs, but by measures which improve general vigour. It ought 
not to be used for blistering ointments, or for setons, nor introduced into the rectum of horses exposed for sale-a barbarous practice, apt to induce serious intestinal irritation.

Doses, ete.-Of black pepper, as a stomachic, horses take about 3 i. ; cattle, 3 ij. ; sheep and swine, grs. x. to grs. xxx. ; dogs, grs. v. to grs. x., repeated two or three times a day, given in bolus, suspended in water or spirit, or in wellboiled gruel. The tincture of capsicum is made with one of capsicum and twenty of alcohol (70 per cent.).

\section{GING ER}

Zingiber. The scraped and dried rhizome of Zingiber officinale (B.P.). Nat. Ord.-Zingiberaceæ.

The Zingiber officinale, grown in many tropical countries, has a biennial, creeping, fleshy, and nodulous rhizome, which gives off numerous descending short radicles, with several ascending annual leafy stems, reaching three or four feet in height, invested with alternate elliptical leaves, and terminated by spikes and racemes of purple flowers. For making green or preserved ginger, the rhizomes are gathered while still soft and juicy, and when about three months old. For other purposes they are taken up when about a year old, when the aerial stems have withered, but while the rhizome is still plump and soft. They are scalded to check vegetation, usually scraped to remove the brown wrinkled epidermis, and dried in the sun.

Properties.-Several sorts are recognised. The Jamaica, in plump, flat, pale pieces or races, the bark stripped of epidermis, producing a light-coloured powder of superior quality; Malabar or Cochin China, a little darker, but usually good; Bengal and African, imported both coated and uncoated, and generally cheap and excellent; Barbados, in short thick races, retaining its brown corrugated epidermis. The unstripped descriptions are sometimes termed black gingers. The several varieties are in flat, irregularlobed, knotted, zigzag pieces, two to four inches in length, externally pale yellow, striated, and fibrous, breaking with a 
mealy, short, somewhat fibrous fracture, having a strong agreeable, aromatic odour, a warm, pungent taste, and dissolving in water and alcohol.

Ginger owes its taste to a pungent resin, its aroma to a volatile oil, and its medicinal and flavouring properties to both constituents, which are chiefly found in the delicate felted layer of skin lying between the starchy, mealy parenchyma and the brown, horny, external covering. As a condiment and medicine Great Britain annually imports about 300 tons of ginger.

Actions AND UsES.-Ginger is an aromatic stimulant, and is used as a stomachic and carminative for all patients, notably for cattle and sheep.

Blown into the nostrils it increases nasal discharge; chewed, it reflexly augments the flow of saliva ; administered internally, it promotes gastric secretion, digestion, and appetite. It is prescribed in atonic dyspepsia, often with antacids and laxatives. Conjoined with purgatives, it diminishes their tendency to nauseate and gripe, and also somewhat hastens their effects.

Doses, etc.-For the horse, Ziv. to Zi. ; for cattle, zi. to Zij. ; for sheep, 3i. to $3 \mathrm{ij}$. ; for pigs, 3 ss. to 3 i. ; for dogs, grs. $\mathrm{x}$. to grs. xxx. A bolus is made with any suitable excipient; an infusion is used sometimes sweetened with treacle or sugar; the B.P. tincture is prepared with two ounces powdered ginger to a pint of rectified spirit by maceration and subsequent percolation. A syrup of ginger, made with a strong tincture and the B.P. simple syrup, is occasionally prescribed. An oleo-resin, obtained from ginger, and known as gingerin may be substituted for ginger in dispensing purgatives.

\section{CIN N A MON}

Cinnamomi Cortex. Cinnamon Bark. The dried inner bark of shoots from the truncated stocks of Cinnamomum zeylanicum. Imported from Ceylon, and distinguished in commerce as Ceylon cinnamon (B.P.) Nat. Ord.-Laurineæ. 
Cinnamomi Oleum. The oil distilled from cinnamon bark (B.P.).

The bark occurs in rolled quills, is thin and brittle, yellowbrown externally, darker brown on its inner surface, with a fragrant odour, and a warm, sweet, aromatic taste. Besides mannite, resin, and other vegetable constituents, the bark contains tannic and cinnamic acids, but its aroma and medicinal properties depend upon the presence of about one per cent. of a volatile oil, cinnamic aldehyde $\left(\mathrm{C}_{9} \mathrm{H}_{8} \mathrm{O}\right)$, which, when fresh, is bright yellow, but becomes cherryred when kept. Its constituents are eugenol (also found in cloves), cinnamic aldehyde, and a terpene. An inferior oil is extracted from the leaves.

ACTIONS AND USES. - Cinnamon bark is aromatic, carminative, and astringent, and is used for flavouring.

The oil resembles that of anise, caraway, coriander, peppermint, and of other Umbelliferæ and Labiatæ. It is antiseptic, carminative, stimulant, and hæmostatic, and is useful in all animals affected with indigestion, flatulence, or diarrhœa. The tincture is made with 4 ounces of bark to a pint of alcohol (70 per cent.).

DosEs, ete.-Of the bark, horses take 3iv. to zi.; dogs, 3 ss. to $3 \mathrm{i}$. Of the oil, horses take $\mathrm{m}_{\mathrm{xx}}$. to $\mathrm{f} 3 \mathrm{i}$.; dogs, $m_{i}$. to miv., administered on sugar, or in syrup, mucilage, or spirit and water.

\section{A SAFGTIDA}

A Gum Resin obtained by incision from the root of Ferula fœtida, and probably other species. Imported from Bombay (B.P.). Nat. Ord.-Umbelliferæ.

The Ferula fœtida, or Narthex asafœtida, has a massive perennial root, black externally, white within. When the plant is four years old, the leaves and stems are removed, and, six weeks later, a slice is cut from the upper part of the root; the slicing is repeated several times at intervals, when the plant is exhausted, after yielding from a half pound to two pounds of a fœtid milky juice, which concretes. The yellow-brown tears are mixed with soft earth and made 
into irregular masses, which are red-brown externally, and within are opaque and milk-white, but gradually change to a dull yellow-brown. Asafœtida has a strong, persistent, alliaceous odour, and a bitter, acrid taste. It is pulverised with difficulty, forms an emulsion with water, is dissolved in rectified spirit, and also in potash and ammonia. Its active constituents are resin, gum, and about 5 per cent. of an acrid volatile oil containing allyl sulphide $\left(\mathrm{C}_{3} \mathrm{H}_{5}\right)_{2} \mathrm{~S}$. A good sample of asafœtida should contain about 65 per cent. of matter soluble in rectified spirit.

Actions AND USES.-Asafœetida is a mild stimulant, expectorant, carminative, antispasmodic, and vermifuge. It is speedily absorbed, its disagreeable odour indicating its general distribution; it is eliminated from the pulmonary mucous surface, the skin, and kidneys, gently stimulating their secretions. Robertson used asafoetida, with aloes and nux vomica, in constipation and torpidity of the bowels in horses, and in flatulent colic prescribed the tincture along with oil of linseed and of turpentine. The spiritus ammoniæ fœtidus, made with $1 \frac{1}{2}$ ounce asafœetida, 2 ounces strong solution of ammonia, and sufficient alcohol (90 per cent.) to make one pint of the spirit; and the tineture of asafœetida, are sometimes prescribed in colic and chronic cough. Like other substances containing odorous volatile oils, asafœtida is a vermifuge, but its action is uncertain. It is allied in some of its actions to valerian, and to sumbul, and closely resembles the two gum-resins, ammoniacum and galbanum, which are scarcely so active, and are chiefly used for making charges and plasters.

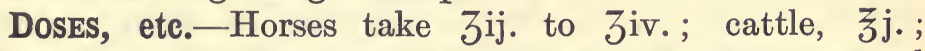
sheep, 3i. ; and dogs, grs. x. to grs. xx. It is given severa! times a day; may be made into bolus with camphor and ammonium carbonate; is frequently prescribed in draught with watery or alcoholic solution of ammonia; and, to prevent their misappropriation, it may be added to alcoholic and ethereal preparations intended for veterinary patients. In colic, asafœetida (3jv.) may be added to a quart of linseed tea and administered per rectum. 


\section{ARNICA}

Arnicer Rhizoma. The dried rhizome and roots of Arnica montana (B.P.). Leopard's Bane. Mountain Tobacco. Nat. Ord.-Compositæ.

Arnica montana is a perennial, growing on the mountainous parts of Central and Southern Europe, and also in Asia and America. It has a hairy stem about one foot high, composite yellow flowers, used in America for making the tincture, obovate leaves, and a cylindrical, contorted, brown root, one to three inches long, two or three lines thick, distinguished by a peculiar, aromatic, offensive odour, and a bitter, peppery, acrid taste. It contains mucin, extractive matter, two volatile oils, and an active, bitter, yellow, amorphous principle, arnicin.

ACTIONS AND USES.-Arnica is irritant and stimulant, has been credited with alterative properties, and is used externally as a stimulant for strains, bruises, and wounds.

Externally, arnica is a popular vulnerary for strains, bruises, and wounds, and especially for broken knees and sore shoulders. An ounce of the tincture is dissolved in twelve to twenty ounces of cold. water. A more efficient lotion is made with a drachm of arnica tincture and one to two drachms of zinc sulphate or lead acetate, diluted with ten or twelve ounces of water. For painful or irritable wounds the tincture is employed with chloroform, belladonna, or laudanum, diluted with water according to circumstances. With liberal feeding and tonics, a drop of arnica tincture placed daily within the eyelids is one of the remedies for those troublesome ulcers of the cornea which affect weakly dogs recovering from distemper. Arnica has, however, been overestimated; the evidence of its value as an internal remedy requires confirmation, while the healing properties ascribed to it appear to depend on the other drugs, spirit, or cold water with which it is generally used.

Doses, etc.-Horses take fziv. to f $z i$. of the tincture; cattle, double that quantity; dogs $m_{\nabla}$. to $m_{\text {viij., mixed }}$ with water, ale, or gruel. The flowers, leaves, and root are occasionally used powdered, especially in poultices; 
the tincture is made with an ounce of the root in powder (No. 40) to a pint of alcohol (70 per cent.). Watery infusions can be of little efficacy, for neither the arnicin nor volatile oil is soluble in water. Arnica opodeldoc consists of white soap four parts, rectified spirit ten, tincture of arnica five and camphor one. It is used as a local stimulant.

\section{A N IS E}

Anisi Fructus. Anise fruit. The dried ripe fruit of Pimpinella anisum. Nat. Ord.-Umbelliferæ.

Oleum Anist. Oil of Anise. The oil distilled from anise fruit; or from the fruit of the Star anise, Illicium verum (B.P.).

The natural order Umbelliferæ yields many aromatic fruits, such as anise, caraway, coriander, dill, and fennel, as well as the aromatic gum-resins, asafœtida, galbanum, and ammoniacum. These aromatic umbelliferous fruits, like the fruits, leaves, and other parts of various plants of such orders as the Myrtaceæ, Labiatæ, and Coniferæ, owe their medicinal value chiefly to their yielding volatile oils. These oils possess certain properties and actions in common, and the student should be familiar with their group characters.

Actions of Volatile OILS.-Volatile oils are readily diffusible; their in-contact effects are produced, not only when they are applied topically, but when they are absorbed and distributed in the blood stream. When concentrated they are local irritants and parasiticides; when diluted they paralyse sensory nerve-endings. Internally, they exert the same actions in the alimentary tract, and hence are anodyne, carminative, and antispasmodic. As a result of this topical action they reflexly stimulate circulation, respiration, and secretion. When absorbed they have little effect on the system in medicinal doses, but large doses first stimulate and later paralyse the central nervous system. As with other stimulants large doses paralyse the heart. They reduce inordinate reflex activity of the spinal cord. They are eliminated chiefly-(1) by the respiratory mucous 
membrane, being stimulant antiseptic expectorants, increasing bronchial secretion, and counteracting septic conditions of the membrane; and (2) by the kidneys, in moderate doses producing diuresis and antisepsis throughout the urinary tract, but in large doses causing overstimulation and strangury.

Anise is chiefly imported from Spain, Germany, and Southern Russia. It is an ovoid, grey-brown fruit, one-fifth of an inch in length, and covered with minute hairs. Like other fruits of this order, it is separable into two symmetrical mericarps, each of which is encircled by five slender ridges, while its transverse section exhibits about fifteen vittæ, which elaborate the oil.

Both anise fruits yield about 5 per cent. of a mixture, in nearly equal proportions, of a fixed oil, and a volatile anethol or camphor-like body, common to the Umbelliferæ, and some other plants, and having the formula $\mathrm{C}_{10} \mathrm{H}_{12} \mathrm{O}$. It is a phenol derivative, with some of its hydrogen atoms displaced by methyl and allyl, and having the rational formula $\mathrm{C}_{3} \mathrm{H}_{5} \cdot \mathrm{C}_{6} \mathrm{H}_{4} \cdot \mathrm{OCH}_{3}$. It is colourless, but becomes yellow on keeping; exhibits intensely the characteristic aromatic odour and taste of the fruit, and is soluble in alcohol and ether. The oil from the pimpinella solidifies at from $50^{\circ}$ to $60^{\circ} \mathrm{Fahr}$.; that from the staranise at about $36^{\circ} \mathrm{Fahr}$.

ACTIONS AND USES.-Anise is an aromatic stimulant, stomachic, and carminative. It is used to relieve indigestion and flatulence, to communicate an agreeable flavour to many medicines, and to diminish the griping of purgatives.

Doses, etc.-Horses receive about $\zeta \mathrm{i}$. ; cattle, $z$ i. to $z \mathrm{ij}$. ; sheep and swine, $3 \mathrm{ij}$. to 3 iij.; dogs, grs. xx. to grs. l., given powdered, repeated several times a day, often conjoined with ginger or other aromatics, and conveniently administered in ale or in spirit and water. Oil of anise, like oil of caraway, or coriander, is a diffusible stimulant, antiseptic, carminative, and antispasmodic. For such purposes cheaper remedies are generally, however, employed; but it is occasionally used as a flavouring ingredient, more especially for ball masses, and, mixed with a little 
spirit and bland oil, for the destruction of lice in pet dogs and other small animals.

Caraway, cardamoms, coriander, fennel, and fenugreek resemble anise in their actions and uses, and may be given in similar doses. These seeds are sometimes used by feeders of pigs, sheep, and cattle, and by waggoners and others, for improving the coat and condition of their charges. Fenugreek especially is prized for such purposes, is a constituent of various 'drinks,' and, with ground peas, maize meal, locust bean, and linseed meal, forms several vaunted condiments or 'nourishing foods.'

\section{EUCALYPTUS}

Oleum Eucalypti. Oil of Eucalyptus. The oil distilled from the fresh leaves of Eucalyptus globulus, and other species of Eucalyptus (B.P.). $\quad \mathrm{C}_{10} \mathrm{H}_{18} \mathrm{O}$.

EUCALYPTI GUMm. Eucalyptus gum, a ruby-coloured exudation from the bark of Eucalyptus rostrata, and some other species of Eucalyptus. Imported from Australia (B.P.). Nat. Ord.-Myrtaceæ.

The leaves and flower-buds of various Myrtaceæ, such as cloves, pimento, myrtle, and cajuput, as well as eucalyptus yield, when distilled, aromatic, antiseptic, volatile oils. The rapidly-growing eucalyptus-trees, indigenous to the Australian colonies, are now largely cultivated in many temperate regions with the view of preventing malarial fevers.

The dried leaves yield about 70 per cent. of eucalyptol, a colourless or pale, straw-coloured, liquid volatile oil, darkening by exposure, of an aromatic odour, and spicy, pungent taste, and soluble in about its own weight of alcohol. Its antiseptic powers increase as it undergoes oxidation by keeping. Other constituents are a crystallisable resin, tannin, and an oil, cineol, which crystallises at $30^{\circ} \mathrm{Fahr}$.

Eucalyptus, or red gum, occurs in grains or masses. It is tough, and has a very astringent taste. Cold water dissolves 80 to 90 per cent., forming a neutral solution. It is soluble in alcohol (90 per cent.). Red gum contains kino-tannic 
acid, catechin, and catechol. It resembles kino, which is less soluble in water.

ACTIONS AND USES.-The oil possesses in marked degree the group characters of volatile oils, well illustrated by turpentine. When freely used in concentrated form it is an in-contact irritant. It is powerfully antiseptic and disinfectant, destroying the lower forms of animal life. Locally applied it first irritates and then impairs sensibility by paralysing sensory nerve terminals. It is readily absorbed, increasing cardiac action, and is hence a stimulant and antispasmodic; and as it passes out of the body it increases the activity of the excreting channels, chiefly the respiratory mucous membrane and the kidneys. Its antiseptic properties confer some anti-malarial action; but it cannot, as has been suggested, take the place of the cinchona alkaloids.

Eucalyptus oil is used as a stimulant and antiseptic gargle, inhalation, and spray to diminish excessive quantity and fotor of nasal, pharyngeal, and bronchial secretions. It is administered in protracted cases of strangles, influenza, and purpura in horses, septicæmia in all animals, and in distemper in dogs-in such cases being usefully combined with quinine, ether, and alcohol. Arloing and Thomas state that solutions containing one $\frac{8}{100}$ th part destroy the virus of black-quarter. As an antiseptic it is three times as powerful as carbolic acid, for which it is sometimes substituted in surgical cases. Eucalyptus gauze is unbleached cotton impregnated with one part oil of eucalyptus, three of dammar resin, and three of paraffin wax. Eucalyptus wool contains 5 per cent. of the oil. In fœtid uterine discharges, eucalyptol injections or pessaries are useful. The gum is astringent, and styptic. As a desiccant, mixed with starch and zinc oxide, it is used for superficial wounds. Occasionally red gum is prescribed for diarrhœa in dogs.

DoSES, etc., of the oil.--Horses and cattle take f $3 i$. to f3iv. ; dogs, $m_{\text {ii. to }} m_{x}$., in diluted spirits, mucilage, or milk. For parasitic skin affections and other external purposes it is dissolved in oil or cacao butter, and is often usefully conjoined with iodoform. Eulyptol, consisting of 
equal parts of eucalyptol, carbolic acid, and salicylic acid, has been recommended as an intestinal antiseptic.

\section{J UN IPER}

Oleum Juniperi. Oil of juniper. The oil distilled from the full-grown, unripe, green fruit of Juniperus communis.

SPIRITUS JUNIPERI. Spirit of Juniper. A solution of the oil in alcohol (90 per cent.) (B.P.). Nat. Ord.Coniferæ.

The junipers are shrubby evergreen trees, growing in most temperate countries. Their leaves are dark-green, linear and arranged three in a whorl. Juniper berries are bluishpurple, furrowed, of the size and appearance of currants ; have an aromatic, terebinthinate odour, and a warm, sweet taste, followed by bitterness. For flavouring gin about two pounds of the berries are added to one hundred gallons of spirit. They owe their distinctive properties to about 2 per cent. of a mixture of two volatile oils, one of which is polymeric with terpene $\left(\mathrm{C}_{10} \mathrm{H}_{16}\right)$.

The fresh and dried tops of Juniperus sabina yield oil of savin, which is sometimes used as an ecbolic. From the wood of the Juniperus oxycedrus, and occasionally from that of the communis, the brown empyreumatic oil of cade is got by dry distillation. It is used in France and other continental countries for most of the purposes of oil of tar, and is recommended in scaly skin diseases.

ACTIONS AND USES.-The fruit, oil, and spirit of common juniper are topical irritants, and when absorbed are stimulant, stomachic, carminative, and diuretic. They resemble the turpentines, and thuja, or arbor vitæ.

Two ounces of the berries given to horses and cattle have little notable effect; but three to four ounces induce diuresis. The fruit and oil are occasionally given as diuretics, stomachics, and carminatives in indigestion and flatulence, and are stated to diminish the evil effects of bad fodder and marshy pastures, and to aid alike the prevention and cure of ascites in sheep. This is probably on account of their 
effects on intestinal parasites and those ingested with the food, such as flukes and strongyles. The oil mixed with lard and applied to exposed wounds prevents irritation from flies.

Doses, etc.-Of the fruit, as a stomachic, horses and

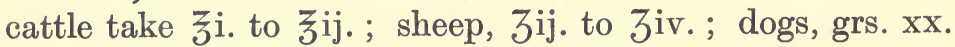
to grs. xl., repeated twice a day, in electuary or bolus. The fruit is readily eaten by most animals, especially by sheep. A decoction, made from the fruit, is occasionally prescribed, and also used as an external stimulant. As a diuretic the oil is the best form. Horses and cattle take $3 \mathrm{i}$. to 3ij.; dogs, $m_{v}$. to $m_{x}$., which may be repeated at intervals of three hours till diuresis is induced. Of the spirit, as a carminative and stomachic, cattle and horses take 3 iv. to そj. ; sheep, 3 ij. to $3 \mathrm{iv}$. ; dogs, $\mathrm{m}_{\mathrm{xx}}$. to $3 \mathrm{j}$., in oil, or mixed with other medicines.

\section{PEPPER MINT}

Oleum Menthe Piperite. Oil of Peppermint. The oil distilled from the fresh flowering peppermint, Mentha piperita (B.P.). Nat. Ord.-Labiatæ.

The natural order Labiatæ furnishes peppermint, spearmint, pennyroyal, lavender, rosemary, marjoram, and thyme; and from these plants, when fresh flowering, aromatic, antiseptic, volatile oils are obtained. Similar oils are extracted from the leaves of various Myrtaceæ, from the petals of roses, from the flowers and fruit of various Rutaceæ, and from the seeds of various Umbelliferæ.

Of the Labiatæ volatile oils peppermint is the most commonly used. The fresh plant yields 1 to $1 \cdot 25$ per cent. of the colourless or pale yellow oil, characterised by its warm aromatic taste and subsequent sensation of coldness. It consists of two isomeric oils - the fluid menthene $\left(\mathrm{C}_{10} \mathrm{H}_{18}\right)$, and the crystalline menthol $\left(\mathrm{C}_{10} \mathrm{H}_{19} \cdot \mathrm{OH}\right)$, which is homologous with thymol, obtained from the volatile oil of thyme.

ACTIONS AND USES.-Oil of peppermint is a typical volatile oil ; it is an antiseptic, topical stimulant and anæsthetic, carminative, antispasmodic, and parasiticide. It is more 
active than the oil from Mentha viridis, or spearmint, or M. pulegium, or pennyroyal. Diluted solutions arrest the development of bacilli as effectually as carbolic acid, or eucalyptus oil, and are hence used as dressings for wounds, and as sprays or gargles for ulcerated throat. It destroys vegetable and animal parasites infesting the skin. After stimulating, it paralyses the ends of sensory nerves with which it is brought into contact, and hence relieves gastrointestinal, neuralgic, and other pains. Painful surfaces are gently rubbed with a cone of menthol, solution being promoted by wetting with a little spirit. Increased anæsthesia is secured by diluting the menthol with eight or ten parts of ether, or mixing it with an equal part of thymol, carbolic acid, or butyl chloral-hydrate. Peppermint oil is used to prevent the nausea and spasms sometimes produced by purgatives ; to flavour medicinal preparations or cover their unpalatable taste. It forms a useful addition to drenches for flatulent colic.

Doses, etc., of the oil.-For horses and cattle, $m_{x x}$. to $m_{x x x}$; for dogs, $m_{\text {iii. to }} m_{v}$., given on a piece of sugar or in spirit and water. Peppermint water contains one of oil in one thousand of water. The spirit consists of one part of oil to nine of alcohol (90 per cent.).

\section{CHAMOMILE FLOWERS}

ANThemidis Flores.-The dried expanded flower-heads of Anthemis nobilis. Collected from cultivated plants (B.P.). Nat. Ord.-Compositæ.

Chamomile flowers are extensively cultivated in the warmer parts of England, are gathered during dry weather, exposed for a short time on trays in the shade, and carefully stored and kept very dry. Both varieties, but especially the single, have a hot, bitter taste, and a strong aromatic odour. They contain bitter extractive matter, soluble both in water and alcohol; a small quantity of tannin; traces of the bitter anthemic acid; a crystallisable, soluble base, anthemine; and 0.60 to 0.80 per cent. of volatile oil, obtained by distillation. The oil is of a pale-blue or green 
colour, gradually becoming yellow-brown, and consisting of a mixture of esters, chiefly of the angelates and valerianates of butyl and amyl.

Actions AND USES.-Chamomile flowers are mildly stimulant, aromatic, stomachic, and carminative ; full doses produce emesis in dogs. The infusion is sometimes given in atonic dyspepsia and diarrhœa. Horses and cattle take one to two ounces ; calves, sheep, and swine, a drachm or more. The flowers are occasionally used for fomentations and poultices. Like other volatile oils, that of chamomile lowers reflex irritability, and hence is useful in dyspepsia, diarrhœa, and spasmodic cough. Dose of the oil for the dog, $\mathrm{m}_{\mathrm{i}}$. to miii. on sugar.

\section{VALERIAN}

VALERIANAe RHIZOMA. The dried rhizome and roots of Valeriana officinalis. Collected in the autumn (B.P.). Nat. Ord.-Valerianaceæ.

The official valerian consists of a short, yellow-brown, tuberous rhizome, about the thickness of the little finger, with attached radicles, shrivelled, brittle, and of an earthybrown colour. It has a penetrating odour, becoming strong and even foetid by keeping, and a camphoraceous, unpleasant, rather bitter taste. It contains 1 to 2 per cent. of a strong-smelling, active volatile oil, isomeric with oil of turpentine $\left(\mathrm{C}_{10} \mathrm{H}_{16}\right)$, and the oily, acrid valerianic acid $\left(\mathrm{C}_{5} \mathrm{H}_{10} \mathrm{O}_{2}\right)$, which is also present in the berries of the guelder rose, in cod-liver oil, and decaying cheese, and may be obtained artificially by distilling amylic alcohol, and treating the distillate with caustic alkali.

ACTIONS AND USES.-Valerian and its volatile oil are topical irritants, stimulants, and antispasmodics. In large doses they paralyse the brain and spinal cord, lower bloodpressure, and slow the pulse. Valerianic acid has no special stimulant action, but is said to resemble acetic acid. The valerianates accordingly do not exhibit the action of valerian or of the volatile oil.

MEDicinal UsEs. - Valerian resembles asafœtida, the other gum-resins, camphor, and sumbul or musk-root imported 
from Russia and India, and produced by an umbelliferous plant. It has little effect on horses or cattle, even in doses of several ounces. It is occasionally given to dogs to allay nervous irritability, and relieve chorea and epilepsy; but little dependence can be placed on it ; used for similar conditions in man its effects are chiefly psychical and due to its nauseous taste and smell. It attracts and excites cats, developing by its suggestive odour their amatory propensities. The volatile oil abates the convulsions of strychnine poisoning, is an anthelmintic, and is excreted by the lungs, skin, and kidneys.

DOSES, etc.-Used for horses or cattle, valerian may be given in doses of $3 \mathrm{jv}$. to $\jmath_{\mathrm{j}}$.; for dogs, grs. viii. to $3 \mathrm{j}$.; for cats, grs. ii. to grs. xx., given in powder or infusion several times daily, conjoined with ginger, gentian, or camphor, or dissolved in spirit of ammonia.

The Valerianates, as above indicated, exhibit the actions of their bases, but not those of valerian. Where it is desired to conjoin the physiological action of valerian with iron, zinc, or other metallic salt, or with quinine, the oil of valerian should be prescribed with a suitable salt of the metallic or vegetable base. The valerianate of zinc has been used for dogs and cats in epilepsy, chorea, and nervous excitability, in doses of grs. ij. to grs. v., but this use is quite irrational, and no good effects ensue.

\section{TURPENTINES}

Terebinthin ж. Nat. Ord.-Coniferæ.

The Coniferæ yield the following drugs-

I. Oleo-resinous juices exuding spontaneously or from incisions made into the trunks or branches, consisting of common and Venice turpentines, Canada balsam, frankincense, and Burgundy pitch.

II. Oil of turpentine $\left(\mathrm{C}_{10} \mathrm{H}_{16}\right)$-the volatile or essential oil procured from turpentines by distillation.

III. Hydrates of turpentine,--terebene, terpene, and terpinol, prepared by acting on oil of turpentine with acids.

IV. Resins - the residue of the distillation of turpentine. 
V. Tar and black pitch-got by subjecting the roots and wood to destructive distillation. Wood wool prepared from pine wood.

\section{The Turpentines or Coniferous Oleo-resins.}

The terebinthinate juices while recently exuded are fluid, or nearly so ; but exposure to the air volatilises and oxidises their essential oil, and they solidify. They have a peculiar, pungent, bitter taste and odour, are scarcely soluble in water, partially soluble in rectified spirit, dissolving readily in oils, ether, and alkaline solutions ; are inflammable, and leave, when burnt, a finely-divided residue of carbon or lamp black. Several of the more important varieties demand notice.

Common TURPEntine is obtained throughout the Southern States of America, from Virginia to the Gulf of Mexico, chiefly from the Pinus Tæda, and P. palustris, australis, or swamp pine, a tree sixty or seventy feet high, having bright green linear leaves about a foot in length, and collected into bundles like those of the Pinus sylvestris, or Scotch fir, from which, throughout Northern Europe, turpentine is also procured.

BORDEAUX TURPENTINe, chiefly produced in the southwest of France, from Pinus maritima and P. pinaster, is got by bleeding or hacking the bark, and conducting the juice into suitable vessels placed at the foot of the tree.

Turpentine from different sources differs somewhat in appearance; the American is dextro-rotatory, the French lævo-rotatory; it is semi-fluid, its consistence varies with the temperature; it gradually solidifies from escape and oxidation of the volatile oil; it has a yellow colour, an aromatic odour, and a warm, pungent taste. Unless melted and strained, it usually contains impurities. Water acquires its flavour, but separates only traces of its active principles. Rectified spirit and ether dissolve it ; eggs and mucilage form with it emulsions convenient for administration. The crude American variety, when recent, yields 15 to 25 per cent. of volatile oil.

Venice Turpentine (Terebinthina Veneta) is chiefly ob- 
tained in the Tyrol, Switzerland, and Piedmont, from the common larch, Larix Europæa-a lofty tree with graceful drooping branches, and leaves at first in fasciculæ, like the pine tribe, but afterwards becoming solitary by elongation of the twigs. Venice turpentine is tenacious, rather opaque, and fluorescent ; less apt than common turpentine to concrete with keeping; has a pale yellow colour, an acrid, bitter taste, a disagreeable terebinthinate odour, and contains 15 per cent. of oil of turpentine. The Venice turpentine of the shops almost invariably consists of about three parts of common resin dissolved in one part of oil of turpentine. This artificial mixture is distinguished by its strong odour, and its more quickly evaporating, and leaving a varnish on a sheet of paper, on which the natural Venice turpentine remains viscid.

CANADA Balsam, chiefly brought from Lower Canada, is obtained by puncturing the vesicles lying between the bark and wood of Abies balsamea. It is a pale, greenish-yellow oleo-resin of the consistence of thin honey, has an agreeable terebinthinate odour, and a slightly bitter, feebly acrid taste. On exposure it dries slowly into a transparent adhesive varnish, and solidifies when mixed with one-sixth of its weight of magnesia and water. It contains 15 to 18 per cent. of oil, is much used by varnish-makers, opticians, and microscopists, and is a constituent, with collodion and castor oil, of flexible collodion. It is sometimes improperly termed Balm of Gilead, which, however, is derived from an Arabian balsamodendron. Strasburg turpentine is a fluid, citron-smelling oleo-resin obtained in the vicinity of the Alps from Abies picea. Chian or Cyprus turpentine, from the island of Scio, nearly resembles Canada balsam in its properties and uses; it is a greenish-yellow, liquid oleo-resin from the Pistacia terebinthus, a tree of the mastic order.

Frankincense, or Thus Americanum, is the semi-opaque, soft, concrete turpentine scraped off the trunks of Pinus palustris, P. Tæda, and other American Coniferæ. On keeping it becomes dry, brittle, and darker in colour. A similar concrete turpentine comes from the south of France under the name of gallipot or barras. 
BuRgundy Pitch is the resinous exudation from the stem of Picea excelsa, or spruce fir, melted and strained. It consists of an amorphous resin, oil of turpentine, and other isomeric oils, and abietic acid. It occurs in semi-opaque red-brown masses, breaks with a shining conchoidal fracture, and has an empyreumatic turpentine odour and aromatic taste. The substance sold as Burgundy pitch is generally made by melting resin and palm oil and stirring in some water. True Burgundy pitch and its imitations spread upon leather are used for stimulant and adhesive plasters, applied in swellings of joints, chest affections, and rheumatism.

ACTIONS AND USES.-The turpentines are topical irritants. When swallowed they are speedily absorbed, act as general stimulants, and are discharged by the kidneys, bronchial membrane, and skin, stimulating these channels of excretion. Their uses resemble those of their more active constituent, oil of turpentine. In percentage of oil, and hence in activity, they stand as follows : Canada balsam, Venice turpentine, common turpentine, and frankincense. They are occasionally used as stimulants in indigestion, colic, and general debility; as laxatives, especially when in combination; and as anthelminties, diureties, and expectorants.

Externally applied, they are stimulants, stypties, and antiseptics, and are used as excipients for making up diuretic and stimulant balls. In the south of France the resinous vapours of the Coniferæ have been employed for the relief of bronchitis, phthisis, and rheumatism in human patients. The growing pine forests, and the oleo-resins extracted from them in presence of oxygen, evolve antiseptic camphoraceous oils and peroxide of hydrogen, which purify the air and destroy disease germs.

Doses, ete.-Horses and cattle take $ろ$ j. to そiij.; sheep, 3 j. to 3 iij.; pigs, 3j. to 3ij.; dogs, grs. xx. to grs. lx. The maximum doses are stimulant and antispasmodic; the minimum, frequently repeated, are diuretic and expectorant. They are administered with milk, oils, linseed gruel, mucilage, eggs, or about $\frac{1}{20}$ th part of magnesia. For external purposes they are made into liniments and ointments. 


\section{OIL of TURPentine. Oleum Terebinthinæ.}

The oil distilled from the oleo-resin, obtained from Pinus sylvestris, and other species of pinus.

The crude turpentines when heated, as they usually are, by steam, yield 15 to 25 per cent. of oil of turpentine, sometimes improperly called spirit of turpentine. It is a mixture of several hydrocarbons (terpenes) having the composition $\mathrm{C}_{10} \mathrm{H}_{16}$. It is limpid, with a strong, peculiar odour and a pungent, bitter taste. It commences to boil at about $320^{\circ}$ Fahr. According to its source, it varies in its odour, specific gravity, boiling point, and effect on polarised light. It is very inflammable, burning with a heavy yellow flame and producing much smoke. It is insoluble in water, soluble in $6 \frac{1}{2}$ of alcohol, and readily dissolved in ether, chloroform, glacial acetic acid, fixed and volatile oils. It is a valuable solvent for wax, resins, fats, many alkaloids, iodine, and phosphorus.

It is the representative of a large group of terpenes, including the volatile oils of chamomile, caraway, juniper, lemons, pepper, savin, thyme, tolu, and valerian-all of which have the formula $\mathrm{C}_{10} \mathrm{H}_{16}$. In common with other terpenes, it is convertible into isomerides, oxidises on exposure to air, forming camphoric peroxide; with water produces crystalline hydrates; and with hydrochloric acid forms crystalline compounds. By this action of hydrochloric acid on turpentine artificial camphor is produced.

Terebene $\left(\mathrm{C}_{10} \mathrm{H}_{16}\right)$, a mixture of dipentene and other hydrocarbons, obtained by agitating oil of turpentine with successive quantities of sulphuric acid, and then distilling in a current of steam; is less disagreeable and acrid to the taste, and optically inactive. It has the same medicinal properties.

Actions AND USES.-Oil of turpentine has the group actions of a volatile oil. It is an antiseptic topical irritant and disinfectant, and is used as a rubefacient and vesicant. Large doses are irritant and narcotic. Medicinal doses are antiseptic, stimulant, especially of mucous and skin surfaces, antispasmodic, hæmostatic, anthelmintic, and anti- 
parasitic: It is also prescribed as an adjuvant cathartic, diaphoretic, and diuretic.

General ACtions.--Like other volatile oils, it is an active antiseptic. In destroying bacteria spores, Koch found it more effective than alcohol, ether, chloroform, or benzol. It poisons lice, acari, entozoa, and other parasites, whether lodged in the skin, bronchial tubes, or bowels. Applied to the skin it irritates, and, if evaporation be prevented, vesicates, and even ulcerates.

When swallowed it is rapidly absorbed, diffused, and excreted, and may be speedily detected in the sweat, breath, and milk, and in the urine, to which it imparts the odour of violets. In the mouth and stomach, its slight irritant action determines an increased flow of blood to those parts, stimulates secretory glands and the nerves of taste, so that digestion is improved. In the intestine the same action, combined with its antiseptic properties, makes it useful in preventing griping, in improving the tone of the bowel wall, and in promoting peristalsis. After absorption the white corpuscles of the blood are increased in number, especially the polynuclear ones. The central nervous system is but little affected by medicinal doses, but reflexly the local irritation and stimulation of function of the alimentary tract cause a general stimulation. Thus the heart is stimulated and the pulse quickened, respiration is generally increased, blood-pressure is raised, and it is not until dangerous doses have been given that the narcotic depressant effect is developed. It is eliminated by the lungs, acting as a stimulating antiseptic expectorant.; by the skin, promoting diaphoresis; by the kidneys, inducing diuresis ; while full doses, especially in combination with laxatives, are cathartic.

Toxic EFFEcTs.-Large doses when inhaled irritate the respiratory mucous membrane, and reflexly cause difficult breathing. Large doses when swallowed cause irritation, and occasionally ulceration of the bowels. A very large dose quickly swallowed is absorbed, and as in the case of alcohol, produces brief primary stimulation and prolonged subsequent paralysis of the central nervous system. Rabbits and kittens were paralysed (narcotised) by injection of 
turpentine emulsion into the stomach. The motor centres are implicated in the same order as in poisoning with members of the alcohol series, those of the brain being first affected, those of the cord later, and those of the medulla last. A dog receiving two drachms, intravenously, staggered, was convulsed, circulation and respiration failed, and death occurred in three minutes (Christison). During excretion large doses cause congestion of the urino-genital organs, diminish or arrest secretion of urine, and induce strangury and sometimes hæmaturia.

Medicinal Uses.-In indigestion, flatulence, and atonic diarrhœa, it checks undue fermentation and acts as a carminative and gastro-intestinal stimulant and astringent. Although an uncertain cathartic when given alone, like many other volatile oils it promotes the action of cathartics, with which it is usefully conjoined in flatulent colic, and in such cases it is also used in enemata. Alike in flatulent and spasmodic colic in horses, it is frequently given combined either with linseed oil or with mucilage and aloes, and in spasmodic cases is conjoined with opium.

As a cardiac and general stimulant it is not so effective as alcohol or ether. But stimulating vaso-motor centres and contracting arterioles, as well as by its local action during excretion, it checks excessive or faulty mucous discharges. Thus, in chronic bronchitis and nasal gleet, terebene gargles and turpentine emulsions and inhalations prove useful, especially when seconded by turpentine liniments applied externally. Turpentine has been credited with astringent hæmostatic properties, and for this purpose it is used in purpura, and in passive hæmorrhage from the lungs, stomach, or bowels, as well as from the kidneys, although in renal cases the drug must be used cautiously and in small doses. That it really possesses hæmostatic properties is doubtful. In purpura in horses, ounce doses are prescribed, with the same quantity of ferric chloride tincture, in milk, twice or thrice daily. This prescription, with two drachms potassium chlorate, is useful in many cases of hæmoglobinuria. Chronic rheumatism in all classes of patients is frequently relieved by conjoining turpentine 
with salines, and in such cases it is also usefully applied externally.

Turpentine, well kept and fully oxidised, as the French variety generally is, contains formic, acetic, and carbonic acids, and is an antidote in poisoning with phosphorus. Phosphorus in repeated doses produces in animals fatty degeneration; but neither this nor other forms of phosphorus poisoning occurred when the drug was given with French turpentine (Köhler). Personne gave phosphorus to five dogs, and all died. To five others, an hour or two after similar lethal doses, he gave turpentine, and only one died. Of five dogs to which he gave turpentine, immediately after deadly doses of phosphorus, only one died (Ringer).

In cattle practice full doses are valuable in hoven. Chronic diarrhœa and dysentery, especially when accompanied by flatulence, are often benefited by small doses conjoined with lime-water, aromatics, or opium. In post-partum hæmorrhage in cows turpentine is prescribed in doses of 3 or 4 ounces, and it is commonly given, along with iron salts, in cases of bovine piroplasmosis or red-water in this country.

For the destruction of intestinal worms oil of turpentine is generally conjoined with a laxative, and given after the bowels have been emptied by a cathartic, and the patient has been fasted. Although it removes round worms, it is not in horses a certain remedy for tape-worms, but its efficacy is increased by combination with male fern. A tolerably good tæniacide for the horse consists of two ounces of turpentine and one ounce of male fern extract, dissolved in a pint of linseed oil. For tape-worms in dogs, areca nut, male fern, and cusso are more effectual than turpentine.

For destroying strongyles infesting the air-passages of calves and lambs, turpentine has been widely used. In some sheep-breeding districts of England, thriftless, coughing lambs, throughout the summer months, at intervals of a week or ten days are given turpentine drenches, with the view of killing both bronchial and intestinal worms : and such treatment certainly greatly diminishes the scouring and mortality to which lambs in some localities are liable. Six-month calves take half an ounce, lambs of the like 
age a drachm, of oil of turpentine, conveniently mixed with milk, and administered by the mouth. Two or three doses, at intervals of two or three days, usually effect a cure. Turpentine inhalations, although fairly effectual, are troublesome to manage. For calves intratracheal injection of turpentine was introduced by Hutton, who made a small incision in the skin, half-way down the neck, and between two rings of the trachea, and with a suitable syringe injected $f 3 i$. to $f 3 i j$. oil of turpentine, with $f 3$ ss. each of carbolic acid, chloroform, and glycerin, which ensures solution of the carbolic acid. No serious irritation resulted. A few paroxysms of coughing occasionally occurred. Brought into actual contact with the parasites, the vermicide promptly destroyed them.

Gapes in poultry, caused by the Syngamus trachealis, is successfully treated by a similar mixture, used diluted with four or five parts of milk or bland oil, two or three drops being placed in the mouth of the ailing fowl. A similar dressing is sometimes applied around the throat, but, although in part absorbed, is not so effectual as when swallowed.

Externally, oil of turpentine is used as an antiseptic, stimulant, and counter-irritant. Rubbed undiluted into the skin of horses, it quickly causes topical irritation, great restlessness, and much excitement, continuing for twenty or thirty minutes, and, if used largely and repeatedly, it vesicates, and may blemish. Cattle are not so sensitive to its irritant effect, and for them it is sometimes employed to increase the activity of other vesicants. A piece of flannel wrung out of hot water, and sprinkled with turpentine oil (turpentine stupes), is frequently applied as a counterirritant. A continuous moderate action is more serviceable than a single violent effect. For inveterate eczema and psoriasis, after removal of the scales with soft soap and water or alkaline dressings, turpentine, diluted with one or two parts of oil or glycerin and water, sometimes beneficially stimulates the hypertrophied, weakened skin, and promotes cure.

It is used as a stimulant for rheumatic swellings, more particularly of cattle and sheep; for sprains and bruises after the first pain and tenderness have been subdued by 
fomentation; for controlling venous congestion and stasis arising from frostbite, which is not uncommon in the limbs of horses used for night work; for promoting absorption of small cysts; for healing the troublesome chronic sores occurring about the heels of draught-horses; and for relieving tedious foot-rot in sheep. For such cases it is usually mixed with two or three parts of vaseline, oil, or glycerin. A smilar mixture destroys lice and other skin vermin, as well as the fungi of ringworm. An occasional sprinkling over dogs' beds keeps them free of fleas. It is often added to stavesacre, tobacco, and other antiparasitic dressings. It enters into the composition of various mixtures used by shepherds to protect their flocks from flies, and to kill maggots. For such purposes three ounces oil of turpentine, one ounce each of sweet oil, common salt, and mucilage, and half a drachm corrosive sublimate, are mixed in a quart of water.

Doses, etc.-For horses and cattle, as a stimulant and anti-spasmodic, the dose is $\mathrm{f} \xi \mathrm{j}$. to $\mathrm{f} \mathrm{j}_{\mathrm{j}}$. ; as a diuretic, $\mathrm{f} \mathrm{zss}_{\text {s. }}$ to $f \xi j$. As an adjuvant cathartic or anthelmintic the dose is about $\mathrm{f} \zeta \mathrm{ij}$., combined with aloes in solution, with castor or linseed oil, with iron salts, quassia, gentian, or other bitters. Full-grown cattle take double these doses. Sheep and pigs receive $\mathrm{f} 3 \mathrm{j}$. to $\mathrm{f} 3 \mathrm{iv}$. ; dogs, $m_{x x}$. to $\mathrm{f} 3 \mathrm{j}$. It is administered dissolved in bland oils, shaken up with linseed gruel or milk, or made into an emulsion with mucilage or eggs. Aromatics, bitters, or ethers are sometimes added. Intratracheally, $3 \mathrm{j}$. to $3 \mathrm{ij}$., mixed with an equal measure of olive oil, may be administered to horses affected with catarrh.

For inhalation half a bucket of boiling water is placed under the patient's nostrils, and an ounce of turpentine placed in it; or it may be introduced into the steam-kettle, which is almost as serviceable in the treatment of bronchitis in animals as in man. For enemata, turpentine is usually diluted with fifty or sixty parts of oil ; or it is mixed with two or three parts of oil or mucilage to ensure solution, and then added to the soap and water. In diarrhœa or dysentery it is conjoined with laudanum and starch gruel.

For external purposes it is usually applied with linseed 
oil, soft soap, or ammonia liniment. Convenient stimulant dressings are made with equal quantities of oil of turpentine, bland oil, and soft soap, or two to three ounces of oil of turpentine are added to a pint of soap liniment. As a stimulant for rheumatism, equal parts of oil of turpentine and laudanum are mixed with two or three parts of linseed oil or soft soap. For dogs, an active embrocation is prepared with an ounce each of oil of turpentine and medicinal ammonia, and six to ten ounces of olive oil.

OIL of ScoTch FIR (oleum pini sylvestris) is prepared by distilling the fresh leaves of the Scotch fir or Pinus sylvestris. It has most of the properties, and is applied for many of the uses of oil of turpentine.

TEREBENE being less acrid than oil of turpentine, and less liable to act on the kidneys, is sometimes substituted for it, especially as an internal stimulant and antiseptic in excessive mucous discharges, and for relieving flatulence. Externally, it is applied as a stimulant, antiseptic, and deodoriser.

OIL of PINE, oleum pini (pinol or pumuline), the oil distilled from the fresh leaves of Pinus Pumilio, is used as an inhalation in bronchial catarrh and in laryngitis.

Terpene Hydrate $\left(\mathrm{C}_{10} \mathrm{H}_{26} 2 \mathrm{HO}\right)$, prepared by passing a current of air through a mixture of four parts oil of turpentine, one part nitric acid, and three parts rectified spirit. It is crystalline, has a slight agreeable balsamic odour and taste; is soluble in 220 parts of cold water, twenty-two parts of warm water, and very soluble in alcohol, ether, turpentine, and oils. Its general actions resemble those of oil of turpentine. One or two drachms may be swallowed by small dogs without causing gastric derangement; two to three drachms quickly produce intoxication with restlessness, uncertain gait, impaired vision, and dreaming, followed in a few hours by sound sleep. It has been prescribed in bronchitis, especially in dogs, to stimulate secretion in the earlier stages, and in the later chronic stages to liquefy and so get rid of excessive discharges. The doses for horses or cattle are grs. xxx. to $3 \mathrm{ij}$. ; for dogs and cats, grs. v. to grs. xxx., repeated two or three times daily, and given with alcohol, glycerin, or syrup, in drench or in the patient's food. 
Terpinol, prepared by boiling terpene in water acidulated with sulphuric acid, is a mobile liquid, with a strong aromatic odour; insoluble in water, but soluble in alcohol and ether. Its actions are similar to those of terpene. Doses for dogs and cats, $m_{\text {iv. }}$ to $m_{x v}$.

\section{Resin, Rosin, Resina.}

The crude turpentines contain 75 to 90 per cent. of resin or colophony, developed by a process of oxidation. Crude turpentine, when distilled with a little water, which the resin retains, leaves a residue of yellow or white resin. When the water is removed, the resin becomes transparent, and when more strongly heated is still clearer, and is known as black or fiddler's resin. These turpentine resins are types of a considerable group of resins, derived chiefly from the vegetable kingdom, distinguished by their appearance, fusibility, inflammability, acidity to test-paper; burning with a smoky flame; insoluble in water, and soluble in alcohol, volatile oils, and alkalies. They unite with fats, wax, and spermaceti, and are largely used in the manufacture of yellow soap. Resin has the formula $\mathrm{C}_{44} \mathrm{H}_{62} \mathrm{O}_{4}$. Coarsely powdered and shaken with warm dilute alcohol, it undergoes hydration, and yields 80 to 90 per cent. of abietic or sylvic acid $\left(\mathrm{C}_{44} \mathrm{H}_{64} \mathrm{O}_{5}\right)$. Bordeaux resin or gallipot contains besides the isomeric pimaric acid.

Actions AND USES. - Resin is a gentle stimulant, astringent, and diuretic. Two to four ounces, swallowed by horses or cattle, cause diuresis. It is added to diuretic masses to increase their consistence. Externally, it is used as a stimulant, astringent, and styptic. In castration, a pinch applied to the severed cord, and melted by the hot iron, helps to seal bleeding vessels. It is largely used to impart firmness and adhesiveness to stimulant plasters. Resin ointment is made with eight parts each of resin, yellow wax, and olive oil, and six of lard, melted with gentle heat, strained while hot through flannel, and stirred constantly while it cools. This simple ointment is much used as a lubricant, and mild stimulant for wounds, ulcers, blistered surfaces, and for giving bulk and consistence to other ointments. 


\section{Tar, OIL of Tar, and Pitch.}

Tar, or Pix liquida, is a thick, viscid, brown-black, aromatic liquid, obtained from the wood of Pinus sylvestris and other pines by destructive distillation. Mineral or Barbados tar has already been noticed. Coal tar (pix carbonis), obtained from the destructive distillation of coal, is a by-product in the manufacture of gas. Two descriptions of wood tar are in use-one got from hard exogens, such as oak, birch, and ash, as a residual product in the making of charcoal for gunpowder ; and the other as an empyreumatic variety imported from Stockholm, Archangel, and America, is got by roasting billets of the roots, branches, and refuse coniferous timber stacked in shallow pits dug on a bank or inclined plane. This old process is now superseded by distillation of the refuse wood in cast-iron stills, whereby nearly double the yield of tar is obtained; 14 per cent. is got from air-dried stems, 16 to 20 per cent. from roots. When wood is thus distilled the condensed products separate into two layers, the upper a mixture of methyl-alcohol, pyroligneous acid, acetone, etc., in water; the lower wood tar.

Stockholm tar is soluble in alcohol, ether, oils, and alkaline solutions, but not in water, which, agitated with it, acquires, however, its odour, taste, and brown colour, and constitutes tar water, once regarded as a valuable medicine. Tar consists of pyroligneous acid, methyl-alcohol, creosote, and various phenols, with toluene, xylene, and other hydrocarbons.

Tar when distilled yields oil of tar (oleum picis liquidæ), an empyreumatic acid liquid, which, although colourless when first distilled, speedily becomes yellow or brown, and is soluble in alcohol. It contains the more volatile hydrocarbons of the tar. There remains in the retorts pitch, or pix nigra, a black, bituminous substance, solid and brittle, with a shining fracture, dissolved by the same solvents as tar, and consisting of modified resin, and a colourless, inodorous, crystalline substance, melting at $194^{\circ} \mathrm{Fahr}$., called retene $\left(\mathrm{C}_{18} \mathrm{H}_{18}\right)$ (Flückiger).

ACTIONS AND USES.- Tar is antiseptic, stimulant, diuretic, diaphoretic, expectorant, and parasiticide. Its active prin- 
ciples being diffusible phenols, it acts not only when applied externally, but produces most of its effects when given internally. The urine of horses receiving tar water keeps unchanged for several days. It is still occasionally prescribed for horses with chronic cough and bronchitis, where the discharges are copious. It is used both internally and externally as a cutaneous stimulant and antiseptic in the squamous stages of grease and other forms of eczema, in psoriasis, and in pityriasis, the scaly surfaces being coated daily with undiluted tar, the dressing after several days washed off with soft soap and water, and any refractory spots dressed with mercurial ointment. In chronic eczema one part of tar is usefully added to four of zinc oxide ointment. Tar water is a popular but serviceable lotion for indolent ulcerations and hæmorrhoids. For thrush and canker of the horse's foot tar is used either alone or with copper sulphate, sulphuric or nitric acid, and other agents.

Mixed with equal parts of fatty matters or soft soap, to impart proper consistence, it forms an excellent stopping for horses' feet, keeping the hoof moist and soft. As a hoof dressing, Miles recommends a quarter of a pound each of tar, beeswax, and honey, a pound and a half of lard, and three ounces of glycerin; the lard and beeswax are melted together, the lard, tar, and glycerin stirred in, and stirring continued until the mass begins to set. For foot-rot in sheep, tar has the several advantages of stimulating healthy growth of horn, deodorising, and preventing attacks of flies. It is used in securing wounds, binding broken horns, and making adhesive plasters.

Oil of tar is sometimes used instead of oil of turpentine. Its empyreumatic constituents confer antiseptic properties ; it cures mange and scab, destroys other parasites, is sometimes added to sheep dips, but has the disadvantage of discolouring the wool, does not mix well with the other ingredients, while large doses or strong solutions are apt to poison. It is applied in both varieties of ringworm, but is seldom so successful as iodine.

Pitch is used as a mild stimulant in thrush, canker, and sand-crack in horses; in foot-rot in sheep; and to give adhesiveness to plasters and charges. 


\section{ARECA-ARECOIINE}

Arec se Semina. The seed of Areca catechu. Betel-Nut. Nat. Ord.-Palmacæ. (Not official.)

The catechu or betel-nut palm is a straight, slender tree, forty or fifty feet high, growing on the Coromandel and Malabar coasts, and throughout the warmer parts of Asia. Within a fibrous fruit lies the hard, ovoid, red-brown seed, of the size and appearance of a nutmeg. When ground, the powder is brown, astringent, and partially soluble in hot water and spirit. It contains besides tannin, the alkaloids, arecoline, arecaine, arecaidine, and guvacine. Arecoline $\left(\mathrm{C}_{8} \mathrm{H}_{13} \mathrm{NO}_{2}\right)$, the chief alkaloid, is strongly alkaline, liquid, colourless, and volatile, soluble in water, alcohol, ether, and chloroform. With acids it forms salts, of which the most important is the hydrobromide.

ACTIONS AND UsEs.-Arecoline is a powerful sialogogue, diaphoretic, intestinal stimulant and vermifuge. In physiological actions it is allied to eserine, pilocarpine, and pelletierine. Like eserine it contracts the pupil and stimulates peristalsis. Exerting more energy than pilocarpine it stimulates the secretory nerves of glands, and under its influence the salivary, skin, and intestinal secretions are much increased. It stimulates unstriped muscle and promotes the discharge of urine. Large doses act on striated muscle, causing twitching and spasm, followed by partial paralysis. Medicinal doses diminish the force and number of the pulsations, and excessive doses paralyse the heart. In horses respiration is increased by small doses, while large and repeated doses lessen the activity of the respiratory nervous centre inducing dyspnœea and suffocation. Arecoline has been used with excellent results in the treatment of acute laminitis and colic in horses and of constipation in cattle. Its value as a remedy for laminitis-first ascertained by Fröhner-has been well attested by Schumacher, Paimans, Gobbels, and others. Schumacher asserts that it shortens the duration of the disease. In colic and fœcal impaction it is almost as powerful as eserine, and stronger and more rapid than pilocarpine though not so lasting in its 
effects. Solutions applied to the eye contract the pupil and diminish ocular tension.

Areca nut is an astringent resembling catechu, and, when freshly powdered, an effective vermicide, especially for dogs, proving destructive alike to tape- and round-worms. Its effects on horses and cattle are less satisfactory. A suitable dose given to a dog which has been fasted for twelve hours often acts effectively within half an hour, but it is usually desirable to conjoin with the areca $m_{x}$. to $m_{x v}$. of male fern extract. This combination is a most effectual remedy for tapeworm in dogs. If the parasites are not removed a second dose of the mixture should be given two or three days later. Tænaline, a registered liquid preparation containing the tæniafuge principles of freshly ground areca nut, is a convenient and efficient vermicide.

Doses, etc.-Areca nut. Dogs, grs. x. to 3ij. ; horses, 3iv. to 3vi.; poultry, gr. j. to grs. x. The dose of the powder for the dog is about 2 grains for every pound of the animal's weight (Mayhew). It is administered in linseed oil, soup, mucilage, butter, or milk.

Arecoline hydrobromide. Horses and cattle, gr. $\frac{2}{3}$ to grs. $1 \frac{1}{2}$; dissolved in $m 60$ to $m 90$ of sterilised water, and injected subcutaneously or into the jugular vein. In laminitis the dose is repeated daily for four or five days. In colic a single injection may suffice. To contract the pupil a 1 per cent. solution of the hydrobromide may be employed.

\section{ARTEMISIA-SANTONIN}

Artemisia Absinthium. Wormwood. (Not official.) Artemisia Maritima. Santonica. Nat. Ord.-Compositæ. Santonin. A crystalline principle prepared from Santonica. (B.P.)

Wormwood and Santonica are low shrubby plants, characterised by their aroma and bitterness. They belong to the natural order Compositæ, which comprises the familiar southernwood and tansy, the mildly anodyne lettuce, and the harmless dandelion.

The dried Artemisia absinthium contains a volatile camphoraceous oil, absinthol, and a bitter extract, yielding the 
neutral crystalline absinthin, which is a narcotic poison and spinal stimulant, causing in dogs and rabbits trembling, stupor, and epileptiform convulsions, which may prove fatal. In medicinal doses, it is an aromatic bitter tonic, and a popular remedy for worms. It is the chief active constituent of the liqueur absinthe.

Santonin. The unexpanded minute flower-heads of Artemisia maritima contain a volatile oil, a resin, and about two per cent. of a crystalline neutral principle, santonin $\left(\mathrm{C}_{15} \mathrm{H}_{18} \mathrm{O}_{3}\right)$. It is soluble in 300 parts cold water, in chloroform, in 40 parts alcohol, fixed oils, and alkaline solutions, and hence in the intestinal juices. It is rendered yellow by sunlight, and gives a violet colour when added to a warm alcoholic solution of potassium. It imparts a blood-red colour to the urine. Large doses cause in dogs grave symptoms and are frequently fatal. At first there is twitching of the head muscles, rolling of the eyes, and grinding of the teeth, rotation of the head, then regular epileptiform convulsions followed by clonic spasms of the limbs and trunk. These are followed by intervals of repose. During the convulsions respiration is irregular and insufficient, and in fatal cases asphyxia results (Cushney). The convulsive effects are probably due to over-stimulation of the cerebral cortex. As antidotes, an emetic and a purgative should first be given to promote excretion. Then such agents as chloroform, potassium bromide, and chloral hydrate to prevent convulsions. Cold water cloths applied to the head are useful, and artificial respiration must be carried out if necessary. Santonin is a vermicide, without effect on tæniæ, but destructive to round and thread worms-given for the former by the mouth, for the latter by enemata, and most active when combined with castor oil. It is less effective in horses than in pigs and dogs, and is given conjoined with aloes or jalap. Half an ounce mixed with the food serves as a vermicide for fifteen young pigs. As in human practice, it is effectual in checking incontinence of urine in young patients, for this purpose being equal to belladonna and superior to nux-vomica. The B.P. santonin lozenge containing 1 grain of the drug, forms a very convenient worm medicine for cats and small dogs. Doses.- 
Pigs, grs. v. to grs. xv. ; dogs, gr. $\frac{5}{6}$ to grs. iii. ; small dogs and cats, gr. $\frac{1}{3}$ to gr. $\frac{5}{6}$.

Santoninoxim is derived from santonin, for which it is sometimes substituted, as larger doses may be given without much risk of poisoning. It is said to kill the worms outright.

Oil of chenopodium, a volatile oil got from chenopodium ambrosioides, the American worm-seed, is a very useful agent for destroying round worms (ascarides) in dogs. The dose is $m_{j}$. to $m_{v}$., given either on sugar, or in an emulsion with castor oil.

\section{STAVESACRE SEEDS}

Staphisagrias Semina. The dried ripe seeds of Delphinium staphisagria (B.P.). Nat. Ord.-Ranunculaceæ.

Stavesacre, or larkspur, is a stout biennial herb, two to four feet high, growing throughout the south of Europe. Its officinal oily seeds are brown, wrinkled, irregularly triangular, about a quarter of an inch long and scarcely so broad, and have a bitter, acrid, and nauseous taste. They contain about one per cent. of several alkaloids, soluble in ether and acetic acid, the most important being delphinine, which resembles aconitine and veratrine, slows the pulse and respiration, and paralyses the spinal cord; and staphisagrine, resembling curare, paralysing the motor nerves and arresting respiration.

ACTIONS AND USES.- The seeds are used for the destruction of lice, and hence have been popularly termed louse seeds. Their action is also exerted on the acari of mange and scab. For such purposes one part of bruised seeds is boiled for two hours with twenty to thirty parts of water, making up the water to the quantity originally used. Such a solution rubbed into the skin not only kills pediculi, but also destroys their eggs. Ointments and liniments are made with one part of powdered seeds heated with six or eight of vaseline or oil. Strong preparations too freely applied, absorbed from denuded surfaces or licked, as they are apt to be by dogs, are liable to nauseate and prostrate. Occasionally they are conjoined with sulphur and tar. 


\section{USSO}

Kousso. Brayera. The dried pannicles of pistillate flowers of Brayera anthelmintica (B.P.) Nat. Ord.Rosaceæ.

Kousso consists of bundles, rolls, or clusters of pannicles of small reddish-brown flowers. Its active principle is koussotoxin - a neutral body, with a bitter taste, insoluble in water, but soluble in alcohol and alkaline solutions. This substance is allied to the active principle of male fern, and its pharmacological action is somewhat similar. It also contains resin, gum, tannic acid, and a volatile oil.

Actions AND UsES.- It narcotises and kills intestinal worms, and in fasting dogs, two consecutive doses, given with an interval of two hours, and followed by a purgative, bring away tape-worms. Although it scarcely causes catharsis, full doses nauseate dogs and cats, and sometimes excite colic. Doses-sheep, 3jv. to そj.; lambs, grs. lxxx. to grs. cl. ; dogs and cats, according to their size, take grs. $x$. to 3iij., usually given in infusion, most effectually used unstrained, sweetened with honey or treacle, and the taste veiled by a little peppermint water.

\section{MALE FERN}

Filix Mas. The rhizome of Aspidium filix-mas. Collected late in the autumn, divested of its roots, leaves, and dead portions, and carefully dried. Should not be kept more than a year (B.P.). Nat. Ord.-Filicinæ.

The male fern grows wild throughout most temperate regions, on the sides of roads and in open woods, especially where the soil is light. Its root stock is perennial, about a foot long and two inches thick ; is scaly, tufted, greenishbrown, and firmly fixed in the ground by numerous black root fibres. The dried root has a disagreeable odour, and a sweet, astringent, nauseous taste. Besides the usual plant constituents, it contains about 4 per cent. of resin, 6 of a green fixed oil, a small amount of volatile oil, with 8 per cent. of the crystalline filicic acid $\left(\mathrm{C}_{14} \mathrm{H}_{18} \mathrm{O}_{5}\right)$, which is one of its active constituents. There are also several neutral and 
acid bodies, of which aspidin is the chief, and these may be the important therapeutic and toxic agents. The root is preserved in stoppered bottles, and the supply renewed annually. Deterioration from keeping, and the substitution of the roots of inactive ferns, in great part explain the depreciatory accounts sometimes given of its efficacy. The green parts are most active, and should alone be used.

ACTIONS AND USES.-Male fern is irritant, vermicide, laxative, and in large doses causes hæmorrhagic gastroenteritis, together with nervous symptoms, drowsiness, and sometimes convulsions, coma, and collapse. In some cases blindness, temporary or permanent, has been caused by large doses. It is one of the most effectual remedies for tape-worm, especially in dogs, and Kuchenmeister considered it quite as poisonous to the genus Bothriocephalus. Doses quite sufficient for this purpose have, as a rule, no other effect, being too small to cause irritation. Harley believed that, like ergot, it stimulates the involuntary muscular fibres of any hollow viscus in which it is placed, and thus explained the vomiting and intestinal peristalsis which full doses produce when swallowed, and the contractions induced when it is injected into the urinary bladder. Fröhner made various experiments with the ethereal extract. He poisoned a small dog with $\mathrm{m}_{\mathrm{xxx}}$, a dog of 40 lbs. with $\mathrm{f} 3 \mathrm{v}$., a sheep of $88 \mathrm{lbs}$. with $\mathrm{f} 3 \mathrm{vi}$., a cow of $660 \mathrm{lbs}$. with about $\mathrm{f}$ 弓iij.

Doses, etc.-The powdered male fern rhizome is given to horses and cattle in doses of そiv. to そvi.; sheep, そj. to そiv.; pigs, 3 iij. to $ろ \mathrm{j}$. ; dogs, $3 \mathrm{ij}$. to $3 \mathrm{jv}$.; and cats, grs. $x x x$. to $3 \mathrm{j}$. But the powder is inconveniently bulky, and less certain than the B.P. ethereal or liquid extract. The dose of the extract for horses and cattle is $\mathrm{f} 3 \mathrm{ij}$. to $\mathrm{f} 3$ vi. ; for sheep and pigs, f3i. to f 3 iij. ; for dogs, $m_{\text {viii. }}$ to $\mathrm{mlx}_{\mathrm{x}}$; and cats, $m_{\mathrm{iv}}$. to $m_{\mathrm{x}}$. It is given sometimes with half a dose of turpentine or calomel, in a little oil, milk, or gruel, flavoured with ginger or peppermint, when the bowels have been emptied by a laxative and several hours' fasting. A dose of the extract, with half a dose of arecanut, constitutes the most effectual remedy for tape-worm in dogs. If the parasite is not expelled, the medicine 
may be repeated in three days. Kaufmann recommends doses to be given in the morning, at noon, and at night. The worms narcotised, relinquish their hold, and are swept out by a laxative, administered a few hours after the last dose of extract.

\section{K A M A L A}

A powder consisting of the minute glands and hairs obtained from the surface of the fruits of Mallotus philippinensis. Nat. Ord.-Euphorbiaceæ. (Not official.)

The granular, brick-red, resinous powder, which constitutes kamala, is obtained from the capsules of an evergreen small tree indigenous to Australia, India, and Abyssinia. It yields an active yellow crystalline substance-rottlerin.

ACTIONS AND USES. - It is a drastic purgative and vermicide. For the destruction of tape-worm it is nearly as active as areca-nut and male shield fern. The dose for a dog is 3ss. to 3i., administered in thick gruel, treacle, or linseed oil, or an alcoholic tincture may be used.

\section{YOHIMBINE}

YohImbine, or ApHrodine $\left(\mathrm{C}_{22} \mathrm{H}_{28} \mathrm{~N}_{2} \mathrm{O}_{3}\right)$, an alkaloid obtained by Spiegel from the bark of the Yohimbehe tree (Corynanthe yohimbi), growing in the Cameroons, and belonging to the natural order, Rubiacece.

Yohimbine occurs in prismatic needles, or as a white amorphous powder, nearly insoluble in water; soluble in alcohol, ether, chloroform, and acids. Exposed to air and light it gradually alters and assumes a yellow tint. It is generally prescribed as the hydrochloride, which keeps well.

ACTIONS AND UsES.-In properly regulated doses yohimbine is a powerful aphrodisiac ; it stimulates the erection centre in the spinal cord, and causes marked congestion of the genital organs. Large doses appear to have no special influence on the sexual system, but they produce a state of general excitement, with restlessness, irregular respiration, fall in blood-pressure, increase of pulse rate, and more or less cardiac depression. Applied to the eye, a one per cent. solution of the hydrochloride induces anæs- 
thesia, lasting for an hour or more, without mydriasis, or alteration of the corneal epithelium. Yohimbine is chiefly used as a remedy for functional impotence and sterility. It appears to be eminently adapted as a means of reviving the sexual powers of apparently healthy animals which have become barren or impotent from prolonged continence or some obscure cause. It has also been employed to arrest obstinate vomiting in dogs, and in the treatment of spinal paralysis in young horses. Holterbach, who first prescribed it in veterinary practice, records the case of a bull which for nine months had refused to cover. Various drugs having been tried without benefit, the bull was given yohimbine in his drinking water for ten days. He then covered twice consecutively, and eleven days later served again quite satisfactorily. Similar treatment of four other bulls, unable or unwilling to cover, was followed by equally good results. Cows, which had ceased to exhibit symptoms of œstrum, and an impotent bull, were successfully treated by the administration of gr. $1 \frac{1}{2}$ to grs. 3 per day. Subcutaneous injections of gr. $\frac{5}{6}$ yohimbine, prescribed by Kogan for a stallion which had been rendered impotent by injury from a falling beam, rapidly restored sexual power. Excellent results have been obtained by Paarl, Simon, Haas, Manuel, and others in stallions, bulls, and cows; by Sewell, Paul, and Cowan in bitches; and by Holterbach in the sow. Yohimbine is non-cumulative, and, unlike cantharides, it does not irritate the urinary organs ; but as dogs have been poisoned with half a grain, care should be exercised in prescribing this drug. The symptoms of poisoning are dyspnœa, depression of the heart, salivation, diarrhœa, falling temperature, partial paralysis, and convulsions.

Doses, etc.-Stallions, gr. $\frac{5}{6}$; bulls, gr. $1 \frac{1}{4}$; cows, gr. $1 \frac{1}{2}$; sheep, gr. $\frac{1}{6}$; small dogs, gr. $\frac{1}{400}$ to gr. $\frac{1}{250} ; \operatorname{dogs}$, weighing 20 to $50 \mathrm{lbs}$, gr. $\frac{1}{60}$; dogs over $50 \mathrm{lbs}$., gr. $\frac{1}{2}$; in food, drinking water, or in bolus or pill. These doses may be repeated three times a day. Tablets containing gr. $\frac{1}{64}$, gr. $\frac{1}{6}$, and gr. $1 \frac{2}{3}$ yohimbine for veterinary patients are now obtainable.

DAmianA.-The liquid extract of damiana prepared 
from the leaves of Turnera aphrodisiaca, growing in California and Mexico, has been employed in the United States as a remedy for functional sterility, and as a tonic and diuretic. The compound damiana mixture (Willows, Francis, Butler and Thompson), containing the liquid extract, with phosphorus and nux vomica, is a useful tonic and aphrodisiac for horses in doses of $\xi \mathrm{j}$., given twice a day for a week, then suspended for five or six days, and repeated if necessary.

\section{E R G O T}

Ergota. Spurred or Horned Rye. Ergot of Rye. The sclerotium of Claviceps purpurea, originating in the ovary of Secale cereale (B.P.).

Ergot attacks not only rye but the other Graminaceæ, the Cyperaceæ, and palms. The earliest symptoms occur about the time of blooming, when the ears of the rye exhibit drops of yellow, sweet, fungous slime, called honey-dew, which attracts ants and beetles, and which after a few days dries up. The soft ovaries of the grains attacked are meanwhile covered and filled by white, spongy, felted-together cellsthe mycelium (or spawn) of the Claviceps purpurea. The grain is disintegrated; at its base the mycelium cells separate, swell, solidify, and form a compact, dark violet body, which, as it grows in a curved, horn-like shape, protrudes from the pales, and constitutes the ergot. The further history of this biennial fungus, investigated by Tulasne, shows that it reaches its fully-developed sclerotium or ergot state in July ; it should be gathered in August or September, before any putrefaction appears; it usually remains in a quiescent state during winter; on moist soil, in March or April, it produces fruit heads of the perfect fungus, the Claviceps purpurea, which, after a few weeks, is again ready to distribute its earlier spores. Close, damp weather and undrained soils favour development and distribution of these ergot spores as of other fungi. The injury done to the rye crop by ergot varies much; sometimes only a few grains in each head are diseased, sometimes scarcely one is altogether sound; five to ten on an average are affected. 
It abounds both in grain and grasses in various parts of the United States of America, where it is stated that as much as $1 \mathrm{lb}$. has been got from $100 \mathrm{lbs}$. of hay. It should be collected before the plants are cut.

Properties.-Ergot of rye is cylindrical, or somewhat triangular, curved, resembling a cock's spur tapering towards the ends; it varies in length from one-third of an inch to an inch and a half, and in breadth from one to four lines; is marked by a longitudinal furrow on each side, often irregularly cracked; has at one end a pale-grey, fragile excrescence, the shrivelled remains of the style, and is covered by the grey, powdery conidia or spores. It is dark violet-black externally and pinkish-white within. Its odour is peculiar and disagreeable; its taste, at first sweet, becomes bitter and slightly acrid. When dry it is inflammable, hard, and brittle; when moist, or long exposed, it becomes soft, darker in colour, and covered with acari. Its structure is made up of felted thread-like cells, amidst which lie drops of oil. Ergot should be free from mustiness ; it deteriorates by keeping, and by exposure to damp (B.P.). Infused in boiling water, it forms a claret-coloured solution, retaining the odour, taste, and actions of ergot.

Ergot consists of about 30 per cent. of a non-drying fixed oil, which has no special action; a peculiar sugar termed mycose ; lactic, acetic, and formic acids ; colouring matters ; and, according to Kobert, of Strasburg, three active principles-an alkaloid, cornutine, and sphacelinic and ergotinic acids. Another base, ergotinin, has been described, but is inert. Amine and ammonia bases are formed normally, and are also produced by decomposition. Jacoby has stated that the action of sphacelinic acid is due to a resinous substance, sphacelotoxin.

ACTIONS AND USES.-Ergot, cornutine, and sphacelinic acid stimulate and contract involuntary muscular fibre, and hence diminish the blood-stream passing through the arterioles. Large or continued doses thus produce ergotism. Medicinal doses are given to contract the uterus, and also the blood-vessels in cases of hæmorrhage.

General Actions.-The physiological effects of the three active principles of ergot may be thus described :- 
Cornutine causes spastic rigidity in frogs, lasting many days, even when given in very minute doses $\left(\frac{1}{32}\right.$ of a milligramme). In warm-blooded animals half a milligramme causes salivation, vomiting, diarrhœa, and active movements of the uterus, which are clonic and not tonic. The vessels are contracted and blood-pressure raised. These clonic convulsions, and the other symptoms probably, are due to stimulation of the medulla oblongata and basal ganglia of the brain. With large doses the stimulation passes into paralysis, and death results from paralysis of respiration.

Sphacelinic acid or Sphacelotoxin especially acts on unstriped muscle through the medium of the sympathetic nerves, increasing its tonus and contraction throughout the body. There is marked contraction of the bloodvessels and a rise of blood-pressure, and in cases of chronic poisoning there is dry gangrene of the extremities. As a result of the insufficient blood supply, a hyaline infiltration of the walls and lumen of the contracted vessels occurs, and this makes the constriction permanent, and so leads to gangrene. This gangrene is especially well seen in fowls, and to a less extent in pigs. In all animals there may be salivation, nausea, vomiting, and purgation, and on postmortem, signs of gastric irritation with blood extravasation. Cornutine and sphacelinic acid are evidently the principles which cause uterine contraction (Kobert).

Ergotinic acid is a saponin, and so is destroyed by digestion, and has little effect except that of a gastric irritant, when given by the mouth. When injected subcutaneously it causes ascending paralysis of the spinal cord and brain both in frogs and mammals, with loss of voluntary motion, paralysis of the vaso-motor centre, and fall of bloodpressure, while respiration and reflex irritability continue. It does not appear to have the power of increasing uterine contractions, and hence cannot be regarded as the most important constituent of ergot.

The actions of ergot are the combined effects of these three agents. It is quickly absorbed, and in the blood soon exerts its specific action. All unstriped muscular fibre is contracted ; the calibre of blood-vessels is hence dimin- 
ished, as may be readily seen in the web of the frog's foot; the iris is contracted; intestinal peristalsis is increased; the urinary bladder is emptied. With moderate doses, the normal uterine contractions are strengthened and improved in tone, but with large doses the contractions of the uterus are continuous and tetanic, are usually produced in fifteen or twenty minutes, and last about an hour. They may cause expulsion of the contents of the pregnant uterus. They result mainly from general contraction of unstriped muscular fibre, but are also believed to be in part determined by stimulation of the uterine centre in the spinal cord. The action of the heart is weakened, and as a rule the pulse rate is slowed. Blood-pressure, after a temporary fall, is raised. Respiration is little affected except in the depression due to toxic doses.

Ergot, given experimentally in large or continued doses, or the protracted use of ergoted grain, causes ergotism, which is characterised by gastro-intestinal derangement, nausea, diarrhœa and vomiting in animals capable of emesis, and from the impaired circulation and nutrition affecting different areas, subsequently assumes two forms(1) dry gangrene, ehiefly involving the extremities, ears, and tail ; (2) inco-ordinate spasms, and sometimes epileptiform convulsions, with contractures of various limb or trunk muscles as sequelæ.

The fresh extract, injected into animals, causes incoordination, cutaneous anæmia, anæsthesia, and paralysis, and in large doses death due to paralysis of respiration. The voluntary muscles are unaffected; the motor nerves are not paralysed, but, on the contrary, have their power somewhat increased; the sensory nerves are paralysed; but it is uncertain whether this action is central or peripheral. The spinal cord is paralysed (Brunton).

Ergot of rye, resembles maize ergot-a fungus occurring on Indian corn, and probably containing the same active principles as ergot. The physiological antagonists of ergot are nitrites.

Toxic EFFEcTs are not so marked on horses, cattle, sheep, and rabbits as on men and dogs. Thirty cows amongst them took daily with impunity $37 \mathrm{lbs}$. for three months; 
two milk cows had between them 9 lbs. daily, with no further evil effect than that the butter was badly tasted. Twenty sheep amongst them ate daily for four weeks $9 \mathrm{lbs}$. without injury (Phœbus and Pereira). Dogs receiving six to twelve drachms suffered from vomiting, tenesmus, prostration of muscular power, enfeebled pulse, convulsive twitchings, spasms, and coma (Tabourin). Three ounces proved fatal to a terrier bitch in twenty hours.

Chronic poisoning occurs especially in patients placed in unfavourable sanitary surroundings. Wright found that ergot, given for several weeks to dogs and rabbits, caused nausea, impaired appetite, a weak, irregular pulse, soon becoming intermittent, diarrhœa; excessive fœtor of the secretions and excretions, paralysis, particularly of the hind extremities, enlargement of the liver, contraction of the spleen, impairment of the special senses, wasting, and general debility. Gangrene of the extremities is not, however, produced so readily as in man. Dogs, cats, and rabbits showed great aversion to the drug, even when it was mixed with sound grain, or considerably diluted with water; and, although pressed by hunger, would scarcely eat it of their own accord. Ergot of maize, according to Roulin, is common in Columbia, and its continued use is stated to cause shedding of the hair, and even of the teeth, both of man and beast. Mules freely fed on it lose their hoofs, and fowls lay eggs without shells.

Abortion attributed to ergoted grasses occurs amongst cows, ewes, and deer in many grass districts of England and Ireland, especially in wet seasons. The hay from pastures subject to ergot is seldom, however, so injurious as the grass, for it is generally cut before the fungus is matured. Cows abort from this cause more frequently than ewes or deer ; for they are more prone to eat the coarser, longer ergoted grasses, and, moreover, are often pregnant in the later months of summer, when ergot occurs. Experimentally, abortion has been produced in guinea-pigs, sows, bitches, cats, cows, and ewes, rabbits, and poultry (Stillé). The negative results obtained by some experimenters may be explained by their having used ergot which had been too long kept. 
Medicinal Uses.-As a parturient, ergot is seldom needed in the lower animals. The foal or calf, coming naturally at the full period, if assistance is requisite, is generally brought away by judicious traction. It is sometimes useful in uterine inertia, where the throes are languid and occurring at long intervals, where the animal has been in labour for some considerable time, where no obstruction is present, and where the os uteri is considerably dilated. It is unsuitable where there is malformation either of the mother or fœtus, where the position of the fœtus prevents its ready expulsion, and sometimes also in first pregnancies, where the uterus, roused to continuous tetanic contractions, is more liable to be injured or torn. After parturition, if the uterus remain flaccid, and especially if hæmorrhage occur, as occasionally happens both in cows and ewes, ergot effectually contracts the organ, and thus arrests the bleeding. In such cases it may be given by the mouth, or, where prompt effects are sought, it is injected subcutaneously, or into the substance of a muscle. It is sometimes prescribed to remove uterine cysts and hasten expulsion of the fotal membranes, which in the lower animals may usually, however, be readily removed by the hand. Given either by the mouth or injection, it is often used in hæmoptysis, and sometimes in hæmatemesis and other hæmorrhages, but it is doubtful whether any beneficial result occurs in the first of these. The pulmonary vessels are quite independent of vaso-motor control ; are not constricted by ergot; but are actually dilated by the general rise of blood-pressure. It is of no avail in purpura. Robertson recommended it in cerebrospinal meningitis in horses ; and several practitioners have tried it, but without much success, in parturient apoplexy in cows. The reduction of fibroid and other tumours has sometimes been effected by injecting them with ergot.

In addition to the powdered drug the following official preparations are used:-Extractum Ergotæ (Ergotin); Extractum Ergotæ Liquidum ; Infusum Ergotæ (1 to 20 of boiling water); Tinctura Ergotæ Ammoniata; and Injectio Ergotæ Hypodermica (made with ergot-extract). It must be noted that the active constituents of ergot are very unstable, and hence many preparations and samples 
of the drug are quite inactive. Proper physiological standardisation is necessary, and without this there is no certainty of action.

DosES, etc.-Ergot freshly powdered, as an ecbolic for the mare or cow, 3 ss. to $\zeta \mathrm{ij}$. ; for sheep about $3 \mathrm{ij}$. ; for swine and bitches 3 ss. to $3 \mathrm{j}$. ; Extract of Ergot (Ergotin), horses and cattle, grs. $\mathrm{xxx}$. to grs. lxxx. ; dogs, grs. v. to grs. x. ; in drench or electuary, and repeated as may be required. The liquid extract may be prescribed for horses and cattle in doses of $\mathrm{mlx}$. to $3 \mathrm{ij}$.; for dogs, $m_{x}$. to $m_{\mathrm{xxx}}$. Of the tincture horses and cattle may be given $\zeta_{\mathrm{iv}}$. to $\xi_{\mathrm{j}}$. or more. The B.P. hypodermic injection of ergot contains 33 grains of the extract in $\mathbf{1 1 0}$ minims. For the mare or cow the dose is $m 60$ to $m 100$ injected subcutaneously or into the substance of the gluteal muscles. The smaller dose should first be tried.

\section{S A VIN}

Sabina Cacumina. Fresh and dried tops of Juniperus sabina, collected in spring from plants cultivated in Britain. Nat. Ord.-Coniferæ. (U.S.P.) Not official in B.P.

Juniperus sabina is an evergreen shrub, common throughout Middle and Southern Europe, and cultivated in this country. The tops of young branches, with their attached leaves, when fresh are green, but become yellow when kept; have a strong, heavy, disagreeable odour, and a bitter, acrid, resinous taste. They communicate their properties to water, spirit, and the fixed oils, and owe their activity to about three per cent. of a colourless or pale yellow volatile oil, prepared from the fresh tops by distillation, isomeric with oil of turpentine $\left(\mathrm{C}_{10} \mathrm{H}_{16}\right)$, and associated, as constantly occurs in plants, with a more oxidised oil $\left(\mathrm{C}_{10} \mathrm{H}_{16} \mathrm{O}\right)$. From the berries ten per cent. of these oils is said to be obtained (Phillips).

ACTIONS AND USES.-Savin is a topical irritant, antiseptic, rubefacient and vesicant. Administered internally, moderate doses are anthelmintic and diuretic; they increase appetite, promote digestion, and stimulate the urino-genital 
organs. Large doses produce gastro-enteritis. Excretion occurs, to a slight extent, by the skin and pulmonary membrane, but especially by the kidneys. Savin resembles the turpentine yielding coniferæ, and especially the other junipers.

Toxic EFFECTs.-Hertwig gave horses half a pound twice daily for six or eight days without effect. Rose records the poisoning of five horses, of which one died immediately, and two after five days; the others recovered, after suffering from diarrhœa, intense thirst, quickened pulse and breathing, with great prostration. Two drachms kill rabbits in a few hours, producing extreme congestion of the intestines, kidneys, and bladder. Orfila records that four drachms destroyed dogs in thirteen hours, when the gullet was tied to prevent vomiting; and similar effects followed when powdered savin was applied to a wound or introduced under the skin. Vomiting, purging, gastro-intestinal inflammation, and collapse were produced. The kidneys and bladder were irritated, usually causing copious discharge of bloody urine.

The uterus is also irritated, and savin has been ignorantly used to produce abortion and hasten parturition. Two cases of abortion in mares heavy in foal have been recorded. In these cases the continued use of savin destroyed both foals, and, being still persevered with, caused their expulsion apparently ten or twelve days later.

Medicinal Uses.-Savin cannot be safely used to produce abortion or hasten parturition. Unlike ergot, it does not directly contract the muscular fibres of the uterus. It stimulates the uterus, and expels its contents only as a result of irritation of the intestines and urinary organs. It is occasionally used chopped with fodder for the destruction of intestinal worms ; but other remedies are safer and more certain. If used at all, the best form is the essential oil. Infusions of the tops in an alkaline ley, and the essential oil, are occasionally applied as antiseptics and stimulants to warts and indolent wounds.

Doses, etc.-Of the volatile oil as an anthelmintic-the only purpose for which savin is administered-horses or cattle, $\mathrm{f}$ Ziij. to $\mathrm{f} 3 \mathrm{iv}$. ; dogs, $\mathrm{m}_{\text {iii. to }} \mathrm{m}_{\mathrm{v}}$. dissolved in any 
mild fixed oil or in mucilage. For external application, infusions and ointments are used. Equal parts of savin and verdigris ointments form a popular stimulant dressing for foot-rot in sheep. An infusion, of one of savin to one hundred of water, is sometimes injected into the uterus to promote expulsion of retained fœtal membranes.

\section{COLCHICUM}

Colchici Cormus. Colchicum Corm. The fresh corm of Colchicum autumnale. Meadow Saffron. Collected in early summer; and the same, stripped of its coats, sliced transversely, and dried at a temperature not exceeding $150^{\circ}$ Fahr.

Colchicr Semina. The dried, ripe seeds of C. autumnale (B.P.). Nat. Ord.-Colchicaceæ.

The meadow saffron grows wild throughout Middle and Southern Europe, and on English lawns and coarse, wet pastures, in mild, moist localities, and is cultivated in gardens. It has an annual stem; lilac or purple flowers, numerous round, red-brown, bitter, acrid seeds about the size of millet; and a bulbous root, which, when about a year old, reaches the size of a walnut, and matures in July.

The corms are used both fresh and dried. Dried slices are kidney-shaped, about one and a half inch long, and an inch broad, are greyish-white, dry, firm, and starchy, with a bitter, acrid taste. They yield their active principles to alcohol and vinegar. They contain about 70 per cent. of water, and 18 of starch and gum, with $\frac{1}{10}$ th of 1 per cent. of a bitter, crystallisable, poisonous alkaloid, colchicine $\left(\mathrm{C}_{17} \mathrm{H}_{19} \mathrm{NO}_{5}\right)$. It is conjoined with gallic acid, is present in other parts of the plant, and is nearly a hundred times more active than the fresh bulb. Soluble in water and alcohol, slightly soluble in glycerin; with acids, it forms crystalline salts.

Actions and Uses.-Colchicum irritates most textures with which it comes into contact. Large doses are gastrointestinal irritants and nervous depressants. Medicinal 
doses are emetic, cathartic, and cholagogue. Its diuretic and diaphoretic actions are uncertain.

Toxic EFFECTs.-The corm, whether used green or dry, the seed, any active preparation, and still more notably colchicine, are in-contact irritants. Owing to the active principle being slightly soluble they have little action on the sound skin. When swallowed, a sense of acridity is produced in the mouth and throat, and the flow of saliva is increased. Passing into the stomach and bowels they cause colic, tenesmus, and diarrhœa, and in carnivora nausea and vomiting. Absorption is slow, and the effect on the central nervous system is not seen for from one to several hours. This action consists in depression, with all the signs of collapse. The circulation and respiration are only affected as part of the general depression. Movement is lessened, and paralysis, commencing in the hind limbs, extends forwards. There is some amount of anæsthesia of the skin in the later stages from paralysis of sensory nerves. Death occurs from paralysis of respiration.

A cow and heifer ate some cut grass containing a considerable amount of meadow saffron. In a few hours they had violent colic, profuse and bloody diarrhœa, tenderness of the abdomen, coldness of the surface, and prostration. The cow recovered; the heifer died from irritation and exhaustion in three days. A number of cows ate small quantities of colchicum, suffered from colic and diarrhœa, but recovered when treated with emollient drenches and mild saline mixtures. Three cattle having eaten colchicum were reported to have suffered from dulness, stupor, grinding of the teeth, dilated pupils, imperceptible pulse, relaxed bowels, cold extremities, and thirst, but no griping pains, or quickened breathing. They were successfully treated by laxatives and stimulants.

Dogs and eats are more susceptible than horses or ruminants. Two drachms of the dried bulb caused in dogs vomiting, bloody evacuations, diuresis, tremors of the limbs, depression of the heart action, and death in five hours. A tenth of a grain of colchicine given to a cat occasioned salivation, vomiting, purging, staggering, extreme languor, colic, and death in twelve hours. Rabbits, as well as frogs 
and other cold-blooded animals, are stated to be less susceptible to the drug, but are very susceptible to an oxidation product, oxydicolchicine. It appears probable that colchicine is oxidised in the blood and tissues of mammals, and then produces its effects. In the frog, oxydicolchicine causes prolongation of muscular contractions in a similar way to veratrine.

As antidotes the stomach must be emptied; full doses of tannin form an insoluble compound with the colchicine; white of egg, oil, or mucilage should be freely given, and stimulants if required.

Medicinal Uses.-Colchicum has little to recommend it as a therapeutic agent. The fresh corm given in large doses to fasting dogs, and its expulsion by vomiting prevented, increased secretion of bile, and also purged powerfully. But action on the liver and gastro-intestinal membrane is more safely effected by other medicines. Small doses, conjoined with alkalies or salines, are occasionally given to horses in rheumatism and influenza, especially in subacute cases in which the inflammation appears to move from joint to joint. Foreign authorities prescribe it in dropsy, hoven, and rheumatism. Williams used it, conjoined with potassium iodide, in pleurisy, in rheumatic pericarditis, and sometimes in pneumonia when the kidneys were torpid. It is excreted in great part by the kidneys, and when not quickly removed by the bowels it increases, alike in health and disease, both the organic and inorganic constituents of the urine.

DosES, etc.-Of the powdered corm or seed as a diuretic for horses, 3 ss. to $3 \mathbf{j}$. ; for cattle, $3 \mathrm{j}$. to $3 \mathrm{ij}$. ; for sheep, grs. $\mathbf{x}$. to grs. xxv. ; for dogs and pigs, gr. i. to grs. vi., given with salines. A convenient solution is made with one part of colchicum, six or eight of vinegar, and a little spirit. Colchicine dissolved in 100 parts of water and glycerin may be given hypodermically or intratracheally in doses of gr. $\frac{1}{3}$ to grs. ij. to horses, and gr. $\frac{1}{120}$ to $\frac{1}{60}$ to dogs. The tincture is made with four ounces of colchicum seeds to the pint of alcohol (45 per cent.). 


\section{A M PHOR}

Camphora. A white crystalline substance obtained from Cinnamomum camphora, purified by sublimation (B.P.). Nat. Ord.-Laurineæ.

The camphor laurel is a tall, handsome evergreen, cultivated in Japan and China, and in many European conservatories. Its wood and leaves evolve a characteristic odour when bruised, and yield about $\frac{1}{500}$ th of their weight of camphor, which is sometimes extracted by dry distillation.

Properties.-Camphor occurs in solid, colourless, transparent masses, of tough consistence. It has a bitter, pungent, cooling taste, and a characteristic, aromatic odour. It floats on water, its specific gravity, varying with the temperature, is about 0.995 . Exposed to the air, it volatilises; heated, it sublimes without residue, and burns readily with a bright but smoky flame. It is difficult to powder, unless when mixed with alcohol, ether, or chloroform. It dissolves readily in ether, in about its own weight of rectified spirit, in one-fourth part of chloroform, four parts olive oil, two parts oil of turpentine, eight times its weight of milk, and in 700 times its weight of water. Camphor $\left(\mathrm{C}_{10} \mathrm{H}_{16} \mathrm{O}\right)$ is a ketone of terpene $\left(\mathrm{C}_{10} \mathrm{H}_{16}\right)$-the chief constituent of oil of turpentine, chamomile, cardamoms, cloves, hops, juniper, savin, and valerian. Most of these volatile oils contain a small amount of a crystalline substance or stearoptine which is deposited on standing. The chief of these bodies is camphor. Continuously heated with nitric acid, camphor oxidises, and is converted into camphoric acid $\left(\mathrm{C}_{10} \mathrm{H}_{16} \mathrm{O}_{4}\right)$. Triturated with chloral hydrate, menthol, phenol, or thymol, it forms a liquid.

Borneo camphor $\left(\mathrm{C}_{10} \mathrm{H}_{18} \mathrm{O}\right)$ is an alcohol derived from the wood of Dryobalanops aromatica, and is distinguished from laurel camphor by its softness, friability, and opacity, its higher density, and its somewhat alliaceous odour. From Borneo, Formosa, and other parts of China, fluid camphor oils are obtained from several different trees. Artificial camphor $\left(\mathrm{C}_{10} \mathrm{H}_{16} \mathrm{HCL}\right)$ is got by the action of hydrochloric 
acid on oil of turpentine. Camphora monobromata $\left(\mathrm{C}_{10} \mathrm{H}_{15}\right.$ $\mathrm{BrO}$ ) resembles bromine rather than camphor in its actions, is sometimes used instead of the bromides, but is not so efficient.

Actions AND USES.-The camphors in large doses are irritant and narcotic. Medicinal doses are antiseptic, stimulant, expectorant, anodyne, and diaphoretic. Externally, they are occasionally employed as antiseptics, parasiticides, and to relieve itching in various skin diseases.

General and Toxic EFFECTs.-The camphors, physiologically, are volatile oils. Like other bodies of the group they are topical irritants, and large doses stimulate and subsequently narcotise the central nervous system, especially the cerebral cortex with its motor areas, and similarly, but to a much less extent, the spinal cord. They frequently produce convulsions. Those which contain the most hydrogen, as the Borneo, the monobromata, as well as menthol $\left(\mathrm{C}_{10} \mathrm{H}_{20} \mathrm{O}\right)$, are least convulsant. In fine powder or solution they are quickly absorbed ; are oxidised in great part into camphoric acid, and quickly produce their effects on the brain and spinal cord. The effect of medicinal doses on the heart is somewhat uncertain, but it is generally slowed. Respiration is not much affected but may be quickened a little. There is, with larger doses, excitement and hyperæsthesia, and in the dog and cat epileptiform convulsions are easily produced, these effects being due to the cerebral stimulation. Subsequently there is depression, inco-ordination of movement, stupor, and collapse. The local action of camphor on the stomach and intestines is that of a volatile oil, namely carminative, antispasmodic, and antiseptic. The drug is excreted chiefly by the kidneys in combination with glycuronic acid, also to a slight extent by the bronchial mucous membrane and the skin. Moiroud records that two ounces produced in horses convulsive movements and acceleration of the pulse, unaccompanied, however, by fatal results. Hertwig mentions that two to four ounces given to horses and cattle, two to four drachms to sheep, and one to three drachms to dogs, accelerate respiration and pulsation, communicate a camphoraceous odour to the breath, heighten sensibility, and occasionally 
induce convulsions. Dogs, besides, exhibit imperfect power of controlling the movements of their limbs, and when the doses amount to three or four drachms insensibility and death ensue. The vapour of camphor destroys fleas, bugs, moths, and spiders, exciting, enfeebling, and stupefying them. It has considerable antiseptic power. Koch found that one part to 2500 of water hindered development of anthrax bacilli.

MEDICINAL USES.-Camphor is used, especially in young animals, as a gastric stimulant and antiseptic. In diarrhœa it is given with aromatics and a few drops of hydrochloric acid, or with ether and laudanum. Robertson prescribed it with opium in enteritis in horses. Many veterinarians give it freely in catarrhal cases presenting increased secretion of bronchial mucus and dyspnœa, conjoining it with salines, ammonia salts, and belladonna. For chronic bronchitis in horses, it may be prescribed with squill, and in convalescence from catarrhal complaints with gentian, ginger, and iron. For exhausting disease, whether in horses or cattle, a stimulating draught is often made with two drachms each of camphor and ammonium carbonate, and an ounce of ether, given in ale or cold gruel. In equine pneumonia, influenza, or other disease associated with increasing prostration, camphor is of great value. Three to five drachms of 25 per cent. camphorated oil or ether may be injected subcutaneously every three hours. To avoid local irritation, the second, third, and following injections should be made a short distance from the preceding puncture, and not more than 75 minims should be injected at one point. Sorethroat and irritable, spasmodic cough are relieved by placing on the tongue, at intervals of two or three hours, or as required, an electuary made with equal parts of belladonna extract, borax, and camphor, reduced to a paste with ammonium acetate solvition, and mixed with eight or ten parts of honey or treacle. Small doses prescribed with belladonna lessen urino-genital irritability, resulting from cantharides or other causes. It does not, as has been popularly believed, diminish the lacteal secretion.

For dogs, a mixture containing grs. $\nabla$. each of camphor and belladonna extract, with $\mathrm{f} \mathbf{3} \mathrm{j}$. of ammonium acetate 
solution, in two ounces of water, is prescribed to relieve cough and bronchial irritation. Camphor and sweet spirit of nitre are useful in modifying the restlessness and convulsions of chorea.

Externally, it is applied either in oil, or weak spirituous solution, to allay itching in chronic eczema and urticaria. Mixed with oil or vaseline, it is used to destroy skin parasites, and to prevent attacks of flies. It is a constituent of soap, opium, belladonna, chloroform, turpentine, and other liniments.

Aqua Camphoræ is made with 70 grains of camphor dissolved in four drachms of alcohol (90 per cent.), and added to one gallon of water.

Camphorated oil for hypodermic use consists of one part of camphor in ten of pure sterilised olive oil.

Compound liniment of Camphor is made with twenty parts camphor, forty strong solution of ammonia, one of oil of lavender, and 120 of rectified spirit.

Spirit of Camphor consists of one part camphor dissolved in nine parts alcohol (90 per cent.).

Doses, etc.-For horses, $3 \mathrm{j}$. to $3 \mathrm{jv}$. ; cattle, $3 \mathrm{ij}$. to $3 \mathrm{vj}$. ; sheep, grs. xxx. to 3ij.; pigs, grs. xv. to grs. xlv. ; dogs, grs. viii. to grs. $x v$. ; and cats, gr. i. to grs. v. When used for anodyne purposes, it is conveniently made into an emulsion with eggs, or dissolved in oil or milk. Subcutaneous injections may be made with camphor one part dissolved in four or ten parts of sterilised olive oil, or ether. Of the stronger solution horses may be given mlxxx. to $\mathrm{m}$ clx.; and of the ten per cent. solution, $m$ clx to $m \operatorname{cccxx}$. For external use, camphor is dissolved in ten parts of alcohol, in diluted acetic acid, rape oil, or oil of turpentine, or mixed with five parts of lard.

\section{SANITAS}

Sanitas occurs in the form of oily and watery fluids, prepared by oxidation of oil of turpentine, and containing camphoraceous bodies and hydrogen peroxide.

A current of air is driven by an engine, for about 120 hours, through a series of Doulton's stoneware receivers, 
surrounded by vats of water, maintained by steam at a temperature of $140^{\circ} \mathrm{Fahr}$. In each receiver are placed thirty gallons of American, Russian, or Swedish oil of turpentine, and about double that amount of water. The oil gradually becomes darker in colour, its specific gravity and boiling point are raised, and it acquires a balsamic odour resembling camphor and peppermint. As the process continues, the turpentine $\left(\mathrm{C}_{10} \mathrm{H}_{16}\right)$ is oxidised, producing camphoric peroxide $\left(\mathrm{C}_{10} \mathrm{H}_{16} \mathrm{O}_{3}\right)$, which is gradually converted into another antiseptic camphoric substance $\left(\mathrm{C}_{10} \mathrm{H}_{16} \mathrm{O}_{2}\right)$, and the soluble hydrogen peroxide which passes into solution in the water.

The several essential oils of the terpene series $\left(\mathrm{C}_{10} \mathrm{H}_{16}\right)$, as well as cymene $\left(\mathrm{C}_{10} \mathrm{H}_{14}\right)$ and menthene $\left(\mathrm{C}_{10} \mathrm{H}_{18}\right)$, undergo similar oxidation, and give rise to the same products. In this way pine forests, especially during sunshine following rain, render the atmosphere not only pleasantly balsamic but antiseptic, more highly oxygenated, and curative for throat and lung complaints. The Eucalyptus globulus in like manner pours forth these antiseptic and highly oxygenated volatile products, which are antidotes to malaria, and sometimes, it is said, even arrest the progress of pulmonary consumption; while, on a smaller scale, every plant or flower producing an essential oil exerts similar oxygenating and purifying effects.

When the manufacture of Sanitas is completed, there floats on the surface of the aqueous solution a yellow-brown dense, oxidised oil of turpentine, consisting chiefly of cam phoric peroxide, which is stated to have an oxidising strength equal to that of a ten volume solution of hydroger peroxide. This Sanitas oil, mixed with a suitable minera or other basis, constitutes a useful disinfecting and deodoran powder. It is introduced into various soaps, conferrin disinfectant properties, and, mixed with vaseline, oils, o fats, forms serviceable antiseptic liniments and ointments Melted with dammar resin and paraffin wax, a mixture $i$ obtained which is used to impregnate muslin, forming a: antiseptic gauze. Disinfectant desiccants are prepared $b$, admixture with chalk or starch. The watery solutior cleared by filtration, and known as Sanitas fluid, consist 
chiefly of thymol, a proportion of the camphoraceous constituents which characterise the oil, and hydrogen peroxide.

Actions AND USES.-Sanitas oil and fluid are volatile, oxidising, non-poisonous antiseptics, disinfectants, and deodorants. Their several constituents in various ways attack and destroy organised ferments, and the lower forms of vegetable and animal life. Sanitas preparations have an agreeable aromatic odour, are not corrosive, and do not stain or injure clothing or other textile fabrics. Their power of checking fermentation has led to the administration of the fluid to calves fed on milk, and suffering from dyspepsia or diarrhœa; an ounce of the fluid is prescribed with six ounces of water, and may be conjoined with spirit, ether, or chloroform.

Useful antiseptic lotions for wounds, ulcers, and bruises are prepared with one part of the fluid, diluted, according to circumstances, with four to ten parts of water. Ointments and liniments are prepared with about the same proportions of oils and fats. When wounds, for ten days or longer, have been treated with carbolic or other irritant dressings, granulation and skin growth often proceed more satisfactorily with the substitution of the milder Sanitas. In sore-throat, catarrh of the sinuses of the head, aphtha, and foot-and-mouth complaint, solutions and sprays are often useful, and being devoid of irritant effects, are also serviceable for rectal, uterine, and vesical injections. Sanitas solutions and soaps not only cleanse and disinfect, but gently stimulate the skin, abate itching, remove scurf, and promote healing in prurigo, chronic eczema, and similar skin complaints. Sanitas fluid, diluted with twenty to fifty parts of tepid water, is serviceable for sponging febrile patients, and for disinfecting animals affected with contagious disease. In canine practice, the fluid diluted with four to six parts of water, is used in canker of the ear, ulceration of the mouth, eczema, and as a uterine injection after parturition. Sanitas oil destroys the parasites of scab and mange, as well as lice, fleas, and maggots, and arrests the cryptogamic growth of ringworm. Even in concentrated form, there is no risk of its exciting undue irritation, or 
inducing from its absorption injurious constitutional effects, such as are apt to follow the free use of strong carbolic preparations.

Sanitas powder and sawdust are used with good effect for disinfecting and deodorising stables, kennels, cowsheds, and piggeries. Sprinkled upon the floors, they also purify the air of slaughter-houses, menageries, manufactories, and exhibitions ; while on shipboard they destroy unpleasant odours, and substitute their own camphoric aroma. These preparations are largely used in hospitals and by medical officers of health.

\section{THYMOL}

ThyмоL-a crystalline substance, obtained from the volatile oils of Thymus vulgaris and Carum copticum. Purified by recrystallisation from alcohol (B.P.).

Thymus vulgaris (Nat. Ord.-Labiatæ) is a bushy evergreen shrub found in dry situations throughout Southern Europe. It derives its aroma from an essential oil separable into two parts-(1) the fluid thymene, which is isomeric with oil of turpentine $\left(\mathrm{C}_{10} \mathrm{H}_{16}\right)$; and (2) the solid thymol.

Thymol $\left(\mathrm{C}_{10} \mathrm{H}_{13} \mathrm{OH}\right)$ occurs in large oblique prisms, with a pungent, aromatic taste. It requires for solution 333 parts of water, 190 of glycerin, or two of olive oil ; and is freely soluble in alcohol, ether, chloroform, and fats. It sinks in cold water, but heated to $110^{\circ}$ to $125^{\circ} \mathrm{Fahr}$., it melts and floats on the surface.

ACTIONS AND UsES.-Thymol is antiseptic, disinfectant, diaphoretic, diuretic, and vermicide. Large doses paralyse the nerve centres of the cord and medulla. It has most of the characteristic properties of a volatile oil. Brunton states that its physiological actions place it between oil of turpentine and carbolic acid.

Solutions of one per cent. destroy bacteria and prevent reproduction of their spores. Applied to the skin or mucous surfaces it causes irritation, followed by anæsthesia. When swallowed it is slowly absorbed. Dogs weighing 20 pounds and rabbits weighing 7 pounds, receiving respectively 60 and 30 grains injected hypodermically, exhibited lowered 
blood-pressure and muscular weakness, paralysis of respiration, and coma; but the fatal effect of full doses was frequently averted by artificial respiration. The respiratory mucous membrane was congested, the lungs were congested and sometimes consolidated, the kidneys inflamed, the urine albuminous, occasionally bloody. In chronic poisoning tissue metabolism appears to be impaired, and there is fatty degeneration of the liver, as in phosphorus poisoning. It is excreted chiefly by the lungs and kidneys, imparting to the urine a green colour by direct, a brown by transmitted, light. Compared with carbolic acid, thymol is not so irritant, caustic, or poisonous ; when absorbed it does not cause preliminary excitement, but from the first paralyses the nerve centres; as an antiseptic it is stated to be more powerful and permanent. Its high price precludes its use as an ordinary antiseptic. Concentrated solutions damage instruments.

MEdicinal UsES.--It has been prescribed in vesical catarrh, and also as an intestinal disinfectant, horses taking

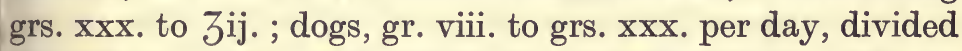
into three doses. As a vermicide in strongylosis of foals, grs. x. to grs. xv., dissolved in glycerin and alcohol, suspended in milk or mucilage, or made into a bolus coated with keratin, are given daily for four or five consecutive days, and followed by a laxative. But its chief use is in antiseptic surgery. Notwithstanding its greater cost, it is sometimes substituted for carbolic, salicylic, and boric acids. For allaying irritation and removing scales in chronic eczema and lichen, 1 to 2 grains are dissolved in an ounce of diluted spirit, or of potassium carbonate solution. For such purposes an ointment is also used, made with 10 to 40 grains to the ounce of vaseline. As a stimulating antiseptic in sore-throat and ozæna, it is used in the form of gargle, spray, or inhalation. It is the active constituent of Volckmann's antiseptic fluid, which, with one part thymol, contains 20 of alcohol, 20 of glycerin, and 960 of water. This solution prevents the development of pyogenic rganisms. 


\section{TOBACCO}

Tabaci Folia. Tobacco leaves. The dried leaves of Nicotiana tabacum. Nat. Ord.-Solanaceæ. (Not official.)

Tobacco derives its name from tabac, the instrument used by the American aborigines for smoking the leaf, from the island of Tobago, or from the town of Tobasco in New Spain. It appears to have been cultivated from time immemorial in America, and is now grown largely in the region watered by the Orinoco, in the United States, and in many temperate and sub-tropical countries of both hemispheres. It was unknown in the Old World - at all events in Europe-until after the discoveries of Columbus ; and was first introduced into England by Sir Francis Drake in 1586.

The Nicotiana tabacum, which yields the Virginian and several commercial tobaccos, is an herbaceous plant, three to six feet in height, with a branching fibrous root, a tall annual stem, funnel-shaped, rose-coloured flowers, and large, moist, clammy, brown leaves, mottled with yellow spots, covered with glandular hairs, and distinguished by a strong, peculiar, narcotic odour, and a nauseous, bitter, acrid taste. The leaves readily communicate their properties to hot water and alcohol. The plant is cut down in August, and the leaves dried, twisted, and carefully packed, with great compression, in hogsheads. For many purposes the midrib is removed, and occasionally the leaf is fermented, in order to remove albuminoids, which, when smoked, give rise to oils and unpleasant products. Sugar and liquorice are sometimes added to impart mellowness and pliability.

Commercial tobaccos contain about 12 per cent. of moisture, 20 to 25 of lignin, and about the same amount of inorganic matters, chiefly salts of potassium and calcium. The chief active principle is nicotine $\left(\mathrm{C}_{10} \mathrm{H}_{14} \mathrm{~N}_{2}\right)$-a colourless, volatile, inflammable, oily alkaloid, with an acrid odour and taste. It occurs in combination with malic and citric acids, constituting 5 to 7 per cent. of the dried leaf. It is soluble in water, alcohol, ether, the fixed and volatile oils. Tobacco also yields, when distilled with water, a crystalline volatile oil-nicotianin, or tobacco camphor- 
produced from oxidation of the nicotine. Tobacco slowly burned, as when smoked, is decomposed, and the smoke contains volatile fatty acids and ethers, traces of hydrocyanic acid and ammonia, while the nicotine in great part is converted into alkaloids of the benzine series-pyridine $\left(\mathrm{C}_{5} \mathrm{H}_{5} \mathrm{~N}\right)$, collidine $\left(\left(\mathrm{CH}_{3}\right) \mathrm{C}_{5} \mathrm{H}_{2} \mathrm{~N}\right)$, picoline $\left(\mathrm{CH}_{3} \mathrm{C}_{5} \mathrm{H}_{4} \mathrm{~N}\right)$, and lutidine $\left(\left(\mathrm{CH}_{3}\right) \mathrm{C}_{5} \mathrm{H}_{3} \mathrm{~N}\right)$.

ACTIONS AND USES.-Tobacco and nicotine are in-contact irritants. They stimulate and then paralyse the spinal cord, the motor nerves of muscles, and the nerve-cells controlling involuntary muscles, and secreting glands. They enfeeble circulation, cause trembling, staggering gait, convulsions, and death from respiratory failure. Tobacco is rarely prescribed internally, but is used externally as an antiparasitic. Nicotine and Nicotianin are antiseptic.

General Actions.-Strong solutions of tobacco are incontact irritants of mucous and denuded skin surfaces. Partly from this topical irritant action and partly from stimulating motor nerves they cause vomiting in carnivora. Large doses in all animals induce gastro-enteritis with collapse. The active principle is quickly taken up from absorbing surfaces. Dogs dressed with concentrated decoctions frequently suffer from salivation, nausea, and vomiting, while human patients have been poisoned by enemata.

Nicotine is somewhat similar in action to conine and lobeline, producing a preliminary stimulation of certain nervecells (especially cells of sympathetic ganglia), and of the central nervous system, followed by depression and paralysis of them. Thus by the stimulation and subsequent depression of vagus ganglion cells, the heart is first slower and even intermittent, then quicker. Similarly, blood-pressure is briefly raised, and then lowered by stimulation and depression of the ganglion cells on the course of the vasoconstrictor nerves. In the same manner the secretion of saliva, sweat, and bronchial mucus is first increased and then lessened. Peristalsis is at first (and briefly) inhibited by stimulation of the peripheral splanchnic ganglion cells, and then is greatly increased and exaggerated by their paralysis. By a combination of local and central action respiration is at first quickened and shallow, later becoming 
somewhat deeper, and then gradually failing both in rhythm and depth, with the central depression.

The action on the brain and spinal cord accounts for the preliminary excitement and hyperæsthesia, the muscular tremors and clonic spasms and then the subsequent paralysis. Death occurs from paralysis of respiration, the heart-beats continuing for some time after breathing has stopped.

TOxic EFFECTS are produced in horses by 9 ounces of tobacco ; in cattle by $1 \mathrm{lb}$; in sheep by 1 ounce; in dogs by 1 to 2 drachms. The poisonous dose of nicotine for horses and cattle is 5 to 6 minims, for dogs 1 to 3 minims. Onetenth part of these doses used hypodermically is dangerous (Fröhner and Kaufmann). Hertwig gave horses half an ounce to an ounce of the powdered leaves, with the effect of lowering the pulse three to ten beats per minute, and rendering it irregular and intermittent; while a repetition of such doses increased evacuation both of fæces and urine. Large doses, especially intravenously injected, accelerated the pulse, increased the action of the bowels and kidneys and caused irritability and restlessness. A healthy middleaged cow received two ounces dissolved in water, in dividec doses, but given within two and a half hours. The tempera. ture of the skin was heightened: the pulse raised from 65 to 70 ; the breathing quickened and somewhat oppressed the pupil dilated, while perspiration was abundant. Nexi day the animal continued dull, but by the third day she was perfectly well. An ox consumed about four pounds 0 : tobacco leaves, and speedily became very restive, grounc his teeth and groaned, lay with outstretched limbs and dis tended rumen, passed quantities of thin fœtid fæces, anc died in eleven hours in convulsions. The leaves wert found in the alimentary canal, and the mucous membrane especially of the fourth stomach, was red and eroded particularly where in contact with the tobacco. Hertwi further mentions that goats are similarly affected by on or two ounces, and generally die in about ten hours.

Orfila administered to a dog five and a half drachm: powdered tobacco (rappee) ensuring its retention by liga ture of the œsophagus. There ensued violent efforts ts vomit, nausea, purging, tremors of the extremities, giddi 
ness, accelerated respiration, quickened pulse, convulsions, stupor interrupted by spasms, and dependent on imperfect oxygenation of the blood, and in nine hours death. A decoction containing half a drachm, injected into the rectum of a dog, produced similar symptoms, but was not fatal. Two and a half drachms, applied to a wound, destroyed a dog in an hour. The pupils are contracted, and in fatal cases are insensible to light. A single drop of nicotine destroys small dogs and rabbits in five minutes, producing convulsions and general paralysis.

Post-mortem discloses appearances of asphyxia ; and in cases where the crude drug has been swallowed, and has not been immediately fatal, the gastro-intestinal tract exhibits evidences of irritation.

The treatment of poisoning, when the crude drug has been swallowed, consists in the use of the stomach-pump or emetics. Tannin renders nicotine insoluble. Keeping the patient warm, and the cautious administration of stimulants, antagonise nausea, depression, and collapse ; while artificial respiration, and the careful hypodermic injection of strychnine or atropine, overcome the tendency to death by asphyxia.

Tobacco is allied to several other motor depressors of the Solanaceæ, notably to dulcamara and belladonna; but it does not produce that peculiar disturbance of the locomotor centres, and consequent irregular movements, which characterise belladonna, while it increases, instead of diminishing, cutaneous and other secretions, and contracts instead of dilating the pupil. It resembles lobelia or Indian tobacco - the dried flowering herb of Lobelia inflata, which is sometimes prescribed for the relief of spasmodic asthma in dogs as well as in human patients. Tobacco is more limited in its paralysant effects than hemlock or prussic acid.

MEdICINAL UsES.-Tobacco is now seldom administered internally. There are many much better emetics than the quid of tobacco sometimes given to the dog, and numerous more effectual remedies for intestinal worms. Tobacco smoke enemas were formerly used to relieve the spasms of colic ; but chloroform, chloral hydrate, opium, and other anodynes are more effectual. A one or two per cent. 
decoction, used as an enema, brings away worms lodged in the rectum. An infusion made with four ounces black tobacco and a pint of boiling water, strained and cooled, has been used successfully as an antidote. in strychnine poisoning.

Externally, it is used to kill the acari of mange and scab, and also lice, fleas, and ticks, but it does not effectually destroy the ova of these parasites. Strong solutions, liberally applied, are apt to cause nausea, trembling, spasms, and sometimes death, but there is no danger in the careful use of decoctions made with thirty or sixty parts of water. For such purposes the leaves are boiled for half an hour with a limited quantity of water, and the decoction diluted as required. For sheep dips and washes two to five per cent. solutions are used, their efficacy being increased by addition of soft soap, potash, tar oils, and occasionally arsenic or corrosive sublimate. Unless, however, the refuse juice of the manufactory can be procured, tobacco is too costly for sheep dips. Law's sheep dip is made with tobacco, $16 \mathrm{lbs}$., oil of tar 3 pints, soda ash 20 lbs., soft soap 4 lbs., water 50 gallons. Macerate the tobacco in three successive portions of water and add the other agents to the fluid.

\section{CATECHU}

Pale Catechu. Catechu pallidum. An extract of the leaves and young shoots of Uncaria gambier (B.P.). Nat. Ord.-Rubiaceæ.

Black or Brown Catechu. Catechu nigrum. The aqueous extract of the wood of Acacia catechu, of Acacia suma, of other Leguminosæ, and of plants of other natural orders. (U.S.P.) Not official in B.P.

The Uncaria gambier, producing the pale catechu (cate, a tree ; chu, juice), is a stout climbing shrub, inhabiting the islands of the Indian Archipelago, and cultivated for its astringent juice. A decoction made of the leaves and young shoots is evaporated, worked into red-brown, earthy-looking masses or cubes, with surfaces about an inch square.

The black or brown catechu, chiefly brought from Bengal 
and Burmah, is derived from several trees, largely from the Acacia catechu, a native of India and Africa. The Acacia suma, a large tree growing in Bengal, Burmah, and Southern India, has a white bark used for tanning, and red heart-wood, from which catechu is also made. The wood of these and of other trees is cut into chips and boiled with water, the decoction concentrated either by fire or the heat of the sun, and the extract cut or moulded into square cakes or masses.

The pale and black catechus are very similar in composition and properties; are porous and opaque; brittle, breaking with a granular fracture; under the microscope exhibit minute, needle-like crystals; are without odour, but have a sweet astringent taste. They are soluble in alcohol and ether, partially soluble in cold water, entirely dissolved by boiling water, with which they form red-brown solutions. They consist of about 40 per cent. of catechutannic acid, which is soluble in cold water ; and of eatechin or eatechuic acid $\left(\mathrm{C}_{13} \mathrm{H}_{12} \mathrm{O}_{5}\right)$, a modification of tannic acid, which deposits in acicular crystals from boiling watery solutions of catechu, and is soluble in alcohol and ether. They further contain the yellow colouring matter quercitin.

ACtions AND Uses.-Catechu is astringent, acting by contact only. It forms insoluble compounds with albumin and gelatin, and, like other tannin-containing substances, is used in making leather. It is less astringent than oak bark or galls, but more astringent than kino, the inspissated juice obtained from incisions made in the trunk of Pterocarpus marsupium; than rhatany, the dried root of Krameria triandra or of K. argentea ; than logwood, the sliced heartwood of Hæmatoxylon campechianum ; or than bearberry or uva-ursi leaves.

Catechu is administered to the several domestic animals for the arrest of chronic catarrhal discharges and hæmorrhage, especially from the throat and alimentary canal. The insoluble catechin beneficially exerts its astringency on the relaxed, over-secreting surfaces alike of the small and large intestines. In persistent diarrhœa and in dysentery it is conjoined with aromatics to allay flatulence; with opium to relieve irritability and spasm; with alkalies, 
magnesia, or chalk, to counteract acidity. A convenient prescription for such cases consists of three ounces each of catechu, prepared chalk, and ginger, and six drachms of opium, made, as is most suitable, into either mass or draught. This will make eight doses for a horse, six for a cow, and eight or ten for a calf or sheep. For the horse the dose is given in bolus ; for the ruminant, suspended in starch gruel. Catechu is occasionally applied to sluggish wounds and ulcers, to excoriations on the udder of cattle, and for the several purposes of a vegetable astringent.

Doses, etc.-For horses, $3 \mathrm{ij}$. to $ろ \mathrm{j}$.; for cattle, $3 \mathrm{iv}$. to そij. ; for sheep and swine, 3 i. to 3 ij. ; and for dogs, grs. v. to grs. 1x. These doses are administered three or four times a day, with sufficient mucilage or gruel to cover their astringent taste. An infusion is readily prepared for veterinary purposes by pouring boiling water over coarselypowdered catechu, digesting by the fire for an hour, and straining. Flavouring ingredients may be added as required. The B.P. orders the tincture to be made with catechu, in coarse powder, four ounces ; cinnamon bark bruised, one ounce; alcohol (60 per cent.), one pint. Compound powder of catechu is composed of catechu, 4 ounces ; kino, 2 ounces ; krameria root, 2 ounces ; cinnamon, 1 ounce ; and nutmeg, 1 ounce. All powdered and mixed. For external purposes the powder, infusion, and an ointment are used.

\section{GALLS}

GALLA. Oak galls. Excrescences on Quercus infectoria resulting from the puncture and deposition of an egg or eggs of Cynips Gallæ tinctoriæ (B.P.). Nat. Ord.Cupuliferæ.

Home-grown galls from the common oak (Quercus robur) are in some seasons abundant throughout the southern and midland counties of England, but seldom contain more than half the tannic acid found in the foreign.

The best commercial variety, known as Levant galls, is imported from Syria, Smyrna, and Constantinople; the light, hollow Chinese, Japanese, or East Indian galls, are 
yielded by the Rhus semialata; large Mecca galls, called Dead Sea apples, are imported from Bussorah.

Galls vary from the size of a bean to that of a hazel-nut, are round, hard, and studded with tubercles; of a bluishgrey colour externally, and yellow within. An inferior variety, from which the larva has escaped, are smoother, of lighter colour, lower density, and less astringency. Galls are easily reduced to a yellow-grey powder, devoid of odour, but having an intensely astringent taste. The active principles are dissolved by forty parts of boiling water and still less of diluted alcohol. Ferric salts, added to a watery solution, slowly precipitate the dark-blue or black iron tannate, the basis of writing ink. An aqueous solution of gelatin throws down a grey flocculent precipitate of tannogelatin. These reactions, and other important properties, depend on the presence of tannin or tannic acid, which, according to the quality of the galls, ranges from 15 to 70 per cent., and is associated with about 3 per cent. of gallic acid.

TANNIC AcID, or tannin $\left(\mathrm{C}_{14} \mathrm{H}_{10} \mathrm{O}_{9} \cdot 2 \mathrm{H}_{2} \mathrm{O}\right)$, is the compound to which oak bark, galls, logwood, and many vegetable astringents owe their properties. The tannic acid from these several sources has, however, somewhat different characteristics, and generally receives such special designations as gallo-tannic, cincho-tannic, catechu-tannic acids. Gallotannic acid is prepared by softening powdered galls by keeping them for two days in a damp place, digesting them for several hours simultaneously with water, which dissolves the tannic acid, and with ether, which dissolves colouring matter and gallic acid. The mixture, filtered and allowed to stand, forms into two layers, and the lower, carefully evaporated, yields tannic acid. It occurs in pale yellow masses, or thin glistening scales ; has a strongly astringent taste and an acid reaction; is readily soluble in water, dilute alcohol, and slowly in glycerin; very sparingly soluble in ether.

GALLIC ACID, or tri-hydroxy-benzoic acid $\left(\mathrm{C}_{6} \mathrm{H}_{2}(\mathrm{OH})_{3 \text { r }}\right.$ $\mathrm{CO}_{2} \mathrm{H}, \mathrm{H}_{2} \mathrm{O}$ ), may be prepared by the action of diluted sulphuric acid on tannic acid or powdered galls. It occurs in acicular prisms, or silky needles, which are colourless or 
pale fawn. It requires for solution about one hundred parts of cold water, three of boiling water, and twelve of glycerin ; but is more soluble than tannic acid in alcohol and ether.

Actions AND USES. - Galls and tannic acid differ only in the degree of their action. They are astringent and antiseptic. Galls have about one half the activity of tannic acid. Gallic acid does not coagulate either gelatin or albumin, and it cannot be considered an astringent. It has no medicinal properties.

Tannic acid may be taken as the type of the group which includes galls, oak bark, catechu, logwood, kino, and rhatany. It has little effect on the unbroken skin ; but on abraded, atonic, and hypersecreting skin and mucous surfaces it coagulates albumin, causes dryness and tanning, with some contraction of the soft textures. But, unlike salts of lead, silver, or other mineral astringents, it does not contract capillary vessels. The precipitate of albumin acts as a protective, and diluted solutions hence relieve irritation. It coagulates blood and arrests bleeding. In the mouth it precipitates albumin on and in the superficial layers of the epithelium, and so causes dryness and roughness. In the stomach it again precipitates proteids forming tannate of albumin, which is slowly digested, setting free the tannic acid again, thus allowing the action on the mucous membrane to continue both in the stomach and small intestine. Pepsin and peptones in acid solution are not affected by tannic acid. In excess, especially when the stomach is empty, tannic acid causes irritation, with vomiting and even diarrhœa in small animals. In moderate doses, the protective coat of albumin and the lessening of glandular secretion, bring about firmness of the fæces and constipation. The drug has some antiseptic action, lessens fermentation, and renders the fæces less offensive. It is slowly and partially absorbed, as gallic acid or as an alkaline tannate, but when thus neutralised it has very little astringent or hæmostatic power. It is excreted as gallic acid, or as some oxidised product thereof.

Medicinal Uses.-Tannic acid and galls, in powder, solution, or spray, are applied in stomatitis, and relaxed conditions of the pharynx and nasal passages. In diarrhœa 
and dysentery the slowly dissolving catechus and kino are sometimes preferred to tannic acid, as they reach the intestines and exert their in-contact effects before they are neutralised. They are frequently prescribed with chalk, acids, aromatics, and opium, and given either in bolus or mucilage. For arrest of internal hæmorrhage, neither tannic nor gallic acid is so effectual as ergot, ferric-chloride, or lead acetate and opium. Stockman's investigations show that gallic acid, even in full doses, has no special general astringent action. Both tannic and gallic acids are used as antidotes in poisoning by alkaloids; but in combating metallic poisoning they are not so serviceable as other chemical antidotes, and as demulcents.

Externally, tannic acid is used with glycerin and water in the weeping stages of eczema; as an astringent wash with opium in prolapsus of the uterus or rectum; while it also checks the discharge and allays the irritability of otorrhœa, which is common in dogs. Tannic acid and antipyrine, ten parts of each, with 100 of alcohol, form an excellent application for soft, ulcerating, bleeding surfaces. For piles in dogs, tannic acid is used in substance, or as an ointment, opium being added if there is much irritability ; and such applications are often advantageously alternated with calomel ointment. Tannic acid mixed with boric ointment and sometimes with opium is useful for burns. For nasal catarrh it may be used mixed with starch, or iodoform, as an insufflation.

DosEs, etc.-Of tannic acid horses take grs. 1xxx. to $3 \mathrm{ij}$. ; cattle, 3iv.; sheep and large pigs, grs. xxx. to 3ij.; dogs and cats, grs. ij. to grs. x. Powdered galls are used in about double these doses. Glycerin of tannic acid, made by stirring one part of acid with five of glycerin, is a soothing antiseptic astringent, used diluted with water as required. Gall and Opium ointment is made with 37 grains powdered galls, 15 grains opium, and 148 grains of benzoated lard, or with vaseline. A styptic colloid may be prepared with one of tannin and eight of alcohol, mixed with four of collodion.

Pyrogallic acid is an astringent, and caustic, recommended in cases of psoriasis and ringworm, and for 
tanning and shrivelling carcinomatous growths. Jarisch's ointment for psoriasis consists of 60 grains pyrogallic acid to 1 ounce of lard.

Tannalbin, a dried albuminate of tannin, has been much used in the treatment of diarrhœa and dysentery in young animals. It is a light brownish powder, without odour or taste, insoluble in water and unaffected by the gastric juice. In the intestine it is slowly dissolved, exerting an astringent-disinfectant action on the mucous membrane. It contains about 50 per cent. of tannin. Doses,-horses and cattle, 3 j. to $3 \mathrm{iv}$.; foals and calves, grs. xx. to grs. xl. ; three times daily, in gruel, milk, or electuary. Tannigen (di-acetyl-tannin), prepared by the action of acetic anhydride on tannin, dissolved in glacial acetic acid, is a yellowish-grey powder, odourless and tasteless, insoluble in water, freely soluble in alcohol. It passes through the stomach unchanged and acts as an intestinal astringent. Recommended as a remedy for parasitic intestinal catarrh in foals. Doses,-grs. xx. to $3 \mathrm{j}$., in boiled milk or linseed tea.

\section{CHRYSAROBIN}

Araroba. Crude Chrysarobin. Goa powder. A substance found in cavities in the trunk of Andira araroba, dried and powdered, and imported from Brazil. Nat. Ord.Leguminosæ (B.P.).

Chrysarobin, obtained from Araroba by extracting with hot chloroform, evaporating and powdering, occurs as a crystalline, brownish-yellow powder, insoluble in water, but soluble in chloroform, and slightly soluble in alcohol. By oxidation it yields chrysophanic acid, $\mathrm{C}_{10} \mathrm{H}_{8} \mathrm{O}_{3}$, which is a constituent of rhubarb, stains yellow, and is less irritant than chrysarobin.

Both chrysarobin and chrysophanic acid are irritant and parasiticide, are seldom given internally, but are applied, usually in the form of a 2 to 5 per cent. ointment, in the second squamous stages of eczema, and in psoriasis and ringworm. Given by the mouth, they are gastro-intestinal 
irritants, causing vomiting and purgation, and also nephritis and albuminuria by the excretion of the small amount absorbed.

\section{BENZOIN}

Benzornum. A balsamic resin obtained from Styrax benzoin and probably from other species of Styrax (B.P.). Nat. Ord.-Styraceæ.

The styrax benzoin abounds in Siam, Sumatra, and Borneo. Incisions are made into the bark, when the thick, white, resinous juice exudes, and concretes in tears, which are subsequently made into larger masses, and imported in wooden cases. The colourless or reddish tears are imbedded in an amber-brown transparent resin. Inferior qualities are dark-brown or nearly black, and devoid of amygdaloid structure.

Benzoin is brittle and easily pulverised, softens readily when warmed, and when further heated yields fumes of benzoic acid. It is slightly heavier than water; soluble in alcohol and in solution of potassium hydroxide. Besides traces of volatile oil, benzoin contains about 80 per cent. of three resins, distinguished by differences of solubility, and from 14 to 20 of the acrid, crystalline, benzoic acid, $\left(\mathrm{C}_{6} \mathrm{H}_{5} \mathrm{CO}_{2} \mathrm{H}\right)$. Some samples contain as much as 10 per cent. of the allied cinnamic acid.

Benzoic acid is obtained from benzoin by sublimation. It may also be obtained from toluene, from hippuric acid, and from other organic compounds (B.P.). It occurs in light, feathery crystals, which are soluble in 400 parts of cold or twelve parts of boiling water, in three parts alcohol (90 per cent.), five parts glycerin, seven of chloroform, and in the fixed and volatile oils; also in solution of the alkalies and of calcium hydroxide forming benzoates. Ammonii benzoas is a crystalline salt produced by neutralising benzoic acid with solution of ammonia. Soluble in six parts of water, twenty-two of rectified spirit, and in eight of glycerin. Sodii benzoas, obtained by neutralising benzoic acid with sodium carbonate, is soluble in two parts cold water, and in twenty-four of rectified spirit. 
ACTIONS AND USES.-Benzoin, benzoic acid, and its salts are very similar in action to salicylic acid. They are stimulant, expectorant, diuretic, antiseptic, and antipyretic. Benzoin, although less frequently employed now than formerly in the treatment of disease of the air passages, is still useful as an antiseptic expectorant in bronchitis and especially in chronic catarrh of aged dogs. It may be administered by the mouth or added to the steam-kettle and used as an inhalation. It is excreted mainly in the urine, part of the benzoic acid being converted in the kidneys into hippuric acid. Freely applied to recent bleeding wounds, it forms an antiseptic coagulum and serves the purpose of a temporary styptic dressing.

Benzoin is extensively used in the form of Friar's balsam, or its pharmaceutical imitation, tinctura benzoini composita, which is thus prepared :- Take of benzoin, 2 ounces, storax, $1 \frac{1}{2}$ ounces, balsam of tolu, $\frac{1}{2}$ ounce, socotrine aloes, 160 grains, alcohol (90 per cent.), 16 ounces: macerate for two days, filter, and add sufficient alcohol to produce one pint. This tincture is an excellent stimulant and antiseptic for wounds, simple ulcers, and various skin complaints in all classes of patients.

Benzoated lard is made with 210 grains of benzoin to each pound of lard.

Benzoic acid is an intestinal antiseptic, lowers abnormal temperature, promotes the elimination of incompletely oxidised matters, increasing the amount of nitrogen excreted, renders alkaline urine acid and disinfects the urinary tract. It is used in the treatment of influenza and similar conditions, and as an antiseptic diuretic in cystitis. W. Rutherford found that twenty grains increased the biliary secretion in dogs. It is as effectual as carbolic acid in arresting the action of enzymes and destroying bacteria. It is eliminated chiefly by the skin and kidneys; in the latter, uniting with glycocoll, it is excreted as hippuric acid.

Ammonium and Sodium benzoates are less irritating and more soluble than the acid, and they are sometimes substituted for salicylate of soda in the treatment of articular rheumatism. Commended as a remedy for joint disease 
in foals, sodium benzoate abates the fever, and reduces the swelling of the limbs in strangles and pneumonia. According to W. Rutherford, it is a powerful hepatic stimulant. It is excreted in the urine as a soluble hippurate. A two per cent. solution is a good preservative for scalpels, forceps, needles, etc., in daily use. Instruments may be kept in the solution for months without oxidising.

DoSES, etc.-of benzoin for horses and cattle, 3iv. or more; dogs, grs. v. to grs. x.; in bolus or electuary. Benzoic acid, horses and cattle, grs. xxx. to grs. lxxv.; dogs, grs. ii. to grs. viii., in bolus, pill, or drench. Hypodermically, horses may be given grs. vi. benzoic acid dissolved in two drachms of a solution of equal parts of alcohol and water. Intratracheally, two drachms to half an ounce of a one per cent. aqueous solution may be used. Sodium benzoate is prescribed in considerably larger doses, and generally in drench.

Styrax or Prepared Storax, a balsam obtained from the trunk of Liquidambar orientalis, and purified by solution in alcohol, filtration and evaporation. Contains styrol, cinnamic acid, styracin and various resins. Storax is a constituent of the compound tincture of benzoin, and is occasionally employed as a mild stimulant, expectorant and parasiticide. For mange, lice, or fleas in dogs, it is used mixed with an equal part of sweet oil.

Balsam of Tolu.-The product of the Myroxylon toluifera, contains a volatile oil, various resins, benzoic and cinnamic acids. Soluble in alcohol, benzol and chloroform. It is stimulant and expectorant. Doses for dogs and cats, $m_{x}$. to $3 \mathrm{j}$. per day.

Balsam of Peru, obtained from Myroxylon Pereiræ, contains about 60 per cent. of a volatile oil, various resins and cinnamic and benzoic acids. It is insoluble in water and olive oil ; soluble in alcohol and in chloroform. It is an expectorant and parasiticide. The volatile oil is toxic to the acari of mange for which a dressing is made with one part Balsam of Peru and eight of lard. Doses for horses and cattle, $3_{\text {iij. to }} \xi_{j}$.; dogs and cats, $m_{v}$. to $m_{x v}$; in pills, capsules, or in emulsion. 


\section{MARSH MALLOW ROOT}

Althӝa Radix. Dried root of Althæa officinalis. Nat. Ord.-Malvaceæ. (U.S.P). Not official in B.P.

The Malvaceæ are rich in mucilage, and several yield tenacious fibres, from which cordage is obtained. The species Gossypium have their seeds surrounded by delicate, flattened, twisted hairs, which constitute raw cotton, and the seeds by expression yield the bland cotton seed oil often substituted for olive oil. The marsh mallow grows both in this country and on the Continent, generally in the neighbourhood of rivers and salt marshes. Mucilage is yielded by most parts of the plant, notably by the two and three year old roots, which contain about 35 per cent. each of mucin and starch, and a little uncrystallisable sugar.

AcTiONS AND USES.-Marsh, and also common mallow roots, are digested with boiling water, and the mucilage thus extracted, which resembles that of linseed, is used as a demulcent. Of the fluid extract of the bark of Gossypium root, horses and cattle may be given $3 \mathrm{jv}$. to $\jmath_{\text {j. }}$; dogs, mxxx. to $3 j$.

\section{BARLEY}

Hordeum. Pearl Barley. Malt. Yeast. Nat. Ord.Graminaceæ.

Barley (Hordeum distichon) is used as food for most of the domesticated animals; and, when stripped of its outer husk, is recognised as pearl barley. Ground to meal, it is used for making poultices and infusions. Good barleymeal contains 68 per cent. of starch, 14 glutin and albumin, 2 fatty matter, 2 saline matter, and 14 water. When moistened and exposed to a temperature of about $100^{\circ}$ Fahr., barley germinates, the starch in great part being converted into dextrin and sugar, and, if the process be arrested by drying, malt is formed. Decoctum Hordei, or barley water, may be made by boiling one part of washed pearl-barley with 15 parts water for twenty minutes, and straining. It is nutrient and demulcent. 
Malt-a sweet, mucilaginous substance, which is more easily digested, but weight for weight is rather less nutritive than barley-forms a palatable and digestible article of diet for sick or convalescent horses, and is used for making poultices and demulcent laxative drinks. Barley-water, infusions of malt, and soft mashes prove especially serviceable in febrile cases, both in horses and cattle. Malt extracts are occasionally prescribed for dyspeptic calves and foals, and for sick horses, and when well prepared are rich in diastase, and hence useful in aiding digestion of starch.

When a solution of malt is fermented, as in the preparation of beer, ale, or porter, there rises to the surface of the liquor a yellow-brown frothy scum, known as yeast or barm, readily putrefying when moist, but when carefully dried remaining for a long time unchanged, and owing its reproductive properties, and its characteristic power of converting cane into grape sugar, and thence into alcohol, to the presence of ovoid, confervoid cells of Torula cerevisiæ, the yeast plant. Yeast is occasionally used as a purgative, especially for cattle, and is given in quantities of about a pint. Fresh or dried yeast, dissolved in tepid water and injected into the vagina, has been successfully used as a remedy for sterility in cows. Antiseptic and deodorising poultices are made by stirring together one part each of boiling water and yeast with two parts of bran or linseed meal, and allowing the mixture to stand near a fire until it rises, when it is fit to use.

Nuclein, an important constituent of animal and vegetable cells, is obtained from yeast, and also from spleen pulp, blood, yolk of egg, milk, etc. The germicidal power of the blood serum is believed to be due to certain phosphorised proteids which also exist in nuclein. It is a body of indefinite composition, containing nucleinic acid and proteid matter rich in phosphorus. Administered, nuclein increases the number of white corpuscles in the blood, and acts as a powerful germicide. It has been employed, with excellent results, in the treatment of pneumonia, pleurisy, strangles, influenza, and purpura hæmorrhagica. Liquor Nucleinicus (Squire \& Sons), a solution of sodium nucleinate of a strength of about 
5 per cent.; or nuclein solution (Parke, Davis, and Co.), containing 5 per cent. of nucleinic acid, may be given hypodermically to horses, in doses of 3ijss., with an equal quantity of physiological salt solution.

\section{GUM ACACIA-TRAGACANTH}

Acacia Gummi. A gummy exudation from the stem and branches of Acacia senegal, and of other species of Acacia (B.P.).

Tragacantha. Tragacanth. A gummy exudation obtained by incision from Astragalus gummifer, and some other species of Astragalus (B.P.). Nat. Ord.-Leguminosæ.

Gum is obtained from many plants, notably from various species of Acacia. These are stunted, withered-looking trees, occurring in tropical countries, most prolific when old and stunted, and during dry, hot seasons. In June and July, from natural cracks or artificial incisions in the bark, a viscid juice exudes, and concretes into round masses or tears varying in size from a pea to a walnut, brittle, usually shining, colourless, yellow or brown, odourless, and of a bland, sweet taste. Gum dissolves in water, forming an adhesive, viscid fluid or mucilage.

Gum acacia, or gum arabic is chiefly collected in Kordofan, in Eastern Africa, and forwarded from Alexandria. When imported, it is picked and sorted, usually into three different qualities, distinguished by the size, colour, and transparency of the tears. It is soluble in about its own weight alike of hot and cold water, is insoluble in and incompatible with alcohol, ether, and oils. Boiled with dilute sulphuric acid, it is converted into gum sugar; oxidised by nitric acid, it is converted into mucic acid. It consists of arabin, or arabic acid, which occurs in gum as arabate of calcium, magnesium, and potassium.

Gum senegal is similar to gum arabic, but less brittle, and requires four or five parts of water to dissolve it. The East Indian gums are generally dark-coloured, more difficult of solution, and less valuable. The gums of Australia and 
the Cape, now imported in considerable quantity, are also inferior to gum arabic.

Tragacanth is collected in Asia Minor, mostly exported from Smyrna, and occurs in thin, semi-transparent, tough, horny, white-grey or yellow lamellæ or plates, and marked with arched or concentric ridges. It is tasteless and odourless. Although readily soluble in boiling water, it is sparingly soluble in cold water, which swells it into a jelly containing starch, as is indicated by the iodine test. Tragacanth contains a neutral gum, bassorin, which, gelatin-like, swells up, is not dissolved either by hot or cold water, but is soluble in alcohol.

British gum or dextrin $\left(\mathrm{C}_{6} \mathrm{H}_{10} \mathrm{O}_{5}\right)$, much used in calico printing, is made by treating starch with dilute nitric acid, drying and heating it to about $240^{\circ} \mathrm{Fahr}$.

Actions AND USES.-Gums are the least nutritive of the carbo-hydrates ; when swallowed, they are dissolved by the alimentary secretions, and in part converted into sugar. They are occasionally prescribed for ensheathing the mucous surfaces in catarrh and diarrhœa, and as demulcent injections in inflammation of the bowels and bladder, but for veterinary purposes are usually superseded by well-boiled linseed or starch gruels. For making emulsions, electuaries, and boluses, gums have the disadvantage of speedily drying and hardening. They are frequently used to suspend powders such as the salts of bismuth, and also to form emulsions with volatile and fixed oils.

Doses, ete.-Gums may be taken almost ad libitum. Horses and cattle may have $\zeta_{\mathrm{ij}}$. to $\boldsymbol{z}_{\mathrm{iij}}$; f foals, calves, and sheep, zi.; and dogs, grs. xx. to grs. xl. An ensheathing mucilage is made with one part gum to six of water.

\section{OLIVE OIL}

OLEUM OLIVÆ. The oil expressed from the ripe fruit of Olea Europæa (B.P.). Nat. Ord.-Oleaceæ.

Several varieties of the evergreen Olea Europæa grow abundantly in the countries bordering the Levant and Mediterranean. From the stem a resinous juice once used 
in medicine can be got ; the leaves are bitter, astringent, and tonic; the olives are oval, succulent, purple drupes, about the size of damsons, and containing a single seed. The ripe pericarp yields about 70 per cent. of oil, of which the finest, imported from Provence and Florence, is obtained by moderate pressure of the freshly-gathered fruit. Inferior qualities are got from stale or damaged fruit, or by extra pressure of the pulp.

Properties.-Olive oil is one of the fixed, fatty, or expressed oils which produce on paper or linen a greasy stain, not removed by heat, and are glycerides of an acid radicle, oleic, palmitic, or stearic acid, and the basic glyceryl. Olive oil contains about 72 per cent. of olein or tri-olein, $\mathrm{C}_{3} \mathrm{H}_{5}\left(\mathrm{C}_{18} \mathrm{H}_{33} \mathrm{O}_{2}\right)_{3}$, together with about 28 of palmitin and allied fatty matters. It is of the consistence of syrup, unctuous, transparent, odourless, and of a bland taste. When pure it is pale greenish-yellow ; when impure, yellow or brown. Specific gravity 0.914 to 0.919 . At $50^{\circ} \mathrm{Fahr}$. it is liable to become of a pasty consistence; and at $32^{\circ}$ Fahr. to form a solid granular mass. It is not miscible with water, is scarcely soluble in alcohol, but dissolves in one and a half parts of ether. It is a capital solvent for cantharidin, yellow phosphorus, atropine, and morphine. Exposed to air, it oxidises, thickens, and slowly becomes rancid, but does not dry up. It is often adulterated with purified cotton-seed oil, in fact this cheaper product is probably often sold as olive oil.

ACTIONS AND USES.-Olive oil is nutrient, laxative, and emollient. Like other bland oils, small quantities are easily digested and assimilated, aid cell development, and by oxidation support animal heat. Larger quantities, such as one to two pints for horses and cattle, and two to three ounces for dogs, are laxative. An ounce each of olive oil and castor oil form a mild laxative for the dog. Like other fluid fats, when injected into the veins, it fatally obstructs capillary circulation. Half an ounce injected into the jugular speedily destroys a dog. As a demulcent and emollient, it is used in poisoning by irritants and corrosives; it antagonises the action of alkalies by forming soaps, and retards solution and absorption of arsenic. Olive oil, in small doses, is 
occasionally given to horses and other animals to soothe the irritable mucous membrane in chronic catarrh and bronchitis, and in cases of gallstone and biliary colic on account of its demulcent action in the duodenum, so indirectly lessening the irritation in the bile duct. Not drying or readily becoming rancid, it is a soothing protective for irritable or abraded surfaces, but for such purposes the cheaper rape oil, lard, or vaseline, is usually substituted. Sterilised olive oil is employed in hypodermic injection of camphor.

\section{LINSEED}

Lindm. Flax or Lint Seeds. The dried ripe seeds of Linum usitatissimum (B.P.). Nat. Ord.-Linaceæ.

Linum Contusum. Crushed linseed. Linseed reduced to a coarse powder (B.P.).

Linseed OIL. Oleum Lini. The oil expressed from linseed at ordinary temperatures (B.P.).

LinseEd CAKE. The residue left after expression of the oil. Linseed MeaL. Farina Lini.

The Linum usitatissimum, or common flax, cultivated in Britain and other European countries, yields several important articles. The stem affords lint and tow ; the seeds, crushed, and subjected to hydraulic pressure, yield linseed oil ; the residual cake is a valuable feeding stuff, and when reduced to powder constitutes linseed meal.

The fibrous stem is utilised by steeping in water, generally used hot; starch and cellulose are got rid of by scutching ; the fibres are hackled and carded-the shorter, coarser portions forming tow ; the finer, when bleached, are made into linen. Soft, loosely woven linen, when scarified, and the cut fibres scraped into gauzy down, constitutes surgeons' lint. Both lint and tow, as well as jute (the prepared fibre of hemp), are employed as protectives for wounds. When saturated with hot or cold water they prove cleanly substitutes for poultices. For cleansing wounds they are preferable to sponges, which are apt to retain and distribute septic germs, while the rag, lint, or tow is thrown away after use. These fibrous materials, saturated with carbolic 
or other antiseptic solutions, are used for antiseptic dressings. Oakum, consisting of detached fibres of old ropes, when treated with Stockholm tar, is also a cheap antiseptic dressing.

Lint seeds are about two lines long, smooth, and shining, of a brown colour and oval shape, flattened laterally, and pointed at one extremity. They are inodorous, but have an oily, mucilaginous taste. They consist of about 20 per cent. of mucilage, wholly present in the envelope of the seed, and hence only properly extracted by prolonged steeping or slow boiling ; 20 of albuminoids, a little sugar, 25 to 30 of oil, contained in the albumin and embryo; 5 to 6 of mineral matters, chiefly phosphates, mostly stored in the husks; 5 to 8 of fibre, and 8 to 10 of water. Crushed linseed should yield not less than 30 per cent. of oil when exhausted by carbon bisulphide. The seeds, ground and pressed without the aid of heat, produce about 25 per cent. of oil of the best quality; steam heat extracts 25 to 35 per cent. The residual linseed cake, or oilcake, contains 10 to 13 per cent. of oil.

Linseed oil is viscid, has a pale-yellow colour, a faint but distinct odour, and a bland taste. Specific gravity 0.930 to 0.940 . It consists largely of olein, or of a variety recognised as linolein. Although it does not solidify until cooled to $-15^{\circ}$ or $-20^{\circ}$ Fahr., at ordinary temperatures it oxidises and becomes viscous, hence receiving the title of a drying oil. This drying property is much increased by boiling, or heating it with litharge or black oxide of manganese. It is insoluble in water, soluble in ten parts alcohol (90 per cent.), in one and a half of ether, and in oil of turpentine. Boiled with alkaline solutions it forms soaps. Mixed with an equal quantity of lime water it forms Carron oil, a useful dressing for burns and scalds. Exposed for some time to a high temperature it becomes a dark, tenacious mass, which, when mixed with lamp-black constitutes printers' ink. It is sometimes adulterated with rapeseed oil, but is more commonly of inferior quality from rancidity, from preparation at a high temperature, or from presence of impurities.

ACTIONS AND UsES.-Linseed and linseed cakes are valu- 
able feeding stuffs for cattle and sheep, and, in restricted amount, for horses. As fat producers they represent about two and a half times the value of starch or sugar. They are emulsified mainly by the pancreatic and biliary fluids ; they are absorbed chiefly by the lacteals, and their combustion develops heat and force. In moderate amount they favour assimilation alike of carbohydrates and proteids, with which they are generally given.

Well-boiled linseed gruel, or crushed linseed cake digested in hot water, is a palatable, digestible nutrient for horses, cattle, and sheep, not only in health, but notably in catarrhal and other inflammatory attacks, in tuberculosis, rheumatism, chronic skin complaints, and during convalescence from reducing disorders. In such cases it proves both food and medicine. In febrile cases many horses will sip cold linseed tea when they will scarcely eat or drink anything else. Where the patient is exhausted, the linseed tea is given with milk, eggs, or beef-tea, or with alcoholic or other stimulants. Horses that are bad feeders, with unthrifty coats, and horses affected with roaring or thick wind, are usually much benefited, especially while living mostly on oats and hay, by about a pound daily of broken linseed cake. For healthy hunters and carriage horses the continued use of linseed cake proves, however, too fattening and often causes itching. Young herbivora reared on skim milk frequently have linseed gruel mixed with it to furnish requisite fatty matters, and also to prevent the formation of tough, indigestible curd. Linseed gruel, or a few ounces of crushed cake given daily to calves or lambs, as soon as they will eat it, not only economically favours growth and early maturity, but is tolerably effectual in warding off attacks of diarrhœa, dysentery, and anæmia.

A mucilaginous, demulcent decoction, made with about one part of steeped seed to fifteen or twenty parts boiling water, is useful in irritable conditions of the throat, respiratory passages, bowels, kidneys, and bladder ; in poisoning with irritants and corrosives; and as a convenient vehicle for the administration of nauseous or acrid medicines.

Crushed linseed makes good poultices, especially when mixed with an equal quantity of bran or oatmeal ; but the 
bruised linseed cake is cheaper, less apt to become rancid, and equally effectual in retaining heat and moisture. The common mass employed for making up balls and pills usually consists of equal quantities of linseed meal and treacle.

LINSEED OIL has been used dietetically; but neither for cattle nor sheep does it serve so well as properly prepared linseed or linseed cake. It has the disadvantage of being too laxative, and it increases rather than diminishes the quantity of ordinary food consumed. As an adjuvant feeding stuff for animals in health, I have found it inferior to linseed cake, beans, or oats. One to two ounces repeated daily are, however, often beneficial, in sore-throat and bronchitis in horses, and especially for subjects that will not take linseed gruel or mashes.

Linseed oil, in quantity too large to be digested, acts as a cathartic ; it is also emollient. It closely resembles rapeseed, almond, and other fixed oils ; but is scarcely so actively cathartic as castor oil.

As a laxative it usually produces tolerably full and softened evacuations, without nausea, griping, or superpurgation. It is prescribed for young and delicate horses, and pregnant mares, and for all subjects in influenza, purpura, and other debilitating disorders ; in diarrhœa, hernia, and irritable states of the intestine, as well as in overloaded, torpid bowels, where aloes and other active purgatives, especially if repeated, might cause dangerous symptoms. It is serviceable in warding off attacks of lymphangitis, hæmoglobinuria, œdema and itching of the limbs, which are liable to occur when hard-worked horses have several days' rest. In the treatment of colic it is generally combined with a stimulant and anodyne. A draught in common use consists of one pint of linseed oil with an ounce each of ether and laudanum, both being doubled in acute cases and in large horses. In colic, aloes, however, generally acts better than linseed oil; but for laxative enemas the oil is preferable.

Two or three ounces of linseed oil, or of a mixture of equal parts of linseed and olive oils, given daily in mash, often suffice, with the use of enemata, to maintain the bowels 
of horses in a sufficiently relaxed state throughout catarrhal and other febrile attacks. This treatment is also specially suitable in inflammation of the kidneys and bladder, when it is ${ }^{-d e s i r a b l e ~ t o ~ r e s t ~ t h e s e ~ o r g a n s, ~ a n d ~ p r o m o t e ~ e x c r e t i o n ~}$ by the bowels and skin. An ounce or two of oil combined with lime-water given daily to broken-winded subjects often advantageously relieves the distressing breathing.

In cattle and canine practice linseed oil is much used as a purgative, especially for young and weakly patients, in advanced pregnancy, in gastro-intestinal derangements, in irritant poisoning, where saline or other active purgatives have been given, and their repetition is inexpedient, and as a convenient menstruum for the administration of croton oil and oil of turpentine. For calves and lambs it is milder and safer than salts. For dogs, especially when young, when the digestive organs are in an irritable state, and exhausting disease has reduced strength, it is a suitable laxative, and more effectual when mixed with an equal amount of castor oil. As a lubricant and emollient linseed oil is useful in choking; mixed with well-boiled starch gruel, and injected into the rectum, it allays irritation; softening the hard, cracked, or scaly skin, it is applied, with an alkaline solution, in psoriasis, impetigo, and eczema. Its soothing effects are increased by admixture with lead acetate solution. For emollient dressings, to be used for a considerable time, vaseline and benzoated almond, or cocoa-nut oil are, however, preferable, as they are not drying or prone to rancidity. The drying properties, possessed in common with poppy and cod-liver oils, render linseed oil less suitable than lard, or olive, almond, rape, or colza oil, for making ointments and liniments. Friction with oil often reduces swollen joints and bursæ. Flannel, soaked in hot linseed oil, is sometimes applied for the relief of rheumatism.

Doses, etc.-As a cathartic, horses take Oss. to Oj.;

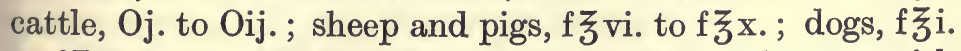
to $\mathrm{f} Z \mathrm{ij}$.; cats, f $3 \mathrm{i}$. to $3 \mathrm{jv}$.; administered shaken up with linseed gruel, mucilage, milk, treacle, lime-water, or spirit and water. For horses or cattle it is sometimes mixed with a well-made bran mash. 


\section{LIQUORICE ROOT}

GLyCyrRhize Radix. The peeled root and peeled subterranean stem of Glycyrrhiza glabra and other species (B.P.). Nat. Ord.-Leguminosæ.

The perennial herbaceous plants yielding liquorice grow in the temperate countries of Continental Europe. Their roots and underground stems arrive at perfection about the third year, and produce a yellow powder having a sweet taste, and soluble in water, and, to a less extent, in alcohol. Besides asparagin, gum, starch, sugar, malic acid, and a resinous oil, to which it owes its sub-acrid taste, liquorice contains about 6 per cent. of a sweet, yellow glucoside, termed glycyrrhizin. The natural juice or watery infusion, concentrated until it becomes solid, forms the extract or black sugar.

ACTIONS AND USES.-Liquorice resembles sugar and treacle in its dietetic and medicinal uses. Powdered, it is occasionally used as a demulcent in irritation of the pulmonary mucous membrane, for making up boluses, electuaries, and covering the disagreeable taste and odour of various drugs. The laxative compound liquorice powder, composed of senna, liquorice root, fennel, sulphur, and sugar, is sometimes prescribed for calves, lambs, and dogs. The dose is $3 \mathrm{j}$. to $3 \mathrm{iv}$.

\section{STARCH}

AмyLum. The starch procured from the grains of common wheat, Triticum sativum ; maize, Zea mays ; and rice, Oryza sativa (B.P.). Nat. Ord.-Graminaceæ.

Starch is an important member of that dietetic series of carbo-hydrates, including gums and sugars, which contains at least six carbon atoms with hydrogen and oxygen in the proportion to form water. Starch is largely present in the cereal grains, in the stems of many plants, and in tubers, being stored in the seeds and tubers for the nourishment of the young plants. Wheat flour contains about 70 per cent. 
of carbo-hydrates, chiefly starch, which receives the special title of amylum, 10 of proteids, with water, and ash. Oatmeal contains 63 of starch and about $12 \cdot 6$ of proteids, with traces of a bitter amorphous alkaloid; barley, 64 starch, 12 proteids ; rice, 83 starch, 5 proteids ; potatoes, 21 starch, 2.8 proteids. From any of these sources pure starch is got by fine division of the grain or root; sometimes facilitating separation of other plant constituents by fermenting; washing the starch granules from fibrous matters, straining, and drying. The white starch used for medicinal and dietetic purposes is dried in powder or granules. The blue preferred for the laundry is in blocks, splits as it dries into columnar masses, is coloured by addition of a little indigo, and generally contains about 18 per cent. of water.

Arrowroot is the starch of the Maranta arundinacea; sago, the granular starch from the sago palm; tous-lesmois, the large ovular granules from the rhizomes of several species of Canna; tapioca or cassava is prepared from the expressed juice of the roots of Manihot utilissima. Corn flour or Oswego is the flour of Indian corn deprived of gluten by a weak solution of soda.

Starch consists of round or oval granules comprising a cell-wall enclosing concentric layers of granulose. The large grains from potatoes are about $\frac{1}{30}$ th of an inch in their long diameter, the small rounded grains of rice measure $\frac{1}{3000}$ th of an inch. Starch grains from various sources differ in appearance when examined under the microscope. Wheat starch presents a mixture of large and small granules, which are lenticular in form, and marked with faint concentric striæ surrounding a nearly central hilum. The maize granules are more uniform in size, frequently polygonal, smaller than those of wheat, having a very distinct hilum, but without evident concentric striæ. Rice granules are extremely minute, and nearly uniform in size, polygonal, the hilum small and without striæ (B.P.).

Starch is insoluble in cold water, has the specific gravity $1 \cdot 5$, and hence is deposited when mixed with water. The cell-wall consisting of cellulose and the contained granulose are isomeric, having the formula usually given as $\left(\mathrm{C}_{6} \mathrm{H}_{10} \mathrm{O}_{5}\right) \mathbf{x}$. 
When mixed with water above $120^{\circ}$ Fahr., the starch grains burst; the granulose, escaping, occupies twenty to thirty times its previous volume, and forms the viscid gelatinous mucilage used by the laundress. A solution of starch when cold gives the characteristic blue compound with solution of iodine. Starch, when boiled with diluted sulphuric or nitric acid, or heated, is converted into the isomeric but more soluble dextrin or British gum, one variety of which is coloured red by iodine. With further action of a weak acid and heat, dextrin takes up water and is converted into maltose $\left(\mathrm{C}_{12} \mathrm{H}_{22} \mathrm{O}_{11} \cdot \mathrm{H}_{2} \mathrm{O}\right)$, and eventually into dextrose $\left(\mathrm{C}_{6} \mathrm{H}_{12} \mathrm{O}_{6}\right)$.

When starch foods are eaten the salivary and intestinal ferments or enzymes gradually resolve the granules, and quickly convert the starch through several forms of dextrin into maltose, and eventually into dextrose. These changes are also readily produced by mixing starch paste with crushed malt, the diastase of which develops the fermentative changes. Animal stareh, or glycogen $\left(\mathrm{C}_{6} \mathrm{H}_{10} \mathrm{O}_{5}\right) \mathrm{x}$., present in the liver, in blood, and in muscle, exhibits most of the characters of vegetable starch.

Actions AND USES.-Starch foods are rapidly digested, especially when cooking or fermentation has cracked the starch cells, or when they have been thoroughly insalivated. Like other such proximate principles, pure starch cannot, however, alone support life for any lengthened period. A properly balanced dietary for horses or cattle should contain one part of proteids and five to eight parts of starch or other carbo-hydrates. Active exertion, as in the case of hard-worked horses, or abnormal secretion, as of heavilymilking cows, causes great expenditure of albuminoids, which must be replaced by the food. Growing animals, in order to build up their tissues, require relatively larger supplies of albuminoids than suffice for adults. The starches - mostly converted into sugar-are consumed in the body more quickly and fully than fats. During their oxidation they are the great source of animal heat, especially in herbivora. They prevent wasteful consumption of the more costly albuminoids and fats. Under favourable conditions, carbo-hydrates, in excess, are also directly concerned 
in the formation of fat, and Pasteur states that they furnish glycerin-the basis of neutral fats. For nutritive purposes seventeen parts (Voit) to twenty-three parts (Rubner) of carbo-hydrates are equivalent to ten parts of fat.

As a demulcent and emollient, starch mucilage protects and softens irritable surfaces. In diarrhœa and dysentery it is used about the consistence of cream, at the temperature of $100^{\circ} \mathrm{Fahr}$., either alone or with laudanum, sugar of lead, or other astringent, and is given both by the mouth and rectum. It is an antidote to excessive doses of iodine. Dry starch readily absorbs water, and hence is a desiccant for wounds, forming a protective covering. Mixed with equal parts of zinc oxide, it dries and soothes the weeping earlier stages of eczema. Conjoined with carbolic acid, or boric acid and iodoform, it forms a convenient desiccant antiseptic. One part of starch, heated with five of glycerin and three of water, make a soothing demulcent. Starch is used for mixing and subdividing medicines, and as a vehicle for their administration. Dextrin and starch are employed to stiffen bandages for fractures and other surgical purposes. A mixture of dextrin 100 parts, water 50, spirit of camphor 50, is used for adhesive plasters.

\section{S U G A R}

Sugar exists in many plants ; is prepared in France and Germany from white beet, in Asia from various palms, and in America from sugar maple (Sorghum saccharatum), and maize. The sugar used in this country is chiefly got from the sugar-cane (Saccharum officinarum), which is extensively cultivated in the West Indies, has a perennial root, and a jointed annual stem six to twelve feet high. These canes are crushed between heavy rollers; the pale green expressed juice, which contains nearly twenty per cent. of sugar, is mixed with a little slaked lime to neutralise acids and precipitate albuminoids, and concentrated in shallow vacuum pans at a temperature not exceeding $140^{\circ}$ Fahr.; the coagulating albumin, entangling impurities, is skimmed off ; the syrup is cooled in wooden vats, and dried 
in the sun, yellow dark-brown crystals of raw sugar are formed, and there drains away a variable quantity of brown uncrystallised molasses. A hundredweight of raw sugar yields about 80 pounds refined sugar and 16 pounds treacle.

There are two classes of sugars-(1) the Sucroses or Saccharoses, which, when dry, have the formula $\mathrm{C}_{12} \mathrm{H}_{22} \mathrm{O}_{11}$, and (2) the Hexoses, with the formula $\mathrm{C}_{6} \mathrm{H}_{12} \mathrm{O}_{6}$.

Sucrose, saccharose, or cane sugar $\left(\mathrm{C}_{12} \mathrm{H}_{22} \mathrm{O}_{11}\right)$, like sulphur and arsenious acid, has an amorphous and a crystalline form, its crystals are monoclinic prisms; specific gravity 1.606 ; it phosphoresces in the dark, and is dextro-rotatory. It is hydroscopic, soluble in one-third of its weight of water at $60^{\circ}$ Fahr., but insoluble in absolute alcohol. A strong solution, evaporated and heated to $320^{\circ} \mathrm{Fahr}$., fuses, and the vitreous mass can be moulded into barley-sugar. Between $356^{\circ}$ and $374^{\circ}$ Fahr. sucrose parts with two molecules of water, loses its sweet taste, acquires a dark colour, and becomes caramel, which is used by confectioners and distillers as a colouring agent.

Sucrose in plants is gradually built up from the simpler hexoses $\left(\mathrm{C}_{6} \mathrm{H}_{12} \mathrm{O}_{6}\right)$, and, conversely, when acted on by dilute acids or by ferments, such as diastase or yeast, it is again converted into glucose and fructose. Sucrose undergoes this change before it yields alcohol.

Maltose $\left(\mathrm{C}_{12} \mathrm{H}_{22} \mathrm{O}_{11} \cdot \mathrm{H}_{2} \mathrm{O}\right)$ is prepared by grinding starch with water, warming it until it gelatinises, and heating with crushed malt, the diastase of which sets up fermentation, causing three molecules of starch to appropriate one of water, and yield one molecule of maltose and one of dextrin. Maltose is also formed during the digestion of starch by the ferments of the salivary, intestinal, and pancreatic juices. It is soluble and readily fermented.

Lactose, or milk sugar $\left(\mathrm{C}_{12} \mathrm{H}_{22} \mathrm{O}_{11}, \mathrm{H}_{2} \mathrm{O}\right)$, is prepared by evaporating whey to a syrup, and crystallising. It occurs in translucent, greyish-white, hard cylindrical masses of rhombic prisms. It is gritty, and, being less soluble, is not so sweet as the vegetable sugars. It is not directly fermentable. Lactose is a very active diuretic, but in practice milk or whey is preferred. 
Glucose, dextrose, or grape sugar $\left(\mathrm{C}_{6} \mathrm{H}_{12} \mathrm{O}_{6}\right)$, is the variety present in grapes and other fruit, and in honey. It is obtained by boiling cane sugar, or acting upon it with alcoholic solution of hydrochloric acid, is formed when starch is boiled with water acidulated with sulphuric acid, and is the variety occurring in blood and urine. It is produced when glucosides, such as salicin, amygdalin, digitalin, etc., are boiled with diluted acid. It is neither so sweet nor so soluble as sucrose, crystallises in six-sided scales, is not charred by sulphuric acid, but forms with it sulphosaccharic acid. It produces a readily crystallisable compound with common salt.

Lævulose, also termed fructose, is isomeric with dextrose, and is associated with it in most fruits. These two sugars are distinguished by the manner in which they turn a ray of polarised light; dextrose to the right, lævulose to the left. Lævulose is sweeter than dextrose, and less fermentable.

Molasses, treacle, theriaca, or sacchari fæx, is the uncrystallised, fermentable, syrupy residue from the preparation and refining of sugar. It has a brown colour, a pleasant sweet taste, and a specific gravity of about 1.4. Molasses is the drainings from the raw sugar; treacle the darker, thicker residue from the moulding process.

Honey or mel, the saccharine secretion deposited in the honeycomb by the hive bee, when first collected is yellow, translucent, and viscid, and consists of variable proportions of sucrose and lævulose. The popular household expectorant oxymel is made of eight parts of honey, liquefied by heat, and mixed with one part each of acetic acid and water.

Actions AND USES.- The sugars are members of the carbo-hydrate series of dietetic substances, are digestible and nutritive ; their important function in all the higher animals is the support of animal heat; they moreover economise the proteids and fats, and directly contribute to the deposits of fat. They are laxatives, demulcents, and antiseptics, and used pharmaceutically as excipients. One or two pounds given to horses or cattle, eight to twelve ounces to sheep or dogs, eight to ten drachms to poultry, increase the amount 
and fluidity of the fæces, and usually also augment secretion of urine. As a demulcent sugar is úsed in the dry stages of common catarrh, in poisoning with salts of mercury and copper, and as a domestic remedy for wounds, and for removing specks from the cornea. Its antiseptic properties recommend it for preserving many vegetable and some soft animal substances, and for making up various medicines. It increases the solubility of calcium salts and retards oxidation of ferrous compounds. The syrupus of the B.P. used for flavouring, preserving, and suspending medicines, is made by dissolving, with the aid of heat, five pounds refined sugar in two pints distilled water, and adding after cooling, sufficient water to make the weight of the product $7 \frac{1}{2}$ lbs. Specific gravity $1 \cdot 330$.

Molasses and treacle are often substituted for sugar. They are palatable, digestible, laxative articles of diet, useful for sick and convalescent animals. They are convenient auxiliary purgatives, and valuable for hastening the action, preventing the nausea, and covering the disagreeable flavour of active cathartics. When full doses of physic have been given, and their repetition is inexpedient, large and repeated doses of treacle encourage the action of the purgative, especially in cattle and sheep. As a soothing antiseptic gargle for horses, three or four ounces of treacle and an ounce of borax or of potassium nitrate or chlorate are dissolved in a pint of water, and a few ounces slowly administered every hour or two. When cough is troublesome an ounce of belladonna extract may be added. Treacle is a convenient antiseptic excipient for electuaries and also for ball masses, imparting a proper consistence, and preventing their becoming dry, hard, or mouldy. The common mass, so largely used as an excipient, is made by thoroughly mixing with gentle heat equal weights of treacle and linseed meal.

Doses, ete.-Of sugar and treacle, as laxatives, horses and

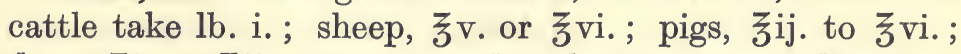
dogs, $z_{i}$. to $\zeta_{i j}$., administered with aromatics and salines, usually dissolved in water, milk, or gruel, or mixed with a mash. 


\section{CANTHARIDES}

Cantharis. Blistering or Spanish Fly. The dried beetle -Cantharis vesicatoria. Class-Insecta. OrderColeoptera.

Cantharides flies are found in most parts of Southern Europe, Germany, and Russia, and occasionally along the south coast of England. They settle on such trees and shrubs as the olive, lilac, privet, ash, elder, honeysuckle, and rose. During May and June, after nightfall or before dawn, the collectors, with their faces protected by masks and their hands by gloves, shake or beat the insects from the trees on which they feed, kill them by exposure to the fumes of oil of turpentine, or by immersion in boiling water or vinegar, and quickly dry them in the sun or by artificial heat. The flies used in this country were formerly brought from Spain (and hence their vernacular name of Spanish flies), but are now chiefly imported from Hungary, St. Petersburg, and Messina, usually packed in barrels or cases containing from 100 to $200 \mathrm{lbs}$.

Properties, etc.-The insect is from three quarters of an inch to an inch long, and a quarter of an inch broad, with two long elytra or wing-sheaths of a shining coppery-green colour, under which are two thin, brownish, gauze-like, membranous wings. The body, especially along its under surface, is covered with grey-white hairs ; the head is large ; the antennæ or horns are black and thread-like. The insect, which lives eight to ten days, deposits its larvæ in the earth, leaving them to be hatched by the heat of the sun. Powdered cantharides has a resinous, acrid taste, and a disagreeable, penetrating, fœtid odour. It is freely soluble in boiling water, alcohol, ether, acetic acid, and fixed and volatile oils. The active principle being volatile, no cantharides preparation should be heated beyond $200^{\circ}$ Fahr. Its distinguishing tests are its vesicant action, and the brilliant green appearance of the wing-sheaths.

Cantharides, besides animal matters, acetic and uric acids, contains a bland oil, a fœtid, acrid, volatile oil, and about 2 per cent. of a fatty crystallisable principle cantharidin 
$\left(\mathrm{C}_{10} \mathrm{H}_{12} \mathrm{O}_{4}\right)$, which is confined to the soft parts of the body, and is present particularly in the blood and female sexual organs. It is slowly deposited, when an alcoholic solution of cantharides is concentrated. When pure, it crystallises in colourless scales or prisms, melts at $482^{\circ} \mathrm{Fahr}$., is insoluble in water, but soluble in alcohol, acetic acid, ether, chloroform, and oils ; $\frac{1}{100}$ of a grain suffices to blister.

IMPORITIES.-As the powdered cantharides sold in the shops sometimes contains euphorbium and various cheap irritants, it is advised that the flies be purchased entire. Other insects are sometimes mixed with them. The species of mylabris sold as Chinese blistering flies have two orangecoloured bands and spots on the wing-covers. Activity is sometimes impaired by damp, long-keeping, and attacks of mites, moths, and beetles-parasitic attacks which are prevented by keeping the fresh flies in closely-stoppered bottles, with a few drops of acetic acid, or a few grains of camphor or ammonium carbonate.

ACTIONS AND USES.-Cantharides is an irritant, and produces its effects on any part with which the free cantharidin is brought into contact. Applied externally, it stimulates and vesicates, and is used as a counter-irritant. When swallowed it irritates the digestive mucous membrane; large doses produce gastro-enteritis. The active cantharidin is absorbed, and in the blood forms a non-irritant albuminoid, but in the kidneys is again liberated, developing its characteristic irritation-small doses stimulating the urino-genital tract, causing diuresis, and in some animals increased sexual desire; full doses inducing nephritis, cystitis, strangury, and hæmaturia.

General Actrons.-According to the strength of the preparation, or the period during which it is applied, cantharides produces redness, vesication, or sloughing of the skin or mucous surfaces. An ordinary vesicant dressing causes congestion, elevation of local temperature, and, usually within three to twelve hours, formation of blisters, which, after a variable but generally short time, burst, and discharge a yellow, serous fluid, which dries into scurfy cicatrices. When freely or continuously used, the deeper-seated skin tissues are inflamed, and ulceration, 
and sloughing with suppuration ensue. When the true skin has thus been seriously inflamed, the hair bulbs are injured; the hair is removed, and permanent baldness and blemishing may result. As a vesicant it is most powerful on horses and dogs, and less powerful on cattle, swine, and poultry.

Toxic EFFEcTs.-Orfila found that ' three drachms of the tincture, with eight grains of powder suspended in it, caused the death of a dog in twenty-four hours, if retained in the stomach by a ligature on the gullet, insensibility being the chief symptom ; and that forty grains of the powder killed another dog in four hours and a half, although he was allowed to vomit. When administered by the stomach, that organ was found much inflamed after death; and if given in the form of powder, fragments of the poison were generally discernible. When applied to a wound, the powder excites surrounding inflammation; and a drachm will, in this way, prove fatal in thirty-two hours, without any constitutional symptom except languor' (Christison). An ounce of powdered cantharides administered to a horse caused death in eighteen hours. In most cases the pulse becomes small and frequent, respiration quick and dyspnœic, there is restlessness and excitement, followed by coma and collapse.

The treatment of the gastro-intestinal or urinary irritation consists in the free use of mucilaginous drinks with opiates. Oils and fats are inadmissible on account of their favouring solution of any unabsorbed poison. When constitutional irritation has resulted from absorption of the cantharidin from a blistered surface, this should be dressed with soothing remedies.

Medicinal Uses.-Small, repeated doses are occasionally prescribed in chronic nasal catarrh. In such cases Robertson gave it with copaiba. It is sometimes serviceable in chronic eystitis; while giving tone to the bladder, small doses prevent involuntary escape of urine. In some parts of Germany it is given to cows which are tardy in coming to service; but its aphrodisiac effects on either sex are uncertain, and seldom produced except by dangerously large doses. When administered for some time, small vesicles 
sometimes appear on the skin, depending on the excretion of cantharidin cutaneously.

External Applications. - Cantharides, in small amount and diluted, stimulates the capillaries and trophic nerves of the part to which it is applied, and thus increases the blood supply and functional activity of the skin and hair bulbs. It hence induces a healthier condition of the dermis in some chronic scaly diseases, and promotes growth of hair; ulcers and tardily-healing wounds are stimulated, and their repair encouraged. Inflammatory products are liquefied and absorbed - an effect familiarly illustrated by the action of a blister on the swelling remaining around a bruise, or on the fulness and thickening resulting from a strain. The beneficial effects of a cantharides blister in arresting inflammation and removing effusion were often exhibited when blood-letting was the rule, and phlebitis of the jugular vein of the horse was not infrequent. Blistering ointment, well rubbed in along the course of the vessel, removed the tense, corded, inflammatory swelling.

Cantharides blister is usefully applied in cases of open joint or bursa, where the wound is small, to prevent escape of synovia. It is also applied in umbilical hernia in foals and calves ; and while the consecutive swelling mechanically prevents the descent of the intestine, the opening in the abdominal wall is gradually obliterated. Similar effects are sometimes obtained by moistening the adjacent skin with sulphuric acid.

Cantharides is much used as a counter-irritant. The external irritation reflexly relieves tension, inflammation, and pain of adjacent or deep-seated parts. Blisters applied experimentally to the chest or loins of dogs and rabbits, while producing external congestion, cause anæmia of the pleura and lungs, or of the deeper-seated muscles of the back. In pleuritic and other cases a blister notably modifies morbid action, and relieves painful tension ; the temperature is reduced $2^{\circ}$ to $3^{\circ} \mathrm{Fahr}$., and the pulse ten beats per minute. Some prefer cantharides to mustard, believing it to cause less irritation and pain, and to produce more permanent curative effects. Others, however, maintain that cantharides and other blisters unnecessarily irritate most 
horses, and, in acute diseases of the respiratory organs, are neither so certain nor so satisfactory as hot fomentations.

In many cases of catarrh and sore-throat, heat and moisture are certainly more effectual than blisters ; but tedious, irritable conditions of the larynx, inducing coughing, are often relieved by a cantharides dressing. In the outset of roaring, counter-irritation is often useful. In acute bronchitis, when mainly affecting the larger tubes, it is serviceable in conjunction with inhalation of steam, and after stuping the parts with hot water. But many think favourably of fly blisters in cases where considerable exudation blocks the smaller tubes. Their efficacy is seldom so obvious in pneumonia, especially when involving a considerable area. In pleurodynia and most stages of pleurisy, cantharides is specially useful ; in the earlier stages it moderates acute inflammation, while later it checks or removes effusion. It is the counter-irritant usually applied in inflammation of the pericardium. Although occasionally used, it is never of much value, in either colic or enteritis. In peritonitis it is seldom so effectual as in pleurisy, but was advised by Robertson in chronic cases. Where acute inflammation extends over a considerable area of the peritoneum, it is desirable that the blister be applied some little distance to the side of and not directly over the closely underlying inflamed spot. Cases of paralysis in cows depending upon parturient apoplexy are usually benefited by moderate counter-irritation, maintained for a week or ten days. In rheumatism, in all patients, advantage frequently results from a fly blister which is maintained active by repeated application.

Irritation and inflammation of joints, bursæ, ligaments, tendons, and bones are combated, and effused products removed by blisters properly used. When external surfaces or comparatively superficial textures are to be directly stimulated, the cantharides application must be mild, and not too long applied. When deeper-seated parts are to be acted on, more powerful preparations are needful, and their effects may be maintained by repetition. It is seldom advisable to apply cantharides directly to any part which 
is hot, tender, or inflamed. In applying blisters to inflamed joints or bursæ, it is sometimes desirable to place them, not immediately upon, but somewhat above or below, the affected spot. Where continued effects are desired, mercury biniodide ointment is alternated with cantharides, or substituted for it, or the actual cautery is used instead of blisters.

Owing to its liability to become absorbed and irritate the kidneys, it is an unsuitable counter-irritant in inflammation of the urinary organs. In common with all other causes of irritation, it must be avoided in tetanus. Unless on a very limited surface, and freely diluted, cantharides must not be used in weakly, exhausted subjects. In dogs, special caution is required, as they are apt to rub the blistered parts. Cantharides sometimes acts with unexpected violence on the skin of well-bred horses, and for such subjects strong blisters are not advisable, and their application over a considerable surface should be avoided. No horse should have all four legs blistered at one time. In some excitable subjects even a moderate blister causes much constitutional disturbance.

DosEs, etc.-For horses, grs. iv. to grs. xx. ; for cattle, grs. $x \times x$. to $3 \mathrm{j}$.; fớr sheep and swine, grs. ij. to grs. viij. ; for dogs, gr. ss. to grs. ij., repeated once or twice a day, usually given with aromatics and bitters, in the form of bolus or tincture; administration suspended if urinary irritation or any untoward effects occur.

Cantharides is used externally in the form of powder tincture, vinegar, ointment, liniment, and plaster.

Powdered cantharides is principally used for maintaining irritation, and for scattering over mustard poultices and other stimulant applications to increase their activity.

Tinctures of cantharides are made of varying strength. The B.P. tincture ( 1 in 80 ), and other alcoholic preparations, used in human medicine are too weak for most veterinary purposes. One ounce of coarsely-powdered flies, macerated for seven days with fifteen or twenty ounces of alcohol, 60 per cent., forms a useful tincture of medium strength. The activity is augmented by addition of liquor ammoniæ, or oil of turpentine. The tinctures in common 
use act speedily, but their effects are less powerful and permanent than those of the ointments. Though producing considerable irritation, they seldom cause blistering, unless applied repeatedly at short intervals. In using them, it is not essential that the hair be removed, nor even that the animal be kept idle. They may be applied repeatedly to the same spot without fear of blemishing.

Vinegar of cantharides made with one part of powdered flies and ten of acetic acid forms a prompt counter-irritant. The B.P. Acetum Cantharidis is prepared with two parts cantharides, and 10 parts each of glacial acetic acid and water.

Ointments of cantharides are much used. Their oleaginous constituents ensure solution of the cantharidin, and render them easy of application. Many contain a number of ingredients, but the simplest are usually the best. A useful ointment of medium strength consists of one part of powdered cantharides to six of benzoated lard, palm oil, or vaseline. A stronger ointment is made with one ounce each of mercury biniodide and cantharides, and eight ounces of vaseline or benzoated lard. Such an ointment, when well made and applied with smart friction, acts effectually. Another excellent ointment is made with one part each of powdered cantharides, Venice turpentine, and resin, with four parts of vaseline, palm oil, or lard. The powdered flies are digested with the oily matters in a covered vessel, over a slow fire or a water-bath, for twelve hours, and the vessel placed in boiling water for fifteen minutes; any wax or resinous matters used to give consistence are then melted and stirred in, any volatile flavouring oil added, and the mixture, if required, strained through muslin.

French, German, and Belgian practitioners frequently, however, add other irritants to their cantharides blisters. Degive states that numerous experiments convince him that the best vesicant is made of ten to fifteen parts each of cantharides and, corrosive sublimate dissolved in one hundred parts of vaseline. The part is prepared by clipping the hair, and washing with soap and water. The ointment is rubbed in for ten minutes, and, if needful, six hours later 
the surface may be simply anointed. Swelling and vesicles appear usually within a few hours; the vesicles are as large as pigeons' eggs; but by the second day inflammation subsides, and blemishing does not occur.

In cattle practice, counter-irritation is generally produced with mustard paste or blister, but some powder, or strong ointment, of cantharides, mixed with the mustard, greatly increases its effects. For dogs, a convenient ointment is made with an ounce each of powdered cantharides and oil of turpentine, and twelve to twenty ounces of lard.

To ensure full vesication, the hair should be removed, the skin washed with soap and water and dried, and the ointment then well rubbed in. The extent of surface to be covered must obviously depend upon the nature, seat, and extent of the malady. "To prevent the blister, when liberally applied, from spreading beyond the desired limits, the blistered area may be surrounded with an edging of resin ointment. The blister, while acting, often causes considerable irritation, and the animal, if permitted, will rub or bite the blistered part. In the horse this should be prevented by securing the head to the rack, putting on a cradle, or, when required, tying up the tail ; in the dog, by the use of the muzzle. On the next, second, or third day, the blistered part should be dressed with zinc oxide ointment, oil, lard, vaseline, or Carron oil. If sufficient effect has not been produced, a little more of the blister may then be applied.

Liniments of cantharides are merely liquefied ointments, and, in respect of activity, usually occupy a place between ointments and tinctures. They generally consist of one part of cantharides and six to ten parts of rape or linseed oil. Oil of turpentine is sometimes added. The B.P. Liquor epispasticus is made with ten of cantharides and twenty of acetic ether.

Plasters of cantharides are made in the same manner as ointments, but rendered more strongly adhesive by the addition of yellow wax, soap plaster, and resin or pitch. To prevent displacement they are usually applied in the melted state, immediately covered by a little tow or teased lint, and enveloped in a suitable bandage. 


\section{COD-LIVER OIL}

Oleum Morrhuse. Oleum Jecoris Aselli. The oil extracted from the fresh liver of the cod, Gadus morrhua, by the application of a temperature not exceeding $180^{\circ} \mathrm{Fahr}$. ; and from which solid fat has been separated by filtration at about $23^{\circ} \mathrm{Fahr}$.

The chief supplies of cod-liver oil come from Newfoundland. An oil called candle-oil, prized by the Indians as a tonic, and used along the Pacific coasts, is obtained from the oslachan or boulican, which inhabits the waters of British Columbia and Vancouver's Island. Good samples of cod-liver oil have a pale yellow colour, and an oily, fishy taste, which becomes, however, less obvious to those accustomed to take it. The dark colour and nauseous flavour of indifferent specimens result from exposure to high temperatures, or from the oil being extracted from stale, putrid livers. Specific gravity, 0.920 to 0.930 ; ether dissolves it readily ; cold alcohol dissolves 2 to 3 per cent.; hot alcohol, 3 to 7 per cent. It consists of olein (85 per cent.), varying proportions of palmitin, myristin, and stearin ; traces of four volatile and two fixed alkaloids, morrhuic acid, with biliary and other organic bodies containing traces of phosphorus, iodine, bromine, and chlorine. A drop of sulphuric acid, added to a few drops of cod-liver oil in a porcelain cup, develops a violet colour, which passes to yellow or brown-red, depends upon the presence of biliary matters, and indicates the source, but not the purity or goodness of the oil.

ACTIONS AND USES.-Cod-liver oil is nutrient, tonic, and alterative. Like other fixed oils, large doses cause nausea, derangement of the bowels, and purgation. For lubricant purposes, vegetable and mineral oils are more convenient and less liable to rancidity.

In experiments with cod-liver oil, made by an Essex agriculturist, on pigs, sheep, and cattle, twenty pigs, separated from a lot of three hundred, averaging from five to fifteen stones, received two ounces of oil daily, with as much meal as they cleared up. The rest of the lot were 
treated in exactly the same manner, but got no oil. Those receiving the oil are stated to have consumed less food, and when killed 'weighed the heaviest and made the most money in the London market, the fat being firm and white. When the daily allowance of oil was increased to four ounces per day, the fat became yellow, and the flesh acquired a fishy taste.' For small pigs, an ounce daily was found the most economical quantity. An ounce given daily to sheep improved the quality both of the fat and flesh; while cattle receiving about half a pint daily are stated to have eaten less food, and paid better, than when treated in the usual way. The oil, it is mentioned, cost from 2s. $8 \mathrm{~d}$. to 3s. per gallon, and in some comparative experiments it is said to have proved superior to sperm oil. These experiments confirm the recognised fact that oleaginous materials are essential to speedy and economical fattening; they do not, however, establish the individual superiority of codliver oil. In healthy animals equally satisfactory results would probably be obtained from the use of linseed, lard, rape, or other mild fixed oil.

MEDICINAL USES.-The biliary constituents of cod-liver oil facilitate its emulsion and digestion. Experiments show that admixture of a little bile hastens absorption of any bland oil included in a loop of intestine. Cod-liver oil is also very readily oxidised. This ready absorption and assimilation render it specially useful not only for children, but for young animals, in cases of malnutrition and convalescence from exhausting disease. Added to skim milk, in quantities of three to four ounces per gallon, it is very useful as a food for calves in place of new milk. Although it has no specific action on any particular organ, it improves general nutrition. Two-ounce doses, given twice daily, usually benefit delicate horses, thriving badly after strangles and influenza. 'In chronic catarrh and bronchitis, it appears to furnish suitable material for the formation of mucous cells and the repair of the inflamed mucous membrane' (Brunton). Like other oils, it materially relieves horses suffering from broken wind. It helps recovery of eattle reduced by diarrhœa, anæmia, or rheumatism, but for many such cases in horses, cattle, and sheep, 
linseed or linseed cake is preferred. For dogs and cats it is useful in protracted cases of distemper, eczema, and other inveterate skin diseases ; in epilepsy, chorea, rickets, and in chronic rheumatism, especially that variety known as kennel lameness, and depending upon bad feeding, faulty nutrition, and damp quarters.

Doses, etc.-Horses take $f z^{i j .}$; cattle, $f z^{i j}$. to $f \xi i v . ;$ sheep, about $f z_{j}$. ; pigs, fZiv. to $f z i$. ; dogs, fZi. to fZiv. ; cats, about $\mathrm{f} 3 \mathrm{i}$. The doses may be repeated twice daily, and persevered with, if required, for weeks ; but if diarrhœea result, they must be reduced or discontinued for a day or two. To remove disagreeable flavour, and prevent nausea or vomiting, it is given mixed with eggs in milk, as an emulsion with mucilage, or in gruel, conjoined with some aromatic, malt extract, or with ether, and is best digested along with or immediately after other food.

\section{A R D}

Adeps. Adeps Preparatus. Axunge. The purified fat of the hog-Sus Scrofa.

To prepare purified lard, the fat from the hog's internal organs is cut into small pieces, triturated in a stone mortar, washed with cold water, drained, melted over a slow fire, strained through flannel or coarse cheese-cloth; is kept stirred in a steam-heated pan at about $130^{\circ} \mathrm{Fahr}$. until it is clear and free from water, strained again through flannel, and preserved in casks, pots, or bladders. When pure, it is white or yellowish-white, granular, neutral to litmus without rancid odour, but with a sweet taste. It melts at about $100^{\circ} \mathrm{Fahr}$., forming a clear, transparent fluid, which is a good solvent for wax and resin, and when boiled with alkalies forms soaps. Like other fats and oils, lard is insoluble in water, slightly soluble in alcohol, but perfectly soluble in ether. Exposed to the air, it becomes rancid, and in this state is unfit for emollient purposes. It contains about 62 per cent. of olein and 38 of palmitin and stearin. Distilled water, in which purified lard has been boiled, when cooled and filtered, gives no precipitate 
with silver nitrate, indicating absence of common salt; and no blue coloration with iodine solution, proving freedom from starch, of which about 20 per cent. is found in some inferior samples; 10 per cent. of water is sometimes incorporated; alum and lime are occasionally added to secure whiteness and increase weight; while many brands of American lard are largely mixed with cotton-seed oil.

Benzoated lard, preferable on account of its agreeable odour and diminished liability to rancidity, is made by melting one pound of purified lard over a water-bath, stirring in two hundred and ten grains of benzoin and afterwards straining. Suet - the fat around the kidneys of sheep or oxen-differs from lard chiefly in being firmer, harder, and more difficult to melt. Horse fat is more easily melted, but firmer than that of swine. Goose grease, much used as a popular remedy for sprains and bruises, is more fluid, from its greater percentage of olein.

Actions AND Uses. - Fats and mild fixed oils, when given without other food, are inadequate to support life ; thus, dogs, receiving only butter and olive oil, with distilled water to drink, died in about thirty-six days. In a well-regulated system of diet, fats serve, however, important purposes; along with albuminoids they form cells ; they build up the nervous structures, so largely composed of fatty matters ; are consumed in the body for the evolution of nervous, muscular, or digestive force, and for the support of animal heat, or, if in excess of these constant requirements, are stored away, investing and protecting internal organs. Fats are emulsified by the alkaline intestinal secretions, more thoroughly dissolved by the bile, and absorbed mainly through the lacteals. Although small doses are easily assimilated, large quantities disorder digestion and cause diarrhœa.

Hog's lard is occasionally used as an internal demulcent, as an antidote for poisoning with alkalies, and as a laxative clyster. It is applied as a lubricant in exploration of the rectum or uterus, and in cases of difficult parturition. In reducing enlarged joints or bursæ by vigorous rubbing, the hand is occasionally moistened with lard to prevent undue skin irritation. When the skin is congested, inflamed, 
thickened, or indurated, the sebaceous and sudoriparous glands being impaired, the application of bland oil, lard, or vaseline usefully replaces the deficient natural oil, and protects abraded surfaces from the action of air or of acrid discharges. Most animal and vegetable fats, freely used, and remaining long in contact with the warm skin, oxidise and become rancid and irritating. Such results are retarded by addition of a little benzoin, and are obviated by the substitution of the mineral vaseline. Lard is occasionally employed as a dressing in mange and scab, but is ineffectual in destroying the acari.

Adeps Lanæ or wool-fat, the purified cholesterin-fat of sheep's wool ; and Adeps Lanæ Hydrosus, hydrous woolfat or 'Lanoline,' promote the absorption of remedies applied to the skin. The official hydrous wool-fat is prepared by melting seven ounces of wool-fat and adding three ounces of distilled water. It is stable, unirritating, not rancid, and accordingly makes a good protecting lubricant. It mixes readily with other fats and oils, with starch, bismuth, zinc oxide, creolin, iodine, salicylic acid and many other antiseptics, and more water may be added without affecting its use as a basis for ointments and liniments. For stock ointments a good combination consists of sixtyfive parts hydrous wool-fat, thirty of liquid paraffin, and five of ceresin (hard white paraffin). Adeps Induratus, or lard deprived of a portion of its oil by pressure, is employed as a substitute for ordinary lard in hot climates (B.P.).

\section{ADRENALIN}

Adrenalin $\left(\mathrm{C}_{9} \mathrm{H}_{13} \mathrm{NO}_{3}\right)$ is the active principle discovered by Takamine in the medulla of the suprarenal gland. SUPRARENIN, a synthetic preparation having similar properties to the animal product is also employed in medicine.

Adrenalin occurs as a white crystalline powder, with a faintly bitter taste ; insoluble in alcohol and ether ; slightly soluble in cold water, more soluble in hot water, and readily dissolved by dilute acids. It is very stable in the dry state, 
and keeps well in weak acidulated solution, but neutral solutions slowly undergo alteration. Adrenalin chloride solution ( $1: 1000)$, containing one part adrenalin chlorideand five parts chloretone in a thousand parts of normal salt solution, is generally used in veterinary practice. This preparation should be kept in well-stoppered bottles, in a cool, dry place, as exposure to air and light, by.inducing oxidation, gradually destroys its value.

Actions AND UsES.--Solution of adrenalin chloride has little or no action on the unbroken skin. When applied to abraded surfaces or mucous membranes, or when injected subcutaneously, it causes local constriction of the arterioles and capillaries, with the result that the part becomes pale and bloodless.

Internally, when injected intravenously, even in minute doses, it causes a general constriction of the smaller bloodvessels throughout the body, with the exception of those of the lungs and brain. This action is believed to be due partly to the excitation of the sympathetic nerves of the part, and partly to direct action on the muscle fibres in the walls of the vessels. Adrenalin also slows and strengthens the heart's action, and causes an extremely rapid rise of blood-pressure, much in the same way as does digitalis. This rise of blood-pressure is chiefly due to the action of the drug in stimulating the sympathetic vaso-constrictor nerves, in constricting the muscular coats of the peripheral arterioles, and to its tonic effects on the heart muscle, strengthening the systole. A very small amount injected intravenously will increase the blood-pressure immediately, but its action is very transient, and the dose requires to be repeated at short intervals. On the other hand, little or no systemic effect is to be expected from its administration orally or per rectum, as it becomes quickly oxidised in the tissues. Besides its effects on the circulatory apparatus, adrenalin affects several other forms of involuntary (unstriated) muscle. Thus, intestinal and stomach movements are inhibited, whilst the ureters, vesiculæ seminales, uterus and vagina all show contractions. Again, the pupil of the eye is dilated, the membrana nictitans is retracted, and the eyeball protruded. All these effects can be ob- 
tained experimentally by stimulation of sympathetic nerves, and it is by such a stimulation that the action of adrenalin can be best explained. Even its action on the heart is in line with this theory, for although it slows the heart when given intravenously, this is due to the rapid rise of blood-pressure. In an isolated heart, or with divided vagi, the first effect of adrenalin is to quicken the heart by sympathetic stimulation.

TOXIC EFFECTs.-Excessive doses cause vomiting in dogs, muscular tremors, rapid breathing, paralysis of hind extremities, dyspnœa, with failing respiration, congestion, with visceral œedema and hæmorrhage, and serous effusion.

MEDicinal Uses.-In veterinary practice adrenalin, though sometimes administered in systemic disease, is mainly employed as a local hæmostatic in minor operations. Injected hypodermically, it renders the part ischæmic and the operation comparatively bloodless. It must be remembered, however, that it will not constrict the larger vessels, but, by the absence of blood, these are more easily detected and ligatured, before being severed. For surgical purposes adrenalin is usually combined with a local anæsthetic such as cocaine, eucaine, or stovaine. Adrenalin intensifies the action of the anæsthetic and limits its toxic effects, for by constricting the arterioles of the part, the entrance of the anæsthetic into the general circulation is retarded or prevented, and its physiological action localised and concentrated.

In red-water in cattle and hæmoglobinuria in horses, Zehl has obtained successful results from the use of a $1: 10,000$ solution, in doses of five to eight fluid drachms injected hypodermically. He has also found this solution beneficial in laminitis, in which he injected 80 minims inside and outside each fore fetlock. In hæmoglobinuria, Budd gave one drachm of adrenalin chloride solution (1:1000) in three ounces of water, and repeated this dose every three hours ; recovery followed. Its use has been suggested in purpura hæmorrhagica to diminish extravasation. Adrenalin is of undoubted value as a hæmostatic in hæmorrhage, but it must be brought in contact with the bleeding part. It is indicated in bleeding from the nose, stomach, bowels, uterus, vagina, 
urethra, etc. In conjunctivitis, iritis, and other inflammatory conditions of the eye, solution of adrenalin chloride $(1: 10,000)$ is very beneficial in controlling congestion, reducing tension and relieving photophobia. In laryngitis, especially in the dog, adrenalin solution, applied as a fine spray, relieves the congestion and diminishes odema and irritation. In cases of shock and collapse, intravenous injections should be used. For this purpose moderate doses of adrenalin chloride solution $(1: 1000)$, diluted with at least twenty volumes of normal salt solution (pure sodium chloride grs. 80 in 20 ounces of boiled water), may be injected repeatedly at short intervals. Crile observed that adrenalin acted upon the blood-vessels after the circulation had ceased, and after experimentally asphyxiating an animal, practised artificial respiration, and at the same time administered adrenalin in saline solution into the jugular vein. By this method animals apparently dead, for various periods up to fifteen minutes, were restored to conscious life. Adrenalin injected intravenously into the femoral or other superficial vein should be valuable in chloroform intoxication, and in cases of sudden heart failure, and collapse from shock.

Doses, etc.-Adrenalin chloride solution (1:1000) by the mouth, horses and cattle, $3 \mathrm{j}$. to $3 \mathrm{jv}$. ; dogs, Mv. to mlx. ; cats, $m_{i}$. to $m_{v}$. Hypodermically, half the above doses may be given. Intravenously (1:10,000 solution), horses and cattle, $m_{x v}$. to $m_{x x x}$; dogs, $m_{i j}$. to $m_{\text {viii. }}$ These doses may be increased in certain cases, but as the maximum doses, which may be administered without danger to the domestic animals, are undetermined, caution should be exercised in prescribing adrenalin.

\section{GELATIN}

Gelatinum.-The air-dried product of the action of boiling water on such animal tissues as skin, tendons, ligaments, and bones (B.P.).

Gelatin is made from damaged hides and skins, and their parings; also from bones, limed, cleaned, and boiled, to remove fatty matters, and then crushed and steamed in a 
partial vacuum. Glue, a coarse variety of gelatin, is made from similar materials, less carefully purified; size is an inferior, weaker variety of glue ; isinglass, a natural colourless gelatin, is prepared from the air-bladder of the sturgeon, and various species of Acipenser ; chondrin is the gelatinous matter extracted from cartilage; ossein, the title given to that obtained from bones. Gelatin, when dried, is hard and tough; varies in colour according to its purity; forms a viscid, tremulous mass, even when one per cent. is dissolved in water and allowed to cool ; and is precipitated from watery solutions by tannic acid. Gelatin is insoluble in alcohol, and ether. It dissolves in acetic acid.

ACTIONS AND USES.-Gelatin, although a product of the disintegration of albuminoid tissues, does not build up the albuminoid or even the gelatinous tissues; but being tolerably easily digested, it appears to economise the more valuable albuminoids. Men, dogs, and even horses, recovering from exhausting diseases, in which disintegration and excretion of proteids is great, exhibit the dietetic value of gelatin when it is given as soup, with fats or carbo-hydrates. As a demulcent it has the disadvantage of becoming hard and dry, and hence is not very suitable as a permanent sheathing for irritable surfaces. Gelatinised serum, consisting of fifty parts sterilised gelatin, ten parts calcium chloride, and a thousand parts of water, is used as a local hæmostatic ; and for internal hæmorrhage a solution of eighty grains white gelatin, in seven to nine ounces of sterilised sodium chloride solution (7 per 1000), may be employed hypodermically in doses of $\bar{\jmath} \mathrm{ij}$. to $\bar{z}$ viij. for horses ; and $3 \mathrm{ij}$. to $\jmath_{\mathrm{j}}$. for dogs.

Glue, mixed with an antiseptic, is employed for securing the broken horns of cattle, and occasionally for making adhesive plasters. For closing wounds, where sutures cannot be used, two pieces of stout cloth are cut so as to leave a number of tails with uncut margins of several inches, and are smeared with melted glue, usually mixed with pitch, and applied, one on either side of the wound, with the uncut margins towards each other. When the plaster is dry, the uncut margins are sewed together, while, to prevent displacement from movement of the skin, narrow strips of 
calico moistened with glue are applied in various directions over the injured spot. Such plasters are sometimes useful in keeping sutured wounds in position, giving support, and preventing annoyance by flies. They are sometimes effectual in the treatment of umbilical hernia in calves and foals.

Gelatin solutions make useful temporary protectives for limited abraded surfaces; hold well for a day or more if applied dry ; are readily washed off with hot water, without irritating the skin, and can be medicated with antiseptics, stimulants, or analgesics. Frick's wound gelatin is made with gelatin soaked in sufficient one per cent. sublimate solution to cover it ; after it has become soft it is dissolved by heat, and glycerin equal in quantity to the tenth part of the dry gelatin is added. It is warmed before use and applied with a brush or a pledget of cotton. The familiar court sticking plaster consists of a strong solution of isinglass painted over thin silk. In pharmacy gelatin is used for clarifying or fining; as a neat and cleanly capsule for pills and boluses; and along with glycerin forms lamellæ or thin dises for enveloping the alkaloids and other active principles used for eye cases and hypodermic injections.

\section{GL Y CER I N}

GLYCERINUM, or Glycerol, is a trihydric alcohol, $\mathrm{C}_{3} \mathrm{H}_{5}(\mathrm{OH})_{3}$, associated with a small percentage of water; it is obtained by the interaction of alkalies, or of superheated steam, with fats and fixed oils (B.P.).

Glycerin was discovered in 1789 , by Scheele, as a product in the manufacture of lead plaster; it occurs in small amount during the fermentation of sugar, and as a byproduct in the manufacture of soap and stearin candles.

Properties.-It is a clear, colourless, syrupy liquid, devoid of odour, sweet to the taste, oily to the touch, with a specific gravity of $1 \cdot 260$. It has a strong affinity for water, and is freely soluble in water and alcohol. Insoluble in ether, chloroform, and fixed oils. It dissolves most substances which are soluble in water, as well as metallic oxides, and alkaloids. It burns with a luminous flame, 
giving off irritating vapours of acrolein. Cautiously added to a mixture of equal measures of nitric and sulphuric acids it produces nitro-glycerin $-\mathrm{C}_{3} \mathrm{H}_{5}\left(\mathrm{NO}_{3}\right)_{3}$.

ACTIONS AND USES.-Glycerin undiluted irritates the mucous membranes, and even the skin by abstracting water. Hence its laxative effect, whether when swallowed or used as an enema. Dogs are purged by $1 \frac{1}{2}$ ounces. Large doses are toxic in animals, causing restlessness, quickened pulse, and respiration, and later tremors and convulsions, ending in coma and death by paralysis of respiration. They break down the red globules and cause hæmoglobinuria. especially if injected subcutaneously. Externally, it is antiseptic, and destroys fleas and other skin parasites. Mixed with water, starch, or other bland materials, it is demulcent and emollient. It is the basis of the emollient, antiseptic, and astringent group of glycerina. It is a solvent for fixed alkalies, for alkaloids and their salts, vegetable acids, iodine, bromine, salicin, etc., a frequent constituent of ointments and lotions, and a convenient menstruum for the administration of nauseous medicines.

It is absorbed fairly rapidly from the intestine and is oxidised in the tissues, but is of little value as a nutrient. Small doses are eliminated by the kidneys, larger by the bowels, producing slight laxative effects, increased by combination with castor oil. As a laxative enema it is not more effectual than oil, or soap and water; indeed, Friedberger states that it has no effect on dogs. Given shortly before meals it has been prescribed to check undue gastric fermentation, acidity, and flatulence, both in calves and dogs. It neither evaporates, nor becomes rancid, and is readily miscible with water, spirit, oils, and other drugs, forming with them emollient, antiseptic, or astringent dressings, used to soften, supple, or soothe dry, rough, scurfy, or irritated skin surfaces. It is the basis of many applications for blisters and burns, for cracked heels, and various forms and stages of eczema. Doses,- -horses and cattle, $ろ$ j. to Zij.; dogs, 3ss. to $3 \mathrm{j}$.

Glycerin of starch is made by stirring and heating gently one of starch, one and a half of water, and six and a half of glycerin, and is used for aphthous and other eruptions about 
the mouth, nostrils and udder, and as a soothing dressing for erythema and the early weeping stages of eczema, especially in dogs. The glycerina of carbolic and tannic acids are prepared with one part of the acid and five of glycerin. Glycerin of alum is made with one of alum, three of distilled water, and six of glycerin. Similar antiseptic and astringent preparations are made with glycerin and borax, and also with glycerin and acetate and oxide of lead, and water to effect thorough solution. A handy antiseptic and astringent application is extemporised by mixing equal parts of glycerin and Goulard's Extract. It is diluted as required, and used for erythematous and eczematous conditions of the skin. Boro-glyceride, prepared by heating ninety-two parts glycerin and sixty-two boric acid, and used diluted with twenty to forty parts water, is an effectual non-poisonous antiseptic, useful for relaxed conditions of the throat, as a dressing for wounds, for the preservation of food, and as a vehicle for applying carbolic acid, iodine, iodoform, and belladonna.

For various itching, erythematous, and eczematous diseases, a soothing, drying protective is prepared by mixing, with the aid of gentle heat, three parts each of glycerin, gelatin, and water, with one of zinc oxide ; and with such a mixture may be incorporated, as required, creosote, carbolic acid, resorcin, naphthol, or dermatol.

\section{SOAPS}

Sodium or Hard Soap. Sapo durus. Sodium Oleate. Potassium or Soft Soap. Sapo mollis. Potassium Oleate. CURd Soap. Sapo animalis. Sodium Stearate, with about 30 per cent. of water. (B.P.)

Soaps consist of alkali salts of higher fatty acids. Hard soaps are made by boiling palm or cocoanut oil or tallow with sodium hydroxide or carbonate, when the alkali unites with the fatty acids, displacing the basic glyceryl. Common salt added to the gelatinous boiling ley separates the soap in flakes, which are collected, run into moulds, and dried. Such soaps are a mixture of sodium stearate, with about 
one-third of oleate, and thirty to sixty per cent. of water. To make yellow soap, the ley, while being concentrated, is treated with considerable quantities of resin. Many soaps are now made with a large percentage of silicates. Mottled and marbled soaps owe their colour to the presence of a little iron. Glycerin soap, prepared by heating the soap ley with water for two or three hours at $400^{\circ} \mathrm{Fahr}$., contains a mixture of soap and glycerin. The costly Castile and some Pharmacopœia soaps are made with purified animal fat, or olive oil, and sodium or potassium hydroxide. Soft or potash soaps are made by boiling seal or whale oil with potassium hydroxide or carbonate, and gradually evaporating to the required consistence. Soaps for medicinal purposes are now made superfatted, neutral, or alkaline, in cake and powder, with admixture of carbolic acid, creolin, resorcin, sulphur, menthol, eucalyptus, balsams, camphor, sanitas, etc.

Soaps have an alkaline, acrid taste, dissolve readily in water and spirit, but should not impart an oily stain to paper. When heated, they fuse, swell up, and leave charcoal and carbonate of their alkali. Calcium and magnesium salts, such as occur in hard waters, decompose soap ; the fatty acids form insoluble flakes of stearate and oleate of calcium and magnesium ; soap is hence used as a test for the hardness of water.

Actions AND Uses.-Soaps contain some free alkali, and hence are slightly irritant. They are mildly laxative, diuretic, emetic, and antacid. They form convenient adjuncts to more active laxatives or diuretics, and are serviceable additions to laxative clysters. Externally, they are used as stimulants, detergents, and lubricants, and in pharmacy as excipients.

Soap and warm water are in everyday use for cleansing the skin, removing scurf, neutralising acrid fatty matters, keeping open the orifices of sebaceous glands, promoting growth of hair, as well as preparing the skin for operations, blisters, and parasiticides. When erythema is produced by badly-fitting harness or other causes, irritation is abated by rubbing the parts with a neutral soap, and subsequently dressing with vaseline, or sugar of lead lotion, or with 
'Sanitas' or other soothing antiseptic powder. Gently rubbed over slight burns or scalds, soap prevents access of air and relieves irritation. In chronic eczema, soft soap, from its lubricant and alkaline properties, is often useful. For such cases a convenient dressing is made with equal parts soft soap and glycerin, half a part of zinc oxide, and six or eight parts water. For eczema and other itching skin diseases, $\mathrm{M}^{\mathrm{C}} \mathrm{Call}$ Anderson prescribed equal parts of soft soap, oil of cade, and rectified spirit. As a stimulant for bruises and strains, or for producing counter-irritation in sore-throat, six ounces of hard soap, cut into small pieces, are macerated with six fluid ounces of dilute liquor ammoniæ and one pint each of alcohol (60 per cent.), and linseed oil; two or three ounces of camphor are sometimes added. Soap and water is much used for clysters. As internal antacids, soaps are less effectual than alkaline carbonates or bicarbonates, but are occasionally administered in poisoning by acids and metallic salts. Soap and water causes emesis in dogs. Soaps are used as excipients for boluses, and as constituents of liniments and plasters.

\section{KERATIN}

A mucilaginous solution which, when dry, is a yellow gumlike substance, insoluble in gastric juice, but soluble in the intestinal juices, and used for coating boluses or pills which are required to pass through the stomach unchanged, and to undergo solution in the intestines.

Keratin is prepared by digesting horn-turnings with acidified pepsin solution, so long as they yield any soluble matters. The residue is then digested in solution of ammonia or acetic acid, and evaporated. Keratin requires to be redissolved before use. The ammonia solution is generally used, but the acetic acid solution is suitable for those drugs which might be decomposed by ammonia. The medicines, made into bolus in the usual way, are generally thinly covered with cacao butter, and then 
coated twice with keratin. This method of dispensing is useful-

(1) For drugs which irritate the gastric mucous membrane - such as anthelmintics, arsenic, creosote, salicylic acid, phosphorus, and the more soluble iron salts.

(2) For such substances as impair digestion in the stomach by precipitating pepsin and peptones-e.g., tannic acid, alum, lead acetate, silver nitrate, corrosive sublimate, etc.

(3) For such substances as are rendered inert by the gastric juice, or are undesirably acted upon by it-e.g., alkalies, soaps, bile, silver nitrate, iodides, intestinal antiseptics, etc.

(4) For medicines which it is desired to introduce into the bowel in as concentrated a form as possible-e.g., kousso, male fern extract, santonin, bile, alkalies, and silver nitrate, lead acetate, or tannin, when their local action is sought to be applied in ulceration or hæmorrhage of the intestines (Brunton).

On pills and boluses for monogastric animals, a coating of fresh mutton fat will be found more convenient than keratin solution, and quite as serviceable.

\section{PEPSIN}

Pepsinum. An enzyme obtained from the mucous lining of the fresh and healthy stomach of the pig, sheep, or calf. It should dissolve 2500 times its weight of hard-boiled white of eggs (B.P.).

Pepsin is prepared by several processes. The stomach is digested in water acidulated with hydrochloric acid, and the pepsin thus extracted is precipitated by sodium chloride. More commonly the mucous surface is cleansed, slightly washed, the surface scraped with a blunt knife, and the viscid pulp thus obtained dried at a temperature not exceeding $100^{\circ}$ Fahr. Pepsin occurs as a light yellowish brown or white powder or in grains or scales, having a faint odour and a saline taste. Soluble in water and in 100 parts of rectified spirit. 
ACTIONS AND UsES.-Pepsin dissolves proteids and converts them into peptones, but does not affect fats or carbohydrates. Its therapeutic value is hence limited to young herbivora while receiving milk, and to dogs living chiefly on animal food. In such patients it is given along with or immediately after meals. When gastric secretion in the domestic animals is at fault, it is more probably from deficiency of the acid than the pepsin, and such a condition is appropriately treated by diluted hydrochloric acid, administered with or after meals.

Pepsin is sometimes used to dissolve fatty and malignant tumours, into which it is injected mixed with a few drops of hydrochloric acid. The B.P. Liquor Pancreatis, containing the digestive principles of the fresh pancreas of the pig, is sometimes given instead of pepsin.

Doses, etc.-Foals, calves, and dogs take of pepsin, grs. $x$. to $3 \mathrm{j}$., usually given in water with a few drops of hydrochloric acid. The pepsin wines and essences seldom contain much of the ferment. The glycerin of pepsin contains five grains of pepsin in each drachm, with a small proportion of hydrochloric acid.

Papain, a vegetable pepsin, is obtained from the unripe fruit and leaves of the Papaw (Carica papaya), a plant found in the East and West Indies. It is a tasteless, greyish-white amorphous powder; soluble in glycerin. While the animal pepsin acts only in acid solutions, the vegetable papain is equally effectual in acid, neutral, and alkaline solutions. A five per cent. solution is stated to dissolve false membranes, and a ten per cent. solution has been employed to destroy small malignant growths. Doses, foals, calves, and dogs, grs. ii. to grs. xii.

\section{ICHTHYOL}

Ichthyol (Ammoniumsulphoichthyolate) is a reddishbrown, tarry liquid, obtained from a Tyrolese bituminous shale- the fossil remains of decomposed aquatic animals, by distillation, and by treating the product with sulphuric acid and ammonia. It is soluble in water, glycerin, alcohol, benzol, and fixed oils. Purified, it yields a clear oil with 
a strong empyreumatic smell. It is antiseptic, causes contraction of the vessels when locally applied, and is a parasiticide. Its properties ally it to the phenols. It has been used by French and German veterinarians for the destruction of the parasites of scab and mange; and as a stimulant and resolvent for swollen joints, strains, and contusions. For such purposes one part is dissolved in ten of oil or lanoline, and rubbed in daily for several days. Ichthyol liniment is made with ten parts dissolved in thirty parts each of alcohol, ether, and distilled water. Thiol is a synthetic preparation of hydrocarbons obtained in a similar manner to ichthyol, for which, in the treatment of skin diseases, it is sometimes substituted. Thiol occurs in powder and in liquid, and is employed in the form of ointment (containing 10 to 50 per cent.), and of dusting powder with starch or other desiccant. The powder is soluble in water and in alcohol. Tumenol (sulphotumenolic acid) is obtained from mineral oils by treating the unsaturated hydrocarbons with sulphuric acid and sodium chloride. The resulting tumenol-sulphonic acid on being further treated yields tumenol-sulphone or tumenol oil, from which is prepared the pharmaceutical powder. Tumenol is dark, odourless, slightly bitter, and soluble in water. In pruritus, eczema, and other skin diseases it is employed in the forms of ointment, dusting powder, and lotion (5 to 10 per cent.). The solution may be made with water, glycerin, or alcohol (Coblentz).

\section{SPERMACETI}

Cetaceum. A concrete, fatty substance obtained, mixed with oil, from the head of the sperm whale (Physeter macro-cephalus). It is separated from the oil by filtration and pressure, and afterwards purified (B.P.).

Spermaceti is found in the cells of the large quadrangular head of the sperm whale, which inhabits the Pacific and Indian Oceans. It is extracted by openings made through the skull, and occasionally by boiling the cellular and adipose tissues. When purified, it is a translucent, pearlywhite, crystalline fat, tasteless, odourless, tough, and diffi- 
cult to powder, unless moistened with a few drops of rectified spirit. It is insoluble in water, sparingly soluble in cold alcohol, readily soluble in hot alcohol, chloroform, and oils, and melts at $114^{\circ}$ to $122^{\circ}$ Fahr. With a little sperm oil, it consists of cetyl palmitate, which, unlike ordinary fats, is saponified with some difficulty, does not yield glycerin, but forms, when heated with an alkali, the crystalline cetyl alcohol $\left(\mathrm{C}_{16} \mathrm{H}_{33} \mathrm{OH}\right)$, and an alkaline palmitate.

Actions AND USES. - It is emollient and demulcent, resembles wax, is rarely given internally, but is used for imparting consistence to ointments and plasters.

\section{W A X}

Cera Flava. Yellow Beeswax.

Cera Alba. White Beeswax.

Prepared from the honeycomb of the hive bee, Apis mellifica. Order.-Hymenoptera.

Beeswax is furnished from the glands on the ventral scales of the bee. After removal of the honey, the comb, pressed, fused in boiling water, strained, and poured into moulds, constitutes yellow wax, which has a dull yellow colour, a granular fracture, a slightly sweet and pleasant taste and odour; it should be free from greasiness; it is insoluble in alcohol, but entirely soluble in oil of turpentine. White wax is made by melting yellow wax with steam, straining, and decolorising it by exposure in thin ribbons to air and sunshine, or by boiling with nitrate of soda and sulphuric acid.

Wax has the specific gravity 960 to $\cdot 965$, is tough and solid, insoluble in water, soluble in fixed and volatile oils, melts at about $145^{\circ} \mathrm{Fahr}$., and readily unites with fat and resins. It consists of nearly two-thirds of cerin, an imperfectly saponifiable waxy substance; about one-third of myricin or myricyl palmitate, a body analogous to spermaceti, and about 5 per cent. of cerolein, a soft acid fat. Chinese wax is the product of an insect of the cochineal tribe. Wax is also produced by several plants. 
Actions And Uses.-Wax, although allied to the fats, is much more difficult of digestion, less nutritive, and less demulcent and emollient. In medicine, its chief use is to increase the consistence and prevent rancidity of ointments, cerates, and plasters. Yellow wax, mixed with hogs' lard, or any of the bland fixed oils, is much used for investing abraded or irritable surfaces, protecting the sound skin from acrid discharges, and preventing corrosives or blisters extending their effects beyond the parts to which their action is to be limited. Unguentum simplex is usually made with one part of yellow wax to four of prepared lard, or one part of wax to one and a half each of almond oil and benzoated lard.

\section{MALLEIN}

Mallein employed for the diagnosis of glanders in animals, is the sterilised and filtered liquid-culture of glanders bacilli. Mallein therefore does not contain even dead bacilli, but it has in solution certain substances which are added to the liquid by the bacilli during their growth (Sir John M'Fadyean).

Directions for using Mallein, as prepared and supplied by the Royal Veterinary College, London :-

1. While under the mallein test horses ought to be left at rest in the stable and protected from draughts. The rectal temperature ought to be taken once or twice on the day before the test is applied.

2. The dose of mallein for a horse is one cubic centimetre, or 18 minims. It ought to be injected about the middle of the side of the neck, with a clean hypodermic syringe. The best form of syringe is one with an asbestos piston, as the whole instrument may then be sterilised by boiling it in water for five minutes before use.

3. The mallein must be injected into the subcutaneous connective tissue, and care must be taken that the whole dose is actually introduced.

4. The temperature must be taken at the time of injection, and at the 9 th, 12th, and 15th hours afterwards.

5. Provided the temperature was normal (under $101^{\circ}$ F.) before the injection, it will rise $2^{\circ}$ or more $\left(103^{\circ}-105^{\circ}\right)$ during the next fifteen hours if the horse is glandered, but it will remain practically unaffected (under $102^{\circ}$ ) if the horse is not glandered.

6. Attention must also be paid to the swelling that forms at the seat of injection. When the horse is glandered this goes on increasing in size during the second twenty-four hours after the injection, and it seldom declines before the third or fourth day. The maximum diameter of this swelling in glandered horses varies from 5 to 10 inches. 
7. In horses that are not glandered the local swelling attains its maximum size during the first fifteen hours, and by the twenty-fourth hour it has almost entirely disappeared. Its maximum diameter is usually about 3 or 4 inches.

8. When the temperature gradually rises from the normal to $104^{\circ}$ during the first fifteen hours, and a large slowly disappearing swelling forms at the seat of injection, the horse may confidently be declared glandered.

9. If, with a normal temperature at the time of injection, a horse displays only the temperature reaction, or only the local reaction, the case must be considered doubtful, and the test repeated after the lapse of a week.

10. When the temperature is $102^{\circ}$ or more, at the time of injection, the temperature reaction is unreliable, but in such a case the diagnosis may be based on the characters of the local swelling.

11. The mallein should be kept in a cool place, and protected from light. Should it lose its transparency, or become cloudy, it must not be used.

\section{TUBERCULIN}

Tuberculin employed for the diagnosis of tuberculosis in animals, is the sterilised and filtered liquid-culture of tubercle bacilli (see Mallein).

\section{Directions for using tuberculin as prepared and supplied} by the Royal Veterinary College, London :-

1. While under the tuberculin test cattle ought to be kept in the shed, fed on their usual food, and protected from draughts. They ought not to be allowed to drink large quantities of cold water between the sixth and fifteenth hours after injection. It is well to take their temperature at least once on the day preceding the test.

2. The dose of tuberculin for a medium-sized cow is 3 cubic centimetres, or 50 minims, and it may be varied above or below that according to the size of the animal. Large bulls ought to receive 4 c.c.

3. It ought to be injected under the skin with a clean hypodermic syringe. The most convenient points are in front of the shoulder, or on the chest wall behind the point of the elbow. The best form of syringe is one with an asbestos piston, as the whole instrument may be sterilised by boiling it in water for five minutes before use.

4. The tuberculin must be injected into the subcutaneous connective tissue, and care must be taken that the whole dose is introduced.

5. The temperature must be taken at the time of injection, and at the 9th, 12th, and 15th hours afterwards.

6. Animals in which the temperature during the fifteen hours following the injection rises gradually to $104^{\circ}$ or more may be classed as tuberculous, and those in which it remains under $103^{\circ}$ as not tuberculous. When the maximum temperature attained is under $104^{\circ}$ but over $103^{\circ}$ the case must be considered doubtful, and the animal may be re-tested after a month.

7. The test is not reliable in the case of animals in the last stage of the disease, or in those in which the temperature is over $103^{\circ}$ before injection.

8. The tuberculin should be kept in a cool place, and protected from light. Should it become turbid or cloudy it must not be used.

9. The tuberculin test does not render the milk in any way injurious. 


\section{BLACK QUARTER VACCINE}

\section{Directions for using Black Quarter Vaccine, as prepared and supplied by the Royal Veterinary College, London :-}

Two vaccines are employed, viz., 1st and 2 nd.

The 1st vaccine is put in the tubes without a black ring.

The 2 nd vaccine is put up in the tubes with a black ring.

Each tube contains vaccine sufficient for at least ten animals.

An interval of eight to ten days ought to be allowed between the first and the second vaccination.

The apparatus necessary for the operation is :-

(1) A small mortar and pestle.

(2) A graduated hypodermic syringe with a capacity of ten c.c. The needle of the syringe ought to be about as thick as an ordinary knitting needle, and have a proportional bore.

(3) A small pointed trocar or exploring needle, which ought to be a little thicker than the needle of the syringe.

Mixing of the vaccine.- Immerse the mortar and pestle for ten minutes in water near the boiling point; have at hand a quantity of water recently boiled and allowed to cool. Rinse out the syringe first with five per cent. carbolic solution (in water), and then two or three times with boiled water.

Drain the mortar and pestle dry, and then turn into the former the contents of one of the small tubes (first vaccine for ten animals). Fill the syringe (ten c.c.) with boiled (and now cold) water. Eject a few drops of this into the mortar, and triturate the powder with it so as to form an uniform paste. Continue the rubbing, and gradually add the whole of the water in the syringe. When the powder has thus been uniformly mixed. with the water, suck the whole back into the syringe.

The operation.-Clip the hair from the under aspect of the tail for about six inches extending upwards from the tip. Wash this part vigorously with five per cent. carbolic lotion. Take the small trocar, previously purified in boiling water, and bury it under the skin on the under surface of the tail, entering it on the middle line about a handbreadth above the tip, and pushing it vertically upwards for three inches. Give the handle of the trocar a side-to-side movement so as to enlarge the gallery at its upper end. Now gently shake the syringe, insert the hypodermic needle, and inject one-tenth of the contents of the syringe ( $=1$ c.c.). Withdraw needle and syringe together, at the same time pressing firmly on the puncture in the skin. The dose mentioned ( 1 c.c. or 18 drops) is sufficient for an animal over one year old. For calves of six months the dose is 12 drops, and for nine months 15 drops.

The second vaccination is carried out after eight or ten days in the same manner, the vaccine being injected under the skin of the tail immediately above the seat of the first operation. After all the animals of one lot have been operater on, the syringe ought to be rinsed out with five per cent. carbolic solution. The operation ought not to be performed when the weather is very hot or very cold.

Every care is taken in the preparation of the vaccine, but no guarantee is given regarding its safety or efficiency.

Tetanus Antitoxin (see p. 7) is supplied in two forms, a liquid serum and a dry powder. The liquid serum, prepared 
by the Pasteur Institute and sent out in sealed vials containing 10 cubic centimetres and 20 cubic centimetres, preserves its properties for a long time when kept in a cool, dark place. Properly administered it confers immunity from tetanus for about six weeks, and this period of protection can be extended by successive injections. As a preventive of tetanus its action is certain, but as a curative it has little value in acute tetanus, though in the slowly developing case it sometimes promotes recovery. As a prophylactic tetanus antitoxin is indicated prior to or soon after dangerous operations, and as early as possible in diseases or wounds which may be considered as particularly exposed to infection by the tetanus bacillus. It is usually administered subcutaneously after careful disinfection of the skin at the point of injection; but intracranial injection, which assures immediate action of the antitoxin on the nervous tissue, should be preferred when practicable. It has been given intravenously ; and occasionally it is injected into a wound which may be suspected as the seat of primary infection.

Doses.-Horses, 10 c.c. ; ponies, 3 c.c. to 5 c.c. ; two injections should be given, the first just before or immediately after the operation, the second in eight or ten days after the first. As a curative, a first injection of 50 c.c. may be given, and followed by injections of 20 c.c. daily for six to eight days.

The dried serum, which keeps indefinitely, is supplied in tubes, each containing a quantity of powder corresponding to 10 c.c. of the liquid serum. This preparation may be sprinkled on operation or accidental wounds, or dissolved. in a little sterilised water and injected subcutaneously in the same way as the liquid antitoxin. Applied to wounds it exerts an antitetanic action and promotes healing (Porcher).

Antistreptococcic polyvalent serum is obtained from horses which have been immunised against various types of streptococci. It is supplied by the Pasteur Institute in sealed vials, each containing 10 c.c. of liquid serum, and in tubes containing an equivalent in powder. Antistreptococcic serum has been used more or less successfully in the early stages of strangles, purpura, and distemper of 
the dog. It is aseptically injected under the skin of the neck. Not more than 10 c.c. should be injected at one point, and the subsequent punctures should be eight to ten inches apart. After injection considerable œedema forms around the punctures, but usually this swelling disappears within thirty-six hours. This serum is of little benefit in advanced or suppurating cases of strangles. Doses.Horses, 30 c.c.; dogs, 3 c.c. to 6 c.c., repeated daily until the symptoms abate; if relapse occur, the injections may be renewed. 


\section{SECTION IV}

\section{VETERINARY PHARMACY}

THIS section presents a brief notice of the art of prescribing the administration of medicines, doses, and of such important preparations and therapeutic agents as alkaloids, baths, boluses, drenches, enemas, fomentations, fumigations, inhalations, injections, plasters, powders, tinctures, etc., arranged for convenience of reference, according to their English names; while appended are the tables of the Pharmacopœia, and of metric weights and measures.

\section{THE ART OF PRESCRIBING}

Successful prescribing obviously necessitates a competent knowledge of the chemical and physiological actions of the drugs used. Those only can be properly conjoined which are chemically, pharmaceutically, and physiologically compatible. Text-books contain lists of medicines which may fittingly be used together under the title of synergists; while lists are given of substances chemically incompatible or physiologically antagonistic.

The prescription should be as brief and simple as possible. It should be explicit and clearly written. It may be expressed either in Latin or in English. The manner in which the medicine is to be used should be specified. Important instructions as to the regimen or diet of the patient are sometimes appended.

Prescriptions usually contain two or more of the following four representative constituents:-(a) The basis or active ingredients. The practice of conjoining several active drugs has wisely been abandoned. Occasionally, however, it may be advantageous to give together two drugs producing their effects in somewhat different ways. Thus, spasms 
of the bowels are more often effectually controlled by the conjunction of a stimulant like ether, and an anodyne like opium, than by either given alone. Pain which is not alleviated by either morphine or atropine is sometimes abated by giving them together. (b) The adjuvant is introduced in order to increase, moderate or modify the action of the basis. Frequently its chief object is to ensure solubility and ready absorption. (c) A corrective is occasionally required to temper the effects of the basis. Thus, a small dose of laudanum is prescribed with oil or other laxative in cases of diarrhœa; ginger is generally added to the aloetic mass for horses. (d) The vehicle generally consists of some comparatively inert substance, added to facilitate administration, such as the treacle, linseed meal, or liquorice powder used as excipients for boluses and pills, the benzoated lard or vaseline used for making ointments, and the water given in drenches.

The prescriber's aphorism, 'Curare cito, tute, et jocunde,' is more easily fulfilled now than formerly. With a widening choice of carefully-prepared drugs, the effects of which are becoming more fully known, the practitioner is better able to cure quickly, safely, and pleasantly. Instead of crude vegetable drugs, often inconveniently bulky, and containing useless and even injurious constituents, extracts, infusions, and tinctures have long been used. But concentration and simplification are being carried still further. Active alkaloids and neutral proximate principles are now prepared, and possess the advantage not only of concentrated form, but usually also of more uniform quality and less liability to spoil.

To guard against impurities and adulterations, the British Pharmacopœia, under the head of each drug, gives characters and tests which enable purchasers to satisfy themselves of the quality and purity of the articles used.

\section{ALKALOIDS}

These alkali-like organic bases are found in various plants, some of which, like the opium poppy, cinchona bark, and jaborandi, contain more than one alkaloid. They probably 
result from the metamorphosis of albuminoid plant constituents. They constitute the active principles of vegetable drugs, and are generally powerful poisons or active medicines. Those chiefly used are morphine, atropine, quinine, cocaine, physostigmine, pilocarpine, and strychnine.

Most alkaloids contain carbon, hydrogen, and oxygen, and all contain nitrogen. But although the molecule may contain twenty or thirty carbon or hydrogen atoms, there are seldom more than two atoms of nitrogen. They are generally crystallisable solids. Three of the natural alkaloids, conine, nicotine, and sparteine, contain, however, no oxygen, and are volatile liquids. Alkaloids are ammonia derivatives, but their sparing solubility in water renders it unlikely that they are ammonium bases ; their not subliming without some decomposition distinguishes them from the amines; their feebly basic character and other properties more nearly ally them with the amides. They may otherwise be defined as substituted ammonias, capable of forming salts by direct addition of acids. Many are closely related to pyridine, and a few may be prepared synthetically from pyridine bases. Most are soluble in alcohol, ether, benzine, and carbon disulphide, and usually in chloroform, which does not, however, dissolve morphine. Most are sparingly soluble in water. Alkaloids readily unite with acids, forming salts, soluble in water; and with radicles such as ethyl and methyl, forming combinations which greatly modify their actions, as is strikingly illustrated in the difference between conine and methyl-conine. Their chemical composition affords no clue to their physiological action. Those of diverse composition have sometimes similar actions; those apparently of like composition differ in their actions. Between the anodyne soporific morphine, and the emetic apomorphine, the formulæ show a difference of only the elements of water. Plants of the same genus usually yield the same or similar alkaloids; thus the atropaceæ yield atropine, the strychnos strychnine. Alkaloids are often associated in plants with some special acid, in combination with which, or with a diluted acid added to the plant juices, they are dissolved out, usually by water, and from such solution the alkaloid may be precipitated by ammonia. 
The neutral organic principles are another group of concentrated active vegetable drugs. They contain carbon, hydrogen, and oxygen, and comport themselves sometimes as bases, sometimes as acids. Under the influence of acids and organic ferments nearly all split up, yielding glucose, and hence are called glucosides. They are represented by aloin, digitalin, santonin, and salicin, and it will be observed have the terminal ' in,' which distinguishes them from the alkaloids, to which the termination 'ine 'or 'ina,' or, in the older works, 'ia,' is given.

The organic animal extracts, prepared from bone marrow, heart, muscle, brain, spinal cord, testicle, liver, and thyroid gland, and occasionally prescribed for the human subject, are seldom employed in veterinary medicine.

\section{BALLS-BOLUSES-PILULA}

Balls correspond in veterinary practice with the pills used in human medicine. Extracts are conveniently moulded into cylindrical balls. Drugs in powder or concentrated solution may be enclosed in gelatin capsules, in cachets, or made into mass or bolus with such excipients as linseed meal and water, oil, lard, soap, mucilage, liquorice, and powdered gentian, treacle, syrup, glycerin, vaseline, and confection of roses, the choice being determined by the nature of the active ingredients. The excipients first mentioned are chiefly used when the bolus or mass is intended for immediate use ; when it is to be kept for any considerable time some of the others are more suitable. To keep a mass in a moist, soft-solid state, it is often advisable to add to it a small quantity of such a deliquescent alkaline salt as potassium acetate, which serves especially well for most diuretic masses. When the active principles are resinous, a little alcohol, glycerin, or oil of turpentine is a useful addition, as in making aloetic masses.

In preparing a ball mass, the various ingredients are sometimes mixed in a mortar or on a slab; but when the materials are waxy or resinous, the aid of heat is necessary. A good ball mass must be soft, and yet possessed of proper consistence and cohesion, must retain these properties 
although kept for a considerable time, and must further be so prepared that each dose shall make a proper-sized ball, which for the horse usually weighs one to two ounces. The ball mass should be preserved in jars covered with rubber sheeting and stout paper, and be made into doses only as required; for when balls are long kept they are apt to become hard, and in this state act slowly and sometimes pass through the alimentary canal unchanged. To prevent spoiling, as well as for cleanliness and to facilitate administration, balls are rolled up in oiled paper, or are coated with keratin, or a solution of one part of gelatin and twenty of water.

The bolus is given to horses with a balling-gun or with the fingers ; and the latter method is preferable, except in infected animals or those in which the mouth cannot be sufficiently opened. The operation, with a little practice and dexterity, is easily performed. The ball is held by one end between the thumb (which supports it below) and the fingers of the right hand. The patient's tongue is gently drawn out a little way by the left hand, and the ball passed rapidly along the roof of the mouth, and dropped on the back of the tongue, which is at once released, the mouth closed, and the head kept slightly elevated for a few seconds. In a similar way balls may be administered to cattle. To dogs pills or cachets may be given enclosed in a piece of meat, or the pill may be carried by forceps or a pill-carrier to the back of the mouth.

\section{BATHS-FOMENTATIONS}

Baths are important, alike for the preservation of health and for the cure of disease amongst the domestic animals. They are used in various ways, at different temperatures, and for the treatment of medical and surgical cases.

Cold baths are of a lower temperature than $70^{\circ} \mathrm{Fahr}$. Judiciously used, they act reflexly on nerve centres, stimulating respiration, circulation, and metabolism. Similar stimulant action is exerted on the sympathetic nervous system, the skin functions are promoted, waste products removed, and abnormal temperature reduced. Owing to 
its saline ingredients, and the constant movement of the waves, a sea bath is more exhilarating than fresh water at rest. Healthful reaction is encouraged by thorough drying, hand-rubbing, clothing, and, if need be, by subsequent administration of stimulants. Cold baths are sometimes used for reducing excessive temperature. The patient may be placed in water at $80^{\circ}$ or $90^{\circ} \mathrm{Fahr}$., and the temperature reduced to $60^{\circ} \mathrm{Fahr}$., or even lower; or he may be placed at once in a bath of $60^{\circ}$ to $50^{\circ} \mathrm{Fahr}$.; or he may have cold water dashed over him. Such baths reduce abnormal temperature from $1^{\circ}$ to $4^{\circ} \mathrm{Fahr}$., and accordingly diminish excessive and dangerous tissue change.

Tepid baths range from $65^{\circ}$ to $85^{\circ} \mathrm{Fahr}$. They cleanse the skin, promote perspiration, allay thirst, and are grateful to heated and overtaxed horses. They are useful not only in promoting comfort, but, after hard work, in removing stiffness alike in horses and dogs.

Warm baths range from $85^{\circ}$ to $97^{\circ} \mathrm{Fahr}$. They soften the epidermis, and hence relieve erythematous and chronic skin disorders. They dilate cutaneous vessels, promote sweating, and thus antagonise internal congestion.

Hot baths range from $97^{\circ} \mathrm{Fahr}$. upwards, and, if the temperature be gradually increased, may be raised to $110^{\circ}$ Fahr. Hot baths elevate the temperature of the body, quicken the pulse and respiration, dilate the skin capillaries, and hasten excretion of waste. They soothe animals which have been subjected to severe muscular exertion, relieve cramps and colic, benefit chronic skin disorders, and check catarrh, rheumatism, and lymphangitis.

Baths are often medicated. Salt or mustard may be added to increase skin stimulation; carbonate of soda or potash is useful in some chronic skin disorders; solutions of arsenious acid, tobacco juice, creolin, and other parasiticides are introduced when skin parasites are to be destroyed. Antiseptic baths are employed for poisoned and offensive wounds, canker, thrush, and other diseases of the foot; and astringent baths form an excellent remedy for cracked heels.

Turkish, vapour, steam, and Russian baths are not used for the lower animals as frequently and systematically as 
for man; but the infirmaries of many veterinarians have capital washrooms provided with steam, hot and cold water, where horses and dogs are conveniently bathed, washed, and dressed; where chronic coughs, chills, rheumatism, dyspepsia, and other ailments are hydropathically treated, and where sprains and enlarged joints can be successfully douched.

Without expensive or special appliances, many of the sanitary and curative advantages of baths can be secured by sponging horses, as their condition or ailment suggests, with tepid, hot, or cold water. Cleansing, tonic, or antipyretic effects may thus be readily obtained. Irritating or noxious matters are removed from the skin, circulation is equalised, and excessive temperature reduced. In acute febrile cases, whether in horses or cattle, the temperature of the water used should not at first be lower than $85^{\circ}$ or $80^{\circ}$ Fahr. A little acetic acid may be advantageously added. The sponging should not occupy more than three or four minutes. The animal should be wisped dry and immediately clothed. Within three or four hours the operation, if necessary, may be repeated, especially if the temperature reduced by the first sponging again rises. After the first or second sponging water at $65^{\circ}$ or $60^{\circ} \mathrm{Fahr}$. may be used. Concurrently with such treatment, antiseptic salines or stimulants may be prescribed. In chorea, especially in tolerably vigorous and short-haired dogs, sponging with cold water is serviceable. Enveloping the patient in a sheet wetted with either tepid or cold water, and covering with two or three blankets, constituting what is entitled the 'wet pack,' is not generally so convenient in veterinary practice as either sponging or douching (see Water, p. 152).

Douches or Affusions of cold water, frequently repeated, are used in the early treatment of bruises, harness galls, bumped knees, and other œdematous swellings. They are often beneficial in cases of prolapsed rectum, or vagina, and in the preliminary treatment of inverted uterus, wrenched joint, periostitis, tendinitis, and synovial dropsy of joint or tendon sheath. Prolonged douching is strongly recommended as a remedy for recent scrotal hernia, and as 
an accessory measure in tympanites, parturient coma of the cow, and partial paralysis of the horse.

Continuous irrigation with cold water, or an antiseptic solution, is employed in the treatment of open and closed arthritis, synovitis of tendon sheath, foot injuries, quittor, abscess cavities, fistulous withers, and accidental wounds of the limbs. It modifies inflammation, lessens pain, and hinders the development of bacteria; but as it somewhat restricts local nutrition, healing is slightly retarded.

Topical baths or fomentations usually consist of water alone, but antiseptics, anodynes, vinegar, salines, and other substances are often added. Unless otherwise specified, they are applied hot. The temperature at which they are used must be determined by the purpose to be served, by the nature and extent of the malady, and the part of the body to which they are applied. For the eye, they should not exceed $100^{\circ} \mathrm{Fahr}$.; for contusions, abscesses, strains, and lymphangitis, they should be as hot as the hand can bear. Up to $110^{\circ} \mathrm{Fahr}$. they generally soften and soothe. At higher temperatures they irritate. To produce active counter-irritation, as in bronchitis, pleurisy, or enteritis, they are used at temperatures ranging from $120^{\circ} \mathrm{Fahr}$. upwards. For such cases, pieces of flannel or horse-cloth saturated with boiling water, are partially dried by a wringer, or by being rolled and pressed between dry coarse towels, and are laid over a considerable extent of surface contiguous to the parts affected. The hot, wet woollen cloth should be covered with oilskin or a piece of mackintosh to retard evaporation and cooling. The pained part to be soothed, or the surface to be stimulated is sometimes covered with several folds of woollen cloth on which hot water is poured at short intervals. Jets of steam mixed with air, to prevent scalding, used either plain or medicated, may be substituted for the ordinary stuping with water. Fomentations are generally made with a sponge, tow or lint. When there is pus or septic discharge, sponges should not be used, as they are apt to retain and convey infective germs, while the piece of lint or tow is thrown away as soon as done with. 
Antiseptic fomentations are used to cleanse wounds and soften hard skin or encrusted discharges. They relax and soothe irritated, congested, inflamed parts to which they are applied, and thus lessen tension and pain. When freely employed for some considerable time, they dilate the capillaries of collateral areas of circulation, withdraw blood from adjacent inflamed parts, and act as mild counterirritants. To obtain their full benefits, they should be continued during several hours; fresh supplies of water, of the requisite temperature, being had in abundance. After the operation is finished, the parts should be dried, and well covered, in order to prevent the rapid diminution of temperature which otherwise ensues from evaporation. Further, to prevent chilling, the fomented surface is sometimes stimulated with a weak mustard paste or mild liniment.

Heat applied to the spine, usually in the form of the hot-water bag, at a temperature of $120^{\circ} \mathrm{Fahr}$., stimulates the cord and sympathetic ganglia, contracts the involuntary muscular fibres of arterioles, and thus lessens the volume and rapidity of blood passing through them. The spinal hot-bag is hence used to arrest hæmorrhage.

The ice-bag applied to the spine, on the other hand, is a sedative to the cord and nerve-centres brought under its paralysing influence, and hence lowers muscular tone, sensibility, and secretion. Applied in the cervical region, it increases afflux of blood to the head; applied over the anterior dorsal region, blood is driven to the chest and anterior extremities ; applied over the posterior dorsal and lumbar regions, blood is moved in larger amount through the abdominal and pelvic organs, and the posterior extremities. Acting upon the spinal and sympathetic centres, the ice-bag controls remote morbid processes ; cramps and spasms are stated to be abated; pains of neuralgia and rheumatism are sometimes arrested; while inordinate discharge, and even hæmorrhage from the lungs, bowels, or kidneys, are sometimes checked (Ringer).

Antiseptic compresses, consisting of several layers of lint or cotton wool thoroughly saturated with an antiseptic solution, are frequently applied to sutured operation wounds. 
They preserve asepsis, support the sutures, and prevent or diminish the consecutive œedema. They are also employed with the object of ensuring asepsis of the operation area prior to neurectomy, tenotomy, oöphorectomy of the bitch and cat, aspiration of bursal distensions, etc. Before application, the hair is removed from skin of the region, which is then washed and disinfected, and the compress is kept in place until the patient is in position for operation.

\section{DECOCTIONS}

Decoctions are solutions prepared by boiling the drug in distilled water. To ensure perfect solution, the substance is bruised or cut into small pieces, and boiled in a glass or earthenware vessel for five to ten minutes. Any insoluble residue is subsequently separated by straining through muslin or calico, or by allowing the solution to settle, and pouring off the supernatant fluid. Decoctions intended to be kept beyond a week or two should be preserved by adding a little spirit or about one part of benzoic acid to 200 of decoction.

\section{DOSES AND THEIR ADMINISTRATION}

The dose, or quantity of the medicine used, affects the degree, and sometimes also the nature of the action produced. Thus, small doses of most salts of potassium, sodium, and magnesium are diuretic, while larger quantities are purgative. Aloes, in small quantity, is tonic, and in large, purgative. Alcohol and opium are examples of medicines in which variation in dose produces difference in effect. With topical remedies, an increase of the time during which the drug is applied is generally equivalent to an increase of dose, as illustrated in the case of mustard, cantharides, and concentrated acids.

The period during which a drug remains in the body determines in like manner its activity. Hence increased action results from rapid absorption and prolonged retention within the body, while diminished action results from tardy absorption and quick excretion. Where prompt and full 
effects are desired, carefully regulated doses are given every hour or oftener. Where continued effects are desired, as in the case of tonics or alteratives, small doses repeated three or four times daily are preferable to larger doses given at longer intervals. Stimulants, which are evanescent in their effects-such as alcohol, ether, and ammonia -are usually beneficially repeated every two or three hours, or, in critical cases, oftener. The dose of a medicine, and the desirability of its repetition, intermission, or suspension, must frequently be determined by the manner in which the patient is affected by the first dose or doses.

The doses mentioned in this work under each drug, unless otherwise stated, are those suitable for adult animals of medium size. But, as already indicated, the size, weight, and environment of the patient require consideration in fixing the dose. In the lower animals, differences of sex do not materially affect dosage ; although, on account of their larger size, extra doses are required for stallions, bulls, and rams. Doses must be adapted to the age of the patient. It is generally estimated that a oneyear-old colt requires one-third the quantity of any medicine given to an adult horse ; a two-year-old, one half ; a threeyear-old, two-thirds. A somewhat similar ratio is applicable to cattle.

Medicinal agents are used to produce either local or general actions, or a combination of both.

LOCAL ACTIONS are produced by applying the agent to the surface of the skin, to the mouth or throat, the eye, ear, or nasal passages, and also by injection into the rectum, bladder, vagina, uterus, udder, and substance of muscles. Agents thus used, besides acting locally, may pass into the general circulation, and produce general effects, or by reflex action develop secondary or remote effects.

General Actions are usually produced by the introduction of the medicine into the circulation. Injection may be made directly into the veins as in the treatment of collapse and acute anæmia, and occasionally into the arteries, for purposes of experiment. When transfusion is undertaken the fluid should be watery, and of the same salt content as blood serum; for example, normal saline solution, a 0.9 per 
cent. solution of sodium chloride. Drugs injected into the peritoneal and other serous cavities are very rapidly absorbed. They are also quickly taken up from abraded skin surfaces.

The channels by which medicines may be administered are :-

1. The digestive tract, generally by the mouth (bolus, pill, drench, electuary), and occasionally by the rectum (evacuant, nutrient, anodyne, or anæsthetic enemata).

2. The pulmonary mucous membrane, by inhalation (anæsthetics, fumigations).

\section{Intratracheal injection.}

4. Intravenous injection.

5. Intraperitoneal injection.

6. The skin: (a) epidermically by inrubbing; endermically by inunction after removal of the epidermis.

7. Hypodermically, by injection into the subcutaneous connective tissues. Intramuscular, intramammary, intraspinal, intrauterine, and subconjunctival injections are also employed, and occasionally injections are made into the nasal chambers, urethra, bladder, and vagina. Medicated spray thrown from an atomizer, or from a steam spray producer, is used for irrigating the throat, when irritable or relaxed, especially in horses.

The mouth is the channel by which medicines are most frequently administered, for they immediately pass into the stomach or intestine, whence they are readily and rapidly absorbed. To avoid their admixture with food, and consequent impaired and delayed effect, they should generally be given after the patient has been fasted for several hours. Nutrient oils, iron salts, arsenic, and other irritants are, however, given with the food, or immediately after eating. When it is desired that they shall be quickly absorbed, and thus act promptly and certainly, they should be given in the fluid state, and this is especially requisite in ruminants. The time, labour, and patience of attendants may be saved, and high-spirited, nervous animals preserved from injurious struggling, if they can be persuaded to take their medicines voluntarily. This may sometimes be accomplished when comparatively concentrated, tasteless, or 
pleasant-tasted drugs are used, by mixing them with palatable food, or disguising them in gruel, milk, or even in water. Dogs and cats will often bolt concentrated drugs rolled up in a piece of meat. Although absorption is not so active from the posterior portions of the digestive tract, nutrients and soluble medicines introduced into the rectum enter the circulation.

The pulmonary mucous membrane has a superficies of fifty times the extent of the skin surface, and actively absorbs substances in the gaseous form. By this channel are administered anæsthetics, when their general effects are required; balsams and anodynes to relieve morbid conditions of the respiratory passages; diluted sulphurous and chlorine gases to destroy bronchial filariæ. But other volatile drugs may thus be introduced into the body, while others, in a finely divided state, can be inhaled with such a readily diffusible volatile body as a chloroform, or ether.

INTRATRACHEAL INJECTION has lately been adopted both in this country and abroad. Tolerably bland fluids in considerable quantity can, with impunity, be introduced into the trachea, and agents too bulky to be used hypodermically can thus be absorbed into the blood more quickly and directly, and with less risk of having their activity impaired than when administered through the digestive tract. Various experiments on dogs, made at St. Petersburg, indicate that the effects of curare, strychnine, and cocaine are frequently produced in ten seconds, and more rapidly than when these drugs are injected subcutaneously. Turpentine by this channel exerts its lethal action very effectually on the bronchial parasites which attack cattle and sheep. The injection is effected with a syringe having a stronger needle than that used for hypodermic purposes. Intratracheal injections intended to produce general effects should be non-irritant and well diluted in water, alcohol, or oil. Beyond a slight gain in time, intratracheal injections, other than those intended to act locally, have no advantage over hypodermic injections. Drugs have occasionally been injected directly into the lungs.

Intravenous injection of medicines is resorted to when immediate effects are desired. In this way sterilised salt- 
solution, barium chloride, silver colloid chloral hydrate, and a few other remedies may be injected into the jugular vein of the horse and ox ; in the saphena vein of the dog. The injection should be diluted, non-irritant, and introduced slowly with strict attention to asepsis. The introduction of air, the formation of clots, and septic contamination must be avoided.

Intraperitoneal injections are serviceable for the administration of physiological salt-solution and other artificial sera, and chloral as a general anæsthetic. The injection is made at the upper region of the left flank, which is punctured with a fine trocar and canula; the trocar having been withdrawn, the nozzle of the syringe is inserted in the canula. The usual aseptic precautions should be observed.

On the skin many medicinal agents are applied, most of them-such as counter-irritants, caustics, and poulticesfor the production of local effects, or of reflex actions exerted on adjacent or distant parts. The skin, protected by epidermis, although it absorbs oxygen and other gases, excretes carbonic acid, and takes up water from baths or from wet clothing, especially if there is a deficiency of fluid in the body, does not absorb drugs unless they are dissolved in chloroform or other agent which promotes penetration. Drugs, whether in alcoholic or watery solution, are absorbed through the unbroken skin only in very small amount and tardily; but when the epidermis is removed by a blister, the true skin readily absorbs drugs placed on it. By this endermic method, morphine was formerly used for the production of its general anodyne effects, but the hypodermic method is more convenient, and is now generally preferred.

The hypodermic administration of drugs, and mallein, tuberculin, the various sera and vaccines, consists in their injection in solution into the subcutaneous cellular tissue. They thus enter the blood-stream unaltered by contact with the contents or secretions of the digestive canal. They escape the changes which many substances undergo in the liver, and hence act more certainly and rapidly. Hypodermic injection is specially indicated-

(1) Where rapid energetic effects are required, as in poisoning, internal hæmorrhage, threatening 
collapse, paroxysms of acute pain, and convulsions.

(2) Where it is desired that the drug shall act promptly and directly on the diseased part, as in neuralgia, rheumatic pains, and mammitis in cows.

(3) Where local and general effects are desired to be conjoined, as in reflex spasms.

(4) Where administration by the mouth is difficult, impossible, or inadvisable.

The drugs thus used should be in neutral, non-irritant, well diluted solution, and preferably dissolved in water or in vegetable oil. Alcohol, chloroform, and glycerin, though often employed, are objectionable, and concentrated solutions should be avoided as they cause pain and are less rapidly absorbed. The quantity injected at one point should seldom exceed two drachms; when larger quantities must be administered, injections should be made at different points of the skin. Many veterinarians use morphine, atropine, ergotin, eserine, arecoline, and other active drugs hypodermically for arresting or controlling the spasms of colic and chronic cough, the sharp twinges of rheumatism, the inflammatory pain of enteritis, pleurisy, and laminitis, as well as for combating the effects of poisons. By this method are administered pilocarpine, apomorphine, adrenalin, atoxyl, sodium cacodylate, caffeine, cocaine and other local anæsthetics, sterilised gelatin, and the various organic and artificial sera. Where local pain is to be counteracted, the injection may be made more deeply near the affected spot, or over the nerve which is believed to be conveying the disordered impression. With active agents it is unwise, without careful trial, to inject subcutaneously more than one-fourth of the dose which would be given by the mouth. Strychnine is too active, and quinine is too irritant for safe administration by this method, but there is less risk in veterinary than in human patients of subsequent local irritation.

The hypodermic syringe has a glass barrel with metal mounts, a hollow needle fitted to the nozzle, and an asbestos piston. It is essential that every part of the syringe be aseptic, and the needle sharp. Hypodermic injection is 
very simple. Choice is generally made of a situation where the skin is thin, and the subcutaneous tissue loose, as behind the elbow, or at the lower part of the neck. A fold of loose skin, after disinfection with 5 per cent. carbolic solution, is taken up between the finger and thumb of the left hand; the needle, detached from the syringe, is passed through the skin, and carried about an inch obliquely under the surface. The nozzle of the filled syringe is then attached to the needle, the piston slowly pushed home, and the instrument cautiously withdrawn. The injection of air can be prevented by seeing that the packing of the piston is sufficient, or by arresting the piston before the contents of syringe have been wholly expelled. The puncture requires no plaster or dressing. Convenient tablets, lamellæ, or pellets of the various drugs used hypodermically are now obtainable.

Intramuscular injections, though very rapidly absorbed, are only occasionally employed. By this method solutions of strychnine and veratrine have been used as stimulants in muscular paralysis, morphine and cocaine as anodynes in rheumatism, compound fractures, and extensive laceration of muscles; and recently fibrolysin has been tried with the object of reducing fibrous indurations. Usually the injections are made into the substance of the gluteal, or scapular muscles. Deep injection of ether and of other drugs occasionally causes temporary motor or sensory paralysis.

Intramammary injections of antiseptic solutions, and insufflations of air, or pure oxygen, are employed in acute and contagious mammitis and parturient toxæmia or ' milk fever' of the cow. Antiseptic solutions are injected by a Higginson's syringe through a teat-syphon or special tube inserted in the teat. Two to four ounces of warm aqueous solution of boric acid ( 3 per cent.), of sodium fluoride (1 to 200-500), or chinosol $(1: 1200)$, are injected in acute and contagious mammitis. In parturient toxæmia filtered air, introduced by a bicycle pump, or pure oxygen from a cylinder, or a solution of potassium iodide may be used. In all cases the udder or diseased quarter should be emptied as completely as possible before injection. After insufflation of air or oxygen, a clamp is applied to the teat, the udder is 
lightly massaged, and after an interval of three or four hours the teats are drawn. In contagious mammitis injections of gradually increasing strength should be repeated three or four times daily; but in acute mammitis usually only one injection is required, though occasionally a second injection is given three or four days after the first. The teats should be drawn at intervals of three or four hours from the first injection until the gland resumes its function.

\section{DRAUGHTS-DRENCHES-DRINKS}

Drenches are generally extemporaneous fluid preparations used in a single dose. Bulky substances which cannot be administered in bolus are necessarily given in draught. They are prescribed for cattle and sheep, horses and dogs, especially when a speedy effect is desired. In ruminants, medicines in the solid state mix with the food always found in the rumen, and thus act tardily and imperfectly. Medicines in liquid form, however, come more immediately into contact with the mucous surface, and pass on more rapidly to the abomasum and intestine. Saline, febrifuge, and tonic draughts, made fairly palatable, are often readily taken by patients in gruel or water, without the trouble of forcible administration.

In preparing drenches, care should be taken that the several ingredients are not incompatible, decomposing or injuriously reacting on each other; and further, that their quantity is not so great as unnecessarily to increase the trouble of administration. Before being given, drenches ought to be thoroughly mixed and well shaken, in order to prevent ether, ammonia, turpentine, or other light constituent floating to the surface, or heavy, partially dissolved substances falling to the bottom.

In giving drenches, the head should be slightly raised, which in horses is conveniently done, with the aid of a mouth-twitch, the noose of which is placed over the animal's upper jaw within the incisor teeth, and the stick held by an assistant, standing on the left side of the patient. In cattle, the head should be steadied by an assistant, who holds either the horn or ears. In dogs, the jaws may be 
kept sufficiently apart by an assistant, or a finger may be placed within the cheek which is then raised to form a pouch into which the medicine can be readily poured. Small dogs are most conveniently dosed when placed on their hind-quarters on a table or bench; but larger dogs should be backed into a corner, the operator holding the head between his knees. Cats can be drenched without doing damage with their claws if rolled in a stout rug or dropped into a capacious top-boot, the head being left out, and the jaws held apart with pieces of tape; or the cheek may be pouched with forceps. In all animals the nostrils must be unobstructed, and the tongue loose, or only gently held down. Drenches should be carefully and slowly given ; and if coughing occur, administration should be stopped, and the animal's head released. Drenches may be given by an ordinary pint syringe. The jaws are fixed by a cord or strap passed round the head, the lips are held closed in front and at the sides; the nozzle of the syringe is then passed into the mouth near one of the commissures, and the injection is directed towards the base of the tongue.

Glass bottles, often used for the administration of drenches, are dangerous, when they break they may injure the mouth of the patient, or the hands of the operator. Veterinarians, and even agriculturists, should therefore only use stout tin drenching bottles. The old-fashioned horn is now generally superseded by the tin bottle.

\section{ENEMAS-ENEMATA-CLYSTERS-SUPPOSITORIES -INJECTIONS}

Enemas are liquid injections into the rectum, and are employed to effect several useful purposes. They soften and remove hard fæces lodged in the rectum, and do so without unnecessarily exciting other parts of the intestine. They accelerate, increase, and maintain the action of purgatives. They destroy and remove worms infesting the posterior part of the canal. They may exert either soothing or stimulating effects ; and, reflexly, such effects may be propagated to adjacent and even to remote organs. They besides prove convenient vehicles for introducing into 
the body food and medicines, in sore-throat, gastritis, tetanus, apoplexy, and other conditions, in which administration by the mouth is contra-indicated or impracticable.

For maintaining the regular action of the bowels, no remedies are so safe and effectual, and when properly used they sometimes supersede the necessity for purgatives. In constipation and torpidity, after hardened, impacted, obstructing fæcal masses have been broken down and removed, laxative enemata are advantageously injected, intermitting the operation whenever straining occurs, raising the hind parts, and by external pressure from behind increasing the retaining capacity of the sphincter. A gallon and a half to five gallons of tepid water may thus be slowly introduced into the rectum of a draught horse; half a pint to a pint into the rectum of a dog $40 \mathrm{lbs}$. weight. When the posterior gut is thus distended, not only is mechanical facility given for the outward movement of the contents of the canal, but by reflex action peristalsis is encouraged far beyond the surface reached by the injection. The effects of tepid water are increased by the addition of soap, bland oil, glycerin, salines, oil of turpentine, or solution of aloes. These copious injections, and the administration of full doses of opium, Indian hemp or atropine, relieve intestinal spasm, and sometimes prove beneficial in cases of suspected invagination and strangulation of the intestines in horses and dogs.

Obstinate torpidity of the bowels in horses, depending on impaction of ingesta, or obstruction from earthy deposits in the colon, may frequently be relieved or removed by the free use of enemata introduced into the large intestine by a special apparatus, an adaptation of O'Beirne's tube. It consists of six feet of one-inch rubber hose, attached to a Read's pump, and provided with a round nozzle, with side perforations. This tube can be passed into the single colon of the horse. Any difficulty in introducing it is overcome, not by force, but by injection of a gallon of water, which, dilating the bowel in advance, facilitates further passage of the tube. Five to fifteen gallons of cold water are injected; and the injection may be repeated at intervals of three or four hours. Unlike ordinary enemata discharged 
into the rectum, these large quantities of fluid are in great part retained, with the effect of softening and mechanically removing obstructions.

Tolerably copious clysters of tepid water or other bland fluid exert local soothing effects, not only on the intestines, but they also reflexly allay irritability, spasm, and pain of the urino-genital organs, and frequently relieve suppression of urine. Their efficacy is sometimes increased by addition of opium and sedatives, which are used in about the same doses as are given by the mouth. By reflex action the kidneys, bladder, and uterus are also sometimes beneficially acted upon by stimulant enemata. For destroying and dislodging the oxyuris curvula, clysters of turpentine and lime water, and of solutions of aloes, quassia, ferric chloride, or common salt, are effectual. In house dogs, troubled with constipation and hæmorrhoids, the cautious daily injection of two or three ounces of cold water proves serviceable.

When intended to be retained and absorbed, enemas should be limited in amount. To allay irritation and spasm in troublesome diarrhœa and dysentery, vegetable astringents, opium, and lead acetate are given, usually with wellboiled tepid starch gruel. In such cases the injection may be repeated every hour or two, so long as straining or diarrhœa continues. Rectal injections intended to produce general effects should be warm and concentrated. Nutrient enemata, useful in tetanus, sore-throat, and debilitating complaints, when patients will not or cannot eat, usually consist of a pint or two of linseed tea, with milk and eggs, or of strong beef tea introduced at a temperature of $100^{\circ}$ Fahr., and repeated not oftener than four or five times in the twenty-four hours. In cases of influenza in horses and distemper in dogs, a little wine or whisky is sometimes added to the nutrient enema.

The apparatus for giving enemata are-Higginson's rubber syringe; Read's patent clyster syringe, improved by Arnold, which is also available as a stomach pump ; the far-reaching apparatus, with six feet of rubber tubing, already described; Gamgee's block-tin funnel, which fills the rectum by gravitation, and obviates the necessity of pumping ; and the common barrel syringe, of tinned copper, 
with a nozzle which unscrews at pleasure, and can be carried in the interior of the instrument. The female catheter may be used for the administration of medicinal injections. In the horse the rectum is generally cleared by the hand before any of these articles are used; and in all animals the part of the apparatus which enters the gut should be smeared with lard or oil, and introduced slowly and carefully.

Suppositories of raw or cooked meat are readily absorbed from the rectum, especially if they are previously moistened with diluted hydrochloric acid and pepsin. Drugs in the form of suppository, usually made up with cacao-butter and a little wax, are occasionally introduced into the rectum, vagina, and uterus. A cylinder of soap introduced within the anus, and held for a minute or two, conveniently encourages the action of the bowels, especially in dogs and cats, and in foals and calves.

Intra-uterine injections of antiseptic, astringent, hæmostatic, anodyne, and saline solutions are used in the treatment of metritis, retention of the fœtal membranes, uterine wounds, hæmorrhage, and leucorrhœa, and after difficult parturition. The solution, varying in quantity from a few ounces to ten or fifteen quarts, should be warm, and slowly injected by a Higginson's syringe or Read's pump, or introduced by gravitation through a double catheter, or halfinch rubber tube, which is passed by the hand through the os uteri for a distance of three to six inches. If the womb contract under the stimulus of the injection, the solution will be completely expelled; but in cases associated with uterine inertia, to prevent distension, poisoning or irritation, and consequent straining, the solution should be withdrawn or syphoned out soon after injection. After manual extraction of the fœtal membranes, the womb is irrigated with a weak, antiseptic solution, and irrigation may be continued until the returning fluid is quite clear.

\section{EXTRACTS-FLUID EXTRACTS-FRESH OR GREEN EXTRACTS}

Extracts consist of the soluble, active principles of plants reduced to a semi-solid paste by evaporation of the natural 
expressed juices, or of decoctions, infusions, or tinctures. Selection is made of the solvent-water, alcohol, or etherwhich most readily removes the active principles. Where two active principles, soluble in different media, are to be dissolved out, the drug is acted upon by the two solvents in succession, and the solutions mixed and evaporated. It is important that evaporation be effected at low temperatures ; heat above $150^{\circ} \mathrm{Fahr}$. is apt to injure or decompose many active principles. Well-made extracts keep for a considerable time without change, especially in a cool, dry place, and if occasionally moistened with rectified spirit; but when twelve months old they should be regarded with suspicion. Of the B.P. extracts, veterinarians chiefly use those of belladonna, hemlock, ergot, and Indian hemp.

The liquid extracts are prepared from infusions, decoctions, or weak tinctures, in the same manner as the semisolid extracts. Most liquid extracts are of such a strength that one fluid ounce represents an ounce of the drug employed. Those chiefly used in veterinary practice are liquid extract of cinchona, ergot, male fern, and opium.

Green extracts, as Extracta belladonnæ viride and hyoscyami viride, are prepared from fresh plants, the leaves and tender stems being generally preferred. They are bruised, the juice expressed, and gently heated to about $130^{\circ} \mathrm{Fahr}$. The green colouring matter is separated by filtration, the strained juice is heated to $200^{\circ} \mathrm{Fahr}$. to coagulate albumin, again filtered, and the filtrate evaporated in a water bath to the consistence of a thin syrup. The green colouring matter previously separated is then added, and evaporation continued, until a suitable consistence is attained.

Abstracts are concentrated extracts, about twice the strength of the corresponding fluid extract, and occur in dry powder mixed with milk sugar. Many of these figure in the U.S.P., including those of aconite, belladonna, hemlock, and digitalis.

\section{INHALATIONS-FUMIGATIONS}

Inhalations or vapours are volatilised applications intended to soothe, stimulate, deodorise, or disinfect the nasal 
chambers, pharynx, larynx, and other portions of the respiratory membrane, though it is doubtful if they have any beneficial effect beyond the nasal passages and the pharynx. The inhalation most frequently used is moist, warm air, produced from a steam kettle, or, in the treatment of horses, from a hot mash, placed in a capacious nose-bag, or in a large bucket brought under the nostrils, the animal's head and the bucket being covered with light waterproof sheeting. Such inhalations are considered serviceable in catarrh and bronchial congestion, and may be rendered more efficacious by mixing with the moist, warm air a little laudanum, eucalyptus oil, or turpentine. Antiseptic properties are conferred by impregnating the air with sulphurous acid, or by the use of creosote, iodine, or iodoform. As in the administration of chloroform for production of anæsthesia, volatile drugs may be conveniently inhaled from a sponge placed in one nostril. Irritability of the larynx and violent coughing are sometimes relieved by spraying the throat with solution of cocaine, or antipyrine with tannin and alcohol.

Fumigations of gas produced in a limited space are used to deodorise and disinfect stables and other premises; and to destroy, or cause the expulsion of, parasites infesting the trachea and bronchial tubes. For these purposes, the agents commonly employed are chlorine, formaldehyde, and sulphur dioxide, which is conveniently obtained by burning sulphur (an ounce to the cubic yard of space) or by using sulphur candles. Fumigations of sulphurous acid gas twice or thrice repeated at intervals of three or four days are usually effectual in destroying bronchial strongyles in calves and lambs. Tar fumes, produced by placing tar on a hot metal plate, are also employed.

\section{GLYCERINA-GLYCEROLES}

Glycerina are solutions of soothing astringent or antiseptic substances in glycerin. They are applied locally to the skin and mucous surfaces. Those of carbolic and tannic acids contain one ounce by weight of the acid, mixed and gently heated with five fluid ounces of glycerin. Glycerinum 
boracis contains one of acid and six of the solvent. Glycerinum amyli is made with one ounce by weight of starch, six and a half fluid ounces of glycerin, and one and a half of water. Glycerina of lead subacetate, tragacanth, boric acid, and salicylic acid are also used.

\section{INFUSIONS}

Infusions are solutions prepared by digesting vegetable substances in hot water. Fourteen of the twenty-two officinal infusions are made with one part of the powdered or cut drug, to twenty of water. To prevent solution of the starch of the root, infusions of calumba and quassia are made with cold water. The process is generally conducted in stoneware jars, provided with a cup having perforated sides and bottom, fitting into the top of the jar, extending about half-way down, and containing the solid matters to be infused. Digestion is effected on a stove, and is continued for fifteen minutes to half an hour. The infusion, when cool, is generally strained, but for veterinary purposes and for immediate use decanting is often sufficient. Unless carefully bottled and corked while hot, infusions soon spoil, especially in warm weather. Their keeping is sometimes improved by concentration, by evaporation, or by addition of alcohol, or about a quarter grain of benzoic acid to the ounce. Examples-infusion of quassia, gentian, ergot, and calumba.

\section{LIQUORS-SOLUTIONS-LOTIONS}

The B.P. enumerates many liquors or solutions, nearly all containing inorganic bodies, or alkaloids, dissolved in water alone, or with other solvents. They vary greatly in strength and dose. The B.P. Liquor Hydrargyri Perchloridi contains gr. $\frac{1}{16}$ th in a fluid drachm. Those containing arsenic, atropine, morphine, and strychnine have about $4 \frac{1}{2}$ grains of the poison in the fluid ounce, or one grain in 110 minims. The liquors chiefly used by veterinarians are liquor ammoniæ, liquor ammonii acetatis, liquor iodi, liquor arsenicalis, liquor ferri perchloridi, liquor 
potassæ, liquor calcis, and liquor zinci chloridi. The B.P. solutions of cocaine, ergot, morphine, and apomorphine, and many unofficial formulæ for hypodermic injection are also used.

Lotions are watery solutions intended for external use. Those for the mouth are called collutoria, and those for the nasal chambers are known as collunaria. Applications to the eye may be dry (powder), soft (pomade), or liquid (collyrium). Collyria may be sprayed or instilled into the eye. In practising instillation in the horse or ox, the eyelids are separated and the lotion is poured into the conjunctival sac at the temporal angle; in the dog and cat the collyrium is dropped on to the surface of the eyeball. Collyria may be anæsthetic (cocaine, novocaine, adrenalin); antiseptic (solution of boric acid, protargol, hydrogen peroxide, etc.); astringent (alum, zinc sulphate, etc.); caustic or stimulant (silver nitrate); mydriatic (atropine, cocaine, etc.); myotic (eserine); and hæmostatic (adrenalin). When frequently applied the salts of lead, zinc, or silver may produce corneal opacity, which does not occur in using boric acid, sodium hyposulphite, or hydrogen peroxide.

\section{MIXTURES-MUCILAGES-EMULSIONS}

Mixtures or misturæ are preparations usually containing insoluble drugs suspended in mucilage or other viscid substances. They are exemplified by camphor, chalk, and catechu mixtures. Insoluble heavy powders, mixed with dissolved gum or starch, are sometimes also termed mucilages.

Emulsions are opalescent mixtures of oil or resin, suspended in aqueous solution of gum, soap, alkali, or white of egg.

\section{OILS, FIXED, VOLATILE, AND MINERAL}

Fixed oils and fats occur in many plants, usually in the seeds or fleshy pulp, frequently associated with mucilage, and are also present in animal bodies. Besides lard, almond, olive, castor, croton, cocoa-nut, linseed, and cod-liver oils, enumerated in the Pharmacopœia, palm, rape, and cotton- 
seed oils are also used in veterinary practice. The fixed oils are obtained by expression. They consist of two or more fatty acids-oleic, margaric, palmitic, and stearic-in combination with the sweet basic principle glycerin. They contain 76-79 parts of carbon, with 11-13 of hydrogen, and 10-12 of oxygen. Their consistence varies according to the proportion of the fluid olein. When fresh, they are generally almost colourless, and are inodorous and tasteless. When exposed to the air, the traces of albuminoids which they contain oxidise, a species of fermentation ensues, as in saponification, resulting in the breaking up of the neutral fatty matters, with the production of disagreeable rancidity, which may, however, be removed by boiling the faulty oil with water, and subsequently washing it with a weak soda solution. Oils and water are mutually but very slightly soluble, but oils and fats are readily dissolved by carbon disulphide, benzine, oil of turpentine, ether, and chloroform. They are miscible, and hence sometimes conveniently administered, in milk. Castor and croton oils are soluble in absolute alcohol. They vary in their combustibility and their melting and freezing points. Their specific gravity ranges from 900-970. The bland oils-such as olive, linseed, palm, and cod-liver-in small quantities are nutrients, but administered in large doses they are purgatives. Croton oil is irritant, whether applied to a mucous surface or to the skin.

The volatile or essential oils are mostly of vegetable origin, being found generally in the flowers, leaves, fruit, or seeds of plants, but they occur in all parts of the coniferæ. The several volatile animal odorous principles-ambergris from the sperm whale, civet, musk, and castoreum are occasionally used. The chemical constitution of the volatile oils differs from that of the fixed oils; most are pure hydrocarbons, with the molecular formula of oil of turpentine $\left(\mathrm{C}_{10} \mathrm{H}_{16}\right)$, and are termed terpenes. With this terpene is generally associated an oxidised product, analogous to the colophony or resin $\left(\mathrm{C}_{20} \mathrm{H}_{30} \mathrm{O}_{2}\right)$ of turpentine. Some, such as oil of garlic, contain sulphur $\left(\left(\mathrm{C}_{3} \mathrm{H}_{5}\right)_{2} \mathrm{~S}\right)$. They produce no permanent grease stain. Unlike the fat oils, they are not unctuous, but make the skin rough or 
brittle. They are mostly colourless, but have a powerful odour, and distinctive-often aromatic-taste. They are insoluble, or only slightly soluble, in water, but are readily soluble in alcohol, ether, fatty and mineral oils. They are mostly lighter than water. Most boil between $302-382^{\circ}$ Fahr., but the boiling point of camphors is about $372^{\circ} \mathrm{Fahr}$. All are acted upon by oxygen, and distil unchanged. They are obtained by distillation, or by extraction. The volatile oils are antiseptic and stimulant, and are used as carminatives, antispasmodics, and parasiticides, and for flavouring.

The Camphors, thymol, menthol, and common camphor are oxygen derivatives of volatile hydrocarbons. In physiological action they are allied to the volatile oils.

Oleo-resins, such as crude turpentine, Canada balsam, and Frankincense, are natural mixtures of volatile oil and resin.

Balsams are vegetable exudations consisting of resins with benzoic or cinnamic acid, dissolved in volatile oils.

Mineral, paraffin, or petroleum oils are a series of paraffin hydrocarbons, obtained from the distillation of shale, coal, and other geological deposits, and having the formula $\mathrm{C}_{\mathrm{n}} \mathrm{H}_{2 \mathrm{n}+2}$. (See Petroleums.) The several members differ in their boiling point, and are separable by distillation. Methane, or marsh gas $\left(\mathrm{CH}_{4}\right)$, and several of the simpler members are gaseous, and used for heating and illumination. Others, such as pentane $\left(\mathrm{C}_{5} \mathrm{H}_{12}\right)$, and hexane $\left(\mathrm{C}_{6} \mathrm{H}_{14}\right)$, are known as petroleum spirit, are solvents for fats, resins, and indiarubber, and are used for making varnishes. Heptane $\left(\mathrm{C}_{7} \mathrm{H}_{16}\right)$, known as benzoline, paraffin oil, or mineral sperm oil, is used for burning. Nonane and dodecane are employed as lubricants. Hexdecane $\left(\mathrm{C}_{16} \mathrm{H}_{34}\right)$ is the chief constituent of vaseline and other soft paraffins. It is used as a bland protective, and for the making of ointments. The solid, hard, or wax paraffins, melting at $110-145^{\circ} \mathrm{Fahr}$., are substituted for wax in the making of ointments.

OINTMENTS-OLEATES-LINIMENTS-CERATESPASTES

Ointments or unguenta are semi-solid mixtures of drugs with fatty matters, and are used externally. The excipients 
generally employed are lard and oils; greater consistence and adhesion are conferred by addition of paraffin wax or resin : rancidity is checked by admixture of benzoin, or by substituting mineral, for animal or vegetable oils. By using hydrous wool fat, or oleic acid as the basis, absorption through the skin is said to be facilitated. When lard or oil is the excipient, the ointment may generally be prepared in a suitable mortar; but when wax or resin is used, it must be melted over a slow fire, the other constituents added, and the mass stirred until it has acquired proper consistence. Ointments, of which forty-four are enumerated in the B.P., should be kept in well-closed pots or jars, which (except when in daily use) should be covered with moistened bladder and strong paper. They are generally dispensed either in chip boxes or earthenware pots, of which the practitioner should have several sizes. In dispensing these and other officinal preparations, spatulæ of steel, bone, wood or horn are essential articles of the pharmacy.

Oleates are solutions of active principles in oleic acid. The B.P. contains directions for making mercuric oleate and the ointments of mercuric and zinc oleates. Oleic acid is used in the preparation of the ointments of cocaine, atropine, aconitine, and veratrine. Oleates are sometimes preferred to the corresponding ointments, on account of their being more readily absorbed. The smart friction employed in the in-rubbing of this class of remedies is itself of considerable therapeutic value. It causes dilatation of cutaneous capillaries, and hence promotes increased circulation through superficial blood and lymph vessels.

Liniments or embrocations are solutions of active principles in oil or spirit; some, besides, contain camphor, several have soap added, to increase their lubricant properties; all are intended for external use. The B.P. enumerates fifteen liniments.

Pastes are topical applications, of which the basis consists wholly or partially of fine silica, magnesium carbonate, or siliceous earths. Zinc oxide and other ointments are sometimes united with ten per cent. of such siliceous matters. The term 'paste' is occasionally also applied to mixtures of farina or flour with such drugs as borax, alum, or zinc oxide. 
The siliceous pastes do not impair skin secretion as fatty bodies do, and, moreover, leave a protective powdery coating on the skin. They are hence indicated. in some cases of moist eczema and other irritable conditions of the skin.

Cerates are stiff ointments containing wax.

Pomades are preparations of soft consistence with a basis of lard, lanoline, vaseline, or glycerin of starch, in which is incorporated various active agents, anæsthetics, antiseptics, astringents, etc.

\section{PLASTERS-EMPLASTRA}

Plasters are adhesive substances, usually containing lead oxide, conjoined with resin, wax, soap, fats, tar, or pitch ; are conveniently kept in rolls ; and are prepared for use by being melted and spread on calico, linen, or leather. The equable pressure of a well-applied plaster gives support and protection; retards evaporation, and hence raises topical temperature ; increases glandular activity; and sometimes also hastens removal of inflammatory products. Plasters are rendered more stimulating by addition of mustard or cantharides; more soothing or anodyne by addition of opium or belladonna.

Ordinary plasters are less useful in veterinary than in human practice ; for in the lower animals they are apt to be displaced by movements, the action of the panniculus carnosus, and by the patient rubbing or gnawing them. Where they are to remain on for several days or weeks, the melted ingredients are applied directly to the skin, and covered first with finely chopped tow or lint, and then with a linen or leather bandage. Plasters of this kind are popularly known as charges, and were formerly much used in some forms of lameness. They are serviceable when properly applied in preventing undue motion of injured parts. For open joint a useful plaster is made by shaking together one lb. shellac and one pint methylated spirit. The hair around the wound is clipped, a small piece of oiled silk is laid on, and the adhesive solution painted on with a camel's hair brush until a firm shield is formed. Adhesive or supporting plasters may be prepared with starch, silicate of potash, 
dextrin, tripoli, gum arabic, plaster of Paris, alum, guttapercha, or flour and white of egg. Velpeau's plaster consists of dextrin 100 parts, camphor tincture and hot water, of each 50 parts. Alum, 16 ounces, and alcohol, 35 ounces, heated until the mixture assumes the consistence of syrup ; or dextrin and alcohol, without the addition of water, solidify quickly and form strong adhesives.

\section{POULTICES-CATAPLASMATA}

Poultices are local baths or semi-solid packs, used for the topical application of heat and moisture. They closely resemble fomentations, and are often employed to soften the horn of the horse's foot. They constitute an important form of emollient. They are made of such farinaceous substances as linseed meal, bran, or oatmeal, stirred into boiling water until the fitting consistence is reached; or of carrots or turnips, either steamed or boiled. Bread and starch make bland porous poultices, and spent hops are indicated where the poultice should be light. Unless nicely prepared, soft, fresh, and changed every two or three hours, they merit Liston's condemnation, that they are associated ' with putrefaction and nastiness.' In order to secure softening of the skin, as well as the soothing of peripheral nerve-endings, medicated poultices are sometimes laid directly on wounds or contusions, or with only a very thin substance intervening. When used, however, either directly or reflexly, to relieve congestion or pain, they are placed in a well-warmed flannel bag, or in folds of flannel, which, conducting heat slowly, justifies their application at a higher temperature than could otherwise be borne, and, moreover, preserves heat longer. Poultices arrest superficial and circumscribed inflammation in the early stages; and in more advanced stages, when white corpuscles have escaped through the vessels, or pus has begun to form, they promote maturation of the abscess. They should not be used for wounds, which, if kept dry or treated antiseptically, will heal by first intention or adhesion, nor for chronic injuries where the parts have become relaxed and deficient in tone, in which cases cold applications are indicated. Heat, 
without moisture, may be applied by the agency of hot bricks, salt, bran, or sand, of hot-water bags, of wellwarmed rugs or flannels, or of the smoothing-iron. A piece of flannel, thoroughly wrung out of boiling water, applied hot, its several folds covered with thin mackintosh, and kept in place by a bandage, in virtue of the heat and equable pressure, relieves strains, and diminishes swelling and pain of the legs of horses worn by much work on hard roads.

Poultices, to be properly and securely applied, require some ingenuity and mechanical tact. To prevent unpleasant sticking, the skin is sometimes covered with a piece of muslin, or moistened with vaseline, oil, or solution of glycerin. Before application of the poultice, the irritable inflamed surface is sometimes dressed with equal parts of belladonna extract, glycerin and water, or an anodyne. To keep the poultice as long as possible at a uniform temperature, it should be of considerable bulk, and usually several inches thick; hot water is poured over the mass every hour or two, or, better still, fresh poultices are supplied as the old ones become dry, lower in temperature, or foul. Such changes should be quickly effected, for exposure chills the moist, warm surface. When the poulticing is done with, the surface should be enveloped in flannel, or in a woollen rug covered with oilskin. In cases of chest or bowel inflammation, dogs, like children, are advantageously placed in jacket or saddle poultices. Poultices are rendered more soothing by addition of opiates or anodynes ; more stimulating by sprinkling with mustard or turpentine; more antiseptic by admixture with boric acid, chlorinated soda, carbolic or salicylic acid, or charcoal.

Poultices too long continued are apt to soften and sodden the skin, and to hinder reparative power. Unwieldy to apply, and troublesome to regulate as to temperature, they are often superseded by fomentations, by antiseptic dressings, by water dressings of moistened and medicated lint or tow, from which evaporation is retarded by a covering of oiled silk or waterproof cloth, or by spongiopiline-a felted wool and sponge, coated on one surface with guttapercha, and when soaked with hot water proving a cleanly, handy substitute for a small poultice. 


\section{POWDERS-PULVERES}

Many medicines may be reduced to a rough powder in a hand-mill such as that used for grinding coffee or pepper ; or in an iron mortar (which should be fixed into a block of wood), with a large, heavy, iron pestle, which ought to be suspended from one end of a flexible rod running along the ceiling, and fixed into the opposite wall. Preparatory to further reduction, many roots and barks are pounded or cut. To effect minuter subdivision, small quantities of the coarse powders are reduced in hand mortars, of marble, or Wedgwood ware, the latter being cheap, easily cleaned, and little affected by acids. When a fine state of division is required, the powder is put through wire-gauze or horsehair sieves, the meshes of which are made of suitable closeness. For light, pungent, or irritant powders, compound sieves, closed in above and below, are used. To facilitate reduction of tough vegetable drugs such as opium, they are sometimes mixed with a hard salt, such as potassium sulphate. To avoid tedious trituration, powders, like calomel and flowers of sulphur, are conveniently obtained by sublimation; others, like magnesium carbonate or mercury red oxide, by precipitation ; other insoluble substances, like prepared chalk, by stirring in water, allowing the coarser particles to settle, and pouring off the solution from which the finely-divided powder is gradually deposited and dried. Nauseous, deliquescent, efflorescent, and volatile substances, and those given in large doses, cannot be conveniently administered in powder. Active drugs are prescribed with such inert substances as starch, gum, liquorice, or sugar of milk. Powders, when not too bulky, are occasionally dropped upon the patient's tongue, or introduced into the mouth or nostrils by insufflation. When free of disagreeable flavour, they are sometimes scattered upon or mixed with the food.

\section{RESIN S}

Resins are amorphous solids consisting of acids formed from the oxidation of terpenes $\left(\mathrm{C}_{10} \mathrm{H}_{16}\right)$. They are insoluble 
in water, soluble in spirit, and melt when heated. They dissolve in alkalies, forming soaps. They are frequently found in plants conjoined with volatile oil, constituting an oleo-resin, such as that of copaiba or of cubebs. Occasionally they are associated with gum, as in the gum-resins, asafœtida, ammoniacum, myrrh, and gamboge. The resins proper are only sparingly soluble in the animal secretions, but they irritate by contact, and hence, when swallowed, are cathartic and diuretic. The oleo-resins are more active; they stimulate mucous surfaces, from which they are absorbed or excreted. Gums or exudations from the stems of plants contain arabin or soluble gum (acacia), bassorin or less soluble gum, and cerasin or insoluble gum. Alcohol precipitates solution of gum.

\section{SYRUPS-CONFECTIONS-ELECTUARIES}

Syrups are saccharine solutions, usually containing flavouring or medicinal substances. Their specific gravity ranges from $1 \cdot 300$ and $1 \cdot 400$. Their consistence is important. If too thin and weak, they become mouldy, and are apt to ferment; if too thick and strong, the sugar crystallises out. But the B.P. ensures uniformity and good keeping by definite instructions as to the proportion of refined sugar and other constituents in the twenty-two syrups. Simple syrup is prepared by dissolving, by the aid of heat, five pounds of refined sugar in two pints of distilled water, and adding sufficient water to make $7 \frac{1}{2} \mathrm{lbs}$. of syrup. The syrups chiefly used in veterinary practice are those of buckthorn, squill, rhubarb, chloral, ginger, and iron iodide, in which the sugar prevents oxidation. Electuaries are made with sugar, mucilage, honey, or treacle. Confections or conserves are soft pastes, largely composed of sugar or honey, and, like syrups and electuaries, are chiefly used as vehicles for administering powders, or disagreeably tasted drugs.

\section{TINCTURES-SPIRITS-WINES}

Tinctures are spirituous solutions of active principles. The usual solvent is ethylic alcohol (45 to 90 per cent.). 
Spirit of ether is used in the preparation of tincture of lobelia, and solution of ammonia, in making the ammoniated tinctures of guaiacum, valerian, ergot, opium, and quinine. Tinctures are prepared without heat by simple solution, by maceration, or by displacement, or sometimes by a combination of these processes. The materials, first reduced by cutting or bruising, are placed with the spirit in a suitable vessel, and usually remain from two to seven days; the solution is poured off, the residue pressed, and the tincture, when filtered, is ready for use. Sometimes the materials, in a state of moderately fine division, are packed in a percolator or cylindrical vessel of glass earthenware, or metal ; the spirit passes gradually through them, displaces and dissolves out their soluble parts, filters through the linen or calico, which is usually stretched across the lower part of the cylinder, and passes off by the stop-cock, which should be attached to the apparatus. Some tinctures are made by macerating the materials in water for a couple of days, obtaining the remaining active principles by percolation with spirit, and mixing the two solutions. More thorough and rapid extraction of active principles is obtained by Burton's process. The drug and solvent are packed in the percolator, on the neck of which an elastic cap is fixed; with an exhausting syringe a partial vacuum is created ; and air being subsequently admitted, the spirit penetrates the drug, and more effectually extracts its active principles.

Tinctures are clear, of a yellow, red, or brown colour, and generally keep well. A few have been standardised, which means that, in accordance with the B.P. 1898, the tincture shall be made to contain a fixed percentage of the chief active principle of the drug. The tinctures of cinchona, jalap, and opium are now standardised, and others are directed to be prepared from standardised extracts. The revisers of the B.P. have endeavoured to reduce dubiety and risk of accident by so adjusting the strength of the tinctures that, for an adult human patient, the average dose of the more potent tinctures is from five to fifteen minims, and of the less potent, from half a drachm to a drachm, while four to eight drachms of the stronger, and one to two ounces of the weaker tinctures may be pre- 
scribed for the horse.-The official tinctures of aconite, digitalis, nux-vomica, cantharides, and iron perchloride, and a few unofficial tinctures are sufficiently concentrated to require special care in their administration.

Medicated Spirits, are solutions of volatile oils, ethers or other substances in alcohol (90 per cent.). The average strength is one in ten. The simple spirits are represented by those of peppermint, anise, camphor ( 1 in 10), juniper, chloroform ( 1 in 20) and ether. The official compound spirits (nitrous ether, sal volatile, etc.) are five in number.

Medicated wines, such as vinum antimoniale and vinum ipecacuanhæ, made with sherry or orange wine, are merely weak tinctures. Elixirs are tinctures mixed with aromatics and syrup.

\section{WEIGHTS AND MEASURES, IMPERIAL AND METRIC}

Two systems of weights - the imperial and the apothecaries'-were formerly employed by medical men, veterinarians, and chemists. The avoirdupois or imperial weight was used by wholesale druggists, and also by retailers in buying their drugs, and usually in selling quantities amounting to or exceeding an ounce. In dealing with smaller quantities, and in making up prescriptions, apothecaries' weight was employed. To avoid the ambiguity occurring from the use of these two systems, the framers of the B.P. in 1864 abolished the apothecaries' weight, adopted the avoirdupois ounce as the standard, divided it into $437 \cdot 5$ grains, and ignored entirely drachms and scruples. But so great is the inconvenience arising from the want of some denomination between the grain and the ounce, that medical and veterinary authorities still use the symbols $\Rightarrow$ (scruple) and 3 (drachm), the former representing 20 and the latter 60 grains.

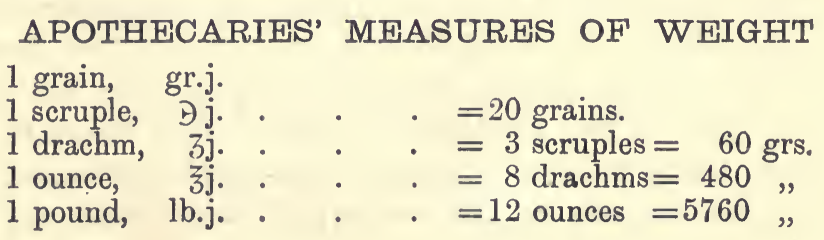




\section{PHARMACOPGIA MEASURES OF MASS}

1 grain, gr.j. $\quad=0.0648$ gramme, or 64.8 milligrammes.

1 ounce, oz.j. $3 \mathrm{j}$. $=437.5$ grains $=28 \cdot 35$ grammes.

1 pound, lbj. $=16$ ounces $=7000$ grains $=453.5825$ grammes.

\section{MEASURES OF CAPACITY}

1 minim, min.,

1 fluid drachm,

1 fluid ounce,

1 pint (octarius),

1 gallon (congius), mj.

f3j. $=60$ minims.

$\mathrm{f} \zeta \mathrm{j} . \quad=8$ fluid drachms

O.j. $\quad=20$ fluid ounces

C.j. $=8$ pints
$=480$ minims.

$=9600$

$=76800 \quad$ "

It is often useful to recollect the weight of different measures. Of water, one minim $\left(m_{j}\right.$.) weighs nine-tenths of a grain ; a fluid ounce at $62^{\circ}$ Fahr. weighs exactly $437 \cdot 5$ grains, or an ounce avoirdupois; hence a pint is equal to a pound and a quarter, and a gallon to ten pounds imperial weight.

To prevent mistakes, medicines for external and internal use should be sent out in differently shaped and differently coloured bottles, properly labelled; while all potent preparations should further be labelled ' Poison.'

When standard measures cannot be obtained, the practitioner has often occasion to use some of the ordinary domestic utensils, with the capacity of which he ought therefore to be familiar. Common tumblers contain from eight to ten fluid ounces; teacups, five to seven fluid ounces; breakfast cups, about eight to ten fluid ounces ; wine-glasses, two to two and a half fluid ounces ; tablespoons, half a fluid ounce ; dessertspoons, two fluid drachms ; and teaspoons, one fluid drachm of sixty minims. Such measurements, however, are merely approximate. The common teaspoon often holds one and a half to two drachms, so that its use for potent and toxic agents is dangerous. The pint and quart bottles, subdivisions of the old wine measure, contain respectively about 13 and 27 fluid ounces, and not, as their names might indicate, 20 and 40 fluid ounces. A Scotch pint contains 60 fluid ounces. Medicines are sometimes measured by the drop, which varies, however, exceedingly with the density and viscidity of the fluid, and the form and size of the vessel from which it falls. 
The metric system of weights and measures, now legalised in this country, is everywhere extensively used in scientific observations, and, from the simplicity of its decimal gradations, is certain to become general. The metric tables of weight, capacity, and length, with their relations to the corresponding English tables, are appended :-

\section{MEASURES OF MASS}

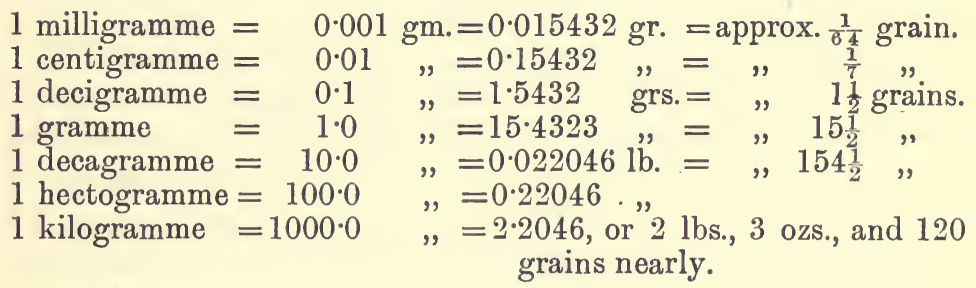

In commerce the kilogramme is calculated as 2 and onetenth pounds.

The gramme, taken as the unit of weight, is a cubic centimetre of water at $4^{\circ} \mathrm{C}$. or $39 \cdot 2^{\circ} \mathrm{Fahr}$.

\section{MEASURES OF CAPACITY}

$1 \mathrm{c.cm}$. , or 1 millilitre $=1$ gramme of water $=0.0610 \mathrm{c.} \mathrm{in.}$ $=$ nearly 17 minims.

$10,, \quad, 1$ centilitre $=10$ gramme of water $=0.610$ c. in. $=$ nearly 170 minims.

$100, \quad, 1$ decilitre $=100$ gramme of water $=6 \cdot 10 \quad$ c. in. $=$ nearly $3 \frac{1}{2}$ ounces.

$1000 ", 1$ litre $\quad=1000$ gramme of water $=61 \cdot 0 \quad$ c. in. $=$ nearly $35 \frac{1}{4}$ ounces.

A litre is equal to 1.75980 pint, or 1 pint, 15 ounces, 1 drachm, and 34 minims nearly.

\section{MEASURES OF LENGTH}

1 millimetre $=0.001$ metre $=0.039370$, or $\frac{1}{25}$ English inch.

1 centimetre $=0.01 \quad, \quad=0.39370$, or $\frac{2}{5}$ English inch.

1 decimetre $=0.1 \quad, \quad=3.9370$, or nearly 4 English inches.

1 metre $=1 \cdot 0 \quad "=39 \cdot 370113$, or 1 yard, 3.37 ins. nearly. 
The Fahrenheit thermometer, the measure of temperature still retained by the B.P., and in many works on human materia medica, is the measure again adopted in this book. The Centigrade scale, however, is also extensively used. It is often requisite to ascertain the corresponding numbers on each scale, and for this purpose the following rule is useful. To convert any number of Centigrade into Fahrenheit degrees, multiply by 9 , divide by 5 , and add 32 . For the converse process, subtract 32 , multiply by 5 , and divide by 9 . 



\section{N D E X E S}

\section{INDEX OF DISEASES AND REMEDIES}

Abortion.

Expulsion of the young before viability; Slipping; Premature birth. (1) Occasional or accidental cases caused by rough treatment, falls, railroad journeys, acute indigestion, ergotised food, occur in all females. (2) Contagious abortion depending upon specific infection of the womb; the microbe is present in the uterine discharges and amnial fluid of aborting cows, ewes, and mares. Contagium may be transmitted by the bull.

Isolate aborted from pregnant animals and burn litter.

Burn, thoroughly disinfect, or deeply bury fotus and its membranes.

Cleanse and thoroughly disinfect premises in which animal has aborted.

Irrigate, with germicides, uterus, vagina, external organs, and tail root of every animal aborting.

One part corrosive sublimate, 40 common salt, 4000 clean rain-water, or one part each mercuric iodide and pot. iodide in 1000 water, safe and effectual.

With this solution irrigate vagina, external genitals, and tail root of animals that exhibit premonitory symptoms.

Postpone breeding with cows that have been affected with contagious vaginitis.

Wash frequently external genitals and tail of gravid animals that have herded with those aborted, with 5 per cent. solution ferrous sulphate.

Animals which have aborted should be fed off, for the microbes are dislodged with difficulty, and fresh generations are liable in subsequent conceptions to repeat their attack (Nocard).

Prohibit use of bulls that have served aborted cows.

\section{Abscess.}

A circumscribed swelling containing pus.

Fomentations, poultices, water dressings relieve tension and pain.

When maturing tardily, apply counter-irritant-mercury oleate or iodide over small area.

Open immediately with knife, and provide drainage.

When deep-seated, open by Hilton's method under local anæsthesia.

After evacuation dress antiseptically.

Healing of chronic abscess cavity hastened by antiseptic injection; as 10 per cent. sol. of iodine.

Belladonna inunction relieves pain.

Acari. Mites. See Mange and Scab.

They produce skin irritation, itching, and eruption.

Soft soap, alkalies, and hot water cleanse skin, remove scales, and lay bare burrows.

Soaking with oil and alkali facilitates removal of crusts. 


\section{ACARI-continued.}

As parasiticides rub in sulphur, or sulphur iodide ointment, mercury oleate, tar oils, stavesacre, or creolin solutions.

For dogs-aniseed or other volatile oil, wood tar oils, or creolin.

For sheep-solutions of tar oils, arsenic, tobacco. Separate affected from healthy. Narrowly watch suspected. Disinfcct premises, racks, rubbing posts, etc.

\section{Actinomycosis.}

A disease caused by the ray fungus found on barley and other plants, thence introduced into the bodies of animals, probably through abrasions in the mucous membranes, producing granulomata, nodules or tumours ; found chiefly in the tongue and jawbones of cattle, udder of sows, etc.

In early stage excise diseased structures.

Scrape and dress with iodine tincture or a mixture of iodine, carbolic acid, and glycerin.

Administer full doses pot. iodide for a month ; iodopin, or small doses of mercury biniodide.

Precautions to prevent transfer of parasite to man or other animals.

Generous diet; tonics.

\section{Acidity of Stomach.}

Pyrosis. Occurs in all animals, notably when under artificial conditions.

Change of food, which should be digestible, unstimulating, and rather restricted in amount.

Half dose of physic will remove any irritant.

Mineral acids, given before or with food.

Alkaline bicarbonates as palliatives.

Lime water and chalk when diarrhœea present.

Magnesia or the carbonate when constipation present.

Place a piece of rock salt in horse's manger.

Conjoin antiseptics with antacids when associated with flatulence.

Bismuth and opium, with liquorice when accompanied by irritation.

Gentian, nux-vomica, and other bitters when resulting from atony.

Silver oxide, tannates, lead acetate, when associated with gastric catarrh.

ACNE.

Inflammation of sebaceous follicles and sweat glands, with retention of secretion and eruption of pimples, usually produced in horses by badly fitting or dirty harness.

Wash and disinfect skin ; hot fomentations; water dressing; alkaline solutions, such as saturated solution sodium bicarbonate, borax, glycerin and water, or sulphur iodide. Touch each pimple with undiluted carbolic acid.

See to fitting and proper lining of harness; zinc shield for collar.

Sulphur, both locally and internally. Belladonna extract, or Goulard's extract relieve local irritation.

Salines and arsenic internally in chronic cases.

After-Pains.

Post-partum pains : Heaving; Septic metritis.

Remove fœtal membranes and clots from uterus; raise hind-quarters.

Syphon into u terus warm solution of antiseptics and anodynes, or solution of carbolic acid, potassium permanganate, or mercuric iodide, with belladonna and opium.

Ergotin hypodermically, if uterus flaccid and dilated.

Administer chloroform or chloral, with cannabis indica or belladonna.

Morphine and atropine hypodermically in persistent cases.

Laxatives and enemata to empty bowels; remove milk from udder.

Disinfect attendants and lambing pens. 
Alopecia.

Baldness depending on faulty nutrition of skin and atrophy of hair bulbs. Often parasitic.

Oleaginous diet, tonics, sulphur iodide. Antiparasitics.

Stimulate skin with ammonia liniment; cantharides tincture, one part, soap or camphor liniment, eight parts; or castor oil.

Shave and rub in vaseline daily, dressing occasionally with above stimulants.

Amaurosis. Buindness.

Paralysis of optic nerve and retina with constantly dilated pupil ; may follow contagious equine pneumonia and canine distemper.

Except when depending upon loss of blood, debility, or lead-poisoning, it is incurable.

Strychnine in traumatic cases and those of nerve atrophy, but unsuitable where there are brain symptoms.

An amia.

Diminution of red corpuscles and other essential constituents of the blood.

Pernicious anæmia, probably dependent on a microbe (Friedberger), frequently infectious, and accompanied by fluidity and redness of bone marrow. In dogs may be due to worms.

Generous diet, containing suitable proteids and fats.

Comfortable quarters, pure air, exercise ; clip horses with heavy coats.

Iron salts and occasional laxatives. Malt extract; hæmoglobin.

Gentian, quinine, and other bitters where appetite faulty; strychnine.

Mineral acids where gastric mucous membrane soft and relaxed.

Arsenic, after iron has been given for ten days, or where iron disagrees.

Calcium phosphate in growing young animals; Squires' syrup, alternated with Donovan's solution, or cod-liver oil.

\section{ANEURISM.}

A localised dilatation with partial rupture of an artery.

Equable pressure, truss, bandages, acupressure, cat-gut ligatures above and below sac.

Aconite relieves pain and lowers circulation.

Ergotin locally injected contracts vessel.

Pot. iodide encourages absorption. Rest, quiet; rather low diet.

Electrolysis, injection of ferric chloride, lead acetate, or sterilised solution of gelatine may cause coagulation within sac.

Radical cure and obliteration of vessel by double ligature or by continuous acupressure.

Argle Berries. See Warts.

Remove by knife, torsion, caustic, or ligature ; dress antiseptically.

Anthrax. Charbon.

Depends upon the Bacillus anthracis, and is communicable by inoculation from one animal and from one species to another. Cattle, horses, and sheep are most frequently attacked. Bacilli and spores are taken up by animals in their food and water; are occasionally inhaled, or may be introduced through skin abrasions. The characteristic lesions are accompanied by acute pyrexia, and are usually localised-

(1) In the subcutaneous structures, corresponding to the malignant pustule of man.

(2) In the pharynx and respiratory passages of pigs, horses, and other animals.

(3) In the intestine of horses.

(4) In the spleen, chiefly of adult cattle and sheep, recognised as splenic apoplexy; very sudden in its onset, and killing sometimes within an hour. 
Anthrax. Charbon-continued.

Curative treatment in animals is dangerous and inadvisable.

Preventive treatment consists in protection from access of bacillus and spores.

Cremate or bury deeply infected discharges and carcases, which should neither be skinned nor opened; fence off graves.

The flesh of animals dead from anthrax has produced by ingestion the disease in pigs and dogs.

Thoroughly disinfect premises.

Keep susceptible animals off pastures where disease has occurred.

Inoculation with attenuated virus ensures temporary protection.

ANuria.

Dysuria; Retention of urine. Results from calculi, or urethral obstruction, paresis or spasm of the bladder, and other causes ; more common and distressing in horses and dogs than in cattle or sheep.

The bladder in the horse may be gently pressed by the hand introdueed into the rectum; warm hip bath for dogs; pass catheter.

Friction and warm applications to the perineum may overcome paralysis or spasm.

Anti-paralysants or antispasmodics administered.

Surgical treatment for calculus ; urinary antiseptics.

Aphtha.

Vesicles in the mouth; Thrush. Associated with epiphytes, oïdium albicans, etc. Most common in young animals.

Alum, hydrogen peroxide, borax, sulphurous or boracic acid, or pot. chlorate solution applied locally.

Oxymel, glycerin and water, or glycerin of starch, or of tannic acid.

When connected with gastric derangement, give laxatives, salines, or grey powder.

When patient reduced prescribe tonics.

Soft digestible food.

Aphthous Fever or Vesicular Epizootic.

Foot-and-mouth-disease; A contagious eruptive fever affecting cattle, sheep, pigs, and occasionally poultry. The infective agent, contained in the discharges from the vesicles and ulcers, is transmissible by direct or by mediate contagion.

Segregate affected; disinfection ; green forage or soft digestible food.

Keep inflamed, abraded surfaces clean, and moisten occasionally with alum, borax, or zinc sulphate, made up with treacle, honey, or glycerin and water.

Lead or zinc acetate solution or ointment, Goulard's extract, or potassium permanganate diluted, applied to udder and feet.

Milk affected cows frequently, preventing lodgment in udder of stale milk.

The milk unsafe unless boiled for fifteen minutes; better destroyed.

Pot. nitrate and chlorate, mixed with mash or drinking water, when fever high.

One attack does not protect the subject exposed to subsequent infection.

Apoplexy, Cerebral.

Rupture of blood-vessel occasionally from atheroma, causing pressure, and sometimes softening of brain substance. Not common in domestic animals.

Blood-letting, pot. iodide, lower arterial pressure where attack threatened or in earlier comatose stage.

Active purgative, laxative enemas, cold water and ice to head relieve congestion.

Nitro-glycerin and bromides also diminish cerebral congestion. 
A POPLEXY, CEREBRAL-continued.

Alcohol and other stimulants may be needful to combat subsequent anæmia.

Massage, electricity, strychnine, relieve paralysis.

Digestible laxative food; avoid over-exertion or exposure to heat of sun.

Iodine and pot. iodide promote absorption.

Recurring attacks in robust subjects prevented by careful diet and regular work.

Apoplexy, Pulmonary.

Hyperæmia and œdema of lungs. Occurs especially in horses out of condition, usually from violent over-exertion.

Cool air to breathe ; smart hand-rubbing of body and limbs, which must subsequently be warmly clothed.

Small repeated doses of alcohol, ether, or ammonia stimulate cardiac and respiratory centres.

Abstraction of blood from jugular relieves congestion of right heart.

Apoplexy, Splenic. See Anthrax.

Appetite, Impaired.

Varied and tempting diet.

Food removed if not eaten, and fresh supply presented at next meal.

Acids, bitters, nux-vomica, quinine, glycerophosphates.

Examine teeth, mouth, and throat, and look for gastric, cardiac, or other cause.

Arthritis. See also Navel Disease.

Inflammation of joint. Occurs in all animals; in young, from pyæmic infection, or rheumatism : in horses, from strains, injuries, or from open joint. Synovial membrane primarily affected, but other structures become involved, and in chronic cases periosteum, ends of bones, and ligaments are attacked.

Rest, anatomical and physiological, fomentations, hot compress.

Antipyretics, with analgesics subcutaneously, to reduce pyrexia and pain; counter-irritation in some cases after abatement of acute symptoms.

Slings, splints, and adhesives needful for horses in acute attacks.

Antiseptics in treatment of open joint. Injection of sterilised glycerin, or solution of hydrogen peroxide.

Sodium salicylate in rheumatic cases.

Ascarides. See Worms.

Ascites.

Abdominal dropsy : Accumulation of fluid in cavity of peritoneum, depending on disease of some important internal organ obstructing return of venous blood to the heart. More common in dogs, sheep, and cattle than in horses.

Remove, if possible, conditions on which it depends.

Diuretics, salines, milk sugar, pot. iodide, pilocarpine.

Digitalis, especially in cardiac complications, infusion of buchu.

Generous diet and tonics in anæmic or tuberculous disease of peritoneum.

Concentrated dietary, iron salts, and turpentine in sanguineous form appearing in impoverished sheep and lambs.

Tapping, even when it does not cure, relieves distressing symptoms. Asthma.

Dyspnœa depending on intermittent bronchial spasm. Rare in horses and cattle, more frequent in dogs.

Paroxysm checked by cautious inhalation of anæsthetics, amyl-nitrite, or nitro-glycerin, or by chloral given by mouth.

Bromides; strong coffee internally.

Emetics relieve many canine cases.

Inhalation of terebene with or without steam : of stramonium with pot. nitrate and chlorate, or amm. chloride. 


\section{Asthma-continued.}

Belladonna, sometimes with eucalyptus, internally or inhaled.

Alkalies, amm. chloride, pot. iodide ; inhalations of sulphur fumes when associated with dry bronchial catarrh.

Oil of turpentine, asafœtida, benzoin, eucalyptus oil when discharges profuse.

Strychnine internally or hypodermically when respiratory centre weak.

Acids and arsenic when complicated with gastric derangement.

Occasional dressing of throat with stimulant embrocation, especially in old dogs with severe bronchial symptoms.

Regular digestible, rather concentrated, diet; exercise.

\section{Atheroma.}

Degeneration of arteries, with calcification. Not common in lower animals.

Avoid over-exertion; prescribe digestible oleaginous diet.

Iron salts, phosphates, Easton's syrup in debilitated patients.

Phosphorus in small doses where brain ressels implicated.

\section{Atrophy.}

Wasting; Emaciation. Results from imperfect nutrition, nervous lesion, pressure, disease, or inaction.

Suitable diet ; fitting use of wasted part ; tonics, arsenic, and strychnine internally.

Friction, massage, electricity locally. Counter-irritation.

Iuunction of oil, cantharides, or mercuric iodide in muscular atrophy.

\section{Azotoria. See Hamoglobinuria.}

\section{Balanitis.}

Inflammation of the glans of penis. Common in dogs ; contagious.

Penis withdrawn, examined, cleansed, and dressed with astringent.

Injection of sheath with mild astringent daily for a week. Zinc chlor. grs. ij. to grs. iv. in an ounce of water ; solution of hydrogen peroxide. Any fungoid or vascular formations scraped or cut off.

In persistent cases horse or bull must be cast and secured, thorough examination made ; inflamed, ulcerated, or fungoid surfaces cauterised or dressed with silver nitrate solution containing grs. $x$. to the ounce of distilled water ; or douched with $2 \frac{1}{2}$ per cent. sol. of lysol.

Zinc sulphate or lead acetate injections repeated daily.

Perfect rest, dose of physic, laxative diet.

Barrenness. Sterility.

Congenital, sometimes from hermaphrodism. Also caused by disease of organs of generation and faulty general health, with acid or septic vaginal discharges. (See Vaginitis.)

Improve condition of debilitated by diet and tonics. Wash out and disinfect vagina; vaginal injections of yeast, one cake in a quart of tepid water.

Dilatation of os uteri if it be constricted; change male. Artificial insemination may be tried.

Alteratives; pot. iodide; yohimbine; or phosphorus and damiana in small doses.

Gradual lowering of fat plethoric subjects.

\section{Bites of INSECTS.}

Ammonia or pot. bicarbonate solution. Eucalyptus oil.

Creolin, carbolic acid, prussic acid, chloroform, cold water dressings.

BLACK-LEG Or BLACK QUARTER.

Quarter evil. Consists in phlegmonous, emphysematous swellings, usually about the hind-quarters, occasionally on the shoulders, neck, or fore-quarters, caused by the Black-quarter bacillus. Cattle, sheep, goats, and rabbits are readily inoculated; the horse, ass, 
BLACK-LEG or BLACK QUARTER-continued.

and white rat exhibit only local swelling at the point of inoculation ; the pig, dog, cat, black rat, and man are immune. The disease is endemic; the bacterium, from the infected pasture or water, probably enters the body by wounds of the legs or feet. Cattle from four to twenty months are most liable to attack.

Curative treatment very unsatisfactory. Free scaritication of limited external swellings and moistening with antiseptics arrest a few slight cases.

Prevention is effected by keeping young cattle and sheep off pastures known to be infected; and by inoculation with special vaccine.

Animals dying from the disease should be burned unskinned and unopened, or deeply buried with lime.

Disinfection of premises is adopted as for contagious diseases.

Setons have been credited with some preventive power.

Administration once or twice weekly of pot. chlorate or other saline antiseptic advised.

Black-quarter vaccine used hypodermically as a preventive in France, Germany, and England; stated to be effectual. See p. 697.

Bladder, Urinary, Inflammation of. See Cystitis.

BLADDER, IRRITABLE.

Diluents, linseed tea, suitable diet. Interdict heated grain or fodder, or other acrid food.

Laxative relieves any gastro-intestinal irritation. Urinary disinfectants.

Belladonna or henbane as anodyne used internally and locally.

Benzoate of soda or ammonium benzoate when urine alkaline.

Alkaline bicarbonate when urine acid; bromide of potash or camphor.

Salol and benzoic acids as antiseptics in chronic cases; urotropine.

Sulphuric and salicylic acids with iron sulphate, where irritation in horses is connected with influenza or purpura.

Anodyne enemata benefit reflexly.

Cleanse with soap and water prepuce and external meatus of male.

Bladder, Paralysis.

Prevent accumulation of urine by use of catheter, or in horse by gentle pressure on viscus from within rectum.

Prescribe cantharides, ergot, or cannabis indica; nux-vomica or eserine when atonic ; and urinary antiseptics.

Bleeding. See Hemorrhage.

\section{Bog Spavix.}

Distension of tibio-tarsal capsule ; in some cases acute synovitis.

Rest; high-heeled shoe.

In acute cases rest best secured by slinging.

Foment when joint hot and tender.

Cold water and refrigerants when acute inflammation abated.

Spring truss sometimes used in simple cases to give equable pressure.

Counter-irritation encourages absorption in chronic cases.

Removal of fluid by aspirator or special trocar may be tried.

BoIl or Furunculus.

A localised infective inflammation, with suppuration and limited necrosis of the cutis, and caused by a staphylococcus. A carbuncle is a boil on a larger scale.

Mercuric nitrate or belladonna ointment, or painting with silver nitrate, sometimes aborts inflammation in early stages.

Fomentations and poultices hasten maturation and relieve pain.

Counter-irritants hasten suppuration.

Anodynes locally relieve irritation and pain. 
BoIL or FURUNCULUS-continued.

Laxative diet, alkaline sulphites and chlorates, and calcium sulphide internally.

When opened, treat antiseptically.

Arsenic internally sometimes prevents recurrence.

Bone Spavin. See Spavin.

Botryomycosis.

A local disease of horses and sometimes of cattle and pigs, caused by a fungus, the Botryomyces equi. Infection leads to formation of cutaneous or subcutaneous suppurating growths of variable size, hard, movable, and not very painful ; occur on shoulder, withers, jaw, scrotum, sheath, tail, etc. ; sometimes inside the body.

Early excision; pot. iodide administered daily for a month or longer.

Bots IN HoRses.

Larvæ of œstrus equi developed in stomach.

Turpentine and oils, bitters, hydrochloric acid, iodine, naphthol, copper and iron sulphates, arsenic, followed by purgatives.

Carbon bisulphide 75 grains, in gelatin capsule, repeat dose in twelve hours, and next day give a dose of physic.

Green fodder; destroy larvæ as they are expelled in spring, and the fly. Bowels, Inflammation of. See Enteritis.

Braxy in SheEP.

An infective gastro-enteritis characterised by patches of inflammation and ecchymoses chiefly affecting the mucous and serous membranes and skin. Pathology undetermined. (Consult Report by Dr. Hamilton on Braxy and Louping-Ill, Board of Agriculture and Fisheries, 1906.)

Most cases prove fatal.

Prevent by carefully regulated dietary.

Removal from exposed, undrained, infected grazings.

Broken KNeEs in Horses.

Where skin not broken, tie up head and apply diluted zinc or lead acetate solution for two or three days.

When skin cut, cleanse thoroughly, dress antiseptically, bring edges together with pin sutures, plaster, styptic colloid, or shellac paste; retain them in position with light calico bandage; dress with mild astringent solutions.

When skin considerably lacerated, the tendon and its sheath bruised and exposed, or the knee-joint opened, the limb should be put in splints and animal in slings ; several folds of antiseptic lint, retained in place by a calico bandage, over which cold water is made to trickle continuously.

When tendon much bruised, knee-joint laid open, bones seriously injured or fractured, anchylosis must ensue, and the animal had better be destroyed.

BROKEN WiNd IN HoRsEs.

Pulmonary emphysema, associated with indigestion and characterised by expiratory dyspnoea and peculiar cough. Of nervous origin and connected with the vagus.

Seldom curable, but relieved by careful dietary; good concentrated food given damped; feed and water frequently, in small quantity at a time, but withhold before hard, fast work.

Reasonable restriction of water specially needful when heart affected.

Laxatives and salines given occasionally.

Rock salt, chalk, or whiting in manger. Two or three ounces linseed oil in the food twice a week.

Arsenic (Fowler's solution) given daily or every second day, may be continued for months.

Sedative cough balls occasionally. 
Bronchial Croup. 'Fat stock show disease.'

Fibrinous exudation on tracheal and bronchial mucous membrane. Frequent in cattle and sheep, and in other animals which have breathed smoke and hot air, especially during fogs.

Rapid progress renders treatment nearly hopeless.

Inhalation of vapour medicated with phenols, terebene, eucalyptus oil, or amm. chloride. Eresh air.

Apomorphine in carnivora.

Tracheotomy essential to prevent asphyxia.

Bronchial Filaria. See Worms.

Bronchitis, Acute.

Inflammation of mucous membrane lining bronchi. Sometimes extends into the bronchioles, alveoli, and lung parenchyma. Horses and dogs attacked more frequently than cattle or sheep. Usually classified as (1) Acute ; (2) Croupous ; (3) Chronic ; (4) Verminous.

Place horse in comfortable box, $60^{\circ}$ to $65^{\circ} \mathrm{Fahr}$, cool, pure air to breathe; body and limbs clothed.

Inhalation of watery vapour from steam.kettle, large mashes, or buckets of boiling water promote exudation in dry stage.

Inhalation, medicated as required by antiseptics, anodynes, or expectorants.

Fomentations and mustard to throat and sides.

Salines in drinking water, and antifebrin or antipyrine relieve fever.

Aconite, a few doses early in robust subjects, where symptoms acute.

Ammonium acetate solution, ipecacuanha, and squill while membrane dry and congested.

Apomorphine, pilocarpine, benzoic acid, eucalyptus oil, terebene, mineral acids diminish excessive secretion.

Soap liniment and laudanum rubbed into throat and down neck twice daily relieve difficult breathing, especially when secretion excessive.

Belladonna eases cough, conjoined with camphor, ether, or chloral hydrate, and in debilitated patients with small repeated doses of alcohol.

Electuaries or gargles of opium, chloral hydrate, with glycerin and liquorice, also relieve cough. Pot. chlorate and amm. chloride promote fluid secretion. Lobelia and opium where there is much discharge and paroxysms of cough.

Ammonium carbonate when mucus abundant and viscid, and patient low.

Mash and milk diet; regulate bowels if possible by enemata; cathartics dangerous in horses.

For dogs, emetic in early stage; apomorphine, ipecacuanha, and antimonial wine, where membrane dry and congested and fever high; in weakly subjects and advanced stages, bronchi cleared by emesis produced by ipecacuanha, squill, and ammonium carbonate; milk and flesh diet.

Bronchitis, Chronic.

Frequently follows acute attacks. Sometimes of verminous origin.

See Worms and Hoose.

Equable temperature; pure fresh air; comfortable clothing which must be removed and patient wisped over night and morning.

Salines, with or without mercurials, relieve congestion and fever.

Terebene and eucalyptus oil as stimulants of bronchial secretion.

Belladonna, balsanıs, and mineral acids diminish excessive secretion.

Ammonium carbonate and chloride useful where secretion viscid and irritating.

Belladonna and ether stimulate respiratory and cardiac centres.

Chloroform, chloral, and opium abate cough.

Mustard and other counter-irritants, carefully used, lessen congestion, irritation, and cough,

Soap liniment, with or without laudanum, frequently relieves cough. 


\section{Bronchitis, Chronic-continued.}

Alcohol, caffeine, ether, volatile oils, digitalis, maintain heart-action in weakly subjects.

Inhalations of sulphurous acid, creosote, eucalyptus, and other antiseptics, when secretions fotid. Intratracheal injections in parasitic cases.

Arsenic occasionally relieves emphysema.

Careful dietary, nutritive oleaginous food, cod liver oil.

Iron and other tonics promote convalescence.

BRONCHOCELE. GoITRE.

Hypertrophy of thyroid gland. Rare in horses and cattle, more common in dogs. Often harmless.

Local stimulation, iodine, pot. iodide. The milk of goats which have been deprived of their thyroid glands.

Puncture and inject with iodine (Cagny).

Liberal dietary ; full proportion of fatty matters. Extirpation may be necessary.

Bruises : Contusions.

Medicated fomentations or poultices, water-dressing, refrigerants, carbolic and other antiseptics. Massage and subsequent inunction with oil.

Lead, zinc, and other astringent solutions limit swelling and promote absorption.

Belladonna, opium, and aconite are useful to relieve the pain.

BruSHING or INTERFERING.

Occurs in horses with faulty action, and in others when leg weary or out of condition.

Careful shoeing. A plain shoe unnailed on the inner branch. A threequarter shoe, or a shoe thin on inside web, without heel on outside.

Well-fitting boot on the fetlock liable to be struck.

Improved condition often the most effectual remedy.

BULLA.

Pemphigus: Herpes: Eruption of large vesicles in irregular patches about junction of skin and mucous membranes, usually connected with gastric derangement, especially in young patients. Rare in animals.

Vaseline, 'Sanitas' solution, carron oil.

Laxatives and salines when connected with gastric derangement.

Adult horses subject to an inflammatory form passing to pustulation, treated by laxative, alkaline wash, and zinc ointment.

\section{Burns and Scalds.}

Protect immediately from air and irritants by layers of cotton wool or application of carron oil.

Liniment of oil and litharge, with two per cent. boric, or carbolic acid, or peppermint oil.

Whiting and water, or fuller's earth and oil, about consistence of cream, applied repeatedly, until tolerable coating formed.

Zinc oxide, with about ten parts of vaseline, or of glycerin and water.

Alkaline solutions, soap lather, saturated solution sodium bicarbonate relieve irritation in slighter cases.

Where discharges are foul, add antiseptics to above dressings.

Where there is irritation or pain, add chloroform or laudanum, or both.

Combat constitutional symptoms with antiseptics and anodynes internally. Anticipate collapse and necrosis in severe cases.

BuRsattee.

Kunkur : A mycosis of the skin and subcutaneous tissue, characterised by the formation of small hard growths which eventually ulcerate and suppurate; affects horses and other animals in India and other tropical countries. 


\section{INDEX OF DISEASES AND REMEDIES}

BURSATTEE-continued.

Improved sanitary conditions; change of food and surroundings.

Kunkur growths excised; wounds and ulcers treated antise ptically.

Calculi, Biliary.

Purgatives, salines, olive oil.

Chloroform, chlorodyne, belladonna internally.

Morphine and atropine hypodermically.

Nitric acid, nitro-hydrochloric acid.

Hot fomentations, counter-irritation.

Calculi, Intestinal.

Dust balls ; Concretions.

Rectal exploration : use long tube for injections; Laparo-enterotomy.

Avoid active cathartics, but give enemas and anodynes. Restrict to soft, concentrated food.

Morphine and atropine hypodermically, chloral or Indian hemp, most prompt and effectual means of relieving spasm and pain.

Calouli, Urinary.

Lithiasis; Gravel.

Dilute mineral acids in horse. Try piperazine or lithium carbonate in dogs.

Alkalies or alkaline bicarbonates diminish tendency to urinary deposits common especially in highly-fed rams and wethers.

Ammonia benzoate helps resolution of phosphatic deposits of sheep.

Diluents, cooling laxative food; raise feeding sheep thrice daily, and drive them a few hundred yards, ensuring their urinating.

Sheep affected must be placed on buttocks, and by manipulation the sabulous matter in urethra is gradually moved.

Where canal hopelessly blocked it must be opened either at the ischial arch or by amputation of appendix.

Lithotomy or lithotrity only means of removing cystic calculi of any considerable size in male animals.

CANCER.

Carcinoma: A malignant growth of epithelial-like cells contained in an alveolar stroma. Affects all classes of animals.

In early .stage, excision of localised accessible growths; when tumour large or diffuse, operation inadvisable.

Destruction by chromic acid or other caustic seldom successful or safe.

Carbolic acid, bromine, or iodoform may retard growth and lessen risk of secondary infection.

Generous diet retards exhaustion caused by absorption from disintegrated tissues.

Analgesic, antiseptic, and deodorant dressings.

Canker of Horse's Foot.

A disease affecting the keratogenous membrane of the foot, and producing disorganisation of the horn of the sole, frog and laminal sheath, and fotid discharge.

Mr. Malcolm, Birmingham, has shown canker to be purely local, occurring in all breeds and descriptions of horses, possibly depending on an epiphyte, and, while confined to the frog and sole, curable (Jour. Comp. Path. and Therap., 1891).

In all cases, the degenerate horn, fungoid growths, and every portion of unhealthy tissue must be excised with the knife or cauterised with the hot iron.

Dress with salicylic acid, chromic acid on carbolised cotton-wool, chinosol, or formalin solution; or with equal parts of sulphates of copper, iron, and zinc, with crude carbolic acid, and vaseline to form a paste. This is covered with tow and a leather or iron sole, and the shoe replaced. 
Canker of Horsk's Foot-continued.

Daily, or every second day, the horse, either standing or cast, examination is made for any canker specks, which are removed and the foot dressed as before.

Mineral acids, iodoform in ether, or silver nitrate sometimes serviceable. Calomel used dry encourages growth of horn.

Examination and dressing repeated at longer intervals.

After first few days many cases are best at work.

Canker of Ear. See Otorrhoea.

Capped Hock, Knee, and Elbow in Horse.

(a) A collection of fluid immediately underneath the skin of the point of the hock.

(b) Chronic synovitis of the bursa of perforatus cap.

Capped Knee-(1) Effusion beneath the skin or annular ligament. (2) Distension of sheath of extensor metacarpi magnus.

Capped Elbow-Subcutaneous infiltration from bruising.

Hot fomentations ; subsequently stimulate with cantharides liniment or mercuric iodide ointment; soft soap rubbed in daily.

If swelling recent and fluctuating puncture with aspirator needle ; inject cavity with iodine solution.

In bursal form of capped hock use shoe raised at heel; apply a charge.

In indurated capped elbow excision is the only satisfactory remedy. Prevent recurrence by heel-pad, ring-pad, or by felt attached to horse-rug.

Carditis. Myocarditis.

Rare in lower animals. Inflammation of muscular fibres of heart with (1) infiltration or induration, or (2) suppuration, as in puerperal metritis in cattle, or omphalo-phlebitis of foals.

Concentrated good food.

Digitalis, caffeine, alcoholic stimulants as heart tonics.

Stimulant embrocations to chest.

Combat pyæmic infection with antiseptics and quinine.

\section{Cataract.}

Definite opacity of the lens.

Extraction of the lens; or needling to promote its absorption.

Chloroform-and locally, atropine and cocaine-for diagnosis and operation; mercurials internally.

Catarrh.

Inflammation of any mucous membrane, with serous or muco-purulent discharge. But the term is specially applied to inflammation of the membrane lining the nasal chambers and upper respiratory passages, constituting Cold in head or Coryza. More frequent in horses than other animals ; contagious.

House comfortably; clothe body and head; bandage legs.

Equable temperature of $60^{\circ}$ to $65^{\circ} \mathrm{Fahr}$.

Steam head with vapour of water alone, or medicated with antiseptics or anodynes.

Warm or vapour bath; patient quickly dried and re-clothed.

Mash diet or green food; laxative enemata; laxatives if required.

Amm. ace tate solution; pot. nitrate or chlorate ; other saline electuaries. Hot fomentations; stimulating embrocations to throat. Antiseptic insufflations.

Catarrh, Chronic, of Horses.

Isolate patient until tested for glanders.

Nasal douche or spray.

Wash out nasal passages, and, if necessary, trephine and disinfect the sinuses.

See to faulty teeth. Examine guttural pouches. 


\section{Catarrh Chronic, of Horses-continued.}

Fresh air, rest, or gentle work ; if coat rough clip or singe.

Green fodder; feed on floor, or graze by day.

Inhalation of sulphurous, carbolic, or iodine vapour.

Administer iron, arsenic, terebene, or copaiba.

Catarrhal Fever, Malignant, of Cattle.

An acute contagious fever, with catarrhal inflammation of the mucous membranes of the eyes and respiratory organs, occasionally involving the gastro-intestinal and urinary membranes, usually of two to four weeks' duration; the mortality 70 to 90 per cent. More frequent in Europe and America than in Great Britain, chiefly affecting young animals in spring (Friedberger and Law).

Antipyretics, saline expectorants, and antiseptics; in Germany intravenous injection of one per cent. solution of colloid silver said to be beneficial.

Combat formidable symptoms and maintain strength.

\section{Cerebral An emia.}

Occurs in all animals.

Stimulants to combat syncope. Sterilised salt solution hypodermically.

Mustard and other cutaneous irritants.

Phosphorus, phosphates, quinine, strychnine.

Cermbral Hamorrhage. See Apoplexy.

Cerebro-spinal Meningitis in Horses.

Inflammation of the cerebro-spinal meninges. Horses and sheep more frequently affected than cattle, hogs, or dogs; horses in America attacked more frequently than in Great Britain; enzootic and epizootic; but special infecting agent has not been discovered.

Slings essential where horse cannot stand.

Half dose of physic, oil, and a few grains calomel.

Salines, laxative enemas, mash diet.

Where urine not freely passed use catheter.

Strychnine thrice daily.

Cold or ice-bag to head and neck ; counter-irritation to spine.

Ergotin and atropine hypodermically.

Pilocarpine, ten grains hypodermically (Friedberger).

Сhoкing.

Usually from obstruction of œsophagus. Common in cattle feeding on roots.

Repeated small quantities slowly given of linseed gruel, oil, or other lubricant.

Secure wooden gag in mouth, which evokes swallowing movements, eructation, and discharge of saliva.

Move foreign body, upwards if possible, by introducing hand into mouth, or by external manipulation of gullet.

Failing, carefully pass the probang, cup end first; emetics and expanding probang for dogs and pigs.

Other means unsuccessful, cut into gullet and extract obstruction.

Where hoven becomes serious puncture rumen with trocar or knife.

In horses symptoms less urgent, and treatment not always satisfactory.

In pharyngeal choking, explore pharynx and remove obstruction.

In cervical cases, manipulate upwards or pass the probang.

In thoracic cases use probang.

Coccidiosis.

A disease of the intestine and liver, caused by Coccidia, cystlike parasites, which invade the epithelial cells of the mucous membrane and biliary canals. Occurs in cattle, sheep, pig, dog, rabbit, fowl, duck, pheasant, etc.

Prevent extension by disinfection of premises, excreta and litter; solution of sulphuric acid ( 3 per cent.) or formalin; pure drinking water. 


\section{Coccidrosis-continued.}

Curative treatment, - disinfect intestine by purgative, followed by antiseptics; glycerin and ferrous sulphate in drinking water ; sodium hyposulphite, sulphur, lysol, cyllin; often incurable.

Colic.

Gripes; Spasm of intestines; Irregular inordinate contractions of muscular walls of intestines. Occurs in all animals; presents two forms-(a) spasmodic; (b) flatulent.

Place horse in spacious, well-littered box.

Purgative to remove irritant : in horse, aloes; in cattle and sheep, oils and salines ; in dog, castor oil.

Catharsis hastened and pain relieved by copious laxative enemas, hot fomentations and friction to abdomen, and gentle exercise.

Barium chloride or eserine and pilocarpine if bowels continue torpid.

Ether, oil of turpentine, other volatile oils, ammonia, and ammonium carbonate combat flatulence.

Ether, alcohol, and chloral hydrate, conjoined with opium, belladonna, cannabis indica, control spasms and pain.

Morphine and atropine hypodermically or inhalation of chloroform quiets violent spasm.

Repeated recurring attacks in influenza in horses, often connected with hepatic derangement, treated with half dose of aloes and a little calomel, spirit of chloroform, and mustard embrocation to abdomen.

In flatulent colic in horse the distended bowel may be punctured by trocar and canula.

Similar treatment in other classes of patients.

Cholera of Fowls and Hogs. See Fowl Cholera and Swine Fever.

Chorea.

Irregular, involuntary, convulsive contractions of voluntary muscles, or groups of muscles. Stated to be sometimes dependent on localised sclerosis of spinal cord.

Occurs in all animals, usually in the young, feeble, or anæmic.

In horse most common in the form of stringhalt, which see.

In dog as sequel of distemper, or from intestinal worms.

Remove gastro-intestinal derangement, worms, or other cause of reflex irritability ; potassium bromide.

In weakly dogs or convalescents from distemper, generous diet, fair proportion of good milk or fatty matters.

Iron, arsenic, other tonics, ether, and spirit of camphor; glycerophosphates.

Sponging or affusion with water, at first tepid, subsequently cold.

Violent spasms relieved by full doses chloral hydrate or Indian hemp internally, or chloroform inhalation.

Counter-irritants over spine in long-standing cases.

Momentary immersion in cold bath and drying quickly with friction; electricity, interrupted current, two to five minutes daily.

Сома.

Stupor, symptomatic of impaired brain function.

Affusion alternately with warm and cold water: ice-bag to head.

Ammonia given by inhalation and subcutaneously.

Mustard to extremities; stimulating enemata, eserine and pilocarpine.

Cautious bleeding; endeavour to promote action of bowels and skin.

Conjunctivitis.

Inflammation of mucous membrane of eye. See also Oрнтнацміа.

Remove any irritant : foment; castor oil between lids relieves irritation. Poppy-head infusion as fomentation.

Boracic acid, zinc sulphate or acetate, with aqueous solution of opium suitably diluted. 
Conjunctivitis-continued.

Shield from light; try cold applications.

Atropine and cocaine as local anodynes.

Mercuric nitrate ointment when lids inflamed and granular.

Ergot fluid extract undiluted relieves vascular engorgement.

Dose of physic ; shade both eyes.

\section{Constipation.}

Torpidity of bowels. Insufficient intestinal secretion and movement.

Laxative diet, diluents, salines, regular exercise.

Purgatives in moderation, especially when liver deranged; repeated laxative clysters; massage of abdomen.

Aloes, oils, calomel, small doses Epsom salt for horses.

Salts, croton, gamboge, veratrine, calomel for cattle.

Calomel and jalap, castor and linseed oils, and emetics for dogs and cats.

Gentian, quinine, and other tonics when associated with debility.

Oil of turpentine by mouth and rectum where there is flatulence.

Physostigmine and pilocarpine in obstinate cases; euonymin.

Soap suppository in young animals.

Nux-vomica, belladonna: electricity or ergot to give tone.

Where bowels obstructed by concretions or twist, cathartics are dangerous, and diluents, laxative enemata, and anodynes are indicated.

In dogs use aloes, or oil and grey powder, or jalap and calomel.

In poultry clear out the rectum and give castor oil.

Consumption, Pulmonary. See Tuberculosis.

Contagious Vaginitis. See Vaginitis.

Convalescence.

Easily digested nutritive food, malt extract, milk and eggs, cod-liver oil, fresh air, exercise.

Alcoholic stimulants, bitters, mineral acids, arsenic.

Pepsin for dyspeptic dogs and young herbivora feeding on milk.

Iron salts, phosphates, baths, cold sponging.

Convulsions.

Fits produced usually by irritation of motor centres of brain or spinal cord; they may be (1) cerebral or (2) spinal, and these again $(a)$ central or $(b)$ reflex.

Chloral hydrate or potassium bromide; chloroform inhaled and swallowed.

Morphine subcutaneously; spinal ice-bag.

When of cerebral origin, bromides or ammonia internally ; cold affusion ; ice to head.

When reflex remove source of irritation.

Corneal Opacities.

Paint spot with silver nitrate, 1 part to 100 distilled water, or corrosive sublimate 1 part, common salt $7 \frac{1}{2}$ parts to 3000 water, and afterwards spray or douche with solution sodium hyposulphite, grs. 5 or 10 to the ounce of boiled water, or 1 per cent. solution of protargol.

Sodium chloride injected under conjunctiva.

Iodine and pot. iodide internally and locally promote absorption.

Corneal Ulcer.

Stimulant and astringent collyrium, silver nitrate grs. iij. to one ounce distilled water, apply with camel-hair brush. When ulcer small, instil 2 per cent. solution fluorescein, which stains ulcer and facilitates its location.

Corns in Foot of Horse.

Bruise of sensitive sole.

Remove shoe and any cause of irritation, pare to relieve pressure, ensure exit of any pus, and disinfect.

Poultices soften horn and abate tenderness.

Use light wide-webbed, bar, or $\frac{3}{4}$ shoe.

Shoe strong feet with tips. 


\section{Cough.}

An expiratory explosion forcing open the glottis and following a deep inspiration. More common and serious in horses than other animals. Comfortable housing and clothing, pure air, careful feeding.

Catarrhal.-Steam head; ammonium acetate solution, salines, ether, mustard to throat.

Bronchial.-Ammonium acetate, ipecacuanha, squill, nitrous ether, counter-irritants.

Dry, with scant secretion. -Amm. acetate or chloride, pot. bicarbonate and chlorate, borax.

With profuse discharge.-Balsams, eucalyptus oil, tar, terebene, creosote, astringent sprays or inhalations.

Irritable.-Demulcents, camphor and belladonna, conium, opium, hydrocyanic acid, cocaine.

Reflex. - Bromides, chloral hydrate ; remove cause of irritation.

Verminous. - Frequent in calves and lambs. See Worms.

Codgh, Chronic, of Horses.

Careful dieting, food damped, linseed mash or oil.

Expectorant and anodyne electuaries.

Intralaryngeal injection of antipyrine solution.

Epsom salt or other salines occasionally.

Belladonna, camphor, alcohol, tar, creosote, arsenic.

Counter-irritants : mustard, mercuric iodide ointment.

Crib-biting and Wind-Sucking.

Iron stable fittings. Feed on the ground.

If manger used turn when empty into recess in wall.

Use muzzle, bib, or spiked neck strap; concentrated digestible food.

Chalk, antacids, and occasional laxative relieve the indigestion from which crib-biter usually suffers.

Crib-biter should be placed by himself, as other horses imitate and acquire the habit.

Crural Paralýsis.

Loss of power in the extensor muscles of the stifle; frequently incurable.

Slings, fomentations, massage ; intramuscular injections of nerve stimulants, strychnine, veratrine; electricity (Faradic current), blisters, setons, increasing exercise.

Curb.

Sprain or injury of calcaneo-cuboid ligament of hock, or of perforatus tendon.

Foment; refrigerants.

Counter-irritants; mercuric iodide ointment; if necessary, firing and blistering.

High-heeled shoe, without toe-piece.

Rest, especially in young horses.

Cow-Pox. See Variola.

Cystitis.

Inflammation of urinary bladder; of several forms-catarrhal, purulent, hæmorrhagic, chronic.

Oleaginous laxatives; aconite, calomel and opium abate acute fever.

Emollient anodyne enemata and suppositories of hyoscyamus, opium or belladonna ; urinary disinfectants.

Rugs wrung out of boiling water to horse's loins.

Mashes, linseed, boiled barley, diluents ; urinary antiseptics, camphoric acid.

Benzoic, boric, or salicylic acid, borax, or pot. chlorate when urine alkaline, fermenting, or offensive.

Pot. bicarbonate or other alkalies internally when urine acid and acrid. 
Cystitis-continued.

Syringe female bladder with alkaline solutions when urine acrid; when fœtid, with benzoic acid or dilute copper sulphate.

Astringents, buchu, eucalyptus oil in chronic vesical catarrh.

Irrigation with solution ferric chloride, adrenalin, alum, or tannin in hæmorrhagic cases.

\section{DEBILITY.}

Weakness. Endeavour to remove cause.

Easily assimilated nutritive food, cod-liver oil, milk, malt extract ; suitable hygiene.

Acids, bitters, quinine when gastric digestion weak.

Alcoholic stimulants when heart action feeble.

Laxatives when elimination of waste defective.

Calcium phosphate and fatty matters useful in young animals.

Nux-vomica and glycerophosphates in nervous debility.

Iron salts when associated with anæmia.

Arsenic, pepsin, or liq. pancreatis when assimilation is at fault.

Cold sponging and baths for dogs.

\section{DELIRIUM.}

Perversion and inco-ordination of brain functions.

For vigorous patients cold affusion applied cautiously.

Ice and refrigerants to head.

Perfect quiet, cathartics, salines, digestible cooling diet.

Chloroform, cannabis indica, bromides, internally.

Alcohol, ammonia, belladonna, camphor, when associated with exhaustion.

Blood-letting in delirium, resulting from injuries, in earlier acute stages of phrenitis, and in robust subjects.

Dentition Fever.

Not infrequent in horses.

Soft laxative food, rest, salines, febrifuges.

Lance gums if absolutely needful.

Remove temporary teeth interfering with eruption of permanent.

When dentition of dogs delayed or defective give calcium phosphate.

Diabetes Insipidus.

Polyuria; Excessive secretion of urine. Peculiar to horses. Caused apparently by some toxic material derived frequently from faulty food, as heated, musty grain or fodder, or produced in connection with such diseases as influenza and glanders; seldom fatal.

Half dose physic, especially when digestion out of order.

Iodine with pot. iodide, either in bolus or solution.

With iodide alternate or conjoin iron salts or sodium bromide.

Chalk or whiting in manger, or sodium bicarbonate in water, counteracts acidity frequently present.

Phosphoric acid and bitters lessen thirst.

Careful feeding; change food. Avoid stale, damp, badly-saved fodder, or musty, unsound grain. Allow moderate supply of water.

Diabetes, SACChariNe.

Diabetes mellitus. Pathology not explained. Occasionally occurs in dogs, very rarely in horses or cattle.

Relief afforded by withholding carbo-hydrates, substituting soup, cooked animal food and milk.

Codeine and iodine; antipyrine; sodium salicylate.

Diaphragmatic Spasm.

Usually resulting from over-exertion or acute gastric derangement; specially observed in horses and dogs; intermittent, seldom lasting many hours.

Chloral hydrate and alcohol per 08 ; rest patient. 


\section{Diaphragmatic Spasm-continued.}

Dogs, an emetic, especially if of gastric origin.

Stimulant embrocation applied to chest.

\section{DiarrheEa.}

Scouring ; frequent discharge of fluid fæces.

Symptomatic of tuberculosis, Johne's disease, strongylosis ; ascertain cause.

Laxatives in first stage to remove irritant.

Perfect rest; keep patient comfortable and warm.

Restrict water; diet carefully ; wheaten flour gruel; boiled milk.

Alkalies; chalk where dejections acid.

Lactic acid, grains 150 to grains 750 , in three pints of boiled water for cattle with profuse thin discharges.

Enemata of starch gruel at $100^{\circ} \mathrm{Fahr}$, with lead acetate and opium.

Volatile oils, ether, chloroform, chlorodyne in moderate, frequently repeated doses relieve flatulence and spasm.

Ammonium carbonate where watery discharges continue and heart action weak. If due to strongyles, carbolic acid, thymol, lysol, iodine, and salol, or other intestinal antiseptic.

Cópper sulphate; corrosive sublimate with creosote and opium when chronic discharges contain mucus and blood.

Ergotin and opium administered with keratin where discharges profuse and continued.

Antiseptics, sodium salicylate, tannalbin, naphthol, lysol, sulphites, sulpho-carbolates where discharges foul.

Nitric acid and nux-vomica when complicated with hepatic derangement. In young animals castor oil with a few drops laudanum.

Grey powder in young patients where discharges pale and fœtid.

While patient fed on milk, if it disagree when given with lime water, in cautiously regulated, restricted amount, substitute cooked starch food, or beef tea and white of egg, with a little wine or spirit if animal' reduced.

\section{Diphtheria.}

A dangerous epizootic of fowls. Attacks poultry, water fowls, turkey, pheasant, partridge, parrot, etc. Caused by two distinct species of fungi (Rivolta). Affects chiefly the mouth, throat, nasal cavities, larynx, bronchi and lungs ; sometimes the eyelids, alimentary canal and skin; incubation period a few days.

Isolate affected birds ; enjoin thorough cleanliness and disinfection.

Moisten external eruptions with 1 part corrosive sublimate, 10 parts salt, and 1000 water, or with dilute sulphurous acid and cover with sulphur.

Where mouth or throat affected give every two hours electuary, 1 part tannoform, 5 each borax, pot. chlorate, glycerin, and 100 simple syrup.

Feed liberally to sustain strength; antiseptics internally.

\section{DisinfeCtion PRIOR TO OPERATION.}

Skin: remove hair by clipping or shaving; wash and scrub, using soap and warm water; then douche the parts with mercuric chloride sol. 1: 1000; carbolic acid sol. 5 per cent.; tincture of iodine; or sol. hydrogen peroxide 3 per cent. In dog and cat, where usually operation area is small, after shaving and washing the seat of operation, douche with alcohol, or sublimate sol. (1:1000).

Eye: Asepsis may be obtained by frequently douching with 4 per cent. sol. boric acid, or 1 to 5000 sublimate, or 2 per cent. sol. hydrogen peroxide.

Foot: remove shoe, trim hoof, wash and brush well, using antiseptic soap, then immerse foot in antiseptic bath, or, better, place foot in antiseptic compress, which is only removed at the time of operation. 
DisinfeCtion PRIOR to OPERATION-continued.

Vagina: wash the mucous lining with 4 per cent. warm sol. of boric acid, then with potassium permanganate (1:2000), and just before operation with 5 per cent. sol. sodium bicarb.

Uterus : irrigate with water boiled and cooled to blood-heat; syphon out fluid if not expelled; and finally irrigate with sol. iodine, grains 8 to the quart of tepid water, or with 2 per cent. sol. carbolic acid.

\section{Dislocations.}

Luxations. Not common in the lower animals.

Bring bones into natural position.

Retain in position by splints, bandages, plasters; sling if necessary.

Abate inflammation by either hot or cold applications, as may be suitable.

\section{Distemper in Dogs.}

A contagious eruptive fever affecting the mucous membranes, skin, and glands, and frequently the cerebro-spinal axis. It attacks dogs, cats, foxes, wolves, hyenas, jackals, and monkeys. Evidently of organismal origin ; cultivations to the seventh generation produce the disease when inoculated in dogs and cats; but the distinctive microbe has not yet been demoustrated.

Prevent spread by early isolation and disinfection.

Good nursing and cleanliness as important as medicines.

An emetic and mild laxative if required in early stage.

Ammonium acetate solution and ipecacuanha, steaming and sponging nose and eyes relieve catarrh.

Boric acid, two per cent. solution in conjunctivitis, which see.

Hot compresses, embrocations, stimulant expectorants in laryngeal and bronchial cases.

Combat fever with antipyrine or quinine.

Injections of polyvalent antistreptococcic serum, recommended by Joly.

Chlorodyne, or bismuth salicylate allays gastro-intestinal irritation.

Boric acid and tannoform powder with ten parts kaolin or starch abate skin irritation ; strophanthus in heart weakness.

Sodium sulphite as antiseptic and antipyretic.

For nervous complications see CHOREA, EPILEPSY, Convulsions.

If food not spontaneously taken give beef tea and milk, beef tea with white of egg, or finely minced lean beef uncooked, and, if required, wine or spirit.

During convalescence give phosphates, and hæmatinics, cod-liver oil ; and a generous diet.

Preventive treatment uncertain : vaccines and antidistemper sera introduced by Copeman, Phisalix and others may be tried.

Dropsy.

Symptomatic serous effusion. See Ascites.

Endeavour to restore functions of heart, kidneys, or liver, the impaired action of which usually causes the effusion; Turkish baths.

Digitalis infusion and strychnine useful in most dropsies, especially in cardiac, in which give with salines or milk sugar.

Copaiba in cardiac and hepatic cases.

Laxatives and pot. iodide in renal dropsy.

Encourage vicarious functions of bowels and skin if kidneys affected.

Iron and salines in cases associated with anæmia.

Restrict quantity of fluid; friction; shampooing; external stimulants.

Trocar and canula, or aspirator for cases requiring tapping.

\section{DYSENTERY.}

Contagious specific inflammation of mucous and glandular structures of intestine, occurring in foals, calves, lambs, and dogs, within a few days after birth. In muco-purulent, blood-stained discharge 


\section{DYSENTERY - continued.}

are numerous bacteria. Mortality 10 to 80 per cent. Observed to concur with epizootic abortion (Friedberger).

Isolation of infected; thorough disinfection.

Antiseptic treatment of females aborting and parturient. See ABortion.

At first, sodium sulphate, then ipecacuanha in linseed tea.

Castor oil and grey powder, intestinal antiseptics, tannates.

Well-boiled starch gruel or other mucilage, with a few drops laudanum.

Salicylic and tannic acids with chamomile infusion.

Rhubarb, magnesium carbonate, and opium.

Chlorodyne, tannoform, or resorcin, with laudanum; acetate of lead.

Drspepsia. Indigestion.

Treatment varies with cause and nature of attack. Suitable dietary. Avoid indigestible food and long fasts. Rectify irregularity of bowels. Enjoin exercise.

Resulting from over-eating, an emetic for dogs; cathartic for animals that do not vomit.

For flatulence, essential oils, ginger, hydrochloric acid, ether; pass probang in cattle.

For gastric catarrh, ammonium chloride, sodium hyposulphite, fragments of ice.

Alkalies, chalk, magnesia, given before feeding or with food.

For atonic forms, in cattle, mineral acids, usually more permanently useful, are given with bitters and pilocarpine.

Pepsin with glycerin for dogs and young animals while living on milk.

Where food irritates, provoking diarrhœea, bismuth salts or arsenic with morphine.

Depending upon worms-appropriate vermicides.

In chronic dyspepsia, obviate errors of diet or management, examine teeth, change food.

Provide horses with whiting and rock salt to lick. Try daily $\frac{1}{2} \mathrm{lb}$. linseed cake or hay-tea.

Restrict cattle to mash diet for two days, and add salt and treacle to drinking water, pilocarpine subcutaneously.

Both in hoven and overloading with dry food rub left flank and belly; soap and water enemata; rumenotomy.

\section{DYSPNEA.}

Difficult breathing.

Discover and, if possible, remove cause.

Fresh air ; chloroform, inhaled or swallowed.

Chloral hydrate in spray or draught.

Belladonna extract and ether.

Amyl-nitrite ; nitro-glycerin in angina pectoris.

Counter-irritants if due to congestion or inflammation of air-passages.

Tracheotomy where obstruction occurs in upper air-passages.

Eclampsia.

Epileptiform spasms, cause unknown. Most common in very young subjects, dogs during teething, and from worms, occasionally in bitches nursing. Cases in milch cows have been recorded.

Remove any exciting cause ; attend to general health.

Pot. bromide, chloral, morphine hydrochloride hypodermically, or chloroform inhalation where excitement considerable.

\section{Естнтма.}

American skin disease ; eruption of papules and subsequently pustules.

Affects horses, sheep, and dogs.

Laxative salines remove gastro-intestinal or other irritants.

Exercise further hastens removal of waste products.

Digestible, rather laxative, dietary. 
Естнума-continued.

Mineral acids, iron salts, bitters, arsenic, act as antiseptics and alteratives.

Pustules treated by water dressing, boric acid, zinc oxide ointment, or by cauterisation.

Contagious; hence patients isolated and disinfection adopted.

ECzema.

Cutaneous catarrh. A dermatitis presenting localised eruption of papules, vesicles, or pustules, appearing consecutively or together, with itching, thickening, and discharge, and subsequently scales or scabs. The earlier or acute stages may continue one to three weeks, the later for months. Affects particular regions, chiefly the back, tail, and extremities. Young, old, and delicate subjects most susceptible. A frequent disease of dogs, in which the several varieties usually well marked. It occurs about the heels of horses, causing ulceration and constituting a variety of grease.

Friedberger records six varieties :-

(1) Erythematous. - The skin hot, tender, red, and swollen.

Cleanse skin with soap and water. Apply vaseline or lead subacetate, glycerin, and olive oil.

Endeavour to prevent rubbing and biting.

Laxative salines, cooling diet, correct gastric derangement.

(2) Papular.-Patches of small, soft swellings, varying in size from a millet to a pea.

With treatment as above, moisten with boro-glycerin or borax solution.

Itching abated by strong solution of pot. bicarbonate, or sod. hyposulphite.

(3) Vesicular.-Eczema simplex. Tetter. Patches of minute, crowded vesicles.

Whether in dogs or horses, trim or shave all hair from irritable spots. Wash with soap and water.

Moisten several times daily with saturated solution pot. bicarbonate.

Zinc oleate or ointment soothes and softens.

Dry dressings, such as zinc oxide and bismuth nitrate, one part each, six or eight parts kaolin or starch, preferable when skin tender or puffy.

(4) Humid. -Eczema rubrum. Red mange of dogs. Inflammation extended and exudation greater.

Remove irritating discharges, and wash skin once with solution of creolin.

Soak limited areas with mercurous oxide wash.

Subsequently dress with zinc oleate or sulphur ointment.

Paint limited inflammatory spots with 5 per cent. silver nitrate solution.

Tannin with ten parts paraffin oil ; dust with boric acid or iodoform.

After abating pyrexia, administer mineral acids, bitters, tonics, arsenic ; linseed for herbivora. In dogs avoid oatmeal and heating animal food, and give cod-liver oil.

Mercuric nitrate or oxide ointment in eczema of eyelids.

(5) Pustulous. - Impetiginous. Inflammation more deeply involving the skin, with free pus formation, as in grease in horses; liable to assume chronic form.

Cleanse and soften scabs with soap and warm water.

Soak suppurating surfaces with zinc chloride in aqueous solution.

Alternate these dressings with mercurous oxide wash, copper sulphate ointment, carbolic acid, naphthol.

Paint inflamed spots with silver nitrate or dilute nitric acid.

Attend to state of bowels and kidneys.

Digestible nutritive food, tonics, arsenic, mineral acids.

(6) Squamous.-Desquamating. Sometimes termed Pityriasis or Psoriasis.

Soak crusts or scabs with oil for some hours and remove them. 


\section{ECzema-continued.}

Salicylic acid in strong borax solution also removes scabs; ichthyol ointment.

Dress with four parts each pot. carbonate and sublimed sulphur, one oleum picis, thirty each lard and olive oil, leaving dressing on for three or four days, then washing off with soap, alkali, and water.

Carbolic acid and paraffin ointment, sulphur iodide ointment.

Mercuric nitrate, or iodine ointments where skin thickened.

A blister in inveterate cases sometimes re-establishes healthy action.

Many cases of eczema in dogs are speedily cured by giving a full dose of purgative medicine, dressing the skin with sulphur ointment or one per cent. lysol solution, and by restricting the diet.

Elephantiasis of Horses.

Chronic dermatitis with dermal and sub-dermal hypertrophy, vascular stasis, and surface deformation. Affects the limbs, and follows repeated attacks of lymphangitis.

When the papillæ are seriously enlarged and skin folds formed cure is hopeless.

Slighter cases cured and others ameliorated by antiseptic treatment. See Grease.

Green food, laxatives, diuretics, salines, iodine, and pot. iodide; exercise.

\section{EMPYеMa.}

Pus in the chest, facial sinus, or other natural cavity.

Remove by operation under antiseptic precautions.

Wash out cavity with warm saline eolution, boric acid, hydrogen peroxide, protargol, or other antiseptic.

Emphysema, Subcutaneous.

Air swelling. Often disappears spontaneously.

May puncture, apply pressure, or counter-irritation.

Prevent entry of air if emphysema due to wound.

Emphysema, Pulmonary. (See Broken Wind.)

Occurring in old hard-worked horses.

Careful dietary, concentrated damped food, occasional linseed mash.

Treatment only palliative.

No water given within an hour of work.

Arsenic and belladonna relieve dyspncea ; laxatives occasionally.

Strychnine stimulates the respiratory centre.

ENDOCARDitis.

Inflammation of membrane lining heart cavities and covering valves.

More frequent than myocarditis ; occurs especially in horses, cattle, and pigs; usually as sequel of contagious fevers depending on infection. Acute cases frequently affect left heart, chronic and infective the right.

Perfect rest and quiet. Seldom curable.

Digitalis to steady heart; quinine and other antipyretics.

Frequently repeated stimulants maintain heart action in exhausted patients.

In rheumatic complications salicylates or pot. bicarbonate.

\section{ENTERITIS.}

Inflammation of the bowels, is produced by irritants, bacteria, toxins, strongyles. In dogs, cattle, and sheep, the small intestines are chiefly affected. Horses, especially hard-worked, irregularly-fed animals of the heavier breeds, are subject to rapid, usually fatal, hæmorrhagic effusion into the submucous tissues of the colon and cæcum, and the subserous structures of the attached mesentery.

In these equine cases morphine and atropine hypodermically every two hours. Ergotin sometimes conjoined with the view of contracting blood-vessels ; antiseptics, salol, tannoform, lysol. 


\section{Enteritis-continued.}

Half drachm each opium, belladonna extract, and camphor in pint of gruel, repeated every two hours.

Where cardiac action violent, one or two small doses of aconite tincture may be added to the anodyne draught.

Occasional laxative enemas. Purgatives aggravate morbid conditions.

From the outset rugs wrung out of hot water placed for two hours around the trunk, and soap linament with opium subsequently rubbed over abdomen give temporary relief.

Enteritis in other animals not so sudden in onset or so rapidly fatal.

Hot fomentations, mustard and soap liniment.

\section{Entropium.}

Inversion of eyelid and eyelashes. Dogs more frequently affected than horses.

Excision of elliptical portion of skin of inturned lid; edges secured by fine sutures; antiseptic adhesive dressing.

EPILEPSy. Fits.

Unconsciousuess associated with spasmodic movements. Excitation of motor centres of cranial cortical substance. Results from $(a)$ tumours, morbid conditions, or parasites in the brain or meninges ; (b) reflexly from irritation of bowels or lesions of peripheral nerves ; (c) fright, anger, excitement. Most common in young dogs.

Hold head of small animal firmly. Protect eyes from light. Maintain normal position.

Take care that animal is not injured during convulsions.

Regulate bowels. Remove worms or irritation connected with teething.

Digestible nutritive diet; healthy surroundings.

Pot. bromide twice daily persisted with.

Iron salts in anæmia ; zinc salts where gastric derangement.

Copper ammonia sulphate; silver nitrate and iodine sometimes useful.

EPISTAXIS.

Bleeding from nostrils.

When from injury rupturing small blood-vessel, plug one nostril, raise head.

If a polypus present remove it.

When from inflammation or ulceration of nasal membrane apply ice to frontal surface and head; spray with ferric chloride tincture, or solution of adrenalin.

When from purpura give by mouth ergot, ferric chloride, or ergotin subcutaneously.

Relieve any determining cerebral, cardiac, or respiratory congestion.

Warm-water bag to spine.

\section{Epizootics.}

Contagious diseases attacking large numbers of animals in a short space of time and spreading widely.

Endeavour to destroy disease germs by carbolic acid, chlorine, formalin, sulphurous acid gas, or other disinfectants.

Isolate infected subjects. Enjoin cleanliness.

Sponge sick and healthy with sulphurous or carbolic solutions.

Administer quinine, sodium sulphite, glycerin carbolate, or other antiseptics.

Epizootic Lymphangitis.

A contagious eruptive disease of the horse, caused by the Cryptococcus farciminosus, and characterised by suppurating nodular swellings of the superficial lymphatics.

Eruption usually appears on the limbs, sometimes on the trunk, or on head and neck.

May be mistaken for farcy or cutaneous glanders. E. lymphangitis and glanders may coexist. Affected horses should be slaughtered. 


\section{Erysipelas.}

A specific febrile disease, characterised by diffuse sloughing inflammation of the skin and subjacent tissues, usually of the head. Occurs occasionally in horses; on head of sheep, spreading as a contagious epizootic (Cagny); and in swine, known in France as mal rouge, and caused by a minute bacillus. See Swine Fever.

Isolate and disinfect; generous diet.

Aperients, salines, pot. chlorate conjoined with spirit of camphor.

In horses hot, medicated fomentations persevered with for several hours, and affected surfaces moistened with borax solution and laudanum.

Subcutaneous peripheral injection of antiseptics sometimes limits swelling and extension.

Abscesses should be opened, but scarification of swellings rarely justifiable.

Milk and eggs, beef tea, alcoholic stimulants sustain strength.

Ferric chloride solution, both locally and internally, especially in weakly patients, for whom also prescribe quinine.

\section{ERYTHema.}

Congestion of the skin with infiltration of its layers. Occurs in all patients from friction, irritants, and exposure to cold winds in wet weather. Intertrigo and mud fever common varieties in horses.

Cleanse thoroughly. Dust with starch, fuller's earth, or zinc oxide, one to six or eight of starch; apply zinc or lead salts in solution or ointment.

Zinc oxide or other antiseptic desiccant with kaolin or starch, in exudative forms, in which moisture to be avoided.

Horses liable to 'mud fever' should not have the hair removed from their legs, and washing should be forbidden. Apply Goulard's extract, glycerin and olive oil.

Salines in drinking water; pot. bicarbonate.

Laxatives, especially when connected with gastro-intestinal irritation.

Chronic forms, such as cracked heels in horses, painted over with silver nitrate, one part to twelve water : or dressed with astringents, copper sulphate, alum, or zinc sulphate.

Arsenic and quinine in persistent cases.

Exostosis.

Outgrowth of bone.

Fomentations or refrigerants allay inflammation and pain of early stages.

Counter-irritants when heat and tenderness have been removed.

Mercuric iodide ointment : needle firing; seton ; periosteotomy.

Laxatives, with iodine and pot. iodide internally, promote absorption.

Fainting.

Syncope. Results from enfeebled action of heart.

Fresh air; remove any pressure from neck; inhalations of oxygen.

If horse down do not attempt to raise him.

Increase blood-pressure by sterilised salt-solution ( $\$ 0$ grains to the pint) subcutaneously.

Ammonia inhaled, or adrenalin solution injected hypodermically.

Alcohol and ether, swallowed or in enema, especially when heart action weak or fluttering.

False Quarter.

A permanent defect of the wall. The deficiency results from injury of secreting coronary band.

Endeavour to restore secretory function of coronary band.

Dress and promote healing of wound in band; dead horn trimmed away; equable pressure applied.

Apply bar shoe to relieve concussion; prevent dirt getting into fissure by filling with guttapercha and ammoniacum or other horn substitute.

Blisters to coronet sometimes useful. 
Farcy. See Glanders.

Favus.

Honeycomb ringworm, produced by various dermatophytes; Achorion Schönleinii (man and rabbit), A. quinckeanum (cat), and Oospora Canina (dog). The so-called favus or whitecomb disease of poultry is due to a special fungus, Lophophyton gallince. Occurs in man, dogs, cats, and poultry. Favus is less common than Trichophytosis. See Ringworm.

Soft soap and warm water, or alkalies with bland oils, soften and remove crusts.

As parasiticides-iodine tincture, iodoform, silver nitrate ointment 1 to 5 per cent., ferric chloride solution, thymol, creosote, copper sulphate, or a mixture of equal parts of carbolic acid, iodine tincture, and chloral hydrate.

In weakly subjects give internally mineral acids, bitters, tonics.

Fever, Acute.

Concurring with acute local inflammation.

Aconite; occasionally blood-letting in earlier stages in robust subjects.

Aperients, salines, magnesia sulphate, quinine, Glauber's salt, laxative enemata.

Remove any cause of irritation; attend to wounds.

Warm clothing, but cool air to breathe.

Warn bath or sponging with tepid water.

Antifebrin and other antipyretics; sodium sulphite and antiseptics.

Alcohol and digitalis sustain cardiac tone.

Salicylic acid and sodium salicylate in rheumatic fever.

Acids and bitters allay thirst and aid digestion.

Light, easily digested food; diluents; salines in drinking water.

Fever, Low.

Occurs in most epizootics, in connection with septicæmia, in inflammation of mucous membranes, and in debilitated subjects.

Mild laxatives and enemata when required.

Antipyretics, antiseptics, salines, acids, bitters.

Quinine, especially in intermittent types; arsenic in infective cases.

Alcohol, ether, or caffeine promote excretion and cardiac action.

Turpentine and iron salt indicated in hæmorrhagic cases.

Suitable clothing, diet, hygiene, sponging, baths.

Fever, Simple.

Continued fever. Occurs independently of local inflammation or septicæmia ; common among horses brought into dealers' stables.

Comfortable box; fresh air and sunlight; perfect quiet.

Clothe body, bandage legs, mild laxative, mash diet, gruel, diluents.

Salines, ammonium acetate solution, pot. chlorate or nitrate, antifebrin, spirit nitrous ether.

Alcohol, ether, bitters, acids, tonics so soon as acute symptoms abate.

Catarrhal, gastro-intestinal, or other specialsymptoms receive appropriate treatment.

Fistula or Sinus.

A chronic discharging wound.

Remove necrosed tissue or other irritant. Render wound aseptic.

Open sinuses with knife; curette and provide drainage by counter opening.

Encourage healing of wound by granulation.

Where knife impracticable, inject solution of corrosive sublimate, sulphate of copper or zinc chloride.

Milder treatment failing, destroy ulcerating surface and fibrous lining with corrosive sublimate or by actual cautery.

Dress with antiseptics.

Flatulence. See Dyspepsia and Hoven. 
Fleas.

Pulex irritans and others. Infest dogs, cats, pigeons, and poultry. Soap and water; cleanliness of skin and surroundings.

Turpentine, aniseed, or other volatile oils. Sassafras oil effectual.

Insect powders; stavesacre decotion; tobacco water; warm lysol solution one per cent.

Pine shavings or sawdust for dog's bed.

\section{Flybow.}

Affecting wounds; specially troublesome in sheep.

Prevent fly striking by dressings of corrosive sublimate solution, turpentine, tar oil, or tar.

\section{Foot-rot in Sheep.}

(1) Abrasions of horn from injuries, rough ground, long journeys; the exposed secreting surfaces, especially of the sole, becoming inflamed by dirt and grit insinuating under the damaged crust. Non-contagious.

(2) A specific contagious inflammation caused by the bacillus necrophorus. It commences at the sole or between the claws and, gradually extending, causes changes in the bones and tendons, with degeneration of horn, suppuration and sloughing. The discharge in contact with sound feet, or introduced by inoculation into the bodies of healthy sheep, produces the disease in fourteen to twenty-one days.

The non-contagious form cured by placing diseased sheep on dry soils, removing loose horn, dressing fungus growths with mild caustics.

The contagious form necessitates separation of affected sheep, dressing them daily or thrice a week.

One part carbolic acid, ten glycerin, applied between the claws; loose and diseased horn removed by knife.

Fungous growths cauterised with mixture of copper sulphate and crude carbolic acid made into paste with vaseline.

Mercuric nitrate, zinc or iron chloride solutions also useful.

Protect surfaces with tar dressing or guttapercha varnish.

Drive sheep twice a week over ground strewn three inches thick with freshly-slaked lime.

Walk sheep through astringent foot-bath, one part copper sulphate to fifty parts water.

Prevent introduction of disease by three weeks' quarantine of fresh purchases.

'Foul in Fent' of Catthe.

A popular, somewhat indefinite term applied to chronic disease with necrosis of the feet of cattle, usually resulting from neglected injuries, or from tuberculous inflammation.

Cleanse, foment; remove loose and diseased horn, and disinfect.

Dress with copper sulphate, carbolic acid, or other antiseptic.

Keep foot dry by tarred bandages.

Where disease is deep-seated, or intractable, amputate claw.

Founder. See Laminitis.

Fowl Cholera.

Affects all species of birds, and by inoculation is produced in rabbits, pigeons, and white mice. Its essential features are croupous and hæmorrhagic inflammation of the mucous membrane and follicles of the intestine, similar conditions usually attacking lungs and heart. It runs its course in thirty-six hours; 90 per cent. die. It is caused by a minute bacterium.

The premises sbould be swept; all litter, manure, and dead birds burned. Walls, woodwork, cribs, repeatedly washed with boiling water, containing carbolic acid, corrosive sublimate, or 5 per cent. sulphuric acid.

Fumigate with sulphurous acid or chlorine. 
Fowl Cholera-continued.

Preventive vaccination advised by Pasteur; but some birds die, and two or three operations necessary to secure immunity.

Ferrous sulphate with a few drops of solution of hydrochloric acid every three hours, sometimes beneficial; or add one drachm of sulphuric acid to four pints of the drinking-water.

Carbolic acid 5 per cent. solution subcutaneously used by Nocard.

Fractures.

Broken bones.

Bones brought into apposition.

Splints of leather, lath, block-tin, poroplastin, or guttapercha.

Encase in plaster of Paris, or starch bandages.

Ends of bones may be kept together by metallic sutures.

A blister, promotes union by increasing reparative materials, and by limiting movement.

Slings advisable in many cases.

Wounds in compound fractures treated antiseptically.

Calcium phosphate internally in weakly subjects.

Frontal and Maxillary Sindses; Inflammation of Lining Membrane.

Caused by nasal catarrh, carious teeth, injuries, tumours, strangles, and glanders; often unilateral. More common in horse than in other patients.

Trephine. Wash out cavities with warm antiseptic: boracic acid, hydrogen peroxide, potassium permanganate, or iodine solution.

Irrigation should if possible be done while animal standing.

Frontal and maxillary sinuses of sheep the seat of the larvæ of œstrus ovis.

These larvæ also sometimes occupy the chambers at base of horns ; producing chronic catarrh and cerebral excitement.

Washing with an antiseptic solution the nostrils of sheep shortly after the ova have been deposited may prevent the mischief.

Fumigations, nasal injections, and insufflations.

Subsequent treatment very hopeless, even when the sinus is trephined, as it is difficult to wash out all the larvæ.

Frost-Bite.

Necrosis of the coronet.

Raise temperature of frozen parts, and inject antiseptics.

Stimulants to affected parts, turpentine and oil, soap liniments.

Treat sores antiseptically.

Fungus Hamatodes.

Vascular sarcomatous growth, usually protruding from orbit. More common in cattle and sheep than horse.

Extirpation of eyeball.

Speedy feeding and slaughter of patient.

GANGRENE.

Mortification. Necrosis with putrefaction, caused by micro-organisms.

Remove textures already dead by knife or silver nitrate, chromic or carbolic acid, iodoform or bromine.

Inject antiseptics at margin of dead part, and endeavour to limit extension of gangrene.

Maintain wounds aseptic. Stimulate circulation in surrounding parts.

Sustain general strength by generous diet; tonics and stimulants.

Gapes in Fowls.

Caused by Syngamus trachealis. See Worms.

Gastritis.

Inflammation of stomach-generally from action of irritants ; occurs in badly-fed foals and calves; occasionally in horses; and in older cattle as abomasitis. In dogs as hæmorrhagic gastritis. 
GASTRITIS-continued.

In dogs foreign bodies or irritants removed by apomorphine solution hypodermically; purgatives contra-indicated. Give enemata.

Ice by the mouth, or morphine hypodermically, relieves irritation and pain ; creosote, magnesia, sodium bicarbonate.

Antacids and bismuth, with or without small doses opium, in young animals and dogs; antiseptics ; hot fomentations to abdomen; milk and soda water.

The brain symptoms and paralysis occurring in cattle usually relieved by full doses of oil, followed by demulcents, treacle, salines, and laxative enemata.

Patients nourished with milk, eggs, well-boiled gruel, and nutritive enemas.

Gastro-enteritis.

Occurs in all the domestic animals. Causes very varied; poisons, foreign bodies, parasites, irritants, defective food or water, etc.

Gentle laxatives; emetic, apomorphine for dog or pig; demulcents.

Allay prominent symptoms with analgesics, antispasmodics, styptics, or special antidotes.

Hot applications externally; soothing enemas; fluid or semi-fluid digestible food.

GiD. See Worms.

GLaNdERs in Horses.

A specific contagious disease of the horse, ass, and mule, caused by the bacillus mallei entering the body with the food, inspired air, or by inoculation through a wound; specially affecting the lungs, respiratory mucous membranes, and lymphatic system, and trans. missible to man and many animals.

Incurable. May exist without nasal discharge, ulcers, or sub-maxillary swelling. Glandered horses should be slaughtered; notification compulsory. Suspected or incontact animals should be isolated, and tested with mallein (p. 695).

Glandular Swellings.

Counter-irritants around or near ; iodine, mercury oleate, or iodide.

Salines, iodine, and iodides, calcium chloride, and sulphide internally. Iron salts in anæmic patients.

Inject with diluted carbolic acid, naphthol, or other antiseptic.

Glaucoma.

Disease of vitreous humour of eye.

Not of ten amenable to treatment; iridectomy affords relief.

Physostigmine diminishes intraocular pressure.

GLossitis.

Inflammation of tongue with or without a wound. Occurs in all animals.

Irrigate with mild astringent solutions.

Electuary of bismuth and oxymel, or treacle and vinegar.

Furnish soft nutritive food. If tongue lacerated, amputation may be necessary.

Grapes in Horses.

Inflammation, œdema, and hypertrophy of the papillary structures of the skin of the pastern and fetlock or shank.

Remove by knife, scissors, hot iron, or caustics.

Dress with mixture of zinc sulphate, carbolic acid, and vaseline.

Dry the discharges with one part corrosive sublimate, five to ten parts hydrochloric acid, 1000 water.

Laxative diet; exercise or suitable work.

Iron salts, arsenic, iodine, salines' internally. 


\section{Grease. Seborrhaic Dermatitis.}

Chronic inflammation of the skin of the horse's fetlock and pastern. By many authorities regarded as pustulous or impetiginous eczema. The skin produces an excessive cell growth, which rapidly degenerates, causing sero-purulent, greasy, fœtid, irritant discharges. The hair follicles, cutaneous glands, and papillæ are involved. Often associated with canker of the foot.

Clip or shave hair, and thoroughly cleanse with soap and warm water.

Wash daily with one part corrosive sublimate, ten parts hydrochloric acid, 1000 water, or with 5 per cent. solution potassium permanganate.

Envelop in wood-wool wadding, which absorbs discharge and protects denuded surfaces, and is kept in place with a bandage.

When, after a few days, discharge thus reduced, apply a pad of jute saturated with one part creosote to six parts spirit to disinfect and repress proliferating growth, and continue to keep dry with wood-wool wadding.

Dust any ulcerating spots with tannoform or iodoform. Dry dressings usually preferred.

With knife, scissors, or hot iron remove grapes or warty growths.

When the discharge is abated, mercuric oxide ointment or tar dressings.

Grogginess. See Navicular Disease.

\section{H XMATEMESIS.}

Hæmorrhage from stomach. Occasional in all animals.

Iced food, fluid and concentrated.

Calcium chloride, adrenalin solution, or gelatin, 10 to 20 per 1000 of physiological salt solution; or lactate of calcium 5 grains to the ounce; lead acetate and opium; ergotin hypodermically.

When bleeding from bowels give styptic in pills coated with keratin.

\section{H AEMATURIA.}

Bloody urine from injury or disease of urinary organs. Occurs in all animals.

Oleaginous laxatives, sulphuric acid, iron salts, lead acetate internally.

Ergotin and belladonna hypodermically; spinal hot-water bag.

\section{HÆMOGLOBINURIA or HAMOGLOBIN eMIA OF HoRSES.}

Azoturia. This disease invariably occurs in horses which have been rested for a day or two. Cause unknown, probably a nerve toxin. The urine, although its specific gravity is unaffected, is albuminous and high-coloured from suspended granular pigment, probably derived from extravasated blood in the voluntary muscles, which are suddenly affected by spasm, and subsequently become pale, flaccid, and wasted; while both the muscles and various internal organs contain after death a crystalline pigment (Sir John $M^{6}$ Fadyean, Jour. of Com. Path. 1888).

Dose of physic ; action seconded by laxative enemata or by venesection. Physostigmine hypodermically, where prompt catharsis required.

Chloral hydrate and opium, or nitrous ether and turpentine used as antispasmodics. Large doses of sodium bicarb. are recommended.

Ammonia and turpentine liniments applied over loins.

Frequently repeated moderate doses of alcohol, ether, or spirit of ammonia, with sulphuric acid and quinine, combat tendency to cardiac failure; adrenalin solution subcutaneously, very useful.

Where urine is not regularly passed use catheter thrice daily.

Attacks prevented by regular work or exercise, and sloppy, unstimulating food when resting. 


\section{HAMOPTYSIS.}

Bleeding from the lungs. Occasionally in horses and in other animals. Abundance of fresh air, and, where lungs congested, friction to skin, embrocation to legs, which subsequently envelop in flannel bandages.

Ice to swallow; cold compresses over chest ; ferric chloride or ergot.

Alcoholic stimulants, repeated every hour or oftener, to restore balance of circulation.

Ergotin hypodermically.

Digitalis or solution adrenalin where heart action weak or irregular.

In dogs and small animals moisten chest externally with chloroform.

Hamorrhage. Bleeding.

Bleeding from superficial wounds, when only small veins are lacerated, usually arrested by pressure, application of cold water, ice, refrigerants, or styptics.

A bleeding artery, as in castration, stopped by hot iron, torsion, or ligature ; sometimes by severing a partially divided vessel.

Bleeding which cannot be got at arrested by cold or heat applied so as to act reflexly ; by administration of such styptics as calcium chloride, lead acetate and opium, sulphuric or tannic acids, ferric chloride, or by ergotin hypodermically. Morphine diminishes blood pressure, and thus favours formation of a firm clot.

Hemorkhage, Post-partum.

Rare in animals.

Promote uterine contraction by removal of fotal membranes and introduction of ice into uterus or rectum, or both.

Contraction also produced by injection of water at about $110^{\circ} \mathrm{Fahr}$.

Injection into uterus of ferric chloride or ergot solution, or ergotin hypodermically.

Raise hind-quarters; hot-water bag to dorsal region.

Alcoholic stimulants with laudanum ward off collapse. Prevent strain. ing by using a binder, and by giving anodynes.

Heart, Fat'ty.

Common in pampered dogs, and in horses that have suffered from serious or repeated attacks of reducing disease.

No over-exertion; suitable dietary.

Iron salts, arsenic, strychnine.

\section{Heart, Hypertrophied.}

Occurs in hard-worked aged horses, generally due to valvular disease.

Discover and mitigate producing conditions.

Aconite and digitalis in small doses if heart action violent.

Moderate, slow work, caffeine, nutritive diet.

Heart, Palpitation.

Rest, perfect quiet, generous diet; iron tonics where there are anæmic murmurs.

Aconite, small doses, where cardiac action violent.

Digitalis or strophanthus where action weak and irregular.

Bromides sometimes useful where action irregular and fluttering.

Laxatives when connected with digestive derangement.

Belladonna where condition associated with strain or over-exertion.

Strychnine acts as heart tonic.

Heart, Valvular Disease.

Endocarditis, in horses, sometimes follows strangles, pneumonia, etc. ;

in dogs rheumatism and distemper, etc.

Treatment only palliative.

Over-exertion and excitement avoided; nourishing diet.

Steady circulation by digitalis and alcoholic stimulants.

Purgatives, nitrites, arsenic, lower vascular tension. 
Heart, Valvular Disease-continued.

Relieve dropsy by caffeine, pilocarpine, and diuretics.

Combat dyspnca and palpitation by morphine hypodermically, and pot. bromide or arsenic by mouth.

\section{HePATITIS.}

Inflammation of the liver, exceedingly rare; congestion more frequent. Cathartic, salines, amm. chloride; aconite if febrile symptoms prominent. Ipecacuanha valuable, especially in dogs.

Fomentation and stimulation over region of liver.

Digestible, laxative diet; suitable exercise when acute symptoms relieved.

Nitro-hydrochloric acid in chronic cases.

Salines and careful dietary safer than more active remedies when hepatic inflammation or congestion associated with epizootic or other debilitating disease.

Chronic cases usually terminating in cirrhosis or amyloid degeneration. Treatment only palliative.

\section{HERNIA.}

Protrusion of organ from natural cavity.

Umbilical. - Exomphalos, chiefly occurs in young animals.

Patient, after fasting for several hours, must be cast, placed on back, and protruding portion of intestine returned.

Retained in position by sutures, clamps of wood or iron, skewers, or elastic ligature.

Cantharides blister, or other irritant, applied to adjacent skin, causes swelling, and in slighter cases occludes opening.

Ventral.-Protrusion of bowel or omentum through rupture in abdominal wall, usually through staking or thrust of a horn.

Unless large, or liable to strangulation, seldom causes inconvenience.

Radical cure effected by returning viscus, and cutting down and suturing opening in abdominal walls.

Inguinal and Scrotal.-Usually occurring in entire horse, sometimes in gelding. Perineal in pig and dog; femoral in bitch.

Cast, and return the bowel by taxis, and if need be by application of ice or refrigerants.

If this fail, enlarge constricting ring.

Covered castration operation in entire animal. Ligation of cord, clamp, or sutures.

Hoose in Calves and Lambs-caused by Strongyles. See Worms.

Hoven and Gastric Impaction in Cattle or Sheep.

When rumen distended, chiefly with gas-

Full dose diluted ammonia, ether, turpentine, alcohol, or sol. barium chloride.

Exercise with friction to abdomen hastens expulsion of gas.

Introduction of hollow probang allows escape of gas when food in stomach limited in amount.

In serious cases puncture rumen through left flank with trocar and canula, or with butcher's, or other large knife.

Administer cathartic to remove any irritant, and feed for several days on sloppy, digestible food.

When rumen distended with food-

A smart purge conjoined with an active stimulant; but repeated purgatives are injurious; pilocarpine subcutaneously.

Solid food interdicted; slops, treacle-water, and ginger, freely given.

Water containing salines offered at short intervals.

Nux-vomica, emetine and veratrine useful when viscus in atonic state.

When distension and distress increase, there should be no delay in emptying the engorged stomach by rumenotomy. 
Hydatid on Brain of Sheep or Cattle. See Worms.

Cœnurus cerebralis ; tapeworm scolex or cyst.

Bore or trephine; remove sac and contained heads. Success of operation depends on position and number of cysts.

Effectually treat all dogs with tapeworm, and prevent their eating hydatid-affected brains.

\section{HYDROCELE.}

Dropsy of scrotum. Rare in veterinary patients.

Evacuate by trocar and canula; suspensory bandage ; observe antiseptic precautions.

Injection of dilute iodine or astringent solution.

\section{HYDROCEPHALUS.}

Chronic cranial dropsy, occasionally in horses, rare in cattle, dogs, or swine; also occurs in fœtus and may hinder parturition. Arrests brain development, producing gradual immobilité, when appearing after third year.

Treatment unsatisfactory. Quiet; light but nutritive diet.

Pilocarpine, calcium phosphates, iron salts.

Trephine or puncture to relieve pressure and facilitate parturition.

Hydrophobia. See Rabies.

HyDROThORAX.

Fluid (sometimes pus) in the chest. Affects all patients.

Digitalis ; pot. nitrate twice daily for a week.

Follow with pot. iodide and iron salts.

Pilocarpine hypodermically.

Iodine ointment and rubefacients externally.

When such measures ineffectual, and no diminution of fluid, or when dyspncea increases, tap with trocar and canula, or aspirator; operate antiseptically.

When pus present antiseptic irrigation of chest cavity may be tried, using one per cent. sol. protargol or lysoform, or one per thousand potassium permanganate.

Indigestion, See Dyspepsia.

Indrgestion, AcUte, of HoRses.

Stomach or grass staggers.

Aloes eserine or calomel and oil to unload stomach and bowels.

Ether or spirit of ammonia every two hours overcomes flatulence and spasm.

Repeated copious laxative enemata, vigorous hand-rubbing of abdomen, and walking exercise, encourage action of bowels and relieve spasm.

Hot fomentations or cloths wrung out of hot water, and in-rubbing of mustard, abate spasm and pain.

Where pain persists, morphine and atropine hypodermically.

One or two small doses of aconite tincture sometimes useful.

Blood-letting sometimes advisable where brain symptoms or dyspncea occur.

Paralysis resulting, give strychnine and apply counter-irritants to spine.

In young animals, where stomach overloaded with curd, prescribe oil, followed by ether or spirit of ammonia, and intestinal antiseptics.

Inflammation, Acute.

Antipyretics, small doses in early stages, repeated three or four times until pulse and temperature reduced.

Place patient in suitable quarters; ensure cool, pure air.

Clothe body, bandage legs.

Hot fomentations or poultices most useful where inflammation is com. paratively superficial. 


\section{Ixflammation, Acute-continued.}

Mustard or other stimulant embrocation applied to warm skin without blistering.

Ice or refrigerants applicable where inflammation limited or superficial, and in pharyngitis and laryngitis.

Cathartics to open bowels and lower arterial tension and temperature.

When more acute symptoms relieved, salines in small repeated dosespot. nitrate and chlorate, magnesium sulphate, ammonium salts, sodium sulphite.

Simple digestible diet; diluents.

Cannabis indica, opium, belladonna; other analgesics internally and topically.

Salicylic acid, salicylates, alkalies, and salines in rheumatic, and with belladonna in cystic inflammations.

\section{Inflammation, Chronic.}

Secure removal of morbid products by keeping excreting channels, when not inflamed, in good working order by clothing, suitable diet, diluents, occasional laxatives, etc.

Salines and antiseptics. See Inflammation, Acute.

Where patient reduced give digestible nutritive food.

Bitters, acids, and alcohol promote appetite.

Alcohol and volatile oils most useful in worn town horses, or where blood-poisoning has occurred.

Iodine and pot. iodide liquefy and remove exudate.

Counter-irritants frequently valuable-mercury oleate, embrocations, mustard occasionally applied and washed off.

Opium, belladonna, other analgesics topically.

When joints or bones affected, mercuric iodide ointment, seton, or firing after subsidence of urgent symptoms.

Influenza. Catarrhal Epizootic. Horse Distemper. Pink Eye.

Infectious febrile epizootic of horse, ass and mule, characterised by sudden onset, rapid development and marked prostration; affects primarily the respiratory tract, but also involves the nerve centres, circulatory system, digestive mucous membrane, eyes, and subcutaneous structures. Communicable chiefly directly, but also indirectly ; very contagious.

Chief Complications :- pneumonia, enteritis, cerebral congestion, paraplegia, ophthalmia, synovitis, laminitis, paralysis of penis.

Isolate patients, for their own comfort and safety of healthy subjects.

Disinfect premises daily. Provide special attendant for sick.

Comfortable box, temperature $60^{\circ}$ to $65^{\circ} \mathrm{Fahr}$, pure air.

Rugs, hood, bandages to legs; good hygienic conditions.

Enemata, linseed mashes, and, if need be, occasional laxative; maintain bowels in regular state. Recovery may take place without medicines.

Liquor amm. acetatis, Epsom salt, and nitre allay slight pyrexia.

More acute fever treated by antifebrin, antipyrine, thallin, digitalis, camphor, alcohol, and ether; hypodermic injections of salt solution; influenza antitoxin may be tried.

Sodium salicylate, pot. iodide, and digitalis conjoined, advised by Trasbot.

Restrict to milk and mash diet, with a little green food, but when pyrexia abates give digestible concentrated nutritive food.

Flannels wrung out of hot water, mustard, or other embrocations to throat, relieve congestion of upper air-passages.

CEdema requires, with salines and antiseptics, early use of alcohol, ether, oil of turpentine, or ferric chloride solution.

Purpura combated by silver colloid, pot. iodide, chlorate, and quinine, alternated with ferric chloride and oil of turpentine.

Cases with lung complications are more difficult to treat. Liquor amm. 
Influenza. Catarrhal Epizootic. Horse Distemper. Pink Eye-con. tinued.

acetatis, other stimulating expectorants, with pot. nitrate or chlorate and camphor, relieve dyspncea; oxygen inhalations.

Hot fomentations to chest; mustard applied, but washed off in fifteen to twenty minutes; reapplied if needful; influenza antitoxin intravenously.

Cardiac asthenia combated by alcohol or ether given early, in moderate doses, every three hours, and conjoined with camphor and digitalis, or with subcutaneous injections of caffeine or camphorated oil.

Diuretics, alkalies, and pilocarpine prescribed by Friedberger.

When lung secretions are fœtid, antiseptics, phenols, per os, or inhaled; benzoates.

Gastro-intestinal complications treated in earlier stages by a few doses of grey powder or calomel, conjoined with laxatives.

In later stages, and with hepatic symptoms, by nitro-hydrochloric acid, sodium salicylate, or hyposulphite.

Abdominal pain relieved by chloral and cannabis indica internally, or by morphine hypodermically.

Rheumatic symptoms treated with salicylates, oil of turpentine, diuretic doses of digitalis and salines, with stimulation of affected muscles and joints.

When strength reduced, give milk, eggs, beef tea, and frequent alcoholic stimulants.

INTUSSUSCEPTION.

Invagination of a portion of intestine.

No medicinal treatment of much avail. Restrict to a limited amount of soft food.

Avoid cathartics.

Opium and cannabis indica allay spasm and pain.

In the horse long enema tube may be tried.

Failing other means, open abdomen and endeavour to rectify the displacement, or practise excision.

\section{IRITIS.}

Inflammation of the iris. Occurs in specific ophthalmia in horse, which see.

Dark box, cathartics, salines : continuous cold irrigation or ice-bag.

Solution of atropine, alternated with eserine, prevent adhesions.

Cannabis indica, opium, and other anodynes internally and locally.

JAUNDICE.

Icterus. Skin discoloration from bile pigments. 1. Catarrhal, affecting the bile ducts. 2. Retention or reabsorption of bile. 3. Hepatic, from congestion, inflammation, or degeneration of liver.

Laxatives clear away excess of bile lodged in the bile tubes or duodenum.

Simple digestible food beneficially diminishes formation of bile.

Massage of liver region, mustard embrocations, and faradisation relieve biliary torpidity and retention; subcutaneous injections of sterilised salt solution.

Amm. chloride and salines in catarrhal cases.

Nitro-hydrochloric acid ; where patient weak conjoin quinine.

Little can be done for cirrhosis or fatty degeneration.

JoHNE's Disease.

A disease of cattle caused by microbes closely resembling tubercle bacilli. The lesions occur in the small intestine and mesenteric glands. Usually fatal.

Kennel Lameness. See Rheumatism.

KerATOMA and KeRAPHYLLOCELE.

Horn tumour, growing from sole or horny laminæ.

Relieve pressure by rasping and poulticing. Excise tumour, dress wound antiseptically. Recurrence frequent. 


\section{LAMiNitis.}

Acute, subacute, and chronic. Inflammation of sensitive laminæ and other vascular parts of the feet of horses. Occasionally in cattle, sheep and goats.

Shoes removed, horn thinned, feet poulticed, loose-box bedded with several inches chaff or cut straw or tan.

Continued hot fomentations or poultices soften horn and relieve tension and pain.

Large cold bran poultices sometimes afford more relief than hot.

Purgative medicines or bleed from jugular in acute cases.

Bleeding more suitable when attacks result from concussion than from gastric derangement; arecoline or pilocarpine hypodermically.

Diuretics in full doses generally beneficial.

Repeated small doses of aconite, nitre, and other salines relieve fever.

Bowels, if need be, regulated by laxative enemata.

Vapour bath frequently serviceable.

When inflammation subdued, removal of exudate hastened by cold applications, by blisters to coronet, and exercise.

Keep heels low and toe short; use stout, wide-webbed, rocker bar shoes.

Neurectomy sometimes subsequently useful where lameness persistent.

\section{LARYNGITIS.}

Inflammation of mucous lining of larynx.

(1) Catarrhal.-Occasionally epizootic and contagious. Occurs especially in horses and dogs.

Comfortable box, suitable clothing, moist atmosphere of $60^{\circ}$ to $65^{\circ}$ Fahr.

Steam head and throat persistently with medicated vapour; heat and moisture externally.

Aconite and laxatives abate fever: emetics in dogs and pigs.

Ammon. acetate, kermes, or camphor and belladonna in electuary.

Counter-irritants of soap liniment, mustard, cantharides.

Intratracheal injection of solution of morphine and hydrocyanic acid used successfully in 200 acute cases by Jelkmann.

Tracheotomy where dyspnœa becomes dangerous.

(2) Fibrinous. - Occurs in cattle and cats; rare in horses and dogs. Usually rapidly fatal.

Steam throat with watery vapour medicated with benzoic acid or eucalyptus.

Emetics for the cat and dog.

Hot compresses ; mustard or other counter-irritant ; tracheotomy.

(3) Edema glottidis.-CEdema of submucous tissue of glottis. From (a) acute laryngitis, from smoke or hot air, operations, choking, corrosive irritant liquids ; $(b)$ infective, as in anthrax, glanders, etc.

If not infective treat with watery medicated vapour; hot fomentations; blister.

Tracheotomy if suffocation threatening.

\section{LEUCORRHCA.}

Fluor albus. Intermittent catarrhal discharge from the uterus; occurs in cow and bitch.

Usually arising from endometritis ; may be tuberculous, or connected with tumour formation. A cause of vaginitis, perhaps of abortion, also of balanitis in male having connection with affected female.

Syphon or syringe daily the uterus and vagina with tepid solution of potassium permanganate, and subsequently with $\frac{1}{2}$ of one per cent. sol. of lysol, and later with one per cent. sol. alum, zinc chloride, or protargol.

Belladonna or iodoform in suppository, or injection, where there is excessive discharge or pain.

Mineral tonics internally, especially in delicate subjects, or in obstinate recurring cases associated with tuberculosis. 
LICE.

Pediculi. Each of the domestic animals infested by its own species.

Horse: Homatopinus macrocephalus, Trichodectes pilosus, and $T$. pubescens.

Cattle : $H$. eurysternus, large ox-louse ; $H$. vituli, calf-louse; and $T$. scalaris, small ox-louse.

Sheep: T. sphærocephalus. The pupiparous dipteran, Melophagus ovinus, ked or fag, also infests the skin of the sheep.

Dog: $H$. piliferus, and T. latus. Cat: T. subrostratus.

Pig : H. urius.

Goat: $H$. stenopsis and T. climax.

Fowls. Goniodes dissimilis, Gonicoctes hologaster, G. gigas, Lipeurus variabilis, $L$. heterographus, Menopon pallidum, $M$. biseriatum.

Cleanse skin with soft soap and warm water, and rub in decotion of stavesacre-one to forty of water. For limited areas, one part stavesacre, twenty vinegar; or creolin solution, 2 per cent.

Oil of tar, one part; oil of rape or other bland oil, 15 parts.

Carbolic soft soap, and rape oil.

Lysoform, 5 per cent., followed by washing with soap and water.

Patients long and seriously infested should have long coarse hair clipped and dressing well rubbed in.

The usual dipping mixtures for sheep.

Diluted solution of chloroform, or oil of sassafras, for pet dogs.

Strong tobacco decoctions and mercurial ointments apt to produce constitutional effects.

Isolate infested subjects and their belongings, and thoroughly cleanse premises occupied by them.

Birds. Dusting skin with sulphur, or one of the insecticide powders may be used.

Disinfect floors, walls, perches and nests; lime dust has been recommended.

\section{LICHEN.}

Chronic papulo.vesicular eczema.

Laxatives, salines, oleaginous diet.

Apply locally, night and morning, an alkaline wash, to which-if there is itching - add a little thymol, chloroform, or belladonna.

Tonics and arsenic, with iodide of sulphur ointment, and tar oils alternated in chronic cases.

Lithiasis : Gravel. See Calculi and Urinary Deposits.

LockJaw. See Tetayds.

Lodping Ill in Sheep. See Meningtitis.

Loxations. See Dislocations.

\section{LYMPHANGITIS IN HoRSES.}

Weed. Inflammatory œdema. Inflammation (infective or toxic) of lymphatic glands and vessels of the limbs; usually affects the inguinal glands; sometimes symptomatic of glanders.

Cathartic, laxative enemata, salines in drinking water remove irritant waste products. Bran mashes and wet food.

Hot fomentations persisted with for several hours, and limb then swathed in woollen or hay bandages.

Where limb tender and painful after fomenting, moisten with soap liniment and laudanum.

When weather cold, clothing needful to promote skin functions.

Stimulants useful where preliminary rigor severe or continued.

Exercise serviceable.

In hard-worked, debilitated subjects, aconite unsuitable. 
LyMPHANGITIS IN HoRses-continued.

Give half dose physic and salines, foment limb, and administer turpentine and alcoholic stimulants.

Aid removal of swelling by exercise, and, subsequently, moderate work ; smart friction of limb daily with oil ; diuretics and tonics; iodine or pot. iodide internally ; laxative diet and green food.

Careful regulation of food, work, and rest prevents recurrence.

LYMPHATICS, INFLAMED.

Occurs specially in horses; from pricks, other injuries, infective as in glanders.

Remove if possible the original cause. Cooling diet, salines, pot. iodide. Foment while heat and tenderness continue; when tenderness abates, apply friction, bandages, iodine ointment ; continue salines in drinking water.

\section{Maladie Du CoÏt. Dourine. Covering Disease.}

A specific equine disease atfecting the male and female genitals, characterised by catarrhal discharges, œdematous and phlegmonous swellings and ulcerations. There supervenes a vaso-nervous urticaria with spinal paralysis. The disease is communicable in coition by affected horse or mare, and is caused by the Trypanosoma equiperdum; the period of incubation varies from eight to sixty days; the mortality in the horse is greater than in the mare, reaching 70 per cent.

Thorough daily irrigation of affected parts with corrosive sublimate one part, common salt ten, water 1000 .

Ulceration treated with strong solution silver nitrate or copper sulphate.

The horse cast and penis thoroughly examined, and treatment adapted to special lesions.

Concentrated nourishing food.

Reduced iron, iodides, atoxyl, arsenic, cacodylate of soda, quinine, mercuric chloride.

Increasing doses of arsenic up to 20 grains per day; atoxyl subcutaneously 2 to 4 grains, gradually increasing to 10 or 12 grains per day; cacodylate of soda subcutaneously grains 10 to grains 40 in 20 per cent. solution, daily for five days, then discontinue for five days and repeat as before.

Months of patient treatment often elapse before cure effected.

In Prussia no affected stallion can be used in the stud until three years after recovery has been reported.

A contagious exanthematous vesicular eruption of a comparatively benign type affects the genital mucous membrane of dogs and cattle, occasionally of horses, and less frequently of goats, sheep, and hogs. The male suffers more than the female. The incubation stage three to six days.

Astringent lotions; ulceration treated as above.

Maggots From Flyblow.

Turpentine and oil ; phenol solutions; oil of tar.

\section{Mallenders and Sallenders.}

Chronic squamous eczema of the skin in the flexures of the horse's knee and hock. The chronic scaly condition preceded by an erythematous and vesicular stage.

Soft soap and water, or carbonated alkali and vaseline remove scales.

Boro-glycerin or zinc oxide ointment relieves irritation.

Mercuric oxide, or iodine ointment abates thickening and infiltration.

Tar oils or decoction of oak bark in chronic cases.

Half dose physic, salines, laxative diet and regular exercise.

In debilitated subjects iron tonics and arsenic. 
Malnutrition.

Liberal oleaginous dietary ; change of food ; cod liver oil.

Acids and bitters, iron salts, calcium phosphate in young animals.

Alcohol, arsenic, nux-vomica, glycerophosphates.

Mammitis.

Garget. Inflammation of the udder: Catarrhal, affecting primarily the mucous membrane; and Lobular or glandular. Occasionally in all milking animals, but most frequent in good dairy cows. May be tubercular.

Cathartic, diuretics in drinking water ; or orange sulphide of antimony, which is excreted in the milk.

Thoroughly milk the affected quarters and hand-rub the udder; inject each teat with warm solution boracic acid ( 3 per cent.); do not repeat injection; draw teats three hours after injection, and repeat the milking every three or four hours until recovery.

In cold weather clothe to promote skin functions.

The teat syphon sometimes facilitates removal of milk, but must be used very carefully. Support udder by bandage.

Apply heat and moisture by persistent fomentations, or poultices of spent hops, supported by suspensory bandage.

Belladonna extract and vaseline applied several times daily lessens congestion, milk secretion, and tenderness.

When inflammation abates encourage removal of exudate by in-rubbing iodine ointment, and by iodine and salines internally.

When abscess forms and matures open it and treat antiseptically.

When gangrene occurs excise dead tissues and dress with antiseptics.

After earlier pyrexia removed sustain strength by concentrated food, tonics, and stimulants.

Until udder again in normal state restrict to dry food, so that production of milk be minimised.

\section{Mammitis, Contagious.}

Common in milch cows; caused by a streptococcus which is transmitted from one cow to another by the milker's hands; infection takes place through the teat. A specific catarrhal inflammation of the milk ducts and sinuses with induration of the affected gland; by extension may involve the whole udder. Milk curdles quickly, is acid, and when mixed with normal milk produces clotting; later milk becomes yellowish, viscous and fœtid.

Prevent by washing and disinfecting udder and teats, and the hands of the milkers, before and after milking.

Curative treatment unsatisfactory ; empty udder and inject teats with 5 per cent. solution boracic acid, 3 per cent. sodium fluoride, 3 per cent. sanitas, or 5 per cent. solution hydrogen peroxide.

Isolate infected cows and destroy milk.

Thoroughly cleanse and disinfect cowsheds.

Newly purchased cows should be kept apart for a month before entering cowshed or herding with unaffected stock.

MaNGe. See ACARI.

Scabies. Skin irritation and itching produced by various acari.

(a) The Psoroptes live on the surface of skin, and are distributed generally over the body. Horse, ox, sheep.

(b) The Sarcoptes burrow in the skin, prefer the head and portions of body sparsely covered with hair. Horse, ox, sheep, pig, and dog.

(c) The Chorioptes are more limited in their distribution, affect chiefly the limbs of horses and sheep, the base of the tail of cattle, and a variety, ch. cynotis invades the ears of the dog and cat.

Isolate infected subjects. 
MaNGE-continued.

Thoroughly cleanse, wash, and disinfect with carbolic, or corrosive sublimate solution, clothing, harness, stable fittings, rubbing-posts, etc.

Clip, collect, and burn hair.

Crusts softened and removed by soap and water, pot. carbonate, with oil, vaseline, or glycerin and water.

Psoroptes and Chorioptes are destroyed without much skin irritation or risk of poisoning by stavesacre ointment or solution, sulphur iodide ointment, or creolin solution, applied daily for a week.

Tar oil one part, palm oil six parts, laid on thickly and allowed to remain undisturbed for four or five days.

Wood tar and sublimed sulphur each one part, soft soap and alcohol each two parts; creolin solution, 3 per cent.

The dressing used must be thoroughly rubbed in, and when washed off should within a week be reapplied.

To kill the burrowing Sarcoptes the parts affected are lathered with soap and water, soaked for some hours with solution of pot. carbonate sulphur and oil, and the parasiticide rubbed in with a brush. To destroy subsequent hatchings a second, and in bad cases a third dressing may be needed, at an interval of a week.

Oil of cade and coal tar, each one part, mixed in a mortar, and three parts benzine added, commended by Trasbot.

In some cases dressing only one half of the skin at a time is advisable.

Persistent spots over limited area dressed with mercurial ointment.

Change dressings repeatedly.

For ears of dogs and cats naphthol ten parts, ether thirty, olive oil 100, or warm solution of cyllin, 2 per cent.

For poultry dust skin thoroughly with pyrethrum or other insect powder (Cagny).

Treatment in sheep specially noticed under ScaB.

\section{Mange, Follicular.}

Caused by the Demodex folliculorum. Inhabits the hair follicles, sebaceous glands and their tubes, usually about the head and extremities; produces erythema of adjacent skin, atrophy of hair roots, muco-purulent discharge, formation of small abscesses ; often assumes a squamous type. Occurs in dogs, cats, and occasionally in pigs.

Separate patient from other animals.

Treatment uncertain and tedious; nature of remedy probably of less importance than its thorough application.

Shave the parts affected; lather with terebene soap, and rub in daily for several weeks balsam of Peru (Siedamgrotzky).

Creosote one part, caustic pot. solution two, olive oil fourteen (Hunting).

Daily rubbing with ointment of cyllin (5) and lanoline (100 parts).

Creosote or oil of cade and caustic pot. are rendered more penetrating by addition of chloroform.

Solutions of benzine, naphthol, or zinc chloride $2 \frac{1}{2}$ per cent., or sulphur iodide ointment.

Creolin and ichthyol are said to be effective if the dog is placed in a bath, containing 2 to 3 per cent. of either remedy, for a quarter of an hour daily for two months (Friedberger).

Mild cases may be cured by shaving and washing the affected skin, squeezing and scraping the pustules, and touching each spot with undiluted iodine tincture.

Daily intradermic injection of one per cent. phenol into and around the lesions, commended by Mollereau.

Subcutaneous injections of vaccine prepared from the staphylococcus pyogenes albus successfully tried by Mettam.

Scrub thoroughly every affected spot with forty grains pot. sulphide in a pint of water, dress limited parts of the legs or body with cantha- 
Mange, Follicular-continued.

rides ointment, and rub the head and remaining portions with balsam of Peru. Repeat daily for a week, applying the cantharides to fresh spots. After a few days' rest resume (Brusasco).

MEGRIMS.

Vertigo; Giddiness; occurring in horses; pathology undetermined.

Remove any pressure interfering with cerebral circulation.

Dash cold water over head and neck.

Walking exercise ; hand-rubbing of body and legs ; pot. iodide to reduce blood-pressure.

Occasional dose of physic sometimes useful.

Horses subject to megrims should be driven carefully.

\section{Melanosis.}

Most frequent in grey horses. A pigmented Sarcoma, usually localised where pigment already exists.

When growth distinctly defined, remove by knife or caustic.

Dress with antiseptics. Recurrence with increased activity of growth frequent after operation.

Meningitis.

Inflammation of the membranes of the brain or spinal cord. The intensity of the attack and the parts specially affected determine excitement or paralysis of the central nervous system. Occurs in all veterinary patients ; frequent and fatal in cattle and sheep, in which often associated with gastric derangement.

Where cerebral excitement prominent, dark box, active cathartic; blood-letting in robust subjects; ice to head. See Phrenitis.

Cathartics, physostigmine, and pilocarpine (Friedberger).

When functional activity of cord exalted hot or cold compresses to spine; laxatives, bromides, or chloral.

Remove retained contents of bladder or rectum.

When acute symptoms relieved, pot. iodide and salines.

Chronic paresis treated by strychnine, and embrocations to poll or spine.

Epizootic form in horses-cerebro-spinal meningitis, which see.

Sheep suffer from chronic spinal paralysis, characterised by hyperæsthesia and convulsive movements, general muscular feebleness, paresis of the hind-quarters, and progressive wasting; no definite pathological lesions; popularly known as Louping-ill, described by Friedberger as Lumbar prurigo, by Trasbot as Maladie des trotteurs.

Treatment unsatisfactory. Remove affected animals from breeding flock. Feed young stock liberally.

Improve poor pastures on which disease occurs.

Metritis. Metro-peritonitis.

Inflammation, usually septic, affecting the uterus and peritoneal membrane. Occurs in all females, usually about three days after difficult parturition. Liable to spread by contagion.

Syphon or syringe uterus and vagina with 1 per cent. creolin, 1 per 1000 corrosive sublimate solution, hydrogen peroxide one part in five of boiled water, or two per cent solution lysoform.

Remove fœetal membranes and blood clots; render aseptic any wounds.

Afterwards, where walls of uterus dilated or flaccid, inject solution of ergot, belladonna tincture or astringent antiseptic.

To facilitate irrigation employ clean horse catheter. See INTRA-UTERINE INJECTIONS.

Woollen cloth or lint wrung out of hot water applied over loins and round abdomen relieves spasms and pain.

As in other septic cases, creolin, ether, phenols, naphthol, or sulphites are given internally. 
Metritis. Metro-Peritonitis-continued.

If bowels torpid half dose physic with laxative enemata.

Remove urine by catheter.

Sustain strength by generous diet, tonics, and stimulants.

Isolate patients from gravid or parturient animals, and use disinfectants.

Mrositis.

Inflammation of muscle, usually from overwork, direct violence, strains, rheumatism. Occurs in all animals.

Rest ; injured muscles if possible kept relaxed.

Fomentation succeeded by application of belladonna.or opium liniment; antiseptics and diuretics internally.

Cathartic and antipyretics, especially when dependent upon rheumatism.

Counter-irritation, galvanism, regular exercise if atrophy supervene.

Free incision if pus form.

Nagana or Tsetse-Fly Disease.

Caused by 'Trypanosoma Brucei, which is transmitted from diseased to healthy animals by the tsetse-fly, Glossina morsitans, and probably other species.

Affects horses, cattle, and dogs. So far arsenical preparations, atoxyl, arrhenal, cacodylate of soda have given the best results in the treatment of this disease.

Nasal Gleet.

Ozæna. Chronic nasal discharge resulting from ulceration of pituitary membrane, necrosis of turbinal bones, impaction of facial sinuses, a carious tooth, or a tumour. Usually occurring in horse. See Frontal AND Nasal Sin USES.

Sulphurous acid, iodine, iodoform inhalations.

Nasal douches of salt and water containing a few drops of iodine tincture. Bleaching powder scattered in box. Insufflation of iodoform or chinosol.

Copper or iron sulphate, arsenic, turpentine, buchu, and copaiba.

Remove faulty teeth.

Trephine sinuses; wash out with antiseptics; solution hydrogen peroxide.

Isolate all horses with suspicious nasal discharges.

\section{Navel Disease. Omphalophlebitis.}

An infective inflammation of the umbilical vein and other structures of the navel, with secondary suppurating lesions of joints (polyarthritis), serous membranes, intestine, liver, kidneys, and lungs ; caused by various microbes (streptococci, staphylococci, necrosis bacillus, ovoid bacteria, etc.); affects foals, calves, and lambs at or soon after birth ; often fatal.

Prevent, by carefully cleansing and disinfecting the navel immediately after birth, aseptic ligation of the cord, and daily dressing of the navel with lysoform, iodoform, or Stockholm tar. Where this disease exists, pregnant animals should be kept separated until after parturition.

Curative treatment rarely successful; douche navel with antiseptics; sol. mercuric chloride, lysol, or carbolic acid. Inject fistula with sol. zinc or copper sulphate, or by introducing sublimate one part in five of collodion; repeat in three or four days. Treating the mare with sodium or potassium iodide recommended by Eckert.

Navicular Disease.

Grogginess. Rarifying ostitis with chronic synovitis of the bursa and ulceration of the fibro-cartilage of the navicular bone. The flexor pedis perforans tendon is sometimes adherent to the navicular bone. Concussion, constant hard work, and rheumatoid diathesis believed to be the chief causes. 
Navicular Disease-continued.

Stand horse in warm water bath, or apply wet swabs to coronets for several hours daily.

Apply at night poultice, but not too unwieldy, or it may prevent patient lying down.

Dose of physic and cooling diet.

After fortnight or three weeks blister coronet.

If marked benefit does not result, relegate horse to slow work and shoe with wedge-heeled shoes.

Lameness sometimes diminished by shoeing with leather, and using shoes thick at quarters and thin at toe.

Where foot and limbs otherwise good, perform neurectomy above the fetlock.

\section{NeOrosis.}

A general term, formerly restricted to death of bone in whole or in part; but now applied to local death of any tissue.

Necrosis bacillus responsible for calf diphtheria; necrosis of gastrointestinal mucous membrane in cattle and sheep, and some cases of navel disease in calves and lambs.

In bone, remove sequestrum; iodoform and other antiseptic dressings.

Encourage reparative process by local and general antiseptic treatment.

Nettle Rash.

Surfeit. See URTICARIA.

\section{NEPHRITIS.}

Inflammation of kidneys. Of several forms.

Absolute rest, light laxative food; diluents, milk diet for dogs.

Maintain activity of other excreting channels, hydrogogue cathartics, sod. sulphate, jalap.

Fomentations, hot compresses; fresh sheep-skin to loins of horses; hot bath for dogs.

Pilocarpine hydrochloride.

When heart weak or irritable, digitalis or caffeine.

Chronic cases require similar treatment.

Digestible nutritive food to sustain strength.

Digitalis, caffeine, and pilocarpine to combat effusion.

In purulent cases alkaline sulphites, copaiba, eucalyptus oil, camphor bromide, and salol.

Where abscess diagnosed pus may be withdrawn by aspirator.

Extirpation of kidney has been successfully practised.

\section{NeUralgia.}

Pain along course of a sensory or mixed nerve.

Fndeavour to discover and remove any cause of nerve irritation.

Any wound likely to cause or aggravate the condition should be fomented and dressed antiseptically.

Laxative beneficially promotes action of bowels and excretory channels.

Analgesics topically; morphine or antipyrine hypodermically along course of nerve.

Administer salicylates, aspirin, or salines, with pot. iodide, quinine, arsenic ; pot. bromide where patient irritable.

Counter-irritants, mustard, iodoform, electricity in chronic cases.

Nerve-stretching by extension of parts or cutting down on nerve.

Generous diet, with fair proportion of oleaginous matters where patient debilitated.

Nedritis or Perinedritis.

Inflammation of the sheath or tissue enclosing the nerve fibres. Occasionally in all patients, from injury, rheumatism, lead-poisoning.

Remove cause if possible.

Rest of parts supplied by nerve. Fomentations, analgesics.

In chronic cases blister along course of nerve. 


\section{INDEX OF DISEASES AND REMEDIES}

CEstrus Equi. See Bots.

Esophageal Obstructions. See Choking.

Mechanical tumours; Peri-œsophageal abscesses.

Pass probang or sound.

Remove obstruction if possible.

Esophageal Dilatations.

Difficult to treat.

Feed frequently in small quantity on concentrated or semi-fluid food.

Partial excision of pouch and insertion of sutures successful in horse.

Esophageal Paralysis.

Pass sound; embrocations externally ; administer strychnine ; counterirritation.

\section{CEsophagitis.}

Inflammation of mucous membrane or muscle of gullet.

Give fluid soft food cold; ice.

Refrigerant compresses.

Antiseptic and mild astringent electuaries.

Stimulant embrocations to absorb hard circumscribed swellings.

OPEN JoInt.

Wound of joint with escape of synovia.

Healing by first intention promoted by fixing joint, cleansing wound, and antiseptic bandage or adhesive.

Inject wound with antiseptics, corrosive sublimate one part to 1000 of boiled water.

If wound suppurating or of several days' standing inject, two or three times a day, sterilised glycerin, or 3 per cent. sol. hydrogen peroxide.

Protect wound by frequently dusting with iodoform or chinosol; or by applying several layers absorbent lint.

Severed surfaces may be sutured, provided movement of joint and tearing can be prevented.

Splints and bandages secure support and protection.

Slinging horse prevents movements which might reopen wound, and removes weight which in injuries of limbs aggravates inflammation.

Inflammation relieved by half doze of physic; cooling diet, and weak antiseptic solution allowed continuously to trickle over a calico bandage lightly laid over the joint. Continuous irrigation with cold water.

When the wound is unlikely to close quickly, or simple treatment has failed, apply synovial styptics or a blister, with view of closing opening, limiting motion, and relieving pain.

Anchylosis apt to result where injury has been serious or case neglected or badly treated.

Ophthalmia.

(a) Simple inflammation of the mucous membrane of the eye or conjunctivitis ; occurring in all animals.

(b) Periodic or Specific ; Specific inflammation of most structures of the eyes of horses; hereditary; liable to recur, and eventually causing cataract, blindness, and atrophy of the eyeball.

Remove any foreign body. Douche eye with warm solution boracic acid, 2 per cent.

Fomentation with tepid water containing aqueous solution of opium abates irritation; four per cent. solution cocaine; half dose physic.

Antiseptics, solution protargol, 2 per cent., mild astringents; vaseline to prevent lids sticking together.

Eserine and atropine, locally and alternately, diminish irritation and prevent adhesion of iris to lens.

Protect the eyes from light.

Periodic form relieved by cathartics, febrifuges, anodynes, belladonna or atropine, locally and internally. 
ORChitis and EPIDIDYMitis.

Inflammation of the testicle and epididymis; sometimes symptomatic of glanders.

Fomentations, scarification, anodynes, dose of physic ; support testicle.

Iodine liniment; pot. iodide internally.

If pus forms must be evacuated by free incision.

Tubercular form intractable; castrate in early stage.

Osteo-malacia or Moluities Ossium.

Fragilitas ossium. Softening, with deformity, in consequence of removal of lime salts, of the bones. Cause unknown; rare in the domestic animals.

Concentrated nutritive food, cod-liver oil, fresh air, exercise; dry healthy surroundings.

Calcium phosphates, bone meal ; remove any gastro-intestinal troubles.

Ostitis.

Chronic or subacute inflammation of bone ; associated with periostitis and producing exostoses; sometimes tuberculous.

Heat and moisture; cold applications; allow escape of any exudate.

Cooling diet, salines, alteratives.

Rest; blister; pyro-puncture.

\section{OTORRHEA.}

Catarrh of external ear. Occurs in dogs, as sequel to distemper, or from being much in water. May be parasitic, or caused by a foreign body.

Cleanse the ear, disinfect with antiseptic dissolved in spirit.

Warm solution of boric acid or glycerin of tannin dropped or syringed into ear. Stop canal with absorbent cotton. Solution of copper sulphate, silver nitrate, or lead acetate, 10 grains in an ounce of alcohol or ether. Ten per cent. sol. iodine in spirit. Dry dressing of ear often beneficial.

Purgative ; restricted laxative diet; soda bicarb., magnesia.

If patient persist in scratching ear, apply net or bandage feet.

Over-Reach in Horse.

Wound or bruise of coronet of fore-limb, caused by the shoe of the hind foot.

Foment; antiseptic dressing.

Protect coronet with pad.

Use light shoes; shorten and round off offending shoe.

Paralysis.

Paresis. Palsy. Impairment or loss of motility or sensation. Impaired motility most common in animals. Classified as-(1) General ; (2) Unilateral or Hemiplegia ; (3) Bilateral or Paraplegia; (4) Local, as supra scapular, radial, or crural paralysis.

Pot. iodide and salines if dependent on pressure from fluid.

Trephine and raise bone if arising from depression of cranial bones.

When caused by embolism or thrombosis treatment almost hopeless.

Remove gastric, uterine, or other local irritation, or blood-poisoning.

Rest, quiet, light digestible food.

When bladder affected remove urine by catheter.

Salines, tonics, and other remedies to improve general vigour.

When chronic, strychnine to stimulate motor centres and nerves.

Friction, kneading, and occasional application of counter-irritants and electricity impart nervous and muscular tone ; intra-muscular injections.

In cattle, paresis when depending on gastric derangement treated by full dose of physic and subsequently nerve tonics. As a sequel of milk fever, pot. iodide followed by nux-vomica, and counter-irritants to spine. Connected with anæmia, liberal dietary and tonics.

In dogs, after dose of castor oil, prescribe pot. iodide, and, if stomach irritable, bismuth and soda bicarb. 
PAROTIDITIS.

Inflammation of parotid gland. 'Iraumatic. May be due to strangles, pharyngitis, salivary calculus, and in the ox to actinomycosis.

Hot moist compresses or poultices.

Infriction as required of mildly stimulating or anodyne ointments.

When chronic or indolent, iodine or cantharides dressings.

Partorient Paralysis. Mammary Toxamia.

Milk fever: A disease of cows (and ewes) occurring at parturition, or a few days thereafter; characterised by loss of copsciousness, and paralysis of motion and sensation; sometimes ushered in by cerebral excitement, spasms, and convulsions; attacking animals in the prime of life, in good condition, and free milkers. Formerly mortality ranged from 40 to 60 per cent. No marked pathological changes. Those recovering do so quickly, and usually perfectly. Cause of disease still unexplained, but probably due to a nerve toxin formed in the udder.

Ever's treatment:- Subcutaneous injection of 50 grains of caffeine dissolved in six drachms of boiled water; then, after emptying the udder, insufflate each quarter with pure oxygen or filtercd air; prevent escape of air by rubber ring on each teat; remove rings in one or two hours later; knead the udder after insufflation.

If swallowing difficult, give cathartic and other medicine with Read's pump.

Prop on sternum: keep up head: turn patient from one side to other every three hours.

Schmidt's treatment:-Dissolve 3ij. to 3iij. pot. iodide in 35 ounces of boiling water; cool to blood heat, empty udder and inject one-fourth of the solution into each teat; knead the udder after injection.

If urine retained, empty bladder by catheter twice daily.

Linseed gruel occasionally by stomach pump and clyster.

Rubefacients to spine; cold pack sometimes beneficial.

Until recovery fairly established, withhold dry food, but allow mashes and diluents.

Purgatives, counter-irritants to the spine, pot. iodide and nux-vomica relieve resulting weakness.

Prevent by sparingly feeding suseeptible subjects for a month before calving.

Cathartic a fortnight before parturition, and another if required immediately after.

Milk cow a fortnight before calving, earlier if milk can be drawn, and empty bag twice daily.

Patella, Partial Luxation of. Arrest of Patelila on Femoral Trochlea. Occurs in colts, occasionally in cattle, and in horses of any age.

After reduction keep limb extended and slightly forward for several hours by cord attached to the fetlock and carried round the neck.

Put on a shoe high at the toe and projecting forward.

Blister stifle.

\section{Pericarditis.}

Inflammation of serous covering of heart. Rare in horses and dogs, in which usually rheumatic or infective; more frequent in cattle and goats, from their swallowing sharp-pointed bodies, which pass through the walls of stomach and diaphragm, and enter the pericardium, producing pericarditis. Frequently fatal.

Morphine hypodermically usually relieves acute pain.

Woollen cloths wrung out of hot water applied to the chest for an hour or two at a time.

Digitalis, strophanthus, caffeine, moderate rapid, violent, irregular heart action. 


\section{Pericarditis-continued.}

Digitalis, pot. iodide, and strychnine assist absorption of fluid by promot. ing diuresis.

In dog prescribe milk diet and diuretics; may puncture pericardium and withdraw exudate.

\section{Periostitis.}

Inflammation beginning in or chiefly affecting the periosteum; may be acute or chronic ; simple, as from kicks or injuries, or diffuse or infective. The vascular structures of the bone are involved. Occurs in all patients.

Rest, cold applications, analgesics, astringent lotions, iodine ointment.

Free incision if pus form; antiseptics; treat enlargements by blisters or firing.

Liberal diet; stimulants and quinine if blood-poisoning imminent.

\section{Peritonitis.}

Inflammation of membrane covering the bowels and lining the abdominal cavity; due to infection and often fatal. May follow castration, operation for hernia, difficult parturition, staking, injury to abdominal wall, perforation of bowel, etc.

Blood-letting or two or three small doses aconite in early stages of acute cases in robust subjects, antiseptics and diuretics internally.

Opium in full doses quiets movement of bowels and relieves pain; morphine and atropine hypodermically prompt and effectual, quinine useful.

Woollen rugs wrung out of hot water applied for two hours continuously, surface thoroughly dried and rubbed with soap liniment and opium tincture.

Light mustard dressing applied for fifteen to twenty minutes; but active counter-irritation injurious.

Milk, eggs, beef tea, and oatmeal gruel support strength; sterilised salt-solution subcutaneously.

When more acute symptoms past, salines in drinking water and enemata maintain natural condition of bowels.

Alcoholic or etherous stimulants and camphor useful in second stages and in young and weakly subjects, and earlier in most influenza cases.

A cantharides blister and pot. iodide where fluid remains unabsorbed.

\section{Pharyngitis.}

Inflammation of lining of pharynx.

(a) Catarrhal or from local irritants ; (b) Extension of neighbouring inflammation; (c) Specific infection. Occurs in all animals, notably horses, under the familiar title of Sore-throat.

Comfortable quarters, pure air, soft nutritive food.

Drinking water impregnated with borax, pot. chlorate, or vinegar.

Sponge lips and nostrils; syringe with mild antiseptics.

Combat acute inflammation by hot compress or poultices.

Belladonna and camphor relieve spasms of pharyngeal muscles.

When swelling hard and circumscribed apply stimulating embrocations.

Glycerin of tannin or ferric chloride solution when throat relaxed.

Medicated inhalations; astringent electuaries.

Pharyngeal abscesses are matured by steaming and fomentation, and may be opened by a guarded knife.

Tracheotomy when swelling produces dangerous dyspnœa.

\section{Phlebitis.}

Inflammation of vein; due to bacterial invasion.

Antiseptic irrigations; double ligation and excision of obstructed vein.

Open abscesses; give cathartic ; laxative diet.

Horse with impervious jugular should not be turned out to grass.

Diffuse phlebitis treated antiseptically, but rarely successfully. 
Phthiriasis. See Lice.

PiLES.

Hæmorrhoids. Swelling inside or around the anus, consisting of dilated or varicose veins. Occur in dogs.

Remove hardened fæces by enema; cooling digestible diet; oily aperients if needful. Glauber's salt in small doses continued for ten days.

Gall and opium ointment or zinc benzoate ointment.

In persistent cases remove by ligature; clamp, cautery, or knife.

Piroplasmosis. Red-Water. Texas Fever. Hamoglobinuria of Cattle.

An infective hæmoglobinæmia produced in cattle, sheep, dogs, and occasionally in horses, by the action of hæmatozoa (piroplasmata), which destroy the red corpuscles of the blood, reducing them to one-third or one-fourth of their normal number, their colouring matters being excreted in the urine. The piroplasmata are transmitted from infected to healthy animals by various ticks.

Salines and oils remedy the earlier constipation.

Subcutaneous injections of sterilised salt-solution.

Intravenous injection of one per cent. solution of formalin, or colloid silver; atoxyl, trypanblue.

Hypodermic injection of five per cent. solution arrhenal recommended by Lignières.

Antiseptics, antipyretics, and tonics.

Prevention consists in inoculation, destroying ticks by spraying, smearing, or dipping, and in keeping cattle off tick-infested pastures.

Plethora.

Defined as a superfluity or hypertrophy of blood. Although not a disease it occasionally predisposes to disease.

Remedied by regulating diet, reducing its quantity or nutritive quality. In horses that, in stable language, are 'gross,' give half dose physic, followed by salines in drinking water.

Reduce amount of corn, especially of beans.

Substitute a little green food for part of hay.

Secure sufficient exercise or work.

Pleurisy.

Inflammation of serous covering of lungs and lining of chest. Caused

by bacteria. May occur as a primary affection or from wounds, foreign bodies; frequently secondary to strangles, glanders, pneumonia, tuberculosis, etc.

Hygienic treatment as in pneumonia; dry nourishing diet.

Emetic and antimonials in animals that vomit.

Two or three doses antipyrine, or calomel and opium, relieve pyrexia.

Salines and antipyretics as in bronchitis and pneumonia.

Pot. iodide and salicylate of soda promote absorption of exudate; hypodermic injection of sterilised salt-solution containing caffeine.

Digitalis and nux-vomica aid removal of fluid.

Rugs wrung out of hot water to sides, or in-rubbing of mustard, washed off in twenty minutes.

Moderate counter-irritation by cantharides, repeated if necessary.

Refrigerant compresses to chest advised in early stage by Friedberger.

Pain reduced by opium, or by morphine hypodermically.

Early tapping requisite where exudate considerable; repeat in twelve hours if required. Antiseptic irrigation of chest cavity of the dog. See Hydrothotrax.

Pleuro-pneumonia Epizootic.

Contagious lung complaint of cattle. An infective inflammation of the lungs and pleuræ of horned cattle, probably caused by an anærobic micro-organism. Spreads generally by direct cohabitation; incubation stage twenty to forty days. 


\section{Pleuro-pnedmonia Epizootic-continued.}

Treatment generally unsatisfactory. Mortality 30 to 50 per cent.

Prevention.-Under British Contagious Diseases (Animals) Act, cattle affected, and those in contact, immediately slaughtered. Slightly affected subjects in good condition are passed for beef ; other carcases, disinfected, used for manure.

Infected premises thoroughly cleansed and disinfected.

The value of protective inoculation doubtful.

\section{Pneumonia. Inflammation of the Lungs.}

The chief distinctive forms are-

(1) Contagious, croupous, or gangrenous pneumonia, with secondary pleurisy and parenchymatous degeneration of principal organs; chest plague. Depends upon a sinall ovoid bacterium (Schiitz), which has been cultivated, and reproduces the disease, not only in horses, but in rabbits, mice, and pigeons. The most common form in horses.

(2) Catarrhal or broncho-pneumonia. Caused by irritants, including worms, and characterised by exudation and epithelial proliferation and desquamation. Of less definite and usually slower progress. Not frequent in horses, but common in cattle, sheep, and dogs.

(3) Interstitial or chronic pneumonia. A sequel to the croupous or catarrhal affection.

Contagious pneumonia of horses is often classed as influenza.

General principles of treatment the same in both types.

Suitable sanitary conditions, diet, and nursing.

Hot compresses changed every twelve or fifteen minutes.

Antipyretics, salines, bowels regulated by enemas; camphorated oil subcutaneously.

If cathartic needful, oil better than aloes or salines.

In neither form is blood-letting required or desirable.

In both, especially in catarrhal, ammonium acetate solution, pot. chlorate or nitrate, given in draught or drinking water.

Two or three small doses aconite where pyrexia acute and horse or cow robust.

Emetic in outset of attack in strong dogs.

Mustard applied to sides for fifteen or twenty minutes, and reapplied as required. More useful in catarrhal than croupous cases.

Frequent doses alcohol, ether, or spiritus ætheris nitrosi where patient feeble.

Digitalis or caffeine if heart weak.

Pot. iodide and moderate counter-irritation encourage absorption of exudate.

\section{Poll Evil. See Abscess and Fistula.}

Prolapse of the Rectum, Uterds, or Vagina.

More common in cows that have had several calves than in other veterinary patients.

Remove any causes still in action.

Raise hind-quarters. Carefully return.

Cleanse and wash organ with mild astringent solution.

If swollen, support on large linen towel or linen web.

Cross and twist in opposite directions ends of web, thus inducing pressure, and keep web soaked with ice-cold water.

Knead repeatedly with hands.

Scarification not advisable. Excision sometimes necessary.

Straining combated by chloral hydrate by nouth, injection of morphine hypodermically and pressure on loins.

Subsequent prolapse prevented by clamp, truss, or sutures; the latter most effectual. 
Prostatitis.

Inflammation of prostate gland, usually from gonorrhœa, cystitis, or calculi. Occurs in dogs.

Hot fomentations. Catheter if needful to remove urine.

Alkalies and pot. iodide, belladonna and eucalyptus oil.

If abscess forms free incision in middle line of perineum.

\section{PruRitus.}

Itching. A cutaneous neurosis occurring in horses and dogs.

When resulting from exposure to sun-heat, the animal is brought into the shade, douched with pot. bicarbonate, any specially irritable spots moistened with lotion of two parts glycerin, one each of sugar of lead and laudanum, and sixty water.

Belladonna in chloroform solution, alternated with alkaline washes, allays hyper-sensibility.

Lotions of resorcin ( 5 per cent.) or chloral (1 per cent.) of ten beneficial.

Corrosive sublimate, thymol, or volatile oils, locally when itching depends upon parasites.

Cathartics, salines, careful dietary, when associated with gastric derangement.

Iron salts, oleaginous food, alkalies, arsenic, internally when patients impoverished.

Psoas Muscle Strain. Occurs in both horses and dogs.

Rugs wrung out of hot water applied over loins and round abdomen.

Anodyne enemata ; rest.

Slings essential when both sides affected.

Psoriasis.

A term applied to the scaly forms of eczema, such as mallenders in horses, which see.

Pumiced Foot in Horses.

Unhealthy horn with convexity and weakness of the sole, the result of laminitis.

Bar shoe with wide web.

Lessen concussion by tar dressing and leather sole.

Stimulate coronet.

Punctured Foot.

Injury from nail in shoeing or from gathered nail.

Remove shoe, search foot, and thin hoof at puncture. May enlarge wound, then disinfect and bandage antiseptically.

Purpura Hamorrhagica in the Horse.

Petechial fever. A fever characterised by circumscribed swellings of the skin and hæmorrhagic petechiæ of the mucous membranes, and their subareolar textures. Similar extravasations appear in the substance of muscles, in the lungs, and other organs. Frequently a sequel of exhausting disease, but etiology not fully explained; probably a toxæmia.

Remove to spacious airy quarters, clothe warmly.

Pot. chlorate, three or four drachms two or three times daily, subsequently half doses; usually given in drinking water.

Early injection of antistreptococcic serum may shorten duration of disease.

Iron salts (sulphate, perchloride), quinine, oil of turpentine, and milk.

Digitalis and cinchona in electuary.

Concentrated nourishment; oatmeal gruel, milk and eggs, alcoholic stimulants. Silver colloid, solution of adrenalin; subcutaneous injections of saline solution containing caffeine.

Bland oil or vinegar diminishes skin tension and tendency to slough.

Limited swellings about head bathed with cold water and refrigerants. 
Purpura Hayorrhagica in the Horse-continued.

Inject intratracheally, 3viii. to 3xii. twice daily of a solution containing one part iodine, six pot. iodide, 100 water (Dieckerhoff).

Small repeated doses of calomel are indicated when there is danger of intestinal infection.

Scrupulous cleanliness and antiseptic dressings essential where portions of skin ulcerating or sloughing; methylene blue ( 2 per cent.) in spirit.

Tracheotomy desirable where dyspnœa distressing.

Pus in Facial Sindses. See Nasal Gleet.

\section{Pyamia.}

A disease caused by infection, and characterised by pyrexia of an intermittent type and formation of secondary abscesses.

Any wound whence infective products may arise must be laid open, cleansed, and rendered aseptic.

Injection of iodine tincture or dilute carbolic acid into inflamed glands sometimes checks destructive suppuration; antistreptococcic serum subcutaneously.

Sanitary conditions must be attended to.

The patient coaxed to take digestible, nutritive, concentrated food to sustain strength and ward off collapse; milk, hay-tea, gelatin.

Moderate doses of alcoholic and etherous stimulants repeated every three or four hours; quinine, salol, or salicylates.

Quarter Evil. See Black Quarter.

\section{QUITTOR.}

See also Fistula. A sinuous wound of the horse's coronet.

Poultice to soften horn, thoroughly cleanse and disinfect, remove dead and detached tissue, provide dependent opening; excision of necrosed cartilage often required.

Inject Villate's solution, or aqueous solution of corrosive sublimate, 1 to 1000. Get perfect asepsis.

Envelop foot in antiseptic tow or jute, soaked in sublimate or other antiseptic solution, and carefully bandage.

In four days to a week remove dressing; with knife and curette remove any necrosing or dead tissue, and dress as before.

Where foot strong no shoe needed; but if weak or broken, bar shoe relieves pressure.

Stimulant embrocations to the coronet promote reparative action.

Radial Paralysis or ' Dropped Elbow.'

Lameness with loss of power to extend the elbow-joint. Caused by injury to the extensor muscles, the brachial plexus, or the nerve supplying the affected muscles. Sometimes attributed to costal fracture. Recovery protracted.

Place horse in slings for a few days, foment extensor region, massage, nerve stimulants, deep injection of sol. strychnine; blisters ; setons, exercise.

RABIES.

A specific febrile disease, occurring especially in the canine and feline races, produced by a virus found in the central nervous system and most organs and secretions, and usually communicated by the bite of a rabid dog or other animal. The incubation period ranges from fifteen to sixty days in the dog, cat and horse, one to three months in the ox, and fifteen to thirty days in the sheep. All warmblooded animals are susceptible. The disease is most certainly and rapidly produced by inoculation, and when developed is incurable.

Under the Contagious Diseases (Animals) Act, rabid dogs and animals bitten by them are destroyed. 
RABIES-continued.

When a person is bitten by a mad dog, circulation through the part should, if practicable, be checked by a ligature, the wound washed with corrosive sublimate solution, and cauterised.

Pasteur has demonstrated that dogs and other animals inoculated with attenuated virus do not develop the disease when bitten by a rabid dog or inoculated with virus which wonld kill unprotected animals.

Like protection is generally secured if the man or animal, within a few days after being bitten, is inoculated with the protective vaccine.

The mortality of persons bitten by rabid dogs previous to 1886 was 16 per cent. Since then many thousands of bitten persons have been treated at the Pasteur Institute, with the result that the mortality has been reduced to less than 1 per cent.

RheUMatisM.

Inflammation of fibrous structures of muscles, tendons, joints, etc.; usually attributed to cold and damp; but sometimes to specific infection; by some believed to depend upon accumulation in body of some product of nutritive derangement (auto-intoxication, possibly lactic acid, but more probably a toxin). Occurs in all classes of patients.

Keep patient warm and dry; dry flannel bandages; pot. iodide internally.

Alkalies, pot. bicarbonate and nitrate in drinking water.

Sodium salicylate, salol, naphthol, antipyrine, aspirin.

Veratrine or morphine hypodermically, repeated daily for a week or ten days. Potass. iodide 3ij daily for a fortnight.

Amm. acetate and colchicum; pilocarpine hypodermically.

When acute symptoms abated, quinine, arsenic, Donovan's solution.

Locally, friction with compound camphor liniment, later blister or fire and blister; exercise beneficial.

In strong dogs an emetic at outset, antipyretics, salicylate of sodium.

Provide warm dry kennel.

Stiffness or swelling subsequently removed by massage; by camphor oil, or soap liniment rubbed in.

Articular rheumatism most frequent in cattle and dogs.

Salicylates, antipyretics; joints enveloped in cotton wool.

Embrocations of ichthyol and lanoline, oil of wintergreen, iodine solution, or mercury oleate.

Cases simulating articular rheumatism in foals, calves, and lambs, sometimes result from infection of navel.

Prevented by aseptic ligation of the cord at birth, and thorough disinfection of adjacent skin, by phenol, or other antiseptic, repeated daily for a week. See Navel Disease.

RICKETS.

Rachitis. Faulty development and softening of the bones of young animals, depending upon malnutrition.

Nourishing diet; milk, crushed oats with linseed for herbivora; milk, meat soup, bone meal, cod-liver oil for dogs; good hygiene and exercise.

In young animals sucking, see that mother's milk sufficient in quantity and quality, and that she is well nourished.

Aperients or antacids to rectify any digestive derangement.

Calcium phosphate; iron salts, lime water, glycerophosphates.

Splints and bandages may be requisite to support the softened bones.

RINDERPEST.

Cattle plague. A specific malignant contagious fever affecting the bovine race, but communicable to sheep and other ruminants, depending upon a specific virus, and characterised by lesions chiefly localised in the mucous membranes and skin. Indigenous to the Asiatic Steppes of Russia and other parts of Asia. 


\section{RINDERPEST-continued.}

Treatment unsatisfactory; protective inoculation by various methods practised in South Africa.

Slaughter of infected animals, and those in contact with them, and effectual disinfection promptly exterminated the disease imported into Great Britain in 1865, as well as the limited outbreaks of 1872 and 1877.

\section{Ringbone, Articular and Non-articular.}

Exostosis on a pastern bone or the coffin bone; sometimes in both positions. Most serious when involving a joint.

When occurring in the fore-limb, use a thin-heeled bar shoe; when in the hind-limb, a high-heeled shoe, thus diminishing pressure and concussion.

Put to slow work on land. When in stable apply wet swabs.

Where there is lameness-rest, give dose physic, and fire and blister, or unnerve.

\section{RingwORM.}

Tinea. Circular elevated spots, becoming itchy, scaly, and hairless, appearing usually on the skin of the head, neck, and body, produced by the action of various species of fungi, which invade especially the hair bulbs. More common in cattle than in horses, dogs, or cats ; rare in sheep and pigs ; may be transmitted from one animal to another.

Horse. Trichophytic ringworm, caused by Trichophyton mentagrophytes, T. flavum, T. equinum, T. verrucosum; and Microsporous ringworm, by Microsporum Audouini.

Cattle. Ringworni always a Trichophytosis and due to T. mentagrophytes.

Dog. Four varieties of ringworm occur: (1) Trichophytic (T. caninum); (2) Microsporous (M. Audouini var. caninum); (3) Eidamellian (Eidamella spinosa); and favus (Oospora canina). See Favos.

Isolate affected animals, and disinfect all brushes, clothing, harness, or whatever the fungus may have lodged on; burn the crusts.

Washing with soap and water, or soaking with oil and pot. carbonate, removes scabs and crusts. Paint spots, after removing crusts, with iodine tincture, or apply solution or ointment of carbolic acid, creosote, naphthol, oil of tar, or sulphur iodide.

If these ineffectual, substitute mercuric nitrate ointment; solutions of mercuric chloride, ferric chloride, or copper sulphate ; or iodoform.

Salines, tonics, arsenic, internally, help to abate irritation and œdema.

Grooming or dressing of affected subjects should be suspended, as it may spread the disease.

\section{RoARING or Whistling.}

May depend on thickening of the mucous lining of the nares, pharynx, or larynx, or on fibrous growths in these regions; but in most instances roaring or whistling is due to paralysis of the left recurrent nerve, with wasting, and fatty degeneration of the dilator muscles of the left side of the larynx. The opening through which the air passes being narrowed, the characteristic noise is produced, especially when inspiration is quickened by excitement or exertion.

Spurious or temporary roaring, depending upon cold, strangles, or influenza, is treated sometimes successfully by stimulation of the throat, and by pot. iodide and arsenic internally.

I'rue roaring or whistling, depending on paralysis and muscular atrophy, is incurable.

Smart blistering, the actual cautery, or galvanism in the earlier stages, sometimes proves beneficial.

A pad fitted on the nostrils regulating the supply of air lessens the noise in bad cases. 


\section{INDEX OF DISEASES AND REMEDIES}

ROARING or WHISTLING-continued.

Tracheotomy also affords relief.

Strychnine persisted with for weeks in the earlier stages appears to arrest and may prevent the muscular wasting.

Of various surgical operations attempted, excision of the left laryngeal ventricle has given the best result.

Rot in Sheep. Distomum Hepaticum. See Worms.

SaddLE Galls.

Bruises from badly-fitting saddles or harness.

Relieve from weight and prevent friction.

Apply hot compress and antiseptic dressings.

Swollen sebaceous follicles in the early stages are reduced by friction with soap liniment; in chronic cases by fomenting, lancing, or cauterising with undiluted carbolic acid.

Portions of dead tissue or exudate constituting sitfasts should be dis. sected out, and the wound treated in the usual way.

\section{SANDCRACK.}

Fissure in horn of horse's foot, usually on inner quarter of a fore- or toe of a hind-foot.

Remove shoe, thin wall on each side of fissure, cleanse and disinfect.

Fomentation, poultice, and rest relieve pressure, inflammation and pain.

When these abated, clamp or wedge the fissure, or groove the wall transversely or obliquely to the direction of the crack, and blister coronet.

Bar shoe, made to relieve fissured horn from pressure and concussion. Dress hoof frequently with wood tar.

SePtic InfeCtion.

A febrile state, the result of the circulation in the blood of pathogenic organisms or the toxins to which they give rise. The organisms multiply at the seat of inoculation, and have the power of entering the circulation, and thus forming secondary infective foci. Infection occurs in puerperal metritis in cows and ewes, in the fever occasionally following castration and other surgical operations, as well as accidental wounds in horses (open joint, gathered nail, etc.).

Arrest if possible the primary source of infection.

Cleanse and render aseptic any wound. Excise necrosing tissue.

Corrosive sublimate solution 1 to 1000 . Zinc chloride 1 to 100 .

Administer antiseptics, antipyretics, creolin, sulphites, quinine, antistreptococcic serum.

Generous dietary; milk, eggs, oatmeal gruel, beef tea.

Alcoholic stimulants, acids, and bitters.

\section{Sarcomatous Tumours.}

Are chiefly composed of embryonic or immature connective tissues; they contain blood-vessels, but neither nerves nor lymphatics have been clearly made out; they differ greatly in appearance, rapidity of growth, and malignancy, and occur in all the lower animals.

In the earlier stages, especially if likely to interfere with any important function, removal by the knife should be attempted.

Scab in Sheep.

Skin irritation, inflammation and scabbing, caused by Psoroptes. See ACari and Mange.

In all bad cases the sheep should be bare shorn and affected parts soaked with pot. carbonate dissolved in twenty parts water before the insecticide is applied. 
SCAB IN SHeEP-continued.

Dress carefully every itching part with creolin and alcohol each one part, soft soap eight parts. Non-poisonous (Fröhner).

Within a few days immerse each sheep for three minutes in bath of two parts each of creolin and pot. carbonate and 100 parts water. Repeat bath in eight days.

Watch for any rubbing, examine each sheep twice a week, and, if required, apply the creolin dressing.

Decoctions of tobacco and stavesacre and solutions of arsenic, tar oil, and creosote are also used, both as lotion and bath.

The following formulae have been selected from a number tested in Germany :- (1) Arsenious acid 10 parts, alum 200, water 1000 parts. (2) Arsenious acid 10 parts, potash 10 parts, water 1000 parts. (3) Arsenious acid 10 parts, alum 10 parts, vinegar 1000 parts, water 1000 parts. To avoid danger from absorption of arsenic by the skin, sheep should not be dipped before the tenth or twelfth day after shearing. Each sheep is immersed twice and brushed all over the body.

All affected sheep should be isolated; while, to prevent the spread of the parasites, which remain alive for at least a week, racks, rubbingposts, and anything on which they may have lodged, are washed with warm lysol solution ( 3 per cent.).

\section{Seedy Toe of Horses.}

A perverted secretion of horn, with excavation between the laminal sheath and outer wall of the foot, the space being partly flled with soft mealy horn. It frequently follows laminitis. A similar condition occurs in sheep.

All diseased horn must be removed and the cavity disinfected.

Healthier growth encouraged by moisture, exercise, and blisters to coronet.

A bar shoe relieves pressure. If space not large, recovery may follow careful disinfection of the cavity. In other cases the outer wall should be excised, and the exposed surface dressed with antiseptics.

To prevent deformation of hoof, the shoe must be removed frequently and the hoof trimmed into shape.

\section{SHIVERING.}

Chronic myelitis with sclerosis of motor tracts of spinal cord, and characterised by a peculiar spasmodic contraction of muscles of hind or fore legs.

Hereditary; occurrence generally preceded by remote attack of strangles, influenza, or pneumonia. Frequently symptoms difficult to discover. Incurable. The horse may do moderate, slow work.

Shoulder Slip (Suprascapular Paralysis).

Injury to nerve, or extensor muscles of the horse's shoulder. Rare in other animals.

Foment, rest, purgatives, massage: induced current to atrophied region.

Blister when tenderness removed.

Douche with cold water; exercise.

\section{Side Bone.}

Ossification of lateral cartilage of horse's foot. When uncomplicated seldom causes lameness.

'The treatment has been bar shoe, cold applications, rest, blisters, firing, with neurectomy in chronic cases if feet otherwise sound. In cases with contraction the wall may be grooved with knife or wedge firingiron, or divided in two or three places, and the foot afterwards shod with a bar shoe. 
Sore Shins. See Ostitis and Periostitis.

\section{Sodth AFrican Horse-sickness.}

An infective disease, peculiar to the equidæ, characterised by tissue dropsy, and proved to be caused by an ultra-visible organism (Sir John M'Fadyean). Believed to be transmitted by an insect; can be produced by inoculation, or ingestion of blood obtained from an infected horse. Very fatal; curative treatment unsatisfactory; prevention by injection of special serum more hopeful.

Spavin, Bog. See Bog Spavin.

Spavin, Bone.

(a) A bony outgrowth on the inner and lower part of the horse's hock, arising from osteo-arthritis of the cuneiform, scaphoid, and metatarsal bones, terminating generally in anchylosis, peripheral and central.

(b) Articular disease, without appreciable enlargement, terminating in anchylosis.

Rest; cathartics and fomentation where there is much lameness.

In young horses blister, pyro-puncture, seton, periosteotomy, or cunean tenotomy. Anterior and posterior tibial neurectomy sometimes

beneficial.

Splenic Apoplexy. See Anthrax.

SPLiNT.

A bony enlargement on a fore or hind canon of the horse; occasionally of the ox.

In slighter cases stop fast work, give half dose physic, foment, and subsequently blister.

In more acute cases subcutaneous periosteotomy, or fire and blister.

Pyro-puncture preferable to line-firing as it blemishes very little.

Mercuric iodide ointment to reduce enlargement.

SPEEDY-CUT.

A bruise or contused wound on the inner aspect of the horse's knee, caused by the opposite foot.

Fomentations : open any abscess; antiseptic dressing.

Prevent by reducing inner crust of offending foot, using nicely-fitting three-quarter shoes, and removing shoes every three weeks.

A boot sometimes worn on the leg liable to cutting.

Avoid over-pacing such horses.

Sprains of Muscles, Tendons, and Ligaments.

The fibres are severely stretched, in serious cases ruptured, causing inflammation and subsequent contraction, and, in case of muscles, atrophy'.

Endeavour to rest horse at once.

Relieve injured parts from strain and weight; in severe cases sling.

In strains connected with back tendons tack on high-heeled shoe.

Apply compress moistened with solution of Epsom salt, or foment until tenderness removed; support with elastic pressure bandage, or with subcutaneous injections of filtered air; close puncture with collodion.

Subsequently cold applications; regular walking exercise.

Blister, or fire and blister, and turn out to grass.

In bad or repeated tendon sprains, contraction occurs which is incurable. Tenotomy in selected cases may be advisable. Median neurectomy may be tried. Subcutaneous injection of fibrolysin may reduce thickening of tendon.

Sterility. See Barrenness.

Stomach Staggers in Horses. See Indigestion. 


\section{Stomatitis.}

Inflammation of buccal mucous membrane, chiefly occurring in young animals- $(a)$ erythematous and catarrhal ; $(b)$ aphthous, vesicular, pustular, frequently contagious ; (c) parasitic (mycosis).

Soft digestible food, laxatives, salines. Remove any gastric derangement. See to any irritation of gums, or faulty teeth.

Give slowly pot. chlorate or borax, 1 to 4 per cent. in glycerin solution.

In sucking foals and calves, besides local treatment, see that mother's milk is sound and that she is properly fed.

Ulcers dressed with glycerin of tannin or painted with silver nitrate, ten grains to ounce distilled water.

A contagious pustular form amongst horses, is described by Friedberger, and treated by pot. permanganate or creolin solution.

Strangles in Horses.

A contagious catarrhal eruptive fever, peculiar to the equine species, caused by a streptococcus. Usually, abscesses develop in the connective tissue in the space between the branches of the lower jaw, and involve adjacent glands : while by extension or secondary infection the disease may attack other glands, the mucous and serous membranes and internal organs, producing pharyngitis, laryngitis, pneumonia, pleurisy, pericarditis, peritonitis, meningitis, synovitis, arthritis, etc. The disease occurs chiefly in young animals.

Good nursing ; perfect sanitary surroundings.

Steam head where catarrhal symptoms troublesome.

Fomentations or poultices to throat hasten formation of abscess.

Sodium sulphite and pot. chlorate dissolved in drinking water.

In early stage antistreptococcic serum, subcutaneously injected, often beneficial.

Horse at grass shelter at night, unless weather warm and case slight.

Supply mash, gruel, grass, sliced roots, malt, steamed oats, or whatever soft digestible food patient will eat.

Milk, eggs, beef tea, with ale or wine if animal weak.

Sweet spirit of nitre or quinine if infection extend.

Abscess should be fully matured before it is opened; indeed many practitioners prefer that it be allowed to burst.

While discharging, keep parts clean; dress daily with antiseptics.

Tracheotomy performed when dyspnœa not relieved by steaming and fomentations ; its timely performance may prevent roaring, and often saves life.

Isolate infected subjects and disinfect premises.

\section{Stringhalt in the Horse.}

Involuntary spasmodic contractions of the muscles of one or both hind, or fore (rare), limbs; not traceable to definite pathological lesion.

Seldom curable; may become worse with hard work and advancing years.

Relieve spavin or other condition which may aggravate reflexly.

Temporary benefit results from a laxative, a course of bromides, and moderation of work. Peroneal tenotomy and aponeurotomy sometimes beneficial.

Stretching or section of the tibial nerves of little value.

\section{Sturdy. Hydatid in Brain. See Worms.}

\section{Surfett. Nettlle-Rash. See Urticaria.}

SURra. Rot.

A specific progressive fever associated with gastric ulceration, anæmia, œdema, emaciation and paralysis, affecting horses and dogs, rarely cattle, and caused by a hæmatozoon (Trypanosoma Evansi). Transmitted by ingestion or by inoculation. 
Surra. Rot-continued.

Prevent by giving pure water, clean grain and forage. Treat disease by increasing doses of arsenic, Fowler's solution, cacodylates or atoxyl. Feed liberally.

Swing Fevers. Swine Plagues.

Under these titles are grouped two specific contagious epizootic fevers of the pig, each depending on its special microbe.

(1) Swine fever proper. In America termed hog cholera. A contagious and infectious disease of the pig, associated with a necrotic and ulcerative condition of the mucous membrane of the intestine, nearly always most marked in the large intestine. The large intestine exhibits ' ringed' patches of necrosis, in more chronic cases nodular elevations; the skin discoloured. The incubation stage is eight to ten days; the mortality 40 to 50 per cent.; higher among young pigs; death occurs in eight to sixteen days. Attacks swine of all ages; is the disease of which the British Contagious Diseases (Animals) Act takes cognisance; notification compulsory.

(2) Swine erysipelas. Mal rouge. An acute septicæmia, characterised by erysipelatous inflammation of the skin, which at first is bright red, becoming blue or brown-red. The gastro-intestinal mucous membrane is acutely inflamed, the spleen is enlarged, there is generally nephritis and endocarditis, but no pneumonia. Incubation stage three to four days; swine three to twelve months chiefly affected; mortality 50 to 75 per cent.

Principles of prevention and treatment the same in both forms.

Slaughter, or deeply bury infected, especially first cases. Isolate incontacts. Disinfect.

In erysipelas form, vaccination with attenuated virus (although causing mortality of 1 or 2 per cent.) is stated to render most subjects immune for a year.

Synovitis.

Inflammation of a synovial membrane from injury, puncture, or localisation of rheumatism or tuberculosis. See also ARTHRITIS.

Fomentations or swathing the joints with cloths wrung out of hot water, persisted with for some hours, parts afterwards rubbed with soap liniment and laudanum.

Anatomical rest may be helped by splints and bandages.

Slings where hock or other large joint of horse's hind limb affected.

If not relieved in a few days, blister ; fire in chronic cases.

Mercury oleate in rheumatic and tubercular cases, which are specially intractable. Internally give potassium iodide, salicylates, alkalies.

Tapeworms. Trenia. See Worms.

Teats, Obstructed.

The teats of cows and ewes are obstructed by curdled milk, calculi, tumours attached to mucous membrane, inflammatory thickening, or stricture.

Where not removable by careful manipulation, concretions may be displaced or broken up by bougie or teat syphon.

Tumours within the teat not reducible by mechanical means are excised.

Warts on the teats removed by scissors or ligature.

Inflammation treated by fomentations and poultices of spent hops.

Stricture, usually a sequel to inflammation, relieved by passing probe, syphon, or teat bistoury.

Tendons or Ligaments, Ruptured.

Occurs chiefly in horses. See also Sprains.

Fomentations allay inflammation. 
Tendons or Ligaments, Ruptured-continued.

Treat as for fractured bones; keep parts perfectly quiescent.

Splints, starch or plaster bandages, slings.

When inflammation moderated stimulate externally.

Tetands.

Lockjaw. Tonic muscular spasms depending on a toxin, produced by an anærobic bristle-shaped microbe. This organism is found in many soils, is inoculable from animal to animal; when brought into contact with a part, especially if bruised or injured, it produces its tetanising toxin, which acts like strychnine on the spinal cord. Horses and sheep are most susceptible, cattle, swine, and dogs less susceptible.

There is hope of recovery if the amount of toxin circulated is limited, if its production by attention to the wound can be arrested, and the patient's strength sustained.

The infected wound when apparent must be rendered aseptic; if closed, may be opened, cauterised, disinfected, or when practicable, excised. In lambs and other young animals cleanse and disinfect the navel cord.

Although the jaws are closed, most patients suck up sloppy food, which is rendered as nutritive as possible and offered frequently.

Action of bowels encouraged by mashes, gruel, treacle, and salines in drinking water, which should be within reach.

Place horse in darkened box, where he will not be disturbed; slings may be required.

Medicines are of little avail. Bromides, prussic acid, and chloral afford temporary relief, especially where excitement considerable.

Powdered opium and cannabis indica extract, thirty grains each in electuary, placed thrice daily within the cheek.

Occasional administration of chloroform relaxes spasms for a time, and may enable food to be swallowed; but this advantage in some patients may be counterbalanced by the irritation caused by giving the anæsthetic.

Tetanus antitoxin renders rabbits, mice, sheep, and horses, resistant to the inoculation of toxic doses of virus; but as a curative its value is doubtful. Further trial desirable. (See pp. 7, 697.)

Thxas Fever. See Piroplasmosis.

Thick Wind in Horses.

May be due to thickening of the mucous lining of the bronchial tubes, or imperfect power of emptying, the air-cells (emphysema).

Seldom curable, but relieved by good, rather concentrated, damped food, and regular exercise.

Allow water in moderate quantity frequently.

Relieve gastro-intestinal irregularity.

\section{THOROUGH-PIN OF THE Hock.}

A bursal enlargement on the lateral aspect of the horse's hock, arising from disease of the sheath of the flexor pedis perforans tendon, or, when co-existing with bog spavin, from dropsy of the true hock joint. Generally more unsightly than troublesome.

Rest, high-heeled shoe, flannel bandages.

Equable pressure from a spring truss.

Where the swelling is not thus reduced apply a blister or fire in lines.

Other treatment failing, the distended bursa may be aspirated.

Thorough-pin of the knee consists in distension of the sheath containing the perforatus and perforans tendons at the back of the knee-joint. Generally caused by injury; lameness well marked.

Treated similarly to thorough-pin of the hock; fomentations, and later by mild blister. 


\section{Thrombosis.}

Coagulation of blood in a vein or artery; causing partial or complete - obstruction.

Absolute rest, in order to diminish risk of a portion of clot being detached and obstructing circulation in the brain or pulmonary vessels.

When affecting main artery of a limb causes intermittent lameness, which is incurable.

The œdema frequently resulting is abated by carefully applied bandages.

Thrush in Mouth. See Aphtha.

Throsh in Horse's Frog.

A fœit discharge from the cleft of the frog.

A leather sole is sometimes placed under the shoe, while the animal is stabled, to keep the frog dry.

Cleanse and disinfect cleft.

Calomel dusted over diseased surface; dressings of tar or wood-tar oil. Regulate feeding, exercise, or work.

Shoe with tips if feet strong and animal works chiefly on land.

Ticks.

Belong to the family Ixodidæ, order Acarina.

They have been proved responsible for the transmission of certain diseases caused by blood parasites.

Ixodes ricinus and $I$. hexagonus attack dogs, cattle, sheep, deer and horses, and Hamaphysalis punctata attacks cattle. The parasite of Redwater or bovine paraplasmosis is conveyed from diseased to healthy cattle by $I$. ricinus, $H$. punctata (United Kingdom), Rhipicephalus annulatus (U.S.A.), Rh. decoloratus and others (South Africa), Rh. Australis or Scrubtick (Australia). Other ticks known to carry disease-producing germs are :-Rh. Evertsi (equine biliary fever), Rh. bursa (ovine piroplasmosis), Rh. appendiculatus (East Coast fever), Hamaphysalis Leachi (canine piroplasmosis), Ambly. omma hebraum (Heart-water of cattle and sheep), Rh. simus, $R h$. capensis, Hyalomma Agyptium, and Argas reflexus, which attacks birds.

Destroy by volatile oils ; by smearing, spraying, or dipping.

When not numerous may be picked off, or snipped with scissors.

Bath of arsenic, potashes, soft soap and water.

Dress with wood-tar, coal-tar, or petroleum oils, or 3 per cent. creolin solution.

Tinea Tonsurans. Trichophytosis. See Ringworm.

\section{TоотнАСне.}

Usually results from caries, attacking chiefly the molar teeth.

Extract diseased tooth with forceps; when this cannot be done, in the horse, the jaw may be trephined and tooth punched out.

In dogs, freely moisten gum with tannin dissolved in alcohol and ether, or with morphine solution; or after scraping and disinfection, stop carious tooth with dental amalgam.

Tread.

A bruise of the horse's coronet, usually caused by the opposite foot, or by another horse. See BRoISE.

Scarification, medicated fomentation, antiseptic dressings.

If sloughing occur, apply antiseptics and bandages.

\section{TUBERCULOSIS.}

A specific disease caused by the bacillus tuberculosis, which develops irritation either directly or by formation of toxins, or in both ways.

The disease is distinctly contagious. The bacillus of one mammal multiplies in the bodies of other mammals; dogs have been infected 


\section{TUBERCULOSIS-continued.}

by the sputa of consumptive human patients ; the milk from tuberculous cows has infected calves and pigs, and probably children. It is doubtful if the bacillus of avian tuberculosis is a distinct species.

Tuberculosis occurs in man and all the domesticated animals ; cattle, swine, and poultry are most frequently affected; horses stand next; sheep and goats are not so susceptible ; dogs and cats still less so.

The tubercule may be (1) localised in particular organs, or (2) generalised, affecting several systems, their serous membranes and lymphatic glands.

Tuberculosis of the lungs. Pulmonary consumption is the prominent form in more than two-thirds of the cattle, dogs, and cats attacked.

Tubercule of the mesenteric glands, the spleen, and liver, is the form most common in horses, pigs, and poultry.

Tuberculosis of the mammary gland of the cov begins as a slowly developing diffused induration of one quarter of the udder; the milk becomes thin or watery and of a blue tint.

Tubercular arthritis, common in human patients, is not so frequent in the lower animals, but sometimes attacks the spinal, knee, hock, digital and other joints of cattle and horses.

Preventive more important than curative treatment. Test with tuberculin.

Affected animals isolated. No suspects used for breeding.

Milk from cows with tuberculous udders or generalised tuberculosis frequently contains the bacillus, and is more likely than the flesh to communicate disease.

Milk from affected subjects should not be used; that from suspects should be boiled before use.

Carcases showing generalised tubercule to be destroyed.

Diseased carcases or organs burned or deeply buried.

Animals in earlier stages stalled by themselves in airy, comfortable quarters, and fattened.

Antiseptic inhalations relieve bronchial irritation.

Tonics, alcoholic stimulants, iodine, creosote, and antiseptics relieve some of the symptoms.

Tympanites. See Hoven and Flatulent Colic.

Udder, J.mplammation of. See Mammitis.

\section{ULCERS.}

A breach of continuity with destruction of tissue, leaving an indented wound, dry or suppurating. Ulcers are classified as Simple, Weak, Indolent, Inflamed, and Specific.

Simple ulcers discharge thick pus, and if kept aseptic and free of irritation, they heal quickly by granulation.

Weak or cedematous ulcers require mild astringent dressings. Dust with calomel. Paint with solution silver nitrate (5 per cent.).

A bandage, where it can be used, affords equable healthy pressure.

Easily digestible nourishing diet; the patient should have suitable exercise.

Indolent or callous ulcers should have their hard edges excised or cauterised, and then be dressed with antiseptics, iodine, calomel, or iodoform.

Pot. iodide sometimes benefits. A purgative generally useful.

Inflamed ulcers require removal of any irritant, with medicated fomentation, and boric acid or mild astringent.

Where there is necrosis use antiseptics freely.

Purgative, and digestible cooling diet.

Specific, occurring in tuberculosis, glanders, actinomycosis, and cancer; also caused by necrosis bacillus. 


\section{INDEX OF DISEASES AND REMEDIES}

ULCERS-continued.

Pare or scrape away unhealthy tissue.

Apply iodoform, iodine, carbolic, and other antiseptics; actual cautery.

Glanders ulcers should not be treated.

Urinary Deposits. See Calculi.

URTICARIA.

Surfeit ; Nettle-rash. An evanescent erythema of the skin, occasionally of the mucous membranes, with circumscribed, itching, serous elevations ; usually appearing and disappearing with equal rapidity. Not infrequent in horses, dogs, and pigs; rare in cattle or sheep.

Purgative medicine; damp the skin repeatedly with an alkaline solution.

When rash persists or recurs give antacids ; attend to diet and cleanliness of clothing, avoid washing with cold water.

URETHRITIS.

Catarrhal inflammation of the urethra; discharge containing bacteria. Prepuce and surface of penis may be affected. Not unconmon in dogs and bulls ; contagious.

Copper sulphate, zinc chloride, or silver nitrate injected in dilute warm solution, 2 to 5 grains to an ounce of water, sol. resorcin 2 to 3 per cent.

Thorough cleanliness. Diluents; alkalies.

Copaiba and eucalyptus are useful anodynes and antiseptics.

Prevent occlusion by cautious introduction of clean catheter.

Persistent obstruction from inflammation or gravel in male sometimes requires perineal opening. See Urinary CaLcUli.

Uterus, Inflammation of. See Metritis.

VAGINITIS.

Infective inflammation of mucous membrane of vagina, sometimes extending to uterus; a frequent cause of sterility and abortion; contagious; occurs in all females.

Isolate affected animals, disinfect cowsheds, loose boxes, and attendants.

Irrigate vagina with antiseptic solutions, corrosive sublimate, protargol, lysoform, or lysol.

Disinfect sheath of bull that has served affected cow ; repeat disinfection after each service, using warm solution of lysol (two per cent).

Cows affected with metritis should be fattened; cows that have recovered should be disinfected before service; sol. soda bicarb.

VARIOLA Ovina.

Sheep-pox. A contagious, inoculable, eruptive fever, probably depending upon a microbe. The incubation stage four to seven days. Papules appear, passing into vesicles and pustules. The mortality ranges from 10 to 20 per cent., but is greater in the confluent malignant form occasionally occurring. Animals attacked are subsequently immune. Goats, swine, and dogs have been infected.

Infected and in-contact subjects should be slaughtered, and the measures adopted in other contagious diseases rigorously carried out.

As the disease runs a definite course, treatment consists in good nursing and guarding against complications.

Inoculation of healthy sheep with attenuated virus is not desirable, for the attack produced is distinctly contagious, rather severe, and the mortality averages 2 per cent.

Variola vaccina, or cow-pox, results from accidental retro-vaccination from recently vaccinated human patients, and sinilar eruptions in like manner occur in swine and occasionally in dogs.

$A$ variola equina may be similarly produced, but the conditions described as horse-pox are not variolous, and appear to be pustular stomatitis and eruptions on the genital mucous membrane (Fröhner, Trasbot). 


\section{Vertigo in Horse. See Megrims.}

\section{VILLITIS.}

Coronitis. Inflammation of the coronary substance in horses. Occurs from standing in cold water or snow. Prevails in America among horses grazed on alkaline marshes, where secretion of crust checked or arrested, and hoof sometimes gradually separates.

Remove shoes; antiseptic fomentations and a laxative abate inflam. mation.

Stimulate coronet with a mild blister so soon as inflammation removed.

Where even one hoof is undergoing separation the horse for months requires attention.

Volvulus.

Ileus. Twist of bowel. Affects small, occasionally large, intestine of horse.

Cannot be rectified by medical treatment, but laparotomy, if undertaken early, may be successful. Rectal exploration useful in twisted large colon.

Warbles in CatTle.

Cystlike swellings on the back due to the presence of the larvæ of the Hypoderma bovis or warble-fly.

To prevent, before turning cattle out, smear their backs once or twice during summer, with Stockholm tar. The larvæ can be removed from the skin by squeezing the swellings. Similar warbles occur sometimes in horses at pasture.

WARTS.

Verrucæ. Excrescences on the skin formed by hypertrophy of the papillæ and epidermis. Occur in all veterinary subjects: most common in young animals.

Remove by excision, torsion or ligature.

Those about the penis liable to reappear unless their site is cauterised.

Acetic, nitric, and chromic acids, and silver nitrate, destroy warts.

Weed. See Lymphangitis.

Whistuing in Horses. See Roaring.

It is a higher-pitched sound than roaring, and is manifested chiefly in the lighter breeds.

WIND-GaLLS.

Distended synovial bursæ; articular and tendinous.

Equable pressure by flannel or wash-leather bandages; bandages wetted with white lotion.

Rest, hand-rubbing, blisters or firing; aseptic aspiration. Recurrence is common.

Withers, Fistulous. See Fistula and Abscess.

WorMs.

Worms. Vermes. The Entozoa most Frequently found infesting the Domestic Animals:-

Round and Thread Worms. Nematoda.

Ascaris equorum, intestine and stomach of horses.

$A s$. vitulorum or calf ascarid, intestine of calves.

As. suis, intestine of swine.

As. marginata and As. mystax, intestines of dogs and cats.

Heterakis papillosa and other species, intestines of fowls and pigeons.

Trichinella spiralis, in immature form, occurring in pork or bacon, which when eaten causes trichiniasis in man and animals. 


\section{Worms-continued.}

Oxyuris compar, intestine of the cat.

Oxyuris curvula or pin-worm, rectum of horses.

Oxyuris mastigodes, large intestine of horses.

E sophagostomum inflatum, large intestine of cattle.

Trichocephalus affinis or whipworm, large intestine of ruminants.

Trichocephalus depressiusculus, cæcum of the dog.

Sclerostomum equinum or palisade worm, intestine and blood-vessels of horses.

Scl. tetracanthum or four-spined sclerostome, large intestine of horses.

Scl. hypostomum, intestine of ruminants.

Spiroptera megastoma, wall of horse's stomach.

Sp. strongylina, stomach of pig.

$S p$. sanguinolenta, stomach and blood-vessels (aorta) of the dog.

Sp. microstoma, stomach of the horse and ass.

Strongylus Axei, stomach of the ass.

St. filicollis, St. vicarius, and St. convolutus, fourth stomach and intestine of cattle, sheep, and goats.

$S t$. contortus, St. cervicornis, St. gracilis, and other species, in abomasum of ruminants ; St. gracilis also occurs in stomach of horse and pig.

Ollulanus triciespis, stomach of the cat.

Strongylus strigosus, stomach of the rabbit and hare.

$S t$. ventricosus, small intestine of cattle, sheep, and deer.

Strongyloides longus, intestine of sheep.

Strongyloides suis, intestine of swine.

Echinorhynchus gigas, small intestine of swine.

Esophagostomum dentatum, cæcum and colon of swine.

Trichocephalus crenatus, intestine of swine.

Anchylostomum trigonocephalum and Anch. stenocephalum, intestine of the dog, cat, and fox.

Strongylus micrurus or pointed-tailed threadworm, air-passages of young cattle causing husk or hoose.

St. filaria, bronchi of sheep, goats, deer, and cattle.

St. rufescens, bronchi and lungs of sheep and goats.

St. pulmonaris, bronchi of calves.

St. Arnfieldi, bronchi of horse and ass.

St. paradoxus, bronchi of swine.

St. pusillus, bronchi of cat.

St. commutatus, causes verminous bronchitis in rabbit and hare.

Syngamus trachealis, air-passages of poultry, causing 'gapes.'

Filaria immitis, heart and pulmonary artery of dog.

Strongylus vasorum, heart and blood-vessels of dog.

Filaria Medinensis or Guinea-worm, subcutaneous connective tissue of man, dog, ox, and horse.

Filaria hamorrhagica, subcutaneous connective tissue of horses, causing hæmorrhage from skin.

In horses and cattle, aloes, oil of turpentine, bitters, creolin, lysol.

Ferric chloride solution, copper sulphate, arsenic, salt in manger.

For dogs and cats, santonin, with extract of male fern, repeated at intervals of two days, and second dose followed by laxative.

For worms in rectum, enemas of weak vinegar, salt solution, infusion of quassia, or creolin solution.

Strongyli infested horses and sheep are fasted, and receive a cathartic conjoined with oil of turpentine, or thymol.

For the $S$. contortus in sheep, give solution of lysol (1 per cent.).

For dogs and cats santonin and male fern extract, or areca nut and linseed oil.

For the $S$. micrurus in air-passages, turpentine in drench, or intra. tracheally for two or three consecutive days.

Sulphurous or chlorine inhalations. Liberal concentrated dietary.

Remove from rough old pastures to seeds, or to closely-cropped, recentlymown dry grass; house calves at night. 


\section{WorMs-continued.}

In poultry, the tip of a partially-stripped feather, introduced into the fauces and twisted round a few times, usually withdraws some of the parasites.

The feather, moistened with oil of turpentine or petroleum and similarly introduced, destroys many worms. Fumigation with tobacco.

Fluke-worms. Trematoda.

Distomum hepaticum, causing liver-rot in sheep, rabbits, and hares, and occasionally cattle.

Furnish affected sheep with concentrated dry food.

Common salt and ferrous sulphate dissolved in water, given daily, mixed with bran or crushed grain.

To prevent affected sheep losing condition and disseminating the disease slaughter as soon as possible.

Keep sound sheep from pastures on which affected sheep have grazed, from low-lying wet land, or from grazings with stagnant poolssituations which nurture the fluke embryo, and the fresh-water snail which constitutes the intermediary host.

Other species of distomata occur in the liver and organs of sheep and other animals.

\section{Tapeworms. Taniz. Cestoda.}

Most animals infested with one or more species, in their mature stage inhabiting the intestine.

Anoplocephala (tania):-perfoliata, plicata, mamillana, in horse.

Moniezia (tania) :-expansa, alba, planissima, in cattle.

Moniezia (tonia):-expansa, alba, planissima, trigonophora. Benedeni, Neumanni; Stilesia (tonia) centripunctata, and Stilesia globipunctata; Thysanosomum actinioides and Th. Giardi, in sheep.

Tanioe:-serrata, marginata, Krabbei, cœnurus, serialis, and echinococcus ; Dipylidium caninum ( $T$. cucumerina), Mesocestoides lineatus ; and Bothriocephalus latus and B. cordatus, in the dog.

Davainea cesticillus ( $T$. infundibuliformis), and seven other tæniæ, in poultry.

The measles of pork, beef, and mutton are tæniæ larvæ, found chiefly in the muscles. Each produces its particulate tapeworm.

The ccenurus cerebralis, the hydatid causing sturdy or gid in sheep, is the encysted larva of Tania conurus, and attains its mature form in the intestines of the dog, which in turn disseminates the ova which undergo further development in the brain of the sheep.

Patients should be fasted twenty-four hours before they receive the tæniacide.

Horses and cattle, eating tolerably clean vegetable food, are not so frequently infested as the dog or pig, are given oil of turpentine and male fern extract in milk for two or three consecutive days, and after the last dose a cathartic.

Dogs swallow the larval forms of ten different tæniæ. Treated with areca nut ten to sixty grains (according to size), aloes, or male fern extract ten to fifteen minims, given with oil, syrup of buckthorn, or mucilage ; dose repeated in two days.

For poultry, areca nut five to thirty grains, repeated in two or three days, followed by a dose of oil.

Tæniasis prevented by isolating and curing infested subjects, burning their excreta, and preventing animals eating uncooked viscera of sheep, hogs, rabbits, or other animals in which tænia larvæ are liable to occur.

\section{Woonds.}

A wound is defined as a breach of continuity of any part of the body. Wounds may be open or subcutaneous. The open are classified as Incised, Punctured, Lacerated, Contused, and Poisoned. 
Wounds-continued.

Every variety requires attention to the following conditions :-

(1) Absolute asepsis.

(2) Perfect arrest of hæmorrhage.

(3) Accurate apposition of severed parts.

(4) Physiological and mechanical rest.

To secure asepsis-

Foreign bodies, grit, sand, etc., are removed from accidental wounds.

Adjacent skin is shaved or closely clipped.

In wounds of soft parts, the edges, if very dirty or damaged, are carefully trimmed.

The wound and adjacent parts are thoroughly and freely washed with an effectual germicide, such as-Carbolic acid, 1 part to 20 of boiled water.

Corrosive sublimate 1 part, common salt $S$ parts, water 1000 to 2000 .

Zinc chloride 1 part, water 40 to 80 parts ; hydrogen peroxide.

When wounds are inflicted several of these germicides should be used in succession.

Wounds may be dusted with a dry dressing of iodoform, tannoform, zinc oxide, boric acid, or chinosol; special cases with dried tetanus antitoxin.

When an aseptic state has been secured, less powerful agents suffice to maintain it, such as solutions of boric, salicylic, or sulphurous acid, or chlorinated soda.

Large, lacerated, contused wounds are provided with drainage, a piece of rubber tubing being introduced into the deepest part, and a dependent opening secured.

Introduction of fermentable, putrefactive, or infective material prevented by covering with several folds of antiseptic lint, cotton wool, or oakum.

Antiseptic precautions to be further observed as to instruments, hands of operator, etc.

Hæmorrhage is arrested temporarily by application of a tourniquet, permanently by ligature, torsion, pressure, water at a temperature of about $120^{\circ}$ Fahr., styptics or cautery.

Accurate apposition of severed parts effected by careful suturing with aseptic catgut, horse-hair, silk, silver wire, etc.

Physiological rest is secured by analgesics; belladonna liniment and tincture equal parts, diluted with 60 to 100 water. Laudanum diluted ; morphine hypodermically.

Anatomical rest obtained by equable pressure of dressings and bandages, support of splints or adhesives, and slinging of the larger animals.

Wounds properly dressed and going on satisfactorily should be disturbed as little as possible, except for cleansing and replacing external dressings; there need be no hurry in removing aseptic sutures.

If a wound become inflamed or painful, the dressings must be removed, sutures cut out, clots or other irritants removed, the wound irrigated or syringed with an antiseptic, and redressed as before.

Opium and belladonna used, with fomentations and antiseptics, when there is much pain.

Excessive granulation checked by pressure, astringents, occasional use of caustic, and exposure to the air.

Attention to state of bowels, cooling digestible diet, and healthful surroundings essential to successful treatment. 


\section{INDEX OF MEDICINES}

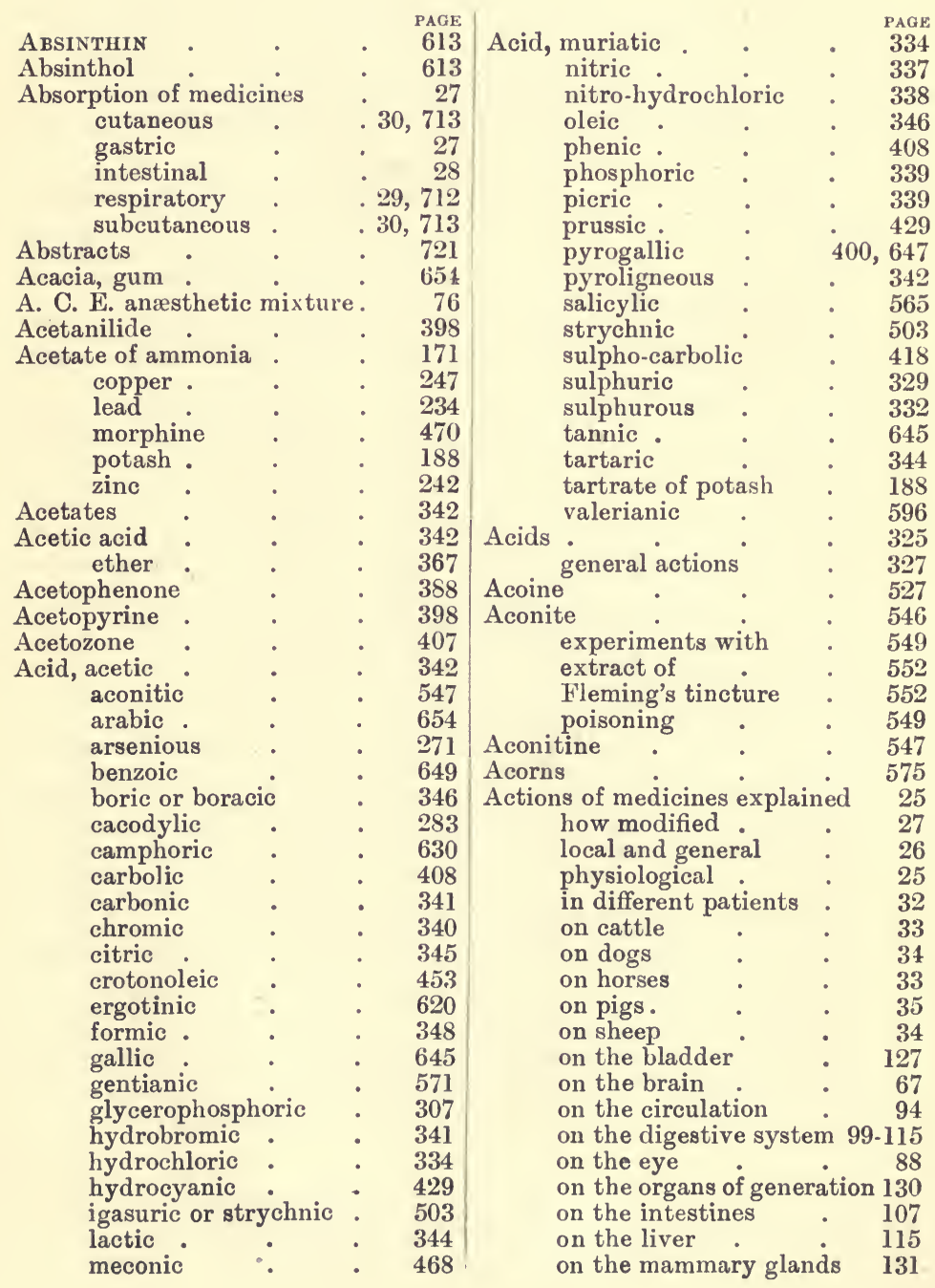




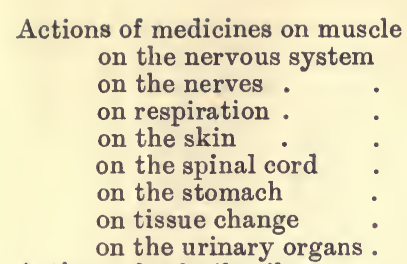

352

352

354

355

353

352

353

353

353

353

359

352

354

173,174

701

38

582

437

437

438

440

438

438

438

445

445

444

\begin{tabular}{|c|c|c|c|c|}
\hline GE & & & & FAUES \\
\hline 63 & Aloin . & & & 445 \\
\hline 66 & experiments $\mathrm{w}$ & vith, & 446 & 447 \\
\hline 85 & Alphol & & & 404 \\
\hline $\begin{array}{r}89 \\
121\end{array}$ & $\begin{array}{l}\text { Alteratives, } 138,181 \\
296,315,319,324 .\end{array}$ & 1,201 & 247, & 278 , \\
\hline 82 & Althæa radix. & & - & 652 \\
\hline 101 & Alum (ammonia) & . & & 22 \\
\hline 133 & (potash) & & . & 22 \\
\hline 124 & Alumen exsiccatum & - & . & 22 \\
\hline 589 & Aluminium chloride & - & & 22 \\
\hline 44 & sulphate & - & 220 & 22 \\
\hline 251 & Ammonia alum & . & & 220 \\
\hline 58 & Ammoni & . & & 587 \\
\hline 3 & Ammonia & & & 301 \\
\hline 679 & are of & inine & & 56 \\
\hline 681 & Ammoniu & mpoun & & 16 \\
\hline 681 & ac & . & & 17 \\
\hline 729 & b) & . & & 172 \\
\hline 70 & ate & - & & 16 \\
\hline 704 & de & . & & 1 \\
\hline 68 & $\operatorname{liq}$ & • & - & 16 \\
\hline 70 & of & • & - & 167 \\
\hline 247 & gdal & • & & 429 \\
\hline 247 & Amylene & - & & 388 \\
\hline 363 & Amyl-nit & - & & 389 \\
\hline 36 & ol . & . & & 35 \\
\hline 379 & Amylofor & & & 392 \\
\hline 36 & Amyloiodoform & & & 39 \\
\hline 363 & Amylum, wheat-fl & & & 662 \\
\hline 368 & Analgesics (Anodyne & & & 341 . \\
\hline 35 & $379,381,385,395$ & & & \\
\hline 225 & $405,480,493,500$ & , 517, & & \\
\hline & & & & \\
\hline
\end{tabular}

$524,534,551,557,570$.

Anaphrodisiacs

Anaphylaxis .

Anæsthesia $\quad 76,78,79$ intraspinal . $\quad 82$ narcosis $\quad . \quad 80$

Anæsthetics, . $\quad$. 74, 376, 380 administration $\quad 79$ local, $75,366,426,526,527$, $528,529$.

Anethol $\quad$. $\quad 590$

Anhydrotics : . $\quad$. 123

Anise, seed . $\quad$ • $\quad$. 589

oil • • 590

Anodynes (see Analgesics) 71, 309, $341,379,381,385,401,405,480$, 493, 522, 534.

Antacids, 102, 168, 170, 179, 191, 205, 207, 212, 225, 291.

Anthelmintics (see Vermicides) 117, $245,258,270,279,296,339,349$, $396,425,443,460,600,604$.

Anthemidis flores . $\quad 595$

Antidotes . . 144, 145, 146

Antifebrin . $\quad$. $\quad 398$

Antilithics : $\quad$ - 128

Antimony and its compounds 262 $\begin{array}{lll}\text { chloride } \quad \text {. } & 264\end{array}$ 
Antimony, oxide sulplide $\quad$. $\quad 263$ tartarised . . 265 wine of $\quad 271$

Antiparasitics (see Insecticides), 192, $194,223,272,321,322,333,396$, $402,403,409,416,421,424,648,651$.

Antipathy

Antiperiodics .

Antipyretics, $141,142,171,181,216$, $344,345,396,398,404,405,406$, 407,650 .

Antipyretics, general actions, $\quad 141$

Antipyrine - • 396

Antiseptics, 42, 44, 46, 160, 185, 192, $194,197,203,209,221,223,224$, $240,241,251,252,262,279,298$, $302,312,315,333,336,347,360$, $386,388,391,393,395,399,400$, $401,403,405,407,409,413,418$, $423,432,529,562,568,592,609$, $635,637,653$.

Antiseptics, general actions 42,46 intestinal, $213,225,226,345$, 393, 400, 401, 402, 403, 424, $425,418,419,648$.

Antiseptic conipresses surgery

Antisialics 708

46,47 100

Antispasmodics, 72, 360, 365, 369, 379 , 380, 383, 385, 389, 487, 500, 521, 5S7, 592, 594, 596.

Antistreptococcic serum

Antitoxins

698

Antitoxin treatment .

Antizymotics .

Aperients (see Laxatives)

5,6

A phrodisiacs 130,617,619

Apomorphine hydrochloride .

Apothecaries' weights, etc.

Aqua .

$$
\text { camphoræ }
$$

chloroformi

fortis regia .

Arabin

\section{Arbutin}

Areca catechu

Areca-nut

Arecoline hydrobromide

Argentum colloidale .

Argenti nitras

Argentol

Argol or crude tartar

Argonin

Aristol

Arnica

Arnicin

Aromatic carbon compounds. oils

470

734

152

633

379

337

338

654

545

611

611

611

251

248

251

188

252

428

588

588

394

589
Arrhenal

PAGF

Arrowroot

Arsenic and its compounds compounds

antidotes

poisoning

Arsenical sheep-dips . solutions

Arsenious acid

Artemisia absinthium maritima

Asafœtida

Ascarides

Aseptic suture twist .

Aseptol

Aspidium filix-mas

Aspirin

Astringents, 60,205, 207, 209, 221, $223,225,232,235,238,239,242$, $245,247,249,262,330,643,645$, 647.

Atoxyl

Atropa belladonna

Atropine

284

486

Autumn crocus

Avoirdupois weight

Axunge or hog's lard .

Bacteria

Bacterial invasion products

486,496

627

734

679

$.3,9,14$

15

$5,18,19$

Bactericides (see also Antiseptics and Gernicides), 42, 46, 48, 251, 252, 283, 284, 402, 407, 409, 422, 426, 653.

Bacteriology .

Balls or boluses $\quad . \quad 703$

Ball mass . $\quad$. $\quad 703$

Balsam, Canada $\quad . \quad 599$

\section{Friar's.}

of Gilead

of Peru.

of Tolu .

650

599

651

Balsams . 651

Balsamodendron myrrha . 580

Barbados aloes . 4 437, 442 tar . . . 436

Barbaloin . . . . 446

Barium chloride $\quad . \quad 218$

Bark, cinchona . $\quad$. 558

574

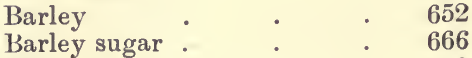

Barm . . . $\quad 653$

Bassorin . . $\quad .655$

Baths . . . . 704

cold . . . . 704

hot . . . 705

medicated . $\quad 705$

sponge . $\quad$. 706

vapour or Turkish $\quad 705$ 


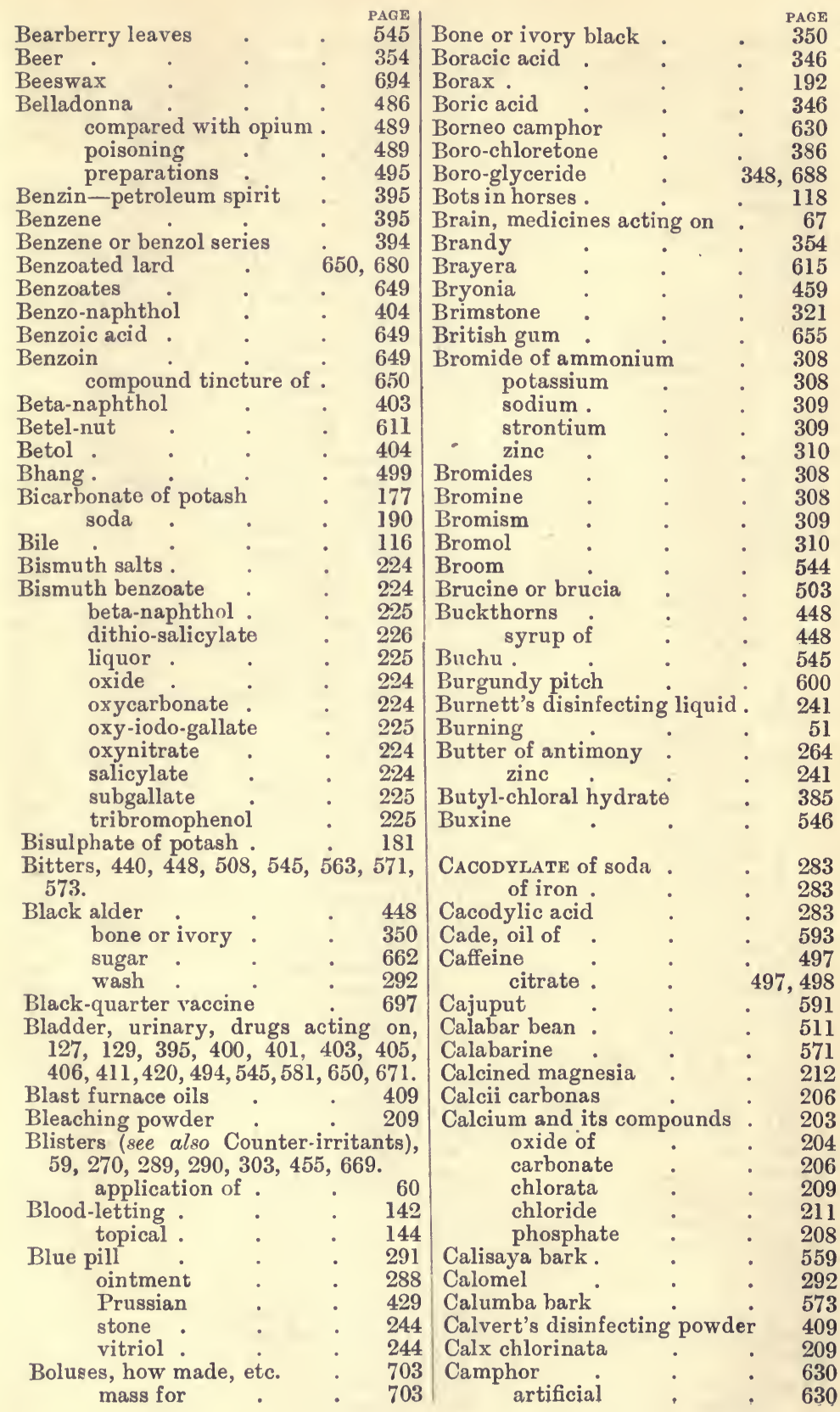


Camphor, Borneo , $\quad 630$ compound liniment oil monobromata . spirit

Camphorated oil

Camphoric acid

Camphors

Canada balsam

Cane sugar

Cannabin

Cannabis indica

Cantharides.

liniments of
ointments of
plasters of
tinctures of
vinegars of

Cantharidin

Cantharis vesicatoria.

Capsaïcin

Capsicum

Caraway

Carbo animalis

hydrates

ligni

Carbolic acid . tow

Carbon wool

Carbonate of ammonia iron

lead

of lime .

magnesia

potash .

soda

zinc

Carbonic acid .

Carbo sapol.

Cardamoms

Cardiac sedatives stimulants tonics, $97,540,543,544$.

48

418

350

349

169

256

232

206

212

177

190

239

341

418

593

98

96

Carminatives (see also Stomachics), $113,207,364,369,563,573,577$, $581,583,585,586,587,590,593$, $596,603$.

Carron oil

Cascara sagrada

evacuant

Cascarilla bark

Cascarillin

Castor oil seeds .

Cataplasmata (poultices)

Catechin

Catechu pale . black.
Catechu infusion

PAGR tincture

644

644

Cathartics, 107, 181, 189, 193, 199, $212,216,218,291,295,440,448$, $449,453,456,457,458,460,463$, 516,660 .

Cathartics for cattle, and sheep, $111,193,199,216,455,458$, 660. dogs, 111, 291, 451, 448, 456, $459,463,465,660$. 458. horses, 110, 193, 199, 216, 455,

pigs $112,442,451,459$.

Caustic ammonia

166

lunar.

248

potash . . . 175

soda . . $\quad 190$

Caustics, 57, 166, 177, 190, 221, 223, $241,247,249,262,265,279,303$, $330,337,340,391$.

Cautery, actual

58

$\begin{array}{lll}\text { Cayenne pepper } \quad \text {. } & \text {. } & \mathbf{5 8 2}\end{array}$

$\begin{array}{llll}\text { Cera alba } & \text {. } & \text {. } & 694\end{array}$

$\begin{array}{rr}\text { flava } & \\ \text { Cerates } & 694\end{array}$

Cerebral sedatives $\quad$. $\quad 69$

$$
\text { stimulants } \quad . \quad 69
$$

Cerevisiæ torula or yeast $\quad$. $\quad 653$

$\begin{array}{llll}\text { Cetaceum } & \text {. } & \text {. } & 693\end{array}$

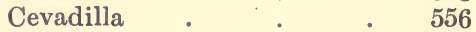

Chalk . $\quad . \quad 206$

207

Chamomile tlowers . $\quad . \quad 595$

Charcoal, animal . $\quad$. 350

wood . . 350

728

Chenopodium oil $\quad . \quad 5614$

Chillies $\quad 582$

Chinaseptol . . . 405

Chinese twist. $\quad . \quad 418$

$\begin{array}{llll}\text { Chinosol } & \text {. } & \text {. } & 407\end{array}$

Chiretta $\quad . \quad$. $\quad . \quad 574$

Chloralamide . $\quad$. $\quad 385$

Chloral hydrate $\quad$. $\quad 76,380$

Chloralum . . . 223

Chlorate of potash . $\quad$. 184

Chloretone . . . 385

$\begin{array}{lll}\text { Chloric ether . } \quad . \quad & 279\end{array}$

Chloride of ammonium $\quad$. 165

antimony . . $\quad 264$

barium. . . 218

lime . . . 211

sodium . $\quad . \quad 196$

zinc . . . 241

449 Chlorides of mercury . $\quad 293,298$

729 Chlorinated lime . . 209

643 soda . . . 202

642 Chlorine $\quad . \quad 5 \quad$. $\quad 310$

642 Chlorodyne : $\quad . \quad$. 380 
Chloroform PAGE

compared with ether precautions in using . spirits of

Cholagogues (see Hepatic stimulants)

$109,115,116,181,337,440,457$, $460,464$.

Chondrin

Chrysarobin

Chrysophan

Chrysophanic acid

Chromic acid .

Churrus

Cinchona

\section{alkaloids}

grey or pale red

tinctures

yellow.

Cinchonidine .

Cinchonine

Cinchonism

Cinnabar

Cinnamon oil

371

376

375

379

685

648

648

648

340

499

558

560

559

559

565

559

561

561

563

285

585

586
Circulation, actions of medicines on 94

Cocaine hydrochloride substitutes

Citrate of iron and quinine

Citric acid

Citrine ointment

Classifications of medicines .

Claviceps purpurea

Climate modifying medicinal action

Clysters or enemata .

Coal tar

Codeine

Cod-liver oil :

Colchicine for feeding purposes

Colchicum autumnale

Cold applications

a natural tonic. sponging

Collargolum

Collodion contractile . flexible.

Collunaria styptic.

Collutoria

Collyria

Colocynth

Colophony-resin

Common mass .

$$
\text { salt }
$$

Compound powder of catechu Confections

523

527, 528

256

345

303

21, 22

619

36

717

436

470

677

677

627

627

157

157

706

251

367

368

368

724

724

724

459

608

668

196

644

732

Conine hydrobromide

Conium maculatum 517
Constipation

PAGE

Continuous irrigation

Convolvulus Scammonia

Copper and its salts.

Copper acetate

iodide

poisoning

resinate

sulphate

- 246

Coriander . . $\quad 593$

Corn flour, Oswego . $\quad$. $\quad 663$

Cornutine . $\quad . \quad 620$

Corrosive sublimate . $\quad 298$

Correctives . . . 701

Corrosives, 175, 248, 271, 298, 325, $334,337$.

antidotes for . . 299

poisoning by . . 285

Counter-irritation : $\quad 55$

Counter-irritants (blisters), 54, 58, $168,270,289,290,303,455,669$

Cough mixtures, 93, 94, 171, 383, $482,541,668$.

Cough, treatment of . $\quad$. 93

Cramp. . . . 73

$\begin{array}{lll}\text { Cream of tartar } \quad \text {. } & 188\end{array}$

Creolin . . . 422

Creosote : $\quad: \quad 419$

Cresol . . . . . 422

Creta præparata . . 206

Croton. . . . 452

453

oil $\quad . \quad$. $\quad . \quad 452$

seeds . . . 452

Croton-oleic acid . . $\quad 452$

Cubebs pepper $\quad . \quad 582$

Cumulative medicines $\quad$. $\quad 31$

$\begin{array}{llll}\text { Cuprea barks . } \quad \text { • } & \text {. } & 559\end{array}$

Cupri acetas . $\quad$. $\quad . \quad 247$

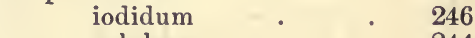

sulphas $\quad \cdot \quad$ • 244

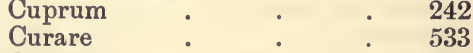

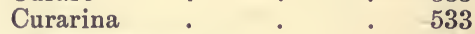

$\begin{array}{lll}\text { Curative systems } \quad . \quad & 38\end{array}$

Cusso . . . . 615

Cyllin . . . . $\quad 425$

Damiana . . $\quad 619$

Danda oil or Assu juice : 449

Datura stramonium . . 522

Deadly nightshade . . . 486

Death during anæsthesia - 78

Decoctions . . . 709

Delphinium staphisagria . 614

Demulcents, 62, 652, 653, 655, 659, $665,668,680,694$.

518, 521 Deodorisors, 43, 53, 187, 194, 210, $311,333,340,350,416,423,636$. 
Dermatol

PAGF

Desiccants, 207, 221, 223, 232, 237, $251,293,350,392,394,418,427$, $428,429,432,570,693$.

Detergents

Dextrin

Dextrose-grape sugar

Diachylon plaster

Diaphoretics .

Diarrhœea

Dieting

Digitalein

Digitalin varieties

Digitalis experiments with

Digitonin

Digitoxin

Diluents

Diphtheria antitoxin .

Diseases treated by inoculation

Diseases modify actions of medicines.

Disinfectants, $43,45,49,5 \mathrm{l}, 187$, $194,210,311,333,340,350,407$, $416,423,581,592,636$.

Dithion

Diuretic mass

570

Diuretics, 124, 165, 171, 179, 183, $185,188,189,192,297,319,370$, $398,498,541,543,544,545,593$, $600,608,628,650$.

Diuretin

Dogs, emetics for, $10 \dot{7}, 237,240,245$, 247, 297, 470, 555, 628 . vermicides for.

Donovan's solution

119

Doses of medicines .

Douches

Dover's powder

Drastic cathartics

Drenches, draughts, or drinks

Drenching horn

Dried alum

Drying milch cows

Duboisine

Duotal

EAsTon's syrup
Ecbolics
Elaterium
Elective affinity
Electricity :
Electrotherapy
Electuaries :
Elemi resin :
Elimination :
Elixir phosphori
Elixirs
Embrocations.

109

716

718

223

132

522

422
Emetics, 103, 170, 237, 239, 240, 245, $247,266,297,460,470,555,597$, 628.

Emetine

556

Emollients, 62, 656, 660, 661, 665, 694.

Emplastra . . . $\quad 728$

Emulsions . . . 724

Endermic application of medicines

713

Enemata . . . 717

Enzymes . $\quad . \quad$. 11, 43

Epsom salt . . . 215

Ergot . . . . 619

Ergotin . . . 619

Ergotinic acid . $\quad . \quad 620$

Errhines . . 91

Erythroxylon coca . . $\quad 523$

Escharotics (see Caustics) . $\quad 57$

Eserine . . 511

Eseridine . . 517

Essential oils . . . $\quad 725$

Ether . . . . 363

acetic . . $\quad 367$

$\begin{array}{llll}\text { chloric . } & \text {. } & \text {. } & 279\end{array}$

nitrous . $\quad . \quad 368$

$\begin{array}{lll}\text { sulphuric } \quad . \quad & 0 & 363\end{array}$

Ethereal oil . . $\quad 367$

Ethyl alcohol . $\quad$. $\quad 352$

$\begin{array}{lll}\text { bromide } & \text {. } & 353\end{array}$

chloride . $\quad 353$

nitrite . $\quad 368$

Eucaine . . $\quad 527$

Eucalyptus globulus . $\quad$. 591

Eucalyptol . . . 591

Eulyptol . $\quad$. $\quad$. 592

Euonymin . , . . 456

Europhen . $\quad$. $\quad .428$

Evacuants . $\quad$. 157

Excipients, 661, 662, 665, 667, 681, $690,703$.

Excitants-stimulants $\quad$. 68

Exalgin . . . 405

Exhilarants . $\quad$ • $\quad 69$

Expectorants, 91, 168, 170, 171, 181, $265,319,519,532,544,545,555$, $581,587,600,650,651$.

Extract, Goulard's . • 234

Extract of belladonna - 495

ergot . . 624

Indian hemp . 502

male fern . $\quad 616$

nux-vomica $\quad$ $\quad 510$

opium . . 485

Extracts . . . 720

Eye, remedies acting on, 88, 222, $236,245,496,516,533,588$.

sacchari 
Fats and fixed oils PAGE

Febrifuges (see Antipyretics) 133, 139, $140,141,181,216,344,345,396$, 398, 404, 406, 407, 562, 567.

Fennel

Fenugreek

Ferments organised unorganised

Ferri carbonas carbonas saccharatus. iodidum peroxidum humidum . pernitratis sulphas

Ferric perchloride

Ferrous salts .

\section{Ferrum} sulphate

Ferrum redactum

Ferratin

Fever medicines (see Antipyretics)

Fibrolysin

Flax, common

Flexible, collodion - $\quad$ - $\quad 368$

Flies, blistering $\quad$. $\quad$. $\quad 669$

Flowers of sulphur . $\quad 321$

Fluid extracts

Fomentations .

Fool's parsley .

Formaldehyde

Formalin

Formates

Formol

Fowler's solution

Foxglove

Frankincense .

Freezing mixtures

Friar's balsam

Frick's wound gelatin

\section{Friction}

Fuller's earth.

Fumigations.

\section{Galactagogues.}

\section{Galbanum}

Gallic acid

Galls or Gallæ

Galvanism

Gamboge

Gargles and sprays

Gastric stimulants or tonics, 103 $240,252,256,260,277,306,330$, $336,337,339,340,349,358,360$, 448, 509, 556, 571, 573, 632 .

\begin{tabular}{|c|c|c|}
\hline sedatives & - & \\
\hline Gelatin & . & \\
\hline solutions & - & \\
\hline Gelsemine & . & . \\
\hline
\end{tabular}

Gentian
593

593

11

256

259

260

261

257

260

252

257

252

256

142

580

57

321
720

707

509

391

391

348

391

283

534

599

157

650

686

56

223

722

132

587

645

644

86

457

93

03,
Gentian infusion

PAGK

573

tincture

573

Gentiana lutea

570

570

Germicides, $4 \dot{3}, 160,251,252,283$, $284,300,333,345,401,402,404$, $407,409,414,418,422,425,426$, $562,592,637,653$.

Ginger

584

$\begin{array}{llll}\text { preserved } & \cdot & \cdot & 584 \\ \text { tincture } & . & \cdot & 585 \\ \text { rin } & . & . & 585 \\ . & & & 354\end{array}$

$\begin{array}{llll}\text { preserved } & . & & 584 \\ \text { tincture } & . & . & 585 \\ \text { rin } & . & . & 585 \\ . & . & . & 354\end{array}$

$\begin{array}{cccc}\text { preserved } & \text {. } & \text {. } & 584 \\ \text { tincture } & \text {. } & \text {. } & 585 \\ \text { Gingerin } & \text {. } & \text {. } & 585 \\ \text { Gins } & & \text {. } & 354\end{array}$

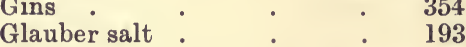

Globulins : $\quad: \quad 17$

Glonoin : : $\quad 391$

Glucose or dextrose : $\quad 667$

Glucosides . . . 703

Glue . • • $\quad 685$

plasters $\quad 0 \quad 685$

Glusidum (saccharin) $\quad$. 400

Glutol . . . 392

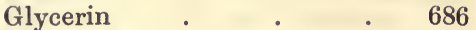

222

496

348

193

417

317

$\begin{array}{ll}\text {, pepsin } & \cdot \\ \text {, starch } & 695,687\end{array}$

$\begin{array}{ll}\text {, pepsin } & \cdot \\ \text {, starch } & 665,687\end{array}$

Glycerina 688

Glycerophosphoric acid : 307

Glycerophosphates . . 307

calcium . $\quad 307$

iron . $\quad 307$

quinine $\quad . \quad 307$

Glycoformal . . $\quad 392$

Glycyrrhizæ radix . $\quad$. 662

Glycyrrhizin . • • 662

Glyphocal emulsion : $\quad$. 307 with formates . $\quad$. $\quad 349$

wium

Goulard's extract . . 234

Grape sugar . . . 667

Green vitriol . • • 257

Gregory's mixture . . . 465

Grey powder . . . $\quad 291$

Guaiacol • . $\quad$ • 422

Guarana . $\quad$. $\quad$. 497

Gum acacia . . $\quad$. 654

587

Arabic . $\quad$. 654

Bassorin . . 655

benzoin . $\quad$. 649

British . . . 655

resins . . $\quad 731$

Senegal . $\quad .654$

tragacanth . $\quad 655$ 
Gun cotton $\quad \begin{array}{r}\text { PAGE } \\ \hline\end{array}$

Gunjah

HABIT modifies the action of medicines

Hæmatinics-blood tonics, 133,138 , $253,256,257,259,261$.

Hæmatoxylon (logwood)

Hæmoglobin .

Hæmostatics, 61, 62, 211, 234, 261, $331,351,407,480,586,603,624$, $682,685$.

Hard paraffin .

433

Hartshorn

Haschisch

166

499

Heart, medicines acting on the, 95,96, $97,98,540,543,544$.

Heat .

$$
\text { and moisture }
$$$$
\text { a stimulant }
$$

10

Hemlock succus .

Henbane or hyoscyamus

Heroin

Hoffmann's anodyne .

Hollands

Holocaine

Homatropine hydrobromide .

Homœopathy .

Honey or mel .

Horse, action of medicines on

Hordeum

Horses do not vomit .

Hot fomentation

Hot iron

How heat is lost

Huile de cade.

Hyderabad-chloroform experiments .

Hydragogue cathartics, 109, 452, 456, 457, 459 .

Hydrargyri bichloridum 298

chloridum .

cum creta . $\quad 291$

emplastrum . $\quad 290$

iodidum . $\quad$. 302

linimentum . $\quad . \quad 290$

nitratis

oleas

unguentum

oxidum flavum

nigrum .

rubrum .

perchloridum .

pilula

subchloridum .

unguentum

Hydrargyrum

Hydrobromic acid

291

292

288

285

341
Hydrochloric or muriatic acid

Hydrocyanic or prussic acid .

Hydrogen peroxide.

Hydronaphthol .

Hydrotherapy

Hydroquinone

Hygienic remedies

Hyoscine

Hyoscyamine .

Hyoscyamus niger

Hypnone

Hypnotics produce sleep, 69,38

produce sleep, 69, 381, $384,385,386,387,388,470$, 500, 523

Hypodermic injections, 27, 145, 284, $307,318,365,367,379,386,390$, $398,402,406,422,471,477,484$, $490,496,498,510,516,521,523$, $527,528,533,534,542,545,523$, $554,556,557,558,565,612,624$, $629,632,651,654,684,685,698,699$ Hypodermic syringe . $\quad 714$ Hypochlorite of calcium ? 209

ICE.$\quad \cdot \quad \cdot \quad \cdot 158$

Ice-bag $\quad . \quad$. $\quad 158,708$

Ichthyol $\quad . \quad$. $\quad . \quad 692$

Idiosyncrasies $\quad$. $\quad$. 37

Indian hemp . $\quad . \quad$. 499 $\begin{array}{llll}\text { tobacco } & . & . & 499\end{array}$

Infusion of catechu $\quad$. $\quad$. 644 cinchona $\quad . \quad 564$ ergot . . . 624 gentian . $\quad 573$ $\begin{array}{llll}\text { tobacco } & \cdot & \cdot & 642 \\ \text { Infusions } & . & . & 723\end{array}$

Inhalations $\quad . \quad 333,375,421,721$

Injectio apomorphinæ hypodermica atropinæ hypodermica $\quad 496$ ergotæ , $\quad 624$ morphinæ ," . 484

Injections, intramammary 711,715 intramuscular . $.27,715$ intraperitoneal . $\quad 711,713$ intra-spinal $\quad 527,528$ intratracheal, $27,315,317,318$, $320,321,384,398,415,484$, $491,517,521,533,542,565$, $605,606,629,651,711$.

intra-uterine . 711,720 intravenous, $27,160,220,383$, $402,415,484,491,517,521$, $534,612,684,711$.

rectal.

subcutaneous (see Hypodermic), 27, 711, 713, 714 .
Intestinal antiseptics and disinfectants (see Antiseptics), 115, 225, $226,345,393,400,401,402,425$, 593,648 . 


\begin{tabular}{|c|c|c|c|c|c|c|c|c|c|c|}
\hline \multirow{2}{*}{\multicolumn{3}{|c|}{ Intestinal astringents }} & \multicolumn{3}{|r|}{ PAGE } & \multirow{2}{*}{\multicolumn{2}{|c|}{ Toutin }} & \multicolumn{3}{|r|}{ PAGE } \\
\hline & & & & & 411 & & & - & & 690 \\
\hline & stimul & ants & . 113 , & , 218, & 511 & Kerm & es mineral & . & . & 263 \\
\hline Iodid & of cop & per & . & . & 246 & Kino & . $\quad$. & . & . & 643 \\
\hline & iron & . & . & . & 259 & Koch' & s experiments & - & . & 45 \\
\hline & lead & . & . & . & 233 & Kouss & 0 , a vermifuge & . & . & 615 \\
\hline & mercu & & . & . & 302 & Kram & eria triandra & . & . & 643 \\
\hline & potass & ium & - & . & 318 & & & & & \\
\hline & starch & & - & 312 , & 664 & LABAI & RRAQUE'S soda & disin & & \\
\hline & sulphu & & - & - & 321 & ing & fluid & . & . & 202 \\
\hline Iodin & : & & . & . & 311 & Lactil & $\operatorname{lin}$ & . & . & 345 \\
\hline & ointme & & . & - & 317 & Lacto & bacilline & - & . & 345 \\
\hline & solutio & & - & . & 317 & Lacto & ne & - & . & 345 \\
\hline & tinctu & es & - & . & 317 & Lacto & se & . & . & 666 \\
\hline Iodip & & - & - & & 317 & Lanol & ine & & . & 681 \\
\hline Iodi & & - & . & & 314 & Lævu & lose-left-hand & ed su & & 667 \\
\hline Iodof & $\mathrm{rm}$ & . & . & & 426 & Lapis & infernalis & . & . & 248 \\
\hline $\begin{array}{l}\text { Iodof } \\
393\end{array}$ & $\begin{array}{l}m \text { sul } \\
418,4\end{array}$ & $\begin{array}{l}\text { stitutes } \\
\text { 28, } 429 \text {. }\end{array}$ & s, 225 , & 251 & 392, & $\begin{array}{l}\text { Lard } \\
\text { Larks }\end{array}$ & pur or stavesac & cre & . & $\begin{array}{l}679 \\
614\end{array}$ \\
\hline Iodof & rmal & . & . & - & 429 & Lauds & num or tinctur & re of 0 & ium & 485 \\
\hline Iodo & rmin & . & . & . & 429 & Laugl & ing gas . & . & - & 75 \\
\hline do & & . & . & . & 428 & Lav & der & . & . & 594 \\
\hline Iodu & & - & . & & 311 & Laxat & ives, 108,189 , & 2 & 323 & 325 , \\
\hline d & alicylic & acid & . & & 428 & 4 & $448,465,656$ & 66 & 68. & \\
\hline Ion & eo & - & . & 160 & 163 & Lead & and its compou & inds & - & 226 \\
\hline Ipe & in & ; & . & . & 554 & & acetate. & - & - & 234 \\
\hline Iron & nd its & salt & - & - & 251 & & ate & - & - & 32 \\
\hline & and & inir & trate & • & 256 & & ioc & $\cdot$ & • & 233 \\
\hline & $\operatorname{ars}$ & & $\cdot$ & - & 255 & & e. & $\cdot$ & $\cdot$ & 233 \\
\hline & cacc & ite & $\cdot$ & . & 283 & & oxide. & - & - & \\
\hline & carl & te & - & - & 256 & & plaster . & b & . & 23 \\
\hline & chlo & & $:$ & & 260 & & poisoning witl & & 227 , & 229 \\
\hline & hyd & ed pero & xide & . & 260 & & antidotes & for & . & 232 \\
\hline & iodi & & . & . & 259 & & sugar of & . & . & 234 \\
\hline & phos & ate & . & . & 255 & & white & - & . & 232 \\
\hline & sacc & rine car & bonate & . & 256 & Lecit & bin & . & . & 306 \\
\hline & sulp & & - & . & 257 & Leopa & rd's bane or ar & nica & . & 588 \\
\hline & tinc & & . & . & 261 & Leucc & cytes & . & . & 10 \\
\hline Irriga & tion, c & ontinuor & & & 707 & Lime, & burnt. & . & . & 204 \\
\hline Isingl & ass & - & . & - & 685 & & carbonates, & . & . & 206 \\
\hline Itrol & . & . & . & - & 251 & & chloride & . & - & 211 \\
\hline Izal & . & - & . & . & 425 & & chlorinated & . & - & 209 \\
\hline $\mathbf{A B C}$ & ANDI & & - & & 529 & & $\begin{array}{l}\text { phate } \\
\text { harated }\end{array}$ & $\dot{.}$ & & 206 \\
\hline$J$ & ine & . & . & . & 529 & & r. & . & . & 205 \\
\hline & & . & . & . & 458 & Linin & ent of ammoni & & . & 169 \\
\hline Jamai & ca ging & & . & - & 584 & & belladonna & . & . & 496 \\
\hline & pepper & & . & - & 582 & & camphor & . & . & 633 \\
\hline Jam & 's powc & ler. & - & . & 263 & & cantharides & . & . & 673 \\
\hline es & s'bark & & . & . & 559 & & croton . & . & . & 456 \\
\hline on & 's gour & & $\because$ & - & 449 & & lime & . & . & 206 \\
\hline & er tol & and ber & cries & . & 59 & & mercury & . & . & 290 \\
\hline Junip & erus & nmunis & . & . & 59 & & $\mathrm{am}$. & - & . & 185 \\
\hline & sabi & & $\cdot$ & . & 593 & & so & - & - & 690 \\
\hline & oxy & rus & - & - & 593 & & turpentine & . & - & 607 \\
\hline Jute & - & - & - & - & 657 & Linim & $\begin{array}{l}\text { ents } \\
\text { ed }\end{array}$ & 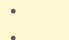 & • & $\begin{array}{l}726 \\
657\end{array}$ \\
\hline & D & & $\mathbf{s}$ & - & 459 & & cake & . & & 657 \\
\hline & & $\operatorname{tas}$ & a sal & . & 173 & & meal & . & & 657 \\
\hline & la, a & rmif & & & 617 & & oil & 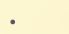 & . & 657 \\
\hline Kaoli & & . & . & . & 223 & Lint & . & . & . & 657 \\
\hline
\end{tabular}


Linum usitatissimum,

Liquid paraffin

Liquor ammoniæ fortis

acetatis

arsenicalis

arsenici et hydrargyri

iodine

atropinæ sulphatis .

calcis

carbonis detergens

chlori

ethyl nitritis

ferri perchloridi

hydrargyri perchloridi

iodi

pancreatis

picis carbonis .

potassæ

strychninæ, .

trinitrini

Liquors

Liquorice root

sugar

Litharge-oxide of lead

Lithontriptics

Liver, drugs acting on

Lobelia-Indian tobacco

Local anæsthetics

Logwood an astringent dye .

Long pepper .

Loretin

Losophan

Lotions, 183, 188, 193, 196, 203, 217 . $222,223,236,238,240,242,246$, $251,252,262,292,301,317,321$, $335,338,344,348,362,392,407$, $408,413,417,418,425,483$.

Lubricants

Lugol's solution

435,680

Lunar caustic .

317

Lysoform

248

Lysol.

392

425

Macdougall's disinfectants .

Magnesium and its compounds calcined carbonate oxide. sulphate

Maize starch.

Male fern : $213,215,217$

Mallein

663

616

Malt extracts .

8. 695

653

Maltose

666

Mammary glands, drugs acting on

Mandrake

Mange and scab dressings (see Parasiticides)

Marjoram
Marsh-mallow PAGE

Mass, common $\quad$. $\quad 660,668$

Massage . . 56

Materia Medica, definition of 1

Matico leaves. $\quad 582$

May apple or podophyllum . 460

Meadow saffron $\quad$. $\quad 627$

Measures, pharmaceutic $\quad$. 734

domestic . . 735

Meconic acid . $\quad$. $\quad 468$

Medicines, absorption of, 26, 27, 28, 29,30

acting on the digestive

system

99

acting by counter-action $\quad 39$

acting generally $\quad$. $\quad 26$

acting hypodermically 27

acting intratracheally . $\quad 29$

acting locally . • 26

acting by mouth, . $\quad 27$

acting by pulmonary membrane . $\quad 29$

acting by rectum $\quad$. $\quad 29$

acting by skin . $\quad$ ?0

circumstances modifying . . . 36,38

classification of $\quad . \quad 21$

curative action of . $\quad 38$

doses . $\quad 700,709$

elective affinity $\quad . \quad 30$

on different patients, $32,34,35$

physiological action of 25,26

therapeutic action of . 25, 26

Mel-honey . . . 667

boracis. $\quad . \quad 193$

Mentha piperita : $\quad . \quad 594$

pulegium $\quad . \quad 595$

viridis . $\quad . \quad 595$

$\begin{array}{lllll}\text { Menthol } & \text {. } & \text {. } & 595\end{array}$

Mercurial compounds . 285

liniments . $\quad 290$

ointments, . 288, 290, 303

pill . . . 291

$\begin{array}{llll}\text { plaster . } & \text {. } & \text {. } & 290\end{array}$

Mercurialism . $\quad . \quad$. 287

Mercuric salts $\quad$. $\quad$. 285

Mercurous salts . . 285

Mercuro-zinc cyrnide . 302

Mercury and its compounds . 285

ammoniated . . 301

$\begin{array}{lll}\text { chlorides } & \text { - } & 298\end{array}$

corrosive sublimate . $\quad 298$

iodides . . 302

liniment . $\quad$. 290

nitrate . $\quad$. 303

nitrate ointment $\quad 303$

ointment . $\quad 288$

oleate . . . 290

oxides . . . 292

pill . . . 291 


\begin{tabular}{|c|c|c|c|c|c|c|}
\hline & & PAGE & & & & 100 \\
\hline lercury poisoning & & 288 & NaPHTHALIN & - & - & 402 \\
\hline plaster & - & 290 & Naphthalol & . & . & 404 \\
\hline with chalk & . & 291 & Naphthol camphor & . & . & 404 \\
\hline Iethylated spirit & . & 354 & Naphthols & . & . & 403 \\
\hline yl a & . & 353 & Narceine & $\cdot$ & & 472 \\
\hline chlo & . & 354 & arcotics & - & 70,472, & 2,500 \\
\hline con & . & 518 & Tarcotine & - & - & 472 \\
\hline ine & & 470 & Nataloin & & 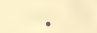 & 445 \\
\hline ate & . & 570 & Natural orders of pla & ants & . & 22 \\
\hline anine & . & 504 & Negri-bodies. & . & . & 3 \\
\hline violet. & . & 401 & Nerve paralysers & . & . & 85 \\
\hline Lethylal & . & 76 & stimulants & . & - & 86 \\
\hline Iethylene & . & 76 & Nervous system, dru & go acti & ting on & 66 \\
\hline blue & . & 402 & Neutral organic prin & ciples & s. & 703 \\
\hline letric weights and & heasures & 736 & Nicotiana tabacum & . & . & 638 \\
\hline icro-organisms & . & . 13,14 & Nicotine & . & . & $63 S$ \\
\hline $\operatorname{gar}$. & . & 666 & $\operatorname{anin}$ & . & . & 638 \\
\hline lphur & . & 322 & Vitrate of Mercury & . & . & 303 \\
\hline spirit & . & 171 & potash. & . & .• & 182 \\
\hline cal Kermes, & . & 263 & silver. & • & 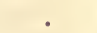 & 248 \\
\hline oils & . & 726 & soda & . & . & 201 \\
\hline Iixtures & . & 724 & Nitre or saltpetre & . & . & 182 \\
\hline freezing & . & 158 & sweet spirit of & & . & 368 \\
\hline sses-treacle & . & $.667-668$ & Nitric acid & . & . & 37 \\
\hline ood . & . & . 546 & te of amyl & . & . & $\$ 9$ \\
\hline hine & . & 465,469 & ethyl. & . & . & 268 \\
\hline acet & . & 470 & sodium . & . & . & 391 \\
\hline hydrochloride & . & 469 & Nitro-glycerin & ${ }^{\circ}$ & . & 391 \\
\hline muri & . & 469 & hydrochloric a & cid & . & 338 \\
\hline $\operatorname{tart}$ & . & 470 & Nitrous ether & . & . & 368 \\
\hline huæ oleum & . & 677 & oxide gas & . & . & 75 \\
\hline . & . & 731 & al saline solutic & & 10,162, & 2, 199 \\
\hline $\mathrm{r}$ depressants & . & - $85, \mathrm{~S} 6$ & aine . & . & . & 528 \\
\hline ds & . & . 13 & Nuc & . & . & 653 \\
\hline ages & . & 654 & n solution & $\therefore$ & . & 654 \\
\hline Iuriate of ammonia & - & 165 & $\mathrm{Nu}$ & . & - & 45 \\
\hline antimony & . & 264 & Nutrients, 134,656 , & , 6 & , 662, & 664 , \\
\hline ry & . & 298 & & & & \\
\hline morphia & . & 469 & enemata, & - & & 719 \\
\hline soda & & 196 & & & . & 502 \\
\hline atic or hydrochl & ori & 334 & alkaloids of & . & . & 503 \\
\hline Iusk . & . & 725 & poisoning & . & . & 505 \\
\hline Muscle stimulants, & - & $.65,516$ & & & & \\
\hline & & - 63,64 & $\mathrm{OAP}_{\mathrm{AP}}$ & & . & 574 \\
\hline alar spasm & • & 64 & galls & . & . & 644 \\
\hline ard & . & 576 & 0 & & . & 658 \\
\hline cations & . & 579 & $\mathrm{Oa}$ & . & . & 663 \\
\hline black . & & 577 & he crocata & . & 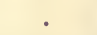 & 509 \\
\hline red wi & h other & & Oil, an & . & & 590 \\
\hline ants & . & 577 & cron. & . & 206, & , 658 \\
\hline oil of & . & 577 & tor & . & . & 449 \\
\hline & & 576 & nomile & . & . & 595 \\
\hline iatics, dilato & of $t$ & upil 89 & -liver & . & & 677 \\
\hline ntracto & of & oupil 89 & n. & • & & 452 \\
\hline . & . & 694 & $\mathrm{ng}$. & . & 658 , & 8,661 \\
\hline in & . & 677 & expressed or fi & ixed & . & 724 \\
\hline in & . & 577 & linseed. & . & . & 658 \\
\hline $\mathrm{rh}$ & - & 580 & olive & - & . & 655 \\
\hline e & • & 581 & ade & . & & 593 \\
\hline Myrrhol & . & 58 & cinnamon & & 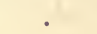 & 586 \\
\hline
\end{tabular}




$$
\text { juniper }
$$

Oil of savin

\section{tar}

thyme.

turpentine

Valerian

vitriol.

wine

Wintergreen

Oils, volatile .

Ointments, how made, etc.

Ointment, blistering .

\section{boric}

cantharides

carbolic

citrine .

iodide of sulphur

iodine.

mercury

red iodide

resin

silver nitrate

simple .

sulphur

Oleates

zinc oxide

Oleate of lead

Oleic acid

Oleo-resins .

Oleum anisi

anthemidis
crotonis
juniperi
lini
menthæ piperi
morrhuæ
olivæ :
picis :
ricini :
terebinthinæ
tiglii

Olibanum or frankincense

Olivæ oleum

Olives

Olive oil

Opodeldoc

Opium

alkaloids

ammoniated tincture

Opium antidotes

camphorated tincture .

Egyptian

extract .

Indian
591

593

580

581

595

607

607

593,625

610

594

601

596

229

367

570

589

727

673

348

673

417

303

321

317

288

302

608

248

695

325

236

727

233

346

597, 732

590

595

452

593

658

595

677

655

609

449

601

452

580

655

656

655

589

465

469

485

479

485

467

485.

467
Opiunt, poisoning by

476 preparations of $\quad . \quad 469$

purity and strength of $\quad 468$

Smyrna . . 467

tinctures of . $\quad$. 485

Turkey . . 467

Organic animal extracts . 703

Orphol . . . 225

Orthoform : : $\quad$ • $\quad 528$

Osmosis, . $\quad . \quad$. 161

Ossein . . . . 685

Oxide of antimony $\quad$. $\quad . \quad 263$ calcium . $\quad 204$

iron . $\quad . \quad 260$

lead : : $\quad 232$

magnesia $\quad . \quad 212$

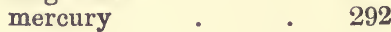

zinc . . . 236

$\begin{array}{llllll}\text { Oxygen } & . & . & . & 158\end{array}$

Oxymel $\quad . \quad 344$

Oxytocics or ecbolics $130,131,619$

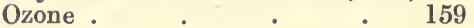

PaIN relievers (see Analgesics) 71

Palma Christi $\quad$. $\quad$. 449

Papain . . $\quad$. 692

Papaver somniferum . $\quad$. 465

$\cdot 406$

Paraffins . . . $\quad 433$

Paraldehyde . $\quad$. $\quad 388$

Paralysants, 85, 86, 430, 519, 524, 534

Paramorphine . . 471

Parasiticides (see also Vermicides), 54, $117,244,257,270,278,296,316$, $321,322,339,349,365,396,402$, $403,408,415,424,557,558,590$, $594,609,610,614,633,635,637$, 639, 648, 65l, 693.

Pareira

546

Parturients . 131

Pastes. $. \quad . \quad . \quad 727$

Pearl ashes . . . $\quad$. 177

barley • • $\quad 652$

Pennyroyal . . . 594

$\begin{array}{llll}\text { Peppers } & \text {. } & \text {. } & 582\end{array}$

Pepper, Cayenne $\quad$. $\quad . \quad 582$ cubebs. $5 \mathrm{S2}$

Peppermint . . . 594 $\begin{array}{lllllll}\text { oil } & . & . & . & 595\end{array}$

spirit . . . $\quad 595$

water ..$\quad 595$

Pepsin • $\quad 691$

733

Perhydrol : $\quad 159,160$

Periodate . . . 428

Permanganate of potash $\quad$. 186

Peroxide of hydrogen $\quad$. $\quad 159$

260

238

Peru balsam . $\quad \cdot \quad \cdot 651$ 
Peruvian bark

Petrolatum

Petroleum benzin vaseline

Petroleums

Pharmacology

Pharmacy

Phenic, or carbolic, acid

Phenacetin

Phenol

$$
\text { -acetamide }
$$$$
\text { -salicylate }
$$

Phenazone (antipyrine)

Phenyform

Phlebotomy

Phosphate of iron lime

Phosphoric acid

Phosphorus

Physic masses

Physiology of vomiting

Physostigmatis semina

Physostigmine sulphate

Picrotoxin

Picric acid

Pill, blue

Pilocarpine nitrate

Pimento or allspice

Pimpinella anisum

Pines .

Pinus Sylvestris

Piper album

Chili

cubeba .

longum

Piperazine

Piperidine

Piperine

Pitch .

Pix Burgundica

liquida .

Plasters, adhesive blistering

glue lead or sticking

Plasters, how made, etc.

Plumbi acetas

$$
\text { emplastrum }
$$
iodidum oxidum

Plumbi carbonas

Plumbism

Plumbum

Podophyllum .

Poisons and antidotes, 144, 145, 146, $149,150$.

Pomades

Poppy heads : oil

559

435

435

435

433

1

700

408

404

408

398

401

396

418

142

255

208

339

304

444

104

511

511

72

339

291

529

582

589

598

601

582

583

582

582

582

404

582

582

598, 610

600

609

$233,234,685$

676

685

233

728

234

233

233

232

232

227

226

460
PAGE

Poultices

bran:

charcoal

linseed .

mustard

yeast

Powder, Dover's

Gregory's

James's

Powders, how made, etc.

Precautions with anæsthetics 79,80

Precipitated chalk

Precipitated sulphur . $\quad$. $\quad 322$

Prepared chalk . . 206

Prescribing . . . 700

Prescriptions . $\quad$. 700

Pressure . . . 56

Preventive inoculation $\quad 4 \quad 4,5$

Proof spirit . $\quad$. $\quad$. 353

Protargol . . . 252

Protective vaccines . $\quad 19$

Protectives 367, 368, 435, 688, 695

Prussic acid . . $\quad 429$ $\begin{array}{lll}\text { antidotes for } & \text {. } & 432 \\ \text { poisoning with } & \text {. } & 431\end{array}$

Purgatives (see Cathartics and Laxatives) 107, 181, 189, 193, 199, 212, $216,218,291,295,442,448,449$, $453,455,456,457,458,460,464$

Pustulants

Pyridine . • . 406

Pyoktanin . . . 401

Pyrocatechin . . . 400

Pyrogallic acid $\quad 4 \quad 400$

Pyroligneous acid . $\quad 342$ 


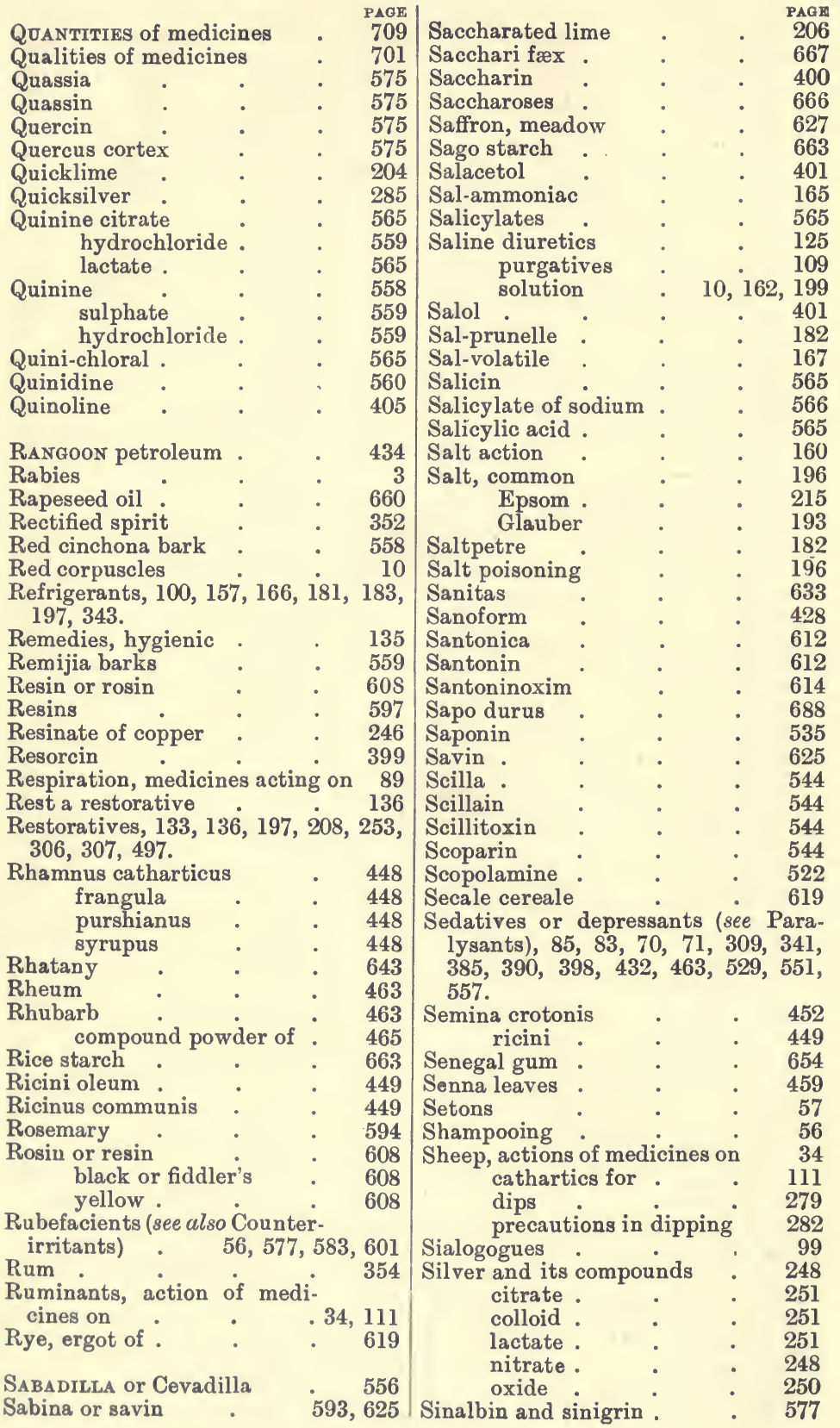




\begin{tabular}{|c|c|c|c|c|c|c|c|}
\hline \multirow{2}{*}{\multicolumn{4}{|c|}{ notond onolipo }} & \\
\hline & & & & Spirit of ammonia & & & $\begin{array}{r}\text { PAGE } \\
167\end{array}$ \\
\hline tions & & . & $.57,579$ & chloroform & . & . & 379 \\
\hline Size-weak g & lue & . & 685 & ether. & . & . & 363 \\
\hline Skin, medicin & es actin & $\mathrm{ng}$ on & 121,713 & salt & . & . & 334 \\
\hline Smelling-salt & & . & 171 & turpentine & . & - & 601 \\
\hline Soaps & & . & 688 & wine & . & . & 352 \\
\hline Castile & & , & 689 & Spirit, proof & $\dot{0}$ & $\dot{0}$ & 353 \\
\hline glyceri & & . & 689 & rectified & . & . & 352 \\
\hline hard o & soda & . & 688 & Spirits or essences & . & . & 734 \\
\hline medici & nal & . & 689 & Spiritus ætheris & . & . & 363 \\
\hline Soap linimen & & . & 690 & ætheris nitrosi & & . & 368 \\
\hline soft or & potash & . & 688 & compositus & . & . & 367 \\
\hline Socaloin & . & . & 445 & Mindereri & . & . & 171 \\
\hline Socins' paste & . & & 241 & rectificatus & . & & 352 \\
\hline Soda salts & . & . & 189 & Spongiopiline . & . & 157, & 730 \\
\hline Soda water & . & . & 191 & Sprays & . & . & 93 \\
\hline Sodium and it & ts comp & oounds & 189 & Spurred rye . & . & . & 619 \\
\hline bicarbc & nate & . & 190 & Squill . & & . & 544 \\
\hline biborat & & 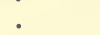 & 192 & Squire's chemical foo & & . & 256 \\
\hline bromid & & . & 308 & Staphisagrine. & . & . & 614 \\
\hline carbola & & - & 418 & Starch & & . & 662 \\
\hline carbon & ates & - & 190 & iodide of & - & . & 664 \\
\hline chlorat & & . & 202 & Stavesacre seeds & & . & 614 \\
\hline chlorid & & . & 196 & Steam a disinfectant & & 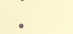 & 51 \\
\hline ethylat & e soluti & ion & 189 & Steaming horse's heac & & . & 91 \\
\hline fluoride & & . & 203 & Steel, tincture of & & . & 261 \\
\hline hydrox & ide or c & caustic & soda 190 & Sternutatories & & . & 91 \\
\hline hyposu & lphite & . & 194 & Stibium (antimony) & & & 262 \\
\hline iodide & & . & 319 & Stimulants, 69,168 , &, 170 & 179 & 289 \\
\hline liquid, & Labar & aque's & 202 & $290,292,360,364$ & 4,36 & & 470 \\
\hline nitr: & & . & 201 & $479,489,497$. & & & \\
\hline perb & tie & . & 193 & aromiatic, 577 , & , 582, & 585, & 586, \\
\hline Sodium nitrit & & . & 391 & $590,592,594$ & 4,596 & & \\
\hline phosph & ate & . & 193 & cerebral & . & - & 69 \\
\hline soap & & . & 688 & glandular & - & - & 531 \\
\hline sul & & . & 193 & spinal & & & 83 \\
\hline sulp & & . & 194 & Stomachics (see also & Carm & ainativ & (ves), \\
\hline thio & phate & . & 194 & $113,197,207,364,36$ & 69,4 & 577 & 581 , \\
\hline Soft paraffin & . & . & 433 & $583,585,586,587,5$ & 590,593 & 3,596 , &, 503 \\
\hline oap & & . & 688 & Stopping for horses' $\mathrm{f}$ & feet & . & 610 \\
\hline Solutio arsen & icalis & . & 283 & ine & - & . & 528 \\
\hline chlo & & . & 310 & monium . & • & . & 522 \\
\hline plumbi & diaceta & atis & 234 & Strongyli & . & . & 118 \\
\hline Solutions & . & . & 723 & Strontium bromide & . & . & 309 \\
\hline ol & . & . & 425 & hanthin . & - & . & 542 \\
\hline ol & . & . & 425 & hanthus Kor & & 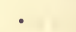 & 542 \\
\hline Soporifics & . & . & 69 & Strychnine & . & . & 503 \\
\hline Sour milk & . & . & 345 & arsenite & - & & 511 \\
\hline Sozoiodol & . & . & 428 & poisoning & . & . & 505 \\
\hline Spanish flies & . & - & 669 & Styptic colloid ${ }^{\circ}$ & & - & 647 \\
\hline teine & . & . & 544 & Styptics (see also A & Astring & gents & and \\
\hline Spearmint & & & 594 & Hæmostatics), 61, & , 211, & 222 & 234, \\
\hline Species of pa & tient $\mathrm{d}$ & liffer & & $261,368,407,600$. & & & \\
\hline affected by & drugs & . & 32 & Styrax & & - & 651 \\
\hline Spermaceti- & cetaceu & & 693 & Subcutane & tions $(s$ & see & \\
\hline Spinal anæstl & nesia & . & 82 & Hypodermic) & . & - & 27 \\
\hline & sants & $\cdot$ & 83 & Sucrose & . & . & 666 \\
\hline stir & ants & - & 83 & Sudorifics & . & - & 121 \\
\hline Spinal ho & & - & 708 & Suet . & - & • & 680 \\
\hline ice-bag & & - & 708 & Sugar. & - & . & 665 \\
\hline
\end{tabular}


grape . $\quad . \quad 667$

liquorice $\quad . \quad 662$

of fruits $\quad . \quad 667$

of lead . $\quad$. $\quad 234$

of milk-lactose $\quad$. $\quad 666$

Sulphate of alumina and potash 221

copper . . . 244

jron . $\quad 257$

magnesia . . 213

potash . $\quad 181$

quinine.

soda

zinc

Sulphates

Sulphite of soda

Sulphur

anhydride

flowers of

iodide .

liniments of

milk of .

ointments of

precipitated

roll or stick

sublimed

vivum.

Sulphide of antimony

potassium

Sulpho-carbolic acid .

carbolates

Sulphonal phenic acid.

Sulphuretted hydrogen

Sulphuric acid

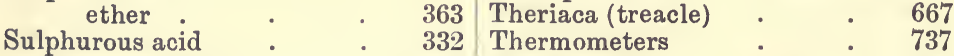

559

193

239

181

194

321

332

322

321

325

322

325

322

322

322

322

263

180

Tannigen

PAGE

Tannin or tannic acid

Tannoform . $\quad . \quad 393$

Tapeworms . . . 118

Tapioca starch

662

Tar . . 598, 609

434,609

Barbados

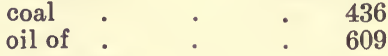

ointment . . 610

Tartar, cream of $\quad$. $\quad . \quad 188$

emetic..$\quad 265$

Tartaric acid . $\quad . \quad$. 344

Tartarised antimony . . $\quad 265$

$\begin{array}{lll}\text { Tartrate of potash } & \text {. } & 188\end{array}$

$\begin{array}{llll}\text { Tæniacides } & \text {. } & \text {. } & 119\end{array}$

Tea . $\quad . \quad 4 \quad 498$

Temperature modifying actions of medicines . high, destroys micro-

Tenaline $\quad . \quad 612$

Terebene . . . 607

Terebinthinæ . $\quad . \quad 598$

Terpene : $\quad \cdot \quad 607$

418 Tetanus antitoxin $\quad . \quad$. 7,697

418 Thalline . . . 406

418 Thebaine $\quad . \quad$. $\quad .471$

386 Theine $\quad$. $\quad . \quad$. 497

323 Theobromine . $\quad$. 498

329 Therapeutic action of medicines 25

Sumbul or musk root. $\quad 587,596$ Thioform $\quad$. $\quad$. $\quad . \quad 226$

Sunlight

Suppositories .

Suprarenin

Surgeon's lint.

Surroundings modify actions of medicines

Sweat glands, action on $\quad 121$

Sweet spirit of nitre . $\quad$. $\quad 368$

Syringes, enema $\quad$. $\quad 719$

Syrups . . . 732

Syrup simple . $\quad$. $\quad$. 668

iodide of iron . $\quad$. $\quad 259$

Easton's . 256

Squire's . . 256

Syrupus chloral $\quad$. $\quad . \quad 385$

rhamni. . $\quad$. 448

rhei . . . 465

TABLES of weights and mea.

sures $\quad . \quad 734,735,736$

Tablets

Tannalbin

648

Thiol . $\quad . \quad 693$

Thus (Frankincense) . $\quad 599$

681 Thyme . 5 594, 636

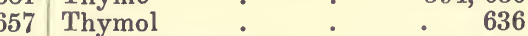

Tinctura aconiti . $\quad$. 552

445

$\begin{array}{lll}\text { arnicæ - } & \cdot & 585 \\ \text { belladonnæ } & \cdot & 495\end{array}$

$\begin{array}{lll}\text { arnicæ : } & \cdot & 583 \\ \text { belladonnæ } & \cdot & 495 \\ \end{array}$

$\begin{array}{ll}\text { benzoini composita } \quad & 650 \\ & 573\end{array}$

$\begin{array}{lll}\text { calumbæ } & \text {. } & 573 \\ \text { cannabis indicæ } & \text {. } & 502\end{array}$

cantharidis . $\quad$. 673

cascarillæ . . . 574

catechu . $\quad . \quad$. 644

chiratæ. $\quad . \quad 574$

chloroformi et morphinæ composita . 380

cinchonæ . $\quad 564$

$\begin{array}{llll}\text { colchici . } & \text {. } & \text {. } & 629\end{array}$

croton . . . $\quad 456$

digitalis $\quad . \quad 542$

ergotæ ammoniata $\quad$. 624 


\begin{tabular}{|c|c|c|c|c|c|c|}
\hline \multicolumn{2}{|c|}{ Tinctura ferri perchloridi } & \multicolumn{2}{|r|}{ PAGE } & Urinary sedatives & & $\begin{array}{l}\text { PAGE } \\
129\end{array}$ \\
\hline gentianæ & . & • & 573 & $\begin{array}{l}\text { tonics. } \\
\text { tons }\end{array}$ & $\dot{.}$ & 129,130 \\
\hline hyoscyamus & - & . & 523 & Urine, secretion of & . & . 124 \\
\hline iodi & . & . & 317 & Urotropine & . & 392 \\
\hline ,, decoloratc & & - & 317 & Uses of antiseptics & . & 46 \\
\hline ", oleosa & - & - & 317 & diuretics & . & 126 \\
\hline myrrhæ & - & - & 581 & Uses of disinfectants & . & 49 \\
\hline nucis vomicæ & • & . & 510 & deodorisers & - & 53 \\
\hline opii . & . & . & 485 & emetics. & . & 106 \\
\hline , ammoniat & & . & 485 & purgatives & . & 112 \\
\hline strophanthus & - & - & 543 & & & \\
\hline zingiberis & - & - & 585 & VACCIN ES & . & - 9,20 \\
\hline Tinctures, how made & e, etc. & - & 733 & Valerian & . & $596-597$ \\
\hline Tobacco & . & - & 638 & Valerianic acid & . & 596 \\
\hline alkaloids & . & . & 638 & Van Swieten's solutio & & 301 \\
\hline enemas. & . & . & 641 & Vapour bath & - & 705 \\
\hline Tolu balsam. & & & 651 & s or inhalation & & 721 \\
\hline lonics, $137,208,25$ & 2,256 & 260 & 277 & Vascular stimulants & - & 96 \\
\hline $\begin{array}{l}306,330,336,337 \\
358,464\end{array}$ & 7,339, & 340 & 349 & tonics $\cdot$ & - & . $\quad 97-99$ \\
\hline $\begin{array}{l}\text { 358, 464, 508, } 563 \text {, } \\
\text { onics, blood, } 138 \text {, }\end{array}$ & $\begin{array}{l}572 . \\
253,2\end{array}$ & 256. & 257. & $\begin{array}{l}\text { Vaso-constrictors } \\
\text { Vaso-dilators }\end{array}$ & • & $\begin{array}{l}99 \\
96\end{array}$ \\
\hline $259,261$. & & & $20 \%$ & $\begin{array}{l}\text { Vaso-dilators } \\
\text { Vaseline }\end{array}$ & $\dot{\bullet}$ & $\begin{array}{r}90 \\
435\end{array}$ \\
\hline gastric, 103 & 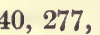 & & 360 & Vehicle & $\dot{.}$ & 701 \\
\hline 448,509 . & & & & Veins, injection into & . & 27 \\
\hline heart and vas & scular, & 98 & 540 , & Venesection & $\dot{.}$ & 142 \\
\hline 543,544 & & & & Venice turpentine & . & 598 \\
\hline nerve, 86,509 . & & & & Ventilation & . & 135 \\
\hline Tow & - & - & 657 & trine & . & 556 \\
\hline Tragacanth & - & . & 655 & igris & 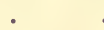 & 247 \\
\hline Treacle & - & . & 667 & Verdigris liniment & & 247 \\
\hline Trilactine & . & . & 345 & Vermicides and verm & nifuges, & 118,119 \\
\hline Trinitroglycerin & . & . & 391 & $245,258,270,279$, & , 296,3 & 339,349 , \\
\hline Tropacocaine . & . & . & 527 & $396,425,587,600$, & & 611,613 \\
\hline Trypanblue . & . & . & 402 & $614,615,616,617,6$ & 626 & \\
\hline ired & - & - & 402 & Vesical sedatives & - & 129 \\
\hline Tuberculin & . & - & 696 & tonics . & . & 130 \\
\hline Tumenol & - & . & 693 & Vesicants (see Counte & er-ir & ants), 56, \\
\hline Turkey opium & - & . & 467 & 577,669 . & & \\
\hline baths & . & & 705 & Veterinary Pharmacy & & 700 \\
\hline Tur & - & 597 & , 598 & te . & - & 175 \\
\hline oil of & . & . & 601 & Vinegar & - & 343 \\
\hline Tuson's disinfectant & . & • & 258 & harides & - & 673 \\
\hline $\mathrm{NC}$ & & & 726 & $n$ & & \\
\hline canthari & dis & & 673 & Virg & $\dot{.}$ & $\begin{array}{l}400 \\
638\end{array}$ \\
\hline cet & & & 247 & Vitriol, b & - & 244 \\
\hline $\mathrm{m}$ & & & 483 & $\mathrm{gr}$ & - & 257 \\
\hline gyri & . & & 28 & oil & . & 329 \\
\hline r-iodidi r & rubri & & 302 & white. & . & 239 \\
\hline is . & . & . & 303 & Volatile oils & & 589 \\
\hline & . & . & 317 & antisepti & & 637 \\
\hline . & . & & 608 & Vor & & 502 \\
\hline & . & 608 , & 695 & ow produc & ced & 104 \\
\hline ris & . & - & 325 & checked & - & 107 \\
\hline & . & & 558 & & & \\
\hline idi & - & 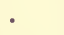 & 23 & WASH, 1 & . & 292 \\
\hline $\mathrm{ra}$ & - & - & 533 & yellow. & - & 292 \\
\hline & * & . & 387 & Water. & - & 49,152 \\
\hline slus & . & . & 128 & ties & . & . 153 \\
\hline disinf & . & - & 129 & actions and uses & & 154 \\
\hline
\end{tabular}




\begin{tabular}{|c|c|c|c|c|c|c|c|c|c|}
\hline \multirow{4}{*}{\multicolumn{2}{|c|}{$\begin{array}{c}\text { Water dressings } \\
\text { lime } \\
\text { mineral } \\
\text { tar }\end{array}$}} & & & $\begin{array}{l}\text { PAGE } \\
157\end{array}$ & \multicolumn{3}{|c|}{ Woorara or curara poison } & . & \multirow{2}{*}{$\begin{array}{r}\text { PAGK } \\
533 \\
680\end{array}$} \\
\hline & & : & . & 205 & Wound gela & & . & & \\
\hline & & . & . & 154 & Wounds & . & . & & 47 \\
\hline & & . & . & 609 & & & & & \\
\hline Watering of $h$ & orses & . & - & 155 & XEROFORM & . & . & • & 225 \\
\hline Wax & . & . & . & 694 & & & & & \\
\hline Weights and & neasure & & . & 734 & YEast & . & . & . & D3 \\
\hline Wet pack & . & . & . & 706 & Yeasts. & . & . & . & 13 \\
\hline Wheat flour & . & . & • & 662 & Yellow cincl & ona & & & 59 \\
\hline Whisky & . & . & . & 354 & resin & . & . & . & 08 \\
\hline White arsenic & & . & . & 271 & wash & . & . & . & 92 \\
\hline lotion & . & . & . & 239 & wax & . & . & . & 94 \\
\hline mustar & seed & - & - & 576 & Yohimbine & - & . & & 17 \\
\hline oil & . & . & . & 169 & & & & & \\
\hline pepper & - & • & . & 582 & $\mathrm{Z}_{\mathrm{INC}}$ and its & com & nds & & 3 \\
\hline vitriol & . & . & . & 239 & acetat & & . & . & 42 \\
\hline wax & . & . & . & 694 & buttes & & . & & \\
\hline Whiting & . & & • & 206 & carbo & ate & . & & \\
\hline Wines . & . & & $\bullet$ & 354 & chlori & & . & . & \\
\hline Wintergreen & . & • & . & 570 & oxide & & . & & 200 \\
\hline Wolfsbane & & . & . & 546 & perox & & . & . & 23 \\
\hline Wood charcoa & & - & . & 350 & sulph & & . & 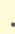 & 23 \\
\hline naphth & & . & . & 353 & sulph & car & & . & 240 \\
\hline $\operatorname{tar}$ & . & . & . & 598 & Zingiber offi & nal & . & & \\
\hline woo & . & - & . & 598 & Zingiberis $\mathrm{ti}$ & ctur & . & . & \\
\hline
\end{tabular}






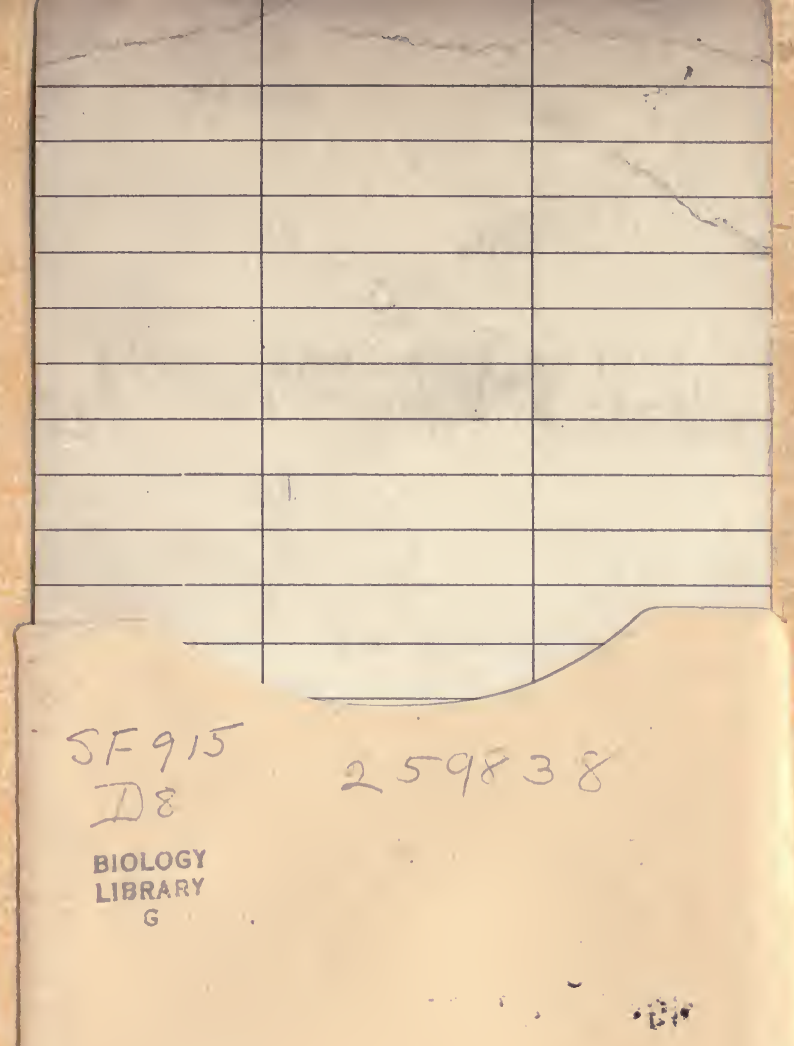

UNIVERSITY OF CALIFORNIA LIBRARY 
